

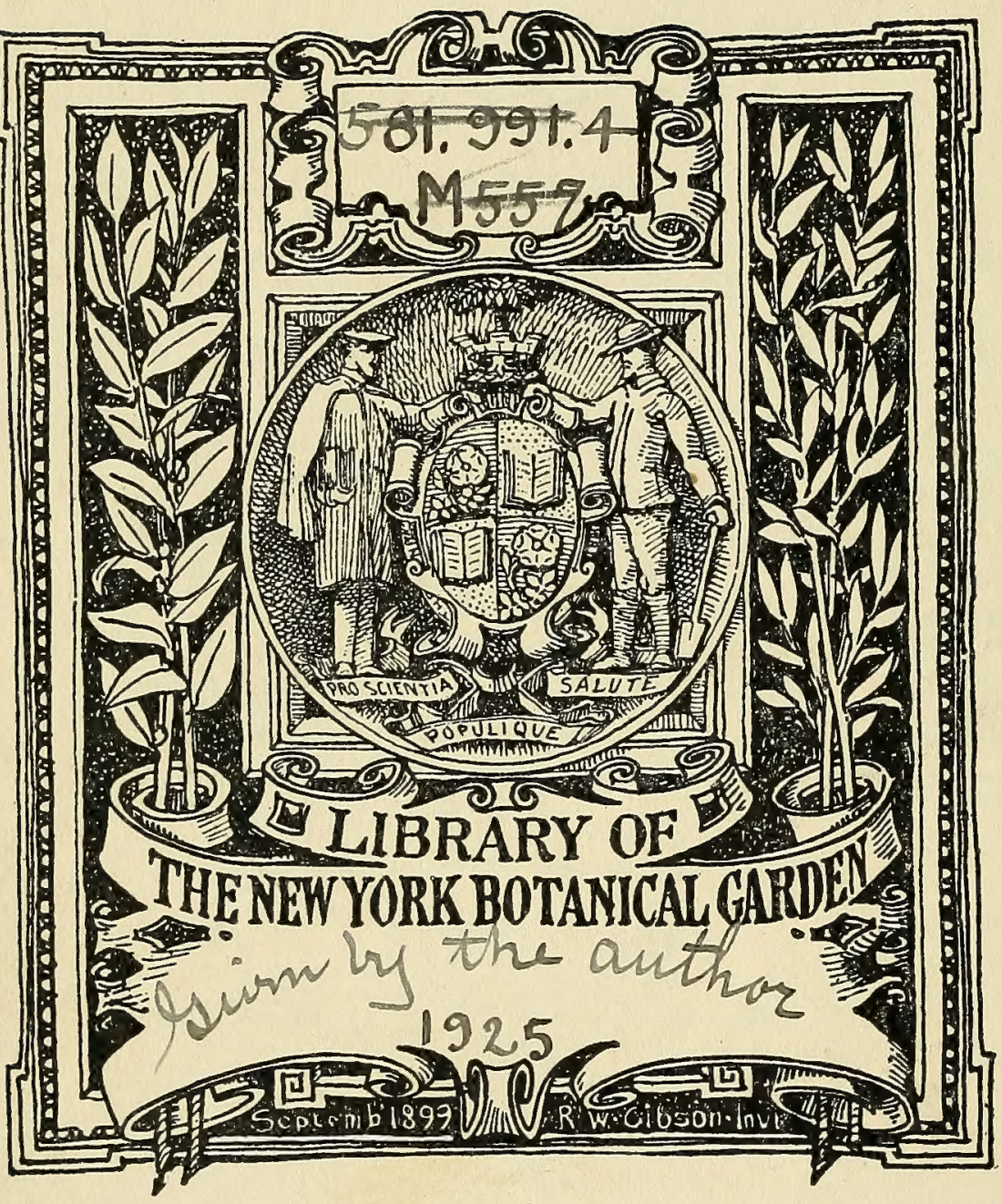


$\cdot$

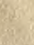





\title{
AN ENUMERATION OF PHILIPPINE FLOWERING PLANTS
}

\author{
BY \\ ELMER D. MERRILL \\ DIRECTOR AND BOTANIST, BUREAU OF SCIENCE
}

VOLUME 1
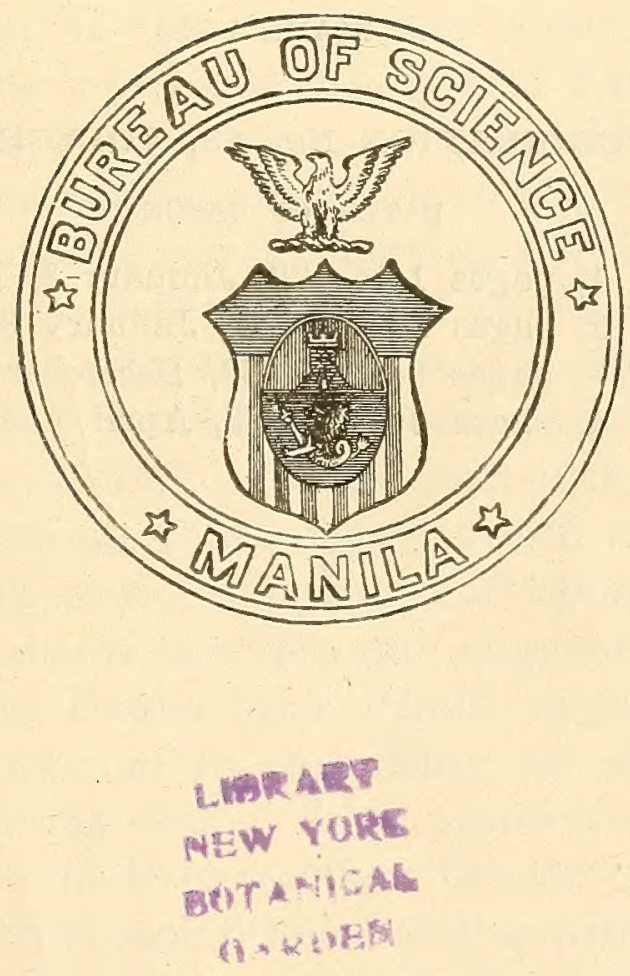

MANILA

BUREAU OF PRINTING 


\section{$Q K 369.95$ , M 412 V.I}

Government of the Philippine Islands Department of Agriculture and Natural Resources BUREAU OF SCIENCE MaNILA

PUBLICATION No. 18, VOLUME 1 DATES OF ISSUE

Fascicle 1, pages 1 to 128, January 3, 1923.

2, pages 129 to 240, January 3, 1923 .

3, pages 241 to 368, December 29, 1924.

4, pages 369 to 463 , April 7, 1925. 


\section{PREFACE}

The object of the present work is to enumerate the known species of flowering plants occurring in the Philippines; to adjust the synonymy; to account for all binomials accredited to the Philippines in botanical literature; to give the most important literature references, especially to the special Philippine publications and to the important extra-Philippine ones; to record the numerous local names for the various species; to give the habitat, occurrence, altitudinal range, Philippine distributions, and approximate extra-Philippine distribution of each species admitted; to cite illustrative specimens when necessary or expedient, especially in critical groups; in brief, to summarize in convenient form for consultation our present knowledge of the Philippine flora.

When publication of this work was commenced in 1921, it was planned to issue it in three volumes, of four or five fascicles each. As publication progressed, it was found necessary to abandon the plan of issuing the work in three volumes and the inclusion of the extensive introductory data in the prefatory part of the present volume and to add a fourth volume to contain the general introduction, bibliography, and indices to local and scientific names. In the individual volumes of the actual enumeration merely brief indices have been included covering the family and generic names that appear in each volume. Complete indices to the entire work form a part of volume 4 .

In the arrangement of families and of genera the sequence in general follows the Engler and Prantl system, but for convenience in indexing and in consulting the species have been arranged alphabetically under their respective genera. While an attempt has been made to compare the very numerous species within each genus with each other and also with extra-Philippine representatives of the same genera, so far as material has been available, much critical work remains to be done; and future studies must of necessity modify the enumeration, not only as to the validity of numerous admitted species but also as to questions of synonymy and geographic distribution. It should be remembered that most of the actual work has been accomplished on the basis of the material and the literature available in Manila, 
and that the local botanical library and the herbarium date only from 1902. While the library is a very excellent working unit, especially for the Indo-Malaysian region, it is far from complete, although practically all the publications appertaining especially to the Philippine flora are represented. The herbarium, containing upward of 250,000 mounted specimens, is especially rich in Philippine material, and contains few local collections made previous to 1902 , except a series of approximately 1,200 numbers from Cuming's collections, made between 1836 and 1840 , and a fairly large set of duplicates representing Warburg's collections. However, nearly all species described from the Philippines of which the types are extant are represented in the herbarium by types, cotypes, fragments of authentic specimens, photographs or sketches of types, or by critically compared specimens. Types of none of the species described by Blanco in his Flora de Filipinas, few of those described by Llanos, or the few described by F.-Villar and Naves are extant. The Blanco and Llanos species have been critically considered in a special publication.*

The extra-Philippine collections are relatively extensive, the herbarium being rich in material from surrounding regions, received in exchange and by collection, from Formosa, China, Indo-China, India, Ceylon, Burma, Siam, the Malay Peninsula, Sumatra, Java, Borneo, Celebes, the Moluccas, New Guinea, New Caledonia, Australia, the Marianne and the Caroline Islands, and Polynesia.

Some idea of the extent and the value of the extra-Philippine botanical material available in the herbarium of the Bureau of Science may be had from the following data: From India, Burma, Siam, Indo-China, and the Malay Peninsula, more or less extensive collections are available from material secured by the following collectors and botanists: Burkill, Buchanan-Hamilton, Burn-Murdoch, Balansa, Bon, Cave, Clarke, Craib, Curtis, Debeaux, Foxworthy, Eberhardt, 'Harmand, Gamble, Godefroy, Hole, Hooker f. and Thomson, Jacquemont, Kurz, Kunstler, King, King's collectors, Kerr, Kloss, Meebold, Petelot, Pierre, Prazer, Rock, Robinson, Ridley, Shaik Mokim, Talmy, Thorel, Thwaites, Walker, Wallich, Wight, and Wray. The flora of China, Japan, and Formosa is well represented by collections made by the following individuals: Bodinier, Callery, Cavalerie, Chun, Chung,

* Merrill, E. D., Species Blancoanae: A critical revision of the Philippine species of plants described by Blanco and by Llanos, Bur. Sci. Publ. 12 (1918) 1-423, map. 
Clemens, Dunn, Ducloux, Delavay, Farges, Faurie, Ford, Groff, Henry, Hu, Ito, Kanehira, Katsumada, Kawakami, Levine, Nakahira, Merrill, McClure, Maire, Steward, Sasaki, Taquet, Ts'oong, Tutcher, Wilson, and Wichura. The flora of some parts of the Malay Archipelago is particularly well represented by botanical material from the following collectors and botanists: Achmad, Agama, Amdjah, Backer, Backhuizen, Blume, Beguin, Beccari, Bartlett, Bünnenmeyer, Clemens, Elmer, Forbes, Foxworthy, Gjellerup, Hallier, Haniff, Hose, Kloss, Koorders, Korthals, C. King, La Rue, Lörzing, Mousset, Moulton, Nieuwenhuis, Ramos, Robinson, Raciborski, Schlechter, Teysmann, Topping, Versteeg, Villamil, Warburg, Winkler, Wood, and Yates. The flora of some parts of Polynesia is fairly well represented by collections made by the following individuals: Heller, Hombron, Le Batard, Le Guillou, McGregor, Nelson, Setchell, Thompson, Vaupel, Vesco, and Volkens. The flora of New Caledonia is particularly well represented by an extensive series of collections made by the following individuals: Balansa, Cribs, Deplanche, Franc, Germain, Lécard, Le Rat, Pancher, and Vieillard.

The duplicates of botanical material secured by the older collectors have in large part been obtained in exchange from European botanical institutions, notably the British Museum (Natural History), the Edinburgh Botanic Garden, the Paris Museum of Natural History, the Berlin Botanic Garden, and the Rijks Herbarium, Leyden; thus, from the Berlin institution more than 8,000 specimens have been secured; from the Paris Museum, more than 12,000 specimens; from the British Museum, about 6,000 specimens; and from the Edinburgh Botanic Garden, 3,500 specimens. An extensive series of exchanges has been carried on with botanic institutions in different parts of India, Malaysia, and Australia; more than 12,000 specimens from the Malay Archipelago have been received from the Buitenzorg Botanic Garden in Java, about 5,000 specimens from the Malay Peninsula have been received from the Botanic Garden at Singapore, and the Australian flora is represented by somewhat over 10,000 specimens secured in exchange with various botanic institutions in Australia. The large exchanges received from the Paris Museum have been particularly rich in material from IndoChina, China, and New Caledonia; the material from the British Museum has been largely from British India, with miscellaneous collections from China and New Guinea; the material received from the Edinburgh Botanic Garden has been largely from India and China. The large amount of extra-Philippine material from 
regions surrounding the Philippines has been invaluable for purposes of direct comparison in the prosecution of our work on the Philippine flora.

Botanical exploration under the auspices of the Bureau of Science has been confined largely to the Philippines, but members of our staff have prosecuted field work in China (Merrill), IndoChina (Robinson), Borneo (Foxworthy, Ramos), Amboina (Robinson), and Guam (McGregor). Large collections have been submitted for identification from various parts of China, Formosa, Hainan, Indo-China, Burma, Siam, the Malay Peninsula, Sumatra, Java, Borneo, New Guinea, Guam, Samoa, and Tahiti.

No attempt has been made to cite all the collections and numbers, as this procedure would have added enormously to the extent of the present publication and would have added very little to its value. In general, well-known and widely distributed species are merely enumerated with their synonyms, occurrence, distribution, etc., without the citation of specimens. In critical groups selected representative specimens only are cited. Generally speaking, when only one or two collections are cited under a species these represent the only collections extant. It should be noted that in approximately 1,800 cases Philippine records for individual species are based solely on single collections, while in about 900 additional cases individual species records are based on two collections only. This will give some idea of the great amount of work still remaining to be done before we can hope to have a reasonably complete knowledge of the very rich Philippine flora. Collections cited in parentheses-for example, (Warburg 14231), (Loher 4250) indicate specimens not examined by me during the actual preparation of this work, although most of these were seen by me in various European herbaria in 1907 and 1908.

While most of the identifications on which this enumeration is based have been made by me, in certain groups much of this work in earlier years was done by former botanists connected with the Bureau of Science; notably, the late Dr. C. B. Sobinson (Myrtaceae, Urticaceae, and certain groups of Euphorbiaceae, especially) and Dr. F. W. Foxworthy (Dipterocarpaceae and Taxaceae). Mr. Oakes Ames has made practically all of the identifications of the Orchidaceae since 1903. Many of the Pandanaceae have been determined by Dr. U. Martelli, while most of the Palmae were identified by the late Dr. O. Beccari. Many identifications in the Gramineae were made by Dr. E. Hackel, especially previous to 1907 ; while in the Cyperaceae the Bureau of Science has had the benefit of the extensive knowledge 
of the late Mr. C. B. Clarke and of the Rev. G. Kükenthal. The Araceae have been largely identified by Dr. A. Engler, and the Dioscoreaceae by Sir David Prain and Mr. I. H. Burkill. The Piperaceae have largely been identified by the late Dr. C. de Candolle. Dr. L. Diels has named many of the Menispermaceae. Dr. J. Perkins in addition to her general work on earlier collections in various families has identified many of the Monimiaceae. The late Mr. O. Focke has named or examined many of the Rubus specimens. Practically all of the Sapindaceae have been named or examined by Dr. L. Radlkofer. The Impatiens material was named by the late Sir J. D. Hooker. Many of the Symplocaceae were determined by Dr. A. Brand. Dr. R. Schlechter has identified or examined most of the species of Asclepiadaceae. Dr. F. Kränzlin accomplished considerable work on the Gesneriaceae. The late Mr. C. B. Clarke identified numerous representatives of the Acanthaceae. Numerous individual identifications and comparisons with type material have been made by other botanists in various European countries, the United States, Japan, Java, Singapore, India, and Australia. 



\title{
AN ENUMERATION OF PHILIPPINE FLOWERING PLANTS
}

\author{
By Elmer D. Merrill \\ Director and Botanist, Bureau of Science
}

GYINOSPERILAE

CYCADACEAE

1. CYCAS Linnaeus

CYCAS CAIRNSIANA F.-Muell. Fragm. 10 (1876-77) 63?

Cycas sp. Foxw. in Philip. Journ. Sci. 6 (1911) Bot. 151.

Culion, Merrill 65\%. In open grasslands at low altitudes. A curious species, of which only the leaves are known, perhaps representing the above Australian species.

CYCAS REVOLUTA Thunb. Fl. Jap. (1784) 229; A. DC. Prodr. $16^{2}$ (1868) 526; F.-Vill. Novis. App. (1880) 212; Vidal Cat. Pl. Prov. Manila (1880) 46; Foxw. in Philip. Journ. Sci. 6 (1911) Bot. 151; Merr. Fl. Manila (1912) 65.

A native of China and Japan, introduced into the Philippines for ornamental purposes and now occasionally cultivated in Manila and in other large towns in the Archipelago. It never produces flowers or fruits in the Philippines so far as our records show.

Local name: Olíva (Sp.).

CYCAS RUMPHIl Miq. in Bull. Soc. Phys. Nat. Néerl. (1839) 45; F.-Vill. Novis. App. (1880) 212; Merr. Interpret. Herb. Amb. (1917) 74, Sp. Blancoanae (1918) 52.

Cycas circinalis Blanco Fl. Filip. (1837) 745, ed. 2 (1845) 513, ed. 3, 3 (1879) 146; F.-Vill. Novis. App. (1880) 212; Vidal Sinopsis Atlas (1883) 43, t. 99; Warb. Monsunia (1900) 178; Merr. in Philip. Journ. Sci. 1 (1906) Suppl. 24, 3 (1908) Bot. 394, Fl. Manila (1912) 65; Foxw. in Philip. Journ. Sci. 6 (1911) Bot. 151 (non Linn.?).

Cycas riuminiana Porte ex Regel in Gartenflora 12 (1863) 16, fig., Ill. Hort. 28 (1881) 32; A. DC. Prodr. $16^{2}$ (1868) 528; F.-Vill. Novis. App. (1880) 212.

Batan Islands and northern Luzon to Palawan and Mindanao. Chiefly along or near the seashore, sometimes in forests; of rather local occurrence. Malaya to Polynesia.

The common coastal form may not be specifically distinct from C. circinalis Linn. A form from the forests of Mt. Mariveles (Merrill 325\%, Whitford 269,1325 , etc.), with small 0 cones and small fruits may be specifically distinct. A second form from Mindoro and Batangas with small fruits may also be distinct (B.S. 22391 Ramos). 
Local names: Báit (Sul.); báyit (Yak.); bitógo (Tag.); olíva (Sp.); patúbo (Tag.); patúgo (Tag.); pitógo (Tag.); pitúgo (P. Bis.); sáuang (Ilk.) ; ulíba (Tag.).

CYCAS sp. Foxw. in Philip. Journ. Sci. 6 (1911) Bot. 152, t. 2\%.

Palawan, F. B. 3842 Curran. Leaves like those of C. rumphii Miq., but the 0 cones are entirely different. Probably an undescribed species.

\section{TAXACEAE}

\section{PODOCARPUS L'Héritier}

PODOCARPUS AMARUS Blume Enum. Pl. Jav. (1827) 88, Rumphia 3 (1847) 213, t. 170; Pilger in Engl. Pflanzenreich 18 (1903) 68, f. B, $A-D$, Warb. Monsunia (1900) 192; Foxw. in Philip. Journ. Sci. 6 (1911) Bot. 159.

Podocarpus euryncha Miq. Fl. Ind. Bat. 2 (1859) 1074; F.-Vill. Novis. App. (1880) 211.

Luzon (Benguet, Lepanto), Mindoro, Mindanao (Davao), Elmer 11682, Merrill 5703, F. B. 10895, 10951 Curran, 18356 Alvarez, 18029 Merritt. In the mossy forest, altitude 1,600 to $2,400 \mathrm{~m}$. Java, Sumatra, Lombok; also reported from tropical Australia.

Local names: Pasuig (Ig.); tumpís (Bag.).

Podocarpus Blumel Endl. Syn. (1847) 208; Parl. in DC. Prodr. 16 " (1868) 508; Pilger in Engl. Pflanzenreich 18 (1903) 60; Merr. in Govt. Lab. (Philip.) Publ. 17 (1904) 5, Philip. Journ. Sci. 1 (1906) Suppl. 24, 2 (1907) Bot. 258; Foxw. op. cit. 6 (1911) Bot. 158.

Podocarpus latifolius Blume Enum. Pl. Jav. (1827) 89; C. B. Rob. in Bull. Torr. Bot. Club. 35 (1908) 63, non R. Br. (1825).

Luzon (Cagayan, Apayar, Bataan), Mindoro, Sibuyan, Panay, Samar. Whitford 1358, Williams 399, 753, 1035, Merrill 5728, Elmer 12360, B. S. 28348 F'énix, F. B. 23937, 23918, 23940 Cortes \& Knapp, 25949 Knapp, 16738, 17200, 17616 Curran, 20888 Hinoban, 194, 14\% Barnes. In primary forests, altitude 300 to $1,800 \mathrm{~m}$. Java, Moluccas, Celebes, New Guinea.

Local name: Makopulá (P. Bis.).

PODOCARPUS BREVIFOLIUS (Stapf) Foxw. in Philip. Journ. Sci. 6 (1911) Bot. 160, t. 29, f. 2.

Podocarpus neriifolius Don var. brevifolia Stapf in Trans. Linn. Soc.

Bot. 4 (1894) 249; Pilger in Engl. Pflanzenreich 18 (1903) 93.

Luzon (Zambales), Mindanao (Bukidnon), B. S. 5002 Ramos, 38500 Ramos \& Edaño, F. B. 9511 Curran \& Merritt. In elfinwood on exposed ridges, altitude $2,000 \mathrm{~m}$. Borneo.

PODOCARPUS COSTALIS Presl. Epim. (1849) 236, p.p. quoad spec. Haenke;

Walp. Ann. 3 (1852-53) 448; F.-Vill. Novis. App. (1880) 211; Miq. Fl. Ind. Bat. 2 (1859) 1074; Ceron Cat. Pl. Herb. (1892) 187; Warb. Monsunia (1900) 193; Pilger in Engl. Pflanzenreich 18 (1903) 161; Foxw. in Philip. Journ. Sci. 6 (1911) Bot. 161.

Podocarpus sp. Vidal Sinopsis Atlas (1883) 43, t. 9\%, f. D.

Luzon (Laguna, Tayabas), Haenke, Merrill 7506, Loher 2140, 7128, B. S. 19581 Ramos, 2393, 2423 Foxworthy, F. B. 7913 Curran \& Merritt. In the mossy forest on Mt. Banahao, altitude 1,700 to 2,200 m. Endemic. 
POdocarpus Glaucus Foxw. in Philip. Journ. Sci. 2 (1907) Bot. 258, 6 (1911) Bot. 159, t. 29, f. 1.

Mindoro, Merrill 5672. Border of an open heath on Mt. Halcon, altitude about $2,400 \mathrm{~m}$. Endemic.

PODOCARPUS JAVANICUS (Burm. f.) Merr. in Philip. Journ. Sci. 19 (1921) 338.

Thuja? javanica Burm. f. Fl. Ind. (1768) 202, t. 64, f. 3.

Podocarpus imbricatus Blume Enum. Pl. Jav. (1827) 89; Pilger in Engl. Pflanzenreich 18 (1903) 56; Foxw. in Philip. Journ. Sci. 6 (1911) Bot. 157; W. H. Br. op. cit. 12 (1917) Bot. 317, t. 17.

Podocarpus cupressina R. Br. ex Mirb. in Mém. Mus. Paris 13 (1825) 75, nomen nudum; Benn. Pl. Jav. Rar. (1838) 35, t. 10; Miq. Fl. Ind. Bat. 2 (1859) 1074; F.-Vill. Novis. App. (1880) 211; Vidal Sinopsis Atlas (1883) 43, t. 9\%, f. B, Phan. Cuming. Philip. (1885) 160, Rev. Pl. Vasc. Filip. (1886) 295; Warb. Monsunia (1900) 191.

Podocarpus cumingii Parl. in DC. Prodr. $16^{2}$ (1868) 521.

Nageia cumingii O. Kuntze Rev. Gen. Pl. 2 (1891) 800.

Podocarpus imbricatus Blume var. cumingii Pilger in Engl. Pflanzenreich 18 (1903) 56, Perk. Frag. Fl. Philip. (1904) 44; Merr. in Philip. Journ. Sci. 5 (1910) Bot. 324.

Luzon (Abra, Lepanto, Bontoc, Benguet, Zambales, Tayabas), Mindoro, Negros, Panay, Mindanao. Common on the higher mountains in the mossy forest, altitude 1,300 to $2,500 \mathrm{~m}$. Burma to Indo-China, Sumatra, Java, Borneo, Celebes, Timor, and New Guinea.

Lacal names: Apilum (Buk.); baginsóloi (Ting.); banyás (Mang.); dionaí (Sub.); haluko (Ig.) ; iguem (Ig.) ; igum (Ig.) ; rorogóñon (Lan.); sarambrum (Bag.).

PODOCARPUS NERIIFOLIUS Don in Lamb. Pin. (1824) 21; Parl. in DC. Prodr. $16^{2}$ (1868) 514; Pilger in Engl. Pflanzenreich 18 (1903) 80, Perk. Frag. Fl. Philip. (1904) 44; Merr. in Philip. Journ. Sci. 1 (1906) Suppl. 24, 2 (1907) Bot. 258; Foxw. op. cit. 6 (1911) Bot. 162 (incl. var. brevipes Blume).

Luzon (Abra, Benguet, Tayabas), Polillo, Mindoro, Sibuyan, Mindanao, Jolo, Merrill 1992, 5768, 5615, F. B. 25285 Somonte, 26834 Hirro, 10894, 10826 Curran, 14422 Darling, B. S. 25522 Yates, 10779 McGregor. In primary forests at low and medium altitudes. India to southern China through Malaya to New Guinea.

Local names: Dílang-butikí (Tag.); pasuik (Ig.).

PODOCARPUS PHILIPPINENSIS Foxw. in Philip. Journ. Sci. 6 (1911)

Bot. $163, t .30$.

Podocarpus muphii Foxw. op. cit. 6 (1911) Bot. 164, non Blume.

Luzon (Ilocos Sur, Bataan, Pampanga), Mindoro, B. S. 1660, 5174, 10844 Foxworthy, F. B. 27786, 13253 Paraiso, 17523, 17594, 6326, 6325, 653\%, 7512, 8987, 17664, 17793 Curran, 2743 Borden, Merrill 5553, 391\%. In primary forests, altitude 400 to $800 \mathrm{~m}$. Endemic.

Local name: Kasírai (Ilk.).

PODOCARPUS PILGERI Foxw. in Philip. Journ. Sci. 2 (1907) Bot. 259, 6 (1911) Bot. 160.

Podocarpus celebicus Warb. Monsunia (1900) 192; Pilger in Engl. Pflanzenreich 18 (1903) 78, non Hemsl. (1896). 
Mindoro, Negros, Mindanao (Agusan, Misamis), Merrill 5754, Phil. Pl. 241, Elmer 14086, F. B. 4673 Mearns \& Hutchinson. In the mossy forest on the higher mountains, altitude 1,400 to $2,500 \mathrm{~m}$. Celebes.

Local names: Lúbang-lúbang (Mbo.) ; tambiáyang (Bag.).

PODOCARPUS POLYSTACHYUS R. Br. ex Mirb. in Mém. Mus. Paris 13 (1825) 75; Endl. Syn. (1847) 215; Parl. in DC. Prodr. $16^{2}$ (1868)

515; Pilger in Engl. Pflanzenreich 18 (1903) 79; Merr. in Philip. Journ. Sci. 3 (1908) Bot. 394; Foxw. op. cit. 6 (1911) Bot. 161.

Batan Islands, Luzon (Ilocos Norte), Palawan, Bucas, B. S. 27146 Ramos, 26902 Edaño, 3586 Fénix, 20771 Escritor, 13202 Foxworthy \& Ramos, 904 Foxworthy, F. B. 24264 Bernardo, 3854 Curran, Merrill 5268. In forests at low and medium altitudes, sometimes along the seashore. Malay Peninsula, Sumatra, Java, Borneo.

Local name: Bantígi (Neg.).

\section{DACRYDIUM Solander}

DACRYDIUM ELATUM (Roxb.) Wall. in Hook. Journ. Bot. 2 (1843) 144, t. 2; Blume Rumphia 3 (1847) 221, t. 172 B, f. 1, t. 172 C, f. 2; de Boer Conif. Archip. Ind. (1868) 29; Parl. in DC. Prodr. $16^{2}$ (1868) 494; Rendle in Journ. Bot. 34 (1896) 355; Merr. in Philip. Journ. Sci. 2 (1907) Bot. 257; Pilger in Engl. Pflanzenreich 18 (1903) 51; Foxw. in Philip. Journ. Sci. 6 (1911) Bot. 154.

Juniperus elata Roxb. Fl. Ind. ed. 2, 3 (1832) 838.

Luzon (Nueva Ecija), Mindoro, Negros, Panay, Mindanao, B. S. 26510, 34497 Ramos \& Pascasio, F. B. 4547, 4548, 4731 Mearns \& Hutchinson, 4227 Everett, 4419, 8527 Merritt, 13612, 13621 Curran \& Foxworthy, Merrill 5789. Chiefly in the mossy forest, altitude 1,200 to $2,400 \mathrm{~m}$. on the east coast of Mindanao occurring as low as $200 \mathrm{~m}$. Indo-China, Malay Peninsula, Sumatra, Borneo, Celebes.

Local names: Hamo-hamo (Neg.); hinubáyan (Buk.); lokínai (Sub.); maglíging (Mbo.).

DACRYDIUM FALCIFORME (Parl.) Pilger in Engl. Pflanzenreich 18 (1903) 45; Merr. in Philip. Journ. Sci. 2 (1907) Bot. 257; Foxw. op. cit. 6 (1911) Bot. 153, t. 28, f. 1.

Podocarpus falciformis Parl. in DC. Prodr. $16^{2}$ (1868) 685; Rendle in Journ. Bot. 34 (1896) 355; Warb. Monsunia (1900) 193.

Nageia falciformis O. Kuntze Rev. Gen. Pl. 2 (1891) 800.

Luzon (Nueva Ecija), Mindoro, Panay, Mindanao (Davao, Surigao), F. B. 27746 Cruz, 4425 Merritt, 26478 Mallonga, 26394 Ramos \& Edaño, Merrill 5744. Chiefly in the mossy forest, altitude 1,000 to $2,000 \mathrm{~m}$. Borneo, Malay Peninsula, Lingga.

Local name: Bináton (Mbo.); magíbus (C. Bis.); maradayónit (Neg.).

3. PHYLLOCLADUS L. C. and A. Richard

PHYLLOCLADUS HYPOPHYLLUS Hook. f. Ic. 9 (1852) t. 889; Carr. Conif. (1867) 706; Parl. in DC. Prodr. $16^{2}$ (1868) 499; Pilger in Engl. Pflanzenreich 18 (1903) 99.

Phyllocladus protractus Pilger in Engl. Pflanzenreich 18 (1903) 99, Perk. Frag. Fl. Philip. (1904) 44; Foxw. ex Merr. in Philip. Journ. Sci. 2 (1907) Bot. 259, 6 (1911) Bot. 165, t. 31. 
Phyllocladus hypophyllus Hook. f. var. proiracta Warb. Monsunia (1911) 194.

Luzon (Isabela, Abra, Ifugao, Lepanto, Bontoc, Benguet), Mindoro, Mindanao (Bukidnon, Lanao, Misamis, Davao), Merrill 4753, 5788, Loher 5203, Elmer 11463, F. B. 27235 Niebert, 18364, 18567 Alvarez, 10957 Curran, 14587 Darling, 4679 Mearns \& Hutchinson, B. S. 37757,38738 Ramos \& $E d a \tilde{n} o$. In the mossy forest on the higher mountains, altitude 1,200 to $2,400 \mathrm{~m}$. Borneo.

The supposed differences between $P$. protractus Pilger and $P$. hypophyllus Hook. f. break down entirely on comparison of a large series of Kinabalu and Philippine specimens.

Local names: Aransisíngit (Ig.); dálung (Ting.) ; galingkínga (Sub.); salumayag (Bag.).

\section{TAXUS Linnaeus}

TAXUS WALLICHIANA Zuce. in Abh. Bayr. Akad. Wiss. 3 (1843) 803, t. 5; Foxw. in Philip. Journ. Sci. 6 (1911) Bot. 166.

Taxus baccata Linn. subsp. wallichiana (Zucc.) Pilger in Engl. Pfianzenreich 18 (1903) 112, Perk. Frag. Fl. Philip. (1904) 44.

Taxus baccata Merr. in Philip. Journ. Sci. 5 (1910) Bot. 324, non Linn.

Cephalotaxus mannii Rendle in Journ. Bot. 34 (1896) 355, non Hook. f.

Luzon (Benguet, Lepanto, Laguna, Tayabas). In the mossy forest, altitude 2,000 to $2,600 \mathrm{~m}$; south of the Mountain Province known in the Philippines only from Mt. Banahao; represented by numerous collections. India to Burma, Sumatra and Celebes.

Local name: Amugáuen (Ig.).

\section{PINACEAE}

\section{AGATHIS Salisbury}

AgAthis ALBA (Lam.) Foxw. in Philip. Journ. Sci. 5 (1910) Gen. Sci. 173, 6 (1911) Bot. 167; Merr. Interpret. Herb. Amb. (1917) 76, Sp. Blancoanae (1918) 52.

Dammara alba Lam. Encycl. 2 (1786) 259; Parl. in DC. Prodr. $16^{2}$ (1868) 374.

Agathis loranthifolia Salisb. in Trans. Linn. Soc. Bot. 8 (1807) 311; Blanco Fl. Filip. ed. 2 (1845) 528, ed. 3, 3 (1879) 170; F.-Vill. Novis. App. (1880) 211; Vidal Sinopsis Atlas (1883) 43, t.98, f. A, Phan. Cuming. Philip. (1885) 160, Rev. Pl. Vasc. Filip. (1886) 295.

Dammara rumphii Presl Epim. (1851) 236.

Podocarpus philippeanus Benth. ex Parl. in DC. Prodr. $16^{2}$ (1868) 375 , in syn.

Agathis philippinensis Warb. Monsunia (1900) 185, Perk. Frag. Fl. Filip. (1904) 36; Merr. in Philip. Journ. Sci.1 (1906) Suppl. 24, 2 (1.907) Bot. 257.

1 1. tachys Warb. Monsunia (1900) 184, 185.

Babuyan Islands and northern Luzon to Palawan and Mindanao, in most islands and provinces. Always in the primary forest, chiefly at medium and higher altitudes; represented by over 60 individual collections. Indo-China through the Malay Peninsula and Archipelago to the Moluccas. 
Local names: Adiáñgau (Bik.); alinságo (Ig.); almáciga (Sp.) ; alintágau (Ig.); anínga (Ig.) ; anano (S. L. Bis.); anting (Neg.); arínga (Klg.) ; bagtík (Kuy.); bálau (C. Bis.); baltík (Tagb.); bidiángau (P. Bis.) ; badiáñgau (P. Bis.); biáyo (Bis.); bunsóg (Ig.); buntóg (Ig.) ; dadiáñau (C. Bis., Tag.) ; dadúñoi (Bik.) ; dinar (Bag.) ; gala-gála (Tag., Tagb.); ladiáñgau (Bik., Tag.) ; makáu (C. Bis.); olinságo (Ig.) ; sálang (Neg.) ; sáleng (Neg.) ; sálong (Tag., Bik.) ; titau (Ting.) ; uli (Sbl.) ; uningát (Ilk.).

\section{PINUS Linnaeus}

PINUS INSULARIS Endl. Syn. Conif. (1847) 157; Presl Epim. (1851) 37; Parl. in DC. Prodr. $16^{2}$ (1868) 390; F.-Vill. Novis. App. (1880) 212; Vidal Sinopsis Atlas (1883) 43, t. 98, f. C, Phan. Cuming. Philip. (1885) 160, Rev. Pl. Vasc. Filip. (1886) 296; Merr. in Forest. Bur. (Philip.) Bull. 1 (1903) 15, Govt. Lab. Publ. (Philip.) 6 (1904) 6, Philip. Journ. Sci. 5 (1910) Bot. 325, Sp. Blancoanae (1918) 53; Foxw. in Philip. Journ. Sci. 6 (1911) Bot. 170; Shaw Gen. Pinus (1914) 60, t. 23, f. 208-210.

Pinus taeda Blanco Fl. Filip. (1837) 767, ed. 2 (1845) 528, ed. 3, 3 (1879) 169, t. 453, non Linn.

Pinus insularis $\dot{x}$ merkusii Perk. Frag. Fl. Philip. (1904) 35.

Pinus kasya Royle ex Parl. in DC. Prodr. $16^{2}$ (1868) 390; F.-Vill. Novis. App. (1880) 212; Merr. in Forest. Bur. (Philip.) Bull. 1 (1903); 15 (khasya).

Luzon (Ifugao, Bontoc, Lepanto, Benguet, Nueva Vizcaya, Nueva Ecija). The dominant species in the Mountain Province, its altitudinal range being from about 1,000 to $2,700 \mathrm{~m}$. Burma and Indo-China.

Local names: Alal (Ig.); balibo (Ig.); bariat (Bon.); bata (Bon.); batáng (Bon.); bebe (Bon.); bolbol (Ig.); bubu (Ig.); bulbúl (Ig.) ; olol (Ig.); paruá (Ilk.); sáhing (Tag.); sáleng (Bon., Ilk., Ig., Ting.); sálit (Sbl.) ; talang (Gad.); tapúlau (Sbl.).

PINUS MERKUSII Jungh. \& DeVr. in Pl. Nov. Ind. Bat. Orient. 5 (1845) t.2, Bot. Zeit. 4 (1846) 13; Miq. Fl. Ind. Bat. 2 (1859) 1069; F.-Vill. Novis. App. (1880) 211; Vidal Sinopsis Atlas (1883) 43, t. 98, f. B, Rev. Pl. Vasc. Filip. (1886) 296; Perk. Frag. Fl. Philip. (1904) 35; Merr. in Forest. Bur. (Philip.) Bull. 1 (1903) 15, Govt. Lab. Publ. (Philip.) 6 (1904) 6; Foxw. in Philip. Journ. Sci. 6 (1911) Bot. 169; Shaw Gen. Pinus (1914) 58, t. 23, f. 198-200.

Luzon (Zambales), Mindoro. Very similar to $P$. insularis Endl. but with only two leaves in a fascicle instead of three; in places it occurs as low as $100 \mathrm{~m}$ altitude. Burma, Indo-China, Sumatra, ? Borneo.

Local names: Agú (Mang.) ; agúu (Mang.) ; sálit (Sbl.) ; sálong (Sbl.); tapúlau (Sbl.).

The following species of Pinaceae have been credited'to the Philippines, but are all introduced and but rarely cultivated and have no claims to be considered as representatives of the Philippine flora:

Araucaria exCersa R. Br.; F.-Vill. Novis. App. (1880) 211.

This does not thrive in the Philippines and never reaches maturity here. Cupressus torulosa Don; F.-Vill. 1. c.

This is rarely cultivated and is commonly known by its Spanish name cipres. 
Juniperus occidentalis Hook.; Usteri Beitr. Ken. Philip. Veg. (1905) 194. Cultivated according to Usteri; I have seen no specimens.

\section{GNETACEAE}

\section{GNETUM Linnaeus}

GNeTUM ARBOREUM Foxw. in Philip. Journ. Sci. 6 (1911) Bot. 174, t. 32.

Luzon (Rizal, Nueva Ecija, Tayabas), B. S. 9439 Robinson, 29635.5, 26.388 Ramos \& Edaño. In the mossy forest, altitude about $900 \mathrm{~m}$. Endemic.

Additional collections show this to be a vine, not a tree, as Robinson and Foxworthy supposed.

Local name: Lapingat (Neg.).

GNETUM GNEMON Linn. Mant. 1 (1867) 125; Blume Rumphia 4 (1848)

3, t. 176, 178 B, f. 5; Parl. in DC. Prodr. $16^{2}$ (1868) 349; Blanco

Fl. Filip. (1837) 747, ed. 2 (1845) 514, ed. 3, 3 (1879) 147; F.-Vill.

Novis. App. (1880) 210; Vidal Phan. Cuming. Philip. (1885) 160,

'Rev. Pl. Vasc. Filip. (1886) 295; Karsten in Ann. Jard. Bot. Buitenz.

11 (1893) 203; Merr. Forestry Bur. (Philip.) Bull. 1 (1903) 16,

Philip. Journ. Sci. 1 (1906) Suppl. 24, Sp. Blancoanae (1918) 53;

Foxw. in Philip. Journ. Sci. 6 (1911) Bot. 173.

Gnetum vinosum Elm. Leafl. Philip. Bot. 7 (1915) 2673.

Luzon (Ilocos Norte, Bataan, Batangas, Laguna, Tayabas, Camarines), Mindoro, Palawan, Sibuyan, Panay, Samar, Leyte, Siargao, Bucas Grande, Mindanao. In forests at low and medium altitudes. Tropical Africa and Asia through Malaya to New Guinea.

Local names: Babayong (Sub.) ; bágo (Buk., Bik., Bag., C. Bis., Mbo., Tag.) ; bágo-sili (C. Bis.) ; banágo (Bis.) ; kugítis (Mbo.) ; kúman (Mand.); lamparan (Tag.) ; magatúñal (Mag.) ; nábo (Bik.).

GNETUM GNEMONOIDES Brongn. in Duperry Voy. Bot. (1829) 12; Merr.

Interpret. Herb. Amb. (1917) 78.

Gnetum rumphianum Becc. Malesia 1 (1877) 182.

Gnetum verrucosum Karst. in Ann. Jard. Bot. Buitenz. 11 (1893) 216.

Funis gnemoniformis Rumph. Herb. Amb. 5 (1747) 12, t. 8.

Luzon (Tayabas, east coast), Basilan, B. S. 15496 Reillo, 29096 Ramos \& Edaño. In forests at low or medium altitudes. Moluccas, New Guinea.

GNETUM INDICUM (Lour.) Merr. Interpret. Herb. Amb. (1917) 77, Sp. Blancoanae (1918) 53.

Abutua indica Lour. Fl. Cochinch. (1790) 630.

Gnetum funiculare Brongn. in Duperry Voy. Bot. (1829) 12; Miq. Fl.

Ind. Bat. 2 (1859) 1068; F.-Vill. Novis. App. (1880) 211.

Gnetum latifolium Blume Fam. Nov. (1833) 30, Rumphia 4 (1848) 5, t. 174; Presl Epim. (1851) 236; Miq. Fl. Ind. Bat. 2 (1859) 1067; Parl. in DC. Prodr. $16^{2}$ (1868) 350; F.-Vill. Novis. App. (1880) 210; Vidal Phan. Cuming. Philip. (1885) 160, Rev. Pl. Vasc. Filip. (1886) 295, Sinopsis Atlas (1883) 42, t. 97, f. A; Warb. Monsunia (1900) 195; Merr. in Philip. Journ. Sci. 1 (1906) Suppl. 25; Foxw. in Philip. Journ. Sci. 6 (1911) Bot. 174.

Thoo pendula Blanco Fl. Filip. (1837) 746.

Thoa edulis Blanco Fl. Filip. ed. 2 (1845) 514, ed. 3, 3 (1879) 146, non Willd. 
Gnetum phitippinense Warb. Monsunia (1900) 196.

Gnetum scandens F.-Vill. Novis. App. (1880) 211, non Roxb.

Gnetum laxifrutescens Elm. Leafl. Philip. Bot. 4 (1912) 1478.

Luzon (Cagayan to Sorsogon), Mindoro, Palawan, Sibuyan, Panay, Leyte, Samar, Siargao, Mindanao. In forests at low and medium altitudes; distinctly variable in vegetative characters. Represented by very numerous collections. India to southern China and Malaya.

Local names: Biás (Tag.); bias-biás (Tag.); bulso (Buk.); dadaotum (Mbo.) ; kadiát (Itn.) ; kalát (Ig., Ilk.) ; kaliát (Ilk.) ; kandiát (Ig.) ; konját (Ig.) ; koliát (Tag.) ; kuliád (Ibn.) ; kuliát (Bik., Tag., Pamp., Mang.) ; lamparahan (Tag.) ; malaígot (S. L. Bis.) ; nonok (P. Bis.) ; tobal (Tag.).

GNeTUm MINUs Foxw. in Philip. Journ. Sci. 6 (1911) Bot. 176, t. 33.

Luzon (Benguet), B. S. 2513 Mearns. In forests or thickets, altitude probably about $1,500 \mathrm{~m}$. Endemic.

EXCLUDFD SPECIES

Gnetum Neglectum Blume; F.-Vill. Novis. App. (1880) 211.

\section{ANGIOSPERMAE \\ MONOCOTYLEDONAE}

TYPHACEAE

1. TYPHA Linnaeus

TYPHA CAPENSIs Rohrb. in Verh. Bot. Ver. Brandenb. 11 (1869) 96; Hallier f. in Lorenz Nova Guinea 8 (1913) 911.

Typha angustifolia Blanco Fl. Filip. (1837) 687, ed. 2 (1845) 477, ed. 3,3 (1879) 91; Naves Novis. App. (1882) 297, non Linn.

Typha angustifolia Linn. subsp. javanica Graebn. in Perk. Frag. Fl. Philip. (1904) 42, Merr. Fl. Manila (1912) 66, Sp. Blancoanae (1918) 53, non Schnizl.

Luzon (Laguna), Mindoro, Negros, Merrill 895, Sp. Blancoanae 3.8, Piper 74, B. S. 9881 Robinson, F. B. 9775 Merritt. In fresh water swamps at low altitudes. Africa and Madagascar, Philippines, New Guinea.

Local names: Balangót (Tag., S. L. Bis.) ; homai-homai (Bis.) ; kaidkéd (Pang.); lampakánai (C. Bis.) ; tubal-tubal (C. 'Bis.).

TYPHA DOMINGENSIS Pers. Syn. 2 (1807) 532; Graebn. in Engl. Pflanzenreich $2^{1}$ (1900) 14; Hallier f. in Lorenz Nova Guinea 8 (1913) 912 , in nota.

Mindoro, Panay, Negros, Cebu, F. B. 13371 Curran, 25893 Tamesis \& $A$ zurin, $B . S .11125$ Ramos. In swamps at low altitudes. Tropical America; Java, Timor.

Local names: Balangót (P. Bis.); buhai-búhai (P. Bis.); lampakánai (C. Bis.).

TYPHA SHUTTLEWORTHII Koch \& Sonder in Koch Syn. ed. 2 (1844) 786; Hallier f. in Lorenz Nova Guinea 8 (1913) 913, in nota.

Typha angustifolia Vidal Rev. Pl. Vasc. Filip. (1886) 280, non Linn.

Typha orientalis Presl Epim. (1851) 239; C. B. Rob. in Bull. Torr. 
Bot. Club 35 (1908) 63; Merr. in Philip. Journ. Sci. 3 (1908) Bot. 394.

Typha shuttleworthii Koch \& Sond. subsp. orientalis Graebn. in Engl. Pflanzenreich $2^{1}$ (1900) 10, quoad syn. Presl.

Luzon (Benguet, Bontoc, Sorsogon), Cebu, Mindanao (Bukidnon, Lanao), Cuming 1767, Elmer 6382, 16.244, Clemens 856, B. S. 24984 Fénix. In swamps at low and medium altitudes, ascending to $1,400 \mathrm{~m}$. Europe to China.

Local names: Aníbung (Bon.); badok-badok (Ilk.); dosi-dosi (Ig.); palabog (Buk.).

I have arbitrarily followed Hallier $f$. in this classification of the Philippine forms but am by no means satisfied that the identifications are correct.

\section{PANDANACEAE}

\section{FREYCINETIA Gaudichaud}

FREYCINETIA ACUTIFOLIA Merr. in Philip. Journ. Sci. 13 (1918) Bot. 267.

Luzon (Camarines), Catanduanes, B. S. 30319 Ramos, 33561 Ramos \& Edaño. In damp primary forests at low altitudes. Endemic.

FREYCINETIA ANGULATA C. B. Rob. ex Martelli in Webbia 3 (1910) 16,309 .

Luzon (Albay), Samar, Panay, B. S. 6221 Robinson, 24813 Edaño, 32393 McGregor. In forests at low altitudes. Endemic.

FREYCINETIA APAYAOENSIS Merr. in Philip. Journ. Sci. 13 (1918) Bot. 269.

Luzon (Apayao, Ilocos Norte), B. S. 28034 F'énix, 33111 Ramos. In forests along streams at low altitudes. Endemic.

FREYCINETIA APOENSis Martelli in Leafl. Philip. Bot. 3 (1911) 1111.

Mindanao (Davao), Elmer 10781. In forests along streams, altitude about 1,200 m. Endemic。

Local name: Rasrás (Bag.).

FREYCINETIA ATOCENSIS Martelli in Webbia 3 (1910) 28, 309.

Luzon (Benguet), (Loher 15\%0). Probably in forests, altitude at or above $1,000 \mathrm{~m}$. Endemic.

FREYCINETIA AURICULATA Merr. in Philip. Journ. Sci. 3 (1908) Bot. 312; Martelli in Webbia 3 (1910) 15, 309.

Palawan, B. S. 876 Foxworthy. In forests at low or medium altitudes. Endemic.

FREYCINETIA BANAHAENSIS Elm. Leafl. Philip. Bot. 1 (1907) 215; Martelli in Webbia 3 (1910) 29, 309.

Luzon (Laguna, Tayabas), Elmer 7908, 18424. In primary forests, altitude 800 to $2,000 \mathrm{~m}$. Endemic.

FREYCINETIA BATANENSIS Martelli in Webbia 3 (1910) 29, 309.

Batan Islands, Luzon (Ilocos Norte), B. S. 3806 F'énix, 33301 Ramos. In forests, altitude about $1,000 \mathrm{~m}$. Endemic.

Local name: Uyód (Iv.). 
FREYCINETIA BOTULIFORMIS Merr. in Philip. Journ. Sci. 13 (1918) Bot. 268.

Luzon (Sorsogon), B. S. 23363 Ramos. In primary forests at medium altitudes. Endemic.

FREYCINETIA BULUSANENSIS Merr. in Philip. Journ. Sci. 13 (1918) Bot. 268.

Luzon (Sorsogon), Panay, B. S. 23686 Ramos, 32571 McGregor, Elmer 14978,16294 as $F$. vulcanica Elm. Apparently in the mossy forest, altitude at or above $900 \mathrm{~m}$. Endemic.

FREYCINETIA CUERNOSENSIS Martelli in Webbia 3 (1910) 26, 310.

Luzon (Sorsogon), Negros, Elmer 967\%, 16314, 14914. In forests at medium altitudes. Endemic.

FREYCINETIA CUMINGIANA Gaudich. Bot. Voy. Bonite (1839-46), t. 3\%, f. 12-14, t. 60; C. B. Rob. in Bull. Torr. Bot. Club 35 (1908) 64; Martelli in Webbia 3 (1910) 19, 310.

Freycinetia luzonensis Presl Epim. (1851) 238 (incl. var. heterophylla Presl); Walp. Ann. 3 (1852-53) 494; Miq. Fl. Ind. Bat. 3 (1856) 172; Vidal Phan. Cuming. Philip. (1885) 154, Rev. Pl. Vasc. Filip. (1886) 280; Naves Novis. App. (1882) 286; Warb. in Engl. Pflanzenreich 3 (1900) 35, f. 10, D-E; Merr. in Philip. Journ. Sci.

3 (1908) Bot. 311; C. B. Rob. op. cit. 6 (1911) Bot. 193.

Luzon (Cagayan, Camarines, Sorsogon), Cuming 1455, Weber 1585, Elmer 15394 as F. membranifolia Elm., 15930, 16016 as F. leuteocarpa Elm., B. S. 13874 Ramos, F. B. 11361 Curran. In forests at low and medium altitudes. Endemic.

Local name: Alapúnti (C. Bis.).

FREYCINETIA CURRANII Merr. in Philip. Journ. Sci. 3 (1908) Bot 312; Martelli in Webbia 3 (1910) 14, 310.

Luzon (Camarines, Sorsogon), Catanduanes, Panay, Camiguin de Misamis, F. B. 11359 Curran, 23789 Alvarez, B. S. 35\%35 Martelino \& Edaño, 14642, 3026\%, 30317 Ramos, Elmer 16590. In primary forests at low and medium altitudes. Endemic.

FREYCINETIA DISCOIDEA Martelli in Leafl. Philip. Bot. 3 (1911) 1113.

Sibuyan, Elmer 1219\%. In forests, altitude about $300 \mathrm{~m}$. Endemic.

FREYCINETIA ENSIFOLIA Merr. in Govt. Lab. Publ. (Philip.) 17 (1904)

5, Philip. Journ. Sci. 1 (1906) Suppl. 25, 3 (1908) Bot. 315; Martelli in Webbia 3 (1910) 32, 310; Elm. Leafl. Philip. Bot. 3 (1911) 1118.

Luzon (Bontoc, Benguet, Bataan, Pampanga), Mindoro, Negros, Merrill 3242, Elmer 6840, Whitford 329, B. S. 1944 Foxworthy, F. B. 6285 Curran, 1347 Borden, 2624 Meyer, B. S. 39472 Ramos. In the mossy forest, altitude 1,200 to $1,600 \mathrm{~m}$. Endemic.

Local names: Kañgingai (Ig.); kakaag (Bon.).

FREYCINETIA FEROX Warb. in Engl. Pflanzenreich 3 (1900) 33; Merr in Philip. Journ. Sci. 3 (1908) Bot. 310; Martelli in Webbia 3 (1910) $13,310$.

Freycinetia lucbanensis Elm. Leafl. Philip. Bot. 1 (1907) 212; Martelli in Webbia $3(1910) 14,312$. 
Luzon (Tayabas), Warburg in herb. Berol., Elmer 8230. In primary forests, altitude about $500 \mathrm{~m}$. Endemic,

FREYCINETIA GITINGIANA Martelli in Leafl. Philip. Bot. 3 (1911) Bot. 1112.

Sibuyan, Elmer 12358. In forests, altitude about $500 \mathrm{~m}$. Endemic.

FREYCINETIA INSIPIDA Martelli in Leaf. Philip. Bot. 3 (1911) 1114.

Sibuyan, Elmer 12426. In forests along streams, altitude 500 to 600 m. Endemic.

Local name: Bagánot (P. Bis.).

FREYCINETIA JAGORII Warb. in Engl. Pflanzenreich 3 (1900) 39; Merr. in Philip. Journ. Sci. 3 (1908) Bot. 313; Martelli in Webbia 3 (1910) 25, 311.

Luzon (Sorsogon), Biliran, Samar, Leyte, Mindanao (Agusan, Lanav, Zamboanga), Elmer 15378, 16210, 15057, 13717, Wenzel 306, Merrill 8300. Clemens, B. S. 18852 McGregor, 17395 Ramos, 37032 Ramos \& Edaño. In forests at low and medium altitudes. Endemic.

Local name: Magalop (Sub.).

FREYCINETIA LEPTOPHYLLA Martelli in Webbia 3 (1910) 20, 312.

Luzon (Rizal, Laguna), (Loher 5450), Bi. S. 15006 Ramos. In forests at medium altitudes. Endemic.

FREYCINETIA LOHERI Martelli in Webbia 3 (1910) 15, 312.

Luzon (Lepanto, Rizal), (Loher 157\%, 1578, 5469). Probably from forests at or above $1,200 \mathrm{~m}$ altitude. Endemic.

FREYCINETIA MAXIMA Merr. in Philip. Journ. Sci. 3 (1908) Bot. 310; Martelli in Webbia 3 (1910) 13, 312, Leaf. Philip. Bot. 3 (1911) 1111.

Luzon (Laguna, Tayabas, Camarines, Albay, Sorsogon), Samar, F. B. 10754,1238 Curran, 253r4 Mabesa, B. S. 23343,17444 Ramos, 6345 Robinson, Elmer $17530,14697,18152$. In primary forests at low and medium altitudes, ascending to $500 \mathrm{~m}$. Endemic.

FReycinetiA MEgAcarpa Merr. in Philip. Journ. Sci. 3 (1908) Bot. 314; Martelli in Webbia 3 (1910) 31, 312, Leafl. Philip. Bot. 3 (1911) 1117.

Mindanao (Agusan, Lanao), Clemens, Elmer 14242. In forests, altitude about $650 \mathrm{~m}$. Endemic.

FREYCINETIA MERRILlII Elm. Leafl. Philip. Bot. 1 (1907) 216; Merr. in Philip. Journ. Sci. 3 (1908) Bot. 314; Martelli in Webbia 3 (1910) 31, 313.

Luzon (Cagayan, Tayabas), Elmer 9010, B. S. 10727 McGregor. In forests along streams, altitude about $600 \mathrm{~m}$. Endemic.

FREYCINETIA MONOCEPHALA Elm. Leafl. Philip. Bot. 1 (1906) 78 bis, (1907) 218; Merr. in Philip. Journ. Sci. 3. (1908) Bot. 314; Martelli in Webbia $3(1910) 31,313$; C. B. Rob. in Philip. Journ. Sci. 6 (1911) Bot. 193.

Luzon (Rizal, Laguna, Tayabas), Elmer \%380, 9012, Whitford 971, Mervill Phil. Pl. 423, F. B. 13094 Curran, B. S. 29372 Ramos \& Edaño. In forests, altitude about $700 \mathrm{~m}$. Endemic. 
FREYCINETIA MONTALBANICA Martelli in Webbia 3 (1910) 18, 313;

C. B. Rob. in Philip. Journ. Sci. 6 (1911) Bot. 193.

Luzon (Rizal), (Loher s. $n_{.}, 5451$ ). Probably in forests at medium altitudes. Of this I have seen only a photograph of Loher 5451. Endemic.

FREYCINETIA MULTIFloRA Merr. in Philip. Journ. Sci. 2 (1907) Bot. 259, 3 (1908) Bot. 312; Elm. Leafl. Philip. Bot. 1 (1907) 212; Martelli in Webbia 3 (1910) 17, 313.

Freycinetia luzonensis Naves in Blanco Fl. Filip. ed. 3 (1877-83) t. 43\%, non Presl.

Luzon (Laguna, Bataan), Mindoro, Mindanao, Merrill 5647, Elmer 8039, 9009, 17974, Clemens 1028, B. S. 16857 Serviñas, 1511\%, 2092 Ramos, 11285 McGregor. In primary forests up to $900 \mathrm{~m}$ altitude. Endemic.

FREYCINETIA NEGROSENSIS Merr. in Philip. Journ. Sci. 3 (1908)' Bot. 313; Martelli in Webbia 3 (1910) 28, 313.

Luzon (Laguna, Sorsogon), Leyte, Negros, Mindanao (Agusan), Whitford 1541, Elmer 16161, 13766, as F. urdanetensis Eim., F. B. 26216 Franco, B. S. 16637 Ramos. In forests at medium altitudes, ascending to 1,200 m. Endemic.

Local name: Pandán-magamoi (C. Bis.).

FREYCINETIA oblongIfoliA Merr. in Philip. Journ. Sci. 3 (1908) Bot. 310; Martelli in Webbia 3 (1910) 16, 313.

Panay, Mindanao (Surigao), Bolster 249, 342, B. S. 32533 McGregor, 31060 Ramos \& Edaño. In primary forests at low altitudes. Endemic.

FREYCINETIA PALAWANENSIS Merr. ex Elm. Leafl. Philip. Bot. 1 (1907) 216, Philip. Journ. Sci. 3 (1908) Bot. 313; Martelli in Webbia 3 (1910) 23, 313.

Freycinetia elmeri Martelli in Webbia 3 (1910) 22, 310, Leafl. Philip. Bot. 3 (1911) 1117.

Luzon (Tayabas, Sorsogon), Palawan, Sibuyan, Panay, Mindanao (Agusan, Lanao), B. S. 706 Foxworthy, 23707 Ramos, 32527, 32231 McGregor, 35753 Martelino \& Edaño, Elmer 12072, 7810, 9386, 14030, Clemens 796. In primary forests, altitude 600 to $1,000 \mathrm{~m}$. Endemic.

FREYCINETIA PERIPIEZOCARPA Martelli in Webbia 3 (1910) 314.

Luzon (Ifugao, Bontoc, Benguet), Negros, F. B. 18020 Merritt, 13638 Curran \& Foxworthy, 10858 Curran, Merrill Phil. Pl. 727. On ridges in thickets and forests, altitude 1,200 to $1,600 \mathrm{~m}$. Endemic.

FREYCINETIA PHILIPPINENSIS Hemsl. in Kew Bull. (1896) 165; Warb. in Engl. Pflanzenreich 3 (1900) 40; Merr. in Philip. Journ. Sci. 3 (1908) Bot. 313; Martelli in Webbia 3 (1910) 25, 314.

Luzon (Cagayan, Tayabas, Camarines), Samar, Biliran, Mindanao (Bukidnon), Gregory 117, Cuming 1898, B. S. 18787 McGregor, 26028 Fénix, 22088, 22167 Ramos. In primary forests at low and medium altitudes. Endemic.

FREYCINeTIA PLATYPHYLLA Merr. in Philip. Journ. Sci. 13 (1918) Bot. 267.

Samar, B. S. 24349 Ramos. In forests along streams at low altitudes. Endemic. 
FREYCINETIA POLYSTACHYA Martelli in Webbia 3 (1910) 14, 314.

Luzon (Ilocos Norte, Rizal, Tayabas), (Loher 5454), B. S. 26642 Ramos \& Edaño, 33299 Ramos, 25540 Yates. In forests at medium altitudes. Endemic.

FREYCINETIA RIGIDA Elm. Leafl. Philip. Bot. 1 (1908) 362; Merr. in Philip. Journ. Sci. 3 (1908) Bot. 313; Martelli, in Webbia 3 (1910) $26,314$.

Freycinetia hemsleyi Elm. Leafl. Philip. Bot. 1 (1907) 214, non Waxb.

Luzon (Tayabas), Elmer 7847. In primary forests, altitude about 800 m. Endemic.

FREYCINETIA ROBINSONII Merr. in Philip. Journ. Sci. 3 (1908) Bot. 311; Martelli in Webbia 3 (1910) 17, 314.

Luzon (Ilocos Norte, Bataan, Pampanga), Leyte, F. B. 752, 2466, 3037 Borden, 2194 Meyer, B. S. 2006 Foxworthy, 27256 Ramos, Whitford 1311, Merrill 3791. In forests at low and medium altitudes. Endemic.

Var. LATIFOLIA Martelli in Webbia 3 (1910) 18, 314.

Luzon (Benguet, Laguna, Rizal), Negros, Elmer 6196. In forests at low and medium altitudes. Endemic.

Local names: Malabálỉng-uái (Tag.); malapandán (Tag.); maloran (Pamp.).

FREYCINetIA RoBusta Elm. Leafl. Philip. Bot. 8 (1919) 3097.

Luzon (Laguna), Elmer 18026. In primary forests at medium altitudes. Endemic.

FREYCINETIA RostratA Merr. in Philip. Journ. Sci. 1 (1906) Suppl. 177, 3 (1908) Bot. 315; Martelli in Webbia 3 (1910) 34, 314, Leafl. Philip. Bot. 3 (1911) 1118.

Samar, Negros, Siargao, Mindanao (Lanao), Merrill 5235, Clemens s. n., B. S. 24787 Edaño, F. B. 19097 Curran. In forests at low and medium altitudes. Endemic.

Local names: Rasrás (Bag.); sagatap (P. Bis.).

FREYCINETIA SCABRIPES Warb. in Engl. Pflanzenreich 3 (1900) 41; Merr. in Philip. Journ. Sci. 3 (1908) Bot. 313, 394; Martelli in Webbia 3 (1910) 27, 314.

Freycinetia dilatata Merr. ex Elm. Leafl. Philip. Bot. 1 (1907) 214, Philip. Journ. Sci. 3 (1908) Bot. 313; Martelli in. Webbia 3 (1910) 310.

Luzon (Cagayan, Bataan, Rizal, Tayabas, Sorsogon), F. B. 4529 Maule, B. S. 99 Foxworthy, Elmer 9008, Merrill 2301. In forests at low and medium altitudes. Endemic.

Local name: Palindán (Tag.).

FREYCINETIA SPHAEROCEPHALA Gaudich. Bot. Voy. Bonite (1843) t. 52; Solms-Laub. in Linnaea 42 (1878) 96; Warb. in Engl. Pflanzenreich 3 (1900) 35; Merr. in Philip. Journ. Sci. 3 (1908) Bot. 314; Martelli in Webbia 3 (1910) 33, 315.

Freycinetia strobilacea Vidal Phan. Cuming. Philip. (1885) 154, Rev. Pl. Vasc. Filip. (1886) 280; F.-Vill. Novis. App. (1882) 285, non Blume. 
Freycinetia globosa Merr. in Philip. Journ. Sci. 2 (1907) Bot. 260;

Elm. Leafl. Philip. Bot. 1 (1907) 217.

Luzon (Ilocos Norte, Apayao, Laguna, Camarines, Albay, Sorsogon), Mindoro, Cuming 839, Merrill 5791, Elmer 14920, Wenzel 511, B. S. 16593, 22016, 20510 Ramos. In forests at low and medium altitudes, ascending to $1,000 \mathrm{~m}$. Endemic.

Local name: Looi-lóoi (Bik.).

The figure of Freycinetia strobilacea Blume given by Vidal, Sinopsis Atlas (1883) 42, t. 95, f. $B$, was copied from Blume's original in Rumphia fide Vidal 1. c. Blume's species does not occur in the Philippines.

FREYCINETIA subFLAGELLATA Elm. Leafl. Philip. Bot. 8 (1919) 3098.

Luzon (Laguna), Elmer 1796\%. In primary forests at medium altitudes. Endemic.

FREYCINETIA SUPERBA Martelli in Leafl. Philip. Bot. 3 (1911) 1115.

Sibuyan, Elmer 1225\%. In forests, altitude 300 to $600 \mathrm{~m}$. Endemic.

FREYCINETIA VIDALII Hemsl. in Kew Bull. (1896) 166; Warb. in Engl. Pflanzenreich 3 (1900) 36; Merr. in Philip. Journ. Sci. 3 (1908) Bot. 311; Martelli in Elm. Leafl. Philip. Bot. 3 (1910) 21, 315.

Freycinetia confusa Elm. Leafl. Philip. Bot. 1 (1907) 213.

Luzon (Cagayan, Apayao, Nueva Ecija, Rizal, Tayabas), Vidal 3964, Elmer 9007, B. S. 16600, 13861 Ramos, 28434 Fénix, F. B.. $195 \% 3$ Curran, 22192 Alvarez. In primary forests at low and medium altitudes. Endemic.

Local name: Takiakad (Ig.).

FREYCINETIA WARBURGII Elm. Leafl. Philip. Bot. 1 (1907) 218; Mierr. in Philip. Journ. Sci. 3 (1908) Bot. 315; Martelli in Webbia 3 (1910) 34, 315; C. B. Rob. in Philip. Journ. Sci. 6 (1911) Bot. 193.

Luzon (Laguna, Tayabas, Sorsogon), Elmer 8229, 15833, as F. sorsogonensis Elm., B. S. 14988, 1660\%, 20608, 10925 Ramos. In forests at medium altitudes. Endemic.

FREYCINETIA WILLIAMSII Merr. in Philip. Journ. Sci. 3 (1908) Bot. 315, 394; Martelli in Webbia 3 (1910) 32, 315.

Batan Islands, Luzon (Ifugao, Bontoc, Benguet, Rizal, Pangasinan, Laguna, Tayabas), Panay, Williams 1013, Elmer 585\%, 18006, Merrill 8611, B. S. 3786, 29862 Fénix, 29737 Ramos \& Edaño, 22233 Catalan, 19755 McGregor, 9380, 17120 Robinson. In forests, extending into the mossy forest, altitude 500 to $1,600 \mathrm{~m}$. Endemic.

Local names: Pandán (Ig.); vayasúbas (Iv.).

\section{DOUBTFUL AND EXCLUDED SPECIES}

Freycinetia Graminea Blume; Naves Novis. App. (1882) 286; Usteri Beitr. Ken. Philip. Veg. (1905) 130.

This Javan species is not known from the Philippines, both of the above records being undoubtedly based on erroneously identified specimens.

Freycinetia insignis Blume; Vidal Cat. Pl. Prov. Manila (1880) 46; Naves Novis App. (1882) 286.

This Javan species certainly does not occur in the Philippines; from the local name cited by Vidal his specimens probably were Pandanus sabotan Blanco. 
Tillandsia PSEUdo-Ananas Blanco Fl. Filip. (1837) 853, ed. 2 (1845) 162, ed. 3, 1 (1877) 292.

This was reduced by Naves to Freycinetia insignis Blume, a species that does not extend to the Philippines. I now consider that Blanco's description applies to Freycinetia, but further identification of the species is impossible from the very imperfect description given by Blanco. It is possibly the same as Pandanus copelandii Merr.

\section{PANDANUS Linnaeus}

PANDANUS AClAdUS Merr. in Philip. Journ. Sci. 13 (1918) Bot. 265. Catanduanes, Mindanao (Bukidnon), B. S. 30462 Ramos, 39172 Ramos \& Edaño. Along streams in forests, altitude about $150 \mathrm{~m}$. Endemic. Local name: Ulañgó (Buk.).

PANDANUS APICULATUS Merr. in Philip. Journ. Sci. 17 (1920) 240.

Mindanao (Surigao), B. S. $345 \% 2$ Ramos \& Pascasio. On ridges, altitude about $650 \mathrm{~m}$. Endemic.

PANDANUS APOENSIS Martelli in Leafl. Philip. Bot. 3 (1911) 1129, Webbia $4^{1}$ (1913) 6, t. 13, f. 12-14.

Mindanao (Davao), Elmer 11313. In primary forests, altitude about 1,200 m. Endemic.

Local names: Baráia (Bag.); maráia (Bag.).

PANDANUS BARAI Martelli in Leafl. Philip. Bot. 3 (1911) 1126, Webbia $4^{1}$ (1913) $7, t .38, f .10-13$.

Mindanao (Davao), Elmer 10924. In damp forests, altiłude about $1,050 \mathrm{~m}$. Endemic.

Local name: Barai (Bag:).

PANDANUS BILIRANENsis Merr. in Philip. Journ. Sci. 13 (1918) Bot. 266.

Biliran, B. S. 18895 McGregor. In forests, altitude about $300 \mathrm{~m}$. Endemic.

PANDANUS BOTRYOIDES Martelli in Philip. Journ. Sci. 3 (1908) Bot. 66, Webbia $4^{1}$ (1913) 8, t. 4, f. 14-16; C. B. Rob. in Philip. Journ. Sci. 6 (1911) Bot. 193.

Polillo, Mindanao (Surigao, Agusan, Lanao), Merrill 5442, Weber 1212 , Clemens 984, B. S. 34570 Ramos \& Pascasio, 10473 Robinson. In forests along tidal streams, and at Lake Lanao along the lake shore, altitude about $650 \mathrm{~m}$. Endemic.

Specifically distinct from $P$. radicans Blanco?

PANDANUS BREVISPATHUS Martelli in Philip. Journ. Sci. 3 (1908) Bot. 69, Webbia $4^{1}$ (1913) 8, t. 26, f. 3-5.

Pandanus polycepha'us Merr. in Govt. Lab. Publ. (Philip.) 17 (1904) 8, non Lour.

Mindanao (Davao), Copeland 44⿱刀口灬. In thickets near the seashore. Endemic.

PANDANUS BREVISTIPES Martelli in Webbia $4^{1}$ (1913) $8,4^{2}$ (1914) $421, t$. 38, f. 4-9. 
Luzon (Batangas), F. B. 7719 Curran \& Merritt. On ridges in forests, altitude about $800 \mathrm{~m}$. Endemic.

Local name: Pandán (Tag.).

PANDANUS CALCEIFORMIS Martelli in Leafl. Philip. Bot. 3 (1911) 1127, Webbia $4^{1}$ (1913) 9, t. 35, f. 3-6.

Mindanao (Bukidnon, Davao), Elmer 11654, B. S. 38580, 39170 Ramos \& Edaño. On forested ridges, altitude about $1,350 \mathrm{~m}$. Endemic.

Local name: Baráui (Bag.).

PANDANUS CAMARINENSIS Merr. in Philip. Journ. Sci. 14 (1919) 367.

Luzon (Camarines), B. S. 33635 Ramos \& Edaño. In forests at low altitudes. Endemic.

PANDANUS CAUdATUS Merr. in Govt. Lab. Publ. (Philip.) 29 (1905) 6; Martelli in Philip. Journ. Sci. 3 (1908) Bot. 64, Webbia $4^{1}$ (1913) 9, t. 4, f. 1-3.

Luzon (Benguet), Elmer 6143. In thickets or forests, altitude about $1,000 \mathrm{~m}$. Endemic.

Local name: Pañihang (Ig.).

PANDANUS CLementIS Merr. in Philip. Journ. Sci. 1 (1906) Suppl. 178, t. 1, 3 (1908) Bot. 68; Martelli in Webbia $4^{1}$ (1913) 10, t. $2^{\%}$, f. $6-10$.

Panay, Mindanao (Lanao), Clemens 436, Merrill 6708. In forests, altitude 100 to $700 \mathrm{~m}$. Endemic.

PANDANUS COPELANDII Merr. in Govt. Lab. Publ. (Philip.) 17 (1904) 7, Philip. Journ. Sci. 3 (1908) Bot. 71; Martelli op. cit. 71, Leafl. Philip. Bot. 3 (1911) 1132, Webbia $4^{1}$. (1913) 11, t. 31, f. 5-\%.

Pandanus muricatus Elm. Leafl. Philip. Bot. 1 (1906) 76.

Luzon (Cagayan, Apayao, Benguet, Pangasinan, Nueva Vizcaya, Nueva Ecija, Zambales, Tayabas, Camarines, Sorsogon), Polillo, Catanduanes, Samar, Bucas Grande, Panay, Leyte, Mindanao (Bukidnon, Zamboanga, Surigao). A very characteristic species, represented by more than 30 collections. In forests at low and medium altitudes, ascending to 1,000 m. Endemic.

Tillandsia pseudo-ananas Blanco is possibly a synonym of this rather than a Freycinetia, see page 15.

Local names: Baléo (Bis.); baléu ( $\mathrm{P}$. Bis.); balíku (C. Bis.); balói (Mbo.) ; bariéu (Bag.); baríu (Bik.); barói (Mbo.); lagutlut (Tag.); pandán (Tag., Ibn.) ; pangdán (Ilk., Pang.); patága (Ibn.) ; sirí (Ibn.). PANDANus CumingIANus Martelli in Philip. Journ. Sci. 3 (1908) Bot. 70, Webbia $4^{1}$ (1913) 11, t. 25, f. 13-15.

Negros, (Cuming. $s . n$. in herb. Mus. Brit.). I have seen only a photograph of the syncarps. The leaves are unknown. Endemic.

PANDANUS DECIPIENS Martelli in Webbia $4^{1}$ (1913) $12,4^{2}$ (1914) 402 , t. $37, f .7-9$.

Palawan, Elmer 12593, Merrill 9246. In thickets and forests at low altitudes, often back of the mangrove swamps. Endemic.

Local name: Olañgó (Tagb.). 
PANDANUS DINAGATENSIS Merr, in Philip. Journ. Sci. 17 (1920) 240.

Dinagat, B. S. 35183 Ramos \& Pascasio. In forests along streams at low altitudes. Endemic.

PANDANUS DUBIUS Spreng. Syst. 3 (1826) 897; Miq. Fl. Ind. Bat. 3 (1855) 159; Naves Novis. App. (1882) 285; Warb. in Engl. Pflanzenreich 3 (1900) 50; Merr. in Govt. Lab. Publ. (Philip.) 17 (1904) 8; Martelli in Philip. Journ. Sci. 3 (1908) Bot.67, Leafl. Philip. Bot. 3 (1911) 1122, Webbia $4^{1}$ (1913) 12.

? Pandanus latifolius Perr. in Mém. Soc. Linn. Paris 3 (1824) 134; Martelli in Philip. Journ. Sci. 3 (1908) Bot. 72; C. B. Rob. op. cit. 306; Warb. in Engl. Pflanzenreich 3 (1900) 88.

Mindanao (Davao), Copeland 613. In thickets or forests along the seashore; Java, Borneo, Celebes, Moluccas, New Guinea to the New Hebrides, Caroline, and Marianne Islands.

Local names: Bákong (P. Bis.); báuang (P. Bis.) ; taboán (Bik.).

PANDANUS ESCULENTUS Martelli in Webbia $4^{1}$ (1913) $13,4^{2}$ (1914) $403, t .25, f .1-3$.

Luzon (Cagayan, Apayao, Ilocos Norte, Bulacan), F. B. 17813, 17817 Curran, 14009 Merritt \& Darling, B. S. 28352 Fénix, 34089 Ramos \& Edaño, 22208 Ramos. In forests at low and medium altitudes, ascending to $1,000 \mathrm{~m}$. Endemic.

Local name: Lígit (Ibn.).

PANDANUS EXALTATÚS Blanco Fl. Filip. (1837) 778, ed. 2 (1845) 536, ed. 3 , 3 (1879) 183; Miq. Fl. Ind. Bat. 3 (1856) 163; Warb. in Engl. Pflanzenreich 3 (1900) 84; Martelli in Philip. Journ. Sci. 3 (1908) Bot. 64, Webbia $4^{1}$ (1913) 13, t. 10, f. 1-3, 9-11; Merr. Sp. Blancoanae (1918) 54.

Pandanus arayatensis Merr. in Govt. Lab. Publ. (Philip.) 17 (1904) 7, t. 3, Philip. Journ. Sci. 1 (1906) Suppl. 25; Martelli in Webbia $4^{1}$ (1913) 6.

Pandanus banahaensis Elm. Leafl. Philip. Bot. 1 (1906) 79; Martelli in Webbia $4^{1}$ (1913) 7 (banahaoensis).

Pandanus vidalii Martelli in Philip. Journ. Sci. 3 (1908) Bot. 65, Webbia $4^{1}$ (1913) 36, t. 8, f. 11-13.

Pandanus fascicularis Naves Novis. App. (1882) 284, non Lam.

Luzon (Apayao, Benguet, Nueva Vizcaya, Pampanga, Rizal, Bataan, Tayabas), Catanduanes, Whitford 1325, Elmer 7574, 90\%4, Merrill 3832, Sp. Blancoanae 4.21, B. S. 30498 Ramos, 28256, 13546 Fénix, 11226 McGregor, 2701 Ahern's collector. In primary forests at medium altitudes, ascending to $800 \mathrm{~m}$. Endemic.

Local names: Alasás (Tag.); bambán (Ibn.); pandán (Tag., Pamp., Ibn.); pangdán (Ilk.).

Forma AHERNIANUS Martelli in Philip. Journ. Sci. 3 (1908) Bot. 64,

Webbia $4^{1}$ (1913) $13, t$ : $10, f .4-8,12-15$.

Luzon (Cagayan, Rizal, Pampanga), Merrill Dec. Philip. Forest Fl. 296, F. B. 17264, 17722 Curran. Habitat of the species from which it is scarcely distinguishable. Endemic. 
PANdAnus glauciphyllus $C^{\circ}$. B. Rob. in Bull. Torr. Bot. Club 35. (1908) 64, 74; Martelli in Philip. Journ. Sci. 3 (1908) Bot. 68, Leafl. Philip. Bot. 3 (1911) 1124, Webbia $4^{1}$ (1913) 15, t. 27, f. 14-16.

Negros, Romblon, Siargao, Mindanao (Zamboanga), Williams 2423, Copeland, F. B. 17372 Curran, B. S. 34855 Ramos \& Pascasio. In forests at low altitudes. Endemic.

Local ǹame: Pandán ( $\mathrm{P}$. Bis.).

PANDANUS GRACILIS Blanco Fl. Filip. (1837) 778, ed. 2 (1845) 536, ed. 3, 3 (1879) 182, t. 446; Warb. in Engl. Pflanzenreich 3 (1900) 84; Merr. in Govt. Lab. Publ. (Philip.) 27 (1905) 89, Philip. Journ. Sci. 2 (1907) Bot. 436, Sp. Blancoanae (1918) อ̄6; Martelli in Philip. Journ. Sci. 3 (1908) Bot. 68, Webbia $4^{1}$. (1913) 16, t. 25, f. 16-24, 25-26.

Pandanus whitfordii Merr. in Govt. Lab. Publ. (Philip.) 17 (1904) 8, Philip. Journ. Sci. 1 (1906) Suppl. 25.

Luzon (Nueva Vizcaya, Zambales, Bataan, Rizal, Laguna, Tayabas, Camarines), Mindoro, Mindanao (Bukidnon, Davao), Whitford 351, Merrill 7612, Sp. Blancoanae 890, 896, B. S. 23860 , 22469, 39493 Ramos, 15783 Fénix, 34844 Ramos \& Pascasio, F. B. 20188 Aguilar, 2944 Borden, 6003. Curran. In primary forests at medium altitudes, ascending to $1,000 \mathrm{~m}$. Endemic.

Local names: Ayasảs (Tag.); lagúloi (Buk.); pandán (Tag.).

PANDANUS JULIFERUS Martelli in Webbia $4^{1}$ (1913) $18,4^{2}$ (1914) $428, t .43, f .10-12$.

Camiguin de Misamis, B. S. 14670, 14671 Ramos. Probably in forests, altitude about $1,000 \mathrm{~m}$. Endemic.

This seems to be very near Pandanus polyglossus Martelli.

PANDANUS LATERAlIS Martelli in Philip. Journ. Sci. 3 (1908) Bot. 68, Leafl. Philip. Bot. 3 (1911) 1125, Webbia $4^{1}$ (1913) 20, t. 27, f. \$1-33.

Mindanao (Davao), Copeland 336, Elmer 11029. In forests at low or medium altitudes. Endemic.

Local names: Bakúling (Bag.) ; pandán (C. Bis., Sul.).

PANDANUS LINNAEI Gaudich. forma PHILIPPINENSIS Martelli in Webbia $4^{1}$ (1913) 21, t. 6, f. 1-2, Philip. Journ. Sci. 3 (1908) Bot. 60 (as $P$. linnaei Gaudich.).

Pandanus exaltatus Merr. in Philip. Journ. Sci. 2 (1907) Bot. 436, non Blanco.

Semirara, Busuanga, Merrill 4140, B. S. 41264 Ramos. In thickets at low altitudes. The species in Celebes, Key, and Aru Islands.

PANDANUS LOHERIANUS Martelli in Webbia $4^{1}$ (1913) $22,4^{2}$ (1914) $423, t .27, f .17-21$.

Luzon (Rizal, Laguna), B. S. 20549 Ramos, 13192 Curran. In forests along streams at low or medium altitudes. Endemic.

PANdANus Luzonensis Merr. in Govt. Lab. Publ. (Philip.) 17 (1904) 6, t. 1, 2, Philip. Journ. Sci. 1 (1906) Suppl. 25; Martelli op. cit. 3 (1908) Bot. 67, Webbia $4^{1}$ (1913) 22, t. 15, f. 5-9.

Pandanus calicarpus Martelli in Webbia 1 (1905) $365,4^{1}$ (1913) 9. 
Luzon (Pangasinan, Zambales, Bulacan, Rizal, Bataan, Laguna). Chiefly in secondary forests at low altitudes; represented by numerous collections, Merrill Phil. Pl. 1931 and 759 are typical. Endemic.

Local names: Alasás (Tag.); dasá (Tag.); pandán (Tag., Ilk., Ibn.).

PANDANUS MAPOLA Martelli in Leafl. Philip. Bot. 3 (1911) 1124, Webbia $4^{1}$ (1913) $22, t .41, f .14-1 \%$.

Sibuyan, Elmer 12139. In thickets or forests at low altitudes; the local name cited, mapulá, merely means red in reference to the color of the fruits. Endemic.

PANDANUS MARTELLII Elm. Leafl. Philip. Bot. 1 (1908) 272; Martelli in Webbia $4^{1}$ (1913) $23, t .11, f .4-7$.

Luzon (Tayabas), Elmer 9082, 9083, 17646, B. S. 23025 McGregor, 23829 Ramos. In forests, altitude about $650 \mathrm{~m}$. Endemic.

PANDANUS MERRILlII Warb. in Perk. Frag. Fl. Philip. (1904) 50; Martelli in Philip. Journ. Sci. 3 (1908) Bot. 71, Webbia $4^{1}$ (1913) $23, t$. 32, f. 35-3\%.

Palawan, Merrill 840, 7250, 9274, 9.375, B. S. 232 Bermejos. In primary forests, sea level to $500 \mathrm{~m}$ altitude. Endemic.

Local name: Pandán (Tagb.).

PANDANUS MINDANAENSIS Martelli in Elm. Leafl. Philip. Bot. 3 (1911) 1121, Webbia $4^{1}$ (1913) 24, t. 36, f. 4-7 (mindanaoensis).

Mindanao (Davao), Elmer 10823. In primary forests, altitude about $1,200 \mathrm{~m}$. Endemic.

PANDANUS MULTIBRACTEATUS Merr. in Philip. Journ. Sci. 17 (1920) 241.

Mindanao (Surigao), B. S. 34571, 34819 Ramos \& Pascasio. In forests along streams, altitude about $650 \mathrm{~m}$. Endemic.

PANDANUS Occultus Merr. in Philip. Journ. Sci. 13 (1918) Bot. 265.

Palawan, Merrill 9361. In primary swamp forests at low altitudes. Endemic.

PANDANUS PALLIDUS Merr. in Govt. Lab. Publ. (Philip.) 29 (1905) 5; Martelli in Philip. Journ. Sci. 3 (1908) Bot. 68, Webbia $4^{1}$ (1913) 26, t. 27, f. $28-30$.

Luzon (Benguet, Nueva Ecija), Elmer 5840, Williams 917, B. S. 26270 Ramos \& Edaño, F. B. 5106, 5069 Curran. In forested stream depressions up to $1, \check{0} 00 \mathrm{~m}$ altitude. Endemic.

Local names: Pangchán (Ig.); tachiáng (Ig.).

PANDANUS PALOENSIS Elm. Leafl. Philip. Bot. 1 (1906) 75; Martelli in Philip. Journ. Sci. 3 (1908) Bot. 68, Leafl. Philip. Bot. 3 (1911) 1123, Webbia $4^{1}$ (1913) 26, t. 27, f. 22-27.

Luzon (Cagayan, Apayao, Camarines, Sorsogon), Leyte, Mindanao (Davao), Elmer 7343, 14858, 11224, Wenzel 1304, Weber 1568, B. S. 33556 Ramos \& Edaño, 28072 Fénix, 13921 Ramos, F. B. 19569 Curran. In forests at low altitudes. Endemic.

Local name: Baróai-latong (Bag.). 
PANDANUS PANAYENSIS Merr. in Philip. Journ. Sci. 14 (1919) 366.

Panay, B. S. 30796 Ramos \& Edaño, 35740 Martelino \& Edaño. In primary forests at low or medium altitudes. Endemic.

PANDANUS PHILIPPINENSIS Merr. in Philip. Journ. Sci. 13 (1918) Bot. 264.

Luzon (Kalinga, Nueva Vizcaya), B. S. 11289 McGregor, 37495 Ramos \& Edaño. On forested slopes at medium altitudes. Endemic.

PANDANUS POLYGLossus Martelli in Leafl. Philip. Bot. 3 (1911) 1130, Webbia $4^{1}$ (1913) 28 , t. 39, f. 16-18.

Mindanao (Davao), Elmer 11335. In primary forests, altitude about $1,350 \mathrm{~m}$. Endemic.

PANDANUS RADICANS Blanco Fl. Filip. (1837) 780, ed. 2 (1845) 537, ed. 3, 3 (1879) 184; Miq. Fl. Ind. Bat. 3 (1856) 166; Warb. in Engl. -Pflanzenreich 3 (1900) 85; Merr. in Govt. Lab. Publ. (Philip.) 27 (1905) 89, Philip. Journ. Sci. 2 (1907) Bot. 436, Sp. Blancoanae (1918) 54; Elm. Leafl. Philip. Bot. 1 (1906) 74; Martelli in Philip. Journ. Sci. 3 (1908) Bot. 66, Webbia $4^{1}$ (1913) 29, t. 6, f. 8-10, Pandanus olango Blanco ex Espejo \& Garcia Suppl. Cat. Seem. Hort. Bot. Manila (1876) 14, nomen nudum.

Luzon (Sorsogon), Leyte, Elmer 7200, 14892. In forests at low altitudes. Endemic.

Local names: Olañó (S. L. Bis.); ouañgó (P. Bis.); oyañgó (Bik.); uañgó (P. Bis.) ; uyañgó (Mbo.).

PANDANUS RAMOSII Merr. in Philip. Journ. Sci. 17 (1920) 242.

Bucas Grande, Dinagat, B. S. 35136 , 35198 Ramos \& Pascasio. In dry forests at low altitudes. Endemic.

PANDANUS RECLINATUS Martelli in Webbia $4^{1}$ (1913) $29,4^{2}$ (1914) $432, t .27, f .3,4$.

Palawan, Elmer 12731. In forests at low altitudes. Endemic.

PANDANUS SABOTAN Blanco Fl. Filip. (1837) 779, ed. 2 (1845) 537, ed. 3, 3 (1878) 184; Miq. Fl. Ind. Bat. 3 (1856) 166; Warb. in Engl. Pflanzenreich 3 (1900) 84; Merr. in Govt. Lab. Publ. (Philip.) 27 (1905) 89, Sp. Blancoanae (1918) 56; Martelli in Philip. Journ. Sci. 3 (1908) Bot. 72, Webbia $4^{3}$ (1913) 30.

Luzon (Laguna, Rizal), Merrill Sp. Blancoanae 185, F. B. 10099 Curran, B. S. 6532, 11934 Robinson, 11239 McGregor. A planted pandan of which the fruits and flowers are unknown. The Filipinos state that it never produces fruit. It is perhaps but a form of $P$. tectorius Solander, and Martelli has so identified some of the specimens cited above. Endemic?

Local names: Parauan (Gad.); sabotán (Tag.); sibután (Tag.).

PANDANUS SIBUYANENSIS Martelli in Leafl. Philip. Bot. 3 (1911) 1120,

Webbia $4^{1}$ (1913) 31, t. 84, f. 4-6 (sibujanensis).

Sibuyan, Elmer 12146. In forests, altitude about $350 \mathrm{~m}$. Endemic.

Local name: Pandan-bugás (P. Bis.).

PANDANUS SIMPLEX Merr. in Govt. Lab. Publ. (Philip.) 29 (1905) 6; Martelli in Philip. Journ. Sci. 3 (1908) Bot. 67, Webbia $4^{1}$ (1913) $31, t .14, f .11-14$.

Pandanus utilissimus Elm. Leafl. Philip. Bot. 1 (1906) 80; Martelli 
in Philip. Journ. Sci. 3 (1908) Bot. 67, Webbia $4^{1}$ (1913) $36, t .14$, f. 3-5; C. B. Rob. in Philip: Journ. Sci. 6 (1911) Bot. 193.

Luzon (Ifugao, Laguna, Tayabas, Sorsogon), Catanduanes, Elmer 7379 , 16366, Whitford 782, F. B. 20166 Aguilar, 8507 Curran, B. S. 20033 McGregor, 6542 Robinson. In forests at low and medium altitudes; also planted. A species remarkable for its enormous syncarps. Endemic.

Local names: Bankoang (Tag.) ; ilohan (Tag.); kalagímai (Tag.) ; karagómoi (Bik.) ; karagúmoi (Bik., Bis., Tag.) ; pandán (Tag.) ; pandantotóo (Tag.).

PANDANUS SUBACAULIS Merr. in Philip. Journ. Sci. 13 (1918) Bot. 263.

Catanduanes, $B$. S. 30489 Ramos. In forests at low or medium altitudes. Endemic.

PANDANUS TECTORIUS Solander in Parkins. Journ. Voy. H. M. S. Endeav. (1773) 46; Warb. in Engl. Pflanzenreich 3 (1900) 46; Perk. Frag. Fl. Philip. (1904) 50; Merr. in Philip. Journ. Sci. 1 (1906) Suppl. 25, 3 (1908) Bot. 394, Fl. Manila (1912) 66, Sp. Blancoanae (1918) 55; Martelli in Philip. Journ. Sci. 3 (1908) Bot. 61, Leafl. Philip. Bot. 3 (1911) 118, Webbia $4^{x}$ (1913) 33.

Pandanus odoratissimus Linn. f. Suppl. (1781)' 424; Vidal Sinopsis Atlas (1883) 42, t. 95, f. A; F.-Vill. Novis. App. (1882) 284.

Pandanus malatensis Blanco Fl. Filip. (1837) 777, ed. 2 (1845) 535, ed. 3, 3 (1879) 181; Warb. in Engl. Pflanzenreich 3 (1900) 84; Merr. in Govt. Lab. Publ. (Philip.) 27 (1905) 89; ? Martelli in Philip. Journ. Sci. 3 (1908) Bot. 70, Webbia $4^{1}$ (1913) 22, t. 26, f. 22.

Pandanus spiralis Blanco Fl. Filip. (1837) 777, ed. 2 (1845) 535, ed. 3, 3 (1879) 181; Merr. in Govt. Lab. Publ. (Philip.) 27 (1905) 89, non $\mathrm{R}$. Br.

Pandanus blancoi Kunth Enum. 3 (1841) 583.

Pandanus tectorius Soland. var. surigaenisis Martelli in Philip. Journ. Sci. 3 (1908) Bot. 64, Webbia $4^{1}$ (1913) 34, t. 8, f. 3-7 (suringousis).

Pandanus tectorius Soland. var. sinensis Warb. in Engl. Pflanzenreich 3 (1900) 48; Martelli in Philip. Journ. Sci. 3 (1908) Bot. 62, Webbia $4^{1}$ (1913) $34, t .9, f .2-10$.

Pandanus tectorius Soland. var. spiralis Martelli in Philip. Journ. Sci. 3 (1908) Bot. 62, Webbia $4^{1}$ (1913) 34, t. 9, f. 1-7, t. 12, f. \&.

Pandanus tectorius Soland. var douglasii Martelli forma philippinensis Martelli in Philip. Journ. Sci. 3 (1908) Bot. 63, Webbia $4^{1}$ (1913) $33, t .8, f .8-10$.

Pandanus vidalii Martelli in Philip. Journ. Sci. 3 (1908) Bot. 65, Webbia $4^{1}$ (1913) 36, p. p., quoad syn. Vidal.

Pandanus coronatus Martelli in Philip. Journ. Sci. 3 (1908) Bot. 65 (incl. f. minor), Webbia $4^{1}$ (1913) 11, t. 4, f. 8-13.

Along the seashore throughout the Philippines, abundant and forming characteristic thickets back of the beach. Indo-Malayan and Polynesian regions generally, extending to southern China and tropical Australia.

Local names: Bároi (Bik.); laha (Sul.) ; ohañgó (Iv.) ; padán (Iv.); pandán (Tag., P. Bis., C. Bis., Sub.) ; pandan-dágat (Tag.) ; pangdán (Ilk., Pang.) ; panglán (Ilk., Sbl.) ; panhakad (Bis.) ; uhañó (Iv.). 
PANDANUS TENUIPEdUnCulatus Merr. in Philip. Journ. Sci. 17 (1920) 243.

Siargao, Mindanao (Surigao), B. S. 34739, 34842 Ramos \& Pascasio. In open forests at low altitudes. Endemic.

PANDANUS ZAMBOANGENSIS Martelli in Webbia $4^{1}$ (1913) $37,4^{2}$ (1914) 426, t. 4.2, f. $7-10$.

Mindanao (Zamboanga, Bukidnon, Lanao), Merrill 8051, 8065, F. B. 9494 Whitford \& Hutchinson, B. S. 15696 Fénix, 3\%428, 374.29 Ramos \& Edaño. In thickets and secondary forests at low altitudes. Endemic.

Local names: Balíu (Sub.); baríu (Sul.); pandán (Sul.).

DOUBTFUL AND EXCLUDED SPECIES

Pandanus Inermis Blanco Fl. Filip. ed. 2 (1845) 537, ed. 3, 3 (1879) 184, non Roxb.

This is unquestionably a Pleomele (Dracaena) and is referable to Pleomele angustifolia (Roxb.) N. E. Br.

PANDANUS KAFU Martelli in Webbia $4^{1}$ (1913) 19, $t .19, f .1-3$.

"Is. Filippine" is an error, the type being from Guam, Marianne Islands.

Pandanus PoRteanus Lescuy. ex Hérincq Hort. Franc. 1 (1866) 16; Warb. in Engl. Pflanzenreich 3 (1900) 90; Martelli in Webbia $4^{1}$ (1913) 28.

A nomen nudum based on a cultivated plant of Philippine origin.

Pandanus bagea Miq., $P$. caricosus Rumph., $P$. ceramicus Rumph., $P$. drupaceus Thouars., $P$. elegans Thouars., $P$. furcatus Roxb., $P$. humilis Rumph., $P$. latifolius Rumph., $P$. littoralis Jungh., $P$. montanus Miq., $P$. moschatus Rumph., $P$. repens Rumph., $P$. spurius Rumph., and $P$. sylvestris Rumph.

The above fourteen species, several of them of entirely doubtful status, were credited to the Philippines by Naves, Novis. App. (1882) 284, 285. With the exception of Pandanus caricosus Rumph. = Scirpiodendron ghaeri (Gaertn.) Merr. none of them are known to occur in the Philippines.

\section{SARARANGA Hemsley}

SARARANGA PHILIPPINENSIS Merr. in Govt. Lab. Publ. (Philip.) 29 (1905) 5; Elm. Leafl. Philip. Bot. 3 (1911) 1110.

Luzon (Tayabas, Camarines, Albay, Sorsogon), Sibuyan, Samar, Dinagat, Mindanao (Surigao, Bukidnon), Merrill 5230, Whitford 749, Elmer 17206, B. S. 6401 Robinson, 35181, 34680 Ramos \& Pascasio, 33710 Ramos \& Edaño, F. B. 26530 Rola. Chiefly in secondary forests, sea level to an altitude of $750 \mathrm{~m}$. Endemic.

A genus represented by one other known species in the Solomon Islands.

Local names: Bagáas (P. Bis.) balúi (Buk.); balur (Buk.); nagsalausáu (Bik.).

\section{POTAMOGETONACEAE}

1. POTAMOGETON Linnaeus

POTAMOGETON ANGUSTIFOLIUS Berchtold \& Presl Rostlinar 2 (1821) 19; A. Benn. in Philip. Journ. Sci. 9 (1914) Bot. 340. 
Luzon (Benguet), Loher 1597.' In shallow water, altitude about 1,400 m. Europe, Asia, and North America.

POTAMOGETON MAACKIANUS A. Benn. in Journ. Bot. 42 (1904) 74, Philip. Journ. Sci. 9 (1914) Bot. 342.

Mindanao (Lanao), Clemens 216. In shallow water of Lake Lanao, altitude about $650 \mathrm{~m}$. Northern Asia, Korea, China, Japan, and Formosa.

POTAMOGETON MALAINUS Miq. Ill. Fl. Archip. Ind. (1871) 46 (malaina) ; Aschers. \& Graebn. in Engl. Pflanzenreich 31 (1907) 83; A. Benn. in Philip. Journ. Sci. 9 (1914) Bot. 341; Merr. Fl. Manila (1912) 67.

Potamogeton mucronatus Presl Epim. (1851) 245; Walp. Ann. 3 (1852-53) 504, non Schrad.

Potamogeton lucens Naves Novis. App. (1882) 297; Vidal Phan. Cuming. Philip. (1885) 155, Rev. Pl. Vasc. Filip. (1886) 282, non Linn.

Luzon (Rizal, Laguna, Sorsogon), Mindanao (Lanao), Merrill 5107, Williams 2041, Elmer 18113, B. S. 22797 McGregor, 11892 Robinson \& Ramos. In shallow water of lakes at low and medium altitudes. India to Korea and Japan southward to Borneo, Java, and Celebes. The Philippine form has been somewhat confused with Potamogeton gaudichaudii C. \& S. which is a form of $P$. lucens Linn.

Local name: Damúng-kulút (Tag.).

POTAMOgETON PERVERSUS A. Benn. in Philip. Journ. Sci. 9 (1914) Bot. 343 .

Luzon (Bontoc), Vanoverbergh 249, 2684. In shallow slow streams and in rice paddies, altitudes about $1,300 \mathrm{~m}$. India to China, Manchuria, Korea, and Formosa.

Local name: Íbas (Bon.).

POTAMOGETON PHILIPPINENSIS A. Benn. in Philip. Journ. Sci. 9 (1914) Bot. 342, hybr. $P$. malainus $\times P$. maackianus.

Potamogeton nipponicus Ostenfeld in Philip. Journ. Sci. 9 (1914)

Bot. 260, non Makino.

Mindanao (Lanao), Clemens. In shallow water of Lake Lanao, altitude about $650 \mathrm{~m}$. Endemic.

POTAMOGETON PUSILLUS Linn. Sp. Pl. (1753) 127; A. Benn. in Philip. Journ. Sci. 9 (1914) Bot. 342.

Luzon (Benguet), Elmer 5951. In shallow water, altitude about 1,300 m. Widely distributed in Europe, Asia, Africa, and North America.

POTAMOGETON TEPPERI A. Benn. in Journ. Bot. 25 (1887) 178; Philip.

Journ. Sci. 9 (1914) Bot. 340.

Luzon (Benguet), Merrill Phil. Pl. 1722, Elmer 5952, Topping 45, B. S. 14110 Robinson. In shallow water, altitude about $1,300 \mathrm{~m}$. Yunnan and Australia.

\section{EXCLUDED SPECIES}

Potamogeton CRispus Linn.; Naves Novis. App. (1882) 297.

Potamogeton Natans Linn.; Naves 1. c.

Potamogeton Javanicus Linn.; Naves 1. c. 


\section{RUPPIA Linnaeus}

RUPPIA MARITIMA Linn. Sp. Pl. (1753) 127; Naves Novis. App. (1882) 297; Aschers. \& Graebn. in Engl. Pflanzenreich 31 (1907) 142; Merr. in Philip. Journ. Sci. 7 (1912) Bot. 228, Fl. Manila (1912) 68.

Luzon (Manila Bay), Panay, Merrill Phil. Pl. 799, Loher 1594, B. S. 20660 Serviñas. In shallow, brackish water. In salt or brackish water in most warm and tropical regions; the Philippine form is referable to the var. rostrata Agardh. (R. maritima Linn. subsp. rostellata Koch).

Local names: Buñgut-búñ̃ut (P. Bis.); damong-pálai (Tag.).

\section{CYMODOCEA Koenig}

CYMODOCEA ROTUNDATA (Ehrb. \& Hempr.) Aschers. \& Schweinf. in Sitzber. Ges. Naturf. Freunde Berlin (1870) 84; Graebner in Engl. Pflanzenreich 31 (1907) 147; Merr. in Philip. Journ. Sci. .10 (1915) Bot. 2.

Phucagrostis rotundata Ehrb. \& Hempr. ex Aschers. in Boiss. Fl. Orient. 5 (1884) 21.

Palawan, Cavilli, Mindanao, Merrill 7180a, Phil. Pl 1264, B. S. 15559 Fénix. On sand about coral reefs in shallow water. In shallow seas from the Red Sea to Australia.

CYMOdOCEA SERRULATA (R. Br.) Aschers. \& Magnus in Sitzber. Ges.

Naturf. Freunde Berlin (1870) 84; Aschers. \& Graebn. in Engl.

Pflanzenreich 31 (1907) 147.

Caulinia serrulata R. Br. Prodr. (1810) 339.

Mindanao, fide Ascherson \& Graebner, 1. c. In shallow seas from the Red Sea and western Africa to Australia.

Cymodocea aequorea Koenig $=C$. nodosa (Ucria) Aschers.! was erroneously credited to the Philippines by Naves Novis. App. (1882) 297.

\section{DIPLANTHERA Thouars}

DIPLANTHERA UNINERVIS (Forst.) Aschers. in Engl. \& Prantl Nat. Pflanzenfam. $2^{1}$ (1897) 37; Graebn. in Engl. Pflanzenreich 31 (1907) 152; Merr. in Philip. Journ. Sci. 10 (1915) Bot. 3.

Zostera uninervis Forsk. Fl. Aeg.-Arab. (1775) 159.

Luzon (Manila Bay), Palawan, Cavilli, Merrill 7179, 9223, Phil. Pl. 1263, Shaw 1148. In sand about reefs. In shallow seas from the Red Sea and western Africa to Australia and Polynesia.

The family Aponogetonaceae has no known representatives in the Philippines; Aponogeton monostachyus Linn. f. and Ouvirandra fenestralis Poir., credited to the Archipelago by Naves, Novis. App. (1882) 298, 299, must be excluded.

\section{NAJADACEAE}

\section{NAJAS Linnaeus}

NAJAS GRAMINEA Delile Fl. Aegypt. (1812) 282, t. 50, f. 3 ; Naves Novis. App. (1882) 298; Rendle in Trans. Linn. Soc. Bot. 5 (1899) 424, 443, t. 3.2, f. 192-201, Engl. Pflanzenreich 7 (1901) 18, f, 5, Q V; Merr. Fl. Manila (1912) 68.

Northern Luzon to Mindanao. In shallow stagnant fresh water, at low and medium altitudes; often common. Northern Africa to Japan southward to New Caledonia and Australia.

Local names: Aragán (Ilk.) ; bangbángi (Bon.) ; lábui (Ig.). 
NAJAS PALUSTRIS Blanco Fl. Filip. (1837) 660; Rendle in Trans. Linn. Soc. Bot. 5 (1899) 427, Engl. Pflanzenreich 7 (1901) 18; Merr. Sp. Blancoanae (1918) 57.

Najas lobata Blanco Fl. Filip. ed. 2 (1845) 459, ed. 3, 3 (1879) 65.

Najas foveolata A. Br. ex Magnus Beitr. (1870) 7; Rendle in Trans.

Linn. Soc. Bot. 5 (1899) 416, t. 31, f. 139-144, Engl. Pflanzenreich 7 (1901) 15.

Najas tenuifolia Naves Novis. App. (1880) 297, non R. Br.

Luzon (Union, Rizal, Laguna, Tayabas), Merrill 5109, Sp. Blancoanae 519, Elmer 14420, Whitford 837, B. S. 24079 Ramos. In shallow stagnant fresh water at low altitudes. India to Japan southward to Timor.

Local names: Aragán-tamnái (Ilk.); rigmáu (Tag.).

DOUBTFUL AND EXCLUDED SPECIES

NAJAS FAlciculata A. Br. in Journ. Bot. 2 (1864) 278, f. 4; Rendle in Trans. Linn. Soc. Bot. 5 (1899) 417, t. 32, f. 145-151, Engl. Pflanzenreich 7 (1901) 15.

Manila, Mertens fide A. Braun 1. c. The type is an Indian specimen and I strongly suspect that the Philippine specimen referred here by $\mathrm{A}$. Braun is Najas palustris Blanco.

NAJAS INDICA Cham.; Schauer in Nov. Act. Acad. Nat. Cur. 19 (1843) Suppl. 1: 425 .

The record was based on a specimen from Laguna de Bay, collected by Meyen. The species is known only from India.

\section{ALISMACEAE}

\section{SAGITTARIA Linnaeus}

SAGITTARIA SAGITTAEFOLIA Linn. Sp. Pl. (1753) 993; Naves Novis. App. (1882) 298; Micheli in DC. Monog. Phan, 3 (1881) 66; Buchenau in Engl. Pflanzenreich 16 (1903) 46; Merr. in Philip. Journ.

Sci. 2 (1907) Bot. 421, Sp. Blancoanae (1918) 58.

Alisma sagittifolium Llanos Frag. Pl. Filip. (1851) 69; F.-Vill. \&

Naves in Blanco Fl. Filip. ed. $3,4^{1}$ (1880) 51, non Willd.

Luzon (Pangasinan, Nueva Ecija, Tayabas, Camarines, Albay, Sorsogon), Catanduanes, Samar, Leyte, Mindanao (Lanao), Merrill Phil. Pl. 982, Elmer 14339, Wenzel 1228, 1521, Clemens 888, B. S. 30161 Ramos, 10703 McGregor. In fresh-water swamps, rice paddies, etc., at low and medium altitudes. Europe through Asia to Japan, southward to Java.

Local names: Gauai-gáuai (S. L. Bis.); tikóg (Bik.).

\section{EXCLUDED GENERA}

Limnophyton obstusifolium Miq.; Naves Novis. App. (1882) 298.

Lophtocarpus GUYANensis Micheli; Naves Novis. App. (1882) 298.

\section{HYDROCHARITACEAE}

\section{HALOPHILA Thouars}

HALOPHILA BECCARII Aschers. in Nuov. Giorn. Bot. Ital. 3 (1871) 302; Merr. in Philip. Journ. Sci. 7 (1912) Bot. 228, Fl. Manila (1912) 70.

Luzon (Rizal), Merrill 8045. In brackish fish ponds. Borneo. 
HALOPHILA OVAlis (R. Br.) Hook. f. Fl. Tasm. 2 (1860) 45; Naves

Novis. App. (1882) 298; Merr. in Philip. Journ. Sci. 10 (1915)

Bot. 2.

Caulinia ovalis R. Br. Prodr. (1810) 339.

Palawan, Bancoran, Biliran, Merrill 7155, Phil. Pl. 1223, B. S. 18755 McGregor. In sand on coral reefs. On sandy bottoms of shallow seas. India to Australia and Polynesia.

HALOPHILA OVATA Gaudich. Bot. Freycinet's Voy. (1826) 430, t. 40, f. 1; Ostenfeld in Philip. Journ. Sci. 4 (1909) Bot. 68; Merr. Fl. Manila (1912) 70.

Luzon (Manila Bay), Merrill 411:, Phil. Pl. 1098, B. S. 9899 Robinson. In shallow water on rather muddy bottom. Marianne Islands.

HALOPHILA SPINUlOSA (R. Br.) Aschers. in Neumayer Anleit. Wissensch. Beob. (1875) 368, ed. 2 (1888) 396; Benth. Fl. Austral. 7 (1878) 183; Merr. in Philip. Journ. Sci. 10 (1915) Bot. 2.

Caulinia spinulosa R. Br. Prodr. (1810) 339.

Luzon (Camarines), Mindanao, F. B. 12275 Curran. In shallow seas on reefs. In shallow seas, eastern and northern coasts of Australia; Java, and probably in other parts of Malaya.

\section{HYDRILLA L. C. Richard}

HYDRILLA VeRTICILLATA (Roxb.) Royle Ill. Bot. Himal. (1839) t. 376; Presl Bemerk. (1842) 112; Casp. in Pringsh. Bot. Jahrb. 1 (1858) 494; Naves Novis. App. (1880) 214; Merr. \& Rolfe in Philip. Journ. Sci. 3 (1908) Bot. 96; Merr. Fl. Manila (1912) 69, Sp. Blancoanae (1918) 58.

Serpicula verticillata Linn. f. Suppl. (1781) 416; Roxb. Pl. Coromandel 2 (1798) t. 164.

Udora verticillata Spreng. Syst. 1 (1825) 170 ; Llanos Frag. Pl. Filip. (1851) 101; F.-Vill. \& Naves in Blanco Fl. Filip. ed. 3, $4^{1}$ (1880) 78.

Northern Luzon to Mindanao, in most or all islands and provinces. In shallow fresh water at low and medium altitudes; often abundant. Central Europe to Asia southward to Australia.

Local names: Gínga (Ibn.) ; ináta (Tag.) ; lomotlomótan (Tag.); lúsai (Mag.).

\section{VALLISNERIA Linnaeus}

VALLISNERIA GIGANTEA Graebn. in Engl. Bot. Jahrib. 49 (1912) 68; Merr. Fl. Manila (1912) 69; Hallier f. Nov. Guinea 8 (1913) 915. Vallisneria spiralis Blanco Fl. Filip. (1837) 781, ed. 2 (1845) 538, ed. 3, 3 (1879) 187; Naves Novis. App. (1880) 214; Vidal Phan. Cuming. Philip. (1885) 148, non Linn.

Luzon (Pangasinan, Bulacan, Nueva Ecija, Laguna, Rizal, Camarines). In lakes and slow streams at low altitudes. Enormously variable in size, apparently depending largely on the depth of the water in which it grows. New Guinea, New South Wales.

Local names: Baláiba (Ilk.) ; lanténg (Ibn.) ; mariu-báriu (Bik.) ; paláipa (Ilk.) sabotán-buáia (Tag.); sintas-sintásan (Tag., from Sp. cinta = ribbon). 


\section{BLYXA Thouars}

BLYXA OCTANDRA (Roxb.) Planch. ex Thwaites Enum. Pl. Ceyl. (1859-64) 332; Merr. in Philip. Journ. Sci. 7 (1912) Bot. 73.

Vallisneria octandra Roxb. Pl. Coromand. 2 (1798) 34, t. 165.

Blyxa roxburghii Rich. in Mém. Inst. France (1811) 77, t. 5; Naves Novis. App. (1880) 214.

Luzon (Bontoc), Palawan, Merrill Phil. Pl. 1244, Vanoverbergh 664. In shallow lakes, rice paddies, etc., from sea level to $1,250 \mathrm{~m}$ altitude; apparently very local. Tropical Asia to Malaya, Australia, and the Caroline Islands.

Local name: Pangpangdán (Bon.).

\section{ENHALUS L. C. Richard}

ENHALUS ACOROIdes (Linn. f.) L. C. Rich. ex Steud. Nomencl. ed. 2, 1 (1840) 554; Chatin Anat. Pl. Aquat. (1862) 15, t. 6; Merr. Interpret. Herb. Amb. (1917) 84, Sp. Blancoanae (1918) ว9. Stratiotes acoroides Linn. f. Suppl. (1781) 268.

Enhalus koenigii L. C. Rich. in Mém. Inst. France 2 (1811) 78; Naves Novis. App. (1880) 214.

Vallisneria sphaerocarpa Blanco Fl. Filip. (1837) 780, ed. 2 (1845) 538, ed. 3, 3 (1879) 186.

Northern Luzon to Palawan and Mindanao, Merrill 8070, Sp. Blancoanae 383, B. S. 18723 MeGregor, 13001, 15558 Fénix. In shallow water of sheltered bays along the seashore; of general occurrence where favorable habitats are found. Ceylon through Malaya to tropical Australia.

Local names: Iaai (Tag.); lamón (Tag., Bik.); mariu-báriu (Bik.); paláipat-baibái (Ilk.) ; bariau-bariau (Kuy.).

\section{THALASSIA Banks}

THALASSIA HEMPRICHII (Ehrenb.) Aschers. in Engl. \& Prantl Nat. Pflanzenfam. $2^{1}$ (1889) 254; Merr. Fl. Manila (1912) 71, Philip. Journ. Sci. 10 (1915) Bot. 2.

Schizotheca hemprichii Ehrenb. in App. Akad. Berl. 1 (1832) 429.

Luzon (Manila Bay), Alabat, Palawan, Cavilli. In tide pools, about coral reefs, etc., probably of general Philippine distribution. In shallow seas, sandy bottom, India to Polynesia.

\section{BOOTTIA Wallich}

BoOtTIA RENIFOlIA Merr. in Philip. Journ. Sci. 4 (1909) Bot. 247.

Luzon (Camarines), Mindanao (Cotabato), F. B. 19274 Curran, B. S. 11593 Robinson. In swamps and slow streams at low altitudes. Endemic.

Local names: Butid-na-ubal (Mag.); gabi-gábi (Bik.).

Boottia cordata Wall., reported from the Philippines by Naves, Novis. App. (1880) 213, was unquestionably admitted on the basis of an erroneous identification.

\section{OTTELIA Persoon}

OTTElIA ALISMoIdes (Linn.) Pers. Syn. 1 (1805) 400; Blanco Fi. Filip. (1837) 461, ed. 2 (1845) 321, ed. 3, 2 (1878) 230; Naves Novis. App. (1880) 213; Vidal Phan. Cuming. Philip. (1885) 148; Merr. Fl. Manila (1912) 70, Sp. Blancoanae (1918) 59.

Stratiotes alismoides Linn. Sp. Pl. (1753) 535. 
Damasonium lancifolium Presl Rel. Haenk. 1 (1827) 89.

Ottelia ensiformis Blanco Fl. Filip. (1837) 460, ed. 2 (1845) 320, ed.

3, 2 (1878) 229; Naves Novis. App. (1880) 214.

Ottelia philippinensis Ostenfeld in Philip. Journ. Sci. 9 (1914) Bot. 259.

Northern Luzon to Mindanao, in most islands and provinces. In shallow fresh water of lakes, pools, and slow streams. India through Malaya to tropical Australia.

A species presenting great variation in its vegetative characters, which largely depends on the depth of the water in which it grows, whether stagnant or not, and whether the plants are crowded or scattered. Two additional synonyms are Ottelia javanica Miq. and $O$. ovalifolia L. C. Rich., credited to the Philippines by Naves, Novis. App. (1880) 214, as distinct species.

Local names: Damong-ilálim (Tag.); espáda (Sp.) ; kalabúa (Tag.) ; lantíng (Bik.); lantén-sápa (Tag.) ; maraduyúk (Ib.); maradúyug (It.); tarabáng (Ilk.).

\section{EXCLUDED GENERA}

HYDROCHARIS MoRsus-RANAE Linn.; Naves Novis. App. (1880) 213.

Nechamandra RoxbUrhir Planch.; Naves Novis. App. (1880) $215=$ Lagarosiphon roxburghii Planch.

TRIURIDACEAE

\section{SCIAPHILA Blume}

SCIAPHILA CLEMENSAE Hemsl. in Hook. Ic. IV 9 (1907) t. 2850, f. 7-14; Fedde Repert. 5 (1908) 265.

Mindanao (Lanao), Clemens 775. In damp primary forests at low altitudes. Borneo.

This species seems to be closely allied to S. tenella Blume. Dr. J. J. Smith states that B. S. 6347 Robinson, F. B. 9350, 9449 Whitford \& Hut-1 chinson, and Clemens 775 p. p. are very similar to Blume's species.

SCIAPHILA MACUlATA Miers in Trans. Linn. Soc. Bot. 21 (1852) 48,

t. 6, f. 19-36; Vidal Phan. Cuming. Philip. (1885) 154, Rev. Pl. Vasc. Filip. (1886) 282.

Luzon (Bataan), Mindanao (Butuan, Zamboanga), F. B. 5537 Curran, 9126 Whitford \& Hutchinson, Weber 1462. In damp primary forests at low and medium altitudes. Endemic.

In addition to these two species what may be Sciaphila tenella Blume occurs in our collections, as noted above, as well as fragmentary material representing two other distinct species.

GRAMINEAE

\section{ZEA Linnaeus}

ZEA MAYS Linn. Sp. Pl. (1753) 971; Blanco Fl. Filip. (1837) 686, ed. 2 (1845) 476, ed. 3, 3 (1879) 90, t. 279; F.-Vill. Novis. App. (1882)

314; Usteri Beitr. Ken. Philip. Veg. (1905) 133; Merr. in Philip. Journ. Sci. 1 (1906) Suppl. 320, Fl. Manila (1912) 74, Sp. Blancoanae (1918) 59.

Extensively cultivated in most parts of the Philippines, in some islands and provinces a staple article of food. Introduced from America. 
Local names: Gahilang (If.) ; igi. (Bon.) ; maís (Sp.) ; maít (It.) ; mañgí (Ibn.); tigi (Bon.).

Teosinte, Euchlaena luxurians Schrad., a native of Mexico, was introduced into the Philippines in the late years of the Spanish occupation and again in the early years of the American occupation and was cultivated experimentally for forage purposes. It has not persisted in cultivation.

\section{POLYTOCA R. Brown}

POLYTOCA HETEROCLITA (Roxb.) Koord. in Exksfl. Java 1 (1911) 99; Merr. in Philip. Journ. Sci. 10 (1915) Bot. 288.

Coix heteroclita Roxb. Fl. Ind. ed. 2, 3 (1832) 572.

Polytoca bracteata R. Br. in Benn. Pl. Jav. Rar. (1838) 20, t. 5.

Busuanga, Mindanao (Bukidnon), Baker 3616, B. S. 39226, 38484, 41267 Ramos \& Edaño. In open grasslands at medium altitudes. India to southern China and Java.

\section{CHIONACHNE R. Brown}

CHIONACHNE BIAURITA Hack. in Philip. Journ. Sci. 1 (1906) Suppl. 263 ; Merr. op. cit. 320.

Luzon (Bontoc, Benguet), Merrill 4282, Vanoverbergh 934. On fresh talus slopes, untilled soil, etc., altitude about 1,200 m. Endemic.

\section{EXCLUDED SPECIES}

Chionachne barbata R. Br.;' F.-Vill. Novis. App. (1882) 314.

\section{4. colX Linnaeus}

COIX LACHRYMA-JOBI Linn. Sp. Pl. (1753) 972; Pilg. ex Perk. Frag. Fl. Philip. (1904) 137; Merr. in Philip. Journ. Sci. 1 (1906) Suppl. 320, 3 (1908) Bot. 394, 5 (1910) Bot. 325, Fl. Manila (1912) 75, Sp. Blancoanae (1918) 59; Usteri Beitr. Ken. Veg. Philip. (1905) 132 (lachrymae-jovis).

Coix lachryma Linn. Syst. ed. 10 (1759) 1261; Blanco Fl. Filip. (1837) 688, ed. 2 (1845) 478, ed. 3, 3 (1879) 92, t. 188; Miq. Fl. Ind. Bat. 3 (1857) 476; F.-Vill. Novis. App. (1882) 314; Vidal Phan. Cuming. Philip. (1885) 157, Rev. Pl. Vasc. Filip. (1886) 288.

Coix agrestis Lour. Fl. Cochinch. (1790) 551; F.-Vill. Novis. App. (1882) 314.

Coix exaltata Jacq. ex Spreng. Syst. 1 (1825) 228; Nees in Hook. Journ. Bot. Kew Miscel. 2 (1850) 97.

Throughout the Philippines in the settled areas at low and medium altitudes, in most or all islands and provinces; common. A rative of the Old World, now pantropic.

Local names: Abúkai (Iv.) ; adlái (Bis.); agágai (Iv.); agdá (Ig.) ; aglái (C. Bis.); alimúdias ( $\mathrm{P}$. Bis.); apági (Ig); atákai (Ilk., Bon.) ; balantákan (Pamp.) ; barubaióko (Bik.) ; bintíkai (Bik.) ; bitógan (Bag.) ; dalái (Sub.) ; damáu (C. Bis.); glias (Sub.); kalabúgau (Buk.) ; kambot (Ting.) ; katáyan (Ig.) ; katigbí (C. Bis.); kibaoung (If.); koldásan (Bik.) ; kudlásan (Tag.) ; lamúdias (P. Bis.) ; liás (Sub.); paiás (P. Bis., Bag.) ; paliás (P. Bis.) ; pintáka (C. Bis.) ; tidbí (S. L. Bis.) ; tigbí (Bik., Tag.); tigbíkai (Bik.); tiguas (Sul.); tikaian (Bon.). 
Var. MA-YUEN (Roman.) Stapf in Hook. f. Fl. Brit. Ind. 7 (1897) 100; Merr. in Philip. Journ. Sci. 1 (1906) Suppl. 321.

Coix ma-yuen Roman. in Bull. Soc. Acclim. Paris III 8 (1881) 442.

Luzon (Rizal), Mindanao (Zamboanga), Jolo, Merrill 5326, F. B. 3394 Ahern's collector. Occasionally cultivated for its edible grain or for use in making fermented drinks. Tropical Asia.

Local name: Ilás (Tag.).

\section{DIMERIA R. Brown}

DIMERIA CILIATA Merr. in Philip. Journ. Sci. 9 (1914) Bot. 262.

Palawan, Merrill 9320. In low open grasslands just above sea level. Endemic.

DIMERIA ORNITHOPODA Trin. Fund. Agrost. (1820) 167, $t$. 14, var. TENERA (Trin.) Hack. in DC. Monog. Phan. 6 (1889) 81; Merr. in Philip. Journ. Sci. 1 (1906) Suppl. 321, Fl. Manila (1912) 75. Dimeria tenera Trin. in Mém. Acad. Sci. St. Pétersb. VI 2 (1833) 335; F.-Vill. Novis. App. (1882) 315.

Luzon (Bontoc, Benguet, Pangasinan, Zambales, Bulacan, Rizal, Camarines), Mindoro, Mindanao (Davao). In open grasslands, on rice-paddy banks, etc., sea level to an altitude of $1,500 \mathrm{~m}$, locally abundant. India to Japan and Malaya.

Haplachne pilosissima Presl and Andropogon chloridiformis Gaudich. are included in the Novissima Appendix by F.-Villar as distinct species. Both are synonyms of Dimeria chloridiformis K. Schum. \& Lauterb. (D. pilosissima Trin.) a very characteristic species known only from the Marianne Islands.

\section{IMPERATA Cyrillo}

IMPERATA CYLINDRICA (Linn.) Beauiv. Agrost. (1912) expl. pl. 5, t. 5, f. 1, var. KOENIGll (Retz.) Benth. ex Pilger in Perk. Frag. Fl. Philip. (1904) 137; Merr. in Philip. Journ. Sci. 1 (1906) Suppl. 322, 3 (1908) Bot. 395, 5 (1910) Bot. 325, Fl. Manila (1912) 76, Sp. Blancoanae (1918) 60 .

Saccharum koenigii Retz. Obs. 5 (1789) 16.

Imperata koenigii Beauv. Agrost. (1812) 165; Nees in Hook. Journ. Bot. Kew Miscel. 2 (1850) 100.

Imperata arundinacea Cyr. Pl. Rar. Ic. 2 (1792) 26, t. 11; Miq. Fl. Ind. Bat. 3 (1857) 514; Vidal Phan. Cuming. Philip. (1885) 158, Rev. Pl. Vasc. Filip. (1886) 289; F.-Vill. Novis. App. (1882) 316. Imperata arundinacea Cyr. var. koenigii Benth.; Hack. in DC. Monog. Phan. 6 (1889) 94; Usteri Beitr. Ken. Phil. Veg. (1905) 132.

Saccharum spicatum Presl Rel. Haenk. 1 (1828) 346; Kunth Enum. 1 (1833) 476; Miq. Fl. Ind. Bat. 3 (1857) 513; F.-Vill. Novis. App. (1882) 317; Scribn. in Rept. Mo. Bot. Gard. 10 (1899) 52, t. 1.

Throughout the Philippines, on open slopes, often gregariously occupying enormous areas, forming characteristic kogonales, the name of these areas being taken from its almost universal local name kogon. It ascends to 2,300 $\mathrm{m}$ altitude. Tropical Asia and Africa to Australia and Polynesia.

Local names: Buchid (Iv.) ; bulum (If.) ; gáon (Ig.) ; gógon (Bik.) ; góon (Bon.) ; ilib (Pamp.) ; kógon (Ilk., Tag., Bik.) ; panáu (Ilk.) ; parrang (Sul.). 
IMPERATA EXALTATA Brongn. Voy. Coq. Bot. (1829) 101, excl. syn. Roxb.; Hack. in DC. Monog. Phan. 6 (1889) 98; Merr. in Philip. Journ. Sci. 1 (1906) Suppl. 322; PiJg. in Perk. Frag. Fl. Philip. (1904) 137.

Saccharum confertum Presl Rel. Haenk. 1 (1828) 346; Kunth Enum. 1 (1833) 476; F.-Vill. Novis. App. (1882) 317.

Imperata ramosa Anders. in Oefvers. Vet. Akad. Forhandl. Stockh. (1855) 158, Rolfe in Journ. Bot. 23 (1885) 216; Vid. Phan. Cuming. Philip. (1885) 158, Rev. Pl. Vasc. Filip. (1886) 289.

Saccharum alopecurus Nees in Hook. Journ. Bot. Kew Miscel. 2 (1850) 100; Walp. Ann. 3 (1853) 792; Miq. Fl. Ind. Bat. 3 (1857)

513; F.-Vill. Novis. App. (1882) 317.

Saccharum negrosense Steud. Syn. Pl. Glum. 1 (1854) 407.

Central Luzon to Palawan and Mindanao. In habitats similar to the preceding species, but much less common. Java to the Moluccas; a variety in tropical America as Hackel interprets it.

Subsp. MERRILLII Hack. in Philip. Journ. Sci. 1 (1906) Suppl. 264; Merr. op. cit. 322 .

Luzon (Ifugao, Benguet), Negros, Merrill 4813, Phil. Pl. 582, B. S. 2768 Mearns, B. S. 19627 McGregor. On exposed ridges, altitude 1,500 to 2,250 m. Endemic.

Local names: Gógon (Bik.); kógon (Tag., Bis., Sul.); padang-lubuk (Sul.); paraugutau (Sub.).

\section{MISCANTHUS Andersson}

MISCANTHUS DEPAUPERATUS Merr. in Philip. Journ. Sci. 5 (1910) Bot. 170 .

Negros, Merrill Phil. Pl. 583. In open places on the crater of Canlaon Volcano, altitude 1,800 to $2,000 \mathrm{~m}$. Endemic.

MISCANTHUS JAPONICUS (Thunb.) Anders. in Oefv. Vet. Akad. Forhandl. Stockh. (1850) 166; Hack. in DC. Monog. Phan. 6 (1889) 107; Merr. in Philip. Journ. Sci. 1 (1906) Suppl. 322, 3 (1908) Bnt. 395.

Saccharum japonicum Thunb. in Trans. Linn. Soc. 2 (1794) 328.

Saccharum praegrande Steud. Syn. Pl. Glum. 1 (1854) 408.

Miscanthus luzoniensis Anders. in Oefv. Vet. Akad. Forhandl. Stockh. (1855) 166; Rolfe in Journ. Bot. 23 (1885) 216; Vidal Phan. Cuming. Philip. (1885) 158, Rev. Pl. Vasc. Filip. (1886) 289.

Saccharum densum Nees in Hook. Journ. Bot. Kew Miscel. 2 (1850) 100 ; Walp. Ann. 3 (1853) 792.

Babuyan Islands to Mindanao, in most islands and provinces. In thickets, on slopes, and along streams at low and medium altitudes, ascending to $1,200 \mathrm{~m}$. Japan to the Moluccas and Polynesia.

Local names: Búyau (If.); loni (Bag.); tanlák (Tag.); tubás (Tag.); rúno (Pang., Ig., Ilk.); víau (Iv.).

MISCANTHUS SINENSIS Anders. in Oefv. Vet. Akad. Forhandl. Stockh. (1855) 166; Hack. in DC. Monog. Phan. 6 (1889) 105; Merr. in Philip. Journ. Sci. 1 (1906) Suppl. 323, 2 (1907) Bot. 260, 3 (1908) Bot. 395, 5 (1910) Bot. 325. 
Eulalia japonica Trin. in Mém. Acad. St. Pétersb. VI 2 (1833) 333; Miq. Fl. Ind. Bat. 3 (1857) 518; F.-Vill. Novis. App. (1882) 316, non Miscanthus japonicus Ariders.

Miscanthus japonicus Pilg. in Perk. Frag. Fl. Philip. (1904) 137, non Anders.

Batan Islands, Luzon (Bontoc, Benguet, Lepanto, Pampanga, Tayabas, Bataan, Zambales), Mindoro, Negros. In open places, on slopes, and in ravines, altitude 800 to $2,700 \mathrm{~m}$, often abundant. Japan to southern China and Celebes.

Local names: Bigáho (Bik.); bígau (Bik.); biídu (Ig.) ; bílau (Ig., If.) ; gáho (Bik.); gísa (Bik.); lólo (Bon.); padéd (Ig.); pao (Bon.); rúno (Ilk.); víau (Iv。).

\section{SACCHARUM Linnaeus}

SACCHARUM ARUNDINACEUM Retz. Obs. 6 (1791) 14; Hack. in DC. Monog. Phan. 6 (1889) 117; Hook. f. Fl. Brit. Ind. 7 (1897) 119: Erianthus sp. Pilger in Perk. Frag. Fl. Philip. (1904) 137.

Luzon (Bontoc, Nueva Vizcaya, Tayabas), Mindoro, Samar, Mindanao, Merrill 1794, 5448, Clemens 809, Williams 2625, B. S. 13279 Ramos, 1420\% McGregor, 20906 Escritor. Along river banks at low and medium altitudes. India to southern China.

Local names: Bagi'-unás (Ilk.); bahí-onás (Isn.); gatbó (Tag.); lakbó (Tag.) ; tanig (Bon.); zaigbau (Sub.).

SACCHARUM OFFICINARUM Linn. Sp. Pl. (1753) 54; Blanco Fl. Filip. (1837) 42; ed. 2 (1845) 29, ed. 3, 1 (1877) 55, t. 18; F.-Vill. Novis. App. (1882) 317; Merr. in Philip. Journ. Sci. 1 (1906) Suppl. 323, F1. Manila (1912) 78, Sp. Blancoanae (1918) 60.

Saccharum violaceum F.-Vill. Novis. App. (1882) 317, non Tussac.

Cultivated throughout the Philippines, in some islands and provinces very extensively, being one of the major crops of the Philippines. Not a native of the Archipelago. Sugar cane.

Local names: Agbó (Ibn.) ; caña dulce (Sp.); tubó (Tag., Bik.) ; tubu (Sul.); uná (Ibn.); unás (Ilk.); unát (It.).

SACCHARUM SPONTANEUM Linn. Mant. 2 (1771) 183 subsp. INDICUM Hack. in DC. Monog. Phan. 6 (1889) 117; Pilger in Perk. Frag. Fl. Philip. (1904) 137; Merr. in Philip. Journ. Sci. 1 (1906) Suppl. 323, 5 (1910) Bot. 325, Fl. Manila (1912) 77, Sp. Blancoanae (1918) 60 .

Saccharum spontaneum Linn.; Miq. Fl. Ind. Bat. 3 (1857) 512; Rolfe in Journ. Bot. 23 (1885) 216; Vidal Phan. Cuming. Philip. (1885) 158, Rev. Pl. Vasc. Filip. (1886) 289.

Saccharum spontaneum Linn. subsp. luzonicum Hack. in DC. Monog. Phan. 6 (1889) 117.

Anthistiria gigantea Blanco Fl. Filip. (1837) 49, ed. 2 (1845) 33, ed. 3, 1 (1877) 62, non Cav.

Saccharum insulare Brongn. var. amplum et var. depauperatum Anders. in Oefv. Vet. Akad. Forhandl. Stockh. 12 (1855) 157.

Throughout the Philippines, in open areas at low and medium altitudes, ascending to $1,500 \mathrm{~m}$, often gregarious and almost exclusively occupying large areas. India to southern China through Malaya to Polynesia. 
Local names: Bogáng (C. Bis.); bugáng (C. Bis.); liddá (Ilk.); šálin (Bon.) ; síkal (Ibn.) ; taláhib (Tag.) ; tibáyo (Bag.); tigbáu (S. L. Bis., P. Bis.).

\section{POLLINIA Trinius}

POLLINIA ARgenteA (Brongn.) Trin. in Miém. Acad. St. Pétersb. VI 4 (1836) 90; Hack. in DC. Monog. Phan. 6 (1889) 162; Pilger in Perk. Frag. Fl. Philip. (1904) 138; Hack. in Philip. Journ. Sci. 1 (1906) Suppl. 265; Merr. op. cit. 325, Fl. Manila (1912) 77.

Eulalia argentea Brongn. Voy. Coqu. Bot. (1829) 92.

Luzon (Cagayan, Isabela, Bontoc, Nueva Vizcaya, Pangasinan, Rizal), Semirara, Merrill 4152, 108, Elmer 6474, B. S. 17850 Otanes, $14216 \mathrm{Mc}$ Gregor, 7815, 8092, 7831 Ramos. In open grasslands at low and medium altitudes. India to Malaya and Australia.

Var. LAGOPUS Hack. in Govt. Lab. Publ. (Philip.) 35 (1906) 79; Merr. in Philip. Journ. Sci. 1 (1906) Suppl. 325.

Pollinia speciosa Pilger in Perk. Frag. Fl. Philip. (1904) 138, non Hack.

Luzon (Ilocos Norte, Lepanto, Bontoc, Benguet, Pampanga), Mindoro, Palawan, Merrill 4222, 3902, 4365, 4535, 472, B. S. 32753, 32779, 5849 Ramos. In open grasslands at low and medium altitudes, ascending to $1,600 \mathrm{~m}$. Endemic.

POLLINIA CONTORTA (Brongn.) comb. nov.

Pogonatherum contortum Brongh. Bot. Voy. Coqu. Bot. (1829) 90, t. 17 .

Andropogon koretrostachys Trin. in Mém. Acad. St. Pétersb. VI 2 (1833) 273; Steud. Syn. Pl. Glum. 1 (1854) 380; Miq. Fl. Ind. Bat. 3 (1857) 484; F.-Vill. Novis. App. (1882) 316.

Pollinia articulata Trin. in Mém. Acad. St. Pétersb. VI 4 (1836) 90; F.-Vill. Novis. App. (1882) 315; Hack. in DC. Monog. Phan. 6 (1889) 153 (subsp. fragilis Hack. var. setifolia Hack.); Merr. in Philip. Journ. Sci. 1 (1906) Suppl. 324; Pilger in Perk. Frag. Fl. Philip. (1904) 138.

Pollinia setifolia Nees in Hook. Journ. Bot. Kew Miscel. 2 (1850) 88; Miq. Fl. Ind. Bat. 3 (1857) 521; F.-Vill. Novis. App. (1882) 315; Vidal Phan. Cuming. Philip. (1885) 158, Rev. Pl. Vasc. Filip. (1886) 290.

Andropogon asthenostachys Steud. Syn. Pl. Glum. 1 (1854) 381; Miq.

Fl. Ind. Bat. 3 (1857) 485.

Eulalia contorta O. Kuntze Rev. Gen. Pl. (1891) 775.

Luzon (Cagayan, Isabela, Abra, Lepanto, Nueva Ecija, Nueva Vizcaya), Mindanao (Bukidnon), Merrill 317, B. S. 20141 McGregor, 7035, 8121, 7809, 4737 Ramos, F. B. 5875 Curran, 22128 Alvarez, 16460 Bacani.- In open grasslands chiefly at medium altitudes. Burma, southern China, Moluccas, Australia.

Local name: Sañgumati (Buk.).

POLLINIA Fulva (R. Br.) Benth. Fl. Austral. 7 (1878) 526.

Saccharum fulvum R. Br. Prodr. (1810) 203.

Pollinia cumingii Nees in Hook. Journ. Bot. Kew Miscel. 2 (1850) $183036-3$ 
98; Steud. Syn. Pl. Glum. 1 (1854) 373; Walp. Ann. 3 (1853) 803; Miq. Fl. Ind. Bat. 3 (1857) 522; Vidal Phan. Cuming. Philip. (1885) 158, Rev. Pl. Vasc. Filip. (1886) 290; Hack. in DC. Monog. Phan. 6 (1889) 167; Pilger in Perk. Frag. Fl. Philip. (1904) 138; Merr. in Philip. Journ. Sci, 1 (1906) Suppl. 325.

Andropogon aureo-fulvus Steud. Syn. Pl. Glum. 1 (1854) 373.

Pollinia aurea F.-Vill. Novis. App. (1882) 315, non Benth.

Eulalia fulva O. Kuntze Rev. Gen. Pl. (1891) 775.

Luzon (Ilocos Norte, Amburayan, Lepanto, Benguet, Pangasinan, Nueva Vizcaya), Mindoro, Cuming 1538, Merrill 260, 4893, B. S. 33254, 482.7 Ramos. In open grasslands, sandy river bars, ete., at low and medium altitudes, ascending to $1,200 \mathrm{~m}$. Bengal, southern China, Timor, Java, Australia.

POllinia glabrata (Brongn.) Trin. in Mém. Acad. St. Pétersb. VI 4 (1836) 90; Hack. in DC. Monog. Phan. 6 (1889) 179.

Eulalia glabrata Brongn. Voy. Coqu. Bot. (1829) 93, t. 19. Andropogon glabratus Steud. Nomencl. ed. 2, 1 (1840) 91. Nemastachys taitensis Steud. Syn. Pl. Glum. 1 (1854) 357.

Pollinia monantha Nees, var. leptathera Hack. in Philip. Journ. Sci. 3 (1908) Bot. 167.

Luzon (Pangasinan, Rizal, Bataan, Laguna, Tayabas), Mindoro, Samar, Panay, Mindanao, Balut, Merrill 5212, 8272, 7323, 5627, 5426, Phil. Pl. 151, B. S. 31411, 29010 Ramos \& Edaño, 21993, 13784 Ramos. In open ravines, thickets, and old clearings at low and medium altitudes. New Caledonia to the Marianne Islands and Polynesia.

Local names: Gulos (Sub.); kapong (Bon.); lusimat (Sub.).

Var. ELMERI (Hack.) comb. nov.

Pollinia monantha Nees var. elmeri Hack. in Philip. Journ. Sci. 3 (1908) Bot. 167.

Luzon (Bontoc, Benguet), Elmer 6524, Merrill 7784, Vanoverbergh 3665. In thickets in damp ravines, altitude 1,200 to $1,500 \mathrm{~m}$. Endemic.

POLLINIA IRRITANS (R. Br.) Hack. in DC. Monog. Phan. 6 (1889) 1う5, Philip. Journ. Sci. 1 (1906) Suppl. 265; Merr. op. cit. 325.

Saccharum irritans R. Br. Prodr. (1810) 203.

Erianthus irritans Kunth Rev. Gram. 1 (1829) 160.

Eulalia irritans O. Kuntze Rev. Gen. Pl. (1891) 775.

Luzon (Bontoc, Benguet), Merrill 4388, Vanoverbergh 3838. In open grasslands, altitude about $1,000 \mathrm{~m}$. Tropical Australia.

POLLINIA MARITIMA Merr. in Philip. Journ. Sci. 1 (1906) Suppl. 326.

Lumbucan, Merrill 527\%, Phil. Pl. 112. On sandy seashores. Closely allied to $P$. fulva Benth. Endemic.

POLliniA NudA Trin. in Mém. Acad. St. Pétersb. VI ₹ (1833) 307; Hack. in DC. Monog. Phan. 6 (1889) 178; Merr. in Philip. Journ. Sci. 1 (1906) Suppl. 265.

Eulalia nuda O. Kuntze Rev. Gen. Pl. (1891) 775.

Luzon (Benguet), Merrill 4727. 4842. In openings in the mossy forest, altitude 2,000 to $2,400 \mathrm{~m}$. India to China and Japan. 
POLLINIA QUADRINERVIS Hack. in DC. Monog. Phan. 6 (1889) 158, Govt. Lab. Publ. (Philip.) 35 (1906) 79; Merr. in Philip. Journ. Sci. 1 (1906) Suppl. 325, 5 (1910) Bot. 325.

Eulalia quadrinervis O. Kuntze Rev. Gen. Pl. (1891) 775.

Luzon (Bontoc, Benguet), Merrill 4354, 4560, 6519, Elmer 5783, B.S. 31980 Santos, F. B. 16183, 16208 Curran, Merritt, \& Zschokke. On open slopes in the pine region, altitude 1,400 to $2,200 \mathrm{~m}$. India to southern China.

Local names: Galakgákan (Ig.) ; taao (Ig.).

POLlinia Tenuis Trin. in Mém. Acad. St. Pétersb. VI 2 (1833) 307; F.-Vill. Novis App. (1882) 315; Miq. Fl. Ind. Bat. 3 (1857) 521; Hack. in DC. Monog. Phan. 6 (1889) 181; Merr. in Philip. Journ. Sci. 1 (1906) Suppl. 326.

Luzon (Ilocos Sur, Bontoc, Benguet, Bataan, Rizal), Merrill 4310, 4382, Kneucker Gram. Exsic. 721, B. S. 5310 Ramos. On dry open slopes, on and about cliffs, etc., from sea level to $1,200 \mathrm{~m}$ altitude. Caroline Islands.

POLLINIA VIMINEA (Trin.) comb. nov.

Andropogon vimineus Trin. in Mém. Acad. St. Pétersb. VI 2 (1833) 268.

Pollinia imberbis Nees in Steud. Syn. Pl. Glum. 1 (1854) 410, var. willderowiana Hack. in DC. Monog. Phan. 6 (1889) 178, forma monostachya Hack. in Philip. Journ. Sci. 1 (1906) Suppl. 265; Merr. op. cit. 326.

Luzon (Bontoc, Benguet, Rizal), Merrill 4ro7, Williams 1960, Vanoverbergh $809,991,3810$. On open wet slopes and in the mossy forest, altitude 1,200 to $2,200 \mathrm{~m}$. India to southern China and Japan.

\section{POLYTRIAS Hackel}

POLYTRIAS AMAUREA (Büse) O. Kuntze Rev. Gen. Pl. (1891) 788; Merr. in Philip. Journ. Sci. 1 (1906) Suppl. 327.

Andropogon amaurus Büse in Miq. Pl. Jungh. (February, 1854) 320.

Andropogon diversiflorus Steud. in Zoll. Syst. Verzeich. (1854) 58, nomen nudum, Syn. Pl. Glum. 1 (July or August, 1854) 370.

Pollinia praemorsa Nees in Steud. Syn. Pl. Glum. 1 (1854) 409.

Polytrias praemorsa Hack. in DC. Monog. Phan. 6 (1889) 189, t. 1, f. 13; Merr. in Govt. Lab. Publ. (Philip.) 6 (1904) 7; Pilger in Perk. Frag. Fl. Philip. (1904) 138.

Polytrias diversiflora Nash in Torreya 5 (1905) 110; Merr. in Philip. Journ. Sci. 11 (1916) Bot. 253.

Luzon (Rizal, Sorsogon), Jolo, Merrill 386, Phil. Pl. 106, Kneucker Gram. Exsic. 602. In open grasslands in and about towns at low altitudes, certainly introduced. Malay Peninsula, Borneo, Java, introduced in the West Indies.

\section{POGONATHERUM Beauvois}

POGONATHERUM PANICEUM (Lam.) Hack. in Allg. Bot. Zeitschr. 12 (1906) 178; Merr. in Philip. Journ. Sci. 3 (1908) Bot. 395, Fl. Manila (1912) 76, Sp. Blancoanae (1918) 60.

Saccharum paniceum Lam. Encycl. 1 (1785) 595, t. 40, f. 1.

Pogonatherum saccharoideum Beauv. Agrost. (1812) 9, t. 11, f. 7 ; 
F.-Vill. Novis. App. (1882) 316; Vidal Phan. Cuming. Philip. (1885) 158, Rev. Pl. Vasc. Filip. (1886) 290; Hack. in DC. Monog. Phan. 6 (1889) 192 (var. monandrum Hack.); Pilger in Perk. Frag. Fl. Philip. (1904) 138; Merr. in Philip. Journ. Sci. 1 (1906) Suppl. 327. Pogonatherum polystachyum R. \& S. Syst. 2 (1825) 497; Nees in Hook. Journ. Bot. Kew Miscel. 2 (1850) 99.

Pogonopsis tenera Presl Rel. Haenk. 1 (1830) 333, t. 46; Scribn. in Rept. Mo. Bot. Gard. 10 (1899) 52, t. 2.

Pogonatherum crinitum Kunth Enum. 1 (1833) 478; Miq. Fl. Ind. Bat. 3 (1857) 516; Usteri Beitr. Ken. Veg. Philip. (1905) 133.

Cinna filiformis Llanos Frag. Pl. Filip. (1851) 9; F.-Vill. \& Naves in Blanco Fl. Filip. 3, $4^{1}$ (1880) 3.

Deyeuxia quadriseta F.-Vill. Novis. App. (1882) 319, non Benth.

Ischaemum crinitum Trin. in Mém. Acad. St. Pétersb. VI 2 (1833) 298; Presl Repert. 1 (1833) 296.

Throughout the Philippines, common on banks of ravines, along streams on cliffs and steep banks, etc., at low and medium altitudes, ascending to $2,000 \mathrm{~m}$. India to China through Malaya to the New Hebrides.

Local names: Dumenéi (Bon.); kisáng (Buk.); lagitlit (C. Bis.); malakauáyan (S. L. Bis.); obán-obán (Mbo.); panaguáyan (C. Bis.); silsilíg (Bon.).

\section{APLUDA Linnaeus}

APluda MUtica Linn. Sp. Pl. (1753) 82; Nees in Nov. Act. Acad. Nat. Cur. 19 (1843) Suppl. 1: 192, Hook. Journ. Bot. Kew Miscel. 2 (1850) 99; Miq. Fl. Ind. Bat. 3 (1857) 501; F.-Vill. Novis. App. (1882) 318; Vidal Phan. Cuming. Philip. (1885) 159, Rev. Pl. Vasc. Filip. (1886) 292; Merr. in Philip. Journ. Sci. 1 (1906) Suppl. 332, Fl. Manila (1912) 79.

Calamina humilis Presl Rel. Haenk. 1 (1828) 344.

Apluda humilis Kunth Enum. 1 (1833) 517; F.-Vill. Novis. App. (1882) 318.

Apluda cumingii Büse in De Vriese Pl. Ind. Bat. Or. (1856) 105;

Miq. Fl. Ind. Bat. 3 (1857) 501; F.-Vill. Novis. App. (1882) 318.

Apluda varia Hack. var. humilis Hack. in DC. Monog. Phan. 6 (1889) 196.

Apluda varia Hack. var. mutica Hack. f. intermedia Hack. in DC.

Monog. Phan. 6 (1889) 197.

Apluda mutica Linn. forma intermedia Merr. in Philip. Journ. Sci. 1 (1906) Suppl. 332.

Apluda varia Hack, var. major Hack. in DC. Monog. Phan. 6 (1889) 198.

Apluda mutica Linn. var. major Hack. in Allgem. Bot. Zeitschr. 12 (1906) 179.

In thickets and open grasslands throughout the Philippines at low and medium altitudes, ascending to $1,800 \mathrm{~m}$. India to southern China, through Malaya to Australia, New Caledonia, and Polynesia.

Local names: Kauakauáyan (Tag.) ; kolokauáyan (Tag.) ; kurukauáyan (Bik) ; magkauáyan (C. Bis.).

Var ARISTATA (Linn.) Rendle ex Forbes \& Hemsl. in Journ. Linn. Soc.

Bot. 36 (1904) 279; Merr. in Philip. Journ. Sci. 1 (1906) Suppl. 332, Fl. Manila (1912) 80. 
Apluda aristata Linn. Amoen. Acad. 4 (1759) 303; F.-Vill. Novis. App. (1882) 318; Usteri Beitr. Ken. Philip. Veg. (1905) 132.

Luzon (Rizal), B. S. 2041 Ramos, Merrill 629, Silva 391. Habitat and range of the species.

\section{ISCHAEMUM Linnaeus}

ISCHAEMUM ANGUSTIFOLIUM (Trin.) Hack. ex Oliv, in Hook. Ic. III 8 (1888) t. 1773, DC. Monog. Phan. 6 (1889) 241; Merr. in Philip. Journ. Sci. 1 (1906) Suppl. 331.

Spodiopogon angustifolius Trin. in Mém. Acad. St. Petersb. VI 2 (1833) 300; Nees in Hook. Journ. Bot. Kew Miscel. 2 (1850) 99; Vidal Phan. Cuming. Philip. (1885) 158, Rev. Pl. Vasc. Filip. (1886) 290.

Andropogon obvallatus Steud. Syn. Pl. Glum. 1 (1854) 373.

Ischaemum notopogon Nees in Miq. Fl. Ind. Bat. 3 (1857) 500; F.-Vill. Novis. App. (1882) 315.

Luzon (Cagayan, Ilocos Norte, Bontoc, Nueva Vizcaya, Pangasinan), Cuming 1002, Merrill 172, Phil. Pl. 171, B. S. 7748, 7919 Ramos, 14214 McGregor, F. B. 16527 Curran \& Merritt. In open grasslands at low and medium altitudes, ascending to $1,400 \mathrm{~m}$. India to southern China and Formosa.

Local names: Kabóot (Ilk., Ibn.) ; poen (Bon.); pueng (Bon.); puenig (Ig.) ; sangumáti (Buk.).

ISCHAEMUM ARISTATUM Linn. Sp. PI. (1753) 1049 var. GIBBUM (Trin.) Hack. in DC. Monog. Phan. 6 (1889) 204; Pilg. in Perk. Frag. Fl. Philip. (1904) 139; Merr. in Philip. Journ. Sci. 1 (1906) Suppl. 330, Fl. Manila (1912) 80.

Ischaemum gibbum Trin. in Mém. Acad. St. Pétersb. VI 2 (1833) 295; Miq. Fl. Ind. Bat. 3 (1857) 498; F.-Vill. Novis. App. (1882) 315. Andropogon gibbus Steud. Syn. Pl. Glum. 1 (1854) 376.

Luzon (Isabela, Ilocos Norte, Nueva Ecija, Nueva Vizcaya, Zambales, Bulacan, Rizal, Camarines), Culion. In open grasslands at low and medium altitudes; often common. Well represented by Merrill Phil. Pl. 179, 197\%, and Kneucker Gram. Exsic. 605. The variety endemic, the species India to China.

ISCHAEMUM ARUNDINACEUM F.-Muell. ex Benth. Fl. Austral. 7 (1878) 519 var. RADICANS Hack. in Philip. Journ. Sci. 1 (1906) Suppl. 266 ; Merr. op. cit. 331.

Luzon (Benguet, Bontoc), Merrill 4264, Phil. Pl. 1000, Williams 1197, Vanoverbergh 861 . On open grassy slopes in the pine regions, altitude 1,200 to $1,600 \mathrm{~m}$. The variety endemic, the species in tropical Australia.

ISCHAEMUM CILIARE Retz. Obs. 6 (1791) 36 ; F.-Vill. Novis. App. (1882)

31̄̄; Hack. in DC. Monog. Phan. 6 (1889) 255 (var. malachophyllum Hack.) ; Merr. in Philip. Journ. Sci. 3 (1908) Bot. 395.

Batan Islands (Batan), B. S. 3169 Mearns. In open grasslands. India to China and Formosa.

ISChAemum GLAuCescens Merr. in Philip. Journ. Sci. 9 (1914) Bot. 263.

Palawan, Merrill 9453. In shallow water, borders of Lake Manguao, at low altitudes. Endemic. 
ISCHAEMUM INTERMEDIUM Brongn. in Duperr. Voy. Coqu. (1829) Bot. 73; Pilg. in Perk. Frag. Fl. Philip. (1904) 139; Merr. in Philip. Journ. Sci. 1 (1906) Suppl. 331, Fl. Manila (1912) 80.

Luzon (Rizal, Laguna, Sorsogon), Polillo, Leyte, Mindanao (Surigao), Merrill 68, 52.2., 5213, Phil. Pl. 102, 1458, Elmer 143.26, 18318, 17408, 16936, B. S. 34749 Ramos \& Pascasio, 12103, 12022 Ramos. In open wet grassy places at low altitudes. Caroline Islands.

ISCHAEMUM MERRILLII Hack. in Philip. Journ. Sci. 1 (1906) Suppl. 266; Merr. op. cit. 331.

Luzon (Lepanto, Benguet), Merrill 4622, B. S. 12917 Fénix. In open rather wet places, altitude about $1,500 \mathrm{~m}$. Endemic.

Local names: Kugañgán (Ig.) ; sinamon (Ig.).

ISCHAEMUM MUTICUM Linn. Sp. PI. (1753) 1049; Miq. Fl. Ind. Bat. 3 (1857) 496; F.-Vill. Novis. App. (1882) 315; Hack. in DC. Monog. Phan. 6 (1889) 212; Pilg. in Perk. Frag. Fl. Philip. (1904) 139; Merr. in Philip. Journ. Sci. 1 (1906) Suppl. 330, 3 (1908) Bot. 395. Ischaemum glabratum Presl Rel. Haenk. 1 (1828) 328; Kunth Enum. 1 (1833) 513; Miq. Fl. Ind. Bat. 3 (1857) 498; F.-Vill. Novis. App. (1882) 315.

Andropogon relictus Steud. Syn. Pl. Glum. 1 (1854) 377.

Ischaemum repens Roxb. Fl. Ind. 1 (1820) 325; Nees in Hook. Journ.

Bot. Kew Miscel. 2 (1850) 100.

Along sandy seashores from northern Luzon to Palawan and Mindanao, in most or all islands. India through Malaya to tropical Australia, New Caledonia, and western Polynesia.

Local name: Karukauáyan (Bik.).

ISChAemum PUBescens Merr. in Philip. Journ. Sci. 9 (1914) Bot. 264.

Dumaran, B. S. 21639 Escritor. In open grasslands at low altitudes. Endemic.

ISCHAEMUM RUGOSUM Salisb. Ic. (1791) $1, t .1$ var. DISTACHYUM (Cav.) Merr. in Philip. Journ. Sci. 1 (1906) Suppl. 330, 2 (1907) Bot. 436, Fl. Manila (1912) 80, Sp. Blancoanae (1918) 61.

Colladoa distachya Cav. Ic. 5 (1799) 37, t. 460; Willd. Sp. Pl. 4* (1805) 496.

Andropogon ramosus Blanco Fl. Filip. (1837) 37, ed. 2 (1845) 25, ed. 3, 1 (1877) 48; Merr. in Govt. Lab. Publ. (Philip.) 27 (1905) 91, non Forsk.

Ischaemum colladoa Spreng. Syst. 1 (1825) 298; Kunth Enum. 1 (1833) 513; Miq. Fl. Ind. Bat. 3 (1857) 498; F.-Vill. Novis. App. (1882) 315.

Ischaemum mugosum Salisb. var. segetum Hack. in DC. Monog. Phan. 6 (1889) 206; Pilg. in Perk. Frag. Fl. Philip. (1904) 139.

Meoschium elegans Nees \& Arn. in Nov. Act. Acad. Nat. Cur. 19 (1843) Suppl. 1: 199; Nees in Hook. Journ. Bot. Kew Miscel. 2 (1850) 100.

Ischaemum rugosum F.-Vill. \& Naves in Blanco Fl. Filip. ed. 3, $4^{1}$ (1880) 105; Miq. Fl. Ind. Bat. 3 (1857) 496; Vidal Phan. Cuming. Philip. (1885) 158, Rev. Pl. Vasc. Filip. (1886) 290; Rolfe in Journ. Bot. (1885) 216, vix Salisb. 
Luzon (Ilocos Norte, Lepanto, Bontoc, Pangasinan, Rizal, Bataan), Polillo, Cuming 565, Merrill 1550, Phil. Pl. 592, Sp. Blancoanae 165. In open usually rather wet 'grasslands. India to China and Malaya.

Local names: Daua (Sub.); gulonglapas (Pang.); tinitrígo (Tag.). ISCHAEMUM TODAYENSE Elm. Leafl. Philip. Bot. 7 (1915) 2678.

Mindanao (Davao), Elmer 10521. In ravines in secondary forests, altitude about $900 \mathrm{~m}$. Endemic.

Local name: Halopos (Bag.).

\section{EXCLUDED SPECIES}

Ischaemum Polystachyum Presl; F.-Vill. Novis. App. (1882) 315.

The type of this was from the Marianne Islands and F.-Villar does not really enter the species as Philippine. It is a synonym of Ischaemum digitatum Brongn.

IsCHAEMUM TIMORENSE Kunth; F.-Vill. Novis. App. (1882) 315.

Ischaemum Minus Presl Rel. Haenk. 1 (1828) 329; F.-Vill. Novis. App. (1882) 315; Kunth Enum. 1 (1833) 514; Miq. Fl. Ind. Bat. 3 (1857) 498.

"Hab. in insulis Philippinis." This is an American species erroneously localized by Presl. It is Ischaemum urvilleanum Kunth; see Hack. in DC. Monog. Phan. 6 (1889) 218. Andropogon minor Steud. Syn. Pl. Glum. 1 (1854) 377 is a synonym.

\section{3a. EREMOCHLOA Büse}

EREMOCHLOA CILIARIS (Linn.) Merr. in Philip. Journ. Sci. 1 (1906) Suppl. 331.

Nardus ciliaris Linn. Sp. Pl. (1753) 53.

Ischaemum leersioides Munro in Proc. Amer. Acad. 4 (1864-65) 363.

Eremochloa leersioides Hack. in DC. Monog. Phan. 6 (1889) 264;

Pilger in Perk. Frag. Fl. Philip. (1904) 139; Merr. in Govt. Lab.

Publ. (Philip.) 6 (1904) 6.

Luzon (Cagayan, Isabela), Merrill 138, B. S. 7813, 7832, 8109 Ramos. In open grasslands at low altitudes. Southern China to Tonkin and Pegu.

\section{3b. ROTTBOELLIA Linnaeus f.}

ROTtBoellia EXALTATA Linn. f. Suppl. (1781) 114; Nees in Nov. Act. Acad. Nat. Cur. 19 (1843) Suppl. 1: 173, Hook. Journ. Bot. Kew Miscel. 2 (1850) 100; Miq. Fl. Ind. Bat. 3 (1857) 407; F.-Vill. Novis. App. (1882) 314; Vidal Phan. Cuming. Philip. (1885) 158, Rev. P1. Vasc. Filip. (1886) 290; Hack. in DC. Monog. Phan. 6 (1889) 293; Merr. in Philip. Journ. Sci. 1 (1906) Suppl. 328, Fl. Manila (1912) 78.

Rottboellia setosa Presl Rel. Haenk. 1 (1828) 329; Kunth Enum. 1 (1833) 466.

Rottboellia denudata Steud. Syn. Pl. Glum. 1 (1854) 362; Miq. Fl.

Ind. Bat. 3 (1857) 408; F.-Vill. Novis. App. (1882) 314.

Rottboellia coelorhachis F.-Vill. Novis. App. (1882) 314, non Forst.

Manisuris exaltata O. Kuntze Rev. Gen. Pl. (1891) 779.

Throughout the Philippines in open places, borders of thickets, etc., 
at low and medium altitudes; common. India to China, tropical Africa, through Malaysia to tropical Australia.

Local names: Agíñai (Tag.); annárai (Iv.); bódo (Bon.); bukal (C. Bis.) ; gaho (Bik.); girun (Bag.) ; nagéi (Bon.) ; sagisi (Ilk.).

Rottboellia glandulosa Trin. in Mém. Acad. St. Pétersb. VI 2 (1833) 250; Hack. in DC. Monog. Phan. 6 (1889) 302; Pilger in Perk. Frag. Fl. Philip. (1904) 138; Merr. in Philip. Journ. Sci. 1 (1906) Suppl. 328.

Coelorhachis muricata Brongn. Bot. Voy. Coqu. 1 (1829) 65, t. 14. Rottboellia muricata Miq. Fl. Ind. Bat. 3 (1857) 407; F.-Vill. Novis. App. (1882) 314; Vidal Phan. Cuming. Philip. (1885) 158, Rev. Pl. Vasc. Filip. (1886) 290, non Retz.

Manisuris glandulosa O. Kuntze Rev. Gen. Pl. (1891) 780.

Culion, Palawan, Balabac, Siargao, Mindanao, Merrill 505, Phil. Pl. 1375, B. S. 34988 Ramos \& Pascasio, 491 Mangubat. In thickets, old clearings, etc., at low altitudes. Burma to Java.

Local name: Murabon (Sul.).

ROTtBoElliA OPHIUROIDES Benth. Fl. Austral. 7 (1878) 514; Hack. in DC. Monog. Phan. 6 (1889) 303; Pilger in Perk. Frag. Fl. Philip. (1904) 138; Merr. in Philip. Journ. Sci. 1 (1906) Suppl. 328, 5 (1910) Bot. 326.

Ischaemum rottboellioides R. Br. Prodr. (1910) 205.

Manisurus rottboellioides O. Kuntze Rev. Gen. Pl. (1891) 779.

Andropogon rottboellioides Steud. Syn. Pl. Glum. 1 (1854) 382.

Luzon (Cagayan, Bontoc, Benguet, Nueva Vizcaya, Tarlac, Pampanga, Pangasinan, Zambales, Rizal), Masbate, Panay, Bohol. In open grasslands at low and medium altitudes, ascending to $2,000 \mathrm{~m}$, often common. New Guinea, tropical Australia.

Local names: Gilon (C. Bis.) ; katalon (Ig.); lagopak (S. L. Bis.) ; talañgo (Tag., Sbl.) ; tanlo (Ig.).

Var. INTERMEDIA Hack. in Philip. Journ. Sci. 1 (1906) Suppl. 265, Merr. op. cit. 329.

Luzon (Benguet), Merrill 4667, Elmer 6393. On dry slopes in the pine region, altitude 1,000 to $1,600 \mathrm{~m}$. Endemic.

ROTtBoellia TRIFlorA Hubbard in Philip. Journ. Sci. 9 (1914) Bot. 257.

Palawan, Ledesma. Borders of thickets at low altitudes. Endemic.

\section{EXCLUDED SPECIES}

Rottboellia myuros Linn.; Ceron Cat. Pl. Herb. Manila (1892) 181.

This record was probably based on an erroneously identified specimen of Ophiurus monostachyus Presl.

Rottboellia mutica Llanos ex F.-Vill. \& Naves in Blanco Fl. Filip. ed. $3,4^{1}$ (1880) 99.

This is an evident misprint for Rottboellia muricata Retz., a species that does not occur in the Philippines. 


\section{MANISURIS Swartz}

MANISURIS GRANULARIS Linn. f. Nov. Gram. Gen. (1779) 37, f. $1-7$; Miq. Fl. Ind. Bat. 3 (1857) 409; F.-Vill. Novis. App. (1882) 314; Hack. in DC. Monog. Phan. 6 (1889) 314; Merr. in Philip. Journ.

Sci. 1 (1906) Suppl. 329, Fl. Manila (1912) 79.

Cenchrus granularis Linn. Mant. 2 (1771) 575.

Hackelochloa granularis O. Kuntze Rev. Gen. Pl. (1891) 776.

Rytilix granularis Skeels in U. S. Dept. Agric. Bur. Pl. Ind. Bull. 282 (1913) 20.

Batan Islands to Mindanao, in most islands and provinces. In disturbed soil, open waste places, old clearings, etc., at low and medium altitudes. Pantropic.

Local name: Gir̃getkaran (Bag.).

\section{OPHIURUS Gaertner}

OPHIURUS EXALTATUS (Linn.) O. Kuntze Rev. Gen. Pl. (1891) 780; Merr: in Philip.Journ. Sci. 1 (1906) Suppl. 329.

Aegilops exaltata Linn. Mant. 2 (1771) 575.

Ophiurus corymbosus Gaertn. Fruct. 3 (1805) 4, t. 181, f. A; F.-Vill.

Novis. App. (1882) 314; Pilger in Perk. Frag. Fl. Philip. (1904) 138.

Rottboellia tongcalingii EIm. Leafl. Philip. Bot. 7 (1915) 2680.

Luzon (Isabela, Nueva Vizcaya, Negros, Mindanao (Bukidnon, Davao), Merrill 127 p. p., Williams 261\%, Elmer 10317, 10521, 16486, B. S. 8076 Ramos. In open rather damp places at low and medium altitudes. India to tropical Australia.

Local names: Girun (Bag.) ; talañgiu (Bon.)

OPHIURUS MONOSTACHYUS Presl Rel. Haenk. 1 (1830) 330; Kunth Enum. 1 (1833) 464; Miq. Fl. Ind. Bat. 3 (1857) 405; F.-Vill. Novis. App. (1882) 314; Vidal Phan. Cuming. Philip. (1885) 158, Rev. Pl. Vasc. Filip. (1886) 290; Hack. in DC. Monog. Phan. 6 (1889) 318; Merr. in Philip. Journ. Sci. 1 (1906) Suppl. 329, Fl. Manila (1912) 79 .

Ophiurus undatus Nees in Hook. Journ. Bot. Kew Miscel. 2 (1850) 100; Walp. Ann. 3 (1853) 791.

Ophiurus undulatus Miq. Fl. Ind. Bat. 3 (1857) 405.

Luzon (Cagayan, Ilocos Norte, Lepanto, Ifugao, Rizal), Mindanao (Bukidnon, Zamboanga, Lanao), Cuming 1399, Merrill Phil. Pl. 124, B. S. 32781 Ramos, 19978 McGregor, 37450, 39203 Ramos \& Edaño. In open grasslands at low and medium altitudes. Southern China, Indo-China.

\section{ARTHRAXON Beauvois}

ARTHRAXON HISPIDUS (Thunb.) Merr. in Philip. Journ. Sci. 7 (1912) Bot. 229.

Phalaris hispida Thunb. Fl. Jap. (1784) 44.

Arthraxon ciliaris Beauv. Agrost. (1812) 111, t. 11, f. 6; F.-Vill.

Novis. App. (1882) 315.

Pleuroplitis langsdorffi Trin. Fund. Agrost. (1820) 174, f. 16.

Arthraxon ciliaris Beauv. subsp. langsdorffi Hack. in DC. Monog.

Phan. 6 (1889) 354, Philip. Journ. Sci. 1 (1906) Suppl. 265;

Merr. op. cit. 333; Perk, Frag. Fl. Philip. (1904) 139. 
Arthraxon citiaris Beauv. subsp. nudus Hack. in DC. Monog. Phan. 6 (1889) 356, Philip. Journ. Sci. 1 (1906) Suppl. 266; Merr. op. cit. 333.

Luzon (Cagayan, Ifugao, Bontoc, Lepanto, Benguet, Nueva Vizcaya), Mindanao (Lanao), Merrill 102, 4427, 4628, 7676, Phil. Pl. 175, Vanoverbergh 3811, 1760, 204, Clemens 100, B. S. 19628 McGregor, 5534, 5926, 5970, 7021, 7912 Ramos. In damp places about streams, in old rice paddies, etc., at medium altitudes, ascending to $1,600 \mathrm{~m}$. Japan, China, Formosa.

Local name: Timi-timí (Bon.).

ARTHRAXON MICROPHYLLUS (Trin.) Hochst. in Flora 39 (1856) 188; Hack. in DC. Monog. Phan. 6 (1889) 351, Philip. Journ. Sci. 1 (1906) Suppl. 265; Merr. op. cit. 333, 5 (1910) Bot. 326.

Andropogon microphyllus Trin. in Mém. Acad. St. Pétersb. VI 2 (1833) 275.

Pleuroplitis microphylla Regel in Bull. Acad. St. Pétersb. 5 (1866) 749.

Luzon (Ilocos Norte, Bontoc, Benguet), Merrill 4363, 4719, Phil. Pl. 193, Clemens 9233, Vanoverbergh 1732, F. B. 12487 Merritt \& Darling. On open slopes in the pine region, altitude 1,200 to $2,000 \mathrm{~m}$. Abyssinia, India, Ceylon.

ARTHRAXON QUARTINIANUS (A. Rich.) Nash in N. Am. Flora 17 (1912, Sept. 18) 99; Merr. in Philip. Journ. Sci. 7 (1912, Sept. 30) Bot. 229, Fl. Manila (1912) 77.

Alectoridia quartiniana A. Rich. Tent. Fl. Abyss. 2 (185̄1) 448, t. 99. Arthraxon ciliaris Beauv. subsp. quartinianus Hack. in DC. Monog.

Phan. 6 (1889) 356, Philip. Journ. Sci. 1 (1906) Suppl. 266; Merr. op. cit. 333,5 (1910) Bot. 326.

Luzon (Ifugao, Bontoc, Benguet, Rizal), Merrill 4704, 467\%, 7370, 9656, 4272, 6514, Phil. Pl. 999, Williams 1189, B. S. 19709 McGregor. On open slopes, in old rice paddies, etc., chiefly at altitudes from 1,100 to $1,600 \mathrm{~m}$, rarely from about sea level. Abyssinia, India, introduced in the West Indies.

\section{ANDROPOGON Linnaeus}

ANDROPOGON ACICULATUS Retz. Obs. 5 (1789) 22; Hack. in DC. Monog. Phan. 6 (1889) 562; Pilger in Perk. Frag. Fl. Philip. (1904) 139; Merr. in Philip. Journ. Sci. 1 (1906) Suppl. 338, Fl. Manila (1912) 83, Sp. Blancoanae (1918) 162.

Chrysopogon aciculatus Trin. Fund. Agrost. (1820) 188; Miq. Fl. Ind. Bat. 3 (1857) 490; F.-Vill. Novis. App. (1882) 316; Vidal Phan. Cuming. Philip. (1885) 158, Rev. Pl. Vasc. Filip. (1886) 291.

Rhaphis trivialis Lour. Fl. Cochinch. (1790) 553; Blanco Fl. Filip. (1837) 4 อ.

Andropogon acicularis Willd. Sp. Pl. $4^{2}$ (1805) 906; Blanco Fl. Filip. ed. 2 (1845) 26, ed. 3, 1 (1877) 49.

Andropogon subulatus Presl Rel. Haenk. 1 (1828) 341; Kunth Enum. 1 (1833) 505.

Chrysopogon subulatus Miq. Fl. Ind. Bat. 3 (1857) 491; F.-Vill. Novis. App. (1882) 317. 
Rhaphis javanica Nees in Hook. Journ. Bot. Kew Miscel. 2 (1850) 99.

Throughout the Philippines in open places at low and medium altitudes; a veritable pest on account of its spikelets adhering to fur, clothing, etc. India to China southward through Malaya to tropical Australia and Polynesia.

Local names: Amor-seco (Sp.); dalekédek (Bon.); dalukdúk (Bon.); lakut-lapas (Sul.); marisékos (Tag.); marískos (Tag.) ; pagippi (Ibn.) ; tinlói (Tag.).

ANDROPOGON BAILEYI F.-Muell. in Vict. Nat. 7 (1891) 16; Hack. in Philip. Journ. Sci. 1 (1906) Suppl. 297; Merr, op. cit. 337.

Sorghum laxiflorum Bailey Syn. Queensl. Fl. Suppl. 3 (1890) 84, Queensl. Fl. 6 (1902) 1869; Rept. Exped. Bellend. Ker Range 25, non Andropogon laxifloms Steud.

Luzon (Bontoc, Lepanto, Benguet, Rizal), Merrill 4399, Vanoverbergh $2131,3839, B$. S. 24082 Ramos. On open grassy slopes at medium altitudes, ascending to $1,200 \mathrm{~m}$. Tropical Australia.

ANDROPOGON BREVIFOLIUS Sw. Prodr. (1788) 26; Hack. in DC. Monog. Phan. 6 (1889) 363; Rolfe in Journ. Bot. 23 (1885) 216; Vidal Phan. Cuming. Philip. (1885) 158, Rev. Pl. Vasc. Filip. (1886) 291; Pilger in Perk. Frag. Fl. Philip. (1904) 139; Merr. in Philip. Journ. Sci. 1 (1906) Suppl. 335.

Luzon (Cagayan, Ifugao, Bontoc, Benguet, Nueva Vizcaya, Bulacan, Zambales, Bataan, Laguna), Mindanao (Lanao, Bukidnon, Davao). In open grasslands at low and medium altitudes. Pantropic.

Local name: Bung-bung (Bag.).

ANDROPOGON CINCTUS Steud. Syn. Pl. Glum. 1 (1854) 398.

Chrysopogon micranthus Benth. var. spicigerus Benth. Fl. Austral. 7 (1878) 538.

Andropogon micranthus Kunth var. spicigerus Hack. in DC. Monog.

Phan. 6 (1889) 489; Merr. in Philip. Journ. Sci. 1 (1906) 336,

3 (1908) Bot. 395.

Batan ahd Babuyan Islands, Luzon (Bontoc, Benguet), Merrill 4836, Elmer 5918, F. B. 15612, 4854 Curran, B. S. 5367 Ramos, 37.26, 3918, 4040 Fénix. In open grasslands up to $1,800 \mathrm{~m}$ altitude. China, Australia, New Caledonia.

I believe this to be specifically distinct from Andropogon micranthus Kunth.

ANDROPOGON CITRATUS DC. Cat. Hort. Monsp. (1813) 78; Merr. in Philip. Journ. Sci. 5 (1910) Bot. 167, Fl. Manila (1912) 82, Sp. Blancoanae (1918) 62.

Andropogon schoenanthus Blanco Fl. Filip. (1837) 39, ed. 2, (1845) 27, ed. 3, 1 (1877) 50; F.-Vill. Novis. App. (1882) 316; Merr. in Philip. Journ. Sci. 1 (1906) Suppl. 339, 2 (1907) Bot. 436, non Linn. Cymbopogon citratus Stapf in Kew Bull. (1906) 257.

Planted here and there about houses in most or all parts of the Philippines for its fragant leaves, but nowhere spontaneous; I have seen only one flowering specimen, Jolo, Link 215. A native of tropical Asia, now widely distributed in cultivation. 
Local names: Balióko (Bis.) ; baráni (Ilk.); paja de méca (Sp.) ; tanglád (Tag., Bik., Bis.) ; sálai (Tag.) ; sái (Mbo., Mand., Sul.) ; sálaid (Tag.).

ANDROPOGON CONTORTUS Linn. Sp. Pl. (1753) 1045; Blanco Fl. Filip. ed. 1 (1837) 38, ed. 2 (1845) 26, ed. 3, 1 (1877) 49; Merr. in Philip. Journ. Sci. 1 (1906) Suppl. 338, Fl. Manila (1912) 83, Sp. Blancoanae (1918) 61.

Heteropogon contortus Beauv. ex R. \& S. Syst. 2 (1817) 836; Nees in Hook. Journ. Bot. Kew Miscel. 2 (1850) 99; F.-Vill. Novis. App. (1882) 315; Miq. Fl. Ind. Bat. 3 (1857) 493; Vidal Phan. Cuming. Philip. (1885) 158, Rev. Pl. Vasc. Filip. (1886) 291.

Heteropogon polystachyus F.-Vill. Novis. App. (1882) 315, non Schultes.

Heteropogon roxburghii Nees in Hook. Journ. Bot. Kew Miscel. 2 (1850) 99, haud Arn.

Luzon (Ilocos Norte, Union, Lepanto, Nueva Ecija, Nueva Vizcaya, Pangasinan, Pampanga, Rizal, Zambales), Mindoro, Mindanao (Bukidnon, Misamis). In open grasslands at low and medium altitudes, often very abundant. Certainly an introduced plant in the Philippines. Pantropic.

Local name: Sibat-sibátan (Tag.).

The Philippine form is mostly referable to the var. hispidissimus Hack. in DC. Monog. Phan. 6 (1889) 587.

ANDROPOGON FASTIGIATUS Sw. Prodr. (1788) 26; Hack. in DC. Monog. Phan. 6 (1889) 393, Philip. Journ. Sci. 1 (1906) Suppl. 267; Merr. op. cit. 335 .

Luzon (Lepanto, Bontoc, Pangasinan), Merrill 4461, B. S. 30055 Ramos. On dry open grassy slopes at low and medium altitudes. Pantropic.

ANDROPOGON FESTUCOIDES Presl Rel. Haenk. 1 (1828) 340; Kunth Enum. 1 (1833) 500; Miq. Fl. Ind. Bat. 3 (1857) 489; F.-Vill. Novis. App. (1882) 316 ; Merr. Sp. Blancoanae (1918) 61.

Andropogon anias Llanos Frag. Pl. Filip. (1851) 29; F.-Vill. \& Naves in Blanco Fl. Filip. ed. 3, $4^{1}$ (1880) 20; F.-Vill. Novis. App. (1882) 316.

Andropogon muricatus Retz. var. aristatus Büse in De Vriese Pl. Ind. Bat. Orient. (1856) 104.

Andropogon nigritanus Benth. in Hook. Niger Fl. (1849) 573.

Andropogon squarrosus Linn. f. var. nigritanus Hack. in DC. Monog.

Phan. 6 (1889) 544.

Vetiveria zizanioides Stapf var. nigritana Camus in Bull. Mus. Hist.

Nat. Paris 25 (1919) 674.

Luzon (Pampanga, Nueva Ecija), Merrill 4231, 4240, Phil. Pl. 389, B. S. 22238 Santos. In open level lands, rice-paddy banks, etc., at low altitudes. Tropical Africa, Java.

Local name: Aniás (Pamp.).

The roots are entirely odorless in striking contrast to those of $A n$ dropogan zizanioides Urban to which it has been erroneously reduced.

ANDROPOGON FILIPENDULUS Hochst. in Flora 29 (1846) 115, var. LACHNATHERUS (Benth.) Hack. in DC. Monog. Phan. 6 (1889)

635, Philip. Journ. Sci. 1 (1906) Suppl. 267; Merr. op. cit. 339. Andropogon lachnatherus Benth. Fl. Austral. 7 (1878) 534. 
Luzon (Bontoc, Lepanto, Benguet), Merrill ry99, 4398, Elmer 639.2. On open grassy slopes, altitude 1,100 to $1,600 \mathrm{~m}$.

Forma BISPICULATA Hack. in Philip. Journ. Sci. 1 (1906) Suppl. 269; Merr. op. cit. 339.

Luzon (Benguet), Merrill 4298. On open slopes, altitude about 1,350 m. The var. lachnatherus Hack. in tropical Australia, other varieties in. tropical Africa and Ceylon.

Local names: Páuai (Ig.); taáu (Bon.).

ANDROPOGON FRAGILIS R. Br. Prodr. (1810) 202, var. LUZONIENSIS Hack. in Philip. Journ.'Sci. 1 (1906) Suppl. 267; Merx. op. cit. 335.

Luzon (Bontoc, Benguet), Merrill 4468, 4386. In dry open grasslands at medium altitudes.

Var. MALAYANUS Merr. var. nov. A typo differt spiculis pedicellisque omnino glabris.

Andropogon fragilis Merr. in Philip. Journ. Sci. 1 (1906) Suppl. 335, Fl. Manila (1912) 82, haud R. Br.

Luzon (Bontoc, Ifugao, Pangasinan, Rizal), Mindanao (Lanao), Merrill 8012, Phil. Pl. 156, Loher 1864, B. S. 19977 McGregor. In dry open grasslands at low and medium altitudes. New Guinea, King 1002. The species in southern China, New Guinea, and in tropical Australia, a variety also in China.

ANDROPOGON GRYLLUS Linn. Amoen. Acad. 4 (1759) 332; Hack. in DC. Monog. Phan. 6 (1889) 550, var. PHILIPPINENSIS Merr. in Philip. Journ. Sci. 14 (1919) 368.

Chrysopogon gryllus Trin.; F.-Vill. Novis. App. (1882) 316 (prob.).

Panay, B. S. 30964 Ramos \& Edaño. On bowlders and ledges along streams at low altitudes. The variety endemic, the species, in various forms, southern Europe to Australia.

ANDROPOGON HALEPENSIS (Linn.) Brot. Fl. Lusit, 1 (1804) 89, var. PROPINQUUS (Kunth) Merr. in Philip. Journ. Sci. 1 (1906)

Suppl. 336, Fl. Manila (1912) 84.

Andropogon propinquus Kunth Enum. 1 (1833) 502.

Andropogon affinis Presl Rel. Haenk 1 (1828) 343, non R. Br.

Sorghum halepense Pers. var. muticum Nees in Hook. Journ. Bot.

Kew Miscel. 2 (1850) 99.

Sorghum halepense Miq. Fl. Ind. Bat. 3 (1857) 503; F.-Vill. Novis. App. (1882) 317; Vidal Phan. Cuming. Philip. (1885) 158; Rev. Pl. Vasc. Filip. (1886) 184, haud Pers.

Andropogon sorghum Pers. subsp. halepensis Hack. var. propinquus

Hack. in DC. Monog. Phan. 6 (1889) 503.

Batan Islands and northern Luzon to Mindanao, in most or all islands and provinces, often common. The species pantropic, the variety Ceylon to New Guinea.

Local names: Aroró (Bik.); baku (If.)-batad-batádan (Tag.); dalapak (Sub.); gingai (Sul.); giñ̄ía (Bag.); riaría (Sul.); sikal (Ibn.); ugínaî (Buk.). 
ANDROPOGON INTERMEDIUS R. Br. Prodr. (1810) 202; Hack. in DC. Monog. Phan. 6 (1889) 485; Merr. in Philip. Journ. Sci. 1 (1906) Suppl. 335 var. HAENKEI (Presl) Hack. in DC. Monog. Phan. 6 (1889) 486; Pilger in Perk. Frag. Fl. Philip. (1904) 139; Merr. in Philip. Journ. Sci. 1 (1906) Suppl. 335, Fl. Manila (1912) 83. Andropogon haenkei Presl Rel. Haenk. 1 (1828) 340; Kunth Enum. 1 (1833) 501; Miq. Fl. Ind. Bat. 3 (18̄̄7) 489; F.-Vill. Novis. App. (1882) 316.

Rhaphis stricta Nees in Hook. Journ. Bot. Kew Miscel. 2 (1850) 99;

Walp. Ann. 3 (1853) 803.

Andropogon leptanthus Steud. Syn. Pl. Glum. 1 (1854) 391; Miq. Fl.

Ind. Bat. 3 (1857) 389 ; Vidal Phan. Cuming. Philip. (1885) 158, Rev. Pl. Vasc. Filip. (1886) 291.

Luzon (Cagayan, Ilocos Norte, Union, Nueva Vizcaya, Nueva Ecija, Pangasinan, Rizal, Batangas), Negros, Mindanao, Cuming 1400, Merrill 6734, 313, 5456, Phil. Pl. 134, 1842, B. S. 30072 Fénix, 8246, 7830, 7658, $7918,7916,1426$ Ramos. In open grasslands at low and medium altitudes. The species in China and Australia, the variety in China and Ceylon, other varieties in Asia and Africa.

Local names: Sálai (Pang.); sálai-párang (Tag.).

ANDROPOGON MICRANTHUS Kunth Rev. Gram 1 (1829) 165; Enum.

1 (1833) 504; Hack. in DC. Monog. Phan. 6 (1889) 488 (var. genuinus); Pilger in Perk. Frag. Fl. Philip. (1904) 140; Merr. in Philip. Journ. Sci. 1 (1906) Suppl. 336.

Andropogon alternans Presl Rel. Haenk. 1 (1828) 342.

Chrysopogon violascens Trin. in Mém. Acad. St. Pétersb. VI 2(1833) 319.

Rhaphis villosulus Nees in Hook. Journ. Bot. Kew Miscel. 2 (1850) 102.

Rhaphis microstachys Nees 1. c., var.

Chrysopogon villosulus Vidal Phan. Cuming. Philip. (1885) 158, Rev. Pl. Vasc. Filip. (1886) 291.

Andropogon parvispica Steud. Syn. Pl. Glum. 1 (1854) 397.

Chrysopogon parviflorus Benth. Fl. Austral. 7 (1878) 537; F.-Vill. Novis. App. (1882) 316.

Luzon (Ilocos Norte, Bontoc, Benguet, Nueva Vizcaya, Nueva Ecija, Zambales), Cuming 980, 1397, Merrill 118, 4434, 4703, Phil. Pl. 176, Elmer 6593, Williams 1959, B. S. 32948 Ramos, 32010 Santos, 26038 Fénix, 14198 McGregor, 8241 Ramos. In open grasslands chiefly at medium altitudes, ascending to $2,300 \mathrm{~m}$. Abyssinia to China through Malaya to tropical Australia.

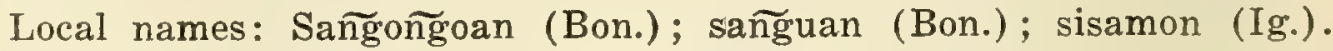

ANDROPOGON NARDUS Linn. Sp. Pl. (1753) 1046 var. TORTILIS (Presl) comb. nov.

Anthistiria tortilis Presl Rel. Haenk. 1 (1828) 347; Kunth Enum. 1 (1833) 483; F.-Vill. Novis. App. (1882) 317.

Andropogon hamatulus Nees in Hook. \& Arn. Bot. Beechy's Voy. (1841) 244.

Andropogon martyni Nees in Hook. Journ. Bot. Kew Miscel. 2 (1850) 99. 
Andropogon nardus Linn. var. hamatulus Hack. in DC. Monog. Phan. 6 (1889) 606; Pilger in Perk. Frag. Fl. Philip. (1904) 140; Merr. in Philip. Journ. Sci. 1 (1906) Suppl. 339, 3 (1908) Bot. 396.

Andropogon nardus Rolfe in Journ. Bot. 23 (1885) 216; Vidal Phan.

Cuming. Philip. (1885) 15̌8, Rev. Pl. Vasc. Filip. (1886) 291, non Linn.

Batan Islands, Luzon (Cagayan, Isabela, Ilocos Norte, Abra, Bontoc, Pangasinan, Zambales, Nueva Ecija), Cagayan Sulu, Merrill 530\%, Cuming 1000, B. S. 7893, 7793, 8047, 32888 Ramos, 10169 McGregor, F. B. 5858, 17037 Curran, 16461 Bacani. In open grasslands at low and medium altitudes. The variety in southern China, the species in India, Africa, and Malaya.

Local name: Balaniu (Bon.).

ANDROPOGON NITIDUS (Vahl) Kunth Rev. Gram. 1 (1829) 166; Merr. Fl. Manila (1912) 84.

Holcus nitidus Vahl Symb. 2 (1791) 102.

Sorghum nitidum Pers. Syn. 1 (1805) 101.

Andropogon fuscus Presl Rel. Haenk. 1 (1828) 342; Kunth Enum. 1 (1833) 503.

Surghum fuscum Miq. Fl. Ind. Bat. 3 (1857) 503; F.-Vill. Novis. App. (1882) 317; Vidal Phan. Cuming. Philip. (1885) 158, Rev. Pl. Vasc. Filip. (1886) 291.

Sorghum tropicum Nees var. muticum Nees in Hook. Journ. Bot. Kew Miscel. 2 (1850) 99.

Andropogon pedicellatus Steud. Syn. Pl. Glum. 1 (1854) 394; Miq.

Fl. Ind. Bat. 3 (1857) 488; F.-Vill. Novis. App. (1882)' 316.

Andropogon serratus Thunb. var nitidus Hack. in DC. Monog. Phan.

6 (1889) 521; Pilger in Perk. Frag. Fl. Philip. (1904) 140; Merr.

in Philip. Journ. Sci. 1 (1906) Suppl. 337, 3 (1908) Bot. 396.

Batan Islands, northern Luzon (Cagayan) to Mindanao, in most islands and provinces. In open grasslands at low arid medium altitudes.

Forma HIRSUTA Pilger in Perk. Frag. Fl. Philip. (1904) 140; Merr. in Philip. Journ. Sci. 1 (1906) Suppl. 337.

Luzon (Isabela), Merrill 13\%. In open grasslands at low altitudes. The form endemic, the species India to southern China and Formosa southward to Java.

ANDROPOGON PHILIPPINENSIS Merr. in Philip. Journ. Sci. 14 (1919) 367.

Luzon (Ilocos Norte), B. S. 32946, 32755 Ramos. On dry open rocky slopes at low altitudes. Endemic.

ANDROPOGON SANGUINEUS (Retz.) Merr. in Philip. Journ. Sci. 12 (1917) Bot. 101; op. cit. 14 (1919) 369.

Rottboellia sanguinea Retz. Obs. 3 (1783) 25.

Thelepogon sanguineus Spreng. Syst. 1 (1825) 299.

Andropogon pseudograya Steud. Syn. Pl. Glum. 1 (1854) 465.

Luzon (Ilocos Norte), Culion, B. S. 3277\%, 41310, 41329 Ramos. On open slopes at low altitudes. Southern China, India, Ceylon, and Madagascar. 
ANDROPOGON SERICEUS R. Br. Prodr. (1810) 201; Nees in Hook. Journ. Bot. Kew Miscel. 2 (1850) 99; Hack. in DC. Monog. Phan. 6 (1889) 575̃; Rolfe in Journ. Bot. 23 (1885) 216; Vidal Phan. Cuming. Philip. (1885) 158, Rev. Pl. Vasc. Filip. (1886) 291; Pilger in Perk. Frag. Fl. Philip. (1904) 140; Merr. in Philip. Journ. Sci. 1 (1906) Suppl. 338, Fl. Manila (1912) 82.

Andropogon tenuiculus Steud. Syn. Pl. Glum. 1 (1854) 371.

Heteropogon tenuiculus Miq. Fl. Ind. Bat. 3 (1857) 494; F.-Vill. Novis. App. (1882) 315.

Luzon (Benguet, Nueva Ecija, Pangasinan, Rizal), Cuming 1398, Merrill 266, Phil. Pl. 163, B. S. 4824 Ramos. In open grasslands at low altitudes. New Guinea, New Caledonia, Australia.

ANDROPOGON SERRATUS Thunb. Fl. Jap. (1784) 41; Hack. in DC. Monog. Phan. 6 (1889) 520; Merr. in Philip. Journ. Sci. 1 (1906) Suppl. 337, Fl. Manila (1912) 84.

Sorghum fulvum Beauv. Agrost. (1812) 164; F.-Vill. Novis. App. (1882) 317.

Luzon (Bontoc, Benguet, Rizal), Mindanao (Lanao, Davao, Bukidnon), Merrill 4297, Phil. Pl. 593, Elmer 5958, Clemens 597, Piper 471, B. S. 5764 Ramos. In open grasslands at low and medium altitudes. Japan and China through Malaya to Australia.

Local names: Girun (Bag.); taáu (Bon.); tígau (Mag.).

ANDROPOGON SORGHUM (Linn.) Brot. Fl. Lusit. 1 (1804) 88; Merr. in Philip. Journ. Sci. 1 (1906) Suppl. 336, Fl. Manila (1912) 84, Sp. Blancoanae (1918) 63 (var. vulgaris Hack.); Pilger in Perk. Frag. Fl. Philip. (1904) 140 (var. sativus Hack.); Usteri Beitr. Ken. Philip. Veg. (1905) 132 (var. niger Kunth).

Holcus sorghum Linn. Sp. Pl. (1753) 1047.

Holcus saccharatus Linn. Sp. Pl. (1753) 1047; Blanco Fl. Filip. (1837) 47, ed. 2 (1845) 32, ed. 3, 1 (1877) 58, t. 436.

Sorghum saccharatum Pers. Syn. 1 (1804) 101; F.-Vill. Novis. App. (1882) 317; Vidal Rev. Pl. Vasc. Filip. (1886) 291.

Sorghum vulgare Pers. Syn. 1 (1804) 101; Miq. Fl. Ind. Bat. 3 (1857) 502.

Andropogon sorghum Brot. subsp. sativus Hack. var. saccharatus Hack. in DC. Monog. Phan. 6 (1889) 505.

Several forms or varieties of sorghum are cultivated in the Philippines, but none are established. A native of Asia or Africa, now cultivated in all warm countries. Sorghum.

Local names: Bakau (Ibn.); bátad (Tag., Bik., Bis.); bátag (Tag.); bukákau (Ilk.); gau (If.); layagan (Sul.).

ANDROPOGON TRITICEUS R. Br. Prodr. (1810) 201; Hack. in DC.

Monog. Phan. 6 (1889) 588; Merr. in Philip. Journ. Sci. 1 (1906)

Suppl. 338.

Andropogon ischyranthus Steud. Syn. Pl. Glum. 1 (1854) 367.

Heteropogon ischyranthus Miq. Fl. Ind. Bat. 3 (1857) 493; F.-Vill.

Novis. App. (1882) 315.

Heteropogon insignis Thwaites Enum. Pl. Zeyl. (1859-64) 437; F.-Vill. Novis. App. (1882) 315. 
Luzon (Abra, Ilocos Norte, Pangasinan), Busuanga, Cuming 100\%, B. S. r216, 4900, 41269 Ramos, F. B. 1247\% Merritt \& Da.limg. In open grasslands at low or medium altitudes. Ceylon and Bruma to tropical Australia.

ANDROPOGON ZIZANIOIDES (Linn.) Urban Symb. Antil. 4 (1903) 79 ; Merr. in Philip. Journ. Sci. 7 (1912) Bot. 228, Fl. Manila (1912) 83, Sp. Blancoanae (1918) 62.

Phalaris zizanioides Linn. Mant. 2 (1771) 183.

Andropogon squarrosus Hack. in DC. Monog. Phan. 6 (1889) 542; Merr, in Philip. Journ. Sci. 1 (1906) Suppl. 337, non Linn. f. (cf. Stapf in Kew Bull. (1906) 348.

Andropogon muricatus Retz. Obs. 3 (1783) 43, 5 (1789) 20; F.-Vill. Novis. App. (1882) 316.

Andropogon nardus Blanco Fl. Filip. (1837) 39, ed. 2 (1845) 27, ed. 3, 1 (1877) 51, non Linn.

Vetiveria zizanioides Stapf in Kew Bull. (1906) 346; Camus in Bull. Mus. Hist. Nat. Paris 25 (1919) 673.

Batan Islands, Luzon (Amburayan, Rizal, Cavite), Merrill Sp. Blancoance 355, Phil. Pl. 118, Loher 7213. Planted about rice-paddy banks, often abundant; certainly an introduced plant in the Philippines. A native of tropical Asia, now pantropic. Vetiver.

Local names: Amóora (C. Bis.) ; amóras (Ilk.) ; anías de móras (Pamp.) ; giron (P. Bis.) ; ílib (Pamp.); móra (Bik., Bis.); móras (Tag., Bis., Bik.) ; móro (Tag.) ; narawastu (Sul.) ; raíz de móras (Sp.) ; rimódas (P. Bis.) ; rimóra (Sbl.); rimóras (Bik.); tres-móras (Bis.).

\section{EXCLUDED SPECIES}

ANdropogon ERIOSTaChys Presl Rel. Haenk. 1 (1830) 339; Kunth Enum. 1 (1833) 496; Miq. Fl. Ind. Bat. 3 (1857) 487; F.-Vill. Novis. App. (1882) 316.

"Hab. in insulis Philippinis" Presl. This is the tropical American Andropogon ternatus Nees var. eriostachys (Presl) Hack. in DC. Monog. Phan. 6 (1889) 425. The type was one of the numerous Malaspina Expedition plants collected by Presl but erroneously localized as Philippine.

Heteropogon teneluus Schultes; F.-Vill. Novis. App. (1882) 315.

\section{ASTHENOCHLOA Büse}

ASTHENOCHLOA TENERA Büse in Miq. Pl. Jungh. (February, 1854) 367. Andropogon leptos Steud. Syn. Pl. Glum. 1 (1854) 397; Merr. in Philip. Journ. Sci. 1 (1906) Suppl. 268, 337, 4 (1909) Bot. 250.

Garnotiella phitippinensis Stapf in Hook. Ic. IV 5 (1896) t. 2494; Merr. in Philip. Journ. Sci. 1 (1906) Suppl. 374.

Garnotiella leptos Stapf in Kew Bull. (1910) 302.

Luzon (Benguet), Panay, Merrill 4322, Phil. Pl. 154, B. S. 5318 Ramos. On steep open slopes up to an altitude of $1,400 \mathrm{~m}$. Originally described from "Andor," a locality I have not been able to locate; perhaps "Ind. or." intended. Java. A monotypic genus.

The alliance of the genus is with Andropogon, not with Garnotia.

$183036-4$ 


\section{THEMEDA Forskål}

THEMEDA GiganteA (Cav.) Hack. in DC. Monog. Phan. 6 (1889) 670 (var. genuina Hack.); Pilger in Perk. Frag. Fl. Philip. (1904) 140 ; Merr. in Philip. Journ. Sci. 1 (1906) Suppl. 340, 3 (1908) Bot. 396,5 (1910) Bot. 326.

Anthistiria gigantea Cav. Ic. 5 (1799) 36, t. 458; Willd. Sp. Pl. $4^{2}$ (1805) 902; F.-Vill. Novis. App. (1882) 317; Vidal Phan. Cuming. Philip. (1885) 159, Rev. Pl. Vasc. Filip. (1886) 292.

Androscepia gigantea Brongn. Bot. in Duperr. Voy. (1829) 78; Miq. Fl. Ind. Bat. 3 (1857) 506; Nees in Hook. Journ. Bot. Kew Miscel. 2 (1850) 99.

Perobachne secunda Presl Rel. Haenk. 1 (1828) 348, t. 48; Kunth Enum. 1 (1833) 485; Miq. Fl. Ind. Bat. 3 (1857) 507; F.-Vill. Novis. App. (1882) 317.

Batan Islands to Mindanao, in most or all islands and provinces. In open grasslands on dry steep slopes at low and medium altitudes. India to China, Malaya, New Caledonia, and Australia.

Local names: Girun (Bag.) ; mañambit (Sub.) ; sai-gaja (Sul.) ; salaisálai (Tag.) ; tanhád (Iv.) ; tanik (Bon.) ; taurar (Ilk.) ; teldak (Pang.); tulango (Tag.) ; talauyo (Tag.).

Var. VULPINA (Anders.) Hack. in DC. Monog. Phan. 6 (1889) 670; Merr. in Philip. Journ. Sci. 1 (1906) Suppl. 340.

Anthistiria vulpina Anders. in Nov. Act. Upsal. III 2 (1856) 423.

Anthistiria arundinacea Rolfe in Journ. Bot. 23 (1885) 216; Vidal

Phan. Cuming. Philip. (1885) 159, Rev. Pl. Vasc. Filip. (1886) 292, haud Roxb.

Luzon (Cagayan), Cuming 1272 and numerous other specimens. India. Scarcely distinguishable from the species.

Subsp. INTERMEDIA Hack. var. DUBIA Hack. in DC. Monog. Phan. 6 (1889) 675; Merr. in Philip. Journ. Sci. 1 (1906) Suppl. 341.

Mindanao (Misamis), Cuming 1609.

The species is variable in its spikelet characters, a number of forms being represented in the Philippines.

THEMEDA TRIANDRA Forsk. Fl. Aegypt.-Arab. (1775) 178; Merr. in Philip. Journ. Sci. 1 (1906) Suppl. 340, Fl. Manila (1912) 81.

Themeda forskalii Hack. in DC. Monog. Phan. 6 (1889) 659; Pilger in Perk. Frag. Fl. Philip. (1904) 140.

Anthistiria ciliata Cav. Ic. 5 (1799) 36, t. 459; F.-Vill. Novis. App. (1882) 317; Nees in Hook. Journ. Bot. Kew Miscel. 2 (1850) 99. (var. subglabra Nees), non Linn. f.

Anthistiria depauperata Anders. in Nov. Act. Upsala III 2 (1856)

343; F.-Vill. Novis. App. (1882) 317.

Anthistiria barbata Presl Repert. 1 (1833) 305, non Desf.

Apluda barbata Llanos in Mém. Acad. Cienc. Madr. 4 (1858) 497;

Fl. Filip. ed. 3, $4^{1}$. (1880) 99 (misprint for Anthistiria barbata).

Batan Islands, Luzon (Cagayan, Isabela, Nueva Vizcaya, Ilocos Norte, Abra, Bontoc, Benguet, Pangasinan, Nueva Ecija, Rizal, Albay), Mindoro, Busuanga, Semirara, Mindanao. In open grasslands at low and medium 
altitudes, ascending to 2,300 m. Frequently abundant and gregarious over large areas. Tropical Asia and Africa through Malaya to Australia.

Local names: Punáu (Bon.); samsamóng (Ilk.); táau (Ig.) ; usímau (Ilk.).

\section{EXCLUDED SPECIES}

Anthistiria Junghuhniana Nees in Hook. Journ. Bot. Kew Miscel. 2 (1850) 99.

This is credited to the Philippines by Nees, on the basis of Cuming 2431, which was from Sumatra, not from the Philippines. It is a synonym of Themeda frondosa (R. Br.) Merr. (T. arguens Hack., not Stipa arguens Linn.), which does not occur in the Philippines.

\section{PEROTIS Aiton}

PEROTIS INDICA (Linn.) O. Kuntze Rev. Gen. Pl. (1891) 787; Merr. in Philip. Journ. Sci. 1 (1906) Suppl. 341.

Anthoxanthum indicum Linn. Sp. Pl. (1753) 28.

Perotis latifolia Ait. Hort. Kew. 1 (1789) 85; - F.-Vill. Novis. App. (1882) 313.

Perotis glabrata Steud. Syn. Pl. Glum. 1 (1854) 186; Miq. Fl. Ind.

Bat. 3 (1857) 479; F.-Vill. Novis. App. (1882) 313; Vid. Phan.

Cuming. Philip. (1885) 158, Rev. Pl. Vasc. Filip. (1886) 289.

Perotis hordeiformis Nees in Steud. Nomencl. ed. 2, 2 (1841) 306,

Hook. Journ. Bot. Kew Miscel. 2 (1850) 102.

Luzon (Cagayan, Nueva Ecija), Mindanao (Agusan), Cuming 1399, Elmer 138\%,$B$. S. 7911 Ramos. In open grasslands at low and medium altitudes. Tropical Asia and Africa.

Local name: Bañgat (Mbo.).

PEROTIS RARA R. Br. Prodr. (1810) 172; Miq. Fl. Ind. Bat. 3 (185̄7) 479; F.-Vill. Novis. App. (1882) 314; Merr. in Philip. Journ. Sci. 1 (1906) Suppl. 341.

Xystidium barbatum Presl Rel. Haenk. 1 (1828) 228; Scribn. in

Rept. Mo. Bot. Gard. 10 (1899) 55.

Xystidium maritimum Trin. Fund. Agrost. (1820) 102, t. 2.

Luzon (Ilocos Norte, Lepanto, Amburayan, Pangasinan, Zambales), Mindoro, Merrill 4447, Phil. Pl. 152, F. B. 997.9 Merritt, 5859 Curran. On dry open slopes at low and medium altitudes. Australia.

\section{ZOISIA Willdenow}

(Zoysioa auct.)

zoIsiA MATRELlA (Linn.) Merr. in Philip. Journ. Sci. 7 (1912) Bot. 230, F1. Manila (1912) 85.

Agrostis matrella Linn. Mant. 2 (1771) 185.

Zoisia pungens Willd. in Ges. Naturf. Fr. Neue Schrift 3 (1801) 441; F.-Vill. Novis. App. (1882) 313; Pilger in Perk. Frag. Fl. Philip. (1904) 140; Merr. in Philip. Journ. Sci. 1 (1906) Suppl. 342.

Osterdammia matrella O. Kuntze Rev. Gen. Pl. (1891) 781.

Northern Luzon to Palawan, in most islands, occurring in open places chiefly along or near tidal streams. Tropical Asia and Africa through Malaya to Australia and western Polynesia.

Local name: Barit-baritan (Tag.). 


\section{ARUNDINELLA Raddi}

ARUNDINELLA AGROSTOIDES Trin. Sp. Gram. Ic. (1836) 23, t. 265 ;

F.-Vill. Novis. App. (1882) 318; Merr. Fl. Manila (1912) 85.

Luzon (Ilocos Norte, Pangasinan, Rizal), Merrill 7399, Phil. Pl. 594, Loher 721\%, F. B. 12482 Merritt \& Darling. In open grasslands and on ledges along small streams at low altitudes. India to Burma.

Var. CILIATA (Roxb.) Hook. f. Fl. Brit. Ind. 7 ,(1897) 71; Hack. in

Philip. Journ. Sci. 1 (1906) Suppl. 268; Merr. op. cit. 243.

Holcus ciliatus Roxb. Fl. Ind. 1 (1820) 321.

Arundinella ciliata Nees in Wall. Cat. (1829) No. 1668.

Luzon (Ilocos Sur, Bontoc, Benguet), Merrill 4328, Williams 1961, F. B. 15940 Bacani, 15671 Merritt \& Darling. On open slopes, about cliffs, etc., altitude 800 to $1,600 \mathrm{~m}$. Range of the species.

ARUNDINELLA MILIACEA Nees in Hook. Journ. Bot. Kew Miscel. 2 (1850)

102; Vidal Phan. Cuming. Philip. (1885) 158.

Arundinella nepalensis F.-Vill. Novis. App. (1882) 318; Vidal Rev.

Pl. Vasc. Filip. (1886) 289; Merr. in Philip. Journ. Sci. 1 (1906)

Suppl. 343, non Trin.

Arundinella hispida Hack. subsp. humilior Hack. in Bull. Herb. Boiss.

II 4 (1904) 527, Allgem. Bot. Zeitschr. 12 (1906) 179.

Arundinella filiformis Jan. ex Mez in Fedde Repert. 17 (1921) 85.

Arundinella caespitosa Jan. 1. c.

Luzon (Apayao, Bulacan, Rizal, Laguna), Panay, Merrill 2262, Phil. Pl. 161, Cuming 667, B. S. 30966 Ramos \& Edaño, 6700 Robinson, 35453 Martelino \& Edaño, 19286 Reillo, 28251 Fénix, 2101 Ramos, 30 Foxworthy, F. B. ${ }^{7 \gamma g}$ Curran, Kneucker. Gram. Exsic. 606. On ledges and banks along swift-running streams at low altitudes. Formosa.

The more-recent collections have been distributed as A. hispida (HBK.) O. Kuntze, following Hackel's identification.

ARUNDINELLA PUBesCenS Merr. \& Hack. in Philip. Journ. Sci. 2 (1907) Bot. 419 .

Palawan, B. S. 856 Foxworthy. On river banks at low altitudes. Endemic.

ARUNdinella SetosA Trin. Diss. 2 (1824) 63, Gram. Pan. (1826) 245; F.-Vill. Novis. App. (1882) 318; Merr. in Philip. Journ. Sci. 1 (1906) Suppl. 342, 5 (1910) Bot. 326.

A mundinella stricta Nees in Hook. Journ. Bot. Kew Miscel. 2 (1850)

102; Walp. Ann. 3 (1853) 804; Miq. Fl. Ind. Bat. 3 (1857) 520.

Danthonia luzonensis Steud. Syn. Pl. Glum. 1 (1854) 245; Miq. Fl.

Ind. Bat. 3 (1857) 427; F.-Vill. Novis. App. (1882) 319; Vidal

Phan. Cuming. Philip. (1885) 159, Rev. Pl. Vasc. Filip. (1886) 292.

Arundinella setosa Trin. var. stricta Pilger in Perk. Frag. Fl. Philip.

(1904) 140.

Luzon (Cagayan, Isabela, Bontoc, Benguet, Nueva Vizcaya, Zambales, Rizal), Mindoro, Mindanao (Bukidnon). In open grasslands, on cliffs, etc., at low and medium altitudes, ascending to $2,000 \mathrm{~m}$. India to China through Malaya to New Guinea.

Local name: Salai-sálai (Sbl.). 


\section{THYSANOLAENA Nees}

THYSANOLAENA MAXIMA (Roxb.) O. Kuntze Rev. Gen. Pl. (1891) 794; Pilger in Perk. Frag. Fl. Philip. (1904) 141; Merr. in Philip. Journ. Sei. 1 (1906) Suppl. 343.

Agrostis maxima Roxb. Fl. Ind. 1 (1820) 319.

Thysanolaena agrostis Nees in Edinb. New Philos. Journ. 18 (1835) 180.

Thysanolaena acarifera Arn. \& Nees in Nov. Act. Acad. Nat. Cur. 19 (1843) Suppl. 1: 181; Vidal Rev. Pl. Vasc. Filip. (1886) 289.

Luzon (Ilocos Norte, Apayao, Bontoc, Benguet, Nueva Vizcaya, Nueva Ecija, Bulacan, Zambales, Bataan, Laguna, Tayabas, Sorsogon), Mindoro. Palawan, Mindanao. On banks of ravines, slopes, about cliffs, etc., at low and inedium altitudes, ascending to $1,600 \mathrm{~m}$. India through Malaya to New Guinea.

Local names: Bugúbui (Pamp.); buybúi (Ilk.); eagadu (Bon.); gatbó (Bik.); lása (Tag.) ; tagádeu (Bon.) ; tagisa (Sub.); tambú (Tag.); talankaran $\left(\mathrm{Ig}_{\circ}\right)$.

\section{DIGITARIA Scopoli}

DIGITARIA CHINENSIS Nees in Hook. \& Arn. Bot. Beechey Voy. (1841) 231.

Paspalum filiculme Nees ex Thwaites Enum. Pl. Zeyl. (1859-64) 358. Digitaria violascens Merr. in Philip. Journ. Sci. 1 (1906) Suppl. 347, 3 (1908) Bot. 396, Fl. Manila (1912) 88, non Link.

Batan Islands and northern Luzon to Palawan and Mindanao, in most islands and provinces, Merrill 4148, 9388, 7107, 767\%, 4788, Phil. Pl. 174, B. S. 21715, 14524, 13939, 8250 Ramos. In open grasslands at low and medium altitudes. India to China and Malaya.

Local names: Timi (Bon.); tumi (Bon.); ualisíbis (Iv.).

DIGITARIA CORYMBOSA (Roxb.) comb. nov.

Panicum corymbosum Roxb. Hort. Beng. (1814) 7, nomen nudum, Fl. Ind. 1 (1820) 292.

Paxicum microbachne Presl Rel. Haenk. 1 (1828) 298; Kunth Enum. 1 (1833) 81.

Digitaria consanguinea Merr. in Philip. Journ. Sci. 1 (1906) Suppl. 347, 3 (1908) Bot. 396, Fl. Manila (1912) 88, Sp. Blancoanae (1918) 64, non Gaudich.

Panicum sanguinale Rolfe in Journ. Bot. 23 (1885) 216; Vidal Phan. Cuming. Philip. (1885) 157, non Linn.

Digitaria lanosa Llanos Fragm. Pl. Filip. (1851) 28; F.-Vill. \& Naves in Blanco Fl. Filip. ed. 3, $4^{x}$ (1880) 19.

Panicum gaudichaudii F.-Vill. Novis. App. (1882) 311, non Kunth.

Panicum pruriens Trin. var. glabrum Nees in Hook. Journ. Bot. Kew Miscel. 2 (1850) 97.

Throughout the Philippines at low and medium altitudes in open waste places, old clearings, etc., usually common, Merrill 4230, 534\%, 7368, 9276 , Sp. Blancoanae 78\%, Phil. Pl. 126, Elmer 18216, 16818, 16417, 9570, B. S. $12036,13334,32736,14746$ Ramos. India to southern China and Formosa through Malaya to Samoa.

Local names: Babanai (Sub.); balíli (C. Bis.) ; balisíbis (Iv.); balud- 
gañgan (Tag.) ; barírid (Bag.) ; gasa (Kuy.); halos (Tag.); maya-máya (S. L. Bis.) ; maraséda (Ilk.) ; sagísi (Ilk.).

This species has been confused with Digitaria sanguinalis Scop. and by me with $D$. consanguinea Gaudich. It is distinguished by its almost obsolete first glume and short second glume, the latter rarely more than one-fourth as long as the spikelet.

DIGITARIA HETERANTHA (Nees \& Mey.) var. PACHYRHACHIS (Hack.) comb. nov.

Panicum heteranthum Nees \& Mey. var. pachyrhachis Hack. in Philip. Journ. Sci. 3 (1908) Bot. 167.

Palmas, Lumbucan, Merrill 5355, 5276. Along sandy seashores. The variety endemic, the species in the Malay Peninsula, Java, China, and Formosa.

DIGITARIA LongiflorA (Gmel.) Pers. Syn. 1 (1805) 85; Merr. in Philip. Journ. Sci. 1 (1906) Suppl. 347, 5 (1910) Bot. 326, Fl. Manila (1912) 88, Sp. Blancoanae '(1918) 64.

Panicum longiflorum Gmel. Syst. (1788) 158.

Panicum parvulum Trin. in Mém. Acad. St. Pétersb. VI 3 (1835) 205; Mez in Perk. Frag. Fl. Philip. (1904) 142.

Paspalum brevifolium Flügge Monogr. (1810) 150; F.-Vill. Novis. App. (1882) 310.

Paspalum fuscescens Presl Rel. Haenk. 1 (1828) '213, ex descr.

Syntherisma fuscescens Scribn. in Rept. Mo. Bot. Gard. 10 (1899) 49, t. 10.

Paspalum fasciculatum Llanos Frag. Pl. Filip. (1851) 23; F.-Vill. \& Naves in Blanco Fl. Filip. ed. $3,4^{12}$ (1880) 16, non Willd.

Northern Luzon to Mindanao, in most islands and provinces, Elmer 16733, Merrill 249, 331, 1129, 6518, Phil. Pl. 167, Sp. Blancoanae 1022, B. S. 32751, 33448 Ramos, 32009 Fénix, 10911 McGregor. In open grasslands, sea level to an altitude of $1,600 \mathrm{~m}$. Warmer parts of the Old World.

Local name: Sasámon (Ig.).

DIGITARIA PEDICELLARIS (Trin.) Prain Bengal Pl. (1903) 1182; Merr. in Philip. Journ. Sci. 1 (1906) Suppl. 348, Fl. Manila (1912) 98. Paspalum pedicellare Trin. ex Steud. Nomencl. ed. 2, 2 (1840) 272. Panicum pedicellare Hack. in Philip. Journ. Sci. 1 (1906) Suppl. 268. Panicum puberulum Mez in Perk. Frag. Fl. Philip. (1904) 143, non (?) Kunth.

Luzon (Nueva Vizcaya, Nueva Ecija, Pampanga, Rizal, Bataan), Culion, Merrill 4225, 3164, 105, Phil. Pl.57\%, B. S. 26273 Ramos \& Edaño, Williams 93. In open grasslands at low and medium altitudes, ascending to $850 \mathrm{~m}$. India to Burma.

DIGITARIA SANGuINAlis (Linn.) Scop. Fl. Carn. ed. 2, 1 (1772) 52; Vidal Rev. Pl. Vase. Filip. (1886) 286; Mez in Perk. Frag. Fl. Philip. (1904) 143; Merr. in Philip. Journ. Sci. 1 (1906) Suppl. 346, Fl. Manila (1912) 88 (var. australis Thw.).

Panicum sanguinale Linn. Sp. Pl. (1753) 57.

Digitaria inaequalis Spreng. Syst. 1 (1825) 271.

Digitaria biformis Willd. Enum. (1809) 92. 
Digitaria radicosa Miq. Fl. Ind. Bat. 3 (1857) 437.

Panicum radicosum Presl Rel. Haenk. 1 (1828) 297; F.-Vill. Novis. App. (1882) 311.

Paspalum inaequale Link Enum. Hort. Berol. 1 (1821) 103; Kunth Enum. 1 (1833) 48; F..Vill. Novis. App. (1882) 310.

Syntherisma sanguinalis Dulac Fl. Hautes Pyr. (1867) 77.

Widely scattered in the Philippines in open waste places, $B$. S. 273.96, 7258, 7092 Ramos, 3681 Fénix, 18737 Otanes, Merrill 7258, Phil. Pl. 114, Williams 1172. All warm countries.

Local names: Dibutbut (Iv.); pagpagai (Bon.); saka-sáka (Ilk.)。

Var. FIM BRIATA (Link.) Stapf in Prain Fl. Trop. Afr. 9 (1919) 440.

Digitaria fimbriata Link. Hort. Berol. 1 (1821) 226.

Digitaria ciliaris Merr. in Philip. Journ. Sci. 5 (1910) Bot. 168, Fl. Manila (1912) 87, non ? Pers.

Panicum ciliare Retz. Obs. 4 (1786) 16 ?

Paspalum sanguinale Lam. var. ciliare Hook. f. Fl. Brit. Ind. 7 (1897) 15.

Northern Luzon to Mindanao, Merrill 40, 330, Phil. Pl. 147, B. S. 7661 Ramos, 2249 Mearns. In open waste places. Pantropic.

Var. AUSTRALIS (Spreng.).

? Panicum australe Spreng. Syst. 1 (1825) 309.

Panicum sanguinale Linn. var. australe Thwaites Enum. Pl. Zeyl. (1864) 358.

? Panicum striatum R. Br. Prodr. (1810) 192, non Lam.

Throughout the Philippines at low and medium altitudes, in thickets, waste places, etc., Merrill 529, 89, 5359, 7259, 9787, Phil. Pl. 578, Elmer $5855,1514 \%$. India through Malaya to Australia.

This identification follows Hackel, but I am by no means certain that our form is the same as the one originally described by $R$. Brown.

\section{DOUBTFUL AND EXCLUDED SPECIES}

Panicum elythroblepharum Steud.; F.-Vill. Novis. App. (1882) 311.

Panicum stipatum Presl Rel. Haenk. 1 (1828) 297; F.-Vill. Novis. App. (1882) 311.

"Hab. in Mexico, Luzon?" Presl. This is the American Digitarla setosa Desv. fide Scribner in Rept. Mo. Bot. Gard. 10 (1899) 49.

PASPAlum FILIForme Sw.; Presl Rel. Haenk. 1 (1828) 297; F.-Vill. Novis. App. (1882) 310.

"Hab. in Luzonia ? Mexico" Presl. This is the American Digitaria filiforme Delile fide Scribner in Rept. Mo. Bot. Gard. 10 (1899) 49.

Paspalum Molle Presl Rel. Haenk. 1 (1828) 213 = Paspalum mollicomum Kunth Enum. 1 (1833) 310; Miq. Fl. Ind. Bat. 3 (1857) 433; F.-Vill.

Novis. App. (1882) $310=$ Syntherisma molle Scribn. in Rept. Mo.

Bot. Gard. 10 (1899) 50.

"Hab. in Luzonia" Presl. This was considered by Scribner to be a valid species, but Presl's description does not apply to any Philippine form known to me and I suspect that the type was an American, not a Philippine plant. 
PASPAlum Fuscum Presl Rel. Haenk. 1 (1828) 214; Kunth Enum. 1 (1833) 46; F.-Vill. Novis. App. (1882) $310=$ Syntherisma fusca Scribn. in Rept. Mo. Bot. Gard. 10 (1899) 49, t. 11.

"Hab. in Luzonia ? in Peruviae montanis huanoccensibus ? Mexico?" Presl. From the figure given by Scribner, drawn from a cotype of Presl's species, this does not appear to represent any known Philippine species. The type was probably from America.

\section{PASPALUM Linnaeus}

PASPALUM CONJUGATUM Berg. in Act. Helvet. 7 (1772) 129, t. 8; Mez in Perk. Frag. Fl. Philip. (1904) 141; Merr. in Govt. Lab. Publ. (Philip.) 6 (1904) 28, Philip. Journ. Sci. 1 (1906) Suppl. 345, Fl. Manila (1912) 87.

Throughout the Philippines in the settled areas, about towns, along trails, streams, etc., often very abundant. A native of tropical America, now pantropic.

Local names: Bantótan (Mbo.) ; kauatkáuat (C. Bis.) ; laau-láau (Tag.).

PASPALUM LONGIFOLIUM Roxb. Hort. Beng. (1814) 7, nomen nudum, Fl. Ind. 1 (1820) 283; Mez in Perk. Frag. Fl. Philip. (1904) 141; Merr. in Philip. Journ. Sci. 1 (1906) Suppl. 345, Fl. Manila (1912) 86.

Paspalum flexuosum Klein in Presl Rel. Haenk. 1 (1828) 216; Kunth Enum. 1 (1833) 54; Miq. Fl. Ind. Bat. 3 (1857) 433; F.-Vill. Novis. App. (1882) 310.

Luzon (Cagayan, Benguet, Bontoc, Nueva Vizcaya, Bulacan, Rizal, Laguna, Albay), Polillo, Palawan, Bohol, Mindanao,.Weber 1063, Merrill 9303, Phil. Pl. 1956, B. S. 26002, 26675 Fénix, 21684, 14512, 29504, 10979 Ramos. In open, usually rather wet lands at low and medium altitudes. India to Malaya.

Local name: Taltal-likód (Ilk.).

Var. TRICHOCOLEUM Hack. in Philip. Journ. Sci. 3 (1908) Bot. 167.

Mindoro, Mindanao (Surigao), B. S. 941 Mangubat, Merrill 5455. In open level grasslands at low altitudes. Endemic.

PASPALUM SCROBICULATUM Linn. Mant. 1 (1767) 29; Nees in Hook. Journ. Bot. Kew Miscel. 2 (1850) 97; F.-Vill. Novis. App. (1882) 310; Vidal Phan. Cuming. Philip. (1885) 156, Rev. Pl. Vasc. Filip. (1886) 286; Mez in Perk. Frag. Fl. Philip. (1904) 141; Merr. in Philip. Journ. Sci. 1 (1906) Suppl. 344, Fl. Manila (1912) 86, Sp. Blancoanae (1918) 63.

Paspalum cartilagineum Presl Rel. Haenk. 1 (1828) 216; Miq. Fl. Ind. Bat. 3 (1857) 432; F.-Vill. Novis. App. (1882) 310; Scribn. in Rept. Mo. Bot. Gard. 10 (1899) 49.

Paspalum kora Willd. Sp. Pl. 1 (1797) 332; Presl Rel. Haenk. 1 (1828) 216.

Paspalum villosum Blanco Fl. Filip. (1837) 40, ed. 2 (1845) 28, ed. 3, 1 (1877) 53, non Thunb.

Paspalum sumatrense Roth Nov. Pl. Sp. (1821) 35; Llanos Frag. Pl. Filip. (1851) 22 ; F.-Vill. \& Naves in Blanco Fl. Filip. ed. $3,4^{1}$ (1880) 15; F.-Vill. Novis. App. (1882) 310. 
Paspalum thunbergii Kunth. ex Steud. Syn. Pl. Glum. 1 (1854) 28; Mez in Perk. Frag. Fl. Philip. (1904) 141.

Throughout the Philippines, in open grasslands at low and medium altitudes, ascending to $1,500 \mathrm{~m}$; common and somewhat variable. I'antropic.

Local names: Ar̃gangsúg (Ig.) ; bias-biásan (S. L. Bis.) ; bubulis (Sub.) ; paragis (Tag.); sabuñg-sabúñgan (Tag.).

Var. PHILIPPINENSE Merr. in Philip. Journ. Sci. 1 (1906) Suppl. 345.

Luzon (Nueva Vizcaya, Rizal), Merrill 140a, B. S. 1449 Ramos. In open, rather wet grasslands at medium altitudes. Endemic.

Var. BISPICATUM Hack. ex Merr. Fl. Manila (1912) 86.

Luzon (Ilocos Norte, Ilocos Sur, Benguet, Rizal, Bataan), Panay, Merrill Phil. Pl. 576, B. S. 10083, 32975 McGregor, 27378 Ramos. In open grasslands at low and medium altitudes. Endemic.

Var: AURICUlatum (Presl) Merr. in Philip. Journ. Sci. 1 (1906) Suppl. 345, Fl. Manila (1912) 86.

Paspalum auriculatum Presl Rel. Haenk. 1 (1828) 217; Kunth Enum. 1 (1833) 54; Miq. Fi. Ind. Bat. 3 (1857) 432; F.-Vill. Novis. App. (1882) 310.

Luzon (Rizal), Palawan, Samar, Leyte, Mindanao, Merrill 5361, 7992, 820, Phil. Pl. 158, B. S. 843 Foxworthy, 24435 Ramos. In open rather wet grasslands at low altitudes. Tropical Asia and Africa.

PASPALUM VAGINATUM Sw. Prodr. Veg. Ind. Occ. (1788) 21; Stapf in Prain Fl. Trop. Afr. 9 (1919) 570.

Paspalum distichum F.-Vill. Novis. App. (1882) 310; Usteri Beitr.

Ken. Veg. Philip. (1905) 133; Merr. in Philip. Journ. Sci. 1 (1906)

Suppl. 346, Fl. Manila (1912) 87, non Linn.

Luzon (Amburayan, Union, Pampanga, Rizal, Laguna), Panay, Samar, Elmer 18140, Merrill 4253, 5229, 7116. In open wet lands, usually in places subject to the influence of salt or brackish water. Pantropic.

Local names: Pagetpet (Ilk.); panluilui (Pamp.).

\section{EXCLUDED SPECIES}

Paspalum Elegans Flügge; F.-Vill. Novis. App. (1882) 310.

PASPALUM CHRYSOTRICHUM Presl Rel. Haenk. 1 (1828) 211; Kunth Enum. 1 (1833) 64; Miq. Fl. Ind. Bat. 3 (1857) 431; F.-Vill. Novis. App. (1882) 310.

"Hab. in Luzonia." The localization is unquestionably erroneous. Presl's species is probably the same as Paspalum aureum HBK. of tropical America.

Paspalum boRyanum Presl Rel. Haenk. 1 (1828) 209; Kunth Enum. 1 (1833) 52; Miq. Fl. Ind. Bat. 3 (1857) 432; F.-Vill. Novis. App. (1882) 310.

"Hab. ad Sorzogon" Presl. This may prove to be a Digitaria, or again it may be an American species of Paspalum.

Paspalum Filiforme Sw.; F.-Vill. Novis. App. (1882) 310. 


\section{ERIOCHLOA Kunth}

ERIOCHLOA RAMOSA (Retz.) O. Kuntze Rev. Gen. Pl. (1891) 775; Hack. in Bull. Acad. Int. Bot. 16 (1906) 19; Merr. in Philip. Journ. Sci. 1 (1906) Suppl. 348, Fl. Manila (1912) 85, Sp. Blancoanae (1918) 64.

Milium ramosum Retz. Obs. 6 (1791) 22.

Paspalus annulatus Flügge Monogr. (1810) 133; Trin. Gram. Pan. (1826) 117, Sp. Gram. 2 (1829) t. 133.

Eriochloa annulata Kunth Enum. 1 (1833) 73; F.-Vill. Novis. App. (1882) 311; Perk. Frag. Fl. Philip. (1904) 141.

Piptantherum annulatum Presl Rel. Haenk. 1 (1828) 221.

Helopus laevis Trin. in Spreng. Neue Entdeck. 2 (1821) 49; Miq. Fl.

Ind. Bat. 3 (1857) 434.

Eriochloa punctata F.-Vill. Novis. App. (1882) 312, non Ham.

Milium zonatum Llanos Frag. Pl. Filip. (1851) 24; F.-Vill. and Naves in Blanco Fl. Filip. ed. $3,4^{1}$ (1880) 16.

Throughout the Philippines at low and medium altitudes, in open usually damp grasslands at low and medium altitudes. Pantropic.

Var. INVOLUCRATA Hack. ex Merr. in Philip. Journ. Sci. 1 (1906) Suppl. 349.

Mindoro, Bohol, Merrill 3333, B. S. 1247 McGregor. Habitat of the species and scarcely distinguishable from it. Endemic.

27. ISACHNE R. Brown

ISACHNE BENECKEI Hack. in Oesterr. Bot. Zeitschr. 51 (1901) 459, Govt. Lab. Publ. (Philip.) 35 (1906) 79; Merr. in Philip. Journ. Sci. 1 (1906) Suppl. 350, 5 (1910) Bot. 327.

Luzon (Ifugao, Bontoc, Lepanto, Benguet, Bataan, Pampanga), Merrill 4680, 5019, 3201, 4544, 4619, 4523, 4522, Phil. Pl. 1467, B. S. 19690, 1979; McGregor, 37736 Ramos \& Edaño, Williams 1193. In open wet places, borders of cold pools and sphagnum swamps, and of narrow forested ridges, altitude 800 to $2,400 \mathrm{~m}$. Java.

ISACHNE CONFERTA Merr. in Philip. Journ. Sci. 9 (1914) Bot. 261.

Luzon (Laguna), B. S. 14914 Ramos. On bowlders and ledges along streams at low or medium altitudes. Endemic.

ISACHNE DEBILIS Rendle ex Forbes \& Hemsl. in Journ. Linn. Soc. Bot. 36 (1904) 322; Hack. in Philip. Journ. Sci. 1 (1906) Suppl. 268; Merr. 1. c. 350 .

Isachne monticola Hack. in Govt. Lab. Publ. (Philip.) 35 (1906) 79, Philip. Journ. Sci. 1 (1906) Suppl. 268, non Büse.

Luzon (Abra, Ifugao, Bontoc, Benguet, Nueva Vizcaya, Nueva Ecija, Bataan, Laguna), Mindanao (Davao), Merrill 5815, 9680, 7675, Phil. Pl. 599, Elmer 5821, 18367. Along streams in damp ravines and on damp forested ridges, altitude 1,000 to $2,000 \mathrm{~m}$. Formosa.

This seems to be close to $I$. myosotis Nees.

ISACHNE DEPAUPERATA (Hack.) comb. nov.

Isachne beneckei Hack. forma depauperata Hack. ex Merr. in Philip. Journ. Sci. 1 (1906) Suppl. 350. 
Luzon (Lepanto, Benguet), Negros, Merrill 4545, 4489, 6978, 6504, B. S. 4249 Mearns. In the mossy forest and in damp thickets, altitude 1,700 to $2,200 \mathrm{~m}$. Endemic.

ISACHNE GLOBOSA (Thunb.) O. Kuntze Rev. Gen. Pl. (1891) 778.

Milium globosum Thunb. Fl. Jap. (1784) 49.

Isachne australis R. Br. Prodr. (1812) 196; F.-Vill. Novis. App. (1882) 321.

Mindanao (Lanao), Clemens 870, 1114. In swamps about Lake Lanao, altitude about $650 \mathrm{~m}$. India to Japan south to Australia.

ISACHNE INCRASSATA (Hack.) Merr, in Philip. Journ. Sci. 5 (1910) Bot. 168.

Isachne debilis Rendle var. incrassata Hack. in Philip. Journ. Sci. 1 (1906) Suppl. 268; Merr. op. cit. 350.

Negros, Mindanao (Davao), Merrill 697\%, De Vore \& Hoover 358. In crevices of ledges along mountain streams, altitude 1,200 to $1,500 \mathrm{~m}$. Endemic.

ISACHNE MAGNA (Merr.) Merr. in Philip. Journ. Sci. 5 (1910) Bot. 327.

Isachne beneckei Hack. var. magna Merr. in Philip. Journ. Sci. 1 (1906) Suppl. 350.

Isachne apoensis Elm. Leafl. Philip. Bot. 7 (1915) 2676.

Luzon (Ifugao, Bontoc, Benguet), Mindanao (Davao), Merrill 4541 , 7679, 7678, 6569, Elmer 11578, B. S. 31686 Santos, 8870, 19731 McGregor. 4483 , 4248 Mearns. In the mossy forest, altitude 1,800 to $2,400 \mathrm{~m}$. Endemic.

Local names: Buyo (Bag.); popoktót (Bon.).

ISACHNE MICRANTHA Merr. in Philip. Journ. Sci. 5 (1910) Bot. 168.

Luzon (Cagayan, Tayabas), F. B. 16837, 16841 Curran, B. S. 28708 Ramos \& Edaño. On siopes, altitude $300 \mathrm{~m}$ and above. Endemic.

Closely allied to $I$. myosotis Nees.

ISACHNE MILIACEA Roth Nov. Pl. Sp. (1821) 58; Merr. Fl. Manila (1912) 89.

Panicum minutulum Gaudich. in Freyc. Voy. (1826) Bot. 410.

Isachne minutula Kunth Rev. Gram. 2 (1829) t. 117, Enum. 1 (1833) 137; Nees in Nov. Act. Acad. Nat. Cur. 19 (1843) Suppl. 1: 172; F.-Vill. Novis. App. (1882) 321; Mez in Perk. Frag. Fl. Philip. (1904) 141; Merr. in Philip. Journ. Sci. 1 (1906) Suppl. 349.

Isachne pulchella Mez in Perk. Frag. Fl. Philip. (1904) 141 p. p. non Roth.

Panicum macilentum Presl Rel. Haenk. 1 (1828) 310; Kunth Enum. 1 (1833) 116; F.-Vill. Novis. App. (1882) 312.

Throughout the Philippines, in low open wet places, old rice paddies, etc., often abundant; Kneucker Gram. Exsic. 609 from Manila well represents the species. India to Malaya and Polynesia.

Local names: Lagitlít (C. Bis.) ; matáng-lamók (Pang.); tagik-tagik (C. Bis.). 
ISACHNE MYOSOTIS Nees in Hook. Journ. Bot. Kew Miscel. 2 (1850) 98; Walp. Ann. 3 (1853) 720; Miq. Fl. Ind. Bat. 3 (1857) 462; F.-Vill. Novis. App. (1882) 321; Vidal Phan. Cuming. Philip. (1885) 156, Rev. Pl. Vasc. Filip. (1886) '286; Merr. in Philip. Journ. Sci. 1 (1906) Suppl. 349, 2 (1907) Bot. 261, 5 (1910) Bot. 327.

Panicum myosotis Steud. Syn. Pl. Glum. 1 (1854) 96.

Isachne pauciflora Hack. var. hirsuta Hack. in Philip. Journ. Sci. 3 (1908) Bot. 167.

Luzon (Abra, Benguet, Rizal, Pampanga), Negros, Mindoro, Mindanac (Zamboanga), B. S. 1765, 13768, 27029 Ramos, 2967\% Ramos \& Edaño, 1921 Foxworthy, F. B. 4405 Merritt, Merrill 616\%, Copeland 162. In open places at medium and higher altitudes, ascending to $2,400 \mathrm{~m}$. Reported also from Australia.

ISACHNE PANGERANGENSIS Z. \& M. Syst. Verz. Zoll. Pfianz. (1845) 102, var. HALCONENSIS Hack. in Philip. Journ. Sci. 3 (1908) Bot. 167, Merr. op. cit. 5 (1910) Bot. 327.

Luzon (Benguet), Mindoro, Merrill 6221, 6203, F. B. 16182 Curran, Merritt, \& Zschokke. In open heaths, altitude 2,400 to $2,600 \mathrm{~m}$. The variety endemic, the species in Java.

ISACHNE PAUCIFLORA Hack. in Govt. Lab. Publ. (Philip.) 35 (1906) 80; Merr. in Philip. Journ. Sci. 1 (1906) Suppl. 350, 5 (1910) Bot. 327.

Luzon (Benguet, Nueva Vizcaya), Elmer 6486, Merrill 4292, 9770, 7673, 7674, Phil. Pl. 195, B. S. 31753 Santos, 14211 McGregor. In wet places, along streams, in rice paddies, etc.; altitude 1,400 to $2,300 \mathrm{~m}$. Endemic.

ISACHNE STRICTA Elm. Leafl. Philip. Bot. 2 (1908) 463.

Leyte, Negros, Mindanao (Lanao), Elmer 10425, Wenzel 781, Clemens 1135a. In crevices of ledges, on seepage slopes, etc., altitude 600 to 1,200 m. Endemic.

ISACHNE VUlCANICA Merr. in Philip. Journ. Sci. 5 (1910) Bot. 169.

Negros, Merrill 6975, Phil. Pl. 585. On bare slopes of the old crater of Canlaon Volcano, altitude 1,800 to $2,100 \mathrm{~m}$. Endemic.

\section{EXCLUDED SPECIES}

ISACHNE JAVANA Nees.

This species was credited to the Philippines by Nees in Hook. Journ. Bot. Kew Miscel. 2 (1850) 97, on the basis of Cuming 2288; this specimen was from Malacca, not from the Philippines. The species has not been found in the Archipelago.

\section{ISACHNE PULCHELLA Roth.}

This species has been credited to the Philippines by F.-Villar, Novis. App. (1882) 321, and by Mez ex Perk. Frag. Fl. Philip. (1904) 141, but one of the specimens cited by the latter is Isachne miliacea Roth, and another is 1 . debilis Rendle. Roth's species is Sphaerocaryum pulchellum (Roth) Merr. (S. elegans Nees) which has not been found in the Philippines. 
28. PANICUM Linnaeus

Panicum Ambiguum Trin. in Mém. Acad. St. Fétersb. VI $3^{2}$ (1895) 243; Steud. Syn. Pl. Glum. 1 (1854) 61; Miq. Fl. Ind. Bat. 3 (1857) 447; F.-Vill. Novis. App. (1882) 311; Mez in Perk. Frag. Fl. Philip. (1904) 141; Merr. in Philip. Journ. Sci。 1 (1906) Suppl. 355.

Urochloa paspaloides Presl Rel. Haenk. 1 (1830) 318; Scribn. in Rept. Mo. Bot. Gard. 10 (1899) 54, t. 14, non Beauv.

Northern Luzon (Cagayan) to Mindanao and Palawan, Merrill 9590, Phil. Pl. 580, 3332, 5369, B. S. 14511, 27128 Ramos, 31536 Ramos \& Edaño. Widely scattered in open more or less waste places, old clearings, etc., at low and medium altitudes. Burma to the Mascarene Islands, Malaya and Polynesia.

PANICUM AMPLeXICAule Rudge Pl. Guian. (1805) 21, t. 27; Merr. in Philip. Journ. Sci. 1 (1906) Suppl. 356, Fl. Manila (1912) 92, Sp. Blancoanae (1918) 66.

Panicum myuros Kunth Enum. 1 (1833) 86; Merr. in Philip. Journ. Sci. 1. (1906) Suppl. 27; Usteri Beitr. Ken. Philip. Veg. (1905) 133, non Lam.

Panicum polygonatum Llanos Frag. Pl. Filip. (1851) 41; F.-Vill. \& Naves in Blanco Fl. Filip. ed. 3, $4^{1,}$ (1880) 30 ; non Kunth.

Panicum acutiglumum Steud. Syn. Pl: Glum. 1 (1854) 66.

Hymenachne serrulata Nees in Hook. Journ. Bot. Kew Miscel. 2 (1850) 98, nomen nudum.

Central. Luzon to Palawan and Mindanao, Merrill 9468, Phil. Pl. 15\%, Sp. Blancoanae 967, Elmer 14561, F. B. 8881 Curran. In open swamps and along swampy margins of lakes and streams at low altitudes. Pantropic.

Local name: Lagtóm (Bik.).

PANICUM AURITUM Presl Rel. Haenk. 1 (1830) 305 (incl. var. procerlus Presl); Kunth Enum. 1 (1833) 113; Steud. Syn. Pl. Glum. 1 (1854) 70; Miq. Fl. Ind. Bat. 3 (1857) 456; Nees in Nov. Act. Acad. Nat. Cur. 19 (1843) Suppl. 1: 172, Hook. Journ. Bot. Kew Miscel. 2 (1850) 97; F.-Vill. Novis. App. (1882) 312; Vidal Phan. Cuming. Philip. (1885) 157, Rev. Pl. Vasc. Filip. (1886) 287; Scribn. in Rept. Mo. Bot. Gard. 10 (1899) 46, t. 15; Mez in Perk. Frag. Fl. Philip. (1904) 142; Merr. in Philip. Journ. Sci. 1 (1906) Suppl. 356, Fl. Manila (1912) 92.

Northern Luzon (Cagayan) to Palawan and Mindanao, in most or all islands and provinces. Common in open, rather wet places at low and medium altitudes. India to southern China and Malaya.

Local name: Mongoto (Sub.).

PANICUM BREVIFOLIUM Linn. Sp. Pl. (1753) 59.

Panicum ovalifolium Poir. in Lam. Encycl. Suppl. 4 (1816) 279; Hook. f. Fl. Brit. Ind. 7 (1897) 44.

Balabac, Merrill 5386, Phil. Pl. 110, B. S. 442 Mangubat. In thickets, along trails in shaded places, etc., at low altitudes. Tropical Africa and Asia to China and Malaya. 
PANICUM CARINATUM Presl Rel. Haenk. 1 (1830) 309; Kunth Enum. 1 (1833) 112; Miq. Fl. Ind. Bat. 3 (1857) 452; F.-Vill. Novis. App. (1882) 312; Scribn. in Rept. Mo. Bot. Gard. 10 (1899) 46, t. 17; Merr. in Philip. Journ. Sci. 1 (1906) Suppl. 362.

Luzon (Ilocos Norte, Pangasinan, Rizal, Laguna, Batangas, Bataan, Camarines), Mindoro, Palawan, Culion, Balabac, Panay, Mindanao, Basilan, Presl! in Herb. Bernhardi, B. S. 30023 Fénix, 29483 Ramos \& Edaño, 16887 Serviñas, Merrill 47\%, 10596, 9241, Phil. Pl. 178. In old clearings, thickets, etc., at low and medium altitudes. Borneo and probably other parts of Malaya.

Perhaps better considered as a variety of $P$. patens Linn.

Local names: Baile (Sul.); gamog-gamog (Sub.); hirabon (Sul.); kauang-káuang (Sul.); malakauáyan (Tag., P. Bis.); tuad-tuáran (Tag.).

PANICUM CAUDIGLUME Hack. in Oesterr. Bot. Zeitschr. 51 (1901) 428,

Govt. Lab. Publ. (Philip.) 35 (1906) 80; Merr. in Philip. Journ.

Sci. 1 (1906) Suppl. 27, 360, Fl. Manila (1912) 92.

Luzon (Ilocos Sur, Bataan), Culion, Palawan, Merrill 832, 3307, 7436 , 11607, Kneucker Gram. Exsic. 731, B. S. 29240, 24083, 41285 Ramos. In open waste places at low altitudes, apparently introduced. Java.

Local name: Kumut-palakâ (Tag.).

PANICUM Coccospermum Steud. Syn. Pl. Glum. 1 (1854) 62.

Panicum villosum Hack. in Philip. Journ. Sci. 1 (1906) Suppl. 269;

Merr. op. cit. 355, 5 (1910) Bot. 327, non Lam.

Luzon (Bontoc, Benguet, Nueva Vizcaya, Rizal), Culion, Mindanao (Lanao), Merrill 7692, 4281, 4396, 4459, 4360, 11606, Phil. Pl. 591, B. S. $5918,8214,41339$ Ramos, 4492 Mearns. On open gxassy slopes and banks chiefly at medium altitudes, ascending to $1,500 \mathrm{~m}$. The identification of our material with Steudel's species was made by Mr. C. A. Backer of Buitenzorg, Java. India to Java.

Local name: Tumi (Bon.).

PANICUM COLONUM Linn. Syst. ed. 10 (1759) 870; Steud. Syn. Pl. Glum. 1 (1854) 46; F.-Vill. Novis. App. (1882) 311; Vidal Phan. Cuming. Philip. (1885) 157, Rev. Pl. Vasc. Filip. (1886) 286; Mez in Perk. Frag. Fl. Philip. (1904) 142; Merr. in Philip. Journ. Sci. 1 (1906) Suppl. 354; Fl. Manila (1912) 91, Sp. Blancoanae (1918) 65.

Panicum colonum Linn. var. pseudocolonum Nees in Nov. Act. Acad. Nat. Cur. 19 (1843) Suppl. 1: 172, Hook. Journ. Bot. Kew Miscel. 2 (1850) 97.

Panicum cumingianum Steud. Syn. Pl. Glum. 1 (1854) 58; Miq. Fl. Ind. Bat. 3 (1857) 447; F.-Vill. Novis. App. (1882) 311.

Echinochloa colona Link Hort. Berol. 2 (1833) 209.

Oplismenus colonus HBK. Nov. Gen. Sp. Pl. 1 (1815) 108; Pres] Rel. Haenk. 1 (1830) 321.

Orthopogon dichotomus Llanos Frag. Pl. Filip. (1851) 38; F.-Vill. \&. Naves in Blanco Fl. Filip. ed. 3, $4^{1}$ (1880) 28.

Throughout the Philippines at low and medium altitudes. In open, rather wet places. Pantropic.

Local names: Búlang (Tag.) ; dakayáng (Ilk.); dukayáng (Ilk.) ; tiribúhan (Tag.); tumi (Bon.). 
PANICUM CORDATUM Büse in Miq. Pl. Jungh. (1854) 376; Merr. in Philip. Journ. Sci. 4 (1909) Bot. 249.

Panicum luxurians Merr. in Philip. Journ. Sci. 1 (1906) Suppl. 359, non Willd.

Panicum montanum Naves Novis. App. (1882) 312; Ceron Cat. Pl. Herb. Manila (1892) 180; Mez in Perk. Frag. Fl. Philip. (1904) 142; Merr. in Philip. Journ. Sci. 1 (1906) Suppl. 27, non Roxb.

Luzon (Bontoc, Union, Bataan, Bulacan), Culion, Palawan, Merrill 9348 , 9318, 554, Phil. Pl. 146, Elmer 5595, 6735, Williams 13.4. In thickets, old clearings, etc., at low and medium altitudes. Java, Borneo.

PANICUM CRASSIAPICULATUM Merr. in Philip. Journ. Sci. 1 (1906) Suppl. 356.

Panicum latifolium Hook. f. Fl. Brit. Ind. 7 (1897) 39, excl. syn. ét var. majus Hook. f., non Linn.

Palawan, Balabac, Mindanao (Agusan), Merrill 5387, 7290, 9240, 9286, Phil. Pl. 111. In thickets and along trails in forests at low altitudes. Malay Peninsula, Java.

Closely allied to the American P. oryzoides Sw.

PANICUM CRUS-GALLI Linn. Sp. Pl. (1753) 56; F.-Vill. Novis. App. (1882) 311; Mez in Perk. Frag. Fl. Philip. (1904) 142; Merr. in Philip. Journ. Sci. 1 (1906) Suppl. 354, 5 (1910) Bot. 327, Fl. Manila (1912) 91, Sp. Blancoanae (1918) 65.

Oplismenus limosus Presl Rel. Haenk. 1 (1830) 321; Kunth Enum. 1 (1833) 144.

Panicum limosum F.-Vill. Novis. App. (1882) 311.

Panicum hispidulum Lam. Ill. 1 (1791) 171; F.-Vill. Novis. App. (1882) 311.

Echinochloa crus-galli Beauv. Agrost. (1812) อ̄3.

Orthopogon hispidus Spreng. Syst. 1 (1825) 307; Llanos Frag. Pl. Filip. (1851) 37; F.-Vill. \& Naves in Blanco Fl. Filip. ed. 3, $4^{1}$ (1880) 27.

Orthopogon subverticillatus Llanos op. cit. 38; F.-Vill. \& Naves op. cit. 28.

Throughout the Philippines at low and medium altitudes, in open wet places, rice paddies, etc. All warm countries.

Local names: Baobao (If.) ; daua-dáua (Tag.); daua-dauáhan (Tag.) ; dauána (Mbo.); lagtom (Bik.); sabsablúg (Bon.); sabsábug (Bon.).

Var. MUTICUM Doell.; Hack. in Philip. Journ. Sci. 1 (1906) Suppl. 268; Merr. op. cit. 354 .

Luzon (Bontoc, Benguet, Rizal), Balabac, Palmas, Merrill 5363, 4307, B. S. 460 Mangubat, 5975 Ramos. Habitat of the species. Pantropic.

PANICUM DISTACHYUM Linn. Mant. 1 (1767) 138; F.-Vill. Novis App. (1882) 311; Merr. Fl. Manila (1912) 91, Sp. Blancoanae (1918) 66. Panicum remotum Retz. Obs. 4 (1786) 71; Merr. in Philip. Journ.

Sci. 1 (1906) Suppl. 354.

Panicum petiveri Trin. Ic. Gram. $t .176, f . C$.

Panicum ramosum Mez in Perk. Frag. Fl. Philip. (1904) 143, non

(?) Linn. 
? Panicum umbrosum F.-Vill. Novis. App. (1882) 311, non Retz.

Panicum miliiforme Presl Rel. Haenk. 1 (1830) 300; Kunth Enum. 1 (1833) 96; Miq. Fl. Ind. Bat. 3 (1857) 488; F.-Vill. Novis. App. (1882) 312; Scribn. in Rept. Mo. Bot. Gard. 10 (1899) 47, t. 20.

Northern Luzon to Palawan and Mindanao, in most or all islands and provinces. In open waste places in and about towns at low and medium altitudes; common. India to China through Malaya to tropical Australia.

PANICUM FLAVIDUM Retz. Obs. 4 (1786) 15; Nees in Hook. Journ. Bot. Kew Miscel. 2 (1850) 97; F.-Vill. Novis. App. (1882) 311; Vidal Phan. Cuming. Philip. (1885) 157, Rev. Pl. Vasc. Filip. (1886) 286; Mez in Perk. Frag. Fl. Philip. (1904) 142; Merr. in Philip. Journ. Sci. 1 (1906) Suppl. 27, 353, Fl. Manila (1912) 90.

Panicum floridum Usteri Beitr. Ken. Philip. Veg. (1905) 133, sphalm.

Northern Luzon to Palawan and Mindanao. A common weed in open waste places in and about towns at low altitudes. Tropical Asia, Africa, and Malaya.

Local names: Giling (C. Bis.); sabuñg-sabúñgan (Tag.); baili itau (Sul.).

PANICUM HUMILE Nees ex Steud. Syn. Pl. Glum. 1 (1854) 84; Hack. in Govt. Lab. Publ. (Philip.) 35 (1906) 80; Merr. in Philip. Journ.

Sci. 1 (1906) Suppl. 358.

Luzon (Bontoc, Lepanto, Tarlac, Pangasinan, Zambales, Rizal), Culion, Mervill 3623, 4466, 11608, B. S. 21998 Ramos, F. B. 5872 Curran. In open waste places, grasslands, etc., at low and medium altitudes. India to southern China and Malaya.

PANICUM INDICUM Linn. Mant. 1 (1767) 184; Nees in Hook. Journ. Bot. Kew Miscel. 2 (1850) 97; F.-Vill. Novis. App. (1882) 311; Vid. Phan. Cuming. Philip. (1885) 157, Rev. Pl. Vasc. Filip. (1886) 287; Mez in Perk. Frag. Fl. Philip. (1904) 142; Merr. in Philip. Journ. Sci. 1 (1906) Suppl. 27, 357, Fl. Manila (1912) 92.

Hymenachne indica Büse ex Miq. Fl. Ind. Bat. 3 (1857) 458.

Sacciolepis indica Chase in Proc. Biol. Soc. Wash. 21 (1908) 8.

Northern Luzon to Palawan and Mindanao. Common in open wet grasslands at low and medium altitudes. India through Malaya to tropical Australia.

Local names: Buntót-pusá (Tag.) ; sabsablúg (Bon.) ; salai-máya (Tag.) ; sangumai·(Tag.) ; sikuán (Ig.).

Var. ANGUSTUM (Trin.) Hook. f. Fl. Brit. Ind. 7 (1897) 42; Merr. in Philip. Journ. Sci. 1 (1906) Suppl. 357.

Panicum angustum Trin. Ic. Gram. 3 (1836) t. 334; Vidal Phan. Cuming. Philip. (1885) 157, Rev. PI: Vasc. Filip. (1886) 287.

Occasional, in the same habitat as the species. India.

PANICUM LUzONENSE Presl Rel. Haenk. 1 (1828) 308; Kunth Enum. 1 (1833) 121; Miq. Fl. Ind. Bat. 3 (1857) 457; F.-Vill. Novis. App.

(1882) 312; Merr. in Philip. Journ. Sci. 1 (1906) Suppl, 358.

Luzon (Amburayan, Nueva Ecija, Bataan, Rizal, Cavite, Laguna), Palawan, Merrill 316, 3107, 4183, Phil. Pl. 1238, B. S. 4600, 21997 Ramos, 
23060 McGregor. In open waste places, old clearings, etc, at low altitudes. Malay. Peninsula.

Local name: Nikníkan (Tag.).

PANICUM MALABARICUM (Linn.) Merr. in Philip. Journ. Sci. 4 (1909)

Bot. 248.

Poa malabarica Linn. Sp. Pl. (1753) 69.

Panicum arnottianum Nees in Steud. Syn. Pl. Glum. 1 (1854) 59.

Mindoro, Palawan, Mindanao, Palmas, F. B. 5349 Merritt, Merrill 5367, $9378,9581, B . S .36687$ Ramos \& Edaño. In old clearings at low altitudes. India to Malaya.

Local name: Salid (Sub.).

PANICUM MAXIMUM Jacq. Ic. Pl. Rar. 1 (1781-86) t. 13; Merr. Fl. Manila (1912) 93.

Cultivated for forage purposes, occasional as an escape or persisting from former cultivation. Cultivated in all tropical countries. Guinea grass.

PANICUM Miliaceum Linn. Sp. Pl. (1753) ō8; Merr. in Philip. Journ.

Sci. 1 (1906) Suppl. 358.

Very rarely cultivated in the Philippines, and nowhere spontaneous. Cultivated in most warm countries. Millet.

Local name: Kabug (C. Bis.).

PANICUM MINDANAENSE Merr. in Philip. Journ. Sci. 1 (1906) Suppl. 360.

Luzon (Ilocos Sur, Rizal, Batangas, Zambales), Mindanao (Lanao), Clemens 99, B. S. 27379, 5103, 29242 Ramos, Merrill Phil. Pl. 168, as $P$. caudiglume Hack. In open places at low and medium altitudes. Endemic.

PANICUM MYOSUROIDES R. Br. Prodr. (1810) 189; Kunth Enum. 1 (1833) 77; Mez in Perk. Frag. Fl. Philip. (1904) 142; Merr. in Philip. Journ. Sci. 1 (1906) Suppl. 357.

Panicum angustissimum Vidal Phan. Cuming. Philip. (1885) 157, Rev. Pl. Vasc. Filip. (1886) 286, non ? Hochst.

Panicum contractum Nees in Hook. Journ. Bot. Kew Miscel. 2 (1850) 97, non ? Trin.

Luzon (Abra, Nueva Vizcaya), Panay, Mindanao (Misamis), Cuming 1668, B. S. 31283 Ramos \& Edaño, 14221 McGregor, F. B. 16459 Bacani. In open wet lands at low and medium altitudes. Tropical Africa and Asia through Malaya to Australia.

PANICUM NOdOSUM Kunth Enum. 1 (1833) 97; Miq. Fl. Ind. Bat. 3 (1857) 448; F.-Vill. Novis. App. (1882) 312; Merr. in Philip. Journ. Sci. 1 (1906) Suppl. 357, Fl. Manila (1912) 92, Sp. Blancoanae (1918) 66.

Panicum multinode Presl Rel. Haenk. 1 (1828) 303, non Lam.

Panicum violaceum Llanos Frag. Pl. Filip. (1851) 42; F.-Vill. \& Naves in Blanco Fl: Filip. ed. $3,4^{1}$ (1880) 31.

Panicum ouonbiense Mez in Perk. Frag. Fl. Philip. (1904) 142, non Balansa.

$183036-5$ 
Northern Luzon to Palawan and Mindanao, in most islands and provinces, Merrill 4228, 527, 7056, 127, 4182, 255, 5385, 5247, Phil. Pl. 113, Sp. Blancoanae 944. In thickets, old clearings, etc., at Iow and medium altitudes; common. India to China and Malaya.

Local names: Baníg-usá (Tag.); kauakauayánan (Tag.); kúmut-u̧sá (Tag.).

PANICUM PALUdosUm Roxb. Fl. Ind. 1 (1820) 310; Usteri Beitr. Ken. Philip. Veg. (1905) 133; Merr. in Philip. Journ. Sci. 4 (1909) Bot. 249, Fl. Manila (1912) 93.

Panicum proliferum Mez in Perk. Frag. Fl. Philip. (1904) 143; Merr.

in Philip. Journ. Sci. 1 (1906) Suppl. 359, non Lam.

Luzon (Cagayan, Rizal, Laguna), Mindanao (Lanao), Merrill 97, Phil. Pl. 159, B. S. 23144 McGregor, 7429 Ramos. In shallow water along margins of streams and lakes at low and medium altitudes. India to southern China and Malaya.

PANICUM PATENS Linn. Sp. Pl. (1753) 86; Mez in Perk. Frag. Fl. Philip. (1904) 142; Merr. in Philip. Journ. Sci. 1 (1906) Suppl. 361, 3 (1908) Bot. 396.

Panicum radicans Retz. Obs. 4 (1786) 18; Steud. Syn. Pl. Glum. 1 (1854) 87; Nees in Hook. Journ. Bot. Kew Miscel. 2 (1850) 97; Miq. Fl. Ind. Bat. 3 (1857) 453; F.-Vill. Novis. App. (1882) 312; Vidal Phan. Cuming. Philip. (1885) 157, Rev. Pl. Vasc. Filip. (1886) 287; Mez in Perk. Frag. Fl. Philip. (1904) 143.

Northern Luzon to Palawan and Mindanao, in most or all islands and provinces. Common in thickets, some secondary forests, old clearings, etc., at low and medium altitudes. India to Malaya.

Local names: Ililít (Ig.) ; malanikník (Tag.); púgad-lagúyo (Tag.).

PANICUM PILIFES Nees in Hook. Journ. Bot. Kew Miscel. 2 (1850) 97; Büse in Miq. Pl. Jungh. (1854) 376; Miq. Fl. Ind. Bat. 3 (1857) 453; Mez in Perk. Frag. Fl. Philip. (1904) 143; Merr. in Philip. Journ. Sci. 1 (1906) Suppl. 27, 361, 3 (1908) Bot. 396, Fl. Manila (1912) 94.

Panicum hermaphroditum Steud. Syn. Pl. Glum. 1 (1854) 67; Rolfe in Journ. Bot. 23 (1885) 216; Vidal Phan. Cuming. Philip. (1885) 157, Rev. Pl. Vasc. Filip. (1886) 287.

Panicum trigonum Nees in Nov. Act. Acad. Nat. Cur. 19 (1843) Suppl. 1: 172; F.-Vill. Novis. App. (1882) 312, non Retz.

Northern Luzon to Palawan and Mindanao, in most or all islands and provinces. In thickets, along streams, in old clearings, etc., at low and medium altitudes. India to the Mascarene Islands, Malaya, tropical Australia, and Polynesia.

Local names: Baitbút (Tagb.) ; baníg-usá (Tag.) ; baririt-pualas (Bag.); marikauáyan (Ig.).

PANICUM PSILOPODIUM Trin. Gram. Pan. (1826) 217; Rolfe in Journ. Bot. 23 (1885) 216; Vidal Phan. Cuming. Philip. (1885) 157, Rev. Pl. Vasc. Filip. (1886) 287; Merr. in Philip. Journ. Sci. 5 (1910) Bot. 168, Fl. Manila (1912) 93, Sp. Blancoanae (1918) 66.

Panicum crispum Llanos Frag. Pl. Filip. (1851) 42; F.-Vill. \& Naves in Blanco Fl. Filip. ed. $3,4^{1}$ (1880) 30. 
Luzon (Benguet, Rizal), Merrill 7079, Phil. Pl. 597, 1764, Elmer 5.97\%, 6589. In open wet places at low and medium altitudes, ascending to $1,400 \mathrm{~m}$; local. India to Burma.

PANICUM PUNCTATUM Burm. f. Fl. Ind. (1768) 26; Hook. f. Fl. Brit. Ind. 7 (1897) 29; Merr. in Philip. Journ. Sci. 1 (1906) Suppl. 353, Fl. Manila (1912) 90.

Panicum fluitans Retz. Obs. 3 (1783) 8; Miq. Fl. Ind. Bat. 3 (1857) 455; Rolfe in Journ. Bot. 23 (1885) 216; Vidal Phan. Cuming. Philip. (1885) 157, Rev. Pl. Vasc. Filip. (1886) 287.

Paspalum pluriracemosum Steud. Syn. Pl. Glum. 1 (1854) 27; Miq. Fl. Ind. Bat. 3 (1857) 431; F.-Vill. Novis. App. (1882) 310.

Panicum brizoides Nees in Hook. Journ. Bot. 2 (1850) 97, Miq. Fl. Ind. Bat. 3 (18517) 445, non Linn.

Luzon (Rizal, Laguna), Leyte, Mindanao (Misamis, Davao, Zamboanga, Agusan), Merrill 7406, 8111, Phil. Pl. 131, Cuming 582, Wenzel 1780. Elmer 12019, B. S. 37364 Ramos \& Edaño. In shallow water and in open muddy places at low altitudes. Northern Africa to Mauritius, India, and Malaya.

Local names: Baririan (Bag.) ; lalabok (Sub.).

PANICUM REPENS Linn. Sp. Pl. ed. 2 (1762) 87; Hook. f. Fl. Brit. Ind. 7 (1897) 49; Ceron Cat. Pl. Herb. Manila (1892) 180; Mez in Perk. Frag. Fl. Philip. (1904) 143; Merr. in Philip. Journ. Sci. 1 (1906) Suppl. 359, 3 (1908) Bot. 396, F́l. Manila (1912) 93, Sp. Blancoanae (1918) 67.

Panicum ischaemoides Retz. Obs. 4 (1786) 17; Nees in Nov. Act. Acad. Nat. Cur. 19 (1843) Suppl. 1: 175; Miq. Fl. Ind. Bat. 3 (1857) 450 ; F.-Vill. Novis. App. (1882) 312.

Panicum convolutum Beauv. ex Spreng. Syst. 1 (1825) 319; Presl Rel. Haenk. 1 (1828) 304; F.-Vill. Novis. App. (1882) 312; Steud. Syn. 1 (1854) 73.

Panicum tuberosum Llanos Frag. Pl. Filip. (1851) 40; F.-Vill. \& Naves in Blanco Fl. Filip. ed. 3, $4^{1}$ (1880) 29.

Panicum miliare Mez in Perk. Frag. Fl. Philip. (1904) 142, non Lam.

Throughout the Philippines in open low lands, especially near the sea, but also inland locally and ascending to $1,500 \mathrm{~m}$; common. Pantropic.

Local names: Kayana (Mbo.); luya-luyáhan (Tag.).

PANICUM REPTANS Linn. Syst. ed. 10 (17ø̄9) 871; Hitchc. in Contr. U. S. Nat. Herb. 12 (1908) 119; Merr. in Philip. Journ. Sci. 4 (1909) Bot. 249.

- Panicum prostratum Lam. Ill. 1 (1791) 171; Miq. Fl. Ind. Bat. 3 (1857) 446; F.-Vill. Novis. App. (1882) 311; Mez in Perk. Frag. Fl. Philip. (1904) 143; Merr. in Philip. Journ. Sci. 1 (1906) Suppl. 355.

Panicum calaccanzense Steud. Syn. Pl. Glum. 1 (1854) 65; Vidal Phan. Cuming. Philip. (1885) 157, Rev. PI. Vasc. Filip. (1886) 287.

Panicum barbatum Lam. Tabl. Encycl. 1 (1791) 171; Presl Rel. Haenk. 1 (1830) 299.

Panicum procumbens Nees Agrost. Bras. (1823) 109, Hook. Journ. Bot. Kew Miscel. 2 (1850) 97. 
Panicum luxurians Willd. ex Nees Agrost. Bras. (1829) 233, non $P$. luxurians Willd. ex Spreng. Syst. 1 (1825) 305.

Throughout the Philippines in open waste places, old clearings, thickets, etc., at low and medium altitudes. Pantropic.

Local names: Babáka-nalabága (Ilk.); marakauáyan (Ilk.).

PANICUM SARMENTOSUM Roxb. FI. Ind. 1 (1820) 311; F.-Vill. Novis. App. (1882) 312; Vidal Phan. Cuming. Philip. (1885) 157, Rev. Pl. Vasc. Filip. (1886) 287; Mez in Perk. Frag. Fl. Philip. (1904) 143; Merr. in Philip. Journ. Sci. 1 (1906) Suppl. 27, 360, 2 (1907) Bot. 261.

Panicum incomptum Trin. Gram. Pan. (1826) 200; Ic. Gram. (1828-36) t. 223; Kunth Enum. 2 (1833) 112; Miq. Fl. Ind. Bat. 3 (1857) 451; Nees in Mart. Fl. Bras. 2: 207.

Panicum extensum Steud. Syn. Pl. Glum. 1 (1854) 72.

Panicum vacillans Steud. op. cit. 75.

Panicum concinnum Nees in Hook. Journ. Bot. Kew Miscel. 2 (1850) 97; Walp. Ann. 3 (1853) 719.

Luzon (Bataan, Cavite, Zambales, Tayabas), Mindoro, Culion, Palawan, Balabac, Panay, Negros. In thickets and secondary forests at low and medium altitudes, often common. India to southern China and Malaya.

Locăl names: Kanubsúban (Tag.) ; kauakauáyan (Tag.); kauáyan-sauák (P. Bis.); kaykauáyan (P. Bis.); kuayan-kuáyan (Sul.).

PANICUM STAGNINUM Retz. Obs. 4 (1786) 17; F.-Vill. Novis. App. (1882) 311; Usteri Beitr. Ken. Philip. Veg. (1905) 133; Merr. in Philip. Journ. Sci. 1 (1906) Suppl. 354, 3 (1908) Bot. 396, Fl. Manila (1912) 91, Sp. Blancoanae (1918) 64.

Echinochloa stagnina Beauv. Agrost. (1812) 57; Miq. Fl. Ind. Bat. 3 (1857) 464.

Aegilops fuviatilis Blanco Fl. Filip. (1837) 47, ed. 2 (1845) 32, ed. 3, 1 (1879) 59.

Orthopogon loliaceus Llanos Frag. Pl. Filip. (1851) 36; F.-Vill. \& Naves in Blanco Fl. Filip. ed. 3, $4^{1}$ (1880) 26, non Spreng.

Panicum crus-galli Linn. var. stagninum O. Kuntze Rev. Gen. Pl. (1891) 783.

Babuyan Islands and northern Luzon to Mindanao, in most islands and provinces. In open wet places, swamps, borders of lakes and streams, at low and medium altitudes. India to Africa and Malaya.

Local names: Balíli (Tag.); banágo (Sub.); lagtóm-na-pulá (Bik.); timsím (Tag.) ; urarói (Bik.).

PANICUM TRICHODES Sw. Prodr. Veg. Ind. Occ. (1788) 24; Hitche.

\& Chase in Contr. U. S. Nat. Herb. 15 (1910) 129, f. 125.

Panicum capillaceum Lam. Tabl. Encycl. 1 (1791) 173.

Luzon (Ilocos Norte, Pangasinan), Culion, Merrill 458, B. S. 7618 Ramos, F. B. 15530 Merritt \& Darling, Gates 567.5. In thickets at low and medium altitudes. Similar in appearance to Panicum brevifolium Linn., but distinguished by its short first glume. I am indebted to Prof. A. S. Hitchcock for indicating to me the error in the original determinations. Widely distributed in tropical America, probably an introduced species in the Philippines. 
PANICUM TRYPHERON Schultes Mant. 2 (1882) 244; Hook. f. Fl.

Brit. Ind. 7 (1897) 47; Mez in Perk. Frag. Fl. Philip. (1904) 143;

Merr. in Philip. Journ. Sci. 1 (1906) SuppI. 358.

Luzon (Cagayan, Lepanto), Semirara, Mindoro, Palawan, Mindanao (Davao), Merrill 678, 4136, 4478, Phil. Pl. 1281, Copeland 396, F. B. 16585 Curran. In open grasslands at low and medium altitudes. Tropical Africa through India to China and Malaya.

PANicum TUBerculatum Presl Rel. Haenk. 1 (1830) 307; Kunth Enum. 1 (1833) 120; Miq. Fl. Ind. Bat. 3 (1857) 454; F.-Vill. Novis. App. (1882) 312.

Panicum caesium Nees in Hook. Journ. Bot. Kew Miscel. 2 (1850) 97; Merr. in Philip. Journ. Sci. 1 (1906) Suppl. 358.

Panicum acroanthum Mez in Perk. Frag. Fl. Philip. (1904) 141, non Steud.

Luzon (Isabela, Nueva Ecija, Pampanga, Rizal, Laguna), Panay, Mindanao (Misamis), Cuming 1667, Merrill 123, 670\%, 4229, 1469, Phil. Pl. $205 \%$, B. S. 23081 McGregor, 8122 Ramos. In open waste places, old clearings, etc., at low and medium altitudes. India to Borneo and New Guinea.

PANICUM UnCINATUM Raddi Agrost. Bras. (1823) 41; Trin. Gram. Pan. (1826) 174, Ic. t. 216; Merr. in Philip. Journ. Sci. 1 (1906) Suppl. 362.

Mindanao (Davao), Clemens 266. In thickets or open lands, altitude about $650 \mathrm{~m}$. Pantropic.

PANicum WARBURgll Mez in Perk. Frag. Fl. Philip. (1904) 143; Merr.

in Philip. Journ. Sci. 1 (1906) Suppl. 362, Fl. Manila (1912) 93.

Panicum patens Linn. var. parvulum Warb. 1. c. in syn.

Luzon (Ilocos Norte, Ifugao, Lepanto, Nueva Vizcaya, Rizal, Zambales, Bataan, Laguna, Tayabas), Mindanao (Lanao), Merrill 303, Kneucker Gram. Exsic. 734, B. S. 28834 Ramos:\& Edaño, 27018, 27394 Ramos, 2000\% McGregor. In thickets and forests, sea level to $1,200 \mathrm{~m}$ altitude; probably but a greatly reduced form of $P$. carinatum Presl, the latter being intermediate between the present species and $P$. patens Linn. Endemic.

\section{EXCLUDED SPECIES}

Panicum BRIZAeforme Presl Rel. Haenk. 1 (1830) 302; Kunth Enum. 1 (1833) 78; Miq. Fl. Ind. Bat. 3 (1857) 445; F.-Vill. Novis. App. (1882) 311; Scribn. in Rept. Mo. Bot. Gard. 10 (1899) 46, t. 16. Panicum paspaloides Pers. Syn. 1 (1805) 81; Kunth Enum. 1 (1833) 77; F.-Vill. Novis. App. (1882) 311.

Hitchcock \& Chase, in Contr. U. S. Nat. Herb. 15 (1910) 30, state that the Haenke specimen of $P$. brizaeforme in the Prague herbarium is labeled "Acapulco, Mexico," and is Panicum geminatum Forsk.

Panicum ElatiUs Kunth; F.-Vill. Novis. App. (1882) 312.

Panicum Paludicola Nees Agrost. Bras. (1823) 179; Steud. Syn. Pl. Glum. 1 (1854) 65; Miq. Fl. Ind. Bat. 3 (1857) 455; F.-Vill. Novis. App. (1882) 312. 
"Brazil ... ins. Manil, Java" Steudel. This is a Brazilian species, Panicum palustre Trin.; see Chase in Proc. Biol. Soc. Wash. 21 (1908) 5, sub Hymenachne palustris (Trin.) Chase.

Panicum mertensil Roth; F.-Vill. Novis. App. (1882) 312.

Panicum Leucophaeum HBK.; Presl Rel. Haenk. 1 (1830) 299; F.-Vill. Novis. App. (1882) 311.

"Hab, in Mexico, in Luzonia" Presl. An American species to which some Australian forms have been referred. The Luzon record was based on an erroneously localized Malaspina Expedition collection.

Panicum helopus Trin.; F.-Vill. Novis. App. (1882) 311.

\section{ICHNANTHUS Beauvois}

ICHNANTHUS VICINUS (F. M. Bail.) comb. nov.

Panicum vicinum F. M. Bail. Syn. Queensl. Fl. Suppl. 3 (1890) 82.

Ichnanthus pallens Munro in Benth. Fl. Hongk. (1861) 414; Merr. in Philip. Journ. Sci. 1 (1906) Suppl. 261, 363; Hack. in Govt. Lab. Publ. (Philip.) 35 (1906) 80, non Panicum pallens Sw.

Panicum paludicolum Miq. Fl. Ind. Bat. 3 (1857) 454; F.-Vill. Novis. App. (1882) 312, non Nees.

Panicum nitens Merr. in Govt. Lab. Publ. (Philip.) 17 (1904) 8.

Luzon (Bontoc, Kalinga, Bataan, Laguna, Sorsogor), Catanduanes, Mindoro, Biliran, Mindanao, Merrill 3756, 3221, 5498, 5538, Phil. Pl. 109, Kneucker Gram. Exsic. 740, B. S. 30226, 23608 Ramos, 18502 McGregor, 37475,38608 Ramos \& Edaño. On forested slopes and ridges up to 1,400 $m$ altitude. India to southern China and Formosa through Malaya to tropical Australia.

Local name: Dóot (Ilk.).

\section{ALLOTEROPSIS Presl}

Alloteropsis Semialata (R. Br.) Hitche, in Contr. U. S. Nat. Herb. 12 (1909) 210; Chase in Proc. Biol. Soc. Wash. 24 (1911) 159.

Panicum semialatum R. Br. Prodr. (1810) 192; Vidal Phan. Cuming. Philip. (1885) 157, Rev. Pl. Vasc. Filip. (1886) 287.

Alloteropsis distachya Presl Rel. Haenk. 1 (1830) 344, t. 47; Scribn. in Rept. Mo. Bot. Gard. 10 (1899) 37, t. 23.

Holosetum philippicum Steud. in Flora 33 (1850) 228, nomen, Syn.

Pl. Glum. 1 (1854) 118; Miq. Fl. Ind. Bat. 3 (1857) 444.

A rundinella nervosa Nees in Hook. Journ. Bot. Kew Miscel. 2 (1850) 102; Steud. Syn. Pl. Glum. 1 (1854) 115; Miq. Fl. Ind. Bat. 3 (1857) 519; F.-Vill. Novis. App. (1882) 318.

Coridochloa semialata Nees in Hook. Journ. Bot. Kew Miscel. 2 (1850) 97.

Panicum philippicum F.-Vill. Novis. App. (1882) 312.

Urochloa semialata Kunth Rev. Gram. 1 (1829) 31; Pax in Perk.

Frag. Fl. Philip. (1904) 144.

Axonopus semialatus Hook. f. Fl. Brit. Ind. 7 (1897) 64; Merr. in

Philip. Journ. Sci. 1 (1906) Suppl. 367.

Luzon (Cagayan, Ilocos Norte, Bontoc, Lepanto, Benguet, Pangasinan, Nueva Vizcaya, Nueva Ecija, Zambales, Laguna). On open grassy slopes 
at low and medium altitudes, ascending to $1,800 \mathrm{~m}$. India to South Africa, China, and tropical Australia.

Local name: Timi (Bon.).

\section{OPLISMENUS Beauvois}

OPLISMENUS BURMANNII (Retz.) Beauv. Agrost. (1812) 54; F.-Vill. Novis. App. (1882) 312; Pilger in Perk. Frag. Fl. Philip. (1904) 144; Merr. in Philip. Journ. Sci. 1 (1906) Suppl. 364, Fl. Manila (1912) 94.

Panicum burmannii Retz. Obs. 3 (1783) 10.

Panicum hirtellum Burm. f. Fl. Ind. (1768) 24, $t$. 12, f. 1, non Linn. Orthopogon burmannii R. Br. Prodr. (1810) 194; Miq. Fl. Ind. Bat. 3 (1857) 442.

Luzon (Pangasinan, Rizal, Bataan), Mindanao (Zamboanga), Merrill 3290, Phil. Pl. 196, Elmer 6645, Williams 135, B. S. 24084 Ramos. In thickets at low altitudes. Pantropic.

OPLISMENUS COMPositus (Linn.) Beauv. Agrost. (1812) 54; F.-Vill. Novis. App. (1882) 312; Merr. in Philip. Journ. Sci. 1 (1906) Suppl. 364, 3 (1908) Bot. 397, Fl. Manila (1912) 94, Sp. Blancoanae (1918) 67.

Panicum compositum Linn. Sp. Pl. (1753) 57.

Panicum loliaceum Lam. Tabl. Encycl. 1 (1790) 170, Encyel. 4 (1798) 743.

Oplismenus loliaceus Beauv. Agrost. (1812) 168; Kunth Enum. 1 (1833) 140 ; F.-Vill. Novis. App. (1882) 312.

Orthopogon sylvaticus Miq. Fl. Ind. Bat. 3 (1857) 443.

Oplismenus sylvaticus R. \& S. Syst. 2 (1817) 481; F.-Vill. Novis. App. (1882) 312.

Orthopogon compositus R. Br. Prodr. (1810) 194; Miq. Fl. Ind. Bat. 3 (1857) 443.

Oplismenus indicus R. \& S. Syst. 2 (1817) 484; Nees in Hook. Journ.

Bot. Kew Miscel. 2 (1850) 97; Vidal Phan. Cuming. Philip. (1885) 157, Rev. Pl. Vasc. Filip. (1886) 288.

Orthopogon setarius Llanos Frag. Pl. Filip. (1851) 35, non Spreng.

Orthopogon hirtellus R. Br.; Llanos op. cit. 37.

Panicum lanceolatum Retz.; F.-Vill. Novis. App. (1882) 311.

Panicum sylvaticum Lam. Encycl. 4 (1798) 733; Steud. Syn. Pl. Glum. 1 (1854) 45.

Throughout the Philippines in all or most islands and provinces, in thickets, old clearings, etc., at low and medium altitudes; often common. Pantropic.

Local names: Balibátong (Sub.); balisíbis (Iv.); baníg-usá (Tag.); kauakauáyan (Tag.) ; litlítun (Bon.) ; malakauáyan (Pang.); marikauáyan (Ig.) ; yamog-yamóg (P. Bis.) ; bailituganalu (Sul.).

Vor. LAsIORHACHIS Hack. in Govt. Lab. Publ. (Philip.) 35 (1906) 81; Merr. in Philip. Journ. Sci. 1 (1906) Suppl. 364.

Oplismenus burmannii Mez in Perk. Frag. Fl. Philip. (1904) 144, non Beauv.

Palawan, Merrill 826. In open dry places at low altitudes. Endemic. 
OPLISMenus Undulatifolius (Ard.) Beauv. Agrost. (1812) 54; Merr. in Philip. Journ. Sci. 1 (1906) Suppl. 364.

Panicum undulatifolium Ard. Anim. Bot. Sp. Alt. (1764) 14, t. 4.

Luzon (Ilocos Norte, Benguet, Bontoc, Ifugao, Union, Bataan, Laguna), Mindoro, Mindanao, B. S. 19708. McGregor, 27397 Ramos, 36693, 37488 , 37108 Ramos \& Edaño, F. B. 2547 Borden, Williams 1179, 1962, 144, Elmer 698\%. In thickets and forests at low and medium altitudes. Europe to tropical Asia and Malaya.

Local names: Lulúpong (Sub.) ; malanikník (Tag.).

Var. IMBECILLIS (R. Br.) Hack. in Govt. Lab. Publ. (Philip.) 35 (1906)

81; Merr. in Philip. Journ. Sci. 1 (1906) Suppl. 364.

Panicum imbecille Trin. Ic. Gram. (1828-36) 16, t. 191.

Orthopogon imbecillis R. Br. Prodr. (1810) 194.

Oplismenus minus Merr. in Govt. Lab. Publ. (Philip.) 17 (1904) 9.

Luzon (Cagayan, Benguet, Bontoc, Rizal, Laguna, Tayabas, Bulacan, Pangasinan, Bataan, Sorsogon), Catanduanes, Leyte, Negros, Mindanao, Merrill 3203, 4511, 5485, 8276, 6976, Kneucker Gram. Exsic. 739, Elmer 17105, B. S. 29923 Fénix, 12281 Foxworthy, 30286, 19381 Ramos, 31692 Santos. Along streams in shaded places and on ridges in the mossy forest, at low and medium altitudes, ascending to 2,400 m. Australia.

\section{SETARIA Beauvois}

SetAriA GeniculatA (Lam.) Beauv. Agrost. (1812) 51, 178.

Panicum geniculatum Lam. Encycl. 4 (1798) 727 (err. typ. 737).

Panicum flavum Nees in Mart. Fl. Bras. 2 (1829) 180.

Setaria flava Kunth Rev. Gram. 1 (1829) 46; Merr. in Philip. Journ. Sci. 1 (1906) Suppl. 365, 5 (1910) Bot. 327, Fl. Manila (1912) 95, Sp. Blancoanae (1918) 68.

Panicum penicillatum Nees in Nov. Act. Acad. Nat. Cur. 19 (1843) Suppl. 1: 173, Hook. Journ. Bot. Kew Miscel. 2 (1850) 97, non Willd.

Panicum chrysanthum Steud. Nomen. ed. 2, 2 (1841) 254, Syn. Pl. Glum. 1 (1854) 50.

Panicum rubiginosum Steud. Syn. Pl. Glum. 1 (1854) 50.

Setaria aurea Hochst. ex A. Br. in Flora 24 (1841) 276.

Setaria pilifera Llanos Frag. Pl. Filip. (1851) 34; F.-Vill. \& Naves in Blanco Fl. Filip. ed. $3,4^{1}$ (1880) 25, non Desv.

Setaria glauca F.-Vill. Novis. App. (1882) 312; Vidal Phan. Cuming. Philip. (1885) 157, Rev. Pl. Vasc. Filip. (1886) 288, non Beauv.

Chaetochloa glauca Scribn. var. aurea W. F. Wight in Contr. U. S. Nat. Herb. 9 (1905) 223.

Setaria glauca Beauv. var. aurea K. Schum. Fl. Deutsch. Schutzegeb. Südsee (1901) 180.

Setaria rubiginosa Miq. Fl. Ind. Bat. 3 (1857) 467; F.-Vill. Novis. App. (1882) 313.

Chaetochloa geniculata Millsp. \& Chase in Field Mus. Bot. 3 (1903)

37; Hitchc. in Contr. U. S. Nat. Herb. 22 (1920) 168, f. 41.

Throughout the Philippines in open grasslands at low and medium altitudes, ascending to $1,800 \mathrm{~m}$. Pantropic.

Local names: Buntót-púsa (Tag.) ; lagilikáran (Bag.) ; putiñán (Ig.) ; sabsablúg (Bon.); samsamáng (Bon.); tirtirígo (Ilk.) ; daua-ambau (Sul.). 
SETARIA ITALICA (Linn.) Beauv. Agrost. (1812) 51; Miq. Fl. Ind. Bat. 3 (1857) 467; F.-Vill. Novis. App. (1882) 312; Vidal. Phan. Cuming. Philip. (1885) 157; Merr. in Philip. Journ. Sci. 1 (1906) Suppl. 365, Sp. Blancoanae (1918) 68; Hubbard in Am. Journ. Bot. 2 (1915) 192 (subsp. stramineo-fructa Hubb. var. brunneoseta Hubb.).

Panicum italicum Linn. Sp. Pl. (1753) 56; Nees in Hook. Journ. Bot. Kew Miscel. 2 (1850) 97.

Panicum miliaceum Blanco Fl. Filip. (1837) 39; ed. 2 (1845) 28; ed. 3, 1 (1877) 52, non Linn.

Panicum comosum Steud. Syn. Pl. Glum. 1 (1854) 53, ex deser.

Setaria comosa Miq. Fl. Ind. Bat. 3 (1857) 468; F.-Vill. Novis. App. (1882) 313.

Chaetochloa italica Scribn. in U. S. Dept. Agr. Div. Agrost. Bull. 4 (1897) 39.

Cultivated here and there throughout the Philippines, occasional as an escape in old clearings. Cultivated in all warm countries. Italian millet.

Local names: Bikákau (Ilk.) ; boróna (Pamp.); bukákau (Ilk.); dáua (C. Bis., P. Bis., Tag.) ; rautnokara (Iv.) ; sábug (Ig.) sammang (Bon.); turai (Sul.).

SETARIA LAXA Merr. in Philip. Journ. Sci. 1 Suppl. (1906) 366, Fl. Manila (1912) 96.

Chamaeraphis gracilis Hack. in Engl. Bot. Jahrb. 6 (1885) 236, non Setaria gracilis HBK.

Panicum chamaeraphoides Hack. ex Koord. Exkursionsfl. Java 1 (1911) 135.

Luzon (Rizal, Laguna), Merrill 7440, Phil. Pl. 595, Hallier. In thickets and fallow lands at low altitudes. Java.

SETARIA PALMIFOLIA (Koenig) Stapf in Journ. Linn. Soc. Bot. 42 (1914) 186.

Panicum palmaefolium Koenig in Naturf. 23 (1788) 208; Miq. Fl. Ind. Bat. 3 (1857) 449; Merr. in Philip. Journ. Sci. 1 (1906) Suppl: 361, 2 (1907) Bot. 261, 5 (1910) Bot. 327.

Panicum plicatum F.-Vill. Novis. App. (1882) 311; Mez in Perk. Frag. Fl. Philip. (1904) 143, non ? Lam.

Panicum amplissimum Steud. Syn. Pl. Glum. 1 (1854) 54; Vidal Phan. Cuming. Philip. (1885) 156, Rev. P1. Vasc. Filip. (1886) 287.

Panicum nepalense Spreng. Syst. 1 (1825) 321; Rolfe in Journ. Bot. 23 (1885) 216.

Panicum neurodes Schultes Syst. Mant. 2 (1824) 321; Vidal Phan. Cuming. Philip. (1885) 157, Rev. Pl. Vasc. Filip. (1886) 287; Usteri Beitr. Ken. Philip. Veg. (1905) 133.

Panicum lene Steud. Syn. Pl. Glum. 1 (1854) 54.

Setaria lenis Miq. Fl. Ind. Bat. 3 (1857) 468; F.-Vill. Novis. App. (1882) 313.

Chamaeraphis. setosa O. Kuntze var. lenis O. Kuntze Rev. Gen. Pl. (1891) 769.

Panicum kleinianum Nees in Hook. Journ. Bot. Kew Miscel. 2 (1850) 97. 
Chaetochloa palmifolia Hitchc. \& Chase in Contr. U. S. Nat. Herb. 22 (1920) 161, f. 38.

Northern Luzon to Palawan and Mindanao, in most or all islands and provinces. Common in thickets, old clearings, abacá plantations, etc., at low and medium altitudes, ascending to $200 \mathrm{~m}$. India to China and Malaya, introduced in tropical America.

Local names: Agusáis (Bik.); asáhas (C. Bis.); bañas (Ig.) ; dumbug (Sul.) ; hagusáhis (Bik.); lalasa (Ig.); liahon (If.) ; lias (Bon.); yas (Ig.).

SETARIA VERTICILlATA (Linn.) Beauv. Agrost. (1812) 51; F.-Vill.

Novis. App. (1882) 312; Merr. in Philip. Journ. Sci. 3 (1908) Bot. 397.

Panicum verticillatum Linn. Sp. Pl. ed. 2 (1762) 82.

Batan Islands, Luzon (Rizal), B. S. 4041 Fénix, Merrill Phil. Pl. 1406. In open waste places. Temperate and tropical regions generally.

SETARIA VIRIDIS (Linn.) Beauv. Agrost. (1812) 51; F.-Vill. Novis. App. (1882) 312; Merr. in Philip. Journ. Sci. 1 (1906) Suppl. 366, Fl. Manila (1912) 95.

Luzon (Cagayan, Bontoc, Rizal). A casual introduction, occurring rarely in open waste places. A temperate and subtropical weed.

Local names: Dapdapígan (Bon.) ; upoan (Bon.).

\section{EXCLUDED SPECIES}

Setaria globularis Presl Rel. Haenk. 1 (1830) 314; Kunth Enum. 1 (1833) 151; Miq. Fl. Ind. Bat. 3 (1857) 314; F.-Vill. Novis. App. (1882) 312; Scribn. in Rept. Mo. Bot. Gard. 10 (1899) 52.

Panicum globutare Steud. Syn. Pl. Glum. 1 (1854) 51.

"Hab. in insulis Philippinis." The species is certainly not Philippine, but the specimens on which it was based were probably of Mexican origin. A sheet of Presl's type material in the herbarium of the Missouri Botanical Garden consists of Setaria caudata Lam. and S. composita Kunth.

Setaria macrostachya HBK.; F.-Vill. Novis. App. (1882) 312.

\section{CENCHRUS Linnaeus}

CENCHRUS VIRIDIS Spreng. Syst. Veg. 1 (1825) 301; Chase in Contr. U. S. Nat. Herb. 22 (1920) 57, f. 11.

Cenchrus inflexus R. Br. Prodr. (1810) 50, non Poir.

Cenchrus echinatus Presl Rel. Haenk. 1 (1830) 317; Miq. Fl. Ind. Bat. 3 (1857) 472; F.-Vill. Novis. App. (1882) 313; Perk. Frag. Fl. Philip. (1904) 145; Merr. in Philip. Journ. Sci. 1 (1906) 367, F1. Manila (1912) 95, Sp. Blancoanae (1918) 68, non Linn.

Cenchrus hexaflorus Blanco Fl. Filip. (1837) 36, ed. 2 (1845) 24, ed. 3,1 (1877) 46.

A widely distributed weed in the settled areas of the Philippines, occurring generally in open waste places in and about towns. A native of tropical America, now also found in Guam, Siam, Indo-China, New Guinea, and northern Australia. Dr. A. B. Rendle has critically compared our material with the type of Cenchrus inflexus $\mathrm{R}$. Br. 
The Philippine form has been confused with C. echinatus Linn.

Local names: Agíngai (Tag.) ; sagisí (Ilk.).

\section{PENNISETUM Persoon}

PENNISETUM ALOPECUROIDEUM (Linn.) Spreng. Syst. 1 (1825) 303.

Panicum alopecuroideum Linn. Sp. Pl. (1753) 55.

Alopecurus hordeiformis Lour. Fl. Cochinch. (1790) 48, non ? Linn.

Pennisetum compressum R. Br. Prodr. (1810) 195; Merr. in Philip. Journ. Sci. 1 (1906) Suppl. 368.

Gymnothrix nigricans Presl Rel. Haenk. 1 (1830) 315.

Pennisetum nigricans. Trin. ex Steud. Nomencl. ed. 2, 2 (1841) 297;

Miq. Fl. Ind. Bat. 3 (1857) 470; F.-Vill. Novis. App. (1882) 312.

Pennisetum cenchroides F.-Vill. Novis. App. (1882) 313, non Spreng.

Luzon (Ilocos Norte, Bontoc, Benguet), Merrill 7704, 4435, Williams 1201, Elmer 5756, B. S. 31898 Santos, 5932, 5365, 27422 Ramos, 12708 Fénix, 8763 McGregor. On open slopes at medium altitudes, chiefly in the pine region, ascending to $2,000 \mathrm{~m}$. India to Indo-China, China, Japan, and Australia.

Local name: Kaibuan (Ig.).

PEnNisetum Macrostachyum Trin. in Mém. Acad. St. Pétersb. VI $3^{2}$ (1835) 177; Ceron Cat. Pl. Herb. Manila (1892) 121; Merr. in Philip. Journ. Sci. 1 (1906) Suppl. 368.

Sericura elegans Hassk. in Flora 25 (1842) Beibl. 2: 2.

Luzon (Laguna, Batangas, Rizal, Tayabas, Sorsogon), Mindoro, Masbate, Panay, Celou, Negros, Mindanao, Basilan, Merrill 3381, Williams 2622, Elmer 9674, 81324, 15327, B. S. 31306 Ramos \& Edaño, 24939 Fénix, 14749, 11079, 5217 Ramos, 15494 Reillo. In old clearings at low and medium altitudes. Malay Peninsula and Archipelago to Polynesia.

Local names: Daráui (C. Bis.); gulálai (Sub.); handaláui (C. Bis.) ; lagóli (Bag.).

\section{CHAMAERAPHIS R. Brown}

CHAMAERAPHIS MURICATA (Linn. f.) comb. nov.

Andropogon muricatus Linn. f. Suppl. (1781) 433; Stapf in Kew Bull. (1906) 348 in nota.

Panicum asperum Koen. in Naturf. 23 (1788) 209.

Panicum spinescens R. Br. Prodr. (1810) 193.

Chamaeraphis spinescens Poir. in Lam. Encycl. Suppl. 2 (1811) 189; F.-Vill. Novis. App. (1882) 313; Hook. f. Fl. Brit. Ind. 7 (1897) 62.

Chamaeraphis aspera Nees in Wall Cat. (1849) No. 8679; Mez in Perk. Frag. Fl. Philip. (1904) 145; Merr. in Philip. Journ. Sci. 1 (1906) 368, Fl. Manila (1912) 96.

Central Luzon to Palawan and Mindanao, Merrill 375, 4194, 7060, 9467, B. S. 29500 Ramos \& Edaño. In shallow stagnant water at low altitudes. India to China through Malaya to tropical Australia.

36. THUAREA Persoon

THUAREA INVOLUTA (Forst.) R. \& S. Syst. 2 (1817) 782; Merr. in Philip. Journ. Sci. 1 (1906) Suppl. 369, Fl. Manila (1912) 97.

Ischaemum involutum Forst. Prodr. (1786) 73. 
Thuarea sarmentosa Pers. Syn. 1 (1805) 110; Nees in Hook. Journ.

Bot. Kew Miscel. 2 (1850) 97 (Thouarea); F.-Vill. Novis. App. (1882) 313; Usteri Beitr. Ken. Phil. Veg. (1905) 133; Merr. in Philip. Journ. Sci. 1 (1906) Suppl. 28.

Luzon (Cagayan, Bataan, Rizal), Mindoro, Palawan, Samar, Mindanao. On sandy beaches and in thickets back of the beach, often common. Ceylon to Madagascar through Malaya to Australia and Polynesia.

\section{SPINIFEX Linnaeus}

SPINIFEX LITTOREUS (Burm. f.) Merr. in Philip. Journ. Sci. 7 (1912) Bot. 229, Fl. Manila (1912) 97, Sp. Blancoanae (1918) 69.

Stipa littorea Burm. f. Fl. Ind. (1768) 29.

Stipa spinifex Linn. Mant. 1 (1767) 84; Blanco Fl. Filip. (1837) 41 ; ed. 2 (1845) 29, ed. 3 , 1 (1877) 54.

Spinifex squarrosus Linn. Mant. 2 (1771) 163; Blanco Fl. Filip. (1837) 46; ed. 2 (1845) 31; ed. 3, 1 (1877) 57; F.-Vill. Novis. App. (1882) 313; Nees in Hook. Journ. Bot. Kew Miscel. 2 (1850) 97; Vidal Phan. Cuming. Philip. (1885) 157, Rev. Pl. Vasc. Filip. (1886) 288; Merr. in Philip. Journ. Sci. 1 (1906) Suppl. 369.

On sandy seashores from the Batan Islands to Palawan and Mindanao. India to China and Malaya. Bis.).

Local names: Baki-báki (Bis.); tartarái (Ilk.) ; tayung-táyung (C.

\section{LEPTASPIS R. Brown}

LEPTASPIS CUMINGII Steud. Syn. Pl. Glum. 1 (1854) 416; Miq. Fl. Ind. Bat. 3 (1857) 375; F.-Vill. Novis. App. (1882) 318; Vidal Phan. Cuming. Philip. (1885) 157, Rev. Pl. Vasc. Filip. (1886) 288; Merr. in Philip. Journ. Sci. 1 (1906) Suppl. 370.

Leptaspis cochleata Hack. in Govt. Lab. Publ. (Philip.) 35 (1906) 81, non Thwaites.

Mindanao (Misamis, Davao), Bongao, Copeland 703', Cuming 1627, B. S. $\$ 6319$ Yates. In forests at low or medium altitudes. Endemic.

LEPTASPIS URCEOlATA (Roxb.) R. Br. in Benn. Pl. Jav. Rar. (1838) 23, t. 6; Miq. Fl. Ind. Bat. 3 (1854) 374; F.-Vill. Novis. App. (1882) 318; Vidal Phan. Cuming. Philip. (1885) 157, Rev. Pl. Vasc. Filip. (1886) 288; Merr. in Philip. Journ. Sci. 1 (1906) Suppl. 370; C. B. Rob. in Philip. Journ. Sci. 6 (1911) Bot. 194.

Pharus urceolatus Roxb. Fl. Ind. ed. 2, 3 (1832) 611.

Leptaspis manillensis Steud. Syn. Pl. Glum. 1 (1854) 8; Miq. Fl. Ind. Bat. 3 (1857) 374; F.-Vill. Novis. App. (1882) 318.

Luzon (Camarines, Sorsogon), Polillo, Palawan, Samar, Leyte, Negros, Mindanao. In thickets and forests at low and medium altitudes; represented by numerous collections. Ceylon, Malay Peninsula, Sumatra, Java, through the Moluccas to New Guinea.

Local names: Alaplap (Mbo.) ; baliotus (Bag.); budhak-budhak (Sul.); handalokot (Mbo.) ; madioug (S. L. Bis.) ; rokot-dókot (S. L. Bis.) ; salindaget (Mbo.) ; talingagito (Sub.). 


\section{ORYZA Linnaeus}

ORYZA MEYERIANA (Zoll. \& Morr.) Benth. ex Pilger in Perk. Frag. Fi. Philip. (1904) 145; Merr. in Philip. Journ. Sci. 1 (1906) Suppl. 370.

Padia meyeriana Zoll. \& Morr. Verz. Ind. Arch. (1854) 103; Steud. Syn. Pl. Glum. 1 (1854) 3; Miq. Fl. Ind. Bat. 3 (18.57) 373.

Oryza granulata Nees \& Arn. in Wall. Cat. (1830) No. 2354, nomen nudum; Hook. f. Fl. Brit. Ind. 7 (1897) 93; Merr. in Govt. Lab. Publ. (Philip.) 6 (1904) 7.

Luzon (Isabela, Ifugao, Nueva Vizcaya, Rizal, Tayabas), Palawan, Panay, Mindanao (Zamboanga), Merrill 116, 6697, 8060, 7244, B. S. 19967 McGregor, 2067y Escritor, 343 Bermejos, 8036 Ramos. In forests, bamboo thickets, etc., at low and medium altitudes. India to Java, Borneo, and Celebes.

ORYZA MINUTA Presl Rel. Haenk. 1 (1830) 208; Kunth Enum. 1 (1833) 7; Miq. Fl. Ind. Bat. 3 (1857) 371; F.-Vill. Novis. App. (1882) 319.

Oryza manillensis Merr. in Philip. Journ. Sci. 3 (1908) Bot. 219, Fl. Manila (1912) 98.

Oryza latifolia F.-Vill. Novis. App. (1882) 319, non Desv.

Luzon (Rizal, Laguna, Tayabas, Sorsogon), Mindanao (Zamboanga), Presl! in Herb. Bernhardi, Merrill 8112, 7402, Phil. Pl. 128, Elmer 17407, B.S. 29492 Ramos \& Edaño, 2194, 12102, 12044, 13269 Ramos, 36714 Ram mos \& Edaño. In open wet places at low and medium altitudes. Borneo, Malay Peninsula.

This is I believe the Malay Peninsula and Bornean form currently referred to Oryza latifolia Desv., but I believe the type of Desvaux's species was an American plant. Of the specimens cited, Elmer 17407, B. S. 36714 Ramos \& Edaño, and Merrill 8112, being more robust than the others, closely match the Malay Peninsula form.

Local names: Palai-máya (Sul.); palai-pálai (Sub.); baili-gaja (Sul.).

ORYZA SATIVA Linn. Sp. Pl. (1753) 333; F.-Vill. Novis. App. (1882) 318; Merr. in Philip. Journ. Sci. 1 (1906) Suppl. 370, Fl. Manila (1912) 97, Sp. Blancoanae (1918) 69, 70.

Oryza aristata Blanco Fl. Filip. (1837) 274; ed. 2 (1845) 190; ed. 3,1 (1877) 339.

Oryza sativa Linn. vars. bixamban, glutinosa, lamuyo, pilosa, praecox, quinanda, rubra, et violacea Blanco 1. c.

Oryza glutinosa Lour. Fl. Cochinch. (1790) 215; F.-Vill. Novis. App. (1882) 319.

Oryza montana Lour. I. c. 215; F.-Vill. I. c.

Oryza praecox Lour. 1. c. 215; F.-Vill. 1. c.

Extensively cultivated throughout the Philippines but not a native of the Archipelago, although of prehistoric introduction. The rice plant is enormously variable and probably several hundred distinct cultural forms occur in the Archipelago. No attempt has been made to compile the very numerous local names currently applied to special varieties. Rice. 
Local names: Ammái (Ibn. It.) ; págai (Il.) ; págei (Bon.) ; pálai (Tag.) ; párai (Bik.) ; pai (Sul.).

\section{LEERSIA Swartz}

LeERsia HEXANDRA Sw. Prodr. (1788) 21; Llanos Frag. Pl. Filip. (1851) 26; F.-Vill. \& Naves in Blanco Fl. Filip. ed. 3, $4^{1}$ (1880) 18; F.-Vill. Novis. App. (1882) 318; Vidal Phan. Cuming. Philip. (1885) 157, Rev. Pl. Vasc. Filip. (1886) 288; Pilger in Perk. Frag. Fl. Philip. (1904) 145; Merr. in Philip. Journ. Sci. 1 (1906) Suppl. 371, Fl. Manila (1912) 98.

Leersia luzonensis Presl Rel. Haenk. 1 (1830) 207; Nees in Hook. Journ. Bot. Kew Miscel. 2 (1850) 97; Miq. Fl. Ind. Bat. 3 (1857) 367; F.-Vill. Novis. App. (1882) 318.

Leersia glaberrima Trin. in Mém. Acad. St. Pétersb. VI 5 (1839) 173; Miq. Fl. Ind. Bat. 3. (1857) 368; F.-Vill. Novis. App. (1882) 318.

Homalocenchrus hexandrus O. Kuntze Rev. Gen. Pl. (1891) 777.

Northern Luzon to Mindanao. In open wet places at low and medium altitudes, ascending to $1,500 \mathrm{~m}$. In the vicinity of Manila this is very extensively cultivated in paddies for green forage.

Local names: Bárit (Tag.); buñgálon (Bis.) ; zacáte (Sp.).

\section{MICROLAENA R. Brown}

MICROLAENA STIPOIDES (Labill.) R. Br. Prodr. (1910) 210; Benth.

Fl. Austral. 7 (1878) 552; Hack. in Philip. Journ. Sci. 1 (1906)

Suppl. 269; Merr. op. cit. 371, 5 (1910) Bot. 328.

Ehrhartia stipoides Labill. Pl. Nov. Holl. 1 (1804) 91, t. 118.

Luzon (Abra, Bontoc, Lepanto, Benguet, Zambales), Merrill 4543, 4696, 4831, 65\% , Phil. Pl. 1343, B. S. $3195 \%$ Santos, 5128, 7064 Ramos. On open slopes and ridges, along mountain trails, etc., altitude 1,800 to $2,300 \mathrm{~m}$. Australia, New Zealand, Hawaii.

\section{PHALARIS Linnaeus}

PHALARIS MINOR Retz. Obs. 3 (1783) 8; Merr. in Philip. Journ. Sci. 2 (1907) Bot. 421.

Luzon (Benguet), B. S. 2785 Mearns. Altitude about $1,500 \mathrm{~m}$; probably a mere casual here. Europe, India, South Africa, Australia.

\section{ANTHOXANTHUM Linnaeus}

ANTHOXANTHUM LUzoniense Merr. in Philip. Journ. Sci. 1 (1906)

Suppl. 178, 372, 5 Bot. (1910) Bot. 328.

Luzon (Benguet), Merrill 4713, 6614, Phil. Pl. 184, B. S. 8890 McGregor, F. B. 16188 Curran, Merritt, \& Zschokke. In open grasslands, altitude 2,000 to $2,700 \mathrm{~m}$. Endemic.

\section{ARISTIDA Linnaeus}

ARIStida CULIONensis Pilg. ex Perk. Frag. Fl. Philip. (1904) 145; Merr. in Philip. Journ. Sci. 1 (1906) Suppl. 372.

Culion, Merrill 471, 515, E. S. 41334 Ramos. In open grasslands at low altitudes. Endemic. 
ARISTIDA CUMINGIANA Trin. \& Rupr. in Mém. Acad. St. Pétersb. VI 7 (1849) 141; Steud. Syn. Pl. Glum. 1 (1854) 140; Miq. Fl. Ind. Bat. 3 (1857) 381; Vid. Phan. Cuming. Philip. (1885) 159, Rev. Pl. Vasc. Filip. (1886) 292; Merr. in Philip. Journ. Sci. 1 (1906) Suppl. 372, 5 (1910) Bot. 328, Fl. Manila (1912) 99.

Aristida capillacea Cav. Ic. 5 (1799) 43, t. 468, f. 1, non Lam.

Chaetaria trichodes Nees in Hook. Journ. Bot. Kew Miscel. 2 (1850) 101.

Aristida trichodes Walp. Ann. 3 (1853) 753.

Luzon (Ilocos Norte, Lepanto, Ifugao, Bontoc, Benguet, Pangasinan, Rizal), Mindanao (Bukidnon). In open dry grasslands at low and medium altitudes, ascending to $1,500 \mathrm{~m}$. India to tropical Africa and southern China.

ARISTIDA TENuISETUlosa (Pilg.) Mez in Fedde Repert. 17 (1921) 147. Aristida stipoides $\mathrm{R}$. Br. var, tenuisetulosa Pilg. ex Perk. Frag. Fl. Philip. (1904) 146; Merr. in Philip. Journ. Sci. 1 (1906) Suppl. 372.

Luzon (Amburayan, Ilocos Norte, Ilocos Sur, Zambales, Pampanga, Bataan), Merrill 329, F. B. 5855, 6361 Curran, 124\%6, 15657 Merritt \& Darling, B. S. 2334 Mearns. In dry open sandy soil at low and medium altitudes. Endemic, but closely allied to the Australian A. stipoides R. Br.

\section{EXCLUDED SPECIES}

Aristida LAXA Cav. Ic. 5 (1799) 44, t. 470, f. 1; Kunth Enum. 1 (1833) 192; Miq. Fl. Ind. Bat. 3 (1857) 381; F.-Vill. Novis. App. (1882) 319.

"Habitat prope Montevideo, et in insulis Philippicis." This is probably a Mexican species, certainly not Philippine, the latter record being based on a wrongly localized specimen of Née's collection.

ARISTIDA LUZoniensis Cav. Ic. 5 (1799) 45, t. 470, f. 2; Spreng. Syst. 1 (1825) 266; Kunth Enum. 1 (1833) 192; Miq. Fl. Ind. Bat. 3 (1857) 381; F.-Vill. \& Naves in Blanco Fl. Filip. ed. 3, $4^{1}$ (1880) 99; Novis. App. (1882) 319.

Chaetaria luzoniensis Beauv. Agrost. (1812) 30.

"Habitat in insula Luzon altera ex Philippicis." Certainly not a Philippine species, but American or Australian.

ARIstida MURInA Cav. Ic. 5 (1799) 44, t. 469, f. 1; Kunth Enum. 1 (1833) 192; F.-Vill. Novis. App. '(1882) 319.

"Habitat in Mindanao insula prope Samboangan [= Zamboanga]." Undoubtedly a wrongly localized species, and Australian or American rather than Philippine.

ARISTIDA RIGIDA Cav. Ic. 5 (1799) 44, t. 469, f. 2; Kunth Enum. 1 (1833) 192; Miq. Fl. Ind. Bat. 3 (1857) 381; F.-Vill. Novis. App. (1882) 319.

"Habitat in insulis Philippicis." Unquestionably àn erroneously 10calized species, and Australian or American rather than Philippine. 
ARIstida sorzogonensis Presl Rel. Haenk. 1 (1830) 224; Kunth Enum. 1 (1833) 192; Miq. Fl. Ind. Bat. 3 (1857) 381; F.-Vill. Novis. App. (1882) 319.

"Hab. in Luzonia ad Sorzogon." This record is probably based on an exroneously labeled specimen, the species in all probability being American.

\section{4a. MUEHLENBERGIA Schreber}

MUEHLENBERGiA ARISANENSIS Hayata Ic. Pl. Formosa 7 (1918) 87, f. 54 .

Luzon (Lepanto, Bontoc, Benguet), Merrill 4716, Clemens 9203, Vanoverbergh 1158, B. S. 40213, 40296, 40360 Ramos \& Edaño. Along small streams, in old clearings, and in the mossy forest, altitude 1,300 to 2,400 m. Formosa. Closely allied to $M$. japonica Steud.

\section{SPOROBOLUS R. Brown}

SPOROBOLUS DIANDRUS (Retz.) Beauv. Agrost. (1812) 26; Miq. Fl. Ind. Bat. 3 (1857) 375; F.-Vill. Novis. App. (1882) 321; Pilger in Perk. Frag. Fl. Philip. (1904) 146; Merr. in Philip. Journ. Sci. 1 (1906) Suppl. 373.

Agrostis diandra Retz. Obs. 5 (1786) 19.

Vilfa diandra Steud. Syn. Pl. Glum. 1 (1854) 155.

Luzon (Ilocos Norte, Pampanga, Rizal, Tayabas, Albay, Sorsogon), Mindoro, Palawan, Panay, Mindanao. In lawns and waste places about towns, perhaps introduced. Tropical Asia through Malaya to Australia.

SPOROBOLUS INDICUS (Linn.) R. Br. Prodr. (1810) 170; F.-Vill. Novis. App. (1882) 321; Merr. in Philip. Journ. Sci. 1 (1906) Suppl. 373, Fl. Manila (1912) 98, Sp. Blancoanae (1918) 71.

Agrostis indica Linn. Sp. Pl. (1753) 63.

Sporobolus elongatus R. Br. Prodr. (1810) 170; Usteri Beitr. Ken. Philip. Veg. (1905) 133.

Spermachiton involutum Llanos Fragm. Pl. Filip. (1851) 25; F.-Vill. \& Naves in Blanco Fl. Filip. ed. 3, $4^{1}$ (1880) 17.

Luzon (Cagayan, Isabela, Bontoc, Benguet), Mindanao (Lanao). Common at medium altitudes in open grasslands, ascending to $2,000 \mathrm{~m}$. Pantropic.

Local names: Bakit (P. Bis.) ; bangkit (P. Bis.) ; lusai (Sub.) ; sa $\widetilde{n g}-$ sañítan (Bon.).

SPOROBOLUS PILIFERUS (Trin.) Kunth Enum. 1 (1833) 611; Merr. in Philip. Journ. Sci. 1 (1906) Suppl. 373.

Vilfa pilifera Trin. Diss. (1828) 157, Sp. Gram. Ic. $t .58$.

Sporobolus ciliatus Presl Rel. Haenk. 1 (1830) 242; Scribn. in Rept.

Mo. Bot. Gard. 10 (1899) 53, t. 30; Hack. in Philip. Journ. Sci.

1 (1906) Suppl. 269.

Luzon (Bontoc, Benguet), Merrill 4362, 4\%18, Phil. Pl. 194, Williams $195 \%$, B. S. 5361, 5905 Ramos. On banks, in open grasslands, etc., at medium altitudes, ascending to $1,800 \mathrm{~m}$. India to the Malay Peninsula. 
SPOROBOLUS VIRGINICUS (Linn.) Kunth Rev. Gram. 1 (1829) 67;

Merr. in Philip. Journ. Sci. 5 (1910) Bot. 171, Fl. Manila (1912) 99. Agrostis virginica Linn. Sp. Pl. (1753) 63.

Sporobolus humilis Presl Rel. Haenk. 1 (1830) 241; Miq. Fl. Ind.

Bat. 3 (1857) 376; F.-Vill. Novis. App. (1882) 321; Scribn. in

Rept. Mo. Bot. Gard. 10 (1899) 53, t. 30; Merr. in Philip. Journ.

Sci. 1 (1906) Suppl. 374.

Vilfa humilis Steud. Syn. Pl. Glum. 1 (1854) 153.

Luzon (Cagayan, Rizal), Merrill Phil. Pl.574, B. S. 7881 Ramos. Along tidal streams within the influence of salt or brackish water. All or most warm countries.

\section{EXCLUDED SPECIES}

ŚPORoBolus SCOPARIUS Presl Rel. Haenk. 1 (1830) 243; Kunth Enum. 1 (1833) 216; Miq. Fl. Ind. Bat. 3 (1857) 376; F.-Vill. Novis. App. (1882) 321; Merr. in Philip. Journ. Sci. 1 (1906) Suppl. 374.

Vilfa scoparia Steud. Syn. Pl. Glum. 1 (1854) 156.

"Hab. ad portum Sorzogon" Presl. Certainly not a Philippine species, but probably based on American material.

\section{GARNOTIA Brongniart}

GARNOTIA STRICTA Brongn. in Bot. Duperr. Voy. (1829) 132, t. 21; Merr. in Philip. Journ. Sci. 1 (1906) Suppl. 374.

Northern Luzon to Mindanao. On talus slopes, along streams, etc., at low and medium altitudes; represented by numerous collections. India to Polynesia and Hawaii.

Var. LoNGISETA (Merr.) Hack. in Allgem. Bot. Zeitschr. 12 (1909) 141.

Garnotia longiseta Merr. ex Hack. 1. c. in syn.

Luzon (Bontoc, Benguet, Nueva Vizcaya, Bulacan, Bataan, Laguna). Chiefly in the mossy forest, altitude up to $2,300 \mathrm{~m}$, but occurring also at low or medium altitudes. Endemic.

Local names: Balahíbong-usá (Tag.); baríri (Bag.); pauai (Ig.).

\section{AGROSTIS Linnaeus}

AGRostis ALBA Linn. Sp. Pl. (1753) 63; Hook, f. Fl. Brit. Ind. 7 (1897) 254.

Luzon (Benguet), B. S. 40496 Ramos \& Edaño. Along trails in open forest, altitude about $1,500 \mathrm{~m}$; probably introduced. A native of temperate Europe and Asia, now widely distributed in temperate and subtemperate countries.

AGRostis ELMERI Merr. in Govt. Lab. Publ. (Philip.) 29 (1905) 7, Philip. Journ. Sci. 1 (1906) Suppl. 375, 5 (1910) Bot. 328.

Luzon (Bontoc, Lepanto, Benguet), Negros. In the mossy forest and in open places, altitude 1,600 to $2,800 \mathrm{~m}$; represented by numerous collections. Southern China. To be compared with A. micrantha Steud.

Local name: Sasamon (Ig.). 


\section{ANISELYTRON Merrill}

ANISELYTRON AGROSTOIDES Merr. in Philip. Journ. Sci. 5 (1910) Bot. 329.

Luzon (Benguet), Merrill 6483. In the mossy forest on Mt. Pulog, altitude about $2,400 \mathrm{~m}$. A monotypic endemic genus known only from the original collection.

\section{CALAMAGROSTIS Roth}

CALAMAGROSTIS ARUNDINACEA (Linn.) Roth var. NIPPONICA (Franch. \& Sav.) Hack. in Bull. Herb. Boiss. 7 (1899) 652, Philip. Journ. Sci. 1 (1906) Suppl. 269; Merr. op. cit. 375.

Calamagrostis nipponica Franch. \& Sav. Enum. Pl. Jap. 3 (1879) 599.

Luzon (Lepanto, Benguet), Mindanao (Davao), Merrill 4564, 4697, 4701, 7766, B. S. 31740 Santos, 4487 Mearns. On open grassy slopes, altitude 1,800 to $2,400 \mathrm{~m}$. Europe to India, China and Japan.*

Local names: Baríri (Bag.) ; sangsangitau (Ig.) ; sasamon (Ig.).

CALAMAgRostIS FILIFOLIA Merr. in Philip. Journ. Sci. 1 (1906) Suppl. 179, 375, 5 (1910) Bot. 330.

Luzon (Benguet), Merrill 4839, 6610, 6611, 4537, 4715, B. S. 31979 Santos, 8891 McGregor. On open slopes, altitude 1,800 to $2,200 \mathrm{~m}$. Endemic.

Local name: Añgangsúg (Ig.).

50. ERIACHNE R. Brown

ERIACHNe PALLesCens R. Br. Prodr. (1810) 184; Benth. Fl. Austral. 7 (1878) 630; F.-Vill. Novis. App. (1882) 321; Pilger in Perk. Frag. Fl. Philip. (1904) 147; Merr. in Philip. Journ. Sci. 1 (1906) Suppl. 376.

Culion, Palawan, Merrill 520, 9582. In open grassy places up to 550 $m$ altitude. India to southern China through Malaya to Australia.

ERIACHNE TRISETA Nees ex Steud. Syn. Pl. Glum. 1 (1854) 237; Pilger in Perk. Frag. Fl. Philip. (1904) 147; Merr. in Philip. Journ. Sci. 1 (1906) Suppl. 376.

Culion, Merrill. In open grasslands at low altitudes. India, Ceylon. EXCLUDED SPECIES

ERIACHNE GRACILIS Brongn; F.-Vill. Novis. App. (1882) 321.

\section{COELACHNE R. Brown}

COELACHNE HACKelil Merr. in Govt. Lab. Publ. (Philip.) 29 (1905) 8, Philip. Journ. Sci. 1 (1906) Suppl. 376.

Luzon (Benguet), Elmer 5762, Merrill 4338, Phil. Pl. 198, Williams 1196. Forming dense mats along small cool streams, altitude about 1,500 m. Endemic.

Coelachne pulchella R. Br. and C. brachiata Munro were both credited to the Philippines by F.-Villar, Novis. App. (1882) 321, but neither has been found in the Archipelago. 
52. DESCHAMPSIA Beauvois

DESCHAMPSIA FleXUOSA (Linn.) Trin. in Bull. Acad. St. Pétersb. 1 (1836) 66; Merr. in Philip. Journ. Sci. 5 (1910) Bot. 330.

Aira flexuosa Linn. Sp. Pl. (1753) 65.

Luzon (Benguet), Merrill 6354, Phil. Pl. 189, B. S. 8904 McGregor, F. B. 16155 Curran, Merritt, \& Zschokke. In open grasslands at the summit of Mt. Pulog, altitude about 2,600 m. Endemic. North Temperate Zone generally.

\section{AVENA Linnaeus}

AVENA FAtUA Linn. Sp. Pl. (1753) 80; F.-Vill. Novis. App. (1882) 318; Merr. in Philip. Journ. Sci. 10 (1915) Bot. 287.

Luzon (Benguet), B. S. 2812 Mearns. Probably merely a casual here, altitude about $1,500 \mathrm{~m}$. A widely distributed weed in temperate regions, of European origin.

AVENA SATIVA Linn. Sp. Pl. (1753) 79; Merr. in Philip. Journ. Sci. 1 (1906) Suppl. 376.

Occasional in the Mountain Province at and above $1,500 \mathrm{~m}$ altitude, here a casual that will probably not persist. Oats.

\section{EXCLUDED SPECIES}

AvenA sterilis Linn.; F.-Vill. Novis. App. (1882) 319.

54. MICROCHLOA R. Brown

MICROCHLOA INDICA (Linn. f.) O. Kuntze Rev. Gen. Pl. 3 (1893) 356;

Hack. in Fedde Repert. 7 (1909) 373; Merr. in Philip. Journ. Sci.

7 (1912) Bot. 74.

Nardus indica Linn. f. Suppl. (1781) 105.

Rottboellia setacea Roxb. Pl. Coromandel 2 (1798) 18, t. 132.

Microchloa setacea R. Br. Prodr. (1810) 208; F.-Vill. Novis. App. (1882) 319.

Luzon (Ilocos Norte, Bontoc, Lepanto, Rizal), B. S. 32889 Ramos, Loher 7179, Vanoverbergh 764. In open dry grasslands at low and medium altitudes. Pantropic.

\section{CYNODON Persoon}

CYNOdON ARCUATUS Presl Rel. Haenk. 1 (1830) 290; F.-Vill. Novis. App. (1882) 320; Scribn. in Rept. Mo. Bot. Gard. 10 (1899) 41, t. 40; Merr. in Govt. Lab. Publ. (Philip.) 17 (1904) 9, Philip. Journ. Sci. 1 (1906) Suppl. 28, 377, Fl. Manila (1912) 103.

Luzon (Ilocos Norte, Bontoc, Tarlac, Bataan, Rizal, Laguna), Merrill 3619, Phil. Pl. 135, Williams 88, B. S. 1392, 32738 Ramos, 23130 Ramos. In open grasslands at low and medium altitudes. Endemic.

CYNODON DACTYLON (Linn.) Pers. Syn. 1 (1804) 85; Miq. Fl. Ind. Bat. 3 (1857) 382; F.-Vill. Novis. App. (1882) 319; Vidal Phan. Cuming. Philip. (1885) 159, Rev. Pl. Vasc. Filip. (1886) 292; Pilg. ex Perk. Frag. Fl. Philip. (1904) 147; Merrr. in Philip. Journ. Sci. 1 (1906) Suppl. 28, 377, Fl. Manila (1912) 103, Sp. Blancoanae (1918) 71. 
Panicum dactylon Linn. Sp. PI. (1753) 58.

Cynodon linearis Willd. Enum. Hort. Berol. (1809) 90; Presl Rel. Haenk. 1 (1830) 290; Nees in Hook. Journ. Bot. Kew Miscel. 2 (1850) 98.

Panicum glumaepatulum Steud. Syn. Pl. Glum. 1 (1854) 41; F.-Vill.

Novis. App. (1882) 312 (glumaepetalum).

Digitaria glumaepatula Miq. Fl. Ind. Bat. 3 (1857) 439.

Cynodon rufescens Llanos Frag. Pl. Filip. (1851) 31; F.-Vill. \&

Naves in Blanco Fl. Filip. ed. 3, $4^{1}$ (1880) 21, non ? Lagasca.

Capriola dactylon O. Kuntze Rev. Gen. Pl. (1891) 764.

Throughout the Philippines, in open grasslands, fallow rice paddies, waste places, at low and medium altitudes; common. Pantropic.

Local names: Balbálut (Bon.) ; galud-gálud (Ilk.) ; gráma (Sp.) ; kolatai (Tag.); palot-gálot (Ilk.).

\section{CHLORIS Swartz}

CHLORIS BARBATA (Linn.) Sw. Fl. Ind. Occ. 1 (1797) 200 ; Miq. Fl. Ind. Bat. 3 (1857) 387; Nees in Hook. Journ. Bot. Kew Miscel. 2 (1850) 102; F.-Vill. Novis App. (1882) 320; Vidal Phan. Cuming. Philip. (1885) 159, Rev. Pl. Vasc. Filip. (1886) 292; Hook. f. Fl. Brit. Ind. 7 (1897) 292; Pilger in Perk. Frag. Fl. Philip. (1904) 147; Merr. in Philip. Journ. Sci. 1 (1906) Suppl. 377, Fl. Manila (1912) 103, Sp. Blancoanae (1918) 72.

Andropogon barbatum Linn. Mant. 2 (1771) 302.

Chloris longifolia Steud. Syn. Pl. Glum: 1 (1854) 205; Miq. Fl. Ind. Bat. 3 (1857) 388; F.-Vill. Novis. App. (1882) 320.

Chloris inflata Llanos Frag. Pl. Filip. (1851) 31; F.-Vill. \& Naves in Blanco Fl. Filip. ed. $3,4^{1}$ (1880) 22, non Link.

A common weed in and about towns in many parts of Luzon, Mindoro, Negros, and Palawan, and probably in other islands. A pantropic weed of American origin.

Local namẹ: Banúko (Ilk.) ; korokorósan (Tag.).

CHLORIS INCOMPLETA Roth Nov. Sp. PI. (1821) 60; Merr. in Philip. Journ. Sci. 10 (1915) Bot. 288.

Luzon (Zambales), Palawan, Mindanao (Zamboanga), Merrill 9275, 9616, 8279, Phil. Pl. 1308, Gates 8145. In dry open sandy soil at low altitudes, sometimes along the seashore. India to Formosa and Malaya.

CHLORIS MEARNSII Merr. in Philip. Journ. Sci. 3 (1908) Bot. 220.

Luzon (Ilocos Norte), B. S. 7606, 27201 Ramos, 2294 Mearns. In dry open places at low altitudes; perhaps but a depauperate form of $C$. incompleta Roth. Endemic.

CHLORIS TENER (Presl) Scribn. in Rept. Mo. Bot. Gard. 10 (1899) 41, t. 40.

Cynodon tener Presl Rel. Haenk. 1 (1830) 291; Kunth Enum. 1 (1833) 260; Miq. Fl. Ind. Bat. 3 (1857) 383; F.-Vill. Novis. App. (1882) 320 .

Luzon (Sorsogon), Negros, (Presl), B. S. 9951 Robinson, as Cynodon. In open places at low altitudes. Hainan, Indo-China. 
Chloris CRINITA Lagasca Varied. Cienc. 4 (1805) 143; Kunth Enum. 1 (1833) 268; Miq. Fl. Ind. Bat. 3 (1857) 389; F.-Vill. Novis. App. (1882) 320.

"Insulae Philippinae" Kunth. This was probably based on a Malaspina expedition collection erroneously localized. Judging from the description it is not a Chloris.

Chloris truncata R. Br. Prodr. (1910) 186; Kunth Enum. 1 (1833) 266;

Miq. Fl. Ind. Bat. 3 (1857) 387; F.-Vill. Novis. App. (1882) 320.

Chloris dolichostachya Lagasca Gen. Sp. Nov.'(1816) 5.

Lagasca's species was credited to the Philippines by him; Link has reduced it to Chloris truncata $\mathrm{R}$. Br., an Australian species. Nothing conforming to it is known from the Philippines.

Chloris INFlata Link Hort. Berol. 1 (1827) 105.

"Calif.; Ins. Philipp." Link. The species was probably based on a Haenke collection, probably erroneously localized, at least as to the Philippine reference.

Chloris Radiata Sw.; F.-Vill. Novis App. (1882) 320.

Chloris RUfescens Lagasca Varied. Cienc. 4 (1805) 143; Kunth Enum. 3 (1833) 268; Miq. Fl. Ind. Bat. 3 (1857) 388; F.-Vill. Novis. App. (1882) 320.

"Insulae Philippinae" Kunth. Probably not a Philippine species but an erroneously localized Malaspina Expedition collection.

CHLORIS RHACHITRICHA Steud. Syn. Pl. Glum. 1 (1854) 205; Miq. Fl. Ind. Bat. 3 (1857) 388; F.-Vill. Novis. App. (1882) 320.

"Cuming no. 111. Ins. Philip." This number of Cuming's Philippine collection is a fern, and none of his Philippine grasses conform to Steudel's description. The reference may be to Cuming's previous South American collection.

\section{TRIPOGON Roth}

TRIPOGON CHINENSIS (Franch.) Hack. in Bull. Herb. Boiss. II 3 (1903)

503; Merr. in Philip. Journ. Sci. 14 (1919) 369.

Nardurus filiformis Steud. var. chinensis Franch. in Nuov. Arch. Mus. Paris II 7 (1894) 149.

Luzon (Ilocos Norte), B. S. 33237 Ramos. On dry open rocky slopes at low altitudes. Korea, China, Formosa.

\section{ELEUSINE Gaertner}

ELEUSINE INDICA (Linn.) Gaertn. Fruct. 1 (1788) 8; Presl Rel. Haenk. 1 (1830) 286; Llanos Frag. Pl. Filip. (1851) 45; Miq. Fl. Ind. Bat. 3 (1857) 386; F.-Vill. Novis. App. (1882) 320; Vidal Phan. Cuming. Philip. (1885) 159; Merr. in Philip. Journ. Sci. 1 (1906) Suppl. 378, Fl. Manila (1912) 104, Sp. Blancoanae (1918) 72. Cynosurus indicus Linn. Sp. Pl. (1753) 72.

Eleusine barbata Vidal Rev. Pl. Vasc. Filip. (1886) 292. 
Eleusine polydactyla Steud. Syn. Pl. Glum. 1 (1854) 211; Miq. Fl. Ind. Bat. 3 (1857) 386; F.-Vill. Novis. App. (1882) 320, ex descr.

An abundant weed in the settled areas throughout the Philippines. All warm countries.

Local names: Apidan (If.) ; bakis-bakísan (Tag.); barañgan (Bik.); bikad-bikad (Sul.); bila-bíla (P. Bis.) ; dinapaiuk (If.) ; gagabútan (Tag.); kabit-kabít (Tag.); palagtíki (Bis.) ; parañís (Ilk.); parañis-sabúngan (Pamp.) ; sabuñ̄-sabúñan (Tag., Pamp.) ; sambáli (Tag.).

\section{EXCLUDED SPECIES}

Eleusine Corocana (Linn.) Gaertn.; F.-Vill. Novis. App. (1882) 320. Eleusine verticillata Roxb.; F.-Vill. Novis. App. (1882) 320.

\section{DACTYLOCTENIUM Willdenow}

DACTYLOCTENIUM AEGYPTIUM (Linn.) Richt. Pl. Europ. 1

68; Merr. Fl. Manila (1912) 104, Sp. Blancoanae (1918) 72.

Cynosurus aegyptius Linn. Sp. Pl. (1753) 72.

Dactyloctenium aegyptiacum Willd. Enum. Hort. Berol. (1809) 1029;

Pilg. ex Perk. Frag. Fl. Philip. (1904) 147; Merr. in Philip. Journ.

Sci. 1. (1906) Suppl. 379.

Eleusine aegyptiaca Desf. Fl. Atl. 1 (1798-1800) 85; F.-Vill. Novis. App. (1882) 320; Vidal Phan. Cuming. Philip. (1885) 159, Rev. Pl. Vasc. Filip. (1886) 293.

Eleusine mucronata Llanos Frag. Pl. Filip. (1851) 46, non Spreng. Dactyloctenium mucronatum Willd. var. aegyptiacum Nees in Hook.

Jouirn. Bot. Kew Miscel. 2 (1850) 102.

Throughout the Philippines. A common weed in the settled areas at low and medium altitudes. Pantropic.

Local names: Alam (Tag.); damúng-bálang (Tag.).

\section{LEPTOCHLOA Beauvois}

LEPTOCHLOA CHINENSIS (Linn.) Nees Syll. Ratisb. 1 (1824) 4 ; Hook. Journ. Bot. Kew Miscel. 2 (1850) 102; Steud. Syn. Pl. Glum. 1 (1854) 209; Miq. Fl. Ind. Bat. 3 (1857) 389; F.-Vill. Novis. App. (1882) 320; Vidal Phan. Cuming. Philip. (1885) 159, Rev. Pl. Vasc. Filip. (1886) 293; Pilger in Perk. Frag. Fl. Philip. (1904) 147; Merr. in Philip. Journ. Sci. 1 (1906) Suppl. 379; Fl. Manila (1912) 105.

Poa chinensis Linn. Sp. Pl. (1753) 69.

Leptochloa tetraquetra Presl Rel. Haenk. 1 (1830) 288; Miq. Fl. Ind. Bat. 3 (1857) 389; F.-Vill. Novis. App. (1882) 320.

Luzon (Cagayan to Camarines, in most provinces), Panay. In open usually wet places along or near streams. India to Japan southward through Malaya to Australia.

Local name: Kurukauáyan (Bik.).

LEPTOCHLOA FILIFORMIS (Pers.) R. \& S. Syst. 2 (1817) 580; Presl Rel. Haenk. 1 (1830) 288; Kunth Enum. 1 (1833) 270; Miq. Fl. Ind. Bat. 3 (1857) 389; F.-Vill. Novis. App. (1882) 320; Merr. in Philip. Journ. Sci. 1 (1906) Suppl. 380.

Eleusine filiformis Pers. Syn. (1805) 87. 
Luzon (Abra, Union), Palawan. In open low lands, along streams, in disturbed soil, etc., at low altitudes. Pantropic.

\section{PHRAGMITES Trinius}

PHRAGMITES KARKA (Retz.) Trin. ex Steud. Nomencl. ed. 2, 2 (1840) 324; Merr. in Philip. Journ. Sci. 1 (1906) Suppl. 381.

Arundo karka Retz. Obs. 4 (1786) 21.

Phragmites roxburghii Steud. 1. c.; Nees in Nov. Act. Acad. Nat. Cur. 19 (1843) Suppl. 1: 173, Hook. Journ. Bot. Kew Miscel. 2 (1850) 101; Miq. Fl. Ind. Bat. 3 (1857) 412; F.-Vill. Novis. App. (1882) 321.

Trichoon roxburghii W. F. Wight in Contr. U. S. Nat. Herb. 9 (1905) 383, 391.

Luzon (Ilocos Norte, Bontoc, Bataan, Laguna, Camarines), Negros, Mindanao, Merrill 31r8, Phil. Pl. 581, Elmer 6851, Williams 201, Clemens 728, B. S. 2213 Mearns, 15910 Fénix. In thickets and along streams at low and medium altitudes. India to Malaya.

Local names: Kanúbong (Bon.) ; lachitlít (Bag.) ; lupí (Bik.) ; sabúnog (P. Bis.); tabúnak (P. Bis.) ; tabúnog (Bis.) ; talúbung (Bon.) ; tantanóbang (Ilk.); tanúbung (Bon.).

PHRAgmites vulgaris (Lam.) Trin. Fund. Agrost. (1820) 134; Pilger in Perk. Frag. Fl. Philip. (1904) 147; Merr. in Philip. Journ. Sci. 1 (1906) Suppl. 381, Fl. Manila (1912) 100, Sp. Blancoanae (1918) 72.

Arundo vulgaris Lam. Fl. Franc. 3 (1778) 615.

Arundo phragmites Linn. Sp. Pl. (1753) 81.

Arundo tecta Blanco Fl. Filip. (1837) 48; ed. 2 (1845) 33; ed. 3, 1 (1877) 60, non Walt.

Arundo donax Llanos in Mem. Acad. Cienc. Madr. 4 (1858) 507; F.-Vill. \& Llanos in Blanco Fl. Filip. ed. $3,4^{1}$ (1880) 105, non Linn.

Phragmites communis Trin. Fund. Agrost. (1820) 134; Usteri Beitr.

Ken. Veg. Philip. (1905) 133.

Arundo madagascariensis F.-Vill. Novis. App. (1882) 321, Vidal

Phan. Cuming. Philip. (1885) 159, Rev. Pl. Vasc. Filip. (1886) 293, non Kunth.

Neyraudia madagascariensis Merr. in Philip. Journ. Sci. 1 (1906) Suppl. 381, non Hook. f.

Northern Luzon to Palawan and Mindanao, often forming dense thickets along the margins of lakes and slow streams. All warm countries.

Local names: Bagang (Bis.) ; bugang (C. Bis.) ; lúpi (Bik.); tabúnak (Bis., P. Bis.) ; tagísi (Ibn.) ; tambó (Tag.) ; uba-uba (Sul.) ; tangbó (C. Bis.) ; tanóbong (Pang., Ilk.) ; tantanúbong (Ibn.); tanúbong (Ilk.).

\section{DIPLACHNE Beauvois}

DIPLACHNE POLYSTACHYA (Forsk.) Backer in Bull. Jard. Bot. Buitenz. III 2 (1920) 325.

Bromus polystachyus Forsk. Fl. Aegýpt.-Arab. (1775) 23.

Diplachne fusca Merr. in Philip. Journ. Sci. 1 (1906) Suppl. 381, Fl. Manila (1912) 99, non Beauv. 
Luzon (Laguna, Rizal), Mindoro, Mindanao (Cotabato), Merrill 5104, Phil. Pl. 183, Kneucker Gram. Exsic. 554a, Elmer 18131, Williams 2027. In swampy often brackish places at low alitudes. Tropical Asia and Africa to Australia:

\section{ERAGROSTIS Beauvois}

ERAGROSTIS AMABILIS (Linn.) W. \& A. in Hook. \& Arn. Bot. Beechy Voy. (1841) 251; Merr. Interpret. Herb. Amb. (1917) 95.

Poa amabilis Linn. Sp. Pl. (1753) 68.

Poa tenella Linn. Sp. Pl. (1753) 69.

Poa plumosa Link. Hort. Berol. 1 (1827) 192; Nees in Hook. Journ. Bot. Kew Miscel. 2 (1850) 102 (f. laxa); Miq. Fl. Ind. Bat. 3 (1857) 394; F.-Vill. Novis. App. (1882) 322; Vidal Phan. Cuming. Philip. (1885) 159, Rev. Pl. Vasc. Filip. (1886) 293; Usteri Beitr. Ken. Philip. Veg. (1905) 132 (f. breviciliata Hack.).

Eragrostis tenella R. \& S. Syst. 2 (1817) 576; Presl Rel. Haenk. 1 (1830) 274; F.-Vill. Novis. App. (1882) 322; Merr. in Philip. Journ. Sci. 1 (1906) Suppl. 383, 3 (1908) Bot. 397, Fl. Manila (1912) 101.

In and about towns throughout the Philippines, a very common weed in open waste places. Old World Tropics generally.

Local names: Bagibuk (Tag.); balisíbis (Iv.); kaliraúrau (Tag.); págai-billít (Ilk.); pulpulút (Bon.); sigsígid (Bon.).

ERAGROSTIS CILIANENSIS (All.) Vig.-Lut. in Malpighia 18 (1904) 386; Hubbard in Philip. Journ. Sci. 8 (1913) Bot. 159.

Briza eragrostis Linn. Sp. Pl. (1753) 70.

Poa cilianensis All. Fl. Pedem. 2 (1785) 246, t. 91, f. 2.

Eragrostis major Host. Gram. Austr. 4 (1809) 14, t. 24.

Eragrostis megastachya Link Hort. Berol. 1 (1827) 187; Nees in Hook. Journ. Bot. Kew Miscel. 2 (1850) 102; Vidal Phan. Cuming. Philip. (1885) 159, Rev. Pl. Vasc. Filip. (1886) 293.

Cebu, Negros, B. S. 11122 Ramos, Eskridge. In open waste places at low altitudes. All warm countries.

ERAGROSTIS DISTANS Hack. in Govt. Lab. Publ. (Philip.) 35 (1906)

81; Merr. in Philip. Journ. Sci. 1 (1906) Suppl. 384, 5 (1910)

Bot. 330, Fl. Manila (1912) 102, Sp. Blancoanae (1918) 74.

Uniola paniculata Llanos Frag. Pl. Filip. (1851) 32; F.-Vill. \& Naves

in Blanco Fl. Filip. ed. $3,4^{1}$ (1880) 23 , non Linn.

Eragrostis pilosa F.-Vill. Novis. App. (1882) 322, non Beauv.

Luzon (Ilocos Norte, Lepanto, Benguet, Pangasinan, Rizal), Palawan, Elmer 6608, Kneucker Gram. Exsic. 761, Merrill 4472, B. S. 4821, 5314 Ramos. In dry open grasslands at low and medium altitudes. Endemic.

ERAGROSTIS ELONGATA (Willd.) Jacq. Eclog. Gram. (1818) 3, t. 3; Presl Rel. Haenk. 1 (1830) 275; Merr. in Philip. Journ. Sci. 1 (1906) Suppl. 384.

Poa elongata Willd. Enum. Hort. Berol. 1 (1809) 108.

Eragrostis elegantula Pilger in Perk. Frag. Fl. Philip. (1904) 148;

Merr. in Philip. Journ. Sci. 1 (1906) Suppl. 384, Fl. Manila (1912) 102, non Steud. 
Eragrostis nigra Usteri Beitr. Ken. Philip. Veg. (1905) 132, non Nees.

Eragrostis luzoniensis Steud. Syn. Pl. Glum. 1 (1854) 266; Miq. Fl.

Ind. Bat. 3 (1857) 393; F.-Vill. Novis. App. (1882) 322.

Luzon (Isabela, Ilocos Norte, Ifugao, Bontoc, Benguet, Nueva Vizcaya, Nueva Ecija, Pangasinan, Rizal), Culion, Palawan, Mindanao (Bukidnon). Common in open grasslands at low and medium altitudes. Pantropic.

Hackel has identified this as Eragrostis atrovirens Trin. Typical specimens are Merrill 4155, 9321 and Phil. Pl. 1460, 130. Cuming 1416, cotype of $E$. luzoniensis Steud., appears to me to be merely a form with pale spikelets.

ERAGRostis INTERRUPTA (R. Br.) Beauv. Agrost. (1812) 71 (non Doell).

Poa interrupta R. Br. Prodr. (1810) 180.

Eragrostis brownei Nees ex Steud. Nomencl. ed. 2, 1 (1841) 562; Hook. Journ. Bot. Kew Miscel. 2 (1850) 102; F.-Vill. Novis. App. (1882) 322; Vidal Phan. Cuming. Philip. (1885) 159, Rev. Pl. Vasc. Filip. (1886) 293.

Eragrostis cumingii Steud. Syn. Pl. Glum. 1 (1854) 266; Miq. Fl. Ind. Bat. 3 (1857) 394; F.-Vill. Novis. App. (1882) 322.

Eragrostis zeylanica Nees in Nov. Act. Acad. Nat. Cur. 19 (1843) Suppl. 1: 204; Hook. Journ. Bot. Kew Miscel. 2 (1850) 102 (var. glomerata et minor); Rolfe in Journ. Bot. 23 (1885) 216; Vidal Phan. Cuming. Philip. (1885) 159, Rev. Pl. Vasc. Filip. (1886) 293; Pilger in Perk. Frag. Fl. Philip. (1904) 148.

Luzon (Abra, Bontoc, Benguet, Union, Nueva Vizcaya, Bataan, Laguna, Zambales), Culion, Cuming 1104, 672, Merrill 686, 4331, Elmer 5707, Williams 1181, 92. In open grasslands at low and medium altitudes. Pantropic.

ERAGRostis JAPONICA (Thunb.) Trin. in Mém. Acad. St. Pétersb. VI 1 (1830) 450; Hack. in Bull. Herb. Boiss. 7 (1899) 705; Merr. in Philip. Journ. Sci. 1 (1906) Suppl. 384, Sp. Blancoanae (1918) 73.

Poa japonica Thunb. Fl. Jap. (1784) 31; Llanos Frag. Pl. Filip. (1851) 47; F.-Vill. \& Naves in Blanco Fl. Filip. ed. 3, $4^{1}$ (1880) 34.

Poa interrupta Lam. Tabl. Encycl. 1 (1791) 185.

Eragrostis aurea Steud. Syn. Pl. Glum. 1 (1854) 267; Miq. Fl. Ind. Bat. 3 (1857) 394; F.-Vill. Novis. App. (1882) 322.

Panicum leptanthum Steud. op. cit. 79.

Vilfa verticillata Steud. op. cit. 158.

Sporobolus verticillatus Nees in Hook. Journ. Bot. Kew Miscel. 2 (1850) 101; Miq. Fl. Ind. Bat. 3 (1857) 375; F.-Vill. Novis. App. (1882) 321.

Eragrostis tenuissima Schrad. ex Nees in Fl. Afr. Austral. (1841) 409; Vidal Phan. Cuming. Philip. (1885) 159, Rev. Pl. Vasc. Filip. (1886) 293.

Eragrostis interrupta (non Beauv.!) var. tenuissima Stapf in Hook. f. Fl. Brit. Ind. 7 (1897) 316.

Poa amboinensis F.-Vill. Novis. App. (1882) 322, ex syn. Llanos, non Linn. 
Eragrostis hapalantha Trin.; Presl Repert. 1 (1833) 252.

Eragrostis minutiflora Presl Rel. Haenk. 1 (1830) 274 (excl. syn. Retz.?).

Eragrostis interrupta Doell in Mart. Fl. Bras. 3: 15̄; Usteri Beitr. Ken. Philip. Veg. (1905) 132; Pilger in Perk. Frag. Fl. Philip. (1904) 148; Merr. in Philip. Journ. Sci. 1 (1906) Suppl. 383, Fl. Manila (1912) 101, non Beauv.

Eragrostis interrupta (non Beauv.!) var. koenigii Stapf in Hook. f. Fl. Brit. Ind. 7 (1897) 316.

Northern Luzon (Cagayan) to Palawan and Mindanao. In open wet grasslands, along streams, etc., at low and medium altitudes. Tropical Asia and Africa to Japan and Malaya:

Thunberg's specific name is accepted for this species as a collective one. Merrill 7322, Phil. Pl. 165, Sp. Blancoanae 709 represent the species as I here interpret it; numerous specimens have been distributed as $E$. interrupta Doell.

ERAGROSTIS LASIOCLAdA Merr. in Philip. Journ. Sci. 1 (1906) Suppl. 382.

Culion, Merrill 461. In fallow rice paddies at low altitudes. Endemic.

ERAGROSTIS MANGALORICA Hochst. ex Steud. Syn. Pl. Glum. 1 (1854) 265; Merr. in Philip. Journ. Sci. 7 (1912) Bot. 299, Fl. Manila (1912) 101.

Luzon (Cagayan, Rizal), Merrill 371, B. S. 7452, 14569 Ramos. In open waste places at low altitudes. India.

ERAGROSTIS PILOSA (Linn.) Beauv. Agrost. (1812) 71; Merr. Fl. Manila (1912) 102.

Poa pilosa Linn. Sp. Pl. (1753) 68.

Luzon (Benguet, Rizal), Merrill 965\%, Phil. Pl. 5\%3. A weed in open waste places, of recent introduction, but now locally abundant. All warm countries.

ERAGRostis REFleXA Hack. in Philip. Journ. Sci. 3 (1908) Bot. 168.

Luzon (Rizal), B. S. 2067 Ramos. 'In fallow lands at low or medium altitudes. Endemic.

ERAGROSTIS SPARTINOIDES Steud. Syn. Pl. Glum. 1 (1854) 265; Pilger in Perk. Frag. Fl. Philip. (1904) 148; Merr. Fl. Manila (1912) 102, Sp. Blancoanae (1918) 74.

Uniola spicata Llanos Frag. Pl. Filip. (1851) 33; F.-Vill. \& Naves in Blanco Fl. Philip. ed. 3, $4^{1}$ (1880) 23, non Linn.

Luzon (Cagayan, Ilocos Norte, Ifugao, Bontoc, Nueva Vizcaya, Rizal, Laguna), Mindoro, Cuming 668, Merrill 7055, Phil. Pl. 107, Sp. Blancoanae 170, 422, etc. In open grasslands at low altitudes. Endemic.

ERAGROSTIS UNIOLOIDES (Retz.) Nees Steud. Syn. Pl. Glum. 1 (1854) 264; F.-Vill. Novis. App. (1882) 322; Merr. in Philip. Journ. Sci.

1 (1906) Suppl. 384, Fl. Manila (1912) 102.

Eragrostis amabilis Auct., non (Linn.) W. \& A.

Eragrostis rubens F.-Vill. Novis. App. (1882) 322, non Hochst.? 
Poa annua Llanos Frag. Pl. Filip. (1851) 47; F.-Vill. \& Naves in Blanco Fl. Filip. ed. 3, $4^{1}$ (1880) 34, non Lam.

Eragrostis polymorpha R. Br. Prodr. (1810) 180; F.-Vill. Novis. App. (1882) 322.

Luzon (Zambales, Rizal, Sorsogon), Elmer 14.531, Merrill Phil. Pl. 160, F. B. 5886 Curran, B. S. 19218 Reillo. In open wet places at low altitudes. Old World Tropics, introduced into the New World.

ERAgrostis ViscosA (Retz.) Trin. in Mém. Acad. St. Pétersb. VI 1 (1831) 397; F.-Vill. Novis. App. (1882) 322; Mexr. in Philip. Journ. Sci. 1 (1906) Suppl. 383, Fl. Manila (1912) 101, Sp. Blancoanae (1918) 73.

Poa viscosa Retz. Obs. 2 (1781) 20.

Eragrostis tenella R. \& S. var. viscosa Stapf in Hook. f. Fl. Brit. Ind. 7 (1897) 315; Pilger in Perk. Frag. Fl. Philip. (1904) 148.

Cyperus paniculatus Blanco Fl. Filip. (1837) 32, ed. 2 (1845) 22, ed. 3, 1 (1877) 42, non aliorum.

Luzon (Zambales, Rizal), Merrill Sp. Blancoanae 229, Phil. Pl. 148. In open waste places in and near towns. India to Malaya.

Local name: Kaliraúrau (Tag.).

EXCLUDED SPECIES

Eragrostis ciliata Nees; F.-Vill. Novis. App. (1882) 322.

\section{CENTOTHECA Desvaux}

CENTOTHECA LATIFOlia (Osbeck) Trin. Fund. Agrost. (1820) 141; Merr. Fl. Manila (1912) 100, Sp. Blancoanae (1918) 74.

Holcus latifolius Osbeck Dagbok Ostind. Resa (1757) 247; Linn. Syst. ed. 10 (1759) 1305.

Cenchrus lappaceus Linn. Sp. Pl. ed. 2 (1763) 1488.

Centotheca lappacea Desv. in Nuov. Bull. Soc. Philom. 2 (1810) 189; Presl Rel. Haenk. 1 (1830) 258; Miq. Fl. Ind. Bat. 3 (1857) 398; Nees in Hook. Journ. Bot. Kew Miscel. 2 (1850) 98; F.-Vill. Novis. App. (1882) 322; Vidal Phan. Cuming. Philip. (1885) 159, Rev. Pl. Vase. Filip. (1886) 293; Merr, in Philip. Journ. Sci. 1 (1906) Suppl. 29, 3 (1908) Bot. 397; Perk. ex Frag. Fl. Philip. (1904) 148. Melica philippinensis Llanos Frag. Pl. Filip. (1851) 44; F.-Vill. \&

Naves in Blanco Fl. Filip. ed. $3,4^{1}$ (1880) 32.

Centotheca malabarica Merr. in Philip. Journ. Sci. 1 (1906) Suppl. 385, non Poa malabarica Linn.

Throughout the Philippines in thickets, forests, and clearings at low and medium altitudes; common. Usually the only grass in some types of forest. Tropical Asia and Africa through Malaya to Polynesia.

Local names: Baili-patung (Sul.); balikbátang (Sub.); kauakauayánan (Tag.) ; lansa-lansa (Sul.); maríga (Sul.); sikúan (Ig.).

65. LOPHATHERUM Brongniart

L.OPHATHERUM GRACILE Brongn. in Bot. Duperr. Voy. (1829) 50, t. 8; F.-Vill. Novis. App. (1882) 323; Pilger in Perk. Frag. Fl. Philip. (1904) 148; Merr. in Philip. Journ. Sci. 1 (1906) Suppl. 386, 2 (1907) Bot. 261; C. B. Rob. op. cit. 6 (1911) Bot. 194. 
Luzon (Nueva Ecija, Laguna, Tayabas, Camarines, Sorsogon), Polillo, Mindoro, Palawan, Leyte, Mindanao, Basilan, Jolo. In forests, along trails, and in old clearings, chiefly at medium altitudes. India to Japan, southward through Malaya to New Guinea.

The Philippine form is referable to var. pilosum Camus in Bull. Mus. Hist. Nat. Paris 25 (1919) 495.

66. POA Linnaeus

POA ANNUA Linn. Sp. Pl. (1753) 68; Merr. in Philip. Journ. Sci. 1 (1906) Suppl. 181, 386.

Luzon (Benguet), Merrill 4288, B. S. 32041 Santos. Naturalized in open waste places, altitude 1,500 to $2,400 \mathrm{~m}$. Temperate and subtemperate regions generally.

The form reported from the Philippines as Poa annua by Llanos, Frag. Pl. Filip. (1851) 47, and by F.-Vill., Novis. App. (1882) 322, was probably. Eragrostis unioloides Nees; there is no description.

POA Luzoniensis Merr. in Philip. Journ. Sci. 1 (1906) Suppl. 180, 386.

Luzon (Benguet), Merrill 4712, B. S. 31911 Santos. Borders of cold marshes and streams, altitude about $2,400 \mathrm{~m}$. Reported by Ridley from New Guinea, but the specimens should be compared.

\section{FESTUCA Linnaeus}

Festuca Megalura Nutt. in Journ. Acad. Sci. Phila. II 1 (1847) 188; Piper in Contr. U. S. Nat. Herb. 10 (1906) 17, t. 5.

Festuca myuros Merr. in Philip. Journ. Sci. 14 (1919) 369, non Linn.

Luzon (Benguet), B. S. 31913 Santos, Hitchcock. In open grassy places, altitude 1,400 to $2,300 \mathrm{~m}$. A native of the west coast of North and South America, introduced in Portugal, and a recent introduction in Benguet. It is very similar to Festuca myuros Iinn. and by some authors is treated as a variety of that species. The identification is by Professor Hitcheock.

\section{MONOSTACHYA Merrill}

MONOSTACHYA CentRolepidoIdes Merr. in Philip. Journ. Sci. 5 (1910) Bot. 331.

Luzon (Benguet), F. B. 16088 Curran, Merritt, \& Zschokke. In open grasslands near the summit of Mt. Pulog, altitude about $2,800 \mathrm{~m}$. A monotypic endemic genus.

\section{RAMOSIA Merrill}

RAMOSIA PHILIPPINENSIS Merr. in Philip. Journ. Sci. 11 (1916) Bot. 2.

Luzon (Sorsogon), B. S. 23607 Ramos. In swampy places, border of Lake Polog, at medium altitudes. A monotypic endemic grenus.

\section{BROMUS Linnaeus}

BROMUS PAUCIFloRUS (Thunk.) Hack. in Bull. Herb. Boiss. 7 (1899) 713; Merr. in Philip. Journ. Sci. 1 (1906) Suppl. 386.

Festuca pauciflora Thunb. Fl. Jap. (1784) 52.

Luzon (Bontoc, Benguet), Merrill 4714, Clemens 9204, 9091, Vanoverlergh 3938. In wet open grassy places, altitude 1,500 to $2,400 \mathrm{~m}$. Japan, China. 
Bromus commutatus Schrad. is represented by B.S. 31966 Santos and Iilerrill 6627, cultivated at Pauai, Benguet; introduced as $B$. inermis Leyss.

\section{DOUBTFUL SPECIES}

Bromus Pallens Cav. Ic. 6 (1801) 66, t. 591, f. 1; Kunth. Enum. 1 (1833) 418; Miq. Fl. Ind. Bat. 3 (1857) 398; F.-Vill. Novis. App. (1882) 323.

"Habitat in Manilae vicinis, ibique Acanthus ilicifolius Née legit." The nigure and description appertain to Bromus, but no representative of the genus is known to occur at low altitudes in the Philippines. The description was probably based on an erroneously localized plant.

Bromus Luzonensis Presl Rel. Haenk. 1 (1828) 262.

Triticum luzonense Kunth Enum. 1 (1833) 446; Miq. Fl. Ind. Bat.

3 (1857) 402; F.-Vill. Novis. App. (1882) 323.

"Hab, in Luzonia" Presl. The type was certainly an erroneously localized specimen, probably from tropical America.

\section{BRACHYPODIUM Beauvois}

BRACHYPODIUM SYLVATICUM Beauv. Agrost. (1812) 146, subsp. LU. ZONIENSE Hack. in Philip. Journ. Sci. 1 (1906) Suppl. 269; Merr. op. cit. 387, 5 (1910) Bot. 332.

Luzon (Benguet), Merrill 4710, 4536, 4830, F. B. 16187 Curran, Merritt, \& Zschokke. On open slopes and in the mossy forest, altitude 1,600 to $2,500 \mathrm{~m}$.

Var. ASPERUM Hack. 1. c.; Merr. 1. c.

Luzon (Benguet), Merrill 4698. On steep slopes in damp shaded ravines, altitude about $1,700 \mathrm{~m}$. The two Philippine forms endemic, the species Europe and northern Africa to India and Ceylon.

72. MONERMA Beauvois

MONERMA REPENS (Forst.) Beauv. Agrost. (1812) 117; Hack. in Govt. Lab. Publ. (Philip.) 35 (1906) 81; Merr. in Philip. Journ. Sci. 1 (1906) Suppl. 387.

Rottboellia. repens Forst. Prodr. (1786) 9.

Lepturus repens R. Br. Prodr. (1910) 207.

Mindoro, Culion, Bancoran, Cajoacan, Palmas, Mindanao, Merrill 606, 3331, 5215, 5354, 11644, Phil. Pl. 153. In sandy soil back of the beach, about sea cliffs, etc. Ceylon through Malaya to tropical Australia and Polynesia.

\section{TRITICUM Linnaeus}

TRITICUM VULGARE Vill. Hist. Pl. Dauph. 2 (1779) 153; Nees in Hook. Journ. Bot. Kew Miscel. 2 (1850) 102; F.-Vill. Novis. App. (1882) 323; Vidal Phan. Cuming. Philip. (1885) 159, Rev. Pl. Vasc. Filip. (1886) 294; Merr. in Philip. Journ. Sci. 1 (1906) Suppl. 388.

Formerly cultivated in certain provinces in Luzon, now planted to a limited extent in northern Luzon. Wheat. 
74. ARUNDINARIA Michaux

ARUNDINARIA NIITAKAYAMENSIS Hayata in Bot. Mag. Tokyo 21 (1907) 49, Journ. Coll. Sci. Tokyo $25^{19}$ (1908) 240; Gamble in Philip. Journ. Sci. 5 (1910) Bot. 267; Merr. op. cit. 332.

Sasa niitakayamensis Camus var. microcarpa Camus Bamb. (1913) 24.

Bambusa aff. B. pygmaea Miq.; Merr. in Philip. Journ. Sci. 2 (1907) Bot. 261.

Luzon (Benguet), Mindoro, Elmer 14279, Merrill 4733, 6489, 6222; Phil. Pl. 17\%, B. S. 8379, 8893 McGregor, 5846 Ramos, F. B. 10823 Curran. In mossy thickets, sphagnum swamps, and open grasslands, altitude 2,200 to 2,700 m. Formosa.

Local name: Útod (Ig.).

75. BAMBUSA Schreber

BAM BUSA CORNUTA Munro in Trans. Linn. Soc. 26 (1868) 113; Gamble in Philip. Journ. Sci. 5 : (1910) Bot. 269; 8 (1913) Bot. 204.

Luzon (Benguet, Nueva Vizcaya), Merrill 124, 7711. In ravines along small streams, altitude 1,000 to $1,600 \mathrm{~m}$. Java.

Local name: Lopa (Isn.).

BAMBUSA MERRILLII Gamble in Philip. Journ. Sci. 5 (1910) Bot. 269; Camus Bamb. (1913) 128.

Luzon (Nueva Vizcaya), Merrill 229. In forests, altitude about $600 \mathrm{~m}$. Endemic.

BAMBUSA MULtiplex (Lour.) Raeusch. Nomencl. ed. 3 (1797) 108; Schultes f. Syst. 7 (1830) 1350; Steud. Syn. Pl. Glum. 1 (1854) 330; Munro in Trans. Linn. Soc. 26 (1868) 119.

Arundo multiplex Lour. Fl. Cochinch. (1790) 58, ed. Willd. (1793) 119.

Ludolphia glaucescens Willd. in Ges. Naturf. Fr. Berl. Mag. 2 (1808) 320.

Bambusa nana Roxb. Hort. Beng. (1814) 25, nomen nudum, Fl. Ind. ed. 2, 2 (1832) 199; F.-Vill. Novis. App. (1882) 323; Gamble in Ann. Bot. Gard. Calc. 7 (1896) 40, t. 38, Philip. Journ. Sci. 5 (1910) Bot. 268; Merr. in Philip. Journ. Sci. 1 (1906) Suppl. 389; Camus Bamb. (1913) 121.

Arundinaria glaucescens Beauv. Agrost. (1812) 144, 152.

Bambusa glaucescens Sieb. ex Munro in Trans. Linn. Soc. 26 (1868)

89, in syn.; Merr. in Philip. Journ. Sci. 7 (1912) Bot. 230, Fl.

Manila (1912) 105.

Planted here and there for ornamental purposes, sometimes for hedges. Probably a native of China, now widely distributed in cultivation.

Local names: Caña de china (Sp.) ; kauáyan-china (Bik., Tag.) ; kauáyansina (Bik., Tag.).

BAMBUSA SPINOSA Roxb. Hort. Beng. (1814) 25 [non Fl. Ind. ed. 2, 2 (1832) 305]; Merr. Interp. Herb. Amb. (1917) 97, Sp. Blancoanae (1918) 75.

Bambusa spinosa Blume ex Nees in Flora 8 (1825) 580.

Bambusa blumeana Schultes f. Syst. $7^{2}(1830)$ 1343; F.-Vill. Novis. 
App. (1882) 323; Gamble in Ann. Bot. Gard. Calc. 7 (1896) 50, t. 47; Philip. Journ. Sci. 5 (1910) Bot. 270, 8 (1913) Bot. 204; Merr. in Philip. Journ. Sci. 1 (1906) Suppl. 29, 388, Fl. Manila (1912) 106, Am. Journ. Bot. 3 (1916) 60; Camus Bamb. (1913) 130.

Bambus pungens Blanco Fl. Filip. (1837) 270; Miq. Fl. Ind. Bat. 3 (1857) 421; Munro in Trans. Linn. Soc. 26 (1868) 119.

Bambus arundo Blanco Fl. Filip. ed. 2 (1845) 188; ed. 3, 1 (1877) $335, t$. 100, non Nees, nec Wight.

Bambusa arundinacea F.-Vill. Novis. App. (1882) 323, non Retz. Bambusa teba Miq. Fl. Ind. Bat. 3 (1857) 418; Munro in Trans. Linn. Soc. 26 (1868) 122.

Northern Luzon to Palawan and Mindanao. A universally planted bamboo at low and medium altitudes throughout the settled areas; the only spiny bamboo in the Philippines. Southern China to the Malay Peninsula and Archipelago to the Moluccas.

The most common and conspicuous bamboo in the settled areas, but in the Philippines apparently always planted. This indicates its probable prehistoric introduction by the early Malay invaders. The species very rarely flowers and then usually only when clumps have been severely injured by fire or by too extensive cutting.

Local names: Aonóo (Bik.); batákan (Bis.) ; baugín (Pamp.) ; dugían (Bik); caña espina (Sp.); kaaóno (Bis.); kauáyan (P. Bis., Bon., C. Bis., Bik., Ilk., Pamp., Sub., Sul., Tag.) ; kauáyan-gid (P. Bis.) ; kauáyanñg-bayóg (Ilk.) ; kauáyan-potog (Sbl.); kauáyan-siitan (Ilk.); kauáyantiník (Tag.); kauáyan-totóo (Tag., Bik.); kabugáuan (Bik.); lamnuan (Is.) ; marurúgi (Bik.) ; pasíngan (Ibn., Is.) ; paua (Bis.) ; rugían (Bik.).

BAMBUSA VULGARIS Schrad. ex Wendl. Collect. Pl. 2 (1808) 26, t. 4\%; Gamble in Ann. Bot. Gard. Calcutta 7 (1896) 43, t. 40, Philip. Journ. Sci. 5 (1910) Bot. 268, 8 (1913) Bot. 204; Merr. Fl. Manila (1912) 105, Am. Journ. Bot. 3 (1916) 61, Sp. Blancoanae (1918) 75; Camus Bamb. (1913) 123.

Bambusa monogyna Blanco Fl. Filip. (1837) 286, ed. 2 (1845) 187, ed. 3, 1 (1877) 333; Miq. FI. Ind. Bat. 3 (1837) 420; Munro in Trans. Linn. Soc. 26 (1868) 119; Merr. in Philip. Journ. Sci. 1 (1906) Suppl. 29, 389; Camus Bamb. (1913) 132.

Bambusa mitis Blanco F1. Filip. (1837) 271, ed. 2 (1845) 188, ed. 3, 1 (1877) 336, non Poir.

Bambusa blancoi Steud. Syn. Pl. Glum. 1 (1854) 331; Miq. Fl. Ind. Bat. 3 (1857) 431; Munro in Trans. Linn. Soc. 26 (1868) 120; Merr. in Philip. Journ. Sci. 1 (1906) Suppl. 389; Camus Bamb. (1913) 134.

Dendrocalamus sericeus F.-Vill. Novis. App. (1882) 324, non Munro.

Dendrocalamus strictus F.-Vill. Novis. App. (1882) 324, non Nees.

Northern Luzon to Palawan and Mindanao, in most islands and provinces, in the settled areas at low and medium altitudes. Probably an introduced plant in the Philippines. Pantropic in cultivation.

Local names: Bolínau (P. Bis.); burírau (P. Bis.); bútong (Bik.); kabalóan (Bik.); kauáyan (Ibn.); kauáyan-china (Tag.) ; kauáyan-kilíng (Ilk., Tag.) ; kauáyan-kitíng (Sbl.) ; kilíng (Ilk., Tag.); lúnas (P. Bis.); 
marobál (Bik.); patong (Tagb.); patung (Sul.); sinámbang (C. Bis.); taiuának (Tag.); taríng (Tagb.); teuának (Tag.); tilíng (P. Bis.).

Var. STRIATA (Lodd.) Gamble in Ann. Bot. Gard. Calc. 7 (1896) 44, Philip. Journ. Sci. 5 (1910) Bot. 269; Merr. Fl. Manila (1912) 105.

Bambusa striata Lodd. ex Penny Cyclop. 3 (1835) 357.

Occasionally cultivated for ornamental purposes; probably originating in China.

\section{EXCLUDED SPECIES}

Bambusa tuldoides Munro; F.-Vill. Novis. App. (1882) 323.

\section{GUADUA Kunth}

GUADUA PHILIPPINENSIS Gamble in Philip. Journ. Sci. 8 (1913) Bot. 203.

Mindanao (Davao), Piper 475. Probably from thickets or secondary forests; collected at Mati. Endemic. The genus is otherwise American.

\section{GIGANTOCHLOA Kurz}

GigantochloA LEVIS (Blanco) Merr. in Am. Journ. Bot. 3 (1916) 61, Sp. Blancoanae (1918) 76.

Bambusa levis Blanco Fl. Filip. (1837) 272, ed. 2 (1845) 189, ed. 3, 1 (1877) 337; Miq. Fl. Ind. Bat. 3 (1857) .421; Munro in Trans. Linn. Soc. 26 (1868) 121; Merr. in Govt. Lab. Publ. (Philip.) 27 (1905) 93, Philip. Journ. Sci. 1 (1906) Suppl. 388; Camus Bamb. (1913) 134.

Gigantochloa scribneriana Merr. in Philip. Journ. Sci. 1 (1906) Suppl. 390; Gamble in Philip. Journ. Sci. 5 (1910) Bot. 270, 8 (1913) Bot. 204; Camus Bamb. (1913) 143.

Dendrocalamus flagellifer F.-Vill. Novis. App. (1882) 324, non Munro. Gigantochloa robusta Gamble in Philip. Journ. Sci. 8 (1913) Bot. 204, non ? Kurz.

Gigantochloa atter C. B. Rob. in Philip. Journ. Sci. 6 (1911) Bot. 194, non Kurz.

Dendrocalamus curranii Gamble in Philip. Journ. Sci. 5 (1910) Bot. 271; C. B. Rob. op. cit. 6 (1911) Bot. 194; Camus Bamb. (1913) 158.

Dendrocalamus latiflorus Gamble in Philip. Journ. Sci. 5 (1910) Bot. 291, non Munro.

Luzon (Bulacan, Bataan, Rizal, Laguna, Tayabas), Culion, Polillo, Leyte, Camiguin de Misamis, Loher 7208, Scribner 14, Merrill Phil. Pl. 586, 590, Sp. Blancoanae 310, B. S. 10414, 10417 McGregor, 6313, 11898 Robinson, 14696 Ramos, F. B. 22771 De Mesa \& Rosario, 10177 Curran, 25405 Labitig, 25418 Duldulao. Usually if not always planted, and probably an introduced species in the Philippines. To be compared critically with the Malayan species.

Local names: Bóko (P. Bis.); bólo (P. Bis.) ; bonsína (Tag.) ; bótong (P. Bis.) ; búhong-china (Tag.); búlung-sína (Tag.); butón (C. Bis.); kabolian (Bik.) ; kauáyan-china (Tag.) ; kauáyan-putí (Tag.) ; kauáyansína (Tag.) ; kayali (C. Bis.) ; patong (Bik., Bis., Sul.). 


\section{DENDROCALAMUS Nees}

DENDROCALAMUS MERRILLIANUS (EIm.) Elm. Leafl. Philip. Bot. 7 (1915) 2675.

Gigantochloa merrilliana Elm. Leafl. Philip. Bot. 1 (Jan., 1908) 273; Camus Bamb. (1913) 139.

Dendrocalamus parviflorus Hack. in Philip. Journ. Sci. 3 (July, 1908) 168; Gamble op. cit. 5 (1910) Bot. 272; Camus Bamb. (1913) 159.

Luzon (Ilocos Sur, Abra, Nueva Ecija, Rizal, Zambales, Pangasinan, Bulacan), Leyte, Cebu, Bohol, Mindanao (Lanao), Elmer r283, Clemens, Barrow 30, F. B. 27932 Lopez \& Reyes, 27319, 27329 Reyes, 27219 Duran, 27177 Maneja \& Bawan, B. S. 22466 Ramos, 27706 Fénix, 17949 Otanes, mostly sterile specimens. In and about towns, chiefly planted. Endemic.

Local names: Bayóg (Ilk., Ibn., Tag., Sbl.) ; bayúgin (Tag.); botóng (C. Bik., Bis.) ; bukáui (Tag.) ; butóng (P. Bis.) ; kauáyan (Tag., C. Bis., Ilk.) ; kauáyan-bayóg (Pang.).

\section{EXCLUDED SPECIES}

Dendrocalamus giganteus Munro; F.-Vill. Novis. App. (1882) 324.

Dendrocalamus Latiflorus Munro; F.-Vill. 1. c.; Vidal Sinopsis Atlas (1883) $42, t .96, f . B$.

Vidal's figure was copied from Munro's plate, fide Vidal 1. c.; see Gigan. tochloa levis Merr.

\section{CEPHALOSTACHYUM Munro}

CEPHALOSTACHYUM MINDORENSE Gamble in Philip. Journ. Sci. 5 (1910) Bot. 272.

Luzon (Camarines), Mindoro, F. B. 11421, 8619, 6213 Merritt, 10667 Curran, 27424 Ablaza \& Ramos. In thickets and forests at low altitudes, locally abundant. Endemic.

This is presumably the form reported from the Philippines by F.Villar, Novis. App. (1882) 324, as C. capitatum Munro, a species that does not occur in the Archipelago. To be compared here is Vidal 4021, reported by Ceron, Cat. Pl. Herb. Manila (1892) 185, as Oxytenanthera sp.

Local names: Bagtók (Bik.); baktó (Tag.); bíkal (Bik.).

\section{SCHIZOSTACHYUM Nees}

SCHIZOSTACHYUM CURRANII Gamble in Philip. Journ. Sci. 5 (1910) Bot. 277; Camus Bamb. (1913) 177.

Luzon (Ifugao, Benguet), F. B. 10849 Curran, 18014 Merritt, B. S. 19778 McGregor, Merrill Phil. Pl. 121\%. In borders of the mossy forest, altitude about $2,000 \mathrm{~m}$. Endemic.

SCHIZOSTACHYUM DIELSIANUM (Pilger) Merr. in Philip. Journ. Sci.

1 Suppì. (1906) 391; Gamble in Philip. Journ. Sci. 5 (1910) Bot.

274, 8 (1913) Bot. 205; Camus Bamb. (1913) 174, t. 96, f. B.

Dinochloa dielsiana Pilger in Perk. Frag. Fl. Philip. (1904) 148.

Palawan, Merrill 711 . In thickets and forests at low altitudes. Endemic.

By no means certainly distinct from S. diffusum Merr. Numerous specimens distributed as $S$. dielsianum Merr. appear to be referable to $S$. diffusum, but many intergrades occur. 
SCHIZOSTACHYUM DIFFUSUM (Blanco) Merr. in Am. Journ. Bot. 3 (1916) 62, Sp. Blancoanae (1918) 78.

Bambus diffusa Blanco Fl. Filip. (1837) 269; ed. 2 (1845) 187, ed. 3, 1 (1877) 334; Miq. Fl. Ind. Bat. 3 (1857) 420; Munro in Trans. Linn. Soc. 26 (1868) 118.

Schizostachyum acutiflorum Munro in Trans. Linn. Soc. 26 (1868) 137; F.-Vill. Novis. App. (1882) 324; Vidal Phan. Cuming. Philip. (1885) 159, Rev. Pl. Vasc. Filip. (1886) 294; Merr. in Philip. Journ. Sci. 1 (1906) Suppl. 391, 3 (1908) Bot. 397; Gamble in Philip. Journ. Sci. 5 (1910) Bot. 273, 8 (1913) Bot. 205; Camus Bamb. (1913) 174, t. 95, f. A.

Dinochloa diffusa Merr. in Govt. Lab. Publ. (Philip.) 29 (1905) 7. ? Schizostachyum blumei F.-Vill. Novis. App. (1882) 324, non Nees. Ischurochiloa floribunda F.-Vill. in Blanco Fl. Filip. ed. 3, 4 (1880) 14, non Büse.

Dinochloa major Pilger in Perk. Frag. Fl. Philip. (1904) 149; Camus Bamb. (1913) 170.

Babuyan Islands, Luzon (Cagayan, Isabela, Apayao, Ilocos Norte, Ilocos Sur, Benguet, Nueva Vizcaya, Pangasinan, Zambales, Bulacan, Nueva Ecija, Pampanga, Rizal, Bataan, Laguna, Tayabas, Camarines), Mindoro, Palawan, Leyte. Common in secondary and primary forests at low and medium altitudes, ascending to $1,700 \mathrm{~m}$. Variable. Endemic.

Local names: Baliáro (Ilk.); balíkau (Ilk.); bíkil (Ilk.); bongbong (Bis.) ; loob (Bis.) ; indi, inri, hindi (Bik.); butor (Isn.); bábui (Bik.); lilit (Pamp.) ; bíkal (Ilk., Ibn., Pamp., Pang., Tag.) ; bíkal-bábi (Pamp.) ; bíkal-bábui (Sbl.); gimak (Tag.); mangnau (Ilk.); tagisí (Tag.) ; usíu (Tag.).

SCHIZOSTACHYUM FENIXII Gamble in Philip. Journ. Sci. 6 (1911) Bot. 289, 8 (1913) Bot. 205.

Luzon (Cagayan, Abra, Ilocos Sur, Benguet), Panay, F. B. 17815 Curran, 23567 Vergara, 25482, 25086 Paraiso, Fénix, McVey. In thickets at low and medium altitudes. Endemic.

Local names: Bólo (Ilk.) ; páua (P. Bis.); púser (Ilk.).

SCHIZOSTACHYUM LIMA (Blanco) Merr. in Am. Journ. Bot. 3 (1916) 62, Sp. Blancoanae (1918) 77.

Bambus lima Blanco Fl. Filip. (1837) 271; ed. 2 (1845) 189; ed. 3, 1 (1877) 336; Miq. Fl. Ind. Bat. 3 (1857) 421; Munro in Trans. Linn. Soc. 26 (1868) 121; Merr. in Philip. Journ. Sci. 1 (1906) Suppl. 388; Camus Bamb. (1913) 134.

Bambusa longinodis F.-Vill. Novis. App. (1882) 323, non ? Miq. Schizostachyum hallieri Gamble in Philip. Journ. Sci. 5 (1910) Bot. 274, 8 (1913) Bot. 205; Camus Bamb. (1913) 175, t. 97, f. B.

Schizostachyum brachycladum Gamble in Philip. Journ. Sci. 8 (1913) Bot. 206, non Kurz.

Schizostachyum longispiculatum Gamble in Philip. Journ. Sci. 8 (1913)

Bot. 206, non Kurz.

Luzon (Nueva Vizcaya, Bataan, Laguna, Sorsogon), Mindoro, Palawan, Leyte, Mindanao, Basilan, Hallier, Merrill 581, 9226, Sp. Blancoanae 41, Phil. Pl. 1472, as S. longispiculatum Kurz, Elmer 7145, 10838, 12958, 12599, 16621, Wenzel 135\%, Weber 1111, Wester 145, B. S. 20120 McGregor, 
F. B. 11374 Merritt. In thickets and secondary forests at low and medium altitudes, sometimes gregarious; a species having unusually long internodes, these being up to $120 \mathrm{~cm}$ in length. Endemic.

Local names: Ános (Tag.); bagákai (C. Bis., P. Bis., Bik.); bitu (Pamp.) ; bolo (Ilk.); buho (P. Bis.); lakap (Buk.) ; nap-nap (C. Bis.) ; sumbiling (Tagb.).

SCHIZOSTACHYUM LUMAMPAO (Blanco) Merr. in Am. Journ. Bot. 3 (1916) 65, Sp. Blancoanae (1918) 77.

Bambus lumampao Blanco Fl. Filip. (1837) 272, ed. 2 (1845) 189; ed. 3, 1 (1877) 338; Miq. F1. Ind. Bat. 3 (1857) 421; Munro in Trans. Linn. Soc. 26 (1868) 118; Merr. in Philip. Journ. Sci. 1 (1906) Suppl. 389; Camus Bamb. (1913) 132.

Dendrocalamus membranaceus F.-Vill. Novis. App. (1882) 324, non Munro.

Schizostachyum mucronatum Hack. in Philip. Journ. Sci. 3 (1908)

Bot. 169; Gamble in Philip. Journ. Sci. 5 (1910) Bot. 276; Camus Bamb。 (1913) 175.

Schizostachyum hirtiflorum Hack. in Philip. Journ. Sci. 2 (1907)

Bot. 420; Gamble in Philip. Journ. Sci. 5 (1910) Bot. 275; Camus

Bamb. (1913) 176.

Luzon (Cagayan, Isabela, Ilocos Norte, Ilocos Sur, Benguet, Pangasinan, Pampanga, Rizal, Bataan, Tayabas, Camarines), Panay, Merrill Sp. Blancoanae 891, Elmer 6179, B. S. 7825 Ramos, 2310 Mearns, 29875 Fénix, F. B. 21926 Alejandro, 26058 Barros, 25829 Paraiso, 22808 Diaz \& Alambra, 24113 Velasco, 23560 Vergara, 20945 De Leon, 20870 Bernardo, 10414 Curran, 3615 Maule. In secondary forests, but more frequently gregarious and occupying extensive areas at low and medium altitudes where forests have been destroyed. Endemic.

Local names: Bakákan (Tagb.) ; bóho (Tag., Bik.) ; bokáui (Tag.) ; bólo (Tag., Ilk., Pang., Bik., P. Bis., Ibn.) ; búlo (Ibn.) ; caña bóho (Sp.); dasó (Bik.) ; lumampáu (Tag.) ; napnáp (Ilk.) ; oras (Bik.); valú (Ibn.); vúlu (It.).

SCHIZOSTACHYUM LUZONICUM Gamble in Philip. Journ. Sci. 5 (1910)

Bot. 277; Camus Bamb. (1913) 177.

Luzon (Zambales), $F$. B. 8411 Curran \& Merritt, 5926 Curran. In forests and in ravines, altitude about $800 \mathrm{~m}$. Endemic.

SCHIZOSTACHYUM PALAWANENSE Gamble in Philip. Journ. Sci. 5 (1910) Bot. 274, 8 (1913) Bot. 205; Camus Bamb. (1913) 175.

Palawan, B. S. 27y Bermejos, Elmer 12618. In forests at low altitudes. Endemic.

Local name: Bugto (Tagb.).

SCHIZOSTACHYUM TEXTORIUM (Blanco) Merr. in Am. Journ. Bot. 3 (1916) 64, Sp. Blancoanae (1918) 76.

Bambus textoria Blanco Fl. Filip. (1837) 270; ed. 2 (1845) 188; ed. 3, 1 (1877) 335; Miq. Fl. Ind. Bat. 3 (1857) 421; Munro in Trans. Linn. Soc. 26 (1868) 122; Merr. in Philip. Journ. Sci. 1 (1906). Suppl. 389; Camus Bamb. (1913) 135.

Gigantochloa atter F.-Vill. Novis. App. (1882) 323, non Kurz.

Schizostachyum merrillii Gamble in Philip. Journ. Sci. 5 (1910) Bot. 278; Camus Bamb. (1913) 174. 
Luzon (Batangas, Rizal), Merrill 1744, Sp. Blancoanae 714. In secondary forests at low altitudes. Endemic.

Local name: Kalbáng (Tag.).

SCHIZOSTACHYUM TOPPINGII Gamble in Philip. Journ. Sci. 5 (1910)

Bot. 276; Merr. Fl. Manila (1912) 106; Camus Bamb. (1913) 178.

Luzon (Nueva Vizcaya, Rizal), Mindoro, B. S. 5222 Topping, F. B. 9500 Merritt, B. S. 39366, 39713 Ramos. In thickets and secondary forests at low and medium altitudes. Endemic.

\section{DINOCHLOA Büse}

DINOCHLOA ELMERI Gamble in Philip. Journ. Sci. 5 (1910) Bot. 280; Camus Bamb. (1913) 170.

Luzon (Benguet), Elmer 6542. In the mossy forests on Mt. Santo Tomas, altitude about 2,200 m. Endemic.

DINOCHLOA LUÇONIAE (Munro) comb. nov.

Bambusa luçoniae Munro in Trans. Linn. Soc. 26 (1868) 115; F.-Vill. Novis. App. (1882) 323 (luzoniae); Merr. in Philip. Journ. Sci. 1 (1906) Suppl. 389; Camus Bamb. (1913) 134.

Dinochloa aguilarii Gamble in Philip. Journ. Sci. 5 (1910) Bot. 280; Camus Bamb. (1913) 170.

Dinochloa ciliata Kurz in Journ. As. Soc. Beng. $42^{2}$ (1873) 253, in nota; Camus Bamb. (1913) 170, t. 95, f. D; Merr. in Philip. Journ. Sci. 11 (1916) Bot. 51.

Dinochloa scandens 0 . Kuntze var. angustifolia Merr. in Philip. Journ. Sci. 1 (1906) Suppl. 392; Gamble in Philip. Journ. Sci. 5 (1910) Bot. 279, 8 (1913) Bot. 206; Camus Bamb. (1913) 169.

Dinochloa tjankorreh Büse var. angustifolia Hack. ex Merr. 1. c. in syn.

Luzon (Cagayan, Ifugao, Ilocos Norte, Nueva Ecija, Bulacan, Zambales, Cavite, Bataan, Laguna, Tayabas), Mindoro, Leyte, Samar, Mindanan, Basilan. In forests at low and medium altitudes. Borneo.

I have examined the type of Bambusa luçoniae Munro, which was from Mt. Banahao, and which is preserved in the United States National Herbarium. I fail to see how $D$. aguilarii Gamble can be distinguished from D. ciliata Kurz, or either from D. luconiae Merr.

Local names: Bíka (Ibn.); bíkal (Ibn.); buloau (Sub.); bolokáui (C. Bis.) ; malilit (Sbl.) ; osíu (Tag.) ; timak (Tag.) ; usíu (Tag.).

DINOChloA PUBIRAMEA Gamble in Philip. Journ. Sci. 5 (1910) Bot. 279; Camus Bamb. (1913) 171.

Dinochloa scandens O. Kuntze var. pubiramea Merr. ex Gamble 1. c. in syn.

Leyte, Samar, Negros, Mindanao (Surigao), Basilan, Bolster 319, Wenzel 1310, F. B. 5589 Everett, 26268 Mallonga, B. S. 17639 Ramos. In. forests at low and medium altitudes. Borneo.

Local name: Bukau (Yak.).

Dinochloa scandens (Blume) O. Kuntze Rev. Gen. Pl. (1891) 773; Merr. in Philip. Journ. Sci. 1 (1906) Suppl. 392; Gamble in Philip. Journ. Sci. 5 (1910) Bot. 278, 8 (1913) Bot. 206; Camus Bamb. (1913) 169. 
Bambusa scandens Blume ex Nees in Flora 7 (1824) 291.

Nastus tjangkorreh Schultes Syst. Veg. $7^{2}$ (1830) 1358.

Dinochloa tjankorreh Büse in Miq. Pl. Jungh. (1854) 388; Miq. Fl.

Ind. Bat. 3 (1857) 415; Munro in Trans. Linn. Soc. 26 (1868)

153; F.-Vill. Novis. App. (1882) 324; Vidal Sinopsis Atlas (1883)

42, t. 96, f. C, Phan. Cuming. Philip. (1885) 157, Rev. Pl. Vasc.

Filip. (1886) 294; Merr. in Philip. Journ. Sci. 1 (1906) Suppl. 29.

Dinochloa macrocarpa Elm. Leafl. Philip. Bot. 7 (1915) 2675.

Luzon (Bulacan, Laguna, Camarines, Sorsogon), Polillo, Mindoro, Palawan, Balabac, Samar, Panay, Sibuyan, Biliran, Leyte, Mindanao, Basilan. Common in primary forests at low and medium altitudes. Malay Peninsula and Archipelago.

Local names: Baká (Tagb.) ; balikau (Tagb.) ; biá (Tagb.) ; biká (Bon.); balilit (Bag.) ; bayokáui (P. Bis.) ; bukáu (Yak.) ; bukáui (Tag.) ; bulukáui (P. Bis.) ; burukáui (S. L. Bis.) ; timak (Mang.); 'usíu (Bik., Tag.).

\section{EXCLUDED GENERA AND SPECIES}

LAPPAgo RACemosa Houck.; F.-Vill. Novis. App. (1882) 313.

Beesha RHeedi Kunth; Vidal Cat. Pl. Prov. Manila (1880) 48; F.-Vill. Novis. App. (1882) 324.

Phyllostachys bambusoldes Sieb.; F.-Vill. Novis. App. (1882) 323.

Phyllostachys NIGRa Munro; F.-Vill. 1. c.

PAPPOPhORUM PHLEOIdes (CaV.) R. \& S.

"Arab. vel ins. Philipp.?" Steud. Syn. Pl. Glum. 1 (1854) 199. This is an American species erroneously credited to the Philippines by Steudel.

Stenotaphrum complanatum Schrank.; F.-Vill. Novis. App. (1882) 313.

Teinostachyum sp.; Gamble in Philip. Journ. Sci. 8 (1913) Bot. 205.

This reference was based on 1 a sterile specimen of what will probably prove to be a species of Schizostachyum.

Several species of the Chlorideae, chiefly of the genus Bouteloud, have been erroneously credited to the Philippines on the basis of American specimens collected by members of the Malaspina Expedition and erroneously localized as Philippine. They are:

Bouteloua curtipendula (Mich.) Torr.; Eutriana curtipendula Trin.; Kunth Enum. 1 (1833) 280; Miq. Fl. Ind. Bat. 3 (1857) 383; F.-Vill. Novis. App. (1882) $320=$ Bouteloua racemosa Lagasca.

Bouteloua BARBata Lagasca; Eutriana barbata Kunth Rev. Gram. 1 (1829) 96; Enum. 1 (1833) 282; Miq. Fl. Ind. Bat. 3 (1857) 284 ; F.-Vill. Novis. App. (1882) 321.

Bouteloua tenuis Griseb.; Chrondrosium tenue Beauv. Agrost. (1812) 41; Kunth Enum. 1 (1833) 76; F.-Vill. Novis. App. (1882) 320.

BoutelouA Simplex Lagasca; Chrondrosium simplex Kunth Enum. 1 276; F.-Vill. Novis. App. (1882) 320. 
Polyschistis paupercula Presl Rel. Haenk. 1 (1830) 294, $t, 41, f$. 2 ; Kunth Enum. 1 (1833) 282; Miq. Fl. Ind. Bat. 3 (1859) 384; F.-Vill. Novis. App. (1882) 321.

"Hab. in insula Luzonia" Presl. The specimens were erroneously localized; a species of tropical America $=$ Pentarrhaphis sp.

Atheropogon PRocumbens Jacq.; R. \& S. Syst. 2 (1825) 292.

"Ins. Philipp. Mexico" Roemer and Schultes. This is one of the American species of Bouteloua.

\section{CYPERACEAE}

\section{LIPOCARPHA R. Brown}

LIPOCARPHA ARgenteA R. Br. in Tuckey's Congo Append. (1818) 459; Miq. Fl. Ind. Bat. 3 (1856) 331; F.-Vill. Novis. App. (1882) 308; Vidal Phan. Cuming. Philip. (1885) 156, Rev. Pl. Vasc. Filip. (1886) 285 ; C. B. Clarke in Philip. Journ. Sci. 2 (1907) Bot. 109.

Lipocarpha lacvigata Nees in Hook. Journ. Bot. Kew Miscel. 6 (1854) 28.

Kyllinga albescens Steud. Syn. Pl. Glum.2 (1855) 68; Miq. Fl. Ind. Bat. 3 (1856) 294; Naves Novis. App. (1882) 300.

Luzon (Cagayan, Lepanto, Bontoc, Benguet, Nueva Vizcaya, Nueva Ecija), Mindanao (Bukidnon), B. S. 26007, 26151 Fénix, 14252 McGregor, 7864 Ramos, Merrill 215, Phil. Pl. 570, Elmer 6484, Williams 1224. In open wet places chiefly at medium altitudes, ascending to $1,800 \mathrm{~m}$. Warmer parts of the Old World.

Local name: Balábak (Buk.).

LIPOCARPHA MICROCEPHALA (R. Br.) Kunth Enum. 2 (1837) 268; Miq. Fl. Ind. Bat. 3 (1856) 331; C. B. Clarke in Hook. f. Fl. Brit. Ind. 6 (1893) 668, Philip. Journ. Sci. 2 (1907) Bot. 110; F.-Vill. Novis. App. (1882) 308; Merr. Fl. Manila (1912) 119.

Hypaelyptum microcephalum R. Br. Prodr. (1810) 220.

Ascolepis kyllingioides Steud. in Zoll. Verz. Ind. Archip. (1854) 61.

Kyllinga squarrosa Steud. Syn. Pl. Glum. 2 (1855) 68.

Luzon (Apayao, Ifugao, Bulacan, Rizal), Merrill 3625, Phil. Pl. 54\%, B. S. 21712, 12177 Ramos, 28174 Fénix. In open wet grasslands at low and medium altitudes. Japan to the Malay Peninsula and tropical Australia.

2. HYPOLYTRUM L. C. Richard

HYPOLYTRUM COMPACTUM Nees in Linnaea 9 (1834) 288, Nov. Act. Acad. Nat. Cur. 19 (1843) Suppl. 1: 73; Steud. Syn. Pl. Glum. 2 (1855) 132; Miq. Fl. Ind. Bat. 3 (1856) 333; Boeck. in Linnaea 37 (1871) 127; F.-Vill. Novis. App. (1882) 309; Merr. in Philip. Journ. Sci. 1 (1906) Suppl. 29; C. B. Clarke op. cit. 2 (1907) Bot. 109.

Luzon (Benguet, Pangasinan, Zambales, Nueva Vizcaya, Bulacan, Bataan), Palawan, B. S. 29983 Fénix, 29537, 29428 Ramos \& Edaño, Elmer 8889, 13041, Merrill 2496, 7597, 5184, Phil. Pl. 1434. In forests at low and medium altitudes, often common. Indo-China, Andaman Islands, Borneo.

Local name: Túhog-dalág' (Tag.). 
HYPOLYTRUM LATIFOLIUM L. C. Rich. in Pers. Syn. 1 (1805) 70; Hook. f. in Curtis's Bot. Mag. III 33 (1877) t. 6282; F.-Vill. Novis. App. (1882) 309; C. B. Clarke in Philip. Journ. Sci. 2 (1907) Bot. 108; Merr. op. cit. 264.

Scirpus anomalus Retz. Obs. 5 (1789) 15, non Hypolytrum anomalum Steud.

Albikia schoenoides Presl Rel. Haenk. 1 (1828) 185, t. 34.

Hypolytrum schoenoides Nees in Linnaea 9 (1834) 288.

Hypolytrum trinervium Kunth Enum. 2 (1837) 272; Miq. F1. Ind. Bat. 3 (1856) 332; F.-Vill. Novis. App. (1882) 308.

Luzon (Tayabas, Pangasinan, Camarines), Palawan, Biliran, Panay, Mindanao, Basilan, Merrill 9449, Phil. Pl. 537, F. B. 20186 Aguilar, B. S. 13115 Foxworthy \& Ramos, 18561 McGregor, 34841 Ramos \& Pascasio, 17r87 Otanes. In forests at low and medium altitudes. India to Formosa through Malaya to Polynesia.

Local names: Baliis (Tag.) ; bubugo (Sub.) ; marakibkib (Neg.); paraibiau (Sul.); root (Neg.).

HYPOLYTRUM SCIRPOIDES (Presl) comb. nov.

Albikia scirpoides Presl Rel. Haenk. 1 (1828) 185, t. 35, excl. syn.

Hypolytrum phitippense C. B. Clarke in Philip. Journ. Sci. 2 (1908) Bot. 109.

Hypolytrum viridinux C. B. Clarke 1 , c.

Throughout the Philippines, Elmer 6223', 15916, 18198, Merrill 7991, 10430, 744, 5126, 8186, B. S. 29589, 28654, 28462 Ramos \& Edaño, 34394 Ramos \& Pascasio, 28164 F'énix, 23601, 13929, 20424, 15314, 1853, 7389, 33162 Ramos, 14238, 32473 McGregor. In forests at low and medium altitudes; often common. Borneo.

Local name: Alisáhis (Buk.).

The Philippine form Presl figured and described is unmistakably the same as $H$. philippense C. B. Clarke; Presl erred in referring to it $H$. nemorum Beauv. and Schoenus nemorum Vahl.

\section{CYPERUS Linnaeus}

CYPERUS COMPRESSUS Linn. Sp. Pl. (1753) 46; Nees in Nov. Act. Acad. Nat. Cur. 19 (1843) Suppl. 1: 56, Hook. Journ. Bot. Kew Miscel. 6 (1854) 27; Naves Novis. App. (1882) 302; Vidal Phan. Cuming. Philip. (1885) 155, Rev. Pl. Vasc. Filip. (1886) 282; C. B. Clarke in Philip. Journ. Sci. 2 (1907) Bot. 84; Merr. op. cit. 3 (1908) Bot. 398, Fl. Manila (1912) 111, Sp. Blancoanae (1918) 79.

Cyperus humitis Llanos Frag. Pl. Filip. (1851) 13; F.-Vill. \& Naves in Blanco Fl. Filip. ed. $3,4^{1}$ (1880) 7 , non Kunth.

Cyperus compressus Linn. var. brachiatus Nees in Linnaea 35 (1868) 518.

Cyperus meyenii Nees in Nov. Act. Acad. Nat. Cur. 19 (1843) Suppl. 1: 57.

Throughout the Philippines in open grasslands, waste places, etc., at low and medium altitudes; common. Pantropic.

Local names: Ananá (Ibn.) ; gísai-kalabáu (Tag.) ; kaptos (Iv.) ; túhogdalág (Tag.); sangsañga (Bon.). 
CYPERUS DIFFORMIS Linn. Amoen. Acad. 4 (1759) 302; Presl Rel. Haenk. 1 (1828) 69; Nees in Nov. Act. Acad. Nat. Cur. 19 (1843) Suppl. 1: 70, Hook. Journ. Bot. Kew Miscel. 6 (1854) 28; Miq. Fl. Ind. Bat. 3 (1856) 269; Naves Novis. App. (1882) 302; Vidal Phan. Cuming. Philip. (1855) 155, Rev. Pl. Vasc. Filip. (1886) 283; C. B. Clarke in Philip. Journ. Sci. 2 (1907) Bot. 82; Merr. Fl. Manila (1912) 110, Sp. Blancoanae (1918) 78.

Cyperus subrotundus Naves Frag. Pl. Filip. (1851) 14; F.-Vill. \& Naves in Blanco Fl. Filip. ed. $3,4^{1}(1880) \cdot 8$.

Cyperus goeringii Steud. Syn. Pl. Glum. 2 (1855) 24; Naves Novis. App. (1882) 303.

Throughout the Philippines in open wet places, ascending to $2,000 \mathrm{~m}$; a characteristic rice-paddy weed. Old World Tropics generally, introduced in Mexico.

Local names: Baki-báki (Bis.); ballayang (Ilk.); bankoan (Bis.); gilamhon (Bis.); pukuugan (Bon.).

CYPERUS DIfFusus Vahl Enum. 2 (1806) 321; Nees in Nov. Act. Acad. Nat. Cur. 19 (1843) Suppl. 1: 58, Hook. Journ. Bot. Kew Miscel. 6 (1854) 28 (var. longifolius Nees); Miq. Fl. Ind. Bat. 3 (1856) 264 ; Naves Novis. App. (1882) 302; Vidal Phan. Cuming. Philip. (1885) 155, Rev. Pl. Vasc. Filip. (1886) 283; C. B. Clarke in Philip. Journ. Sci. 2 (1907) Bot. 83; Merr. op. cit. 262, Fl. Manila (1912) 110.

Cyperus scirpoideus Presl Rel. Haenk. 1 (1828) 178, non Vahl.

Cyperus sorzogonensis Presl op. cit. 351.

Cyperus longifolius Dene. in Nuov. Ann. Mus. Paris 3 (1834) 359;

Nees in Hook. Journ. Bot. Kew Miscel. 6 (1854) 28.

Throughout the Philippines, in thickets, secondary and primary forests, and in old clearings at low and medium altitudes; common. Pantropic.

Local names: Barsa ñgá-bákir (Ilk.); gumi-gúmi (Tag.); haras (Tag.) ; nipinpin-di-gúbat (Sbl.); túhog-dalág (Tag.).

Var. PUBESQUAMA (Steud.) Kükenth. in herb. comb. nov.

Cyperus pubesquama Steud. in Zoll. Verz. Ind. Archipel. (1854) 62; Miq. Fl. Ind. Bat. 3 (1856) 266; Naves Novis. App. (1882) 302; C. B. Clarke in Philip. Journ. Sci. 2 (1907) Bot. 83.

Cyperus lagorensis Steud. Syn. Pl. Glum. 2 (1855) 36; Miq. Fl. Ind. Bat. 3 (1856) 275.

Cyperus bancanus Naves Novis. App. (1882) 302; Merr. in Philip. Journ. Sci. 1 (1906) Suppl. 30; C. B. Clarke op. cit. 2 (1907) Bot. 83, non Miq.

Cyperus calacaryensis Steud. Syn. Pl. Glum. 2 (1855) 34; Miq. F1. Ind. Bat. 3 (1856) 275; Naves Novis. App. (1882) 304.

Luzon (Cagayan, Isabela, Bulacan, Rizal, Bataan, Laguna), Mindoro, Leyte, Mindanao, F. B. 2581 Meyer, B. S. 13951, 8015 Ramos, Merrill 5054, 5692, Phil. Pl. 523. Habitat of the species from which it is scarcely distinguishable. India to Malaya.

Var. CELEBICA Miq. Fl. Ind. Bat. 3 (1856) 283.

Throughout the Philippines, Elmer 18286, 6674, 8306, Williams 85, B. S. 28839 Ramos \& Edaño, 16166 Reillo, F. B. 20172 Aguilar. Habitat of the species. Malaya. 
CYPERUS DIGITATUS Roxb. Hort. Beng. (1814) 81, nomen nudum, Fl. Ind. 1 (1820) 209; C. B. Clarke in Philip. Journ. Sci. 2 (1907) Bot. 86.

Cyperus auricomus Naves Novis. App. (1882) 305; Ceron Cat. Pl. Herb. Manila (1892) 177, non Sieber.

Babuyan Islands, Luzon (Cagayan, Isabela, Nueva Vizcaya, Rizal, Pampanga, Laguna), Leyte, Mindanao, B. S. 1450, 8075, 8151, 13516 Ramos, Weber 10.83, Merrill $231 \%$. In open wet places at low and medium altitudes; widely scattered. Pantropic.

CYPERUS DISTANS Linn. f. Suppl. (1781) 103; Nees in Hook. Journ. Bot. Kew Miscel. 6 (1854) 27; Naves Novis. App. (1882) 303; Vidal Phan. Cuming. Philip. (1885) 155, Rev. Pl. Vasc. Filip. (1886) 283; C. B. Clarke in Philip. Journ. Sci. 2 (1907) Bot. 84; Merr. op. cit. 3 (1908) Bot. 398, 5 (1910) Bot. 333, Fl. Manila (1912) 111.

Cyperus kurrii Steud. Syn. Pl. Glum. 2 (1855) 38; Miq. Fl. Ind. Bat. 3 (1856) 279; Naves Novis. App. (1882) 305.

Throughout the Philippines. Common in open rather wet places at low and medium altitudes. Pantropic.

Local names: Amúsan (Tag.); bakabakáhan (Tag.); barisañgá (Ilk.); burabongdai (S. L. Bis.); gagauan (Bon.); hañganga (If.); langalang (Bik.) ; mutháng-kalabáu (Tag.); pulukgalo (Buk.) ; talúbak (Buk.).

CYPERUS ELATUS Linn. Amoen. Acad. 4 (1759) 301; Naves Novis. App. (1882) 304; C. B. Clarke in Philip. Journ. Sci. 2 (1907): Bot. 86.

Cyperus bispicatus Steud. in Zoll. Verzeich. Ind. Archipel. (1854) 62; Naves Novis. App. (1882) 303.

Luzon (Laguna), Biliran, Leyte, Wenzel 1279, B. S. 1495.5 Ramos, 23140, 18897 McGregor, distributed as C. radiatus Vahl, and not certainly distinct from Vahl's species. In swamps and open wet places at low altitudes. India to Malaya.

Local name: Kobong-kóbong (Bis.).

CYPERUS ELEUSINOIDES Kunth Enum. 2 (1837) 39; Naves Novis. App. (1882) 303; C. B. Clarke in Philip. Journ. Sci. 2 (1907) Bot. 84.

Luzon (Cagayan, Bontoc, Benguet, Nueva Vizcaya), Mindanao (Bukidnon), Merrill 226, B. S. 24950 Fénix, 22728 Castillo, 8747, 14264 McGregor. In open wet places at low and medium altitudes, not common. Tropical Asia and Africa through Malaya to tropical Australia.

Local names: Gauang (Bon.); salingánga (Bon.).

CYPERUS FlABELliformis Rottb. Descr. Nov. Pl. (1773) 42; Merr.

Fl. Manila (1912) 110.

Cultivated for ornamental purposes, occasional in waste places in Manila but scarcely established. A native of Africa and southwestern Asia.

CYPERUS HASPAN Linn. Sp. Pl. (1753) 45; Miq. Fl. Ind. Bat. 3 (1856) 267; Naves Novis. App. (1882) 302; C. B. Clarke in Philip. Journ. Sci. 2 (1907) Bot. 82; Merr. op. cit. 3 (1908) Bot. 398, Fl. Manila (1912) 110, Sp. Blancoanae (1918) 79.

Cyperus caespitosus Llanos Frag. Pl. Filip. (1851) 14 (caespitorus); F.-Vill. \& Naves in Blanco Fl. Filip. ed. 3, $4^{1}$ (1880) 8; C. B. Clarke in Philip. Journ. Sci. 2 (1907) Bot. 86, non Poir. 
Cyperus dehiscens Naves Novis. App. (1882) 303, non Kunth.

Cyperus flavidus C. B. Clarke in Philip. Journ. Sci. 2 (1907) Bot. 82 , non Retz.

In open wet places throughout the Philippines; a common and characteristic rice-paddy weed. Pantropic.

I fail to see how Philippine specimens referred to C. flavidus Retz. can be distinguished from C. haspan Linn.

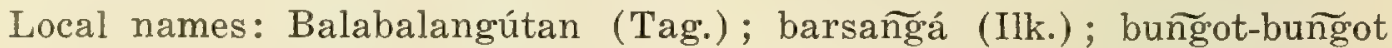
(P. Bis.) ; manik-maníkan (Tag.) ; misai-kalabau (Tag.) ; zaingal (Sub.).

CYPERUS IRIA Linn. Sp. Pl. (1753) 45; Presl Rel. Haenk. 1 (1828) 174; Nees in Nov. Act. Acad. Nat. Cur. 19 (1843) Suppl. 1: 70, Hook. Journ. Bot. Kew Miscel. 6 (1854) 28; Naves Novis. App. (1882) 303; Vidal Phan. Cuming. Philip. (1885) 155, Rev. Pl. Vasc. Filip. (1886) 283; C. B. Clarke in Philip. Journ. Sci. 2 (1907) Bot. 83 (incl. var. paniciformis C. B. Clarke); Merr. Fl. Manila (1912) 111, Sp. Blancoanae (1918) 79.

Cyperus nuttallii Llanos Frag. Pl. Filip. (1851) 14; F.-Vill. \& Naves in Blanco Fl. Filip. ed. 3, $4^{1}$ (1880) 9, non Torr.

Chlorocyperus iria Rikli in Jahrb. Wissensch. Bot. 27 (1895) 564; Palla in Allgem. Bot. Zeitschr. 17 (1911) Beil. 6; Kneucker Cyp. Exsic. No. 237.

In open wet places throughout the Philippines; a common and characteristic rice-paddy weed. Old World Tropics generally.

Local names: Alinang (Bik); okokiang (Bon.); paiung-paiung (Bik.); sud-sud (Bik.); taga-taga (Bik.).

CYPERUS LEUCOCEPHALUS Retz. Obs. 5 (1789) 11; Nees in Hook. Journ. Bot. Kew Miscel. 6 (1854) 28; Vidal Phan. Cuming. Philip. (1885) 155, Rev. Pl. Vasc. Filip. (1886) 283; C. B. Clarke in Philip. Journ. Sci. 2 (1907) Bot. 82.

Sorostachys kyllingioides Steud. in Flora 33 (1850) 229, nomen nudum, Syn. Pl. Glum. 2 (1855) 71; Miq. Fl. Ind. Bat. 3 (1856) 296. Cyperus sorostachys Boeck. in Linnaea 35 (1868) 588; Scheff. in Nat. Tijdschr. Nederl. Ind. 34 (1874) 48.

Cyperus pulchellus R. Br. Prodr. (1810) 213; Naves Novis. App. (1882) 301.

Mindanao (Misamis), Caming 1617. This does not seem to have been found in the Philippines since Cuming collected it, between 1836 and 1840 . Pantropic.

CYPERUS MALACCENSIS Lam. Tabl. Encycl. 1 (1791) 146; Usteri Beitr. Ken. Philip. Veg. (1905) 131; Merr. in Philip. Journ. ISci. 1 (1906) Suppl. 30, Fl. Manila (1912) 111; C. B. Clarke in Philip. Journ. Sci. 2 (1907) Bot. 84.

Chlorocyperus malaccensis Palla in Allgem. Bot. Zeitschr. 17 (1911) Beìl. 6, Kneucker Cyp. Exsic. No, 238.

Central Luzon to Palawan and Mindanao, on the muddy margins of tidal streams within the influence of salt or brackish water. Tropical Asia through Malaya to Australia and Polynesia.

Local names: Baga-as (P. Bis.); balangót (Tag., Bik., Bis., Pamp.) ; balongát (Pamp.); barangót (Bik.); talaid (Bag.); tíkog (Mbo.). 
CYPERUS PILOSUS Vahl Enum. 2 (1806) 354; Naves Novis. App. (1882) 303; Vidal Phan. Cuming. Philip. (1885) 155, Rev. Pl. Vasc. Filip. (1886) 283; C. B. Clarke in Philip. Journ. Sci. 2 (1907) Bot. 84; Merr. Fl. Manila (1912) 111.

Cyperus marginellus Nees in Wight Contr. (1834) 83, Hook. Journ. Bot. Kew Miscel. 6 (1854) 28.

Cyperus pauciflorus Steud. Syn. P1. Glum.2 (1855) 34; Miq. Fl. Ind. Bat. 3 (1856) 275; Naves Novis. App. (1882) 304.

Cyperus obliquus Nees in Wight Contr. (1834) 86; Naves Novis. App. (1882) 305.

Duvaljouvea pilosa Palla in Koch Syn. ed. 3 (1905) 2555; Kneucker Cyp. Exsic. No. 245.

In open wet places throughout the Philippines; common. Tropical Asia and Africa through Malaya to Australia.

Local name: Paragi (Sub.).

CYPERUS RADIATUS Vahl Enum. 2 (1806) 369; Naves Novis. App. (1882) 305; Vidal Phan. Cuming. Philip. (1885) 155 (var. elongata Boeck.), Rev. Pl. Vasc. Filip. (1886) 283; C. B. Clarke in Philip. Journ. Sci. 2 (1907) Bot. 85; Merr. in Philip. Journ. Sci. 3 (1908) Bot. 398, Fl. Manila (1912) 112, Sp. Blancoanae (1918) 78.

Cyperus racemosus Nees in Hook. Journ. Bot. Kew Miscel. 6 (1854) 27; Naves Novis. App. (1882) 303, ? non Retz.

Cyperus macrosciadion Steud. Syn. Pl. Glum. 2 (1855) 37; Miq. Fl. Ind. Bat. 3 (1856) 277; Naves Novis. App. (1882) 305.

Cyperus anabaptistus Steud. Syn. Pl. Glum. 2 (1855) 37; Miq. Fl. Ind. Bat. 3 (1856) 277; Naves Novis. App. (1882) 305; C. B. Clarke in Philip. Journ. Sci, 2 (1907) Bot. 86, ex descr.

Cyperus cumingii Steud. 1. c. in syn.

Cyperus imbricatus Llanos Frag. Pl. Filip. (1851) 17; F.-Vill. \& Naves in Blanco Fl. Filip. ed. $3,4^{1}$ (1880) 11, non Retz.

Cyperus spicatus Presl Rel. Haenk. 1 (1828) 173; Steud. Syn. Pl. Glum. 2 (1855) 52; Miq. Fl. Ind. Bat. 3 (1856) 287; Naves Novis. App. (1882) 306; C. B. Clarke in Philip. Journ. Sci. 2 (1907) Bot. 87 , ex descr.

Dichostylis radiatus Palla in Allgem. Bot. Zeitschr. 17 (1911) Beil. 8, Kneucker Cyp. Exsic. No. 247.

Throughout the Philippines in open wet places at low and medium altitudes. Pantropic.

Local names: Alinang (P. Bis.); balabalañgútan (Tag.); balayang (Ilk.) ; dagko (Bis.) ; obod-obod (Bis.) ; upopi (Ibn.).

CYPERUS ROTUNDUS Linn. Sp. Pl. (1753) 45; Presl Rel. Haenk. 1 (1828) 175; Blanco Fl. Filip. (1837) 31, ed. 2 (1845) 21, ed. 3, 1 (1877) 40; Miq. Fl. Ind. Bat. 3 (1856) 274; Naves Novis. App. (1882) 304; Vidal Phan. Cuming. Philip. (1885) 155, Rev. Pl. Vasc. Filip. (1886) 282; C. B. Clarke in Philip. Journ. Sci. 2 (1907) Bot. 85; Merr. Fl. Manila (1912) 112, Sp. Blancoanae (1918) 79.

Cyperus hexastachyus Rottb. Descr. Ic. (1773) 28, t. 14 f. 2; Nees in Nov. Act. Acad. Nat. Cur. 19 (1843) Suppl. 1: 60, var. castaneus Nees in Hook. Joürn. Bot. Kew Miscel. 6 (1854) 27.

Cyperus curvatus Llanos Frag. Pl. Filip. (1851) 15; F.-Vill. \& Naves in Blanco Fl. Filip. ed. 3, $4^{1}$ (1880) 9, non Vahl. 
Throughout the Philippines in open areas at low and medium altitudes. Common in lawns, along roads, and in waste places. Pantropic.

Local names: Barsañgá (Ilk.) ; boto-botónes (Bik.) ; galonálpas (Pamp.) ; kusung (Pamp.); malaapúlid (Pamp.); motá (Pamp.); muthá (Tag.); omadiung (Pamp.); onoran (Pamp.); sur-sur (Pamp.); tarugug (Bik.). CYPERUS STOLONIFERUS Retz. Obs. 4 (1786) 10; Presl Rel. Haenk. 1 (1828) 169; Boeck. in Linnaea 35 (1868) 489; Naves Novis. App. (1882) 302; C. B. Clarke in Philip. Journ. Sci.' 2 (1907) Bot. 85.

Luzon (Rizal), Panay, McGregor 41, Copeland. In open places at low altitudes; rare and local. Tropical Asia to Mauritius, Formosa, and through Malaya to tropical Australia.

CYPERUS UNCINATUS Poir. in Lam. Encycl. 7 (1806) 247; C. B. Clarke in Philip. Journ. Sci. 2 (1907) Bot. 82.

Cyperus cuspidatus HBK. Nov. Gen. Sp. Pl. 1 (1815) 204; Naves Novis. App. (1882) 301; Vidal Phan. Cuming. Philip. (1885) 155, Rev. Pl. Vasc. Filip. (1886) 282.

Cyperus solutus Steud. Syn. Pl. Glum. 2 (1855) 14; Miq. Fl. Ind. Bat. 3 (1856) 263; Naves Novis. App. (1882) 302.

Cyperus pusillus Nees in Hook. Journ. Bot. Kew Miscel. 6 (1854) 27, non ? Vahl.

Luzon (Bontoc, Benguet, Bulacan, Rizal, Laguna), Mindanao (Lanao), Merrill Phil. Pl. 1488, Williams 1975bis, 1969bis, B. S. 19213 Ramos. In open lands, old clearings, etc., at low and medium altitudes. Pantropic.

Local name: Salasa (Tag.).

CYPERUS ZOLLINGERI Steud. in Zoll. Verz. Ind. Archipel. (1854) 62; Syn. Pl. Glum. 2 (1855) 17; Miq. Fl. Ind. Bat. 3 (1856) 264; Naves Novis. App. (1882) 302; C. B. Clarke in Philip. Journ. Sci. 2 (1907) Bot. 85; Merr. Fl. Manila (1912) 112.

Cyperus tenuiculmis Boeck. in Linnaea 36 (1869) 286; Scheff. in Nat. Tijdschr. Nederl. Ind. 34 (1874) 50; Naves Novis. App. (1882) 306.

Luzon (Cagayan, Ilocos Norte, Benguet, Rizal, Sorsogon), Palawan, Panay, Mindanao, Elmer 16488, 6473, Merrill 7667, 9310, Phil. Pl. 530, B. S. 32583 McGregor, 29513 Ramos \& Edaño, 1106, 4636, 7672, 7820 Ramos. In open grasslands at low and medium altitudes, ascending to $1,600 \mathrm{~m}$. Tropical Asia and Africa through Malaya to tropical Australia.

Local names: Dáat-láua (Tag.); pakama (Bag.).

\section{DOUBTFUL AND EXCLUDED SPECIES}

Cyperus bulbosus Vahl; Naves Novis. App. (1882) 304.

CyPerus Castaneus Willd.; Naves Novis. App. (1882) 301.

Cyperus Cephalotes Vahl; Naves Novis. App. (1882) 301.

Cyperus corymbosus Rottb.; Naves Novis. App. (1882) 303.

Cyperus esculentus Linn.; Naves Novis. App. (1882) 304.

CyPerus eumorphus Steud.; Naves Novis App. (1882) 303.

Cyperus laevigatus Linn.; Naves Novis. App. (1882) 301.

Cyperus. Longus Linn.; Naves Novis. App. (1882) 304. 
Cyperus ornatus R. Br.; Naves Novis. App. (1882) 303.

Cyperus Pangorei Rottb.; Naves Novis. App. (1882) 304.

Cyperus radians Nees; Naves Novis. App. (1882) 305.

Cyperus sexflorus R. Br.; Naves Novis. App. (1882) 304.

Cyperus Tegetiformis Roxb.; Naves Novis. App. (1882) 303.

Cyperus clavatus Lam.; Usteri Beitr. Ken. Philip. Veg. (1905) 131.

A lapsus calami; no such name occurs in botanical literature.

CyPerus CRUentus Rottb.; Boeck. in Linnaea 36 (1869) 338; Scheff. in Nat. Tijdschr. Nederl. Ind. 34 (1874) 51; Naves Novis. App. (1882) 305.

The Philippine record was based on Cuming 2372, which was from $\mathrm{Ma}$ lacca, not from the Philippines.

Cyperus Haematodes Endl.; Steud. Syn. Pl. Glum. 2 (1855) 44; Miq. Fl. Ind. Bat. 3 (1856) 282; Naves Novis. App. (1882) 305.

The type was from Norfolk Island; the Philippine record is due to Steudel's error in transcribing the type locality as "Ins. Norf. Philipp."

Cyperus iamprocarpus Nees in Hook. Journ. Bot. Kew Miscel. 6 (1854)

27; Boeck. in Linnaea 35 (1868) 490 ; Naves Novis. App. (1882) 306.

The type is Cuming 2437, which was from Sumatra, not from the Philippines. It is supposed to be a synonym of Cyperus stoloniferus Retz.

Cyperus tuberosus Rottb.; Miq. Fl. Ind. Bat. 3 (1856) 265.

"Luzon ?" fide Miquel 1.c. The record must be an erroneous one as the species is not known from the Philippines."

Cyperus minutiflorus Presl Rel. Haenk. 1 (1830) 351; Steud. Syn. PI. Glum. 2 (1855) 52; Miq. Fl. Ind. Bat. 3 (1856) 287; Naves Novis. App. (1882) 306; C. B. Clarke in Philip. Journ. Sci. 2 (1907) Bot. 87.

Cyperus micranthus Presl Rel. Haenk. 1 (1828) 178, non Nees.

Cyperus breviflorus Dietr. Sp. Pl. 2 (1833) 316.

Cyperus multiflorus Kunth Enum. 2 (1837) 562 err. typ.

"Hab. in insula Luzon."

Cyperus philippensis Presl Rel. Haenk. 1 (1828) 174; Steud. Syn. Pl.

Glum 2 (1855) 52; Miq. Fl. Ind. Bat. 3 (1856) 287; Naves Novis.

App. (1882) 306; C. B. Clarke in Philip. Journ. Sci. 2 (1907) Bot. 87.

"Hab. in insula Luzon." It is desirable that the types of the last two be examined as from the description I cannot place either of these satisfactorily. Both are probably referable to some of the admitted species listed above.

CyPerus exaltatus Retz.; Naves Novis. App. (1882) 304; Ceron Cat. Pl. Herb. Manila (1892) 178; C. B. Clarke in Philip. Journ. Sci. 2 (1907) Bot. 86.

Vidal's specimen on which Ceron's record is based is C. elatus Linn. fide C. B. Clarke in Philip. Journ. Sci. 2 (1907) Bot. 86. I suspect that Llanos's plant cited by Clarke as C. exaltatus Retz. is either C. radiatus Vahl or C. elatus Linn. 


\section{PYCREUS Beauvois}

PYCREUS ERAGROSTIS (Vahl) Merr. Fl. Manila (1912) 108.

Cyperus eragrostis Vahl Enum. 2 (1806) 322; Naves Novis. App. (1882) 300; Suringar Geslacht. Cyp. (1898) 65.

Pycreus sanguinolentus Nees in Linnaea 9 (1834) 283; C. B. Clarke in Philip. Journ. Sci. 2 (1907) Bot. 79.

Cyperus sanguinolentus Vahl Enum. 2 (1806) 351.

Cyperus atratus Steud. in Zoll. Verz. Ind. Archip. (1854) 62; Naves Novis. App. (1882) 301.

Luzon (Ilocos Norte, Bontoc, Benguet, Pangasinan, Rizal, Laguna, Sorsogon), Mindanao, Elmer 14336, 14323, Merrill 7354, 9792, Phil. Pl. 514, Williams 1972, B. S. 27620, 4893, 12039 Ramos. In open wet places at low and medium altitudes, ascending to $1,600 \mathrm{~m}$. Widely distributed in the warmer parts of the Old World.

PYCREUS GLoBosus (All.) Reichb. Fl. Germ. Exkurs. (1830-32) $140{ }^{10}$; C. B. Clarke in Philip. Journ. Sci. 2 (1907) Bot. 81 (incl. var. nilagiricus C. B. Clarke).

Cyperus globosus All. Fl. Pedem. Auctuar. (1789) 49; Naves Novis. App. (1882) 301; Suringar Geslacht. Cyp. (1898). 58.

Cyperus vulgaris Kunth Enum. 2 (1837) 4; Miq. Fl. Ind. Bat. 3 (1856) 256.

Cyperus lanceolatus Presl Rel. Haenk. 1 (1828) 167, non ? Poir.

Luzon (Bontoc, Lepanto, Benguet, Nueva Vizcaya), Leyte, Mindanao (Lanao), Merrill 237, 4630, Phil. Pl. 556, Elmer 6483, Williams 1242, B. S. 31810 Santos, 14261, 14257 McGregor, 5972, 8238 Ramos. In open usually wet places at medium altitudes, ascending to $2,000 \mathrm{~m}$. Warmer parts of the Old World.

Local name: Tantanud (Bon.).

PYCREUS NITENS (Retz.) Nees in Nov. Act. Acad. Nat. Cur. 19 (1843) Suppl. 1: 53; Merr. Fl. Manila (1912) 108.

Cyperus nitens Retz. Obs. 5 (1789) 13; Nees in Hook. Journ. Bot. Kew Miscel. 6 (1854) 28; Vidal Phan. Cuming. Philip. (1885) 155, Rev. Pl. Vasc. Filip. (1886) 283; Suringar Geslacht. Cyp. (1898) 55. Cyperus pumilus Linn. Amoen. Acad. 4 (1759) 302; Naves Novis. App. (1882) 300, non Pycreus pumilus Nees.

Pycreus pulvinatus Nees in Linnaea 9 (1834) 283; C. B. Clarke in Philip. Journ. Sci. 2 (1907) Bot. 79.

Cyperus gymnoleptus Steud. Syn. Pl. Glum. 2 (1855) 3; Miq. Fl. Ind. Bat. 3 (1856) 255.

Northern Luzon to Mindanao, in most or all islands and provinces. In open usually damp places at low and medium altitudes. Common in the warmer parts of the Old World; Florida (probably introduced).

PYCREUS ODORATUS (Linn.) Urban Symb. Antil. 2 (1900) 164; Merr. FI. Manila (1912) 108, Interpret. Herb. Amb. (1917) 104, Sp. Blancoanae (1918) 80.

Cyperus odoratus Linn. Sp. Pl. (1753) 46, excl. syn. Sloane.

Pycreus polystachyus Beauv. Pl. Oware et Benin 2 (1807) 48, t. 86, f. 2; C. B. Clarke in Philip. Journ. Sci. 2 (1907) Bot. 80; Merr. op. cit. 3 (1908) Bot. 397. 
Cyperus polystachyus R. Br: Prodr. (1810) 214; Miq. Fl. Ind. Bat. 3 (1856) 258; Naves Novis. App. (1882) 301.

Cyperus strigosus Llanos Frag. Pl. Filip. (1851) 16; F.-Vill. \& Naves in Blanco Fl. Filip. ed. $3,4^{1}$ (1880) 10, non Linn.

Throughout the Philippines. Common in open usually damp grasslands, fallow rice paddies, etc., at low altitudes. Pantropic.

Local names: Alúsang-pasígan (Tag.) ; haná (Iv.).

Var. PANiculatus (Rottb.) Merr. Fl. Manila (1912) 109.

Cyperus paniculatus Rottb. Descr. Ic. (1773) 40.

Pycreus polystachyus Beauv. var. laxiflorus Benth. Fl. Austral. 7

(1878) 261; C. B. Clarke in Philip. Journ. Sci. 2 (1907) Bot. 80.

Luzon (Isabela, Pampanga, Rizal), Negros. Occasional; habitat of the species.

Var. HOLOSERICEUS (Link) Merr. in Journ. Str. Branch Roy. As. Soc. 76 (1917) 79.

Cyperis holosericeus Link Hort. Berol. 1 (1827) 317.

Luzon (Zambales, Laguna), Palmas, Merrill 5343, Bolster 11, Baker 983, B. S. 22986 McGregor. In open wet places; rare and local. Borneo, its other range uncertain.

PYCREUS SULCINUX C. B. Clarke in Hook. f. FI. Brit. Ind. 6 (1893) 593; Philip. Journ. Sci. 2 (1907) Bot. 80.

Cyperus sulcinux C. B. Clarke in Journ. Linn. Soc. Bot. 21 (1884) 593 ; Suringar Geslacht. Cyp. (1898) 63.

Luzon (Benguet, Bulacan), Palawan, Mindanao (Lanao), Merrill 4289, 9278, Phil. Pl. 548, Williams 1969, Elmer 6579, B. S. 21742 Ramos. In open places, in dry or damp soil, sea level to $1,200 \mathrm{~m}$ altitude; local. Warmer parts of the Old World.

PYCREUS UNIOLOIDES ( $R$. Br.) comb. nov.

Cyperus unioloides R. Br. Prodr. (1810) 216.

Cyperus angulatus Nees in Wight Contrib. (1834) 73.

Pycreus angulatus Nees in Linnaea 9 (1834) 283.

Luzon (Bontoc, Benguet, Nueva Vizcaya), Mindanao (Lanao), Williams 1971, B. S. 14228 McGregor, 8262 Ramos. In boggy places at medium altitudes. Pantropic.

\section{DOUBTFUL AND EXCLUDED SPECIES}

Cyperus albus Presl Rel. Haenk. 1 (1828) 175; Miq. Fl. Ind. Bat. 3 (1856) 287; Steud. Syn. Pl. Glum. 2 (1855) 52; Naves Novis. App. (1882) 306; C. B. Clarke in Philip. Journ. Sci. 2 (1907) Bot. 81.

"Hab. in insula Luzon" Presl. This is a Pycreus judging from Presl's description of the styles as 2-fid. It is necessary to examine the type to determine its status.

Cyperus Luzonensis Presl Rel. Haenk. 1 (1828) 174; Steud. Syn. Pl. Glum.

2 (1855) 52; Miq. Fl. Ind. Bat. 3 (1856) 287; Naves Novis. App.

(1882) 306 ; C. B. Clarke in Philip. Journ. Sci. 2 (1907) Bot. 81.

"Hab. in insula Luzon" Presl. The type should be examined as the status of the species is hardly determinable from the description alone.

Cyperus flavescens Linn.; Naves Novis. App. (1882) 301.

Cyperus flavicomus Michx.; Naves 1. c. 


\section{MARISCUS Gaertner}

MARISCUS CYPERINUS (Retz.) Vahl Enum. 2 (1806) 377; Nees in Hook. Journ. Bot. Kew Miscel. 6 (1854) 28; C. B. Clarke in Philip. Journ. Sci. 2 (1907) Bot. 87; Merr. op. cit. 3 (1908) Bot. 398, 5 (1910) Bot. 333, Fl. Manila (1912) 113.

Kyllinga cyperinus Retz. Obs. 6 (1791) 21.

Mariscus sundaicus Miq. Fl. Ind. Bat. 3 (1856) 289.

Cyperus sundaicus Naves Novis. App. (1882) 306.

Mariscus umbellatus Moritzi Verz. Zoll. Pflanz. (1845) 98, vix Vahl. Cyperus umbellatus Naves Novis. App. (1882) 305, vix Benth.

Cyperus manilensis Boeck. in Engl. Bot. Jahrb. 5 (1884) 501.

Mariscus philippensis Steud. Syn. Pl. Glum. 2 (1855) 66; Miq. F1. Ind. Bat. 3 (1856) 290; C. B. Clarke in Philip. Journ. Sci. 2 (1907) Bot. 88.

Cyperus steudelianus Naves Novis. App. (1882) 306; Boeck. in/ Engl. Bot. Jahrb. 5 (1884) 91.

Mariscus umbellatus Presl Rel. Haenk. 1 (1828) 191, non Vahl.

Cyperus cylindrostachys Boeck. in Linnaea 36 (1869) 383, p. p.; Vidal Phan. Cuming. Philip. (1885) 155, Rev. Pl. Vasc. Fïlip. (1886) 283.

Throughout the Philippines at low and medium altitudes, in open waste places, old clearings, etc. India to Malaya and Polynesia.

Local names: Alúsang (Tag.) ; alúsang-párang (Tag.); bubuyangikat (Sub.) ; busikad-dako (C. Bis.); haná (Iv.); saka-án (Ig.) ; silal (Buk.); ubud-úbud (Tag.).

MARISCUS DILUTUS (Vahl) Nees in Wight Contrib. (1834) 90; Merr. in Philip. Journ. Sci. 7 (1912) Bot. 231, Fl. Manila (1912) 113, Sp. Blancoanae (1918) 80, 81.

Cyperus dilutus Vahl Enum. 2 (1806) 357; Nees in Hook. Journ. Bot. Kew Miscel. 6 (1854) 27; Nees in Nov. Act. Acad. Nat. Cur. 19 (1843) Suppl. 1: 65; Miq. Fl. Ind. Bat. 3 (1856) 285; Naves Novis. App. (1882) 305; Vidal Phan. Cuming. Philip. (1885) 155; Vidal Rev. Pl. Vasc. Filip. (1886) 283.

Mariscus microcephalus Presl Rel. Haenk. 1 (1828) 182; Steud. Syn. Pl. Glum. 2 (1855) 66; Miq. Fl. Ind. Bat. 3 (1856) 290; C. B. Clarke in Philip. Journ. Sci. 2 (1907) Bot. 88.

Cyperus haenkeanus Kunth Enum. 2 (1837) 93.

Cyperus quadriflorus Naves Frag. Pl. Filip. (1851) 17 (cuadriflorus); F.-Vill. \& Naves in Blanco Fl. Filip. ed. 3, $3^{1}$ (1880) 12.

Cyperus luzoniensis Llanos 1. c.; F.-Vill. \& Naves op. cit. 11.

Cyperus septatus Steud. Syn. Pl. Glum. 2 (1855) 46; Naves Novis. App. (1882) 305.

Cyperus microcephalus Naves Novis. App. (1882) 304, non R. Br.

Sphaeromariscus microcephalus Camus in Not. Syst. 1 (1910) 239.

Duvaljouvea diluta Palla in Allgem. Bot. Zeitschr. 17 (1911) Beil.

8; Kneucker Cyp. Exsic. No. 246.

Throughout the Philippines, in all or most islands and provinces. Common in open wet places at low and medium altitudes. Tropical Asia to Malaya and the Mascarene Islands.

Local names: Baki-báking-pulá (S. L. Bis.) ; durugi (Sub.); giron (Bag.) kadang-kádang (Bik.); tíkai (Tag.). 
MARISCUS MERRILLII C. B. Clarke in Philip. Journ. Sci. 2 (1907) Bot. 87.

Luzon (Cavite), Merrill 4170. On wet banks in ravines, altitude about $300 \mathrm{~m}$. Endemic.

MARISCUS NIVEUS (Murr.) Merr. in Philip. Journ. Sci. 14 (1919) $36 \%$.

Schoenus niveus Murr, in Linn. Syst. Veg. ed. 13 (1774) 81.

Scirpus glomeratus Linn. Sp. Pl: (1753) 52, excl. syn. Gronov,, non Mariscus glomeratus Barton.

Schoenus coloratus Linn. var. $\beta$ Linn. Sp. Pl. ed. 2 (1762) 64.

Cyperus kyllingiaeoides Vahl Enum. 2 (1806) 312.

Mariscus dregeanus Kunth Enum. 2 (1837) 120; C. B. Clarke in Hook.

f. Fl. Brit. Ind. 6 (1893) 620.

Cyperus irroratus Nees in Hook. Journ. Bot. Kew Miscel. 6 (1854) 28.

Luzon (Ilocos Norte), 32826 Ramos. On dry open rocky slopes at low altitudes. India to tropical Africa, the Malay Peninsula, Singapore, and Borneo.

MARISCUS PENNATUS (Lam.) comb. nov.

Cyperus pennatus Lam. Tabl. Encycl. 1 (1791) 144; Miq. Fl. Ind. Bat. 3 (1856) 281; Naves Novis. App. (1882) 304; Vidal Phan. Cuming. Philip. (1885) 155, Rev. Pl. Vasc. Filip. (1886) 283.

Cyperus stuppeus Forst. f. Prodr. (1786) 89, nomen nudum.

Mariscus stuppeus Merr. in Philip. Journ. Sci. 3 (1908) Bot. 398, Fl. Manila (1912) 113, Sp. Blancoanae (1918) 80.

Cyperus canescens Vahl Enum. 2 (1806) 355; Nees in Hook. Journ. Bot. Kew Miscel. 6 (1854) 27; Boeck. in Linnaea 36 (1869) 340; Scheff. in Nat. Tijdschr. Nederl. Ind. 34 (1874) 51.

Mariscus albescens Gaudich. Bot. Freyc. Voy. (1826) 415; C. B. Clarke in Philip. Journ. Sci, 2 (1907) Bot. 88.

Cyperus holciflorus Presl Rel. Haenk. 1 (1828) 173; Steud. Syn. Pl. Glum. 2 (1855) 43; Miq. Fl. Ind. Bat. 3 (1856) 282; Naves Novis. App. (1882) 305.

Cyperus bracteolatus Steud. Syn. Pl. Glum. 2 (1855) 49; Miq. Fl. Ind. Bat. 3 (1856) 286; Naves Novis. App. (1882) 305.

Cyperus bracteatus Vidal Phan. Cuming. Philip. (1885) 155.

Cyperus ovatus Naves Frag. P1. Filip. (1851) 15; F.-Vill. \& Naves in Blanco Fl. Filip. ed. 3, 3 (1880) 10.

Cyperus nitidulus Boeck. in Linnaea 36 (1869) 363; Naves Novis. App. (1882) 305; Scheff. in Nat. Tijdschr. Nederl. Ind. 34 (1874) 51; Vidal Phan. Cuming. Philip. (1885) 155, Rev. Pl. Vasc. Filip. (1886) 283.

Cyperıs anomalus Steud. Syn. Pl. Glum. 2 (1855) 37; Miq. Fl. Ind. Bat. 3 (1856) 277; Naves Novis. App. (1882) 305.

Cyper?s stigmatosus Steud. in Zoll. Verz. Ind. Archip. (1854) 96, Syn. Pl. Glum. 2 (1855) 38; Naves Novis. App. (1882) 305.

Along shores of tidal streams, inner borders of mangrove swamps, etc., within the influence of salt or brackish water throughout the Philippines. Tropical Africa to southeastern Asia, Malaya, and Polynesia.

Local names: Kai-kai (Mbo.); obod-óbod (Ibn.); tuhog-dalág (Tag.). $183036-8$ 
MARISCUS SIEBERIANUS Nees in Linnaea 9 (1834) 286; C. B. Clarke in Philip. Journ. Sci. 2 (1907) Bot. 88.

Northern Luzon to Palawan and Mindanao, in most or all islands and provinces. In old clearings, open grasslands, etc., at low and medium altitudes. Pantropic; considered by Kükenthal to be a variety of $M$. umbellatus Vahl.

Local names: Kupiúpi (Sub.) ; okokiang (Bon.) ; mañgilang (Sub.).

MARISCUS TENUIFOLIUS Nees in Mart. F1. Brazil $2^{1}$ (1843) 46; C. B.

Clarke in Philip. Journ. Sci. 2 (1907) Bot. 87.

Mariscus flabelliformis C. B. Clarke in Philip. Journ. Sci. 2 (1907)

Bot. 89; Merr. Fl. Manila (1912) 113, non ? HBK.

Northern Luzon to Palawan and Mindanao. Scattered in thickets and secondary forests at low and medium altitudes. India to the Malay Peninsula.

Local name: Bakis-bakísan (Tag.).

\section{JUNCELLUS C. B. Clarke}

JUNCELLUS PYGMAEUS (Rottb.) C. B. Clarke in Hook. f. Fl. Brit. Ind. 6 (1893) 596, Philip. Journ. Sci. 2 (1907) Bot. 81; Merr. Fl. Manila (1912) 109.

Cyperus pygmaeus Rottb. Descr. Ic. (1773) 20, t. 14, f. 4, 5; Miq. Fl. Ind. Bat. 3 (1856) 261; Naves Novis. App. (1882) 301.

Dichelostylis pygmaea Nees in Linnaea 9 (1834) 289; Kneucker Cyp. Exsic. No. 248.

Luzon (Cagayan, Rizal, Laguna), Mindanao (Lanao), Merrill Phil. Pl. 509, B S. 22984, 2684 Ramos, 22714 Castillo. In open wet places, river banks, etc., at low and medium altitudes. Africa, India to Korea southward to Australia.

\section{TORULINIUM DesvauX}

TORULINIUM FERAX (L. C. Rich.) Ham. Prodr. Pl. Ind. Occ. (1825) 15 (ferox) ; Merr. Fl. Manila (1912) 114.

Cyperus ferax L. C. Rich. in Act. Soc. Hist. Nat. Paris 1 (1792) 106; Naves Novis. App. (1882) 305; Vidal Phan. Cuming. Philip. (1885) 15อ̃, Rev. Pl. Vasc. Filip. (1886) 283.

Torulinium confertum Desv. ex Ham. Prodr. Pl. Ind. Occ. (1825) 15; Usteri Beitr. Ken. Philip. Veg. (1905) 131; C. B. Clarke in Philip. Journ. Sci. 2 (1907) Bot. 89.

Diclidium elatum Nees in Hook. Journ. Bot. Kew Miscel. 6 (1854) 27. Cyperus calopterus Miq. F1. Ind. Bat. 3 (1856) 282; Naves Novis. App. (1882) 305.

Cyperus holophyllus Miq. op. cit. 283; Naves 1. c.

Mariscus ferax C. B. Clarke in Hook. f. Fl. Brit. Ind. 6 (1893) 624. Cyperus haenkei Presl Rel. Haenk. 1 (1828) 172.

Throughout the Philippines. In open wet places at low and medium altitudes. Pantropic.

Local names: Biliran (S. L. Bis.); pulakgalau (Sub.).

\section{KYLLINGA Rottboell}

KYLLINGA BREVIFOLIA Rottb. Descr. Ic. (1773) $13, t$. 4, f. 3 ; Naves Novis. App. (1882) 300; Nees in Hook. Journ. Bot. Kew Miscel. 6 (1854) 28; C. B. Clarke in Philip. Journ. Sci. 2 (1907) Bot. 78; Merr. Fl. Manila (1912) 107.

Kyllinga rigidula Steud. Syn. Pl. Glum. 2 (1855) 71; Miq. Fl. Ind. Bat. 3 (1856) 294; Naves Novis. App. (1882) 300, quoad pl. Philip. 
Kyllinga caespitosa Nees var. robusta Boeck. in Linnaea 35 (1868) 413; Scheff. in Nat. Tijdschr. Nederl. Ind. 34 (1874) 45; Vidal Phan. Cuming. Philip. (1885) 155, Rev. Pl. Vasc. Filip. (1886) 283. Kyllinga longiculmis Miq. Fl. Ind. Bat. 3 (1856) 292; Naves Novis. App. (1882) 300.

Kyllinga fuscata Miq. 1. c. 294; Naves 1. c.

Kyllinga squarrosa Steud. Syn. Pl. Glum. 2 (1855) 68; Naves 1. c. Kyllinga sororia Kunth Enum. 2 (1837) 131; Naves 1. c.

Throughout the Philippines in open grasslands, waste places, etc., at low and medium altitudes; common. Pantropic.

Local names: Bibi-inok (Bon.); kadkadot (Ig.) ; pugo-púgo"(C. Bis.).

KYLLINGA CYLINDRICA Nees in Wight Contrib. (1834) 91; C. B. Clarke in Philip. Journ. Sci. 2 (1907) Bot. 79.

Luzon (Benguet, Laguna), Mindanao (Bukidnon, Lanao), Elmer 6500, Merrill Phil. Pl. 571, Williams 1225, B. S. 26039 Fénix, 14033 Robinson, 10980 Ramos. In open grasslands at medium altitudes, ascending to $1,600 \mathrm{~m}$ Warmer parts of Africa and Asia, through Malaya to Australia. Considered by Kükenthal to be a variety of $K$. odorata Vahl.

KYLLINGA INTERMEDIA R. Br. Prodr. (1810) 219; C. B. Clarke in Philip. Journ. Sci. 2 (1907) Bot. 78; Merr. op. cit. 262, 5 (1910) Bot. 333.

Kyllinga oligostachya Boeck. in Linnaea 35 (1868) 407.

Luzon (Ifugao, Bontoc, Lepanto, Benguet, Nueva Vizcaya, Laguna). Mindoro, Negros, Mindanao, B. S. 14249 McGregor, 14125 Robinson, Elmer 6494, 10021, 9675, Merrill 6589, 4618, 4624, 4871, 4421, 4695, Phil. Pl. 539; Williams 1228. In open wet places, on slopes, about cliffs, etc., at medium altitudes, ascending to 2,200 m. Formosa, Australia, Samoa. Considered by Kükenthal to be a variety of $K$. brevifolia Rottb.

Local names: Busíkad (C. Bis.); tabtabúlog (Buk.).

KYLLINGA MELANOSPERMA Nees in Wight Contrib. (1834) 91; C. B. Clarke in Hook. f. Fl. Brit. Ind. 6 (1893) 588.

Kyllinga pungens C. B. Clarke in Philip. Journ. Sci. 2 (1907) Bot. 78 , non Link.

Kyllinga bifolia Miq. Fl. Ind. Bat. 3 (1856) 293; Naves Novis. App. (1882) 300.

Luzon (Ilocos Norte, Ifugao, Kalinga, Benguet, Nueva Vizcaya), Mindanao (Lanao), B. S. 27159 Ramos, 14296, 19995 McGregor, Merrill 216, 4649, Phil. Pl. 1761, 572, Williams 1235, Elmer 6292, 6495. In open wet places at medium altitudes, ascending to $2,000 \mathrm{~m}$. India to Madagascar and Malaya.

KYLLINGA MONOCEPHALA Rottb. Descr. Ic. (1773) 13, t. 4, f. 4; Miq. Fl. Ind. Bat. 3 (1856) 291; Naves Novis. App. (1882) 199; C. B. Clarke in Philip. Journ. Sci. 2 (1907) Bot. 77; Merr. op. cit. 3 (1908) Bot. 397, F1. Manila (1912) 108, Sp. Blancoanae (1918) 81. Kyllinga triceps Blanco Fl. Filip. (1837) 34, ed. 2 (1845) 23, ed. 3, 1 (1877) 44; Steud. Syn. Pl. Glum. 2 (1855) 72; Naves Novis. App. (1882) 300 (Linn. f., p. p.). 
Kyllinga mindorensis Steud. Syn. Pl. Glum. 2 (1855) 67; Miq. Fl. Ind. Bat. 3 (1856) 292; Naves Novis. App. (1882) 300.

Kyllinga monocephala Rottb. var. mindorensis Boeck. in Linnaea 35 (1868) 424; Vidal Phan. Cuming. Philip. (1885) 155, Rev. Pl. Vasc. Filip. (1886) 284.

Kyllinga gracilis Kunth Enum. 2 (1837) 134; Nees in Hook. Journ.

Bot. Kew Miscel. 6 (1854) 28; Naves Novis. App. (1882) 300.

Throughout the Philippines. Common in waste places, open grasslands, etc., at low and medium altitudes. Pantropic.

Local names: Anúang (Tag.); barubotónes (Bis.); bolobotónes (Bis.) ;

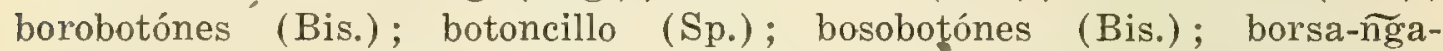
dadakkel (Ilk.) ; baki-báki (S. L. Bis.) ; bosíkad (C. Bis.) ; boskad (Bis.) ; busíkad (P. Bis.) ; katutu (Mag.) ; kurukamóting-orig" (Bik.) ; malabotónes (Bis.) ; muthá (Tag.) ; mustra (Tag.) ; puñós (S. L. Bis.) ; sangsangítan (Bon.) ; sud-sud (Bis.); uli-uli (Bag.).

\section{EXCLUDED SPECIES}

KYlinga Pumila Steud.; Naves Novis. App. (1882) 300.

\section{FUIRENA Rottboell}

FUIRENA CILIARIS (Linn.) Roxb. Hort. Beng. (1814) 81, Fl. Ind. 1 (1820) 184; Merr. Fl. Manila (1912) 119, Sp. Blancoanae (1918) 81.

Scirpus ciliaris Linn. Mant. 2 (1771) 182.

Fuirena glomerata Lam. Tabl. Encycl. 1 (1791) 150; F.-Vill. Novis. App. (1882) 309; C. B. Clarke in Philip. Journ. Sci. 2 (1907) Bot. 101.

Fuirena striata Llanos Frag. Pl. Filip. (1851) 21; F.-Vill. \& Naves in Blanco Fl. Filip. ed. 3, $4^{\mathrm{I}}$ (1880) 14.

Throughout the Philippines, in rice paddies, open wet lands, etc., at low and medium altitudes, often abundant. Old World Tropics generally.

Local name: Pugápuk (Tag.).

FUIRENA UMBELLATA Rottb. Descr. Ic. (1773) 70, t. 19, f. 3; F.-Vill. Novis. App. (1882) 308; Vidal Phan. Cuming. Philip. (1885) 156, Rev. Pl. Vasc. Filip. (1886) 284; C. B. Clarke in Philip. Journ. Sci. 2 (1907) Bot. 101.

Fuirena paniculata Lam. Tabl. Encycl. 1 (1791) 150, t. 39.

Fuirena pentagona Nees in Wight Contrib. (1834) 93, Hook. Journ. Bot. Kew Miscel. 6 (1854) 28.

Throughout the Philippines in open wet lands at low and medium altitudes. Pantropic.

\section{SCIRPUS Linnaeus}

SCIRPUS ARTICULATUS Linn. Sp. Pl. (1753) 47; Rolfe in Journ. Bot. 23 (1885) 216; Ceron Cat. Pl. Herb. Manila (1892) 178; Clarke in Philip. Journ. Sci. 2 (1907) Bot. 99; Merr. Fl. Manila (1912) 118, Sp. Blancoanae (1918). 81.

Carex glomerata Blanco Fl. Filip. ed. 2 (1845) 24, ed. 3, 1 (1877) 45; Merr. in Govt. Lab. Publ. (Philip.) 27 (1905) 90, non Thunb. Scirpus incurvatus Roxb. Fl. Ind. 1 (1820) 216; Llanos ex F.-Vill. \& Naves in Blanco Fl. Filip. ed. 3, $4^{1}$ (1880) 105. 
Luzon (Cagayan, Ilocos Norte, Pangasinan, Nueva Ecija, Rizal, Laguna), Merrill Sp. Blancoanae 268, Phil. Pl. 51:3, B. S. 2295, 2298 Mearns, 4949, 7447 Ramos. In open wet places in settled areas at low altitudes. Old World Tropics generally.

Local name: Apúrau (Pang.).

SCIRPUS ERECTUS Poir. in Lam. Encycl. 6 (1804) 761; Usteri Beitr. Ken. Philip. Veg. (1905) 132; C. B. Clarke in Philip. Journ. Sci. 2 (1907) Bot. 99; Merr. Fl. Manila (1912) 118.

Scirpus juncoides Roxb. Fl. Ind. 1 (1820) 216; Ceron Cat. Pl. Herb. Manila (1892) 178.

Scirpus luzonensis Presl Rel. Haenk. 1 (1828) 193; Nees in Nov. Act. Acad. Nat. Cur. 19 (1843) Suppl. 1: 93; Steud. Syn. Pl. Glum. 2 (1855) 84; Miq. Fl. Ind. Bat. 3 (1856) 304.

Luzon (Ifugao, Bontoc, Benguet, Nueva Vizcaya, Rizal, Bulacan, Sorsogon), Polillo, Leyte, Biliran, Mindanao. In open wet places, rice paddies, etc., at low and medium altitudes. Warmer parts of both hemispheres.

Local names: Bitubituínan (Tag.) ; gúmi (Tag.) ; paratupit (Ilk.).

SCIRPUS GROSSUS Linn. f. Suppl. (1781) 104; Presl Rel. Haenk. 1 (1828) 195; Miq. Fl. Ind. Bat. 3 (1856) 307; F.-Vill. Novis. App. (1882) 308; Merr. Fl. Manila (1912) 117, Sp. Blancoanae (1918) 82.

Cyperus difformis Blanco Fl. Filip. (1837) 32, ed. 2 (1845) 22, ed. 3, 1 (1877) 41, Merr. in Govt. Lab. Publ.' (Philip.) 27 (1905) 90, non Linn.

Scirpus kysoor Roxb. Hort. Beng. (1814) 6; Llanos Frag. Pl. Filip. (1851) 20 (kisoor) ; F.-Vill. \& Naves in Blanco Fl. Filip. ed. 3, $4^{2}$ (1880) 14 (kisoor).

Scirpus grossus Linn. f. var. kysoor (Roxb.) C. B. Clarke in Hook. f. Fl. Brit. Ind. 6 (1893) 660, Philip. Journ. Sci. 2 (1907) Bot. 100. Schoenoplectus grossus Palla in Allgem. Bot. Zeitschr. 17-(1911) Beil. 3.

Central Luzon to Mindanao, in most islands and provinces, Merrill Sp. Blancoanae 564, 692, Phil. Pl. 515, Kneucker Cyp. Exsic. 220. In freshwater swamps at low altitudes. India to Indo-China and Malaya.

Local names: Agas (Bik.); baga-ás (P. Bis.); baki-báki. (P. Bis.) ; balakbák (Pang.) ; balañgót (P. Bis.); bangkúang (Bik.); ragiudíu (Bik.); tikúg (Mbo.); tíkiu (Tag.); títiu (Tag.).

SCIRPUS LACUSTRIS Linn. Sp. P1. (1753) 48; Merr. in Philip. Journ. Sci. 5 (1910) Bot. 172.

Luzon (Càgayan, Zambales, Benguet), F. B. 17g90 Curran, IIerrill Phil. Pl. 1792. In fresh-water swamps, sea level to $1,300 \mathrm{~m}$ altitude. In most warm countries except Malaya and South America.

Local name: Tiker (Ilk.).

SCIRPUS MERRILLII (Palla) Kükenth. in herb. comb. nov.

Schoenoplectus merrillii Palla in Allgem. Bot. Zeitschr. 17 (1911) Beil. 3.

Cyperus tenellus C. B. Clarke in Philip. Journ. Sci. 2 (1907) Bot. 81, non Linn. f.

Scirpus inundatus C. B. Clarke op. cit. 99, non Poir. 
Luzon (Bontoc, Benguet), Negros, Mindanao (Davao), Merrill $4 r_{40}$, 6639, 7680, Phil. Pl. 511, Kneucker Cyp. Exsic. 223, Elmer 11379a, B. S. 31748 Santos, 8331 McGregor. In open wet places on the higher mountains, altitude 1,600 to $2,700 \mathrm{~m}$. Endemic.

Local name: Salaisoi (Bag.).

SCIRPUS. MUCRONATUS Linn. Sp. Pl. (1753) 50; F.-Vill. Novis. App. (1882) 308; C. B. Clarke in Philip. Journ. Sci. 2 (1907) Bot. 100; Merr. Fl. Manila (1912) 118.

Scirpus acutus Presl Rel. Haenk. 1 (1828) 192, non Muhl.

Scirpus preslii Dietr. Sp. Pl. 2 (1833) 175 (presslii); Steud. Syn.

Pl. Glum. 2 (1855) 84; Miq. Fl. Ind. Bat. 3 (1856) 305; F.-Vill.

Novis. App. (1882) 308 (preslei).

Northern Luzon to Mindanao, in most islands and provinces. In open wet places at low and medium altitudes, ascending to $1,500 \mathrm{~m}$. Warmer parts of the Old World.

Local names: Kanubsúban (Tag.); libíran (Tag.); parapipit (Ilk.) ; pulutapit (Bon.); pupuegan (Bon.).

SCIRPUS PULOGENSIS Merr. in Philip. Journ. Sci. 5 (1910) Bot. 333.

Luzon (Benguet), Merrill 7789, 6616, 6550, B. S. 31750 Santos, F. B. 16134 Curran, Merritt, \& $Z$ schokke. In very damp ravines and on open grassy slopes on the highest mountains, altitude 2,400 to 2,700 m. Endemic.

SCIRPUS SUPINUS Linn. Sp. Pl. (1753) 49; F.-Vill. Novis. App. (1882) 308; C. B. Clarke in Philip. Journ. Sci. 2 (1907) Bot. 99 (incl. var. uninodis C. B. Clarke); Merr. Fl. Manila (1912) 118.

Luzon (Ilocos Norte, Rizal), Merrill 3654, Phil. Pl. 504, B. S. 7654, 21970 Ramos. In open wet places, rice paddies, etc., at low altitudes. Europe to southern Africa and Australia.

SCIRPUS TERNATANUS Reinw. ex Miq. Fl. Ind. Bat. 3 (1856) 307; C. B. Clarke in Philip. Journ. Sci. 2 (1907) Bot. 100 (ternatensis).

Scirpus chinensis Munro in Seem Bot. Voy. Herald (1857) 423.

Luzon (Ifugao, Kalinga, Benguet, Nueva Vizcaya, Rizal, Tayabas), Mindanao (Davao, Bukidnon, Zamboanga), B. S. 31934 Santos, 8173, 26750 Ramo8, 29113, 37489, 3919.2 Ramos \& Edaño, 19737 McGregor, Merrill 2315 , Phil. Pl. 559, Williams 1249, Elmer 6287, 5790. In open wet places and also in thickets chiefly at medium altitudes, ascending to $2,200 \mathrm{~m}$. India to China and the Riu Kiu Islands, Celebes, and the Moluccas.

Local names: Daátan (Tag.); sagari (Bag.); sañgaña (Klg.); siilak (Ig.).

SCIRPUS TRIQUETER Linn. Mant. 1 (1767) 29; C. B. Clarke in Philip.

Journ. Sci. 2 (1907), Bot. 100 (var. segregata C. B. Clarke).

Luzon, (Loher 802), fide C. B. Clarke. I have seen no specimens of this species. Europe to Japan, also in South Africa and New Guinea.

EXCLUDED SPECIES

ScirPus FluCtans Linn.; F.-Vill. Novis. App. (1882) 308.

SCIRPUS MARITIMUS Linn.; F.-Vill. 1. c.

ScIRPUS MAXimus Linn.; F.-Vill. 1. c. 


\section{ELEOCHARIS R. Brown}

(Heledcharis auct.)

ELEOCHARIS ACICULARIS (Linn.) R. \& S. Syst. 2 (1817) 154; Merr. in Philip. Journ. Sci. 9 (1914) Bot. 264.

Scirpus acicularis Linn. Sp. Pl. (1753) 48.

Luzon (Bontoc, Benguet), Merrill 7665, Vanoverbergh 293. About rice paddies, altitude 1,200 to $1,500 \mathrm{~m}$. North Temperate Zone generally, extending southward to southern China.

ELEOCHARIS AFFLATA Steud. in Zoll. Verz. Ind. Archip. (1854) 62; Syn. Pl. Glum. 2 (1855) 76; Naves Novis. App. (1882) 307; C. B. Clarke in Philip. Journ. Sci. 2 (1907) Bot. 90; Merr. op. cit. 3 (1908) Bot. 398.

Eleocharis microcarpa C. B. Clarke in Philip. Journ. Sci. 2 (1908) Bot. 91, non Torr.

? Eleocharis pellucida Presl Rel. Haenk. 1 (1828) 196; Steud. Syn. Pl. Glum. 2 (1855) 80; Miq. Fl. Ind. Bat. 3 (1856) 301; Naves Novis. App. (1882) 307 (Heleocharis).

Eleocharis subprolifera Steud. in Zoll. Verz. Ind. Archip. (1854) 62; Naves Novis. App. (1882) 307 (Heleocharis).

Babuyan Islands, Luzon (Bontoc, Lepanto, Benguet), Elmer 6299, 5751, Williams 1230, 1243, 1247, Merrill 4621, 7703, Phil. Pl. 555. In shallow water, swampy places, rice paddies, etc., altitude 1,000 to $1,600 \mathrm{~m}$. Japan to China and India, southward to Borneo.

ELEOCHARIS ATROPURPUREA (Retz.) Presl Rel. Haenk. 1 (1828) 196; Kunth Enum. 2 (1837) 151; C. B. Clarke in Philip. Journ. . Sci. 2 (1907) Bot. 90.

Scirpus atropurpureus Retz. Obs. 5 (1789) 14.

Admitted on the authority of C. B. Clarke. I have seen no Philippine material representing the species. Warmer parts of the world.

ELEOCHARIS CARIBAEA (Rottb.) Blake in Rhodora 20 (1918) 24; Merr. Sp. Blancoanae (1918) 83.

Scirpus caribaeus Rottb. Descr. Pl. Rar. Progr. (1772) 24, Descr. Ic. Nov. Pl. (1772) 46, t. 24.

Eleacharis capitata Miq. Fl. Ind. Bat. 3 (1856) 299; Naves Novis. App. (1882) 307; C. B. Clarke in Philip. Journ. Sci. 2 (1907) Bot. 90 ; Merr. Fl. Manila (1912) 114, non R. Br.

Eleocharis retroflexus Llanos Frag. Pl. Filip. (1851) 19; F.-Vill. \&

Naves in Blanco Fl. Filip. ed. 3, $4^{1}$ (1880) 13, non Poir.

Luzon (Cagayan, Ilocos Norte, Bataan, Rizal, Laguna), Cebu. Common in open wet lands at low altitudes. Pantropic.

Blake has shown that the type of Scirpus capitatus Linn. is identical with the plant commonly known as Eleocharis tenuis (Schultes) Willd., which should henceforth bear the name Eleocharis capitata (Linn.) R. Br.

ELEOCHARIS DULCIS (Burm. f.) Trin. ex Henschel Vita Rumph. (1833) 186; Merr. Interpret. Herb. Amb. (1917) 104, Sp. Blancoanae (1918) 82.

Andropogon dulcis Burm. f. Fl. Ind. (1768) 219.

Hippuris indica Lour. Fl. Cochinch. (1790) 16. 
Eleocharis plantaginea R. Br. Prodr. (1810) 224; Naves Novis. App. (1882) 306.

Carex tuberosa Blanco Fl. Filip. (1837) 35, ed. 2 (1845) 24, ed. 3, 1 (1877) 45, t. 15, non Degl.

Scirpus plantaginoides Rottb. Descr. Nov. Pl. (1773) 45.

Scirpus plantagineus Retz. Obs. 5 (1789) 14.

Eleocharis plantaginoidea W. F. Wight in Contrib. U. S. Nat. Herb. 9 (1905) 268.

Eleocharis tuberosa Naves Novis. App. (1882) 306, non ? Schultes. Eleocharis tumida R. \& S. Syst. Mant.'2 (1824) 86; Naves Novis.

App. (1882) 306.

Luzon (Cagayan, Bulacan, Laguna, Camarines), Negros, B. S. 7448 Pamos, 12332 McGregor, Merrill. Sp. Blancoanae 395, Phil. Pl. 521. In cpen wet lands, shallow swamps, etc., at low altitudes. India to Malaya.

Local names: Apúlid (Tag., Bik.); cabezas de negrito (Sp.) ; kalañüub (C. Bis) ; potok (Tag.).

ELEOCHARIS EQUISETINA Presl Rel. Haenk. 1 (1828) 195; Steud. Syn.

Pl. Glum. 2 (1855) 82; Miq. Fl. Ind. Bat. 3 (1856) 302; Navés Novis. App. (1882) 306; C. B. Clarke in Philip. Journ. Sci. 2 (1907)

Bot. 89; Merr. Fl. Manila (1912) 114.

Heleocharis plantaginea Vidal Phan. Cuming. Philip. (1885) 156,

Rev. Pl. Vasc. Filip. (1886) 284, non R. Br.

Luzon (Rizal, Sorsogon), Mindanao (Lanao), Elmer 14341, Kneucker Cyp. Exsic. 224, Merrill Phil. Pl. 531. In open wet lands at low altitudes. Ceylon to Madagascar, Malaya, and New Caledonia.

ELEOCHARIS FISTUlosa (Poir.) Schultes fo in Roem. \& Schultes Syst. Mant. 2 (1824) 89; C. B. Clarke in Hook. f. Fl. Brit. Ind. 6 (1893) 626; Naves Novis. App. (1882) 306; Camus in Lecomte Fl. Gén. Indo-Chine 7 (1912) 84, f. 13, 3, 4.

Scirpus fistulosus Poir. in Lam. Encycl. 6 (1804) 749.

Eleocharis planiculmis Steud. Syn. Pl. Glum. 2 (1855) 80; Naves Novis. App. (1882) 306.

Luzon (Rizal), Mindanao (Davao, Lanao), Merrill 9y90, Copeland 441. In open wet places at low altitudes. Pantropic.

ELEOCHARIS OCHREATA Nees in Linnaea 9 (1834) 294; C. B. Clarke in Philip. Journ. Sci. 2 (1907) Bot. 90.

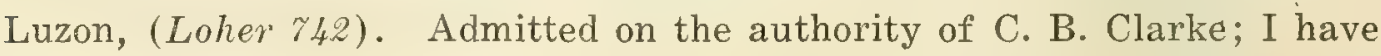
seen no specimens. Pantropic.

ELEOCHARIS RETROFLEXA (Poir.) Urban Symb. Antil. 2 (1900) 165 (Heleocharis).

Scirpus retroflexus Poir. in Lam. Encycl. 4 (1804) 753.

Eleocharis chaetaria R. \& S. Syst. 2 (1817) 154; Naves Novis. App. (1882) 307; C. B. Clarke in Philip. Journ. Sci. 2 (1907) Bot. 90.

Luzon (Laguna, Sorsogon), Polillo, Negros, Mindanao, Elmer 14558, Clemens 845, F. B. 19276 Curran, B. S. 9029 Robinson, 10049 Ramos. Along streams and in rice paddies at low and medium altitudes. Pantropic. 
ELEOCHARIS SPIRALIS (Rottb.) Steud. Syn. Pl. Glum. 2 (1855) 81; C. B. Clarke in Hook. f. Fl. Brit. Ind. 6 (1893) 627; Merr. in Philip. Journ. Sci. 14 (1919) 370.

Scirpus spiralis Rottb. Descr. Ic. (1772) $45, t .15, f .1$.

Luzon (Rizal), Merrill 9788. In open wet lands at low altitudes. India to Ceylon, Burma, and Indo-China.

ELEOCHARIS TETRAQUETRA Nees in Wight Contrib. (1834) 113; Naves Novis. App. (1882) 307.

Luzon (Bontoc, Benguet), Mindanao (Lanao), Merrill Phil. Pl. 5.5, 1\% 6, Clemens 1105, Vanoverbergh 461. In swamps, altitude 650 to $1,800 \mathrm{~m}$. India to China and tropical Australia.

ELEOCHARIS VARIEGATA Presl Oken Isis 21 (1828) 269, var. LAXIFLORA (Thw.) C. B. Clarke in Hook. f. F1. Brit. Ind. 6 (1893) 626, Philip. Journ. Sci. 2 (1907) Bot. 90; Merr: Fl. Manila (1912) 114.

Luzon (Buiacan, Rizal, Camarines), Biliran, Mindanao, Ierrill Phil. Pl. 520, 1461, B. S. 33928 Ramos \& Edaño, 18515 McGregor, 26129 Fénix, 1112 Ramos. In open wet places at low altitudes. India to Madagascar, China, Malaya, and Polynesia.

\section{FIMBRISTYLIS Vahl}

FIMBRISTYLIS ACUMINATA Vahl Enum. 2 (1806) 285; Nees in Hook. Journ. Bot. Kew Miscel. 6 (1854) 29; Naves Novis. App. (1882) 307; C. B. Clarke in Philip. Journ. Sci. 2 (1907) Bot. 91; Merr: Fl. Manila (1912) 115.

Luzon (Bulacan, Rizal, Zambales, Albay, Sorsogon), Merrill 9798, 7355, Plit. Pl. 546, Kneucker Cyp. Exsic. 2334, Elmer 14322. In open wet places, cld rice paddies, etc, at low and medium altitudes. Tropical Asia, Malaya, and tropical Australia.

Local names: Puyóng-usá (Tag.); surusibúyas (Bik.).

FIMBRISTYLIS AESTIVALIS (Retz.) Vahl Enum. 2 (1806) 288; F.-Vill. Novis. App. (1882) 308; C. B. Clarke in Philip. Journ. Sci. 2 (1907) Bot. 94.

Scirpus aestivalis Retz. Obs. 4 (1786) 12.

Fimbristylis dichotoma Presl Rel. Haenk. 1 (1828) 191, non Vahl.

Luzon (Cagayan, Isabela, Bontoc, Benguet, Pangasinan, Pampanga, Rizal), Mindanao (Lanao), Merrill 1465, 4292, Phil. Pl. 554, Elmer 60ro, B. S. 17847 Otañes, 7473, 8066, 3358, 5971 Ramos. In open damp places, rice paddies, etc., at low and medium altitudes.

Var. MACROSTACHYA Benth. Fl. Austral. 7 (1878) 310; C. B. Clarke in Philip. Journ. Sci. 2 (1907) Bot. 94.

Leyte, (Jagor 1008). The species India to Amurland southward to Australia, the variety in Australia.

Local names: Boboók (Bon.) lamlamsit (Bon.); sirau-sirau (Ilk.).

FIMBRISTYLIS ANNUA (All.) R. \& S. Syst. 2 (1817) 95.

Scirpus annuus All. Fl. Pedem. 2 (1785) 277.

Scirpus diphyllus Retz. Obs. 5 (1789) 15.

Fimbristylis diphylla Vahl Enum. 2 (1806) 289; F.-Vill. Novis. App. (1882) 307, Vidal Rev. Pl. Vasc. Filip. (1886) 284; Merr. in Philip. Journ. Sci. 1 (1906) Suppl. 30, 3 (1908) Bot. 398, Fl. Manila (1912) 116; C. B. Clarke in Philip. Journ. Sci. 2 (1907) Bot. 93. 
Fimbristylis tomentosa Vahl Enum. 2 (1806) 290; Nees in Nov. Act. Acad. Nat. Cur. 19 (1843) Suppl. 1: 81.

Fimbristylis brizoides 'Sm. var., Nees in Hook. Journ. Bot. Kew Miscel. 6 (1854) 28.

Fimbristylis philippica Steud. Syn. Pl. Glum. 2 (1855) 116; Miq. Fl. Ind. Bat. 3 (1856) 324; F.-Vill. Novis. App. (1882) 308.

Fimbristylis communis Kunth Enum. 2 (1837) 234; Miq. F1. Ind. Bat. 3 (1856) 323.

Fimbristylis affinis Presl Rel. Haenk. 1 (1828) 191; Kunth Enum. 2 (1837) 234; F.-Vill. Novis. App. (1882) 308.

? Scirpus falcatus Llanos Frag. Pl. Filip. (1851) 20; F.-Vill. \& Naves in Blanco Fl. Filip. ed. 3, $4^{1}$ (1880) 13; Merr. Sp. Blancoanae (1918) 83.

Fimbristylis dichotoma C. B. Clarke in Philip. Journ. Sci. 2 (1907) Bot. 93, non Vahl.

Throughout the Philippines in open waste places at low and medium altitudes, abundant and variable; in the Philippines various forms or varieties occur that have been characterized as species, such as $F$. podocarpa Nees, $F$. tomentosa Vahl, $F$. diphylla Vahl, $F$. calocarpa Steud., and $F$. royeniana Nees. Pantropic.

Local names: Baliótas (Bag.); bubáging (Sub.) ; gilal (Sub.) ; tabtábin (Sbl.) ; talágig (Bag.); tayok-táyok (Sbl.).

FIMBRISTYLIS CAPITULIFERA Merr. in Philip. Journ. Sci. 9 (1914) Bot. 265.

Batan and Babuyan Islands, B. S. 4042, 3575, 3926 Fénix, 3172, 3171, 3173 Mearns, 10203 McGregor. In open places, usually near the sea. Endemic.

FIMBRISTYLIS COMPLANATA (Retz.) Link. Hort. Berol. 1 (1827) 292; Usteri Beitr. Ken. Philip. Veg. (1905) 131 (var. kraussiana Hochst.) ; C. B. Clarke in Philip. Journ. Sci. 2 (1907) Bot. 96.

Scirpus complanatus Retz. Obs. 5 (1789) 14.

Trichelostylis complanata Nees in Hook. Journ. Bot. Kew Miscel. 6 (1854) 29.

Fimbristylis amblyphylla Steud. Syn. Pl. Glum. 2 (1855) 117; Miq. Fl. Ind. Bat. 3 (1856) 324; F.-Vill. Novis. App. (1882) 308.

Fimbristylis autumnalis Vidal Phan. Cuming. Philip. (1885) 156, Rev. Pl. Vasc. Filip. (1886) 284, non R. \& S.

Isolepis willdenowii R. \& S. Syst. 2 (1817) 320 ; Presl Rel. Haenk. 1 (1828) 189.

Luzon (Ilocos Norte, Bontoc, Pampanga, Rizal, Laguna, Camarines, Sorsogon), Mindoro, Palawan, Negros, Panay, Mindanao, Merrill Phil. Pl. 1425, Elmer 18096, 14324, B. S. 31445, 30941, 33500 Ramos \& Edaño, 27619, 32956 Ramos. In open wet places at low and medium altitudes. All warm countries.

Local name: Baki-báki (P. Bis.).

FIMBRISTYLIS CORNICULATA Merr. in Philip. Journ. Sci. 7 (1912) Bot. 231, Fl. Manila (1912) 116.

Luzon (Rizal), Merrill 7359. In open sterile grasslands at low altitudes. Endemic. 
FIMBRISTYLIS DIPSACEA (Rottb.) Benth. ex C. B. Clarke in Hook. $\mathfrak{l}$ Fl. Brit. Ind. 6 (1893) 635, Philip. Journ. Sci. 2 (1907) Bot. 93; Merr. Fl. Manila (1912) 116.

Scirpus dipsaceus Rottb. Descr. Ic. (1773) 56, t. 12, f. 1.

Echinolytrum dipsaceum Desv. Joum. Bot. 1 (1808) 21, t. 1.

Luzon (Rizal, Laguna), Mindanao (Lanao), B. S. 3321 Ramos, 22985 McGregor, Merrill 5105. On muddy lake and stream margins. Tropical Africa and Asia north to the Amur region.

FIMBRISTYLIS FERRUGINEA (Linn.) Vahl Enum. 2 (1806) 291; F.-Vill.

Novis. App. (1882) 307; Vidal Phan. Cuming. Philip. (1885) 156, Rev. Pl. Vasc. Filip. (1886) 284; C. B. Clarke in Philip. Journ. Sci. 2 (1907) Bot. 94; Merr. op. cit. 1 (1906) Suppl. 30, Fl. Manila (1912) 94.

Scirpus ferrugineus Linn. Sp. Pl. (1753) 50.

Widely distributed in the Philippines in open wet lands, especially those subject to the influence of brackish water, Kneucker Cyp. Exsic. 196a, Merrill 546, 4252, Phil. Pl. 549. Pantropic.

FIMBRYSTYLIS FUSCA (Nees) Benth. ex C. B. Clarke in Hook. f. Fl. Brit. Ind. 6 (1893) 649, Philip. Journ. Sci. 2 (1907) Bot. 98.

Abildgaardia fusca Nees in Wight Contrib. (1834) 95.

Gussonea pauciflora Brongn. in Bot. Duperr. Voy. 2 (1829) 171, t. 34, $B$, non Fimbristylis pauciflora R. Br.

Abildgaardia pauciflora Kunth Enum. 2 (1837) 298.

Cladium cyperoides Merr. in Philip. Journ. Sci. 7 (1912) Bot. 74.

Luzon (Cagayan, Bontoc, Ilocos Norte, Rizal), Palawan, Merrill 2785, 9389, Vanoverbergh 273, B. S. 32809, 32742, 7859, 7836. Ramos. In open, rather dry grasslands at low and medium altitudes, ascending to $1,300 \mathrm{~m}$. India to Malaya.

FIM BRISTYLIS GLOBULOSA (Retz.) Kunth Enum. 2 (1837) 231; F.-Vill. Novis. App. (1882) 308; Usteri Beitr. Ken. Philip. Veg. (1905) 132; C. B. Clarke in Philip. Journ. Sci. 2 (1907) Bot. 96.

Scirpus globulosus Retz. Obs. 6 (1791) 19.

Fimbristylis torresiana Gaudich. Bot. Freyc. Voy. (1826) 413; F.-Vill. Novis. App. (1882) 308.

Fimbristylis utilis Elm. Leafl. Philip. Bot. 3 (1910) 855.

Luzon (Ilocos Norte, Lepanto, Bontoc, Tayabas, Sorsogon), Polillo, Panay, Leyte, Samar, Negros, Bohol, Mindanao, Elmer 12571, 15584, Wenzel 1592, Merrill Phil. Pl. 1446, B. S. 34777 Ramos \& Pascasio, 30672 Ramos \& Edaño. In open wet places at low altitudes. India to Malaya and Polynesia.

Local names: Anahiúan. (Mbo., Sub., C. Bis.) ; badang-badang (Ilk.) ; pilokong-kabo (Mbo.); sud-sud (Buk.); tayok-táyok (P. Bis.); tíkog (C. Bis., P. Bis.) .

FIMBRISTYLIS JUNCIFORMIS (Retz.) Kunth Enum. 2 (1837) 239; C. B. Clarke in Philip. Journ. Sci. 2 (1907) Bot. 97.

Scirpus junciformis Retz. Obs. 6 (1791) 19.

Fimbristylis brevifolia Presl Rel. Haenk. 1 (1828) 193, non R. Br. Fimbristylis brachyphylla Presl op. cit. 351, non Schultes. 
Fimbristylis haenkei Dietr. Sp. P1. 2 (1833) 161.

Fimbristylis falcata Kunth Enum. 2 (1837) 239; F.-Vill. Novis. App. (1882) 308.

Admitted on the authority of C. B. Clarke who apparently examined Presl's specimen, which was not from Monte Rey, California, but possibly from the Philippines, and also a specimen collected by Callery. India to Indo-Chinà.

FIMBRISTYLIS LEPTOCLADA Benth. Fl. Hongk. (1861) 647; C. B. Clarke in Hook. f. Fl. Brit. Ind. 6 (1893) 647.

Luzon (Bulacan), Culion, Palawan, Merrill 9285, Phil. Pl. 1426, B. S. 41314 Ramos. In dry open sandy soil at low altitudes. Ceylon, Malay Peninsula, Borneo, southern China.

FIMBRYSTYLIS MARIANA Gaudich. in Bot. Freyc. Voy. (1826) 413, var. FOENEA Kükenth. in Fedde Repert. 16 (1920) 432.

Luzon (Cagayan, Isabela, Ilocos Norte), B. S. 7894, 8074, 27625 Ramos, F. B. 16756 Curran. In open wet places. Marianne and Caroline Islands, the variety endemic.

The specimens look very much like $F$. schoenoides Vahl, and three of those cited were originally referred by Kükenthal to $F$. mariana Gaudich var. maxima (K. Schum.) Kükenth.

FIMBRISTYLIS MILIACEA (Linn.) Vahl Enum. 2 (1806) 287; F.-Vill. Novis. App. (1882) 308; Vidal Phan. Cuming. Philip. (1885) 156, Rev. Pl: Vasc. Filip. (1886) 284; C. B. Clarke in Philip. Journ. Sci. 2 (1907) Bot. 96; Merr. op. cit. 1 (1906) Suppl. 30, 3 (1908)

Bot. 398, FI. Manila (1912) 117, Sp. Blancoanae (1918) 83.

Scirpus miliaceus Linn. Syst. ed. 10 (1759) 868.

Scirpus niloticus Blanco Fl. Filip. (1837) 33, ed. 2 (1845) 23, ed. 3, 1 (1877) 43, non Gmel.

Trichelostylis miliacea Nees \& Arn. in Nov. Act. Acad. Nat. Cur.

19 (1843) Suppl. 1: 84, Hook. Journ. Bot. Kew Miscel. 6 (1854) 29. Isolepis miliacea Presl Rel. Haenk. 1 (1828) 189.

Throughout the Philippines in open wet places; a characteristic ricepaddy weed. Pantropic.

Local names: Agor (Tag.); gúmi (Pang.); sirau-sirau (Ilk.); sirisibúyas (Bik.); taulat (Tag.) ; ubod-úbod (Tag.).

FIMBRISTYLIS MONOSTACHYA (Linn.) Hassk. Pl. Jav. Rar. (1848) 61; F.-Vill. Novis. App. (1882) 307; C. B. Clarke in Philip. Journ.

Sci. 2 (1907) Bot. 97; Merr. Fl. Manila (1912) 117.

Cyperus monostachyus Linn. Mant. 2 (1771) 180.

Abildgaardia monostachya Vahl Enum. 2 (1806), 296.

Abildgaardia compressa Presl Rel. Haenk. 1 (1828) 179; Nees in

Nov. Act. Acad. Nat. Cur. 19 (1843) Suppl. 1: 74; Miq. Fl. Ind.

Bat. 3 (1856) 297; Steud. Syn. Pl. Glum. 2 (1855) 72.

Fimbristylis compressa F.-Vill. Novis. App. (1882) 307.

Throughout the Philippines in open grasslands at low and medium altitudes. Pantropic. 
FIMBRISTYLIS PALUdOSA Merr. in Philip. Journ. Sci. 9 (1914) Bot. 265.

Luzon (Bontoc, Benguet), Williams 122.9, Merrill Phil. Pl. 551, Elmer 6497. In cold open swamps, altitude 1,300 to $1,600 \mathrm{~m}$. Endemic, unless referable to $F$. salbundia Kunth.

FIMBRISTYLIS PINETORUM Merr. in Philip. Journ. Sci. 9 (1914) Bot. 266.

Luzon (Benguet), Merrill. $6664, P h i l . P l .558$. On open grassy slopes in thin pine forests, altitude about $1,500 \mathrm{~m}$. Endemic.

FIMBRISTYLIS PODOCARPA Nees in Wight Contrib. (1834) 98, Nov. Act. Acad. Nat. Cur. 19 (1843) Suppl. 1: 77; C. B. Clarke in Philip. Journ. Sci. 2 (1907) Bot. 94.

Luzon, (Loher 766). Admitted on the authority of C. B. Clarke; I have seen no specimens. India to Malaya and the Marianne Islands.

FIMBRISTYLIS POLYTRICHOIDES (Retz.) Vahl Enum, 2 (1806) 248 (polythricoides); F.-Vill. Novis. App. (1882) 307; C. B. Clarke in Philip. Journ. Sci. 2 (1907) Bot. 92; Merr. Fl. Manila (1912) 115. Scirpus polytrichoides Retz. Obs. 4 (1786) 11.

Luzon (Pampanga, Rizal), Kneucker Cyp. Exsic. 233, Merrill 9803, 4249, Phil. Pl. 522. In open wet places, especially near the sea or brackish streams. Tropical Africa and Asia to Japan, through Malaya to Australia.

FIMIBRISTYLIS QUINQUANGULARIS Kunth Enum. 2 (1837) 229; C. B. Clarke in Philip. Journ. Sci. 2 (1907) Bot. 96.

Luzon (Cagayan, Ilocos Norte, Ilocos Sur, Rulacan, Sorsogon), Negros, Panay, Mindanao, Merrill Phil. Pl.1423, B. S. 14558, 7625, 7673, 7684 Ramos, 15656 Merritt \& Darling. In open wet lands, cultivated areas, etc., at low and medium altitudes. India to the Riu Kiu and Marianne Islands.

FIMBRISTYLIS RIGIDULA Nees in Wight Contrib. (1834) 99; Nees in Hook. Journ. Bot. Kew Miscel. 6 (1854) 29; C. B. Clarke in Philip. Journ. Sci. 2 (1907) Bot. 95.

Luzon (Cagayan, Nueva Vizcaya), Panay, Negros, B. S. 14226, 32233 McGregor, 7856, 32811 Ramos. In open grasslands at low and medium altitudes. India to China.

FIMBRISTYLIS SCHOENOIDES (Retz.) Vahl Enum. 2 (1806) 286; Naves Novis. App. (1882) 307; Ceron Cat. Pl. Herb. Manila (1892) 178; C. B. Clarke in Philip. Journ. Sci. 2 (1907) Bot. 92; Merr. Fl. Manila (1912) 116.

Fimbristylis bispicata Nees in Linnaea 9 (1834) 290, var. monostachya Nees in Hook. Journ. Bot. Kew Miscel. 6 (1854) 29, F.-Vill. Novis. App. (1882) 307.

Abildgaardia nervosa Presl Rel. Haenk. 1 (1828) 180; Steud. Syn. Pl. Glum. 2 (1855) 73.

Fimbristylis nutans Naves Novis. App. (1882) 307; Vidal Phan. Cuming. Philip. (1885) 156, Rev. Pl. Vasc. Filip. (1886) 284, non Vahl. 
Luzon (Ilocos Norte, Nueva Ecija, Bulacan, Rizal, Albay), Palawan, Merrill 9322, 9796, 7110, Phil. Pl. 1447, 507, B. S. 32690, 32954 Ramos. In cpen grasslands, fallow rice paddies, etc., at low altitudes. India through Malaya to Australia.

Local name: Gumi-gúmi (Tag.).

FIMBRISTYLIS SETACEA Benth. in Hook. Lond. Journ. Bot. 2 (1843) 239 ; C. B. Clarke in Philip. Journ. Sci. 2 (1907) 91.

Fimbristylis acuminata Vahl var. minor Nees in Hook. Journ. Bot. Kew Miscel. 6 (1854) 29; Miq. Fl. Ind. Bat. 3 (1856) 314; Boeck. in Linnaea 37 (1871) 4; Vidal Phan. Cuming. Philip. (1885) 156, Rev. P1. Vasc. Filip. (1886) 284.

Fimbristylis bursifolia Vidal Phan. Cuming. Philip. (1885) 156, Re $\%$ Pl. Vasc. Filip. (1886) 284, sphalm.

Abildgaardia brevifolia Steud. Syn. Pl. Glum. 2 (1855) 72; Miq. Fl. Ind. Bat. 3 (1856) 297.

Luzon (Bulacan, Zambales, Rizal, Laguna, Sorsogon), B. S. $21 \% 40$ Ramos, F. B. 3990 Ahern's collector, 8156 Curran \& Merritt, 5873, 12388 Curran. In open wet lands at low altitudes. Burma to Tropical Australia.

FIMBRISTYLIS SPATHACEA Roth. Nov. Sp. P1. (1821) 24; C. B. Clarke in Philip. Journ. Sci. 2 (1907) Bot. 95; Merr. op. cit. 3 (1908) Bot. 398.

Fimbristylis glomerata Nees in Linnaea 9 (1834) 290; Boeck. in Linnaea 37 (1871) 47.

Scirpus glomeratus Retz. Obs. 4 (1786) 11, non Linn.

Fimbristylus rigida Kunth Enum. 2 (1837) 246; F.-Vill. Novis. App. (1882) 308.

Isolepis haenkei Presl Rel. Haenk. 1 (1828) 187.

Luzon (Cagayan, Ilocos Norte, Union, Zambales, Batangas, Laguna, 'Tayabas), Panay, Negros, B. S. 32274 McGregor, 33444, 32737, 32795. 29245, 7469 Ramos, 29123 Ramos \& Edaño. In open wet or dry places, usually near the sea. Pantropic.

FIMBRISTYLIS SQUARROSA Vahl Enum. 2 (1806) 289; C. B. Clarke in Hook. f. Fl. Brit. Ind. 6 (1893) 635.

Palawan, Mindanao (Bukidnon), Merrill 9566, Phil. Pl. 2093, B. S. 26093 Fénix. Shores of lakes and in wet soil at low altitudes. Pantropic.

FIMBRISTYLIS subBispicata Nees in Nov. Act. Acad. Nat. Cur. 19 (1843) Suppl. 1: 75; F.-Vill. Novis. App. (1882) 307; Miq. Fl. Ind. Bat. 3 (1856) 315 (var. caesia Miq.); C. B. Clarke in Philip. Journ. Sci. 2 (1907) Bot. 92.

Luzon, (Loher 747). Admitted on the authority of C. B. Clarke and, as he suggests, perhaps best referable to $F$. schoenoides Vahl. India to China and Japan.

FIMBRISTYLIS TENERA R. \& S. Syst. Mant. 2 (1824) 57; C. B. Clarke in Philip. Journ. Sci. 2 (1907) Bot. 95.

Luzon, (Loher 740). Admitted on the authority of C. B. Clarke. Tropical Asia, Africa, and Malaya. 
FIMBRISTYLIS TETRAgonA R. Br. Prodr. (1810) 226 ; F.-Vill. Novis. App. (1882) 307; C. B. Clarke in Philip. Journ. Sci. 2 (1907) Bot. 91; Merr. Fl. Manila (1912) 115.

Scirpus tetragonus Poir. in Lam. Encycl. Suppl. 5 (1804) 98.

Luzon (Pangasinan, Rizal), Merrill 7360, 9793, Loher 749, B. S. 4892 Ramos. In open rather wet places at low altitudes. India through Malaya to Australia.

FIMBRISTYLIS THOMSONII Boeck. in Linnaea 37 (1871) 37; C. B. Clarke in Hook. f. Fl. Brit. Ind. 6. (1893) 646.

Palawan, Merrill 9350. On dry open slopes bordering thickets at low altitudes. India to Indo-China.

FIMBRISTYLIS THOUARSII (Kunth) comb. nov.

Arthrostylis thouarsii Kunth Enum. 2 (1837) 284.

Arthrostylis chinensis Benth. Fl. Hongk. (1861) 397.

Actinoschoenus filiformis Benth. in Hook. Ic. III 4 (1881) 33, t. 1346.

Fimbristylis actinoschoenus C. B. Clarke in Hook. f. Fl. Brit. Ind. 6

(1893) 650, Philip. Journ. Sci. 2 (1907)' Bot. 98.

Actinoschoenus thouarsii Benth. in Hook. Ic. III 4 (1881) 33.

Culion, Busuanga, Merrill 553, B. S. 41184 Ramos, back of the mangrove swamp. Ceylon to Madagascar, Malay Peninsula, southern China, and the Riu Kiu Islands.

\section{DOUBTFUL AND EXCLUDED SPECIES}

Fimbristylis obtusifolia Kunth; F.-Vill. Novis. App. (1882) 308.

Fimbristylis Pauciflora R. Br.; F.-Vill. Novis. App. (1882) 307.

Fimbristylis PIlosa Vahl; Presl Rel. Haenk. 1 (1828) 191; Steud. Syn. Pl. Glum. 2 (1855) 117.

"Hab. in insula Luzon ad portum Sorsogon." Vahl's species is from Africa; the Luzon specimens referred here by Presl possibly represent a form of $F$. annua R.\& S.

Fimbristylis PRESLII Kunth Enum. 2 (1837) 228; Steud. Syn. Pl. Glum. 2 (1855) 108; F.-Vill. Novis. App. (1882) 308.

Abildgaardia pubescens Presl Rel. Haenk. 1 (1828) 180.

"Hab. in Mexico et in Luzon" Presl. Probably not Philippine.

Fimbristylis cymosa R. Br. Prodr. (1810) 228; C. B. Clarke in Philip. Journ. Sci. 2 (1907) Bot. 97.

"'Toubonia' (1433 Cuming) in hb. Kew." This was from Cuming's earlier collections, probably from Polynesia. The printed label bears the date 1831, so that it cannot possibly be from Cuming's Philippine collections.

\section{BULBOSTYLIS Kunth}

BULBOSTYLIS BARBATA (Rottb.) Kunth Enum. 2 (1837) 208; C. B. Clarke in Philip. Journ. Sci. 2 (1907) Bot. 98; Merr. op. cit. 3 (1908) Bot. 398.

Scirpus barbatus Rottb. Descr. Ic. (1773) 52, t. 1\%, f. 4; Vidal Phan. Cuming. Philip. (1885) 156, Rev. Pl. Vasc. Filip. (1886) 284.

Isolepis barbata R. Br. Prodr. (1810) 212; Presl Rel. Haenk. 1 (1828) 187. 
Oncostylis barbata Nees in Hook. Journ. Bot. Kew Miscel. 6 (1854) 29. Fimbristylis barbata Benth. Fl. Austral. 7 (1878) 32 ; F.-Vill. Novis. App. (1882) 308; Merr. F1. Manila (1912) 116.

Isolepis cumingii Steud. Syn. Pl. Glum. 2 (1855) 101; Miq. Fl. Ind. Bat. 3 (1856) 310.

Fimbristylis cumingii F.-Vill. Novis. App. (1882) 308.

Batan Islands, Luzon (Cagayan to Sorsogon, in most or all provinces), Negros. In open dry sandy places at low and medium altitudes, locally abundant, ascending to $1,500 \mathrm{~m}$. All or most warm countries.

Local names: Humot (Iv。); kulilis (Tag.) ; tirtiris (Ilk.).

BULBOSTYLIS CAPILLARIS (Linn.) Kunth Enum. 2 (1837) 211; Usteri Beitr. Ken. Philip. Veg. (1905) 131; C. B. Clarke in Philip. Journ. Sci. 2 (1907) Bot. 98 (var. trifida C. B. Clarke); Merr. op. cit. 5 (1910) Bot. 334.

Scirpus capillaris Linn. Sp. Pl. (1753) 49.

Isolepis capillaris R. \& S. Syst. 2 (1817) 118; Miq. Fl. Ind. Bat. 3 (1856) 311.

Luzon (Cagayan, Lepanto, Bontoc, Benguet). In open places, chiefly at medium altitudes, ascending to $2,000 \mathrm{~m}$. Pantropic.

\section{SCHOENUS Linnaeus}

SCHOENUS AXILlARIS (R. Br.) Poir. in Lam. Encycl. Suppl. 2 (1811) 251; Merr. in Philip. Journ. Sci. 5 (1910) Bot. 334.

Chaetospora axillaris R. Br. Prodr. (1810) 233.

Luzon (Benguet), F. B. 16141 Curran, Merritt, \& Zschokke. On Mt. Pulog, probably from the summit grasslands, altitude about $2,800 \mathrm{~m}$. Australia, New Zealand.

SCHOENUS FALCATUS R. Br. Prodr. (1810) 232; Merr. in Philip. Journ. Sci. 9 (1914) Bot. 268.

Cladium filiforme Merr. in Philip. Journ. Sci. 5 (1910) Bot. 172.

Luzon (Ilocos Norte, Zambales), Palawan, B. S. 5036, 3271\% Ramos \& Pascasio, 508, 717 Foxworthy. In meadows and along streams at low altitudes ascending to $600 \mathrm{~m}$. Formosa, Borneo, northeastern Australia.

SCHOENUS MELANOSTACHYS R. Br. Prodr. (1810) 231; Merr. in Philip. Journ. Sci. 2 (1907) Bot. 263.

Mindoro, Merrill 617\%. In an open heath on Mt. Halcon, altitude about 2,400 m. Borneo, Queensland, New South Wales.

SCHOENUS PHILIPPINENSIS (Palla) Kükenth. in herb. comb. nov.

Helothrix philippinensis Palla in Allgem. Bot. Zeitschr. 17 (1911)

Beil. 1.

Schoenus apogon C. B. Clarke in Philip. Journ. Sci. 2 (1907) Bot. 102; Merr. op. cit. 5 (1910) 334, non R. \& S.

Luzon (Benguet, Tayabas, Laguna), Negros, Mindanao (Davao), Kneuc.ker Cyp. Exsic. 211, Merrill 6508, 69\%3, Phil. Pl. 540, Elmer 11370. On the highest mountains, in the mossy forest and in open grassy places, altitude 1,800 to $2,700 \mathrm{~m}$. Endemic.

Local name: Kalesi (Bag.). 
15. CLADIUM P. Browne

CLADIUM CRINITUM (Stapf) comb. nov.

Vincentia.crinita Stapf in Philip. Journ. Sci. 19 (1921) 65.

Luzon (Albay), B. S. 2935 Mearns. On slopes at medium altitudes on Mt. Mayon. Endemic.

CLADIUM DISTICHUM C. B. Clarke in Philip. Journ. Sci. 2 (1907) Bot. 102.

Luzon (Tayabas, east coast), Merrill 1123. Probably along streams at low altitudes. Endemic.

Local name: Barokíbok (Tag.).

CLADIUM FAlcatum (Nees) C. B. Clarke in Kew Bull. Add. Series 8 (1908) 46.

Baumea falcata Nees in Hook. Journ. Bot. Kew Miscel. 6 (1854) 29.

Vincentia falcata Stapf in Journ. Linn. Soc. Bot. 42 (1914) 179.

Luzon (Albay), Cuming 932. I have seen merely a fragment of the type, and Kloss's New Guinea specimen. New Guinea, a variety in Sumatra.

CLADIUM JUNCOIDES Elm. Leafl. Philip. Bot. 3 (1910) 854.

Luzon (Isabela, Ilocos Norte, Tayabas), Palawan, Sibuyan, Panay, Dinagat, Mindanao (Surigao), Elmer 112150, 13145, B. S. 34593, 35209, 35446, 34605 Ramos \& Pascasio, 32846 Ramos, 25428 Yates, 21172 Escritor, 13209 Foxworthy \& Ramos, Merrill Phil. Pl. 1258. In thickets and forests at low and medium altitudes, along streams, etc. Endemic.

Local name: Magodtañiád (P. Bis.).

CLADIUM LATIFOLIUM Merr. in Philip. Journ. Sci. 2 (1907) Bot. 262, 3 (1908) Bot. 399.

Vincentia malesica Stapf. in Journ. Linn. Soc. Bot. 42 (1914) 178.

Batan Islands, Luzon (Laguna, Tayabas, Sorsogon, Albay), Mindoro, Negros, Mindanao (Davao), Elmer 17638, Williams 2614, B. S. 23499 Ramos, 6497 Robinson, 3822 Fénix, 2386 Foxworthy. On ridges in the mossy forest, and in open places, altitude 1,500 to $2,400 \mathrm{~m}$. Sumatra, Borneo.

Local name: Samalang (Bag.).

CLADIUM PHILIPPINENSE Merr. in Philip. Journ. Sci. 5 (1910) Bot. 171.

Luzon (Zambales, Nueva Ecija, Tayabas, Camarines), Mindoro, Palawan, Sibuyan, Panay, Bucas Grande, Mindanao (Surigao), F. B.6724 Merritt, B. S. 34619, 35097 Ramos \& Pascasio, 35429 Martelino \& Edaño, 33476, 26508 Ramos \& Edaño, 20678 Escritor, 13161 Foxworthy \& Ramos, Elmer 1283.2, 12401, Weber 1053, Merrill 758. On ledges and bowlders along streams at low and medium altitudes. Endemic.

Local name: Kumpai (Bag.).

CLADIUM RIPARIUM. (Nees) Benth. Fl. Austral. 7 (1878) 405; C. B. Clarke Ill. Cyp. (1909) t. 85, f. 5-8; Merr. in Philip. Journ. Sci. 10 (1915) Bot. 288.

Chapelliera riparia Nees in Lehm. Pl. Preiss. 2 (1846-48) 76.

Baumea riparia Boeck. in Linnaea 38 (1874) 246.

Mindanao (Lanao), Clemens 916. In swamps, altitude about $650 \mathrm{~m}$. Australia, with a variety in British India.

$183036-9$ 


\section{EXCLUDED SPECIES}

Cladium Mariscoides F.-Vill. Novis. App. (1882) 309.

This was based on Baumea mariscoides Gaudich. non Torr. and is the Marianne Islands Cladium gaudichaudii W. F. Wight in Contr. U. S. Nat. Herb. 9 (1905) 230. It is allied to the Philippine Cladium juncoides Elm.

Cladium Glomeratum F.-Vill. Novis. App. (1882) 309, non R. Br.

This was based on Baumea glomerata Gaudich. It is a Moluccan species.

Cladium mariscus R. Br.; F.-Vill. Novis. App. (1882) 309.

\section{REMIREA Aublet}

REMIREA MARITIMA Aubl. Pl. Guin. (1775) 45, t. 16; Naves Novis. App. (1882) 309; Vidal Phan. Cuming. Philip. (1885) 156, Rev. Pl. Vasc. Filip. (1886) 285; C. B. Clarke in Philip. Journ. Sci. 2 (1907) Bot. 103; C. B. Rob. op. cit. 6 (1911) Bot. 195.

Mariscus pungens Steud. Syn. Pl. Glum. 2 (1855) 60; Miq. Fl. Ind.

Bat. 3 (1856) 288.

Mariscus capitatus Steud. in Zoll. Verz. Ind. Archip. (1854) 63.

Cyperus capitatus Naves Novis. App. (1882) 306, non Retz.

Remirea wightiana Nees in Hook. Journ. Bot. Kew Miscel. 6 (1854) 29.

Luzon (Cagayan, Camarines), Polillo, Mindoro, Panay, Mindanao, B. S. 32220 McGregor, 6305, 9133 Robinson, F. B. 16680 Bacani, 5534 Merritt, Merrill 881, 11542, Copeland 85\%. On sandy seashores. Pantropic.

\section{RYNCHOSPORA Vahl}

RYNCHOSPORA CORYMBOSA (Linn.) Britton in Trans. N. Y. Acad. Sci.

11 (1892) 84; W. F. Wight in Contr. U. S. Nat. Herb. 9 (1905) 366. Scirpus corymbosus Linn. Cent. Pl. 2 (1756) 7.

Rynchospora aurea Vahl Enum. 2 (1806) 229; Presl Rel. Haenk. 1 (1828) 200; Miq. Fl. Ind. Bat. 3 (1856) 336; F.-Vill. Novis. App. (1882) 309; Vidal Phan. Cuming. Philip. (1885) 156, Rev. Pl. Vasc. Filip. (1886) 285; C. B. Clarke in Philip. Journ. Sci. 2 (1907) Bot. 102.

Luzon (Laguna, Tayabas, Camarines, Sorsogon), Mindoro, Palawan, Leyte, Biliran, Samar, Basilan. In open swampy places at low altitudes. Pantropic.

Local names: Agas (Bik., Bis.) ; bariu-báriu (Bik.) ; ragiudíu (Bik.); rakido (Bik.) ; piso-piso (Bik.).

RYNCHOSPORA GLAUCA Vahl Enum. 2 (1806) 233, var. CHINENSIS

(Boeck.) C. B. Clarke in Hook. f. Fl. Brit. Ind. 6 (1893) 672, Philip. Journ. Sci. 2 (1907) Bot. 102; F.-Vill. Novis. App. (1882) 309 (as $R$. glauca Vahl).

Luzon (Bontoc, Lepanto, Benguet), Mindanaa (Lanao), Clemens 1203, Merrill 4623, Phil. Pl. 569, Williams 1245, Elmer 5757, B. S. 2493, 2781 Mearns. On open seepage slopes, along small streams, etc., at medium altitudes, ascending to $1,800 \mathrm{~m}$. Pantropic.

RYNCHOSPORA RUBRA (Lour.) Makino in Bot. Mag. Tokyo 17 (1903) 180; Merr. Fl. Manila (1912) 119.

Schoenus ruber Lour. F1. Cochinch. (1790) 41.

Rhynchospora haenkei Presl Rel. Haenk. 1 (1828) 199; Miq. Fl. Ind. Bat. 3 (1856) 336. 
Rhynchospora wallichiana Kunth Enum. 2 (1837) 289; F.-Vill. Novis. App. (1882) 309.

Rynchospora wallichiana C. B. Clarke in Hook. f. Fl. Brit. Ind. 6 (1893) 668; Philip. Journ. Sci. 2 (1907) Bot. 101.

Northern Luzon to Palawan and Mindanao, in most islands and provinces. In open grasslands at low and medium altitudes. Tropical Africa and Asia to Japan, southward through Malaya to Australia.

RYNCHOSPORA WIGHTIANA C. B. Clarke in Hook. f. Fl. Brit. Ind. 6 (1893) 669; Philip./Journ. Sci. 2 (1907) Bot. 101.

Rhynchospora wightiana Steud. Syn. Pl. Glum. 2 (1855) 148.

Luzon (Pangasinan, Bulacan, Rizal), Merrill Phil. Pl. 2099, Loher 79.9, $B$. S. 4822, 21743 Ramos. In open grasslands at low and medium altitudes; local. India to Indo-China and southern China.

\section{GAHNIA Forster}

GAHNIA JAVANICA Moritzi Verz. Zoll. Pflanz. (1845-46) 98; Miq. Fl. Ind. Bat. 3 (1857) 340; F.-Vill. Novis. App. (1882) 309; Vidal Rev. Pl. Vasc. Filip. (1886) 285; C. B. Clarke in Philip. Journ. Sci. 2 (1907) Bot. 103; Merr. op. cit. 263, 5 (1910) Bot. 334.

Luzon (Benguet, Laguna, Tayabas), Mindoro, Negros, Mindanao, Witliams 2621, Merrill 6162, 6596, Phil. Pl. 538, Elmer 11589, B. S. 19571 Ramos; 38538 Ramos \& Edaño. In the mossy forest, in open heaths, etc., altitude 1,700 to $2,700 \mathrm{~m}$. Malay Peninsula to western China, south to New Guinea and Polynesia.

Local name: Tamauan (Buk.).

\section{EXXCLUDED SPECIES}

Gahnia Rawacensis Miq.; F.-Vill. Novis. App. (1882) 309.

\section{SCIRPODENDRON Zippel}

SCIRPODENDRON GHAERI (Gaertn.) Merr. in Philip. Journ. Sci. 9 (1914) Bot. 268, Interpret. Herb. Amb. (1917) 106.

Chionanthus ghaeri Gaertn. Fruct. 1 (1781) 190, t. 29, f. a-c; Boerl. in Journ. Linn. Soc. Bot. 31 (1896) 246.

Scirpodendron costatum Kurz in Journ. As. Soc. Beng. $38^{2}$ (1869)

85; Merr. in Philip. Journ. Sci. 2 (1907) Bot. 422; C. B. Rob. op. cit. 6 (1911) Bot. 195.

Scirpodendron pandaniforme Zipp. ex Kurz 1. c. in syn.

Scirpodendron sulcatum Miq. Illustr. Fl. Archip. Ind. (1871) 65, t. 28. Pandanus caricosus Spreng. Syst. 3 (1826) 897.

Luzon (Cagayan, Tayabas), Polillo, Palawan, Biliran, Mindanao (Zamboanga), Merrill 525\%, 8114, 11218, Phil. Pl. 1295, B. S. 10249, 18592 McGregor, F. B. 17828, 19612 Curran. In wet places near the seashore and along streams at low altitudes. Ceylon through Malaya to Australia and Polynesia.

Local names: Bilis (Sub.); gáas (Bik.).

\section{MAPANIA Aublet}

MAPANIA AfFinis Merr. in Journ. Str. Branch Roy. As. Soc. 85 (1922) 157.

Jolo, Clemens 9330. On forested slopes, altitude about $800 \mathrm{~m}$. Borneo. 
MAPANIA GRACILLIMA Kükenth. \& Merr. in Philip. Journ. Sci. 9 (1914) Bot. 267.

Luzon (Tayabas), Mindanao (Zamboanga), Merrill 8215, B. S. 26519 Ramos \& Edaño. On forested ridges, altitude about 1,100 m. Endemic.

MAPANIA HUMILIS (Hassk.) F.-Vill. Novis. App. (1882) 309; C. B. Clarke in Philip. Journ. Sci. 2 (1907) Bot. 109; C. B. Rob. op. cit. 6 (1911) Bot. 194.

Pandanophyllum humile Hassk. in Tijdschr. Nat. Vereen. 10 (1843) 119 ; Miq. Fl. Ind. Bat. 3 (1856) 334.

Lipironia humilis Miq. Illustr. Fl. Archip. Ind. (1871) 61, $t .23$.

Luzon (Apayao, Rizal, Nueva Vizcaya, Laguna, Tayabas, Sorsogon), Polillo, Mindoro, Palawan, Sibuyan, Samar, Negros, Panay, Mindanao, incl. Elmer 14864 as $M$. alpina Elm. In damp primary forests at low and medium altitudes. Malay Peninsula and Archipelago.

Local names: Babakal (Sub.); lubigan (P. Bis.); maribari (S. L. Bis.).

MAPANIA KURZII C. B. Clarke in Hook. f. Fl. Brit. Ind. 6 (1894) 681; Merr. in Philip. Journ. Sci. 2 (1907) Bot. 422; C. B. Rob. op. cit. 6 (1911) Bot. 194.

Mapania banahaensis Elm. Leafl. Philip. Bot. 2 (1909) 574.

Luzon (Laguna, Tayabas, Camarines, Sorsogon), Alabat, Polillo, Catanduanes, Palawan, Panay, Negros, Elmer 7639, 16222, 15519, Merrill 10505, 7220, 4001, Whitford 1569, B. S. 31414 Ramos \& Edaño, 30318, 13347 Ramos, $10248 \mathrm{McGregor}$. In primary forests at low and medium altitudes. Malay Peninsula.

MAPANIA MACROCEPHALA (Gaudich.) K. Sch. ex Warb. in Engl. Bot. Jahrb. 13 (1891) 265; Merr. in Philip. Journ. Sci. 2 (1907) Bot. 422.

Hypolytrum macrocephalum Gaudich. Bot, Freyc. Voy. (1826) 414.

Lepironia macrocephala Miq. Illustr. Fl. Archip. Ind. (1871) 64, t. $2 \%$.

Mindanao (Davao), Balut, Merrill 5409, Elmer 11978. In primary forests, altitude about $650 \mathrm{~m}$. Moluccas to the Bismarck Archipelago.

Local name: Barongis (Bag.).

MAPANIA PALUSTRIS (Hassk.) F.-Vill. Novis. App. (1882) 309; C. B. Clarke in Hook. f. Fl. Brit. Ind. 6 (1894) 681; Merr. in Philip. Journ. Sci. 9 (1914) Bot. 267.

Pandanophyllum palustre Hassk. in Tijdschr. Nat. Vereen. 10 (1843) 119.

Lepironia palustris Miq. Illustr. Fl. Archip. Ind. (1871) 63, t. 25.

Mindanao (Zamboanga, Agusan), Merrill 7298, B. S. 37892 Ramos \& Edaño. In muddy places along small streams in forests at low altitudes. Malay Peninsula and Archipelago.

Local name: Kulibang (Sub.).

\section{THORACOSTACHYUM KurZ}

THORACOSTACHYUM LUCBANENSE (Elm.) Kükenth. ex Merr. in Philip. Journ. Sci. 11 (1916) Bot. 258.

Mapania lucbanensis Elm. Leafl. Philip. Bot. 2 (1909) 573.

Luzon (Laguna, Tayabas), Panay, Mindanao (Zamboanga), Elmer B. S. 28469, 2917\%, 31136 Ramos \& Edaño, 22929 McGregor, 20493, 10029 Ramos, 
36625, 37414 Ramos \& Edaño, Elmer 9116. In primary forests at low and medium altitudes. Amboina.

Local names: Payong-payong (Sub.) ; tiñgol (Sub.).

\section{SCLERIA Bergius}

SCLERIA ANNULARIS (Nees) Kunth Enum. 2 (1837) 359; C. B. Clarke in Philip. Journ. Sci. 2 (1907) Bot. 104.

Hypopyrum annulare Nees in Linnaea 9 (1834) 303.

Luzon, (Loher 80\%), fide C. B. Clarke. Not seen. India and China.

SCLERIA BANCANA Miq. Fl. Ind. Bat. Suppl. (1862) 693; C. B. Clarke in Hook. f. Fl. Brit. Ind. 6 (1894) 693, excl. syn. Presl.

Scleria malaccensis Boeck. in Linnaea 38 (1874) 507.

Busuanga, Palawan, Merrill 9202, B. S. 41175 Ramos. In old clearings, borders of thickets, etc., at low altitudes. Tenasserim through Malaya to New Guinea.

SCLERIA CORYM BOSA Roxb. Hort. Beng. (1814) 103, nomen nudum, Fl. Ind. ed. 2, 3 (1832) 574; C. B. Clarke in Philip. Journ. Sci. 2 (1907) Bot: 104.

Culion, Palawan, Merrill 656, 9402. In wet places at low altitudes. India to the Malay Peninsula.

SCLERIA HEBECARPA Nees in Wight Contrib. (1834) 117; C. B. Clarke

in Philip. Journ. Sci. 2 (1907) Bot. 105.

Scleria wichurai Boeck. in Engl. Bot, Jahrb. 5 (1884) 510.

Luzon (Rizal, Laguna), Palawan, Mindanao (Bukidnon, Zamboanga, Cotabato), B. S. 23136 McGregor, 12042 Ramos, 29679 Ramos \& Edaño, $11 \% 03$ Robinson. In thickets and open places at low and medium altitudes. India to Japan and Polynesia.

Var. PUBESCENS (Steud.) C. B. Clarke in Hook. f. Fl. Brit. Ind. 6 (1894) 689.

Scleria pubescens Steud. Syn. Pl. Glum. 2 (1855) 168.

Luzon (Laguna), Panay, Mindanao (Lanao), Clemens, B. S. 23796 Ramos, 30957 Ramos \& Edaño. In dry open places at low altitudes. India to China and Malaya.

Local name: Dáat (Tag.).

SCLERIA LEVIS Retz. Obs. 4 (1786) 13; C. B. Clarke in Hook. f. F1. Brit. Ind. 6 (1894) 694.

Mindoro, Palawan, Negros, Merrill 9244, 8880, F. B. 5529 Merritt. In open wet places, ravines, etc., sea level to $1,200 \mathrm{~m}$ altitude. India to southern China, southward to Java.

SCLERIA LITHOSPERMA (Linn.) Sw. Prodr. (1788) 18; F.-Vill. Novis. App. (1882) 310; Vidal Phan. Cuming. Philip. (1885) 156, Rev. Pl. Vase. Filip. (1886) 285; C. B. Clarke in Philip. Journ. Sci. 2 (1907)

Bot. 103; Merr. Fl. Manila (1912) 120.

Scirpus lithospermus Linn. Sp. Pl. (1753) 51.

Scleria glaucescens Presl Rel. Haenk. 1 (1828) 202.

Northern Luzon to Mindanao, in most islands and provinces. In dry thickets at low and medium altitudes. Pantropic.

Var. ROXBURGHII Thw. Enum. Pl. Zeyl. (1859-64) 354; C. B. Clarke in Philip. Journ. Sci. 2 (1907) Bot. 104. 
Luzon, Mindanao, B. S. 16409 Reillo, Merrill 11543, (Loher 805, 806). In thickets at low altitudes. India and Ceylon.

Local names: Dáat (Tag.) ; katábad (Tag.) ; taláid (Bag.).

SCLERIA LUzONENSIS Palla in Ailgem. Bot. Zeitschr. 13 (1907) 49.

Scleria chinensis C. B. Clarke in Philip. Journ. Sci. 2 (1907) Bot. 105; Merr. op. cit. 1 (1906) Suppl. 31, non Nees.

Luzon (Bontoc, Benguet, Nueva Vizcaya, Pampanga, Rizal, Laguna, Bataan, Tayabas), Mindoro, Palawan, Negros, Panay, Mindanao, Merrill 4834, 3958, 3964, 3921, 9523, Phil. Pl.560, Kneucker Cyp. Exsic. 167, Elmer 8401, Whitford 151\%, 1518, Williams 1227, 730. In primary forests, on ridges, etc., altitude 800 to $2,200 \mathrm{~m}$. Endemic.

Thought by Kükenthal to be but a variety of $S$. multifoliata Boeck.

Local names: Agagedán (Bon.) ; egegedán (Bon.) ; mankot (Ig.) ; papan (Bon.).

SCLERIA MERRILlII Palla in Allgem. Bot. Zeitschr. 17 (1911) Beil. 8.

Luzon (Rizal), Kneucker Cyp. Exsic. 249, Merrill Phil. Pl. 525 (as S. zeylanica Poir.), B. S. 2029 Ramos. In fallow rice paddies at low altitudes. Endemic.

This is perhaps the same as the form referred by. C. B. Clarke to S. annularis Kunth.

SCLeRIA MOTLEYI C. B. Clarke in Philip. Journ. Sci. 2 (1907) Bot. 104. Scleria trigona Merr. in Philip. Journ. Sci. 8 (1913) Bot. 363.

Scleria trigonocarpa Ridl. in Journ. Str. Branch Roy. As. Soc. 46 (1906) 228, nomen, Mater. Fl. Malay. Pen. (Monocot.) 1 (1907) 110.

Luzon (Pangasinan, Bulacan, Rizal, Tayabas, Camarines, Sorsogon), Catanduanes, Busuanga, Palawan, Panay, Leyte, B. S. 1854, 30426 Ramos, 20681 Escritor, 29706, 33583, 34110 Ramos \& Edaño, 35496 Martelino \& Edaño, 25500 Yates, 29896 F'énix, Elmer 15411 as S. sorsogonensis Elm., Wenzel 158. In forests at low and medium altitudes. Borneo, Malay Peninsula.

Var. DENSISPICATA C. B. Clarke 1. c.

Luzon (Ilocos Norte, Nueva Vizcaya, Pampanga), B. S. 20997 Santos, 22441, 32845 Ramos. In forests at low and medium altitudes, ascending to $800 \mathrm{~m}$. Endemic.

SCLERIA ORYZOIDES Presl Rel. Haenk. 1 (1828) 201; Miq. Fl. Ind. Bat. 3 (1857) 342; F.-Vill. Novis. App. (1882) 310; C. B. Clarke in Philip. Journ. Sci. 2 (1907) Bot. 105.

Luzon (Cagayan, Isabela, Camarines), Palawan, Merrill 144, Phil. Pl. 1294, F. B. 11621 Curran, B. S. 7777,8063 Ramos. In fresh-water swamps, old wet rice paddies, etc., at low altitudes. India and tropical East Africa to tropical Australia.

Local name: Agáas (Bik.).

SCLERIA PERGRACILIS (Nees) Kunth Enum. 2 (1837) 354; C. B. Clarke in Hook. f. Fl. Brit. Ind. 6 (1894) 685; Merr. in Philip. Journ. Sci. 7 (1912) Bot. 75.

Hypophorum pergracile Nees in Edinb. Philos. Journ. 17 (1834) 267.

Luzon (Bontoc), Mindanao (Cotabato), Vanoverbergh 889, Copeland. On open slopes, altitude 300 to $1,400 \mathrm{~m}$. Tropical Africa through India to Yunnan and Indo-China.

Local name: Bangbangló (Bon.). 
SCLERIA PURPUREO-VAGINATA Boeck. in Engl. Bot. Jahrb. 5 (1884)

513 ; C. B. Clarke in Philip. Journ. Sci. 2 (1907) Bot. 105.

Scleria sumatrensis Vidal Rev. Pl. Vasc. Filip. (1886) 285, non Retz.

Batan Islands, Luzon (Cagayan, Abra, Pangasinan, Nueva Vizcaya, Bataan, Rizal, Laguna, Sorsogon), Polillo, Mindoro, Palawan, Leyte, Panay, Negros, Mindanao, F. B. 1929 Borden, B. S. 31169 Ramos \& Edaño, 13992, 26971 Ramos, 29881 Fénix, 14248 McGregor, Wenzel 1691, Elmer 16063, Copeland 95. In thickets and in open places along streams at low and medium altitudes; scarcely distinguishable from $S$. scrobiculata Nees except by its winged sheaths. Ceram Laut.

Local name: Tañílad (Tag.).

SCLERIA SCROBICULATA Nees in Wight Contrib. (1834) 117, Nov. Act. Acad. Nat. Cur. 19 (1843) Suppl. 1: 119; Miq. Fl. Ind. Bat. 3 (1856) 342; F.-Vill. Novis. App. (1882) 310 ; C. B. Clarke in Philip. Journ. Sci. 2 (1907) Bot. 106; Merr. op. cit. 1 (1906) Suppl. 81, 3 (1908) Bot. 399, Fl. Manila (1912) 121, Sp. Blancoanae (1918) 83. Scleria foveolata Llanos Frag. Pl. Filip. (1851) 103; F.-Vill. \& Naves in Blanco Fl. Filip. ed. $3,4^{1}$ (1880) 79 , non Cav.1

Scleria multifoliata C. B. Clarke in Philip. Journ. Sci. 2 (1907) Bot. 106, p. p., non Boeck.

Throughout the Philippines, in thickets, open places, old clearings, etc., at low and medium altitudes; usually common. Andaman Islands to the Riu Kiu Islands, southward to New Guinea.

Of two of the three specimens referred by Clarke to S. multifoliata Boeck. I consider Topping 460 to be S. scrobiculata Nees and Merrill 4834 to be $S$. merrillii Palla.

Local names: Agagidán (Bon.); aladán (Ilk.) ; amamgid (Ilk.); amgid (Tag.) ; árat (Pamp., Tag.) ; dáat (Tag.) ; dáhat (Bik.) ; dat (Pamp., P. Bis.) ; dáut (Tag.) ; gáat (Iv.) ; haras (P. Bis.) ; katábad (Tag.) ; mangked (Iv.); tangra (Ilk.) ; ulat (Pang., Tag.).

SCLERIA SUMATRENSIS Retz. Obs. 5 (1789) 19, t. 2; Rolfe in Journ. Bot. 23 (1885) 215; Usteri Beitr. Ken. Philip. Veg. (1905) 132; C. B. Clarke in Philip. Journ. Sci. 2 (1907) Bot. 106.

Scleria purpurascens Steud. Syn. Pl. Glum. 2 (1855) 169; F.-Vill. Novis. App. (1882) 310.

Palawan, Mindanao, Basilan, B. S. 16349 Reillo, Merrill 9750, De Vore \& Hoover $\%$. In open places and in forests at low and medium altitudes. India to Sumatra, Java, and Borneo.

Local names: Balbalíli (Bon.) ; bulálo (Sub.) ; pangpáyung (C. Bis.).

SCLERIA TESSELLATA Willd. Sp. Pl. 4 (1805) 315, excl. syn. Rumph.; F.-Vill. Novis. App. (1882) 310; C. B. Clarke in Philip. Journ. Sci. 2 (1907) Bot. 104; Merr. Fl. Manila (1912) 120.

Luzon (Lepanto, Bontoc, Benguet, Pangasinan, Bulacan, Rizal), Panay, B.S.30025 Fénix, 12221 Ramos, Merrill 4370, 4617, Phil. Pl. 1444, Williams 1970. In open grasslands at low and medium altitudes, ascending to 1,500 m. India to Japan southward to tropical Australia.

Local name: Katábad (Tag.). 
SCLERIA ZEYLANICA Poir. in Lam. Encycl. 7 (1806) 3 p. p.; C. B. Clarke in Philip. Journ. Sci. 2 (1907) Bot. 104; Merr. Fl. Manila (1912) 120.

Luzon (Ilocos Norte, Bulacan, Rizal), Merrill 3665, Phil. Pl. 1451, B. S. $2 \% 626,10905,21688$, 22001 Ramos. In open rather wet places at low altitudes. India through Malaya to New Caledonia.

Local ṅames: Dáat-babáe (Tag.); dáat-párang (Tag.); pugad-púgad (Tag.).

\section{DOUBTFUL SPECIES}

Scleria Macrophylia Presl Rel. Haenk. 1 (1828) 200; Miq. Fl. Ind. Bat. 3 (1857) 343; F.-Vill. Novis. App. (1882) 310.

"Hab. in insula Luzon" Presl. This has been reduced to Scleria bancana Miq. with which Presl's description does not remotely agree. In its leaves being 16 lines broad, its panicle a foot long and much branched, and in its fruits being the size of a pea Scleria macrophylla Presl does not agree with any of the known Philippine or Indo-Malayan species, but apparently belong's in the group of Scleria macrocarpa Nees. Presl's type was doubtless from South America or Mexico, not from the Philippines.

\section{DIPLACRUM R. Brown}

DIPLACRUM CARICINUM R. Br. Prodr. (1910) 241; C. B. Clarke in Philip. Journ. Sci. 2 (1907) Bot. 106; Merr. Fl. Manila (1912) 121. Scleria caricina Benth. Fl. Austral. 7 (1878) 426; F.-Vill. Novis. App. (1882) 309.

Luzon (Abra, Tarlac, Rizal, Camarines), Panay, Bucas Grande, Mindanao, Merrill 3626, 8236, Loher 715\%, B. S. 35034 Ramos \& Pascasio, 30954, 33507 Ramos \& Edaño. In open grasslands at low and medium altitudes. India to southern China through Malaya to tropical Australia.

\section{UNCINIA Persoon}

UNCINIA RUPESTRIS Raoul in Ann. Sci. Nat. III 2 (1844) 117 var. CAPILLACEA Kükenth. in Engl. Pflanzenreich 38 (1909) 64, Philip. Journ. Sci. 6 (1911) Bot. 58; Merr. op. cit. 5 (1910) Bot. 334.

Luzon (Benguet, Laguna), Mindanao (Davao), B. S. 19560 Ramos, F. B. 16140 Curran, Merritt, \& Zschokke. In the mossy forest on Mits. Pulog, Banahao, and Apo, altitude 2,200 to 2,500 m. New Zealand, Tasmania.

Local name: Loniai (Bag.).

\section{CAREX Linnaeus}

CAREX BACCANS Nees in Wight Contrib. (1834) 122; C. B. Clarke in Curtis's Bot. Mag. III 49 (1893) $t$. 7288, Journ. Linn. Soc. Bot. 37 (1904) 14, Philip. Journ. Sci. 2 (1967) Bot. 108; Merr. op. cit. 5 (1910) Bot. 334; Kükenth. in Engl. Pflanzenreich 38 (1909) 258, f. $39 E-H$, Philip. Journ. Sci. 6 (1911) Bot. 59.

Luzon (Bontoc, Lepanto, Benguet). In damp thickets and in the mossy forest on the higher mountains, altitude 1,600 to $2,400 \mathrm{~m}$. Himalayan region to southern China, Formosa, Sumatra, and Java.

Local names: Gihidsan (Bon.); silak (Ig.) ; mankat (Ig.). 
CAREX BREVICULMIS R. Br. Prodr. (1810) 242, subsp. ROYLEANA Nees var. KINGIANA (Lév. \& Van.) Kükenth. in Engl. Pflanzenreich 38 (1909) 469, Philip. Journ Sci. 6 (1911) Bot. 62.

Carex kingiana Lév. \& Van. in Bull. Acad. Internat. Geog. Bot. 10 (1901) 272.

Luzon (Benguet), Merrill 6609, 6628, Phil. Pl.561, B. S. 317 ry Santos, 8853 McGregor. In open grassy meadows and on damp open slopes, altitude 2,200 to $2,700 \mathrm{~m}$. The species in Australia, the subspecies India to Japan, the variety in Japan.

CAREX BREVISCAPA C. B. Clarke in Hook. f. Fl. Brit. Ind. 6 (1894) 736; Kükenth. in Engl. Pflanzenreich 38 (1909) 474.

Luzon (Sorsogon), Palawan, Merrill 9521; Elmer 15353. On forested ridges, altitude about $800 \mathrm{~m}$. Ceylon.

CAREX BRUNNEA Thunb. Fl. Jap. (1784) 38; C. B. Clarke in Philip. Journ. Sci. 2 (1907) Bot. 107; Merr. op. cit. 1 (1906) Suppl. 31, 5 (1910) Bot. 335; Kükenth. in Engl. Pflanzenreich 38 (1909) 599, Philip. Journ. Sci. 6 (1911) Bot. 63.

Luzon (Ilocos Norte, Abra, Bontoc, Benguet, Lepanto, Kalinga, Zambales, Pampanga, Rizal, Laguna, Tayabas, Bataan), Negros, Mindanao. In the mossy forest, on forested ridges and slopes, altitude 800 to $2,400 \mathrm{~m}$.

Var. SUBTEIOGYNA Kükenth. in Fedde Repert. 8 (1910) 8, Philip. Journ. Sci. 6 (1911)' Bot. 63.

Luzon (Benguet), Merrill 4731, 6505, B.S.8866 McGregor. Borders of the mossy forest, altitude about $2,200 \mathrm{~m}$. The species Japan to southern Asia, Malaya, Australia, and the Mascarene Islands, the variety endemic.

Local names: Silak (Ig.) ; tayalid (Bag.).

CAREX CRYPTOSTACHYS Brongn. in Bot. Duperr. Voy. (1828) 152, t. 25; F.-Vill., Novis. App. (1882) 310; Merr. in Philip. Journ. Sci. 3 (1908) Bot. 220; Kükenth. in Engl. Pflanzenreich 38 (1909) 471, Philip. Journ. Sci. 6 (1911) Bot. 62.

Luzon (Nueva Vizcaya, Rizal, Laguna, Tayabas, Sorsogon), Mindanao (Zamboanga), Merrill 8218, Elmer 7306, 14595, 16110, B. S. 1760 Ramos, 29384 Ramos \& Edaño. In forests at medium altitudes, ascending to 1,000 m. Formosa and southern China to the Malay Peninsula, Java, and Waigiou.

CAREX ELMERI Kükenth. in Fedde Repert. 8 (1910) 326, Leaf. Philip. Bot. 3 (1910) 853, Philip. Journ. Sci. 6 (1911) Bot. 64.

Luzon (Bontoc, Benguet, Laguna), Mindoro, Negros, Vanoverbergh 868, Merrill 6972, 80:25. In forests, altitude 1,500 to 2,200 m. Endemic.

Doubtfully distinct from C. loheri C. B. Clarke.

CAREX FILICINA Nees in Wight Contrib. (1834) 123, var. CEYLANICA (Boeck.) Kükenth. in Philip. Journ. Sci. 6 (1911) Bot. 59; C. B. Clarke in Journ. Linn. Soc. Bot. 37 (1904) 11 (C. filicina), Philip. Journ. Sci. 2 (1907) Bot. 107; Merr. op. cit. 2 (1907) Bot. 263, 5 (1910) Bot. 335; Kükenth. in Engl. Pflanzenreich 38 (1909) 274. Carex ceylanica Boeck. in Linnaea 40 (1876) 341; Kükenth. in Engl. Pflanzenreich 38 (1909) 279. 
Luzon (Cagayan, Ifugao, Abra, Lepanto, Bontoc, Benguet, Laguna, Tayabas, Rizal), Negros, Mindanao (Bukidnon, Davao), B. S. 19559, 7213, 13986 Ramos, 19732, 19695, McGregor, Kneucker Caric. Exsic. 372, Elmer $858 \%$, 10728, Merrill 7496, Phil. Pl. 54.4, Loher 7155. In the mossy forest, altitude 1,400 to $2,500 \mathrm{~m}$. India to China, southward to Java.

Local names: Silak (Ig.) ; táan (Ig.); taláyig (Bag).

Forma SATURATA (C. B. Clarke) Kükenth. in Engl. Pflanzenreich 38 (1909) 275 (as var. of filicina), Philip. Journ. Sci. 6 (1911) Bot: 59.

Carex saturata C. B. Clarke in Journ. Linn. Soc. Bot. 37 (1904) 12.

Luzon (Laguna, Tayabas), Whitford 949, F. B. 866 Klemme. In the mossy forest on Mt. Banahao, altitude about $1,800 \mathrm{~m}$. Java, Sumatra, Borneo.

Fiorma DEPAUPERATA Kükenth. 1. cc.

Luzon (Lepanto, Bontoc), Mindoro, Merrill 4513, 4743, 6200. In the mossy forest, altitude 2,200 to $2,400 \mathrm{~m}$. India to China, Java, and Sumatra.

CAREX FUIRENOIDES Gaudich. Bot. Freyc. Voy. (1826) 412, var. CIRRHULOSA (Nees) Kükenth. in Engl. Pflanzenreich 38 (1909) 287, Philip. Journ. Sci. 6 (1911) Bot. 61.

Carex fuirenoides F.-Vill. Novis. App. (1882) 310; C. B. Clarke in Philip. Journ. Sci. 2 (1907) Bot. 107, Journ. Linn. Soc. Bot. 37 (1904) 11, p. p., vix Gaudich.

Carex cirrhulosa Nees in Hook. Journ. Bot. Kew Miscel. 6 (1854) 29.

Carex fibrata Boott ex Vidal Phan. Cuming. Philip. (1885) 156, Rev.

Pl. Vasc. Filip. (1886) 286, nomen nudum.

? Carex densiftora Presl Rel. Haenk. 1 (1828) 204; F.-Vill. Novis. App. (1882) 310.

Luzon (Nueva Vizcaya, Rizal, Laguna), Merrill 109, B. S. 26203 Ramos, 29388 Ramos \& Edaño. In forests at medium altitudes. The variety endemic, the species in the Marianne Islands.

CAREX GRAEFFEANA Boeck. in Flora 58 (1875) 22; C. B. Clarke in Journ. Linn. Soc. Bot. 37 (1904) 5, Philip. Journ. Sci. 2 (1907) Bot. 107; Kükenth. in Engl. Pflanzenreich 38 (1909) 403, Philip. Journ. Sci. 6 (1911) Bot. 62; Merr. op. cit. 5 (1910) Bot. 335.

Luzon (Bontoc, Benguet, Nueva Vizcaya), Negros, Leyte, Mindanao (Bukidnon, Davao), B. S. 817\%, 8174 Ramos, 31742, 32076 Santos, 8370, 14401 McGregor, 37710, 38642 Ramos \& Edaño, Merrill 6622, 9652, Phil. Pl. 566, Elmer 9842, 8532, Wenzel 625. On slopes in open places, along streams, and in the mossy forest, altitude 1,600 to $2,200 \mathrm{~m}$. Samoa.

Local names: Alasas (Buk.) ; bagibi (Bag.) ; giron (Bag.) ; kigid (Bon.) ; sidak (Ig.) ; silak (Ig.).

CAREX INDICA Linn. Mant. 2 (1771) 574, var. FISSILIS (Boott) Kükenth. in Engl. Pflanzenreich 38 (1909) 264, Philip. Journ. Sci. 6 (1911) Bot. 59; F.-Vill. Novis. App. (1882) 310 (indica) ; Usteri Beitr. Ken. Philip. Veg. (1905) 132 (indica).

Palawan, B. S. 844 Foxworthy. On river banks, probably at low or medium altitudes. The species India to Java, the variety Borneo to Australia and Samoa. 
CAREX JAPONICA Thunb. var. CHLOROSTACHYS (D. Don) Kükenth. in Engl. Pflanzenreich 38 (1909) 620.

Carex chlorostachys D. Don in Trans. Linn. Soc. 14 (1825) 330.

Carex doniana Spreng. Syst. 3 (1826) 825.

Luzon (Benguet), B.S. 40359 Ramos \& Edaño. In openings in the mossy forest on Mt. Simacoco, altitude about $1,900 \mathrm{~m}$. India to Korea, Japan, and Formosa.

CAREX LIGATA Boott in Benth. Fl. Hongk. (1861) 402, var. NEXA (Boott) Kükenth. in Engl. Pflanzenreich 38 (1909) 474, Philip. Journ. Sci. 6 (1911) Bot. 63.

Carex nexa Boott. 1. c.

Luzon (Kalinga, Bontoc), Vanoverbergh 496, 1139, B. S. 37491,37516 Ramos \& Edaño, $40 \% 65$ Ramos. In forests, altitude about $1,600 \mathrm{~m}$. Central and southern China to the Riu Kiu Islands and Formosa.

Var. FORMOSENSIS (Lév. \& Van.) Kükenth. in Engl. Pflanzenreich 38 (1909) 474.

Carex formosensis Lév. \& Van. in Mém. Soc. Sci. Nat. Cherbourg 25 (1906) 216.

Luzon (Cagayan, Bontoc, Benguet), Merrill Phil. Pl. 562, 1763 as C. subtransversa Clarke, B. S. 31958, 31684 Santos, 13987 Ramos. Along: streams and trails, on cliffs and steep banks, etc., in and near the mossy forest, altitude 1,300 to $2,400 \mathrm{~m}$. Formosa.

Local names: Egegedán (Bon.); silak (Ig.).

CAREX LOHERI C. B. Clarke in Journ. Linn. Soc. Bot. 37 (1904) 14, Philip. Journ. Sci. 2 (1907) Bot. 108; Merr. in Philip. Journ. Sci. 5 (1910) Bot. 335; Kükenth. in Engl. Pflanzenreich 38 (1909) 487, Philip. Journ. Sci. 6 (1911) Bot. 64.

Luzon (Lepanto, Benguet, Zambales, Laguna, Tayabas), Merrill 4488, 6506, 6607, 6605, Phil Pl.563, Elmer 8582, 8444, B. S. 5133, 19565 Ramos. In the mossy forest, altitude 1,300 to $2,400 \mathrm{~m}$. Endemic.

Forma GRANDIMAscula Kükenth. in Philip. Journ. Sci. 6 (1911) Bot. 64.

Luzon (Benguet), Merrill 4729. In clearings near the mossy forest, altitude about $2,200 \mathrm{~m}$. Endemic. Scarcely distinguishable from the species.

Local name: Silak (Ig.).

CAREX MERRILLII Kükenth. in Fedde Repert. 8 (1910) 7, Philip. Journ. Sci. 6 (1911) Bot. 63.

Luzon (Ifugao, Benguet), Merrill 6623, 7806, Phil. Pl. 568, Yates. In the mossy forest and in open places along trails, altitude about $2,250 \mathrm{~m}$. Endemic.

CAREX MYOSURUS Nees in Wight Contrib. Bot. Ind. (1834) 122; Kükenth. in Engl. Pflanzenreich 38 (1909) 258, var. EMINENS (Nees) Boeck. in Linnaea 39 (1875) 335; Kükenth. op. cit. 259.

Carex eminens Nees in Wight Contrib. Bot. Ind. (1834) 122. India.

Luzon (Bontoc), Vanoverbergh 992. On slopes, altitude about $1,700 \mathrm{~m}$. 
CAREX NIKKOENSIS Franch. \& Savat. Enum. Pl. Japon. 2 (1879) 132, 558; Kükenth. in Engl. Pflanzenreich 38 (1909) 252, f. A-E, Philip. Journ. Sci. 6 (1911) Bot. 59.

Luzon (Benguet), Merrill 6631. Along trails in the mossy forest, altitude about $2,300 \mathrm{~m}$. Japan.

CAREX NODIFLORA Boeck. in Engl. Bot. Jahrb. 5 (1884) 516; Kükenth. in Engl. Pflanzenreich 38 (1909) 288, Philip. Journ. Sci. 6 (1911) Bot. 61.

Carex cumingii Vidal Phan. Cuming. Philip. (1885) 156, Rev. Pl. Vasc. Filip. (1886) 286; C. B. Clarke in Journ. Linn. Soc. Bot. 37 (1904) 11, Philip. Journ. Sci. 2 (1907) Bot. 107.

Luzon (Isabela, Ilocos Norte, Rizal, Batangas, Laguna, Sorsogon), Alabat, Mindanao (Agusan), Elmer 6449, 15328, 17\%81, 8304, Merrill 10470, Phil. Pl. 1481, B. S. 33013, 22411, 3350, 8006 Ramos. In primary forests at low and medium altitudes. Endemic.

CAREX PALAWANENSIS Kükenth. in Leafl. Philip. Bot. 4 (1911) 1169.

Palawan, Elmer 13146. In damp soil in thickets along streams, altitude àbout $150 \mathrm{~m}$. Endemic.

CAREX PHACOTA Spreng. Syst. 3 (1826) 826 ; Kükenth. in Engl. Pflanzenreich 38 (1909) 350, f. 56 A-C, Philip. Journ. Sci. 6 (1911) Bot. 62 .

Luzon (Bontoc, Benguet), Merrill Phil. Pl. 565, Williams 1246, B. S. 2505 Mearns. In open damp places, altitude about $1,500 \mathrm{~m}$. Himalayan region to China, southern Japan, and Java:

CAREX PYCNOTHYRSOS Kükenth. in Philip. Journ. Sci. 6 (1911) Bot. 60.

Luzon (Bontoc, Nueva Ecija), Negros, Mindanao (Agusan), Merrill Phil. Pl. 543, Weber 1113, B. S. 26309, 37881 Ramos \& Edaño. In primary forests, altitude 250 to $1,200 \mathrm{~m}$. Endemic.

Local name: Harakhaches (Neg.).

CAREX RAFFLESIANA Boott in Trans. Linn. Soc. 20 (1846) 132, Illustr. 1 (1858) 12, t. 33; Kükenth. in Engl. Pflanzenreich 38 (1909) 282, Philip. Journ. Sci: 6 (1911) Bot. 59.

Luzon (Benguet, Pampanga, Bataan, Laguna, Sorsogon), Palawan, Mindanao, B. S. 8378 McGregor, 665, 679, 554 Foxworthy, 6076 Robinson, Whitford 1121, Merrill 4221, 4742, 4669, Elmer 6039. In and near the mossy forest, altitude 800 to $2,200 \mathrm{~m}$. Sumatra and Java to northeastern Australia.

Var. SCABERRIMA (Boeck.) Kükenth. in Engl. Pflanzenreich 38 (1909) 283, Philip. Journ. Sci. 6 (1911) Bot. 60; Merr. op. cit. 5 (1910) Bot. 335.

Carex bengalensis Roxb. var. scaberrima Boeck. in Linnaea 40 (1876) 347; Vidal Phan. Cuming. Philip. (1885) 156, Rev. Pl. Vasc. Filip. (1886) 285 (bengalensis).

Carex scaberrima C. B. Clarke in Journ. Linn. Soc. Bot. 37 (1904) 10, Philip. Journ. Sci. 2 (1907) Bot. 107, Kew Bull. Add. Series 8 (1908) 72.

Carex cruciata Merr. in Philip. Journ. Sci. 3 (1908) Bot. 399, non Wahl. 
Batan Islands, Luzon (Cagayan to Sorsogon, in most provinces), Leyte, Mindanao, Elmer 11607, 17692, 15522, 1154, Merrill 7599, 4795, B. S. 3801, 12704 Fénix, 27035 Ramos. Common on ridges in and near the mossy forest, altitude 1,000 to $2,000 \mathrm{~m}$. Sumatra to the Moluccas.

Local names: Chidak (Ig.) ; ikidsan (Klg.) ; taláyid (Bag.) ; tamalang (Bag.).

Var. ConTINUA (C. B. Clarke) Kükenth. in Philip. Journ. Sci. 6 (1911) Bot. 60.

Carex continua C. B. Clarke in Hook. f. Fl. Brit. Ind. 6 (1894) 717, Journ. Linn. Soc. Bot. 36 (1903) 281, 37 (1904) 11, Philip. Journ. Sci. 2 (1907) Bot. 107; Merr. op. cit. 1 (1906) Suppl. 31; Kükenth. in Engl. Pflanzenreich 38 (1909) 281.

Luzon (Ilocos Norte, Bontoc, Kalinga, Benguet, Nueva Vizcaya, Pampanga, Bataan, Laguna, Tayabas), Mindoro, Negros, Mindana (Zamboanga), Merrill 3197, 6971, 8189, 8208, Phil. Pl. 564, Whitford 189, 1145, B. S. 20999 Santos, $39143,8221,13495,15141$ Ramos, 14246 McGregor, F. $B$. 4384 Merritt. On ridges in and near the mossy forest, altitude 800 to 2,000 m. India to southern China and the Philippines.

CAREX RAMOSII Kükenth. in Fedde Repert. 8 (1910) 8, Philip. Journ. Sci. 6 (1911) Bot. 63.

Luzon (Kalinga, Pangasinan, Rizal), B. S. 1434, 20990, 40738, 40798 Ramos, 11859 Robinson \& Ramos, 17866 Otanes, Merrill Phil. Pl. 536. In forests at medium altitudes. Endemic.

CAREX RARA Boott in Proc. Linn. Soc. 1 (1845) 284, Trans. Linn. Soc. 20 (1846) 139 subsp. CAPILLACEA (Boott), Kükenth. in Engl. Pflanzenreich 38 (1909) 102, Philip. Journ. Sci. 6 (1911) Bot. 58; Merr. op. cit. 5 (1910) Bot. 335.

Carex capillacea Boott Illustr. 1 (1858) 44, t. 110; C. B. Clarke in Journ. Linn. Soc. Bot. 37 (1904) 7, Philip. Journ. Sci. 2 (1907) Bot. 107.

Luzon (Benguet), Merrill 7715, 6626, 6632, 6612, 4732, Phil. Pl. 505, B. S. 31744 Santos, $8333 \mathrm{McGregor}$. In open wet borders of small streams, altitude 2,200 to $2,600 \mathrm{~m}$. Japan and northeastern Asia to the Himalayen region and New South Wales.

CAREX RHIzOMATOSA Steud. in Zoll. Verz. Ind. Archip. (1854) 60, Syn. Pl. Glum. 2 (1855) 206; Miq. Fl. Ind. Bat. 3 (1857) 384; C. B. Clarke in Journ. Linn. Soc. Bot. 37 (1904) 12, Philip. Journ. Sci. 2 (1907) Bot. 108; Kükenth. in Engl. Pflanzenreich 38 (1909) 289, f. 4.4, Philip. Journ. Sci. 6 (1911) Bot. 61.

Carex cumingiana Steud. Syn. Pl. Glum. 2 (1855) 206; Miq. Fl. Ind. Bat. 3 (1857) 349 ; Boott Illustr. 3 (1862) 107, t. 324, 325; Boeck. in Linnaea 40 (1876) 367; F.-Vill. Novis. App. (1882) 310; Vidal Phan. Cuming. Philip. (1885) 156, Rev. Pl. Vasc. Filip. (1886) 286. Carex oligostachya Nees in Hook. Journ. Bot. Kew Miscel. 6 (1854) 29.

Luzon (Bontoc, Rizal), Mindanao (Davao, Lanao), B. S. 2700 Ramos, Clemens 1096, Williams 2615, Vanoverbergh 323. On open slopes, altitude 800 to $1,400 \mathrm{~m}$. India to Indo-China, Java, and the Moluccas. 
CAREX RHYNCHACHAENIUm C. B. Clarke ex Merr. in Govt. Lab. Publ. (Philip.) 35 (1906) 5, Philip. Journ. Sci. 1 (1906) Suppl. 31, 2 (1907) Bot. 108, Kew Bull. Add. Series 8 (1908) 79; Kükenth. in Engl. Pflanzenreich 38 (1909) 480, Philip. Journ. Sci. 6 (1911) Bot. 62 .

Luzon . Kalinga, Pampanga, Bataan, Laguna), Mindanao (Bukidnon, Zamboanga), Elmer 6983, Merrill 8024, 8213, Phil. Pl. 512, B. S. 37575, 3900\%, 39081 Ramos \& Edaño. On forested ridges in and near the mossy forest, altitude 800 to $2,100 \mathrm{~m}$. Endemic.

CAREX subtransversa C. B. Clarke in Philip. Journ. Sci. 2 (1907)

Bot. 108, Kew Bull. Add. Series 8 (1908) 92; Kukenth. in Engl.

Pflanzenreich 38 (1909) 614, Philip. Journ. Sci. 6 (1911) Bot. 63.

Luzon (Benguet), Merrill 4730, 7714. On dry open slopes of recent clearings, along trails, etc., altitude 1,900 to 2,300 m. Endemic.

CAREX TRISTACHYA Thunb. Fl. Jap. (1784) 38 var. POCILLIFORMIS

(Boott) Kükenth. in Engl. Pflanzenreich 38 (1909) 471, Philip.

Journ. Sci. 6 (1911) Bot. 62 ; Merr. op. cit. 5 (1910) 335.

Carex pocilliformis Boott Illustr. 4 (1867) 175, t. 593.

Luzon (Benguet), Merrill 6606, 6630, 6629, B. S. 8856 McGregor. In open meadows and on grassy slopes, altitude 2,200 to $2,500 \mathrm{~m}$. Japan to Formosa, the species also in eastern and central China.

CAREX TURRITA C. B. Clarke in Journ. Linn. Soc. Bot. 37 (1904) 13, Philip. Journ. Sci. 2 (1907) Bot. 108, Kew Bull. Add. Series 8 (1908) 74; Kükenth. in Philip. Journ. Sci. 6 (1911) Bot. 63.

Carex walkeri Arn. var. turrita Kükenth. in Engl. Pflanzenreich 38 (1909) 546.

Luzon (Abra, Bontoc, Ifugao, Benguet), Loher 700, B. S. 7288 Ramos, 40354 Ramos \& Edaño. Apparently in open places, altitude probably above $1,400 \mathrm{~m}$. Endemic.

Local name: Tangtaño (Bon.).

\section{EXCLUDED SPECIES}

Carex longebracteata Steud.; F.-Vill. Novis. App. (1882) 310.

Carfix Haenkeana Presl Rel. Haenk. 1 (1828) 205; Steud. Syn. P1. Glum. 2 (1855) 241; Miq. Fl. Ind. Bat. 3 (1857) 354; F.-Vill. Novis. App. (1882) 310.

"Hab, in insulis Philippinis" Presl. This is the South American Carex pseudocyperus Linn. var. haenkeana Kükenth., the record as Philippine being based on an erroneously localized Malaspina Expedition specimen.

\section{PALMAE}

\section{PHOENIX Linnaeus}

PHOENIX HANCEANA Naud, var. PHILIPPINENSIS Becc. in Philip. Journ. Sci. 3 (1908) Bot. 339, 14 (1919) 339.

Batan Islands (Sabtan), B. S. 3744 Fénix. Along mountain streams at low or medium altitudes. The species in southeastern China, Hainan, and Formosa.

Local name: Voyáyoi (Iv.). 


\section{EXCLUDED SPECIES}

Phoentx DACTylifera Linn.; Naves Novis، App. (1882) 282.

The date palm is very rarely found in gardens in the larger towns of the Philippines. It is not adapted to Philippine climatic conditions.

PHOENIX FARINIFERA Roxb.; Naves 1. c.

PhoEnIX sylVestris Roxb.; Naves 1. c.

\section{CORYPHA Linnaeus}

CORYPHA ELATA Roxb. Fl. Ind. ed. 2, 2 (1832) 176; Merr. F1. Manila (1912) 123, Sp. Blancoanae (1918) 84; Becc. in Philip. Journ. Sci. 14 (1919) 341, Leafl. Philip. Bot. 8 (1919) 3023; Brown \& Merr. in Philip. Bur. Forestry Bull. 18 (1919) 68, t. 24-28.

Corypha umbraculifera Blanco Fl. Filip. (1837) 228, ed. 2 (1845) 160, ed. 3, 1 (1877) 290; Naves Novis App. (1882) 281; Vidal Sinopsis Atlas (1883) 41, t. 93, f. A; Merr. in Govt. Lab. Publ. (Philip.) 27 (1905) 88, non' Linn.

Corypha sylvestris Mart. Hist. Nat. Palm. 3 (1849) 233, saltem quoad. cit. Philip.

Sagus rumphii Perr. in Mém. Soc. Linn. Paris 3 (1824) 143, non Willd.

Livistona vidalii Becc. in Webbia 1 (1905) 343.

Throughout the Philippines in most islands and provinces, in some regions widely scattered, in others subgregarious and abundant at low and medium altitudes. India to Malaya.

Local names: Bagátai (Is.) ; burí (Bis., Bik., Pamp., Tag.) ; busí (Pamp., Bis., Tag.) ; ípus (Tag.) ; piét (Tag., Pang.) ; sílad (Bik.) ; sílag (Ilk., Pang.) ; sílal (Sub.) ; sírair (Bag.) ; takták (Is.).

CORYPHA MICROCLADA Becc. in Webbia 5 (1921) 7.

Biliran, B. S. 18720 McGregor. In open places at low altitudes. Endemic.

\section{LICUALA Wurmb}

LICUALA SPINOSA Wurmb in Verh. Bat. Genoots, 2 (1780) 469; Miq. Fl. Ind. Bat. 3 (1855) 53; Naves Novis. App. (1882) 281; Becc. in Perk. Frag. Fl. Philip. (1904) 45, Webbia 1 (1905) 338, Philip. Journ. Sci. 14 (1919) 339; Brown \& Merr. in Philip. Bur. Forestry Bull. 18 (1919) 88, t. 33.

Culion, Palawan, Balabac, Merrill 543, Phil. Pl. 1463, B. S. 836 Foxworthy, 269 Bermejos, 448 Mangubat, F. B. 3611 Curran. In thickets at low altitudes, sometimes immediately back of the mangrove in brackish mud; now cultivated in Manila for ornamental purposes. Malay Peninsula and Archipelago to the Moluccas.

Local names: Balatbát (P. Bis.); ugsáng (Sul.).

\section{EXCLUDED SPECIES}

Licuala Elegans Mart.; Naves Novis. App. (1882) 281.

LiCUAlA RUMPHII Blume; Naves 1. c.

Licuala spectabilis Miq.; Naves 1. c. 


\section{LIVISTONA R. Brown}

LIVISTONA MERRILLII Becc. in Perk. Frag. Fl. Philip. (1904) 45, Webbia 1 (1905) 339, Philip. Journ. Sci. 14 (1919) 340.

Livistona whitfordii Bece. in Philip. Journ. Sci. 4 (1909) Bot. 615.

Luzon (Cagayan, Zambales, Tayabas, Camarines), Merrill 20\%1, Whitford 731, F. B. 17811, 10409, 17278, 10189, 5876 Curran, 18451 Alvarez, B. S. 33759 Ramos \& Edaño. In forests and in thickets at low altitudes. Endemic.

Local names: Anáhau (Ibn., Bik., Tag.) ; bal-láng (Ibn.) ; labik (Neg.) ; magálai (Bik.); tekis (Sbl.).

LIVISTONA RobinsoniAnA Becc. in Philip. Journ. Sci. 6 (1911) Bot. 230, 14 (1919) 341.

Luzon (Laguna), Polillo, B. S. 9265 Robinson, 10471 McGregor, Loher 7056. In forests at low altitudes. Endemic.

Local name: Pilíg (Tag.).

LIVISTONA ROTUNDIFOLIA (Lam.) Mart. var. LUZONENSIS Becc. in Leafl. Philip. Bot. 8 (1919) 3025, Philip. Journ. Sci. 14 (1919) 340;

Brown \& Merr. in Philip. Bur. Forestry Bull. 18 (1919) 92, t. 36.

Livistona rotundifolia Becc. in Leafl. Philip. Bot. 2 (1919) 647; Merr.

Fl. Manila (1912) 122; Naves Novis. App. (1882) 281, vix Mart.

Corypha minor Blanco Fl. Filip. (1837) 229, ed. 2 (1845) 161, ed.

3, 1 (1877) 290, non Jacq.

Livistona blancoi Merr. Sp. Blancoanae (1918) 84.

Luzon (Benguet, Union, Pangasinan, Zambales, Pampanga, Laguna, Tayabas, Camarines, Albay), Negros, Mindanao, Merrill 37.99, Sp. Blancoanae 919, Elmer 18054, 9293, F. B. 5834, 17344, 17689, 10631 Curran, 1532. Aguilar, $135 \% 6$ Meyer \& Foxworthy, 26228 Mabesa. In forests at low and medium altitudes; sometimes planted for ornamental purposes.

Local names: Abiáng (Pamp., Pang.); aná-au (Ilk.) ; anáhau (Bik., P. Bis., Tag.) ; anáu (Pamp., Ibn.) ; bagsáng (S. L. Bis.); báhi (Bis.) ; bal-láng (Ibn.); balak (Mag.); bolong-úlong (Pamp.); bulús (Bik.); buláu (Bik.) ; bulnó (Bik.) ; iñgaing (Ig.) ; labígi (Ilk.); lúyong (Sbl.); palma brava (Sp.); pilíg (Tag.); tikis (Sbl.).

Var. MICROCARPA Becc. in Leafl. Philip. Bot. 8 (1919) 3024, Philip.

Journ. Sci. 14 (1919) 341.

Livistona microcarpa Becc. in Philip. Journ. Sci. 2 (1907) Bot. 231.

Palawan, Mindanao (Davao), F. B. 3784 Curran, Elmer 1196\%. In forests at low altitudes. The species widely distributed in Malaya, the two varieties endemic.

Local name: Bal-la (Bag.).

Var. MINDORENSIS Becc. in Philip. Journ. Sci. 14 (1919) 341.

Livistona mindorensis Becc. in Philip. Journ. Sci. 4 (1909) Bot. 615.

Mindoro, F. B. 4108 Merritt. Common in forests at low altitudes on Bungabong River. Endemic.

Local name: Panobau (Tag.). 
LIVISTONA SARIBAS (Lour.) Merr. ex A. Chev. in Bull. Econ. Indochine 22 (1919) 501.

Corypha saribas Lour. Fl. Cochinch. (1790) 212, ed. Willd. (1793) 263, excl. syn. Rumph.

Livistona cochinchinensis Mart. Hist. Nat. Palm. 3 (1838) 319; Becc. Malesia 3 (1886) 69, Philip. Journ. Sci. 14 (1919) 340; Becc. \& Hook. f. in Hook. f. Fl. Brit. Ind. 6 (1892) 434, Brown \& Merr. in Philip. Bur. Forestry Bull. 18 (1919) 92, t. 35.

Livistona spectabilis Griff. in Calcutta Journ. Nat. Hist. 5 (1845) 336, Palms Brit. Ind. (1845) 130, t. 266 C.

Livistona inaequisecta Becc. in Philip. Journ. Sci. 4 (1909) Bot. 616.

Luzon (Cagayan, Laguna), F. B. 17145, 17302, 17156, 10079 Curran, 14762 Darling. In open, somewhat swampy regions at low altitudes, in Cagayan gregarious. Malay Peninsula, Indo-China.

Local names; Taráu (Ibn.) ; tíkal (Tag.).

EXCLUDED SPECIES

Livistona Papuana Becc.; Naves Novis. App. (1882) 282.

\section{METROXYLON Rottboell}

METROXYLON SAgU Rottb. in Nye Samml. Dansk. Vidensk. Selsk. Skrift. 2 (1783) 527; Naves Novis. App. (1882) 276; Merr. Interpret. Herb. Amb. (1917) 112; Brown \& Merr. in Philip. Bur. Forestry Bull. 18 (1919) 96, t. 3\%.

Metroxylon rumphii Mart. Hist. Nat. Palm. 3 (1838) 214, 313, $t$. 102, 159; Naves 1. c.; Becc. in Leafl. Philip. Bot. 8 (1919) 3026, Phil. Journ. Sci. 14 (1919) 342, Ann. Bot. Gard. Calcutta $12^{2}$ : t. 105 ined.

Cebu, Bohol, Siquijor, Mindanao (Agusan, Surigao, Misamis, Zamboanga, Cotabato, Davao), Basilan, Sulu. Abundant in fresh-water swamps at low altitudes, in some places planted. Widely distributed in Malaya. The sago palm.

Local names: Ambolong (Mbo.); bagsáng (Bis.); langdáng (Bis.); lumbái (Bis.); lumbiá (C. Bis., Bag.) ; lumbiág (Sul.); sagú (Mbo., Bis.).

\section{EXCLUDED SPECIES}

Metroxylon sylvestre Mart.; Naves Novis. App. (1882) 276.

METRoXylon LONGisPinum Mart.; Naves 1. c.

METROXYLON FILARE Mart.; Naves 1. c.

6. COELOCOCCUS H. Wendland

COElococcus AmICARUM (Wendl.) W. F. Wight in Contr. U. S. Nat. Herb. 9 (1905) 244, t. 45, 46.

Sagus amicarum Wendl. in Bot. Zeit. 36 (1878) 115.

Coelococcus carolinensis Dingl. in Bot. Centralbl. 32 (1887) 349.

Rarely planted, Panay, Mindanao (Zamboanga). Introduced from the Caroline Islands. Polynesian ivory palm.

$183036-10$ 


\section{ZALACCA Reinwardt}

ZALACCA CLemensianA Becc. in Philip. Journ. Sci. 4 (1909) Bot. 618, 14 (1919) 342, Leaff. Philip. Bot. 8 (1919) 3028, Ann. Bot. Gard. Calcutta $12^{2}: t .49$, ined.

Mindanao (Lanao, Zamboanga, Davao), Clemens 1109, Elmer 11879, B. S. 37427, 37431, 37430 Ramos \& Edaño. In forests, altitude 600 to $900 \mathrm{~m}$. Endemic.

Local names: Dalúbi (Sub.) ; kaúbi (Mag.) ; lakaúbi (Bag.) ; lubo (Sub.).

\section{EXCLUDED SPECIES}

ZalACCA EDUlis Reinw.; Naves Novis. App. (1882) 274.

This has not as yet been found in the Philippines except as a recently introduced and cultivated palm.

\section{KORTHALSIA Blume}

KORTHALSIA LACINIOSA Mart. Hist. Nat. Palm. 3 (1838) 211, 343 ; Becc. in Philip. Journ. Sci. 14 (1919) 343, Leafl. Philip. Bot. 8 (1919) 343, Malesia 2 (1884) 74.

Luzon (Tayabas, Camarines, Sorsogon), Elmer 15634, 166r2, F. B. 24537 Aguilar, Klemme, (Vidal 4066); possibly also B. S. 10464 McGregor from Polillo. In primary forests at low and medium altitudes. Burma and Andaman and Nicobar Islands.

Local name: Dánan (Bik.).

Korthalsia MerRillII Becc. in Ann. Bot. Gard. Calcutta $12^{2}:$ t. 81, ined., Philip. Journ. Sci. 14 (1919) 342.

Palawan, Merrill 9410. In primary forests at low altitudes. Endemic.

KORTHALSIA SCAPHIGEROIDES Becc. in Philip. Journ. Sci. 4 (1909)

Bot. 619, 14 (1919) 342, Ann. Bot. Gard. Calcutta 12:: t. 67, ined.

Mindanao (Agusan, Surigao, Zamboanga), Basilan, Merrill 7313, F. B. 6106,4816 Hutchinson, 23913 Ponce. In primary forests at low altitudes. Endemic.

Local name: Kapnigid (C. Bis.).

KORTHALSIA SQUARROSA Bece. in Philip. Journ. Sci. 4 (1909) Bot. 620, 14 (1919) 343, Ann. Bot. Gard. Calcutta 12 ${ }^{2}:$ t. 103, ined.

Palawan, Balabac, Merrill 5384, F. B. 4185 Curran. In primary forests at low altitudes. Endemic.

\section{EXCLUDED SPECIES}

Korthalsia Angustifolia Blume; Naves Novis. App. (1882) 273.

Korthalsia Robusta Blume; Naves 1. c.

9. PLECTOCOMIA Martius and Blume

PLECTOCOMIA ELMERI Becc. in Leafl. Philip. Bot. 8 (1919) 3029, Philip. Journ. Sci. 14 (1919) 342, Ann. Bot. Gard. Calcutta 12: t. 49 ined. Mindanao (Davao), Elmer 1188\%. In forests, altitude about $900 \mathrm{~m}$. Endemic.

Local name: Uñgáng (Bag.). 


\section{CALAMUS Linnaeus}

CALAMUS ARUgDA Bece. in Philip. Journ. Sci. 4 (1909) Bot. 622, 14 (1919) 352.

Luzon (Cagayan), F. B. 6649 Klemme, 26618 Velasco. In primary forests at low altitudes. Endemic.

Local names: Arúgda (Ibn.); uái (Ilk.).

CALAMUS BICOLOR Bece. in Leafl. Philip. Bot. 8 (1919) 3055, Philip. Journ. Sci. 14 (1919) 356, Ann. Bot. Gard. Calcutta 11 Suppl. 126, t. 73 ined.

Mindanao (Davao), Elmer 10541, 10618. In primary forests, altitudes 1,200 to $1,800 \mathrm{~m}$. Endemic.

Local names: Lási (Bag.); rási (Bag.); sambonótan (Bag.).

CALAMUS BLANCOI Kunth Enum. 3 (1841) 595; Mart. Hist. Nat. Palm. 3 (1849) 343; Walp. Ann. 3 (1853) 492, 5 (1858) 832; Miq.' Fl. Ind. Bat. 3 (1855) 139; Becc. Rec. Bot. Surv. India 2 (1902) 204, Webbia 1 (1905) 346, Philip. Journ. Sci. 4 (1909) Bot.635, 14 (1919) 349, Ann. Bot. Gard. Calcutta 11 (1908) 82, 216, t. 64, Leafl. Philip. Bot. 8 (1919) 3048; Merr. in Govt. Lab. Publ. (Philip.) 27 (1905) 88, Sp. Blancoanae (1918) 86.

Calamus gracilis Blanco Fl. Filip. (1837) 267, ed. 2 (1845) 186, ed. 3,1 (1877) 332, non Roxb.

Calamus buroensis Naves Novis. App. (1882) 275, Vidal Cat. Pl. Prov. Manila (1880) 47, non Mart.

Calamus brevifrons Mart. Hist. Nat. Palm. 3 (1849) 338; Walp. Ann. 3 (1853) 488; Miq. Fl. Ind. Bat. 3 (1855) 127; Naves Novis. App. (1882) 275.

Calamus parvifolius Vidal Phan. Cuming. Philip. (1885) 154, non Roxb.

Luzon (Ilocos Norte!, Cuming 1225, Zambales, Bataan, Bulacan), Leyte, Mindanao (Zamboanga), Elmer 7282, (Loher 1376), Merrill Sp. Blancoanae 791, F. B. 4819 Hutchinson, 387 Maule, 24697 Lazaro, B. S. 33047 Ramos. In forests at low altitudes. Endemic.

Local names: Abit (Sbl.); alip (Sul.); talóla (Tag.); u'-uái (Ibn.); yantók (Tag.).

CALAMUS CUMINGIANUS Bece. in Rec. Bot. Surv. Ind. 2 (1902) 210,

Webbia 1 (1905) 346, Ann. Bot. Gard. Calcutta 11 (1908) 99, 348, t. 142, Leafl. Philip. Bot. 8 (1919) 3050, Philip.Journ. Sci. 14 (1919) 351.

Luzon (Tayabas), Mindanao (Agusan), Cuming 762, Elmer 13646. In primary forests at low and medium altitudes. Endemic.

Local name: Douung-douung (Mbo.).

CALAMUS DIEPENHORSTII Miq. Fl. Ind. Bat. Suppl. (1860-61) 594 ; Becc. in Ann. Bot. Gard. Calcutta 11 (1908) 96, 322, t. 126, var. EXULANS Becc. in Philip. Journ. Sci. 4 (1909) Bot. 627, 6 (1911) Bot. 230, 14 (1919) 350.

Polillo, Palawan, Merrill 935\%, B. S. 756 Foxworthy, 9111 Robinson (Loher 7054 from Rizal Province, Luzon, originally referred here by Beccari is C. filispadix Becc. fide Beccari). In primary forests at low altitudes. The variety endemic, the species in the Malay Peninsula, Sumatra, and (?) Borneo. 
CALAMUS DIMORPHACANTHUS Becc. in Rec. Bot. Surv. India 2 (1902) 214, Webbia 1 (1905) 355, Ann. Bot. Gard. Calcutta 11 (1908) 117, 479, t. 219, Philip. Journ. Sci. 4 (1909) Bot. 631, 14 (1919) 355, Leafl. Philip. Bot. 8 (1919) 3055.

Luzon (Benguet, Union, Laguna), Panay, Elmer 6238, 18103, 1833\%, (Loher 1370, 13\%1, Vidal 3956). In forests at medium altitudes, ascending to $1,700 \mathrm{~m}$. Endemic.

Var. MONTALBANICUS Becc. in Philip. Journ. Sci. 4 (1909) Bot. 631, 14 (1919) 355

Luzon (Rizal, Bataan), (Loher 7085), B. S. 29599 Ramos \& Edaño. In primary forests at medium altitudes. Endemic.

Var. ZAMBALENSIS Becc. in Philip. Journ. Sci. 4 (1909) Bot. 632, 14 (1919) 355.

Luzon (Zambales), F. B. 8412 Curran \& Merritt. In mossy forests on exposed ridges, altitude about $2,000 \mathrm{~m}$. Endemic.

CALAMUS DISCOLOR Mart. Hist. Nat. Palm. 3 (1849) 341; Kunth Enum. 3 (1841) 212; Miq. Fl. Ind. Bat. 3 (1855) 136; Naves Novis. App. (1882) 275; Becc. in Webbia 1 (1905) 355, Ann. Bot. Gard. Calcutta 11 (1908) 120, 495, t. 228, Philip. Journ. Sci. 4 (1909) Bot. 635, 14 (1919) 350, Leafl. Philip. Bot. 2 (1909) 649, 8 (1919) 3048 .

Calamus lindenii Rod. Ill. Hort. 30 (1883) 157, t. 489; Ridley in Journ. Str. Branch Roy. As. Soc. 44 (1905) 200.

Cycas? hypoleuca Presl Epim. (1851) 238; Walp. Ann. 3 (1852-53) 453; F.-Vill. Novis. App. (1880) 212; Foxw. in Philip. Journ. Sci. 6 (1911) Bot. 152, ex descr.

Luzon (Laguna, Tayabas, Sorsogon), Elmer 9299, 168\%1, B. S. 12035 Ramos, F. B. 25744 Mabesa. In primary forests at low or medium altitudes. Endemic.

Young plants are commonly cultivated in Manila for ornamental purposes.

Local names: Hamlís (Tag.) ; kumabói (Tag.) ; likbón (C. Bis.) ; simurán (P. Bis) ; ubánon (C. Bis.) ; ulisí (C. Bis.) ; yamíng (P. Bis.).

Var. NEGRosensis Bece. in Philip. Journ. Sci. 4 (1909) Bot. 635.

Negros, Siargao, Mindanao (Surigao), F. B. 12432 Danao, 20893 Tamesis, 24024 Razon. In primary forests at low and medium altitudes. Endemic.

CALAMUS ELMERIANUS Becc. in Leafl. Philip. Bot. 2 (1909) 647, 8 (1919) 3051, Philip. Journ. Sci. 14 (1919) 353, Ann. Bot. Gard. Calcutta 11 Suppl. 69, t. 37 ined.

Luzon (Tayabas), Dinagat, Mindanao (Agusan, Davao), Elmer 14166, 11756, 9298, Merrill 7289, F. B. 24021 Razon, B. S. 34909 Ramos \& Pascasio. In primary forests; sometimes at low altitudes, more often in the mossy forest at 1,300 to $1,800 \mathrm{~m}$.

Local names: Sababai (Mbo.); samánid (Bag.).

CALAMUS FILISPADIX Bece. in Philip. Journ. Sci. 6 (1911) Bot. 230, 14 (1919) 350, Leaff. Philip. Bot. 8 (1919) 3048.

Calamus hookerianus Becc. op. cit. 4 (1909) Bot. 621, non Becc. in Ann. Bot. Gard. Calcutta (1908). 
Luzon (Rizal, Tayabas, Camarines, Sorsogon), Polillo, Masbate, Samar, Palawan, Mindanao (Agusan), Elmer 13949, 16846, 12769, Merrill 7251, F. B. 26219 Mariano, 24824 Valencia, 10630 Curran, B. S. 13128 Foxworthy \& Ramos, 10467 McGregor, 33761 Ramos \& Edaño, 24427 Ramos. In forests at low and medium altitudes, ascending to $1,200 \mathrm{~m}$. Endemic.

Local names: Kangnobnób (Mbo.); nókut (S. L. Bis.); pañganpañgánan (Tag.).

CALAMUS FOXWORTHYI Becc. in Philip. Journ. Sci. 14 (1919) 352, nomen nudum, Ann. Bot. Gard. Calcutta 11 Suppl. 81, t. 45 ined.

Palawan, B. S. 690 Foxworthy. On forested rocky slopes along streams, altitude about $1,000 \mathrm{~m}$. Endemic.

CALAMUS GRANDIFOLIUS Bece. in Philip. Journ. Sci. 4 (1909) Bot. 629,14 (1919) 352.

Luzon (Laguna, Tayabas), B. S. 9448 Robinson, (Loher 7088).

Local name: Uái (Tag., Neg.).

CALAMUS HALCONENSIS Becc. in Philip. Journ. Sci. 4 (1909) Bot. 633, 14 (1919) 356, Ann. Bot. Gard. Calcutta 11 Suppl. 116, t. 65 ined.

Luzon (Laguna), Mindoro, F. B. 4399 Merriti, 25742 Mabesa, B. S. 39471 Ramos. In primary forests, altitude 450 to $1,500 \mathrm{~m}$. Endemic.

Local name: Lambútan (Tag.).

CALAMUS JENNINGSIANUS Becc. in Philip. Journ. Sci. 4 (1909) Bot. 623, 14 (1919) 353.

Mindoro, F. B. 4400 Merritt. In forests, altitudes about $1,500 \mathrm{~m}$. Endemic.

CALAMUS MANILLENSIS (Mart.) Wendl. in Kerch. Palm. (1878) 237; Becc. in Rec. Bot. Surv. India 2 (1902) 215, Webbia 1.(1905) 349, Ann. Bot. Gard. Calcutta 11 (1908) 108, 413, t. 226, f. 1, Leafl. Philip. Bot. 8 (1919) 3052, Philip. Journ. Sci. 14 (1919) 352.

Daemonorops ? manillenis Mart. Hist. Nat. Palm. 3 (1849) 330, t. 175, f. 1-3; Walp. Ann. 3 (1853) 480, 5 (1858) 829; Naves Novis. App. (1882) 275.

Luzon (Nueva Vizcaya, Tayabas), Dinagat, Mindanao (Agusan, Davao, Surigao), Elmer 11714, 10560a, 14011, 14133, 14173, 16811, 17077, B. S. 18994 McGregor, 35255, 34693 Ramos \& Pascasio, 26605 Ramos \& Edaño. In forests, altitude 600 to $1,000 \mathrm{~m}$. Endemic.

Local names: Bayábong (Mbo.) ; bunlak (Mbo.) ; lintókan (Bag.). saráni (Bag.) ; tumárom (Mbo.).

CALAMUS MAXIMUS Blanco Fl. Filip. (1837) 266, ed. 2 (1845) 185, ed. 3 1 . (1877) 331; Mart. Hist. Nat. Palm. 3 (1849) 343; Miq. Fl. Ind. Bat. 3 (1855) 139; Becc. in Perk. Frag. Fl. Philip. (1904) 45; Merr. in Govt. Lab. Publ. (Philip.) 27 (1905) 88, Sp. Blancoanae (1918) 85.

Calamus merrillii Becc. in Webbia 1 (1905) 347, Ann. Bot. Gard. Calcutta 11 (1908) 105, 390, t. 16\%, Philip. Journ. Sci. 4 (1909) Bot. 629, 14 (1919) 351, Leaf. Philip. Bot. 8 (1919) 3057. 
Luzon (Rizal, Laguna, Tayabas), Masbate, Mindanao (Agusan, Lanao, Davao), Basilan, Merrill 1893, Sp. Blancoanae 879, Clemens 1112, 1124, Elmer 11885, 18926, F. B. 2065, Vitlamil, 25373, 23988 Mabesa, 25353 Bawan, 23977 Maneja, 26225 Mariano, 24822 Valencia, 24326 Acedillo. In primary forests at medium altitudes, ascending to 1,200 m. Endemic.

Local names: Akab-ákab (Bag.) ; ápas (Tag.); buai-magi (Yak.) ; kalapí (Bik.) ; labni (Pamp.); lokuán (Tag.); palásan (Tag., Bik., Mbo.); parásan (S. L. Bis.) ; súpot (Tag.) ; tumalím-bábui (Tag.).

Var. NANGA (Becc.) comb. nov.

Calamus merrillii Becc. var. nanga Becc. in Philip. Journ. Sci. 14 (1919) 351, Leafl. Philip. Bot. 8 (1919) 3057, Ann. Bot. Gard. Calcutta 11 Suppl. 78, t. 42 ined.

Mindanao (Davao), Elmer 11110, 11874. In primary forests, altitude 600 to $1,000 \mathrm{~m}$. Endemic.

Local name: Nañga (Bag.).

Var. MERRITTIANUS (Becc.) comb. nov.

Calamus merrittianus Becc. in Philip. Journ. Sci. 2 (1907) Bot. 233, 4 (1909) Bot. 629.

Calamus merrillii Bece. var. merrittianus Becc. in Philip. Journ. Sci. 14 (1919) 351, Ann. Bot. Gard. Calcutta 11 Suppl. 78, $t .42$ ined.

Luzon (Tayabas), Mindoro, F. B. 3912 Merritt, 26218 Mariano. In primary forests at low altitudes. Endemic.

CALAMUS MEGAPHYLLUS Becc. in Philip. Journ. Sci. 14 (1919) 353, Leaff. Philip. Bot. 8 (1919) 3060, Ann. Bot. Gard. Calcutta 11 Suppl. 66, $t .55$ ined.

Mindanao (Agusan, Davao), Elmer 11878, 13542. In forests up to 900 $m$ altitude. Endemic.

Local names: Banakbo (Mbo.) ; lintókan (Bag.).

CALAMUS MELANORHYNCHUS Becc. in Philip. Journ. Sci. 14 (1919) 350, Leafl. Philip. Bot. 8 (1919) 3058, Ann. Bot. Gard. Calcutta 11 Suppl. $30, t .16$ ined.

Mindanao (Davao), Elmer 11708. In primary forests, altitude about $1,200 \mathrm{~m}$. Endemic.

Local name: Dalimban (Bag.).

CALAMUS MEYenIANUS Schauer in Nov. Act. Acad. Nat. Cur. 19 (1843) Suppl. 1: 425; Becc. in Rec. Bot. Surv. India 2 (1902) 217, Webbia 1 (1905) 346, Ann Bot. Gard. Calcutta 11 (1908) 82, 215, t. 63, Philip. Journ. Sci. 14 (1919) 349.

Luzon (Pangasinan, Nueva Vizcaya), B. S. 17755 Otanes, 11442 McGregor. In forests at low and medium altitudes. Endemic.

Local name: Bárit (Ilk.).

CALAMUS MICROCARPUS Becc. in Rec. Bot. Surv. India 2 (1902) 213, Webbia 1 (1905) 355, Ann. Bot. Gard. Calcutta 11 (1908) 117, 477, t. 218, Philip. Journ. Sci. 4 (1909) Bot. 627, 6 (1911) Bot. 230, 14 (1919) 356, Leafl. Philip. Bot. 8 (1919) 3055. 
Luzon (Rizal, Laguna, Tayabas, Camarines, Sorsogon), Polillo, Mindanao (Davao, Agusan, Lanao), Elmer 10676, 13551, 15573, 16810, 16991, B. S. 9131 Robinson, 10465 McGregor, 12326 Foxworthy, F. B. 13305 Tumesis, 20667, 20668 Villamil, 27031 Villaflor, 24522 Cortez \& Valderrama. In primary forests at low and medium altitudes, ascending to $1,200 \mathrm{~m}$. Endemic.

Local names Agúsi (Mbo.); kalapít (Bik.); lambútan (Tag.); pagípi (Mbo.) ; paríti (Bag.) ; sipai (Tag.); tandúlang-gúbat (Tag.).

Var. DIMINUTUS Becc. in Philip. Journ. Sci. 14 (1919) 356.

Luzon (Laguna), B. S. 16599 Ramos. In primary forests at low altitudes. Endemic.

CALAMUS MICrosphaerion Becc. in Perk. Frag. Fl. Philip. (1904) 45, Webbia 1 (1905) 349, Ann. Bot. Gard. Calcutta 11 (1908) 114, 453, t. 204, Philip. Journ. Sci. 4 (1909) Bot. 627, 14 (1919) 353.

Luzon (Bataan), Culion, Merrill 50\%, Whitford, F. B. 20917 De Leon. In forests at low altitudes. Endemic.

Local names: Kulaklíng (Tag.); lábit (Tag.).

Var. SPINOSIOR Becc. in Philip. Journ. Sci. 14 (1919) 354.

Palawan, Merrill 9358, 924\%. In forests at low atitudes. Endemic.

CALAMUS MINdoRensis Becc. in Philip. Journ. Sci. 2 (1907) Bot. 235, 4 (1909) Bot. 625, 14 (1919) 352, Ann. Bot. Gard. Calcutta 11 Suppl. 88, t. 49 ined.

Luzon (Laguna), Mindoro, McGregor 309, F. B. 6217 Merritt, F. B. 25745 Mabesa. In forests at low altitudes. Endemic.

Local name: Tumalín (Tag.).

CALAMUS MITIS Becc. in Philip. Journ. Sci. 3 (1908) Bot. 341, 14 (1919) 353, Ann. Bot. Gard. Calcutta 11 Suppl. 68, t. 36 ined.

Batan and Babuyan Islands; Luzon (Ilocos Norte), B. S. 3817, 4075 Fénix, F. B. 24810 Paraiso. In forests at low and medium altitudes. Endemic.

Local names: Matkóng (Ilk., Ting.) ; tebdas (Iv.).

CALAMUS Moseleyanus Becc. in Bot. Surv. India 2 (1902) 211 , Webbia 1 (1905) 348, Ann. Bot. Gard. Calcutta 11 (1908) 105, 396, t. 1\%1, Leafl. Philip. Bot. 8 (1919) 3053, Philip. Journ. Sci. 14 (1919) 352.

Mindanao (Zamboanga, Davao), Malanipa, Hallier, Elmer 11886. In forests at low altitudes, ascending to 1,000 m. Endemic.

Local name: Saráni (Bag.).

CALAMUS MULTINERVIS Becc. in Philip. Joum. Sci. 14 (1919) 352, Leafi. Philip. Bot. 8 (1919) 3060, Ann. Bot. Gard. Calcutta 11 Suppl. 88, t. 49 ined.

Mindanao (Davao), Elmer 11791, 11955. In primary forests, altitude about $1,200 \mathrm{~m}$. Endemic.

Local names: Balála (Bag.); úbli (Bag॰). 
CALAMUS ORNATUS Blume ex Schultes f. Syst. $7^{2}$ (1830) 1326, var. PHILIPPINENSIS Becc. in Webbia 1 (1905) 346, Ann. Bot. Gard. Calcutta 11 (1908) 102, 370, Philip. Journ. Sci. 6 (1911) Bot. 230 (excl. syn. Blanco), 14 (1919) 350, Leafl. Philip. Bot. 8 (1919) 3054 .

Luzon (Cagayan, Bataan, Laguna, Tayabas, Rizal, Sorsogon), Polillo, Mindoro, Negros, Mindanao (Davao), Surigao, Basilan, Elmer 76.25, 9086, 11236, 15607, 16886, 18425, 17999, Whitford 343, 502, Williams 337, F. B. 212 Barnes, 2854 Meyer, 2439 Borden, 7154, 17252 Curran, 23973, 25041 Maneja, 3911, 3913 Merritt, 6089 Everett, 6107 Hutchinson, B. S. 9266 Robinson, 10461 MeGregor, 16619, 23364 Ramos, 947 Mangubat.

In primary forests at low and medium altitudes. Endemic, the species in Malay Peninsula, Sumatra, Borneo, and Java.

Local names: Agúbak (Tag.) ; alimúran (Ibn.) ; kalapí (S. L. Bis., P. Bis., Bik.) ; kayapí (C. Bis.) ; likúto (Tag.) ; limúran (Tag., Pamp., Sbl.) ; lukuán (Tag.) ; mayañgyang (C. Bis.) ; palaklakánin (Tag.) ; tubo (Bag.) ; uái (Tag.).

CAlamus RAMulosus Becc. in Perk. Frag. Fl. Philip. (1904) 46, Webbia 1 (1905) 349, Ann. Bot. Gard. Calcutta 11 (1908) 114, 454, t. 205, Philip. Journ. Sci. 14 (1919) 354.

Luzon (Tayabas), Merrill 20\%0. In forests at low altitudes. Endemic. Local name: Paulís (Tag.).

CALAMUS REYESIANUS Becc. in Philip. Journ. Sci. 2 (1907) Bot. 237, 14 (1919) 353, Ann. Bot. Gard. Calcutta 11 Suppl. 86, t. 36 ined.

Luzon (Laguna, Tayabas), Reyes, B. S. 13312 Ramos, F. B. 25746 Mabesa. In forests at low and medium altitudes. Endemic.

Local names: Ápas (Tag.); lukuán (Tag.); samúlid (Tag.).

CALAMUS SAMIAN Becc. in Philip. Journ. Sci. 14 (1919) 353, Leafl. Philip. Bot. 8 (1919) 3059, Ann. Bot. Gard. Calcutta 11 Suppl. 92, t. 52 ined.

Luzon (Sorsogon), Mindanao (Davao), Elmer 11336, 17255. In primary forests, altitude about $1,300 \mathrm{~m}$. Endemic.

Local name: Samian (Bag.).

CALAMUS SIPHONOSPATHUS Mart. Hist. Nat. Palm. 3 (1849) 342 ; Walp. Ann. 3 (1853) 491, 5 (1858) 832; Miq. Fl. Ind. Bat. 3 (1855) 137; Naves Novis. App. (1882) 275; Usteri Beitr. Ken. Philip. Veg. (1905) 130 (tiphonolpathus); Ceron Cat. Pl. Herb. Manila (1892) 175; Becc. in Rec. Bot. Surv. India 2 (1902) 213, Webbia 1 (1905) 350, Ann. Bot. Gard. Calcutta 11 (1908) 116, 471, Philip. Journ. Sci. 14 (1919) 354.

Calamus inflatus Warb. in Perk. Frag. Fl. Philip. (1904) 45.

Luzon (Cagayan, Isabela, Pampanga, Rizal, Laguna, Tayabas), Mindoro, Mindanao, Merrill 1891, 1643, Elmer 11652, F. B. 18558 Alvarez, 20633 Villamil, 23975 Maneja, 24321 Acedillo, 26223 Mariano, 11625 Fischer, 23825 Mabesa. In primary forests at low and medium altitudes, ascending to $1,200 \mathrm{~m}$. Endemic. 
Local names: Bejúco (Sp., Tag.); bíri (Tag.); dagdág (Ilk.); hamlís (Tag.) ; husi (Neg.) ; lambútan (Tag.); malaúban (Tag.); oban-óban (Bag.) ; palimanók (Pamp.) ; papákin (Tag.) ; sipai (Tag.) ; súkol (Tag.) ; susúko (Tag.); talóla (Tag.) ; valit (Iv.).

Var. BATANENSIS Becc. in Philip. Journ. Sci. 3 (1908) Bot. 342, 14 (1919) 355, Ann. Bot. Gard. Calcutta 11 Suppl. 115 ined.

Batan Islands (Batan), B. S. 3611 Fénix. In forests along streams. Endemic.

Var. FARINosus Becc. in Ann. Bot. Gard. Calcutta 11 (1908) 474, Philip. Journ. Sci. 14 (1919) 355.

Luzon (Rizal), (Loher 7083), F.B. 23972 Maneja. In forests at medium altitudes. Endemic.

Var. OLIGolePIS Becc. (minor et major) in Webbia 1 (1905) 353, Ann. Bot. Gard. Calcutta 11 (1908) 475, 476, Philip. Journ. Sci. 14 (1919) 355.

Luzon (Rizal, Tayabas), (Loher, Warburg). In forests at low or medium altitudes. Endemic.

Var. POLYLEPIS Becc. in Webbia 1 (1905) 354, Ann. Bot. Gard. Calcutta 11 (1908) 477, t. 216.

Luzon (Rizal), (Vidal 4068). In forests at low or medium altitudes. Endemic.

Var. SUBLEVIS Becc. 1. cc. Philip. Journ. Sci. 14 (1919) 355.

Luzon (Ilocos Norte, Zambales, Rizal, Bataan, Laguna, Tayabas), Whitford 308, F. B. 15 7'y Borden, 2635 Meyer, 6225, 6514 Curran, 25739 Mabesa, 13985 Merritt \& Darling, 25100 Paraiso, B. S. 26643,29601 Ramos \& Edaño, 4713, 33182 Ramos, 10787 McGregor. In forests at low and medium altitudes. Endemic.

CALAMUS SPINIFOLIUS Becc. in Rec. Bot. Surv. India 2 (1902) 202, Webbia 1 (1905) 348, Ann. Bot. Gard. Calcutta 11 (1908) 107, 410, t. 178, Philip. Journ. Sci. 14 (1919) 353.

Luzon (Pampanga, Bataan), Palawan, Panay, F. B. 17309 Curran, 20947 De Leon, 1454 Ahern's collector. In forests at low altitudes. Endemic. Local names: Kuraklíng (Pamp., Tag.) ; síka (Tagb.); yantók (Tag.).

CALAMUS SYMPHYSIPUS Mart. in Hist. Nat. Palm. 3 (1849) 336; Benc. in Ann. Bot. Gard. Calcutta 11 (1908) 346, t. 141, Leafl. Philip. Bot. 8 (1919) 3049, Philip. Journ. Sci. 14 (1919) 351.

Luzon (Sorsogon), Bucas Grande, Mindanao (Agusan), Elmer 16701, 13902, B. S. 35035 Ramos \& Edaño. In primary forests up to $900 \mathrm{~m}$ altitude. Celebes.

Local name: Bolánog (Mbo.).

CAlamus TRISPERMUS Becc. in Perk. Frag. Fl. Philip. (1904) 46, Webbia 1 (1905) 349, Ann. Bot. Gard. Calcutta 11 (1908) 108, 412, t. 180, Philip. Journ. Sci. 4 (1909) Bot. 625, 14. (1919) 352.

Luzon (Rizal), Merrill 1645, (Loher rori). In forests at medium altitudes. Endemic. 
CALAMUS USITATUS Blanco Fl. Filip. (1837) 265, ed. 2 (1845) 185, ed. 3, 1 (1877) 330, t. 9.9 (as C. mollis); Mart. Hist. Nat. Palm. 3 (1849) 340; Miq. Fl. Ind. Bat. 3 (1855) 131; Vidal Sinopsis Atlas (1883) 42, t. 93, f. D; Merr. Sp. Blancoanae (1918) 85; Brown \& Merr. in Philip. Bur. Forestry Bull. 18 (1919) t. 11, 12.

Calamus mollis Naves in Blanco Fl. Filip. ed. 3 (1877-83) t. 99; Vidal Rev. Pl. Vasc. Filip. (1886) 280; Becc. in Rec. Bot. Surv. India 2 (1902) 204, Perk. Frag. Fl. Philip. (1904) 46, Ann. Bot. Gard. Calcutta 11 (1908) 82, 212, t. 61, 62, Webbia 1 (1905) 345, Philip. Journ. Sci. 3 (1908) Bot. 342, 14 (1919) 348, Leafl. Philip. Bot. 8 (1919) 3046; Merr. in Govt. Lab. Publ. (Philip.) 27 (1905) 88, Philip. Journ. Sci. 3 (1908) Bot. 399, Fl. Manila (1912) 124, non Blanco.

Calamus haenkeanus Mart. Hist. Nat. Palm. 3 (1849) 337; Kunth Enum. 3 (1841) 211; Walp. Ann. 2 (1852) 488, 5 1858) 831; Miq. Fl. Ind. Bat. 3 (1855) 127; Naves Novis. App. (1882) 275.

Batan and Babuyan Islands, Luzon (Ilocos Norte, Bontoc, Apayao, Nueva Ecija, Nueva Vizcaya, Bulacan, Rizal, Bataan, Zambales, Laguna, Tayabas, Camarines, Sorsogon), Palawan, Biliran, Samar, Mindanao (Surigao), Elmer 11969, 17052, 17727, 18201, Merrill 7407, 1642, 1743, 1411, 2077, Williams 561, B. S. 28131 Fénix, 11218, 18634 McGregor, 9571 Robinson, 4032 Fénix, 381 Ramos, F. B. 23981 Maneja, 24536 Aguilar, 17034, 595\%, 6297, 5982, 6338, 17273, 8489 Curran, 2480, 2481, 3026 Borden, 24195 Bawan, 25102 Paraiso. In secondary forests and thickets at low and medium altitudes. Endemic.

Local names: Abét (Tag.) ; abít (Tag.) ; arit (Bag.); babúyan (Sbl.) ; bálit (Ilk.) ; bári (Ibn.) ; bárit (Ibn., Ilk.) ; digtan (Tag.) ; hanápas (Bik.); kakalsán (Tag.); pagípe (C. Bis.) ; ritoko (Ting.); tindaróra (Bik.); tandúlang-párang (Tag.) ; tagalóa ( S. L. Bis.) ; tagókan (P. Bis.) ; tolólanglutúkan (Tag.) ; uéi (Pang.) ; uái (Tag., Ilk.) ; uhói (Tagb.) ; válet (Iv.) ; yantók (Tag., Sbl.).

Var. MAJOR (Bece.) comb. nov.

Calamus mollis var. major Becc. in Webbia 1 (1905) 345, Philip. Journ. Sci. 14 (1919) 349.

Luzon (Bataan), F. B. 2499 Meyer, Whitford 80. In forests at low altitudes.

Local name: Uái (Tag.).

Var. PALAWANICUS (Becc.) comb. nov.

Calamus mollis var. palawanicus Becc. in Philip. Journ. Sci. 2 (1907)

Bot. 233, 14 (1919) 345, Leafl. Philip. Bot. 8 (1919) 3047.

Palawan, Elmer 12607, F. B. 3613 Curran, B. S. 609 Foxworthy, 191, 196 Bermejos. In forests at low altitudes. Endemic.

CALAMUS VIDALIANUS Becc. in Rec. Bot. Surv. India 2 (1902) 212, Webbia 1 (1905) 350, Ann. Bot. Gard. Calcutta 11 (1908) 115, 464, t. 211, Philip. Journ. Sci. 4 (1909) Bot. 634.

Calamus horrens Vidal Rev. Pl. Vasc. Filip. (1886) 280; Ceron Cat. Pl. Herb. Manila (1902) 175, non Blume. 
Luzon (Nueva Ecija, Rizal, Bataan, Tayabas, Camarines), F. B. 20948 De Leon, 22162 Alvarez, B. S. 13:14, 222sli Rumos, sirri.) Ramos \& Edaño. In primary forests at low and medium altitudes.

Local names: Butárak (Ilk.); yantók (Tag.).

CALAMUS VINOSUS Becc. in Philip. Journ. Sci. 14 (1919) 352, nomen nudum, Leafl. Philip. Bot. 8 (1919) 3061.

Mindanao (Agusan), Elmer 14158. On forested ridges, altitude about $1,450 \mathrm{~m}$. Endemic.

CALAMUS VIRIDISSIMUS Bece. in. Philip. Journ. Sci. 14 (1919) 353, Leaf. Philip. Bot. 8 (1919) 3059, Ann. Bot. Gard. Calcutta 11 Suppl. 84, $t .47$ ined.

Mindanao (Davao), Elmer 11938. In forests, altitude about $300 \mathrm{~m}$. Endemic.

Local name: Akal (Bag.).

\section{DOUBTFUL AND EXCLUDED SPECIES}

Calamus albls Pers.; Naves Novis. App. (1882) 276; Vidal Cat. Pl. Prov. Manila (1880) 47.

Calamus concinnus Mart.; Naves op. cit. 275.

Calamus curag Blanco ex Miq. Fl. Ind. Bat. 3 (1855) 113; Becc. in Ann. Bot. Gard. Calcutta 11 (1908) 498.

Blanco published no such species, but casually mentions a form known locally as kurag, or purag.

Calamus Equestris Willd.; Naves Novis. App. (1882) 276.

Calamus Javensis Blume; Naves op. cit. 275.

Calamus nitidus Mart.; Linden Ill. Hort. 28 (1881) 16.

"Philippines." This is an Indian species, Linden's specimen being either erroneously named or erroneously localized.

Calamus PISICARPUS Blume; Naves op. cit. 275.

Calamus Philippensis Linden Ill. Hort. 28 (1881) 16, nomen nudum, "Philippines."

Calamus RHomboideus Blume; Naves op. cit. 276.

CALAmUS SCIPIONUm Lour.; Naves 1. c.

Calamus verus Blanco Becc. in Ann. Bot. Gard. Calcutta 11 (1908) 503.

This is merely a lapsus calami, Blanco being erroneously cited instead of Loureiro. The page reference given conforms to Loureiro, Flora Cochinchinensis, not to Blanco, Flora de Filipinas.

\section{DAEMONOROPS Blume}

DAEMONOROPS AFFINIS Becc. in Leafl. Philip. Bot. 8 (1919) 3042, Philip. Journ. Sci. 14 (1919) 359.

Mindanao (Agusan), Elmer 13978. In the mossy forests on exposed peaks and ridges, altitude about $1,200 \mathrm{~m}$. Endemic.

Local name: Bag-bág (Mbo.). 
DAEMONOROPS CLEMENSIANUS Becc. in Philip. Journ. Sci. 4 (1909) Bot. 636, 14 (1919) 359, Ann. Bot. Gard. Calcutta $12^{1}$ (1911) 163, t. 67 .

Mindanao (Lanao), Clemens 122\%. This is possibly a mixture of the fruits of $D$. ochrolepis Becc. and the leaves of some other species of doubtful status. Endemic.

DAEMONOROPS CURRANII Becc. in Philip. Journ. Sci. 2 (1907) Bot. 238, 4 (1909) Bot. 636, 14 (1919) 359, Ann. Bot. Gard. Calcutta 12 (1911) 142, t. 58, Leafl. Philip. Bot. 8 (1919) 3033.

Palawan, Elmer 12663, F. B. 3791 Curran. In forests at low altitudes. Endemic.

As later noted by Beccari the portion of leaf-sheath and the fragment of the spadix on the left of the original plate do not belong here but appertain to $D$. pedicellaris Becc.

Local names: Pit-pit (Tagb.); saranoi (Tagb.).

DAEMONOROPS GRACILIS Becc. in Leafl. Philip. Bot. 8 (1919) 3014, Philip. Journ. Sci. 14 (1919) 359.

Palawan, (Elmer 12945). In forests. Endemic.

DAEMONOROPS LOHERIANUS Becc. in Philip. Journ. Sci. 4 (1909) Bot.

637, 14 (1919) 358, Ann. Bot. Gard. Calcutta $12^{1}$ (1911) 104, t. 41.

Luzon (Rizal), (Loher rorg). In forests at low or medium altitudes. Endemic.

DAEMONOROPS MARGARITAE (Hance) Becc. var. PALAWANICUS Becc. in Philip. Journ. Sci. 4 (1909) Bot. 636, 14 (1919) 358, Leaf. Philip. Bot. 8 (1919) 3030, Ann. Bot. Gard. Calcutta $12^{1}$ (1911) 57.

Palawan, Merrill 9359, Elmer 12943, B. S. 899 Foxworthy. In forests at low altitudes. The variety endemic, the species in southeastern China.

DAEMONOROPS MOLLIS (Blanco) Merr. Sp. Blancoanae (1918) 86.

Calamus mollis Blanco Fl. Filip. (1837) 264, ed. 2 (1845) 184, ed. 3, 1 (1877) 329; Mart. Hist. Nat. Palm. 3 (1849) 336; Kunth Enum. 3 (1841) 594; Walp. Ann. 3 (1853) 486, 5 (1858) 831; Miq. Fl. Ind. Bat. 3 (1855) 123.

Daemonorops gaudichaudii Mart. Hist. Nat. Palm. 3 (1849) 331; Walp. Ann. 3 (1853) 481; Naves Novis. App. (1882) 275; Becc. in Perk. Frag. Fl. Philip. (1904) 47, Rec. Bot. Surv. India 2 (1902) 226, Webbia 1 (1905) 355, Philip. Journ. Sci. 3 (1908) Bot. 342, 14 (1919) 359, Leafl. Philip. Bot. 8 (1919) 3031, Ann. Bot. Gard. Calcutta $12^{1}$ (1911) $157, t .64$.

Daemonorops fuscus Mart. Hist. Nat. Palm. 3 (1849) 331; Walp. Ann. 3 (1853) 481, Becc. Rec. Bot. Surv. India 2 (1902) 226; Naves Novis. App. (1882) 274.

Calamus usitatus Merr. in Govt. Lab. Publ. (Philip.) 27 (1905) 88, non Blanco.

Babuyan Islands to Mindanao, in most islands and provinces, Whitford 289, 1371, Elmer 11880, 12494, Merrill Sp. Blancoanae 685, B. S. 1212, 24394 Ramos, 948 Mangubat, 11358, 1419.2 MaGregor, 4066 Fénix, 29368, 38720 Ramos \& Edaño, 24876, 24777 Edaño, 22577 Ramos \& Deroy, 28401 
Fénix, F. B. 21713 Abellanosa, 3741, 6218 Merritt, 22163 Alvarez, 2601 Meyer, 17310, 17218, 17252, 10190 Curran, 25040, 23974 Maneja, 24108 Bernardo, 25519 Cenabre, 24825 Valencia, 20892 Tamesis. In primary forests at low and medium altitudes, often common. Endemic.

Local names: Alimúran (Ibn.); alundayág (Ibn.); babúyan (Tag.); bag-bág (Tag.) ; barítau (Ibn.) ; batárag (Ilk.) ; batásan (P. Bis.) ; digtán (Tag.) ; dí́tan (Tag.) ; ditán (Ibn., Tag.) ; gatásan (Tag., S. L. Bis., Bik., P. Bis.) ; hamlís (Tag.) ; lakaóan (Bik.); labnig (Bik.); lisan (Sub.) ; mamuktóng (Tag.) ; nákat (Bag.) ; núkot (S. L. Bis.) ; samúlig (Bik.); sumúlid (Tag.); tabánga (Tag.); tákol (Bik.); tamarúra (Tagb.) ; tapnikid (C. Bis.) ; uái (Tag., Ilk.) ; uban-úban (P. Bis.); yantók (Tag.).

DAEMONOROPS OCHROLEPIS Becc. in Perk. Frag. Fl. Philip. (1904) 47, Webbia 1 (1905) 356, Philip. Journ. Sci. 6 (1911) Bot. 230, 14 (1919) 358, Leafl. Philip. Bot. 8 (1919) 3032, Ann. Bot. Gard. Calcutta $12^{1}$ (1911) 160 , t. 65, incl. var. radulosus Becc. in Philip. Journ. Sci. 4 (1909) Bot. 636, Ann. Bot. Gard. Calcutta $12^{1}$ (1911) $162, t .66$.

Luzon (Ilocos Norte, Nueva Ecija, Rizal, Laguna, Tayabas, Sorsogon), Polillo, Mindana, Merrill 2069, Elmer 11875, 14120, 14838, 16869, B. S. 13101 Foxworthy \& Ramos, 13235 Ramos, 9039 Robinson, 26644 Ramos \& Edaño, 33048 Ramos, F.B. 8490, 10190 Curran, 16221 Mariano, 25740 Ma besa. In primary forests at low and medium altitudes, ascending to 1,200 m. Endemic.

Local names: Ditán (Tag.) ; ligid-lígid (Tag.); mayáñan (Mbo.); palaklakánin (Tag.); saladíngan (Bag.) ; sumúlid (Tag.).

DAEMONOROPS OLIGOLEPIS Berc. in Leafl. Philip. Bot. 8 (1919) 3035, Philip. Journ. Sci. 14 (1919) 359.

Mindanao (Davao), Elmer 1175\%. In primary forests, altitude about $1,100 \mathrm{~m}$. Endemic.

Local name: Rogmán (Bag.).

DAEMONOROPS PANNOSUS Becc. in Leafl. Philip. Bot. 8 (1919) 3033 , Philip. Journ. Sci. 14 (1919) 359.

Mindanao (Davao), Elmer 11600. In forests along streams, altitude about $1,200 \mathrm{~m}$. Endemic.

Local name: Sabilóg: (Bag.).

DAEMONOROPS PEDICELLARIS Becc. in Leafl. Philip. Bot. 8 (1919) 3040, Philip. Journ. Sci. 14 (1919) 358.

Mindanao (Agusan, Lanao, Davao), Elmer 11896, 14132, 13853, Clemens 1230. In primary forests, altitude 650 to $1,250 \mathrm{~m}$. Endemic.

Local names: Hiyod (Mbo.); oban-óban (Mbo.) ; rogmán (Bag.).

DAEMONOROPS URDANETANUS Becc. in Leafl. Philip. Bot. 8 (1919) 3038, Philip. Journ. Sci. 14 (1919) 358.

Mindanao (Agusan), Elmer 14201. In primary forests, altitude about $1,600 \mathrm{~m}$. Endemic.

Local name: Saháan (Mbo.).

DAEMONOROPS VIRESCENS Bece. in Perk. Frag. Fl. Philip. (1904) 47,

Webbia 2 (1905) 357, Ann. Bot. Gard. Calcutta $12^{1}$ (1911) 201, t. 91, Philip. Journ. Sci. 14 (1919) 358.

Palawan, Merrill 868. In forests at low altitudes. Endemic. 


\section{EXCLUDED SPECIES}

Daemonorops ACCedens Blume; Naves Novis. App. (1882) 274.

DaEmonorops ANGustifolius Griff.; Naves op. cit. 274.

DAEMONOROPS CALAPPARIUS Blume; Náves op. cit. 275.

DaEMonorops idRACo Mart.; Naves op. cit. 274.

Daemonorops melanochaetes Blume; Mart. Hist. Nat. Palm. 3 (1849) 203; Vidal Cat. Pl. Prov. Manila (1880) 47; Naves Novis. App. (1882) 275.

The Haenke specimen cited by Martius as representing Blume's species was doubtless wrongly identified.

DAEMONOROPS NIGER Blume; Naves op. cit. 274.

DaEMONOROPS OBLONGUS Mart; Naves 1. c.

LARMONOROPS RUMPHII Mart.; Naves 1. c.

\section{CARYOTA Linnaeus}

CARYOTA CUMINGII Lodd. ex Mart. Hist. Nat. Palm. 3 (1849) 315; Walp. Ann. 3 (1853) 466; Miq. Fl. Ind. Bat. 3 (1855) 41; Hook. f. in Curtis's Bot. Mag. III 25 (1869) t. 5762; Naves Novis. App. (1882) 279; Vidal Sinopsis Altas (1883) 42, t. 93, f. B; Becc. in Webbia 1 (1905) 331, Perk. Frag. Fl. Philip...(1904) 48, Philip. Journ. Sci. 14 (1919) 338; Merr. Fl. Manila (1912) 123, Sp. Blancoanae (1918) 87.

Caryota urens Blanco Fl. Filip. (1837) 740, ed. 2 (1845) 510, ed. 3, 3 (1879) 142, t. 349; Naves Novis. App. (1882) 279, Vidal Cat. Pl. Prov. Manila (1880) 47, non Linn.

Caryota merrillii Becc. in Webbia 1 (1905) 333, Philip. Journ. Sci. 14 (1919) 338.

Luzon (Apayao, Union, Rizal, Nueva Ecija, Pangasinan, Nueva Vizcaya, Laguna, Tayabas), Mindoro, Palawan, Guimaras, Panay, Mindanao, Mermill 2880, 8036, 9360, Sp. Blancoanae 736, Elmer 5657, 18286, F. B. 10348, 8490 Curvan, 6216 Merritt, 119 Gammill, 9077 Whitford \& Hutchinson, 19961, 2049.2 Villamil, B. S. 873 Foxworthy, 28160 Fénix, 11243 McGregor. In forests at low and medium altitudes; sometimes planted in towns for ornamental purposes. Endemic.

Local names: Anívung (Ibn.) ; aníbong (Ibn., Ilk., Tag.) ; bahi (Man.) ; batíkan (C. Bis.) ; datípan (Tag.); hagol (Bik.); lagípan (Pamp.); lulúg (Tag.) ; pugáhan (Tag.) ; pugúhan (Mbo.); patíkan (P. Bis., Sul.); pola (Bag.); takípan (Sul.); tagípan (Pamp.).

CARYOTA MAJESTICA Linden in Ill. Hort. 28 (1881) 16, nomen nudum; Bece. in Perk. Frag. Fl. Philip. (1904) 48, Webbia 1 (1905) 335, Philip. Journ. Sci. 14 (1919) 338.

Luzon (Rizal), Merrill 1892, 8490. In forests at low and medium altitudes. Endemic.

Local name: Aníbong (Tag.).

This is perhaps C. rumphiana Mart. var. philippinensis Becc.; the material is imperfect. 
CARYOTA MITIS Lour. Fl. Cochinch. (1790) 569, ed. Willd. (1793) 697; Becc. \& Hook. f. Fl. Brit. Ind. 6 (1892) 423; Becc. in Philip. Journ. Sci. 14 (1919) 338, Leafl: Philip. Bot. 8 (1919) 3020.

Caryota: sobolifera Wall. Cat. (1848) No. 8594; Naves Novis. App. (1882) 279.

Palawan, Elmer 12606, F. B. 3838,4148 Curran. In forests near streams at low altitudes; cultivated in Manila for ornamental purposes. Burma and Indo-China to the Malay Peninsula, Sumatra, Java, and Borneo.

Local name: Bató (Tagb.).

CARYOTA RUMPHIANA Mart. var. PHILIPPINENSIS Becc. in Philip. Journ. Sci. 14 (1919) 337, Leafl. Philip. Bot. 8 (1919) 3019.

Caryota rumphiana Naves Novis. App. (1882) 274; Becc. in Webbia 1 (1905) 331, Perk. Frag. Fl. Philip. (1904) 48, vix Mart.

Caryota maxima Blume in Mart. Hist. Nat. Palm. 3 (1838) 195;

Merr. Fl. Manila (1912) 123.

Luzon (Cagayan, Rizal, Laguna, Tayabas, Sorsogon), Mindoro, Negros, Mindanao (Agusan, Davao, Cotabato), Elmer 10940, 13623b, 9301, 14929, 15572, 15931, Merrill 2006, 803\%, Whitford 1373, F. B. 10064, 19403, 17157, 17764 Curran, 19961 Villamil, 7.850 Curran \& Merritt, B. S. 13471 Foxworthy \& Ramos, 11579'Robinson. In primary forests at low and medium altitudes.

Var. OXYODONTA Becc. in Philip. Journ. Sci. 14 (1919) 337.

Luzon (Laguna), F. B. 10045 Curran. In forested valleys at medium altitudes. The two varieties endemic, the species widely distributed in Malaya.

Local names: Aníhung (It.) ; anívung (Ibn.) ; bagsang (Bis.); kalalios (Bis.) ; lumang (Ibn.) ; pogáhan (Mbo.) ; pola (Bag.); pugáhan (Tag.); pulá (Mag.).

\section{DOUBTFUL AND EXCLUDED SPECIES}

Caryota griffithil Becc.; Naves Novis. App. (1882) 279.

CARYota PROPINQUA Blume; Naves 1. c.

CARYoTa NANA Linden Ill. Hort. 28 (1881) 16, nomen nudum "Philippines." Caryota speciosa Linden 1. c. nomen nudum "Philippines."

\section{ARENGA Labillardière}

ARENGA AMBONG Becc. in Philip. Journ. Sci. 2 (1907) Bot. 229, 14 (1919) 335.

Arenga tremula Becc. op. cit. 4 (1909) Bot. 612, 14 (1919) 335, quoad descr., excl. syn., non Caryota tremula Blanco.

Wallichia oblongifolia Becc. in Webbia 1 (1905) 328, non Griff.

Luzon (Tayabas, Sorsogon), Palawan, Basilan, Balabac, Cebu, Mindanao (Zamboangra), Sulu Archipelago, Merrill.51379, 8367, Elmer 12596, 16237, Ahern 653, Copeland 1681, F. B. 10213, 10280, 3542 Curran, 4032 Hutchinson, B. S. 735, 10816 Foxworthy, 1737 McGregor. In forests at low altitudes. Endemic.

Local names: Ambúng (Sul.); banísan (Sul.); bat-bát (Tagb.).

ARENGA PINNATA (Wuirmb) Merr. Interpret. Herb. Amb. (1917) 119, Sp. Blancoanae (1918) 88; Brown \& Merr. in Philip. Bur. Forestry Bull. 18 (1919) 26, t. 78. 
Saguerus pinnatus Wurmb in Verh. Bat. Genoots. 1 (1779) 351.

Borassus gomutus Lour. F1. Cochinch. (1790) 618.

Arenga saccharifera Labill. in Mém. Inst. Paris 4 (1801) 209; F.-Vill.

Novis. App. (1882) 280; Vidal Sinopsis Atlas (1883) 42, t. 93, f. C;

Becc. in Perk. Frag. Fl. Philip. (1904) 48, Webbia 1 (1905) 328;

Merr. Fl. Manila (1912) 126.

Sagus gomutus Perr. in Mém. Soc. Linn. Paris 3 (1824) 142; C. B.

Rob. in Philip. Journ. Sci. 3 (1908) Bot. 306.

Caryota onusta Blanco Fl. Filip. (1837) 741, ed. 2 (1845) 511, ed. 3,3 (1879) 143, t. 419.

Arenga gamuto Merr. in Philip. Journ. Sci. 9 (1914) Bot.63. (There

is no "Saguerus gamuto Houtt." as indicated in literature.)

Luzon (Rizal, Cavite, Bataan, Laguna, Tayabas), Polillo, Biliran, Mindanao. Planted here and there about towns, and abundant in some forested areas but never at any great distance from settled areas; generally planted in most islands and provinces. It has all the appearance of an introduced palm that has become naturalized. Sugar palm.

Local names: Bagátbat (C. Bis.) ; bagóbat (C. Bis.) ; bat-bát (C. Bis.) ; hibiók (P. Bis.) ; hidiók (Bik., P. Bis.); hiliók (Mbo.); ibiók (C. Bis.); idióg (C. Bis.) ; idiók (C. Bis.) ; igók (P. Bis.) ; irók (Sbl.) ; kábo-négro (Tag.) ; káong (Tag.); káuing (Tag.) ; onau (C. Bis.) ; rapítan (Ilk.) ; unau (C. Bis.).

AREnga TREMulA (Blanco) Becc. in Philip. Journ. Sci. 4 (1909) Bot. 612, quoad syn. excl. descr.; Merr. Sp. Blancoanae (1918) 87; Brown \& Merr. in Philip. Bur. Forestry Bull. 18 (1919) 34, t. 9.

Caryota tremula Blanco Fl. Filip. (1837) 744, ed. 2 (1845) 512, ed. 3, 3 (1879) 144; Kunth Enum. 3 (1841) 549; Merr. in Govt. Lab. Publ. (Philip.) 27 (1905) 88.

Wallichia tremula Mart. Hist. Nat. Palm. 3 (1849) 315; Miq. Fl. Ind. Bat. 3 (1855) 34; Naves Novis. App. (1882) 280; Vidal Rev. Pl. Vasc. Filip. (1886) 279.

Arenga mindorensis Becc. in Perk. Frag. Fl. Philip. (1904) 48, Webbia 1 (1905) 329, Philip. Journ. Sci. 14 (1919) 355.

Saguerus mindorensis O. F. Cook in Invent. Seeds Import. U. S. Dept. Agr. 33 (1915) 10.

Didymosperma tremulum Wendl. \& Drude in Kerch. Palm. (1878) 243.

Luzon (Bataan, Batangas, Laguna, Tayabas), Mindoro, Mindanao (Davao), Merrill 1790, Sp. Blancoanae 828, Elmer 18472, 11192, Baker 1807. F. B. 10203,5469 Curran, 8768 Merritt, B. S. 12395 McGregor, 13102 Foxworthy \& Ramos, 19468 Ramos. In thickets and secondary forests at low altitudes. Endemic.

Local names: Abígi (Bik.) ; abíki (Bik.); báris (Bag.); bat-bát (Tagb.) ; bilis (Bik.) ; dayáka (Tag.); dumáka (Tag.) ; dumayáka (Tag.); gumáka (Bik.) ; gumayáka (Tag.) ; rumáka (Bik.); tipon-tipon (Bik.).

\section{DOUBTFUL AND EXCLUDED SPECIES}

Arenga manillensis Wendl. Index Palm. (1864) 3, nomen nudum; Naves Novis. App. (1882) 280.

Saguerus manillensis Lodd. 1. c. in syn.

A nomen nudum and hence indeterminable; probably the same as one of the three species listed above. 
AREnGA obtusifolia Mart.; Naves Novis. App. (1882) 280.

A species of the Malay Peninsula allied to A. pinnata (Wurmb) Merr.; not known from the Philippines.

\section{ORANIA ZippeI}

ORANIA DECIPIENS Becc. in Philip. Journ. Sci. 4 (1909) Bot.614, 14 (1919) 333.

Mindoro, F. B. 4120 Merritt. In primary forests at low altitudes. Endemic.

Var. MINDANAOENSIS Becc. in Philip. Journ. Sci. 14 (1919) 333.

Mindanao (Zamboanga), F. B. 9179 Whitford \& Hutchinson. In primary forests at low altitudes. Endemic.

Var. MONTANA Becc. in Leafl. Philip. Bot. 8 (1919) 3018, Philip. Journ. Sci. 14 (1919) 333.

Mindanao (Agusan, Davao), Elmer 11881, 13970. In forests, altitude 800 to $1,000 \mathrm{~m}$. Endemic.

Local name: Báñga (Tag., P. Bis., Bag., Mbo.).

ORANIA PALINDAN (Blanco) Merr. in Govt. Lab. Publ. (Philip.) 27 (1905) 88, Philip. Journ. Sci. 1 (1906) Suppl. 32, Sp. Blancoanae (1918) 88; Brown \& Merr. in Philip. Bur. Forestry Bull. 18 (1919) 110 , t. 42.

Caryota palindan Blanco Fl. Filip. ed. 2 (1845) 513, ed. 3, 3 (1879) 145.

Orania regaiis Naves Novis. App. (1882) 279, non Blume.

Orania philippinensis Scheff. ex Becc. in Ann. Jard. Bot. Buitenz. 2 (1885) 156; Becc. in Webbia 1 (1905) 335, Philip. Journ. Sci. 14 (1919) 332.

Luzon (Cagayan, Union, Benguet, Zambales, Bataan, Rizal, Batangas, Laguna, Tayabas, Camarines), Samar, F. B. 1610 Borden, 5818, 10410, 17068, 10708 Curran, 8413 Curran \& Merritt, 2183 Meyer, 20988 Villamil, B. S. 12391 McGregor, 24107, 24131 Ramos, Elmer 61, 74, 18248, Merrill Sp. Blancoanae 144, Phil. Pl. 1900. In forests at low and medium altitudes. Endemic.

Var. SIBUYANENSIS (Becc.) comb. nov.

Orania philippinensis Scheff. var. sibuyanensis Becc. in Leafl. Philip.

Bot. 8 (1919) 3017, Philip. Journ. Sci. 14 (1919) 332.

Sibuyan, Elmer 12066. In primary forests, altitude about $225 \mathrm{~m}$. Endemic.

Local names: Ambobá đga (Ibn.); báñga (Buk., P. Bis.); barangói (Tag., Pamp., Sbl.); niogniógan (Tag.); palịndán (Tag.).

ORANIA PARAGUANENSIS Becc. in Webbia 1 (1905) 335; Philip. Journ. Sci. 14 (1919) 332.

Palawan, Merrill 869, 9269, Phil. Pl. 1316. In primary forests at low altitudes; probably not distinct from $O$. palindan Merr., which it exactly resembles in habit.

Local name: Báñga (Tagb.). 183036-11 
ORANIA RUBIgINosA Becc. in Philip. Journ. Sci. 14 (1919) 333.

Luzon (Cagayan, Isabela, Tayabas), B. S. 10658, 10575 McGregor, 13390 Ramos, F. B. 17259,17192 Curran. In forests at low altitudes. Endemic.

Local name: Kalahán (Ibn.).

\section{EXCLUDED SPECIES}

Orania MaCrocladus Mart.; Naves Novis. App. (1882) 280.

\section{HETEROSPATHE Scheffer}

Heterospathe ElATA Scheff. in Ann. Jard. Bot. Buitenz. 1: (1876) 141, 162; Becc. Webbia 1 (1905) 328, Philip. Journ. Sci. 14 (1919) 325, Leafl. Philip. Bot. 8 (1919) 3013; Brown \& Merr. in Philip. Bur. Forestry Bull. 18 (1919) 86, t. 32.

Luzon (Cagayan, Laguna, Tayabas, Camarines), Mindoro, Masbate, Cebu, Samar, Panay, Siquijor, Dinagat, Bucas Grande, Mindanao (Davao, Zamboanga), F. B. 10352, 10481, 13238, 17064 Curran, 9684 Merritt, 19749 Villamil, 9030 Whitford \& Hutchinson, B. S. 35243, 34624 Ramos \& Pascasio, Whitford 1395, Elmer 11968, 18222, Merrill 2770. In forests at low altitudes, sometimes planted. Moluccas; introduced in Guam.

Local names: Balaniúg (Sub.); dagumáka (Ibn.); dayumáka (Ibn.); dumayáka (Ibn.) ; niogniógan (Tag.); sagasí (Tag.); sagisí (Bik., Tag., S. L. Bis.) ; salanióg (Bag.); tagisí (Bik.).

Heterospathe NegRosensis Becc. in Philip. Journ. Sci. 4 (1909) Bot. 611, 14 (1919) 326, Leafl. Philip. Bot. 8 (1919) 3014.

Luzon (Tayabas, Sorsogon), Negros, Panay, Elmer 9439, 10147, 17297, Whitford 1539, F. B. 13639 Curran \& Foxworthy, B. S. 9443,9358 Robinson, 30752, 28684 Ramos \& Edaño. In forests at medium altitudes. Endemic.

Heterospathe PHILIPPINENSIS Becc. in Philip Journ. Sci. 4 (1909)

Bot. 610, 14 (1919) 326, Leafl. Philip. Bot. 8 (1919) 3013.

Ptychoraphis phitippinensis Becc. in Ann. Jard. Bot. Buitenz. 2 (1885) 90, Malesia 3 (1886) 109, Webbia 1 (1905) 47.

Rhopaloblaste sp. Vidal Phan. Cuming. Philip. (1885) 153.

Luzon (Apayao, Ifugao, Benguet, Nueva Vizcaya, Rizal, Laguna, Camarines, Sorsogon), Leyte, F. B. 10856, 10892 Curran, 18019 Merritt, 21752 Fischer, B. S. 19784, 19785 McGregor, 8198, 15260 Ramos, 283.91 Fénix, 33606 Ramos \& Edaño, Elmer 16519. In primary forests at medium altitudes, ascending to $1,600 \mathrm{~m}$ or higher. Endemic.

Local names: Sanakti (Ig.); tagisí (Bik.).

HeTERospathe sibuyAnensis Elm. Leafl. Philip. Bot. 8 (1919) 3014, Philip. Journ. Sci. 14 (1919) 325.

Sibuyan, Elmer 12350. In forests, altitude about $225 \mathrm{~m}$. Endemic. Local name: Bilis (C. Bis.).

\section{ONCOSPERMA Blume}

ONCOSPERMA GRAcILIPES Bece. in Philip. Journ. Sci. 2 (1907) Bot. 228, 4 (1909) Bot. 610, 14 (1919) 330, Leafl. Philip. Bot. 8 (1919) 3017. 
Luzon (Laguna, Tayabas, Sorsogon), Biliran, Merrill 4010, Elmer 15223, Baker 3704, B. S. 18490, 22876 McGregor, 13213 Ramos, F. B. 10148, 10396 Curran, 26801 Mabesa. In forests at low altitudes. Endemic.

Local name: Aníbong (Tag.).

ONCOSPERMA HORRIDUM (Grifü.) Scheff. in Nat. Tijdschr. Nederl. Ind. 32 (1871) 189; Becc. in Philip. Journ. Sci. 4 (1909) Bot. 610, 6 (1911) Bot. 230, 14 (1919) 330, Leafl. Philip. Bot. 8 (1919) 3017.

A reca horrida Griff. in Calcutta Journ. Nat. Hist. 5 (1845) 465.

Polillo, Mindanao (Agusan, Zamboanga, Davao), Elmer 118\%6, 13886, Copeland 1626, B. S. 3277 Robinson. In forests at low and medium altitudes, ascending to $800 \mathrm{~m}$. Malay Peninsula and Archipelago.

Local names: Aníbong (Tag., Mbo.); tanáian (Bag.).

ONCOSPERMA PLATYPHYLLUM Becc。in Philip. Journ. Sci. 4 (1909) Bot. 609, 14 (1919) 330.

Negros, Whitford $1670, F . B .17347$ Curran. In forests at low and medium altitudes. Endemic.

Local name: Aníbong (P. Bis.).

ONCOSPERMA TIGILLARIA (Jack) Ridl. in Journ. Str. Branch Roy. As. Soc. 33 (1900) 173.

Areca tigillaria Jack Malay Miscel. $2^{7}$ (1820) 88.

Oncosperma filamentosa Blume Rumphia 2 (1836) 97, t. 82, 103; Becc. in Philip. Journ. Sci. 4 (1909) Bot. 610, 14 (1919) 330, Leafl. Philip Bot. 8 (1919) 3016.

Areca nibung Mart. Hist. Nat. Palm. 3 (1838) 173, t. 153; Naves Novis. App. (1882) 279.

Palawan, Elmer 12662, F. B. 3790 Curran. In somewhat swampy places immediately back of the mangrove more or less subject to the influence of brackish water. Malay Peninsula and Archipelago.

Local name: Aníbong (Bis., Tagb.).

17. ACTINORHYTIS Wendland and Drude

ACTINORHYTIS CALAPPARIA (Blume) Wendl. \& Drude in Linnaea 39 (1875) 184; Becc. in Leafl. Philip. Bot. 8 (1919) 3010, Philip. Journ. Sci. 14 (1919) 324.

Areca calapparia Blume Rumphia 2 (1836) 70, in nota, t. 100, f. 2.

Seaforthia calapparia Mart. Hist. Nat. Palm. 3 (1849) 313.

Ptychosperma calapparia Miq. Fl. Ind. Bat. 3 (1855) 20; Naves Novis. App. (1882) 377.

Mindanao (Davao), Elmer 11238. Isolated trees in settled areas, altitude about $500 \mathrm{~m}$, undoubtedly introduced. Widely distributed in the Malay Archipelago.

Local name: Tañgálo (Bag.).

\section{PTYCHORAPHIS Beccari}

PTYCHORAPHIS CAGAYANENSIS Becc. in Philip. Journ. Sci. 14 (1919) 326.

Heterospathe cagayanensis Becc. op. cit. 4 (1909) Bot. 611.

Luzon (Cagayan), F. B. 12286 Klemme, 16757 Curran. In primary forests at low altitudes. Endemic.

Local name: Dagumáka (Ibn.). 
PTYCHORAPHIS ELMERI Becc. in Philip. Journ. Sci. 14 (1919) 328, Leafl. Philip. Bot. 8 (1919) 3010.

Heterospathe elmeri Becc. in Leafl. Philip. Bot. 2 (1909) 646.

Negros, Elmer 9669. In forests, altitude about 1,000 m. Endemic.

Local name: Bilísan (C. Bis.).

PTYCHORAPHIS INTERMEDIA Becc. in Leafl. Philip. Bot. 8 (1919) 3011,

Philip. Journ. Sci. 14 (1919) 328.

Samar, Biliran, Mindanao (Agusan), Elmer 13663, B. S. 18107 McGregor, 24753 Ramos. In forests 600 to $1,000 \mathrm{~m}$ and above. Endemic.

Local names: Lubilúbi (S. L. Bis.); marighoi (Mbo.).

PTYCHORAPHIS MICROCARPA Becc. in Philip. Journ. Sci. 14 (1919) 327.

Camiguin de Misamis, B. S. 14678 Ramos. In forests at medium altitudes. Endemic.

\section{ADONIDIA Beccari}

ADONIDIA MERRILLII (Becc.) Becc. in Philip. Journ. Sci. 14 (1919) 329; Brown \& Merr. in Philip Bur. Forestry Bull. 18 (1919) 15, t. 2, 3 .

Normanbya merrillii Becc. in Philip. Journ. Sci. 14 (1909) Bot. 606, t. 30, 31, Leafl. Philip. Bot. 8 (1919) 3009; Merr. Fl. Manila (1912) 127.

Actinorhytis calapparia Vidal Sinopsis Atlas (1883) 42, t. 94, f. B, non Wendl. \& Drude.

Coron, Palawan, Elmer 12708, Merrill 9415. On limestone formations in open places, often subgregarious, and in sandy soil near the seashore at low altitudes. Cultivated in Manila for ornamental purposes since about 1875. A monotypic, endemic genus.

Local names: Búnga de chína (Tag.) ; búnga de jolo (Tag.) ; oring-óring (Tagb.).

\section{PINANGA Blume}

PINANGA BARNESII Becc. in Webbia 1 (1905) 320, Philip. Journ. Sci. 3 (1908) Bot. 340, 6 (1911) Bot. 229, 14 (1919) 320, Leaf. Philip. Bot. 8 (1919) 3002.

Pinanga barnesii Becc. var. macrocarpa Becc. in Philip. Journ. Sci. 2 (1907) Bot. 227.

Babuyan Islands, Luzon (Cagayan, Union, Pampanga, Rizal, Bataan, Laguna, Camarines, Tayabas), Polillo, Catanduanes, Mindoro, Panay, Siargao, Mindanao, F. B. 122 Barnes, 2762 Meyer, 6309, 10757, 17756 Curran, 6853, 11385 Merritt, B. S. 13248, 13419 Ramos, 6937 Robinson, 10466, 23202 McGregor, 4144 Fénix, 28525, 3120\%, 33700 Ramos \& Edaño, 13248, 13419, 13858, 30528 Ramos, 34862 Ramos \& Pascasio, 2003 Foxworthy, McGregor 275, Elmer 7924, 9297, Whitford 799. In primary forests chiefly at medium altitudes, ascending to $1,600 \mathrm{~m}$. Endemic.

Local names: Abisi (Tag.); bubulin (Sub.); búñgang-matsíng (Tag.); gasígan (Neg.); katidi (Ig.) ; sauag (Sub.).

PINANGA BASILANENSIS Bece. in Philip. Journ. Sci. 14 (1919) 322.

Mindanao (Zamboanga), Basilan, B. S. 16119 Reillo, 3662, 36623 Ramos \& Edaño. In primary forests at low or medium altitudes. Endemic.

Local name: Buburis (Sub.). 
PINANGA BATANENSIS Bece. in Philip. Journ. Sci. 3 (1908) Bot. 340, 14 (1919) 322.

Batan Islands (Batan), B. S. 3841 Fénix. Along mountain streams in forests. Endemic.

Local name: Dapiau (Iv.).

PINANGA COPELANDII Becc. in Webbia 1 (1905), 317, 14 (1919) 320, Leafl. Philip. Bot. 8 (1919) 3002.

Luzon (Camarines, Sorsogon), Negros, Mindanao (Agusan, Davao), Weber 1134, Copeland 1283, Elmer 10467, 13875, Whitford 1669, Merrill 7281, Phil. Pl.1594, B. S. 15918 F'énix, 23619 Ramos, F. B. 2415a Cortez \& Valderrama. In damp primary forests at low and medium altitudes, ascending to $1,200 \mathrm{~m}$. Endemic.

Local names: Abikí (Bik.); bagtoan (Mbo.); habikí (Bik.); hiliuásiu (P. Bis.) ; tambiñálan (Mbo.) ; timbañgalan (Bag.).

PINANGA CURRANII Becc. in Philip. Journ. Sci. 2 (1907) Bot. 226, 14 (1919) 320.

Palawan, F. B. 3515 Curran. In primary forests at low altitudes. Endemic.

PINANGA GeONOMAEFORMIS Becc. in Philip. Journ. Sci. 4 (1909) Bot. 602, 14 (1919) 318.

Luzon (Rizal, Laguna, Tayabas), F. B. 10155 Curran, 26797 Mabesa, B. S. 20474 Ramos, 28565 Ramos \& Edaño. In damp primary forests at low and medium altitudes. Endemic.

PINANGA HeterophyLLA Becc. in Philip. Journ. Sci. 14 (1919) 319.

Luzon (Sorsogon), Negros, F. B. 19346 Curran, 23635 Ramos. In primary forests, altitude 100 to $700 \mathrm{~m}$. Endemic.

PINANGA INSIGNIS Bece. in Philip. Journ. Sci. 2 (1907) Bot. 223, 14 (1919) 322, Leafl. Philip. Bot. 8 (1919) 3004.

Luzon (Apayao, Pangasinan, Nueva Vizcaya, Rizal, Laguna, Camarines, Tayabaș, Sorsogon), Mindoro, Leyte, Mindanao (Agusan, Zamboanga, Surigao, Bukidnon), Elmer 8028, 13950, 14909, 15445, 17656, Whitford 1388, Bolster 222, Wenzel 534, Merrill Phil. Pl. 1135, B. S. 11233 McGregon, 33512 Ramos \& Edaño, 15111, 13236 Ramos, 26067, 29969, 28237 Fénix, 25566 Yates, F. B. 9502 Merritt, 10491 Curran, 7791 Curran \& Merritt. In primary forests at low and medium altitudes. Endemic.

Var. GASTERoCARPA Becc. in Philip. Journ. Sci. 14 (1919) 322.

Masbate, Negros, F.B. 17848 Curran, 11555 Rosenbluth, 13560 Meyer \& Foxworthy. In primary forests at low and medium altitudes. The Luzon material placed here by Beccari I refer to the typical form of the species. Endemic.

Var. LePTOCARPA Bece. 1. c.

Negros, Whitford 1621. In primary forests at low and medium altitudes. Endemic. 
Subsp. LOHERIANA Becc. 1. c.

Luzon (Laguna), (Loher 7092), B. S. 9789 Robinson. In forests on Mt. Banahao, altitude about $1,000 \mathrm{~m}$. Endemic.

Local names: Bañga (Tag.) ; saláuak (Sub.) ; sarámau (Tag.) ; saráuag (P. Bis., Mbo.) ; takón (Ibn.) ; tibanglán (Tag.) ; tibanglóan (Bik., S. L. Bis.).

PINANGA ISABELENSIS Becc. in Philip. Journ. Sci. 14 (1919) 318.

Luzon (Isabela), B. S. 10660 McGregor. In forests near the sea at low altitudes. Endemic.

PINANGA MACULATA Porte in Ill. Hort. 10 (1863) 92, t. 361 ; Drude in Bot. Zeit. 35 (1877) 636, t. 5, f. 12-13; Becc. Malesia 3 (1886) 145, Webbia 1 (1905) 325, Perk. Frag. Fl. Philip. (1904) 48, Philip. Journ. Sci. 14 (1919) 317; C. H. Wright in Curtis's Bot. Mag. IV 1 (1905) t. 8011.

Palawan, Merrill 712 . In primary forests at an altitude of about $500 \mathrm{~m}$, a juvenile sterile specimen, its identity with Porte's species problematical.

I have observed juvenile forms of Pinanga with mottled leaves in Laguna and Pampanga Provinces, Luzon; and in Mindoro. Endemic.

PINANGA MODESTA Becc. in Philip. Journ. Sci. 2 (1907) Bot. 225, 14 (1919) 318.

Bucas Grande, Mindanao (Davao, Bukidnon, Lanao, Agusan, Zamboanga), Basilan, Clemens 48\%, Copeland, Merrill 8302, B. S. 15751 Fénix, 35098 Ramos \& Pascasio, 39002 Ramos \& Pascasio, F. B. 3987 Hutchinson, 4583 Mearns \& Hutchison. In primary forests at low and medium altitudes, ascending to $1,200 \mathrm{~m}$. Endemic.

Local names: Lampigi (Lan.); mambuding (Yak.); tigahui (Buk.).

PINANGA NEGRosensIS Becc. in Leafl. Philip. Bot. 2 (1909) 642, 8 (1919) 3006, Philip. Journ. Sci. 14 (1919) 322.

Negros, Dinagat, Elmer 10030, B. S. 35182 Ramos \& Pascasio. In damp forested ravines, altitude about $900 \mathrm{~m}$. Endemic.

PINANGA PHILIPPINENSIS Becc. in Malesia 3 (1887) 180, Perk. Frag. Fl. Philip. (1904) 48, Webbia 1 (1905) 324; Merr. in Philip. Journ. Sci. 1 (1906) Suppl. 32; Becc. in Philip. Journ. Sci. 14 (1919) 320, Leafl. Philip. Bot. 8 (1919) 3003; Brown \& Merr. in Philip. Bur. Forestry Bull. 18 (1919) 112, t. 44.

Pinanga elmeri Becc. in Webbia 1 (1905) 322, Philip. Journ. Sci. 3 (1908) Bot. 341, 14 (1919) 320, Leafl. Philip. Bot. 8 (1919) 3004; Merr. Philip. Journ. Sci. 1 (1906) Suppl. 32, 2 (1907) Bot. 264.

Babuyan Islands, Luzon (Cagayan, Ilocos Norte, Ifugao, Bontoc, Benguet, Pangasinan, Nueva Vizcaya, Bulacan, Rizal, Bataan, Zambales, Laguna, Tayabas, Sorsogon), Mindoro, Leyte, Negros, Panay. Common on exposed ridges in the mossy forest, sometimes in ravines, altitude 800 to $1,800 \mathrm{~m}$; represented by numerous collections. Endemic.

Local names: Búa-ti-báked (Ilk.); búa-ti-bákis (Ilk.); buñgañó-dakígan (Ilk.) ; gatílei (Bon.) ; katídai (Ig.); katíldi (Ig.); lubiá (Tag.) ; mamá (Ilk.); matsing (Tag.). 
PINANGA RIGIDA Becc. in Leafl: Philip. Bot. 2 (1909) 644, 8 (1919) 3005, Philip. Journ. Sci. 14 (1919) 321.

Luzon (Tayabas), Negros, Elmer 10187, B. S. 28732 Ramos \& Edaño. In the mossy forest, altitude 1,200 to' $1,800 \mathrm{~m}$. Endemic.

Elmer 10147 is not cited by Beccari in the original description; this is pretty clearly referable to Heterospathe negrosensis Becc. The type, Elmer 10187, is clearly a Pinanga. This note is published in reference to Elmer's statement, Leafl. Philip. Bot. 8 (1919) 3006, that Pinanga rigida Becc. was probably referable to Heterospathe or Ptychoraphis.

PINANGA SAMARANA Becc. in Philip. Journ. Sci. 14 (1919) 321.

Samar, B. S. 17535 Ramos. In forests at low or medium altitudes. Endemic.

PINANGA SCLEROPHYLLA Becc. in Philip. Journ. Sci. 4 (1909) Bot. 603, 14 (1919) 322.

Mindoro, F. B. 4468 Merritt. In primary forests, altitude about 1,500 m. Endemic.

PINANGA SIBUYANENSIS Becc. in Philip. Journ. Sci. 14 (1919) 324, Leaf. Philip. Bot. 8 (1919) 3006.

Sibuyan, Elmer 12425. In forests along streams, altitude about $600 \mathrm{~m}$. Endemic.

Local name: Tibañán (C. Bis.).

PINANGA SPECIOSA Becc. in Webbia 1 (1905) 316, Philip. Journ. Sci. 14 (1919) 323, Leafl. Philip. Bot. 8 (1919) 3005.

Mindanao (Agusan, Lanao, Zamboanga, Davao), Copeland 1265, Elmer 10484, 13739, 13941, 14023, Clemens 374, F. B. 9271 Whitford \& Hutchinson, 28206 Elumir. In primary forests, altitude 400 to $1,200 \mathrm{~m}$. Endemic.

Local names: Banísan (Sub.); sakolon (Mbo.); saráuag (Bag:); sadáuag (Bag.).

PINANGA URDANETANA Becc. in Leafl. Philip. Bot. 8 (1919) 3008, Philip. Journ. Sci. 14 (1919) 321.

Mindanao (Agusan), Bukidnon, Elmer 14137, B. S. 38612, 38611, 38610 , $385 \% 0$ Ramos \& Edaño. On forested slopes, altitude about $1,400 \mathrm{~m}$. Endemic.

Local names: Sagasí (Buk.); sagisí (Buk.); salang-ísog (Mbo.).

PINANGA URosPeRmA Becc. in Philip. Journ. Sci. 3 (1908) Bot. 341.

Babuyan Islands (Camiguin), Luzon (Apayao), B. S. 4044, 28038 Fénix. On forested slopes at medium altitudes. Endemic.

Local name: Lagás (Ibn.).

PINANGA WOODIANA Becc. in Philip. Journ. Sci. 4 (1909) Bot. 604, 14 (1919) 322, Leafl. Philip. Bot. 8 (1919) 3003.

Luzon (Bontoc), Mindoro, Mindanao (Davao), Merrill 5680, Elmer $10485,11334, B . S .38078,40651$ Ramos \& Edaño. In primary forests, altitude 1,050 to $1,350 \mathrm{~m}$. Endemic:

Local names: Irár (Bag.) ; mumuing (Mang.). 


\section{ARECA Linnaeus}

ARECA CATECHU Linn. Sp. Pl. (1753) 1189 (err. cathecu); Blanco Fl. Filip. (1887) 714, ed. 2 (1845) 494, ed. 3,3 (1879) 129, t. 350 ; Miq. Fl. Ind. Bat. 3 (1855) 8; Vidal Sinopsis Atlas (1883) 42, t. 9.4, f. $A$; Naves Novis. App. (1882) 278; Becc. in Philip. Journ. Sci. 3 (1908) Bot. 340, 6 (1911) Bot. 229; Merr. in Philip. Jour'n. Sci. 1 (1906) Suppl. 32, F1. Manila (1912) 128, Sp. Blancoanae (1918) 89 ; Becc. in Leafl. Philip. Bot. 8 (1919) 2997, Philip. Journ. Sci. 14 (1919) 304; Brown \& Merr. in Philip. Bur. Forestry Bull. 18 (1919) 20,t. 4, 5.

Areca alba "Rumph;" Naves Novis. App. (1882) 278.

Cultivated throughout the settled areas of the Philippines, in some places spontaneous; possibly a native of the Philippines, having been found once spontaneous in primary forests in Palawan. Old World Tropics generally, introduced in the New World.

Var. BATANENSIS Becc. in Philip. Journ. Sci. 14 (1919) 304.

Batan Islands, B. S. 3824 Fénix. Along a mountain stream. Endemic.

Var. LoNGICARPA Becc. in Philip. Journ. Sci. 6 (1911) Bot. 229, 14 (1919) $304, t .2, f .2$.

Polillo, B. S. 10470 McGregor. Probably from planted trees. Endemic.

Local names: Boá (Ilk., Ig., Ibn.) ; buá (Ibn.) ; buñga (Tag., Bis., Sul.) ; dapiau (Iv.) ; huá (It.) ; lúgos (Sul.) ; lúyos (Pamp.); pasa (Yak.) takobtob (Bik.); vuá (Ibn.); va (It.).

Var. SilvaticA Becc. 1. c. $p l .1, f .2$.

Palawan, Merrill 944\%. In primary forests at a place where an old trail crosses a small stream near Lake Manguao. Endemic.

ARECA CALIso Becc. in Leafl. Philip. Bot. 8 (1919) 2998, Philip. Journ. Sci. 14 (1919) 310.

Luzon (Sorsogon), Biliran, Leyte, Samar, Mindanao (Agusan, Davao), Elmer 11898, 13814, 14839, B. S. 18875 McGregor, 24130, 15388 Ramos, 24881 Edaño. In forests, altitude 350 to $850 \mathrm{~m}$. Endemic.

Local names: Kalíso (Bag.); sakolon (Mbo.).

ARECA CAMARINENSIS Becc. in Philip. Journ. Sıi. 14 (1919) 309, $t$. \&, f. \&.

Luzon (Camarines), F. B. 14268 Aguilar, B. S. 33829 Ramos \& Edaño. In forests at low altitudes. Endemic.

Local name: Monó (Bik.).

ARECA CostulatA Becc. in Philip. Journ. Sci. 14 (1919) 312.

Leyte, Dinagat, B. S. 15236 Ramos, 35242 Ramos \& Pascasio. In primary forests at low and medium altitudes. Endemic.

ARECA HUtChinsoniAnA Becc. in Philip. Journ. Sci. 14 (1919) 312.

Areca mammillata Bece. var. mindanaoensis Becc. op. cit. 4 (1909) Bot. b02. 
Mindanao (Lanao, Zamboanga), Basilan, F. B. 9141 Whitford \& Hutchinson, 4019 Hutchinson, 23159 Agama, 25242 Alvarez, B. S. 36953,37288 Ramos \& Edaño, 38364 Brown. In primary forests at low altitudes. Endemic.

Local names: Pisa (Yak.); pitha (Sub.); sambuláyan (Sub.).

ARECA IPOT Becc. in Leafl. Philip. Bot. 2 (1909) 639, 8 (1919) 2998, Philip. Journ. Sci. 6 (1911) Bot. 229 (var. polillensis Becc.) 14 (1919) 308, t. 3, f. 4; Merr. Sp. Blancoanae (1918) 89.

Areca catechu Linn. var. humilis Blanco Fl. Filip. (1837) 716, ed. 2 (1845) 495, ed. 3, 3 (1879) 120; Merr. in Govt. Lab. Publ. (Philip.) 27 (1905) 88.

Areca glandiformis Naves Novis. App. (1882) 278 (prob.!), non Lam.

Luzon (Laguna, Tayabas), Polillo, Merrill Sp. Blancoanae 844, $F . B$. 10178 Curran, B.S.6938, 9712 Robinson, 10469, 10463 McGregor. Planted in and about towns, and along streams in forests at low and medium altitudes. Endemic.

Local names: Buñgañípot (Tag.) ; ípod (Tag.) ; ípot (Tag.) ; mañipod (Tag.) ; saksíg (Tag.) saksík (Tag.).

ARECA MACRocARPA Becc. in Philip. Journ. Sci. 4 (1909) Bot. 601, 14 (1919) 306, t. $3, f .2$.

Mindanao (Zamboanga), F. B. 9103 Whitford \& Hutchinson. Probably but a form or variety of Areca catechu Linn. Endemic.

ARECA MAM MILLATA Bece. in Philip. Journ. Sci. 2 (1907) Bot. 220.

A reca vidaliana Becc. op. cit. 222, 14 (1919) 312.

Palawan, Merrill Phil. Pl. 1464, F'. B. 3816 Curran, B. S. 21575 Escritor, 619 Foxworthy. In swampy forests at low altitudes, and also on forested slopes. Endemic.

Local names: Boga (Tagb.); pita (Tagb.).

ARECA PARENS Becc. in Philip. Journ. Sci. 14 (1919) 307, t. $s$, f. 1.

Luzon (Camarines), F. B. 21712 Peñas, Soriano, \& Abellanosa. Along streams at low altitudes. Endemic.

Local name: Takobtób (Bik.).

ARECA WHITFORDII Becc. in Philip. Journ. Sci. 2 (1907) Bot. 219, 14 (1919) 306, Leafl. Philip. Bot. 8 (1919) 2997.

Luzon (Sorsogon), Mindoro, Whitford 1872, Elmer 16240. In semiswampy forests at low altitudes. Endemic.

Var. LUZONENSIS Becc. in Philip. Journ. Sci. 14 (1919) 306.

Luzon (Cagayan, Nueva Vizcaya), B. S. 11384 McGregor, F. B. 17770 Curran. Wild in forests and in river swamps at low and medium altitudes. Endemic.

Local name: Buñgañ-gúbat (Tag.).

\section{EXCLUDED SPECIES}

Areca triandra Roxb.; Naves Novis. App. (1882) 278. 


\section{ELAEIS Jacquin}

ELAEIS GUINEENSIS Jacq. Select. Am. (1763) 280, $t .172 ;$ Merr. Fl. Manila (1912) 126; Brown \& Merr. in Philip. Bur. Forestry Bull. 18 (1919) 84, t. 30, 31.

Cultivated for ornamental purposes, and producing fruits abundantly. A native of tropical Africa. African oil palm.

23. cocos Linnaeus

cocos NucIFERA Linn. Sp. P1. (1753) 1188; Blanco Fl. Filip. (1837) 716, ed. 2 (1845) 495 (nucigera), ed. 3, 3 (1879) 123, t. 364; Naves Novis. App. (1882) 282; Vidal Sinopsis Atlas (1883) 42, t. 94, f. $D$; Miq. Fl. Ind. Bat. 3 (1855) 64 (incl. var. macrocarpa Miq. var. saccharina Miq.); Merr. Fl. Manila (1912) 125, Sp. Blancoanae (1918) 89, 90.

Cocos mamillaris Blanco Fl. Filip. (1837) 722 (mammilaris), ed. 2 (1845) 499, ed. 3, 3 (1879) 123.

Very extensively cultivated in the Philippines, especially in those regions where the dry season is not too prolonged; one of the most important agricultural crops of the Archipelago. Pantropic in cultivation, probably a native of Polynesia, certainly not a native of the Philippines. Coconut.

Local names: Coco (Sp.) ; cocotéro (Sp.) ; gira-gíra (Sbl.) ; i-ing (It.) ; iniúg (Ibn.) ; lobí (S. L. Bis.) ; lubí (C. Bis.) ; nióg (Ilk., Bik., Bis., Ibn.,

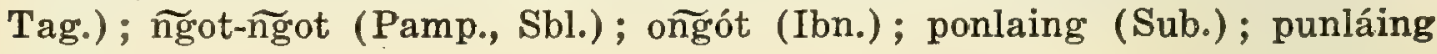
(Yak.).

\section{NYPA Wurmb}

\section{(Nipa Thunb.)}

NYPA FRUTICANS Wurmb in Verh. Bat. Genoots. 1 (1779) 350; Miq. Fl. Ind. Bat. 3 (1855) 150; Naves Novis. App. (1882) 284; Vidal Sinopsis Atlas (1883) 42, t. 94, f. C; Blanco Fl. Filip. ed. 2 (1845) 461 , ed. 3, 3 (1879) 68, t. 386; Becc. in Webbia 1 (1905) 357; Merr. Fl. Manila (1912) 125, Sp. Blancoanae (1918) 90; Brown \& Merr. in Philip. Bur. Forestry Bull. 18 (1919) 98, t. 38-41 (mostly as Nipa).

Nipa fructicans Thunb. in Vet. Akad. Nya Handl. Stockh. 3 (1782) 231.

Nipa fruticosa Lam. ex Perr. in Mem. Soc. Linn. Paris 3 (1824) 132; C. B. Rob. in Philip. Journ. Sci. 3 (1908) Bot. 306.

Nipa litoralis Blanco Fl. Filip. (1837) 662.

Along tidal streams in brackish swamps throughout the Philippines, in favorable habitats gregarious over large areas. India, through Malaya to tropical Australia.

Local names: Anípa (Ibn.) ; lasá (Tag.) ; nipá (Sp.-Fil.) ; paúid (Tag.) ; pinóg (It.) ; pinók (Ibn.) ; sagá (Sbl.) ; sasá (Pamp., Tag.), tatá (Ibn.).

\section{EXCLUDED GENERA AND SPECIES}

Bentinckia RendA Mart.; Naves Novis. App. (1882) 280.

Borassus dichotoma White; Naves op. cit. 281.

BorassUS FLABELLIFORMIS Linn.; Naves op. cit. 280. 
Calyptrocalyx spicatus Blume; Naves op. cit. 280.

Chamaerops excelsa Thunb.; Naves op. cit. 282.

Ceratolobus glaucescens Blume; Naves op. cit. 273.

Drymophloeus appendiculatus Becc.; Naves op. cit. 277.

Iguanura sp.; Vidal Rev. Pl. Vasc. Filip. (1886) 279; Ceron Cat. Pl. Herb. (1892) 174.

Kentia molucCANa Becc.; Naves 1. c.

LODOICEA SEYCHELLARUM Labill.; Naves op. cit. 281.

Nenga latisecta Blume; Naves op cit. 277.

PholidocarPus IHUR Blume; Naves op. cit. 281.

Plectocomia elongata Mart.; Naves op. cit. 274.

Ptychosperma cochinchinensis Miq.; Naves op. cit. 277.

Ptychosperma kuhlit Miq.; Naves 1. c.

Ptychosperda PUNicea Miq.; Naves 1. c.

PTYCHOSPERMA RUMPHII Blume; Naves 1. c.

Ptychosperia saxatilis Blume; Naves 1. c.

Ptychosperma vestiaria Miq.; Naves 1. c.

Rhapis arundinacea Llanos in Mem. Acad. Cienc. Madrid III 2 (1857) 499; F.-Vill. \& Naves in Blanco Fl. Filip. ed. 3, $4^{1}$ (1880) 100 ; Naves Novis. App. (1882) 282, nomen nudum.

Rhapis Flabelliformis L'Hérit.; Naves Novis. App. (1882) 282.

This is occasionally cultivated in Manila gardens as a pot plant, but it never produces flowers here.

RhaPis JavanicA Blume; Naves 1. c.

The above twenty-two species were credited to the Philippines by Naves, but none of the genera has known indigenous representatives in the Archipelago. A few of the species in the list now occur as recently introduced and cultivated forms.

Since about 1905 a large number of palms have been introduced into Manila for cultivation as ornamental species. Some of these are now mature and flower and fruit freely; others have not as yet produced flowers. None of them occurs outside of cultivation, and for the most part they are as yet rare in cultivation. The list of these recently introduced and cultivated palms includes such species as: Acoelorrhaphe wightii Wendl., Archontophoenix alexandrae H. Wendl. \& Drude, Attalea cohune HBK., Chrysalidocarpus lutescens Wendl., Cocos plumosa Hook., Coccothrinax garberi Sarg., Cyrtostachys lakka Becc., Dictyosperma alba Wendl. \& Drude, Dypsis madagascariensis Nichols, Howea belmoreana Becc., Hyophorbe amaricaulis Mart., Latania commersonii Gmel., L. loddigesii Mart., Livistona australis Mart., L. chinensis R. Br., Martinezia caryotaefolia HBK., Oreodoxia regia HBK., Phoenix pusilla Gaertn., P. roebelenii O'Brien, P. rupicola T. And., Pinanga kuhlii Blume, Pritchardia gaudichaudii Wendl., P. pacifica Seem. \& H. Wendl., Ptychosperma macarthurii 
H. Wendl., Raphia ruffia Mart., Sabal adansonii Guerns., S. blackburneanum Glazebr., S. mauritiiforme Griseb. \& Wendl., S. palmetto Lodd., Thrinax argentea Lodd., $T$. robusta Wendl., and Washingtonia filifera Wendl.

\section{ARACEAE}

\section{POTHOS Linnaeus}

POThos ACUMINAtissimus Merr. in Philip. Journ. Sci. 11 (1916) Bot. 175.

Samar, B. S. 24125 Ramos. In primary forests at low altitudes. Endemic.

POTHOS CYLINDRICUS Presl Epim. (1851) 243; Walp. Ann. 3 (1853) 501; Schott Aroid. 1 (1853) 23, t. 48, Prodr. (1860) 569; Miq. Fl. Ind. Bat. 3 (1855) '180; Engl. in DC. Monog. Phan. 2 (1879) 86, Pflanzenreich 21 (1905) 36, f. 17, D, E; Vidal Phan. Cuming. Philip. (1885) 154, Rev. P1. Vasc. Filip. (1886) 282; Naves Novis. App. (1882) 287.

Luzon (Camarines, Albay), Leyte, Panay, Mindanao (Davao), Cuming 914, Williams 2937, Piper 481, Wenzel 387, B. S. 22143 Ramos, 32451 McGregor. In primary forests at low and medium altitudes. Endemic.

Local name: Palipí (Bik.).

POTHOS DOLICHOPHYLLUS Merr. in Philip. Journ. Sci. 11 (1916) Bot. 4.

Luzon (Sorsogon), Samar, B. S. 17628, 23708 Ramos. In primary forests at low altitudes. Endemic.

POTHOS HERMAPHRODITUS (Blanco) Merr. Sp. Blancoanae (1918) 90.

Batis hermaphrodita Blanco Fl. Filip. (1837) 791, ed. 2 (1845) 541, ed. 3, 3 (1879) 197.

Pothos longifolius Presl Epim. (1851) 242; Schott Aroid. 1 (1853) 22, t. 34, Prodr. (1860) 563; Walp. Ann. 3 (1853) 501; Miq. Fl. Ind. Bat. 3 (1856) 180; Naves Novis. App. (1882) 262; Engl. in DC. Monog. Phan. 2 (1879) 82, Pflanzenreich 21 (1905) 25, f. 9; Vidal Phan. Cuming. Philip. (1885) 154, Rev. Pl. Vasc. Filip. (1886) 282; Merr. Interpret. Herb. Amb. (1917), 124.

Pothos chapelieri Naves Novis. App. (1882) 286, non Schott.

Luzon (Pangasinan, Rizal, Laguna, Tayabas), Samar, Leyte, Panay, Siargao, Mindanao (Davao), Cuming 682, Merrill 2792, Sp. Blancoanae 57, Phil. Pl. 4, Elmer 18005, Wenzel 265, Loher 7045, B. S. 348\%1 Ramos \& Pascasio, 13622, 24475 Ramos, 19211 Reillo, 16859 Serviñas, F. B. 24911 Mabesa. In primary forests at low and medium altitudes. Sumatra, Java, Celebes, Moluccas.

Local names: Apis (Tag.); hipan (Bik.); oro-óla (Bik.).

POTHOS INAEQUILATERUS (Presl) Engl. in DC. Monog. Phan. 2 (1879) 88, Pflanzenreich 21 (1905) 37; Naves Novis. App. (1882) 287.

Scindapsus inaequilaterus Presl Epim. (1851) 241; Walp. Ann. 3 (1853) 500.

Pothos cummingianus Schott in Oester. Bot. Wochenbl. (1855) 19, Prodr. (1860) 570; Walp. Ann. 5 (1858) 910 (cumingianus); Miq. Fl. Ind. Bat. 3 (1856) 181; Vidal Rev. Pl. Vasc. Filip. (1886) 282, Phan. Cuming. Philip. (1885) 154. 
Luzon (Albay, Sorsogon), Samar, Leyte, Cuming 908, Wenzel 35\%, 1110, Elmer 17050 as $P$. peninsularis Elm., B. S. 24474 Ramos. In primary forests at low and medium altitudes. Endemic.

POTHOS LUzONENSIS (Presl) Schott in Oester. Bot. Wochenbl. (1855) 19, Aroid. 1 (1853) 25, t. 56, Prodr. (1860) 574; Miq. Fl. Ind. Bat. 3 (1856) 182; Walp. Ann. 5 (1858) 910; Naves Novis. App. (1882) 287; Engl. in DC. Monog. Phan. 2 (1879) 93, Pflanzenreich 21 (1905) 42; Vidal Rev. Pl. Vasc. Filip. (1886) 282, Phan. Cuming. Philip, (1885) 154.

Goniurus luzonensis Presl Epim. (1851) 244; Walp. Ann. 3 (1853) 500.

Luzon (Zambales, Rizal, Bataan, Camarines), Cuming 1045, Merrill Phil. Pl. 1529, Loher 7036, r03\%, F. B. 5948 Curran. In primary forests at. low and medium altitudes. Endemic.

Local name: Uarat-uárat (Bik.).

POTHOS OVATIFOLIUS Engl. in Pflanzenreich 21 (1905) 40.

Luzon (Tayabas, Camarines, Sorsogon), Samar, Mindanao (Agusan), Ahern 155, Elmer 14014, 15204, B. S. 24543 Ramos, 28549 Ramos \& Edaño, $F$. B. 12247 Curran. In primary forests at low or medium altitudes. Endemic.

Local names: Kalípkip (Tag.) ; kalot-kágot (Bik.) ; maláang-lakop-lákop (S. L. Bis.).

POTHOS PHILIPPINENSIS Engl. in Pflanzenreich 21 (1905) 315; Merr. in Philip. Journ. Sci. 1 (1906) Suppl. 32.

Luzon (Apayao, Nueva Ecija, Bulacan, Bataan, Tayabas), Samar, $F . B$. 369 Barnes, B.S. 34031,36414 Ramos \& Edaño, 20742 Escritor, 28190 Fénix, 13054 Ramos, 1640 Foxworthy, Whitford 341, 1046bis, Williams 486. In primary forests at low and medium altitudes. Endemic.

Local names: Bagubalának (S. L. Bis.) ; baladaúan (Bik., Tag.) ; bayámi (Tag.) ; malagayámon (Tag.) ; oroalaypan (Bik.) ; tibátib (Tag.) ; tagoápi (Bik.).

POTHOS RUMPHII Schott Melet. 1 (1832) 21; Engl. Pflanzenreich 21 (1905) 38, f. 16; Naves Novis. App. (1882) 287; Merr. Interpret. Herb. Amb. (1917) 124.

Scindapsus mumphii Presl Epim. (1851) 241.

Luzon (Rizal, Laguna, Tayabas, Camarines, Albay, Sorsogon), Negros, Samar, Panay, Leyte, Mindanao, Basilan, B. S. 310\%8, 28585 Ramos \& Edaño, 24144, 22132, 19367 Ramos, 15435 Reillo, F. B. 9656 Curran, Loher 7033, Wenzel 22, Clemens 685, Merrill 7309, Weber 1044, Elmer 15854 as $P$. clemensii Elm. Celebes, Moluccas, New Guinea.

Local names: Amlóng (Bik., Bis., Tag.) ; bislag (Mbo.) ; dílang-báka (Tag.) ; takúling (C. Bis.).

EXCLUDED SPECIES

Pothos barberianus Schott; Naves Novis. App. (1882) 287.

Pothos becCarianus Engl:; Naves 1. c.

Pothos gracilis Roxb.; Naves 1. c.

Pothos LePtostachyus Schott; Naves 1. c. 
Pothos Longipes Schott; Naves 1. c.

Pothos LOUREIRI Hook.; Naves 1. c.

Ротноs MACRophyllus De Vriese; Naves op. cit. 286.

Pothos oxyphyllus Miq.; Naves op. cit. 287.

Pothos RoXbURghi De Vriese; Naves op. cit. 286.

Pothos sumatranus Miq.; Naves op cit. 287.

Pothos vRIESEanus Schott; Naves op. cit. 286.

Pothos zollingerI Schott; Naves op. cit. 287.

Pothos celatocaulis N. E. Br. in Gard. Chron. 13 (1880) 200; Engl. in Bot. Jahrb. 25 (1898) 5, Pflanzenreich 37 (1908) 44.

This was described from Bornean material, and Engler, 1. c., referred to it Warburg 14603, from Davao, Mindanao. Later, Pflanzenreich 21 (1905) 44, Engler admits the species as one of doubtful status from Borneo only. It is probably a Rhaphidophora, $R$. celatocaulis v. A. v. R. in Bull. Jard. Bot. Buitenz. 1 (1920) 382.

Pothos scandens Linn. Engl. Pflanzenreich 21 (1905) 26, quoad Philip.; Naves Novis. App. (1882) 286; Vidal Rev. Pl. Vasc. Filip. (1886) 177.

Vidal 1990 is referred here by Engler, who gives the range of the species as from India to Indo-China, Java, Sumatra, and Borneo. I suspect the Philippine specimen to be referable to $P$. hermaphroditus Merr. in which species the relative length and width of the petiole and lamina are exceedingly variable.

\section{ANADENDRON Schott}

ANADENDRON MONTANUM (Blume) Schott in Bonplandia 5 (1857) 45, Prodr. (1860) 391; Engl. in DC. Monog. Phan. 2 (1879) 97, Pflanzenreich 21 (1905) 49; Naves Novis. App. (1882) 287.

Calla montana Blume Cat. Gew. Buitenz. (1823) 62.

Mindanao (Zamboanga), B. S. 37023 Ramos \& Edaño. In primary forests at low altitudes. Siam and Indo-China to the Malay Peninsula, Sumatra, Java, and Borneo.

\section{EXCLUDED SPECIES}

Anadendron AFFine Schott; Naves Novis. App. (1882) 287.

\section{POTHOIDIUM Schott}

POTHOIDIUM LOBBIANUM Schott in Oester. Bot. Wochenbl. 7 (1857) 70, Aroid. 1 (1857) 26, t. 57, Gen. Aroid. (1858) 96, t. 96, Prodr. (1860) 576; Naves Novis. App. (1882) 287; Engl. in DC. Monog. Phan. 2 (1879) 95, Pflanzenreich 21 (1905) 46; Merr. in Philip. Journ. Sci. 1 (1906) Suppl. 32.

Northern Luzon (Cagayan) to Palawan and Mindanao, in most or all islands and provinces. In primary forests at low and medium altitudes. A monotypic genus. Celebes, Ternate.

Local names: Aríuan (Ibn.); balong-kahínai (P. Bis.) ; barálta (Tag.) ; magutapílak (P. Bis.) ; malagayáman (Sbl.) ; pakokaláñgan (Mbo.) ; vara alta (Sp.). 


\section{ACORUS Linnaeus}

ACorus CALAMUS Linn. Sp. Pl. (1753) 324; Miq. Fl. Ind. Bat. 3 (1856) 175; Naves Novis. App. (1882) 287; Engl. in DC. Monog. Phan. 2 (1879) 216, Pflanzenreich 21 (1905) 309; Merr. Fl. Manila (1912) 130.

Luzon (Bontoc, Benguet), Merrill Phil. Pl. 1789, Vanoverbergh 2585. In swamps, altitude about $1,300 \mathrm{~m}$, here native or thoroughly naturalized; cultivated here and there at low altitudes. North temperate regions generally.

Local names: Acóro (Sp.) ; bueng (Pamp.) ; dálau (Ilk.) ; dárau (Ilk.) ; déngau (Bon.) ; lubígan (Tag.).

ACORUS GRAMINEUS Soland. in Ait. Hort. Kew 1 (1789) 474; Engl. Pflanzenreich 21 (1905) 312; Merr. in Philip. Journ. Sci. 10 (1915) Bot. 288.

Luzon (Laguna), B. S. 14949 Ramos. On rocks or ledges in beds of small streams at low or medium altitudes. Japan to southern China.

\section{RHAPHIDOPHORA Hasskarl}

RHAPHIDOPHORA ACUMINATA Merr. in Philip. Journ. Sci. 10 (1915) Bot. 265.

Luzon (Batangas, Sorsogon), Leyte, Wenzel 1139, B. S. 23300 Ramos, 22669 Ramos \& Deroy. In primary forests at low altitudes. Endemic.

RHAPHIDOPHORA COPELANDII Engl, in Bot. Jahrb. 37 (1905) 115;

Engl. ex Krause in Pflanzenreich 37 (1908) 49.

Rhaphidophora trinervia Elm. Leafl. Philip. Bot. 8 (1919) 3073.

Luzon (Benguet, Ifugao, Laguna), Mindanao (Davao), Copeland 1193, Merrill 7700, Williams 2605, Elmer 18.057, B. S. 19706 McGregor. In forests and on ledges in thickets, altitudes 400 to $1,600 \mathrm{~m}$. Endemic.

RHAPHIDOPHORA ELMERI Engl. \& Krause in Engl. Bot. Jahrb. 44 (1910) Beibl. 101: 11.

Luzon (Tayabas, Sorsogon), Negros, Elmer (9286, 9268), 10135 a, 15116. In forests at medium altitudes. Endemic.

RHAPHIDOPHORA GRANDIFOLIA Krause in Engl. Bot. Jahrb. 44 (1910) Beibl. 101: 11.

Negros, Elmer 9464. In forests. Endemic.

Local name: Dakoling (C. Bis.).

RHAPHIDOPHORA LAGUNENSIS Elm. Leafl. Philip. Bot. 8 (1919) 2072.

Luzon (Laguna), Elmer 17812, Pascasio. In the mossy forest, summit of Mt. Maquiling, altitude about 1,100 m. Endemic.

RHAPHIDOPHORA MERRILLII Engl. Bot. Jahrb. 37 (1905) 115; Engl. \& Krause in Engl. Pflanzenreich 37 (1908) 51; Merr. in Philip. Journ. Sci. 1 (1906) Suppl. 32, 5 (1910) Bot. 335, Fl. Manila (1912) 129, Sp. Blancoanae (1918) 90.

Pothos pinnata Blanco Fl. Filip. (1837) 646, ed. 2 (1845) 450, ed. 3,3 (1879) 48, t. 339, non Linn.

Epipremnum merrillii Engl. \& Krause in Engl. Pflanzenreich 37 (1908) 137. 
Luzon (Bontoc, Benguet, Union, Nueva Vizcaya, Zambales, Bataan, Rizal, Laguna, Tayabas, Sorsogon), Polillo, Palawan, Leyte, Negros, Mindanao, Merrill 9434, Sp. Blancoanae 715, Elmer 5539, 7296, 15135 as Epipremnum glaucocephalum Elm., B. S. 11292, 10370 McGregor, 6004, 5883 Curran. In thickets and forests at low and medium altitudes. Endemic.

Local names: Amíling (Is.) ; amlóng (Bik., Bis.) ; amólong (Ilk.) ; amúling (Is.) ; bagák (S. L. Bis.) ; bakág (Bik.) ; balikupkúp (Bis.) ; balision (Is.) ; bisako (Bis.) ; daila (Bis.) ; dibátib (Bis.); dukúp (Bon.); garban (Bis.) ; garusíba (Ibn.) ; gátgati (Bon.); gayáman (Sbl.); horoa (Bis.) ; kígau (Bon.); kilat (Sbl.); malapakpák-baláuai (Tag.); mamisi (Ig.); maragayáman (Sbl.); tabátib (Tag.); takóling (C. Bis.); takótin (Bis.); tampinbanal (Tag.); tibátib (Tag.).

RHAPHIDOPHORA MONTICOLA Krause in Engl. Bot. Jahrb. 44 (1910) Beibl. 101: 12.

Luzon (Laguna, Sorsogon), Negros, Leyte, Elmer 10098, 16790 as $R$. bulusanensis Elm., Merrill 7154, Wenzel 1160, B. S. 23455 Ramos, F. B. 21387 Villamil. In primary forests ascending into the mossy forest, altitude 700 to $1,200 \mathrm{~m}$. Endemic.

RHAPHIDOPHORA PERKINSIAE Engl. in Engl. Bot. Jahrb. 37 (1905)

115; Engl. \& Krause in Engl. Pflanzenreich 37 (1908) 37.

Luzon (Benguet, Bataan, Rizal, Laguna, Tayabas), Elmer 6305, Loher 7035, Whitford 1169, Merrill 9667, F. B. 26747 Mabesa, 7826 Curran \& Merritt. In primary forests on trees and in thickets about limestone cliffs and bowlders, altitude 1,000 to $1,600 \mathrm{~m}$. Endemic.

Local names: Ámlong (Tag., Ig.); madulúnang (Ig.).

RHAPHIDOPHORA PHILIPPINENSIS Engl. \& Krause in Engl. Pflanzenreich 37 (1908) 137.

Samar, Mindanao (Agusan, Lanao), Clemens 1229, Elmer 13776, B. S. 24405 Ramos. In forests at low and medium altitudes. Endemic.

Local name: Mananáu (S. L. Bis.).

RHAPHIDOPHORA RIGIDA Krause in Leafl. Philip. Bot. 6 (1914) 2284.

Luzon (Sorsogon), Negros, Elmer 10220, 15056. In primary forests, altitude about $1,250 \mathrm{~m}$; apparently very near $R$. philippinensis Engl. \& Krause.

RHAPHIDOPHORA STENOPHYLLA Elm. Leafl. Philip. Bot. 8 (1919) 3073.

Luzon (Laguna), Elmer 18449. Margins of forests at low altitudes. Endemic.

RHAPHIDOPHORA TODAYENSIS Krause in Leafl. Philip. Bot. 6 (1914) 2284.

Mindoro, Mindanao (Davao), Elmer 10849, B. S. 39735 Ramos. In forests at low altitudes. Endemic.

RHAPHIDOPHORA WARBURGII Engl. Bot. Jahrb. 37 (1905) 116; Engl.

\& Krause in Engl. Pflanzenreich 37 (1908) 51.

Luzon (Cagayan or Isabela), Warburg in herb. Berol. Probably from primary forests at low altitudes. Endemic. 
EXCLUDED SPECIES

RHAPHIDOPhora decursiva Schott; Naves Novis. App. (1882) 288.

RhaPhidophora fallax Schott; Usteri Beitr. Ken. Philip. Veg. (1905) 130.

RHAPHIDOPHORA LANCIfolra Schott; Naves Novis. App. (1882) 288.

RHAPHIDOPHORA MONTANA Schott; Naves 1. c.

RHAPHIDOPHORA PEEPla Schott; Naves 1. c.

RHAPHIDOPHORA PERTÚSA Schott; Naves 1. c.

RHAPHIDOPHORA SYLVESTRIS Engl.; Naves 1. c.

\section{EPIPREMNUM Schott}

EPIPREMNUM ELMERIANUM Engl. ex Engl. \& Krause in Pflanzenreich 37 (1908) 66.

Leyte, Elmer 7295. In forests at low and medium altitudes. Endemic. EPIPREMNUM PHILIPPINENSE Engl. \& Krause in Engl. Pflanzenreich 37 (1908) 137.

Luzon (Laguna, Tayabas, Sorsogon), Elmer 7623, 9253, 14522, 15113 as E. miniatum Elm., 16422 as E. sorsogonense Elm., B. S. 10052, 20548, 23299 Ramos, 35419 Martelino \& Edaño. In forests, altitude about $650 \mathrm{~m}$. Endemic.

EPIPREMNUM PINNATUM (Linn.) Engl. Pflanzenreich 37 (1908) 60, f. 2.5 .

Pothos pinnata Linn. Sp. Pl. ed. 2 (1763) 1374.

Epipremnum robinsonii Krause in Notizbl. Kgl. Bot. Gart. Berlin 5 (1912) 266.

Luzon (Tayabas), Polillo, Mindanao (Davao), Whitford 746, B. S. 15866 Fénix, Robinson 9181, Elmer 16061 as E. multicephalum Elm. In forests near the seashore. Tenasserim to New Guinea and Polynesia.

Local names: Lukmói (Tag.); tibátib (Tag.).

EPIPREMNUM TRUNCATUM Engl. \& Krause in Engl. Pflanzenreich 37 (1908) 63.

Luzon (Camarines, Sorsogon), Biliran, Leyte, Mindanao (Agusan), Elmer 7291, 13729, 14721, Merrill 7349, F. B. 27244 Palma, B. S. 18925 McGregor. In primary forests at low altitudes. Endemic.

Jocal names: Lukmói ('Tag.) ; pasagianit (Mbo.).

EXCLUDED SPECIES

EPIPREMNUm gIGANTEUm Schott; Naves Novis. App. (1882) 288.

\section{EPIPREMNOPSIS Engler}

EPIPREMNOPSIS HUEGELIANA (Schott) Engl. in Engl. \& Krause Pflanzenreich 37 (1908) 138.

Rhaphidophora huegeliana Schott in Bonplandia 5 (1857) 45, Prodr. (1860) 384.

Epipremnum mirabile Naves Novis. App. (1882) 288; Vidal Rev. Pl. Vasc. Filip. (1886) 281; Engl. in Bot. Jahrb. 37 (1905) 119; Usteri Beitr. Ken. Philip. Veg. (1905) 130, non Schott. $183036-12$ 
Epipremnum medium Engl. in DC. Monog. Phan. 2 (1879) 250, quoad Philip.; Naves Novis. App. (1882) 289.

Epipremnopsis media Engl. ex Engl. \& Krause in Pflanzenreich 37 (1908) 1, quoad Philip.

Batan and Babuyan Islands, Luzon (Bataan, Laguna, Camarines, Sorsogon), Catanduanes, Panay, Mindanao (Agusan, Zamboanga, Cotabato), B.S. 35662 Ramos \& Edaño, 22852 McGregor, 11716 Robinson, 3902 Fénix, Elmer 16052, 18223, Merrill 8071. In forests at low and medium altitudes. Endemic.

Local names: Lusígut (Ilk.) ; takhib (Iv.).

\section{SCINDAPSUS Schott}

SCINDAPSUS AUREUS (Lind. \& André) Engl. in Pflanzenreich 37 (1908) 80; Merr. Fl. Manila (1912) 139.

Pothos aurea Lindl. \& André in Ill. Hort. (1880) 69, t. 381.

Cultivated for ornamental purposes, but never observed in flower. A native of the Solomon Islands.

SCINDAPSUS CURRANII Engl. \& Krause in Pflanzenreich 37 (1908) 138.

Luzon (Cagayan, Laguna, Tayabas, Sorsogon), Mindoro, Negros, Camiguin de Misamis, F. B. 8014, 8027 Curran \& Merritt, 13711 Curran, 11376 Merritt, 26916 Mabesa, 25707 Foxworthy, B. S. 24064 Ramos, Elmer 18475, 14590, 16556. In primary forests at low altitudes. Endemic.

Local name: Lukmói (Tag.).

SCINDAPSUS FALCIFOLIUS Engl: Bot. Jahrb. 37 (1905) 119; Engl. \& Krause in Pflanzenreich 37 (1908) 79.

Jolo, Warburg in herb. Berol. Celebes.

SCINDAPSUS HEDERACEUS Schott in Bonplandia 5 (1857) 45; Naves Novis. App. (1882) 289; Engl. \& Krause in Pflanzenreich 37 (1908) $76,138, f .32, E-N$.

Scindapsus pictus Hassk. var. argyraeus Engl. Bot. Jahrb. 25 (1898) 13, Pflanzenreich 37 (1908) 80.

Scindapsus argyraea Engl. in DC. Monog. Phan. 2 (1879) 255; Naves Novis. App. (1882) 289 (argyracea).

Luzon (Laguna, Sorsogon), Catanduanes, Negros, Leyte, F. B. 4274 Everett, 26436 Catalan, B. S. 15019, 12028, 30383 Ramos, 23151 McGregor, 29239 Ramos \& Edaño, Elmer 15230. In primary forests at low altitudes. Malay Peninsula and Cochinchina to Borneo, Sumatra, and Java.

Scindapsus argyraea Engl. as represented by Engl. Araceae exsic. 265 is a juvenile form with mottled leaves; this is common in Laguna Province, Luzon. In mature plants the leaves are usually not mottled.

Local names: Maragayáman (Ilk.); putoputóhan (Tag.).

\section{EXCLUDED SPECIES}

Scindapsus Pertusus Schott; Vidal Cat. Pl. Prov. Manila (1880) 47.

\section{SPATHIPHYLLUM Schott}

SPATHIPHYLLUM COMMUTATUM Schott in Oester. Bot. Wochenbl. 7 (1857) 158, Prodr. (1860) 424, Ann. Mus. Bot. Lugd.-Bat. 3 (1863) 79; Engl. in DC. Monog. Phan. 2 (1879) 230; Vidal Phan. Cuming. Philip. (1885) 154, Rev. Pl. Vasc. Filip. (1886) 281; Naves 
Novis. App. (1882) 288; Merr. in Philip. Journ. Sci. 2 (1907) Bot.

264; Engl. \& Krause in Pflanzenreich 37 (1908) 133, f. 55, A-G.

Luzon (Tayabas, Camarines, Albay, Sorsogon), Polillo, Biliran, Mindoro, Leyte, Mindanao, B. S. 33596, 38523 Ramos \& Edaño, 10401, 18707 McGregor, 23571, 15385 Ramos, 6476 Robinson, F. B. 11415 Merritt, Wenzel 235, Merrill 5486, 4080, Copeland 1263, Williams 2364. In damp primary forests at low and medium altitudes. Celebes, Amboina.

All other species of this genus are confined to tropical America.

\section{CYRTOSPERMA Griffith}

CYRTOSPERMA MERKUSII (Hassk.) Schott in Oester. Bot. Wochenbl. (1859) 61, Prodr. (1860) 403; Engl. in DC. Monog. Phan. 2 (1879) 271, Pflanzenreich 48 (1911) 20, f. $7, G-K$.

Lasia merkusii Hassk. Cat. Hort. Bogor. (1844) 59, Pl. Jav. Rar. (1848) 161.

Cyrtosperma griffithii Merr. in Philip. Journ. Sci. 2 (1907) Bot. 422 sphalm.

Luzon (Sorsogon), Mindoro, Samar, Leyte, Mindanao, Elmer 15234, Merrill 5218, B. S. 1515 Bermejos, 24267 Ramos, 6678 Robinson. In ravines along streams, probably often planted. Java and Borneo to New Guinea. Bis.).

Local names: Galiáng (Bik) ; palau (C. Bis.) ; palauán (S. L. Bis., P.

\section{AMORPHOPHALLUS Blume}

AMORPHOPHALLUS CAMPANULATUS (Roxb.) Blume ex Decne. in Ann. Mus. Hist. Nat. Paris 3 (1834) 366; Miq. Fl. Ind. Bat. 3 (1856) 201; Engl. in DC, Monog. Phan. 2 (1879) 309, Pflanzenreich 48 (1911) 76; Naves Novis. App. (1882) 289; Merr. Fl. Manila (1912) 130, Philip. Journ. Sci. 10 (1915) Bot. 290, Interpret. Herb. Amb. (1917) 127, Sp. Blancoanae (1918) 91.

Arum campanulatum Roxb. Pl. Coromandel 3 (1819) 69, t. 272.

Arum decurrens Blanco Fl. Filip. (1837) 656, ed. 2 (1845) 457, ed. 3,3 (1879) 62.

Amorphophallus decurrens Kunth Enum. 3 (1841) 581; Miq. F1. Ind.

Bat. 3 (1856) 202; Engl. in DC. Monog. Phan. 2 (1879) 319, Pflanzenreich 48 (1911) 108; Merr. in Philip. Journ. Sci. 10 (1915) Bot. 290.

Luzon, most or all provinces, Mindoro. In thickets and secondary forests, along roads, trails, etc., at low and medium altitudes in the settled areas, often common. India through Malaya to Polynesia.

Local names: Ánto (Bis.) ; ápon (Tag.); apong-ápong (Tag.); bagáng (Ibn.) ; bagóng (Bik., Sul.) ; oroi (Bis.) ; pamangkílon (Bis.) ; puñgápuñ

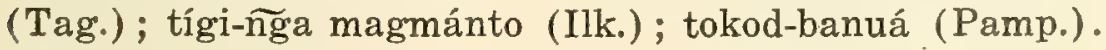

AMORPHOPHALLUS LONGISPATHACEUS Engl. \& Gehrm. in Engl. Pflanzenreich 48 (1911) 91.

Mindanao (Davao), Williams 2684. In thickets or forests at medium altitudes. Endemic.

AMORPHOPHALLUS LUZONIENSIS Merr. in Philip. Journ. Sci. 10 (1915) Bot. 289.

Luzon (Cagayan),F.B. 19560 Curran, Weber. In crevices of limestone ledges at low altitudes. Endemic. 
AMORPHOPHALLUS MERRILLII Krause in Notizbl. Kgl. Bot. Gart. Berlin 5 (1912) 266.

Panay (Batbatan Island), Cavilli, Merrill 7177, McGregor. Abundant in primary forests on Cavilli, a small island in Sulu Sea. Endemic.

AMORPHOPHALLUS RIVIERI Durieu Cat. Jard. Bordeaux (1869) 12, var. KONJAC (C. Koch) Engl. in DC. Monog. Phan. 2 (1879) 313, Pflanzenreich 48 (1911) 85.

Amorphophallus konjac C. Koch in Berl. Allg. Gartenz. (1858) 166.

Luzon (Rizal, Bataan, Laguna), Mindoro, Palawan, Balabac, Panay, Mindanao, McGregor 274, Elmer 18185, Merrill 9419, Whiford 1338, F. B. 17649 Curran, B. S. 26211 Ramos, 15641 Fénix. In thickets and forests at low altitudes. Indo-China, Japan.

Local names: Bulañg-an (Mang.); pungápuñg (Tag.).

AMORPHOPHALLUS VARIABILIS Blume in Rumphia 1 (1835) 146, $t$. 35, 37; Kunth Enum. 3 (1841) 33; Miq. Fl. Ind. Bat. 3 (1856) 200 ; Naves Novis. App. (1882) 290; Engl. in DC. Monog. Phan. 2 (1879) 313, Pflanzenreich 48 (1911) 80; Usteri Beitr. Ken. Philip. Veg. (1895) 130.

Luzon (Union), Mindanao (Davao), Basilan, Copeland 1264, B. S. 15444 Reillo, 9999 Robinson. In light forests at low altitudes. Java.

Local name: Bagóng (Bag.).

EXCLUDED SPECIES

Amorphophallus BUlbifer Blume; Naves Novis. App. (1882) 290.

AmorPhophallus dubius Blume; Naves op. cit. 289.

Amorphophallus giganteus Blume; Naves op. cit. 290.

AMORPHophallus titanum Becc.; Naves op. cit. 289.

AMORPHOPHALLUS TUBERCULIGERA Schott; Naves 1. c.

12. HOMALOMENA Schott

HOMALOMENA ELMERI Engl. ex Engl. \& Krause in Pflanzenreich 55 (1912) 42.

Palawan, Elmer 13229, F. B. 21586 Danao. In forests along streams at low altitudes; scarcely distinct from $H$. palawanensis Engl. \& Krause. Endemic.

homalomena PALAWANENSIS Engl, ex Engl. \& Krause in Pflanzenreich 55 (1912) 39, f. 19.

Palawan, B. S. 912 Foxworthy. Margins of pools at low altitudes. Endemic.

HOMALOMENA PHILIPPINENSIS Engl. ex Engl. \& Krause in Pflanzenreich 55 (1912) 55, f. 33.

Homalomena rubescens Kunth var. latifolia Engl. Bot. Jahrb. 55 (1898) 18. 
Luzon (Isabela, Union, Pangasinan, Pampanga, Rizal, Bataan, Laguna, Sorsogon), Mindoro, Palawan, Panay, Leyte, Biliran, Negros, Mindanao. Along small streams in primary forests at low altitudes; common. Endemic.

Local names: Alipaiu (Tag.); alupayi (Tag.); hayau (Sub.); payau (Tag.) ; salet-r̃ga-nalabága (Ilk.); tahíg (Bik.).

HOMALOMENA PYGMAEA (Hassk.) Engl. in Bot. Jahrb. 25 (1898) 18;

Engl. \& Krause in Engl. Pflanzenreich 55 (1912) 34, f. 15.

Aglaonema pygmaeum Hassk. in Hoev. \& De Vriese Tijdschr. 9 (1842)

161, Cat. Hort. Bogor. (1844) 57.

Chamaecladon lanceolatum Miq. Fl. Ind. Bat. 3 (1856) 212, t. 40;

Schott Gen. Aroid. (1858) t. 60.

Negros, Whitford 1591. Along stream beds, in cañons, altitude about $175 \mathrm{~m}$. Malay Peninsula, Sumatra, Java, Borneo, and Celebes.

\section{EXCLUDED SPECIES}

Homalomena AlbA Hassk.; Naves Novis. App. (1882) 290.

Homalomena aromatica Schott; Naves 1. c.

Homalomena COERULESCEns Jungh.; Naves 1. c.

Homalomena Minor Griff.; Naves 1. c.

Homalomena miqueliana Schott; Naves 1. c.

Homalomena Propinqua Schott; Naves 1. c.

Homalomena RUBescens Kunth; Naves 1. c.

Homalomena RUbra Hassk.; Naves 1. c.

Homalomena sagitTifolia Jungh.; Naves 1. c.

Homalomena cordatum Schott; Usteri Beitr. Ken. Philip. Veg. (1905) 130.

Homalomena paludosum Hook. f.; Usteri 1. c.

Homalomena rostratum Griff.; Usteri 1. c.

The last three species were credited to the Philippines on the basis of specimens collected in Negros by Usteri and determined by Engler. The latter author, however, does not credit them to the Philippines in his recent monographic treatment of the group.

\section{SCHISMATOGLOTTIS Zollinger and Moritzi}

SCHISMATOGLOTTIS CALYPTRATA (Roxb.) Zoll. \& Mor. Syst. Verz. (1854) 83; Naves Novis. App. (1882) 291; Usteri Beitr. Ken. Philip. Veg. (1905) 130; Merr. Interpret. Herb. Amb. (1917) 129; Engl. Pflanzenreich 55 (1912) 115 (var. concolor Hallier f.).

Luzon (Bataan, Cavite, Laguna, Tayabas, Albay), Samar, Leyte, Panay, Mindanao, Merrill 7603, 7273 (forma grandifolia Engl.), 7975, 6703, B. S. 24952 Fénix. In damp shaded ravines usually along streams on banks and cliffs at low and medium altitudes. Burma, through Malaya to New Guinea.

Local name: Alapáyi (Tag.). 
SCHISMATOGLOTTIS LATIFOLIA Miq. Fl. Ind. Bat. 3 (1856) 214, var. VIRIDIS Engl. ex Engl. \& Krause in Pflanzenreich 55 (1912) 117. Schismatoglottis luzonensis Engl. Pflanzenreich 55 (1912) 121, non Engl. op. cit. 88.

Alocasia merrillii Engl. \& Krause in Engl. Bot. Jahrb. 54 (1916)

Beibl. 118: 124, Pflanzenreich 71 (1920) 79.

Luzon (Pampanga, Nueva Vizcaya, Laguna, Camarines), Mindanao, B. S. 2015 Foxworthy, 33830, 33783 Ramos \& Edaño, 11248 McGregor, 6736 Robinson, F. B. 1243 Borden Copeland 1310, Baker 1034, Elmer 17829, Merrill 8072. In shaded ravines along streams on banks and cliffs at low and medium altitudes. Java, Celebes.

SCHISMATOGLOTTIS LUZONENSIS Engl. in Pflanzenreich 55 (1912) 88.

Luzon (Rizal), Panay, Merrill 5047, B. S. 30875 Ramos \& Edaño. On wet ledges in small stream beds at low altitudes. Endemic.

SCHISMATOGLOTTIS MERRILLII Engl. in Pflanzenreich 55 (1912) 89.

Luzon (Bontoc, Benguet), B. S. 12679 Fénix, 8772 McGregor, F. B. 5099 Curran. On ledges and cliffs in ravines along streams, altitude 1,200 to $1,600 \mathrm{~m}$. Endemic.

Local names: Atúped (Ilk.); píhau (Ig.); píkau (Bon.); picháu (Ig.). SCHISMATOGLOTTIS MINDANAOANA Engl. in Pflanzenreich 55 (1912) 103.

Mindanao (Agusan, Lanao, Davao), Merrill 7320, Elmer 13489, Clemens 1225. In damp primary forests along small streams at low and medium altitudes. Endemic.

SCHISMATOGLOTTIS PUSILLA Engl. in Bot. Jahrb. 1 (1881) 184, Pflanzenreich 55 (1912) 103,f.64.

Leyte, Jagor 1018a in herb. Berol. Endemic.

SCHISMATOGLOTTIS RAMOSII Engl, in Pflanzenreich 55 (1912) 98.

Schismatoglottis rizalensis Engl. op. cit. 100.

Luzon (Bulacan, Rizal), B. S. 3351, 13023 Ramos. On cliffs and bowlders along streams in shaded ravines at low altitudes. Endemic.

Local name: Talísig (Tag.).

SCHISMATOGLOTTIS WARBURGIANA Engl, in Bot. Jahrb. 55 (1898) 20, Pflanzenreich 55 (1912) 90, f. $5 \%$.

Mindanao (Davao), Warburg 14605 in herb. Berol. In forests up to $600 \mathrm{~m}$ altitude. Endemic.

\section{EXCLUDED SPECIES}

Schismatoglottis PICTA Schott; Naves Novis. App. (1882) 291.

SchismatoglotTis RUPestris Zoll. \& Mor.; Naves 1. c.

\section{AGLAONEMA Schott}

AGLAONEMA COMMUTATUM Schott Syn. (1856) 123, Prodr. (1860) 304, Gen. Aroid. (1859) t. 59; Regel Gartenflora (1865) t. 470; Engl. Pflanzenreich 64 (1919) 27, f. 10, G.

Aglaonema marantaefolium Blume var. maculatum Hook. in Curtis's Bot. Mag. III 21 (1865) t. 550a.

Aglaonema marantifolium Blume var. commutatum Engl. in DC. Monog. Phan. 2 (1879) 441. 
Not uncommon in cultivation in Manila as an ornamental on account of its mottled leaves. Widely cultivated in tropical botanical gardens, probably a native of the Moluccas.

For some years I thought that this might be Aglaonema maculatum Blume. Blume's species is based entirely on Dracunculo Luzonis primo seu indorum Gavay Sinbuga Camel in Ray Hist. Nat. 3 App. (1704) 36, and Camel's specimens were from Leyte and apparently from wild plants. I now suspect Aglaonema maculatum Blume to be a form of $A$. oblongifolium Kunth. There is no evidence that $A$. commutatum Schott is of ancient cultivation in the Philippines.

AGLAONEMA CORDIFOLIUM Engl. Pflanzenreich 64 (1915) 29, f. 14, $A-C$.

Mindanao (Davao), (Warburg 14604). In primary forests at low altitudes. Endemic.

AGLAONEMA DENSINERVIUM Engl. Bot. Jahrb. 37 (1905) 134, Pflanzenreich 64 (1915) 13.

Negros, Leyte, Siquijor, Mindanao, Basilan, Copeland 418, Piper 466, Clemens 476, Wenzel 1128, Williams 2429, Merrill 7347, 8204, F. B. 4774 Mearns \& Hutchinson, B. S. 26139 Fénix, 15452 Reillo. In primary forests at low and medium altitudes. Endemic.

AGLAONEMA HAENKEI (Presl) Schott in Bonplandia 7 (1859) 30, Prodx. (1860) 301; Naves Novis. App. (1882) 291; Engl. in DC. Monog. Phan. 2 (1879) 439, Pflanzenreich 64 (1915) 17; Vidal Phan. Cuming. Philip. (1885) 154, Rev. Pl. Vasc. Filip. (1886) 281; Merr. in Philip. Journ. Sci. 3 (1908) Bot. 400.

Scindapsus haenkei Presl Epim. (1851) 240; Walp. Ann. 3 (1853) 500; Miq. F1. Ind. Bat. 3 (1856) 183.

Caladium seguinum Presl Rel. Haenk. 1 (1827) 148, non Vent.

Babuyan Islands, Luzon (Apayao, Laguna, Cavite), Panay, Merrill 4186, Elmer 17668, B. S. 4089 Fénix, 35350 Martelino \& Edaño, 12404, 32590 McGregor, 10008 Ramos, F. B. 26047 Mabesa. In primary forests chiefly along streams at low and medium altitudes. Celebes.

AGLAONEMA OBLONGIFOLIUM (Roxb.) Kunth Enum. 3 (1841) 55; Merr. Interpret. Herb. Amb. (1917) 129.

Calla oblongifolia Roxb. Hort. Beng. (1814) 65, Fl. Ind. ed. 2, 3 (1832) 516.

Aglaonema marantifolium Blume Rumphia 1 (1835) 153, t. 66; Engl. in DC. Monog. Phan. 2 (1879) 441, Pflanzenreich 64 (1915) 27, t. 10, f. A-F; Naves Novis. App. (1882) 291; Merr. in Philip. Journ. Sci. 1. (1906) Suppl. 33.

? Aglaonema maculatum Blume Rumphia 1' (1835) 154; Kunth Enum. 3 (1841) 56; Miq. Fl. Ind. Bat. 3 (1856) 217; Schott Syn. (1856) 123, Prodr. (1860) 306, Engl. in DC. Monog. Phan. 2 (1879) 442, Pflanzenreich 64 (1915) 34.

Northern Luzon (Cagayan) to Mindanao, in most or all islands and provinces, incl. Elmer 16520 as $A$. alpinum Elm. In primary forests chiefly along streams; the leaves are sometimes mottled. Amboina, Banda.

Local names: Dampíng-banál (Tag.) ; pamitágen (Mang.) ; pauangkílon (C. Bis.). 
AGLAONEMA PHILIPPINENSE Engl. in Bot. Jahrb. 25 (1898) 21, Pflanzenreich 64 (1915) 29.

Aglaonema latifolium Engl. Bot. Jahrb. 37 (1905) 134.

Luzon (Laguna, Tayabas, Camarines), Panay, Mindanao (Davao), Gregory 86, B. S. 18139 Robinson, 23084, 23088, 22847, 23077 McGregor. In forests at low altitudes. Endemic.

\section{EXCLUDED SPECIES}

Aglaonema modestum Schott ex Engl. in DC. Monog. Phan. 2 (1879) 442, Pflanzenreich 64 (1915) 29, f. 13.

The type of this is a specimen collected by Gaudichaud supposedly in Luzon, but which in all probability came from Macao; the species occurs in southern China, but has not been detected in the Philippines. See Merrill in Philip. Journ. Sci. 13 (1918) Bot. 133.

Aglaonema PICtum Kunth; Naves Novis. App. (1882) 291.

Aglaonema simplex Schott; Naves Novis. App. (1882) 291; ? Vidal Rev. Pl. Vasc. Filip. (1886) 281.

Vidal's doubtful record for this Bornean species was based on his No. 1996 from San Mateo, Rizal Province, Luzon. I have not seen the specimen but suspect it to be the same as $A$. haenkei Schott or $A$. oblongifolium Kunth.

\section{ALOCASIA Necker}

ALOCASIA ATROPURPUREA Engl. Pflanzenreich 71 (1920) 132.

Luzon (Ifugao, Mt. Polis), B. S. 19663 McGregor. In the mossy forest at medium altitudes. Endemic.

ALOCASIA CULIONENSIS Engl. in Engl. Bot. Jahrb. 37 (1905) 135; Engl. \& Krause Pflanzenreich 71 (1920) 102.

Luzon (Pangasinan), Mindoro, Culion, Palawan, Panay, Merrill 555, 9819, 9824, 9507, B. S. 29883 Fénix, 31264 Ramos \& Edaño, 39602 Ramos. In primary forests at low altitudes. Endemic.

To be compared with $A$. longiloba Miq.

Alocasia Heterophylla (Pres1) Merr. in Philip. Journ. Sci. 3 (1908) Bot. 220, Fl. Manila (1912) 133; Engl. \& Krause Pflanzenreich 71 (1920) 101, f. 22.

Caladium heterophyllum Presl Rel. Haenk. 1 (1827) 148.

Colocasia? heterophylla Kunth Enum. 3 (1841) 40; Miq. Fl. Ind.

Bat. 3 (1856) 204; Schott Prodr. (1860) 139.

Alocasia warburgii Engl. in Engl. Bot. Jahrb. 25 (1898) 25; Merr.

in Philip. Journ. Sci. 1 (1906) Suppl. 33.

Alocasia manilensis Engl. Bot. Jahrb. 25 (1898) 23; Engl. \& Krause

Pflanzenreich 71 (1920) 95, f. 19, E, F.

Luzon (Ilocos Norte, Pampanga, Rizal, Bataan, Laguna, Tayabas, Camarines, Sorsogon), Polillo, Alabat, Panay, Negros, Samar, Jolo. In primary forests at low and medium altitudes. Alocasia manilensis Engl. is merely a juvenile form. Celebes.

Local name: Naroknarok (Mbo.). 
ALOCASIA MACRORRHIZA (Linn.) Schott Melet. (1832) 18; Engl. in DC. Monog. Phan. 2 (1879) 502; Naves Novis. App. (1882) 293; Merr. Interpret. Herb. Amb. (1917) 130, Sp. Blancoanae (1918) 91; Engl. \& Krause Pflanzenreich 71 (1920)' 84, f. 15. Arum macrorrhizon Linn. Sp. Pl. (1753) 965.

Calla maxima Blanco Fl. Filip. (1837) 658.

Arum grandifolium Blanco op. cit. ed. 2 (1845) 458, ed. 3, 3 (1879) $63, t .17 \%$.

Calla badian Blanco Fl. Filip. (1837) 658.

Alocasia indica Naves Novis. App. (1882) 293, Usteri Beitr. Ken. Philip. Veg. (1905) 130; Merr. Fl. Manila (1912) 133, Engl. \& Krause Pflanzenreich 71 (1920) 87, quoad Philip., non Schott.

Throughout the Philippines in old clearings and secondary forests at low and medium altitudes (incl. Elmer 17381 as A. talipan Elm.), the older plants often with a tall stout caudex. India to Malaya; planted for ornamental purposes in other tropical countries.

Local names: Abá (Ibn.) ; aba-ába (Ig.) ; badiáng (Tag., Bis.) ; bagiáng (Bis.) ; bíra (Ilk.) ; bíga (Tag., Ilk., Bis., Pamp.); bilbíla (Bon.) ; gabi (Bik.); galiáng (Bis.); gandus (Pamp.); malabíga (Tag.); ragiáng (Bis.); sininába (Ilk.); talípan (Bik.); taliáng (Bis.).

ALOCASIA MAQUILINGENSIS Merr. in Philip. Journ. Sci. 13 (1918) Bot 3.

Luzon (Laguna, Sorsogon), Baker 868, Merrill 7153, Elmer 17955, 16181 as $A$. vulcanica Elm., B. S. 6737 Robinson, 16926 Servinas. On slopes in primary forests, on Mt. Maquiling, altitude 100 to $600 \mathrm{~m}$. Endemic.

Alocasia MICHOLITZIANA Sander in Gard. Chron. 51 (1912) Suppl.

XV, f. 9; N. E. Br. in Curtis's Bot. Mag. IV 9 (1913) t. 8522;

Engl. \& Krause Pflanzenreich 71 (1920) 89.

Luzon (Apayao, Benguet), Merrill 9744, B. S. 28024, 28421 Fénix. In thickets about limestone bowlders, altitude about 1,300 m. Endemic.

AlocasiA PORTEI (Schott) Engl. \& Bece. in DC. Monog. Phan. 2 (1879)

645; Naves Novis. App. (1882) 294; Merr. Fl. Manila (1912) 132.

Schizocasia portei Schott in Bonplandia 10 (1862) 148; Engl. in DC.

Monog. Phan. 2 (1879) 495; Engl. \& Krause Pflanzenreich 71 (1920)

117.

Alocasia indica Schott var. heterophylla Engl. in DC. Monog. Phan. 2 (1879) 502.

Alocasia indica Schott var. diversifolia Engl. Pflanzenreich 71 (1920) 88.

Luzon (Bataan, Laguna, Camarines), Lyon, Holman. In old clearings at medium altitudes. Endemic.

Local name: Badiáng (Bik.).

Alocasia ReVersa N. E. Br. in Gard. Chron. III 8 (1890) 38; Hook.

f. in Curtis's Bot. Mag. III 52 (1896) t. 7498; Usteri Beitr. Ken.

Philip. Veg. (1905) 130; Engl. \& Krause Pflanzenreich 71 (1920) 95.

This was based on cultivated specimens supposedly of Philippine origin. I have seen no specimens. Endemic. 
Alocasia SANDERIANA Bull. Cat. (1894) 8, fig.; Merr. Fl. Manila

(1912) 132; Engl. \& Krause Pflanzenreich 71 (1920) 104.

Schizocasia sanderiana Engl. in Bot. Jahrb. 25 (1898) 26.

Mindanao (Misamis, Agusan), Merrill 7276, F. B. 4762 Mearns \& Hutchinson. In damp primary forests at low altitudes. A very beautiful species common in greenhouse cultivation in Europe and America. Endemic.

Alocasia sinuata N. E. Br. in Gard. Chron. (1885) 678; Engl. \& Krause Pflanzenreich 71 (1920) 102.

This was described from cultivated specimens supposedly of Philippine origin; Merrill 9568 from Palawan possibly represents it. Endemic.

ALocasia WenzelII Merr. in Philip. Journ. Sci. 13 (1918) Bot. 2.

Biliran, Samar, Leyte, Mindanao (Agusan), Wenzel 97, B. S. 24266 Ramos, 18929 McGregor, F. B. 7615 Hutchinson. In forests at low altitudes; perhaps a gigantic form of $A$. zebrina C. Koch \& Veitch. Endemic.

Local name: Handúroi (S. L. Bis.).

ALOCASIA ZEBRINA C. Koch \& Veitch in Wochenschr. Gärtn. Pflanzenk. 6 (1863) 331, Fl. des Serres 15: t. 1541; Naves Novis. App. (1882) 294; Engl. in DC. Monog. Phan. 2 (1879) 507; Merr. Fl. Manila (1912) 133; Engl. \& Krause Pflanzenreich 71 (1920) 105.

Luzon (Laguna, Tayabas, Sorsogon), Whitford 645, Elmer 17790, 14911, B. S. 6731 Robinson. In primary forests at low and medium altitudes. Endemic.

Local name: Badiáng (Bik.).

EXCLUDED SPECIES

Alocasia Alba Schott; Naves Novis. App. (1882) 293.

Alocasia cucullata Schott; Naves 1. c.

Alocasia longiloba Miq.; Naves op. cit. 294.

Alocasia Lowir Hook. f.; Naves l. c.

Alocasia montana Schott; Naves op. cit. 293.

Alocasia Odora C. Koch; Naves op. cit. 294.

Alocasia PUBERA Schott; Naves op. cit. 293.

16. COLOCASIA Schott

COlocasia ESCULENTUM (Linn.) Schott Melet. 1 (1832) 18; Merr. Fl. Manila (1912) 134, Interpret Herb. Amb. (1917) 131, Sp. Blancoanae (1918) 92.

Arum esculentum Linn. Sp. Pl. (1753) 965.

Arum colocasia Linn. 1. c.

Colocasia antiquorum Schott Melet. 1 (1832) 18, Naves Novis. App.

(1882) 292; Engl. in DC. Monog. Phan. 2 (1879) 491; Hook. f. in Curtis's Bot. Mag. III 50 (1894) t. 7364; Engl. \& Krause in Engl. Pflanzenreich 71 (1920) 65. 
Calla gaby Blanco Fl. Filip. (1837) 659.

Caladium esculentum Vent. Jard. Cels. (1800) sub. t. 30; Blanco Fl. Filip. ed. 2 (1845) 459, ed. 3, 3 (1879) 64.

Caladium colocasia W. F. Wight in Contr. U. S. Nat. Herb. 9 (1905) 208.

Caladium violaceum Desf. Cat. Hort. Paris ed. 3 (1829) 7; Naves Novis. App. (1882) 292.

Generally cultivated throughout the Philippines, but not a native of the Archipelago. It rarely produces flowers in the Philipines. A number of varieties are recognized by Filipinos. Pantropic in cultivation. Taro.

Local names: Abá (Ilk.) ; áua (Ilk.) ; abálong (Bis.) ; amóang (Bon.) ; gábi (Tag.); pising (Bon.); dagmái (Bis.) ; kimpói (Bis.); lagbái (Tag., (Bis.) ; linsá (Bik.) ; lubíngan (If.) ; natóñg (Bik.).

\section{EXCLUDED SPECIES}

Colocasia AfFinis Schott; Naves Novis. App. (1882) 293.

Colocasia Fallax Schott; Naves 1. c.

Colocasia Indica Engl.; Naves 1. c.

17. CALADIUM Ventenat

CALADIUM BICOLOR (Ait.) Vent. Jard. Cels. (1800) t. 30 ; Naves Novis. App. (1882) 291; Merr. Fl. Manila (1912) 131; Engl. \& Krause in Engl. Pflanzenreich 71 (1920) 31.

Arum bicolor Ait. Hort. Kew 3 (1789) 316.

Cultivated for its variegated leaves; occasional in the settled areas as an escape. A native of tropical America.

Local names: Corazón de maría (Sp.); linsáng-pulá (Bik.).

18. TYPHONIUM Schott

TYPHONIUM DIVARCATUM (Linn.) Decne. in Nouv. Ann. Mus. Paris 3 (1834) 367; Engl. in DC. Monog. Phan. 2 (1879) 611; Naves Novis. App. (1882) 295 et in Blanco Fl. Filip. ed. 3 (1877-83) t. 390, var. MOTLEYANUM (Schott) Engl. in DC. Monog. Phan. 2 (1879) 612.

Typhonium motleyanum Schott Prodr. (1860) 106; Usteri Beitr. Ken. Philip. Veg. (1905) 130; Engl. Pflanzenreich 73 (1920) 115.

Introduced and cultivated only; occasional in old gardens, but scarcely established. India to the Moluccas.

TYPHONIUM FLAGELLIFORME (Lodd.) Blume in Wall. Cat. (1832) No. 8928; Engl. Pflanzenreich 73 (1920) 112, f. 16.

Arum fiagelliforme Lodd. Bot. Cab. (1819) t. 396.

Arum cuspidatum Blume Cat. Gew. Buitenz. (1823) 101.

Typhonium cuspidatum Decne. in Nouv. Ann. Mus. Paris 3 (1834) 367; Engl. in DC. Monog. Phan. 2 (1879) 616; Naves Novis. App. (1882) 2.95; Merx. Fl. Manila (1912) 131, Sp. Blancoanae (1918) 92.

Arum divaricatum Blanco Fl. Filip. (1837) 657, ed. 2 (1845) 458, ed. 3 , 3 (1879) 62, non Linn. 
Luzon (Ilocos Norte, Cavite, Rizal), Merrill 4171, 71.28, 7111 , Sp. Blancoanae 676, 970, B. S. 1369 Robinson. In waste places about settlements, along trails, roads, etc., at low altitudes. India to Indo-China through Malaya to Timor, and the Marianne Islands.

\section{EXCLUDED SPECIES}

Typhonium Trilobatum Schott; Naves Novis. App. (1882) 295.

This, like T. divaricatum Decne, has apparently been cultivated in Manila, as it is represented in the Kew herbarium by Loher 2428, from Manila. It is not a native of the Philippines.

\section{ARISAEMA Martius}

ARISAEMA CUSPIDATUM (Roxb.) Engl. in DC. Monog. Phan. 2 (1879) 536, Pflanzenreich 73 (1920) 158.

Arum cuspidatum Roxb. Fl. Ind. ed. 2, 3 (1832) 506.

Arisaema roxburghii Kunth Enum. 3 (1841) 18; Hook. f. Fl. Brit. Ind. 6 (1893) 497.

Palawan, Merrill 9339. In primary forests at low altitudes. Assam, Malay Peninsula, Penang.

ARISAEMA POLYPHYLLUM (Blanco) Merr. in Govt. Lab. Publ. (Philip.) 27 (1905) 90, Philip. Journ. Sci. 1 (1906) Bot. 33, 2 (1907) Bot. 264, 5 (1910) Bot. 336, Sp. Blancoanae (1918) 92; Engl. Pflanzenreich 73 (1920) 182.

Calla polyphylla Blanco Fl. Filip. (1837) 659.

Caladium digitatum Blanco op. cit. ed. 2 (1845) 459, ed 3, 3 (1879) 64.

Arisaema cumingii Schott Syn. Aroid. (1856) 28 (cummingii), Prodr. (1860) 52; Miq. Fl. Ind. Bat. 3 (1856) 198; Engl. in DC. Monog. Phan. 2 (1879) 558; Naves Novis. App. (1882) 294; Vidal Phan. Cuming. Philip. (1885) 154, Rev. Pl. Vasc. Filip. (1886) 281.

Arisaema sarasinorum Engl. Bot. Jahrb. 37 (1905) 143.

Luzon (Ilocos Norte, Ifugao, Bontoc, Benguet, Rizal, Bataan, Laguna, Tayabas), Mindoro, Panay, Negros, Mindanao. In forests and in open places, altitude 1,000 to $2,400 \mathrm{~m}$, in the mossy forest often growing as an epiphyte on mossy trunks. Celebes.

Local names: Bía (Ibn.); bíla (Bon.) ; igigin-di-gáyang (Bon.) ; takindi-gáyang (Bon.); tigtigki (Ig.).

Var. ANGustifolium Merr. in Philip. Journ. Sci. 5 (1910) Bot. 336; Engler 1. c.

Luzon (Bontoc, Benguet, Zambales), Merrill 6472, Elmer 6330, F. B. 18387 Alvarez. In the pine region and in forests, altitude 800 to $2,000 \mathrm{~m}$. Endemic.

\section{EXCLUDED SPECIES}

Arisaema Fimbriatum Mast. in Gard. Chron. II 2 (1884) 680, 689, f. 119; Hook. f. in Curtis's Bot. Mag. III 46 (1890) t. 7150.

This was originally described from cultivated specimens supposed to have come from the Philippines; it is a native of the Langkawi Islands, north of Penang, and nothing remotely resembling it is known from the Philippines.

Arisaema laminatum Blume; Naves Novis. App. (1882) 294. 
20. CRYPTOCORYNE Fischer

CRYPTOCORYNE APONOGETIFOLIA Merr. in Philip. Journ. Sci. 14 (1919) 370.

Negros, Panay, B. S. 31119 Ramos \& Edaño, F.B. 19411 Curran. Submerged in shallow water of streams at low altitudes. Endemic.

CRYPTOCORYNE PYGMAEA Merr. in Philip. Journ. Sci. 14 (1919) 371.

Mindanao (Zamboanga), Merrill 814\%, Copeland. In crevices of ledges in stream beds in damp shaded ravines, altitude about $300 \mathrm{~m}$. Endemic.

CRYPTOCORYNE USTERIANA Engl. ex Usteri Beitr. Ken. Philip. Veg. (1905) 130, Engl. Bot. Jahrb. 54 (1916) Beibl. 118: 125; Pflanzenreich 73 (1920) 240, f. 60.

Guimaras, (Usteri). I have seen only the type, which is preserved in the Berlin herbarium. Endemic.

\section{EXCLUDED SPECIES}

Cryptocoryne ciliata Fisch.; Naves Novis. App. (1882) 295.

\section{PISTIA Linnaeus}

PISTIA STRATIOTES Linn. Sp. Pl. (1753) 963; Blanco Fl. Filip. (1837) 651, ed. 2 (1845) 454, ed. 3, 3 (1879) 55, t. 468; Presl Epim. (1851) 240 ; Miq. F1. Ind. Bat. 3 (1856) 218; Engl. in DC. Monog. Phan. 2 (18794) 634; Naves Novis. App. (1882) 296; Vidal Phan. Cuming. Philip. (1885) 154, Rev. Pl. Vasc. Filip. (1886) 281; Merr. Fl. Manila (1912) 130, Sp. Blancoanae (1918) 93; Engl. Pflanzenreich 73 (1920) 259, f. 63.

Pistia cumingii Klotz. in Abh. Akad. Berlin (1854) 354; Walp. Ann. 5 (1858) 913.

Pistia stratiotes Linn. var. cuneata Engl. in DC. Monog. Phan. 2 (1879) 634.

Northern Luzon to Mindanao. Floating in shallow water of lakes and slow streams at low altitudes; often abundant. Pantropic.

Local names: Alaluán (Ibn.); dagáylo (Mbo.); darahíro (Bik.); daraido (Bik.) ; daraíro (Bik.) ; kaiápo (Bis.) ; kiápo (Tag.) ; kiúpu (Mag.) ; loloán (Ilk.).

\section{EXCLUDED GENERA AND SPECIES}

Chamaecladon ovalifolium Schott; Naves Novis. App. (1882) 290.

Chamaecladon pygmaeum Engl.; Naves op. cit. 291.

CUSUARIA MARANTIFolia Schott; Naves op. cit. 289.

DiefFenbachia seguine Schott; Naves op. cit. 291.

LAGENANDRA TOXICARIA Dalz.; Naves op. cit. 295.

LASIA SPINOSA Thwaites; Naves op. cit. 289.

TheIrophonIUM Crenatum Blume; Naves op. cit. 295.

TheIrophonIUM wightil Engl.; Naves 1. c.

Piptospatha sp.; Usteri Beitr. Ken. Philip. Veg. (1905) 130. 


\section{LEMNACEAE}

\section{SPIRODELA Schleiden}

SPIRODELA POLYRRHIZA (Linn.) Schleid. in Linnaea 13 (1839) 392; Hegelm. Monogr. Lemn. (1868) 151, 156; Engl. Bot. Jahrb. 21 (1895) 284; Merr. Fl. Manila (1912) 134.

Lemna polyrrhiza Linn. Sp. Pl. (1753) 970 ; Naves Novis. App. (1882) 296 ; Usteri Beitr. Ken. Philip. Veg. (1905) 131.

Northern Luzon to Mindanao, F. B. 11622 Curran, Merrill 5121, 4445. Floating on stagnant fresh water at low and medium altitudes. Temperate and tropical regions of both hemispheres.

\section{LEMNA Linnaeus}

LEMNA PAUCicostatA Hegelm. Lemn. (1868) 294, Engl. Bot. Jahrb. 21 (1895) 294; Usteri Beitr. Ken. Philip. Veg. (1905) 131; Merr. Fl. Manila (1912) 134, Sp. Blancoanae (1918) 93.

Lemna gibba Blanco Fl. Filip. (1837) 672, ed. 2 (1845) 468, ed. 3, 3 (1879) 78; Naves Novis. App. (1882) 296, non Linn.

Lemna oligorrhiza Naves Novis. App. (1882) 296, non Kurz.

Luzon to Mindanao, floating on stagnant fresh water at low and medium altitudes, often abundant. Widely distributed in the warmer parts of both hemispheres.

Local names: Inaliá (Tag.); liá (Tag.); liyá (Tag.).

LEMNA TRISUlCA Linn. Sp. Pl. (1753) 970; Naves Novis. App. (1882) 296; Hegelm. Lemn. (1868) 134, Engl. Bot. Jahrb. 21 (1895) 293.

Luzon (Benguet), Mindanao (Lanao), Clemens, B. S. 8782 McGregor. Floating on stagnant fresh water at medium altitudes. Widely scattered in bath hemispheres.

\section{EXCLUDED SPECIES}

LEMNA MINOR Linn.; Naves Novis. App. (1882) 296.

3. WOLFFIA Horkel

WOLFFIA ARRHIZA (Linn.) Wimm. Fl. Schles. (1857) 140; Hegelm. Monogr. Lemn. (1868) 124, Engl. Bot. Jahrb. 21 (1895) 301; Naves Novis. App. (1882) 296.

Lemna arrhiza Linn. Mant. 2 (1771) 294.

Luzon (Pampanga, Bulacan, Rizal), Merrill 4244. Floating on stagnant fresh-water pools at low altitudes. Warmer parts of both hemispheres.

Local names: Dugmán (Bis.); liá (Tag.). EXCLUDED SPECIES

WolfFiA DELILII Schleid.; Naves Novis. App. (1882) 296.

Wolffia shleideni Miq.; Naves 1. c.

\section{FLAGELLARIACEAE}

\section{JOINVILLEA Gaudichaud}

JOINVILLEA BORNeENSIS Becc. Nelle Foreste di Borneo (1902) 198. Joinvillea malayana Ridley in Journ. Str. Branch Roy. As. Soc. 44 (1905) 199; Merr. in Philip. Journ. Sci. 1 (1906) Suppl. $181_{1}$ 
Palawan, Jolo, B. S. 575 Foxworthy, Merrill 9515, Clemens 9374. In forests and on ridges, altitude 650 to $1,000 \mathrm{~m}$. Malay Peninsula, Borneo.

Local name: Odyúng : (Tagb.).

\section{FLAGELLARIA Linnaeus}

FLAGELLARIA INDICA Linn. Sp. Pl. (1753) 333; Blanco Fl. Filip. ed. 2 (1845) 196, ed. 3, 1 (1877) 347; Miq. Fl. Ind. Bat. 3 (1856) 249; Naves Novis. App. (1880) 263; Vidal Rev. Pl. Vasc. Filip. (1886) 279 ; Merr. in Govt. Lab. Publ. (Philip.) 27 (1905) 87, Sp. Blancoanae (1918) 93.

Flagellaria philippinensis Elm. Leafl. Philip. Bot. 1 (1908) 274.

Batan Islands to Mindanao and Palawan, in all or most islands and provinces. In secondary forests at low and medium altitudes; common. Tropical Africa, tropical Asia through Malaya to tropical Australia and the Marianne Islands.

Flagellaria philippinensis Elm. is a form with very large leaves; all intergrades between it and the typical form occur.

Local names: Anuád (Ilk.) ; aráyan (Tag.); auái (Iv.) ; auái-sji-gáyang (Is.) ; baling-uái (Tag., Pamp.) ; boboáya (Mbo.); hoág-uái (Bik.); huág (S. L. Bis., Mbo); húak (Bis.); iñúal (Ilk.); ingúla (Tag.); inúal (Pang.) ; kala-uái (Ibn.) ; kala-uaiuai (Ibn.) ; ouág-oái (Bik.) ; ouag-ouág (Mbo.); páua (P. Bis.); sagákap (P. Bis.); tauá (P. Bis.); tinúung (Ibn.) ; uág (Sul., Bis., Bag., Bik.) ; uái-ti-uák (Ilk.) ; uák (Bis.) ; venagaíang (Is.).

\section{HANGUANA Blume}

HANGUANA MALAYANA (Jack) Merr. in Philip. Journ. Sci. 10 (1915) Bot. 3.

Veratrum? malayanum Jack Malay Miscel. 1 (1820) 25; Hook. Bot. Miscel. 2 (1831) 74.

Hanguana kassintu Blume Enum. Pl. Jav. (1827) 15, ed. 2 (1830) 15.

Veratonia malayana Miq. F1. Ind. Bat. 3 (1859) 553.

Susum malayanum Planch. ex Hook. f. Fl. Brit. Ind. 6 (1892) 391.

Mindoro, Palawan, Mindanao (Lanao, Surigao), Merrill 7221, 9498, Piper 529, Clemens 1134a. In thickets back of the mangrove swamps, ascending to $900 \mathrm{~m}$ in forests. Malay Peninsula and Archipelago.

\section{CENTROLEPIDACEAE}

\section{CENTROLEPIS Labillardière}

CENTROLEPIS PHILIPPINENSIS Merr. in Philip. Journ. Sci. 2 (1907) Bot. 264.

Mindoro, Merrill 6160, Phil. Pl. 8\%. In open heaths, altitude about 2,400 m. Endemic.

This was also credited to Borneo, by Turrill, but Miss Gibbs later described the Bornean form as C. kinabaluensis Gibbs, Phytogeog. Dutch New Guinea (1917) 99. Only one other species of the family is known north of Australia, this being a species of Centrolepis in Indo-China. 


\section{XYRIDACEAE}

1. XYRIS Linnaeus

XYRIS COMPLANATA R. Br. Prodr. (1810) 256; Naves Novis. App. (1880) 268; Hallier f. in Nova Guinea 8 (1913) 903, Beihefte Bot. Centralbl. $34^{2}$ (1916) 45 .

Xyris anceps Merr. in Philip. Journ. Sci. 5 (1910) Bot. 172, non Lam.

Luzon (Isabela), Semirara, B. S. 8065 Ramos, Merrill 4151. In open grasslands at low altitudes. Malay Archipelago to Australia.

Local name: Gumi-gúmi (Tag.).

XYRIS PAUCIFloRA Willd. Phytogr. (1794) 2, $t$. 1; Miq. Fl. Ind. Bat. 3 (1859) 529; Naves Novis. App. (1880) 268; Merr. Fl. Manila (1912) 135.

Luzon (Pangasinan, Tarlac, Bulacan, Rizal), Loher 6985, Merrill 3628, Phil. Pl. 463, B. S. 4911 Ramos. In open grasslands at low altitudes. India to China and Formosa through Malaya to Australia.

EXCLUDED SPECIES

XYRIS INDICA Linn.; Naves Novis. App. (1880) 268.

XYRIS OPERCULATA Labill.; Naves 1. c.

\section{ERIOCAULONACEAE}

\section{ERIOCAULON Linnaeus}

ERIOCAULON ALATUM H. Lecomite in Journ. de Bot. 21 (1908) 104, f. 2; Merr. in Philip. Journ. Sci. 7 (1912) Bot. 232, Fl. Manila (1912) 136.

Luzon (Rizal), Merrill 7362, B. S. 1831 Ramos, 19270 Reillo. In open wet grasslands at, low altitudes. Indo-China.

ERIOCAULON ALPESTRE Hook. f. \& Th. ex Koern. in Miq. Ann. Mus. Bot. Lugd.-Bat. 3 (1867) 163; Ruhl. in Engl. Pflanzenreich 13 (1903) 95; Merr. in Philip. Journ. Sci. 10 (1915) Bot. 291.

Mindanao (Davao), Copeland 1431. In open wet places, altitude about $1,800 \mathrm{~m}$. India to Japan south to Indo-China.

ERIOCAULON BREVIPEDUNCULATUM Merr, in Philip. Journ. Sci. 2 (1907) Bot. 265.

Mindoro, Merrill 6214. On seepage slopes in open heaths on Mt. Halcon, aititude about $2,400 \mathrm{~m}$. Borneo.

ERIOCAULON CINEREUM R. Br. Prodr. (1810) 254; Benth. Fl. Austral. 7 (1878) 193; Merr. in Philip. Journ. Sci. 10 (1915) Bot. 291, F1. Manila (1912) 136.

Eriocaulon sieboldianum Sieb. \& Zucc. ex Steud. Syn 2 (1855) 272; Ruhl. in Engl. Pflanzenreich 13 (1903) 111, f. 15 A-G.

Luzon (Rizal), Merrill Phil. Pl. 293, 1276, B. S. 24072 Ramos. In fallow wet rice lands at low altitudes. Africa to Japan, Malaya, and Australia.

Local name: Dundunsúg (P. Bis.). 
ERIOCAULON DEPAUPERATUM Merr. in Philip. Journ. Sci. 5 (1910) Bot. 336.

Luzon (Lepanto, Benguet), Merrill 4520, 6590, B. S. 40528 Ramos \& Edaño. In pools and small shallow ponds, altitude 2,200 to $2,800 \mathrm{~m}$. Endemic.

ERIOCAULON ECHINULATUM Mart. in Wall. Pl. As. Rar. 3 (1832) 28; Ruhl. in Engl. Pflanzenreich 13 (1903) 106.

Culion, B. S. 41325 Ramos. In open grassy places at low altitudes. Tanasserim to Indo-China and southern China.

ERIOCAULON MERRILLII Ruhl. in Perk. Frag. Fl. Philip. (1904) 136; Merr. in Philip. Journ. Sci. 10 (1915) Bot. 291, Fl. Manila (1912) 136.

Luzon (Nueva Vizcaya, Nueva Ecija, Tarlac, Zambales, Rizal, Sorsogon), Polillo, Culion, Sibuyan, Mindanao (Lanao), Merrill,572, 7122, 3624, Elmer 14350, 12248, B. S. 14149 McGregor, 9031 Robinson. In open wet places, old rice paddies, along small streams, etc., at low altitudes. Endemic. Distinct from $E$. truncatum Ham.?

Local name: Boibotánis (P. Bis.).

ERIOCAULON NIGRICEPS Merr. in Philip. Journ. Sci. 10 (1915) Bot. 290.

Luzon (Lepanto, Benguet), B. S. 5544 Ramos, 31747 Santos, Merrill 4646, $7 \gamma_{48}$, Elmer 6617, Williams 946, 1004. In open wet places, altitude 1,400 to $2,300 \mathrm{~m}$. Endemic.

ERIOCAULON TRUNCATUM Mart. in Wall. Pl. As. Rar. 3 (1832) 29; Steud. Syn. 2 (1859) 270; Ruhl. in Engl. Pflanzenreich 13 (1903) 107; Naves Novis. App. (1882) 299.

Luzon (Abra, Ifugao, Bontoc, Nueva Vizcaya, Rizal, Camarines), B. S. 39791 Ramos \& Edaño, 24089 Ramos, 19980, 20172, 90149 McGregor, 19267 Reillo. In open wet places, rice paddies, etc., at low and medium altitudes. India to southern China and Malaya.

Cuming 2326, credited to the Philippines by Ruhland, was from Malacca.

Local names: Bauañg-bauángan (Bik.); dasdasnuk (Bon.).

\section{EXCLUDED SPECIES}

Eriocaulon longissimum Usteri Beitr. Ken. Philip. Veg. (1905) 131.

This is a misprint for $E$. longifolium Nees, a species unknown from the Philippines.

Eriocaulon sexangulare Linn.; Naves Novis. App. (1882) 299; Miq. Fl.

Ind. Bat. 3 (1857) 523; Ruhl. in Engl. Pflanzenreich 13 (1903) 110.

Eriocaulon SETACeum Linn.; Naves 1. c.

\section{BROMELIACEAE}

\section{ANANAS Tournefort}

ANANAS Comosus (Linn.) Merr. Interpret. Herb. Amb. (1917) 133, Sp. Blancoanae (1918) 94.

Bromelia comosa Linn. in Stickman Herb. Amb. (1754) 21, Amoen. Acad. 4 (1759) 130.

$183036-13$ 
Bromelia ananas Linn. Sp. Pl. (1753) 285; Blanco Fl. Filip. (1837) 230, ed. 2 (1845) 162, ed. 3,1 (1877) 291, t. 458.

Bromelia pigna Perr. in Mém. Soc. Linn. Paris 3 (1824) 103; C. B. Rob. in Philip. Journ. Sci. 3 (1908) Bot. 304.

Ananas sativus Schultes f. Syst. $7^{2}$ (1830) 1283; Merr. Fl. Manila (1912) 137.

Ananassa sativa Lindl. Bot. Reg. (1827) sub. t. 1068; Miq. Fl. Ind.

Bat. 3 (1859) 584; Naves Novis. App. (1880) 256; Merr. in Govt.

Lab. Publ. (Philip.) 6 (1904) 24.

Ananassa ananas Karst. Deutsch. Fl. (1880-83) 466.

Widely cultivated in the Philippines; in some regions, Palawan, thoroughly naturalized. A native of tropical America, now cultivated in all tropical countries. Pineapple.

Local names: Apangdán (Bon.); pangdán (Bon.); piña (Sp.).

\section{EXCLUDED GENERA}

Billbergia zebrina Lindl.; Naves Novis. App. (1880) 257.

Pitcairnia brachiata Cham.; Naves 1. c.

\section{COMMELINACEAE}

\section{POLLIA Thunberg}

POLLIA MACROPHYLLA Benth. Fl. Austral. 7 (1878) 90; C. B. Clarke in DC. Monog Phan. 3 (1881) 128; Naves Novis. App. (1880) 269 ; Ceron Cat. Pl. Herb. (1892) 173.

Philippines, fide C. B. Clarke, who cites specimens from Cuming and Llanos. These, however, need critical comparison with $P$. sorzogonensis (E. Meyer) Steud. New Guinea, Solomon Islands, tropical Australia.

POLLIA SORzOGONENSIS (E. Meyer) Steud. Nomencl. ed. 2, 2 (1841) 368; Miq. Fl. Ind. Bat. 3 (1859) 541; C. B. Clarke in DC. Monog. Phan. 3 (1881) 126; Naves Novis. App. (1880) 269; Vidal Phan. Cuming. Philip. (1885) 153, Rev. Pl. Vasc. Filip. (1886) 278; Merr. in Philip. Journ. Sci. 1 (1906) Suppl. 34, 3 (1908) Bot. 400, Sp. Blancoanae (1918) 94.

Aclisia sorzogonensis E. Meyer in Presl Rel. Haenk. 1 (1827) 138, t. 25.

Lechea minor Blanco Fl. Filip. (1837) 52, ed. 2 (1845) 35, ed. 3, 1 (1877) 65, non Linn.

Aclisia cumingiana Klotz. in Hassk. Commel. Ind. (1870) 47.

Northern Luzon (Cagayan) to Palawan and Mindanao. Common in primary forests at low and medium altitudes. Southern China to Java and the Moluccas.

Local names: Alikbángon (Tag.) ; lohod-lóhod (Bis.) ; Iuho-lúho (S. L. Bis.); salibáñung (Bag.).

POllia sumatranA Hassk. Commel. Ind. (1870) 56; C. B. Clarke in DC. Monog. Phan. 3 (1881) 125; Naves Novis. App. (1880) 269; Vidal Rev. Pl. Vasc. Filip. (1886) 278.

Luzon (Rizal, Bulacan, Laguna), Polillo, Biliran, Samar, Leyte, Mindanao, Piper 483, Elmer 7153, B. S. 6724 Robinson, 15702 Fénix, 18667 McGregor, 1088, 24425 Ramos. In primary forests at low and medium altitudes. Sumatra. 
Clarke's description is based on Cuming's Philippine plant. I do not know whether or not it is identical with Hasskarl's type.

Local names: Aregbabáñon. (S. L. Bis.) ; idog (Mbo.); mantauási (Buk.).

POLLIA THYRSIFLORA (Blume) Steud. Nomencl. ed.2, 2 (1841) 368; C. B. Clarke in DC. Monog. Phan. 3 (1881) 124; Naves Novis. App. (1880) 269; Vidal Rev. Pl. Vasc. Filip. (1886) 278; Hallier f. in Nova Guinea 8 (1913) 907, Beihefte Bot. Centrabl. 34 ${ }^{2}$ (1916) 51.

Tradescantia thyrsiflora Blume Enum. Pl. Jav. 1 (1827) 6.

Pollia philippinensis Elm. Leafl. Philip. Bot. 1 (1908) 275.

Luzon (Cagayan to Sorsogon) to Palawan and Mindanao, Elmer 9162, 13545,15329 , Wenzel 1166, Merrill 9268, 4007, Loher 6981, F. B. 19623 Curran, B. S. 25857 Yates, 12651 Fénix. In primary forests at low and medium altitudes. Andaman Islands to Java and Celebes.

Local names: Talibáñung (Bag.); sapilko (Ig.).

EXCLUDED SPECIES

Poldia AClisia Hassk.; Naves Novis. App. (1880) 268.

Pollita crispata Benth.; Naves op. cit. 269.

Pollia JAPONICA Thunb.; Naves op. cit. 268.

\section{COMMELINA Plumier}

COMMELINA BENGHALENSIS Linn. Sp. Pl. (1753) 41; Miq. Fl. Ind. Bat. 3 (1859) 533; C. B. Clarke in DC. Monog. Phan. 3 (1881) 159; Naves Novis. App. (1880) 269; Merr. in Philip. Journ. Sci. 3 (1908) Bot. 400, Fl. Manila (1912) 138, Sp. Blancoanae (1918) 94. Commelina polygama Blanco Fl. Filip. (1837) 25 (poligama), ed. 2 (1845) 18, ed. 3, 1 (1877) 34, t. 13, non Roth.

Throughout the Philippines in open grasslands and waste places in the settled areas at low and medium altitudes, often common. Tropical Africa and Asia to Japan and Malaya.

Local names: Alibáñon (Tag.) ; bias-bias (Pamp.); kabiláu (Bis.) ; kuhási (Iv.) ; kulkul-lási (Ilk.); sabiláu (Bis.) ; sambiláu (Bis.) ; uligbóñgon (Tag.).

COMMELINA CHAMISSONIS Klotz. ex C. B. Clarke in DC. Monog. Phan. 3 (1881) 186; Naves Novis. App. (1880) 270.

The type is a specimen from Luzon, collected by Chamisso, probably from either Cavite or Batangas Province or possibly from the vicinity of Manila. I have seen no specimens I can with certainty refer to it, but some may have been distributed sub $C$. nudiflora Linn. Australia.

COMMELINA NUdiflora Linn. Sp. Pl. (1753) 4; C. B. Clarke in DC. Monog. Phan. 3 (1881) 144; Naves Novis. App. (1880) 269; Merr. in Philip. Journ. Sci. 3 (1908) Bot. 400, Fl. Manila (1912) 138.

Tradescantia cristata Naves in Blanco Fl. Filip. ed. 3 (1877-83) t. 83, non Linn.

Throughout the Philippines in open grasslands, waste places, etc., at low and medium altitudes, often common. Pantropic.

Local names: Alikbáñon (Tag.); bañgar-an-laláki (If.) ; gatiláng (Bon.) katkataúang (Bon.); kitkit-aúang (Bon.); kohási (Iv.); kolási (Ilk.) ; kulkul-lási (Ilk.). 
COMmelina undulata R. Br. Prodr. (1810) 270; C. B. Clarke in DC. Monog. Phan. 3 (1881) 179; Naves Novis. App. (1880) 270; Ceron Cat. Pl. Herb. Manila (1892) 173.

Philippines fide C. B. Clarke 1. c. India to southern China, Riu Kiu Islands, Moluccas, tropical Australia.

\section{EXCLUDED SPECIES}

Commelina barbata Lam.; Naves Novis. App. (1880) 270.

Commelina communis Linn.; Naves 1. c.

Commelina obliqua Ham.; Naves 1. c.

CoMmelina SALICIFolia Roxb.; Naves op. cit. 269.

Commelina subulata Roth; Naves 1. c.

Commelina suffruticosa Blume; Naves op. cit. 270.

\section{ANEILEMA R. Brown}

ANeilemA AzUReUm Merr. in Philip. Journ. Sci. 10 (1915) Bot. 292.

Luzon (Bulacan), Semirara, Merrill 4134, B. S. 21669 Ramos.' In open wet grasslands at low altitudes. Endemic.

ANEILemA GIGANTeum (Vahl) R. Br. Prodr. (1810) 271; Naves Novis. App. (1880) 271; C. B. Clarke in DC. Monog. Phan. 3 (1881) 212; Merr. in Philip. Journ. Sci. 10 (1915) Bot. 294.

Commelina gigantea Vahl Enum. 2 (1806) 177.

Semirara, Mindanao (Bukidnon), Merrill 4132, B. S. 31385 Escritor, 39210 Ramos \& Edaño, 26138 F'énix, F. B. 26553 Rola. In open grasslands at low and medium altitudes. Tropical Africa and Asia through Malaya to tropical Australia.

ANEILEMA HUMILE Merr. in Philip. Journ. Sci. 13 (1918) Bot. 4.

Alabat, Merrill 10459. On wet slopes in primary forests at low altitudes. Endemic.

ANeIlemA MALABARICUM (Linn.) Merr. in Philip. Journ. Sci. 7 (1912) Bot. 232, Fl. Manila (1912) 138.

Tradescantia malabarica Linn. Sp. Pl. ed. 2 (1762) 412.

Commelina nudicaulis Burm. f. Fl. Ind. (1768) 17, t. 8, f. 1.

Commelina nudiflora Linn. Mant. 1 (1767) 177, non Sp. Pl. (1753). Aneilema nudiflorum R. Br. Prodr. (1810) 271; Miq. Fl. Ind. Bat.

3 (1859) 537; Naves Novis. App. (1880) 271, t. 467; C. B. Clarke in DC. Monog. Phan. 3 (1881) 210; Vidal Phan. Cuming. Philip. (1885) 153, Rev. Pl. Vasc. Filip. (1886) 278.

Commelina trichocolea Schauer in Nov. Act. Acad. Nat. Cur. 19 (1843)

Suppl. 1: 448; Walp. Ann. 1 (1849) 885.

Throughout the Philippines. Common in open grasslands, waste places, etc., at low and medium altitudes. India to China, the Riu Kiu Islands, and Malaya.

Local names: Aligbáñon (Pang.); aligbáñgon-laláki (Tag.); alitbañun (Tag.) ; fañgau (Bon.) ; olikbáñgon (Tag.); takdáyan (Ig.).

ANeILema PLATyPHYLlum Merr. in Philip. Journ. Sci. 10 (1915) Bot. 293.

Luzon (Pangasinan, Rizal), B. S. 985, 3342, 20985 Ramos, 17900 Otanes. Along streams in shaded places at low altitudes. Endemic. 
ANEILEMA SPIRATUM (Linn.) R. Br. ex C. B. Clarke in DC. Monog. Phan. 3 (1881) 207; Naves Novis. App. (1880) 270.

Commelina spirata Linn. Mant. 2 (1771) 176.

This is credited to the Philippines by C. B. Clarke on the basis of a specimen from Lake Bay, Luzon, collected by Baume and preserved in the Paris herbarium. This specimen may be A. malabaricum (Linn.) Merr., or I may have included $A$. spiratum $\mathrm{R}$. Br. in my conception of $A$. malabaricum (Linn.) Merr. India to Indo-China, China, and Java.

ANEILEMA VERSICOLOR Dalz. in Hook. Journ. Bot. Kew Miscel. 3 (1851) 136; C. B. Clarke in DC. Monog. Phan. 3 (1881) 208; Merr. in Philip. Journ. Sci. 7 (1912) Bot. 232, Fl. Manila (1912) 139.

Luzon (Rizal), Culion, Merrill 3644, 518, Phil. Pl. 1980, 763, Loher $6986, B$. S. 10877 Ramos, 19171 Reillo. In open wet grasslands, fallow rice paddies, etc., at low altitudes. India.

ANEILEMA VITIENSE Seem. Fl. Vit. (1865-73) 314, t. 96 ; C. B. Clarke in DC. Monog. Phan. 3 (1881) 220; Naves Novis. App. (1880) 271.

Philippines, Moseley, fide C. B. Clarke. Fiji, Samoa.

Var. PETIOLATA C. B. Clarke 1. c.

Northern Luzon (Cagayan) to Palawan and Mindanao, in most islands and provinces, Merrill 4862, 5332, 5119, 7134, Phil. Pl. 33, Weber 1022, Elmer 1355\%, 15366, 18053, Wenzel 4, Whitford 973, B. S. 12409 McGregor, 18081 Robinson, 15701 Fénix, 2905\%, 30928 Ramos \& Edaño. In damp shaded ravines usually along small streams at low and medium altitudes, ascending to $1,400 \mathrm{~m}$. The variety endemic, the species in Samoa and Fiji.

Aneilema scaberrima (Blume) Kunth has been credited to the Philippines by Hallier f., Beihefte Bot. Centralbl. $34^{2}$ (1916) 50, as A. protensum Wall., but I suspect that the specimen he examined is A. vitiense Seem.

Local names: Balúkid (Buk.); dalidígan (Mbo.).

\section{EXCLUDED SPECIES}

ANeilema dimorPhum Dalz.; Naves Novis. App. (1880) 270.

Aneilema glaucum Thw.; Naves 1. c.

ANEILEMa hamiltonianum Wall.; Naves 1. c.

ANEILEMA HERBACEUM Wall.; Naves op. cit. 270.

ANEILEMA LOUREIRII Hance; Naves l. c.

ANEILEMa Medium R. Br.; Naves 1. c.

ANEILEMA MONADELPHUM Kunth; Naves op. cit. 271.

ANEILEMA SCAPIFLORUM Wight; Naves op. cit. 270.

ANEILEMA SINICUM Lindl.; Naves op. cit. 271.

\section{FORRESTIA A. Richard}

FORRESTIA HISPIDA Less. \& A. Rich. Sert. Astrolab. (1834) 2, t. 1, var. PHILIPPINENSIS (Merr.) comb. nov.

Forrestia philippinensis Merr. in Govt. Lab. Publ. (Philip.) 35 (1906) 5, Philip. Journ. Sci. 2 (1907) Bot. 266.

Forrestia hispida Less. \& A. Rich. var. glabrior Hallier f. in Nova Guinea 8 (1913) 906, Beihefte Bot. Centralbl. $34^{2}$ (1916) 46. 
Luzon (Laguna, Tayabas, Camarines, Albay, Sorsogon), Polillo, Catanduanes, Mindoro, Leyte, Negros, Panay, Samar, Mindanao (Agusan, Bukidnon, Lanao), Basilan. In thickets and old clearings in the vicinity of primary forests and in primary forests at low and medium altitudes; represented by numerous collections. The variety in Borneo, the species in New Guinea.

Local names: Arigbabáñon (S. L. Bis.); lohod-lohód (C. Bis.); malitágo (Mbo.); mani-utús (Lan.); salimbágun (Bag.); tahig-tahíg (Bik.).

\section{EXCLUDED SPECIES}

Forrestia glabrata Hassk.; Naves Novis. App. (1880) 271.

Forrestia marginata Hassk.; Naves 1. c.

FORRESTIA MOLLIS Hassk.; Naves 1. c.

\section{CYANOTIS D. Don}

CYANOTIS AXILlaRIS (Linn.) D. Don Prodr. Fl. Nepal. (1825) 46; R. \& S. Syst. $7^{2}$ (1830) 1154; Schauer in Nov. Act. Acad. Nat. Cur. 19 (1843) Suppl. 1: 447; C. B. Clarke in DC. Monog. Phan. 3 (1881) 244; Naves Novis. App. (1880) 272; Vidal Phan. Cuming. Philip. (1885) 153, Rev. Pl. Vasc. Filip. (1886) 278; Merr. Fl. Manila (1912) 140.

Commelina axillaris Linn. Sp. Pl. (1753) 42.

Northern Luzon to Palawan and Mindanao, in most islands and provinces. In clearings, open places along streams, rice paddies, etc., at low and medium altitudes; often common. India to China through Malaya to Australia.

Local names: Alikbáñơn (Tag.) ; alitbáñon (Tag.); kulásin-marinték (Pang.) ; sabiláu (P. Bis.).

CYANOTIS CAPITATA (Blume) C. B. Clarke in DC. Monog. Phan. 3 (1881) 243; Naves Novis. App. (1880) 272.

Tradescantia capitata Blume Enum. Pl. Jav. (1827-28) 6.

Northern Luzon to Palawan and Mindanao, Clemens 778 , Copeland 740, Merrill 803, 4426, 8154, 5462, 1804, F. B. 3453 Hutchinson, B. S. 26076 Fénix, 23850 Ramos, 32555 McGregor. Along streams in shaded ravines, in damp thickets, etc., at low and medium altitudes; really distinct from $C$. moluccana (Roxb.) Merr.? Japan to Indo-China and Java.

CYANOTIS CRISTATA (Linn.) R. \& S. Syst. $7^{2}$ (1830) 1150 ; Miq. F1. Ind. Bat. 3 (1859) 544; C. B. Clarke in DC. Monog. Phan. 3 (1881) 247; Naves Novis. App. (1880) 272; Vidal Phan. Cuming. Philip. (1885) 153, Rev. Pl. Vasc. Filip. (1886) 278; Merr. Fl. Manila (1912) 140, Sp. Blancoanae (1918) 95.

Commelina cristata Linn. Sp. Pl. (1753) 42.

Tradescantia critata Linn. Syst. ed.'12 (1767) 233; Blanco Fl. Filip. (1837) 231, ed. 2 (1845) 163, ed. 3, 1 (1877) 293.

Zygomenes cristata W. F. Wight in Contr. U. S. Nat. Herb. 9 (1905) 404.

Luzon (most or all provinces), Basilan. In old clearings, open places along streams, about cliffs, etc, at low and medium altitudes. India to Mauritius and Malaya.

Local names: Alikbáñgon (Tag.); kulásing-akabaleg (Pang.). 
CYANOTIS MOLUCCANA (Roxb.) Merr. in Philip. Journ. Sci. 2 (1907)

Bot. 266, Fl. Manila (1912) 139.

Commelina moluccana Roxb. F1. Ind. 1 (1820) 176.

Cyanotis uniflora Hassk. Commel. Ind. (1870) 104; C. B. Clarke in DC. Monog. Phan. 3 (1881) 242; Naves Novis. App. (1880) 272;

Merr. in Philip. Journ. Sci. 1 (1906) Suppl. 34.

Luzon (Bontoc, Pampanga, Rizal, Bataan, Laguna, Sorsogon), Mindoro, Leyte, Mindanao, Baker 500, Wenzel 542, Merrill 4206, 6227, r132, Williams 249. On bowlders in ravines, along small streams, etc, at low and medium altitudes. Java, Borneo, Moluccas.

Not always readily distinguishable from $C$. capitata Clarke.

CYANOTIS PEDUNCULATA Merr. in Philip. Journ. Sci. 10 (1915) Bot. 266.

Leyte, Wenzel 1024. In forests, altitude about $500 \mathrm{~m}$. Endemic.

EXCLUDED SPECIES .

Cyanotis Papilionacea R. \& S.; Naves Novis. App. (1880) 272.

Cyanotis tuberosa R. \& S.; Naves 1. c.

CYaNotis vaga R. \& S.; Naves 1. c.

\section{FLOSCOPA Loureiro}

FLOSCOPA SCANDENS Lour. Fl. Cochinch. (1790) 193; C. B. Clarke in DC. Monog. Phan. 3 (1881) 265; Naves Novis. App. (1880) 272; Vidal Phan. Cuming. Philip. (1885) 153, Rev. Pl. Vasc. Filip. (1886) 278; Merr. Sp. Blancoanae (1918) 95.

Tradescantia rufa Presl Rel. Haenk. 1 (1827) 138.

Floscopa rufa Hassk. in Miq. Pl. Jungh. (1852) 151; Miq. Fl. Ind. Bat. 3 (1859) 542.

Tradescantia geniculata Blanco Fl. Filip. (1837) 232, ed. 3, 1 (1877) 294, non Jacq.

Northern Luzon (Cagayan) to the Calamian Islands and Mindanao, in most or all islands and provinces. In wet places along small streams at low and medium altitudes. India to China through Malaya to tropical Australia.

Local names: Aligbángon (Tag.); babiláu (S. L. Bis.) ; kúmpai (P. Bis.) ; púgad-labúyo (Tag.); sambiláu (S. L. Bis.).

\section{RHOEO Hance}

RHOEO DISCOLOR (L'Hérit.) Hance in Walp. Ann. 3 (1853) 660; Naves Novis. App. (1882) 273, Blanco Fl. Filip. ed. 3 (1877-83) t. 84; Merr. Fl. Manila (1912) 139, Sp. Blancoanae (1918) 94.

Tradescantia discolor L'Hérit. Sert. Angl. (1788) 8, t. 12; Blanco Fl. Filip. (1837) 232, ed. 2 (1845) 163, ed. 3, 1 (1877) 294, t. 84.

Planted for ornamental purposes, sometimes long-persistent after cultivation but scarcely established. A native of tropical America.

\section{ZEBRINA Schnizlein}

ZEBRINA PENDULA Schnizl. in Bot. Zeit. 7 (1849) 870; C. B. Clarke in DC. Monog. Phan. 3 (1881) 318; Merr. in Philip. Journ. Sci. 7 (1912) Bot. 232, Fl. Manila (1912) 140. 
Introduced from tropical America and cultivated as an ornamental, now more or less established in coconut groves, etc., at medium altitudes. Wandering Jew.

\section{PONTEDERIACEAE}

\section{EICHORNIA Kunth}

EICHORNIA CRASSIPES (Mart.) Solms in DC. Monog. Phan. 4 (1883) 527.

Pontederia crassipes Mart. Nov. Gen. Sp. Pl. 1 (1824) 9, t. 4.

Eichornia speciosa Kunth Enum. Pl. 4 (1843) 131.

Piaropus crassipes Britton in Ann. N. Y. Acad. Sci. 7 (1893) 241.

Introduced about 1912 as an ornamental, now very abundant in shallow water of lakes and in slow streams in central Luzon, occurring also in northern Luzon and in Negros. Within a few years to be expected throughout the Philippines. A native of tropical America, now pantropic. Water hyacinth.

\section{MONOCHORIA Presl}

MONOChORIA HASTATA (Linn.) Solms in DC. Monog. Phan. 4 (1883) 523; Merr. Fl. Manila (1912) 141, Sp. Blancoanae (1918) 95.

Pontederia hastata Linn. Sp. Pl. (1753) 288.

Monochoria hastaefolia Presl Rel. Haenk. 1 (1827) 128; Miq. Fl. Ind. Bat. 3 (1859) 548; Naves Novis. App. (1880) 267.

Pontederia vaginalis Blanco Fl. Filip. (1837) 255, ed. 2 (1845) 178, ed. 3,1 (1877) $320, t .466$, non Burm. f.

Monochoria, dilatata Kunth Enum. 4 (1843) 134; Naves Novis. App. (1880) 268.

Luzon to Mindanao, Elmer 14319, Merrill 4088, Phil. Pl. 1121, Sp. Blancoanae 419, McGregor 93, Clemens 179, B. S. 1456 Mangubat. In open wet lands, swamps, etc., at low and medium altitudes. India to southern Chins and Malaya.

Local names: Gabi-gábi (Bis) ; gabi-gabíhan (Tag.); kosol-kósol (Bis.) ; payau-páyau (Bis).

MONOCHORIA VAGINALIS (Burm. f.) Presl Rel. Haenk. 1 (1827) 128; Miq. Fl. Ind. Bat. 3 (1859) 548; Naves Novis. App. (1880) 267; Solms in DC. Monog. Phan. 4 (1883) 524; Vidal Phan. Cuming. Philip. (1885) 153, Rev. Pl. Vasc. Filip. (1886) 278; Merr. Fl. Manila (1912) 141, Interpret. Herb. Amb. (1917) 135.

Pontederia vaginalis Burm. f. Fl. Ind. (1768) 80; Linn. Mant. 2 (1771) 222; Schauer in Nov. Act. Acad. Nat. Cur. 19 (1843) Suppl. 1: 446. Monochoria ovata Kunth Enum. 4 (1843) 665; Naves Novis. App. (1880) 268.

Throughout the Philippines at low and medium altitudes; common in open wet places, rice paddies, swamps, etc. Tropical and subtropical Asia to Malaya.

Local names: Biga-bigáan (Tag.) ; bil-lagut (Ilk.); gabi-gábi (P. Bis., C. Bis.) ; gabing-uák (Tag.) hahalung (If.); hakhaklung (If.) ; kalabúa (Tag.); lagtáng (Bik.); lapalápa (Ilk.) ; saksaklung (Ig.); saksakong (Bon.); upi-úpi (Bik.). 
Var. PAUCIFLORA (Blume) comb. nov.

Pontederia pauciflora Blume Enum. Pl. Jav. 1 (1827) 32.

Pontederia plantaginea Roxb. Fl. Ind. ed. 2, 2 (1832) 123.

Monochoria pauciflora Kunth Enum. 4 (1843) 135; Naves Novis.

App. (1880) 268.

Monochoria plantaginea Kunth 1. c.; Naves 1. c.

Monochoria vaginalis Presl, var. plantaginea Solms in DC. Monog.

Phan. 4 (1883) 524.

Luzon (Ilocos Norte, Bontoc, Benguet, Nueva Vizcaya), Merrill 4616 , Phil. Pl. 1723, Elmer 5953, B. S. 31813 Santos, 11371 McGregor, 14109 Robinson, F. B. 16531 Curran \& Merritt, 15516 Merritt \& Darling. In swamps, rice paddies, etc., altitude 1,000 to $2,000 \mathrm{~m}$. India to China and Java.

The family Philydraceae has been credited to the Philippines on the basis of a Cuming specimen so labeled; Caruel in DC. Monog. Phan. 3 (1881) 3; Naves Novis. App. (1880) 268. Cuming's specimen was from the Malay Peninsula, not from the Philippines. No representative of the family is known from the Archipelago.

\section{JUNCACEAE}

\section{JUNCUS Linnaeus}

JUNCUS BUFONIUS Linn. Sp. Pl. (1753) 328; Buchenau in Engl. Pflanzenreich 25 (1906) 105; Merr。in Philip. Journ. Sci. 10 (1915) Bot. 294.

Luzon (Benguet), Merrill 7794. Along the bridle trails in the mossy forest on Mt. Santo Tomas, altitude $1,900 \mathrm{~m}$; probably introduced. Warmer parts of both hemispheres.

JUNCUS EFFusus Linn. Sp. Pl. (1753) 326; Buchenau in Engl. Pflanzenreich 25 (1906) 135.

Juncus communis E. Meyer Junc. Gen. Monogr. Spec. (1819) 20; Naves Novis. App. (1882) 273.

Luzon (Benguet, Bontoc), Mindanao (Davao). In open swampy places, altitude 1,400 to $2,300 \mathrm{~m}$. Warmer parts of both hemispheres, especially common in the North Temperate Zone.

Local names: Badíli (Ig.) ; balíli (Bon.); piñót (Bon.) ; sudsúd (Ig.). JUNCUS PRISMATOCARPUS R. Br. Prodr. (1810) 259, var. LESCHENAULTII (J. Gay) Buchenau in Engl. Bot. Jahrb. 6 (1885) 137, Engl. Pflanzenreich 25 (1906) 180.

Juncus leschenaultii J. Gay ex Laharpe in Mém. Soc. Linn. Paris

3 (1827) 205; C. B. Rob. in Philip. Journ. Sci. 3 (1908) Bot. 176.

Luzon (Lepanto, Benguet), Merrill 4517, 4739, Williams 1974bis, B. S. 2791, 4261 Mearns. In cold open swamps, altitude 1,500 to $2,300 \mathrm{~m}$. India and Ceylon to Kamchatka and Japan, to southern China, Australia, and New Zealand.

\section{LUZULA de Candolle}

LUZULA CAMPESTRIS (Linn.) DC. Fl. Fr. 3 (1805) 161; Naves Novis. App. (1882) 273; Merr. in Philip. Journ. Sci. 5 (1910) Bot. 337, in nota; Buchenau in Engl. Pflanzenreich 25 (1906) 83, f. $52-55$. Juncus campestris Linn. Sp. Pl. (1753) 329. 
Luzon (Benguet), B. S. 8426 McGregor, 31787 Santos. On slopes in pine forests, at Pauai, extending into the mossy forest along trails, altitude 2,000 to $2,400 \mathrm{~m}$. Temperate regions in both hemispheres.

LUzUlA EFFuSA Buchenau Krit. Verz. Junc. (1880) 53, 88, Engl. Pflanzenreich 25 (1906) 61; Merr. in Philip. Journ. Sci. 5 (1910) Bot. 337.

Luzon(Benguet), Merrill 6490. In the upper border of the mossy forest and in the summit grasslands on Mt. Pulong, altitude about $2,700 \mathrm{~m}$. Himalayan region, China.

\section{STEMONACEAE}

\section{STEMONA Loureiro}

StEMONA PHILIPPINENSIS Merr. in Govt. Lab. Publ. (Philip.) 6 (1904) 16.

Masbate, Merrill 3061. On ridges in thickets of forests at low altitudes. Endemic.

Local name: Sigíd (P. Bis.).

STEMONA TUBEROSA Lour. Fl. Cochinch. (1790) 404; C. H. Wright in Journ. Linn. Soc. Bot. 32 (1896) 495; Merr. in Govt. Lab. Publ. (Philip.) 6 (1904) 16, Interpret. Herb. Amb. (1917) 135.

Roxburghia gloriosoides Roxb. Pl. Coromandel 1 (1795) . 29, t. 32.

Luzon, without definite locality, Palawan, Leyte, Negros, Merrill 792, Loher 6969, Wenzel 1746, Eskridge. In thickets and forests at low altitudes; rare or local. India to Formosa and southward to the Moluccas.

Local name: Lubilubí (C. Bis.).

\section{LILIACEAE}

\section{IPHIGENIA Kunth}

IPHIGENIA INDICA (Linn.) Kunth Enum. 4 (1843) 213; Hook. f. Fl. Brit. Ind. 6 (1892) 357; C. B. Rob. in Philip. Journ. Sci. 3 (1908) Bot. 176.

Melanthium indicum Linn. Mant. 2 (1771) 226.

Luzon (Cavite, Rizal, Laguna), Mindanao (Bukidnon, Davao), Weber 1462, Williams 2962, Loher 1913. In open grasslands at low and medium altitudes; rare or local. India to Australia.

\section{THYSANOTUS R. Brown}

THYSANOTUS CHINENSIS Benth. Fl. Hongk. (1861) 372; Hallier $f$. in Nova Guinea 8 (1914) 991.

Thysanotus chrysantherus F. Muell. Fragm. 5 (1866) 202; Naves Novis. App. (1880) 266; Vidal Phan. Cuming. Philip. (1885) 153, Rev. Pl. Vasc. Filip. (1886) 277.

Luzon (Ilocos Norte, Ilocos Sur, Bontoc, Lepanto, Benguet, Pangasinan), Cuming 981, Merrill 4451, 7750, Williams 971, 1455, B. S. 4905. 27527 Ramos, F. B. 4836, 1561.8 Curran. On open grassy slopes at medium altitudes, ascending to $1,600 \mathrm{~m}$. Malay Peninsula, Indo-China, southern China, New Guinea, tropical Australia.

\section{DIANELLA Lamarck}

DiANELLA CAERULeA Sims in Curtis's Bot. Mag. 15 (1801) t. 505; Red. Lil. 2 (1805) t. 79; Merr. in Philip. Journ. Sci. 2 (1907) Bot. 266, 5 (1910) Bot. 337; Hallier f. in Nova Guinea 8 (1914) 993. 
Luzon (Cagayan, Ilocos Norte, Bontoc, Benguet, Pangasinan, Bulacan, Rizal, Bataan, Nueva Vizcaya, Laguna, Tayabas, Camarines, Sorsogon), Catanduanes, Polillo, Mindoro, Leyte, Negros, Mindanao. In forests at medium and higher altitudes, ascending to $2,200 \mathrm{~m}$; represented by numerous collections. New Guinea, New Caledonia, Queensland, Fiji.

Local names: Abláas (Bag.) ; bariu-báriu (P. Bis.) ; duñgau (Ig.) ; oyonóyon (P. Bis.).

DIANELLA ENSIFOLIA (Linn.) DC. in Red. Lil. 1 (1802) t. 1; Naves Novis. App. (1880) 264; Vidal Rev. Pl. Vasc. Filip. (1886) 277, Phan. Cuming. Philip. (1885) 153; Merr. in Philip. Journ. Sci. 2 (1907) Bot. 266, 3 (1908) Bot. 401, 5 (1910) Bot. 337; Hallier f. in Nova Guinea 8 (1914) 992.

Dracaena ensifolia Linn. Mant. 1 (1767) 63.

Dianella robusta Elm. Leafl. Philip. Bot. 5 (1913) 1806.

Palawan, Panay, Jolo, Bucas Grande, Mindanao, B. S. 35067, $34801 R a-$ mos \& Pascasio, 30995 Ramos \& Edaño, 21562 Escritor, Elmer 12900, Merrill 531\%, 9338, Clemens 937\%. In light forests at low altitudes. India to the Mascarene Islands, China, Formosa, Malaya, tropical Australia, and Polynesia.

DIANELLA JAVANiCA (Blume) Kunth Enum. 5 (1850) 52; Hallier f. in Nova Guinea 8 (1914) 992.

Rhuacophila javanica Blume Enum. 1 (1827) 14; Ridl. in Journ. Linn. Soc. Bot. 42 (1914) 166.

Dianella austro-caledonica Seem. Fl. Vit. (1865-73) 312; Lauterb. in Engl. Bot. Jahrb. 50 (1913) 294.

Batan Islands, Luzon (Ifugao, Bontoc, Lepanto, Benguet, Zambales, Tayabas, Camarines, Albay), Mindoro, Mindanao (Davao), B. S. 31837 Santos, 8478, 17671, 8873 McGregor, 5408 Ramos, 37636 Ramos \& Edaño, Cuming 929, Merrill 5504, 6585, Williams 2556, 1274, 1533, Elmer 5797, Copeland 1035. On open slopes, in ravines, and in forests, altitude 1,000 to $2,400 \mathrm{~m}$. Malay Peninsula, Sumatra, Java, Borneo, Celebes, Moluccas, New Guinea, New Caledonia.

Local names: Apilug (Ig.); sapiláu (Ig.); talobatub (Bon.); uráya (Ig.).

\section{DOUBTFUL AND EXCLUDED SPECIES}

Dianelta Bancana Miq.; Naves Novis. App. (1880) 264.

Dianella montana Blume; Naves 1. c.

Dianella Philippensis Perr. in Mém. Soc. Linn. Paris 3 (1824) 112; C. B. Rob. in Philip. Journ. Sci. 3 (1908) Bot. 305.

"Espece nouvelle de Mindanao" Perrottet. A nomen nudum.

Dianella Revoluta R. Br.; Schauer in Nov. Actp Acad. Nat. Cur. 19 (1843)

Supp. 1: 445 .

Meyen's Jala Jala specimen, so identified by Schauer, is probably referable to the species I have interpreted as $D$. caerulea Sims. 


\section{ALOE Tournefort}

ALOE VERA Linn. Sp. Pl. (1753) 320; Berger in Engl. Pflanzenreich 33 (1908) 228, f. 84, A-D; Merr. Sp. Blancoanae (1918) 95.

Aloe humilis Blanco Fl. Filip. (1837) 256, ed 2 (1845) 179, ed. 3, 1 (1877) 321, t. 95, non Linn.

Aloe barbadensis Mill. Gard. Dict. ed. 8 (1768) No. 2; Naves Novis. App. (1880) 266.

Cultivated for ornamental and medicinal purposes, Merrill Sp. Blancoanae 1005. Subtemperate and tropical regions generally, often cultivated.

Local names: Dílang-boáia (Bis.); dílang-hálo (Bis.); sábila (Tag.) ; sábila-píña (Tag.).

\section{EXCLUDED SPECIES}

Aloe umbellata DC.; Naves Novis. App. (1880) 266.

\section{ALLIUM Tournefort}

No species of this genus occurs naturally in the Philippines, although several are cultivated. These, include the onion, Allium cepa Linn., locally known as cebollas or sibuyas, lasoná (Ilk.); the garlic, Allium sativum Linn., locally known as ájos and báuang; and $A$. tuberosum Roxb., a form locally known as cuchái or ganda. The last is the form described by Blanco as Allium tricoccum (non Ait.) and by Loureiro as Allium angulosum (non Linn.). Other species credited to the Philippines by Naves Novis. App. (1880) 267 are Allium fistulosum Linn., A. jacquemontii Kunth, $A$. ascalonicum Linn., and A. uliginosum Don $=A$. tuberosum Roxb.; I have seen no Philippine specimens of the first three, and it is probable that they do not occur in the Philippines even in cultivation.

\section{LILIUM Tournefort}

LILIUM LONGIFLORUM Thunb. in Trans. Linn. Soc. 2 (1794) 333; Merr. in Philip. Journ. Sci. 3 (1908) Bot. 400.

Y'Ami and Batan Islands, Worcester, B. S. 3774 Fénix. On open grassy slopes at low altitudes. Japan, China, Formosa.

Local name: Vonítan (Iv.).

LILIUM PHILIPPINENSE Baker in Gard. Chron. (1873) 1141, f. 243, Journ. Linn. Soc. Bot.' 14 (1874) 228 (philippense); Curtis's Bot. Mag. III 32 (1876) t. 6250; Naves Novis. App. (1880) 266; Vidal Rev. Pl. Vasc. Filip. (1886) 277; Merr. Philip. Journ. Sci. 5 (1910) Bot. 337.

Luzon (Bontoc, Benguet, Pangasinan), B. S. 31851 Santos, 8791 McGregor, 43449, 3493 Mearns, 8291 Ramos, Merrill 4802, Williams 1132, Elmer 6389. On open slopes in the pine region, altitude 1,100 to $2,300 \mathrm{~m}$. Formosa.

Local names: Kanyon (Ilk.); luplúpak (Ig.) ; soyásoi (Ig.); tuktukpáu (Bon.); tubtubkau (Ig.).

\section{YUCCA Dillenius}

YUCCA ALOIFOLIA Linn. Sp. Pl. (1753) 319; Naves Novis. App. (1880) 266; Merr. in Govt. Lab. Publ. (Philip.) 6 (1904) 32,-Fl. Manila (1912) 145.

Planted here and there for ornamental purposes, but not naturalized. A native of tropical America. 


\section{EXCLUDED SPECIES}

YUCCA gloriosa Linn.; Naves Novis. App، (1880) 266.

YUCCA SUPERBA Haw.; Naves 1. c.

\section{TAETSIA Medicus}

\section{(Cordyline Commerson)}

TAETSIA FRuticosA (Linn.) Merr. Interpret. Herb. Amb. (1917) 137, Sp. Blancoanae (1918) 96.

Convallaria fruticosa Linn. in Stickman Herb. Amb. (1754) 16, Amoen. Acad. 4 (1759) 126, Syst. ed. 10 (1759) 894.

Asparagus terminalis Linn. Sp. Pl. ed. 2 (1762) 450.

Dracaena terminalis Rich. in Lam. Encycl. 2 (1786) 324; Blanco Fl. Filip. (1837) 263, ed. 2 (1845) 183, ed. 3, 1 (1877) 328, t. 9.8.

Cordyline terminalis Kunth in Abh. Acad. Berlin (1820) 30, Enum. 5 (1850) 25; Miq. Fl. Ind. Bat. 3 (1855) 558; Naves Novis. App. (1880) 265; Merr. Fl. Manila (1912) 145.

Widely cultivated for ornamental purposes, but not a native of the Archipelago. Pantropic in cultivation, probably a native of Malaya.

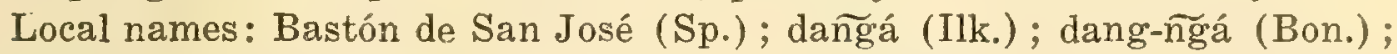
donglá (If.); kiláa (Bis.); kilála (Bik.); sagilála (Tag.); tókor-pári (Pamp.); tónkod-obíspo (Tag.); túngkod-pári (Tag.).

\section{PLEOMELE Salisbury}

Pleomele ANGUStifolia (Roxb.) N. E. Br. in Kew Bull. (1914) 277; Merr. Interpret. Herb. Amb. (1917) 137, Sp. Blancoanae (1918) 96.

Dracaena angustifolia Roxb. Hort. Beng. (1814) 24, Fl. Ind. ed. 2, 2 (1832) 155; Naves Novis. App. (1880) 264; Merr. in Philip. Journ. Sci. 1 (1906) Suppl. 35, 3 (1908) Bot. 400.

Pandanus inermis Blanco Fl. Filip. ed. 2 (1845) 537, ed. 3, 3 (1879) 184, non Roxb.

Batan Islands and northern Luzon to Palawan and Mindanao, in most or all islands and provinces. Chiefly in primary forests at low and medium altitudes; represented by more than 50 individual collections. India through Malaya to Australia.

Local names: Gigiman (Pang.); malasambál (Tag.) ; pasiú (Ibn.) ; saranakatmano (Bag.); taligbúhuk (Tag.); uhañgo-nu-kurang (Iv.).

PLEOMELE MULTIFLORA (Warb.) comb. nov.

Dracaena multiflora Warb. ex Sarasin Reisen in Celebes 1 (1905) $136, t .4$.

Batan Islands, Luzon (Nueva Ecija, Tayabas), Polillo, Mindoro, Palawan, Ticao, Masbate, Sulu Archipelago, Merrill 9424, 5402 B. S. 26434 Ramos \& Edaño, 10407 McGregor, 39508 Ramos, F. B. 18815 Foxworthy, De Mesa, \& Villamil. Chiefly on limestone formations along the seashore. Celebes.

\section{EXCLUDED SPECIES}

Dracaena acuminata Thunb.; Naves Novis. App. (1880) 264.

Dracaena draco Linn.; Naves op. cit. 265. 


\section{CORDYLINE Royen ex Adanson}

(Sansevieria Thunberg)

CORDYLINE ROXBURGHIANA (Schultes) comb. nov.

Sansevieria roxburghiana Schultes Syst. 7 (1829) 357; Naves Novis. App. (1880) 265; N. E. Br. in Kew Bull. (1915) 227, f. 12, D. E.

Sansevieria zeylanica Roxb. Pl. Coromandel. 2 (1798) 43, t. 184, quoad

fig.; Naves Novis. App. (1880) 265, Fl. Filip. ed. 3 (1877-83)

t. 422; Miq. Fl. Ind. Bat. 3 (1859) 559; Merr. Fl. Manila (1912)

144, non Willd.

Cordyline hyacinthoides W. F. Wight in Contr. U. S. Nat. Herb. 9 (1905) 249, non Aloe hyacinthoides Linn.

Often cultivated, but in many regions naturalized, occurring in thickets and hedges at low and medium altitudes. A native of tropical Asia, now pantropic in cultivation. Bowstring hemp.

Local names: Aspi-aspi (Pamp.); baniat (Is.); bontót-pálos (Tag.) ; dildíla (Ilk.); kakaróhai (Ibn.); pakaróhai (Ibn.); rabo de león (Sp.); rabo de tígre (Sp.); sígre (Is.); sináua (Tag.); tígi (Ibn.); tígre (Sp.).

\section{EXCLUDED SPECIES}

SANSEVIERIA GRANDICUSPIS Haw.; Naves Novis. App. (1880) 265.

SANSEVIERIA guineEnsis Willd.; Naves 1. c.

\section{ASPARAGUS Tournefort}

ASPARAgus cochinchInensis (Lour.) Merr. in Philip. Journ. Sci. 15 (1919) 230.

Melanthium cochinchinense Lour. Fl. Cochinch. (1790) 216.

Asparagus lucidus Lindl. Bot. Reg. (1844) Misc. 29, var. dolichocladus Merr. \& Rolfe in Philip. Journ. Sci. 3 (1908) Bot. 96.

Luzon (Benguet), Loher 1928, without data, but probably from above $1,200 \mathrm{~m}$ altitude. Japan, China, Formosa.

The above is the only indigenous species of the genus. Introduced and occasionally cultivated species include the common asparagus (Sp. espárrago) Asparagus officinalis Linn., A. plumosus Baker, and A. sprengeri Regel; see Merrill, Fl. Manila (1912) 143. Naves has credited Asparagus declinatus Linn. and $A$. racemosus Willd. to the Philippines, Novis. App. (1880) 264, but both were apparently admitted on erroneous identifications.

\section{DISPORUM Salisbury}

DISPORUM LUZONIENSE Merr. in Philip. Journ. Sci. 5 (1910) Bot. 338. Disporum pullum Merr. in Philip. Journ. Sci. 1 (1906) Suppl. 182, non Salisb.

Luzon (Lepanto, Bontoc, Benguet), Merrill 485\%, 6619, Phil. Pl. 899, B. S. 31675 Santos, 594.2 Ramos, 8483 McGregor, 4316 Mearns. In the mossy forest, altitude 1,700 to $2,400 \mathrm{~m}$. Endemic.

\section{LIRIOPE Loureiro}

LIRIOPE BRACHYPHYLLA Merr. in Philip. Journ. Sci. 2 (1907) Bot. 266.

Mindoro, Merrill 5710. On seepage slopes in open heath on Mt. Halcon, altitude about $2,400 \mathrm{~m}$. Endemic. 
LIRIOPE GRAMINIFOLIA (Linn.) Baker in Journ. Linn. Soc. Bot. 17 (1879) 499.

Dracaena graminifolia Linn. Syst. ed. 12 (1767) 246.

Liriope spicata Lour. Fl. Cochinch. (1790) 201; C. H. Wright in Journ. Linn. Soc. Bot. 36 (1903) 79.

Ophiopogon spicatus Ker in Bot. Reg. t. 593; Naves Novis. App. (1880) 264.

Luzon (Ilocos Norte, Benguet), F. B.968 Barnes, B. S. 33196 Ramos. On open slopes, altitude about $1,400 \mathrm{~m}$. Japan to Manchuria, southward to Hongkong and Formosa.

\section{OPHIOPOGON Ker}

OPHIOPOGON JAPONICUS (Linn. f.) Ker in Curtis's Bot. Mag. (1807) t. 1063; Merr. in Philip. Journ. Sci. 1 (1906) Suppl. 35, 5 (1910) Bot. 338.

Convallaria japonica Linn. f. Suppl. (1781) 204.

Luzon (Bontoc, Lepanto, Benguet, Nueva Ecija, Zambales, Pampanga, Bataan, Laguna, Tayabas), Mindanao (Bukidnon, Davao). Chiefly in the mossy forest, altitude 850 to $2,400 \mathrm{~m}$; represented by numerous collections. Japan to southern China and Formosa.

Local names: Langigit (Ig.); takaáu (Bon.); uli-úli (Bag.).

OPHIOPOGON MALAYANUS Ridl. in Journ. Str. Branch Roy. As. Soc. 41 (1904) 34.

Jolo, Clemens 9874. On forested slopes, altitude about $700 \mathrm{~m}$. Malay Peninsula, Borneo.

\section{ALETRIS Linnaeus}

ALETRIS SPICATA (Thunb.) Franch. in Journ. de Bot. 10 (1896) 199; Merr. in Philip. Journ. Sci. 1 (1908) Suppl. 182, 5 (1910) Bot. 338. Hypoxis spicata Thunb. Fl. Jap. (1784) 136.

Aletris japonica Lamb. in Trans. Linn. Soc. 10 (1811) 407, non Thunb.

Luzon (Abra, Bontoc, Benguet), Merrill 4685, 4801, Clemens 9178, Vanoverbergh 568, B. S. 7242 Ramos, 31668 Santos. On open grassy slopes in the pine region, altitude 1,600 to $2,300 \mathrm{~m}$. Japan, China, Formosa.

Local name: Salenganga (Ig.).

\section{SMILAX Tournefort}

SMILAX BRACTEATA Presl Rel. Haenk. 1 (1827) 131; Kunth Enum. 5 (1850) 251; Miq. Fl. Ind. Bat. 3 (1859) 566; A. DC. Monog. Phan. 1 (1878) 197; Merr. in Govt. Lab. Publ. (Philip.) 27 (1905) 87, Fl. Manila (1912) 142, Sp. Blancoanae'(1918) 97.

Smilax pseudochina Blanco Fl. Filip. (1837) 795, ed. 2 (1845) 548, ed. 3,3 (1879) 204, non Linn.

Smilax fistulosa Blanco op. cit. 796, 549, 205; A. DC. Monog. Phan. 1. (1878) 211.

Smilax divaricata Blanco op. cit. 795, 548, 206, p. p.; Merr. in Govt. Lab. Publ. (Philip.) 27 (1905) 87.

Smilax blancoi Kunth Enum. 5 (1850) 262; A. DC. Monog. Phan. 1 (1878) 211.

Smilax indica Naves Novis. App. (1880) 262, non Vitm. 
Luzon (Benguet, Pangasinan, Bulacan, Rizal, Bataan, Laguna, Tayabas), Merrill 2548, Sp. Blancoanae 572, Phil. Pl. 313, B. S. 17777 Otanes. Common in thickets and secondary forests at low and medium altitudes. Endemic.

Local names: Banág (Ilk., Tag., Ig.) ; banál (Ig.); barág (Pamp.) ; kamágsa-óbat (Tag.); hampás-tigbálang (Tag.); kolót-bábui (Tag.); rónas (Pamp.).

SMILAX CHINA Linn. Sp. Pl. (1753) 1029; Naves Novis. App. (1880) 261; Merr. in Philip. Journ. Sci. 2 (1907) Bot. 267, 5 (1910) Bot. 339.

Luzon (Bontoc, Lepanto, Benguet, Zambales), Mindoro, Negros, $B . S$. \$1754 Santos, 5413, 5733 Ramos, 8411 McGregor, F. B. 17382 Curran, Merrill 4749, 4497, 6126, 6211, 6140, 6552, 6494, 7043, Williams 1340, 2008. In the mossy forest, altitude 1,600 to $2,400 \mathrm{~m}$. Japan, China.

Local names: Buánal (Ig.); palitpit (Bon.).

SMILAX ELMERI Merr. in Philip. Journ. Sci. 13 (1918) Bot. 6.

Smilax reticulata Elm. Leafi. Philip. Bot. 8 (1915) 2740, non Desv., nec Heer.

Luzon (Sorsogon), Bucas Grande, Mindanao (Bukidnon, Agusan), Elmer $13684,16132, B$. S. 35065 Ramos \& Pascasio, 38761 Ramos \& Edaño. On forested ridges up to $1,000 \mathrm{~m}$ altitude. Endmic.

Local name: Simbanag (Mbo.).

SMILAX ERECTA Merr, in Philip. Journ. Sci. 13 (1918) Bot. 5.

Luzon (Tayabas, east coast), B. S. 28560, 28472 Ramos \& Edaño. On forested slopes at low or medium altitudes. Endemic.

SMILAX LEUCOPHYLLA Blume Enum. Pl. Jav. 1 (1827) 18; Naves Novis. App. (1880) 263; Stapf in Trans. Linn. Soc. Bot. 4 (1894) 242; Ceron Cat. Pl. Herb. Manila (1892) 172; Merr. in Philip. Journ. Sci. 3 (1908) Bot. 96, Sp. Blancoanae (1918) 97.

Smilax latifolia Blanco Fl. Filip. ed. 2 (1845) 548, ed. 3, 3 (1879) 204, non R. Br.

Smilax vicaria Kunth Enum. 5 (1850) 262; A. DC. Monog. Phan. 1

(1878) 212; Merr. in Philip. Journ. Sci. 1 (1906) Suppl. 35, 2 (1907) Bot. 267, 436.

Smilax macrophylla Naves Novis. App. (1880) 262, non Roxb.

Luzon (Benguet, Pangasinan, Pampanga, Rizal, Bataan, Laguna), Mindoro, Culion, Palawan, Mindanao, Merrill 3771, 1713, 659, 5579, Sp. Blancoanae 951, 690, Elmer 12613, Williams 1555, B. S. 20554, 10888 Ramos, 22776 McGregor, 12664, 15865 Fénix, 199.2 Foxworthy, F. B. 1877 Ahern's collector. Chiefly in primary forests at medium altitudes. Malay Peninsula, Java, Borneo, Moluccas.

Local names: Banág (Tagb.); banál (Ig.); kaguno (Neg.); kámotkabág (Tag.) ; sarsaparíllang-putí (Tag.) ; sípit-uláng (Tag.).

SMILAX LOHERI Merr. in Philip. Journ. Sci. 10 (1915) Bot. 294.

Luzon (Nueva Ecija, Rizal), Loher 6968, B. S. 26506 Ramos \& Edaño, 40708 Ramos. In the mossy forest at or above $900 \mathrm{~m}$ altitude.

Local name: Kiting-kiting (Neg.). 
SMILAX LUCIDA Merr. in Philip. Journ. Sci. 13 (1918) Bot. 5.

Luzon (Rizal, Tayabas, east coast), B. S. 26611 Ramos \& Edaño, 40787

Ramos. In forests at low or medium altitudes. Endemic.

Local name: Báru (Ibn.).

SMILAX LUZONENSIS Presl Rel. Haenk. 1 (1827) 131; Kunth Enum. 5 (1850) 261; Miq. Fl. Ind. Bat. 3 (1859) 566; Merr. in Philip. Journ. Sci. 10 (1915) Bot. 295.

Smilax helferi A. DC. Monog. Pharp. 1 (1878) 176, quoad. syn. Presl; Naves Novis. App. (1880) 262.

Luzon (Ilocos Norte), Panay, Catanduanes, Mindanao (Bukidnon, Lanao), B. S. 33327, 30228 Ramos, 35611 Martelino \& Edaño, 38849 Ramos de Edaño, Clemens 835, 905, Presl! in herb. Prague. In forests at medium altitudes. Endemic.

SMILAX PYGMAEA Merr. in Philip. Journ. Sci. 5 (1910) Bot. 339.

Luzon (Benguet), Merrill 6598, B. S. 8902. In the summit grasslands on Mt. Pulog, altitude about $2,800 \mathrm{~m}$. Endemic.

SMILAX VerRuCUlosA Merr. in Philip. Journ. Sci. 5 (1910) Bot. 173.

Luzon (Ifugao, Bontoc, Benguet), Topping 13, Merrill ry42, Elmer 5820, 8572, Williams 1046, B. S. 19702 McGregor, 3767\% Ramos \& Edaño, 2810 Mearns, F. B. 15617 Curran. In thickets about limestone cliffs, in forests and ravines, altitude 1,300 to $1,600 \mathrm{~m}$. Endemic.

Local names: Baná (Bon.); pauá (Bon.).

SMILAX WILliamsil Merr. in Philip. Journ. Sci. 5 (1910) Bot. 173.

Luzon (Tayabas, east coast), Mindanao (Lanao, Bukidnon, Davao), Williams 2579, Clemens 751, B. S. 29139, 38760 Ramos \& Edaño. In thickets or forests at medium altitudes. Endemic.

Local name: Banúg (Bag.).

\section{EXCLUDED SPECIES}

Smilax ASPERA Linn.; Naves Novis. App. (1880) 262.

Smilax barbata Wall.; Naves op. cit. 263.

Similax Bauhinioides Kunth; Naves op. cit. 262.

Smilax BLumei A. DC.; Naves op. cit. 263.

SMIlAX FERox Wall.; Naves op. cit. 262.

Smilax Javeñsis A. DC.; Naves 1. c.

Smilax GRANDIFOLIA Regel; Naves 1. c.

Smilax KLotzschiI Kunth; Naves 1. c.

Smilax LAevis Wall.; Naves op. cit. 261.

Smilax MaCrabucha Duch.; Naves op. cit. 263.

SmilaX MaCrocarpa Blume; Naves op. cit. 262.

Smilax odoratissima Blume; Naves 1. c.; Stapf in Trans. Linn. Soc. Bot. 4 (1894) 242.

Smilax ovalifolia Roxb.; Naves op. cit. 263.

$183036-14$ 
SmilaX PolyaCantha Wall.; Naves op. cit. 262.

Smilax wightir A. DC.; Naves 1 . c.

SMilaX zeYlanica Linn.; Naves 1. c.

\section{EXCLUDED GENERA}

Heterosmilax borneensis A. DC.; Naves Novis. App. (1880) 261.

Scilla sp.; Naves op. cit. $266 . \quad$.

TUlipa GeSNERIANA Linn.; Naves op. cit. 266.

Xerotes longifolia R. Br.; Naves op. cit. 273.

\section{AMARYLLIDACEAE}

\section{ATAMOSco Greene}

ATAMosco RoseA (Lindl.) Greene Pittonia 3 (1897) 188.

Zephyranthes rosea Lindl. in Bot. Reg. 10 (1824) t. 821; Merr. Fl. Manila (1912) 147.

Habranthus versicolor Herb. in Curtis's Bot. Mag. t. 2485; Naves

Novis. App. (1880) 255, Fl. Filip. ed. 3 (1877-83) t. $271,375$.

Widely distributed in cultivation in and about towns, sometimes persisting from previous cultivation but scarcely naturalized. A native of tropical America.

\section{CRINUM Linnaeus}

CRINUM ASIATICUM Linn. Sp. Pl. (1753) 292; Blanco Fl. Filip. (1837) 251, ed. 2 (1845) 175, ed. 3, 1 (1877) 314, t. 168; Miq. Fl. Ind. Bat. 3 (1859) 580; Naves Novis. App. (1880) 254; Merr. in Philip. Journ. Sci. 1 (1906) Bot. 35, Fl. Manila (1912) 147, Sp. Blancoanae (1918) 98.

Crinum giganteum Blanco Fl. Filip. ed. 2 (1845) 175, ed. 3, 1 (1877) 315, non Andr.

Haemanthus pubescens Blanco Fl. Filip. (1837) 253, non Linn. f.

Throughout the Philippines along sandy seashores, sometimes planted inland. India to Malaya and western Polynesia.

Local names: Agabáhan (Bis.) ; agákong (Bon.) ; bákong (Tag., Bag., Ilk., Sbl.); bilíba (Sub.) ; kábong (Bik.) ; palagúkon (Bis.) ; sakníb (Bon.); salibángbang (Bis.); tebá (Bon.).

CRINUM GRACILE E. Meyer in Presl Rel. Haenk. 1 (1827) 120; Kunth Enum. 5 (1850) 566; Naves Novis. App. (1880) 255; Baker Handb. Amaryl. (1888) 81.

Crinum cumingii Baker in Gard. Chron. II 16 (1881) 72, Handb. Amaryl. (1888) 81; Rolfe in Journ. Bot. 23 (1885) 215; Vidal Phan. Cuming. Philip. (1885) 152, Rev. Pl. Vasc. Filip. (1886) 275.

Luzon (Nueva Ecija, Laguna), Mindoro, Mindanao, Cuming 1382, Presl! in herb. Prague, Merrill 4063, McGregor, 152, 228, Elmer 17667, Baker 8725, Bolster 303. In primary forests at low altitudes. Endemic.

CRINUM LATIFOLIUM Linn. Sp. Pl. (1753) 291; Merr. Fl. Manila (1912) 148.

Cultivated in towns for ornamental purposes, but scarcely naturalized. A native of India. 
CRINUM ZEYLANICUM Linn. Syst. ed. 12 (1767) 236; Baker Handb. Amaryl. (1888) 87; Merr. Fl. Manila (1912) 148.

Crinum ornatum Herb. Amaryll. (1837) 262; Naves Novis. App. (1880) 255.

Cultivated for ornamental purposes but nowhere naturalized. A native of tropical Asia.

Local names: Lílio (Sp.); lírio (Tag.).

\section{EXCLUDED SPECIES}

Crinum amabile Donn; Naves Novis. App. (1880) 254.

Crinum amoenum Roxb.; Naves 1. c.

CRINUm Defixum Ker; Naves 1. c.

Crinum Pratense Hub.; Naves 1. c.

Crinum sumatranum Roxb.; Naves 1. c.

\section{HYMENOCALLIS Salisbury}

HYMENOCALLIS LITTORALE (Jacq.) Salisb. in Trans. Hort. Soc. 1 (1812) 338; Merr. Sp. Blancoanae (1918) 98.

Pancratium littorale Jacq. Select. Stirp. Amer. (1763) 99.

Pancratium illyricum Blanco Fl. Filip. (1837) 251, ed. 2 (1845) 176, ed. 3,1 (1877) 316, t. 411 (as Hymenocallis adnata Herb.), non Linn.

Pancratium maritimum Blanco op. cit. 252, 177, 316; Naves Novis. App. (1880) 256; Merr. in Govt. Lab. Publ. (Philip.) 27 (1905) 86 , non Linn.

Hymenocallis adnata Herb. Amaryll. (1837) 215; Naves Novis. App. (1880) 256.

Hymenocallis tenuiflora Herb. Amaryll. Súppl. (1837) 44; Merr. Fl. Manila (1912) 149.

Cultivated in Manila and other towns, occasional in waste places, etc., but apparently propagating only by its bulbs. A native of tropical. America.

\section{EUCHARIS Planchon and Linden}

EUCHARIS GRANDIFLORA Planch. \& Linden Fl, des Serres I 9 (185354) 255 ; Merr. Fl. Manila (1912) 149.

This occurs in the Philippines only as a cultivated ornamental in Manila and in a few of the larger towns. A native of South America. The Amazon lily.

\section{EURYCLES Salisbury}

EURYCLES AMBOINENSIS (Linn.) Lindl. in Loud. Encycl. Pl. (1829) 242; Miq. Fl. Ind. Bat. 3 (1859) 582; Naves Novis. App. (1880) 255; Vidal Phan. Cuming. Philip. (1885) 152, Rev. Pl. Vasc. Filip. (1886) 276; Merr. Fl. Manila (1912) 149, Sp. Blancoanae (1918) 99. Pancratium amboinense Linn. Sp. Pl. (1753) 291; Blanco Fl. Filip. (1837) 252, ed. 2 (1845) 177, ed. 3,1 (1877) 317, t. 406.

Eurycles sylvestris Salisb. in Trans. Hort. Soc. 1 (1812) 337.

Luzon (Cavite, Rizal, Laguna), Mindoro, Palawan. In secondary forests at low altitudes, never far from settlements and hence probably introduced; often cultivated for ornamental purposes. Malay Peninsula and Archipelago to tropical Australia. 
Local names: Abod (Bis.) ; abur (Bis.) ; dausum (Bis.) ; katañgal (Bis.); kosol (Bis.); panabor (Bis.); taliunúd (Bik.); talaonor (Bis.); tambal (Tag.) ; tanual (Bis.).

6. PANCRATIUM Linnaeus

PANCRATIUM ZEYLANICUM Linn. Sp. Pl. (1753) 290; Blanco Fl. Filip. (1837) 253, ed. 2 (1845) 177, ed. 3, 1 (1877) 317, t. 3.21; Naves Novis. App. (1880) 256; Merr. Fl. Manila (1912) 148, Sp. Blancoanae (1918) 99.

Luzon (Union, Camarines), Palawan, Biliran, Merrill Sp. Blancoanae 378, B. S. 18534 McGregor, 881 Foxworthy. In coconut and abaca plantations, thickets, etc., sometimes planted. Certainly not a native of the Philippines. India to Malaya.

Local names: Baoang-báoang (Bis.); hagobaóa (Bis.); katoñăal (Tag.).

\section{EXCLUDED SPECIES}

Pancratium malabathricum Herb.; Naves Novis. App. (1880) 256.

\section{HIPPEASTRUM Herbert}

HIPPEASTRUm MINIATUM Herb. Amaryll. Suppl. (1837) 31; Naves Novis. App. (1880) 255; Merr. Sp. Blancoanae (1918) 99.

Amaryllis atamasco Blanco Fl. Filip. (1837) 254, ed. 2 (1845) 178, ed. 3, 1 (1877) 319, t. 359; Merr. in Govt. Lab. Publ. (Philip.) 27 (1905) 85, non Linn.

Luzon (Union, Rizal, Laguna, Tayabas), Panay, Mindanao, Siassi, Merrill Sp. Blancoanae 1048, Elmer 17438b. Introduced and planted for ornamental purposes, but more or less naturalized in coconut groves, etc. A native of tropical America.

Local name: Lírio (Tag.).

\section{POLIANTHES Linnaeus}

POLIANthes tuberosa Linn. Sp. Pl. (1753) 316; Blanco Fl. Filip. (1837) 259, ed. 2 (1845) 181, ed. 3, 1 (1877) 323; Naves Novis. App. (1880) 254; Merr. in Govt. Lab. Publ. (Philip.) 27 (1905) 85, Fl. Manila (1912) 146, Sp. Blancoanae (1918) 99.

Cultivated for its fragrant flowers but nowhere naturalized. A native of tropical America. Tuberose.

Local names: Azucéna (Sp.); nárdo (C. Bis.).

\section{AGAVE Linnaeus}

AGAVE CANTAlA Roxb. Hort. Beng. (1814) 25; Berger Agav. (1915) 236; Merr. Interpret. Herb. Amb. (1917) 144, Sp. Blancoanae (1918) 99.

Furcraea cantala Haw. Syn. Pl. Succul. Suppl. (1819) 42.

Agave cantula Roxb. Fl. Ind. ed. 2, 2 (1832) 167; Naves Novis. App. (1880) 253; Merr. Fl. Manila (1912) 146.

Agave americana Blanco Fl. Filip. (1837) 258, ed. 2 (1845) 180, ed. 3, 1 (1877) 322, t. 96; Naves Novis. App. (1880) 253; Merr. in Govt. Lab. Publ. (Philip.) 6 (1904) 23; Usteri Beitr. Ken. Philip. Veg. (1905) 129, non Linn. 
Introduced from Mexico at an early date in colonial history, now widely distributed in the Philippines, in some provinces extensively grown for its fiber. Pantropic.

Local names: magéi (all dialects); píta (C. Bis.).

AGave sisalana Perrine ex Engelm. in Trans. Acad. Sci. St. Louig 3 (1875) 314; Berger Agav. (1915) 230. f. 66.

Introduced in the early part of the present century, and now widely planted for its fiber. A native of tropical America. Sisal.

EXCLUDED SPECIES

Agave mexicana Lam.; Naves Novis. App. (1880) 253.

10. CURCULIGo Gaertner

CuRCuligo Agusanensis EIm. Leafl. Philip. Bot. 5 (1913) 1645.

Luzon (Sorsogon), Mindanao (Zamboanga, Agusan), Elmer 13584, $17005, B$. S. 36910 Ramos \& Edaño. In forests at low altitudes. Endemic.

Local names: Kuyabáng (Sub.); pagátpat (Mbo.); tayábong (Sub.).

CURCUligo BREVIPEdUnCUlata Elm. Leafl. Philip. Bot. 5 (1913) 1647.

Palawan, Balabac, Panay, Elmer 18164, Merrill 5373, Phil. Pl. 1318, B. S. 32576 McGregor. In forests at low altitudes. Endemic.

CURCUligo CAPITUlatA (Lour.) O. Kuntze Rev. Gen. Pl. (1891) 703; Merr. Interpret. Herb. Amb. (1917) 142.

Leucojum capitulatum Lour. Fl. Cochinch. (1790) 199.

Curculigo recurvata Dry. in Ait. Hort. Kew ed. 2, 2 (1811) 253;

Naves Novis. App. (1880) 253; Merr. in Philip. Journ. Sci. 3 (1908)

Bot. 401; Elm. Leafl. Philip. Bot. 5 (1913) 1645.

Batan Islands, Luzon (Bontoc, Benguet, Laguna, Tayabas, Sorsogon, Biliran, Leyte, Panay, Siargao, Mindanao), Merrill 9670, Phil. Pl. 847, Elmer 6202, 7771, 18196, Williams 1150, B. S. 35389 Ramos \& Edaño, 31962 Santos, 15299, 23571 Ramos, 18867 McGregor, 14006 Robinson, F. B. 4893 Curran. In thickets and forests, chiefly at medium altitudes, ascending to $1,800 \mathrm{~m}$. India to southern China through Malaya to tropical Australia.

Local names: Abang-ábang (Bik.); atukgan (Ig.); katuágan (Ig.); talauáñgit (Bag.); tayúbang (Bon.); tokán (Ig.) ; tolábang (If.).

CuRculigo Glabra Merr. in Philip. Journ. Sci. 2 (1907) Bot. 267; C. B. Rob. op. cit. 6 (1911) Bot. 196; Elm. Leafl. Philip. Bot. 5 (1913) 1645.

Luzon (Sorsogon), Polillo, Alabat, Mindoro, Merrill 5750, 10449, Elmer $14990, B$. S. 10366 McGregor. In primary forests at low and medium altitudes. Endemic.

CURCULIGO ORCHIOIDES Gaertn. Fruct. 1 (1788) 63, t. 16; Elm. Leafl. Philip. Bot. 5 (1913) 1645; Merr. Sp. Blancoanae (1918) 100.

Gethyllis acaulis Blanco Fl. Filip. (1837) 260, ed. 2 (1845) 181, ed. 3,1 (1877) 324. 
Luzon (Bontoc, Pangasinan, Rizal, Sorsogon), Mindoro, Semirara, Palawan, Biliran, Panay, Mindanao, Merrill 4144, Sp. Blancoanae 260, Elmer 16484, Williams 2927, Copeland 472, B. S. 32413, 18637 McGregor, 26122 Fénix, 1520 Bermejos, 6rзg Robinson, 21440 Escritor, 17858 Otanes. In open grasslands, chiefly in cogonales (Imperata areas), at low and medium altitudes. India to Java.

Local names: Sulsulitík (Bon.); taloáñ̃gi (Bag.); tataluañõi (Buk.).

CURCULIGo WEBERI EIm. Leafl. Philip. Bot. 5 (1913) 1646.

Samar, Mindanao (Agusan, Lanào), Elmer 14304, Weber 1081, Clemens 1225, Taylor $285, B$. S. 17657 Ramos. In forests at low and medium altitudes. Endemic.

Local name: Bongbón (C Bis.).

\section{EXCLUDED SPECIES}

Curculigo ensifolia R. Br.; Naves Novis. App. (1880) 253.

Curculigo latifolia Dry.; Naves 1. c.

\section{HYPOXIS Linnaeus}

HYPOXIS AUREA Lour. Fl. Cochinch. (1790) 200.

Hypoxis franquevillei Miq. Fl. Ind. Bat. 3 (1859) 586; Naves Novis. App. (1880) 253.

Hypoxis flava Merr. in Govt. Lab. Publ. (Philip.) 27 (1905) 85 sphalm.

Luzon (Bontoc, Benguet, Nueva Ecija), Mindanao (Lanao, Bukidnon), Elmer 6585, Merrill 247, 4453, Phil. Pl. 848, Williams 1063, B. S. 15775 F'énix, 31818 Santos. In open grasslands at medium and higher altitudes, ascending to $2,300 \mathrm{~m}$. India to Japan southward to Java.

Local name: Kitkitlí (Ig.).

\section{EXCLUDED GENERA AND SPECIES}

AMArYlis CARNea Schultes; Naves Novis. App. (1880) 255.

AMARYLlis FORMOSISSIMA Linn.; Naves 1. c.

AMaryllis vitTata Ait.; Naves 1. c.

Furcraea gigantea Vent.; Naves op. cit. 253.

Furcraea Rigida Haw.; Naves op. cit. 254.

Hermione tazetta Haw.; Naves op. cit 256.

\section{TACCACEAE}

\section{TACCA Forster}

TACCA ELMERI Krause in Leafl. Philip. Bot. 6 (1914) 2283.

Palawan, Leyte, Elmer 12679, Weber 1522. In light forests at low altitudes. Endemic.

Loca name: Odang-ódang (Tagb.).

TACCA PAlMATA Blume Enum. Pl. Jav. 1 (1827) 83; Miq. Fl. Ind. Bat. 3 (1859) 577; Naves Novis. App. (1880) 257; Merr. in Philip. Journ. Sci. 1 (1906) Suppl. 35, Fl. Manila (1912) 150, Sp. Blancoanae (1918) 100.

Tacca montana Schultes Syst. $7^{1}$ (1829) 168; Vidal Phan. Cuming. Philip. (1885) 152, Rev. Pl. Vasc. Filip. (1886) 276. 
Tacca vesicaria Blanco Fl. Filip. (1837) 261.

Tacca palmata Blanco Fl. Filip. ed. 2 (1845) 182, ed. 3, 1 (1877) 325.

Tacca rumphii Schauer in Nov. Act. Acad. Nat. Cur. 19 (1843)

Suppl. 1: 442; Miq. Fl. Ind. Bat. 3 (1859) 577; Naves Novis. App. (1880) 257.

Luzon (Pangasinan, Nueva Ecija, Bulacan, Rizal, Bataan, Laguna), Semirara, Panay. In thickets and secondary forests at low altitudes. Malay Archipelago.

Local names: Kanálong (Bis.); magsalóro (Bis.); payung-payúñ̆an (Tag.).

TACCA PINNATIFIDA Forst. Char. Gen. (1776) 70, $t$. 35; Presl Rel. Haenk. 1 (1827) 148; Blanco Fl. Filip. ed. 2 (1845) 182, ed. 3, 1 (1877) 327; Miq. Fl. Ind. Bat. 3 (1859) 577; Naves Novis. App. (1880) 257; Hook. f. in Curtis's Bot. Mag. III 49 (1893) t. 7299, 7300; Merr. Fl. Manila (1912) 150, Sp. Blancoanae (1918) 101.

Tacca gaogao Blanco Fl. Filip. (1837) 262, 856.

Luzon (Ilocos Norte) to Palawan and Mindanao, in most islands and provinces. Chiefly in sandy soil in thickets near the sea. Tropical Africa and Asia through Malaya to Australia and Polynesia.

Local names: Gau-gáu (Tag.) ; kanóbong (Bis.); panaríen (Ilk.) ; tambóbon (Sbl.); tayóbong (Bis.); yabyában (Tag.).

\section{EXCLUDED SPECIES}

TACCA ASPERA Roxb.; Naves Novis. App. (1880) 258.

TACCA LANCAEFolia Zoll.; Naves 1. c.

\section{DIOSCOREACEAE}

\section{DIOSCOREA Plumier}

DIoscoreA ALATA Linn. Sp. Pl. (1753) 1033; Blanco Fl. Filip. (1837) 799, ed. 2 (1845) 550, ed. 3, 3 (1879) 207; Miq. Fl. Ind. Bat. 3 (1859) 572; Naves Novis. App. (1880) 259; Merr. Fl. Manila (1912) 151, Interpret. Herb. Amb. (1917) 146, Sp. Blancoanae (1918) 102; Prain \& Burkill in Journ. As. Soc. Beng. II 10 (1914) 39.

Cultivated throughout the Philippines for its edible tubers, but certainly not a native of the Archipelago. Pantropic in cultivation, a native of the old World Tropics.

Local names: Ípoi (Ig.) ; kinampái (Bis.) ; lúktu (If.) ; úbi (Tag.), Bik., Ibn., Ilk., It., Bis.).

DIOSCOREA BULb!feRA Linn. Sp. Pl. (1753) 1033; Schauer in Nov. Act. Acad. Nat. Cur. 19 (1843) Suppl. 1:445; Miq. Fl. Ind. Bat. 3 (1859) 574; Naves Novis. App. (1880) 258; Ceron Cat. Pl. Herb. Manila (1892) 171; Merr. Fl. Manila (1912) 151; Prain \& Burkill in Leafl. Philip. Bot. 5 (1913) 1596, Journ. As. Soc. Beng. II 10 (1914) 26.

Luzon (Bontoc, Union, Nueva Ecija, Batangas, Bulacan, Rizal, Bataan, Zambales, Sorsogon), Masbate, Leyte, Mindanao. In thickets at low 
and medium altitudes; represented by numerous collections. India to China and Malaya.

Local names: Aribúkbuk (Ilk.) ; bayag-kabáyo (Tag.); dadákan (Bag.) ; pulúgan (Bik.) ; ubí-ubíhan (Tag.) ; utóñg-utóñan (Tag.).

DIOSCOREA CUMINGII Prain \& Burkill in Journ. As. Soc. Beng. II 4 (1908) 449, 10 (1914) 25, Leafl. Philip. Bot. 5 (1913) 1595.

Dioscorea inaequifolia Elmer ex Prain \& Burkill in Leafl. Philip.

Bot. 5 (1913) 1595, Journ. As. Soc. Beng. II 10 (1914) 24.

Luzon (Apayao, Bontoc, Benguet, Lepanto, Rizal, Bataan, Laguna, Nueva Vizcaya, Rizal, Camarines, Sorsogon), Mindoro, Mindanao, Cuming 1469, Elmer 15333, 8692, Merrill 4455, 7683, Phil. Pl. 2034, F. B. 22956bis Caly$\cos a, 3376$ Ahern's collector, 69 Barnes, B. S. 8207 Ramos. In thickets and forests at low and medium altitudes, ascending to 1,400 m. Endemic.

Local names: Lima-limá (Tag.); pari (Bag.).

DIOSCOREA DIVARICATA Blanco Fl. Filip. (1837) 797, ed. 2 (1845) 550, ed. 3, 3 (1879) 207; Merr. in Philip. Journ. Sci. 1 (1906) Suppl. 35, 2 (1907) Bot. 436, Sp. Blancoanae (1918) 101.

Dioscorea batatas Naves Novis. App. (1880) 258, non Decne.

Dioscorea foxworthyi Prain \& Burkill in Journ. As. Soc. Beng. II 10 (1914) 34.

Luzon (Ilocos Norte, Pangasinan, Pampanga, Rizal, Bataan, Laguna), B. S. 22425, 33185 Ramos, 29960, 30092 Fénix, 1558 Foxworthy, F. B. 2572 Borden, Merrill Sp. Blancoanae 391. In thickets and secondary and primary forests at low and medium altitudes. Endemic.

Local names: Baliákag (Bis.) ; bulói (Tag.) ; dulián (Ilk.) ; durián (Ilk.) ; cuúyan (Pang.); kirói (Tag.) ; kobág (Tag.) ; pakít (Tag.) ; ubág (Tag.).

DIOScoreA ELMERI Prain \& Burkill in Leafl. Philip. Bot. 5 (1913) 1594, Journ. As. Soc. Beng. II 10 (1914) 24 (incl. var. vera and var. dubia).

Batan Islands, Luzon (Laguna, Tayabas), Samar, Elmer 9156, 8265, B. S. 28717,24196 Ramos \& Edaño, 3659 F'énix. In forests at low altitudes; really distinct from $D$. cumingii Prain \& Burkill? Endemic.

DIOSCOREA EsculentA (Lour.) Burkill in Gard. Bull. Straits Settl. 1 (1917) 396; Merr. Interpret. Herb. Amb. (1917) 147, Sp. Blancoanae (1918) 102.

Oncus esculentus Lour. Fl. Cochinch. (1790) 194.

Dioscorea papillaris Blanco Fl. Filip. (1837) 801, ed. 2 (1845) 552, ed. 3, 3 (1879) 200.

Dioscorea tugui Blanco Fl. Filip. (1837) 800.

Dioscorea sativa Blanco Fl. Filip. ed. 2 (1845) 551, ed. 3, 3 (1879) 209, Naves Novis. App. (1880) 259, non Linn.

Dioscorea fasciculata Roxb. Fl. Ind. ed. 2, 2 (1832) 801; Naves Novis. App. (1880) 260; Merr. Fl. Manila (1912) 151.

Dioscorea tiliaefolia Kunth Enum. 5 (1840) 401; Miq. Fl. Ind. Bat. 3 (1859) 576; Naves Novis. App. (1880) 260; Vidal Phan. Cuming. Philip. (1885) 153, Rev. Pl. Vasc. Filip. (1886) 276.

Dioscorea aculeata Naves Novis. App. (1880) 260; Prain \& Burkill in Journ. As. Soc. Beng. II 10 (1914) 19, Leafl. Philip. Bot. 5 (1913) 1593 (var. tiliaefolia Prain \& Burkill), non Linn. 
Batan Islands, Luzon (Cagayan, Benguet, Union, Pangasinan, Nueva Vizcaya, Bulacan, Rizal, Bataan, Laguna), Semirara, Merrill 3805, 2727, 2709, 4147, Sp. Blancoanae 6r7, 202, Phil. Pl. 325, Elmer 6421, B. S. 32265, 14381, 23076 McGregor, 21120 Escritor, 21720 Ramos, F. B. 3280 Ahern'8 collector, 24879 Mabesa. In thickets and secondary forests at low altitudes, also often cultivated. India to Malaya and Polynesia.

Local names: Anég (Ibn.) ; bóga (Ilk.) ; dúkai (Iv.); kamigíng (Bik.); lúttu (Ibn.); tóngo (Tag.) túgi (Tag., Ilk.); túñgo (Tag.).

DIOSCOREA FILIFORMIS Blume Enum. Pl. Jav. 1 (1827) 22; Prain \& Burkill in Journ. As. Soc. Beng. II 10 (1914) 38.

Dioscorea myriantha Kunth Enum. 5 (1850) 382; Miq. Fl. Ind. Bat. 3 (1859) 571; Naves Novis. App. (1880) 258; Vidal Phan. Cuming. Philip. (1885) 153, Rev. Pl. Vasc. Filip. (1886) 276; Prain \& Burkill in Journ. As. Soc. Beng. II 10 (1914) 38.

Luzon (Nueva Ecija, Rizal, Bataan, Batangas, Laguna), Culion, Palawan, Mindanao (Jolo), Merrill 466, 85\%, 8058, 3167, 8120, 8244, Baker 460, B. S. 22266 Ramos, 209, 330 Bermejos, F. B. 6036 Zschokke. In thickets and in ravines at low altitudes. Java, Celebes, Moluccas.

DIOSCOREA FLABELLIFOLIA Prain \& Burkill in Leafl. Philip. Bot. 5 (1913) 1593, Journ. As. Soc. Beng. II 10 (1914) 12.

Luzon (Laguna, Sorsogon), Catanduanes, Mindoro, Loher 7027, Elmer 9095, 1667\%, B. S. 30278, 23564, 39412 Ramos, 22830 McGregor. In forests at low and medium altitudes. Borneo.

DIOScoReA GRATA Prain \& Burkill in Journ. As. Soc. Beng. II 10 (1914) 35.

Luzon (Rizal), Loher 7012, 7017, without data as to habitat. Endemic.

DIOScoreA HISPIDA Dennst. Schlüssel Hort. Malabar. (1818) 33; Merr. Interpret. Herb. Amb. (1917) 148, Sp. Blancoanae (1918) 103.

Dioscorea hirsuta Blume Enum. Pl. Jav. 1 (1827) 21; Miq. Fl. Ind. Bat. 3 (1859) 575; Naves Novis. App. (1880) 260.

Dioscorea daemona Roxb. Hort. Beng. (1814) 72, nomen nudum. Fl. Ind. ed. 2, 3 (1832) 805; Merr. in Govt. Lab. Publ. (Philip.) 27 (1905) 86, Fl. Manila (1912) 151.

Dioscorea triphylla Blanco Fl. Filip. (1837) 799, ed. 2 (1845) 551, ed. 3, 3 (1879) 208; Miq. F1. Ind. Bat. 3 (1859) 573; Naves Novis. App. (1880) 259; Prain \& Burkill in Journ. As. Soc. Bengal II 10 (1914) 25, Leafl. Philip. Bot. 5 (1913) 1595, non Linn.

Throughout the Philippines in thickets and forests at low and medium altitudes. India to southwestern China and Formosa through Malaya to New Guinea.

Local names: Bagai (Mbo.); gáyos (Bis.); kalút (Tag., Pamp., Sbl.); karót (Ilk.) ; karóti (Sul.) ; káyos (Tag.) ; kolót (Bís.) ; korót (S. L. Bis.) ; kulót (Sbl.) ; mamó (Bik.) ; namí (Tag.) ; orkót (Bis.).

DIOSCOREA LOHERI Prain \& Burkill in Journ. As. Soc. Beng. II 10 (1914) 33; Merr. Sp. Blancoanae (1918) 101.

Dioscorea sp. (cobag na quiroi) Blanco Fl. Filip. (1837) 798, ed. 2 (1845) 550, ed. 3, 3 (1879) 206. 
Luzon (Rizal), Merrill Sp. Blancoanae 634, Phil. Pl. 6, 1498, B. S. 12529 Ramos. In thickets at low altitudes. This is perhaps referable to $D$. divaricata Blanco, the plants from not fully grown tubers. Endemic.

Local names: Kirói (Tag.); kobág (Tag.).

DIOSCOREA LUzONENSIS Schauer in Nov. Act. Acad. Nat. Cur. 19 (1843) Suppl. 1: 444; Miq. Fl. Ind. Bat. 3 (1859) 571; Naves Novis. App. (1880) 258; Vidal Phan. Cuming. Philip. (1885) 153, Rev. Pl. Vasc. Filip. (1886) 276; Merr. in Philip. Journ. Sci. 5 (1910) Bot. 339; Prain \& Burkill in Leafl. Philip. Bot. 5 (1913) 1597. Tourn. As. Soc. Beng. II 10 (1914) 28.

Luzon (Bontoc, Lepanto, Ilocos Norte, Union, Nueva Vizcaya, Bulacan, Rizal, Batangas, Laguna, Tayabas), Cuming r79, Elmer 18290, 18466, Merrill Sp. Blancoanae 117, Phil. Pl. 1070, F. B. 24909 Mabesa, 13218 Curran, 13336 Tamesis, 3293 Ahern's collector, B. S. 13734, 37240, 21752, 1380 Ramos. In thickets at low and medium altitudes. Endemic.

Local names: Aribúbu (Ilk.) ; kamáñgeg (Ilk.) ; kiríni (Tag.) ; mayatbáng (Tag.) ; pakít (Tag.) ; ubág (Tag.).

DIOSCOREA MERRILLII Prain \& Burkill in Leafl. Philip. Bot. 5 (1913) 1598, Journ. As. Soc. Beng. II 10 (1914) 35.

Mindoro, Mindanao (Davao), Merrill 565\%, Elmer 11924. In old clearings and in thickets, altitude about $700 \mathrm{~m}$. Endemic.

DIOSCOREA NUMmUlariA Lam. Encycl. 3 (1789) 231; Naves Novis. App. (1880) 258; Merr. in Philip. Journ. Sci. 2 (1907) Bot. 276; Prain \& Burkill in Leafl. Philip. Bot. 5 (1913) 1599, Journ. As. Soc. Beng. II 10 (1914) 35.

Luzon (Bontoc to Sorsogon, in most or all provinces), Polillo, Leyte, Camiguin de Misamis, Mindanao, Basilan. In thickets and forests at low and medium altitudes.

Var. GLAuCA Prain \& Burkill in Journ. As. Soc. Beng. II 10 (1914) 35.

Luzon (Benguet), Elmer 5638. Scarcely distinguishable from the type. The species in Celebes, Moluccas, New Guinea to Prince of Wales Island.

Local names: Banán (Bag.); malabalukbúk-dágis (Pamp.); pakít (Tag.) ; pinót (Tag.) ; úbing-básol (Tag.).

DIOscorea PENTAPHYLla Linn. Sp. PI. (1753) 1032; Blanco Fl. Filip. (1837) 802, ed. 2 (1845) 552, ed. 3, 3 (1879) 210; Miq. Fl. Ind. Bat. 3 (1859) 574; Naves Novis. App. (1880) 259; Merr. in Philip. Journ. Sci. 1 (1906) Suppl. 35, 3 (1908) Bot. 401, Fl. Manila (1912) 152, Sp. Blancoanae (1918) 103; Prain \& Burkill in Journ. As. Soc. Beng. II 10 (1914) 23.

Luzon (Rizal, Bataan, Laguna), Panay, Merrill Sp. Blancoänae 458, $F$. B. 1971 Ahern's collector, B. S. 12176 Ramos. In thickets at low and medium altitudes. India to Yunnan, southward through Malaya to New Guinea.

Local names: Kasi (Ig.) ; lima-limá (Tag.) sapáng (Bis.).

The Philippine form is apparently referable to var. malaica Prain \& Burkill 1. c. 
DIoscoreA PePeroldes Prain \& Burkill in Elm. Leafl. Philip. Bot. 5 (1913) 1597, Journ. As. Soc. Beng. II 10 (1914) 28 (var. vera Prain \& Burkill).

Luzon (Benguet, Rizal), Golo, Palawan, Elmer 6399, 6400, Merrill 9441, 6512, 11552, 11555, Loher 7007, 7010. In thickets up to 1,300 m altitude, in Palawan about limestone formations at sea level.

Var. SAGITTIFOLIA Prain \& Burkill in Journ. As. Soc. Beng. II 10 (1914) 28.

Luzon (Nueva Vizcaya), B. S. 8178 Ramos. In thickets at low or medium altitudes. The species and this variety endemic, another variety in Indo-China.

DIOscoreA SORoR Prain \& Burkill in Leafl. Philip. Bot. 5 (1913) 1598, Journ. As. Soc. Beng. II 10 (1914) 34 (var.vera Prain \& Burkill).

Luzon (Lepanto, Bataan), Elmer 6737, Bona, B. S. 5941 Ramos. In thickets at low and medium altitudes. Endemic. Probably the same as D. divaricata Blanco.

Var. GLAuCA Prain \& Burkill in Journ. As. Soc. Beng. II 10 (1914) 34.

Luzon (Bulacan), Yoder. In thickets at low altitudes. Endemic.

\section{EXCLUDED SPECIES}

Dioscorea eburnea Lour.; Naves Novis. App. (1880) 260.

DIOSCOREA GLABRA Roxb.; Naves op. cit. 258.

Dioscorea JAPONICA Thunb.; Naves op. cit. 259.

DIOSCOREA oppositifolia Linn.; Naves op. cit. 258.

DIOSCOREA PYRIFOLIA Kunth Enum. 5 (1850) 384; Miq. Fl. Ind. Bat. 3 (1859) 571; Naves Novis. App. (1880) 258.

This record was based on Cuming 2314 as Philippine, but this number was from Malacca; the species does not occur in the Philippines.

Dioscorea SATIVA Linn.; Naves op. cit. 259.

Dioscorea spiculata Blume; Naves 1.c.

DIOSCOREA VUlgaRIs Miq.; Naves 1. c.

\section{STENOMERIS Planchon}

STENOMERIS CUMINGIANA Becc. in Nuov. Giorn. Bot. Ital. 2 (1870) 8, t. 2; Scheff. in Nat. Tijdschr. Nederl. Ind. 34 (1874) 70; Rolfe in Journ. Bot. 23 (1885) 215, Rev. Pl. Vasc. Filip. (1886) 278; Taubert in Engl. Bot. Jahrb. 15 (1893) Beibl. 38: 2.

Luzon (Laguna, Sorsogon), Mindoro, Sibuyan, Leyte, Mindanao (Zamboanga), Cuming 1739 in herb. Webb.?, Wenzel 1210, 962, 718, Elmer 12064, F. B. 26909 Mabesa, B. S. 39429 Ramos. In forests at low and medium altitudes. Endemic.

STENOMERIS DIOSCOREAEFOLIA Planch. in Ann. Sci. Nat. Bot. III 18 (1852) 320; Walp. Ann. 6 (1860) 42; Becc. in Nuov. Giorn. Bot. Ital. 2 (1870) 8; Scheff. in Nat. Tijdschr. Nederl. Ind. 34 (1874) 70 ; Vidal Phan. Cuming. Philip. (1885) 153, Rev. Pl. Vasc. Filip. (1886) 276; Rolfe in Journ. Bot. 23 (1885) 216; Taubert in Engl. Bot. Jahrb. 15 (1893) Beibl. 38: 2; Merr. in Philip. Journ. Sci. 2 (1907) Bot. 268. 
Luzon (Rizal, Laguna, Tayabas, Albay, Sorsogon), Catanduanes, Mindoro, Leyte, Biliran, Dinagat, Mindanao, Cuming 875, Merrill 5775, 8301, Phil. Pl. 427, Elmer 9098, Baker 3383, B. S. 1005, 30339, 23514 Ramos, 35192 Ramos \& Pascasio. In thickets, old clearings, etc., at low and medium altitudes. Endemic.

STENOMERIS WALLISII Taubert in Engl. Bot. Jahrb. 15 (1893) Beibl. 38: 2 .

Luzon, Wallis! in herb. Berol. Endemic.

The genus has one other known species in Borneo and the Malay Peninsula.

EXCLUDED GENUS

RaJana sp.; Naves Novis. App. (1880) 260.

\section{IRIDACEAE}

\section{BELAMCANDA Adanson}

BELAMCANDA CHINENSIS (Linn.) DC. in Red. Lil. 3 (1802) $t .121$; Usteri Beitr. Ken. Philip. Veg. (1905) 129; C. B. Rob. in Philip. Journ. Sci. 6 (1911) Bot. 196; Merr. Fl. Manila (1912) 152.

Ixia chinensis Linn. Sp. Pl. (1753) 36.

Pardanthus chinensis Ker in Konig \& Sims Ann. Bot. 1 (1805) 247;

Naves Novis. App. (1880) 252, Fl. Filip. ed. 3 (1877-83) t. 376.

Planted for ornamental purposes but nowhere naturalized. A native of southeastern Asia, now cultivated in most warm countries.

Local name: Abanico (Sp.).

\section{PATERSONIA R. Brown}

PATERSONIA LOWII Stapf in Trans. Linn. Soc. Bot. 4 (1894) 241, t. 20, f. $7 \rightarrow 9$; Merr. in Philip. Journ. Sci. 2 (1907) Bot. 268.

Mindoro, Merrill 550\%. In an open heath on Mt. Halcon, altitude about 2,400 m. Borneo. The genus a typical Australian one.

\section{ELEUTHERINE Herbert}

ELEUTHerine PALMIfolia (Linn.) Merr. in Philip. Journ. Sci. 7 (1912) Bot. 233, Fl. Manila (1912) 153, Sp. Blancoanae (1918) 104. Sisyrinchium palmifolium Linn. Mant. 1 (1767) 122; Naves Novis. App. (1880) 252.

Eleutherine plicata Herb. in Bot. Reg. 29 (1843) sub t. $5 \%$

Antholyza meriana Blanco Fl. Filip. (1837) 24, ed. 2 (1845) 18, ed.

3, 1 (1877) 33, t. 100; Merr. in Govt. Lab. Publ. (Philip.) 27 (1905) 85, non Linn.

A native of tropical America, introduced and now naturalized in some parts of the Philippines, occurring in hemp plantations and, occasionally, in waste places about towns, etc.; also planted for ornamental purposes.

Local names: Ahos-áhos (C. Bis.) ; hagusáhis (S. L. Bis.).

\section{EXCLUDED GENERA AND SPECIES}

ANTHOLYzA AEThIOPICA Linn.; Naves Novis. App. (1880) 252.

IRIS SUSIANA Linn.; Naves op. cit. 253.

Gladiolus SEgEtum Ker; Naves 1. c.

WATSONIA MERIANA Ker; Naves 1. c. 


\section{MUSACEAE}

\section{MUSA Linnaeus}

MUSA ERRANS (Blanco) Teodoro in Philip. Journ. Sci. 10 (1915) Bot. 390, t. 1\%, f. 6-8; Merr. Sp. Blancoanae (1918) 105.

Musa troglodytarum Linn. var. errans Blanco Fl. Filip. (1837) 247, ed. 2 (1845) 172, ed. 3, 1 (1877) 310.

Musa amboinensis Naves Novis. App. (1880) 218, non Rumph.

Luzon (Apayao, Bulacan, Bataan, Rizal, Laguna), Merrill Sp. Blancoanae 873, Teodoro, B. S. 28268 F'énix. In forests at low and medium altitudes. Endemic.

Local names: Pau-uá (Ibn.); ságing-matsíng (Tag.); ságing-na-ligau (Tag.).

Var. BOTOAN Teodoro in Philip. Journ. Sci. 10 (1915) Bot. 391, t. $\%, f$. 5-10; Merr. Sp. Blancoanae (1918) 106.

Musa troglodytarum Blanco Fl. Filip. (1837) 246, ed. 2 (1845) 172, ed. 3,1 (1877) 310, t. 89, non Linn.

Luzon (Rizal), Merrill Sp. Blancoanae 21\%, Teodoro. Planted in most parts of the Philippines; really referable to $M$. errans as a variety?

Local names: Baláiang (Ilk.); butúan (Tag.); butúhan (Tag); vulluñgán ' (Ibn., It.).

MusA GLAucA Roxb. Pl. Coromandel 3 (1819) t. 300; Naves Novis. App. (1880) 218; Teodoro in Philip. Journ. Sci. 10 (1915) Bot. 387; Merr. Sp. Blancoanae (1918) 104.

Musa troglodytarum Linn. var. dolioformis Blanco Fl. Filip. (1837) 855, ed. 2 (1845) 174, ed. 1 (1877) 312.

Luzon (Cavite, Laguna), Merrill Sp. Blancoanae 946, 537. In ravines, etc., at low and medium altitudes. Burma, Pegu.

Local name: Virgen (Sp.).

MUSA PARADISIACA Linn. Sp. Pl. (1753) 1043; Miq. Fl. Ind. Bat. 3 (1859) 588; Naves Novis. App. (1880) 215; Merr. Fl. Manila (1912) 154; Teodoro in Philip. Journ. Sci. 10 (1915) Bot. 412;

K. Schum. in Engl. Pflanzenreich 1 (1900) 19, f. 3.

The plantains are those bananas which are edible after being cooked and very inferior or inedible raw. A number of forms or varieties occur in the Philippines, which from a species standpoint are scarcely to be distinguished from Musa sapientum Linn., the bananas. Following custom the forms are distributed between the two Linnean binomials. It is by no means certain that the numerous varieties which have been proposed by Blanco, Teodoro, and Quisumbing are correctly distributed as between Musa paradisiaca Linn. and $M$. sapientum.

I am of the opinion that no more weight should be placed on the numerous forms of the banana than on similar forms of other exceedingly variable cultivated plants such as the orange, the apple, rice, the common bean, sugar cane, etc., because relatively speaking the differences in cultivated bananas are certainly no gireater than those in the cultivated forms of the other plants mentioned above. No attempt has been made to correlate these Philippine forms or varieties with extra-Philippine ones although some, perhaps many, of our cultivated bananas are certainly identical with 
those of tropical Asia and Malaya. Quisumbing, Phil. Agr. Review 123 (1919) 1-73 t. 1-30, described and figured a number of varieties from extraPhilippine plants, cultivated at Los Baños, which have not been included in this enumeration. They are Musa sapientum Linn. var. gladiata Quis., origin unknown; var. oanga Quis., from New Guinea; var. klum Quis., from India; var. misi Quis., from Samoa; var. saloor Quis., from Borneo; var. chuntara Quis., from Siam; var. pop Quis., from Borneo; var. maori Quis., from Raratonga; var. sicsec Quis., from New Guinea; var. languma Quis., from New Guinea; var. radja Quis., from Java (=Musa regia Rumph.!); var. nam Quis., apparently from Indo-China; var. yanaikonban Quis., from India; var. rachidis Quis., from the Malay Peninsula; var. cochinchinensis Quis., from Indo-China (=Musa odorata Lour.!); var. katali Quis., from India; var. khai Quis., from Siam; var. krie Quis., origin unknown; var. yan Quis., origin unknown; var. hazara Quis., from India; var. maduranga Quis., from India; Musa paradisiaca Linn. var. enosa Quis., from Java (=Musa corniculata Rumph.!) ; and var. tiparot Quis., origin unknown.

Var. MAGNA Blanco Fl. Filip. (1837) 244, ed. 2 (1845) 171, ed. 3, 1 (1877) 307; Teodoro in Philip. Journ. Sci. 10 (1915) Bot. 413; Merr. Sp. Blancoanae (1918) 108; Quis. in Philip. Agr. Review $12^{3}$ (1919) 67, t. 12, f. 212, 213.

Planted here and there for its fruit. Endemic?

Local name: Tundók (Tag.).

Var. MAXIMA Blanco Fl. Filip. (1837) 245, ed. 2 (1845) 171, ed. 3, 1 (1877) 308; Teodoro in Philip. Journ. Sci. 10 (1915) Bot. 414; Merr. Sp. Blancoanae (1918) 109.

Planted here and there for its fruit, Merrill Sp. Blancoanae 920. Endemic?

Local names: Batábia (Tag.); matábia (Tag.).

Var. SUBRUBEA Blanco Fl. Filip. (1837) 245, ed. 2 (1845) 171, ed. 3, 1 (1877) 307; Teodoro in Philip. Journ. Sci. 10 (1915) Bot. 414, t. 16, f. 1-5; Merr. Sp. Blancoanae (1918) 109.

Planted here and there. Endemic?

Teodoro's figure and description were apparently based on material of Cuban origin and hence may well not represent the form Blanco described.

MUSA SAPIENTUM Linn. Syst. ed. 10 (1759) 1303; Teodoro in Philip. Journ. Sci. 10 (1915) Bot. 393.

Musa paradisiaca Linn. subsp. sapientum O. Kuntze Rev. Gen. Pl. 1 (1892) 692; K. Schum. in Engl. Pflanzenreich 1 (1900) 20.

The bananas, distinguishable from the plantains (Musa paradisiaca) chiefly in the consistency and characters of the mature fruits. A number of forms are found in the Philippines in cultivation, many in all probability introduced from other parts of Malaya and from tropical Asia.

Var. ANGAO Quis. in Philip. Agr. Review $12^{3}$ (1919) 58, $t .5, f .30 ; t$. \%, f. 69 ; t. 9, f. $126 ;$ t. 11, f. 192, 193; t. 26, f. 2.

Mindanao (Misamis). Cultivated at Los Baños.

Local name: Añgau (C. Bis.). 
Var. BACA Quis. in Philip. Agr. Review $12^{3}$ (1919) 51, t. 5, f. 23; t. 7, f. $60 ;$ t. $9, f .118 ; t .11, f .176,17 \%$.

Luzon (Cavite). Cultivated at Los Baños.

Local name: Súñgai-báka (Tag.).

Var. BINUTIG Teodoro in Philip. Journ. Sci. 10 (1915) Bot. 401, t. 10 , f. $1-5$.

Teodoro's specimens were apparently from Leyte, according to material prepared by him from plants cultivated at Los Baños.

Local name: Binutig (S. L. Bis.).

Var. CANARA Teodoro in Philip. Journ. Sci. 10 (1915) Bot. 406, t. 10 , f. $6-10$.

Cultivated at Los Baños, origin unknown but probably Philippine, and very likely from Camarines Province, where kanára is a well-known banana.

Var. CANAYA Quis. in Philip. Agr. Review $12^{3}$ (1919) 44, t. 4, f. 18; t. $6, f .54$; t. 9, f. 111 ; t. 10, f. 162,$163 ;$ t. 20, f. 2 .

Mindanao (Surigao). Cultivated at Los Baños.

Local name: Kanáya (C. Bis.):

Var. CINEREA (Blanco) Teodoro in Philip. Journ. Sci. 10 (1915) Bot. 397, t. 13, f. 1-5; Merr. Sp. Blancoanae (1918) 106.

Musa paradisiaca Linn. var. cinerea Blanco Fl. Filip. (1837) 250, ed. 2 (1845) 175, ed. 3, 1 (1877) 313.

One of the commonest bananas in the Philippines, Merrill Sp. Blancoanae 926.

Local names: Latúndan (Tag.) ; letóndal (Tag.) ; tórdan (Tag.).

Var. COMPRESSA (Blanco) Teodoro in Philip. Journ. Sci. 10. (1915)

Bot. 408, t. 7, f. 1-5; Merr. Sp. Blancoanae (1918) 108.

Musa paradisiaca Linn. var. compressa Blanco Fl. Filip. (1837) 240, ed. 2 (1845) 168, ed. 3, 1 (1877) 304.

A very common banana in most parts of the Philippines.

Local names: Bísco (Tag.) ; obíspo (Tag.) ; sabá (Tag.).

This is probably better placed under $M$. paradisiaca Linn.

Var. DINALAGA Quis. in Philip. Agr. Review $12^{3}$ (1919) 55, t. 5, f. 31 ; t. 7,$66 ;$ t. 9, f. 122; t. 11, f. 184, 185; t. 26, f. 1 .

Luzon (Tayabas). Cultivated at Los Baños.

Local name: Dinalága (Tag.).

Var. DOOL Quis. in Philip. Agr. Review $12^{3}$ (1919) 35, t. 4, f. 6; t. 6, f. $44 ;$ t. 9, f. $101 ;$ t. $10, f .142,143 ;$ t. f. $15, f .1$.

Mindanao (Bukidnon). Cultivated at Los Baños.

Local name: Duol (Buk.).

Var. EDA Quis. in Philip. Agr. Review $12^{3}$ (1919) 37, t. 4, f. 9; t. 6, $f$. $47 ;$ t. 9, f. $104 ;$ t. 10, f. 148,$149 ;$ t. $15, f .1$.

Mindanao (Surigao). Cultivated at Los Baños.

Local name: Eda-án (C. Bis.). 
Var. Flabellata Quis. in Philip. Agr. Review $12^{3}$ (1919) 66, $t .5, f$. $35 ;$ t. $8, f .78,87 ; t .12, f .208,209 ; t .29, f .2$.

Luzon (Laguna, Bulacan). Cultivated at Los Baños.

Local names: Binendíto (Tag.) ; inabaníko (Tag.).

Perhaps better placed under $M$. paradisiaca Linn.

Var. GALATAYAN Quis. in Philip. Agr. Review. $12^{\text {s3 }}$ (1919) 40, t. 4, f. 13 ; t. $6, f .50 ;$ t. 9, f. 108 ; t. 10, f. 154,$155 ;$ t. $18, f .1$.

Luzon (Cavite). Cultivated at Los Baños.

Local name: Galatáyan (Tag.).

Var. DARYAO Teodoro in Philip. Journ. Sci. 10 (1915) Bot. 403, t. 8, f. $1-5$.

Leyte, Teodoro, cultivated at Los Baños.

Local name: Daryau (S. L. Bis.).

Var. GARANGAO Teodoro in Philip. Journ. Sci. 10 (1915) Bot. 401, t. 10, f. $6-10$.

Leyte, Teodoro, cultivated at Los Baños.

Local name: Garañgau (S. L. Bis.).

Var. GLABERRIMA (Blanco) Teodoro in Philíp. Journ. Sci. 10 (1915)

Bot. 399, t. 15, f. 1-5; Merr. Sp. Blancoanae (1918) 106.

Musa paradisiaca Linn. var. glaberrima Blanco Fl. Filip. (1837) 245, ed. 2 (1845) 171, ed. 3, 1 (1877) 308.

Luzón (Rizal, Laguna), Merrill Sp. Blancoanae 865, Teodoro.

Local names: Dinuguan (Tag.) ; durugó (Tag.) ; galámai-señora (Tag.).

Var. GlaucA (Blanco) Teodoro in Philip. Journ. Sci. 10 (1915) Bot. 402, t. 11, f. 6-10; Merr. Sp. Blancoanae (1918) 107.

Musa paradisiaca Linn. var. glauca Blanco Fl. Filip. (1837) 250, ed.

2 (1845) 175, ed. 3, 1 (1877) 312.

Luzon (Rizal), Merrill Sp. Blancoanae 912.

Local names: Bingti-kohol (Tag.) ; tinalong (Tag.) ; veinte-kohol (Tag.).

Var. GRANDIS Teodoro in Philip. Journ. Sci. 10 (1915) Bot. 410, t. 14, f. 1-3.

Luzon (Laguna). Occasionally planted.

Local name: Sabáng-ilóko (Tag.).

Var. HUMILIS (Perr.).

Musa humilis Perr. in Mém. Soc. Linn. Paris 3 (1824) 131; C. B.

Rob. in Philip. Journ. Sci. 3 (1908) 306; Teodoro in Philip. Journ.

Sci. 10 (1915) Bot. 393, t. 18, f. 1-5.

Mindanao (Zamboanga, Cotabato). A peculiar form with small obovoid fruits.

Local name: Pula-pulá (Mag.).

Var. KINAMAY Quis. in Philip. Agr. Review $12^{8}$ (1919) 57. t. 5, f. 32; t. 7, f. 67 ; t. 9, f. $125 ;$ t. 11, f. 190,$191 ;$ t. 23, f. 1 .

Luzon (Bulacan). Cultivated at Los Baños.

Local name: Kinamái (Tag.). 
Var. INARNIBAL Teodoro in Philip. Journ. Sci. 10 (1915) Bot. 406, t. 9, f. 1-5.

Luzon (Laguna), Teodoro.

Local name: Inarníbal (Tag.).

Var. LACATAN (Blanco) Teodoro in Philip. Journ. Sci. 10 (1915) Bot. 405, t. 11, f. 1-5; Merr. Sp. Blancoanae (1918) 107.

Musa paradisiaca Linn. var. lacatan Blanco Fl. Filip. (1837) 243, ed. 2 (1845) 170, ed. 3,1 (1877) $305, t .88$.

A widely cultivated banana and one of the most popular varieties in the Philippines.

Local names: Bañáran (Bik.); lakatán (Tag., S. L. Bis.).

Var. LONGA (Blanco) Teodoro in Philip. Journ. Sci. 10 (191う) Bot. 407; Merr. Sp. Blancoanae (1918) 108.

Musa paradisiaca Linn. var. longa Blanco Fl. Filip. (1837) 245, ed. 2. (1845) 171, ed. 3, 1 (1877) 308.

Known only from Blanco's description.

Local name: Kinauáyan (Tag.).

Var. PUMILA (Blanco).

Musa paradisiaca Linn. var. pumila Blanco Fl. Filip. (1837) 244, ed. 2 (1845) 171, ed. 3, 1 (1877) 306.

Musa cavendishii Lamb. var. pumila Teodoro in Philip. Journ. Sci. 10 (1915) Bot. 412.

Musa sapientum Linn. var.; Merr. Sp. Blancoanae (1918) 108.

Occasionally planted; perhaps a dwarfed form of the buñulan.

Local name: Tampóhin (Tag.)。

Var. PADILAT Quis. in Philip. Agr. Review $12^{3}$ (1919) 63, t. 5, f. 38; t. $7, f .71 ; t .8, f .88 ; t .12, f .202,203 ; t .28, f .1$.

Babuyan Islands (Dalupiri). Cultivated at Los Baños.

Local name: Padílat (Ilk.).

Var. PAMOTION Quis. in Philip. Agr. Review $12^{3}$ (1919) 49, t. 5, f. 21; t. $7, f .59 ; t .9, f .116 ; t .11, f .172,173 ; t .22, f .1$.

Mindanao (Surigao). Cultivated at Los Baños.

Local name: Pamotion (C. Bis.).

Var. PELIPIA Quis. in Philip. Agr. Review $12^{3}$ (1919) 42, t. 4, f. 14; t. 6, f. $51 ;$ t. $9, f .109 ; t .10, f .158,159 ; t .18, f .2$.

Mindanao (Surigao). Cultivated at Los Baños.

Local name: Pelípia (C. Bis.).

Var. PRINCIPE Quis, in Philip. Agr. Review $12^{3}$ (1919) 50, t. 5, f. 22; $t$. $7, f .61 ; t .9, f .117 ; t .11, f .174,175 ; t .22, f .2$.

Philippines, without locality. Cultivated àt Los Baños.

Var. PUTIAN Quis. in Philip. Agr. Review $12^{3}$ (1919) 36, t. 4, f. 8; t. 6, f. $46 ; t .9, f .103 ;$ t. $10^{\circ}, f .146,147 ; t .15, f .2$.

Cultivated at Los Baños, from the Visayan Islands.

Local name: Putían (Bis.).

$183036-15$ 
Var. RAINES Quis. in Philip. Agx. Review $12^{3}$ (1919) 55, t. 5, f. 27; t. 7, f. $65 ;$ t. 9, f. $124 ;$ t. $11, f .186,187 ;$ t. $25, f .1$.

Polillo: Cultivated at Los Baños.

Local name: Raines-na-putí (Tag.).

Var. SARocsoc Quis, in Philip. Agr. Review $12^{3}$ (1919) 39, t. 4, f. 11; t. $6, f .49 ;$ t. $9, f .104 ;$ t. $10, f .152,153 ;$ t. $17, f .1$.

Luzon (Camarines). Cultivated at Los Baños.

Local names: Saroksók (Bik.); sinaroksók (Bik.).

Var. SIsION Quis. in Philip. Agr. Review $12^{3}$ (1919) 35, t. 4, f. 7 ; t. 6, f. $45 ;$ t. 9, f. $102 ;$ t. 10, f. 144,145 .

Panay (Capiz). Cultivated at Los Baños.

Local name: Sision (P. Bis.).

Var. SUAVEOLENS (Blanco) Teodoro in Philip. Journ. Sci. 10 (1915) Bot. 400, t. 14, f. 6-10; Merr. Sp. Blancoanae (1918) 106.

Musa paradisiaca Linn. var. suaveolens Blanco Fl. Filip. (1837) 244, ed. 2 (1845) 171, ed. 3, 1 (1877) 306.

One of the most popular bananas in the Philippines, Merrill Sp. Blancoanae 928.

Local name: Buñgúlan (Tag.).

Var. TERnAtensis (Blanco) Teodoro in Philip. Journ. Sci. 10 (1915) Bot. 404, t. 7, f. 1-5; Merr. Sp. Blancoanae (1918) 107.

Musa paradisiaca Linn. var. suaveolen's Blanco Filip. (1837) 243, ed. 2 (1845) 170, ed. 3, 1 (1877) 305.

Occasionally cultivated, Merrill Sp. Blancoanae 216.

Local names: Glória (Tag., Ilk., Ibn.) ; ternáte (Tag.).

Var. Tом BAK (Blanco) Teodoro in Philip. Journ. Sci. 10 (1915) Bot. 407; Merr. Sp. Blancoanae (1918) 108; Quis. in Philip. Agr. Review $12^{3}$ (1919) 33, t. 4, f. 4; t. 6 , f. 42 ; t. 9, f. 99 ; t. $10, f .138,139 ; t$. 14, f. 2 .

Musa paradisiaca Linn. var. tombak Blanco Fl. Filip. (1837) 246, ed. 2 (1845) 171, 3, 1 (1877) 307.

Cultivated here and there, Merrill Sp. Blancoanae 953.

Local names: Goyóran (Tag.) ; tinumbága (Tag.) ; tómbak (Tag.).

Var. TULDOC Teodoro in Philip. Journ. Sci. 10 (1915) Bot. 407, t. 10, f. $1-5$.

Luzon (Rizal). Cultivated by Teodoro at Los Baños.

Local name: Tuldók (Tag.).

Var. TUDLONG Teodoro in Philip. Journ. Sci. 10 (1915) Bot. 402, t. 11, f. $6-10$.

Leyte, cultivated by Teodoro at Los Baños.

Local name: Tudlong (S. L. Bis.).

Var. VIOLACEA (Blanco) Teodoro in Philip. Journ. Sci. 10 (1915) Bot. 398, t. 5, f. 6-10; Merr. Sp. Blancoanae (1918) 106.

Musa paradisiaca Linn. var. violacea Blanco Fl. Filip. (1837) 245, ed. 2 (1845) 171, ed. 3, 1 (1877) 307.

Cultivated here and there.

Local name: Morádo (Sp.). 
MUSA TEXTILIS Née in Anal. Cienc. Nat. 4 (1801) 123; Hook. Lond. Journ. Bot. 7 (1848) 268; Baker in Ann. Bot. 7 (1893) 211; K. Schum. in Engl. Pflanzenreich 1 (1900) 19; Ridl. in Philip. Journ. Sci. 4 (1909) Bot. 199; Teodoro in Philip. Journ. Sci. 10 (1915) Bot. 388, t. 18, f. 6-10; Merr. Sp. Blancoanae (1918) 105.

Musa abaca Perr. in Mém. Soc. Linn. Paris 3 (1824) 130; C. B. Rob. in Philip. Journ. Sci. 3 (1908) Bot. 305.

Musa troglodytarum Linn. var. textoria Blanco Fl. Filip. (1837) 247, ed. 2 (1845) 173, ed. 3,1 (1877) 311.

Musa mindanaensis Rumph.; Miq. Fl. Ind. Bat. 3 (1859) 588; Naves Novis. App. (1880) 218.

Abacá or Manila hemp of commerce. Very extensively cultivated in those provinces from central Luzon to Mindanao where the dry season is not pronounced. In Luzon the chief abacá provinces are Camarines, Albay, and Sorsogon. The islands of Samar and Leyte are very important producing centers, as are also parts of Mindanao, especially Davao. Endemic.

Local name: Abaká (most dialects). The species is variable, and sundry forms have distinctive local names.

MUSA URANOScopos Lour. F1. Cochinch. (1790) 645, excl. syn. Rumph. Musa coccinea Andr. Bot. Rep. t. 47; Naves Novis. App. (1880) 218; Ridl. in Philip. Journ. Sci. 4 (1909) Bot. 199; Teodoro op. cit. 10 (1915) Bot. 388.

Planted here and there for ornamental purposes on account of its very showy red bracts. A native of southeastern Asia.

\section{DOUBTFUL AND EXCLUDED SPECIES}

Musa Alphurica Rumph.; Naves Novis. App. (1880) 217.

Musa CORniculata Rumph.; Naves l. c.

MUSA ornata Roxb.; Naves op. cit. 218.

MUSA SIMIARUM Rumph.; Naves op. cit. 217.

Musa URanoscopos 'Rumph.; Naves op. cit. 218.

Musa Chapara Perr. in Mém. Soc. Linn. Paris 3 (1825) 131; C. B. Rob. in Philip. Journ. Sci. 3 (1908) Bot. 305; Teodoro op. cit. 10 (1915) Bot. 415.

Unrecognizable except that it is a form or variety of Musa sapientum Linn.

Musa EnSeTe Gmel.; Teodoro in Philip. Journ. Sci. 10 (1915) Bot. 387.

This occurs on the grounds of the College of Agriculture at Los Baños as a recently introduced and cultivated species and has no claims to be considered as Philippine.

Musa NIGra Perr. in Mém. Soc. Linn. Paris 3 (1824) 131; Teodoro in Philip. Journ. Sci. 10 (1915) Bot. 415. Linn.

Unrecognizable except that it is a form or variety of Musa sapientum

MUSA PARAdisiaca Linn. var. Ulnaris Blanco Fl. Filip. (1837) 246, ed. 2 (1845) 172, ed. 3, 1 (1877) 309; Teodoro in Philip. Journ. Sci. 10 (1915) Bot. 414; Merr. Sp. Blancoanae (1918) 107. 
A purely imaginary variety, the description based on hearsay data, probably inspired by one of the tundók varieties.

Musa Cavendishir Lamb.; Teodoro in Philip. Journ. Sci. 10 (1915) Bot. 410 , incl. var. hawaiiensis Teodoro 1. c. $t$. 15, f. 6-10.

This is generally considered as a distinct species, but I believe it to be merely a variety of Musa sapientum Linn. Teodoro's descriptions were based on recently introduced and cultivated specimens.

Musa sapientum Linn, var. Cubensis Teodoro in Philip. Journ. Sci. 10 (1915) Bot. 397, t. 11, f. 6-10.

The description was based on material originating in Cuba.

Musa SAPIEntum Linn. var. AMERICANA Teodoro in Philip. Journ. Sci. 10 (1915) Bot. 398, t. 7, f. 6-10.

This variety was based on material of Cuban origin.

\section{RAVENALA Adanson}

RAVENALA MAdAGASCARIENSIS Gmel. Syst. (1791) 567; Naves Novis. App. (1880) 219; Ridl. in Philip. Journ. Sci. 4 (1909) Bot. 199; Merr. Fl. Manila (1912) 155; K. Schum. in Engl. Pflanzenreich 1 (1900) $29, f .6$. '

Planted in Manila and in other large towns for ornamental purposes, a native of Madagascar. The so-called traveler's palm.

\section{HELICONIA Linnaeus}

HELICONIA BIHAI Linn. Mant. 2 (1771) 211; Naves Novis. App. (1880) 215; K. Schum. in Engl. Pflanzenreich. 1 (1900) 36.

Heliconiopsis amboinensis Miq. Fl. Ind. Bat. 3 (1859) 590; Naves 1. c.

Planted occasionally for ornamental purposes. A native of tropical America, naturalized in some parts of the Old World.

\section{EXCLUDED GENUS}

Strelitzia Reginae Banks Naves Novis. App. (1880) 219.

\section{ZINGIBERACEAE}

\section{ZINGIBER Adanson}

ZINGIBER APOENSE Elm. Leafl. Philip. Bot. 8 (1915) 2914.

Mindanao (Davao), Elmer 10545, 11750. In forests, 1,000 to $1,300 \mathrm{~m}$. Endemic.

Local name: Bog’óbog (Bag.).

ZINGIBER BREVIFOLIUM N. E. Br. in Gard. Chron. 26 (1886) 390, non K. Schum. (1904).

Described from cultivated specimens said to have originated in the Philippines. Otherwise unknown. Endemic.

ZINGIBER BULUSANENSE Elm. Leafl. Philip. Bot. 8 (1919) 2993.

Luzon (Sorsogon), Elmer 16914, 15178. In primary forests, altitude about $450 \mathrm{~m}$. Endemic. 
ZINGIBER CASSUMUNAR Roxb. in Asiat. Research 11 (1810) 347, t. 5 , Fl. Ind. 1 (1820) 49; Vidal Phan. Cuming. Philip. (1885) 152, Rev. Pl. Vasc. Filip. (1886) 274; Naves Novis. App. (1880) 220; Ridl. in Govt. Lab. Publ. (Philip.) 35 (1906) 84.

Mindanao (Misamis), (Cuming 1605). I have seen no Philippine material representing this species. Pantropic in cultivation, a native of tropical Asia.

ZINGIBER MOLLE Ridl. in Leafl. Philip. Bot. 2 (1909) 607, nomen nudum, Philip. Journ. Sci. 4 (1909) Bot. 168.

Luzon (Bontoc, Lepanto, Benguet, Pampanga, Tayabas), F. B. 4886, 14914 Curran, 14490 Darling, B. S. 5364 Ramos, 1991, 1985 Foxworthy, Merrill 4843. In ravines along streams and in forests, altitude 1,100 to $1,600 \mathrm{~m}$. Endemic.

Local name: Kaláuing (Bon.).

ZINGIBER NEGROSENSE Elm. Leafl. Philip. Bot. 8 (1915) 2917.

Negros, Mindanao (Davao), Elmer 10421, 11768. In forested ravines, altitude about $1,050 \mathrm{~m}$. Endemic.

ZINGIBER OfFICINALE Rose. in Trans. Linn. Soc. 7 (1807) 348, Monandr. Pl. (1828) t. 83; Miq. Fl. Ind. Bat. 3 (1859) 593; Naves Novis. App. (1880) 220; K. Schum. in Engl. Pflanzenreich 20 (1904) 170, f. 23; Merr. F1. Manila (1912) 159, Sp. Blancoanae (1918) 110. Amomum zingiber Linn. Sp. Pl. (1753) 1; Blanco Fl. Filip. (1837) 2, ed. 2 (1845) 2, ed. 3, 1 (1877) 3, t. 181.

Zingiber blancoi Hassk. in Flora 47 (1864) 20.

Widely cultivated although not on an extensive scale, and nowhere naturalized. Pantropic in cultivation, a native of tropical Asia. Ginger.

Local names: Agát (Pang.) ; baseng (Ilk.) ; gengibre (Sp.) ; laíal (Sbl.) ; laíya (If.); layá (Ilk., Bon., Ibn., It.); lúya (Tag.).

ZINGIBER PUBISQUAMA Ridl. in Philip. Journ. Sci. 4 (1909) Bot. 169.

Mindanao (Lanao), Clemens 1163a. In open grasslands, cogon (Imperata) type, altitude about $650 \mathrm{~m}$. Endemic.

ZINGIBER SYLVATICUM Elm. Leafl. Philip. Bot. 8 (1915) 2919.

Luzon (Cagayan, Laguna, Tayabas, Camarines, Sorsogon), Polillo, Catanduanes, Negros, Elmer 9843, 18004, 14992, 14936, B. S. 19456, 30521, 22048 Ramos, 13076 Foxworthy, 10237, 10238 McGregor, 9198 Robinson. In forests chiefly at medium altitudes, ascending to $1,300 \mathrm{~m}$. Endemic.

ZINGIBER ZERUMBET (Linn.) Sm. Exot. Bot. 2 (1804) 103, t. 112; Miq. Fl. Ind. Bat. 3 (1859) 593; Naves Novis. App. (1880) 220; K. Schum. in Engl. Pflanzenreich 20 (1904) 172, f. 24; Ridl. in Philip. Journ. Sci. 4 (1909) Bot. 199; Merr. Fl. Manila (1912) 159, Sp. Blancoanae (1918) 111.

Amomum zerumbet Linn. Sp. Pl. (1753) 1; Blanco Fl. Filip. (1837) 2 , ed. 2 (1845) 2 , ed. 3,1 (1877) $3, t .370$.

Zingiber cassumunar Naves in Blanco Fl. Filip. ed. 3 (1877-83), $t .370$, non Roxb.

Zingiber zerumbet Sm. var. magnum Elm. Leafl. Philip. Bot. 8 (1919) 2922. 
Northern Luzon to Mindanao, in most or all islands and provinces. In thickets in and near settlements at low and medium altitudes. A native of tropical Asia, now pantropic.

Local names: Balauág (Bik.); barik (Tag.); lampúyang (Buk.); langkauás (Tag.); layág sa-súlug (Bik.); luíang-ósiu (Tag.); tamohílang (Buk.); tumbóng-áso (Tag.).

\section{EXCLUDED SPECIES}

Zingtber AMaricans Blume; Naves Novis. App. (1880) 221.

ZINGIBER AQUOSUM Blume; Naves 1. c.

ZiNgiber ELATUM Roxb.; Naves l. c.

ZINGIBER GRACILE Jack; Naves 1. c.

ZINGIBER GRAMINEUM Blume; Naves $1 . c$.

ZiNGIBER INFLEXUM Blume; Naves 1. c.

Zingiber Marginatum Roxb.; Naves 1. c.

ZINGIBER ODORIFERUM Blume; Naves 1 . c.

ZINGIBER ovoIDeum Blume; Naves op. cit. 220.

ZINGIBER PURPUREUM Rosc.; Naves 1. c.

ZiNGIBER ROSEUM Roxb.; Naves op. cit. 221.

ZingIBER SQUARRosUm Roxb.; Naves 1. c.

\section{LANGUAS Koenig}

(Alpinia auctt., non Linnaeus*)

LANGUAS APOENSIS (Elm.) comb. nov.

Alpinia apoensis Elm. Leafl. Philip. Bot. 8 (1915) 2888.

Catanduanes, Mindanao (Davao), Elmer 11889, B. S. 30490 Ramos. In primary forests up to an altitude of $1,200 \mathrm{~m}$. Endemic.

Local name: Otin-ótin (Bag.).

LANGUAS BRACHYANTHA (Merr.) comb. nov.

Alpinia brachyantha Merr. in Philip. Journ. Sci. 10 (1915) Bot. 296.

Luzon (Cagayan), Weber 1571, B. S. 13920 Ramos, F. B. 19617 Curran. In forests at low or medium altitudes. Endemic.

LANGUAS BREVILABRIS (Presl) comb. nov.

Alpinia brevilabris Presl Rel. Haenk. 1 (1827) 110, t. 17; Naves

Novis. App. (1880) 225; Ridl. in Philip. Journ. Sci. 4 (1919) Bot. 190; K. Schum. in Engl. Bot. Jahrb. 27 (1899) 276, Pflanzenreich 20 (1904) 314.

Alpinia fraseriana Usteri Beitr. Ken. Philip. Veg. (1905) 128, non Oliv.

* The genus Alpinia of Linnaeus was based wholly on a tropical American plant, Alpinia racemosa Linn. = Renealmia racemosa $\mathrm{A}$. Rich., and is thus identical with Renealmia Linn. f. (1781), which it should replace. As between the two generic names, no matter which one be applied to the American group, Alpinia is clearly not applicable to the Indo-Malayan genus long known under this name. 
Batan Islands and northern Luzon to Mindanao. In most islands and provinces. Common in primary forests at low and medium altitudes.

Local names: Ápat (Ig.) ; bagongbóng (Bis.); bánai (Pamp.) ; gagadno-kóran (Iv.) ; katkátan (Pamp).; katólan (Bis.); katótang (Bis.) ; luyalúya (Tag.); malatalbák (Tag.); panáuil (Bon.); parapád (Ig.); tagbák (Bik., Tag., C. Bis.) ; tagbák-gúbat (Tag.) ; tagbák-matsíng (Tag.) ; tagbás (C. Bis.) ; talbák (Tag.) ; tugís (S. L. Bis.).

LANGUAS COPELANDII (Ridl.) comb. nov.

Alpinia copelandii Ridl. in Philip. Journ. Sci. 4 (1909) Bot. 164.

Mindanao (Zamboanga), Copeland. In forests at low altitudes. Endemic.

LANGUAS CUMINGII (K. Schum.) comb. nov.

Alpinia cumingii K. Schum. in Engl. Pflanzenreich 20 (1904) 315; RidI. in Philip. Journ. Sci. 4 (1909) Bot. 187.

Alpinia chinensis Vidal Phan. Cuming. Philip. (1885) 152, Rev. Pl. Vasc. Filip. (1886) 274, non Rosc.

Luzon (Albay), Cuming 859. Of this I have seen only a few detached flowers. Endemic.

LANGUAS DIVERSIFOLIA (Elm.) comb. nov.

Alpinia diversifolia Elm. Leafl. Philip. Bot. 8 (1915) 2919.

Vanoverberghia diversifolia EIm. op. cit. 2913.

Luzon (Benguet), Elmer 8853. In damp ravines, altitude about 1,000 m. Endemic.

LANGUAS FLABELLATA (Ridl.) comb. nov.

Alpinia flabellata Ridl. in Philip. Journ. Sci. 4 (1909) Bot. 188.

Batan Islands and northern Luzon to Mindanao, Merrill Phil. Pl. 831, B. S. 31885 Santos, 2718, 2871 Mearns. Not always easily distinguishable from $A$. brevilabris Presl. In forests at medium altitudes ascending to $2,000 \mathrm{~m}$. Endemic.

Var. MAJOR (Ridl.) comb. nov.

Alpinia flabellata Ridl. var. major Ridl. 1. c.

Range and habitat of the species and doubtfully distinct even as a variety.

Local names: Biláu (Sub.) ; tagbák-gúbat (Bik.) ; tagbák-laláki (Tag.) ; tuká-máya (Tag.).

LANGUAS FOXWORTHYI (Ridl.) comb. nóv.

Alpinia foxworthyi Ridl. in Philip. Journ. Sci. 4 (1909) Bot. 189;

Elm. Leafl. Philip. Bot. 8 (1915) 2887.

Palawan, B. S. 7r2 Foxworthy, Elmer 12930. In forests at low altitudes. Endemic.

LANGUAS GLABRESCENS (Ridl.) comb. nov.

Alpinia glabrescens Ridl. in Philip. Journ. Sci. 4 (1909) Bot. 185.

Batan Islands (Batan, Sabtan), B. S. 3679 Fénix, 3141 Mearns, 10156 McGregor. On slopes at low altitudes. Endemic unless identical with some Formosan species represented by Faurie 975 of his 1913 collection.

Local name: Gagád (Iv.). 
LANGUAS GRAMINEA (Ridl.) comb. nov.

Alpinia graminea Ridl. in Philip. Journ. Sci. 4 (1909) Bot. 189; Elm. Leafl. Philip. Bot. 8 (1919) 2966.

Luzon (Cagayan, Nueva Ecija, Laguna, Tayabas, Sorsogon), Catanduaines, Negros, Panay, F. B. 7267, 11208 Everett, B. S. 26359, 30678 Ramos \& Edaño, 25411 Yates, 28375 Fénix, 30239 Ramos, Elmer 16228. In forests up to $800 \mathrm{~m}$ altitude. Endemic.

Perhaps but a narrow-leaved form of L. brevilabris (Presl) Merr.

Local names: Barañgin (Ibn.); lampíñgit (Neg.); tagbák (P. Bis.).

LANGUAS HAENKEI (Presl) comb. nov.

Alpinia haenkei Presl Symb. 1 (1832) 66, t. 43; K. Schum. in Engl. Pflanzenreich 20 (1904) 341; Ridl. in Govt. Lab. Publ. (Philip.)

35 (1906) 86, Philip. Journ. Sci. 4 (1909) Bot. 184.

Alpinia malaccensis Presl Rel. Haenk. 1 (1827) 110; Naves Novis. App. (1880) 225; Vidal Phan. Cuming. Philip. (1885) 152, Rev. Pl. Vasc. Filip. (1886) 275, non Rosc.

Alpinia philippinensis Ridl. in Govt. Lab. Publ. (Philip.) 35 (1906) 86; Merr. in Philip. Journ. Sci. 1 (1906) Suppl. 37.

Batan Islands and northern Luzon to Palawan and Mindanao, in most or all islands and provinces. In primary forests at low and medium altitudes, often common, represented by over 60 individual collections. Endemic.

Local names: Bagumbúng (Tag.); barápat (Ig.) ; birao-bírao (Sul.); púnan (C. Bis.) ; sakiápag (Neg.) ; simiónan (Buk.) ; tagbák-babáe (Tag.) ; tagbák-laláki Tag.) ; tagusáhis (P. Bis.) talbák (Tag.); tamotámo (Sbl.) ; tukáng-máya (Tag.); kalaueg (Ig.).

LANGUAS ILLUSTRIS (Ridl.) comb. nov.

Alpinia illustris Ridl. in Philip. Journ. Sci. 4 (1909) Bot. 185.

Mindoro, Palawan, B. S. 514 Foxworthy, 39485 Ramos. In forests along stream at low altitudes; very similar to $A$. malaccensis Rose. Endemic.

LANGUAS LONGIPETIOLATA (Elm.) comb. nov.

Alpinia longipetiolata Elm. Leafl. Philip. Bot. 8 (1919) 2969.

Luzon (Sorsogon), Elmer 16167, 16954, B. S. 23441 Ramos. In forests up to $600 \mathrm{~m}$ altitude. Endemic.

LANGUAS MACROSCAPHIS (K. Schum.) comb. nov.

Alpinia macroscaphis K. Schum. in Engl. Bot. Jahrb. 27 (1899) 284,

Pflanzenreich 20 (1904) 337, f. 40, B. C.

Mindanao (prob. Davao), (Warburg). The type is preserved in the Berlin herbarium. I have seen no specimens. Endemic.

LANGUAS MOLLIS (Presl) comb. nov.

Alpinia mollis Presl Rel. Haenk. 1 (1827) 110; Naves Novis. App.

(1880) 225; K. Schum. in Engl. Pflanzenreich 20 (1904) 368;

Ridl. in Philip. Journ. Sci. 4 (1909) Bot. 191.

"Hab. in insula Luzon." The type was collected by Haenke. It is known only from the original description and is a species of doubtful status. Endemic.

LANGUAS MUSAEFOLIA (Ridl.) comb. nov.

Alpinia musaefolia Ridl. in Leafl. Philip. Bot. 2 (1909) 604, Philip. Journ. Sci. 4 (1909) Bot. 186. 
Negros, Elmer 9539. In forests, altitude about 1,200 m. Endemic. Local name: Lunhás (C. Bis.).

LANGUAS PENDULIFLORA (Ridl.) comb. nov.

Alpinia penduliflora Ridl. in Leafl. Philip. Bot. 2 (1909) 570, Philip. Journ. Sci. 4 (1909) Bot. 187.

Leyte, Elmer 7288. Probably from forests at low altitudes. Endemic.

LANGUAS PUBIFLORA (Benth.) comb. nov.

Hellenia pubiflora Benth. in Hook. Lond. Journ. Bot. 2 (1843) 235.

Alpinia pubiflora K. Schum. in Engl. Pflanzenreich 20 (1904) 313, f. 99, F, G; Ridl. in Philip. Journ. Sci. 4 (1909) Bot. 188; Elm. Leafl. Philip. Bot. 8 (1919) 2965.

Luzon (Tayabas, Sorsogon), Guimaras, Camiguin de Misamis, Mindanao, B. S. 34699 Ramos \& Pascasio, 14728 Ramos, F. B. 9100 Whitfordh \& Hutchison, 34 Ritchie, Elmer 10456, 16546, Copeland 319, Piper 606. In forests at low and medium altitudes. New Guinea, Caroline Islands.

Local names: Tagbág (Sul.); tagbák (P. Bis.).

LANGUAS PULCHELLA (K. Schum.) comb. nov.

Alpinia pulchella K. Schum. in Engl. Bot. Jahrb. 27 (1899) 276, t.2, f. E, Pflanzenreich 20 (1904) 315; Ridl. in Philip. Joum. Sci. 4 (1909) Bot. 189.

Mindanao (Davao), (Warburg). I have seen no material representing this species. New Guinea.

LANGUAS PYRAMIDATA (Blume) comb. nov.

Alpinia pyramidata Blume Enum. Pl. Jav. 1 (1827) 58; Naves Novis. App. (1880) 225; Ridl. in Elm. Leafl. Philip. Bot. 2 (1909) 604, Philip. Journ. Sci. 4 (1909) Bot. 186; Elm. Leafl. Philip. Bot. 8 (1919) 2967.

Alpinia galanga Naves Novis. App. (1880) 225, vix Sw.

Alpinia galanga Sw. var. pyramidata K. Schum. in Engl. Pflanzenreich 20 (1904) 316.

Luzon (Rizal, Laguna, Camarines, Sorsogon), Panay, Leyte, Mindanao (Lanao, Agusan), Palawan, Elmer 16352, Wenzel 140\%, Clemens 612, B. S. 21556 Escritor, 23865 Fénix, 35279 Martelino \& Edaño. In old clearings, etc., sometimes planted. Himalayan region through Malaya to the Moluccas.

Local names: Langkauás (Tag., Bis., Bik.); lankauás (Tag., Pamp., Bis., Mbo.) ; pal-la (Mand.).

LANGUAS ROMBLONENSIS (EIm.) comb. nov.

Alpinia romblonensis Elm. Leafl. Philip. Bot. 8 (1915) 2889.

Romblon, Samar, Elmer 12050, B. S. 21238 Escritor (from Romblon, not from Panay as labeled), 17548 Ramos. Along streams at low altitudes. Endemic.

Local name: Tagbák (P. Bis.).

LANGUAS ROSEA (Elm.) comb. nov.

Alpinia rosea Elm. Leafl. Philip. Bot. 8 (1915) 2890.

Sibuyan, Elmer 12509. In dense forests, altitude about $1,350 \mathrm{~m}$. Endemic. 
LANGUAS RUFA (Presl) comb. nov.

Hellenia rufa Presl Rel. Haenk 1 (1827) 114, t. 21; Miq. Fl. Ind. Bat. 3 (1859) 604.

Alpinia rufa Naves Novis. App. (1880) 226; K. Schum, in Engl. Bot. Jahrb. 27 (1899) 293, Pflanzenreich 20 (1904) 361; Ridl. in Philip. Journ. Sci. 4 (1909) Bot. 190, Leafl. Philip. Bot. 2 (1909) 604, 8 (1915) 2887.

Luzon (Laguna, Camarines, Tayabas, Sorsogon), Polillo, Catanduanes, Samar, Leyte, Panay, Negros, Bucas, Mindanao, Basilan. In forests at low and medium altitudes. Endemic.

Local names: Bihai (C. Bis.); kaukos (Bag.); tagbág (Mbo.) ; tagbák (Tag.).

LANGUAS SIBUYANENSIS (Elm.) comb. nov.

Alpinia sibuyanensis Elm. Leafl. Philip. Bot. 8 (1915) 2891.

Sibuyan, Elmer 12316. In forests along streams, altitude about 225 m. Endemic.

Local name: Bobonan (P. Bis.).

LANGUAS SPECIOSA (Wendl.) comb. nov.

Zerumbet speciosum Wendl. Sert. Hann. (1798) t. 19.

Alpinia nutans Rosc. in Sm. Exot. Bot. 2 (1805) t. 106; Naves Novis. App. (1880) 226.

Alpinia cernua Naves Novis. App. (1880) 226, Fl. Filip. ed. 3 (1877-83) t. 464, non Sims.

Alpinia speciosa K. Schum. Fl. Kaiser-Wilhelmsl. (1887) 29, Engl. Bot. Jahrb. 27 (1899) 284, t. 3, f. A, B, Pflanzenreich 20 (1904) $339, f .40 D-F$.

Occasionally cultivated for ornamental purposes, but not a native of the Archipelago and nowhere naturalized. China, Japan; cultivated in other warm countries.

LANGUAS SUBFUSICARPA (Elm.) comb. nov.

Alpinia subfusicarpa Elm. Leafl. Philip. Bot. 8 (1915) 2892.

Mindanao (Davao), Elmer 10.506. In damp primary forests, altitude about $1,200 \mathrm{~m}$. Endemic.

Local name: Bosadak (Bag.).

LANGUAS TRACHYASCUS (K. Schum.) comb. nov.

Alpinia trachyascus K. Schum. in Engl. Pflanzenreich 20 (1904) 336; Ridl. in Philip. Journ. Sci. 4 (1909) Bot. 185.

Mindanao (Davao), Warburg 14514 in herb. Berol. I have seen only the type which has the facies of Alpinia haenkei Presl. Endemic.

LANGUAS VANOVERBERGHII (Merr.) comb. nov.

Alpinia vanoverberghii Merr. in Philip. Journ. Sci. 7 (1912) Bot. 75.

Luzon (Bontoc), Vanoverbergh 573. On slopes, altitude about 1,350 m. Endemic.

Local names: Kalaúin (Bon.); kalaúing (Bon.); takáng (Bon.).

LANGUAS VULCANICA (Elm.) comb. nov.

Alpinia vulcanica Elm. Leafl. Philip. Bot. 8 (1919) 2971.

Luzon (Camarines, Sorsogon), Bucas Grande, Elmer 16168, B. S. 33864 Ramos \& Edaño, 35051 Ramos \& Pascasio, 23428 Ramos. In primary forests at low and medium altitudes, ascending to $800 \mathrm{~m}$. Endemic. 
LANGUAS WENZELII (Merr.) comb. nov.

Alpinia wenzelii Merr. in Philip. Journ. Sci. 9 (1914) Bot. 353.

Leyte, Wenzel 623. In forests, altitude about $500 \mathrm{~m}$. Endemic.

EXCLUDED SPECIES

Alpinia ARCTIflora F.-Muell.; Naves Novis. App. (1880) 227.

AlPinia CAPITELlatia Jack; Naves op. cit. 226.

Alpinia chinensis Rosc.; Naves op. cit. 225.

AlPiNia COERUlea Benth.; Naves op. cit. 227.

AlPINIA ELATIOR Jack; Naves op. cit. 226.

AlPiNIA gigantea Blume; Naves op. cit. 225.

Alpinia Javanica Blume; Naves op, cit. 226.

Alpinia Mutica Roxb.; Naves 1. c.

Alpinia RUbra (Miq.) Naves l. c.

Alpinia scabra (Blume) Naves 1. c.

Alpinia SPICATa Roxb.; Naves 1. c.

Alpinia stachyoides Hance; Naves 1. c.

3. KOLOWRATIA PresI

KOLOWRATIA CONGESTA (EIm.) comb: nov.

Alpinia congesta Elm. Leafl. Philip. Bot. 8 (1919) 2968.

Luzon (Sorsogon), Elmer 16144. On forested slopes, altitude about $450 \mathrm{~m}$. Endemic.

KOLOWRATIA ELEgANS Presl Rel. Haenk. 1 (1827) 113, t. 20; Miq. Fl. Ind. Bat. 3 (1859) 610; Naves Novis. App. (1880) 228; Ridl. in Govt. Lab. Publ. (Philip.) 35 (1906) 86, Philip. Journ. Sci. 4 (1909) Bot. 181; Merr. Fl. Manila (1912) 159, Sp. Blancoanae (1918) 110.

Renealmia gracilis Blanco Fl. Filip. (1837) 1.

Renealmia exaltata Blanco op. cit. ed. 2 (1845) 1, ed. 3, 1 (1877) 2, t. 1, non Linn.

Hellenia gracilis Hassk. in Flora 47 (1864) 19.

Alpinia gracilis Rolfe in Journ. Linn. Soc. Bot. 21 (1884) 316; Vidal

Phan. Cuming. Philip. (1885) 152, Rev. Pl. Vasc. Filip. (1886), 275.

Alpinia elegans K. Schum. in Engl. Bot. Jahrb. 27 (1899) 288, Pflanzenreich 20 (1904) 352, f. 41 C; Merr. in Govt. Lab. Publ. (Philip.)

27 (1905) 84, Philip. Journ. Sci. 1 (1906) Suppl. 37.

Luzon (Apayao, Amburayan, Lepanto, Nueva Vizcaya, Pampanga, Bulacan, Nueva Ecija, Bataan, Rizal, Laguna, Tayabas, Sorsogon), Polilio, Mindoro, Leyte. In thickets along streams at low and medium altitudes; represented by numerous collections. Endemic.

Local names: Bagombón (Tag.); katkátan (Bis.); katótang (Bis.); salbák (Tag.) ; tagbák (Tag.); tugbák (Tag.).

KOLOWRATIA ERUCAEFORMIS Ridl, in Philip. Journ. Sci. 4 (1909) Bot. 182.

Bucas, Merrill 5258. On dry slopes at the borders of forests at low altitudes. Endemic.

The genus is confined to the Philippines. 


\section{Leptosolena Presl}

LePTOSOLENA HAENKEI Presl Rel. Haenk. 1 (1827) 111, t. 28; Naves Novis. App. (1880) 227; Ridl. in Philip. Journ. Sci. 4 (1909) Bot. 181.

Alpinia leptosolenia K. Schum. in Engl. Pflanzenreich 20 (1904) 312. Leptosolena insignis Ridl. in Govt. Lab. Publ. (Philip.) 35 (1906)

84, Philip. Journ. Sci. 4 (1909) 181; Elm. Leafl. Philip. Bot. 8 (1915) 2907.

Luzon (Ilocos Norte, Bontoc, Benguet, Union, Nueva Vizcaya, $B . S$. 8211, 8172, 32863 Ramos, 21093 Escritor, 37896 Ramos \& Edaño, Elmer 6428, Lete 84, Vanoverbergh 384. In thickets and on open slopes at low and medium altitudes. A monotypic endemic genus.

Local names: Bánai (Ilk.); kaláuin (Bon.); panáuil (Bon.).

\section{ADELMERIA Ridley}

(Elmeria Ridley, non Rydberg)

ADELMERIA ALPINA Elm. Leafl. Philip. Bot. 8 (1915) 2885.

Mindanao (Davao), Elmer 10534, 10642. In forests, altitude 1,200 to 2,100 m. Endemic.

Local names: Lurasísing (Bag.); pusadók (Bag.).

ADELMERIA GIGANTIFOlIA (Elm.) Elm. Leafl. Philip. Bot. 8 (1919) 2963.

Zingiber gigantifolium Elm. op. cit. 8 (1915) 2916.

Luzon (Tayabas, Sorsogon), Elmer 9282, 14603. In forests on talus slopes and along small streams, altitude 500 to 700 . Endemic.

ADELMERIA OBLONGA Merr. in Philip. Journ. Sci. 9 (1914) Bot. 443.

Adelmeria albida Elm. Leafl. Philip. Bot. 8 (1919) 2964.

Luzon (Bontoc, Benguet, Sorsogon), Panay, Negros, Elmer 6255, 15912, Vanoverbergh 3108, Eskridge, B. S. 30884 Ramos \& Edaño. In forests at low and medium altitudes, ascending to $1,500 \mathrm{~m}$. Endemic.

ADELMERIA PARADOXA (Ridl.) Merr. in Philip. Journ. Sci. 9 (1914) Bot. 444.

Hornstedtia paradoxa Ridl. in Govt. Lab. Publ. (Philip.) 35 (1905) 85. Elmeria bifida Ridl. in Leafl. Philip. Bot. 2 (1909) 568.

Adelmeria bifida Ridl. op. cit. 603; Philip. Journ. Sci. 4 (1909) Bot. 179.

Luzon (Apayao, Bontoc, Benguet, Nueva Ecija, Pangasinan, Rizal, Laguna, Tayabas), Panay, Mindanao (Lanao), Elmer 6629, 18145, 8568, 77706, Merrill 4786, 7517, Loher 7001, B. S. 29652, 26458, 30675, 38032 Ramos \& Edaño, F. B. 15854 Merritt. In the mossy forest and in damp forested ravines, altitude 1,000 to $1,800 \mathrm{~m}$. Endemic.

Local name: Parápat (Ig.)

ADELMERIA PINETORUM (Ridl.) Ridl. in Philip. Journ. Sci. 4 (1909) Bot. 180 .

Elmeria pinetorum Ridl. in Leafl. Philip. Bot. 2 (1909) 569. 
Luzon (Bontoc, Benguet), Elmer 8548, Vanoverbergh 453. In thickets and on open slopes about limestone bowlders, altitude 1,300 to $1,500 \mathrm{~m}$. Endemic.

Local names: Alauín (Bon.); barápat (Ig.).

\section{PLAGIOSTACHYS Ridley}

PLAGIOSTACHYS CORRUGATA Elm. Leafl. Philip. Bot. 8 (1915) 2908.

Mindanao (Davao), Elmer 11130. In forests, altitude about $450 \mathrm{~m}$. Endemic.

The flowers are unknown; probably no Plagiostachys.

PLAGIOSTACHYS ELEGANS Ridl. in Philip. Journ. Sci. 4 (1909) Bot. 178.

Mindanao (Lanao), Clemens. In forests, altitude about $900 \mathrm{~m}$. Endemic.

PLAGIOSTACHYS ESCRITORII Elm. Leafl. Philip. Bot. 8 (1915) 2910, (1919) 2989.

Luzon (Laguna, Sorsogon), Samar, Mindanao, Elmer 10516, 10544, 17501, 18071,16216, 18477\%a, 16062, B. S. 15919 Fénix, 24242 Ramos, 21504 Escritor, 37990, 37262 Ramos \& Edaño. In forests at medium altitudes, ascending to $1,300 \mathrm{~m}$. Endemic.

Local names: Bosadak (Bag.); pupundia (Sub.).

PLAGIOSTACHYS PARVIFLORA (Presl) Ridl. in Philip. Journ. Sci. 4 (1909) Bot. 178.

Amomum parviflorum Presl Rel. Haenk. 1 (1827) 112, t. 19; Naves Novis. App. (1880) 224.

Alpinia parviflora Rolfe in Journ. Linn. Soc. Bot. 21 (1884) 316 quoad syn. Presl, Vidal Phan. Cuming. Philip. (1885) 152, Rev Pl. Vasc. Filip. (1886) 275, p. p.; K. Schum. in Engl. Bot. Jahrb. 27 (1899) 299, Engl. Pflanzenreich 20 (1904) 368.

Luzon (Sorsogon, Leyte, Panay, Mindanao (Agusan), Elmer 16215, Wenzel 305, Merrill 7318, B. S. 31256 Ramos. In primary forests at low altitudes. Endemic.

PLAGIOSTACHYS PHILIPPINENSIS Ridl. in Leafl. Philip. Bot. 2 (1909) 571, Philip. Journ. Sci. 4 (1909) Bot. 177; C. B. Rob. op. cit. 6 (1911) Bot. 197; Elm. Leaf. Philip. Bot. 9 (1919) 2989.

Plagiostachys ridleyi Elm. Leafl. Philip. Bot. 8 (1915) 2911, (1919) 2989.

Luzon (Laguna, Tayabas, Camarines), Polillo, Catanduanes, Sibuyan, Panay, Samar, Mindanao, B. S. 33'614, 31245, 31239, 29272 Ramos \& Edaño, 24157 Ramos, 11253 McGregor, Baker 3457, Clemens 855, Elmer 7915, Williams 2402, Elmer 12209. In primary forests at low and medium altitudes. Endemic.

Local name: Tagbák (S. L. Bis., Mbo.).

Plagiostachys Rolfei (K. Schum.) Ridl, in Philip. Journ. Sci. 4 (1909) Bot. 179.

Alpinia rolfei K. Schum. in Engl. Pflanzenreich 20 (1904) 368.

Alpinia parviflora Rolfe in Journ. Linn. Soc. Bot. 21 (1884) 316,

p. p. quoad Cuming 1827.

Luzon (Cagayan, not Albay!), Cuming 132\%. Not seen. Endemic. 


\section{VANOVERBERGHIA Merrill}

VANOVERBERGHIA SePulchreI Merr. in Philip. Journ. Sci. 7 (1912)

Bot. 76; Elm. Leafl. Philip. Bot. 8 (1915) 2913, (1919) 2990.

Luzon (Ifugao, Bontoc, Benguet, Sorsogon), Panay, Vanoverbergh 956, Sandkuhl 257, Elmer 8560, 17383, F. B. 947 Barnes, B. S. 12913 Fénix, 30734, 30134 Ramos \& Edaño. In ravines along streams at medium altitudes, ascending to $1,500 \mathrm{~m}$. A monotypic endemic genus.

Local names: Agbáb (Bon.); barápat (Ig.); chakchákil (Ig.).

\section{AMOMUM Linnaeus}

AMOMUM BULUSANENSE Elm. Leafl. Philip. Bot. 8 (1919) 2973.

Luzon (Sorsogon), Elmer 16395. In primary forests, altitude about 600 m. Endemic.

AMOMUM CONOIDEUM (Ridl.) Elm. Leafl. Philip. Bot. 8 (1919) 2972. Hornstedtia conoidea Ridl. in Leafl. Philip. Bot. 2 (1909) 605, Philip. Journ. Sci. 4 (1909) Bot. 175.

Luzon (Laguna, Sorsogon), Negros, Mindanao (Davao, Lanao), Elmer 10246, 16924, 17896, 11181, Clemens, Barrett. In damp forested ravines at medium altitudes. Endemic.

Valeton, in Bull. Jard. Bot. Buitenz. III 3 (1921) 174, 176, cites Elmer 10246, the type of this species, as possibly representing Hornstedtia alliacea Valeton and as $H$. deliana Valeton, the former definitely known from Java and Sumatra, the latter from Sumatra. Ridley's specific name is the oldest.

AMOMUM DALICAN (Elm.) comb. nov.

Hornstedtia dalican EIm. Leafl. Philip. Bot. 8 (1915) 2906.

Mindanao (Davao), Elmer 11626, 11759. Along small streams in forests, altitude about $1,100 \mathrm{~m}$. Endemic

Local name: Dalikán (Bag.).

AMOMUM DEUTERAMOMUM K. Schum. in Engl. Bot. Jahrb. 27 (1899) 313, Pflanzenreich 20 (1904) 237; Ridl. in Philip. Journ. Sci. 4 (1909) Bot. 173.

Mindanao (Davao), (Warburg 15735). In forests. Not seen. Endesnic.

AMOMUM ELEgans Ridl. in Govt. Lab. Publ. (Philip.) 35 (1906) 84, Philip. Journ. Sci. 4 (1909) Bot. 172.

Luzon (Bataan), Mindanao (Lanao), Whitford 300, 20\%, Clemens 1061, $F$. B. 3033 Borden. In primary forests at low and medium altitudes. Endemic.

Local names: Luya-luyáhan (Tag.); tagbák-gúbat (Tag.).

AMOMUM FUSIFORME Ridl. in Leafl. Philip. Bot. 2 (1909) 604, nomen nudum, Philip. Journ. Sci. 4 (1909) Bot. 171.

Negros, Samar, Mindanao (Zamboanga, Surigao), Bolster 331, 224, Eilmer 9509, B. S. 24159 Ramos, 36690, 37001 Ramos \& Edaño. In primary forests at low and medium altitudes. Endemic.

Local names: Loya-lóya (C. Bis.) ; mali-máli (Sub.) ; satagbák (C. Bis.). 
AMOMUM IROSINENSIS (EIm.) comb. nov.

Hornstedtia irosinensis Elm. Leafl. Philip. Bot. 8 (1919) 2981.

Hornstedtia peninsula Elm. op. cit. 2983.

Luzon (Sorsogon), Elmer 16832, 16263, 16455. In forested ravines, altitude 350 to $500 \mathrm{~m}$. Endemic.

AMOMUM LEPICARPUM Ridl. in Leafl. Philip. Bot. 2 (1909) 605, Philip.

Journ. Sci. 4 (1909) Bot. 174.

Hornstedtia lepicarpa Elm. Leafl. Philip. Bot. 8 (1919) 2979.

Hornstedtia subviridis Elm. op. cit. 2987.

Luzon (Laguna, Sorsogon), Negros, Mindanao (Davao), Elmer 10044, $17176,17869,10835,1643 \%, 17788$. In forests at low and medium altitudes. Endemic.

AMOMUM LINEARIFOLIUM Elm. Leafl. Philip. Bot. 8 (1919) 2975.

Luzon (Sorsogon), Elmer 16926. In thickets bordering forests, altitude about $300 \mathrm{~m}$. Endemic.

AMOMUM LOHERI K. Schum. in Engl. Pflanzenreich 20 (1904) 247;

Ridl. in Philip. Journ. Sci. 4 (1909) Bot. 171.

Luzon, without locality, Loher 675. I saw Loher's specimen at Kew in 1907 and at that time made a note that it was very similar to $A$. trilobum Ridl. Endemic.

AMOMUM LOPHOPHORUM (Ridl.) Elm. Leafl. Philip. Bot. 8 (1919) 2973.

Hornstedtia lophophora Ridl. in Leafl. Philip. Bot. 2 (1909) 607, Philip. Journ. Sci. 4 (1909) Bot. 176.

Negros, Mindanao (Davao), Elmer 10365, 10519. In forested ravines, altitude about $1,200 \mathrm{~m}$. Endemic.

AMOMUM LUZONENSE Elm. Leafl. Philip. Bot. 8 (1919) 2976.

Luzon (Sorsogon), Biliran, (Elmer 15645), B. S. 18696 McGregor, det. Elmer. In secondary forests at low or medium altitudes. Endemic.

AMOMUM MICROCHEILA (Ridl.) comb. nov.

Hornstedtia microcheila Ridl. in Leafl. Philip. Bot. 2 (1909) 606, Philip. Journ. Sci. 4 (1909) Bot. 176.

Negros, Elmer 10279. On seepage banks along streams, altitude about $900 \mathrm{~m}$. Endemic.

AMOMUM MINDANAENSE Elm. Leafl. Philip. Bot. 8 (1915) 2894.

Mindanao (Davao), Elmer 10822. In primary forests, altitude about $1,100 \mathrm{~m}$. Endemic.

Local name: Tanaulak (Bag.).

AMOMUM MURICARPUM Elm. Leafl. Philip. Bot. 8 (1915) 2896.

Hornstedtia muricarpa Elm. op. cit. 8 (1919) 2980.

Mindanao (Cotabato, Zamboanga, Davao), Elmer 10947, B. S. 15846 Fénix, 11555 Robinson, 36603 Ramos \& Edaño. In primary forests at low and medium altitudes. Endemic.

Local names: Lalañgod (Sub.); panikabán (Bag.); túgis (Buk.).

AMOMUM PALAWANENSE Elm. Leafl. Philip. Bot. 8 (1915) 2897.

Palawan, Elmer 12795. In forests along streams at low altitudes. Very similar to $A$. mindanaense Elm. Endemic. 
AMOMUM PANDANICARPUM Elm. Leafl. Philip. Bot. 8 (1915) 2899.

Hornstedtia pandanicarpa Elm. op. cit. 8 (1919) 2979.

Hornstedtia sorsogonensis Elm. op. cit. 2985.

Luzon (Sorsogon), Panay, Mindanao (Bukidnon, Davao), Elmer 10508, 16223, $16755, B$. S. 31184,38758 Ramos \& Edaño. In primary forests at low and medium altitudes, ascending to $1,100 \mathrm{~m}$. Endemic.

Local name: Dalikán (Bag.).

AMOMUM PHILIPPINENSE (Ridl.) comb. nov.

Hornstedtia philippinensis Ridl. in Govt. Lab. Publ. (Philip.) 35 (1906) 86 p. p., Leafl. Philip. Bot. 2 (1909) 605, Philip. Journ. Sci. 4 (1909) Bot. 175; Elm. Leafl. Philip. Bot. 8 (1915) 2905, (1919) 2981.

Luzon (Laguna, Tayabas, Sorsogon), Mindoro, Palawan, Biliran, Negros, Panay, Leyte, Mindanao, Elmer 9281, 9.280, 11973, 16713, 17903, 7285, Merrill 9762, Williams 2888, B. S. 804 Foxworthy, 34554 Ramos \& Pascasio, 31123 Ramos \& Edaño, F. B. 3737 Merritt. In forests at low and medium altitudes. Endemic.

The fruit described by Ridley does not belong to this species; see Elmer, Leafl. Philip. Bot. 8 (1919) 2980, for the true fruit characters.

Local names: Dalikán (Mbo.) ; lulutúkoan (Sub.) ; parápad (Ig.) ; tagbák (P. Bis.) ; tumbák (Neg.).

AMOMUM PROPINQUUM Ridl. in Govt. Lab. Publ. (Philip.) 35 (1906) 84, Philip. Journ. Sci. 4 (1909) Bot. 172; Elm. Leafl. Philip. Bot. 8 (1915) 2894; Merr. Sp. Blancoanae (1918) 111.

Amomum echinatum Blanco Fl. Filip. ed. 2 (1845) 3, ed. 3, 1 (1877) 4 , non Willd.

Amomum aculeatum Naves Novis. App. (1880) 224, non Roxb.

Hornstedtia propinqua Elm. Leafl. Philip. Bot. 8 (1919) 2979.

Luzon (Benguet, Batangas, Laguna, Tayabas, Sorsogon), Masbate, Biliran, Mindanao (Davao), Elmer 6284, 15900, 15614, 18456, Merrill Sp. Blancoanae $925, F . B .1704$ Clark. In forests at low and medium altitudes. Endemic.

Local name: Túgis (S. L. Bis.).

AMOMUM PUBESCENS (Ridl.) comb. nov.

Amomum lepicarpum Ridl. var. pubescens Ridl. in Leafl. Philip. Bot. 2 (1909) 604.

Hornstedtia pubescens Elm. op. cit. 8 (1919) 2979.

Negros, Elmer 10384. In forests, altitude about 1,100 m. Endemic.

AMomum pubimarginatum Elm. Leafl. Philip. Bot. 8 (1915) 2900.

Mindanao (Davao), Elmer 10546. In primary forests, altitude about 1,300 m. Endemic.

Local name: Tabák-bukalod (Bag.).

AMOMUM PURPUREUM (Elm.) comb. nov.

Hornstedtia purpurea Elm. Leafl. Philip. Bot. 9 (1919) 2984.

Luzon (Sorsogon), Elmer 16819, 17023. In densely forested ravines, altitude about $450 \mathrm{~m}$. Endemic. 
AMOMUM TRILobUM Ridl. in Govt. Lab. Publ. (Philip.) 35 (1906) 85, Philip. Journ. Sci. 4 (1909) Bot. 173.

Luzon (Ilocos Norte, Bontoc, Bulacan, Pampanga, Laguna, Rizal), Palawan, Panay, Bolster 67, Elmer 17566, B. S. 4602, 4655 Ramos, 817 Foxworthy, F. B. 12698 Rosenbluth \& Tamesis. In primary forests at low and medium altitudes. Endemic.

Local name: Tagbák (Tag.).

AMOMUM WARBURGII K. Schum. in Engl. Pflanzenreich 20 (1904) 257;

Ridl. in Philip. Journ. Sci. 4 (1909) Bot. 171.

Costus warburgii K. Schum. in Engl. Bot. Jahrb. 27 (1899) 346.

Mindanao (probably Davao), Warburg. The type, a mere scrap, has distinctly the appearance of Costus; to be compared with Costus clemensiae Ridl. A species of very indefinite status. Endemic.

\section{EXCLUDED SPECIES}

Amomum CARDAMomum Linn.; Naves Novis. App. (1880) 223.

Amomum ciliatum Blume; Naves 1. c.

Amomum Dealbatum Roxb.; Naves 1. c.

AMomum GRacile Blume; Naves 1. c.

Amomum Hatuanum (Rumph.) Naves op. cit. 224.

Amomum heteranthum Blume; Naves op. cit. 223.

AMOMUM MAXimum Roxb.; Naves op. cit. 224.

Amomum villosum Lour.; Naves op. cit. 223.

\section{PHAEOMERIA Lindley}

PHAEOMERIA SPECIOSA (Blume) comb. nov.

Elettaria speciosa Blume Enum. Pl. Jav. (1827) 51.

Alpinia magnifica Rosc. Monandr. Pl. (1828) t. 75.

Phaeomeria magnifica K. Schum. in Engl. Pflanzenreich 20 (1904) 262.

Phaeomeria imperialis Lindl. Nat. Syst. ed. 2 (1836) 446; Ridl. in

Philip. Journ. Sci. 4 (1909) Bot. 177; Elm. Leafl. Philip. Bot. 8 (1915) 2907.

Mindanao (Davao, Bukidnon), Elmer 10652, Williams 2943, Wester 49. In ravines, probably introduced. Malay Peninsula, Java, Celebes.

Local name: Tikála (Buk.).

\section{HEDYCHIUM Koenig}

HEDYCHIUM CORONARIUM Koenig in Retz. Obs. 3 (1783) 73; Miq. Fl. Ind. Bat. 3 (1859) 608; Naves Novis. App. (1880) 227; Vidal Phan. Cuming. Philip. (1885) 152, Rev. Pl. Vasc. Filip. (1886) 274; K. Schum. in Engl. Pflanzenreich 20 (1904) 44, f. 8, A, C; Ridl. in Philip. Journ. Sci. 4 (1909) Bot. 164; Merr. Fl. Manila (1912) 157; Elm. Leafl. Philip. Bot. 8 (1919) 2978.

Hedychium lingulatum Hassk. P1. Jav. Rar. (1848) 135; Naves Novis. App. (1880) 227. 
Introduced and cultivated for ornamental purposes, in some regions in the southern Philippines naturalized. In Mindanao certainly of prehistoric introduction, in Luzon of recent introduction. A native of India, now pantropic, cultivated and naturalized.

Local names: Bánai (Bis.) ; dansúli (Buk.) ; kámia (Tag., Bik., Sp., C. Bis.); katkátan (Bis.) ; katotang (Bis.).

HEDYCHIUM PHILIPPINENSE K. Schum. in Engl. Pflanzenreich 20 (1904) 47; Ridl. in Philip. Journ. Sci. 4 (1909) Bot. 164; Elm. Leafl. Philip. Bot. 8 (1915) 2903.

Hedychium mindanaense Elm. Leafl. Philip. Bot. 8 (1915) 2904, (1919) 2978.

Luzon (Rizal, Laguna, Tayabas, Sorsogon), Polillo, Negros, Leyte, Mindanao, Jolo, Elmer 10673, 7909, 15968, Loher 6996, Clemens 747, B. S. 22897 McGregor, 35015 Ramos \& Pascasio, 279.47 Ocampo, 20777 Escritor, 15206, 10962, 10003 Ramos, F. B. 4252 Everett. In damp forests at low and medium altitudes, ascending to $1,100 \mathrm{~m}$; often epiphytic or pseudoepiphytic. Endemic.

Local names: Dainsúli (Sub.) ; kaláuag (Bag.); tagbák (P. Bis.).

\section{EXCLUDED SPECIES}

Hedychium hasselti Blume; Naves Novis. App. (1880) 227.

\section{KAEMPFERIA Linnaeus}

KAEMPferia Galanga Linn. Sp. Pl. (1753) 2; Naves Novis. App. (1880) 222; Ridl. in Philip. Journ. Sci. 4 (1909) Bot. 165; K. Schum. in Engl. Pflanzenreich 20 (1904) 77; Merr. Fl. Manila (1912) 157, Sp. Blancoanae (1918) 110.

Kaempferia rotunda Blanco Fl. Filip. (1837) 5, ed. 2 (1845) 4, ed. 3, 1 (1877) 7; Naves Novis. App. (1880) 222; Merr. in Govt. Lab. Publ. (Philip.) 27 (1905) 84, non Linn.

Kaempferia latifolia Hornem. Hassk. in Flora 47 (1864) 22, quoad syn. Blanco.

Luzon (Bontoc, Rizal), Mindanao (Bukidnon), Merrill Sp. Blancoanae 942, B. S. 26075 Fénix, 4560 Ramos. In open grasslands at low and medium altitudes. India through Malaya to the Moluccas.

Local names: Disól (Ilk.); dosó (Bon.); dosól (Sbl.); dotó (Bon.); dusó (Tag.); dusóg (Tag.); dusól (Tag.); gisól (Tag., Pamp.) ; kisól (Buk., Bis.) ; kosól (Bis.); kusól (Pamp.).

KAEMPFERIA PHILIPPINENSIS Merr. in Philip. Journ. Sci. 10 (1915) Bot. 296.

Luzon (Laguna), B. S. 14952 Ramos. In forests along streams at low or medium altitudes. Endemic.

\section{EXCLUDED SPECIES}

KaEMPFERIA LATIFolia Don; Naves Novis. App. (1880) 223.

KaEMPFERIA PANDURATa Roxb.; Naves 1. c. 
12. BOESENBERGIA O. Kuntze

(Gastrochilus Wallich, non Don)

BOESENBERGIA LONGIPETIOLATA (Ridl.) comb. nov.

Gastrochilus longipetiolatus Ridl. in Philip. Journ. Sci. 4 (1909) Bot. 166.

Mindanao (Zamboanga), F. B. 9110 Whitford \& Hutchinson, B. S. 36798 Ramos \& Edaño. In primary forests at low altitudes. Endemic.

Local name: Bubúnad (Sub.).

\section{CURCUMA Linnaeus}

CURCUMA LONGA Linn. Sp. Pl. (1753) 2; Blanco Fl. Filip. (1837) 5, ed. 2 (1845) 4, ed. 3, 1 (1877) 6, t. 3; Naves Novis. App. (1880) 221; K. Schum. in Engl. Pflanzenreich 20 (1904) 108; Ridl. in Philip. Journ. Sci. 4 (1909) Bot. 167.

Curcuma xanthorrhiza Naves in Blanco Fl. Filip. ed. 3 (1877-83) t. 3, Novis. App. (1880) 222, non Roxb.

Widely distributed in the Philippines in and about towns, sometimes in open waste places, sometimes planted, Merrill Sp. Blancoanae 91\%, Elmer 11933. A native of India, now pantropic. Turmeric.

Local names: Ađ̃gai (Pamp.); barák (Kuy.); diláu (Tag.) ; duláu (S. L. Bis.) ; kalabága (Bis.) ; kaláuag (Mbo., Bis.) ; kinambói (Bis.) ; kulálo (Pamp.) ; kulyáu (Ilk.) ; kúnig (Ilk.) ; kúnik (Ibn.) ; lampúyang (P. Bis.) ; láuag (Sub.) ; lúyang-dilaú (Tag.) ; pañgas (Pamp.); salampauyan (Bag.). CURCUMA ZEDOARIA (Berg.) Rosc. Monandr. Pl. (1828) t. 109; K. Schum. in Engl. Pfianzenreich 20 (1904) 110; Ridl. in Govt. Lab. Publ. (Philip.) 35 (1906) 84, Philip. Journ. Sci. 4 (1909) Bot. 166; Merr. in Philip. Journ. Sci. 2 (1907) Bot. 433; F1. Manila (1912) 158, Sp. Blancoanae (1918) 109; Elm. Leafl. Philip. Bot. 8 (1919) 2977 (err. zeodaeria).

Amomum zedoaria Berg. Mat. Med. (1788) 41.

Curcuma zerumbet Roxb. Pl. Coromandel 2 (1798) t. 101; Naves Novis. App. (1880) 222.

Costus nigricans Blanco Fl. Filip. (1837) 3, ed. 2 (1845) 3, ed. 3, 1 (1877) 5.

Costus luteus Blanco Fl. Filip. (1837) 4, ed. 2 (1845) 3, ed. 3, 1 (1877) 6.

Roscoea nigro-ciliata Hassk. in Flora 47 (1864) 21.

Roscoea lutea Hassk. 1. c.

In open waste places in and near towns throughout the Philippines, often abundant; certainly introduced. India to Malaya.

Local names: Alimpuyás (C. Bis.) ; alimpuyíng (C. Bis.) ; barák (Tag.) ; bolon (Tag.); gánda (Sbl.); kónik (Ilk.); konikó (Bon.); lampóyang (P. Bis.) ; langkúas (Ilk.) ; luya-luyáhan (Tag.); tamahíba (Tag.) ; tamahílan (Bik.) ; tamó (Pamp., Tag.) ; tamokánsi (Tag.) ; únig (If.).

\section{EXCLUDED SPECIES}

Curcuma Aeruginosa Roxb.; Naves Novis. App. (1880) 222.

Curcuma australasica Hook. f.; Naves op. cit. 221.

Curcuma CAEsia Roxb.; Naves op. cit. 222.

Curcuma purpurascens Blume; Naves 1. c.

CURCUMA VIRIDIFLora Roxb.; Naves op. cit. 221. 


\section{GLOBBA Linnaeus}

\section{GLobBa AUREA Elm. Leafl. Philip. Bot. 8 (1915) 2902.}

Busuanga, Palawan, Elmer 13243, Merrill 7199, 9342, B. S. 21569 Escritor, 19339 Weber. In thickets and in rock crevices along streams at low and medium altitudes. Endemic.

GLobBA BREVIFOLIA K. Schum. in Engl. Pflanzenreich 20 (1904) 145; Ridl. in Philip. Journ. Sci. 4 (1909) Bot. 160.

Globba parviflora Presl var. brevifolia K. Schum. in Engl. Bot. Jahrb. 27 (1899) 330.

Luzon (Zambales), Corregidor, Warburg 14066, F. B. 13216 Curran. In thickets or forests at low or medium altitudes. Endemic.

Apparently very close to $G$. campsophylla K. Schum.

GLOBBA CAMPSOPHYLLA K. Schum. in Engl. Pflanzenreich 20 (1904) 145; Ridl. in Govt. Lab. Publ. (Philip.) 35 (1906) 83, Philip. Journ. Sci. 4 (1909) Bot. 159.

Globba parvifora Vidal Phan. Cuming. Philip. (1885) 152; K. Schum. in Engl. Bot. Jahrb. 27 (1899) 330, non Presl.

Luzon (Nueva Ecija, Rizal, Tayabas, Bulacan, Bataan), Catanduanes, Panay, Samar, Mindanao, Cuming 1090, Elmer 11284, Merrill 4177, 5207, Phil. Pl. 334, F. B. 1461 Ahern's collector, 7208 Curran, B. S. 1110, 2427\%, 19444, 30296 Ramos, 6132 Robinson, 24975 Fénix, 21392 Escritor. In damp thickets and forests, on and about cliffs, etc., at low and medium altitudes. Endemic.

Local names: Luya-luyáhan (Tag.) ; malasabután (S. L. Bis.) ; tangbák (Tag.).

GLOBBA GRACILIS K. Schum. in Engl. Pflanzenreich 20 (1904) 145; Ridl. in Philip. Journ. Sci. 4 (1909) Bot. 161, Leafl. Philip. Bot. 2 (1909) 605.

Mindanao (Misamis, Davao), Warburg 14580 in herb. Berol., $F . B$. 4752 Mearns \& Hutchinson. In primary forests, altitude 300 to $1,000 \mathrm{~m}$. Endemic.

GLobBA LATIFoliA Ridl. in Philip. Journ. Sci. 4 (1909) Bot. 161.

Mindoro, Panay, Mindanao (Zamboanga), Merrill 4064, Williams 2334 , B. S. 30976 Ramus \& Edaño, 32197 McGregor. In damp primary forests at low and medium altitudes. Endemic.

GLobBA LeucocARPA Ridl. in Philip. Journ. Sci. 4 (1909) Bot. 161.

Luzon (Benguet), Polillo, Mindoro, Leyte, Elmer 6465, 7298, Merriul 1789, McGregor 201. In damp primary forests, on shaded cliffs, etc., at low and medium altitudes. Endemic.

GlobBa MARANTINA Linn. Mant. 2 (1771) 170; Schauer in Nov. Act. Acad. Nat. Cur. 19 (1843) Suppl. 1: 427; Presl Rel. Haenk. 1 (1827) 115; Llanos Frag. Pl. Filip. (1851) 7; F.-Vill. \& Naves in Blaneo Fl. Filip. ed. $3,4^{1}$ (1880) 2, t. 351; Vidal Phan. Cuming. Philip. (1885) 151, Rev. Pl. Vasc. Filip. (1886) 274 ; Naves Novis. App. (1880) 219; K. Schum. in Engl. Pflanzenreich 20 (1904) 156; Ridl. in Philip. Journ. Sci. 4 (1909) Bot. 162; Merr. Fl. Manila (1912) 156, Sp. Blancoanae (1918) 111. 
Globba barthei Gagnep. in Bull. Soc. Bot. France 48 (1901) 208, t. 7, f. 1-4; K. Schum. in Engl. Pflanzenreich 20 (1904) 159; Ridl. in Govt. Lab. Publ. (Philip.) 35 (1906) 83.

Globba ectobolus K. Schum. in Engl. Pflanzenreich 20 (1904) 156, f. $19, L$.

Globba heterobracteata K. Schum. op. cit. 159, f. 19, H.

Luzon (Union, Nueva Ecija, Pangasinan, Pampanga, Bulacan, Cavite, Rizal, Bataan), Palawan, Panay, Negros. In thickets in and near towns at low and medium altitudes, often abundant. Certainly introduced. Malay Peninsula and Archipelago to New Guinea and the Bismarck Archipelago.

Local names: Banglíu (Ilk.) ; barák (Tag.) ; luyan-luyáan (Pang.).

GLOBBA MERRILLII Ridl. in Govt. Lab. Publ. (Philip.) 35 (1906) 83, Philip. Journ. Sci. 4 (1909) Bot. 160.

Luzon (Bataan), Merrill 3869, Whitford 481, F. B. 1598 Borden. Along streams in primary forests and on exposed forested ridges up to $1,000 \mathrm{~m}$ altitude; not easily distinguishable from $G$. campsophylla $K$. Schum. Endemic.

GlobBa PARVIflora Presl Rel. Haenk. 1 (1827) 115; Miq. Fl. Ind. Bat. 3 (1859) 592; Naves Novis. App. (1880) 220; K. Schum. in Engl. Pfianzenreich 20 (1904) 146; Ridl. in Govt. Lab. Publ. (Philip.) 35 (1906) 83, Philip. Journ. Sci. 4 (1909) Bot. 159; EIm. Leafl. Philip. Bot. 8 (1919) 2977.

Luzon (Rizal, Albay, Sorsogon), Leyte, Mindanao, Merrill 2783, 278?, Elmer 15666, 15381, B. S. 16503 Reillo, F. B. 2910 Mearns, 12453 Danao. In forests at low and medium altitudes. Endemic.

GlobBa PYRAmidatA Gagnep. in Bull. Soc. Bot. France 48 (1901) 204, t. 4, f. 7-10; K. Schum. in Engl. Pflanzenreich 20 (1964) 146; Ridl. in Philip. Journ. Sci. 4 (1909) Bot. 158.

Mindanao (Agusan), Weber 1128. In primary forests at low altitudes. Indo-China.

GLobBA USTUlAtA Gagnep, in Bull. Soc. Bot. France 48 (1901) 208, t. $\%$, f. 5-\%; K. Schum. in Engl. Pflanzenreich 20 (1904) 158; Ridl. in Philip. Journ. Sci. 4 (1909) Bot. 163.

Balabac (Marche 227 $B$ ). The type is preserved in the Paris Museum; I have seen no specimens. A variety in Indo-China fide Gagnepain.

EXCLUDED SPECIES

Globba Aurantiaca Miq.; Naves Novis. App. (1880) 219.

GlobBa Careyana Roxb.; Naves op. cit. 220.

Globba CILIATA Jack; Naves op. cit. 219.

Globba leucantha Miq.; Naves 1. c.

Globba maculata Blume; Naves 1. c.

Globba Patens Mia.; Naves 1. c.

GlobBa saltatoria Rose.; Naves 1. c.

Globba strobilifera Zoll.; Naves 1. c. 


\section{5. costus Linnaeus}

Costus CLemensAe Ridl. in Philip. Journ. Sci. 4 (1909) Bot. 193.

Mindanao (Zamboanga, Lanao), Clemens 1173, B. S. 36578 Ramos \& Edaño. On forested slopes, altitude about $700 \mathrm{~m}$. Endemic.

Local name: Tiuasi-tiuasi (Sub.).

COSTUS GLABRA (K. Schum.) comb. nov.

Costus speciosus Sm. var. glabra K. Schum. in Engl. Bot. Jahrb. 27 (1899) 343, Pflanzenreich 20 (1904) 398.

Herba spiralis II laevis Rumph. Herb. Amb. 6: 143, t. 64, f. 2.

Costus speciosus Elm. Leafl. Philip. Bot. 8 (1919) 2977, non Sm.

Luzon (Tayabas, Albay, Sorsogon), Polillo, Samar, Leyte, Panay, Wenzel 227, Elmer 14707, Whitford 830, B. S. 6299, 6926 Robinson, 35478 Martelino \& Edaño, 24820, 24811 Edaño, 15302 Ramos. In forests, old clearings, abacá plantations, etc., at low and medium altitudes. Moluccas.

COSTUS HIRSUTUS Blume Enum. Pl. Jav. 1 (1827) 61.

Costus speciosus Sm. var. hirsuta K. Schum. in Engl. Bot. Jahrb. 27 (1899) 343, Pflanzenreich 20 (1904) 398.

Luzon (Bontoc, Benguet, Union, Batangas), Vanoverbergh 1392, Cuzner 21, Lete 264. In thickets and forests, altitudes 300 to $1,200 \mathrm{~m}$. India to southern China and Formosa through. Malaya to New Guinea.

Costus SERICEUS Blume Enum. PI. Jav. 1 (1827) 62; Naves Novis. App. (1880) 227.

Costus speciosus Sm. var. argyrophyllus Wall. ex Baker in Hook. f. Fl. Brit. Ind. 6 (1892) 249; Ridl. in Govt. Lab. Publ. (Philip.) 35 (1906) 84, Philip. Journ. Sci. 4 (1909) Bot. 192.

Costus speciosus Vidal Phan. Cuming. Philip. (1885) 152, Rev. Pl. Vasc. Filip. (1886) 274; Ridl. in Philip. Journ. Sci. 4 (1909) Bot. 192, non Sm.

Luzon (Benguet, Nueva Vizcaya, Bataan, Rizal, Cavite, Laguna, Tayabas, Camarines, Sorsogon), Polillo, Masbate, Negros, Panay, Cebu, Leyte, Mindanao, Basilan, Jolo. Common in primary forests, old clearings, etc., at low and medium altitudes. Burma, through Malaya to New Guinea.

Local names: Tiuási (Sub.) ; tubong-usá (Bik.).

Costus SPEcIosus (Koenig) Sm. in Trans. Linn. Soc. 1 (1791) 249; Miq. Fl. Ind. Bat. 3 (1859) 610; Naves Novis. App. (1880) 227, in Blanco Fl. Filip. ed. $3(1877-83)$ t. 442; $\mathrm{K}$. Schum. in Engl. Pflanzenreich 20 (1904) 398; Merr. Fl. Manila (1912) 160.

Banksia speciosa Koenig in Retz. Obs. 3 (1783) 75.

Occasionally cultivated for ornamental purposes. This very largeflowered form is a native of the Malay Peninsula and is, I believe, specifically distinct from the common wild Philippine form. It is cultivated in various parts of Malaya and in some other tropical countries.

\section{EXCLUDED SPECIES}

Costus Potierae F.-Muell.; Naves Novis. App. (1880) 227.

\section{EXCLUDED GENERA}

Elettaria Alba Blume; Naves Novis. App. (1880) 224.

Elettaria ANTHoldes Teysm.; Naves 1. c. 
Elettaria cardamomum Maton; Naves 1. c.

Elettaria coccinea Blume; Naves 1. c.

Elettaria foetens Blume; Naves 1. c.

Elettaria hemisphaerica Blume; Naves 1. c.

Elettaria macrocephala Miq.; Naves op. cit. 225.

Elettaria minuta Blume; Naves op. cit. 224.

EleTtaria mollis Blume; Naves 1. c.

Elettaria PinANGa Miq.; Naves op. cit. 225.

EletTARia PUnicea (Roxb.) Naves op. cit. 224.

ELETTARIA RUBRA Miq.; Naves op. cit. 225.

EletTaria solaris Blume; Naves op. cit. 224.

EleTtARIA' SPECIOSA Blume; Naves 1. c.

Elettaria tomentosa Miq.; Naves op. cit. 225.

Elettaria villosa Miq.; Naves 1. c.

Roscoea Purpurea Spreng.; F.-Vill. \& Naves in Blanco Fl. Filip. ed. $3,4^{1}$ (1880) 99; Naves Novis. App. (1880) 223.

\section{CANNACEAE}

\section{CANNA Linnaeus}

CANNA COCCINEA Mill. Gard. Dict. ed. 8 (1768) No. 3; Miq. Fl. Ind. Bat. 3 (1859) 613; Naves Novis. App. (1880) 229; Merr. Fl. Manila (1912) 161; Krånzl. in Engl. Pflanzenreich 56 (1912) 60.

Cultivated for ornamental purposes, but nowhere naturalized. A native of Central and South America.

CANNA FlACCidA Salisb. Ic. Stirp. Rar. (1791) t. 2; Merr. Fl. Manila (1912) 162 (C. flaccida Salisb. x iridiflora Ruiz \& Pav.) ; Kränzl. in Engl. Pflanzenreich 56 (1912) 49.

Widely cultivated for its showy flowers, but not naturalized and apparently never or very rarely producing seeds. A native of tropical America.

Local names: Bandera española (Sp.) ; kambút bandera española (Ilk.).

CANNA GLAuCA Linn. Sp. Pl. (1753) 1; Ridl. in Philip. Journ. Sci. 4 (1909) Bot. 198; Merr. Fl. Manila (1912) 162; Kränzl. in Engl. Pflanzenreich 56 (1912) 54.

Cultivated for ornamental purposes, Merrill 648,6690, 6691. A native of tropical America.

CANNA INDICA Linn. Sp. Pl. (1753) 1; Blanco Fl. Filip. (1837) 6, ed. 2 (1845) 5, ed. 3, 1 (1877) 8, t. 4; Naves Novis. App. (1880) 229; Ridl. in Philip. Journ. Sci. 4. (1909) Bot. 198; Merr. op. cit. 5 (1910) Bot. 340, Fl. Manila (1912) 161, Sp. Blancoanae (1918) 111. Canna orientalis Rose. Monandr. Pl. (1828) t. 12; Kränzl. in Engl. Pflanzenreich 56 (1912) 47. ?

Throughout the Philippines in the settled areas, occurring in waste places in and near settlements. A native of tropical America, now pantropic 
Local names: Balunsáying (Bis.) ; bañali (Bik.) ; kakuentásan (Tag.); kiuíngan (If.) ; kolintásan (Bis.) ; kuentas-kuentásan (Tag.) ; lása (Iv.); saging-ságing (Tag.) ; tapuránga (Bis.) ; tíkas (Tag.); tikas-tíkas (Tag., P. Bis.) ; tikis-tíkis (Tag.); tukas-túkas (Tag.).

CANNA REEVESII Lindl. Bot. Reg. 23 (1837) t. 2004; Kränzl. in Engl. Pflanzenreich 56 (1912) 50, f. 10, A-C.

Cultivated for ornamental purposes, but nowhere spontaneous, Merrill 6692, det. Kränzlin. A native of tropical America.

CANNA SPECIOSA Rosc. Monandr. Pl. (1828) $t$. 1\%; Naves Novis. App. (1880) 229; Merr. Fl. Manila (1912) 161; Kränzl. in Engl. Pflanzenreich 56 (1912) 44, f. 8 D.

Occasional in open waste places, naturalized, Merrill 6689. A native of tropical America.

Local name: Kambút-r̃ganalabága (Ilk.).

CANNA WARCEWICZII Dietr. in Allg. Gart. Zeit. 19 (1851) 290, fig.; Merr. Fl. Manila (1912) 162; Kränzl. in Engl. Pflanzenreich 56 (1912) 64.

Cultivated for ornamental purposes, but nowhere established. A native of tropical America.

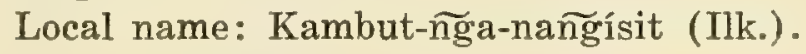

\section{EXCLUDED SPECIES}

Canna montana Blume; Naves Novis. App. (1880) 229.

Canna Edulis Ker.; Naves 1. c.

Canna Pedunculata Sims; Naves 1. c.

Canna RUbricaulis Link; Naves 1. c. $=$ C. edulis Ker.

CanNa Flavescens Link; Naves 1. c.

All our forms of Canna are introduced plants. in the Philippines. Two or three characteristic species are thoroughly naturalized, especially Canna indica Linn., which was introduced at an early date in colonial history. A very large number of garden forms, mostly hybrids, have been introduced from Europe and America since the beginning of the present century, but it is impossible to determine their botanical origins, as the necessary data are lacking; few of these hybrids ever produce seeds here, but are reproduced by means of their rhizomes.

\section{MARANTACEAE}

\section{DONAX Loureiro}

DONAX CANNAEFORMIS (Forst. f.) K. Schum. in Engl. Bot. Jahrb. 15 (1893) 440; Rolfe in Journ. Bot. 45 (1907) 243; Ridl. in Philip. Journ. Sci. 4 (1909) Bot. 193; Valeton ex Merr. Interpret. Herb. Amb. (1917) 166; Merr. Sp. Blancoanae (1918) 112.

Thalia cannaeformis Forst. f. Prodr. (1786) 1.

Maranta arundinacea Blanco F1. Filip. (1837) 7, ed. 2 (1845) 5, ed. 3, 1 (1877) 9, t. 5, non Linn. 
Clinogyne grandis Benth. \& Hook: f. ex Rolfe in Journ. Bot. 23 (1885) 465; Vidal Phan. Cuming. Philip. (1885) 152, Rev. Pl. Vasc. Filip. (1886) 275.

Donax arundastrum K. Schum. in Engl. Pflanzenreich 11 (1902) 3:; Merr. in Philip. Journ. Sci. 1 (1906) Suppl. 37, Forestry Bureau Bull. 1 (1903) 16; Perk. Frag. Fl. Philip. (1904) 68, t. 1 f. G-J, non Lour.

Actoplanes cannaeformis K. Schum. in Engl. Pflanzenreich 11 (1902) 34 p. p. excl. syn. Miq.

Phrynium dichotomum Roxb. in Asiat. Research 11 (1810) 324; Schauer in Nov. Act. Acad. Nat. Cur. 19 (1843) Supp1. 1: 427.

Maranta dichotoma Wall. Cat. (1832) No. 6615; Naves Novis. App. (1880) 228; Miq. Fl. Ind. Bat. 3 (1859) 611; Usteri Beitr. Ken. Philip. Veg. (1905) 128.

Maranta grandis Miq. Fl. Ind. Bat. Suppl. (1860-1) 616; Naves Novis. App. (1880) 228.

Batan Islands and northern Luzon to Palawan and Mindanao, in all or most islands and provinces. Common in secondary forests, especially along streams at low and medium altitudes. Java and Borneo to New Guinea, the Aru and Admiralty Islands, New Hebrides, and the Marianne Islands.

Local names: Alaró (Bis.) ; aratán (Gad.); babán (Chab.); bambán (Ilk., Bis., Mbo., Tag., Sul., Buk.) ; banbán (Tag., Bik., Bis., Bag., Ibn.) : bankólid (Mbo.) ; baras-barásan (Tag.) ; bonbón (Tag.) ; buabán (Bag.); daromáka (Ilk.); darumáka (Ilk.); garomáka (Ilk.); lankuás (Ilk.); mambán (Bik., S. L. Bis.) ; manbán (Tag.) ; matalbák (Tag.) ; matapál (Isn.) ; miní (Ig.); niní (Ig.) ; niník (Iv.).

\section{PHRYNIUM Willdenow}

PHRYNIUM PHILIPPINENSE Ridl. in Leafl. Philip. Bot. 2 (1909) 569, Philip. Journ. Sci. 4 (1909) Bot. 195.

Phrynium capitatum Presl Rel. Haenk. 1 (1827) 109; Naves Novis. App. (1880) 228, non Willd.

Phrynium densiforum Naves Novis. App. (1880) 228; Vidal Phan.

Cuming. Philip. (1885) 152, Rev. Pl. Vasc. Filip. (1886) 275, non Blume.

Babuyan Islands, Luzon (Isabela, Nueva Vizcaya, Rizal, Laguna, Tayabas, Albay, Sorsogon), Alabat, Polillo, Mindoro, Palawan, Panay, Leyte, Negros, Mindanao. In forests at low and medium altitudes; scarcely distinguishable from $P$. capitatum Willd. Endemic.

Local names: Bogigkík (Mbo.); hagikhík (Bis.); hagithít (Tag.); lutatában (P. Bis.) ; okos (Bag.).

\section{EXCLUDED SPECIES}

Phrynium latifolium Blume; Naves Novis. App. (1880) 228.

PhRYNIUM MAXIMUM Blume; Naves 1. c.

Phrynium Pubinerve Blume; Naves 1.c.

Some species of Calathea have been introduced into the Philippines and are occasionally found in cultivation for ornamental purposes. 


\section{MARANTA Linnaeus}

MARANTA ARUNDINACEA Linn. Sp. Pl. (1753) 2; Naves Novis. App. (1880) 228; K. Schum. in Engl. Pflanzenreich 11 (1902) 125; Perk. Frag. Fl. Philip. (1904) 72, t. 2; Merr. in Govt. Lab. Publ. (Philip.) 6 (1904) 29, Fl. Manila (1912) 163; Ridl. in Philip. Journ. Sci. 4 (1909) Bot. 197.

Maranta ramosissima Wall. Pl. As. Rar. 3 (1832) 51, t. 286; Naves Novis. App. (1880) 228.

Widely distributed in the Philippines in cultivation for its starchy rhizomes. A native of tropical America, now pantropic. Arrowroot.

Local names: Ararú (Ibn., It.); arorú (Tag.); arurú (Tag.); bái (Iv.) ; galamáka (Bon.) ; sagú (Bik., Ilk.).

Var. VARIEGATA (N. E. Br.) Ridl. in Gard. Chron. III 9 (1891) 73; Merr. Fl. Manila (1912) 163.

Phrynium variegatum N. E. Br. in Ill. Hort. 33 (1886) 125, t. 606.

Cultivated for ornamental purposes.

\section{MONOPHRYNIUM K. Schumann}

MONOPHRYNIUM CONGESTUM Ridl. in Philip. Journ. Sci. 4 (1909) Bot. 197.

Luzon (Apayao, Tayabas, Laguna), F. B. 9548 Curran, B. S. 28082, 28245 Fénix. In primary forests at low or medium altitudes. Endemic.

Local names: Aláuan (Ibn.); arúpi (Ibn.).

MONOPHRYNIUM FAsciculatum (Presl) K. Schum. in Engl. Pfianzenreich 11 (1902) 68; Perk. Frag. Fl. Philip. (1904) 69, t. 1, f. $A-F$; Ridl. in Philip. Journ. Sci. 4 (1909) Bot. 196.

Calathea fasciculata Presl Rel. Haenk. 1 (1827) 108, t. 10, f. 1; Naves Novis. App. (1880) 229.

Phrynium fasciculatum Horan. Monogr. (1862) 11.

Luzon (Cagayan, Laguna, Tayabas), Samar, Leyte, Biliran, Mindanao (Surigao), B. S. 34797 Ramos \& Pascasio, 28589 Ramos \& Edaño,24136, 13356 Ramos, 22837 McGregor, Elmer 9148, Wenzel 378. In primary forests at low and medium altitudes. Halmahera. The only representative of the genus known outside of the Philippines.

Local names: Hagithít (Tag.) ; hagithít-na-daytoi (S. L. Bis.).

MONOPHRYNIUM SIMPLEX Elm. Leafl. Philip. Bot. 1 (1908) 276; Ridl. in Philip. Journ. Sci. 4 (1909) Bot. 196.

Luzon (Laguna, Tayabas), Elmer $7611,914 \%, 17502$. On forested slopes near streams, altitude about $750 \mathrm{~m}$. Endemic.

\section{PHACELOPHRYNIUM K. Schumann}

PHACELOPHRYNIUM BRACTEOSUM (Warb.) K. Schum. in Engl. Pflanzenreich 11 (1902) 123; Perk. Frag. Fl. Philip. (1904) 71, t. 3, f. F-O; Merr. in Philip. Journ. Sci. 2 (1907) Bot. 268; Ridl. op. cit. 4 (1909) Bot. 195.

Phrynium bracteosum Warb. ex K. Schum. 1. c. in syn.

Luzon (Sorsogon), Mindoro, Samar, Leyte, Biliran, Mindanao (Agusan, Lanao), Merrill 7288, 5202, 5232, Elmer 16145, 15240, Clemens 423, Wenzel 
239, B. S. 34564 Ramos \& Pascasio, 24732 Edaño, 15229, 17456,39780 Ramos, 18818 McGregor. In primary forests at low and medium altitudes. Eorneo.

PHACELOPHRYNIUM CYLINDRICUM Merr. in Philip. Journ. Sci. 13 (1918) Bot. 269.

Catanduanes, Panay, B. S. 30503 Ramos, 31271 Ramos \& Edaño. In primary forests at low altitudes. Endemic.

PHACELOPHRYNIUM INTERRUPTUM (Warb.) K. Schum, in Engl. Pflanzenreich 11 (1902) 121; Perk. Frag. Fl. Philip. (1904) 70, t. 8, f. A-E; Ridl. in Philip. Journ. Sci. 4 (1909) Bot. 194.

Phrynium interruptum Warb. ex K. Schum. l. c. in syn.

Luzon (Ilocos Norte, Benguet, Bulacan, Rizal, Laguna, Tayabas, Camarines, Sorsogon), Polillo, Samar, Panay, Leyte, Mindanao (Agusan, Davao). In forests at low and medium altitudes, represented by about fifty individual collections. Endemic.

Local names: Bambán (Tag.) ; hagikhík (S. L. Bis.) ; hagitít (Tag., Bik.) ; kokos (Bag.) ; nítni (Ig.).

\section{BURMANNIACEAE}

\section{BURMANNIA Linnaeus}

BURMANNIA CLEMENTIS Schltr. in Philip. Journ. Sci. 1 (1906) Suppl. 305.

Mindanao (Lanao), Clemens 24. In grasslands, altitude about $650 \mathrm{~m}$. Endemic.

BURMANNIA COELESTIS D. Don Prodr. Fl. Nepal. (1825) 44; Naves Novis. App. (1880) 252; Merr. in Philip. Journ. Sci. 4 (1909) Bot. 250.

Burmannia azurea Griff. Notul. 3 (1851) 236, Ic. Pl. As. (1851) t. 272, f. 1; Becc. Malesia 1 (1878) 242, t. 15, f. 1-3.

Luzon (Pangasinan), B. S. 4918 Ramos. Probably in open places at low altitudes. India to Borneo and the Caroline Islands.

BURMANNIA LONGIFOLIA Becc. Malesia 1 (1878) 244; Naves Novis. App. (1880) 252; Rendle in Journ. Bot. 34 (1896) 355; Merr. in Philip. Journ. Sci. 2 (1907) Bot. 268.

Mindoro, Negros, Camiguin de Misamis, Mindanao (Agusan, Davao), Merrill 5741, Elmer 13690, B. S. 14428 Ramos, F. B. 13611 Curran \& Foxworthy. On ridges in wet mossy forests, altitude 1,200 to $1,600 \mathrm{~m}$. Malay Peninsula, Borneo, Amboina, New Guinea.

Local names: Bagumbáan (Mbo.); pamaláuan (Bag.).

EXCLUDED GENERA AND SPECIES

BurmanNia disticha Linn.; Naves Novis. App. (1880) 252.

Burmannia sumatrana Miq.; Naves 1. c.

Burmannia tuberosa Becc.; Naves 1. c.

GYMNOSIPHON APHYLLUM Blume; Naves 1. c.

SARCOSIPHON CLANDESTINUM Blume; Naves I. c. 


\section{APOSTASIACEAE*}

\section{NEUWIEDIA Blume}

NEUWIEDIA VERATRIFOLIA Blume in Hoev. \& De Vriese Tijdschr. Nat. Gesch. 1 (1834) 142, in Ann. Sci. Nat. II 2 (1834) 94; Reichb. f. in Bonpl. 5 (1857) 58, in nota; Miq. Fl. Ind. Bat. 3 (1859) 748; Naves Novis. App. (1882) 251; Rolfe in Journ. Linn. Soc. 25 (1889) 231, 241; Hook. f. in Hook. Icon. Pl. 20 (1891) t. 1987; Rolfe in Orch. Rev. 4 (1896) 329; Kränzl. Orch. Gen. et Sp. 1 (1897) 3, p. p.; Pfitz. in Engl. Pflanzenreich IV. 50 (1903) 5, fig. 3; J. J. Sm. Fl. Buitenz. 6 (Orch. Jav.) (1905) 15; Ames in Philip. Journ. Sci. 2 (1907) Bot. 311; J. J. Sm. in Bull. Jard. Buitenz. II 14 (1914) 1.

Mindoro, Merrill 5681. Mt. Halcon, in forest at $750 \mathrm{~m}$ altitude. In Java and Borneo.

\section{EXCLUDED OR SYNONYMOUS SPECIES}

Neufimda zollingeri Reichb. f. apud Naves Novis. App. (1882) 251; Rolfe in Journ. Linn. Soc. 25 (1889) 231; Pfitz. in Engl. Pflanzenreich IV. 50 (1903) 5.

This Javan and Bornean species is credited to the Philippines solely on the basis of Naves's record.

\section{APOSTASIA Blume}

APOSTASIA WALLICHII R. Br. in Wall. Pl. Asiat. Rar. 1 (1830) 75, t. 84; Miq. Fl. Ind. Bat. 3 (1859) 748; Rolfe in Journ. Linn. Soc. 25 (1889) 239; Hook. f. Fl. Brit. Ind. 6 (1890) 175; Ridl. in Journ. Linn. Soc. 32 (1896) 415; Kränzl. Orch. Gen. et Sp. 1 (1897) 7; Trim. Handb. Fl. Ceyl. 4 (1898) 238; Pfitz. in Engl. Pflanzenreich IV. 50 (1903) 7, fig. 4; J. J. Sm. Fl. Buitenz. 6 (Orch. Jav.) (1905) 18, Fig.-Atlas (1908) fig. 3, in Bull. Dept. Agr. Ind. Néerl. 13 (1907) 1; Ridl. Mat. Fl. Mal. Penin. 1 (1907) 232; Ames in Philip. Journ. Sci. 2 (1907) Bot. 311.

Mesodactylus deflexa Wall. Pl. Asiat. Rar. 1 (1830) 74.

Luzon (Camarines), Sibuyan, Mindoro, Palawan, F. B. 18750 Darling, Merrill 5521, 5639, Elmer 13141. Terrestrial, up to 1,000 $\mathrm{m}$ altitude. In India, Ceylon, Malay Peninsula, Sumatra, Java, Borneo, and New Guinea.

\section{EXCLUDED OR SYNONYMOUS SPECIES}

Apostasia odorata Blume apud Naves Novis. App. (1882) 251; Rolfe in Journ. Linn. Soc. 25 (1889) 239.

The Philippine record, repeated by Rolfe, is based wholly on the earlier one of Naves; the species is known only from Java.

* The enumeration of the Apostasiaceae and the Orchidaceae was prepared by Prof. Oakes Ames, who was materially assisted in the bibliographic research connected therewith by Mr. F. Tracy Hubbard.- E. D. M. 


\section{ORCHIDACEAE *}

\section{CORDULA Rafinesque}

CORDULA ARGUS (Reichb. f.) Rolfe in Orch. Rev. 20 (1912) 2.

Cypripedium argus Reichb. f. in Gard. Chron. (1873) 608, (1874) 710, III 7 (1890) 259; Hook. f. in Bot. Mag. 101 (1875) t. 6175; Naves Novis. App. (1882) 251; Veitch Man. Orch. Pl.pt. 4 (1889) 11; Sander Reichenbachia I 2 (1890) 77, t. 83; Ceron Cat. Pl. Herb. Manila (1892) 169; Kränzl. Orch. Gen. et Sp. 1 (1897) 56.

Cypripedium barbatum Lindl. var. argus Vos. in Belg. Hort. 25 (1875) 57.

Cypripedium pitcherianum Manda in Am. Flor. 3 (1887)' 178.

Cypripedium moensianum Hort. ex L. Linden in Lindenia 3 (1887) 69, t. 129.

Cypripedium argus Reichb. f. var. moensii Hort. ex Veitch Mar. Orch. Pl. pt. 4 (1889) 11.

Cypripedium argus Reichb. f. subvar. nigricans Veitch Man. Orch. pl, pt. 4 (1889) 11.

Paphiopedilum argus Stein Orehideenb. (1892) 453; Pfitz. in Engl. Bot. Jahrb. 19 (1894) 40, in Engl. Pflanzenreich IV. 50 (1903) 90; Ames Orch. 1 (1905) 63, 2 (1908) 23, 5 (1915) 10.

Paphiopedilum argus Stein var. moensii Stein Orchideenb. (1892) 450 ; Pfitz. in Engl. Pflanzenreich IV. 50 (1903) 91; Ames Orch. 2 (1908) 24.

Paphiopedilum argus Stein var. nigricans Hort. ex Stein Orchideenb. (1892) 454; Pfitz. in Engl. Pflanzenreich IV. 50 (1903) 91; Ames Orch. 2 (1908) 24.

Luzon (Bontoc, Benguet, Tayabas), Negros (Oriental Negros), Vanoverbergh 262, 1186, 4042, Elmer 6296, 9433, Lyon 158, B. S. 32015 Santos, 20735 Escritor. Grassy slopes at 1,300 to $2,200 \mathrm{~m}$ altitude.

CORDULA BARBATA (Lindl.) Rolfe in Orch. Rev. 20 (1912) 2.

Cypripedium barbatum Lindl. in Bot. Reg. 27 (1841) Misc. 53, 28 (1842) t. 17; Hook. in Bot. Mag. 72 (1846) t. 4234; Miq. Fl. Ind. Bat. 3 (1859) 737; Vidal Phan. Cuming. Philip. (1885) 151, Rev. Pl. Vasc. Filip. (1886) 237; Veitch Man. Orch. Pl. pt. 4 (1889) 12, text cut; Hook. f. Fl. Brit. Ind. 6 (1890) 174; Ridl. in Journ. Linn. Soc. 32 (1896) 414; Kränzl. Orch. Gen. et Sp. 1 (1897) 62; Ridl. Mat. Fl. Mal. Penin. 1 (1907) 229.

Paphiopedilum barbatum Pfitz. in Pringsh. Jahrb. 19 (1888) 159, in Engl. \& Prantl Pflanzenfam. 2, Abt. 6 (1888) 84, in Engl. Bot. Jahrb. 19 (1894) 40, in Engl. Pflanzenreich IV. 50 (1903) 91; Ames Orch. 2 (1908) 24, 5 (1915) 10.

Palawan, B. S. 640, 645 Foxworthy. Mt. Victoria, in rocky forest at 930 to $1,000 \mathrm{~m}$ altitude. In Malay Peninsula and western Siam.

\footnotetext{
* See preceding footnote.
} 
CORDULA CILIOLARIS (Reichb. f.) Rolfe in Orch. Rev. 20 (1912) 2.

Cypripedium ciliolare Reichb. $x$. in Gard. Chron. II 18 (1882) 488, 20 (1883) 46; Rodigas in Ill. Hort. 31 (1884) 127, t. 530; Veitch Man.. Orch. Pl. pt. 4 (1889) 16; Kränzl. Orch. Gen. et. Sp. 1 (1897) 64.

Paphiopedilum ciliolare Stein Orchideenb. (1892) 462, fig. 145; Pfitz. in Engl. Bot. Jahrb. 19 (1894) 40, in Engl. Pflanzenreich IV. 50 (1903) 89; Ames Orch. 2 (1908) 23, 5 (1915) 11, in Philip. Journ. Sci. 6 (1911) Bot. 39.

Luzon (Bontoc), Dinagat, Mindanao (Surigao, Agusan), B. S. 37996 Ramos \& Edaño, 34586 Ramos \& Pascasio, Lyon 142, Weber 110. Forest slopes at 310 to $1,830 \mathrm{~m}$ altitude.

CORDULA HAYNALDIANA (Reichb. f.) Rolfe in Orch. Rev. 20 (1912) 2.

Cypripedium haynaldianum Reichb. f. Xen. Orch. 2 (1874) 222, 3 (1881) 33, t. 212, in Gard. Chron. II 7 (1877) 272, 752; Hook. f. in Bot. Mag. 103 (1877) t. 6296; Naves Novis. App. (1882) 251; Veitch Man. Orch. Pl. pt. 4 (1889) 28; Kränzl. Orch. Gen. et Sp. 1 (1897) 71.

Paphiopedilum haynaldianum Stein Orchideenb. (1892) 470; Pfitz. in Engl. Bot. Jahrb. 19 (1894) 41, in Engl. Pflanzenreich IV. 50 (1903) 66, fig. 31 A-C; Ames Orch. 2 (1908) 22, 5 (1915) 10, in Philip. Journ. Sci. 6 (1911) Bot. 39; Memml. in Orchis 14 (1920) 18.

Luzon (Kalinga, Tarlac, Rizal), Wallis s. n., Lyon 49, Boettcher s. n., Ramos s. n. Sea level to $1,000 \mathrm{~m}$ altitude.

CORDULA PHILIPPINENSIS (Reichb. f.) Rolfe in Orch. Rev. 20 (1912) 2.

Cypripedium philippinense Reichb. f. in Bonpl. 10 (1862) 335; Veitch Man. Orch. Pl. pt. 4 (1889) 42, text cut; Kränzl. Orch. Gen. et Sp. 1 (1897) 46; Rolfe in Orch. Rev. 22 (1914) 253, fig. 29.

Cypripedium laevigatum Batem. in Bot. Mag. 91 (1865) t. 5508; Dombrain in Paxt. Floral Mag. 5 (1866) t. 298; Morr. in Belg. Hort. 17 (1867) 102, t. 6; Van Houtte in Fl. des Serres 17 (1869) 83, t. 1760-61; Batem. Second Cent. Orch. Pl. (1867) t. 101; Naves Novis. App. (1882) 251; Ceron Cat. Pl. Herb. Manila (1892) 169.

Cypripedium roebbelenii Reichb. f. in Gard. Chron. II 20 (1883) 684; Kränzl. Xen. Orch. 3 (1893) 113, t. 265, Orch. Gen. et Sp. 1 (1897) 47.

Selenipedium laevigatum May in Rev. Hort. 57 (1885) 301.

Cypripedium cannartianum Hort ex L. Linden in Lindenia 3 (1887) $93, t .141$.

Cypripedium roebbelenii Reichb. f. var. cannartianum L. Linden in Lindenia 3 (1887) 93, in syn.

Paphiopedilum laevigatum Pfitz. in Engl. \& Prantl Pflanzenfam. 2, Abt. 6 (1888) 84.

Cypripedium philippinense Reichb. f. var. platytaenium Desbois Bull. Orchidéene (1890), Cypriped. (1898) 285. 
Phapiopedilum philippinense Stein Orchideenb. (1892) 480; Pfitz. in Engl. Bot. Jahrb. 19 (1894) 41, in Engl. Pflanzenreich IV. 50 (1903) 61, fig. 28 A-E; Ames Orch. 2 (1908) 19, 5 (1915) 10.

Paphiopedilum roebelinii Stein Orchideenb. (1892) 480, 482, in syn.; Pfitz. in Engl. Bot. Jahrb. 19 (1894) 41 (as röbbelinii), in Engl Pflanzenreich IV. 50 (1903) 62.

Paphiopedilum philippinense Stein var. platytaenium Pfitz. in Engl. Pflanzenreich IV. 50 (1903) 61; Ames Orch. 2 (1908) 20.

Paphiopedilum philippinense Stein var. cannartianum Pfitz. in Engl. Pflanzenreich IV. 50 (1903) 62 (as cannaertianum); Ames Orch. 2 (1908) 20; Memml. in Orchis 14 (1920) 18.

Paphiopedilum philippinense Stein var. roebbelenii Kerchove apud Pfitz. in Engl. Pflanzenreich IV. 50 (1903) 62, in syn.; Ames Orch. 2 (1908) 20.

Cypripedium philippinense Reichb. f. var. roebbelenii Veitch apud Pfitz. in Engl. Pflanzenreich IV. 50 (1903) 62, in syn.

Palawan (Apulit Island), Merrill 94.23.

\section{HERMINIUM R. Brown}

HERMINIUM ANGUSTIFOLIUM (Lindl.) Benth. ex Hook. f. Fl. Brit. Ind. 6 (1890) 129; King \& Pantl. in Ann. Roy. Bot. Gard. Calc. 8 (Orch. Sik.-Himal.) (1898) 332, t. 434; Kränzl. Orch. Gen. et Sp. 1 (1901) 553; J. J. Sm. Fl. Buitenz. 6 (Orch. Jav.) (1905) 25, Fig.-Atlas (1908) fig. 7; Duthie in Ann. Roy. Bot. Gard. Calc. 9 (Orch. North-west. Himal.) (1906) 197; Ames Orch. 2 (1908) 25, 5 (1915) 12; Merr. in Philip. Journ. Sci. 13 (1918) Bot. 135. Aceras angustifolia Lindl. in Wall. Cat. (1832) No. 7061, nomen; Lindl. in Bot. Reg. 18 (1832) sub t. 1525; Lindl. Gen. \& Sp. Orch. (1835) 282; Wight Icon. $5^{1}$ (1852) 11, t. 1691.

Aceras longicruris C. Wright ex A. Gray in Mem. Am. Acad. II 6 (1858) 411.

Luzon (Bontoc, Lepanto, Benguet). Reasonably common in this region at about 2,000 to 2,300 $\mathrm{m}$ aititude. In British India, China, Japan, and Java.

\section{HABENARIA Willdenow}

HABENARIA ALAgensis Ames in Philip. Journ. Sci. 2 (1907) Bot. 312, Orch. 5 (1915) 12.

Habenaria luzonensis Ames in Elm. Leafl. Philip. Bot. 5 (1912) 1550.

Luzon (Tayabas, Sorsogon), Catanduanes, Mindoro, Leyte, Mindanao (Camiguin Island), Elmer 7254, 7636a, 15474, 15748, B. S. 28510 Ramos \& Edaño, 14440 Ramos, Merrill 5803, Wenzel 0971, 0972, 0984, Ramos s. n. Terrestrial, apparently near streams, up to $3,000 \mathrm{~m}$ in Mindoro.

habenaria Angustata (Blume) Ktze. Rev. Gen. Pl. 2 (1891) 664; Ames in Philip. Journ. Sci. 2 (1907) Bot. 312; Orch. 2 (1908) 26, in Merr. in Journ. Roy. Asiat. Soc. Straits Branch, Special No. (1921) 137.

Mecosa angustata Blume Bijdr. (1825) 404, fig. 1. 
Platanthera angustata Lindl. Gen. \& Sp. Orch. (1835) 290; Reichb. f. in Bonpl. 5 (1857) 34; Miq. Fl. Ind. Bat. 3 (1859) 714; Naves Novis. App. (1882) 251; Kränzl. Orch. Gen. et Sp. 1 (1899) 647; J. J. Sm. Fl. Buitenz. 6 (Orch. Jav.) (1905) 29, Fig.-Atlas (1908) fig. 12.

Platanthera amesiana Schltr. in Fedde Repert. 9 (1911) 212.

Habenaria amesiana Ames Orch. 5 (1915) 12.

Luzon (Benguet, Bataan, Laguna, Albay), Negros (Oriental Negros), Mindanao (Camiguin Island), B. S. 31983 Santos, 14442 Ramos, 2948 Mearns, Ramos \& Edaño s. n., Whitford 162, Elmer 10002, Merrill 7494. In Java and Borneo.

HABENARIA ARISTULIFERA Reichb. f. in Bonpl. 3 (1855) 213; Vidal Phan. Cuming. Philip. (1885) 151, Rev. Pl. Vasc. Filip. (1886) 273; Kränzl. in Engl. Bot. Jahrb. 16 (1892) 84, Orch. Gen. et Sp. 1. (1897) 231; Ames Orch. 2 (1908) 32, 5 (1915) 13.

Habenaria cumingii Kränzl. Orch. Gen. et Sp. 1 (1898) 262; Ames Orch. 5 (1915) 13.

Luzon (Rizal, Laguna), Alabat, Mindanao (Davao), Cuming 2091, Wilkes Exped., B. S. 12530, 14590, 16957 Ramos, Weber 283, Merrill 10435, Taylor 9, 10.

HABENARIA BISAETA Ames Orch. 2 (1908) 30, text cut, 5 (1915) 13.

Habenaria tipulifera Par. \& Reichb. f. apud Vidal Phan. Cuming. Philip. (1885) 151; Vidal Rev. Pl. Vasc. Filip. (1886) 273; Ames Orch. 5 (1915) 15.

Luzon (Bontoc, Benguet), Cuming 2095, Vanoverbergh 788, 1359, 1480, R. S. Williams 1936, Merrill 4325, F. B. 4887 Curran, B. S. 4461 Mearns, Ramos \& Edaño 57, 73. Terrestrial in mountain regions at about 1,300 to $2,300 \mathrm{~m}$ altitude.

HABENARIA BOADANENSIS Ames Orch. 7 (1922) 153.

Luzon (Benguet), Ramos \& Edaño 78 . Mt. Boadan, on open slopes at $2,000 \mathrm{~m}$ altitude.

HABENARIA CONGESTA Ames Orch. 2 (1908) 38, text cut, 5 (1915) 13; Merr. Fl. Manila (1912) 167.

Luzon (Lepanto), Panay (Antique), Guimaras, Busuanga, Culion, Mindanao (Zamboanga), Jolo, Merrill 4479, Yoder 26, s. n., B. S. 18008 Robinson, 41397 Lopez, 41333 Ramos, Hallier s. n., F. Link s. n. Apparently in dry open situations at low altitude.

HABENARIA COPELANDII Ames Orch. 2 (1908) 29, text cut, 5 (1915) 13.

Luzon (Bontoc, Nueva Ecija, Bataan, Rizal, Cavite, Laguna, Batangas), Panay, Culion, Coron, Vanoverbergh 1320, 3664, 4050, Copeland 1403, Merrill 4172, F. B. 3243 Ahern's collector, B. S. 4588, 22398, 41147, 41321 Ramos, 6189 Robinson, 1248 Mangubat, 26316, 26489 Ramos \& Edaño, 32444 McGregor, Native collector s. n. At about 15 to $1,250 \mathrm{~m}$ altitude.

HABENARIA CURRANII Ames in Philip. Journ. Sci. 7 (1912) Bot. 2, Orch. 5 (1915) 13.

Luzon (Cagayan, Benguet), F. B. 17138 Curran, B. S. 12578 Fenix. Lalloc, edge of forest at $50 \mathrm{~m}$ altitude, and Sablan. 
HABENARIA DELESSERTIANA Kränzl. in Ann. Conserv. Jard. Bot. Genêve 1 (1897) 108, Orch. Gen. et Sp. 1 (1897) 233; Ames in Philip. Journ. Sci. 7 (1912) Bot. 2, Orch. 5 (1915) 13.

Luzon (Laguna), Mindanao (Lanao), Cuming 2086, pro parte, B. S. 12407 McGregor, M. S. Clemens 865. Calauan and Camp Keithley.

HABENARIA DIPHYLLA (Nim.) Dalz. in Hook. Kew Journ. Bot. 2 (1850) 262; Reichb. f. in Walp. Ann. 3 (1852) 588; Hook. f. Fl. Brit. Ind. 6 (1890) 151; Kränzl. Orch. Gen. et Sp. 1 (1898) 346; Duthie in Ann. Roy. Bot. Gard. Calc. 9 (Orch. North-west. Himal.) (1906) 185, t. 137; Ames Orch. 2 (1908) 37, 5 (1915) 13.

Liparis diphyllos Nim. in Grah. Cat. Bomb. Pl. (1839) 252.

Habenaria jerdoniana Wight Icon. $5^{1}$ (1852) 14, t. 1715; Kränzl. in Engl. Bot. Jahrb. 16 (1892) 154.

Habenaria sutteri Reichb. f. in Linnaea 25 (1852) 229.

Habenaria cordata Naves Novis. App. (1882) 250; Merr. Sp. Blancoanae (1918) 21, non R. Br.

Habenaria ahernii Schltr. in Engl. Bot. Jahrb. 53 (1915) 502.

Luzon (Ilocos Norte, Rizal, Laguna), $F^{\prime}$. B. 3241 Ahern's collector, Ramos 1071, 4668, B. S. 21331, 32754 Ramos, Native collector s. n. In India.

HABENARIA ELMERI Ames in Elm. Leafl. Philip. Bot. 5 (1912) 1550, Orch. 5 (1915) 14.

Mindanao (Davao), Elmer 11845a. Todaya, Mt. Apo.

HABENARIA FALCIGERA Reichb. f. in Linnaea 41 (1876) 100; Kränzl. in Engl. Bot. Jahrb. 16 (1892) 153, Orch. Gen. et Sp. 1 (1898) 345; Ames Orch. 2 (1908) 36, 5 (1915) 14.

Luzon (Bontoc, Benguet), Wallis s. n., Vanoverbergh 1239, B. S. 8792 McGregor, 4332 Mearns, F. B. 4884 Curran.

HABENARIA GOODYEROIDES D. Don Prodr. Fl. Nepal. (1825) 25 ; Hook. f. Fl. Brit. Ind. 6 (1890) 161 (excl. syn. P. grandis Blume); King \& Pantl. in Ann. Roy. Bot. Gard. Calc. 8 (Orch. Sik.-Himal.) (1898) 326, t. 430; Schltr. in K. Schum. \& Lauterb. Nachtr. Fl. Deutsch. Schutzgeb. (1905) 79; Ames Orch. 2 (1908) 27, 5 (1915) 14, 6 (1920) 8; Schltr. in Engl. Bot. Jahrb. 45, Beibl. No. 104 (1911) 3, in Fedde Repert. Beihefte 1 (Orch. Deutsch-Neu-Guin.) (1911) 5; Merr. Fl. Manila (1912) 167; Ames in Merr. in Journ. Roy. Asiat. Soc. Straits Branch, Special No. (1921) 138.

Herminium goodyeroides Lindl. in Wall. Cat. (1832) No. 7066, nomen. Peristylus goodyeroides Lindl. Gen. \& Sp. Orch. (1835) 299, in Bot. Reg. 26 (1840) Misc. 81; Ceron Cat. Pl. Herb. Manila (1892) 169; Grant Orch. Burma (1898) 329 (excl. syn. P. grandis Blume); Kränzl. Orch. Gen. et Sp. 1 (1898) 503 (excl. syn. P. grándis Blume) ; J. J. Sm. Fl. Buitenz. 6 (Orch. Jav.) (1905) 32, Fig.Atlas (1908) fig. 14, in Nova Guinea 12 (1915) Bot. 178.

Platanthera goodyeroides Vidal Phan. Cuming. Philip. (1885) 151.

Habenaria lauterbachii Kränzl. in K. Schum. \& Lauterb. Fl. Deutsch.

Schutzgeb. (1901) 239, nomen.

Luzon (Bontoc, Union, Benguet, Bataan, Rizal, Cavite, Laguna), Panay (Antique), Mindanao (Bukidnon, Lanao). A fairly common species in $200823-17$ 
the Philippines, especially in Luzon, up to $1,300 \mathrm{~m}$ altitude in Bontoc. In India, Yunnan, Assam, Burma, Andaman Islands, Sumatra, Java, Borneo, New Guinea, and Formosa.

HABENARIA HALCONENSIS Ames in Philip. Journ. Sci. 2 (1907) Bot. 312, Orch. 5 (1915) 14.

Platanthera halconensis Schltr. in Fedde Repert. 9 (1911) 212.

Mindoro, Mervill 5835. Mt. Halcon, terrestrial on open heath at 2,650 $m$ altitude.

HABENARIA HYSTRIX Ames Orch. 2 (1908) 35, 5 (1915) 14.

Dissorhynchium muricatum Schauer in Nov. Act. Nat. Cur. 19, Suppl. 1 (1843) 434, t. 1, fig. A.

Habenaria muricata Vidal Phan. Cuming. Philip. (1885) 151, Rev. Pl. Vasc. Filip. (1886) 273; Rolfe in Journ. Bot. 24 (1886) 59; Kränzl. in Engl. Bot. Jahrb. 16 (1892) 140, Orch. Gen. et Sp. 1 (1898) 326; Ames Orch. 1 (1905) 64, non Rodr. (1882).

Luzon (Bataan, Rizal, Cavite, Laguna, Batangas, Tayabas, Sorsogon), Siquijor, Dumaran, Mindanao (Zamboanga). A rather widely distributed species in the Philippines and fairly common in Luzon at about 120 to 300 $m$ altitude. Also in British North Borneo.

HABENARIA INTRUDENS Ames Sched. Orch. 6 (1923) 1, fig. 1.

Habenaria lacertifera Ames Orch. 2 (1908) 39, 5 (1915) 14, non Benth.

Luzon (Benguet, Bulacan, Rizal), F. B. 5085 Curran, R. S. Williams 1933, B. S. 21706,21999 Ramos. At 90 to $100 \mathrm{~m}$ altitude.

HABENARIA LEIBERGII Ames Orch. 2 (1908) 34, text cut, 5 (1915) 14, in Philip. Journ. Sci. 4 (1909) Bot. 600.

Luzon (Ilocos Norte, Bontoc, Bataan, Rizal), B. S. 32866 Ramos, 5266 Topping, Vanoverbergh 620, Leiberg 6112, Reillo s. $n$. Mountain regions of Luzon.

HABENARIA LINGUlosA Ames Orch. 2 (1908) 33, text cut, 5 (1915) 14.

Luzon (Bontoc, Benguet), Vanoverbergh 900, 1525, Merrill 4845. Near streams in mountain regions up to $1,400 \mathrm{~m}$ altitude.

HABENARIA MALINTANA (Blanco) Merr. Sp. Blancoanae (1918) 112; Ames Orch. 6 (1920) 276.

Thelymitra malintana Blanco Fl. Filip. (1837) 642, ed. 2 (1845) 447, ed. 3, 3 (1879) 40; Merr. in Govt. Lab. Publ. (Philip.) 27 (1905) 85.

Habenaria pelorioides Par. \& Reichb. f. in Trans. Linn. Soc. 30 (1874) 135, 139, t. 27, fig. A, 1-3; Reichb. f. Otia Bot. Hamb. 1 (1878) 39; Hook. f. Fl. Brit. Ind. 6 (1890) 166; Kränzl. in Engl. Bot. Jahrb. 16 (1892) 85, Orch. Gen. et Sp. 1 (1897) 234; Grant Orch. Burma (1898) 340; Ames Orch. 2 (1908) 31, 5 (1915) 15. Habenaria trinervis Naves Novis. App. (1882) 250, non Wight.

Luzon (Bontoc, Benguet, Nueva Vizcaya, Pangasinan, Tarlac, Zambales, Bataan, Rizal). A common species in Luzon, in the forest, up to $1,300 \mathrm{~m}$ altitude in Bontoc. In Burma. 
HABENARIA MEARNSII Ames in Philip. Journ. Sci. 7 (1912) Bot. :, Orch. 5 (1915) 15.

Luzon (Benguet), B. S. 4313 Mearns, 8815, 8835 Mcliregor. Pauai and Mt. Pulog, at about 2,300 $\mathrm{m}$ altitude.

HABENARIA MILITARIS Reichb. f. var. PHILIPPINENSIS Ames ()rch. 2 (1908) 40, 5 (1915) 15.

Mindanao (Zamboanga, Cotabato, Davao), Copeland 1425, 1586, Merrill 8299, Weber 214, 287. Terrestrial in damp soil at 60 to $1,000 \mathrm{~m}$ altitude. The variety endemic, the species a native of Cochinchina and China.

HABENARIA PONEROSTACHYS Reichb. f. in Bonpl. 3 (1855) 213; Kränzl. in Engl. Bot. Jahrb. 16 (1892) 179, Orch. Gen. et Sp. 1 (1898) 385; Ames in Philip. Journ. Sci. 7 (1912) Bot. 4, Orch. 5 (1915) 15.

Leyte, Negros, Mindanao (Camiguin Island, Cotabato), Cuming 20.5.5, Wenzel 0636, Merrill 7022, B. S. 14415 Ramos, Weber 200. Terrestrial in forest at 230 to $1,600 \mathrm{~m}$ altitude.

HABENARIA RETICULATA Ames Orch. 7 (1922) 154.

Luzon (Benguet), Ramos \& Edaño 108. Mt. Pulogloko, on open pine slopes, at $2,000 \mathrm{~m}$ altitude.

HABENARIA ROBINSONII Ames in Philip. Journ. Sci. 7 (1912) Bot. 5 , Orch. 5 (1915) 15.

Luzon (Laguna, Batangas, Tayabas), Merrill 1095, 6306, B. S. 9660 Robinson, 25541 Yates, F. B. 29030 Sulit.

HABenaria RosulatA Ames in Philip. Journ. Sci. 7 (1912) Bot. 5, Orch. 5 (1915) 15.

Luzon (Tayabas), B. S. 13203 Foxworthy \& Ramos. Quinatacutan. HABENARIA VANOVERBERGHII Ames Orch. 5 (1915) 15.

Luzon (Bontoc), Vanoverbergh $368 \%$. In wet soil, at $1,300 \mathrm{~m}$ altitude.

HABENARIA WARBURGIANA Kränzl. Orch. Gen. et Sp. 1 (1898) 467;

Ames Orch. 5 (1915) 16.

Luzon (Ilocos Norte, Bontoc, Zambales), Warburg s. n., Vanoverbergh $567,3654,3923, B . S$. 32841 Ramos. Up to $1,300 \mathrm{~m}$ altitude in Bontoc.

HABENARIA ZEPHYRICA Ames Orch. 5 (1915) 16.

Mindanao (Lanao), M. S. Clemens s. n., Lake Lanao, Camp Keithley, terrestrial.

EXCLUDED OR SYNONYMOUS SPECIES

HABENARIA AHERNII Schltr. in Engl. Bot. Jahrb. 53 (1915) $502=$ Habenaria diphylla (Nim.) Dalz.

HABENARIA AMEsiana Ames Orch. 5 (1915) 12 = Habenaria angustata (Blume) Ktze.

Habenaria Cordata Naves Novis. App. (1882) 250; Merr. Sp. Blancoanae (1918) 21 = Habenaria diphylla (Nim.) Dalz.

Habenaria CUmingi Kränzl. Orch. Gen. et Sp. 1 (1897) 262; Ames Orch. 5 (1915) 13 = Habenaria aristulifera Reichb. f. 
Habenaria graminea Lindl. apud Naves Novis. App. (1882) 251.

Habenaria lacertifera Ames Orch. 2 (1908) 39, 5 (1915) 14, non Benth. $=$ Habenaria intrudens Ames.

Habenaria Luzonensis Ames in Elm. Leafl. Philip. Bot. 5 (1912) $1550=$ Habenaria alagensis Ames.

Habenaria muricata Vidal Phan. Cuming. Philip. (1885) 151, Rev. Pl. Vasc. Filip. (1886) 273; Rolfe in Journ. Bot. 24 (1886) 59; Kränzl. in Engl. Bot. Jahrb. 16 (1892) 140, Orch. Gen. et Sp. 1 (1898) 326; Ames Orch. 1 (1905) 64, non Rodr. (1882) = Habenaria hystrix Ames.

Habenaria peilorioldes Par. \& Reichb. f. in Trans. Linn. Soc. 30 (1874) 135, 139, t. 27, fig. A, 1-3; Kränzl. in Engl. Bot. Jahrb. 16 (1892) 85, Orch. Gen. et Sp. 1 (1897) 234; Ames Orch. 2 (1908) 31, 5 (1915) $15=$ Habenaria malintana (Blanco) Merr.

Habenaria Rumphit Lindl. apud Naves Novis. App. (1882) 250.

Habenaria thisee Vidal Phan. Cuming. Philip. (1885) $151=$ Oberonia thisbe Reichb. f.

Habenaria tipulifera Par. \& Reichb. f. apud Vidal Phan. Cuming. Philip. (1885) 151; Vidal Rev. Pl. Vasc. Filip. (1886) 273; Ames Orch. 5 (1915) $15=$ Habenaria bisaeta Ames.

Habenaria Trinervis Naves Novis. App. (1882) 250 , non Wight $=\mathrm{Ha}$ benaria malintana (Blanco) Merr.

\section{DISPERIS Swartz}

DISPERIS PHILIPPINENSIS Schltr. in Fedde Repert. 9 (1911) 436; Ames Orch. 5 (1915) 17.

Luzon (Pampanga, Batangas, Sorsogon), Merrill 4215, Curran \& Merritt s. n., Elmer 17355. Terrestrial at 300 to $700 \mathrm{~m}$ altitude.

\section{THELYMITRA Blume}

THELYMITRA JAVANICA Blume Bijdr. (1825) 419; Lindl. Gen. \& Sp. Orch. (1840) 519; Blume Fl. Jav. Orch. (1858) 116 (Orch. Arch. Ind. 137), t. 44, fig. 3, t. 48 A; Miq. Fl. Ind. Bat. 3 (1859) 737; J. J. Sm. Fl. Buitenz. 6 (Orch. Jav.) (1905) 45, Fig.-Atlas (1908) fig. 25 ; Ames Orch. 2 (1908) 41, 5 (1915) 18.

Luzon (Bontoc, Benguet), Vanoverbergh 1357, 1476, R. S. Williams 1944, Merrill 4848, F. B. 4998 Curran, B. S. 4325 Mearns, 31929 Santos, Ramos \& Edaño 61,63. On open slopes at 1,350 to $2,300 \mathrm{~m}$ altitude.

\section{EXCLUDED OR SYNONYMOUS SPECIES}

Thelymitra Malintana Blanco Fl. Filip. (1837) 642, ed. 2 (1845) 447, ed. 3, 3 (1879) 40; Merr. in Govt. Lab. Publ. (Philip.) 27 (1905) $85=$ Habenaria malintana (Blanco) Merr. 


\section{MICROTIS R. Brown}

MICROTIS UNIFOLIA (Forst. f.) Reichb. f. Beitr. Syst. Pflanzenk. (1871) 62; Kränzl. in Engl. Bot. Jahrb. 6 (1885) 55; Ames Orch. 1 (1905) 65, 2 (1908) 41, 5 (1915) 18; J. J. Sm. Orch. Jav. Fig.-Atlas (1908) fig. 26; Merr. \& Merritt in Philip. Journ. Sci. 5 (1910) Bot. 340 .

Ophrys unifolia Forst. f. Fl. Ins. Austral. Prodr. (1786) 59.

Epipactis porrifolia Sw. in Kongl. Svensk. Vet.-Akad. Nya Handl. 21 (1800) 233.

Microtis rara R. Br. Prodr. Nov. Holl. (1810) 321; Lind1. Gen. \& Sp. Orch. (1840) 396; Hook. f. Fl. Tasm. 2 (1860) 24; Reichb. f. Beitr. Syst. Pflanzenk. (1871) 22.

Microtis porrifolia R. Br. Prod. Nov: Holl. (1810) 320, by inference only; Spreng. Syst. Veg. 3 (1826) 713; Hook. f. Fl. N. Zeal. 1 (1853) 245; Benth. Fl. Austral. 6 (1873) 347; J. J. Sm. Fl. Buitenz. 6 (Orch.' Jav.) (1905) 47.

Microtis banksii A. Cunn. ex Hook. in Bot. Mag. 62 (1835) sub t. $337 \%$.

Microtis pulchella Lindl. Gen. \& Sp. Orch. (1840) 395; Hook. f. Fl. Tasm. 2 (1860) 24, t. 118, non R. Br.

Microtis arenaria Lindl. Gen. \& Sp. Orch. (1840) 396; Hook. f. Fl. Tasm. 2 (1860) 24.

Microtis frutetorum Schlechtendal in Linnaea 20 (1847) 568; Reichb. f. in Walp. Ann. 1 (1849) 807.

Microtis javanica Reichb. $f$. in Bonpl. 5 (1857) 36.

Luzon (Bontoc, Lepanto, Benguet), Vanoverbergh 743, 1356, 1479, R. S. Williams 1935, Merrill 4345, Topping 97, B. S. 31930a Santos, Ramos \& Edaño 98,F.B. 16344 Curran, Merritt, \& Zschoktie. Grassy slopes up to $1,300 \mathrm{~m}$ altitude in Bontoc. In Java and Australia.

\section{CORYBAS Salisbury}

CORYBAS MERRILLII Ames Orch. 2 (1908) 42, 5 (1915) 19; Schltr. in Fedde Repert. 19 (1923) 20.

Corysanthes merrillii Ames Orch. 1 (1905) 65, text cut.

Luzon (Bataan, Laguna), Merrill 3871, Whitford 451, Leiberg 60\%4, $B$. S. 26664 Brown, Elmer 17939. Mt. Mariveles and Mt. Maquiling, terrestrial on mossy cliffs.

\section{CRYPTOSTYLIS R. Brown}

CRYPTOSTYLIS ARACHNITES (Blume) Hassk. Cat. Hort. Bogor. (1844) 48; Reichb. f. in Bonpl. 5 (1857) 36; Blume Fl. Jav. Orch. (1858) 112 (Orch. Arch. Ind. 133), t. 45, fig. 2; Hook. f. Fl. Brit. Ind. 6 (1890) 118; Ridl. in Journ. Linn. Soc. 32 (1896) 407; Trim. Handb. Fl. Ceyl. 4 (1898) 209; J. J. Sm. Fl. Buitenz. 6 (Orch: Jav.) (1905) 59, Fig.-Atlas (1908) fig. 37; Ridl. Mat. Fl. Mal. Penin. 1 (1907) 225; Ames Philip. Journ. Sci. 2 (1907) Bot. 313, Orch. 2 (1908) 44, 5 (1915) 19, in Merr. in Journ. Roy. Asiat. Soc. Straits Branch, Special No. (1921) 139. 
Zosterostylis arachnites Blume Bijdr. (1825) 419, fig. 32; Lindl. Gen. \& Sp. Orch. (1840) 446; Miq. Fl. Ind. Bat. 3 (1859) 721; Hook. in Bot. Mag. 89 (1863) t. 5381.

Zosterostylis zeylanica Lindl. Gen. \& Sp. Orch. (1840) 446; Thw. Enum. Pl. Zeyl. (1864) 312.

Zosterostylis walkerue Wight Icon. $5^{1}$ (1852) 20, t. 1748 IV.

Cryptostylis philippinensis Schltr. in Engl. Bot. Jahrb. 58 (1922) 54.

Luzon (Apayao, Rizal, Tayabas, Sorsogon), Polillo, Mindoro, Biliran, Leyte, Negros (Occidental Negros), Mindanao (Camiguin Island, Davao). A common, variable, and widely distributed species in the Philippines. Terrestrial, in forest at 30 to $1,000 \mathrm{~m}$ altitude. In India, Ceylon, Malay Peninsula, Java, and Borneo (Sarawak).

J. J. Smith, in Bull. Jard. Bot. Buitenz. III 3 (1921) 239, expresses doubt as to $B$. S. 20462 being referable to Cryptostylis arachnites. A reexamination of all available specimens from the Philippines indicates that there is only one species present. I cannot separate this from ${ }^{\circ}$. arachnites. It is probable that Smith in studies of living material has been able to detect differences among the species of Cryptostylis allied with C. arachnites which are evanescent in herbarium specimens. Cryptostylis arachnites is a very variable species, flowers on the same raceme exhibiting a wide range of variation in the labellum.

EXCLUDED OR SYNONYMOUS SPECIES

Cryptostylis Philippinensis Schltr. in Engl. Bot. Jahrb. 58 (1922) $54=$ Cryptostylis arachnites (Blume) Hassk.

\section{GALEOLA Loureiro}

GALEOLA ALTISSIMA (Blume) Reichb. f. Xen. Orch. 2 (1865) 77; Hook. f. Fl. Brit. Ind. 6 (1890) 98; Grant Orch. Burma (1895) 349; Ridl. in Journ. Linn. Soc. 32 (1896) 395; J. J. Sm. Fl. Buitenz. 6 (Orch. Jav.) (1905) 67, Fig.-Atlas (1908) fig. 44; Ridl. Mat. Fl. Mal. Penin. 1 (1907) 202; Ames in Merr. in Journ. Roy. Asiat. Soc. Straits Branch, Special No. (1921) 139.

Cyrtosia altissima Blume Bijdr. (1825) 396, Rumphia 1 (1835) t. 70;

Lindl. Gen. \& Sp. Orch. (1830) 26.

Erythrorchis altissima Blume Rumphia 1 (1835) 200; Lindl. Gen. \& Sp. Orch. (1840) 438, p. p.; Reichb. f. in Walp. Ann. 3 (1852) 602; Miq. Fl. Ind. Bat. 3 (1859) 720.

Haematorchis altissima Blume Rumphia 4 (1848) t. $200 \mathrm{~B}$.

Luzon (Cagayan), F. B. 17812 Curran, B. S. 13985 Ramos. In Malay Peninsula, Burma, and Java.

GALEOLA KUHLII Reichb. f. Xen. Orch. 2 (1865) 78; Naves Novis. App. (1882) 247.

Vanilla pterosperma Lindl. in Wall. Cat. (1832) No. 7402, nomen.

Erythrorchis altissima Lindl. Gen. \& Sp. Orch. (1840) 438, p. p., non Blume.

Erythrorchis kuhlii Reichb. f. Xen. Orch. 2 (1862) t. 119.

Galeola hydra Reichb. f. Xen. Orch. 2 (1865) 77; Hook. f. Fl. Brit. Ind. 6 (1890) 89; Grant Orch. Burma (1895) 350; King \& Pantl. in Ann. Roy. Bot. Gard. Calc. 8 (Orch. Sik.-Himal.) (1898) 264, t. 351; J. J. Sm. F1. Buitenz. 6 (Orch. Jav.) (1905) 68, Fig.-Atlas 
(1908) fig. 45; Ames in Philip. Journ. Sci. 2 (1907) Bot. 313; Ridl. Mat. Fl. Mal. Penin. 1 (1907) 202; Ames. Orch. 2 (1908) 46.

Galeola pterosperma Schltr. in Engl. Bot. Jahrb. 45 (1910) 386; J. J. Sm. in Bull. Jard. Bot. Buitenz. II 9 (1913) 10; Ames Orch. 5 (1915) 19.

Babuyan Islands (Camiguin Island), Luzon (Rizal, Laguna, Tayabas, Sorsogon), Mindoro, Samar, Panay (Capiz), Leyte, Mindanao (Lanao, Davao). A common and widely distributed species in the Philippines at 75 to $180 \mathrm{~m}$ altitude. In Sikkim, Himalaya, Burma, Malay Peninsula, Java, and Sumatra.

The valid name of this species has been a source of difference of opinion. It has been most widely known as Galeola hydra Reichb. f., but in 1910 Schlechter proposed the combination Galeola pterosperma (Lindl.) Schltr. In doing so he overlooked the fact that the name-bringing synonym Vanilla pterosperma Lindl. is a nomen nudum and has no standing. The earliest valid name is Galeola kuhlii Reichb. f., published in Xen. Orch. 2 (1865) 78, in which Reichenbach cites, in synonymy, the plate published prior to the description [Xen. Orch. 2 (1862) t. 119]. This plate was published as Erythrorchis kuhlii and has full analytical drawings. According to the international rules adopted at Vienna in 1905 (Sect. 4, Art. 37) this plate constitutes valid publication.

GALEOLA PHILIPPINENSIS Ames Sched. Orch. 6 (1923) 5.

Luzon (Sorsogon), Elmer 16122.

\section{EXCLUDED OR SYNONYMOUS SPECIES}

Galeola HYdRA Reichb. f. Xen. Orch. 2 (1865) 77; Ames in Philip. Journ. Sci. 2 (1907) Bot. 313, Orch. 2 (1908) 46 = Galeola kuhlii Reichb. f.

Gateola PTERosPerma Schltr. in Engl. Bot. Jahrb. 45 (1910) 386; Ames Orch. 5 (1915) 19 = Galeola kuhlii Reichb. f.

\section{VANILLA Swartz}

VANILLA CALOPOGON Reichb. f. in Otia Bot. Hamb. 1 (1878) 40; Rolfe in Journ. Linn. Soc. 32 (1896) 459; Ames Orch. 5 (1915) 21, 6 (1920) 277.

Luzon (Ilocos Norte), Cuming 20\%0, Loher 630, B. S. 32928 Ramos.

VANILLA OVALIS Blanco Fl. Filip. ed. 2 (1845) 448, ed. 3,3 (1879) 42; Ames Orch. 5 (1915) 21; Merr. Sp. Blancoanae (1918) 112. Epidendrum vanilla Blanco Fl. Filip. (1837) 643, non Linn. Vanilla majaijensis Blanco Fl. Filip. ed. 2 (1845) 593, ed. 3, 3 (1879) 43.

Vanilla planifolia Ait. apud Naves Novis. App. (1882) 248.

Vanilla aromatica Sw. apud Naves Novis. App. (1882) 248.

Vanilla philippinensis Rolfe in.Journ. Linn. Soc. 32 (1896) 459; Ames Orch. 2 (1908) 47.

Luzon (Bulacan, Rizal, Laguna, Tayabas, Sorsogon), Sibuyan, Biliran, Mindanao (Dapitan, Lanao, Butuan), Cuming 2132, Merrill Sx. Blancoanae 665, Holman s. n., Serrato s. n., Elmer 9259, 12534, 16035, F. B. 4585 Mearns \& Hutchinson, 28614 Sulit, B. S. 19346 Wester, 18952 McGregor, M. S. Clemens s. $n$. 


\section{EXCLUDED OR SYNONYMOUS SPECIES}

VANilla AlBidA Blume apud Naves Novis. App. (1882) 248.

VANILla APHYLLA Blume apud Naves Novis. App. (1882) 248.

Vanilla aromatica Sw. apud Naves Novis. App. (1882) $248=$ Vamilla ovalis Blanco.

VANILla PHILIPPINENSIS Rolfe in Journ. Linn. Soc. 32 (1896) 459; Ames Orch. 2 (1908) $47=$ Vanilla ovalis Blanco.

VANilia Planifolia Ait. apud Naves Novis. App. (1882) $248=$ Vanilla ovalis Blanco.

VANILla MaJAIJEnsis Blanco Fl. Filip. ed. 2 (1845) 593, ed. 3,3 (1879) 43 = Vanilla ovalis Blanco.

Vanilla speciosa Boxall ex Vidal ex Naves Novis. App. (1882) 248, nomen.

\section{LECANORCHIS Blume}

LECANORCHIS JAVANICA Blume Mus. Bot. Lugd.-Bat. 2 (1856) 188, Fl. Jav. Orch. (1858) 150 (Orch. Arch. Ind. 178), t. 63, fig. 1, $t$. $66 \mathrm{~A}$; Miq. Fl. Ind. Bat. 3 (1859) 718; J. J. Sm. in Nova Guinea 8 (1909) Bot. 136, 12 (1913) 7, 12 ${ }^{3}$ (1915) 185, in Bull. Dept. Agr. Ind. Néerl. 43 (1910) 1; Ames Orch. 6 (1920) 277.

Lecanorchis triloba J. J. Sm. in Bull. Dept. Agr. Ind. Néerl. 19 (1908) 26, in Nova Guinea 8 (1909) Bot. 10, t. 3, fig. 9; Ames Orch. 5 (1915) 21.

I.uzon (Laguna, Sorsogon), Leyte, Mindanao (Agusan), B. S. 22961 McGregor, Elmer 16369, Wenzel 0120, 0282, 0851, Weber 71. Terrestrial, in damp forest, at 150 to $320 \mathrm{~m}$ altitude. In Java and New Guinea.

J. J. Smith, in Nova Guinea $12^{3}$ (1915) 185, suggests that Lecanorchis papuana Schltr. is the same as this species.

\section{EXCLUDED OR SYNONYMOUS SPECIES}

Lecanorchis triloba J. J. Sm. in Bull. Dept. Agr. Ind. Néerl. 19 (1908) 26; Ames Orch. 5 (1915) 21 = Lecanorchis javanica Blume.

\section{APHYLLORCHIS Blume}

APHYLLORCHIS BENGUETENSIS Ames Orch. 2 (1908) 49, text cut, 5 (1915) 22.

Luzon (Benguet, Laguna), Mindanao (Agusan, Davao), F. B. 5086 Curran, B. S. 16565 Ramos, Serrato s. n., Elmer 11848, 13841.

APHYLLORCHIS HALCONENSIS Ames Sched. Orch. 6 (1923) 7.

Mindoro, Ramos \& Edaño s. n. Terrestrial at $1,000 \mathrm{~m}$ altitude.

APHYLLORCHIS PALLIDA Blume Bijdr. (1825) fig. $7 \gamma$ (without description); Lindl. Gen. \& Sp. Orch. (1840) 536 (mention only); Blume Mus. Bot. Lugd.-Bat. 1 (1849) 30, Fl. Jav. Orch. (1858) 43 (Orch. Arch. Ind. 52), t. 13, fig. 1 and $t .17 D$; Miq. Fl. Ind. Bat. 3 (1859) 721; Hook. f. Fl. Brit. Ind. 6 (1890) 117; Ridl. in Journ. Linn. Soc. 32 (1896) 405; J. J. Sm. Fl. Buitenz. 6 (Orch. Jav.) (1905) 71, Fig.-Atlas (1908) fig. 47; Ridl. Mat. Fl. Mal. Penin. 
1 (1907) 205; Ames in Philip. Journ. Sci. 2 (1907) Bot. 313, Orch. 2 (1908) 50, 5 (1915) 22, 6 (1920) 11, in Merr. in Journ.

Roy. Asiat. Soc. Straits Branch; Special No. (1921) 139.

Mindoro, Merrill 5810, 5811. Terrestrial in humid forest, Mt. Halcoit and vicinity, at 330 to $930 \mathrm{~m}$ altitude. In Malay Peninsula, Java, and Borneo.

\section{EPIPOGUM Gmelin}

EPIPOGUM ROSEUM (D. Don) Lindl. in Journ. Linn. Soc. 1 (1857) 177; Ames Orch. 2 (1908) 48, 5 (1915) 22.

Limodorum roseum D. Don Prodr. Fl. Nepal. (February, 1825) 30. Galera nutans Blume Bijdr. (July, 1825) 416, fig. 3; Lindl. Gen. \& Sp. Orch. (1840) 500; Blume Mus. Bot. Lugd.-Bat. 2 (1856) 187, Fl. Jav. Orch. (1858) 117 (Oxch. Arch. Ind. 139) t. 52, fig. 3, t. $54 E$.

Ceratopsis rosea Lindl. Gen. \& Sp. Orch. (1840) 384.

Podanthera pallida Wight Icon. $5^{1}$ (1852) $22, t .1759$.

Galera rosea Blume Mus. Bot. Lugd.-Bat. 2 (1856) 188, Fl. Jav. Orch. (1858) 117 (Orch. Arch. Ind. 139).

Galera pallida Blume Fl. Jav. Orch. (1858) 117 (Orch. Arch. Ind. 139), in obs.

Epipogum nutans Reichb. f. in Bonpl. 5 (February, 1857) 36; Lindl. in Journ. Linn. Soc. 1 (1857) 177; Miq. Fl. Ind. Bat. 3 (1859) 718; Thw. Enum. Pl. Zeyl. (1864) 311; Benth. Fl. Austral. 6 (1873) 308; Hook. f. Fl. Brit. Ind. 6 (1890) 124; Fitzger. Austral. Orch. $2^{5}$ (1896) $t$. ; Rolfe in Dyer. Fl. Trop. Afr. 7 (1897) 188; Trim. Handb. F1. Ceyl. 4 (1898) 222; King \& Pantl. in Ann. Roy. Bot. Gard. Calc. 8 (Orch. Sik.-Himal.) (1898) 253, t. 335 ; Ames Orch. 1 (1905) 68; J. J. Sm. Fl. Buitenz. 6 (Orch. Jav.) (1905) 61, Fig.-Atlas (1908) fig. 39; Schltr. in Fedde Repert. Beihefte 1 (Orch. Deutsch-Neu-Guin.) (1911) 37; J. J. Sm. in Nova Guinea $12^{3}$ (1915) 185.

Epipogum guilfoylii F. v. Muell. Fragm. 8 (1873) 30, comb. non faciet, ex Benth. Fl. Austral. 6 (1873) 308, in syn.

Luzon (Nueva Vizcaya, Laguna), Mindoro, Leyte, Negros (Oriental Negros), Mindanao (Lanao, Davao), Merrill 231, Copeland 1241, 5623 , Gates 6317, B. S. 17915 Brown, Wenzel 0284, 0289, 0427, Elmer 946\%, $10536, M$. S. Clemens s. n., Weber 27\%, Ramos s. n. Terrestrial in forest, at 500 to $700 \mathrm{~m}$ altitude. In India, Ceylon, Java, New Guinea, Australia, and Africa.

\section{EXCLUDED OR SYNONYMOUS SPECIES}

EPIPOGUM NUTANS Reichb. f. in Bonpl. 5 (February, 1857) 36; Ames Orch. 1 (1905) $68=$ Epipogum roseum (D. Don) Lindl.

\section{NERVILIA Commerson ex Gaudichaud}

NERVILIA ARAGOANA Gaudich. in Freyc. Voy. Uranie et Physic. Bot. (1829) 422, t. 35; Schltr. in Engl. Bot. Jahrb. 45 (1911) 405 (excl. syn. Pogonia gracilis Blume), in Engl. Bot. Jahrb. 45, Beibl. 104 (1911) 3, in Fedde Repert. Beihefte 1 (Orch. Deutsch-NeuGuin.) (1911) 43; J. J. Sm. in Nová Guinea 123 (1915) 182; Ames Orch. 5 (1915) 23. 
Pogonia flabelliformis Lindl. in Wall. Cat. (1832) No. 7400, nomen, Gen. \& Sp. Orch. (1840) 415; Miq. Fl. Ind. Bat. 3 (1859) 715; Naves Novis. App. (1882) 248; Hook. f. Fl. Brit. Ind. 6 (1890) 121; Grant Orch. Burma (1895) 353; Ridl. in Journ. Linn. Soc. 32 (1896) 408; J. J. Sm. Fl. Buitenz. 6 (Orch. Jav.) (1905) 57, Fig.-Atlas (1908) fig. 36, Orch. Amb. (1905) 10; Duthie in Ann. Roy. Bot. Gard. Calc. 9 (Orch. North-west. Himal.) (1906) 158, t. 125; Ridl. Mat. Fl. Mal. Penin. 1 (1907) 203.

Pogonia carinata Wight Icon. $5^{1}$ (1852) $15, t$. 1720, non Lindl.

Luzon (Zambales, Rizal, Laguna), Mindanao (Lanao), Sandkuhl s.n., B. S. 22683 Ramos, 17918 Brown, M. S. Clemens s. n., 546. Terrestrial, in forest. In India, Burma, Malay Peninsula, Java, Sumatra, and New Guinea, and said to extend to Samoa.

Pogonia nervilia Blume is commonly referred to this species as a synonym, but J. J. Smith [in Nova Guinea 8 (1809) 10] treats it as a valid species. Further study is needed to determine its status.

Pogonia gracilis Blume is treated by J. J. Smith [Fl. Buitenz. 6 (Orch. Jav.) (1905) 58] as a distinct species, but in his notes Smith states that he regards it as a weak form of Pogonia flabelliformis Lindl. which is conspecific with Nervilia aragoana Gaudich.

NERVILIA CRISPATA (Blume) Schltr. in K. Schum. \& Lauterb. Nachtr.

Fl. Deutsch. Schutzgeb. (1905) 82, in nota, in Engl. Bot. Jahrb. 45 (1911) 402, in Fedde Repert. Beihefte 1 (Orch. Deutsch-NeuGuin.) (1911) 38; Ames Orch. 5 (1915) 23.

Pogonia crispata Blume Mus. Bot. Lugd.-Bat. 1 (1849) 32, Fl. Jav. Orch. (1858) 127 (Orch. Arch. Ind. 151), t. 52, fig. 4A-C; Miq. Fl. Ind. Bat. 3 (1859) 716; Reichb. f. Xen. Orch. 2 (1863) t. 126, V, VI, 10, (1865) 87; Naves Novis. App. (1882) 248; J. J. Sm. Fl. Buitenz. 6 (Orch. Jav.) (1905) 56, Fig.-Atlas (1908) fig. 35.

Bolborchis javanica Zoll. \& Mor. Syst. Verz. Java (1845-46) 89; Reichb. f. in Walp. Ann. 1 (1849) 775.

Coelogyne javanica Lindl. Fol. Orch. Coelog. (1854) 17.

Nervitia fimbriata Schltr. in K. Schum. \& Lauterb. Nachtr. Fl. Deutsch. Schutzgeb. (1905) 82.

Palawan, Merrill 9288. Taytay, in forests on dry slopes. In Java and German New Guinea.

Pogonia prainiana King \& Pantl. has been referred to this species, which would extend the range to India, if this reduction is correct.

NERVILIA DILATATA (Blume) Schltr. in Engl. Bot. Jahrb. 45 (1911) 402; Ames Orch. 5 (1915) 23, in Merr. in Journ. Roy. Asiat. Soc. Straits Branch, Special No. (1921) 140.

Pogonia dilatata Blume F1. Jav. Orch. (1858) 127 (Orch. Arch. Ind. 151), t. 10, fig. 4, 1 and 2; Ames Orch. 1 (1905) 66.

Luzon (Benguet), Bancalan, Elmer 6259, Merrill 7741, Weber 1574. Grassy hillsides at about $1,600 \mathrm{~m}$ altitude. In Borneo.

Although the determination has been carefully made, there is room for uncertainty as to the identity of the Philippine material with the Bornean species. In twenty years but three incomplete specimens have been obtained. 
NERVILIA DISCOLOR (Blume) Schltr. in Engl. Bot. Jahrb. 45 (1911) 403; Ames Orch. 5 (1915) 23.

Cordyla discolor Blume Bijdr. (1825) 417.

Rophostemon discolor Blume Fl. Jav. Praef. (1828) VI; Lindl. Gen. \& Sp. Orch. (1840) 453.

Pogonia discolor Blume Mus. Bot. Lugd.-Bat. 1 (1849) 32, Fl. Jav. Orch. (1858) 128 (Orch. Arch. Ind. 152.), t. 5\%, fig. 1; Miq. Fl. Ind. Bat. 3 (1859) 716; Hook. f. in Bot. Mag. 100 (1874) t. 6125; Naves Novis. App. (1882) 248.

Pogonia pudica Ames Orch. 2 (1908) 44, text cut.

Pogonia plicata Ames Orch. 2 (1908) 43, non Lindl.

Luzon (Bontoc, Laguna, Batangas), Panay (Antique), Mindanao (Davao), Vanoverbergh 459, 1201, 1460, Elmer s. n., 8180, 9346, B. S. 17917 Brown, F. B. 21501 Tamesis, 21331 Foxworthy \& Catalan, Copeland 427, R. S. Williams 3088, McLean s. n., McGregor 5748. Grassy hillsides, at 350 to $1,240 \mathrm{~m}$ aititude. In Java.

\section{STEREOSANDRA Blume}

STEREOSANDRA JAVANICA Blume Mus. Bot. Lugd.-Bat. 2 (1856) 176, Fl. Jav. Orch. (1858) 27 (Orch. Arch. Ind. 31), t. 10, fig. 3, $t$. 11G; Miq. Fl. Ind. Bat. 3 (1859) 717; J. J. Sm. Fl. Buitenz. 6 (Orch. Jav.) (1905) 63, Fig.-Atlas (1908) fig. 41; Ames Orch. 1 (1905) 67, 2 (1908) 46, 5 (1915) 24; Schltr. in Engl. Bot. Jahrb. 45 (1911) 406.

Luzon (Benguet, Bataan, Rizal, Sorsogon), Mindanao (Zamboanga, Agusan), Elmer 6458, 17302, Merrill s. n., B. S. 12124 Ramos, 38349 Brown. Weber 300. In Java.

\section{DIDYMOPLEXIS Griffith}

DIDYMOPLEXIS PALLENS Griff. in Calc. Journ. Nat. Hist. 4 (1844) 383, t. 17; Kurz in Journ. Bot. 4 (1866) 40 (excl. syns. Epiphanes and Gastrodia); Hemsl. in Journ. Linn. Soc. 20 (1884) 308; Hook. f. Fl. Brit. Ind. 6 (1890) 122; Ridl. in Journ. Linn. Soc. 32 (1896) 408; King \& Pantl. in Ann. Roy. Bot. Gard. Calc. 8 (Orch. Sik.-Himal.) (1898) 260, t. 346; J. J. Sm. in Icon. Bogor. 2 (1903) 5, t. 101B, Fl. Buitenz. 6 (Orch. Jav.) (1905) 77, Fig.Atlas (1908) t. 51; Ridl. Mat. Fl. Mal. Penin. 1 (1907) 206; Ames Orch. 2 (1908) 51, 5 (1915) 24; Schltr. in Engl. Bot. Jahrb. 45 (1911) 408; Merr. Fl. Manila (1912) 165.

? Leucorchis sylvatica Blume Mus. Bot. Lugd.-Bat. 1 (1849) 31, Fl. Jav. Orch. (1858) 124 (Orch. Arch. Ind. 147) text cut; Miq. Fl. Ind. Bat. 3 (1859) 722.

? Arethusa ecristata Griff. Notul. 3 (1851) 378, Icon. Pl. Asiat. 3 (1851) $343,344$.

? A petalon minutum Wight Icon. $5^{1}$ (1852) 22, t. 1758.

Epiphanes pallens Reichb. f. in Seem. Fl. Vit. (1868) 266, in nota. ? Arethusa bengalensis Hort. Bot. Calc. ex Hemsl. in Journ. Linn.

Soc. 20 (1884) 311, in syn.

Luzon (Pampanga, Rizal, Laguna), Bolster 27, Loher 6002, F. B. 21333 Foxworthy \& Catalan. Saprophyte, near brooks. In India, Malay Peninsula, and Java. 
DIDYMOPLEXIS PHILIPPINENSIS Ames Orch. 5 (1915) 24.

Luzon (Camarines), Palawan, Leyte, B. S. 22083 Ramos, Merrill 9409, Wenzel 0362. In forest at 6 to $300 \mathrm{~m}$ altitude.

\section{GASTRODIA R. Brown}

GASTRODIA JAVANICA (Blume) Lindl. Gen. \& Sp. Orch: (1840) 384; Blume Mus. Bot. Lugd.-Bat. 2 (1856) 175, Fl. Jav. Orch. (1858) 121 (Orch. Arch. Ind. 145) t. 52, fig. 1; Miq. Fl. Ind. Bat. 3 (1859) 717; Thw. Enum. Pl. Zeyl. (1864) 311; Naves Novis. App. (1882) 247; Hook. f. Fl. Brit. Ind. 6 (1890) 123; Trim. Handb. Fl. Ceyl. 4 (1898) 221; J. J. Sm. Fl. Buitenz. 6 (Orch. Jav.) (1905) 75, Fig.-Atlas (1908) fig. 50; Schltr. in Engl. Bot. Jahrb. 45 (1911) 410; Ames in Philip. Journ. Sci. 7 (1912) Bot. 6, Orch. 5 (1915) 25.

Epiphanes javanica Blume Bijdr. (1825) 421, fig. 4.

Luzon (Pampanga, Tayabas), Mindoro, Palawan, Leyte, Mindanao (Davao), Merrill 4211, 7233, Elmer 7276, Weber 274, B. S. 28718 Ramos \& Edaño, Ramos s. $n$. Terrestrial, apparently more general in mountainous regions. In Ceylon (?) and Java.

18. SPIRANTHES L. C. Richard

SPIRANTHES SINENSIS (Pers.) Ames Orch. 2 (1908) 53, 5 (1915) 26, 6 (1920) 11; Schltr. in Fedde Repert. Beihefte 4 (Orch. Sino-Jap. Prodr.) (1919) 160, in Beihefte Bot. Centralbl. 37, Abt. 2 (1920) 357; Ames in Merr. in Journ. Roy. Asiat. Soc. Straits Branch, Special No. (1921) 140.

Ophrys spiralis Georgi Bemerk. Reise Russ. Reich. 1 (1775) 232, non Linn.

Aristotelea spiralis Lour. Fl. Cochinch. 2 (1790) 522, non Spiranthes spiralis Koch.

Epidendrum aristotelia Raeusch. Nomencl. Bot. ed. 3 (1797) 265, nomen.

Neottia spiralis $\delta$ Willd. Sp. Pl. 4 (1805) 74.

Neottia sinensis Pers. Syn. Pl. 2 (1807) 511; Poir. in Lam. Encyc. Suppl. 4 (1816) 85.

Neottia australis R. Br. Prodr. Nov. Holl. (1810) 319; Poir. in Lam. Encyc. Suppl. 4 (1816) 86; D. Don Prodr. Fl. Nepal (1825) 27.

Neottia flexuosa Sm. in Rees. Cycl. 24 (1819) No. 9.

Neottia parviflora Sm. in Rees Cycl. 24 (1819) No. 10, non Blume.

Neottia amoena Bieb. Fl. Taur. Cauc. 3 (1819) 606.

Spiranthes pudica Lindl. Collect. Bot. (1821) t. 30, in Bot. Reg. 10 (1824) sub t. 823; Spreng. Syst. Veg. 3 (1826) 708, p. p.

Neottia australis R. Br. $\beta$ chinensis Lindl. in Bot. Reg. 7 (1822) t. 602 .

Spiranthes australis Lindl. in Bot. Reg. 10 (1824) sub t. 823; Spreng. Syst. Veg. 3 (1826) 708; Lindl. Gen. \& Sp. Orch. (1840) 464 (excl. syns. Neottia strateumatica R. Br., Spiranthes strateumatica Lindl., and Orchis strateumatica Linn.); A. Rich. in Ann. Sci. Nat. 
II 15 (1841) 78, Monogr. Orch. Nil.-Gher. (1841) 10, 30; Reichb. f. Icon. Fl. Germ. 13-14 (Orch. Eur.) (1851) 152, t. 124 (476); Wight Icon. $5^{1}$ (1851) 15, t. 1724; Hook. f. Fl. Tasm. 2 (1855) 15; Lindl. in Journ. Linn. Soc. 1 (1857) 178; Miq. Fl. Ind. Bat. 3 (1859) 722; Benth. Fl. Hongk. (1861) 360; Miq. Sumatra (1862) 275; Thw. Enum. Pl. Zeyl. (1864) 312; Hook. f. Handb. N. Zeal. Fl. (1864) 272; Miq. Prol. Fl. Jap. (1866-67) 141; Benth. Fl. Austral. 6 (1873) 314; Franch. \& Sav. Enum. Pl. Jap. 2 (1879) 37; Fitzger. Austral. Orch. $1^{2}$ (1880) t. 1; Naves Novis. App. (1882) 249; Kränzl. in Engl. Bot. Jahrb. 6 (1885) 55; Ridl. in Journ. Linn. Soc. 24 (1888) 392; Hook. f. Fl. Brit. Ind. 6 (1890) 102; Kränzl. in Notizbl. Bot. Gart. Berl. 2 (1898) 105; King \& Pantl. in Ann. Roy. Bot. Gard. Calc. 8 (Orch. Sik.-Himal.) (1898) 278, t. 369; Trim. Handb. Ceyl. Fl. 4 (1898) 217; Palibin Consp. Fl. Korea pt. 3 (1901) 4 [in Act. Hort. Petrop. 19 (1901) 104]; Kränzl. in K. Schum. \& Lauterb. Fl. Deutsch. Schutzgeb. (1901) 240; Ames Orch. 1 (1905) 69; J. J. Sm. Fl. Buitenz. 6 (Orch. Jav.) (1905) 81, Fig.-Atlas (1908) fig. 55; Duthie in Ann. Roy. Bot. Gard. Calc. 9 (Orch. North-west. Himal.) (1906) 163; Nakai in Journ. Coll. Sci. Imp. Univ. Tokyo 31 (Fl. Korea 2) (1911) 225.

Spiranthes parviflora Lindl. in Bot. Reg. 10 (1824) sub t. 823.

Spiranthes flexuosa Lindl. in Bot. Reg. 10 (1824) sub t. 823; Spreng. Syst. Veg. 3 (1826) 708; Schltr. in Beihefte Bot. Centralbl. 37, Abt. 2 (1920) 352.

Neottia crispata Blume Bijdr. (1825) 406.

Spiranthes amoena Spreng. Syst. Veg. 3 (1826) 708 (excl. syn. Spiranthes congesta Lindl.); Lindl. in Wall. Cat. (1832) No. 7377; Bunge Enum. Pl. Chín. (1831) 63; Ledeb. Fl. Alt. 4 (1833) 173; Schltr. in Fedde Repert. Beihefte 4 (Orch. Sino-Jap. Prodr:) (1919) 160, in Beihefte Bot. Centralbl. 37, Abt. 2 (1920) 349.

Neottia pudica Sweet Hort. Brit. ed. 2 (1830) 485.

Spiranthes wightiana Lindl. in Wall. Cat. (1832) No. 7378, nomen; Schltr. in Beihefte Bot. Centralbl. 37, Abt. 2 (1920) 359.

Calanthe australis Ait. ex Loud. Hort. Brit. Suppl. 2 (1839) 615, nomen.

Spiranthes longispicata A. Rich. in Ann. Sci. Nat. II 15 (1841) 78, Monogr. Orch. Nil.-Gher. (1841) 10, 30.

Spiranthes densa A. Rich. in Ann. Sci. Nat. II 15 (1841) 78, Monogr. Orch. Nil.-Gher. (1841) 10, 31.

Spiranthes indica Steud. Nomencl. Bot. ed. 2, 2 (1841) 625.

Spiranthes crispata Zoll. \& Mor. Syst. Verz. Java (1845-46) 89; Schltr. in Beihefte Bot. Centralb. 37, Abt. 2 (1920) 352.

Spiranthes Griff. Notul. 3 (1851) 384, Icon. Pl. Asiat. 3 (1851) t. 348.

Spiranthes novaezelandiae Hook. f. Fl. N. Zeal. 1 (1853) 243; Schltr. in Beihefte Bot. Centralbl. 37, Abt. 2 (1920) 355; Ames Orch. 7 (1922) 128, in obs.

Spiranthes australis Lindl. var. pudica Lindl. in Hook. Kew Journ. Bot. 7 (1855) 38. 
Spiranthes stylites Lindl. in Journ. Linn. Soc. 1 (1857) 178; Schltr. in Fedde Repert. Beihefte 4 (Orch. Sino-Jap. Prodr.) (1919) 160, in Beihefte Bot. Centralbl. 37, Abt. 2 (1920) 358.

Gyrostachys australis Blume Fl. Jav. Orch. (1858) 108 (Orch. Arch. Ind. 129).

Gyrostachys australis Blume var. amoena Blume Fl. Jav. Orch. (1858) 108 (Orch. Arch. Ind. 129).

Gyrostachys australis Blume var. wightiana Blume Fl. Jav. Orch. (1858) 108 (Orch. Arch. Ind. 129).

Gyrostachys australis Blume var. flexuosa Blume Fl. Jav. Orch. (1858) 109 (Orch. Arch. Ind. 130) t. 38, fig. 2.

Gyrostachys australis Blume var. parviflora Blume Fl. Jav. Orch. (1858) 109 (Orch. Arch. Ind. 130).

Gyrostachys australis Blume var, crispata Blume Fl. Jav. Orch. (1858) 109 (Orch. Arch. Ind. 130) t. 37, fig. 3.

Gyrostachys amoena Blume Fl. Jav. Orch. (1858) 108 (Orch. Arch. Ind. 129), text cut only.

Spiranthes australis Lindl. var. wightiana Lindl. ex Hook. f. Fl. Brit. Ind. 6 (1890) 102.

Gyrostachys stylites Ktze.' Rev. Gen. Pl. 2 (1891) 664.

Spiranthes chinensis Ames apud Schltr. in Notes Roy. Bot. Gard. Edinb. 24 (1912) 105.

Spiranthes australis Lindl. var. suishanensis Hayata Icon. Fl. Formosa 6 (1916) 86.

Spiranthes suishanensis Schltr. in Fedde Repert. Beihefte 4 (Orch. Sino-Jap. Prodr.) (1919) 161.

Gyrostachys crispata Blume apud Schltr. in Beihefte Bot. Centralbl. 37, Abt. 2 (1920) 352, in syn.

Gyrostachys flexuosa Blume apud Schltr. in Beihefte Bot. Centralbl. 37, Abt. 2 (1920) 352, in syn.

Gyrostachys parviflora Blume apud Schltr. in Beihefte Bot. Centralbl. 37, Abt. 2 (1920) 352, in syn.

Luzon (Bontoc, Benguet), Mindanao (Agusan), Vanoverbergh 1269, 3668, 3920, Elmer 6623, R. S. Williams 1923, Merrill 4344, F. B. 4951 Curran, Weber 80. Terrestrial, at 360 to $1,500 \mathrm{~m}$ altitude. In India, Afghanistan, Ceylon, China, Formosa, Japan, Manchuria, Siberia, Java, Sumatra, Borneo, Australia, Tasmania, and New Zealand.

Spiranthes novaezelandiae Hook. $f$. does not appear to be a distinct species, but is probably a good geographic variety of Spiranthes sinensis differing in the structure of the column.

\section{EXCLUDED OR SYNONYMOUS SPECIES}

Sptranthes AUstralis Lindl. in Bot. Reg. 10 (1824) sub t. 823; Naves Novis. App. (1882) 249; Ames Orch. 1 (1905) 69, Spiranthes sinensis (Pers.) Ames.

Spiranthes Pusilla Miq. apud Naves Novis. App. (1882) 249.

Possibly a form of Spiranthes sinensis (Pers.) Ames. 
19. EPIPACTIS (Haller) Boehmer, non Zinn *

EPIPACTES CLAUSA A. A. Eaton ex Ames Orch. 3 (1908) 41, t. ;8; Ames in Philip. Journ. Sci. 4 (1909) Bot. 663.

Goodyera clausa Schltr. in Engl. Bot. Jahrb. 45 (1911) 392; Ames Orch. 5 (1915) 26.

Luzon (Lepanto, Benguet, Tayabas), Negros (Occidental Negros), Merrill 4591, 7759, Elmer 8052, Banks s. n.

EPIPACTIS ELMERI Ames Sched. Orch. 6 (1923) 12, fig. 3.

Luzon (Sorsogon), Elmer 14884.

EPIPACTIS LUZONENSIS Ames comb. nov.

Goodyera luzonensis Ames Orch. 5 (1915) 26.

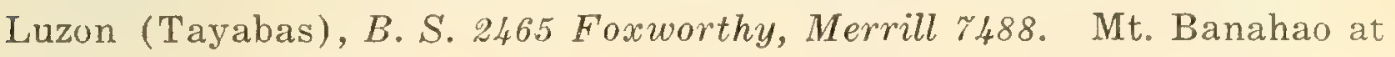
about $2,000 \mathrm{~m}$ altitude.

EPIPACTIS PHILIPPINENSIS Ames Orch. 2 (1908) 60, text cut.

Goodyera philippinensis Schltr. in Engl. Bot. Jahrb. 45 (1911) 392;

Ames Orch. 5 (1915) 27.

Luzon (Bontoc, Benguet, Zambales, Bataan, Cavite, Laguna), Mindoro. Reasonably common in Luzon at 100 to $1,100 \mathrm{~m}$ altitude.

EPIPACTIS RAMOSII Ames Sched. Orch. 6. (1923) 14.

Goodyera ramosii Ames in Philip. Journ. Sci. 8 (1913) Bot. 409, Orch. 5 (1915) 27.

Mindanao, (Camiguin Island), B. S. 14421, 14446 Ramos. On a dead tree, in mossy forest.

EPIPACTIS RUBICUNDA (Blume) A. A. Eaton in Proc. Biol. Soc. Wash. 21 (1908) 65; Ames in Philip. Journ. Sci. 4 (1909) Bot. 664.

Neottia rubicunda Blume Bijdr. (1825) 408.

Neottia grandis Blume Bijdr. (1825) 407; Lindl. Gen. \& Sp. Orch. (1840) 494.

Goodyera rubicunda Lindl. in Bot. Reg. 25 (1839) Misc. 61, Gen. \& Sp. Orch. (1840) 493, in Journ. Linn. Soc. 1 (1857) 184; Miq. Fl. Ind. Bat. 3 (1859) 728; Naves Novis. App. (1882) 248; J. J. Sm. Fl. Buitenz. 6 (Orch. Jav.) (1905) 121, Fig.-Atlas (1909) fig. 88; Ames Orch. 5 (1915) 27.

Speiranthes grandis Hassk. Cat. Pl. Bogor. (1844) 47.

Goodyera rubens Blume Fl. Jav. Orch. (1858) 36 (Orch. Arch. Ind.

43) t. 9c, figs. 17-26, t. 9d, fig. 1; Hook. f. Fl. Brit. Ind. 6 (1890)

111; Ridl. in Journ. Linn. Soc. 32 (1896) 403, Mat. Fl. Mal. Penin. 1 (1907) 219.

Goodyera grandis Blume Fl. Jav. Orch. (1858) 36 (Orch. Arch. Ind. 43); Miq. Fl. Ind. Bat. 3 (1859) 728; Naves Novis. App. (1882) 248.

* Zinn's Epipactis as to apparent source of name is this, but as to majority of species given is Serapias Linn. 
Goodyera celebica Blume Fl. Jav. Orch. (1858) 36 (Orch. Arch. Ind. 43); Miq. Fl. Ind. Bat. 3 (1859) 728.

Orchiodes rubicundum Ktze. Rev. Gen. Pl. 2 (1891) 675.

Orchiodes celebicum Ktze. Rev. Gen. Pl. 2 (1891) 675.

Orchiodes grande Ktze. Rev. Gen. Pl. 2 (1891) 675.

Luzon (Laguna, Sorsogon), Panay (Capiz), Negros (Oriental Negros), Mindanao (Lanao, Cotabato, Davao), Elmer 10173, 11150, 14867, B. S. 31096, 31455 Ramos \& Edaño, 6049 Robinson, Weber 201, Cuming s. n., M. S. Clemens s. n. Terrestrial in damp forest at 110 to $260 \mathrm{~m}$ altitude. In Malay Peninsula and Java.

EPIPACTIS VIRIDIFLORA (Blume) Ames Orch. 2 (1908) 61.

Neottia viridiflora Blume Bijdr. (1825) 408; Lindl. Gen. \& Sp. Orch. (1840) 494.

Physurus viridiflorus Lindl. in Journ. Linn. Soc. 1 (1857) 180; Miq. F1. Ind. Bat. 3 (1859) 734.

Goodyera viridiflora Blume Fl. Jav. Orch. (1858) 34 (Orch. Arch: Ind. 41), t. 9c, fig. 2, 12-16; Ames Orch. 5 (1915) 27.

Orchiodes viridiflorum Ktze. Rev. Gen. Pl. 2 (1891) 675.

Luzon (Benguet, Bataan), Negros (Oriental Negros), Mindanao (Davao), R. S. Williams 1085, B. S. 6191 Robinson, F. B. 13657 Curran, Elmer 9820, Copeland s. $n$. Epiphyte, up to $2,000 \mathrm{~m}$ altitude in Davao. In Java.

20. LEPIDOGYNE Blume

LEPIDOGYNE LONGIFOLIA Blume Fl. Jav. Orch. (1858) 78 (Oreh. Areh. Ind. 94) t. 25; Miq. Fl. Ind. Bat. 3 (1859) 745; Naves Novis. App. (1882) 249; Ridl. in Journ. Linn. Soc. 32 (1896) 405; J. J. Sm. Fl. Buitenz. 6 (Orch. Jav.) (1905) 129, Fig.-Atlas (1909) fig. 95; Ridl. Mat. Fl. Mal. Penin. 1 (1907) 224; J. J. Sm. in Nova Guinea 8 (1909) 18, t. 6, fig. 17, $8^{3}$ (1912) 526, 12 (1913) 10; Ames in Elm. Leafl. Philip. Bot. 5 (1912) 1553, Orch. 5 (1915) 28.

Neottia longifolia Blume Bijdr. (1825) 406; Lindl. Gen. \& Sp. Orch. (1840) 476.

Speiranthes longifolia Hassk. Cat. Pl. Bogor. (1844) 47.

Lepidogyne sceptrum Schltr. in Fedde Repert. Beihefte 1 (Orch. Deutsch-Neu-Guin.) 55.

Negros (Oriental Negros), Leyte, Elmer 9579, Wenzel 0399, 0789, 0850, 0904. Terrestrial in mountain regions, at 500 to $600 \mathrm{~m}$ altitude in Leyte. In Malay Peninsula, Java, and New Guinea.

Lepidogyne minor Schltr. in Fedde Repert. Beihefte 1 (Orch. DeutschNeu-Guin.). (1922) 56 is probably conspecific.

\section{DICEROSTYLIS Blume}

DICEROSTYLIS LANCEOLATA Blume Fl. Jav. Orch. (1858) 98 (Orch. Arch. Ind. 116) t. 38, fig. 1, 1-11; J. J. Sm. Fl. Buitenz. 6 (Orch. Jav.) (1905) 117, Fig.-Atlas (1908) fig. 85; Ames Orch. 5 (1915) 28. Hylophila lanceolata Miq. Fl. Ind. Bat. 3 (1859) 746; Hook. f. Fl. Brit. Ind. 6 (1890) 110; Ridl. in Journ. Linn. Soc. 32 (1896) 403,

Mat. Fl. Mal. Penin. 1 (1907) 223.

Batan Islands, B. S. 3781 Fenix. Santo Domingo de Basco, near Mt. Iraya, in mossy forest. In Malay Peninsula and Java. 
DICEROSTYLIS RUBRA (Ames) Schltr. in Engl. Bot. Jahrb. 45 (1911) 392; Ames Orch. 5 (1915) 28.

Hylophila rubra Ames in Philip. Journ. Sci. 2 (1907) Bot. 315, nomen, Orch. 2 (1908) 65, text cut.

Luzon (Laguna), Mindoro, Panay (Antique), Sibuyan, F. B. 4378 Merritt, Elmer 12517, Baker 3573, B. S. 32201 McGregor. Mt. Halcon and Mt. Giting-giting, terrestrial, up to $1,450 \mathrm{~m}$ altitude.

\section{ERYTHRODES Blume}

ERYTHRODES BOETTCHERI Ames Sched. Orch. 6 (1923) 8.

Luzon (Kalinga), Boettcher s. $n$. At 1,000 m altitude.

ERYTHRODES MERRILLII Ames Orch. 3 (1908) 79, t. 54,5 (1915) 29) Herpysma merrillii Ames in Philip. Journ. Sci. 2 (1907) Bot. 313.

Mindoro, Merrill 5836. Mt. Halcon, in damp ravine at $730 \mathrm{~m}$ altitude.

ERYTHRODES VRYDAGZYNOIDES Ames Orch. 5 (1915) 29.

Leyte, Wenzel 0150, 0225. Dagami, in forest at $60 \mathrm{~m}$ altitude.

ERYTHRODES WEBERI Ames Orch. 5 (1915) 30.

Mindanao (Lanao, Agusan), Jolo, M. S. Clemens s. n., Weber 156, 156 A, $J$. Clemens s. n. Lake Lanao and Talacogon, terrestrial in shady forest at $10 \mathrm{~m}$ altitude. In Jolo at $830 \mathrm{~m}$ altitude.

ERYTHRODES WENZELII Ames Orch. 5 (1915) 31.

Palawan, Leyte, Merrill 7218, Wenzel s. $n$.

23. KUHLHASSELTIA J. J. Smith

KUHLHASSELTIA WHITEHEADII (Rendle) Ames Orch. 5 (1915) 32.

Zeuxine whiteheadii Rendle in Journ. Bot. 34 (1896) 358.

Haemaria merrillii Ames in Philip. Journ. Sci. 2 (1907) Bot. 315; Orch. 3 (1908) 21, t. 30.

Kuhlhasseltia merrillii Schltr. in Fedde Repert. 9 (1911) 437.

Luzon (Laguna), Mindoro, B. S. 6567 Robinson, 19577 Ramos, Copeland s. n., Merrill 5819, 5840. Mt. Banahao and Mt. Halcon, terrestrial in mossy forest at 1,400 to $2,000 \mathrm{~m}$ altitude.

EXCLUDED OR SYNONYMOUS SPECIES

KuHlHAsseltiA MeRRILiI Schltr. in Fedde Repert. 9 (1911) $437=$ Kuhlhasseltia whiteheadii (Rendle) Ames.

\section{CYSTORCHIS Blume}

CYSTORCHIS APHYLLA Ridl. in Journ. Linn. Soc. 32 (1896) 400; J. J. Sm. Fl. Buitenz. 6 (Orch. Jav.) (1905) 93, Fig.-Atlas (1908) fig. 64; Ridl. Mat. Fl. Mal. Penin. 1 (1907) 212; Ames in Philip.

Journ. Sci. 2 (1907) Bot. 313, Orch. 2 (1908) 56, 5 (1915) 33.

Luzon (Benguet, Pampanga, Bataan), Merrill s. n., 3135, R. S. Williams 1921, Elmer 7012, F. B. 7496 Curran. Terrestrial, in forest. In Malay Peninsula and Java. 
CYSTORCHIS JAVANICA Blume Fl. Jav. Orch. (1858) 73 (Orch. Arch. Ind. 87) $t$. 24, fig. 1, 1-16; Hook. f. Fl. Brit. Ind. 6 (1890) 97; Ames in Philip. Journ. Sci. 6 (1911) Bot. 39, Orch. 5 (1915) 33. Etaeria javanica Blume Bijdr. (1825) 410.

Aetheria javanica Blume apud Lindl. Gen. \& Sp. Orch. (1840) 491. Hetaeria javanica Miq. Fl. Ind. Bat. 3 (1859) 725.

Cystorchis variegata Blume var. purpurea Ridl. in Journ. Linn. Soc. 32 (1896) 399; J. J. Sm. Fl. Buitenz. 6 (Orch. Jav.) (1905) 93, Fig.Atlas (1908) fig. 63; Ridl. Mat. Fl. Mal. Penin. 1 (1907) 212; J. J. Sm. in Nova Guinea 8 (1909) 14; Schltr. in Engl. Bot. Jahrb. 45, Beibl. 104 (1911) 3; Ames in Merr. in Journ. Roy. Asiat. Soc. Straits Branch, Special No. (1921) 140.

Mindanao (Davao), Weber 286. Magum-Compostela Trail, in dense forest at $45 \mathrm{~m}$ altitude. In Malay Peninsula, Sumatra, Java, Borneo, and Dutch New Guinea.

CYSTORCHIS LUZONENSIS Ames Orch. 5 (1915) 33.

Luzon (Laguna), Mindoro, Merrill 5146, B. S. 17910 Brown, Quisumbing, 1221, Ramos \& Edaño s. n. Mt. Maquiling and Mt. Halcon, terrèstrial in forest at 800 to $1,600 \mathrm{~m}$ altitude.

\section{CYSTOPUS Blume}

CYSTOPUS MINDANAENSIS Ames Orch. 5 (1915) 34.

Mindanao (Camiguin Island), B. S. 14422 Ramos. Mt. Mahinog, on tree top.

CYSTOPUS PHILIPPINENSIS Ames Orch. 5 (1915) 35.

Luzon (Tayabas, Sorsogon), B. S. 9446 Robinson, 23636 Ramos. Infantas and Lake Pulog, the latter in moss at $500 \mathrm{~m}$ altitude.

\section{ANOECTOCHILUS Blume}

ANOECTOCHILUS sp. Ames Orch. 5 (1915) 36.

Leyte, Wenzel 0286.

\section{EXCLUDED OR SYNONYMOUS SPECIES}

Anoectochilus ARgYroneurum Boxall ex Naves Novis. App. (1882) 250 , nomen.

Anoectochilus bullenji Boxall ex Naves Novis. App. (1882) 250, nomen. Anoectochilus dayi Boxall ex Naves Novis. App. (1882) 250, nomen.

Anoectochilus Intermedium Boxall ex Naves Novis. App. (1882) 250 , nomen.

Anoectochilus ReINWARDTi Blume apud Naves Novis. App. (1882) 250.

A Javan, Sumatran, and Malayan species misdetermined by Naves.

Anoectochilus roxburghil Lindl, apud Naves Novis. App. (1882) 250.

A native of India and China, wrongly determined by Naves. 


\section{MACODES Lindley}

MACODES PETOLA (Blume) Lindl. Gen. \& Sp. Orch. (1840) 497; Reichb. f. Xen. Orch. 1 (1858) 227, t. 96, fig. I, 1-5; Blume Fl. Jav. Orch. (1858) 100 (Orch. Arch. Ind. 119) t. 31, fig. 2, t. 36D; Naves Novis. App. (1882) 249; Ridl. in Journ. Linn. Soc. 32 (1896) 399; J. J. Sm. Fl. Buitenz. 6 (Orch. Jav.) (1905) 103, Fig.Atlas (1908) fig. 73; Ridl. Mat. Fl. Mal. Penin. 1 (1907) 215; Ames Orch. 5 (1915) 36.

Neottia petola Blume Bijdr. (1825) 407, fig. 2.

Luzon (Laguna, Tayabas), Mindanao (Agusan), B. S. 13905 Ramos, 94 fit Robinson, Elmer 14180, 18108, 18436. Mt. Maquiling and Mit. Binuang, forest slope, the latter at $300 \mathrm{~m}$ altitude. In Malay Peninsula and Java.

\section{EXCLUDED OR SYNONYMOUS SPECIES}

Macodes setaceus Boxall ex Naves Novis. App. (1882) 249, nomen.

Macodes striatus Boxall ex Naves Novis. App. (1882) 249, nomen.

Macodes Veitchil Boxall ex Naves Novis. App. (1882) 249, nomen.

Macodes Xanthophyllus Boxall ex Naves Novis. App. (1882) 249, nomen.

\section{CHEIROSTYLIS Blume}

CHEIROSTYLIS OCTODACTYLA Ames in Philip. Journ. Sci. 2 (1907) Bot. 314 , Orch. 3 (1908) 46, t. 40, 5 (1915) 37.

I on (Laguna), Mindoro, Merrill 5834, 7495. Mt. Banahao and Mt. Hallon, in forest at 2,500 to $2,700 \mathrm{~m}$ altitude.

CHEIROSTYLIS PHILIPPINENSIS Ames Orch. 2 (1908) 59, 5 (1915) 37.

Luzon (Kalinga, Benguet, Tayabas), Panay (Iloilo), Negros (Oriental Negros), Elmer 6035, 9701, Topping 19, Boettcher s. n., Merrill 4015, B. S. 18196 Robinson. In mountainous regions at 330 to $830 \mathrm{~m}$ altitude.

29. PHILIPPINAEA Ames and Schlechter.

PHILIPPINAEA WENZELII (Ames) Àmes \& Schltr. in Ames Orch. 6 (1920) 278, t. 100.

Adenostylis wenzelii Ames Orch. 5 (1915) 41.

Luzon (Sorsogon), Leyte, Mindanao (Agusan), Elmer 13884, 16668, Wenzel 0119, 0210, 0510, 0562. Terrestrial at 60 to $500 \mathrm{~m}$ altitude.

\section{ADENOSTYLIS Blume}

AdenOSTylis Benguetensis Ames in Elm. Leafl. Philip. Bot. 5 (1912) 1551, Orch. 5 (1915) 39.

Luzon (Benguet), Elmer 8856. Baguio.

ADENOSTYLIS ELMERI Ames in Elm. Leafl. Philip. Bot. 5 (1912) 1552, in Philip. Journ. Sci. 8 (1913) Bot. 408, Orch. 5 (1915) 39.

Luzon (Bontoc, Benguet, Bataan), Palawan, Vanoverbergh 69, 1099, 2259, 4054, Elmer $8648,12634, F . B .6240$ Curran. Terrestrial, on woody hillocks up to $1,300 \mathrm{~m}$ altitude. 
ADENOSTYLIS LEYTENSIS Ames Orch. 5 (1915) 39.

Luzon (Sorsogon), Leyte, Elmer 15672, Wenzel 0349. Terrestrial.

ADENOSTYLIS LUZONENSIS Ames Orch. 2 (1908) 57, text cut, 5 (1915) 39.

Zeuxine luzonensis Ames in Philip. Journ. Sci. 2 (1907) Bot. 314, nomen.

Luzon (Bontoc, Lepanto), Mindoro, Vanoverbergh 787, Merrill 4604, 5841. Terrestrial on mountains, in the forest, at 930 to $1,300 \mathrm{~m}$ altitude.

ADENOSTYLIS MARIVELENSIS Ames Orch. 2 (1908) 58, text cut, 5 (1915) 39.

Luzon (Bataan), Negros (Occidental Negros), Merrill 798, 3963, F. B. 6517 Curran, Weber 1\%, Merrill 7019, Banks s. n., R. S. Williams 62\%.

ADENOSTYLIS PHILIPPINENSIS Ames Sched. Orch. 6 (1923) 9.

Leyte, Wenzel 0375 . Terrestrial in forest at $60 \mathrm{~m}$ altitude.

ADENOSTYLIS STRATEUMATICA (Linn.) Ames Orch. 2 (1908) 59, 5 (1915) 40.

Orchis strateumatica Linn. Sp. P1. (1753) 943, ed. 2 (1763) 1336.

Spiranthes strateumatica Lindl. in Bot. Reg. 10 (1824) sub t. 823.

Adenostylis emarginata Blume Bijdr. (1825) 414.

Adenostylis integerrima Blume Bijdr. (1825) 414, fig. 1\%.

Pterygodium sulcatum Roxb. Fl. Ind. ed. 2, 3 (1832) 452.

Tripleura pallida Lindl. in Wall. Cat. (1832) No. 7391, nomen, Gen. \& Sp. Orch. (1840) 452.

Zeuxine sulcata Lindl. in Wall. Cat. (1832) No. 7392, nomen, Gen. \& Sp. Orch. (1840) 485; Griff. Notul. 3 (1851) 396, Icon. P1. Asiat. 3 (1851) t. 349; Blume Fl. Jav. Orch. (1858) 55 (Orch. Arch. Ind. 67) ; Miq. Fl. Ind. Bat. 3 (1859) 723; Benth. Fl. Hongk. (1861) 360; Thwaites Enum. Pl. Zeyl. (1861) 312; Hook. f. Fl. Brit. Ind. 6 (1890) 106; King \& Pantl. in Ann. Roy. Bot. Gard. Calc. 8 (Orch. Sik.-Himal.) (1898) 286, t. 381; Trim. Handb. Fl. Ceyl. 4 (1898) 215; Matsum. Ind. Pl. Jap. 2 (1905) 264; J. J. Sm. Fl. Buitenz. 6 (Orch. Jav.) (1905) 108, Fig.-Atlas (1908) fig. 76, Orch. Amb. (1905) 12; Duthie in Ann. Roy. Bot. Gard. Calc. 9 (Orch. North-west. Himal.) (1906) 168; Ridl. Mat. Fl. Mal. Penin. 1 (1907) 217.

Zeuxine emarginata Lindl. Gen. \& Sp. Orch. (1840) 485; Reichb. f. in Bonpl. 5 (1857) 36.

Zeuxine integerrima Lindl. Gen. \& Sp. Orch. (1840) 486; Blume Fl. Jav. Orch. (1858) 55 (Orch. Arch.' Ind. 67), t. 19, fig. 1, t. 23c; Miq. Fl. Ind. Bat. 3 (1859) 723.

Zeuxine membranacea Lindl. Gen. \& Sp. Orch. (1840) 486; Blume Fl. Jav. Orch. (1858) 56 (Orch. Arch. Ind. 68), t. 22, fig. 2, t. $23 A$.

? Corycium humile Herb. Ham. ex. Lindl. Gen. \& Sp. Orch. (1840) 485 , in syn.

Neottia strateumatica R. Br. ex Steud. Nom. Bot. ed. 2, 2 (1841) 190. Zeuxine bracteata Wight Icon. $5^{1}$ (1852) 16, t. 1724bis.

Zeuxine brevifolia Wight Icon. $5^{1}$ (1852) $16, t .1725$.

Zeuxine robusta Wight Icon. $5^{1}$ (1852) $16, t$. 1726 .

Zeuxine tripleura Lindl. in Journ. Linn. Soc. 1 (1857) 186. 
Zeuxine procumbens Blume Fl. Jav. Orch. (1858) 56 (Orch. Arch. Ind. 68) t. 22, fig. 3, t. $23 B$.

Zeuxine sp. Vidal Rev. Pl. Vasc. Filip. (1886) 273.

Zeuxine strateumatica Schltr. in Fedde Repert. Beihefte 1 (Orch. Deutsch-Neu-Guin.) (1911) 77.

Mindanao (Misamis, Agusan), M. S. Clemens 6, 679a, Weber s. n. Texrestrial in the forest, apparently at low altitude. In Afghanistan, India, Ceylon, Malay Peninsula, China, Assam, Japan, Java, and Amboina.

Adenostylis VANOVERBerghII Ames in Philip. Journ. Sci. 8 (1913) Bot. 408, Orch. 5 (1915) 40.

Luzon (Bontoc, Lepanto), Vanoverbergh 1490, 3936, Ramos \& Edaño ci, 84. In wet or mossy forest at 930 to $2,540 \mathrm{~m}$ altitude.

ADENOSTYLIS WEBERI Ames Orch. 5 (1915) 40.

Mindanao (Davao), Weber 280. Mt. Binatun, in bamboo forest at $580 \mathrm{~m}$ altitude.

ADENOSTYLIS ZAMBOANGENSIS Ames Sched. Orch. 6 (1923) 10.

Mindanao (Zamboanga), Copeland 156\%. Terrestrial, in forest at $130 \mathrm{~m}$ altitude.

\section{EXCLUDED OR SÝNONYMOUS SPECIES}

-Adenostylis Wenzeli Ames Orch. 5 (1915) $41=$ Philippinaea wenzelii (Ames) Schltr. \& Ames.

\section{MYRMECHIS Blume}

MYRMECHIS GRACILIS Blume Fl. Jav. Orch. (1858) 64 (Orch. Arch. Ind. 77), t. 21, fig. 2; Franch. \& Sav. Enum. Pl. Jap. 2 (1879) 39; J. J. Sm. Fl. Buitenz. 6 (Orch. Jav.) (1905) 105, Fig.-Atlas (1908) fig. 7\%; Matsum. Ind. Fl. Jap. 2 (1905) 254; Ames in Philip. Journ. Sci. 2 (1907) Bot. 315, Orch. 2 (1908) 63, 5 (1915) 42. Anecochilus gracilis Blume Bijdr. (1825) 413.

Anaectochilus gracilis Blume apud Lindl. Gen. \& Sp. Orch. (1840) 500. Rhamphidia alsinefolia Lindl. in Journ. Linn. Soc. 1 (1857) 182;

Miq. F1. Ind. Bat. 3 (1859) 730.

Rhamphidia japonica Reichb. f. in Bot. Zeit. 36 (1878) 75.

Myrmechis japonica Rolfe in Journ. Linn. Soc. 36 (1903) 44, sub Myrmechis chinensis Rolfe.

Luzon (Benguet, Bataan), Mindoro, Mindanao (Misamis, Bukidnon, Davao), B. S. 8422 McGregor, 38520 Ramos \& Edaño, Merrill 3865, F. B. 4432, 4460 Merritt, 4622 Mearns \& Hutchinson, Elmer 11372. In mountain regions, up to $2,100 \mathrm{~m}$ altitude in Benguet, and $3,300 \mathrm{~m}$ altitude in Mindanao. In Java and Japan.

MYRMECHIS PERPUSILLA Ames Sched. Orch. 6 (1923) 15.

Luzon (Benguet), Copeland 124.

MYRMECHIS PHILIPPINENSIS Ames Orch. 2 (1908) 64, text cut, 5 (1915) 42.

Luzon (Bataan), Leiberg 6066. Mt. Mariveles, terrestrial at $800 \mathrm{~m}$ altitude. 


\section{VRYDAGZYNEA Blume}

VRYDAGZYNEA ALBIDA Blume Fl. Jav. Orch. (1858) 62 (Orch. Arch. Ind. 75), t. 19, fig. 2; Hook. f. Fl. Brit. Ind. 6 (1890) 97; Ridl. in Journ. Linn. Soc. 32 (1896) 397; J. J. Sm. Fl. Buitenz. 6 (Orch. Jav.) (1905) 89, Fig.-Atlas (1908) fig. 61; Ridl. Mat. Fl. Mal. Penin. 1 (1907) 211; Ames in Philip. Journ. Sci. 2 (1907) Bot. 313; Orch. 2 (1908) 55, 5 (1915) 42.

Etaeria albida Blume Bijdr. (1825) 410.

Aetheria albida Blume apud Lindl. Gen. \& Sp. Orch. (1840) 491.

Hetaeria albida Blume apud Miq. Fl. Ind. Bat. 3 (1859) 726; Naves

Novis. App. (1882) 249.

Luzon (Rizal, Tayabas, Sorsogon), Mindoro, Samar, Negros (Oriental Negros), Leyte, Mindanao (Zamboanga, Davao), Jolo. A rather common and widely distributed species in the Philippines. Terrestrial in dense forest at 137 to $1,000 \mathrm{~m}$ altitude. In Malay Peninsula and Java.

VRYDAGZYNEA WEBERI Ames Orch. 5 (1915) 42.

Luzon (Apayao, Rizal, Laguna, Tayabas, Sorsogon), Polillo, Leyte, Weber 333, Elmer 14616, 14685, 17374, 18028, B. S. 9191, 9489 Robinson, 2978 Ramos \& Edaño, 25401 Yates, Wenzel 0244, 0630. Terrestrial, up to $600 \mathrm{~m}$ altitude in Leyte.

\section{HETAERIA Blume}

HETAERIA BLACKII Ames Orch. 5 (1915) 43.

Mindanao (Lanao, Davao), M. S. Clemens 489, Black s. n.

HETAERIA LANCIFOLIA Ames Orch. 5 (1915) 44 (as lancifolium).

Luzon (Apayao), Weber 32\%. Abulug-Narig Trail, terrestrial in forest at $76 \mathrm{~m}$ altitude.

HetAeRIA LeYTENSIS Ames Sched. Orch. 6 (1923) 14.

Leyte, B. S. 42081b Edaño. At $300 \mathrm{~m}$ altitude.

HETAERIA MINDANAENSIS Ames Orch. 5 (1915) 45.

Mindanao (Agusan) Weber 31\%. Sangaan-Cabadbaran Trail, terrestrial in shady forest at $22 \mathrm{~m}$ altitude.

HETAERIA OBLongIFOLIA Blume Fl. Jav. Orch. (1858) 85 (Orch. Arch. Ind. 102), t. 32, fig. 3; Miq. Fl. Ind. Bat. 3 (1859) 725; Kränzl. in K. Schum. \& Lauterb. Fl. Deutsch. Schutzgeb. (1901) 241; J. J. Sm. Fl. Buitenz. 6 (Orch. Jav.) (1905) 113, Fig.-Atlas (1908) fig. 80; Ames Orch. 1 (1905) 70, 2 (1908) 62, 5 (1915) 46; J. J. Sm. in Nova Guinea 8 (1909) $17,8^{3}$ (1912) 525.

Etaeria oblongifolia Blume Bijdr. (1825) 410, fig. 14.

Aettieria oblongifolia Lindl. Gen. \& Sp. Orch. (1840) 491.

Etaeria rubicunda Reichb. f. in Bonpl. 3 (1855) 214.

Rhamphidia tenuis Lindl. in Journ. Linn. Soc. 1 (1857) 182; Miq. F1. Ind. Bat. 3 (1859) 730; Naves Novis. App. (1882) 248.

Hetaeria tenuis Vidal Phan. Cuming. Philip. (1885) 151, Rev. Pl. Vasc. Filip. (1886) 273; Rolfe in Journ. Bot. 24 (1886) 59; Ceron Cat. Pl. Herb. Manila (1892) 169.

Goodyera rubicunda J. J. Sm. Fl. Buitenz. 6 (Orch. Jav.) (1905) 121 (as to syn. Aetheria mbicunda Reichb. f.). 
Luzon (Ilocos Norte, Cagayan, Benguet, Rizal, Laguna, Tayabas), Ticao, Mindoro, Negros (Oriental Negros), Palawan, Balabac, Mindanao (Zam. boanga, Lanao). A common and widely distributed species in the Philippines. Terrestrial in forest at 30 to $90 \mathrm{~m}$ altitude. In Java, Molucca Islands, New Guinea, and possibly Samoa.

HETAERIA WENZELII Ames Orch. 5 (1915) 46.

Leyte, Wenzel 0227. Dagami, terrestrial in forest at $60 \mathrm{~m}$ altitude.

EXCLUDED OR SYNONYMOUS SPECIES

Hetaeria Albida Blume apud Miq. Fl. Ind. Bat. 3 (1859) 726; Naves Novis. App. (1882) $240=$ Vrydagzynea albida Blume.

Hetaeria Javanica (Blume) Miq. Fl. Ind. Bat. 3 (1859) $725=$ Cystorchis javanica Blume.

Hetaeria tenuis Vidal Phan. Cuming. Philip. (1885) 151, Rev. Pl. Vasc. Filip. (1886) 273; Rolfe in Journ. Bot. 24 (1886) 59; Ceron Cat. Pl. Herb. Manila (1892) $169=$ Hetaeria oblongifolia Blume.

Hetamia variegata Miq. apud Naves Novis. App. (1882) 249.

This is probably referable to Cystorchis javanica Blume.

\section{TROPIDIA Lindley}

TROPIDIA CALCARATA Ames in Philip. Journ. Sci. 7 (1912) Bot. 7, Orch. 5 (1915) 47.

Luzon (Rizal, Laguna), B. S. 13603 Ramos, Merrill 7144, Catalan 223, Elmer 18188.

TROPIDIA MINDANAENSIS Ames in Elm. Leafl. Philip. Bot. 5 (1912) 1553, Orch. 5 (1915) 47.

Mindanao (Davao), Elmer 11785. Todaya, Mt. Apo.

TROPIDIA MINDORENSIS Ames in Philip. Journ. Sci. 2 (1907) Bot. 315, Orch. 5 (1915) 47.

Mindoro, Merrill 5552. Binabay River, terrestrial in humid forest at $300 \mathrm{~m}$ altitude.

TROPIDIA ROBINSONII Ames Sched. Orch. 6 (1923) 16.

Luzon (Tayabas), B. S. 9496 Robinson.

TROPIDIA SEPTEMNERVIS (Schauer) Reichb. f. in Linnaea 25 (1852) 230 ; Ames Orch. 5 (1915) 47, in textu.

Ptychochilus septemnervis Schauer in Nov. Act. Nat. Cur. 19, Suppl. 1 (1843) 431, t. 12, fig. B.

Tropidia minor Ames Orch. 2 (1908) 65, text cut, 5 (1915) 47.

Luzon (Nueva Ecija, Pampanga, Bataan, Laguna), Mindoro, Leyte, Merrill 4204, Whitford 1368, B. S. 16558 Ramos, 1542 Bermejos, 2626\% Ramos \& Edaño, 17927 Brown, Wenzel 055\%, F.B. 7501 Curran. Terrestrial in forest at $500 \mathrm{~m}$ in Leyte.

EXCLUDED OR SYNONYMOUS SPECIES

Tropidia curculigoides Lindl. apud Naves Novis. App. (1882) 249.

A native of Java, Borneo, and Malacca, wrongly attributed to the Philippines. Cf. Ames Orch. 5 (1915) 47, in textu. 
Tropidia graminea Blume apud Naves Novis. App. (1882) 249.

T'ropidia Grandis Hance apud Naves Novis. App. (1882) 249.

Tropidia Minor Ames Orch. 2 (1908) 65, text cut, 5 (1915) 47 = Tropidia septemnervis (Schauer) Reichb. $\mathrm{f}$.

\section{CORYMBORCHIS Thouars}

CORYMBORCHIS CONFUSA Ames Orch. 5 (1915) 48.

Corymbis disticha Lindl. Fol. Orch. Corymbis (1854) 1, quoad Philip.; Thou. apud Miq. Fl. Ind. Bat. 3 (1859) 736, quoad Philip.

Chloidia confusa Ames Orch. 2 (1908) 67, text cut.

Corymbis confusa Schltr. in Engl. Bot. Jahrb. 45 (1911) 395.

Luzon (Ilocos Norte, Apayao), Leyte, Bancalan, Mindanao (Agusan, Zamboanga), Jolo, B. S. 32723, 32752 Ramos, 37295 Ramos \& Edaño, 28227 Fenix, Cuming s. n., Weber 320, 339, Wenzel 0209, Elmer 13433, r. S. Williams 2153, J. Clemens s. n. Terrestrial, in forest at sea level to $830 \mathrm{~m}$ altitude.

\section{CHRYSOGLOSSUM Blume}

CHRYSOGLOSSUM VILLOSUM Blume Bijdr. (1825) 338, fig. \%; Lindl. Gen. \& Sp. Orch. (1830) 14; De Vriese Ill. Orch. Ind. Neerl. (1854) t. 12, fig. 4; Blume Fl. Jav. Orch. (1858) 137 (Orch. Arch. Ind. 162), t. 47; Miq. Fl. Ind. Bat. 3 (1859) 626; Reichb. f. in Walp. Ann. 6 (1861) 206; Hook. f. Fl. Brit. Ind. 5 (1890) 783; Ridl. in Journ. Linn. Soc. 32 (1896) 316; J. J. Sm. Fl. Buitenz. 6 (Orch. Jav.) (1905) 173, Fig.-Atlas (1909) fig. 131; Ridl. Mat. Fl. Mal. Penin. 1 (1907) 112; Ames in Philip. Journ. Sci. 2 (1907) Bot. 316, Orch. 2 (1908) 67, 5 (1915) 48, in Merr. in Journ. Roy. Asiat. Soc. Straits Branch, Special No. (1921) 143.

Mindoro, Merrill 5838. Binabay River, terrestrial at about $300 \mathrm{~m}$ altitude. In Malay Peninsula, Java, and Borneo.

\section{NEPHELAPHYLLUM Blume}

NEPHELAPHYLLUM MINDORENSE Ames in Philip. Journ. Sci. 2 (1907) Bot. 316, Orch. 3 (1908) 83, t. 55, 5 (1915) 48.

Luzon (Nueva Ecija), Mindoro, B. S. 26319, 26494, 26652 Ramos \& Edaño, Merrill 5623. Terrestrial on dry slopes and in humid forest at 200 to $300 \mathrm{~m}$ altitude.

38. DIGLYPHOSA Blume

DIGLYPHOSA ELMERI Ames in Elm. Leafl. Philip. Bot. 5 (1912) 1555, Orch. 5 (1915) 48.

Mindanao (Davao), Elmer 11815. Todaya, Mt. Apo.

39. COELOGYNE Lindley

COELOGYNE ASPERATA Lindl. in Journ. Hort. Soc. 4 (July, 1849) 221, Fol. Orch. Coelog. (1854) 3; J. Linden Pescat. 1 (1854) text to t. 7; Miq. Fl. Ind. Bat. 3 (1859) 666; Reichb. f. in Walp. Ann. 6 (1861) 224; Warner \& Will. Orch. Alb. 7 (1888) t. 311; Hook. f. Fl. Brit. Ind. 5 (1890) 835; Veitch. Man. Orch. Pl. pt. 6 (1890) 31; Ridl. in Journ. Linn. Soc. 31 (1896) 287, 32 (1896) 326; L. Linden in Lindenia 13 (1897) 15, t. 582; Ridl. in Journ. Bot. 36 (1898) 214, Mat. Fl. Mal. Penin. 1 (1907) 129; Pfitz. in 
Engl. Pflanzenreich IV. 50. II. B. 7 (1907) 76; Ames Orch. 2 (1908) 68, 5 (1915) 49; J. J. Sm. in Nova Guinea 8 (1909) 20, $8^{3}$ (1912) 527, 12 (1913) 10, $12^{3}$ (1915) 196; Ames in Merr. in Journ. Roy. Asiat. Soc. Straits Branch, Special No. (1921) 143.

Coelogyne lowii Lind1. in Paxt. Mag. Bot. 16 (September, 1849) 225, t. and text cut; J. Linden Pescàt. 1 (1854) t. 7 ; Boxall ex Naves Novis. App. (1882) 237.

Pleione asperata Ktze. Rev. Gen. Pl. 2 (1891) 680.

Samar, Leyte, Negros (Oriental Negros), Mindanao (Agusan, Surigao. Davao), Basilan, B. S. 17624 Ramos, 15500, 16257 Reillo, Wenzel s. n., Elmer 10008, Weber 55, 250, Bolster 294,F. B. 6103 Hutchinson. Epiphyte in forest at 75 to $430 \mathrm{~m}$ altitude. In Malay Peninsula, Sumatra, Borneo, and New Guinea.

Coelogyne Bilamellata Lind1. Fol. Orch. Coelog. (1854) 14; Miq. Fl. Ind. Bat. 3 (1859) 668; Naves Novis. App. (1882) 237; Ridl. in Journ. Linn. Soc. 31 (1896) 287; Pfitz. in Engl. Pflanzenreich IV. 50. II. B. 7 (1907) 24; Ames Orch. 2 (1908) 73, text cut, 5 (1915) 94.

Panisea bilamellata Reichb. f. in Walp. Ann. 6 (1861) 240.

Pleione bilamellata Ktze. Rev. Gen. Pl. 2 (1891) 680.

Luzon (Laguna, Tayabas), Leyte, Mindanao (Zamboanga, Agusan, Bukidnon, Surigao), B. S. 15131 Ramos, 39046 Ramos \& Edaño, Elmer r7z\%, 13900, Wenzel 0412, 0494. 0513, Merrill 8202, Bolster 288. Epiphyte in forest, up to $1,000 \mathrm{~m}$ altitude in Mindanao. Reported from Borneo.

COELOGYNE CANDOONENSIS Ames Sched. Orch. 6 (1923) 18, $\mathrm{fg} .4$.

Mindanao (Bukidnon), B. S. 38894 Ramos \& Edaño. In forest at 1,830 $m$ altitude.

COELOGYNE CHLOROPTERA Reichb. f. in Gard. Chron. II 19 (1883) 466; Kränzl. Xen. Orch. 3 (1890) 69, t. 235, fig. I. 1-6; Pfitz. in Engl. Pflanzenreich IV. 50. II. B. 7 (1907) 55, fig. 18L; Ames Orch. 2 (1908) 71, t., 5 (1915) 49.

Coelogyne sparsa Ames Orch. 1 (1905) 72, p. p. (as to Elmer 5842), non Reichb. f.

Luzon (Cagayan, Kalinga, Bontoc, Benguet, Pampanga, Bataan, Tayabas), Negros (Oriental Negros). A fairly common species in Luzon, on trees and rocks at 1,000 to $1,530 \mathrm{~m}$ altitude.

COELOGYNE CONFUSA Ames Orch. 5 (1915) 49.

Mindanao (Camiguin Island), B. S. 14430 Ramos. Epiphyte.

COELOGYNE ELMERI Ames in Elm. Leafl. Philip. Bot. 5 (1912) 1556, Orch. 5 (1915) 50.

Mindanao (Bukidnon, Davao), B. S. 38583 Ramos \& Edaño, Elme: 10694. Mt. Lipa and Todaya, Mt. Apo, up to $1,300 \mathrm{~m}$ altitude.

CoElogyne INTEgerRima Ames in Philip. Journ. Sci. 4 (1909) Bot. 665, 6 (1911) Bot. 40; Merr. \& Merritt in Philip. Journ. Sci. 5 (1910) Bot. 340; Ames Orch. 5 (1915) 50.

Luzon (Bontoc, Benguet, Tayabas), Vanoverbergh 3646, Merrill 635n, B.S. 8454, 8519, 8822 McGregor, 31711 Santos, Elmer 9258. Mountain regions at 1,750 to $2,450 \mathrm{~m}$ altitude. 
COELOGYNE LOHERI Rolfe in Kew Bull. (1908) 414; Ames Orch. 5 (1915) 50, 6 (1920) 279.

Luzon (Bontoc, Benguet), Loher 549, Vanoverbergh 1197, 2867, 3906, 4041, Boettcher s. n., B. S. 31909 Santos. Mountain regions up to $1,700 \mathrm{~m}$ altitude.

COELOGYNE LONGIRACHIS Ames Orch. 7 (1922) 88.

Mindanao (Bukidnon), B. S. 38671 Ramos \& Edaño. Mahilucot River, along streams in forest at $1,300 \mathrm{~m}$ altitude.

COELOGYNE MARMORATA Reichb. f. in Linnaea 41 (1877) 116; Pfitz.

in Engl. Pflanzenreich IV. 50. II. B. 7 (1907) 85; Ames Orch. 5

(1915) 50, Sched. Orch. 6 (1923) 20.

Coelogyne zahlbrucknerae Kränzl. in Fedde Repert. 17 (1921) 389.

Luzon (Benguet), Wallis s. n., Schultze s. n., Loher s. n. Sablan, May, 1909.

COELOGYNE MERRILLII Ames in Philip. Journ. Sci. 6 (1911) Bot. 40, Orch. 5 (1915) 51.

Luzon (Benguet, Rizal), Merrill 6620, Phil. Pl. 716, Haight 14, B. S. 31814 Santos, 29764 Ramos \& Edaño, Lyon 164. Mountain regions at 300 to $2,300 \mathrm{~m}$ altitude.

COELOGYNE MINUTISSIMA Kränzl. in Fedde Repert. 17 (1921) 390.

Philippines, probably Luzon, Loher s. $n$.

No specimen seen.

COELOGYNE PALAWANENSE Ames Orch. 5 (1915) 51.

Palawan, Merrill 949.9. Mt. Capoas at 1,000 $\mathrm{m}$ altitude.

COELOGYNE QUINQUELAMELLATA Ames Orch. 6 (1920) 280.

Mindanao (Surigao), Wenzel 01175, 01179, 01182, 01183, 01206. Epiphyte in forest at $150 \mathrm{~m}$ altitude.

COELOGYNE RAMOSII Ames Orch. 7 (1922) 90.

Mindanao (Bukidnon, Agusan), B. S. 38892, 38944 Ramos \& Edaño, Elmer 14130. Mt. Candoon and Cabadbaran (Mt. Urdaneta) up to 1,850 $m$ altitude.

COELOGYNE ROCHUSSENII De Vriese Ill. Orch. Ind. Neerl. (1854) t. 2, t. 11, fig. 6; Miq. Fl. Ind. Bat. 3 (1859) 666; Reichb. Xen. Orch. 1 (1856) 212, t. 85; Ridl. in Journ. Linn. Soc. 32 (1896) 321; J. J. Sm. Fl. Buitenz. 6 (Orch. Jav.) (1905) 144, Fig.-Atlas (1909) fig. 107; Ridl. Mat. Fl. Mal. Penin. 1 (1907) 128; Pfitz. in Engl. Pflanzenreich IV. 50. II. B. 7 (1907) 70, figs. 22E, 23; Ames in Philip. Journ. Sci. 4 (1909) Bot. 664; Schltr. in Engl. Bot. Jahrb. 45, Beibl. 104 (1911) 7; Ames Orch. 5 (1915) 52, in Merr. in Journ. Roy. Asiat. Soc. Straits Branch, Special No. (1921) 145.

Coelogyne macrobulbon Hook. f. Fl. Brit. Ind. 5 (1890) 830.

Pleione rochusenii Ktze. Rev. Gen. Pl. 2 (1891) 680.

Pleione macrobulbon Ktze. Rev. Gen. Pl. 2 (1891) 680.

Luzon (Pampanga), Mindanao (Lanao), M. S. Clemens s. n., Lyon s. $n$. Camp Keithley, September, 1907. In Malay Peninsula, Sumatra, Java, Celebes, and Borneo. 
COELOGYNE SPARSA Reichb. f. in Gard. Chron. II 19 (1883) 306;

Veitch Man. Orch. Pl. pt. 6 (1890) 49; Kränzl. Xen. Orch. 3 (1890) 70, t. 235, fig. II. 7-12; Wittmack in Gartenfl. 46 (1897) 449, t. 1442; Ames Orch. 1 (1905) 71 (excl. Elmer 5842), 2 (1908) 70, text cut, 5 (1915) 53; Pfitz. in Engl. Pflanzenreich IV. 50. II. B. 7 (1907) 54, fig. 18, J. K.

Pleione sparsa Ktze. Rev. Gen. Pl. 2 (1891) 680.

Luzon (Rizal, Bataan), Panay (Antique), Negros (Oriental Negros), Palawan, B. S. 2176 Ramos, 647 Foxworthy, Weber 11, 15, 16, Merrill 3961. Whitford 1090, Lyon 133, R. S. Williams 626, r59, Yoder s. n., Elmer. 10210, McGregor 5806. Mountain regions, epiphytic at 900 to $1,050 \mathrm{~m}$ altitude.

COELOGYNE SWANIANA Rolfe in Kew Bull. (1894) 183, in Orch. Rev. 2 (1894) 198, in Sander Reichenbachia II 2 (1894) 105, t. 92; Hook. f. in Bot. Mag. 124 (1898) t. 7602; Pfitz. in Engl. Pflanzenreich IV. 50. II. B. 7 (1907) 68, fig. 22C; Ames Orch. 5 (1915) 53.

Only horticultural specimens of this species have been seen.

COELOGYNE VANOVERBERGHII Ames Orch. 5 (1915) 53.

Bontoc, Vanoverbergh 2865. Bauco at $1,700 \mathrm{~m}$ altitude.

EXCLUDED OR SYNONYMOUS SPECIES

Comlogyne ciliata Boxall ex Naves Novis. App. (1882) 237, nomen.

CoelogYNe CONCHOIDEA Reichb. f. in Walp. Ann. 6 (1861) 237; Naves Novis. App. (1882) 237 = Pholidota imbricata Lindl.

Conlogyne cumingi Lindl. apud Naves Novis. App. (1882) 237.

Cuming's specimen, on which this species was based, was from Singapore, not from the Philippines.

Coklogyne fimbriata Lindl. apud Naves Novis. App. (1882) 237.

Comlogyne imbricata (Lindl.) Reichb. f. in Walp. Ann. 6 (1861) 238 $=$ Pholidota imbricata Lindl.

Coelogyne LowiI Lindl. in Paxt. Mag. Bot. 16 (September, 1849) 225, $t$. and text cut; J. Linden Pescat. 1 (1854) $t .7$; Boxall ex Naves Novis. App. (1882) $237=$ Coelogyne asperata Lindl.

Coelogyne Palidda (Lindl.) Reichb. f. in Walp. Ann. 6 (1861) $238=$ Pholidota imbricata Lindl.

Coelogyne Pandurata Lindl. apud Naves Novis. App. (1882) 237.

Coelogyne pumila Reichb. f. in Walp. Ann. 6 (1861) 236 = Acoridium pumilum (Reichb. f.) Rolfe.

CoElogyne RHOMBopHora Reichb. f. in Linnaea 41 (1877) $116=$ Acoridium rhombophorum (Reichb. f.) Ames.

Comigyne Rumphit Lindl. apud Naves Novis. App. (1882) 237.

COELOGYNE SPARSA Ames Orch. 1 (1905) 72, p. p. (as to Elmer 5842), non Reichb. $\mathrm{f}$. $=$ Coelogyne chloroptera Reichb. $\mathrm{f}$.

Comlogyne speciosa Lindl. apud Naves Novis. App. (1882) 237.

Comlogyne trifida Reichb. f. apud Naves Novis. App. (1882) 237. 
Coelogyne triotos Reichb. f. in Walp. Ann. 6 (1861) 238, in Hamb. Gartenz. 18 (1862) 34; Naves Novis. App. (1882) $237=$ Pholidota imbricata Lindl.

CoELogyne ventricosa (Blume) Reichb. f. in Walp. Ann. 6 (1861) $237=$ Pholidota ventricosa (Blume) Reichb. f.

Coelogyne viscosa Boxall ex Vidal ex Naves Novis. App. (1882) 237, nomen.

Coelogyne zahlbrucknerae Kränzl. in Fedde Repert. 17 (1921) $389=$ Coelogyne marmorata Reichb. f.

\section{PSEUDACORIDIUM Ames}

PSEUDACORIDIUM WOODIANUM Ames Orch. 7 (1922) 79.

Dendrochilum woodianum Ames in Philip. Journ. Sci. 2 (1907) Bot. 318, nomen, Orch. 2 (1908) 80, text cut, 5 (1915) 66.

Mindoro, Merrill 5816. Mt. Halcon, at 2,260 to $2,830 \mathrm{~m}$ altitude.

41. ACORIDIUM Nees and Meyen

ACORIDIUM AFFINE Ames Orch. 7 (1922) 80.

Dendrochilum affine Ames Orch. 2 (1908) 95, 5 (1915) 55.

Luzon (Bataan), Merrill 3218, Whitford 1354, B. S. 5184 Topping.

ACORIDIUM ANFRACTOIDES Ames Orch. 7.(1922) 80.

Dendrochilum anfractoides Ames Orch. 3 (1908) 13, t. 28, 5 (1915) 55, in Philip. Journ. Sci. 4 (1909) Bot.665; Merr. \& Merritt in Philip. Journ. Sci. 5 (1910) Bot. 340.

Luzon (Benguet), F. B. 4312a Mearns, B. S. 8358, 8834, 8858.McGregor, Haight 10, Ramos \& Edaño 11, 48, 48a, 49. In mossy forest at 1,930 to $2,160 \mathrm{~m}$ altitude.

ACORIDIUM ANFRACTUM Ames in Proc. Biol. Soc. Wash. 19 (1906) 148.

Dendrochilum anfractum Pfitz. in Engl. Pflanzenreich IV. 50. II. B. 7 (1907) 118, fig. 38P; Ames Orch. 2 (1908) 91, t. 21, fig. I, 1, 5 (1915) 55.

Luzon (Lepanto, Benguet), Merrill 4482, Ramos \& Edaño 10, 39, 40, 47. In mossy forest at 2,100 to $2,290 \mathrm{~m}$ altitude.

ACORIDIUM AURICULARE Ames Orch. 7 (1922) 80.

Dendrochilum auriculare Ames in Philip. Journ. Sci. 4 (1909) Bot. 595 , Orch. 5 (1915) 55.

Luzon (Benguet), B. S. 57\%1, 5818 Ramos, Ramos \& Edaño 9. In mossy forest at $2,060 \mathrm{~m}$ altitude.

ACORIDIUM BINUANGENSE Ames Orch. 7 (1922) 80.

Dendrochilum binuangense Ames Orch. 6 (1920) 281.

Luzon (Tayabas), B. S. 28620 Ramos \& Edaño. Summit of Mt. Binuang at $500 \mathrm{~m}$ altitude. 
ACORIDIUM CINNABARINUM (Pfitz.) Ames Orch. 7 (1920) 80.

Dendrochilum cinnabarinum Pfitz. in Engl. Pflanzenreich IV. 50. II. B. 7 (1907) 104, fig. $35 F$; Ames Orch. 3 (1908) 10, 5 (1915) 56, 6 (1920) 282, in Philip. Journ. Sci. 4 (1909) Bot. 597; Merr. \& Merritt in Philip. Journ. Sci. 5 (1910) Bot. 340.

Luzon (Benguet, Rizal), Merrill 6475, Boettcher s. n., Ramos \& Edaño 43, B. S. 8844 McGregor, F. B. 16348 Curran, Merritt, \& Zschokke. In mossy forest at 2,160 to $2,930 \mathrm{~m}$ altitude.

ACORIDIUM CONFUSUM Ames Orch. 7 (1922) 80.

Dendrochilum confusum Ames in Philip. Journ. Sci. 6 (1911) Bot. 41, Orch. 5 (1915) 56.

Luzon (Laguna, Sorsogon), Leyte, F. B. 19124 Tamesis, B. S. 5640 Curran, Elmer 15068, Wenzel 0853. In forest at $700 \mathrm{~m}$ altitude.

ACORIDIUM CURRANII Ames Orch. 7 (1922) 80.

Dendrochitum curranii Ames Orch. 3 (1908) 15, t. 29, 5 (1915) 57.

Luzon (Rizal, Laguna), B. S. 3053, 16570, 19561 Ramos, 19152 Reillo, 3077 Curran, 6575, 6580 Robinson, Disdon s. n.

ACORIDIUM ELMERI Ames Orch. 7 (1922) 80.

Dendrochilum elmeri Ames in Elm. Leafl. Philip. Bot. 5 (1912) 1558, Orch. 5 (1915) 57.

Mindanao (Davao), Elmer 10635. Todaya, Mt. Apo, epiphyte in mossy forest at $2,500 \mathrm{~m}$ altitude.

ACORIDIUM EXASPERATUM Ames Sched. Orch. 6 (1923) 21, fig. 6.

Luzon (Rizal), B. S. 42338 Ramos. In mossy forest at $1,300 \mathrm{~m}$ altitude. ACORIDIUM EXILE Ames Orch. 7 (1922) 80.

Dendrochilum exile Ames in Philip. Journ. Sci. 2 (1907) Bot. 317, nomen, Orch. 2 (1908) 84, text cut, 5 (1915) 57.

Mindoro, Merrill 5721. Mt. Halcon.

ACORIDIUM FOXWORTHYI Ames Orch. 7 (1922) 80.

Dendrochilum foxworthyi Ames Orch. 3 (1908) 8, t. 26, 5 (1915) 57.

Luzon (Zambales), B. S. 2542 Foxworthy. Mt. Pinatubo at $1,800 \mathrm{~m}$ altitude.

ACORIDIUM GRAMINIFOLIUM Ames in Proc. Biol. Soc. Wash. 19 (1906) 144.

Dendrochilum graminifolium Pfitz. in Engl. Pflanzenreich IV. 50. II. B. 7 (1907) 114, fig. 38 Q, R; Ames Orch. 2 (1908) 12, 83, t. 22, fig. II, 2, 5 (1915) 57.

Luzon (Lepanto, Benguet), Merrill 4594, 4764, F. B. 5734 Klemme, 15907 Bacani, Ramos \& Edaño 32, B. S. 8487 McGregor.

ACORIDIUM HASTATUM Ames Orch. 7 (1922) 80.

Dendrochilum hastatum Ames in Philip. Journ. Sci. 2 (1907) Bot. 317, nomen, Orch. 2 (1908) 99, text cut, 5 (1915) 58.

Mindoro, F. B. 4441 Merritt, Merrill 5759. Mt. Halcon at $2,260 \mathrm{~m}$ altitude. 
ACORIDIUM HUTCHINSONIANUM Ames comb. nov:

Dendrochilum hutchinsonianum Ames in Philip. Journ. Sci. 2 (1907)

Bot. 317, nomen, Orch. 2 (1908) 96, text cut, 5 (1915) 58.

Acoridium hutchinsonii Ames Orch. 7 (1922) 81, sphalm.

Dendrochilum hutchinsonii Ames Orch. 7 (1922) 81, in syn.

Mindoro, Merrill 5813. Mt. Halcon, in mossy thickets at 2,660. $\mathrm{m}$ altitude.

ACORIDIUM IRIGENSE Ames Orch. 7 (1922) 81.

Dendrochilum irigense Ames Orch. 5 (1915) 58.

Luzon (Camarines), B. S. 22186 Ramos, Santos s. n. Mt. Iriga at 500 $m$ altitude.

ACORIDIUM LOHERI Ames Orch. 7 (1922) 81.

Dendrochilum loheri Ames Orch. 3 (1908) 12, t. 2\%, fig. I, A, 5 (1915) 59, 6 (1920) 283; Merr. \& Merritt in Philip. Journ. Sci. 5 (1910) Bot. 340.

Luzon (Benguet), Loher 461A, Merrill 64\%6, Boettcher s. n.

ACORIDIUM LONGIBULBUM Ames Orch. 7 (1922) 81.

Dendrochilum longibulbum Ames in Philip. Journ. Sci. 7 (1912) Bot.

26, Orch. 5 (1915) 59.

Luzon (Bontoc, Lepanto), Vanoverbergh 782, 3830, Ramos \& Edaño 25, 37, 46. At high altitudes.

ACORIDIUM LUCBANENSE Ames Orch. 7 (1922) 81.

Dendrochilum lucbanense Ames in Elm. Leafl. Philip. Bot. 5 (1912) 1559, Orch. 5 (1915) 60.

Luzon (Tayabas), Elmer $75 \% 1$. Lucban, in mossy forest at $530 \mathrm{~m}$ altitude.

ACORIDIUM LUZONENSE Ames Orch. 7 (1922) 81.

Dendrochilum luzonense Ames Orch. 2 (1908) 121, text cut, 5 (1915) 60.

Luzon (Lepanto, Benguet), F. B. 5735 Klemme, 15729 Curran \& Merritt, Ramos \& Edaño 18, B. S. 5\%rg Ramos. At 1,600 to $2,000 \mathrm{~m}$ altitude.

ACORIDIUM MACGREGORII Ames Orch. 7 (1922) 81.

Dendrochilum sp. Merr. \& Merritt in Philip. Journ. Sci. 5 (1910) Bot. 341.

Dendrochilum macgregorii Ames in Philip. Journ. Sci. 6 (1911) Bot. 42 , Orch. 5 (1915) 60.

Luzon (Benguet), B. S. 8849 McGregor. Mt. Pulog.

ACORIDIUM MALEOLENS (Kränzl.) Ames Orch. 7 (1922) 81.

Dendrochilum maleolens Kränzl. in Orchis 2 (1908) 63; Ames Orch. 5 (1915) 54, 60

Luzon (Rizal), Loher s. $n$.

Described from a plant collected by Loher at Manila, and said to be near Acoridium oliganthum Ames. No specimen of this species has been seen. 
ACORIDIUM MARGINATUM Ames Sched. Orch. 6 (1923) 23, fig. :

Luzon (Rizal), Ramos.s: $n$. In forest at $1,630 \mathrm{~m}$ altitude.

ACORIDIUM MERRILLII Ames in Proc. Biol. Soc. Wash. 19 (1906) 151. Dendrochilum merrillii Pfitz. in Engl. Pflanzenreich IV. 50. II. B. 7 (1907) 119, fig. 38A, B; Ames Orch. 2 (1908) 12, 102, t. 23, fig. I, 1, text cut, 5 (1915) 60.

Luzon (Lepanto, Benguet), Merrill 4585, 4858, B. S. 8353 McGregor, Fimos \& Edaño 1, 21, 44. At 2,100 to $2,800 \mathrm{~m}$ altitude.

ACORIDIUM MICROCHILUM (Schltr.) Ames Orch. 7 (1922) 81.

Platyclinis microchila Schltr. in Bull. Herb. Boiss. II 6 (April, 1906) 302.

Acoridium venustulum Ames in Proc. Biol. Soc. Wash. 19 (September, 1906) 147.

Dendrochilum venustulum Pfitz. in Engl. Pflanzenreich IV. 50. II. B. 7 (1907) 116, fig. 38U; Ames Orch. 2 (1908) 12, 88, t. 22, fig. III, 3, in Philip. Journ. Sci. 4 (1909) Bot. 595; Merr. \& Merritt in Philip. Journ. Sci. 5 (1910) Bot. 341.

Dendrochilum microchilum Ames Orch. 2 (1908) 87, in textu, 5 (1915) 60, in Philip. Journ. Sci. 7 (1912) Bot. 26.

Luzon (Bontoc, Lepanto, Benguet, Nueva Vizcaya, Laguna). A rather common species in the above region, especially so in Benguet at about 1,800 to $2,400 \mathrm{~m}$ altitude.

ACORIDIUM MINDANAENSE Ames Sched. Orch. 6 (1923) 25, fig. 6.

Mindanao (Agusan), Elmer 14095.

ACORIDIUM MINDORENSE Ames Orch. 7 (1922) 81.

Dendrochilum mindorense Ames in Philip. Journ. Sci. 2 (1907) Bot. 317, nomen, Orch. 2 (1908) 91, text cut, 5 (1915) 60.

Mindoro, Merrill 5729,5795. Mt. Halcon at 2,000 $\mathrm{m}$ altitude.

ACORIDIUM MONTANUM Ames Sched. Orch. 5 (1923) 1.

Acoridium linearifolium Ames Orch. 7 (1922) 141, non Rolfe.

Luzon (Benguet), Ramos \& Edaño 56. Mt. Boadan, in mossy forest at about $2,000 \mathrm{~m}$ altitude.

ACORIDIUM OCELLATUM Ames in Proc. Biol. Soc. Wash. 19 (1906) 151.

Dendrochilum ocellatum Pfitz. in Engl. Pflanzenreich IV. 50. 11. B. 7 (1907) 117, fig. 38C; Ames Orch. 2 (1908) 12, 101, t. 23, fig. II, 2, 5 (1915) 61, in Philip. Journ. Sci. 4 (1909) Bot. 596.

Luzon (Lepanto), Merrill 4481a, Ramos \& Edaño \%. Mt. Data at $2,300 \mathrm{~m}$ altitude.

ACORIDIUM OLIGANTHUM Ames in Proc. Biol. Soc. Wash. 19 (1906) 150

Dendrochilum oliganthum Pfitz. in Engl. Pflanzenreich IV. 50. II. B. 7 (1907) 117, fig. 38E; Ames Orch. 2 (1908) 12, 98, t. 23, fig. IV, 4, 5 (1915) 61.

Luzon (Lepanto), Merrill 4481b, Ramos \& Edaño 35. Mt. Data and Mt. Sinapsapan, at about $2,300 \mathrm{~m}$ altitude. 
ACORIDIUM PARVULUM Ames in Proc. Biol. Soc. Wash. 19 (1906) 146.

Dendrochilum parvulum Pfitz. in Engl. Pflanzenreich IV. 50.11. B. 7

(1907) 116, fig. 38N; Ames Orch. 2 (1908) 12, 87, t. 21, fig. II, 2, 5 (1915) 61.

Luzon (Bataan, Rizal, Laguna), Merrill 3217b, Whitford 141, $B . S$. 29724, 29732, Ramos \& Edaño, F. B. 8024 Curran \& Merritt. Lamao River, Mt. Mariveles.

ACORIDIUM PERPLEXUM Ames Orch. 7 (1922) 142.

Luzon (Lepanto), Ramos \& Edaño 55. In mossy forest, Mt. Sinapsapan at $2,000 \mathrm{~m}$ altitude.

ACORIDIUM PHILIPPINENSE Ames in Proc. Biol. Soc. Wash. 19 (1906) 149.

Dendrochilum philippinense Pfitz. in Engl. Pflanzenreich IV. 50. II. B. 7 (1907) 118, fig. 38F; Ames Orch. 2 (1908) 12, 97, t. 23, fig. $V$,

5, 5 (1915) 61, in Philip. Journ. Sci. 4 (1909) Bot. 595.

Luzon (Bontoc, Lepanto, Ifugao, Benguet). A fairly common species in the above region at about 2,000 to $2,300 \mathrm{~m}$ altitude.

ACORIDIUM PULCHERRIMUM Ames Orch. 7 (1922) 144.

Luzon (Benguet), Ramos \& Edaño 45. In mossy forest, Mt. Natoo, at $1,930 \mathrm{~m}$ altitude.

ACORIDIUM PULOGENSE Ames Orch. 7 (1922) 81.

Dendrochilum pulogense Ames in Philip. Journ. Sci. 4 (1909) Bot. 594; Merr. \& Merritt in Philip. Journ. Sci. 5 (1910) Bot. 340; Ames Orch. 5 (1915) 61.

Luzon (Benguet), F. B. 16347A Curran, Merritt, \& Zschokke, Merrill 6478, B. S. 8355, 8842, 8843 McGregor, Haight 2, Boettcher s. n., Ramo: \& Edaño 24, 33.

ACORIDIUM PUMILUM (Reichb. f.) Rolfe in Orch. Rev. 12 (1904) 220; Ames Orch. 7 (1922) 81.

Dendrochilum pumilum Reichb. $\mathrm{f}$. in Bonpl. 3 (1855) 222, in Walp. Ann. 6 (1864) 927; Naves Novis. App. (1882) 231; Vidal Phan. Cuming. Philip. (1885) 81, 149, Rev. Pl. Vasc. Filip. (1886) 268; J. J. Sm. in Rec. Trav. Bot. Néerl. 1 (1904) 58; Ames in Philip. Journ. Sci. 2 (1907) Bot. 317, 4 (1909) Bot. 596; Pfitz. in Engl. Pflanzenreich IV. 50. II. B. 7 (1907) 162; Ames Orch. 2 (1908) 93, text cut, 3 (1908) 6, 5 (1915) 61.

Coelogyne pumila Reichb. f. in Walp. Ann. 6 (1861) 236.

Luzon (Bataan, Laguna, Tayabas). Fairly frequent, especially in Laguna, on the mountains at 950 to $2,000 \mathrm{~m}$ altitude.

ACORIDIUM PURPUREUM Ames Orch. 7 (1922) 81.

Dendrochilum purpureum Ames Orch. 6 (1920) 286.

Luzon (Apayao), Boettcher s. n. Mt. Pasoan at 2,000 m altitude.

ACORIDIUM QUADRILOBUM Ames Orch. 7 (1922) 81.

Dendrochilum quadrilobum Ames Orch. 5 (1915) 61.

Luzon (Apayao, Ifugao), Boettcher s. n., B. S. 19894, 19896 McGregor. 
ACORIDIUM RECURVUM Ames in Proc. Biol. Soc. Wash. 19 (1906) 148. Dendrochilum recurvum Ames in Philip. Journ. Sci. 2 (July, 1907) Bot. 318; Pfitz. in Engl. Pflanzenreich IV. 50. II. B. 7 (November, 1907) 117, fig. 38T; Ames Orch. 2 (1908) 12, 90, t. 22, fig. IV, 4, 5 (1915) 62.

Luzon (Lepanto, Benguet), Mindoro, Merrill 4584, 5.509, 5831, Ramos ¿Edaño 2, 4, 23, 27. In mossy forest, in mountain regions at 2,230 to $2,730 \mathrm{~m}$ altitude.

ACORIDIUM RENIFORME Ames Orch. 7 (1922) 81.

Dendrochilum reniforme Ames Orch. 5 (1915) 63.

Luzon (Rizal, Laguna), B. S. 13759,20440 Ramos, Calica s. n. Mt. Canumay and San Antonio.

ACORIDIUM RHOMBOPHORUM (Reichb. f.) Ames Sched. Orch. 5 (1923) 1.

Coelogyne rhombophora Reichb. f. in Linnaea 41 (1877) 116.

Pholidota rhombophora Reichb. f. in Linnaea 41 (1877) 117, in textu;

Pfitz. \& Kränzl. in Engl. Pflanzenreich IV. 50. II. B. 7 (1907) 153.

Acoridium whitfordii Rolfe ex Ames Orch. 1 (1905) 73.

Dendrochilum whitfordii Pfitz. in Engl. Pflanzenreich IV. 50. II. B. 7

(1907) 162; Ames Orch. 2 (1908) 100, text cut, 5 (1915) 64.

Drendrochilum rhombophorum Ames Orch. 3 (1908) 7, 5 (1915) 64.

Luzon (Bataan, Rizal, Laguna, Tayabas), Wallis s. n., Whitford 139, Topping 360, Elmer 7395, 7679, F. B. 6516 Curran, B. S. 27935 Ocampo, 28619, 28620 Ramos \& Edaño. On mountains, usually at a fairly high altitude.

ACORIDIUM SACCOLABIUM (Kränzl.) Ames Sched. Orch. 5 (1923) 2. Dendrochilum saccolabium Kränzl. in Ann. k. k. Hofmus. Wien 30 (1916) 56.

Without locality, Loher s. n. A fragment from the living type plant cultivated at Nymphenburg, near Munich, Bavaria, is in the Ames Herbarium.

ACORIDIUM SERRATOI Ames Sched. Orch. 6 (1923) 27, fig. 6.

Luzon (Laguna), Serrato s. n. In dense forest.

ACORIDIUM SIMULACRUM Ames Orch. 7 (1922) 82.

Dendrochilum simulacmu Ames Orch. 2 (1908) 92, text cut, 5 (1915) 64.

Luzon (Benguet, Tayabas), F. B. 4981 Curran, Elmer 7586. Mt. Tooglon and Lucban.

ACORIDIUM SPHACELATUM Ames Orch. 1 (1905) 1, $t$. 1, figs, 1, $4-6,8,10,12,14,16,18,20$.

Dendrochilum sphacelatum Pfitz, in Engl. Pflanzenreich IV. 50. II.

B. 7 (1907) 112, fig. 37 A, B, D, F, H, K; Ames Orch. 2 (1908)

82,5 (1915) 64, 6 (1920) 287.

Luzon (Benguet), Elmer 6554, Boettcher s. n., Sandkuhl 9\%. Mt. Santo Tomas at $2,300 \mathrm{~m}$ altitude.

$200823-19$ 
ACORIDIUM STRICTIFORME Ames in Proc. Biol. Soc. Wash. 19 (1906) 147.

Dendrochilum strictiforme Pfitz. in Engl. Pflanzenreich IV. 50. II. B. 7 (1907) 116, fig. 38L; Ames Orch. 2 (1908) 12, 89, t. 21, fig. III, 3, 5 (1915) 64, in Philip. Journ. Sci. 4 (1909) Bot. 597.

Luzon (Lepanto, Benguet, Tayabas), Leyte. A fairly common species in the mountains at 660 to $2,300 \mathrm{~m}$ altitude.

ACoridium Tenellum Nees \& Meyen in Nov. Act. Nat. Cur. 19, Suppl. 1 (1843) 131; Rolfe in Orch. Rev. 12 (1904) 219, 220; Ames Orch. 1 (1905) 3, t. 1, figs. 2, 3, $7,11,13,15,1 \%, 19$.

Dendrochilum junceum Reichb. f. in Otia Bot. Hamb. 1 (1878) 54; Reichb. f. Xen. Orch. 3 (1881) 30; Naves Novis. App. (1882) 245; J. J. Sm. in Rec. Trav. Bot. Néerl. 1 (1904) 78.

Dendrochilum tenellum Ames in Philip. Journ. Sci. 2 (July, 1907) Bot. 318; Pfitz. in Engl. Pflanzenreich IV. 50. II. B. 7 (November, 1907) 112, fig. 37C, E, G, J, L; Ames Orch. 2 (1908) 81, 5 (1915) 64.

Babuyan Islands (Camiguin), Luzon (Apayao, Kalinga, Bontoc, Ifugao, Benguet, Nueva Vizcaya, Zambales, Bataan, Laguna, Sorsogon), Mindoro, Leyte. Probably the commonest and most widely distributed species of the genus in the Philippines, growing at fairly high altitudes on the mountains.

ACORIDIUM TENUE Ames in Proc. Biol. Soc. Wash. 19 (1906) 145.

Dendrochilum tenue Pfitz. in Engl. Pflanzenreich IV. 50. II. B. 7 (1907) 116, fig. 38M; Ames Orch. 2 (1908) 12, 86, t. 21, fig. V, 5, 5 (1915) 64.

Luzon (Bontoc, Ifugao, Laguna), Negros, Leyte, Mindanao (Misamis, Bukidnon, Agusan). One of the commoner and widely distributed species of the genus, growing in mossy forest at 500 to $2,160 \mathrm{~m}$ altitude.

ACORIDIUM TENUIFOLIUM Ames in Proc. Biol. Soc. Wash. 19 (1906) 145.

Dendrochilum tenuifolium Pfitz. in Engl. Pflanzenreich IV. 50. II. B. 7 (1907) 114, fig. 380; Ames Orch. 2 (1908) 12, 86, t. 21, fig. IV, 4, 5 (1915) 64; Merr. \& Merritt in Philip. Journ. Sci. 5 (1910) Bot. 340.

Luzon (Lepanto, Benguet, Albay). Rather common in Lepanto and Benguet, in mossy forest at 2,060 to $2,260 \mathrm{~m}$ altitude.

ACORIDIUM TURPE Ames in Proc. Biol. Soc. Wash. 19 (1906) 149.

Dendrochilum turpe Pfitz. in Engl. Pflanzenreich IV. 50. 11. B. 7 (1907) 1:18, fig. 38D; Ames Orch. 2 (1908) 12, 98, t. 23, fig. III, 3, 5 (1915) 64.

Luzon (Benguet), Merrill 4758, Ramos \& Edaño 30, 42. Mountain, regions at 500 to $2,260 \mathrm{~m}$ altitude.

ACORIDIUM UNICORNE Ames Orch. 7 (1922) 145.

Luzon (Benguet), Ramos \& Edaño 43a, 54. Mt. Simakoko, in mossy forest at $2,160 \mathrm{~m}$ altitude.

ACORIDIUM VANOVERBERGHII Ames Orch. 7 (1922) 82.

Dendrochilum vanoverberghii. Ames in Philip. Journ. Sci. 7 (1912) Bot. 27, Orch. 5 (1915) 65.

Luzon (Bontoc, Ifugao), Vanoverbergh 1046, B. S. 19893 McGregor. 
ACORIDIUM WENZELII Ames Orch. 7 (1922) 82.

Dendrochilum wenzelii Ames Orch. 5 (1915) 65.

Luzon (Kalinga, Camarines), Leyte, B. S. 22161 Ramos, 42088 Edañn, Wenzel 0264, 0398, 0787, 0803, 0862, 0876, 0880, 0886, Boettcher s. n. In forest at 500 to $700 \mathrm{~m}$ altitude.

ACORIDIUM WILLIAMSII Ames in Proc. Biol. Soc. Wash. 19 (1906) 143. Dendrochilum williamsii Pfitz. in Engl. Pflanzenreich IV. 50. 11. B. 7 (1907) 114, fig. 38S; Ames Orch. 2 (1908) 12, 82, t. 22, fig. I, 1, 5 (1915) 66.

Luzon (Lepanto, Benguet), Ramos \& Edaño 5, 38, R. S. Williams 1999. Mountain region at fairly high altitude.

EXCLUDED OR SYNONYMOUS SPECIES

ACORIDIUM ARACHNITES Rolfe in Orch. Rev. 12 (1904) $219=$ Dendrochilum arachnites Reichb. f.

ACORIDIUM COBBIANum Rolfe in Orch. Rev. 12 (1904) $220=$ Dendrochilum cobbianum Reichb. f.

ACORIDIUM CONVALLARIAEFORME Rolfe in Orch. Rev. 12 (1904) $220=$ Dendrochilum convallariaeforme Schauer.

Acoridium copelandi Ames in Proc. Biol. Soc. Wash. 19 (1906) $152=$ Dendrochilum copelandii Ames.

Acoridium cucullatum Ames in Proc. Biol. Soc. Wash. 19 (1906) $152=$ Dendrochilum cucullatum (Ames) Pfitz.

ACORIDIUM CUCUMERINUM Rolfe in Orch. Rev. 12 (1904) $220=$ Dendrochilum cucumerinum Reichb. f., spec. excl.

ACORIDIUM FILIFORME Rolfe in Orch. Rev. 12 (1904) $220=$ Dendrochilum filiforme Lindl.

ACORIDIUM GLUMACEUM Rolfe in Orch. Rev. 12 (1904) $220=$ Dendrochilum glumaceum Lindl.

ACORIDIUM GRACILISCAPUM Ames in Proc. Biol. Soc. Wash. 19 (1906) $152=$ Dendrochilum graciliscapüm (Ames) Pfitz.

ACORIDIUM HUTCHINsonil Ames Orch. 7 (1922) 81, sphalm $=$ Acoridium hutchinsonianum Ames.

ACORIDIUM LATIFOLIUM Rolfe in Orch. Rev. 12 (1904) $220=$ Dendrochitum latifolium Lindl.

ACORIDIUM LINEARIFOLIUM Ames Orch. 7 (1922) 141, non Rolfe $=$ Acoridium montanum Ames.

Acoridium longILABRe Ames in Proc. Biol. Soc. Wash. 19 (1904) $152=$ Dendrochilum longilabre (Ames) Pfitz.

ACORIDIUM Magnum Rolfe in Orch. Rev. 12 (1904) $220=$ Dendrochilum magnum Reichb. f.

Acoridium parvulum Ames in Proc. Biol. Soc. Wash. 19 (1904) $146=$ Dendrochilum parvulum (Ames) Pfitz.

ACoRidium PhilipPinense Ames in Proc. Biol. Soc. Wash. 19 (1904) $149=$ Dendrochilum philippinense (Ames) Pfitz. 
Acoridium uncatum Rolfe in Orch. Rev. 12 (1904) $220=$ Dendrochilum uncatum Reichb. f.

Acoridium venustulum Ames in Proc. Biol. Soc. Wash. 19 (September, 1906) $147=$ Acoridium microchilum (Schltr.) Ames.

Acoridium WHITFondi Rolfe ex Ames Orch. 1 (1905) $73=$ Acoridium rhombophorum (Reichb. f.) Ames.

\section{DENDROCHILUM Blume}

DENDROCHILUM ARACHNITES Reichb. f. in Gard. Chron. II 17 (1882) 256 ; J. J. Sm. in Rec. Trav. Bot. Néerl. 1 (1904) 65; Ames in Philip. Journ. Sci. 2 (1907) Bot. 316; Pfitz. in Engl. Pflanzenreich IV. 50. II. B. 7 (1907) 92; Ames Orch. 2 (1908) 119, text cut; 5 (1915) 55; Merr. \& Merritt in Philip. Journ. Sci. 5 (1910) Bot. 340 .

Platyclinis arachnites Rolfe in Kew Hand-list Orch. (1896) 189, nomen.

Acoridium arachnites Rolfe in Orch. Rev. 12 (1904) 219.

Luzon (Lepanto, Benguet, Rizal), Mindoro, Leyte, Mindanao (Zamboanga, Bukidnon, Agusan, Davao). A common and rather widely distributed species. Epiphyte in forest at 660 to $2,300 \mathrm{~m}$ altitude.

DENDROCHILUM BICALLOSUM Ames in Philip. Journ. Sci. 2 (1907) Bot. 317, nomen, Orch. 2 (1908) 117, text cut, 5 (1915) 56, in Philip. Journ. Sci. 4 (1909) Bot. 596.

Luzon (Laguna, Camarines), Mindoro, F. B. 7797 Curran \& Merritt, B. S. 21983 Ramos, 26674 Brown, 26438 Catalan, Santos s. n., Merrill 5812. Mountain regions, epiphyte in trees at 300 to $830 \mathrm{~m}$ altitude.

Var. MINOR Ames in Philip. Journ. Sci. 2 (1907) Bot. 317, nomen, Orch. 2 (1908) 118.

Mindoro, Merrill 5663. Mt. Halcon.

DENDROCHILUM CAGAYANENSE Ames in Philip. Journ. Sci. 6 (1911) Bot. 41, Orch. 5 (1915) 56.

Luzon (Cagayan, Apayao, Rizal, Laguna), Leyte, Mindanao (Davao), F. B. 16722 Curran, B. S. 28425 Fenix, 15392, 16583 Ramos, Reillo s. n., Wenzel 0512, 0532, 0602, Elmer 11844. Epiphyte, in forest, up to $700 \mathrm{~m}$ altitude in Leyte.

DENDROCHILUM CLEMENSIAE Ames Orch. 2 (1908) 109, text cut, 5 (1915) 56.

Mindanao (Lanao), M. S. Clemens s. n., 642.

DENDROCHILUM COBBIANUM Reichb. f. in Gard. Chron. II 14 (1880) 748; J. J. Sm. in Rec. Trav. Bot. Néerl. 1 (1904) 75; Pfitz. in Engl. Pflanzenreich IV. 50. II. B. 7 (1907) 101; Ames Orch. 2 (1908) 112, text cut, 5 (1915) 56.

Platyclinis cobbiana Hemsl. in Gard. Chron. II 16 (1881) 656; Veitch Man. Orch. Pl. pt. 5 (1889) 80.

Acoridium cobbianum Rolfe in Orch. Rev. 12 (1904) 220.

Luzon (Bontoc, Benguet), Mindanao (Agusan). A fairly common species in Luzon at 1,400 to $2,400 \mathrm{~m}$ altitude. 
DENDROCHILUM CONVALLARIAEFORME Schauex in Nov. Act. Nat. Cur. 19, Suppl. 1 (1843) 429; Pfitz. in Engl. Pflanzenreich IV. 50. II. B. 7 (1907) 97 (excl. syn. Acoridium copelandii Ames); Ames Orch. 5 (1915) 56.

Acoridium convallariaeforme Rolfe in Orch. Rev. 12 (1904) 220.

Manila, Meyen.

No specimens of this species have been seen.

DENDROCHILUM COPELANDII Ames Orch. 2 (1908) 12, 115, 1 . 竹, firg. I, 1,5 (1915) 56.

Acoridium copelandii Ames in Proc. Biol. Soc. Wash. 19 (1906) 153. Mindanao (Zamboanga), Copeland s. $n$.

DENDROCHILUM CUCULLATUM (Ames) Pfitz. in Engl. Pflanzenreich IV. 50. II. B. 7 (1907) 98, fig. 38J; Ames Orch. 2 (1908) 12, 115, t. 24, fig. III, 3, 5 (1915) 56.

Acoridium cucullatum Ames in Proc. Biol. Soc. Wash. 19 (1906) 153.

Mindanao (Davao), Copeland s. n. Mt. Apo, on mossy trees at 2,000 m. altitude.

DENDROCHILUM CYMBIFORME Ames in Philip. Journ. Sci. 6 (1911) Bot. 41, Orch. 5 (1915) 57.

Luzon (Abra, Kalinga), B. S. 7133,7135 Ramos, Boettcher s. n. Mt. Bawagan and Mt. Pastan, at 550 to $2,000 \mathrm{~m}$ altitude.

DENDROCHILUM ECALLOSUM Ames Orch. 6 (1920) 282.

Catanduanes, B. S. 30240 Ramos. Mt. Mariquidon, on tree in forest at $140 \mathrm{~m}$ altitude.

DENDROCHILUM FILIFORME Lindi. in Bot. Reg. 26 (1840) Misc. 52; Regel in Gartenfl. 18 (1869) 33, t. 604; Naves Novis. App. (1882) 231; J. J. Sm. in Rec. Trav. Bot. Néerl. 1 (1904) 68; Pfitz. in Engl. Pflanzenreich IV. 50. II. B. 7 (1907) 97; Ames Orch. 2 (1908) 113, text cut, 5 (1915) 57.

Dendrochilum glumaceum André in Ill. Hort. 25 (1878) t. 323, non Lindl.

Platyclinis filiformis Benth. ex Hemsl. in Gard. Chron. II 16 (1881) 656; Veitch Man. Orch. Pl. pt. 5 (1889) 80; Cogn. in Dict. Icon. Orch. (1902) Platyclinis t. 1.

Acoridium filiforme Rolfe in Orch. Rev. 12 (1904) 220.

Luzon (Rizal), Cuming s.n., Ramos s. n. Up to $660 \mathrm{~m}$ altitude.

DENDROCHILUM GLUMACEUM Lindl. in Bot. Reg. 27 (1841) Misc. 23; Hook. in Bot. Mag. 81 (1855) t. 4853; Miq. Fl. Ind. Bat. 3 (1859) 627; Reichb. f. in Walp. Ann. 6 (1861) 218; Batem. Second Cent. Orch. Pl. (1867) t. 134; Naves Novis. App. (1882) 231; J. J. Sm. in Rec. Trav. Bot. Néerl. 1 (1904) 76; Ames in Philip. Journ. Sci. 2. (1907) Bot. 317; Pfitz. in Engl. Pflanzenreich IV. 50. II. B. 7 (1907) 104; Ames Orch. 2 (1908) 110, text cut, 5 (1915) 57.

Platyclinis glumacea Benth. in Journ. Linn. Soc. 18 (1881) 295, by inference only; ex Hemsl. in Gard. Chron. II 16 (1881) 656; Vidal Phan. Cuming. Philip. (1885) 148, Rev. Pl. Vasc. Filip. (1886) 267; Pfitz. in Engl. \& Prantl Nat. Pflanzenfam. 2, Abt. 6 (1888) 128, 
fig. 127 B-F; Veitch Man. Orch. Pl. pt. 5 (1889) 81, t.; Cogn. in

Dict. Icon. Orch. (1902) Platyclinis t. 2; Ames Orch. 1 (1905) 74. Acoridium glumaceum Rolfe in Orch. Rev. 12 (1904) 220.

Luzon (Isabela, Bontoc, Lepanto, Benguet, Nueva Vizcaya, Pampanga, Bataan, Rizal, Laguna, Tayabas, Camarines), Catanduanes, Leyte, Mindanao (Davao). The commonest species of the genus in the Philippines, especially so in Luzon. Epiphyte in forest at 30 to $2,300 \mathrm{~m}$ altitude.

Var. VALIDUM (Rolfe) Pfitz. in Engl. Pflanzenreich IV. 50. II. B. 7 (1907) 104; Ames Orch. 2 (1908) 112.

Platyclinis glumacea Benth. ex Hemsl. var. valida Rolfe in Orch. Rev. 1 (1893) 115, 12 (1904) 73, fig. 18.

DENDROCHILUM GRACILISCAPUM (Ames) Pfitz. in Engl. Planzenreich IV. 50. II. B. 7 (1907) 99, fig. 38H; Ames Orch. 2 (1908) 12, 118, t. 24, fig. II, 2, 5 (1915) 57.

Acoridium graciliscapum Ames in Proc. Biol. Soc. Wash. 19 (1906) 152.

Mindanao (Davao), Copeland 1019, Elmer 10633, 1064\%, 11359. Mt. Apo, epiphyte on mossy trees at $1,800 \mathrm{~m}$ altitude.

DENDROCHILUM LATIFOLIUM Lindl. in Bot. Reg. 29 (1843) Misc. 56; J. J. Sm. in Rec. Trav. Bot. Néerl. 1 (1904) 71; Pfitz. in Engl. Pflanzenreich IV. 50. II. B. 7 (1907) 102, fig. B, C; Ames Orch. 2 (1908) 104, text cut, 5 (1915) 59.

Platyclinis latifolia Hemsl. in Gard. Chron. II 16 (1881) 656; Rendle in Journ. Bot. 34 (1896) 355; Ames Orch. 1 (1905) 74.

Acoridium latifolium Rolfe in Orch. Rev. 12 (1904) 220.

Luzon (Pampanga, Bataan, Tayabas, Camarines), Panay (Antique, Capiz), Negros (Occidental Negros), Mindanao (Lanao, Davao). A common species, especially in Luzon. Epiphyte in mountain regions at 300 to $1,000 \mathrm{~m}$ altitude.

DENDROCHILUM LONGILABRE (Ames) Pfitz. in Engl. Pflanzenreich IV.

50. II. B. 7 (1907) 99, fig. 38K; Ames Orch. 2 (1908) 12, 119, t. 24, fig. IV, 4, 5 (1915) 59.

Acoridium longilabre Ames in Proc. Biol. Soc. Wash. 19 (1906) 152.

Mindanao (Davao), Copeland 1025, ? Elmer 11419. Mt. Apo, epiphyte on mossy trees at $1,530 \mathrm{~m}$ altitude.

DENDROCHILUM LONGISPICATUM Ames Orch. 2 (1908) 105, text cut, 5 (1915) 59.

Luzon (Bontoc, Benguet), Sibuyan, Panay (Capiz), Negros (Oriental Negros), Vanoverbergh 899, 1533, 1729, 3871, 3940, 4043, R. S. Williams 1942, Merrill 4336, Elmer 9909, 12436, B. S. 35586, 35605 Martelino \& Edaño, 4462 Mearns. Epiphyte, in mountain regions up to $1,700 \mathrm{~m}$ altitude in Bontoc.

DENDROCHILUM MAGNUM Reichb. f. in Walp. Ann. 6 (1861) 240 ; Naves Novis. App. (1882) 231; J. J. Sm. in Rec. Trav. Bot. Néerl. 1 (1904) 74; Ames in Philip. Journ. Sci. 2 (1907) Bot. 317; Pfitz. in Engl. Pflanzenreich IV. 50. II. B. 7 (1907) 100, fig. $33 \mathrm{H}, J$;

Ames Orch. 2 (1908) 106, text cut, 5 (1915) 60.

Acoridium magnum Rolfe in Orch. Rev. 12 (1904) 220. 
Luzon (Rizal), Mindoro, Panay (Antique), Cuming s. n., Merrill 5611, 5730, Ramos s. n., B. S. 32383 McGregor. Mt. Halcon, in mossy forest at 2,000 $\mathrm{m}$ altitude, and Mt. Iriga at $1,630 \mathrm{~m}$ altitude.

DENDROCHILUM MALINDANGENSE Ames Orch. 2 (1908) 114, text cut, 5 (1915) 60.

Mindanao (Dapitan), F. B. 4597 Mearns \& Hutchinson. Mt. Malindang at $1,230 \mathrm{~m}$ altitude.

DENDROCHILUM MEARNSII Ames Orch. 2 (1908) 116, text cut, 5 (1915) 60 .

Camiguin de Misamis, Mindanao (Misamis, Bukidnon), B.S.14426 Ramos, 38523 Ramos \& Edaño, F. B. 4605 Mearns \& Hutchinson. Up to 3,060 m altitude on Mt. Malindang.

DENDROCHILUM NIVEUM Ames Orch. 6 (1920) 284.

Luzon (Kalinga), Boettcher s. $n$. Balbalan at $1,000 \mathrm{~m}$ altitude.

DENDROCHILUM PALAWANENSE Ames Orch. 2 (1908) 103, text cut, 5 (1915) 61, in Elm. Leafl. Philip. Bot. 5 (1912) 1559.

Palawan, B. S. 553, 557 Foxworthy, Elmer 13195. In mossy scrub at $1,250 \mathrm{~m}$ altitude.

DENDROCHILUM PANGASINANENSE Ames Sched. Orch. 6 (1923) 29.

Luzon (Pangasinan), B. S. 8286 Ramos.

DENDROCHILUM PRODIGIOSUM Ames Orch. 7 (1922) 98.

Mindanao (Bukidnon), B. S. 38521 Ramos \& Edaño. Mt. Lipa, in mossy forest at $2,160 \mathrm{~m}$ altitude.

DENDROCHILUM PROPINQUUM Ames Orch. 6 (1920) 285.

Leyte, Wenzel 0279, 0329, 0376, 0398, 0449, 0497, 0548, 0582, 0682, 0805, 0809, 085\%, 0868, 0911, 0920. Jaro, epiphyte in forest at 500 to $700 \mathrm{~m}$ altitude.

DENDROCHILUM RAMOSII Ames in Philip. Journ. Sci. 8 (1913) Bot. 410 , Orch. 5 (1915) 62.

Luzon (Laguna), B. S. 15003 Ramos, Reillo 4. San Antonio.

DENDROCHILUM UNCATUM Reichb. f. in Bonpl. 3 (1855) 222, in Walp. Ann. 6 (1864) 927, in Gard. Chron. II 16 (1881) 780; Naves Novis. App. (1882) 231; J. J. Sm. in Rec. Trav. Bot. Néerl. 1 (1904) 72 ; Pfitz. in Engl. Pflanzenreich IV. 50. II. B. 7 (1907) 103, fig. 35 G, H; Ames Orch. 2 (1908) 108, text cut, 5 (1915) 65; Merr. \& Merritt in Philip. Journ. Sci. 5 (1910) Bot. 340.

Dendrochilum uncatum Reichb. f. var. lancifolium Reichb. f. in Gard. Chron. II 16 (1881) 780; Pfitz. in Engl. Pflanzenreich IV. 50. II. B. 7 (1907) 104.

Platyclinis uncata Rolfe ex Vidal Phan. Cuming. Philip. (1885) 148;

Vidal Rev. Pl. Vasc. Filip. (1886) 267.

Acoridium uncatum Rolfe in Orch. Rev. 12 (1904) 220.

Luzon (Benguet, Nueva Vizcaya, Pampanga, Bataan, Rizal, Laguna); Panay (Antique). A fairly common species in the mountains of Luzon: especially of Benguet Subprovince, at 1,000 to $2,300 \mathrm{~m}$ altitude. 
DENDROCHILUM Weberi Ames in Philip. Journ. Sci. 8 (1913) Bot. 410, Orch. 5 (1915) 65.

Mindanao (Agusan), Basilan, Weber 59, B. S. 16240 Reillo. Type from Cabadbaran River at $80 \mathrm{~m}$ altitude.

\section{EXCLUDED OR SYNONYMOUS SPECIES}

Dendrochilum AFFine Ames Orch. 2 (1908) 95, 5 (1915) $55=$ Acoridium affine Ames.

Dendrochilum ANFractoides Ames Orch. 3 (1908) 13, t. 28, 5 (1915) 55, in Philip. Journ. Sci. 4 (1909) Bot. 665; Merr. \& Merritt in Philip. Journ.'Sci. 5 (1910) Bot. $340=$ Acoridium anfractoides Ames.

Dendrochilum anfractum (Ames) Pfitz. in Engl. Pflanzenreich IV. 50. II. B. 7 (1907) 118, fig. 38P; Ames Orch. 2 (1908) 91, t. 21, fig. I. 1, 5 (1915) $55=$ Acoridium anfractum Ames.

Dendrochilum AURICUlare Ames in Philip. Journ. Sci. 4 (1909) Bot. 595, Orch. 5 (1915) $55=$ Acoridium auriculare Ames.

Dendrochilum AURItum Reichb. f. apud Naves Novis. App. (1882) 231.

DendRochilum BINUANGEnse Ames Orch. 6 (1920) $281=$ Acoridium binuangense Ames.

Dendrochilum Bistortum (Wendl. \& Kränzl.) J. J. Sm. in Rec. Trav. Bot. Néerl. 1 (1904) 64; Pfitz. in Engl. Pflanzenreich IV. 50. II. B. 7 (1907) 96, fig. 34 A-D; Ames Orch. 5 (1915) 54.

Platyclinis bistorta Wendl. \& Kränzl. Xen. Orch. 3 (1900) 169, t. 299, fig. I. 1-5.

"Indischer Archipel. Insel Maschate (?) leg. W. Micholitz, imp. F. Sander."-Wendland and Kränzlin. Merrill writes: "I do not know of any Malayan island by this name and suspect that Masbate of the Phil. ippine Archipelago was intended; Micholitz has carried on extensive explorations in the Philippines." It has not been possible to refer this to any species of Philippine origin.

Dendrochilum cinnabarinum Pfitz. in Engl. Pflanzenreich IV. 50. II. B. 7 (1907) 104, fig. 35F; Ames Orch. 3 (1908) 10, 5 (1915) 56, 6 (1920) 282, in Philip. Journ. Sci. 4 (1909) Bot. 597; Merr. \& Merritt in Philip. Journ. Sci. 5 (1910) Bot. $340=$ Acoridium cinnabarinum (Pfitz.) Ames.

Dendrochilum confusum Ames in Philip. Journ. Sci. 6 (1911) Bot. 41, Orch. 5 (1915) $56=$ Acoridium confusum Ames.

Dendrochilum cucumerinum Reichb. f. in Gard. Chron. II 22 (1884) 56, 649; J. J. Sm. in Rec. Trav. Bot. Néerl. 1 (1904) 71; Pfitz. in Engl. Pflanzenreich IV. 50. II. B. 7 (1907) 103, fig. 35D, E; Ames Orch. 5 (1915) 54, 57.

Platyclinis cucumerina Rolfe in Kew Hand-list Orch. (1896) 189, nomen.

Acoridium cucumerinum Rolfe in Orch. Rev. 12 (1904) 220.

This was described from cultivated specimens of unknown origin and has been ascribed to the Philippines; it may prove to be a native of some other region. 
DENDROCHILUM CURRANiI Ames Orch. 3 (1908) 15, t. 29, 5 (1915) $57=$ Acoridium curranii Ames.

Dendrochilum ElMeri Ames in Elm. Leafl. Philip. Bot. 5 (1912) 1558, Orch. 5 (1915) $57=$ Acoridium elmeri Ames.

Dendrochilum exile Ames in Philip. Journ. Sci. 2 (1907) Bot. 317, nomen, Oxch. 2 (1908) 84, text cut, 5 (1915) $57=$ Acoridium exile Ames.

DENDROCHILUM FOXWORTHYI Ames Orch. 3 (1908) 8, t. 26, 5 (1915) 57 $=$ Acoridium foxworthyi Ames.

Dendrochilum GLUMACEum André in Ill. Hort. 25 (1878) t. 323, non LindI. $=$ Dendrochilum filiforme Lindl.

Dendrochilum GRAminifolium (Ames) Pfitz. in Engl. Pflanzenreich IV. 50. II. B. 7 (1907) 114, fig. 38 Q, R; Ames Orch. 2 (1908) 12, 83, t. 22, fig. II, 2, 5 (1915) $57=$ Acoridium graminifolium Ames.

DENDROCHILUM GRANDIFLORUM Schltr. in Fedde Repert. 8 (1910) 563; Ames Orch. 5 (1915) 54, 58, non Pfitz.

The geographic origin of this species is not certainly known; Schlechter" stated "Philippinen?," but a drawing received from him is marked "India."

This species is very close to Dendrochilum latifolium Lindl.

Dendrochilum Hastatum Ames in Philip. Journ. Sci. 2 (1907) Bot. 317, nomen, Orch. 2 (1908) 99, text cut, 5 (1915) $58=$ Acoridium hastatum Ames.

Dendrochilum hutchinsonianum Ames in Philip. Journ. Sci. 2 (1907) Bot. 317, nomen, Orch. 2 (1908) 96, text cut, 5 (1915) 58, 7 (1922) 81, in syn. (as D. hutchinsonii) $=$ Acoridium hutchinsonianum Ames.

Dendrochilum hymenanthum Reichb. f. apud Vidal Phan. Cuming. Philip. (1885) 149; Vidal Rev. Pl. Vasc. Filip. (1886) 268, sphalm = Dendrobium hymenanthum Reichb. $f$.

DENDROCHILUM IRIGENSE Ames Orch. 5 (1915) $58=$ Acoridium irigense Ames.

Dendrochilum Junceum Reichb. f. Otia Bot. Hamb. 1 (1878) 54, Xen. Orch. 3 (1881) 30 ; Naves Novis. App. (1882) 231; J. J. Sm. in Rec. Trav. Bot. Néerl. 1 (1904) 78 = Acoridium tenellum Nees \& Meyen.

DENDROCHILUM LOHERI Ames Orch. 3 (1908) 12, t. 27, I, A, 5 (1915) 59, 6 (1920) 283; Merr. \& Merritt in Philip. Journ. Sci. 5 (1910) Bot. $340=$ Acoridium loheri Ames.

Dendrochilum longibulbum Ames in Philip. Journ. Sci. 7 (1912) Bot. 26, Orch. 5 (1915) $59=$ Acoridium longibulbum Ames.

Dendrochilum longifolium Reichb. f. apud Pfitz. in Engl. Pflanzenreich IV. 50. II. B. 7 (1907) 108, fig. $36 \mathrm{~B}$ (as to Jagor 680).

Jagor 680 is a specimen from Luzon, probably incorrectly identified by Pfitzer who had not seen the type of the species, which is a native of the Malay Peninsula.

Dendrochilum Lucbanense Ames in Elm. Leafl. Philip. Bot. 5 (1912) 1559. Orch. 5 (1915) $60=$ Acoridium lucbanense Ames. 
Dendrochilum LUzonense Ames Orch. 2 (1908) 121, text cut, 5 (1915) $60=$ Acoridium luzonense Ames.

Dendrochilum MacgregoriI Ames in Philip. Journ. Sci. 6 (1911) Bot. 42, Orch. 5 (1915) $60=$ Acoridium macgregorii Ames.

Dendorochilum MaLeolens Kränzl. in Orchis 2 (1908) 63; Ames Orch. 5 (1915) 54, $60=$ Acoridium maleolens Ames.

Deñrochilum Merrillit (Ames) Pfitz. in Engl. Pflanzenreich IV. 50. II. B. 7 (1907) 119, fig. 38 A, B; Ames Orch. 2 (1908) 12, 102, t. 23, fig. I, 1, 5 (1915) $60=$ Acoridium merrillii Ames.

Dendrochilum Microchilum (Schltr.) Ames Orch. 2 (1908) 87, in textu, 5 (1915) 60, in Philip. Journ. Sci. 7 (1912) Bot. $26=$ Acoridium microchilum (Schltr.) Ames.

Dendrochilum mindorense Ames in Philip. Journ. Sci. 2 (1907) Bot. 317, nomen, Orch. 2 (1908) 91, text cut, 5 (1915) 60 = Acoridium mindorense Ames.

Dendrochilum ocellatum (Ames) Pfitz. in Engl. Pflanzenreich IV. 50. II. B. 7 (1907) 117, fig. 38 C; Ames Orch. 2 (1908) 12, 101, t. 23, fig. II, 2, 5 (1915) 61, in Philip. Journ. Sci. 4 (1909) Bot. $596=$ Acoridium ocellatum Ames.

Dendrochilum oliganthum (Ames) Pfitz. in Engl. Pflanzenreich IV. 50. II. B. 7 (1907) 117, fig. 38 E; Ames Orch. 2 (1909) 12, 98, t. 23, fig. IV, 4, 5 (1915) $61=$ Acoridium oliganthum Ames.

Dendrochilum Parvulum (Ames) Pfitz. in Engl. Pflanzenreich IV. 50. II. B. 7 (1907) 116, fig. $98 \mathrm{~N}$; Ames Orch. 2 (1908) 12, 87, t. 21, fig. II, 2, 5 (1915) $61=$ Acoridium parvulum Ames.

Dendrochilum Philippinense (Ames) Pfitz. in Engl. Pflanzenreich IV. 50. II. B. 7 (1907) 118, fig. $38 \mathrm{~F}$; Ames Orch. 2 (1908) 12, 97, t. 23, fig. $V, 5,5$ (1915) 61, in Philip. Journ. Sci. 4 (1909) Bot. $595=$ Acoridium philippinense Ames.

Dendrochilum Pulogense Ames in Philip. Journ. Sci. 4 (1909) 594; Merr. \& Merritt in Philip. Journ. Sci. 5 (1910) Bot. 340; Ames Orch. 5 (1915) $61=$ Acoridium pulogense Ames.

Dendrochilum Pumilum Reichb. f. in Bonpl. 3 (1855) 222, in Walp. Ann. 6 (1864) 927; Naves Novis. App. (1882) 231; Vidal Phan. Cuming. Philip. (1885) 81, 149, Rev. Pl. Vasc. Filip. (1886) 268; J. J. Sm. in Rec. Trav. Bot. Néerl. 1 (1904) 58; Ames in Philip. Journ. Sci. 2 (1907) Bot. 317, 4 (1909) Bot. 596; Pfitz. in Engl. Pflanzenreich IV. 50. 11. B. 7 (1907) 162; Ames Orch. 2 (1908) 93, text cut, 3 (1908) 6, 5 (1915) $61=$ Acoridium pumilum (Reichb. f.) Rolfe.

Dendrochilum PuRPureum Ames Orch. 6 (1920) 286 = Acoridium purpureum Ames.

Dendrochilum QUADRILOBum Ames Orch. 5 (1915) $61=$ Acoridium quadrilobum Ames. 
Dendrochilum Recurvum Ames in Philip. Journ. Sci. 2 (July, 1907) Bot. 318; Pfitz. in Engl. Pflanzenreich IV. 50. II. B. 7 (November, (1907) 117, fig. 38 T; Ames Orch. 2 (1908) 12, 90, t. 22, fig. IV, 4, 5 (1915) $62=$ Acoridium recurvum Ames.

DeNDROCHILUM RENIFORME Ames Orch. 5 (1915) 63 = Acoridium reniforme Ames.

Dendroehilum RHOMBophoRum (Reichb. f.) Ames Orch. 3 (1908) 7, 5 (1915) $64=$ Acoridium rhombophorum (Reichb. f.) Ames.

Dendrochilum SACColabium Kränzl, in Ann. k. k. Hofmus. Wien 30 (1916) $56=$ Acoridium saccolabium (Kränzl.) Ames.

Dendrochilum simulacrum Ames Orch. 2 (1908) 92, text cut, 5 (1915) 64 $=$ Acoridium simulacrum Ames.

Dendrochilum Sfhacelatum (Ames) Pfitz. in Engl. Pflanzenreich IV. 50. II. B. 7 (1907) 112, fig. 37 A, B, D, F. H. K; Ames Orch. 2 (1908) 82,5 (1915) 64, 6 (1920) $287=$ Acoridium sphacelatum Ames.

Dendrochilum strictiforme (Ames) Pfitz. in Engl. Pflanzenreich IV. 50. II. B. 7 (1907) 116, fig. $38 \mathrm{~L}$; Ames Orch. 2 (1908) 12, 89, t. 21, fig. III, 3, 5 (1915) 64, in Philip. Journ. Sci. 4 (1909) Bot. $597=$ Acoridium strictiforme Ames.

Dendrochilum tenellum (Nees \& Meyen) Ames in Philip. Journ. Sci. 2 (July, 1907) Bot. 318; Pfitz. in Engl. Pflanzenreich IV. 50. II. B. 7 (November, 1907) 112, fig. 37 C, E, G, J, L; Ames Orch. 2 (1908) 81, 5 (1915) $64=$ Acoridium tenellum Nees \& Meyen.

Dendrochilum tenue (Ames) Pfitz. in Engl. Pflanzenreich IV. 50. 11. B. 7 (1907) 116, fig. $38 \mathrm{M}$; Ames Orch. 2 (1908) 12, 86, t. 21, fig. $V, 5,5$ (1915) $64=$ Acoridium tenue Ames.

Dendrochilum tenuifolium (Ames) Pfitz. in Engl. Pflanzenreich IV. 50. II. B. 7 (1907) 114, fig. 38 O; Ames Orch. 2 (1908) 12, 85, t. 21, fig. IV, 4, 5 (1915) 64; Merr. \& Merritt in Philip. Journ. Sci. 5 (1910) Bot. $340=$ Acoridium tenuifolium Ames.

Dendrochilum turpe (Ames) Pfitz, in Engl. Pflanzenreich IV. 50. II. B. 7 (1907) 118, fig. 38 D; Ames Orch. 2 (1908) 12, 98, t. 23, fig. III, 3,5 (1915) $64=$ Acoridium turpe Ames.

Dendrochilum vanoverberghil Ames in Philip. Journ. Sci. 7 (1912) Bot. 27, Orch. 5 (1915) $65=$ Acoridium vanoverberghii Ames.

Dendrochilum venustulum (Ames) Pfitz. in Engl. Pflanzen reich IV. 50. II. B. 7 (1907) 116, fig. $38 \mathrm{U}$; Ames Orch. 2 (1908) 12, 88, t. 22, fig. III, 3, in Philip. Journ. Sci. 4 (1909) Bot. 595; Merrr. \& Merritt in Philip. Journ. Sci: 5 (1910) Bot. $341=$ Acoridium microchilum (Schltr.) Ames.

Dendrochilum Wenzelit Ames Orch. 5 (1915) $65=$ Acoridium wenzelii Ames. 
Dendrochilum whitfordi (Rolfe) Pfitz. \& Kränzl. in Engl. Pflanzenreich IV. 50. 11. B. 7 (1907) 162; Ames Orch. 2 (1908) 100, text cut, 5 (1915) $66=$ Acoridium rhombophorum (Reichb. f.) Ames.

Dendrochilum williamsil Pfitz. in Engl. Pflanzenreich IV. 50. II. B. 7 (1907) 114, fig. $38 \mathrm{~S}$; Ames Orch. 2 (1908) 12, 82, t. 22, fig. 1, 1, 5.(1915) $66=$ Acoridium williamsii Ames.

Pendrochilum woodianum Ames in Philip. Journ. Sci. 2 (1907) Bot. 318, nomen, Orch. 2 (1908) 80, text cut, 5 (1915) $66=$ Pseudacoridium woodianum Ames.

\section{PHOLIDOTA Lindley}

PHOLIDOTA CARNEA (Blume) Lindl. Gen. \& Sp. Orch. (1830) 37; Miq. Fl. Ind. Bat. 3 (1859) 669; J. J. Sm. Fl. Buitenz. 6 (Orch. Jav.) (1905) 158, Fig.-Atlas (1909) fig. 118; Ames Orch. 6 (1920) 66, in Merr. in Journ. Roy. Asiat. Soc. Straits Branch, Special No. (1921) 149, Sched. Orch. 6 (1923) 20.

Crinonia carnea Blume Bijdr. (1825) 339.

Coelogyne carnea Reichb. f. in Walp. Ann. 6 (1861) 237.

Crinonia elmeri Ames Orch. 5 (1915) 67, nomen.

Mindanao, Elmer 13834.

PHOLIDOTA IMBRICATA Lindl. in Hook. Exot. Fl. 2 (January, 1825) t. 138, in Bot. Reg. 14 (1829) t. 1213, 21 (1835) t. 17\%7, Gen.\& Sp. Orch. (1830) 36; Lodd. Bot. Cab. 20 (1833) t. 1934; Wight Ícon. $3^{2}$ (1849) 9, t. 907; Dalz. \& Gibs. Bomb. Fl. (1861) 262; Thw. Enum. Pl. Zeyl. (1864) 300; Benth. Fl. Austral. 6 (1873) 290; Naves Novis. App. (1882) 237; Vidal Phan. Cuming. Philip. (1885) 149, Rev. P1. Vasc. Filip. (1886) 269; Fitzger. Austral. Orch. ${ }^{4}$ (1893) t.; Hook. f. Fl. Brit. Ind. 5 (1890) 845; Grant Orch. Burma (1895) . 161; Ridl. in Journ. Linn. Soc. 31 (1896) 287, 32 (1896) 328; King \& Pantl. in Ann. Roy. Bot. Gard. Calc. 8 (Orch. Sik.-Himal.) (1898) 144, t. 201; Trim. Handb. F1. Ceyl. 4 (1898) 162; J. J. Sm. Fl. Buitenz. 6 (Orch. Jav.) (1905) 151, Fig.-Atlas (1909) fig. 112; Ames Orch. 1 (1905) 72, 2 (1908) 75, 5 (1915) 66; Duthie in Ann. Roy. Bot. Gard. Calc. 9 (Orch. North-west. Himal.) (1906) 115; Pfitz. \& Kränzl. in Engl. Pflanzenreich IV. 50. II. B. 7 (1907) 154; Ridl. Mat. Fl. Mal. Penin. 1 (1907) 135; J. J. Sm. in Nova Guinea 8 (1909) Bot. 21, $8^{3}$ (1912) Bot. 527, 12 (1913) Bot. 11; Schltr. in Engl. Bot. Jahrb. 45, Beibl. 104 (1911) 11, in Fedde Repert. Beihefte 4 (Orch. Sino-Jap. Prodr.) (1919) 188; Ames in Merr. in Journ. Roy. Asiat. Soc. Straits Branch, Special No. (1921) 149.

Cymbidium imbricatum Roxb. Hort. Beng. (1814) 63, nomen, Fl. Ind. ed. 2,3 (1832) 460.

Ptilocnema bracteatum D. Don Prodr. Fl. Nepal. (February, 1825) 33. Onithidium imbricatum Wall. ex Lindl. in Bot. Reg. 14 (1829) sub t. 1213, in syn.

Pholidota pallida Lindl. in Bot. Reg. 21 (1835) sub t. 1777; Pfitz. \& Kränzl. in Engl. Pflanzenreich IV. 50. II. B. 7 (1907) 153. 
Pholidota conchoidea Lindl. in Bot. Reg. 26 (1840) Misc. 84; Pfitz. \& Kränzl, in Engl. Pflanzenreich IV. 50. II. B. 7 (1907) 154, fig. $50 \mathrm{~K}$.

Pholidota loricata Reichb. f. in Bonpl. 5 (1857) 43; Pfit\%. \& Kränzl. in Engl. Pflanzenreich IV. 50. II. B. 7 (1907) 153.

Coelogyne conchoidec Reichb. f. in Walp. Ann. 6 (1861) 237; Naves Novis. App. (1882) 237.

Coelogyne imbricata Reichb. f. in Walp. Ann. 6 (1861) 238.

Coelogyne pallida Reichb. f. in Walp. Ann. 6 (1861) 238.

Coelogyne triotos Reichb. $f$. in Walp. Ann. 6 (1861) 238, in Hamb.

Gartenz. 18 (1862) 34; Naves Novis. App. (1882) 237.

Pholidota triotos Reichb. f. in Walp. Ann. 6 (1861) 238, in syn.; Pfitz.

in Engl. Pflanzenreich IV. 50. II. B. 7 (1907) 154.

Luzon (Bontoc, Benguet, Bulacan, Bataan, Rizal), Polillo, Panay (Capiz), Samar, Leyte, Palawan, Mindanao (Zamboanga, Lanao, Davao), Basilan. A common and widely distributed species in the Philippines at 15 to $1,600 \mathrm{~m}$ altitude. In India, Burma, Assam, Siam, Malay Peninsula, China, Sumatra, Java, Celebes, Borneo, and New Guinea.

PHOLIDOTA VENTRICOSA (Blume) Reichb. f。 in Bonpl. 5 (1857) 43 ; Rolfe in Gard. Chron. III 5 (1889) 585; J. J. Sm. Fl. Buitenz. 6 (Orch. Jav.) (1905) 154, Fig.-Atlas (1909) fig. 114; Pfitz. in Engl. Pflanzenreich IV. 50. II. B. 7 (1907) 148, fig. 51; Ames Orch. 2 (1908) 75, 5 (1915) 66, in Philip. Journ. Sci. 4 (1909) Bot. 665; J. J. Sm. in Bull. Dept. Agr. Ind. Néerl. 43 (1910) 13; Ames in Merr. in Journ. Roy. Asiat. Soc. Straits Branch, Special No. (1921) 150.

Chelonanthera ventricosa Blume Bijdr. (1825) 383, fig. 51; Lindl. Gen. \& Sp. Orch. (1833) 178; Miq. Fl. Ind. Bat. 3 (1859) 673.

Coelogyne ventricosa Reichb. f. in Walp. Ann. 6 (1861) 237.

Pholidota sesquitorta Kränzl. Xen. Orch. 3 (1893) 114, t. 266, fig. I. 1-7; Pfitz. \& Kränzl. in Engl. Pflanzenreich IV. 50. II. B. 7 (1907) 146, fig. $50 \mathrm{~F}$.

Luzon (Lepanto), Mindanao (Zamboanga), F. B. 5715 Klemme, Copeland 1621. At about $300 \mathrm{~m}$ altitude in Mindanao. In Sumatra, Java, and introduced into Borneo.

\section{EXCLUDED OR SYNONYMOUS SPECIES}

Pholidota conchoidea Lindl. in Bot. Reg. 26 (1840) Misc. 84; Pfitz. \& Kränzl. in Engl. Pflanzenreich IV. 50. 11. B. 7 (1907) 154, fig. $50 K=$ Pholidota imbricata Lindl.

Pholidota elmeri Ames in Elm. Leaf. Philip. Bot. 5 (1912) $1557=$ Thecostele alata (Roxb.) Par. \& Reichb. f.

Pholidota grobosa Lindl. apud Naves Novis. App. (1882) 237.

Pholidota membranacea Teijsm. \& Binn. apud Naves Novis. App. (1882) 237.

Pholidota RHOMbophora Reichb. f. in Linnaea 41 (1877) 116, in textu; Pfitz. \& Kränzl. in Engl. Pflanzenreich IV. 50. II. B. 7 (1907) 153 = Acoridium rhombophorum (Reichb. f.) Ames. 
Pholidota triotos Reichb. f. in Walp. Ann. 6 (1861) 238, in syn.; Pfitz. in Engl. Pflanzenreich IV. 50. II. B. 7 (1907) $154=$ Pholidota imbricata Lindl.

44. MALAXIS Solander ex Swartz

MALAXIS ACUMINATA D. Don Prodr. Fl. Nepal. (1825) 29.

Microstylis wallichii Lindl. in Wall. Cat. (1828) No. 1938, nomen. Gen. \& Sp. Orch. (1830) 20; Ridl. in Journ. Linn. Soc. 24 (1888) 338.

Var. BILOBA (Lindl.) Ames comb. nov.

Microstylis biloba Lindl. in Wall. Cat. (1828) No. 1940, nomen, Gen. \& Sp. Orch. (1830) 20; Par. \& Reichb. f. in Trans. Linn. Soc. 30 (1874) 139.

Dienia carinata Reichb. f. in Bonpl. 3 (1855) 223.

Microstylis carinata Reichb. f. in Walp. Ann. 6 (1861) 207; Naves Novis. App. (1882) 230; Vidal Phan. Cuming. Philip. (1885) 148, Rev. Pl. Vasc. Filip. (1886) 266; Ridl. in Journ. Linn. Soc. 24 (1888) 338.

Microstylis wallichii Lindl. var. biloba Hook. f. Fl. Brit. Ind. 5 (1888) 686; Grant Orch. Burma (1895) 18; King \& Pantl. in Ann. Roy. Bot. Gard. Calc. 8 (Orch. Sik.-Himal.) (1898) 16, t. 19; Duthie in Ann. Roy. Bot. Gard. Calc. 9 (Orch. North-west. Himal.) (1906) 88.

Malaxis biloba Ames Orch. 2 (1908) 122, 5 (1915) 69.

Luzon (Bontoc, Bataan, Rizal, Cavite, Batangas, Laguna), Cuming 2144, Loher s. n., B. S. 1468, 3244 Ahern's collector, 1472, 4562, 13555 , 2133\%, 22384a Ramos, 29515 Ramos \& Edaño, Merrill 4175, Leiberg 6081, Native collector s. n., Vanoverbergh 1349, F. B. 24298, 24298bis Bawan \& Borromeo. Terrestrial in forest. In India and Burma. The species proper does not occur in the Philippines.

MALAXIS ALAGENSIS Ames Orch. 2 (1908) 122, text cut, 5 (1915) 68.

Microstylis alagensis Ames in Philip. Journ. Sci. 2 (1907) Bot. 318.

Luzon (Tayabas), Mindoro, B. S. 25392, 25432, 25543 Yates, Merrill, 5801,5807, Ramos s. $n$. Terrestrial in humid forest at 130 to $400 \mathrm{~m}$ altitude.

MALAXIS ARIETINA Ames in Philip. Journ. Sci. 8 (1913) Bot. 411, Orch. 5 (1915) 68.

Mindanao (Camiguin Island, Agusan), B. S. 14418, 14443 Ramos, Wéber 44, 69. Terrestrial up to $315 \mathrm{~m}$ altitude in Agusan.

MALAXIS ATRosanguinea Ames Sched. Orch. 6 (1923) 30, fig. 7.

Mindanao (Agusan), Elmer 13815.

MALAXIS BALABACENSIS Ames in Philip. Journ. Sci. 6 (1911) Bot. 42, Orch. 5 (1915) 68.

Luzon (Cavite, Laguna, Sorsogon), Leyte, Palawan, Balabac, Mindanao (Camiguin Island, Lanao, Bukidnon, Agusan, Surigao), Sibutu. A fairly common and rather widely distributed species. Terrestrial, in the forest at relatively low altitude, up to $130 \mathrm{~m}$ in Agusan and at $800 \mathrm{~m}$ in Cavite. 
MALAXIS BANCANOIDES Ames Orch. 2 (1908) 129, text cut, 5 (1915) 68.

Sibuyan, McGregor 14. Mountains back of San Fernando, terrestrial at about $660 \mathrm{~m}$ altitude.

MALAXIS BATAANENSIS Ames in Philip. Journ. Sci. 6 (1911) Bot. 43, Orch. 5 (1915) 69:

Malaxis flavescens Ames Orch. 2 (1908) 127, 5 (1915) 70 (excl. syn.).

Luzon (Bataan), B. S. 1674 Foxworthy, 6211 Robinson, Merrill 3885. Lamao Forest Reserve, Mt. Mariveles, at 800 to $1,000 \mathrm{~m}$ altitude.

MALAXIS BENGUeTENSIS Ames in Philip. Journ. Sci. 6 (1911) Bot. 43, Orch. 5 (1915) 69.

Luzon (Benguet), B. S. 8362 McGregor, Boettcher s.n. Pauai at 2,100 $m$ altitude.

MALAXIS BINABAYENSIS Ames Orch. 2 (1908) 124, text cut, 5 (1915) 69.

Microstylis binabayensis Ames in Philip. Journ. Sci. 2 (1907) Bot. 318.

Mindoro, Merrill 5804. Binabay River, terrestrial in forest at $330 \mathrm{~m}$ altitude.

MALAXIS BRACTEOSA Ames Orch. 7 (1922) 111.

Luzon (Sorsogon), Mindanao (Bukidnon, Dapitan), B. S. 38658, 386\%.? Ramos \& Edaño, F. B. 4575 Mearns \& Hutchinson, Elmer 15698. Mahilucot River, terrestrial along streams, in forest at 100 to $1,400 \mathrm{~m}$ altitude.

MALAXIS BULUSANENSIS Ames Sched. Orch. 6 (1923) 33.

Luzion (Sorsogon), Elmer 14830.

MALAXIS COPELANDII Ames Orch. 2 (1908) 124, text cut, 5 (1915) 69.

Mindanao (Zamboanga), Copeland s. $n$.

MALAXIS CUNEIPETALA Ames Orch. 6 (1920) 288.

Leyte, Wenzel 0724. Jaro, terrestrial in forest at $800 \mathrm{~m}$ altitude.

MALAXIS CURRANII Ames in Philip. Journ. Sci. 6 (1911) Bot. 44, Orch. 5 (1915) 69.

Luzon (Bontoc, Benguet), Vanoverbergh 3919, F. B. 5105 Curran, B. S. 12721 Fenix. Terrestrial, up to $1,300 \mathrm{~m}$ altitude in Bontoc.

MALAXIS DAVAENSIS Ames Orch. 5 (1915) 69.

Mindanao (Davao), Weber 243. Terrestrial in shade of forest at $1,075 \mathrm{~m}$ altitude.

MALAXIS DENTATA Ames Orch. 2 (1908) 125, text cut, 5 (1915) 70.

Microstylis dentata Ames in Philip. Journ. Sci. 2 (July, 1907) Bot. 319.

Microstylis philippinensis Kränzl. in Orchis 2 (November, 1907) 16. Malaxis philippinensis Ames Orch. 5 (1915) 71.

Luzon (Rizal, Sorsogon, Camarines), Polillo, Mindoro, Leyte, Mindanao (Agusan), Jolo. A fairly common species, terrestrial or in trees at 60 to $1,000 \mathrm{~m}$ altitude. 
MALAXIS ELMERI Ames Orch. 2 (1908) 126, text cut, 5 (1915) 70.

Luzon (Benguet), Elmer 6621, R. S. Williams 1110.

MALAXIS EPIPHYTICA Ames Sched. Orch. 6 (1923) 34.

Mindanao (Cotabato), Weber 223. At $900 \mathrm{~m}$ altitude.

MALAXIS HUTCHINSONIANA Ames Orch. 2 (1908) 128, text cut, 5 (1915) 70.

Microstylis hutchinsoniama Ames in Philip. Journ. Sci. 2 (1907)

Bot. 319 .

Mindoro, Merrill 5809. Mt. Halcon, terrestrial in forest at $760 \mathrm{~m}$ altitude.

MALAXIS LATIFOLIA Sm. in Rees Cycl. 22 (1819) No. 3; Ames in Philip. Journ. Sci. 6 (1911) Bot. 44, Orch. 5 (1915) 70, in Merr. in Journ. Roy. Asiat. Soc. Straits Branch, Special No. (1921) 151.

Malaxis plicata Roxb. Hort. Beng. (1814) 63, nomen; Roxb. Fl. Ind. ed. 2,3 (1832) 456.

Dienia congesta Lindl. in Bot. Reg. 10 (1824) sub t. 825, in Wall. Cat. (1828) No. 1936, Gen. \& Sp. Orch. (1830) 22; Reichb. f. in Bonpl. 3 (1855) 250 ; Benth. Fl. Hongk. (1861) 352.

Gastroglottis montana Blume Bijdr. (1825) 387.

Dienia fusca Lindl. Gen. \& Sp. Orch. (1830) 22.

Microstylis congesta Reichb. f. in Walp. Ann. 6 (1861) 206; Hance Suppl. Fl. Hongk. (1872) 39; Naves Novis. App. (1882) 230; Ridl. in Journ. Linn. Soc. 24 (1888) 334, 32 (1896) 223; Hook. f. FI. Brit. Ind. 5 (1890) 689; Grant Orch. Burma (1895) 16 (excl. syn. in part); King \& Pantl. in (Ann. Roy. Bot. Gard. Calc. 8 (Orch. Sik.-Himal.) (1898) 19, t. 23; Trim. Handb. Fl. Ceyl. 4 (1898) 141; Ridl. Mat. Fl. Mal. Penin. 1 (1907) 14.

Microstylis trilobulata Kurz Rept. Veg. Andam. Isles App. B (1870) xix, nomen; N. E. Br. in Gard. Chron. II 18 (1882) 393.

Microstylis bernaysii F. v. Muell. Fragm. 11 (1878) 21.

Liparis bernaysii F. v. Muell. Fragm. 11 (1878) 21, in syn.; Bernays Fl. Queensl. (?) 507; F. M. Bail. Syn. Queensl. Fl. (1883) 508.

Microstylis congesta Reichb. f. var. fusca Ridl. in Journ. Linn. Soc. 24 (1888) 335.

Microstylis latifolia J. J. Sm. Fl. Buitenz. 6 (Orch. Jav.) (1905) 248, Fig.-Atlas (1910) fig. 185; Schltr. in Fedde Repert. Beihefte 1 (Orch. Deutsch-Neu-Guin.) (1911) 124; J. J. Sm. in Nova Guinea 12 (1913) 15.

Microstylis latifolia J. J. Sm. var. fusca J. J. Sm. FI. Buitenz, 6 (Orch. Jav.) (1905) 249.

Malaxis latifolia Sm. var. fusca Ames in Philip. Journ. Sci. 6 (1911)

Bot. 45.

Luzon (Bontoc, Benguet, Rizal, Laguna, Sorsogon), Mindanao (Surigao). A fairly common species, though apparently localized. Terrestrial. in forest at 150 to $1,300 \mathrm{~m}$ altitude. In India, Ceylon, Burma, Malay Peninsula, Siam, China, Sumatra, Java, Celebes, Borneo, New Guinea, and Australia. 
MALAXIS LILACINA Ames Sched. Orch. 6 (1923) 35.

Panay (Antique), Leyte, McGregor 6205, Wenzel 0641, 066\%. At 600 to $900 \mathrm{~m}$ altitude.

MALAXIS MACGREgORII Ames in Philip. Journ. Sci. 6 (1911) Bot. 45, Orch. 5 (1915) 71.

Malaxis longipedunculata Ames in Philip. Journ. Sci. 8 (1913) Bot. 411, Orch. 5 (1915) 70.

Luzon (Tayabas), Polillo, Leyte, Mindanao (Agusan, Davao), B. S. 10440 McGregor, 25539 Yates, Wenzel 033, 052, 0132, 023\%, 0238, 0503, $084 \%$, Weber 294, 296,313 . Terrestrial in forest at 60 to $600 \mathrm{~m}$ altitude.

MALAXIS MERRILL!I Ames Orch. 2 (1908) 128, text cut, 5 (1915) 71. Microstylis merrillii Ames in Philip. Journ. Sci. 2 (1907) Bot. 319.

Mindoro, Merrill 5820. Mt. Halcon, terrestrial, at base of cliffs at $1,930 \mathrm{~m}$ altitude.

MALAXIS MINDANAENSIS Ames in Elm. Leafl. Philip. Bot. 5 (1912) 1560, Orch. 5 (1915) 71.

Mindanao (Davao), Elmer 11855. Todaya, Mt. Apo, terrestrial in dense forest at $1,330 \mathrm{~m}$ altitude.

MALAXIS MINDORENSIS (Rendle) Ames Orch. 2 (1908) 131, 5 (1915) 71. Microstylis mindorensis Rendle in Journ. Bot. 34 (1896) 356.

Mindoro, Mt. Dulangan, Whitehead. Known only from a photograph and a sketch of the type.

MALAXIS NEGROSIANA Ames in Elm. Leafl. Philip. Bot. 5 (1912) 1561, Orch. 5 (1915) 71, 6 (1920) 288.

Negros (Oriental Negros), Leyte, Elmer 9600, Wenzel 0641, $066 \%$. Terrestrial or epiphytic in forest at 600 to $1,300 \mathrm{~m}$ altitude.

MALAXIS PROPINQUA Ames Orch. 6 (1920) 289.

Luzon (Bontoc), Vanoverbergh 3916. Terrestrial, on hills at 1,200 m altitude.

MALAXIS QUADRIDENTATA Ames Orch. 2 (1908) 130, text cut, 5 (1915) 72.

Microstylis quadridentata Ames in Philip. Journ. Sci. 2 (1907) Bot. 320 .

Mindoro, Merrill 5818, 5805. Mt. Halcon, terrestrial in forest at 800 to $1,000 \mathrm{~m}$ altitude.

MALAXIS QUADRILOBA Ames Orch. 2 (1908) 131, text cut, 5 (1915) 72.

Luzon (Bontoc, Benguet, Bataan), Panay, (Antique), Vanoverbergh r86, $1348,3680,3944, R$. S. Williams 1108, B. S. 32381 McGregor, Merrill, 3886 in part.

MALAXIS RAMOSII Ames in Philip. Journ. Sci. 6 (1911) Bot. 45, Orch. 5 (1915) 72.

Luzon (Rizal, Laguna), B. S. 4567 Ramos, 17040 Robinson, Baker 3573. $\mathrm{Up}$ to $700 \mathrm{~m}$ altitude in Laguna.

200823-20 
MALAXIS RIZALENSIS Ames in Philip. Journ. Sci. 6 (1911) Bot. 46, Orch. 5 (1915) 72.

Luzon (Rizal), B. S. 4561 Ramos. Bosoboso, in thickets.

MALAXIS TAYLORI Ames Orch. 6 (1920) 290.

Mindanao (Agusan), Elmer 13495, Taylor s. n.

MALAXIS UNCATA Ames in Philip. Journ. Sci. 6 (1911) Bot. 46, Orch. 5 (1915) 72.

Luzon (Laguna), F. B. 9552 Curran. Paete-Piapi.

MALAXIS WENZELII Ames in Philip. Journ. Sci. 8 (1913) Bot. 412, Orch. 5 (1915) 72.

Panay (Capiz), Leyte, B. S. 31141 Ramos, Wenzel 034, 0130, 0144, 0211, 0239, 0503a. Terrestrial, in forest at 60 to $500 \mathrm{~m}$ altitude.

MALAXIS WILLIAMSII Ames Orch. 2 (1908) 131, text cut, 5 (1915) 72. Luzon (Benguet), R. S. Williams 1109. Baguio.

\section{EXCLUDED OR SYNONYMOUS SPECIES}

MaLAXIS ANCEPS Reichb. f. in Walp. Ann. 6 (1861) $214=$ Oberonia anceps Lindl.

Malaxis angustifolia Blume Bijdr. (1825) $393=$ Liparis caespitosa. (Thou.) Lindl.

MALAXIS APOROPHYLIA Reichb. f. in Walp. Ann. 6 (1861) $214=$ Oberonia aporophylla Reichb. f.

MataXis bILoba Ames Orch. 2 (1908) 122, 5 (1915) 69 = Malaxis acu minata D. Don var. biloba Ames.

Mataxis Caespitosa Thou. Orch. Isles Afr. (1822) t. $89=$ Liparis caespitosa (Thou.) Lindl.

Malaxis COMPRessa Blume Bijdr. (1825) 390, fig. 54 = Liparis compressa (Blume) Lindl.

MALAXIS CYLINDRICA Reichb. f. in Walp. Ann. 6 (1861) 208 = Oberonia cylindrica Lindl.

MALAXIS DISTICHA Thou. Orch. Isles Afr. (1822) t. $88=$ Lipanis disticha (Thou.) Lindl.

Malaxis flavescens Ames Orch. 2 (1908) 127, 5 (1915) 70 (excl. sym.) $=$ Malaxis'bataanensis Ames.

MALAXIS IRIDIFOLIA Reichb. f. in Walp. Ann. 6 (1861) $208=$ Oberonia iridifolia (Roxb.) Lindl.

MalaXis LATIFolia Blume Bijdr. (1825) 393 = Liparis latifolia (Blume) Lindl.

Malaixis longipedunculata Ames in Philip. Journ. Sci. 8 (1913) Bot. 411, Orch. 5 (1915) $70=$ Malaxis macgregorii Ames.

Malaxis Miniata Reichb. f. in Walp. Ann. 6 (1861) $215=$ Oberonia miniata Lindl. 
MaLAXIS MrNima Blume Bijdr. (1825) 391 = Ciparis cuespitosa, (Thou.) Lindl.

MaLAXIs Monstruosa Blume Bijdr. (1825) 395 - Oberonia monstruosa (Blume) Lindl.

MALAXIS PALLIDA Blume Bijdr. (1825) 391 = Liparis pallidu (Blume) Lindl.

MALAXIS PARVIFLORA Blume Bijdr. (1825) 392 = Liparis parvifloro, (Blume) Lindl.

Malaxis PhilipPinensis Ames Orch. 5 (1915) $71=$ Malaxis dentuth Ámes.

MALAXIS THISBE Reichb. f. in Walp. Ann. 6 (1861) 213 = Oberonio thisbe Reichb. f.

\section{OBERONIA Lindley}

OBERONIA ANCEPS Lindl. Sert. Orch. (1838) sub t. 8, Fol. Orch. Oberonia (1859) 6; Hook. f. Fl. Brit. Ind. 5 (1888) 685; Ridl. in Trans. Linn. Soc. II 3 (1893) 359; Grant Orch. Burma (1895) 28; Ridl, in Journ. Linn. Soc. 32 (1896) 219; Schltr. in Perk. Frag. Fl. Filip. (1904) 43; J. J. Sm. F'l. Buitenz. 6 (Orch. Jav.) (1905) 238, Fig.-Atlas (1910) t. 174; Ames Orch. 1 (1905) 78, 5 (1915) 72; Ridl. Mat. Fl. Mal. Penin. 1 (1907) 18; J. J. Sm. in Bull. Jard. Bot. Buitenz. II 26 (1918) 18.

Oberonia imbricata Wight Icon. $5^{1}$ (1852) 3, t. 1629.

Oberonia griffithii Wight Icon. $5^{1}$ (1852) 4 .

Malaxis anceps Reichb. f. in Walp. Ann. 6 (1861) 214.

Oberonia ancipita Naves Novis. App. (1882) 230; Vidal Phan. Cuming. Philip. (1885) 148, Rev. Pl. Vasc. Filip. (1886) 266; Ceron Cat. Pl. Herb. Manila (1892) 166, sphalm.

Iridorchis anceps Ktze. Rev. Gen. Pl. 2 (1891) 669.

Luzon (Bataan, Laguna, Tayabas), Polillo, Samar, Culion, Mindanao (Agusan), Serrato s. n.; B. S. 16580, 24156 Ramos, Merrill 139, 569, s. n., Weber s. $n$.

OBERONIA A'POROPHYLLA Reichb. f. in Bonpl. 3 (1855) 223; Lindl. Fol. Orch. Oberonia (1859) 6; Naves Novis. App. (1882) 230; Ames Orch. 2 (1908) 140, 5 (1915) 72, in Philip. Journ. Sci. 4 (1909) Bot. 666.

Malaxis aporophylla Reichb. f. in Walp. Ann. 6 (1861) 214.

Oberonia imbricata Vidal Phan. Cuming. Philip. (1885) 148, Rer. Pl. Vasc. Filip. (1886) 266, non Lindl.

Iridorchis aporophylla Ktze. Rev: Gen. P1. 2 (1891) 669.

Luzon (Bontoc, Benguet), Mindanao (Lanao), Cuming 2113, Vanoverbergh 822, 1474, 3827, B. S. 8319, 8508 McGregor, M1. S. Clemens 699. Mountainous regions at 1,700 to $2,100 \mathrm{~m}$ altitude.

OBERONIA BASILANENSIS Ames Orch. 5 (1915) 72.

Basilan, B. S. 16247 Reillo.

oberoniA BENGUetensis Ames in Philip. Journ. Sci., 7 (1912) Bot 8, Orch. 5 (1915) 73.

Luzon (Benguet), Merrill 4855. Suyoc to Pauai, epiphyte in mossy forest at $2,200 \mathrm{~m}$ altitude. 
OBERONIA CYLINDRICA Lindl. in Bot. Reg. 26 (1840) Misc. 20, Fol. Orch. Oberonia (1859) 1; Naves Novis. App. (1882) 230; Ames Orch. 1 (1905) 77, 5 (1915) 73; Merr. \& Merritt in Philip. Journ. Sci. 5 (1910) Bot. 341; Ames in Philip. Journ. Sci. 6 (1911) Bot. 47.

Malaxis cylindrica Reichb. f. in Walp. Ann. 6 (1861) 208.

Iridorchis cylindrica Ktze. Rev. Gen. Pl. 2 (1891) 669.

Luzon (Bontoc, Lepanto, Union, Benguet), Leyte, Bohol, Palawan. A fairly common species in the Philippines. Epiphyte at 6 to $2,650 \mathrm{~m}$ altitude.

oberoniA ELMERI Ames in Elm. Leafl. Philip. Bot. 5 (1912) 1564, Orch. 5 (1915) 73.

Luzon (Benguet), Elmer 8434,F.B.5057 Curran. Baguio, epiphyte at $1,580 \mathrm{~m}$ altitude.

OBERONIA HISPIDULA Ames in Philip. Journ. Sci. 7 (1912) Bot. 8, Orch. 5 (1915) 73.

Luzon (Benguet, Rizal, Tayabas), Mindanao (Agusan), F. B. 5124 Curran, B. S. 19240 Reillo, Elmer 7406, 9261, 14084. Epiphytic, at 800 to $1,230 \mathrm{~m}$ altitude.

OBERONIA IRIDIFOLIA (Roxb.) Lindl. in Wall. Cat. (1828) No. 1948, p. p., Gen. \& Sp. Orch. (1830) 15 (excl. syn. Malaxis ensiformis Sm.), Fol. Orch. Oberonia (1859) 1; Benth. Fl. Austral. 6 (1873) 274; Naves Novis. App. (1882) 230; Hook. f. Fl. Brit. Ind. 5 (1888) 675; Grant Orch. Burma (1895) 31; King \& Pantl. in Ann. Roy. Bot. Gard. Calc. 8 (Orch. Sik.-Himal.) (1898) 8, t. 8; J. J. Sm. Fl. Buitenz, 6 (Orch. Jav.) (1905) 239, Fig.-Atlas (1910) fig. 175; Ames Orch. 1 (1905) 77, 5 (1915) 73; Ridl. Mat. Fl. Mal. Penin. 1 (1907) 16; Schltr. in Fedde Repert. Beihefte 4 (Orch. Sino-Jap. Prodr.) (1919) 194; Ames in Merr. in Journ. Roy. Asiat. Soc. Straits Branch, Special No. (1921) 152.

Cymbidium iridifolium Roxb. Hort. Beng. (1814) 63, nomen, Fl. Ind. ed. 2,3 (1832) 458.

? Cymbidium flavescens Llanos Fragm. Pl. Filip. (1851) 96; F.-Vill. \& Naves in Blanco Fl. Filip. ed. 3, $4^{1}$ (1880) 74; Merr. Sp. Blancoanae (1918) 113.

Malaxis iridifolia Reichb. f. in Walp. Ann. 6 (1861) 208.

Iridorchis iridifolia Ktze. Rev. Gen. Pl. 2 (1891) 669.

Luzon (Bataan, Rizal, Sorsogon), Palawan, Mindanao (Zamboanga, Davao), Basilan, F. B. 130 Barnes, 51\%4 Hutchinson, B. S. 5643, 24100 Ramos, Weber 269, 60\%. Epiphytic, up to $400 \mathrm{~m}$ in Mindanao. In India, Burma, Malay Peninsula, China, Sumatra, Java, Borneo, and Australia. OBERONIA LEYTENSIS Ames Orch. 6 (1920) 291, t. 98.

Leyte, Wenzel 0945. Epiphyte, in forest at sea level. OBERONIA LIPENSIS Ames Orch. 7 (1922) 114.

Mindanao (Bukidnon), B. S. 38535 Ramos. Mt. Lipa, epiphytic in mossy forest at $2,200 \mathrm{~m}$ altitude. 
OBERONIA LUZONENSIS Ames Orch. 5 (1915) 74.

Luzon (Laguna, Tayabas), Mindanao (Surigao), Elmer 1\%558, B. S. 20809 Escritor, 34489 Ramos \& Pascasio.

OBERONIA MCGREgORII Ames in Philip. Journ. Sci. 2 (1907) Bot. 321, Orch. 3 (1908) 65, t. 48, 5 (1915) 74.

Luzon (Laguna), Mindoro, Mindanao (Agusan), Reillo 16, 5’. S. 15009 Ramos, Weber 168. Epiphytic apparently at low altitudes.

OBERONIA MERRILLII Ames in Philip. Journ. Sci. 7 (1912) Bot. 9, Orch. 5 (1915) 74; Merr. Fl. Manila (1912) 168.

Luzon (Nueva Ecija, Rizal, Tayabas), Busuanga, Mindanao (Zamboanga, Davao), B. S. 5298 MeGregor, Merrill 7348, 11219, Ramos s. n., Weber s.n.,? F. B. 9022 Whitford \& Hutchinson, $2658 \%$ Reyes, Copelund 1309. Epiphyte at 18 to $400 \mathrm{~m}$ altitude.

OBERONIA MINDORENSIS Ames in Philip. Journ. Sci. 2 (1907) Bot. 322, Orch. 3 (1908) 67, t. 49, 5 (1915) 75.

Mindoro, Leyte, Mindanao (Agusan), Merrill 5619, Wenzel 0420, 0425, 0559; Weber 316. Epiphyte at 400 to $500 \mathrm{~m}$ altitude.

OBERONIA MINIMA Ames Orch. 5 (1915) 75.

Leyte, Wenzel 046. Dagami, epiphyte at $60 \mathrm{~m}$ altitude.

OBERONIA MINUTISSIMA Ames Orch. 7 (1922) 115.

Mindanao (Surigao) Wenzel 01030, 01032. Placer, epiphytic in forest at $150 \mathrm{~m}$ altitude.

OBERONIA MONSTRUOSA (Blume) Lindl. Gen. \& Sp. Orch. (1830) 17; Miq. Fl. Ind. Bat. 3 (1859) 624; J. J. Sm. Fl. Buitenz. 6 (Orch. Jav.) (1905) 235, Fig.-Atlas (1910) fig. 171; Ames Orch. 5 (1915) 76.

Malaxis monstruosa Blume Bijdr. (1825) 395. Java.

Mindanao (Agusan), Weber 173, 312. Epiphytic at low altitude. In

OBERONIA OBESA Ames Orch. 5 (1915) 76.

Luzon (Bontoc), Vanoverbergh $40 \%$.

OBERONIA REILLOI Ames Sched. Orch. 6 (1923) 40.

Luzon (Laguna), Reillo 20. Epiphyte.

OBERONIA SETIGerA Ames in Philip. Journ. Sci. 7 (1912) Bot. 10, Orch. 5 (1915) 77.

Luzon (Bataan), Whitford 1122. Mt. Mariveles, epiphyte, at 1,200 m altitude.

OBERONIA SURIGAENSIS Ames Orch. 6 (1920) 292.

Mindanao (Surigao), Wenzel 01159. Epiphyte, in forest at $150 \mathrm{~m}$ altitude. 
OBERONIA THISBE Reichb. f. in Bonpl. 3 (1855) 223; Lindl. Fol. Orch. Oberonia (1859) 5; Naves Novis. App. (1882) 230; Ames Orch. 2 (1908) 141, 5 (1915) 77.

Malaxis thisbe Reichb. f. in Walp. Ann. 6 (1861) 213.

Habenaria thisbe Vidal Phan. Cuming. Philip. (1885) 151.

Iridorchis thisbe Ktze. Rev. Gen. Pl. 2 (1891) 669.

Oberonia mufilabris Ames Orch. 1 (1905) 78, non Lindl.

Luzon (Kalinga, Benguet, Laguna), Polillo, Mindanao (Surigao), Boettcher s. n., Elmer 5839, Reillo 52, B. S. 15039 Ramos, 9255 Robinson, Cuming 2134, Piper 19\%. Epiphyte at sea level to 2,000 $\mathrm{m}$ altitude.

OBERONIA TOPPINGII Ames in Philip. Journ. Sci. 8 (1913) Bot. 413, Orch. 5 (1915) 77.

Luzon (Rizal), Topping s. n. Wawa.

OBERONIA WENZELII Ames Orch. 5 (1915) 77.

Leyte, Wenzel 0386, 0388, 0414, 0426,0436. Jaro, epiphyte in forest at $500 \mathrm{~m}$ altitude.

\section{EXCLUDED OR SYNONYMOUS SPECIES}

Oberonia ANCIPITA Naves Novis. App. (1882) 230; Vidal Phan. Cuming. Philip. (1885) 148, Rev. Pl. Vasc. Filip. (1886) 266; Ceron Cat. Pl. Herb. Manila (1892) 166, sphalm = Oberonia anceps Lindl.

Oberonia Imbricata Vidal Phan. Cuming. Philip. (1885) 148, Rev. Pl. Vasc. Filip. (1886) 266, non Lindl. = Oberonia aporophylla Reichb. $f$.

OBERONIA MINIATA Lindl. in Bot. Reg. 29 (1843) Misc. 6, Fol. Orch. Oberonia (1859) 6; Naves Novis. App. (1882) 230; Ames Orch. 5 (1915) 75.

Malaxis miniata Reichb. f. in Walp. Ann. 6 (1861) 215.

This species was based on material from Singapore. It has been credited to the Philippines, but there is little reason for considering it Philippine. It has not appeared in our modern collections.

OBERONIA RUFILABRIS Ames Orch. 1 (1905) 78, non Lindl. = Oberonia thisbe Reichb. f.

Oberonia spatulata Lindl. apud Naves Novis. App. (1882) 230.

46. HIPPEOPHYLLUM Schlechter

HIPPEOPHYLLUM WENZELII Ames in Philip. Journ. Sci. 8 (1913) Bot. 413 , Orch. 5 (1915) 78.

Leyte, Wenzel 020, 0158. Dagami, epiphyte, at $60 \mathrm{~m}$ altitude.

47. LIPARIS L. C. Richard

LIPARIS AMESIANA Schltr. in Fedde Repert. Beihefte 1 (Orch. DeutschNeu-Guin.) (1911) 210; Ames Orch. 5 (1915) 78.

Cestichis gracilis Ames Orch. 2 (1908) 136, text cut, non Liparis gracilis Hook. f.

Luzon (Bataan, Rizal), Mindanao (Bukidnon), Copeland 274, Merril s. n., Weber 28, B. S. 1782, 38632 Ramos. Epiphyte in forest at 930 to $1,460 \mathrm{~m}$ altitude. 
LIPARIS BENGUETENSIS (Ames) Schltr. in Fedde Repert. Beihefte 1 (Orch. Deutsch-Neu-Guin.) (1911) 210; Ames Orch. 5 (1915) 78. Cestichis benguetensis Ames Orch. 1 (1905) 9, 75, t. 3, fig. I, 1-6, 2 (1908) 132.

Luzon (Bontoc, Benguet), Negros. Fairly common in Luzon in mountainous regions at 700 to $2,300 \mathrm{~m}$ altitude.

LIPARIS BONTOCENSIS Ames Sched. Orch. 6 (1923) 38.

Luzon (Bontoc), Vanoverbergh 1245, 3.947a. At 1,650 to $1,700 \mathrm{~m}$ altitude.

LIPARIS CAESPITOSA (Thou.) Lindl. in Bot. Reg. 11 (1825) sub t. 8.8; A. Rich. Monogr. Orch. Isles France et Bourb. (1827) [in Mém. Soc. Hist. Nat. Par. 4 (1828) 47]; Lindl. Gen. \& Sp. Orch. (1830) 32; Bojer Hort. Maur. (1837) 321; S. Moore in Baker Fl. Maur. \& Seych. (1877) 342; Ridl. in Journ. Linn. Soc. 22 (1886) 290; Cordem. Fl. Réun. (1895) 180; J. J. Sm. Fl. Buitenz. 6 (Orch. Jav.) (1905) 266, Fig.-Atlas (1910) fig. 204, in Nova Guinea 8 (1909) 35, $12^{3}$ (1915) 221; Schltr. in Engl. Bot. Jahrb. 45 Beibl. 104 (1911) 17; Ames Orch. 5 (1915) 78, in Merr. in Journ. Roy. Asiat. Soc. Straits Branch, Special No. (1921) 153.

Malaxis caespitosa Thou. Orch. Isles Afr. (1822) $t .89$.

Malaxis minima Blume Bijdr. (1825) 391.

Malaxis angustifolia Blume Bijdr. (1825) 393.

Liparis minima Lindl. Gen. \& Sp. Orch. (1830) 32; Miq. Fl. Ind. Bat. 3 (1859) 622; Naves Novis. App. (1882) 230; Ridl. in Journ. Linn. Soc. 22 (1886) 297; Schltr. in Fedde Repert. 10 (1911) 28. Liparis angustifolia Lindl. Gen. \& Sp. Orch. (1830) 31; Miq. Fl. Ind. Bat. 3 (1859) 622; Thw. Enum. Pl. Zeyl. (1864) 296.

Liparis auriculata Reichb. f. in Flora 55 (1872) 277, non Blume.

Liparis pusilla Ridl. in Journ. Linn. Soc. 22 (1886) 294; Hook. f. in Hook. Icon. Pl. 19 (1889) t. 1856 A, Fl. Brit. Ind. 5 (1890) 701; King \& Pantl. in Ann. Roy. Bot. Gard. Calc. 8 (Orch. Sik.-Himal.) (1898) 32, $t .41$.

Liparis prainii Hook. f. in Hook. Icon. Pl. 19 (1889) t. 1857 A, Fl. Brit. Ind. 5 (1890) 700.

Liparis duthiei Hook. f. in Hook. Icon. Pl. 19 (1889) t. 1857 B, Fl. Brit. Ind. 5 (1890) 701; King \& Pantl. in Ann. Roy. Bot. Gard. Calc. 8 (Orch. Sik.-Himal.) (1898) 32, t. 42; Duthie in Ann. Roy. Bot. Gard. Calc. 9 (Orch. North-west. Himal.) (1906) 93.

Liparis obscura Hook. f. in Hook. Icon. Pl. 19 (1889) t. 1886, Fl. Brit. Ind. 5 (1890) 701; Trim. Handb. Fl. Ceyl. 4 (1898) 147.

Leptorchis caespitosa Ktze. Rev. Gen. Pl. 2 (1891) 671.

Leptorchis duthiei Ktze. Rev. Gen. Pl. 2 (1891) 671.

Leptorchis minima Ktze. Rev. Gen. Pl. 2 (1891) 671.

Leptorchis obscura Ktze. Rev. Gen. Pl. 2 (1891) 671.

Leptorchis prainii Ktze. Rev. Gen. Pl. 2 (1891) 671.

Leptorchis pusilla Ktze. Rev. Gen. Pl. 2 (1891) 671.

Cestichis caespitosa Ames Orch. 2 (1908) 132, $t$.

Luzon (Benguet, Rizal), Leyte, Mindanao (Cotabato, Davao), R. S. Williams 428, 1954, Merrill 4684, B. S. 13558 Ramos, 19238 Reillo, Wenzel 0734, Weber 232, Elmer 11858. Epiphyte in forest at 800 to $1,160 \mathrm{~m}$ 
altitude. In India, Ceylon, Sumatra, Java, Celebes, Borneo, New Guinea, Bourbon, Mauritius, Reunion, and Madagascar.

LIPARIS CLEMENSIAE Ames Orch. 5 (1915) 79.

Cestichis clemensiae Ames in Philip. Journ. Sci. 4 (1909) Bot. 666. Mindanao (Lanao), M. S. Clemens 129. Camp Keithley.

LIPARIS COMPRESSA (Blume) LindI. Gen. \& Sp. Orch. (1830) 32; Miq. Fl. Ind. Bat. 3 (1859) 621; Naves Novis. App. (1882) 230; Ridl. in Journ. Linn. Soc. 22 (1886) 291, 32 (1896) 230; Hook. f. Fl. Brit. Ind. 5 (1890) 706; J. J. Sm. Fl. Buitenz. 6 (Orch. Jav.) (1905) 280, Fig.-Atlas (1910) fig. 215; Ridl. Mat. Fl. Mal. Penin. 1 (1907) 25; Schltr. in Engl. Bot. Jahrb. 45, Beibl. 104 (1911) 17; Ames Orch. 5 (1915) 79.

Malaxis compressa Blume Bijdr. (1825) 390, fig. 54.

Leptorchis compressa Ktze. Rev. Gen. Pl. 2 (1891) 670.

Cestichis compressa Ames Orch. 1 (1905) 76, text cut, 2 (1908) 134.

Luzon (Lepanto, Bataan), Borden 1816, Merrill 45\%\%. Mt. Data and Mt. Mariveles, epiphyte in forest at 1,060 to $2,300 \mathrm{~m}$ altitude. In Malay Peninsula, Sumatra, Java, and Celebes.

LIPARIS CONFUSA J. J. Sm. Fl. Buitenz. 6 (Orch. Jav.) (1905) 275 , Fig.-Atlas (1910) fig. 211; Schltr. in Engl. Bot. Jahrb. 45, Beibl. 104 (1911) 17, in Fedde Repert. 10 (1911) 28, in Fedde Repert. Beihefte 1 (Orch. Deutsch-Neu-Guin.) (1911) 205; J. J. Sm. in Bull. Jard. Buitenz. II 14 (1914) 32; Ames Orch. 5 (1915) 79, in Merr. in Journ. Roy. Asiat. Soc. Straits Branch, Special No. (1921) 153.

Liparis flaccida Schltr. in K. Schum. \& Lauterb. Nachtr. Fl. Deutsch. Schutzgeb. (1905) 103, non Reichb. f.

Cestichis vestita Ames Orch. 2 (1908) 139, p. p., non Liparis vestita Reichb. f.

Luzon (Apayao, Bontoc, Benguet, Nueva Vizcaya, Rizal, Laguna, Tayabas, Sorsogon), Mindoro, Leyte, Samar, Mindanao (Zamboanga, Lanao, Agusan, Butuan, Davao), Basilan. Common and widely distributed in the Philippines. Epiphyte in forest at 15 to $1,430 \mathrm{~m}$ altitude. In Sumatra, Java, Celebes, Borneo, and New Guinea.

LIPARIS CUMINGII Rid1. in Journ. Linn. Soc. 22 (1886) 292; Hook. f. Fl. Brit. Ind. 5 (1890) 707; Ames Orch. 5 (1915) 79.

Cestichis cumingii Ames Orch. 1 (1905) 13, in Philip. Journ. Sci. 7 (1912) Bot. 7.

Luzon (Bontoc, Bataan, Laguna, Sorsogon), Leyte, Bohol,* Mindanao (Zamboanga), Weber 26, B. S. 17037 Robinson, Elmer 14r03, 16296, Wenzel 0680, 0808, Copelands. n.,Vanoverbergh 710. Epiphyte in forest at 600 to $1,400 \mathrm{~m}$ altitude.

LIPARIS DUMAGUETENSIS Ames Orch. 5 (1915) 80.

Liparis elmeri Ames in Elm. Leafl. Philip. Bot. 5 (1912) 1561, nec Cestichis elmeri Ames, nec Liparis elmeri (Ames) Schltr.

Luzon (Tayabas), Panay (Antique), Negros (Oriental Negros), Leyte, Mindanao (Camiguin Island), Elmer 7566, 7579, 9445, 10236, Wenzel 0298, 0299, McGregor 5805, B. S. 14444 Ramos, 41401 Herre. Terrestrial in forest at 600 to $1,300 \mathrm{~m}$ altitude.

* Bopol, according to Hook. f. in Fl. Brit. Ind. 5 (1890). 
LIPARIS ELMERI (Ames) Schltr. in Fedde Repert. Beihefte 1 10rch. Deutsch-Neu-Guin.) (1911) 210; Ames Orch. 5 (1915) 80.

Cestichis elmeri Ames Orch. 1 (1905) 10, 75, t. \&, fig. II, 7-12, 2 (1908) 135.

Luzon (Benguet, Laguna, Tayabas), Elmer 655.3, R. S. Williams 1928, Ramos \& Edaño 107, F. B. 5065 Curran, B. S. 26528 Kamns \& Edaño, 6085 Robinson, 27940 Ocampo. Epiphyte, 260 to 2,230 m altitude.

LIPARIS FORBESII Ridl. in Journ. Linn. Soc. 22 (1886) 28.; ; J. J. Sm. Fl. Buitenz. 6 (Orch. Jav.) (1905) 268, Fig.-Atlas (1910) fig. 205; Ames Orch. 5 (1915) 80.

Leptorchis forbesii Ktze. Rev. Gen. Pl. 2 (1891) 671.

Cestichis forbesii Ames Orch. 2 (1908) 135.

Luzon (Bontoc, Benguet), Mindanao (Lanao), Vanoverbergh 711, 138: $1478,3947, R$. S. Williams 1941, M. S. Clemens 446.

LIPARIS FRAGILIS Ames Orch. 5 (1915) 80.

Cestichis fragitis Ames in Philip. Journ. Sci. 8 (1913) Bot. 412.

Mindanao (Zamboanga), Merill 8184, 8289. Epiphyte at about $900 \mathrm{~m}$ altitude.

LIPARIS HALCONENSIS Ames Orch. 5 (1915) 80.

Cestichis halconensis Ames in Philip. Journ. Sci. 2 (1907) Bot. 321 , Orch. 3 (1908) 63, t. $4 \%$.

Luzon (Ifugao), Mindoro, Panay (Antique), Merrill 5799, McGregor 6182, B. S. 19882 McGregor. Terrestrial at 400 to $730 \mathrm{~m}$ altitude.

LIPARIS JARENSIS Ames Orch. 6 (1920) 293.

Leyte, Wenzel, 0628, 0708,0799, 0813. Jaro, epiphyte in forest at 600 to $700 \mathrm{~m}$ altitude.

LIPARIS LEYTENSIS Ames Orch. 5 (1915) 80.

Panay (Capiz), Leyte, Mindanao (Agusan), Wenzel 0216, 035., 0.21. Weber 160, B. S. $3560 \%$ Martelino \& Edaño. Epiphyte in forest at 12 to $800 \mathrm{~m}$ altitude.

LIPARIS LINEARIFOLIA Ames Orch. 5 (1915) 81.

Cestichis linearifolia Ames in Elm. Leafl. Philip. Bot. 5 (1912) 1563.

Mindanao (Davao), Elmer 11494, Todaya, Mt. Apo, epiphyte in dense forest at $2,000 \mathrm{~m}$. altitude.

LIPARIS LONGIPES Lindl. in Wall. Pl. Asiat. Rar. 1 (1830) 31 , t. 35, Gen. \& Sp. Orch. (1830) 30; Wight Icon. Pl. $3^{2}$ (1849) 9, t. 906; Benth. in Hook. Kew Journ. Bot. 7 (1855) 33; Reichb. f. in Bonpl. 5 (1857) 57; Benth. Fl. Hongk. (1861) 352; Reichb. f. in Walp. Ann. 6 (1861) 218; Thw. Enum. Pl. Zeyl. (1864) 295; Reichb. f. in Seem. Fl. Vit. (1868) 302; Ridl. in Journ. Linn. Soc. 22 (1886) 293 (excl. syn. L. elegans Lindl.), 32 (1896) 229; Hook. f. Fl. Brit. Ind. 5 (1890) 703; Grant Orch. Burma (1895) 21; King \& Pantl. in Ann. Roy. Bot. Gard. Calc. 8 (Orch. Sik.-Himal.) (1898) 29, t. 37; Trim. Handb. Fl. Ceyl. 4 (1898) 147; Forbes \& Hemsl. in Journ. Linn. Soc. 36 (1903) 7; Matsum. Ind. Pl. Jap. 2 (1905) 252; Duthie in Ann. Roy. Bot. Gard. Calc. 9 (Orch. North-west. Himal.) 
(1906) 93; Ridl. Mat. Fl. Mal. Penin. 1 (1907) 25; Schltr. in Fedde Repext. Beihefte 4 (Orch. Sino-Jap. Prodr.) (1919) 200; Ames Orch. 6 (1920) 94, in Merr. in Journ. Roy. Asiat. Soc. Straits Branch, Special No. (1921) 154.

Liparis pendula Lindl. in Bot. Reg. 24 (1838) Misc. 94; Regel Ind. Sem. Hort. Petrop. (1868) 79; Kränzl. in Orchis 2 (1907) 32.

Liparis spathulata Lindl. in Bot. Reg. 26 (1840) Misc. 81.

Sturmia longipes Reichb. $f$. in Bonpl. 3 (1855) 250.

? Liparis boothii Regel Ind. Sem. Hort. Petrop. (1864) SuppI. 14.

? Liparis nesophila Reichb. f. Otia Bot. Hamb. 1 (1878) 56, Xen. Orch. 3 (1881) 31.

Liparis longipes Lindl. var. spathulata Ridl. in Journ. Linn. Soc. 22 (1886) 294; King \& Pantl. in Ann. Roy. Bot. Gard. Calc. 8 (Orch. Sik.-Himal.) (1898) 30, t. 38.

Liparis triloba Ridl. in Journ. Linn. Soc. 22 (1886) 295; Ames Orch. 5 (1915) 84.

Cestichis pendula Pfitz. in Engl. \& Prantl Nat. Pflanzenfam. 2, Abt. 6 (1889) 131, fig. $128 \mathrm{C}-\mathrm{E}, \mathrm{G}$.

Leptorchis longipes Ktze. Rev. Gen. Pl. 2 (1891) 670.

Leptorchis triloba Ktze. Rev. Gen. Pl. 2 (1891) 671.

Stichorchis pendula Pfitz. in Engl. \& Prantl Nat. Pflanzenfam. Nachtr. 1 (1897) 103.

Cestichis longipes Ames Orch. 1 (1905) 75 (excl. Luzon specimen), 2 (1908) 137.

Luzon (Ilocos Norte, Laguna), Mindoro, Mindanao (Zamboanga), Cuming s. n., B. S. 27138 Ramos, 9679 Robinson, Ramos \& Edaño s. n., R. S. Williams 21\%6, F. B. 9218 Whitford \& Hutchinson. Epiphyte at 40 to $1,650 \mathrm{~m}$ altitude. In India, Ceylon, Burma, Malay Peninsula, China, Formosa, and Samoa.

LIPARIS LYONII Ames Orch. 5 (1915) 81.

Cestichis lyonii Ames in Philip. Journ. Sci. 6 (1911) Bot. 47.

Luzon (Bontoc, Benguet), Vanoverbergh 3885, Lyon 155, Elmer 8463.

LIPARIS MAGNICALLOSA Ames Orch. 7 (1922) 108.

Mindanao (Bukidnon), B. S. 38739 Ramos \& Edaño. Mt. Candoon, epiphyte in forest at $1,600 \mathrm{~m}$ altitude.

LIPARIS MERRILLII (Ames) Schltr. in Fedde Repert. Beihefte 1 (Orch. Deutsch-Neu-Guin.) (1911) 210; Ames Orch. 5 (1915) 81.

Cestichis merrillii Ames Orch. 1 (1905) 11, 75, t. 3, fig. III, 18-18, 2 (1908) 138, in Philip. Journ. Sci. 2 (1907) Bot. 321.

Luzon (Bontoc, Ifugao, Bataan, Rizal, Tayabas), Mindoro, Leyte, Mindanao (Misamis). A fairly common and widespread species, in moun tainous regions, epiphyte in mossy forest at 300 to $3,060 \mathrm{~m}$ altitude.

LIPARIS MONOPHYLLA Ames Orch. 6 (1920) 294.

Leyte, Wenzel 0660, 067\%. Jaro, epiphyte in forest at $600 \mathrm{~m}$ altitude.

LIPARIS NEGROSIANA Ames in Elm. Leafl. Philip. Bot. 5 (1912) 1562, Orch. 5 (1915) 81.

Negros (Oriental Negros), Elmer 9606. Dumaguete, terrestrial at 1,300 $m$ altitude. 
LIP.ARIS NUTANS Ames Orch. 5 (1915) 81.

Cestichis nutans Ames in Philip. Journ. Sci. 4 (1909) Bot. 597.

Palawan, Mindanao (Lanao, Agusan, Surigao), B. S. 638 Foxworthy, M. S. Clemens s. n., Weber 70, Bolster 289. In forest at 60 to $1,050 \mathrm{~m}$ altitude.

LIPARIS PALAWANENSIS Ames Sched. Orch. 6 (1923) 3.9.

Palawan, Weber 616. Alfonso XIII at sea level.

LIPARIS PALLIDA (Blume) Lindl. Gen. \& Sp. Orch. (1830) 31; Miq. Fl. Ind. Bat. 3 (1859) 622; Naves Novis. App. (1882) 230; Ridl. in Journ. Linn. Soc. 22 (1886) 296; J. J. Sm. Fl. Buitenz. 6 (Orch.Jav.) (1905) 272, Fig.-Atlas (1910), fig. 209; Ames Orch. 6 (1920) 295.

Malaxis pallida Blume Bijdr. (1825) 391.

Leptorchis pallida Ktze. Rev. Gen. Pl. 2 (1891) 671.

Liparis latifolia Ames in Elm. Leafl. Philip. Bot. 5 (1912) 1562, Orch. 5 (1915) 80, non Lindl.

Leyte, Mindanao (Bukidnon, Davao), Wenzel 0775, 0908, 0930, B. S. 39120 Ramos \& Edaño, Elmer 10648. Epiphyte in forest at 500 to 2,250 m altitude. In Java.

LIPARIS PARVIFLORA (Blume) Lindl. Gen. \& Sp. Orch. (1830) 31; Miq. Fl. Ind. Bat. 3 (1859) 622; J. J. Sm. Fl. Buitenz. 6 (1905) 276, Fig.-Atlas (1910) fig. 212; Schltr. in Engl. Bot. Jahrb. 45, Beibl. 104 (1911) 17; Ames Orch. 5 (1915) 81, 6 (1920) 95, in Merr. in Journ. Roy. Asiat. Soc. Straits Branch, Special No. (1921) 155. Malaxis parviflora Blume Bijdr. (1825) 392.

Liparis flaccida Reichb. f. in Linnaea 41 (1876) 45; Ridl. in Journ. Linn. Soc. 22 (1886) 296, '32 (1896) 228; Hook. f. Fl. Brit. Ind. 5 (1890) 703; Ridl. Mat. Fl. Mal. Penin. 1 (1907) 24.

Leptorchis flaccida Ktze, Rev. Gen. Pl. 2 (1891) 670.

Leptorchis parviflora Ktze. Rev. Gen. Pl. 2 (1891) 671.

Cestichis vestita Ames Orch. 2 (1908) 139, p. p., non Liparis vestita Reichb. f.

Luzon (Apayao, Bontoc, Rizal, Laguna, Tayabas), Polillo, Leyte, Busuanga, Mindanao (Cotabato, Davao), Basilan. A fairly common and widely distributed species in the Philippines. Epiphyte from about sea level to $1,450 \mathrm{~m}$ altitude. In Malay Peninsula, Sumatra, Java, Borneo, and Siam.

LIPARIS PECTINATA Ridl. in Journ. Linn. Soc. 22 (1886) 277; Ames Orch. 5. (1915) 82.

Leptorchis pectinata Ktze. Rev. Gen. Pl. 2 (1891) 671.

Luzon (Laguna), Biliran, Leyte, Mindanao (Davao), Reillo 55, Cuming 8. n., B. S. 18934 McGregor, 21450 Escritor, Wenzel 0207, 0223, 0380, 0481, 0578 , Black s. n., Weber 379 . Terrestrial in forest at 60 to $500 \mathrm{~m}$ altitude.

LIPARIS PHILIPPINENSIS (Ames) Schltr. in Fedde Repert. Beihefte 1 (Orch. Deutsch-Neu-Guin.) (1911) 210; Ames Orch. 5 (1915) 83.

Cestichis philippinensis Ames Orch. 1 (1905) 7, 75, t. 2, 2 (1908) 138, in Philip. Journ. Sci. 2 (1907) Bot. 321.

Luzon (Abra, Bontoc, Lepanto, Benguet, Bataan, Pampanga, Rizal, Laguna), Mindoro, Panay (Antique), Negros (Oriental Negros), Leyte, Min. 
danao (Agusan, Bukidnon, Davao). A common and widely distributed species, mostly in mountain regions at 300 to $2,900 \mathrm{~m}$ altitude.

LIPARIS PRAVA Ames Orch. 7 (1922) 109.

Leyte, Wenzel 0718, 0749, 0804, 0834, 0842. Jaro, epiphyte in forest at 500 to $800 \mathrm{~m}$ altitude.

LIPARIS PROPINQUA Ames Orch. 7 (1922) 110.

Liparis gregaria Rolfe in Journ. Bot. 23 (1885) 215; Vidal Phan. Cuming. Philip. (1885) 148, Rev. Pl. Vasc. Filip. (1886) 266, non Lindl.

Cestichis disticha Pfitz. in Engl. in Engl. \& Prantl Nat. Pflanzenfam. 2 Abt. 6 (1888) 131, quoad Philip.; Ames in Philip. Journ. Sci. 2 (1907) Bot. 320.

Liparis disticha Ames Orch. 5 (1915) 79, non Lindl.

Luzon (Laguna), Mindoro, Palawan, Bancalan, Mindanao (Davao), Cuming 2099, B. S. 6546 Robinson, Merrill 5638, 5643, F. B. 8598 Merritt, 28185 Elumir, Weber s. n., 011, Elmer 18305. Epiphyte at sea level to $600 \mathrm{~m}$ altitude.

LIPARIS RIZALENSIS Ames Orch. 6 (1920) 295.

Luzon (Rizal, Tayabas), B. S. 24070 Ramos, 2689.2 Edaño, 26533 Rames Edaño. Epiphyte on forest slopes at about $300 \mathrm{~m}$ altitude.

LIPARIS STRICTA J. J. Sm. in Bull. Dept. Agr. Ind. Néerl. 5 (1907) 3; Ames Orch. 6 (1920) 95, in Merr. in Journ. Roy. Asiat. Soc. Straits Branch, Special No. (1921) 155.

Basilan, Mindanao (Agusan), B. S. 16250 Reillo, Weber \%'4. Terrestrial in shaded forest at $400 \mathrm{~m}$ altitude in Mindanao. In Borneo.

LIPARIS TRICALLOSA Reichb. f. in Gard. Chron. II 11 (1879) 684; Ridl. in Journ. Linn. Soc. 22 (1886) 275; Hook. f. in Bot. Mag. 127 (1901) t. 7804; Ames Orch. 5 (1915) 83.

Sulu Archipelago (Buut Island), Jolo, Burbidge s. n., J. Clemens s. $n$. Wooded hillside at $660 \mathrm{~m}$ (?) altitude.

LIPARIS TRICHOGLOTTIS (Ames) Schltr. in Fedde Repert. Beihefte 1 (Orch. Deutsch-Neu-Guin.) (1911) 203; Ames Orch. 5 (1915) 83. Cestichis trichoglottis Ames Orch. 2 (1908) 139, text cut.

Luzon (Laguna, Tayabas), Mindanao (Lanao), Basilan, B. S. 1656s Ramos, 16253 Reillo, Elmer 7612, M. S. Clemens 131.

LIPARIS WENZELII Ames Orch. 5 (1915) 84.

Leyte, Wenzel 0403 . Epiphyte in forest at $500 \mathrm{~m}$ altitude.

EXCLUDED OR SYNONYMOUS SPECIES

LIPARIS AFFinIS Lindl. apud Naves Novis. App. (1882) 230.

LIPARIS DIPHyllos Nim. in Grah. Cat. Bomb. Pl. (1839) $252=$ Habenaria diphylla (Nim.) Dalz.

LIPARIS DISTICHA Ames Orch. 5 (1915) 79, non Lindl. = Liparis propinqua Ames. 
LiPARIS ELMERI Ames in Elm. Leafl. Philip. Bot. 5 (1912) 1561, nec Cestichis elmeri Ames, nec Liparis elmeri (Ames) Schltr. = Liparis dumaguetensis Ames.

LIPARIS GREGARIA Rolfe in Journ. Bot. 23 (1885) 215; Vidal Phan. Cuming. Philip. (1885) 148, Rev. Pl. Vasc. Filip. (1886;) 266, non Lindl. =

Liparis propinqua Ames.

LIPARIS HABENARIA F.-Muell. apud Naves Novis. App. (1882) 230.

Liparis laAtifolia Ames in Elm. Leaf. Philip. Bot. 5 (1912) 1562, Orch. 5 (1915) 80, non Lindl. = Liparis pallida (Blume) Lindl.

LIPAßIS MINIMA Lindl. Gen. \& Sp. Orch. (1830) 32; Naves Novis. App. (1882) 230 = Liparis caespitosa (Thou.) Lindl.

LIPARIS MUCRONATA Lindl. apud Naves Novis. App. (1882) 230.

LIPARIS REFLEXA Lindl. apud Naves Novis. App. (1882) 229.

LIPARIS RHEEDII Lindl, apud Naves Novis. App. (1882) 230.

LIPARIS TRILOBA Ridl, in Journ. Linn. Soc. 22 (1886) 295; Ames Orch. 5 (1915) $84=$ Liparis longipes Lindl.

LIPARIS VIRIDIFLORA Lindl. apud Naves Novis. App.. (1882) 230.

48. DILOCHIA Lindley

DILOCHIA ELMERI Ames in Elm. Leafl. Philip. Bot. 5 (1912) 1554, Orch. 5 (1915) 8 อ.

Luzon (Rizal), Leyte, Mindanao (Davao), Reillo s. n., Wenzel 0446, 0579, Elmer 10664. Epiphyte, up to $500 \mathrm{~m}$ altitude in Leyte.

49. CERATOSTYLIS Blume

CERATOSTYLIS DATAENSIS Ames Orch. 7 (1922) 151.

Luzon (Lepanto), Ramos \& Edaño \%6. Mt. Data, epiphyte in mossy forest at $2,540 \mathrm{~m}$ altitude.

CERATOSTYLIS ELMERI Ames Sched. Orch. 6 (1923) 49.

Mindanao (Davao), Elmer 11358. Epiphyte at 2,580 m altitude.

CERATOSTYLIS LATIPETALA Ames in Philip. Journ. Sci. 4 (1909) Bot. 671 , Orch. 5 (1915) 86.

Luzon (Rizal), Mindanao (Zamboanga, Misamis, Bukidnon, Agusan, Davao), B. S. 19232 Reillo, 38815, 38953 Ramos \& Edaño, F. B. 4661 Mearns \& Hutchinson, Elmer 10614, 13801, Merrill 8134, Copeland s. n. Mountainous regions up to $1,400 \mathrm{~m}$ altitude in Misamis.

CERATOSTYLIS MINDANAENSIS Ames Sched. Orch. 6 (1923) 51.

Mindanao (Bukidnon), B. S. 39015 Ramos \& Edaño. At 1,600 m altitade.

CERATOSTYLIS PHILIPPINENSIS Rolfe ex Ames Orch. 1 (1905) 79 , text cut, 2 (1908) 149, 5 (1915) 86; Ames in Philip. Journ. Sci. 6 (1911) Bot. 50.

Luzon (Bontoc, Lepanto, Benguet, Bataan, Camarines), Leyte. A rather common species in the mountainous regions of Luzon, at 700 to $2,100 \mathrm{~m}$ altitude. 
CERATOSTYLIS RAMOSA Ames \& Rolfe in Philip. Journ. Sci. 2 (1907)

Bot. 323, nomen; Ames Orch. 2 (1908) 149, text cut, 5 (1915) 86.

Luzon (Bontoc, Bataan), Mindoro, Mindanao (Zamboanga), Vanoverbergh 785, 1390, Whitford 45\%, Leiberg 6039, Merrill 8130, Merritt 4418.

CERATOSTYLIS RETISQUAMA Reichb. f. in Bonpl. 5 (1857) 53; Rolfe in Journ. Bot. 23 (1885) 215; Vidal Phan. Cuming. Philip. (1885) 149, Rev. Pl. Vasc. Filip. (1886) 269; Ames Orch. 5 (1915) 86. Philippines, Cuming 2152.

CERATOSTYLIS RUBRA Ames in Philip. Journ. Sci. 4 (1909) Bot.670, Orch. 5 (1915) 86.

Luzon (Ilocos Norte, Rizal, Bataan, Camarines), Mindanao (Lanao), B. S. 3042, 26220, 39231 Ramos, 3070 Foxworthy, 384, 3079. M. S. Clemens, Santos s. n., Lyon $J$.

CERATOSTYLIS SENILIS Reichb. 1. Otia Bot. Hamb. 1 (1878) 54, Xen. Orch. 3 (1881) 30; Ames Orch. 2 (1908) 150, 5 (1915) 86.

Luzon (Bataan, Rizal, Laguna, Tayabas, Sorsogon), Mindanao (Surigao), R. S. Williams 506, B. S. 3017, 13761, 1936:-Ramos, Elmer 9262, 14964, Didson s. n., F. B. 7788 Curran \& Merritt, Bolster 215, Taylor 28. Epiphyte up to $900 \mathrm{~m}$ altitude in Tayabas and $1,000 \mathrm{~m}$ in Laguna.

CERATOSTYLIS subulatA Blume Bijdr. (1825) 306; Lindl. Gen. \& Sp. Orch. (1833) 231; Reichb. f. in Bonpl. 5 (1857) 53; Miq. Fl. Ind. Bat. 3 (1859) 706; Reichb. f. Xen. Orch. 2 (1863) t. 12\%, fig. II, 2, 2 (1865) 93; Naves Novis. App. (1882) 245; J. J. Sm. Fl. Buitenz. 6 (Orch. Jav.) (1905) 299, Fig.-Atlas (1910) fig. 229; Ames in Philip. Journ. Sci. 2 (1907) Bot. 323, Orch. 2 (1908) 151, text cut, 5 (1915) 86, 6 (1920) 138, in Merr. in Journ. Roy. Asiat. Soc. Straits Branch, Special No. (1921) 176.

Appendicula teres Griff. Notul. 3 (1851) 359, Icon. Pl. Asiat. 3 (1851) t. 332.

Ceratostylis teres Reichb. f. in Bonpl. 2 (1854) 89, in Walp. Ann. 6 (1862) 470; Hook. f. Fl. Brit. Ind. 5 (1890) 825; King \& Pantl. in Ann. Roy. Bot. Gard. Calc. 8 (Orch. Sik.-Himal.) (1898) 247, t. 329.

Ceratostylis cepula Reichb. $f$. in Bonpl. 5 (1857) 53.

Ceratostylis gracilis Reichb. f. Xen. Orch. 2 (1863) t. 127, fig. I, 1 , 2 (1865) 92; Naves Novis. App. (1882) 245; Vidal Rev. PI. Vasc. Filip. (1886) 269; Ridl. in Journ. Linn. Soc. 32 (1896) 307, p. p., Ames Orch. 1 (1905) 80; Ridl. Mat. Fl. Mal. Penin. 1 (1907) 109, non Blume.

Corymbis gracilis Vidal Rev. Pl. Vasc. Filip. (1886) 272; Ceron Cat. Pl. Herb. Manila (1892) 168, non Blume.

Ceratostylis malaccensis Hook. f. Fl. Brit. Ind. 5 (1890) 825, in Hook. Icon. Fl. 21 (1892) t. 2098; Ridl. in Journ. Linn. Soc. 32 (1896) 308.

Luzon (Ifugao, Benguet, Nueva Vizcaya, Bataan, Pampanga, Rizal), Mindoro, Panay (Capiz), Mindanao (Lanao, Zamboanga, Bukidnon, Davao). A fairly common species in the Philippines. Epiphyte at 1,600 
to $2,160 \mathrm{~m}$ altitude. In India, Assam, Malay Peninsula, Sumatra, Java, and Borneo.

CERATOSTYLIS Wenzelil Ames in Philip. Journ. Sci. 8 (1913) Bot. 421, Orch. 5 (1915) 87.

Leyte, Basilan, Wenzel 013, 0175, 0661, 0678, 0745, B. S. 16256 Reilln. Epiphyte at 60 to $800 \mathrm{~m}$ altitude.

\section{EXCLUDED OR SYNONYMOUS SFECIES}

CeratostYus Gigas Reichb. f. apud Naves Novis. App. (1882) 245.

Ceratostylis Graculis Reichb. f. Xen. Orch. 2 (1863) t. 127, fig. I, 1, 2 (1865) 92; Naves Novis. App. (1882) 245; Vidal Rev. Pl. Vasc. Filip. (1886) 269; Ridl. in Journ. Linn. Soc. 32 (1896) 307, p. p., Ames Orch. 1 (105) 80; Ridl. Mat. Fl. Mal. Penin. 1 (1907) 109, non Blume $=$ Ceratostylis subulata Blume.

Ceratostylis graminea Blume apud Naves Novis. App. (1882) 245.

\section{AGROSTOPHYLLUM Blume}

AGROSTOPHYLLUM: ELMERI Ames Sched. Orch. 6 (1923) 48.

Luzon (Laguna), Elmer $1804 \%$.

AGROSTOPHYLLUM HASSELTII (Blume) J. J. Sm. in Icon. Bogor. 2 (1903) 55, in textu, Fl. Buitenz. 6 (Orch. Java) (1905) 288, Fig.Atlas (1910) fig. 220; Ames Orch. 5 (1915) 87.

Appendicula hasseltii Blume Bijdr. (1825) 304; Lindl. Gen. \& Sp. Orch. (1833) 230; Miq. Fl. Ind. Bat. 3 (1859) 704.

Appendicula graminifolia Teijsm. \& Binn. in Nat. Tijdschr. Ned. Ind. 24 (1862) 322.

Appendicula elongata Ridl. in Trans. Linn. Soc. II 3 (1893) 375;

Ridl. in Journ. Linn. Soc. 32 (1896) 389.

Podochilus hasseltii Schltr. in Mém. Herb. Boiss. 21 (1900) 61; Ridl.

Mat. Fl. Mal. Penin. 1 (1907) 198.

Leyte, Mindanao (Agusan), Wenzel 0610, 0719, 0742, 0763, 0798, Weber $30 \%$. In the forest at 500 to $800 \mathrm{~m}$ altitude. In Malay Peninsula, Sumatra, and Java.

AGROSTOPHYLLUM INOCEPHALUM (Schauer) Ames Orch. 2 (1908) 148,5 (1915) 87.

Diploconchium inocephalum Schauer in Nov. Act. Nat. Cur. 19, Suppl. 1 (1843) 428, t. 12, fig. A.

Luzon (Benguet, Bataan), Mindanao (Lanao, Agusan), R. S. Williams 1926, Lyon T., M. S. Clemens s. n., 130, Weber 6, 161, Elmer 13852. On rocks or in trees from sea level to $500 \mathrm{~m}$ altitude.

AGROSTOPHYLLUM LEYTENSE Ames Orch. 6 (1920) 298.

Leyte, Wenzel 0597, 0632, 0654. Jaro, in forest at 600 to $700 \mathrm{~m}$ altitude. AGROSTOPHYLLUM LONGIVAgINATUM Ames in Philip. Journ. Sci. 8 (1913) Bot. 420, Orch. 5 (1915) 87.

Luzon (Apayao, Rizal, Laguna), Mindoro, Leyte, Weber 345, Serrato s. n., B. S. 16584 Ramos, 5656 Merrill, 42061 Lopez, Wenzel 0170, 0171, $0240,0619,0646,0825$. In the forest at 30 to $600 \mathrm{~m}$ altitude. 
AgRostophyLLUM MALINDANGense Ames in Philip. Journ. Sci. 6 (1911) Bot. 50, Orch. 5 (1915) 88.

Mindanao (Misamis, Bukidnon, Davao), F. B. 4610, Mearns \& Hutchinson, B. S. 38836 Ramos \& Edaño, Elmer 10868. On mountains.

AGROSTOPHYLLUM MEARNSII Ames in Philip. Journ. Sci. 8 (1913) Bot. 420 , Orch. 5 (1915) 88.

Luzon (Bataan), Mindanao (Misamis, Agusan), with Whitford 457, F. B. $460 \%$ Mearns \& Hutchinson, Elmer 13700. On mountains at 1,060 to $1,560 \mathrm{~m}$ altitude.

AGROSTOPHYLLUM MERRILLII Ames in Philip. Journ. Sci. 2 (1907) Bot. 323, Orch. 5 (1915) 88.

Mindoro, Merrill 5844. Mt. Halcon, in forest at $1,000 \mathrm{~m}$ altitude.

AGROSTOPHYLLUM MINDANAENSE Ames Orch. 5 (1915) 88.

Agrostophyllum pelorioides Ames in Philip. Journ. Sci. 8 (1913) Bot. 421, non Schltr.

Mindanao (Cotabato, Davao), Weber 231, Copeland 1119. On mountains at about $860 \mathrm{~m}$ altitude.

AgRostophyLLUM PHILIPPINENSE Ames in Philip. Journ. Sci. 4 (1909) Bot.669, Orch. 5 (1915) 88.

Babuyan Islands (Camiguin Island), Luzon (Bataan, Rizal, Sorsogon), Leyte, Negros. Apparently fairly common, especially in Leyte, and widespread. On mountains, in the forest at 60 to $700 \mathrm{~m}$ altitude.

AGROSTOPHYLLUM WENZELII Ames Orch. 5 (1915) 88.

Leyte, Mindanao (Agusan), Wenzel 028, 017\%, 0212, 0752, 0824, Weber 104, Elmer 1396\%. At 60 to $800 \mathrm{~m}$ altitude.

EXCLUDED OR SYNONYMOUS SPECIES

Agrostophyllum Pelorioides Ames in Philip. Journ. Sci. 8 (1913) Bot. 421, non Schltr. = Agrostophyllum mindanaense Ames.

51. GLOMERA Blume

GLOMERA MERRILLII Ames in Philip. Journ. Sci. 8 (1913) Bot. 419, Orch. 5 (1915) 89.

Mindanao (Zamboanga), Merrill 8290. Sax River, epiphyte in damp shaded ravine at about $200 \mathrm{~m}$ altitude.

LXXLUDED OR SYNONYMOUS SPECIES

Glomera erythrostoma Blume apud Naves Novis. App. (1882) 245.

52. PODOCHILUS Blume

PODOCHILUS BICAUDATUS Schltr. in Fedde Repert. 3 (1906) 19; Ames in Philip. Journ. Sci. 4 (1909) Bot. 669, 8 (1913) Bot. 415, Orch.

5 (1915) 90.

Palawan, Basilan, Usteri s. n., Merrill 775, B. S. 16255, 16259A Reillo. Up to $560 \mathrm{~m}$ altitude on Palawan. 
PODOCHILUS CUMINGII Schltr. in Fedde Repert. 3 (1906) 19; Ames in Philip. Journ. Sci. 4 (1909) Bot. 668, 8 (1913) Bot. 415, Orch. 5 (1915) 89.

Podochilus zollingeri Schltr. in Mém. Herb. Boiss. 21 (1900) 23, non Reichb. f.

Catanduanes, Luzon (Rizal), B. S. 30310 Ramos, Reillo s. n. Epiphyte in forest at about $100 \mathrm{~m}$ altitude.

PODOCHILUS HYSTRICINUS Ames Sched. Orch. 6 (1923) 42, fig. 8.

Leyte, Wenzel 0262, 0878. At 700 to $750 \mathrm{~m}$ altitude

PODOCHILUS INTRICATUS Ames in Philip. Journ. Sci. 8 (1919) Bot. 415,416 , Orch. 5 (1915) 90.

Luzon (Tayabas), Mindanao (Agusan, Davao), Basilan, Disdon s. n., Elmer 13704, Copeland 1164, Weber 244, B. S. 16263 Reillo s. n., 19406 Ramos. Epiphyte at 600 to $1,030 \mathrm{~m}$ altitude.

PODOCHILUS LONGILABRIS Ames in EIm. Leaff. Philip. Bot. 5 (1912) 1565, in Philip. Journ. Sci. 8 (1913) Bot. 415, Orch. 5 (1915) 90.

Luzon (Laguna, Tayabas, Camarines), Quisumbing s. n., Whitford 939, Elmer 7585, B. S. 22038 Ramos. Epiphyte at 1,160 to $1,500 \mathrm{~m}$ altitude.

POdochILUS PLUmosus Ames in Philip. Journ. Sci. 4 (1909) Bot. 668, 8 (1913) Bot. 415, Orch. 5 (1915) 90.

Luzon (Nueva Ecija, Zambales, Laguna, Tayabas), Panay (Capiz), Leyte, Mindanao (Zamboanga, Cotabato, Agusan, Surigao). A fairly common species, especially in Luzon. Epiphyte at 80 to $960 \mathrm{~m}$ altitude.

PODOCHILUS RAMOSII Ames in Philip. Journ. Sci. 8 (1913) Bot. 415, 417, Orch. 5 (1915) 90.

Mindanao (Camiguin Island), B. S. 14441 Ramos.

PODOCHILUS SCIUROIDES Reichb. f. in Bonpl. 5 (1857) 41; Schltr. in Mém. Herb. Boiss. 21 (1900) 20; J. J. Sm. Fl. Buitenz. 6 (Orch. Jav.) (1905) 511, Fig.-Atlas (1912) fig. 388; Ridl. Mat. Fl. Mal. Penin. 1 (1907) 192; Ames Orch. 6 (1920) 140, in Merr. in Journ. Roy. Asiat. Soc. Straits Branch, Special No. (1921) 177.

Cryptoglottis serpyllifolia Reichb. f. in Bonpl. 5 (1857) 42, non Blume.

Podochilus microphyllus Hook. f. Fl. Brit. Ind. 6 (1890) 81; Grant Orch. Burma (1895) 320; Ridl. in Journ. Linn. Soc. 31 (1896) 301, 32 (1896) 386; Ames in Merr. in Journ. Roy. Asiat. Soc. Straits Branch, Special No. (1921) 177, non Lindl.

Podochilus curviunguis Schltr. in Fedde Repert. 10 (1911) 34; Ames Orch. 5 (1915) 89.

Luzon (Pampanga, Bataan, Tayabas), Mindanao (Zamboanga, Lanao), Basilan, Merrill 4260, 8311, B. S. 1678 Foxworthy, 16259 Reillo, 28652 Ramos \& Edaño, M. S. Clemens s. n. Epiphyte in mossy forest at 450 to $850 \mathrm{~m}$ altitude. In Burma, Malay Peninsula, Sumatra, Java, Celebes, and Borneo. 
PODOCHILUS SERPYLLIFOLIUS (Blume) Lindl, in Journ. Linn. Soc.

3 (1859) 37, in nota (by inference only); ex Miq. Fl. Ind. Bat.

3 (1859) 688; Naves Novis. App. (1882) 244; Schltr. in Mém.

Herb. Boiss. 21 (1900) 20; J. J. Sm. Fl. Buitenz. 6 (Orch. Jav.) (1905) 508, Fig.-Atlas (1912) fig. 385; Ames Orch. 5 (1915) 90, 6 (1920) 140, in Merr. in Journ. Roy. Asiat. Soc. Straits Branch, Special No. (1921) 177.

Cryptoglottis serpyllifolia Blume Bijdr. (1825) 297, fig. 42; Lindl.

Gen. \& Sp. Orch. (1833) 230; Reichb. f. in Walp. Ann. 6 (1864) 894. Hexameria disticha R. Br. in Benn. Pl. Jav. Rar. (1838) 26, t. 7;

Schauer in Nov. Act. Nat. Cur. 19, Suppl. 1 (1843) 431; Miq.

Fl. Ind. Bat. 3 (1859) 656.

Luzon, Meyen s. $n$.

PODOCHILUS STRICTUS Ames in Philip. Journ. Sci. 4 (1909) Bot. 669, 8 (1913) Bot. 415, Orch. 5 (1915) 90.

Podochilus robinsonii Ames in Philip. Journ. Sci. 6 (1911) Bot. 49, 8 (1913) Bot. 415.

Podochilus lucescens Ames Orch. 5 (1915) 90, non Blume.

Luzon (Cagayan, Rizal, Laguna, Tayabảs, Camarines, Sorsogon), Catanduanes, Panay, Negros, Leyte, Samar, Mindanao (Zamboanga, Lanao, Agusan, Surigao, Davao), Basilan. A common, variable, and widely distributed species. Epiphyte at 10 to $1,500 \mathrm{~m}$ altitude.

\section{EXCLUDED OR SYNONYMOUS SPECIES}

Podochilus ANceps Schltr. in Mém. Herb. Boiss. 21 (1900) 33; Ames Orch. 2 (1908) $142=$ Appendicula anceps Blume.

Podochilus clemensiae Ames in Philip. Journ. Sci. 4 (1909) Bot. $667=$ Appendicula clemenside Ames.

Podochilus cornutus Schltr. in Mém. Herb. Boiss. 21 (1900) 34; Ridl. Mat. Fl. Mal. Penin. 1 (1907) 194; Ames in Philip. Journ. Sci. 2 (1907) Bot. 322, Orch. 2 (1908) $143=$ Appendicula cornuta Blume.

Podochilus crotalinus Ames in Philip. Journ. Sci. 4 (1909) Bot. $667=$ Appendicula crotalina (Ames) Schitr.

Podochilus curviunguis Schltr. in Fedde Repert. 10 (1911) 34; Ames Orch. 5 (1915) $89=$ Podochilus sciuroides Reichb. $\mathrm{f}$.

PoDochILUs ELMERI Ames in Elm. Leafl. Philip. Bot. 5 (1912) $1565=$ Appendicula elmeri Ames.

PoDochilus FenIXII Ames in Philip. Journ. Sci. 6 (1911) Bot. $48=$ Appendicula fenixii (Ames) Schltr.

PoDochilus Fruticosus Ames in Philip. Journ. Sci. 6 (1911) Bot. $48=$ Appendicula buxifolia Blume.

PoDochilus LONGICALCARATUs Rolfe in Kew Bull. (1904) $186=$ Appen dicula undulata Blume var. longicalcarata (Rolfe) Ames.

Podochilus Lucbanensis Ames in Elm. Leafl. Philip. Bot. 5 (1912) 1566 $=$ Appendicula lucbanensis Ames. 
Podochilus Lucescens Ames Orch. 5 (1915) 90, non Blume = Podochilus strictus Ames.

Podochilus Luzonensis Ames in Elm. Leafl. Philip. Bot. 5 (1912) 1567 $=$ Appendicula luzonensis Ames.

Podochilus Malindangensis Ames in Philip. Journ. Sci. 6 (1911) Bot. $49=$ Appendicula malindangensis (Ames) Schltr.

Podochilus Micranthus Schltr. in Mém. Herb. Boiss. 21 (1900) 36, 39; Ames Orch. 2 (1908) $144=$ Appendicula mierantha Lindl.

Podochilus negrosianus Ames in Elm. Leafl. Philip. Bot. 5 (1912) $1568=$ Appendicula negrosiana Ames.

Podochilus Pendulus Schltr. in Mém. Herb. Boiss. 21 (1900) 48, quoad Philip.; Ames in Philip. Journ. Sci. 2 (1907) Bot. 322, 8 (1919) Bot. 415, Orch. 2 (1908) $145=$ Appendicula philippinensis (Schltr.) J. J. Sm.

Podochilus Perplexus Ames in Elm. Leafl. Philip. Bot. 5 (1912) $1569=$ Appendicula perplexa Ames.

Podochilus PHilippinensis Schltr. in Mém. Herb. Boiss. 21 (1900) 49; Ames in Philip. Journ. Sci. 2 (1907) Bot. $322=$ Appendicula philippinensis (Schltr.) J. J. Sm.

PoDochIlus REFlexus Ames in Philip. Journ. Sci. 2 (1907) Bot. 322, non Schltr. = Appendicula micrantha Lindl.

Podochilus Robinsonil Ames in Philip. Journ. Sci. 6 (1911) Bot. 49, 8 (1913) Bot. $415=$ Podochilus strictus Ames.

PODOCHILUS SCALPELliformis Blume apud Naves Novis. App. (1882) 244.

This is probably Podochilus strictus Ames.

Podochilus tenuis Lindl. apud Naves Novis. App. (1882) 244.

Podochilus undulatus Schltr. in Mém. Herb. Boiss. 21 (1900) $42=$ Appendicula undulata Blume.

Podochilus undulatus Schltr. var. LONGICALCARATUS Schltr. in Mém. Herb. Boiss. 21 (1900) 43; Ames Orch. 2 (1908) $146=$ Appendicula undulata Blume var. longicalcarata (Rolfe) Ames.

Podochilus XYTRIophorus Schltr. in Mém. Herb. Boiss. 21 (1900) 47; Ames in Philip. Journ. Sci. 2 (1907) Bot. 322, Orch. 2 (1908) 147 = Appendicula xytriophora Reichb. $\mathrm{f}$.

Podochilus zollingeri Schitr. in Mém. Herb. Boiss. 21 (1900) 23, non Reichb. f. = Podochilus cumingii Schltr.

53. APPENDICULA Blume

APPENDICULA ANCEPS Blume Bijdr. (1825) 299; Lindl. Gen. \& Sp. Orch. (1833) 228; Miq. Fl. Ind. Bat. 3 (1859) 702; J. J. Sm. Fl. Buitenz. 6 (Orch. Jav.) (1905) 518, Fig.-Atlas (1912) fig. 393; Ames in Philip. Journ. Sci. 8 (1913) Bot. 415, Orch. 5 (1915) 91.

Metachilum cyathiferum Lindl. in Wall. Cat. (1829) No. 2022, nomen; Lindl. Gen. \& Sp. Orch. (1830) 74. 
Appendicula lewisii Griff. in Calc. Journ. Nat. Hist. 4 (1844) 378, t. 19, Notul. 3 (1851) 360, Icon. Pl. Asiat. 3 (1851) t. 337"; Reichb. f. in Walp. Ann. 6 (1864) 893; Hook. f. Fl. Brit. Ind. 6 (1890) 83; Ridl. in Journ. Linn. Soc. 32 (1896) 389.

Appendicula cyathifera Reichb. f. MSS ex Hook. f. Fl. Brit. Ind. 6 (1890) 83.

Dendrobium vaginatum Wall. MSS ex Hook. f. Fl. Brit. Ind. 6 (1890) 83.

Appendicula complanata Ridl. in Journ. Linn. Soc. 32 (1896) 389.

Podochilus anceps Schltr. in Mém. Herb. Boiss. 21 (1900) 33; Ames Orch. 2 (1908) 142; Schltr. in Engl. Bot. Jahrb. 45, Beibl. 104 (1911) 19.

Mindanao (Zamboanga), Basilan, Merrill 8122, 8128, Copeland s. n., B. S. 16238, 16245 Reillo, 37006, 37361 Ramos \& Edaño. Terrestrial in forest at $560 \mathrm{~m}$ altitude. In Malay Peninsula, Java, Borneo, and Sumatra.

APPENDICULA BUXIFOLIA Blume Bijdr. (1825) 300; Lindl. Gen. \& Sp. Orch. (1833) 228; Miq. Fl. Ind. Bat. 3 (1859) 702; J. J. Sm. Fl. Buitenz, 6 (Orch. Jav.) (1905) 532, Fig.-Atlas (1912) fig. 406; Ames Orch. 5 (1915) 93.

Appendicula frutex Ridl. in Journ. Linn. Soc. 31 (1896) 302.

Podochilus buxifolius Schltr. in Mém. Herb. Boiss. 21 (1900) 52.

Podochilus fruticosus Ames in Philip. Journ. Sci. 6 (1911) Bot. 48. Appendicula fruticosa Ames in Philip. Journ. Sci. 8 (1913) Bot. 415.

Luzon (Laguna, Sorsogon), Mindanao (Misamis, Cotabato), B. S.16905 Serviña, Elmer 16138, M. S. Clemens s. n., Weber 216. Apparently in the mountain regions at about $600 \mathrm{~m}$ altitude. In Java.

APPENDICUla CLemensiae Ames in Philip. Journ. Sci. 8 (1913) Bot. 415, Orch. 5 (1915) 91.

Podochilus clemensiae Ames in Philip. Journ. Sci. 4 (1909) Bot. 667.

Luzon (Laguna, Tayabas, Sorsogon), Leyte, Samar, Mindanao (Lanao). B. S. $13670,19514,24254$ Ramos, 24909 Edaño, Disdon s. n., Elmer 15097, 15609, 16593, Wenzel 023, 096, 0187, 0741, M. S. Clemens s. n., Boettcher s. n. Mountain regions usually, but at from 60 to $1,700 \mathrm{~m}$ altitude.

APPENDICULA CORNUTA Blume Bijdr. (1825) 302; Lindl. Gen. \& Sp. Orch. (1833) 229; Miq. Fl. Ind. Bat. 3 (1859) 704; J. J. Sm. Fl. Buitenz. 6 (Orch. Jav.) (1905) 522, Fig.-Atlas (1912) fig. 396; Ames Orch. 5 (1915) 91.

Appendicula congenera Blume Bijdr. (1825) 303; Lindl. Gen. \& Sp. Orch. (1833) 229; Miq. Fl. Ind. Bat. 3 (1859) 704.

Dendrobixm manillense Schauer in Nov. Act. Nat. Cur. 19, Suppl. 1 (1843) 430.

Appendicula manillensis Reichb. f. ex Miq. Fl. Ind. Bat. 3 (1859) 703; Naves Novis. App. (1882) 245.

Appendicula bifaria Lindl. in Hook. Kew Journ. 7 (1855) 35; Benth. Fl. Hongk. (1861.) 358; Reichb. f. in Walp. Ann. 6 (1864) 893; Hook. f. Fl. Brit. Ind. 6 (1890) 82; Ridl. in Journ. Linn. Soc. 31 (1896) 301, 32 (1896) 387; King \& Pantl. in Ann. Roy. Bot. 
Gard. Calc. 8 (Orch. Sik.-Himal.) (1898) 248, t. 330 ; Rolfe in Journ. Linn. Soc. 36 (1903) 39.

Appendicula reduplicata Par. \& Reichb. f. Otia Bot. Hamb. 1 (1878) 45.

Podochilus cornutus Schltr. in Mém. Herb. Boiss. 21 (1900) 34; Ridl. Mat. Fl. Mal. Penin. 1 (1907) 194; Ames in Philip. Journ. Sci. 2 (1907) Bot. 322, Orch. 2 (1908) 143; Schltr. in Engl. Bot. Jahrb. 45, Beibl. 104 (1912) 19.

Podochilus brachiatus Schltr. in Mém. Herb. Boiss. 21 (1900) 38.

Podochilus congener Schltr. in Mém. Herb. Boiss. 21 (1900) 40.

Appendicula brachiata Schltr. in Fedde Repert. Beihefte 1 (Orch. Deutsch-Neu-Guin.) (1912) 337.

Luzon (Laguna), Mindoro, Negros (Oriental Negros), Leyte, Palawan Mindanao (Agusan, Davao), B. S. 16573 Ramos, 641 Foxworthy, 41399 Herre, Merrill 5541, 5843, Wenzel 0106, 0302, 0310, 0311, Weber 192, Elmer 11329. In humid forest at 210 to $2,000 \mathrm{~m}$ altitude. In India, Malay Peninsula, Java, Borneo, and China.

APPENDICULA CROTALINA (Ames) Schltr. in Fedde Repert. Beihefte 1 (Orch. Deutsch-Neu-Guin.) (1912) 336, in Philip. Journ. Sci. 8 (1913) Bot. 416, Orch. 5 (1915) 93.

Podochilus crotalinus Ames in Philip. Journ. Sci. 4 (1909) Bot. 667.

Mindanao (Misamis, Cotabato, Davao), M. S. Clemens s, n., Weber 230 , Elmer 10695. Mountainous regions up to $860 \mathrm{~m}$ altitude.

APPENDICULA CUNEATA Ames Sched. Orch. 6 (1923) 44, fig. 9, habit and flower.

Luzon (Tayabas), Leyte, Samar, Mindanao (Bukidnon, Cotabato, Da'vao), B. S. 28750 , 38644 Ramos \& Edaño, 24236, 24524 Edaño, Wenzel 0635, 0668, 0685, 0702, Weber 237, Elmer 10696.

APPENDICULA ELMERI Ames in Philip. Journ. Sci. 8 (1913) Bot. 416, Orch. 5 (1915) 92.

Podochilus elmeri Ames in Elm. Leafl. Philip. Bot. 5 (1912) 1565.

Mindanao (Agusan, Bukidnon, Cotabato, Davao), Weber 86, 226, B. S. 386.30 , 39048 Ramos \& Edaño, Elmer 10718. Along streams at 400 to $1,300 \mathrm{~m}$ altitude.

APPENDICULA FENIXII (Ames) Schltr. in Fedde Repert. Beihefte 1 (Orch. Deutsch-Neu-Guin.) (1912) 336; Ames in Philip. Journ. Sci. 8 (1913) Bot. 415, Orch. 5 (1915) 91.

Podochilus fenixii Ames in Philip. Journ. Sci. 6 (1911) Bot. 48.

Batan Islands (Batan), B. S. 3794 Fenix. Mt. Iraya, near the summit. APPENDICULA IRIGENSIS Ames Sched. Orch. 5 (1923) 5.

Luzon (Rizal), B. S. 41941 C Ramos. Mt. Iriga, epiphyte in dense forest at $1,000 \mathrm{~m}$ altitude.

APPENDICULA LEYTENSIS Ames Sched. Orch. 6 (1923) 47, fig. 9, flower.

Leyte, Wenzel 0251, 0283, 0359; 0364, 041\%, 0482. Epiphyte in forest at $500 \mathrm{~m}$ altitude. 
Appendicula lucbanensis Ames in Philip. Journ. Sci. 8 (1913) Bot. 415, Orch. 5 (1915) 92.

Podochilus lucbanensis Ames in Elm. Leafl. Philip. Bot. 5 (1912) 1566.

Luzon (Apayao, Abra, Bontoc, Rizal, Tayabas), Panay (Capiz), Mindanao (Camiguin Island, Agusan), B. S. 28350 Fenix, 7143, 14427, 419416, 8. n., Ramos, 30778, 37888 Ramos \& Edaño, Elmer 8086, 13688, 13743. Apparently restricted to mountain regions.

APPENDICUla LUZONensis Ames in Philip. Journ. Sci. 8 (1913) Bot. 415, Orch. 5 (1915) 92.

Podochilus luzonensis Ames in Elm. Leafl. Philip. Bot. 5 (1912) 1567. Luzon (Tayabas), Elmer 7930.

APPENDICULA MALINDANGensis (Ames) Schltr. in Fedde Repert. Beihefte 1 (Orch. Deutsch-Neu-Guin.) 337; Ames in Philip. Journ. Sci. 8 (1913) Bot. 415, Orch. 5 (1915) 91.

Podochilus malindangensis Ames in Philip. Journ. Sci. 6 (1911) Bot. 49, in Elm. Leafl. Philip. Bot. 5 (1912) 1567.

Negros (Oriental Negros), Mindanao (Misamis, Bukidnon), Elmer 9543, F. B. 4733 Mearns \& Hutchinson, B. S. 38563 Ramos \& Edaño. Mountainous regions up to $1,560 \mathrm{~m}$ altitude.

APPENDICULA MAQUILINGENSIS Ames in Philip. Journ. Sci. 8 (1913) Bot. 417, Orch. 5 (1915) 92.

Luzon (Laguna, Camarines, Sorsogon), Catanduanes, Panay (Antique, Capiz), Leyte, Negros (Oriental Negros), Mindanao (Camiguin Island, Bukidnon, Davao), Jolo. A fairly common and widely distributed species at 40 to $2,000 \mathrm{~m}$ altitude, on rocks or on trees in mossy forest.

APPENDICULA MERRILlII Ames in Philip. Journ. Sci. 8 (1913) Bot. $415,418$.

Chilopogon merrillii Ames Orch. 5 (1915) 91.

Mindanao (Zamboanga, Cotabato), Merrill 8135, Weber 219, B. S. 36974 Ramos \& Edaño. Epiphyte at 700 to $1,160 \mathrm{~m}$ altitude.

APPENDICULA MICRANTHA Lindl. in Ann. Mag. Nat. Hist. 15 (1845) 386; Rolfe in Journ. Bot. 23 (1885) 216; Vidal Phan. Cuming. Philip. (1885) 151, Rev. Pl. Vasc. Filip. (1886) 272; Ames Orch. 5 (1915) 91.

Podochilus micranthus Schltr. in Mém. Herb. Boiss. 21 (1900) 36, 39; Ames Orch. 2 (1908) 144.

Podochilus reflexus Ames in Philip. Journ. Sci. 2 (1907) Bot. 322, non Schitr.

Appendicula wenzelii Ames in Philip. Journ. Sci. 8 (1913) Bot. 419, Orch. 5 (1915) 91.

Luzon (Pampanga, Bataan, Rizal, Laguna, Tayabas), Polillo, Mindoro, Panay, (Capiz, Antique), Negros, Leyte, Palawan, Mindanao (Bukidnon, Agusan, Davao). A common and widely distributed species in the Philippines. Terrestrial or epiphytic at 15 to $600 \mathrm{~m}$ altitude. 
APPENDICULA Negrosiana Ames in Philip. Journ. Sci. 8 (1913) Bot. 416, Orch. 5 (1915) 93.

Podochilus negrosianus Ames in Elm: Leaff. Philip. Bot. 5 (1912) 1568.

Negros (Oriental Negros), Elmer 9636. In the Cuernos Mountains.

APPENDICULA PERPLEXA Ames in Philip. Journ. Sci. 8 (1913) Bot. 415, Orch. 5 (1915) 92.

Podochilus perplexus Ames in Elm. Leafl. Philip. Bot. 5 (1921) 1569.

Luzon (Tayabas), Elmer 7407 . Mt. Banahao, in shaded forest at 800 in altitude.

APPENDICULA PHILIPPINENSIS (Schltr.) J.J. Sm. Orch. Amb. (1905) 91, in textu; Ames in Philip. Journ. Sci. 8 (1913) Bot. 415, Orch. 5 (1915) 92.

Appendicula pendula Blume apud Naves Novis. App. (1882) 245; Ames Orch. 5 (1915) 92.

Podochilus pendulus Schltr. in Mém. Herb. Boiss. 21 (1900) 48, quoad Philip.; Ames in Philip. Journ. Sci. 2 (1907) Bot. 322, 8 (1913) Bot. 415, Orch. 2 (1908) 145.

Podochilus philippinensis Schltr. in Mém. Herb. Boiss. 21 (1900) 49; Ames in Philip. Journ. Sci. 2 (1907) Bot. 322.

Luzon (Tayabas, Laguna, Sorsogon), Mindoro, Leyte, Palawan, Mindanao (Zamboanga, Bukidnon). A variable and fairly common species at 115 to $1,600 \mathrm{~m}$ altitude.

APPENDICULA POLYANTHA Ames Sched. Orch. 5 (1923) 6.

Luzon (Rizal), B. S. 42058 Lopez, 29733 Ramos \& Edaño. Mt. Angilog, at $2,200 \mathrm{~m}$ altitude.

APPENDICULA TAGALENSIUM Kränzl. in Ann. k. k. Nat. Hofmus. Wien 30 (1916) 65.

Philippines, Loher s. $n$.

No specimens of this species have been seen.

APPENDICULA UNDULATA Blume Bijdr. (1825) 301; Lindl. Gen. \& Sp. Orch. (1833) 229; Miq. Fl. Ind. Bat. 3 (1959) 703; J. J. Sm. Fl. Buitenz. 6 (Orch. Jav.) (1905) 527, Fig.-Atlas (1912) t. 402; Ames in Merr. in Journ. Roy. Asiat. Soc. Straits Branch, Special No. (1921) 179.

Appendicula purpurascens De Vriese Ill. Orch. Ind. Neerl. (1854) t. 12, fig. 1, non Blume.

Appendicula peruligera Reichb. $f$. in Bonpl. 5 (1857) 41.

Podochilus unciferus Hook. f. Fl. Brit. Ind. 6 (1890) 81, in Hook. Icon. Pl. 22 (1893) t. 2145; Ridl. in Journ. Linn. Soc. 32 (1896) 385, Mat. Fl. Mal. Penin. 1 (1907) 194.

Podochilus undulatus Schltr. in Mém. Herb. Boiss. 21 (1900) 42.

The species proper is a native of Malay Peninsula, Java, Sumatra, and Borneo, represented in the Philippines by the following variety: 
Var. LongicAlCARATA (Rolfe) Ames in Merr. in Journ. Roy. Asiat. Soc. Straits Branch, Special No. (1921) 179.

Podochilus longicalcaratus Rolfe in Kew Bull. (1894) 186.

Podochilus undulatus Schltr. var. longicalcaratus Schltr. in Mém.

Herb. Boiss. 21 (1900) 43; Ames Orch. 2 (1908) 146.

Appendicula undulata Blume var. calcarata Ames in Philip. Journ.

Sci. 8 (1913) Bot. 416, Orch. 5 (1915) 93, sphalm.

Luzon (Rizal), Leyte, Mindanao (Agusan, Lanao, Zamboanga, Cotabato, Surigao, Davao), Cuming s.n., Reillo s. n., B. S. 15389 Ramos, 3702,, 37963 Ramos \& Edaño, 42084 Edaño, Elmer 13622, M. S. Clemens 516, $R$. S. Williams 2331, Copeland 1584, Weber 207, 292, Wenzel 01095. In mossy forest, apparently in mountainous regions; also in Borneo (Sarawak).

APPENDICULA WEBERI Ames in Philip. Journ. Sci. 8 (1913) Bot. 418, Orch. 5 (1915) 93.

Mindanao (Agusan, Cotabato), Weber 88, 217, Elmer 13639. On mountains, in damp shady forest at 430 to $690 \mathrm{~m}$ altitude.

APPENDICULA XYTRiOPHORA Reichb. f. in Seem. Fl. Vit. (1868) 299 ;

Rolfe in Journ. Bot. 23 (1885) 215; Vidal Phan. Cuming. Philip. (1885) 151, Rev. Pl. Vasc. Filip. (1886) 272; Hook. f. Fl. Brit. Ind. 6 (1890) 84; Ridl. in Journ. Linn. Soc. 31 (1896) 301, 32 (1896) 391; Ames in Philip. Journ. Sci. 8 (1913) Bot. 415, Orch. 5 (1915) 92, in Merr. in Journ. Roy. Asiat. Soc. Straits Branch, Special No. (1921) 179.

Podochilus xytriophorus Schltr. in Mém. Herb. Boiss. 21 (1900) 47;

Ames in Philip. Journ. Sci. 2 (1907) Bot. 322, Orch. 2 (1908) 147.

Luzon (Benguet, Rizal, Laguna, Tayabas, Sorsogon), Polillo, Mindoro, Panay (Capiz), Leyte, Mindanao (Lanao, Cotabato, Davao). A common. and widely distributed species in the Philippines; in mossy forest at 60 to $1,330 \mathrm{~m}$ altitude. In Malay Peninsula and Borneo.

EXCLUDED OR SYNONYMOUS SPECIES

APPEndicula AlBA Blume apud Ames Orch. 5 (1915) 91.

This species does not occur in the Philippines.

Appendicula Fruticosa Ames in Philip. Journ. Sci. 8 (1913) Bot. $425=$ Appendicula buxifotia Blume.

APPENDICUla KUHLII Reichb. f. apud Naves Novis. App. (1882) 244.

Appendicula longifolia Blume apud Naves Novis. App. (1882) 245.

Appendicula Manillense Reichb. f. ex Miq. Fl. Ind. Bat. 3 (1859) 703;

Naves Novis. App. (1882) $245=$ Appendicula cornuta Blume.

Appendicula Pendula Blume apud Naves Novis. App. (1882) 245; Ames

Orch. 5 (1915) $92=$ Appendicula philippinensis (Schltr.) J. J. Sm.

Appendicula Reflexa Blume ex Ames in Philip. Journ. Sci. 2 (1907) Bot. 322, in syn. = Appendicula micrantha Lindl.

APPEndicula RHODiola Reichb. f. apud Naves Novis. App. (1882) 245.

ApPendicula torta Blume apud Naves Novis. App. (1882) 244.

Appendicula Wenzeli Ames in Philip. Journ. Sci. 8 (1913) Bot. 419, Orch. 5 (1915) $91=$ Appendicula micrantha Lindl. 


\section{POLYSTACHYA Hookex}

POLYSTACHYA LUTEOLA (Sw.) Hook. Exot. Fl. 2 (1824) t. 103; Spreng. Syst. Veg. 3 (1826) 742; Lindl. Gen. \& Sp. Orch. (1830) 73; Boj. Hort. Maurit. (1837) 320; Lindl. in Bot. Reg. 24 (1838) Misc. 78; Mutel Mém. sur. plus. Orch. (in Mém. Strassb. Soc. Hist. Nat. 3) (1840) 3, t. 1, fig. 2; Focke in Tijdschr. Nat. Wetensch. 2 (1849) 203; A. Rich. in Sagra Hist. Isla Cuba 11 (F1. Cub. Faner. 2) (1850) 247 (var. angustifolia); Reichb. f. in Nederl. Kruidk. Arch. 4 (1858) 331, in Walp。Ann. 6 (1863) 639; Thw. Enum. Pl. Zeyl. (1864) 308; Griseb. Fl. Brit. W. Ind. (1864) 628, Cat. Pl. Cub. (1866) 265; Sauv. Fl. Cub. (1873) 230; Reichb. f. in Linnaea 41 (1877) 128; Eggers Fl. St. Croix \& Virg. Is. (U. S. Nat. Mus. Smithson. Inst. Bull. 13) (1879) 113, No.970; Hemsl. in Godm. \& Salv. Biol. Centr. Am. 3 (1883) 248; Chaipm. Fl. So. U. S. ed. 2 (1883) 653, ed. 3 (1897) 482; Reichb. f. in Ber. Deutsch. Bot. Gesell. 3 (1885) 276; Rolfe in Journ. Bot. 23 (1885) 215; Corỏem. Fl. l'Ile Réun. (1895) 190; Cogn. in Mart. F1. Bras. $3^{4}$ (1895) 312; Dur. \& Schinz Consp. Fl. Afr. 5 (1895) 35; Duss Fl. Phan. Antill. Franc. (in Ann. Inst. Col. Marseille 3) (1897) 597; Urb. Symb. Antill. 4 (F1. Portor.) (1903) 170; Ames Contr. Knowl. Orch. F1. So. Fla. (1904) 16; Cogn. in Bull. Soc. Roy. Bot. Belg. 43 (1906) 304; Pulle Enum. Vasc. Pl. Surinam (1906) 119; Cogn. in Urb. Symb. Antill. 6 (1909) 381; Ames in Philip. Journ. Sci. 4 (1909) Bot. 666; Boldingh Fl. Dutch. W. Ind. 1 (St. Eust., Saba \& St. Mart.) (1909) 41.

Epidendrum minutum Aubl. Hist. Pl. Guian. Franc. 2 (1775) 824.

Dendrobium polystachyon Sw. in Kongl. Svensk. Vet.-Akad. Nya Handl. 21 (1800) 247; Willd. Sp. Pl. 4 (1805) 137; Sw. in Schrad. Neues Journ. Bot. 1 (1806) 95, Fl. Ind. Occ. 3 (1806) 1978, 1998; Pers. Syn. 2 (1807) 523; Lodd. Bot. Cab. 5 (1820) t. 458; Lindl. Coll. Bot. (1821) t. 20; Thou. Orch. Iles Austr. Afr. (1822) Troisième Tabl. Espec. (as polystachion), t. 84 (as polystachys); A. Rich. Monogr. Orch. Iles Fr. et Bourb. (1827) 50 [in Mém. Soc. Nat. Hist. Par. 4 (1828) 57], t. 8, fig. 4 (as polystachyum).

Cranichis luteola Sw. Fl. Ind. Occ. 3 (1806) 1433.

Onychium flavescens Blume Bijdr. (1825) 325.

Polystachya mauritiana Spreng. Syst. Veg. 3 (1826) 742; Rolfe in Elliot in Journ. Linn. Soc. 29 (1891) 53; Dur. \& Schinz. Consp. Fl. Afr. 5 (1895) 35; Palacky Cat. Pl. Madag. Fase. 1 (1906) 7; Hochreut. Sert. Madag. (in Ann. Conserv. Jard. Bot. Genève 11 \& 12) (1908) 55 (21); Schltr. in Beihefte Bot. Centralbl. 33, Abt. 2 (1915) 414.

Dendrobium flavescens Lindl. Gen. \& Sp. Orch. (1830) 85, in Bot. Reg. 30 (1844) Misc. 57; Kränzl. in Engl. Pflanzenreich IV: 50. II. B. 21 (1910) 302.

Epidendrum parviforum Pav. ex Lindl. Gen. \& Sp. Orch. (1830) 73 , in syn.

Polystachya zeylanica Lindl. in Bot. Reg. 24 (1838) Misc. 78; Reichb. f. in Walp. Ann. 6 (1863) 639; S. Moore in Baker Fl. Maurit. \& Seych. (1877) 362; Hook. f. Fl. Brit. Ind. 6. (1890) 21; Trim. Handb. Fl. Ceyl. 4 (1898) 183. 
Polystachya zollingeri Reichb. f. in Bonpl. 5 (1857) 39, in Walp. Ann. 6 (1863) 640.

Polystachya extinctoria Reichb. f. in Walp. Ann. 6 (1863) 638.

Callista flavescens Ktze. Rev. Gen. Pl. 2 (1891) 654.

Dendrorchis extinctoria Ktze. Rev. Gen. Pl. 2 (1891) 659.

Dendrorchis minuta Ktze. Rev. Gen. Pl. 2 (1891) 658.

Dendrorchis zollingeri Ktze. Rev. Gen. Pl. 2 (1891) 659.

Epidendrum resupinatum Sessé \& Moc. Fl. Mex. ed. 2 (1894) 202, fide Cogn. in Urb. Symb. Antill.

Polystachya minuta Frapp. ex Cordem. Fl. l'Ile Réun. (1895) 190, in nota; Britton in Small Fl. SE. U. S. (1903) 328, ed. 2 (1913) 324; Fawc. \& Rendle Fl. Jam. 1 (1910) 48, t. \%, figs. 4 \& 5; Small Fl. Miami (1913) 55; Ames Orch. 5 (1915) 93; Schltr. in Beihefte Bot. Centralbl. 36, Abt. 2 (1918) 479; Britton \& Millsp. Bahama Fl. (1920) 89; Schltr, in Urb. Symb. Antill. 8 (Fl. Doming.) (1920) 122; Britton in Addisonia 7 (1922) 19, t. 234; G. Wilson in Orch. Rev. 30 (1922) 324; Musgrove in Orch. Rev. 31 (1923) 167, in textu, non A. Rich. \& Gal.

Polystachya flavescens J. J. Sm. Fl. Buitenz. 6 (Orch. Jav.) (1905) 284, Fig.-Atlas (1910) fig. 218; Schltr. in Engl. Bot. Jahrb. 45, Beibl. 104 (1911) 24; Ames in Merr. in Journ. Roy. Asiat. Soc. Straits Branch, Special No. (1921) 180.

Luzon (Bontoc, Benguet, Pangasinan, Zambales, Rizal, Tayabas), Mindanao (Lanao), Vanoverbergh 760, 1332, 3670, Elmer 852\%, Fenix s. n., Boettcher s. n., B. S. 13550 Ramos, 20781 Escritor, M. S. Clemens s. n. At sea level to $1,200 \mathrm{~m}$ altitude. In India, Ceylon, Sumatra, Java, Borneo, Madagascar, Mauritius, Bourbon, and also in Florida, Central America, the West Indies, and northern South America.

The oldest name for the species is Epidendrum minutum Aubl. and although the combinations Polystachya minuta (Aubl.) Frapp. ex Cordem. and (Aubl.) Britton have been made, it seems inadvisable to use either on account of the already existing Polystachya minuta A. Rich. \& Gal. in Ann. Sci. Nat. III 3 (1845) 27. The last named is presumably not a Polystachya, but its status is at present (and probably will remain) unsettled.

\section{EXCLUDED OR SYNONYMOUS SPECIES}

Polystachya minuta Frapp. ex Cordem. Fl. l'Ile Réun. (1895) 190, in nota; Britton in Small Fl. SE. U. S. (1903) 328; Ames Orch. 5 (1915) 93, non A. Rich. \& Gal. = Polystachya luteola (Sw.) Hook.

\section{ACANTHOPHIPPIUM Blume}

ACANTHOPHIPPIUM MANTINIANUM L. Linden \& Cogn. in Journ. des Orch. 7 (1896) 138; Ames in Philip. Journ. Sci. 6 (1911) Bot. 50, Orch. 5 (1915) 93.

Luzon (Bontoc, Sorsogon), Vanoverbergh 1203, Elmer 16409. In wet places. 


\section{PHAJUS Loureiro}

PHAJUS FLAVUS (Blume) Lindl. Gen. \& Sp. Orch. (1831) 128; Blune" Mus. Bot. Lugd.-Bat. 2 (1856) 179; Miq. Fl. Ind. Bat. 3 (1859) 671; J. J. Sm. Fl. Buitenz. 6 (Orch. Jav.) (1905) 192, Fig.-Atlas (1909) fig. 144; Ames in Philip. Journ. Sci. 6 (1911) Bot. 50; Schltr. in Engl. Bot. Jahrb. 45 Beibl. 104 (1911) 24; Ames Orch. 5 (1915) 94.

Limodorum flavum Blume Bijdr. (1825) 375.

Bletia woodfordii Hook, in Bot. Mag. 54 (1827) t. 2719.

Phaius maculatus Lind1. in Wall. Cat. (1831) No. 3748, nomen, Gen. \& Sp. Orch. (1831) 127; Lodd. Bot. Cab. 19 (1832) t. 1808; Hook. in Bot. Mag. 68 (1842) t. 3960; Blume Mus. Bot. Lugd.Bat. 2 (1856) 180, Fl. Jav. Orch. (1858) 9 (Orch. Arch. Ind. 9) t. 5, E; Reichb. f. in Walp. Ann. 6 (1862) 460; Veitch Man. Orch. Pl. pt. 6 (1890) 12; Hook. f. Fl. Brit. Ind. 5 (1890) 817, 6 (1890) 192; King \& Pantl, in Ann. Roy. Bot. Gard. Calc. 8 (Orch. Sik.-Himal.) (1898) 107, t. 149.

Bletia flava Wall. in Bibl. Anglo-Indica ic. 1147 ex Lindl. Gen. \& Sp. Orch. (1831) 127, in syn.

Phajus flexuosus Blume Mus. Bot. Lugd.-Bat. 2 (1856) 179, Fl. Jav. Orch. (1858) 7 (Orch. Arch. Ind.6); Miq. Fl. Ind. Bat. 3 (1859) 671.

Phajus crispus Blume Mus. Bot. Lugd.-Bat. 2 (1856) 180, Fl. Jav. Orch. (1858) 8 (Orch. Arch. Ind. 8) t. 4, fig. 2, A, B; Miq. Fl. Ind. Bat. 3 (1859) 671.

Phajus platychilus Reichb. f. Xen. Orch. 1 (1856) 201, t. 76 , fig. III, 4-6, in Bonpl. 5 (1857) 42; Miq. Fl. Ind. Bat. 3 (1859) 740. Phajus indigoferus Reichb. f. Xen. Orch. 1 (1856) 202, t. 76, fig. IV, 7 \& 8 (excl. syn.); Blume Fl. Jav. Orch. (1858) 7 (Orch. Arch. Ind. 7) t. 2, fig. 2; Naves Novis. App. (1882) 246, non Hassk.

Phajus bracteosus Reichb. f. in Bonpl. 5 (1857) 42; Miq. Fl. Ind. Bat. 3 (1859) 740; Naves Novis. App. (1882) 249.

Luzon (Bontoc, Lepanto, Sorsogon), Panay (Antique), B. S. 5616 Worcester, 32593 McGregor, Elmer 16530. At 1,200 to $1,500 \mathrm{~m}$ altitude in Panay. In India, Java, and Sumatra.

PHAJUS HALCONENSIS Ames in Philip. Journ. Sci. 2 (1907) Bot. 323, Orch. 5 (1915) 94.

Phaius calanthoides Ames Orch. 2 (1908) 153, text cut.

Luzon (Bontoc, Benguet, Nueva Vizcaya, Laguna, Tayabas), Mindoro, Negros. Common in Luzon at fairly high altitudes, up to $2,130 \mathrm{~m}$ on $\mathrm{Mt}$. Halcon.

PHAJUS LINEARIFOLIUS Ames in Philip. Journ. Sci. 7 (1912) Bot, 10, Orch. 5 (1915) 94.

Luzon (Bontoc), Vanoverbergh 1288, 3654. Terrestrial on hillocks and in brooks at 1,180 to $1,650 \mathrm{~m}$ altitude. 
PHAJUS LYONII Ames Orch. 5 (1915) 94.

Luzon (Bontoc, Lepanto, Ifugao, Zambales, Laguna, Sorsogon), Leyte, Mindanao (Cotabato, Davao), B. S. 5613 Worcester, 22926 Brown, Lyon s. n., Elmer 11798, 16524, Wenzel 0118, Weber 225, Wester s. n. Terrestrial in the open or shade at 600 to $850 \mathrm{~m}$ altitude.

PHAJUS MINDORENSIS Ames in Philip. Journ. Sci. 2 (1907) Bot. 324, Orch. 5 (1915) 95.

Luzon (Laguna) Mindoro, Merrill 5131, 5612, B. S. 17115 Robinson. Epiphyte or terrestrial in mossy forest at 1,000 to $1,430 \mathrm{~m}$ altitude.

PHAJUS PHILIPPINENSIS N. E. Br. in Gard. Chron. III 6 (1889) 239;

Ames in Philip. Journ. Sci. 8 (1913) Bot. 422, Orch. 5 (1915) 95.

Luzon (Bontoc, Nueva Vizcaya), Vanoverbergh 3886, B. S. 11139 McGregor. Up to $1,300 \mathrm{~m}$ altitude in Bontoc.

PHAJUS RAMOSII Ames Orch. 5 (1915) 96.

Calanthe ramosii Ames in Philip. Journ. Sci. 7 (1912) Bot. 12.

Luzon (Zambales), B. S. 4987 Ramos.

\section{EXCLUDED OR SYNONYMOUS SPECIES}

Phajus amboinensis Blume apud Naves Novis. App. (1882) 246.

Phajus BRacteosus Reichb. f. in Bonpl. 5 (1857) 42; Naves Novis. App. (1882) 249 = Phajus flavus (Blume) Lindl.

PhaiUs CALANTHOIDES Ames Orch. 2 (1908) 153, text cut = Phajus halconensis Ames.

Phasus Callosus Lindl. apud Naves Novis. App. (1882) 246.

PhAJUS GRANDIFolius Lour. Fl. Cochinch. (1790) 529; Naves Novis. App. (1882) 246; Ames in Philip. Journ. Sci. 4 (1909) Bot. 671, Orch. 5 (1915) 94.

There seems no real foundation for believing that this species occurs in the Philippines; the material referred to it is presumably Phajus flavus (Blume) Lindl.

PhaJUS INDIGOFERUs Reichb. f. Xen. Orch. 1 (1856) 202, t. $76, f i g . I V$, 7 \& 8 (excl. syn.) ; Naves Novis. App. (1882) 246, non Hassk. = Phajus flavus (Blume) Lindl.

Phajus KUhlII Reichb. f. apud Naves Novis. App. (1882) 246.

PhaJus Wallichil Lindl. apud Naves Novis. App. (1882) 246.

57. CALANTHE R. Brown

CALANTHE ANGUSTIFOliA (Blume) Lindl. Gen. \& Sp. Orch. (1833) 251, Fol. Orch. Calanthe (1854) 5; Miq. Fl. Ind. Bat. 3 (1859) 710 ; Reichb. f. in Walp. Ann. 6 (1864) 914; Hook: $f$. Fl. Brit. Ind. 5 (1890) 854; Ridl. in Journ. Linn. Soc. 32 (1896) 339; J. J. Sm. Fl. Buitenz. 6 (Orch. Jav.) (1905) 205, Fig.-Atlas (1909) fig. 152; Ames in Philip. Journ. Sci. 2 (1907) Bot. 324; Ridl. Mat. Fl. Mal. Penin. 1 (1907) 121; Ames Orch. 2 (1908) 154, 5 (1915) 96, 7 (1922) 150; J. J. Sm. in Bull. Jard. Bot. Buitenz. II 14 (1914) 30. 
Ambyglottis angustifolia Blume Bijdr. (1825) 369.

Calanthe phajoides Reichb. f. Xen. Orch. 1 (1856) 207, t. 79, fig. II, 4 \& 5, in Bonpl. 5 (1857) 37; Hook. f. in Hook. Ic. Pl. 19 (1889)

t. 1864; Naves Novis. App. (1882) 246.

Alismorchis angustifolia Ktze. Rev. Gen. Pl. 2 (1891) 650.

Alismorchis phajoides Ktze. Rev. Gen. Pl. 2 (1891) 650.

Luzon (Benguet), Mindoro, Ramos \& Edaño 82, 109, Merrill 567\%. Terrestrial in mossy forest, at 2,130 to $2,260 \mathrm{~m}$ altitude in Mindorr. In Malay Peninsula, Sumatra, and Java.

CALANTHE CONSPICUA Lindl. Fol. Orch. Calanthe (1854) 4; Miq. Fl. Ind. Bat. 3 (1859) 710; Reichb. f. in Walp. Ann. 6 (1864) 913; Naves Novis. App. (1882) 246; Vidal Phan. Cuming. Philip. (1885) 149, Rev. Pl. Vasc. Filip. (1886) 269; Ames Orch. 5 (1915) 96. Calanthe lilacina Loher in Gard. Chron. III 47 (1910) 66, text cut, in Fedde Repert. 11 (1912) 96.

Luzon (Albay, Sorsogon), Palawan, Cuming 209\%, B. S. 64.5. Robinson, 23561 Ramos, Foxworthy 5\%6. At 290 to $485 \mathrm{~m}$ altitude.

CALANTHE DAVAensis Ames in Philip. Journ. Sci. 8 (1913) Bot. 422, Orch. 5 (1915) 97.

Mindanao (Davao), Copeland s. n. Mt. Apo, terrestrial at 2,000 m altitude.

CALANTHE ELMERI Ames Orch. 2 (1908) 155, text cut, 5 (1915) 97.

Luzon (Abra, Bontoc, Benguet), B. S. 27003 Ramos, 12936 Fenix, Vanoverbergh 2195, 2210, Lyon 153, Elmer 5886, 8382, Topping s. n., Weber 406. Terrestrial, sea level to $1,800 \mathrm{~m}$ altitude.

CALANTHE FURCATA Batem. ex Lindl. in Bot. Reg. 24 (1838) Misc. 28; Knowles \& Westc. Floral Cab. 2 (1838) 46; Lindl. in Paxt. Flow. Gard. 3 (1852) 37, Fol. Orch. Calınthe (1854) 25; Moore Ill. Orch. Pl. (1857) Calanthe 5; Miq. Fl. Ind. Bat. 3 (1859) 711; Walp. Ann. 6 (1864) 916; Naves Novis. App. (1882) 246; Vidal Phan Cuming. Philip. (1885) 149, Rev. Pl. Vasc. Filip. (1886) 269; Ames Orch. 1 (1905) 81.

Calanthe veratrifolia R. Br. apud Naves Novis. App. (1882) 246; J. J. Sm. Fl. Buitenz. 6 (Orch. Jav.) (1905) 211, quoad syn. C. furcata Batem. et quoad Philip.; J. J. Sm. Orch. Amb. (1905) 23, quoad syn. C. furcata Batem. et quoad Philip.

Alismorchis furcata Ktze. Rev. Gen. Pl. 2 (1891) 650.

Calanthe triplicata Ames in Philip. Journ. Sci. 2 (1907) Bot. 326, Orch. 2 (1908) 159, non Orchis triplicata Willem.

Luzon (Apayao, Kalinga, Bontoc, Benguet, Nueva Vizcaya, Pampanga, Bataan, Rizal, Laguna, Tayabas, Albay, Sorsogon), Mindoro, Panay (Capiz, Antique), Negros (Oriental Negros), Leyte, Mindanao (Camaguin Island, Zamboanga, Dapitan, Lanao, Bukidnon, Cotabato, Davao), Sibutu. A common and very widely distributed species in the Philippines. Terrestrial at 60 to $3,100 \mathrm{~m}$ altitude.

It has been deemed advisable to adopt the name $C$. furcata for the Philippine material as there is no question as to its identity. As pointed out by J. J. Smith, in Merr. Interp. Rumph. Herb. Amb. (1917) 170, the combination $C$. triplicata Ames is untenable as Orchis triplicata Willem.. 
the name-bringing synonym, is not conspecific. While $C$. veratrifolia $R$. Br., as to plant figured and described, is very probably conspecific with C. furcata Batem., it is open to grave doubt, as pointed out by Smith, 1. c., whether Limodorum veratrifolium Willd, is the same as the plant that Brown figured and described. Should Limodorum veratrifolium Willd. be Calanthe sylvatica Lindl., as seems probable, the combination Calanthe veratrifolia $\mathrm{R}$. Br., (as to name) would apply to that species. In view of the uncertain status of Calanthe veratrifolia $\mathrm{R}$. Br. no attempt has been made to cite its bibliography, synonymy, or distribution.

CALANTHE HALCONENSis Ames in Philip. Journ. Sci. 2 (1907) Bot. 324 , Orch. 5 (1915) 97.

Luzon (Laguna), Mindoro, Raymundo s. n., Merrill 5489. Terrestrial, in forest at 760 to $1,260 \mathrm{~m}$ altitude.

CALANTHE HENNISII Loher in Gard. Chron. III 46 (1909) 34, in Fedde Repert. 8 (1910) 384; Ames in Philip. Journ. Sci. 8 (1913) Bot. 423, Orch, 5 (1915) 97.

Luzon (Rizal, Tayaủas), Loher s. n., Lyon 41, B. S. 13111 Foxworthy \& Ramos, 41916 Ramos. At 500 to $1,000 \mathrm{~m}$ altitude, according to Loher.

CAlanthe LAcerata Ames in Philip. Journ. Sci. 7 (1912) Bot. 11, Orch. 5 (1915) 97.

Luzon (Lepanto, Benguet, Tayabas, Camarines), Merrill 4582, 4756, Ramos \& Edaño 74, 114, F. B. 11355 Curran, Whitford 960. Terrestrial or epiphytic ?, in forest at 1,000 to $2,500 \mathrm{~m}$ altitude.

CAlANTHE LYRoglossa Reichb. f. Otia Bot. Hamb. 1 (1878) 53, Xen. Orch. 3 (1881) 30; Naves Novis. App. (1882) 246; Ames Orch. 2 (1908) 156, text cut, 5 (1915) 97.

Alismorchis lyroglossa Ktze. Rev. Gen. Pl. 2 (1891), 650.

Luzon (Apayao, Cagayan, Zambales, Bataan, Laguna), Negros (Oriental Negros), Palawan, Wilkes, s. n., Weber 343, s. n., B. S. 4782, 19558 Ramos, F. B. 6306 Curran, Elmer 10083, Foxworthy 637. Epiphyte at 50 to $1,100 \mathrm{~m}$ altitude.

CALANTHE MAQUILINGENSIS Ames Orch. 5 (1915) 97.

Luzon (Laguna), Negros (Oriental Negros), Leyte, B. S. 17246 Brown, Elmer 10063, Wenzel 0658. Terrestrial at 600 to $1,000 \mathrm{~m}$ altitude.

CALANTHE MCgRegorII Ames in Philip. Journ. Sci. 2 (1907) Bot. 325 , Orch. 5 (1915) 98.

Polillo, Mindoro, Mindanao (Lanao), McGregor 17\%, B. S. 10452 McGregor, M. S. Clemens s. n. Balete, Rio Baco.

CALANTHE MINDORENSIS Ames in Philip. Journ. Sci. 2 (1907) Bot. 325 , Orch. 5 (1915) 98.

Mindoro, Merrill 5525. Terrestrial, ridge forest, Mt. Halcon at $760 \mathrm{~m}$ altitude. 
CALANTHE PULChRA (Blume) Lindl. Gen. \& Sp. Orch. (1833) 250; J. J. Sm. F1. Buitenz. 6 (Orch. Jav.) (1905) 202, Fig.-Atlas (1909) fig. 150; Ames in Philip. Journ. Sci. 2 (1907) Bot. 326, Orch. 2 (1908) 158, 5 (1915) 98, 6 (1920) 161, in Merr. in Journ. Roy. Asiat. Soc. Straits Branch, Special No. (1921) 181.

Ambyglottis pulchra Blume Bijdr. (1825) 371.

Styloglossum nervosum Breda Gen. \& Sp. Orch. Java (1827) t. 10. Calanthe curculigoides Lindl. in Wall. Cat. (1832) No. 7340, nomen, Gen. \& Sp. Orch. (1833) 251, in Bot. Reg. 33 (1847) t. 8; Reichb. f. in Walp. Ann. 1 (1849) 792, 6 (1864) 913; Lindl. Fol. Orch. Calanthe (1854) 4; Miq. Fl. Ind. Bat. 3 (1859) 710; Hook. f. in Bot. Mag. 100 (1874) t. 6104; Hook. f. Fl. Brit. Ind. 5 (1890) 854; Veitch Man. Orch. Pl. pt. 6 (1890) 63; Ridl. in Journ. Linn. Soc. 32 . (1896) 330, Mat. Fl. Mal. Penin. 1 (1907) 122.

Alismorchis pulchra Ktze. Rev. Gen. Pl. 2 (1891) 650.

Calanthe curantigiodes Ktze. Rev. Gen. Pl. 2 (1891) 650, sphalm, in syn:

Luzon (Sorsogon), Mindoro, Negros (Oriental Negros), Leyte, Elmer 9576, 14883, Merrill 5849, 5850, Wenzel 0648, 0689, mixed with 0704. Ter. restrial at 130 to $760 \mathrm{~m}$ altitude.

\section{EXCLUDED OR SYNONYMOUS SPECIES}

Calanthe Australis Ait. ex Loud. Hort. Brit. Suppl. 2 (1839) 615, nomen $=$ Spiranthes sinensis (Pers.) Ames.

Calanthe emarginata Lindl. apud Naves Novis. App. (1882) 246.

CALANTHe Jusneril Boxall ex Naves Novis. App. (1882) 247, nomen.

CALANTHE LILACINA Loher in Gard. Chron. III 47 (1910) 66, text cut, in Fedde Repert. 11 (1912) 96 = Calanthe conspicua Lindl.

Catanthe Masuca Boxall ex Naves Novis. App. (1882) 246, nomen.

Calanthe nivalis Boxall ex Naves Novis. App. (1882) 246, nomen.

Calanthe PhajoIdes Reichb. f. Xen. Orch. 1 (1856) 207, t. 79 , fig. $I I$, $4 \& 5$; Naves Novis. App. (1882) $246=$ Calanthe angustifolia (Blume) Lindl.

CALANTHe RAMosil Ames in Philip. Journ. Sci. 7 (1912) Bot. $12=$ Phajus ramosii Ames.

Calanthe speciosa Lindl. apud Naves Novis. App. (1882) 246.

Calanthe Triplicata Ames in Philip. Journ. Sci. 2 (1907) Bot. 326, Orch. 2 (1908) 159, non Orchis triplicata Willem. = Calanthe furcata Batem.

Calanthe veratrifolia R. Br. apud Naves Novis. App. (1882) 246 ; J. J. Sm. Fl. Buitenz. 6 (Orch. Jav.) (1905) 211, quoad syn. C. furcata Batem. et quoad Philip.; J. J. Sm. Orch. Amb. (1905) 23, quoad syn. C. furcata Batem. et quoad Philip. = Calanthe furcata Batem. 


\section{ASCOTAINIA Ridley}

ASCOTAINIA ELMERI Ames Orch. 5 (1915) 99; Schltr. in Fedde Repert. Beihefte 5 (Orch. Sino-Jap. Prodr.) (1919) 246; Ames Sched. Orch. 6 (1923) 79.

Tainia elmeri Ames in Elm. Leafl. Philip. Bot. 5 (1912) 1570.

Tainia inamoena Kränzl. in Fedde Repert. 17 (1921) 387.

Luzon (Bontoc, Benguet), Mindanao (Agusan, Davao), Vanoverbergh 1110, 2434, 4052, Elmer 8526, Weber 042, 061, 075, 0263, Loher s. n. Terrestrial in forest, at 3 to $1,300 \mathrm{~m}$ altitude.

\section{SPATHOGLOTTIS Blume}

SPATHOGLOTTIS CHRYSANTHA Ames Orch. 2 (1908) 161, text cut, 5 (1915) 99.

Luzon (Bontoc, Lepanto, Ifugao, Benguet, Nueva Vizcaya, Albay). Vanoverbergh 378, 1211, 4039, F. B. 14494 Darling, Ramos \& Edaño 79, B. S. 14387, 19884 McGregor, 5374, 5546, 8179 Ramos, Sandkuhl 295, Elmer 57\%9, 8815. Lyon 156, R. S. Williams 1064, Mearns 2905. At 1,400 to $1,650 \mathrm{~m}$ altitude.

SPATHOGLOTTIS ELMERI Ames in Elm. Leafl. Philip. Bot. 5 (1912) 1572, Orch. 5 (1915) 99.

Spathoglottis aurea Lindl. apud Naves Novis. App. (1882) 246; Ames in Philip. Journ. Sci. 2 (1907) Bot. 327, Orch. 5 (1915) 99.

Mindoro, Negros (Oriental Negros), Merritt 4375, Elmer 10080. At $1,415 \mathrm{~m}$ altitude on Mt. Halcon and from Dumaguete (Cuernos Mountains).

SPATHOGLOTTIS KIMBALIANA Hort. Sander ex Gartenflora 41 (1892) 184, nomen; Rolfe in Sander Reichenbachia II 2 (1894) 95, $t .88$, text cut; Hook. f. in Bot. Mag. 121 (1895) t. 7443.

Var. ANGUSTIFOLIA Ames Orch. 5 (1915) 99.

Luzon (Ilocos Norte, Isabela, Albay), Sibuyan, B. S. 32872 Ramos, 21149 Escritor, 6284 Robinson, Elmer 12556. The species proper is from Borneo.

SPATHOGLOTTIS PLICATA Blume Bijdr. (1825) 401, fig. 76; Lindl. Gen. \& Sp. Orch. (1831) 119, in Wall. Cat. (1831) No. 3734; Hassk. in Tijdschr. Nat. Gesch. 9 (1842) 139; Lindl. in Bot. Reg. 30 (1844) Misc. 45; Hassk. Pl. Jav. Rar. (1848) 126; De Vriese Ill. Orch. Ind. Neerl. (1854) t.; Moore Ill. Orch. Pl. (1857) Spathogl. 2; Miq. Fl. Ind. Bat. 3 (1859) 673; Reichb. f. in Walp. Ann. 6 (1862) 455; Hemsl. in Gard. Chron. II 18 (1882) 532; Naves Novis. App. (1882) 245; Vidal Phan. Cuming. Philip. (1885) 148, Rev. Pl. Vasc. Filip. (1886) 269; Linden \& Rodigas in Lindenia 2 (1886) 15, t. 54; Kränzl. in Engl. Bot. Jahrb. 7 (1886) 438, in K. Schum. \& Hollr. Fl. Kais. Wilhelmsland (1889) 33; Hook, f. Fl. Brit. Ind. 5 (1890) 813; Kränzl. Xen. Orch. 3 (1893) 112; Grant Orch. Burma (1895) 192; Ridl. in Journ. Linn. Soc. 31 (1896) 285, 32 (1896) 311; Kränzl. in Reinecke in Engl. Bot. Jahrb. 25 (1898) 603, in K. Schum. \& Lauterb. Fl. Deutsch. Schutzgeb. (1901) 244; Volkens in Engl. Bot. Jahrb. 31 (1902) 461; Schltr. in Perk. Frag. Fl. Philip. (1904) 43; J. J. Sm. Fl. Buitenz. 6 (Orch. Jav.) (1905) 219, Fig.-Atlas (1909) fig. 162, Orch. Amb. (1905) 24; Ames Orch. 
1 (1905) 83, 2 (1908) 61, 5 (1915) 100, 6 (1920) 164; Ridl. Mat. Fl. Mal. Penin. 1 (1907) 117; Ames. in Philip. Journ. Sci. 2 (1907) Bot. 327; J. J. Sm. in Nova Guinea 8 (1909) 25, $8^{3}$ (1912) 529, 12 (1913) 14; Schltr. in Fedde Repert. 10 (1911) 70, in Engl. Bot. Jahrb. 45, Beibl. 104 (1911) 27; J. J. Sm. in Philip. Journ. Sci. 12 (1917) Bot. 255, in Merr. Interp. Rumph. Herb. Amb. (1917) 172; Schltr. in Fedde Repert. Beihefte 4 (Orch. Sino-Jap. Prodr.) (1919) 245; Ames in Merr. in Journ. Roy. Asiat. Soc. Straits Branch, Special No. (1921) 182.

Bletia angustata Gaudich. in Freyc. Voy. Uran. et Physic. Bot. (1826) 421.

Bletia angustifolia Gaudich. in Freyc. Voy. Uran. et Physic. Bot. (1826) $t$. 32.

Paxtonia rosea Lindl. in Bot. Reg. 24 (1838) Misc. 61, t. 60; Moore Ill. Orch. PI. (1857) Paxtonia 4, t. 1; Reichb. f. in Walp. Ann. 6 (1862) 456; Naves Novis. App. (1882) 237.

Spathoglottis lilacina Griff. Notul. 3 (1851) 323 and Icon. Pl. Asiat. 3 (1851) t. 311, fig. III.

Phajus rhumphii Blume Mus. Bot. Lugd.-Bat. 2 (1856) 179.

Spathoglottis spicata Lindl. in Journ. Linn. Soc. 3 (1859) 9.

Batan Islands, Luzon (Bontoc, Lepanto, Ifugao, Benguet, Nueva Vizcaya, Tarlac, Pampanga, Rizal, Laguna, Tayabas, Camarines, Sorsogon), Mindoro, Panay (Capiz), Negros (Occidental Negros), Samar, Leyte, Palawan, Dinagat, Mindanao (Zamboanga, Lanao, Cotabato, Bukidnon, Agusan, Surigao). A common and widely distributed species in the Philippines. Terrestrial at 160 to $1,500 \mathrm{~m}$ altitude. In Malay Peninsula, Formosa, Sumatra, Java, Amboina, Celebes, Borneo, New Guinea, and the Caroline Islands.

SPATHOGLOTTIS TOMENTOSA Lindl. in Bot. Reg. 31 (1843) sub t. 19; Reichb. f. in Walp. Ann. 6 (1862) 455; Naves Novis. App. (1882) 246 ; Vidal Phan. Cuming. Philip. (1885) 149; Kränzl. Xen. Orch. 3 (1893) 112; Ames Orch. 2 (1908) 163 (excl. Luzon spec.), 5 (1915) 100.

Mindanao (Misamis, Lanao, Agusan, Surigao), Cuming 2108, M. S. Clemens 803, Weber 50, Bolster 256, (?) F. B. 7590 Hutchinson. Terrestrial at 200 to $430 \mathrm{~m}$ altitude.

SPATHOGLOTTIS VANOVERBERGHII Ames Orch. 6 (1920) 299.

Spathoglottis tomentosa Ames Orch. 2 (1908) 163, non Lindl., as to specimens from Luzon.

Luzon (Bontoc, Lepanto, Benguet), Vanoverbergh 163, 1109, 1112, 2106, 3943, 4046, Merrill 4287, Elmer 8478. At 1,000 to 1,300 m altitude.

\section{EXCLUDED OR SYNONYMOUS SPECIES}

Spathoglottis affinis De Vriese apud Naves Novis. App. (1882) 245.

Spathogrottis AUrea Lindl. apud Naves Novis. App. (1882) 246; Ames in Philip. Journ. Sci. 2 (1907) Bot. 327, Orch. 5 (1915) $99=$ Spathoglottis elmeri Ames.

Spathoglottis tomentosa Ames Orch. 2 (1908) 163, non Lindl., as to specimens from Luzon $=$ Spathoglottis vanoverberghii Ames. $200823-22$ 


\section{PLOCOGLOTTIS Blume}

PlocoglotTIS BICALlosa Ames in Elm. Leafl. Philip. Bot. 5 (1915) 1571, Orch. 5 (1915) 100.

Luzon (Sorsogon), Negros (Oriental Negros), Mindanao (Camiguin Island), Elmer $957 \%, 1711 \%$, B. S. 14437 Ramos. Terrestrial at 1,300 m altitude in Negros.

PLOCOGLOTTIS COPELANDII Ames in Philip. Journ. Sci. 2 (1907) Bot. 326, Orch. 5 (1915) 100.

Plocoglottis acuminata Ames Orch. 1 (1905) 82, non Blume.

Luzon (Laguna, Tayabas, Camarines, Sorsogon), Polillo, Mindoro, Panay (Capiz), Biliran, Leyte, Palawan, Mindanao (Camiguin Island, Zamboanga, Cotabato, Agusan, Davao). A common and widely distributed species. Terrestrial in forest at 10 to $580 \mathrm{~m}$ altitude.

Plocoglottis LUCBANENSIS Ames in Elm. Leaf. Philip. Bot. 5 (1912) 1572, Orch. 5 (1915) 100.

Babuyan Islands (Camiguin Island), Luzon (Laguna, Tayabas, Sorsogon), B. S. 4147 Fenix, F. B. 28938 Willie \& Salvosa, Elmer 7ro7, 17123. Terrestrial in damp forest at $915 \mathrm{~m}$ altitude in Tayabas.

PLOCOGLOTTIS MCGREGORII Ames Orch. 7 (1922) 121.

Panay (Antique), B. S. 32189 McGregor. East of Culasi, in mossy forest at $1,000 \mathrm{~m}$ altitude.

PLOCOGLOTTIS MINDORENSIS Ames in Philip. Journ. Sci. 2 (1907) Bot. 327, Orch. 5 (1915) 101.

Mindoro, Sibuyan, Mindanao (Surigao), Merrill 5837, Elmer 12147, Bolster 278. Terrestrial in forest at 10 to $330 \mathrm{~m}$ altitude.

PLOCOGLOTTIS WENZELII Ames Orch. 5 (1915) 101.

Leyte, Wenzel 067,0385, 0453. Ormoc and Jaro, terrestrial in forest at 200 to $500 \mathrm{~m}$ altitude.

EXCLUDED OR SYNONYMOUS SPECIES

Plocoglotis acuminata Ames Orch. 1 (1905) 82, non Blume = Plocoglottis copelandii Ames.

Plocoglottis latifolia Blume apud Naves Novis. App. (1882) 247.

Plocoglottis Lowil Reichb. f. apud Naves Novis. App. (1882) 247.

Plocoglottis moluccana Blume apud Naves Novis. App. (1882) 247.

\section{PACHYSTOMA Blume}

PACHYSTOMA PUBESCENS Blume Bijdr. (1825) 376, fig. 29; Reichb. f. in Bonpl. 3 (1855) 251; Miq. Fl. Ind. Bat. 3 (1859) 675; Reichb. f. in Walp. Ann. 6 (1862) 463; Naves Novis. App. (1882) 247; J. J. Sm. Fl. Buitenz. 6 (Orch. Jav.) (1905) 186, Fig.-Atlas (1909) fig. 140; Ames in Philip. Journ. Sci. 6 (1911) Bot. 51, Orch. 5 (1915) 102.

Pachychilus pubescens Blume Mus. Bot. Lugd.-Bat. 2 (1856) 173,

Fl. Jav. Orch. (1858) 24 (Orch. Arch. Ind. 27) t. 10, fig. 1, t. 11E.

Luzon (Cagayan, Bontoc, Lepanto, Benguet, Nueva Vizcaya, Rizal), Mindoro, Vanoverbergh 1102, 2435, 4040, B. S. 7050, 7969 Ramos, 11135 
McGregor, 6774 Robinson, F. B. 1100 s ilerrit, 10912 Curran, Rumos s. n. Grassy hillsides up to $1,200 \mathrm{~m}$ altitude in Bontoc.

62. EULOPHIA R. Brown

EULOPHIA DENTATA Ames in Philip. Journ. Sci. 6 (1911) Bot. 51, Orch. 5 (1915) 102.

Eulophia graminea Naves Novis. App. (1882) 241 (?); Vidal Phan. Cuming. Philip. (1885) 150 (?), Rev. Pl. Vasc. Filip. (1886) 269 (?); Ames Orch. 2 (1908) 166, non Lindl.

Luzon (Bontoc, Isabela, Pangasinan), F. B. 17035 Curran, B. S. 7977 Ramos, Alberto 36, Cuming 2059.

EULOPHIA MACRORHIZA Blume Fl. Jav. Orch. (1858) 155 (Orch. Arch. Ind. 183) t. 63, fig. 2, t. 66B; Miq. Fl. Ind. Bat. 3 (1859) 742; J. J. Sm. Fl. Buitenz. 6 (Orch. Jav.) (1905) 227, Fig.-Atlas (1909) fig. 166; Ames Orch. 2 (1908) 167, 5 (1915) 104.

Graphorchis macrovhiza Ktze. Rev. Gen. P1. 2 (1891) 662.

Luzon (Bataan, Tayabas, Sorsogon); Leyte, Whitford 621, Lyon 16j, Elmer 16118, Wenzel 0121. Terrestrial at 60 to $1,000 \mathrm{~m}$ altitude. In Java.

EULOPHIA MACROSTACHYA Lindl. Gen. \& Sp. Orch. (1833) 183, in Bot. Reg. 23 (1837) t. 1972; Wight Icon. $5^{1}$ (1852) 8, t. 1667-68; Reichb. f. in Walp. Ann. 6 (1863) 646; Thw. Enum. Pl. Zeyl. (1864) 301; Hook. f. in Bot. Mag. 102 (1876) t. 6246, Fl. Brit. Ind. 6 (1890) 4; Trim. Handb. Fl. Ceyl. 4 (1898) 176; J. J. Sm. Fl. Buitenz. 6 (Orch. Jav.) (1905) 224, Fig.-Atlas (1909) fig. 164; Ames Orch. 2 (1908) 169, 5 (1915) 104; J. J. Sm. in Nova Guinea 8 (1909) 25, $12^{3}$ (1915) 215; Rolfe in Gibbs in Journ. Linn. Soc. 42 (1914) 157; Ames in Merr. in Journ. Roy. Asiat. Soc. Straits Branch, Special No. (1921) 191.

Eulophia emarginata Blume Fl. Jav. Orch. (1858) 152 (Orch. Arch. Ind. 180) ; Miq. Fl. Ind. Bat. 3 (1859) 741; Miq. Sumatra (1862) 274, non Lindl.

Graphorchis macrostachya Ktże. Rev. Gen. Pl. 2 (1891) 662.

Graphorchis blumeana Ktze. Rev. Gen. Pl. 2 (1891) 663.

Luzon (Laguna, Camarines, Sorsogon), Leyte, Mindanao (Camiguin Island, Zamboanga, Lanao). Fairly common in the Philippines. Ter. restrial in forest at low altitudes. In India, Ceylon, Sumatra, Java, Ternate, Borneo, and New Guinea.

EULOPHIA MERRILLII Ames Orch. 5 (1915) 104.

Mindanao (Surigao), Merrill 5452. Caraga, open grassy slopes at about $100 \mathrm{~m}$ altitude.

EULOPHIA SQUALIDA Lindl. in Bot. Reg. 27 (1841) Misc. 77; Hook. f. Fl. Brit. Ind. 6 (1890) 6; Ridl. in Journ. Linn. Soc. 31 (1896) 289, 32 (1896) 332, 36 (1898) 214; J. J. Sm. Fl. Buitenz. 6 (Orch. Jav.) (1905) 225, Fig.-Atlas (1909) fig. 165; Ridl. Mat. Fl. Mal. Penin. 1 (1907) 137; Ames in Philip. Journ. Sci. 4 (1909) Bot. 671, Orch. 5 (1915) 105, 6 (1920) 210; Merr. \& Merritt in Philip. Journ. Sci. 5 (1910) Bot. 341; Rolfe in Gibbs 
in Journ. Linn. Soc. 42 (1914) 157; Ames in Merr. in Journ. Roy. Asiat. Soc. Straits Branch, Special No. (1921) 192.

Cyrtopera squalida Reichb. f. in Bonpl. 5 (1857) 38; Lindl. in Journ.

Linn. Soc. 3 (1859) 31; Miq. Fl. Ind. Bat. 3 (1859) 686; Reichb.

f. in Walp. Ann. 6 (1863) 668; Naves Novis. App. (1882) 242.

? Eulophia lutea Blume Fl. Jav. Orch. (1858) 153 (Orch. Arch. Ind.

181); Miq. Fl. Ind. Bat. 3 (1859) 742.

? Eulophia celebica Blume Fl. Jav. Orch. (1858) 154 (Orch. Arch.

Ind. 182); Miq. Fl. Ind. Bat. 3 (1859) 742.

Eulophia elongata Blume Fl. Jav. Orch. (1858) 154 (Orch. Arch. Ind. 182); Miq. Fl. Ind. Bat. 3 (1859) 742; Naves Novis. App. (1882) 241.

Eulophia mucronata Blume Fl. Jav. Orch. (1858) 154 (Orch. Arch.

Ind. 182); Miq. Fl. Ind. Bat. 3 (1859) 742; Naves Novis. App. (1882) 242.

Cyrtopodium squalidum Vidal Phan. Cuming. Philip. (1885) 150, Rev.

Pl. Vasc. Filip. (1886) 270.

Eulophia elata Hook. f. Fl. Brit. Ind. 6 (1890) 3, fide Ridl.

Graphorchis elata Ktze. Rev. Gen. Pl. 2 (1891) 662.

? Graphorchis lutea Ktze. Rev. Gen. Pl. 2 (1891) 662.

Graphorchis squalida Ktze. Rev. Gen. Pl. 2 (1891) 662.

? Eulophia squalida Lindl. var. celebica Schltr. in Fedde Repert. 10 (1911).

Luzon (Bontoc, Lepanto, Benguet, Zambales), Leyte, Bancalan, Palawan, Mindanao (Davao). A fairly common and rather widespread species in the Philippines. Terrestrial at 5 to $1,300 \mathrm{~m}$ altitude. In Malay Peninsula, ? Sumatra, Java, Celebes, and Borneo.

EULOPHIA STRICTA (Presl) Ames Orch. 5 (1915) 103.

Bletia stricta Presl Rel. Haenk. 1 (1827) 98; Naves Novis. App. (1882) 247.

Eulophia exaltata Reichb. f. in Bonpl. 5 (1857) 38;.J. J. Sm. Fl. Buitenz. 6 (Orch. Jav.) (1905) 228, Fig.-Atlas (1910) fig. 167; Ames Orch. 1 (1905) 85, 2 (1908) 166, 5 (1915) 103; J. J. Sm. in Bull. Dept. Agr. Ind. Néerl. 13 (1907). 22.

Eulophia leschenaultii Blume Fl. Jav. Orch. (1858) 154 (Orch. Arch. Ind. 182); Naves Novis. App. (1882) 242.

Cyrtopera ensiformis Lindl. in Journ. Linn. Soc. 3 (1859) 31; Miq. Fl. Ind. Bat. 3 (1859) 686; Naves Novis. App. (1882) 242.

Cyrtopodium ensiforme Vidal Phan. Cuming. Philip. (1885) 80, 150, Rev. P1. Vasc. Filip. (1886) 270; Schltr. in Perk. Frag. Fl. Philip. (1904) 43.

Graphorchis exaltata Ktze. Rev. Gen. Pl. 2 (1891) 662.

Eulophia warburgiana Kränzl. Xen. Orch. 3 (1893) 115, t. 266, fig. II, 8-12.

Eulophia ensiformis Schltr. in Perk. Frag. Fl. Philip. (1904) 202.

Luzon (Bontoc, Nueva Vizcaya, Rizal, Camarines), Mindanao (Zamboanga, Bukidnon, Lanao, Butuan, Davao). Not uncommon in the Philippines, terrestrial at 80 to $1,400 \mathrm{~m}$ altitude. In Java and Celebes. 
EULOPHIA VANOVERBERGHII Ames in Philip. Journ. Sci. 7 (1912) Bot. 13, Orch. 5 (1915) 105.

Luzon (Bontoc), Vanoverbergh 396, 1188, 3876, 4051. Terrestrial at about $1,300 \mathrm{~m}$ altitude.

\section{EXCLUDED OR SYNONYMOUS SPECIES}

EUlophia BICOLOR Blume apud Naves Novis. App. (1882) 241.

Eulophia elongata Blume Fl. Jav. Orch. (1858) 154 (Orch. Arch. Ind. 182) ; Naves Novis. App. (1882) $242=$ Eulophia squalida Lindl.

Eulophia ENSIFORMis Schltr. in Perk. Frag. Fl. Philip. (1904) $202=$ Eulophia stricta (Presi) Ames.

Eulophia exaltata Reichb. $f$. in Bonpl. 5 (1857) 38; J. J. Sm. Fl. Buitenz. 6 (Orch. Jav.) (1905) 228, Fig.-Atlas (1910) fig. 167; Ames Orch. 1 (1905) 85, 2 (1908) 166, 5 (1915) 103; J. J. Sm. in Bull. Dept. Agr. Ind. Néerl. 13 (1907) $22=$ Eulophia stricta (Presl) Ames.

Eulophia Graminea Naves Novis. App. (1882) 241; (?); Vidal Phan. Cuming. Philip. (1885) 150 (?), Rev. Pl. Vasc. Filip. (1886) 269 (?); Ames Orch. 2 (1908) 166, non Lindl. = Eulophia dentata Ames.

Eulophia leschenaulti Blume Fl. Jav. Orch. (1858) 154 (Orch. Arch. Ind. 182); Naves Novis. App. (1882) $242=$ Eulophia stricto (Presl) Ames.

Eulophia MUcronata Blume Fl. Jav. Orch. (1858) 154 (Orch. Arch. Ind. 182); Naves Novis. App. (1882) $242=$ Eulophia squalida Lindl.

Eulophia SAUNDERSIANA Reichb. f. apud Naves Novis. App. (1882) 242. Eulophia sumatrana Blume apud Naves Novis. App. (1882) 242.

Closely allied to Eulophia squalida Lindl. The Philippine material is presumably referable to that species.

Eulophia venosa Reichb. f. apud Naves Novis. App. (1882) 242.

Eulophia Warburgiana Kränzl. Xen. Orch. 3 (1893) 115, t. 266, fig. 11 . 8-12 = Eulophia stricta (Presl) Ames.

\section{GEODORUM Jackson}

GEOdORUM NUTANS (Presl) Ames Orch. 2 (1908) 164, 5 (1915) 106, 6 (1920) 306; Merr. Fl. Manila (1912) 166, Sp. Blancoanae (1918) 113; Wells in Philip. Journ. Sci. 14 (1919) 4.

Dendrobium nutans Presl Rel. Haenk. 1 (1827) 102, Symb. Bot. 1 (1832) 34, t. 23; Naves Novis. App. (1882) 234.

Arethusa glutinosa Blanco Fl. Filip. (1837) 641, ed. 2 (1845) 446, ed. 3,3 (1879) 38, t. 429bis.

Dendrobium haenkeanum Steud. Nomencl. Bot. ed. 2, 1 (1840) 490. Geodorum semicristatum Lindl. Fol. Orch. Geodorum (1854) 2; Miq. Fl. Ind. Bat. 3 (1859) 712; Reichb. f. in Walp. Ann. 6 (1863) 
633; Naves Novis. App. (1882) 241; Vidal Phan. Cuming. Philip. (1885) 150, Rev. Pl. Vasc. Filip. (1886) 270; Merr. in Govt. Lab. Publ. (Philip.) 8 (1903) 45, 52, 61, 75, 88, 152, 27 (1905) 84. ? Geodorum pallidum Llanos in Mem. Real. Acad. Cienc. Madrid 4 (1859) 507; F.-Vill. \& Naves in Blanco Fl. Filip. ed. $3,4^{1}$ (1880) 105.

Babuyan Islands (Dalupiri Island), Luzon (Nueva Vizcaya, Bataan, Rizal, Cavite, Laguna, Tayabas, Camarines, Sorsogon), Polillo, Masbate, Panay (Antique), Negros (Oriental Negros), Biliran, Samar, Palawan, Bancalan, Mindanao (Bukidnon, Davao). A common and widely spread, endemic species; terrestrial at sea level to $300 \mathrm{~m}$ altitude.

\section{EXCLUDED OR SYNONYMOUS SPECIES}

Geodorum Citrinum Jacks. apud Naves Novis. App. (1882) 241.

Geodorum dilatatum R. Br. apud Naves Novis. App. (1882) 241.

GEOdorum PALLidum Llanos in Mem. Real. Acad. Cienc. Madrid 4 (1859) 507; F.-Vill. \& Naves in Blanco Fl. Filip. ed. 3, $4^{1}$ (1880) $105=$ ? Geodorum nutans (Presl) Ames.

Geodorum PICTum Lindl. apud Naves Novis. App. (1882) 241.

Geodorum purpureum R. Br. apud Naves Novis. App. (1882) 241.

Geodorum semicristatum Lindl. Fol. Orch. Geodorum (1854) 2; Miq. Fl. Ind. Bat. 3 (1859) 712; Reichb. f. in Walp. Ann. 6 (1863) 633; Naves Novis. App. (1882) 241; Vidal Phan. Cuming. Philip. (1885) 150, Rev. Pl. Vasc. Filip. (1886) 270; Merr. in Govt. Lab. Publ. (Philip.) 8 (1903) 45, 52, 61, 75, 88, 152, 27 (1905) 84 = Geodorum nutans (Presl) Ames.

\section{DENDROBIUM Swartz}

DENDROBIUM ACLINIA Reichb. f. in Bonpl. 4 (1856) 329.

Dendrobium pseudaclinia Lindl. in Journ. Linn. Soc. 3 (1859) 9; Kränzl. in Engl. Pflanzenreich IV. 50. II. B. 21 (1910) 297; Ames Orch. 5 (1915) 134.

Luzon (Isabela, Zambales, Rizal), B. S. 5618, 19065 (cult.) Curran, Lyon 63, 148, 150, B. S. 19151 Reillo \%. Epiphyte up to $330 \mathrm{~m}$ altitude in Rizal.

Reichenbach states that his specimen came from "Ostindien," a very indefinite localization. Lindley in 1859 renamed the species, without justification, Dendrobium pseudaclinia, citing $D$. aclinia Reichb. $f$. as a synonym, and then made a new $D$. aclinia based on the genus Aclinia of Griffith. This proceeding is certainly unjustified and, moreover, the $D$. aclinia of Lindley is considered by J. D. Hooker, Fl. Brit. Ind. 5 (1890) 718, to be a peloric form of $D$. incurvum Lindl. Consequently $D$. aclinia Reichb. $f$. is reinstated in place of $D$. pseudactinia Lindl.

DENDROBIUM aCuminatissimum (Blume) Lindl. Gen. \& Sp. Orch. (1830) 86; Lindl. in Bot. Reg. 30 (1844) Misc. 50; Miq. Fl. Ind. Bat. 3 (1859) 636; J. J. Sm. Fl. Buitenz. 6 (Orch. Jav.) (1905) 350, Fig.-Atlas (1910) fig. 265, in Nova Guinea 8 (1909) 71; 
Kränzl. in Engl. Pflanzenreich iv. 50, I1. B. 21 (1910) 170; Schltr. in Engl. Bot. Jahrb. 45, Beibl. 104 (1911) 35; Ames Orch. 5 (1915) 114; J. J. Sm. in Philip. Journ. Sci. 12 (1917) Bot. 256. Grastidium acuminatissimum Bl. Bijdr. (1825) 333.

Dendrobium caudatum Teijsm. \& Binn. var. javanica Teijsm. \& Binn. in Nat. Tijdschr. Ned. Ind. 24 (1862) 315.

Callista acuminatissima Ktze. Rev. Gen. P1. 2 (1891) 654.

Leyte, Wenzel 04\%. Dagami, epiphyte at $60 \mathrm{~m}$ altitude. In Sumatra, Java, Amboina, and New Guinea.

DENDROBIUM ACUMINATUM Rolfe ex Ames Orch. 1 (1905) 86, 2 (1908) $1,170, t .17,5$ (1915) 114.

? Dendrobium cymbidioides Blume apud Naves Novis. App. (1882) 231. Srrcopodium cymbidioides Kränzl. in Engl. Pflanzenreich IV. 50.

II. B. 21 (1910) 325, quoad Philip.

Sarcopodium acuminatum Rolfe in Orch. Rev. 18 (August, 1910) 239; Kränzl. in Engl. Pflanzenreich IV. 50. II. B. 21 (November, 1910) $329, f i g .32 D, E$.

Luzon (Kalinga, Bataan), Lyon 47, Boettcher s. n., Whitford 223, F. B. 738 Borden, Topping 461, Weber 23, Copeland s. n. Epiphyte at 1,000 to $2,000 \mathrm{~m}$ altitude.

DENDROBIUM AGUSANENSE Ames Sched. Orch. 6 (1923) 79.

Mindànao (Agusan), Elmer 12462. At $330 \mathrm{~m}$ altitude.

DENDROBIUM ALBAYENSE Ames in Philip. Journ. Sci. 7 (1912) Bot. 14, Orch. 5 (1915) 115.

Luzon (Laguna, Tayabas, Albay), Negros (Occidental Negros), Leyte, Mindanao (Butuan, Agusan, Davao). A fairly common species; epiphytic at sea level to $900 \mathrm{~m}$ altitude.

DENDROBIUM AMETHYSTOGLOSSUM Reichb. f. in Gard. Chron. (1872) 109; Hook. f. in Bot. Mag. 98 (1872) t. 5968; Veitch Man. Orch. Pl. pt. 3 (1888) 16; Kränzl. in Engl. Pflanzenreich IV. 50. II. B. 21 (1910) 115; Ames Orch. 5 (1915) 115.

Callista amethystoglossa Ktze. Rev. Gen. Pl. 2 (1891) 654.

Luzon (Benguet), Lyon $15 \%$.

DENDROBIUM ANOSMUM Lindl. in Bot. Reg. 31 (1845) Misc. 32; Naves Novis. App. (1882) 234; J. J. Sm. in Winkler in Engl. Bot. Jahrb. 48 (1912) 101, in Nova Guinea 12 (1913) Bot. 74, 12 (1916) Bot. 351, in Merr. Interp. Rumph. Herb. Amb. (1917) 175; Merr. Sp. Blancoanae (1918) 114; Ames in Merr. in Journ. Roy. Asiat. Soc. Straits Branch, Special No. (1921) 156.

Dendrobium macrophyllum Lindl. in Bot. Reg. 25 (1839) Misc. 36, 28 (1842) Misc. 84, 30 (1844) Misc. 47, Sert. Orch. (1840) t. 35; Paxt. Mag. Bot. 8 (1841) 97, t.; Hook. Cent. Orch. Pl. (1851) t. 12; Planch. Hort. Donat. (1855) 156, Atlas t. 1; Reichb. f. in Linden Pescat. 1 (1860) 40, t. 40, non A. Rich.

Dendrobium macranthum Hook. in Bot. Mag. 69 (1842) t. 3970; Paxt. Mag. Bot. 9 (1842) 238; Planch. in Fl. des Serres 8 (1852) 21, t. 757; Miq. Fl. Ind. Bat. 3 (1859) 642; Naves Novis. App. (1882) 233; Ceron Cat. Pl. Herb. Manila (1892) 166, non A. Rich. 
Epidendrum ruibarbarum redolens Blanco Fl. Filip. ed. 2 (1845) 593. Dendrobium macrophyllum Lindl. var. giganteum Lindl. in Gard. Chron. (1854) 219; Warner Sel. Orch. Pl. I (1862-65) t. 26.

Dendrobium macranthum Hook. var. purpureo-marginatum De Vriese Ill. Orch. Ind. Neerl. (1854) t. 17.

Dendrobium macrophyllum Lindl. var. pallidum Planch. Hort. Donat. (1855) 156.

?Dendrobium retusum Llanos in Mem. Real Acad. Cienc. Madr. 4 (1857) 498; F.-Vill. \& Naves in Blanco Fl. Filip. ed. $3,4^{1}$ (1880) 100.

Dendrobium macranthum Hook. var. marginatum De Vriese ex Miq. Fl. Ind. Bat. 3 (1859) 643; Naves Novis. App. (1882) 233.

Dendrobium superbum Reichb. f. in Walp. Ann. 6 (1861) 282, in Gard. Chron. II 17 (1882) 776; Warner \& Will. Orch. Alb. 1 (1882) t. 42; Vidal Phan. Cuming. Philip. (1885) 149, Rev. Pl. Vasc. Filip. (1886) 267; Veitch Man. Orch. PI. pt. 3 (1888) 77; Du Buyss, in l'Orchidoph. 12 (1892) 70; Ridl. in Journ. Bot. 36 (1898) 211; Cogn. in Dict. Icon. Orch. (1899) Dendrob. $t .20$; Finet in Bull. Mus. Hist. Nat. Par. 6 (1903) 300; J. J. Sm. Orch. Amb. (1905) 67; Ames Orch. 1 (1905) 90, 2 (1908) 186, 5 (1915) 139; Rolfe in Orch. Rev. 14 (1906) 177, fig. 21; Ridl. Mat. Fl. Mal. Penin. 1 (1907) 56; Kränzl. in Engl. Pflanzenreich IV. 50. II. B. 21 (1910) 33; O'Brien in Gard. Chron. III 72 (1922) 9.

Dendrobium superbum Reichb. f. var. anosmum Reichb. f. in Walp. Ann. 6 (1861) 283; Veitch Man. Orch. Pl. pt. 3 (1889) 77; Rolfe in Lindenia 6 (1890) 51, t. 264; Kränzl. in Engl. Pflanzenreich IV. 50. II. B. 21 (1910) 33.

Dendrobium superbum Reichb. f. var. giganteum Reichb. f. in Walp. Ann. 6 (1861) 283; Ames Orch. 2 (1908) 187; Kränzl. in Engl. Pflanzenreich IV. 50. II. B. 21 (1910) 33.

Dendrobium superbum Reichb. f. var. purpureo-marginatum Reichb. f. in Walp. Ann. 6 (1861) 283; Kränzl. in Engl. Pflanzenreich IV. 50. II. B. 21 (1910) 33.

Dendrobium macrophyllum Lindl. var. purpureo-marginatum De Vriese ex Reichb. $f$. in Walp. Ann. 6 (1861) 283, in syn.

Dendrobium superbum Reichb. f. var. huttonii Reichb. f. in Gard. Chron. (1869) 206; Rolfe in Lindenia 6 (1890) 52, sub t. 264; Ames Orch. 2 (1905) 187; O'Brien in Gard. Chron. III 72 (1922) 9, fig. 6 .

Epidendrum rhabarbarum redolens Blanco Fl. Filip. ed. 3, 3 (1879) $45, t .389$.

Dendrobium macranthum Hook. var. giganteum Naves Novis. App. (1882) 233; Roman in Journ. des Orch. 6 (1895) 284.

? Dendrobium macranthum Hook. var. album Naves Novis. App. (1882) 233.

Dendrobium superbum Reichb. f. var. burkei Reichb. f. in Gard. Chron. II 21 (1884) 306; Kränzl. in Engl. Pflanzenreich IV. 50. II. B. 21 (1910) 34; O'Brien in Gard. Chron. III 72 (1922) 9.

Dendrobium scortechinii Hook. f. Fl. Brit. Ind. 5 (1890) 741; Ridl. in Journ. Linn. Soc. 32 (1896) 264.

Callista anosma Ktze. Rev. Gen. Pl. 2 (1891) 653. 
Callista scortechinii Ktze. Rev. Gen. Pl. 2 (1891) 655.

Dendrobium superbum Reichb. f. var. dearei Day ex Rolfe in Lindenia 6 (1890) 52, sub t. 264; Rolfe in -Orch. Rev. 14 (1906) 177, fig. 22; O'Brien in Gard. Chron. III 72 (1922) 9.

Dendrobium leucorhodum Schltr. in Fedde Repert. Beihefte 1 (Orch. Deutsch-Neu-Guin.) (1912) 499.

Luzon (Abra, Bontoc, Lepanto, Benguet, Nueva Vizcaya, Rizal). Fairly frequent in Luzon, up to $760 \mathrm{~m}$ altitude in Bontoc. In Malay Peninsula, Indo-China (fide Finet), Celebes, Amboina, Borner, and New Guinea.

DENDROBIUM APOROIDES (Lindl.) Merr. Sp. Blancoanae (1918) 113. Aporum incrassatum Brongn. in Duperr. Voy. Coquille (1834) 204, t. $42 B$, nor Blume.

Epidendrum equitans Blanco Fl. Filip. (1837) 645, ed. 2 (1845) 449, ed. 3, 3 (1879) 44, non Dendrobium equitans Kränzl.

Eria aporoides Lindl. in Journ. Linn. Soc. 3 (1859) 60; Miq. Fl. Ind: Bat. 3 (1859) 657; Naves Novis. App. (1882) 236; Merr. in Govt. Lab. Publ. (Philip.) 27 (1905) 84.

Pinalia aporodes Ktze. Rev. Gen. Pl. 2 (1891) 678.

Dendrobium brongniartii Kränzl. in Engl. Pflanzenreich IV. 50. II. B. 21 (1910) 210; Ames in Philip. Journ. Sci. 7 (1912) Bot. 15, Orch. 5 (1915) 116.

Dendrobium equitans Kränzl. in Engl. Pflanzenreich IV. 50. 11. B. 21 (1910) 228, quoad Philip.

Luzon (Ilocos Norte, Cagayan, Nueva Vizcaya, Zambales, Rizal, Tayabas, Camarines, Sorsogon), Leyte, Basilan, Mindanao (Camiguin Island, Agusan, Surigao, Davao). A fairly common and widely distributed species in the Philippines at sea level to $1,000 \mathrm{~m}$ altitude.

DENDROBIUM APPENDICULOIDES Ames Orch. 7 (1922) 93.

Mindanao (Bukidnon), B. S. 38940 Ramos \& Edaño. Mt. Candoon; epiphyte in forest at $1,650 \mathrm{~m}$ altitude.

DENDROBIUM BASILANENSE Ames in Philip. Journ. Sci. 7 (1912) Bot. 14, Orch. 5 (1915) 116.

Panay (Iloilo), Basilan, Mindanao (Zamboanga, Agusan), B. S. $1818 \gamma$ Robinson, F. B. 3968 Hutchinson, Merrill 8068, Weber s. n., 45, 48, 132. Epiphyte at sea level to $400 \mathrm{~m}$ altitude.

DENDROBIUM BULLENIANUM Reichb. f. in Bot. Zeit. 20 (1862) 214; KränzI. in Engl. Pflanzenreich IV. 50. II. B. 21 (1910) 133; Ames Orch. 5 (1915) 117.

Dendrobium erythroxanthum Reichb. f. in Gard. Chron. II 2 (1874) 162; Boxall ex Naves Novis. App. (1882) 234; Kränzl. in Engl. Bot. Jahrb. 25 (1898) 604.

No specimens seen, but drawings have been studied.

Regarding Dendrobium bullenianum Reichenbach states: "Sie soll aus Manilla stammen." Kränzlin gives in distribution "Luzon?... SamoaInseln." Reichenbach's specimen may have come from Polynesia rather than from the Philippines. Dendrobium erythroxanthum is supposed to have come from the Philippines. 
It seems probable that Dendrobium chameleon Ames is conspecific, but sufficient evidence of this fact is at present lacking.

DENDROBIUM BUSUANGENSE Ames Orch. 6 (1920) 296, t. 101.

Busuanga, Taylor s. $n$. (cult. in Manila).

DENDROBIUM CABADBARENSE Ames Orch. 5 (1915) 117.

Mindanao (Agusan, Davao), Weber 157, 192, 297, with 310, Black s. $n$. Epiphyte at sea level to $100 \mathrm{~m}$ altitude.

DENDROBIUM CANDOONENSE Ames Orch. 7 (1922) 94.

Mindanao (Bukidnon), B. S. 38716 Ramos \& Edaño. Epiphyte on forest slope, Mt. Candoon, at $1,650 \mathrm{~m}$ altitude.

DENDROBIUM CARINATUM (Linn.) Willd. Sp. Pl. 4 (1805) 133; Lindl. Gen. \& Sp. Orch. (1830) 92; Kränzl. in Engl. Pflanzenreich IV. 50. II. B. 21 (1910) 298; Ames Orch. 5 (1915) 118.

Bontia luzonica, geniculis inferioribus carinulatis Petiver Gazophyll. Decas 5 (1702) 70, t. 44, fig. 10.

Epidendrum carinatum Linn. Sp. Pl. (1753) 953, ed. 2 (1763) 1350.

Dendrobium robinsonii Ames in Philip. Journ. Sci. 8 (1913) Bot. 425, Orch. 5 (1915) 138.

Luzon (Zambales) Mindoro, F. B. 6315 Curran, B. S. 3059 Foxworthy, 5642 Robinson. Epiphyte in open sandy spots.

DENDROBIUM CERAULA Reichb. f. in Linnaea 41 (1876) 40; Kränzl. in Engl. Pflanzenreich IV. 50. II. B. 21 (1910) 104; Ames Orch. 5 (1915) 118.

Luzon, Jagor 895, 896 (in Herb. Berol.).

DENDROBIUM CHAMELEON Ames Orch. 2 (1908) 174, text cut, 5 (1915) 119; Kränzl. in Engl. Pflanzenreich IV. 50. II. B. 21 (1910) 134.

Luzon (Benguet), R. S. Williams 1940. Baguio; epiphyte, hanging from the trees.

DENDROBIUM CHRYSOGRAPHATUM Ames Orch. 5 (1915) 119.

Dendrobium angustifolium Ames in Philip. Journ. Sci. 7 (1912) Bot. 14, non Lindl.

Luzon (Bontoc, Benguet, Zambales, Bataan, Rizal), Vanoverbergh 708, 709, 1346, 1522, Ramos \& Edaño 95, F. B. 6972 Curran, R. S. Williams í64, B. S. 29588 Ramos \& Edaño. Epiphyte at 1,450 to 2,000 m altitude.

DENDRoBIUM CLemensiAe Ames in Philip. Journ. Sci. 7 (1912) Bot.

16, Orch. 5 (1915). 120.

Negros (Occidental Negros), Leyte, Mindanao (Lanao), Lyon 40, Wenzel 0530, 0591, 0599, 0906, M. S. Clemens 508. Epiphyte at 500 to $700 \mathrm{~m}$ altitude in Leyte.

DENDROBIUM CRUMENATUM Sw. in Schrad. Journ. Bot. 2 (1799) 237, in Kongl. Svensk. Vet.-Akad. Nya Handl. 21 (1800) 246; Willd. Sp. Pl. 4 (1805) 137; Lindl. in Trans. Hort. Soc. 7 (1830) 40, Gen. \& Sp. Orch. (1830) 88; Roxb. Fl. Ind. ed. 2, 3 (1832) 480; 
Lindl. in Bot. Reg. 25 (1839) t. 22; Paxt. Mag. Bot. 6 (1839) 90, 10 (1843) 189; Edit. in Allg. Berl. Gartenz. 7 (1839) 254, 11 (1843) 175; Knowles \& Westc. Floral Cab. 3 (1840) 47; Hook. in Bot. Mag. 69 (1843) t. 4013 ; Griff. Notul. 3 (1851) 315; De Vriese Ill. Orch. Ind. Neerl. (1852) t. 3, fig. VI; Miq. Fl. Ind. Bat. 3 (1859) 638; Reichb. f. in Walp. Ann. 6 (1861) 291; Miq. Sumatra (1862) 274, Choix des Pl. (1863) sub t. 22, p. p. (excl. fig.); Thw. Enum. Pl. Zeyl. (1864) 297; Naves Novis. App. (1882) 232; Vidal Phan. Cuming. Philip. (1885) 148, Rev. Pl. Vasc. Filip. (1886) 267; Rolfe in Lindenia 5 (1889) 33, t. 207; Hook. f. Fl. Brit. Ind. 5 (1890) 729; Ridl. in Trans. Linn. Soc. II 3 (1893) 362; Grant Orch. Burma (1895) 80; Ridl. in Journ. Linn. Soc. 31 (1896) 270, 32 (1896) 254; Trim. Handb. Fl. Ceyl. 4 (1898) 151; Finet in Bull. Mus. Hist. Nat. Par. 6 (1903) 297; J. J. Sm. Fl. Buitenz. 6 (Ol'ch. Jav.) (1905) 330, Fig.-Atlas (1910) fig. 249, Orch. Amb. (1905) 42; Ames Orch. 1 (1905) 88, 2 (1908) 175, 5 (1915) 121, 7 (1922) 95; Ridl. Mat. Fl. Mal. Penin. 1 (1907) 48; J. J. Sm. in Bull. Dept. Agr. Ind. Néerl. 43 (1910) 45; Kränzl. in Engl. Pflanzenreich IV. 50. II. B. 21 (1910) 235, fig. 19 C, D, 6-8; Schltr. in Engl. Bot. Jahrb. 45, Beibl. 104 (1911) 34; Merr. Fl. Manila (1912) 168; J. J. Sm. in Winkler in Engl. Bot. Jahrb. 48 (1912) 98; Ames in Merr. in Journ. Roy. Asiat. Soc. Straits Branch, Special No. (1921) 158.

Onychium crumenatum Blume Bijdr. (1825) 326.

Callista crumenata Ktze. Rev. Gen. Pl. 2 (1891) 653.

Dendrobium cumulatum Kränzl. in Engl. Pflanzenreich IV. 50. II. B. 21 (1910) 115, quoad Philip.; Ames Orch. 5 (1915) 121.

Dendrobium caninum Merr. in Philip. Journ. Sci. 19 (1921) 344; Ridl. in Journ. Bot. 60 (1922) 274; Merr. in Journ. Bot. 61 (1923) 173, non Epidendrum caninum Burm, f.

Luzon (Benguet, Rizal, Zambales, Tayabas), Polillo, Samar (Basiao Island), Biliran, Leyte, Palawar, Mindanao (Camiguin Island, Zamboanga, Lanao, Agusan, Surigao, Davao). A common and widely distributed species in the Philippines, at sea level to $500 \mathrm{~m}$ altitude. In Ceylon, Burma, Malay Penirsula, Andaman Islands, Cochinchina, Sumatra, Java, Amboina, Timor, and Borneo.

DENDROBIUM CULTRATUM Schltr. in Fedde Repert. 10 (1911) 71; Ames Orch. 5 (1915) 121.

Dendrobium sinuatum Schltr. in Perk. Frag. Fl. Philip. (1904) 43; Kränzl. in Engl. Pflanzenreich IV. 50. II. B. 21 (1910) 216, quoad Philip., non Lindl.

Dendrobium atropurpureum Ames Orch. 1 (1905) 86, 2 (1908) 172; Kränzl. in Engl. Pflanzenreich IV. 50. II. B. 21 (1910) 217, quoad Philip., non Miq.

Iuzon (Apayao, Ifugao, Pangasinan, Rizal, Tayabas), Polillo, Mindoro, Panay (Capiz), Negros (Occidental Negros), Samar, Palawan, Mindanao (Agusan, Surigao, Davao), Sibutu. A fairly common and widely distributed species in the Philippines, at sea level to $150 \mathrm{~m}$ altitude. In Celebes. 
DENDROBIUM DEAREI Reichb. $f$. in Gard. Chron. II 18 (1882) 361; Warner \& Will. Orch. Alb. 3 (1884) t. 120; Veitch Man. Orch. Pl. pt. 3 (1888) 37, text cut; Cogn. in Dict. Icon. Orch. (1905) Dendrob. t. 36; Ames in Philip. Journ. Sci. 4 (1909) Bot. 599; Kränzl. in Engl. Pflanzenreich IV. 50. II. B. 21 (1910) 89; Rolfe in Orch. Rev. 19 (1911) 261; Ames Orch. 5 (1915) 122.

Mindoro, Dinagat, Mindanao, F. B. 8525 Merritt, Lyon 121.

DENDROBIUM DISTICHUM (Presl) Reichb. f. in Linnaea 41 (1876) 39; Naves Novis. App. (1882) 232; Kränzl. in Engl. Pflanzenreich IV. 50. II. B. 21 (1910) 211 (excl. syn. in part); Ames Orch. 5 (1915) 122.

Schizmoceras disticha Presl Rel. Haenk. 1 (1827) 96, t. 19, fig. 2. Dendrobium indivisum Naves Novis. App. (1882) 231; Vidal Phan. Cuming. Philip. (1885) 148, Rev. Pl. Vasc. Filip. (1886) 267; Kränzl. in Engl. Pflanzenreich IV. 50. II. B. 21 (1910) 217, quoad Philip.; Ames in Philip. Journ. Sci. 6 (1911) Bot. 52, non Miq.

Luzon (Benguet, Zambales, Bataan, Rizal, Camarines, Sorsogon), Poliilo, Sulu Archipelago. A fairly common species in Luzon, at sea level to $900 \mathrm{~m}$ altitude.

DENDROBIUM ELMERI Ames in Elm. Leafi. Philip. Bot. 5 (1912) 1573, Orch. 5 (1915) 123.

Luzon (Laguna, Tayabas), B. S. 16568, 20458 Ramos, 20851 Escritor, Serrato s. n., Calica s.n., Elmer 9249. Epiphyte, in forest at $830 \mathrm{~m}$ altitude in Tayabas.

DENDROBIUM EPIDENDROPSIS Kränzl. in Orchis 2 (1908) 79, fig. 12; Ames in Philip. Journ. Sci. 4 (1909) Bot. 598; Kränzl. in Engl. Pflanzenreich IV. 50. 1I. B. 21 (1910) 106; Ames Orch. 5 (1915) 123.

Luzon (Benguet, Bataan, Rizal), Loher s. n., Lyon 118, 161, F. B. 2109 Borden, Elmer 6839, B. S. 3060 Ramos.

DENDROBIUM ESCRITORII Ames Orch. 5 (1915) 123.

Luzon (Tayabas, Camarines), Mindanao (Surigao), B. S. 20683 Escritor, 22019 Ramos, 27405 Alambra, Wenzel 01091. Epiphyte at 35 to 150 $\mathrm{m}$ altitude.

DENDROBIUM EURORUM Ames Orch. 5 (1915) 124.

Leyte, Wenzel 065, 0612. Epiphyte in forest at 120 to $500 \mathrm{~m}$ altitude.

DENDROBIUM GERLANDIANUM Kränzl. in Fedde Repert. 6 (1909) 317, in Engl. Pflanzenreich IV. 50. II. B. 21 (1910) 238; Ames Orch. 5 (1915) 125.

The species was described from a Loher plant from Manila. The type has not been seen, but from the description it would seem to approach D. philippixense Ames. 
DENDROBIUM HERCOGLOSSUM Reichb. f. in (xaxd. Chron. II 26 (1886) 487; Edit. in Hamb. Gartenz. 42, (1886) 558; Hook. f. Fl. Brit. Ind. 6 (1890) 185; Kränzl. in Engl. Pflanzenreich IV. 50. II. B. 21 (1910) 75.

Dendrobium aduncum Hook. f. in Bot. Mag. 110 (1884) t. 6784, non, Lindl.

Mindanao (Lanao) (so reported), Lyon 138.

In view of the above cited (reported Philippine) specimen it does not seem improbable that the material referred to $D$. aduncum Lindl. by Naves and by Ceron is in reality this species.

DENDROBIUM HETEROCARPUM Wall. Cat. (1828) No. 20, nomen, ex Lindl. Gen. \& Sp. Orch. (1830) 78, Pl. Asiat. Rar. 2 (1831) 84, t. 196; Lindl. in Bot. Reg. 30 (1844) Misc. 49; Paxt. Mag. Bot. 14 (1847) 68; Hook. in Bot. Mag. 79 (1853) t. 4708; Planch. in Fl. des Serres 8 (1854?) 253, t. 842; Lem.'Jard. Fleur. 4 (1854) t. 386; Batem. Second Cent. Orch. Pl. (1867) t. 150; Douglas in Gard. Chron. II 25 (1886) 397; Hook. f. Fl. Brit. Ind. 5 (1890) 737; Berkl. in Orch. Rev. 1 (1893) 82; Grant Orch. Burma (1895) 97; King \& Pantl. in Ann. Roy. Bot. Gard. Calc. 8 (Orch. Sik.Himal.) (1898) 53, t. 74; Trim. Handb. Fl. Ceyl. 4 (1898) 154; Cogn. in Dict. Icon. Orch." (1898) Dendrob. t. 10; J. J. Sm. Fl. Buitenz. 6 (Orch. Jav.) (1905) 368, Fig.-Atlas (1911) fig. 281; Ames Orch. 1 (1905) 89, 2 (1908) 176, text cut; Kränzl. in Engl. Pflanzenreich IV. 50. 11. B. 21 (1910) 45; Merr. \& Merritt in Philip. Journ. Sci. 5 (1910) Bot. 341; Schltr. in Fedde Repert. 10 (1911) 37, Orchideen (1915) 260.

Dendrobium aureum Lindl. Gen. \& Sp. Orch. (1830) 77, in Bot. Reg. 30 (1844) Misc. 51; Wight Icon. $5^{1}$ (1852) 6, t. 1646; Lindl. in Fl. des Serres 7 (1852) 210; Reichb. f. in Walp. Ann. 6 (1861) 287: Thw. Enum. P1. Zeyl. (1864) 297; Hemsl. in Gard. Chron. II 16 (1881) 688; Veitch Man. Orch. Pl.pt. 3 (1888) 19, text cut; Reichb. f. in Sander Reichenbachia I 2 (1890) 31, t. 63; Stein Orchideenb. (1892) 186; Du Buyss. in l'Orchidoph. 12 (1892) 184; Ridl. Mat. Fl. Mal. Penin. 1 (1907) 56; Ames Orch. 5 (1915) 115. Dendrobium aureum Lindl. var. pallidum Lindl. in Bot. Reg. 25 (1839) t. 20; Knowles \& Westc. Floral Cab. 3 (1840) 48; Reichb. f. in Walp. Ann. 6 (1861) 287; Veitch Man. Orch. Pl. pt. 3 (1888) 19; Stein Orchideenb. (1892) 186.

Dendrobium rhombeum Lindl. in Bot. Reg. 29 (1843) t. 17, 30 (1844) Misc: 56; Reichb. f. in Bot. Zeit. 1 (1843) 333; Lindl. in Paxt. Flow. Gard. 3 (1852-53) 52; Naves Novis. App. (1882) 233; Reichb. f. in Sander Reichenbachia I 2 (1890) 31, in obs.

Lendrobium heterocarpum Wall. var. henshallii Hook. in Bot. Mag. 83 (1857) t. 49\%0; Kränzl. in Engl. Pflanzenreich IV. 50. II. B. 21 (1910) 45.

Dendrobium aureum Lindl. var, philippinense Reichb. f. in Gard. Chron. II 13 (1880) 72; Veitch Man. Orch. Pl. pt. 3 (1888) 19; Stein Orchideenb. (1892) 187. 
Dendrobium atractodes Ridl. in Journ. Linn. Soc. 23 (1885) 123, fide Kränzl.

Dendrobium aureum Lindl. var. henshallii Veitch Man. Orch. Pl. pt. 3 (1888) 19; Stein Orchideenb. (1892) 186.

Callista aurea Ktze. Rev. Gen. Pl. 2 (1891) 653.

Callista heterocarpa Ktze. Rev. Gen. Pl. 2 (1891) 654.

Dendrobium aureum Lindl, var. rhombeum Stein Orchideenb. (1892) 187.

Dendrobium heterocarpum Wall. var. pallidum Kränzl. in Engl. Pflanzenreich IV. 50. 11. B. 21 (1910) 45.

Dendrobium heterocarpum Wall. var. philippinense Kränzl. in Engl. Pflanzenreich IV. 50. II. B. 21 (1910) 45.

Dendrobium hildebrandii Kränzl. in Engl. Pflanzenreich IV. 50. II. B. 21 (1910) 34, p. p., non Rolfe, fide Schltr.

Dendrobium minahassae Kränzl. in Engl. Pflanzenreich IV. 50.11. B. 21 (1910) 107, fide Schltr.

Luzon (Bontoc, Benguet, Bataan, Rizal, Albay, Sorsogon), Mindanao (Davao). Fairly common in the Philippines, especially in Luzon at 1,300 to $1,500 \mathrm{~m}$ altitude. In India, Ceylon, Burma, Malay Peninsula, Java, and Celebes.

It has seemed advisable to adopt the name $D$. heterocarpum in place of $D$. aureum Lindl., because the first definite union of the species occurs in Bot. Mag. 79 (1853) t. 4708 (cf. International Rules adopted at Vienna, Sect. 6, Art. 46) and also because it is the earliest name, although a nomen, and still further because it has been more commonly accepted by reputable authors.

DENDROBIUM HYMENANTHUM Reichb. f. in Bonpl. 3 (1855) 222, in Walp. Ann. 6 (1861) 302; Naves Novis. App. (1882) 234; Ames in Philip. Journ. Sci. 4 (1909) Bot. 598; Kränzl. in Engl. Pfianzenreich IV. 50. II. B. 21 (1910) 288; Ames Orch. 5 (1915) 125. Dondrochilum hymenanthum Vidal Phan. Cuming. Philip. (1885) 149, Rev. Pl. Vasc. Filip. (1886) 268, sphalm.

Luzon (Rizal, Tayabas), Polillo, Cuming 2135, Lyon 147, B. S. 3035 Ramos, 5647, 10453 McGregor, F. B. 28368 Mabesa.

DENDROBIUM INTERJECTUM Ames Orch. 5 (1915) 125.

Leyte, Wenzel 065a, 0534, 05\% .059\%, 067\%, 0691. Epiphyte in forest at 120 to $700 \mathrm{~m}$ altitude.

DENDROBIUM LEYTENSE Ames Orch. 5 (1915) 126.

Leyte, Wenzel 082, 0203, 0415. Epiphyte in forest at 60 to $500 \mathrm{~m}$ altitude.

DENDROBIUM LOHERIANUM Kränzl, in Ann. k. k. Nat. Hofmus. Wien 30 (1916) 62.

Philippines, probably Luzon, Loher $s . n$.

Known only from the type collection. 
DENDROBIUM LUNATUM Lindl, in Journ. Linn. Soc. 3 (1859) 4; Miq. Fl. Ind. Bat. 3 (1859) 630; Naves Novis. App. (1882) 231; Vidal Phan. Cuming. Philip. (1885) 148, Rev. Pl. Vasc. Filip. (1886) 267; Kränzl. in Engl. Pflanzenreich IV. 50. 11. B. 21 (1910) 218; Ames in Philip. Journ. Sci. 7 (1912) Bot. 17, in Elm. Leafl. Philip. Bot. 5 (1912) 1574, Orch. 5 (1915) 127.

Palawan, Cuming s. n, 2136, B. S. 793, 907 Foxworthy, Elmer 12764. Weber 606, Merrill 9337, 9623, Ledesma s. n. Epiphyte at sea level to $500 \mathrm{~m}$ altitude.

DENDROBIUM LUZONENSE Lindl. in Bot. Reg. 30 (1844) Misc. 54; Reichb. f. in Walp. Ann. 6 (1861) 290; Naves Novis. App. (1882) 234 ; Vidal Phan. Cuming. Philip. (1885) 148, Rev. Pl. Vasc. Filip. (1886) 267; Kränzl. in Engl. Pflanzenreich IV. 50. II. B. 21 (1910) 172, fig. 12 A-D; Ames Orch. 5 (1915) 127.

Dendrobium alagense Ames in Philip. Journ. Sci. 2 (1907) Bot. 328.

Luzon (Kalinga, Benguet, Sorsogon), Catanduanes, Mindoro, Leyte, Mindanao (Lanao, Agusan, Davao). A fairly common species; epiphyte at sea level to $1,300 \mathrm{~m}$ altitude.

DENDROBIUM LYONII Ames Orch. 2 (1908) 177, 5 (1915) 127, in Philip. Journ. Sci. 4 (1909) Bot. 672.

Dendrobium acuminatum Lyon in Gard. Chron. III 42 (1907) 210, fig. 88; Rolfe in Orch. Rev. 15 (1907) 312, as to plant spoken of; Kränzl, in Orchis 2 (1908) 73, t. 16, non Rolfe ex Ames; Edit. in Gard. Chron. III 46 (1909) 150, fig. 64, in Gardners' Mag. 52 (1909) 659, 660, text cut, non Rolfe ex Ames.

Sarcopodium lyonii Rolfe in Orch. Rev. 18 (1910) 240; Ames in Philip. Journ. Sci. 6 (1911) Bot. 53, in Addisonia 8 (1923) 17, t. 265.

Sarcopodium acuminatum Rolfe var. lyonii Kränzl. in Engl. Planzenreich IV. 50. II. B. 21 (1910) 329, fig. $32 A-C$.

Luzon (Bataan), Lyon s.n., 10, F. B. 7153 Curran. Epiphyte at 660 to $1,100 \mathrm{~m}$ altitude.

DENDROBIUM MACGREgORII Ames in Philip. Journ. Sci. 7 (1912) Bot. 17, Orch. 5 (1915) 128.

Polillo, B. S. 10457 McGregor.

DENDROBIUM MARIVELENSE Ames Orch. 2 (1908) 180, text cut, 5 (1915) 128.

Dendrobium parciflorum Kränzl. in Engl. Pflanzenreich IV. 50. II. B. 21 (1910) 222, quoad Philip.; Ames in Philip. Journ. Sci. 7 (1912) Bot. 18, non Reichb. $f$.

Luzon (Bataan), Mindanao (Lanao), Merrill s. n., Whitford 1127, M. S. Clemens 8. n. 
DENDROBIUM MERRILLII Ames Orch. 2 (1908) 181, text cut, 5 (1915) 126, in Philip. Journ. Sci. 4 (1909) Bot. 599; Kränzl. in Engl. Pflanzenreich IV. 50. II. B. 21 (1910) 299.

Dendrobium aloifolium Reichb f. apud Kränzl. in Engl. Pflanzenreich IV. 50. II. B. 21 (1910) 206, quoad syn. D. merrillii; Ames Orch. 5 (1915) 115 (D. aloefolium).

Luzon (Ifugao, Pangasinan, Rizal, Tayabas), Samar, B. S. 19989 McGregor, 29863 Fenix, 24355 Ramos, Loher 601\%, Merrill 335\%. Epiphyte at apparently rather low altitude $(350 \mathrm{~m})$.

DENDROBIUM MINDANAENSE Ames in Philip. Journ. Sci. 8 (1913) Bot. 423, Orch. 5 (1915) 128.

Luzon (Rizal), Polillo, Negros (Occidental Negros), Leyte, Culion, Palawan, Mindanao (Zamboanga, Agusan, Davao). A fairly common species of rather wide distribution. Terrestrial ? or epiphyte at low altitudes.

DENDROBIUM MODESTUM Reichb. f. in Bonpl. 3 (1855) 222, in Walp. Ann. 6 (1861) 281; Naves Novis. App. (1882) 234; Kränzl. in Engl. Pflanzenreich IV. 50. II. B. 21 (1910) 220; Ames Orch. 5 (1915) 128.

Llanos s. $n$.

Reichenbach described this species from a Llanos specimen from Manila and ascribed to it affinity with $D$. aciculare Lindl. Kränzlin in his monograph cites a Cuming specimen in the Lindley Herbarium. This species has not been recognized in any of the material collected during the past twenty years.

DENDROBIUM MULTIRAMOSUM Ames Orch. 5 (1915) 129.

Luzon (Benguet, Rizal), Williams 1934, Lyon 42.

DENDROBIUM O'BRIENIANUM Kränzl. in Gard. Chron. III 11 (1892) 266; Ames Orch. 2 (1908) 182, text cut, 5 (1915) 130; Kränzl. in Engl. Pflanzenreich IV. 50. II. B. 21 (1910) 105, fig. 5 N-O.

Dendrobium striatum Hort. ex Gard. Chron. III 38 (1905) 382;

Kränzl. in Engl. Pflanzenreich IV. 50. 11. B. 21 (1910) 298.

Known only from a cultivated specimen sent from Luzon by Micholitz to F. Sander Co.

DENDROBIUM ORNITHOFLORUM Ames Orch. 3 (1908) 60, t. 46, 5 (1915) 130.

Mindanao (Misamis), F. B. 4627 Mearns \& Hutchinson. Mt. Malindang at $1,900 \mathrm{~m}$ altitude.

DENDROBIUM PAPILIO Loher in Gard. Chron. III 21 (1897) 416;

Kränzl. in Engl. Pflanzenreich IV. 50. II. B. 21 (1910) 231, excl. Williams 1934; Rolfe in Orch. Rev. 22 (1914) 272; Ames Orch. 5 (1915) 130.

Dendrobium vanoverberghii Ames in Philip. Journ. Sci. 8 (1913) Bot. $425, t .13$.

Luzon (Bontoc, Benguet), Negros (Oriental Negros), Mindanao (Davao), Vanoverbergh 704, 1345, B. S. 31846 Santos, Weber 246, Herre s. n., Boettcher s. $n$. Epiphyte at 1,450 to $2,200 \mathrm{~m}$ altitude. 
DENDROBIUM PERGRACILE Ames in Philip. Journ. Sci. 8 (1913) Bot. 423, Orch. 5 (1915) 131.

Mindanao (Lanao), M. S. Clemens 99.). Camp Keithley, Lake Lanao.

DENDROBIUM PHILIPPINENSE Ames in Philip. Journ. Sci. 8 (1913) Bot. 424, Orch. 5 (1915) 131.

Dendrobium aciculare Lindl. apud Vidal Phan. Cuming. Philip. (1885) 148; Vidal Rev. Pl. Vasc. Filip. (1886) 267; Hook. f. Fl. Brit. Ind. 5 (1890) 725, quoad Philip.; Kränzl. in Engl. Pflanzenreich IV. 50. 11. B. 21 (1910) 241, quoad Philip.; Ames Orch. 5 (1915) 113.

Luzon (Laguna, Tayabas), Mindoro, Leyte, Cuming 2112, Loher 48\%, 6022, Lyon 67, Reillo s. n., B. S. 5641 Grifin, 20684 Escritor, 5658 Merrill, Wenzel 06, 07\%, 086, 0142. Epiphyte, apparently at low altitudes.

DENDRobium PLANIBULbe Lindl. in Bot. Reg. 29 (1843) Misc. 54; Reichb. f. in Walp. Ann. 6 (1861) 301; Naves Novis. App. (1882) 232; Ames Orch. 5 (1915) 132.

Dendrobium blumei Lindl. in Journ. Linn. Soc. 3 (1859) 14; Miq. Fl. Ind. Bat. 3 (1859) 638; Naves Novis. App. (1882) 232; Kränzl. in Engl. Pflanzenreich IV. 50. II. B. 21 (1910) 234, quoad syn. D. planibulbe Lindl, et quoad Philip.

Samar, Mindanao (Zamboanga, Agusan), Cuming s. n., B. S. 24434 Ramos, Reillo s. n., Sablaya s. n., F. B. 5176 Hutchinson, Weber 174. Epiphyte at low altitude.

DENDROBIUM PLATYCAULON Rolfe in Kew Bull. (1892) 139, in Gard.

Chron. III 12 (1892) 29, fig. 212; Kränzl. in Engl. Pflanzenreich IV. 50 II. B. 21 (1910) 267; Ames in Philip. Journ. Sci. 6 (1911) Bot. 52, Orch. 5 (1915) 132.

Dendrobium lamellatum Lindl. apud Kränzl. in Engl. Pflanzenreich IV. 50. II. B. 21 (1910) 269, quoad Philip.; Ames Orch. 5 (1915) ix. Merrill (cult.).

Known only from cultivated specimens.

DENDROBIUM PLICATILE Lindl. in Bot. Reg. 26 (1840) Misc. 10; Reichb. f. in Walp. Ann. 6 (1861) 307; Naves Novis. App. (1882) 234; Ames Orch. 5 (1915) 132.

Dendrobium macraei Ames Orch. 1 (1905) 89, 2 (1908) 176, non Lindl.

Desmotrichum fimbriatum Blume apud Kränzl. in Engl. Pflanzenreich

IV. 50. II. B. 21 (1910) 354, quoad Philip.

Luzon (Tarlac, Pampanga, Bataan, Rizal, Laguna, Tayabas), Panay (Capiz), Leyte, Samar, Mindanao (Lanao, Cotabato, Agusan). A common and rather widely distributed species; epiphyte at 60 to $800 \mathrm{~m}$ altitude.

DENDROBIUM POLYTRICHUM Ames in Philip. Journ. Sci. 2 (1907)

Bot. 328, nomen, Orch. 2 (1908) 183, 5 (1915) 133, 6 (1920) 297;

Kränzl. in Engl. Pflanzenreich IV. 50. 11. B. 21 (1910) 240.

Luzon (Cagayan, Isabela, Bataan, Rizal, Laguna, Tayabas), Mindoro. A fairly common species, especially in Luzon; up to $260 \mathrm{~m}$ altitude in Mindoro. 
DENDROBIUM PRISTINUM Ames Orch. 5 (1915) 133.

Luzon (Rizal), B. S. 3041 Ramos.

DENDROBIUM PROFUSUM Reichb. f. in Gard. Chron. II 21 (1884) 510; Kränzl, in Engl. Pflanzenreich IV. 50. II. B. 21 (1910) 259; Ames Orch. 5 (1915) 134.

Roebelen s. $n$.

Known only from the description and a tracing of the analytical drawings in the Reichenbach Herbarium.

DENDROBIUM PSEUDOCONVEXUM Ames Orch. 5 (1915) 135.

Luzon (Bataan, Rizal, Laguna, Sorsogon). A number of collections have been made in Luzon. Epiphyte in forest at 600 to $700 \mathrm{~m}$ altitude.

DENDROBIUM PTEROCARPUM Ames Sched. Orch. 6 (1923) 8C.

Mindanao (Davao), Elmer 11528. Epiphyte at 2,080 m altitude.

DENDROBIUM PURPUREOSTELIDIUM Ames Orch. 5 (1915) 136.

Luzon (Laguna, Sorsogon), Leyte, Mindanao (Agusan), Basilan, B. S. 15103 Ramos, 16258 Reillo, Elmer 16203, Wenzel 0193, 0228, Weber 191. Epiphyte at sea level and low altitudes.

DENDROBIUM RAMOSII Ames in Philip. Journ. Sci. 7 (1912) Bot. 18, Orch. 5 (1915) 138.

Luzon (Bontoc, Lepanto, Benguet), Vanoverbergh 9, 1715, B. S. 7049 Ramos, Lyon 154, Merrill 4682, Ramos \& Edaño 115. Apparently terrestrial in rather open ground in mountainous regions at 1,200 to $1,300 \mathrm{~m}$ altitude.

DENDROBIUM SANDERAE Rolfe in Gard. Chron. III 45 (1909) 374, fig. 163, in Orch. Rev. 17 (1909) 209, fig. 17; Ames in Philip. Journ. Sci. 4 (1909) Bot. 673; Fedde Repert. 8 (1910) 382; Rolfe in Bot. Mag. 136 (1910) t. 8351; Ames Orch. 5 (1915) 138.

Luzon (Bontoc, Lepanto, Benguet), Vanoverbergh 560, 1263, 3112, B. S. 5614 Worcester, 3497 Mearns, Lyon 114. Epiphyte at 1,000 to $1,650 \mathrm{~m}$ altitude.

DENDROBIUM SCHUETZEI Rolfe in Orch. Rev. 19 (1911) 224, 20 (1912) 289, 308, 337, fig. 47, in Gard. Chron. III 50 (1911) 42, III 52 (1912) 229, fig. 102; G. Wilson in Orch. World 3 (1912) 19, t.; Rolfe in Bot. Mag. 139 (1913) t. 8495; Ames Orch. 5 (1915) 138, 7 (1922) 96.

Mindanao (Surigao), Lyon s. $n$.

DENDROBIUM SCOPA Lindl. in Bot. Reg. 28 (1842) Misc. 55; Reichb. $\mathbf{f}$. in Walp. Ann. 6 (1861) 303; Naves Novis. App. (1882) 234; Ames in Philip. Journ. Sci. 6 (1911) Bot. 52, 9 (1914) Bot. 13, Orch. 5 (1915) 139.

Dendrobium thysanochilum Schltr. in K. Schum. \& Lauterb. Nachtr. Fl. Deutsch. Schutzgeb. (1905) 152, fide Kränzl.

Desmotrichum scopa Kränzl. in Engl. Pflanzenreich IV. 50. 11. B. 21 (1910) 349.

Luzon (Tarlac, Rizal, Laguna), Mindoro, Leyte, Mindanao (Agusan), Loddiges 273, Lyon s. n., 110, Reillo 53, Merrill 916\%, Wenzel 064, Weber 
163, Ramos 8. $n$. Epiphyte at sea level to $350 \mathrm{~m}$ altitude. In Guam and also probably in Formosa, Celebes (fide Kränzlin), New Guinea, and Samoa.

DENDROBIUM SECUNDUM Wall. Cat. (1828) No. 1996, nomen; Lindl. in Bot. Reg. 15 (1829) t. 1291, 30 (1844) Misc. 65, Gen. \& Sp. Orch. (1830) 81; Hook. in Bot. Mag. 74 (1848) t. 4.352; De Vriese Ill. Orch. Ind. Neerl. (1854) t. 6; Miq. Fl. Ind. Bat. 3 (1859) 641 , Reichb. f. in Walp. Ann. 6 (1861) 296; Miq. Sumatra (1862) 274; Naves Novis. App. (1882) 233; Veitch Man. Orch. Pl. pt. 3 (1888) 73; Hook. f. Fl. Brit. Ind. 5 (1890) 730; Stein Orchideenb. (1892) 207; Grant. Orch. Burma (1895) 120; Ridl. in Journ. Linn. Soc. 31 (1896) 271, 32 (1896) 259; J. J. Sm. Fl. Buitenz. 6 (Orch. Jav.) (1905) 358, Fig.-Atlas (1910) fig. 272; Kränzl. in Engl. Pflanzenreich IV. 50. 11. B. 21 (1910) 98; Ames Orch. 5 (1915) 139, in Merr. in Journ. Roy. Asiat. Soc. Straits Branch, Special No. (1921) 165.

Pedilonum secundum Blume Bijdr. (1825) 322.

Dendrobium bursigerum Lindl. in Journ. Linn. Soc. 3 (1859) 17; Miq. Fl. Ind. Bat. 3 (1859) 642; Edit. in l'Orchidoph. 2 (1882) 421; Reichb. f. in Gard. Chron. II 17 (1882) 424; Naves Novis. App. (1882) 233; Vidal Phan. Cuming. Philip. (1885) 148, Rev. Pl. Vasc Filip. (1886) 267; Ames Orch. 2 (1908) 173, 5 (1915) 117; Kränzl. in Engl. Pflanzenreich IV. 50. II. B. 21 (1910) 119, fig. $10 \mathrm{G}-\mathrm{O}$; Ames in Merr. in Journ. Roy. Asiat. Soc. Straits Branch, Special No. (1921) 157.

Callista bursigera Ktze. Rev. Gen. Pl. 2 (1891) 654.

Callista secunda Ktze. Rev. Gen. Pl. 2 (1891) 653.

Dendrobium secundum, Wall. var. bursigem Ridl. in Journ. Linn. Soc. 31 (1896) 271.

Luzon (Bataan, Rizal, Camarines), Mindanao (Lanao, Agusan), R. S. Williams 765, B. S. 30.97, 22155 Ramos, Weber 4, 144, F. B. 12287 Curran, Merrill 9821 (cult.). Epiphyte at 15 to $230 \mathrm{~m}$ altitude. In Burma, Malay Peninsula, Sumatra, Java, and Borneo.

DENDROBIUM SINUOSUM Ames Orch. 7 (1922) 96.

Leyte, Wenzel 0996. Tacloban, Tigbao, at sea level.

DENDROBIUM SPURIUM (Blume) J. J. Sm. Fl. Buitenz. 6 (Orch. Jav.) (1905) 343, Fig.-Atlas (1910) fig. 260; Ames Orch. 2 (1908) 185, text cut, 5 (1915) 139; Kränzl. in Engl. Pflanzenreich IV. 50. II. B. 21 (1910) 264, figs. 34 A, B, 35 E-H; Ames in Merr. in Journ. Roy. Asiat. Soc. Straits Branch, Special No. (1921) 165. Dendrocolla spuria Blume Bijdr. (1825) 290; Miq. Fl. Ind. Bat. 3 (1859) 697.

Aërides spurium Lindl. Gen. \& Sp. Orch. (1833) 241.

Dendrobium unguiculatum Teijsm. \& Binn. in Tijdschr. Ned. Ind. 5 (1853) 491; Miq. Fl. Ind. Bat. 3 (1859) 634; Reichb. f. in Walp. Ann. 6 (1861) 303; Naves Novis. App. (1882) 231.

Dendrobium euphlebium Reichb. f. ex Lindl. in Journ. Linn. Soc. 3 (1859) 7; Miq. Fl. Ind. Bat. 3 (1859) 633; Reichb. f. Xen. Orch. 2 (1862) 26, t. 110, II 6; Naves Novis. App. (1882) 232; 
Ridl. in Journ. Linn. Soc. 31 (1896) 268, 32 (1896) 258, Mat.

Fl. Mal. Penin. 1 (1907) 49.

Sarcochilus spurius Reichb. f. in Walp. Ann. 6 (1863) 500.

Thrixspermum spurium Reichb. f. Xen. Orch. 2 (1867) 122.

Callista euphlebia Kate. Rev. Gen. Pl. 2 (1891) 654.

Callista unguiculata Ktze. Rev. Gen. Pl. 2 (1891) 655.

Luzon (Cagayan, Bataan, Sorsogon), F. B. 16727 Curran, Merrill 3692, Whitford 1118, Lyon 43, Weber 31, B. S. 23427 Ramos, Elmer 16384, 17311. Epiphyte at 100 to $930 \mathrm{~m}$ altitude. In Malay Peninsula, Java, and Borneo.

DENDROBIUM STELLA-SILVAE (Loher \& Kränzl.) Ames Orch. 5 (1915) 139.

Sarcopodium stella-silvae Loher \& Kränzl. in Fedde Repert. 7 (1909) 40; Rolfe in Orch. Rev. 18 (August, 1910) 240; Kränzl. in Engl. Pflanzenreich IV. 50. II. B. 21 (1910) 326, fig. 31 A-C; Ames in Philip. Journ. Sci. 8 (1913) Bot. 426.

Luzon (Abra, Bontoc, Rizal), B. S. 7134 Ramos s. n., Tanoverbergh 2418,2868 , Loher s. $n$. On forest slope at $1,000 \mathrm{~m}$ altitude.

DENDROBIUM TAURINUM Lindl. in Bot. Reg. 29 (1843) t. 28, 30 (1844) Misc. 62; Paxt. Mag. Bot. 10 (1843) 143, 217, t. \& text cut; G. K. in Bot. Zeit. 1 (1843) 511; Reichb. f. in Walp. Ann. 6 (1861) 298; Van Houtte in Fl. des Serres 18 (1870) 133, t. 1904; Anderson in Gard. Chron. (1872) 974; Blanco Fl. Filip. ed. 3, Laminas (1877-83) t. 429; Naves Novis. App. (1882) 234; Hemsl. in Gard. Chron. II 17 (1882) 776; Veitch Man. Orch. Pl. pt. 3 (1888) 78; Du Buyss. in l'Orchidoph. 12 (1892) 37; Stein Orchideenb. (1892) 210; L. Linden in Lindenia 13 (1898) 93, t. 621; Ames Orch. 1 (1905) 90, 2 (1908) 187, 5 (1915) 139; Kränzl. in Engl. Pflanzenreich IV. 50. II. B. 21 (1910) 145, fig. 10A.

? Dendrobium venatrifolium Naves Novis. App. (1882) 233, non Lindl.

Callista taurina Ktze. Rev. Gen. Pl. 2 (1891) 655.

Luzon (Rizal, Laguna), Guimaras, Mindanao (Davao), Lyon s. n., 24, E. S. 2018, 21383, 22245 Ramos, Reillo s. n., 41, Foxworthy s. n., Loher 480, Everett 4206, Black s. n., 3, Copeland 505.

DENDROBIUM TERnAtense J. J. Sm. in Bull. Dept. Agr. Ind. Néerl. 22 (1909) 26; Kränzl. in Engl. Pflanzenreich IV. 50. II. B. 21 (1910) 246, fig. 20 A-C; Ames Orch. 5 (1915) 139.

Dendrobium macrophyllum Ames Orch. 2 (1908) 179, non A. Rich.; Kränzl. in Engl. Pflanzenreich IV. 50. II. B. 21 (1910) 244, quoad Philip.

Luzon (Bataan), Whitford 1083. Mt. Mariveles, Lamao River. Apparently rare in the Philippines. Epiphyte at $900 \mathrm{~m}$ altitude. In Ternate Island. 
DENDROBIUM TOPAZIACUM Ames Orch. 5 (1915) 140.

Leyte, Wenzel 0202. Dagami, epiphyte at $60 \mathrm{~m}$ altitude.

DENDROBIUM UNICORNE Ames Orch. 5 (1915) 140.

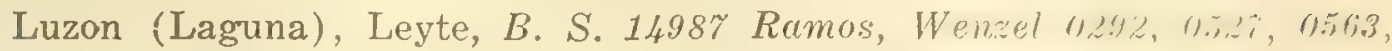
0872. Epiphyte at 500 to $700 \mathrm{~m}$ altitude in Leyte.

DENDROBIUM UNIFLORUM Griff. Notul. 3 (1851) 305, Icon. Pl. Asiat. 3 (1851) t. 303; Ridl. in Trans. Linn. Soc. II 3 (1893) 363, in Journ. Linn. Soc. 31 (1896) 271, 32 (1896) 256, Mat. F1. Mal. Penin. 1 (1907) 46; Ames Orch. 1 (1908) 91; Kränzl. in Engl. Pflanzenreich IV. 50. II. B. 21 (1910) 185, quoad bibliog. et spec. (excl. descr.); Ames in Merr. in Journ. Roy. Asiat. Soc. Straits Branch, Special No. (1921) 166.

Dendrobium revolutum Hook. f. Fl. Brit. Ind. 5 (1890) 732, quoad syn. D. uniflorum Griff. et spec. Malacea; Grant Orch. Burma (1895) 118, quoad syn. D. uniflorum Griff. et spec. Malacca; Ames Orch. 2 (1908) 184, quoad syn. D. uniflorum Griff. et spec., 5 (1915) 138; Kränzl. in Engl. Pflanzenreich IV. 50. II. B. 21 (1910) 185, quoad descr. (excl. bibliog. et spec.), non Lindl.

Luzon (Ilocos Norte, Bataan, Laguna, Sorsogon), Sibuyan, Negros (Occidental Negros), Leyte, Dinagat, Mindanao (Lanao, Agusan, Surigao). Fairly common and of wide distribution in the Philippines. Epiphyte at 60 to $700 \mathrm{~m}$ altitude. In Malay Peninsula and Borneo (Sarawak).

Further study of the Philippine material has proved that the species concerned is $D$. uniflomu Griff. and not its near ally $D$. revoiutum Lindl. with which it has been much confused.

DENDROBIUM USTERI Schltr. in Usteri Viert. Naturf. Gesell. Zurich 56 (1906) 450, in Bull. Herb. Boiss. II 6 (1906) 458; Kränzl. in Engl. Pflanzenreich IV. 50. II. B. 21 (1910) 300; Ames Orch. 5 (1915) 141.

Negros, Guimaras, Usteri s. n., Lyon $13 \%$.

DENDROBIUM USTERIOIDES Ames Orch. 5 (1915) 142.

Luzon, Mindoro, Lyon 22, F. B. 3693 Merritt 75.

DENDROBIUM VENTRICOSUM Kränzl. in Engl. Pflanzenreich IV. 50. 11. B. 21 (1910) 209; Ames in Philip. Journ. Sci. 7 (1912) Bot. 19, Orch. 5 (1915) 144.

Luzon (Zambales, Sorsogon), Mindanao (Agusan, Surigao, Davao), Loher s.n., 6004, Elmer 14441, 14rr9, 16423, Weber 119, 1\%6, 1\%月, WTenzel 01156, Black s. $n$. Epiphyte at low altitudes in Mindanao.

DENDROBIUM VERRUCUlosum Ames in Philip. Journ. Sci. 8 (1913)

Bot. 426, Orch. 5 (1915) 144.

Luzon (Ilocos Norte), Leyte, Lyon s. n., 143, B. S. 33236 Ramos, Wenzel 0140. Epiphyte. 
DENDROBIUM VICTORIAE REGINAE Loher in Gard. Chron. III 21 (1897) 399, 22 (1897) 121, fig. 34; Cogn. Dict. Icon. Orch. (1899) Dendrob. t. 21; Ames Orch. 1 (1905) 92, 5 (1915) 144; Kränzl. in Engl. Pflanzenreich IV. 50 II. B. 21 (1910) 109, fig. 6 A, B; Rolfe in Orch. Rev. 20 (1912) 17, fig. 6.

Dendrobium coeleste Loher in Gard. Chron. III 22 (1897) 1; Ames Or̀ch. 5 (1915) 120.

Dendrobium victoriae reginae Loher var. exile Ames in Philip. Journ.

Sci. 2 (1907) Bot. 328.

Luzon (Benguet), Mindoro, Negros, Mindanao (Camiguin Island), Elmer 6272, B. S. 4326 Mearns, 31797 Santos, 14429 Ramos, Lyon 122, Boettcher s. n., Merrill 5503, 7011, Loher 483. Epiphyte at 1,300 to 2,650 m altitude.

DENDROBIUM WENZELII Ames Orch. 5 (1915) 144.

Leyte, Mindanao (Lanao, Surigao), Wenzel 039, 0139, M. S. Clemens 43. Hutchinson 7551. Epiphyte at 30 to $60 \mathrm{~m}$ altitude.

DENDROBIUM ZAMBOANGENSE Ames Orch. 5 (1915) 145.

Mindanao (Zamboanga, Agusan), Merrill 8174, Elmer 1369\%. Epiphyte at about $1,100 \mathrm{~m}$ altitude.

\section{EXCLUDED OR SYNONYMOUS SPECIES}

Dendrobium aciculare Lindl. apud Vidal Phan. Cuming. Philip. (1885) 148; Vidal Rev. Pl. Vasc. Filip. (1886) 267; Hook. f. Fl. Brit. Ind. 5 (1890) 725, quoad Philip.; Kränzl. in Engl. Pflanzenreich IV. 50. II. B. 21 (1910) 241, quoad Philip.; Ames Orch. 5 (1915) $113=$ Dendrobium phitippinense Ames.

Dendrobium acuminatum Lyon in Gard. Chron. III 42 (1907) 210, fig. 88; Rolfe in Orch. Rev. 15 (1907) 312, as to plant spoken of; Kränzl. in Orchis 2 (1908) 73, t. 16; Edit. in Gard. Chron. III 46 (1909) 150, fig. 64, in Gardners' Mag. 52 (1909) 659, 660, text cut, non Rolfe ex Ames = Dendrobium lyonii Ames.

DENDROBIUM ADUNCUM Wall. ex Lindl. apud Boxall ex Naves Novis. App. (1882) 234; Ceron Cat. Pl. Herb. Manila (1892) 166; Ames Orch. 5 (1915) 114, in nota.

This is a Himalayan species, and there is no sufficient reason for considering that it grows in the Philippines; cf. Ames 1. c. for a critical note. It is not improbable that the plant reported as growing there may in reality be the closely related species Dendrobium hercoglossum Reichb. $f$.

Dendrobium Alagense Ames in Philip. Journ. Sci. 2 (1907) Bot. $328=$ Dendrobium luzonense Lindl.

Dendrobium ALbum Boxall ex Naves Novis. App. (1882) 234, nomen; Ceron Cat. Pl. Herb. Manila (1892) 166, nomen.

Boxall probably intended Dendrobium album Wight, which is a synonym of D. aqueum Lindl., an Indian species.

Dendrobium aloifolium Reichb. f. apud Kränzl. in Engl. Pflanzenreich IV. 50. 11. B. 21 (1910) 206, quoad syn. D. merrillii; Ames Orch. 5 (1915) $115(D$. aloefolium $)=$ Dendrobium merrillii Ames. 
Dendrobium Angustifolium Ames in Philip. Journ. Sci. 7 (1912) Bot. 14, non Lindl. = Dendrobium chrysographatum Ames.

Dendrobium antennatum Lindl. apud Naves Novis. App. (1882) 233.

DeNDROBIUM ATROPURPUREUM Ames Orch. 1 (1905) 86, 2 (1908) 172; Kränzl. in Engl. Pflanzenreich IV. 50. II. B. 21 (1910) 217, quoad Philip., non Miq. = Dendrobium cultratum Schltr.

DendRobium AUREOROSEUM Reichb. f. apud Kränzl. in Engl. Pflanzenreich IV. 50. II. B. 21 (1910) 125, p. p.; Ames Orch. 5 (1915) 115.

This is a Javan species to which Kränzlin has referred a Philippine specimen collected by Cuming. J. J. Smith considers it to be a synonym of Dendrobium nudum Lindl.

Dendrobium AUREUm Lindl. Gen. \& Sp. Orch. (1830) 77; Ames Orch. 5 (1915) $115=$ Dendrobium heterocarpum Lindl.

Dendrobium BIFARIUM Lindl, apud Naves Novis. App. (1882) 233.

DENDROBIUM BINNENDIJKII Reichb. f.' apud Naves Novis. App. (1882) 232.

DENDROBIUM BLUMET Lindl. in Journ. Linn. Soc. 3 (1859) 14; Miq. Fl. Ind. Bat. 3 (1859) 638; Naves Novis. App. (1882) 232; Kränzl. in Engl. Pflanzenreich IV. 50. II. B. 21 (1910) 234, quoad syn. D. planibulbe Lindl. et quoad Philip. = Dendrobium planibulbe Lindl.

DENDROBIUM BRONGNIARTII Kränzl. in Engl. Pflanzenreich IV. 50. II. B. 21 (1910) 210; Ames in Philip. Journ. Sci. 7 (1912) Bot. 15, Orch. 5 (1915) 116 = Dendrobium aporoides (Lindl.) Merr.

DeNDRobium BURSigerum Lindl, in Journ. Linn. Soc. 3 (1859) 17; Miq. Fl. Ind. Bat. 3 (1859) 642; Naves Novis. App. (1882) 233; Vidal Phan. Cuming. Philip. (1885) 148, Rev. Pl. Vasc. Filip. (1886) 267; Ames Orch. 2 (1908) 173, 5 (1915) 117; Kränzl. in Engl. Pflanzenreich IV. 50, I1. B. 21 (1910) 119, fig. $10 \mathrm{G}-\mathrm{O}=$ Dendrobium secundum Wall.

DENDROBIUM CAICEOLUM Roxb. apud Naves Novis. App. (1882) 231.

DENDROBIUM CALOPHYLLUM Reichb. f. apud Naves Novis. App. (1882) 232. DendRobIUm CALOPOgon Reichb. f. apud Naves Novis. App. (1882) 232.

Dendrobium Canaliculatum R. Br, apud Naves Novis. App. (1882) 232. Dendrobium CANinum Merr, in Philip. Journ. Sci. 19 (1921) 344, non Epidendrum caninum Burm. $\mathrm{f}$. $=$ Dendrobium crumenatum Sw.

DENDRoBIUM CAPILliPES Reichb. f. apud Naves Novis. App. (1882) 233.

Dendrobium CARnosum Teijsm. \& Binn. apud Naves Novis. App. (1882) 234.

Dendrobium cerinum Reichb. f. in Gard. Chron. II 12 (1879) 554; Ridl. in Journ. Linn. Soc. 32 (1896) 263, in textu sub D. sanguinolentum Lindl.; Kränzl. in Engl. Pfianzenreich IV. 50, II. B. 21 (1910) 121; Ames Orch. 5 (1915) 119.

Reichenbach merely states that the type material came from the Malay Archipelago; Ridley, under $D$. sanguinolentum Lindl., doubts its being a 
distinct species and states that Burbidge's specimen came from the Sulu Archipelago. No material comparable with this species has been seen from the Philippines; for discussion cf. Ames 1. c.

DENDRobIUM cobbianum Reichb. f. in Gard. Chron. II 14 (1881) 748; Kränzl. in Engl. Pflanzenreich IV. 50. II. B. 21 (1910) 297.

The origin of this plant is unknown; it was sent to Reichenbach without localization by the firm of Hugh Low \& Co. Why it is included among the Philippine species is unknown, as there seems no ground upon which to assume that it came from the Philippines.

DENDROBIUM COELESTE Loher in Gard. Chron. III 22 (1897) 1; Ames Orch. 5 (1915) $120=$ Dendrobium victoriae reginae Loher.

Dendrobium CONCINNUM Miq. apud Naves Novis. App. (1882) 234.

Dendrobium connatum Lindl. apud Naves Novis. App. (1882) 231.

DENDROBIUM CONOSTALIX Reichb. f. in Walp. Ann. 6 (1861) 292; Usteri Beitr. Ken. Philip. Veg. (1905) 128; Kränzl. in Engl. Pflanzenreich IV. 50. II. B. 21 (1910) 162; Ames Orch. 5 (1915) 120.

This is a Malayan species which has been reported from the Philippines. No material from the Philippines matches it and the reports seem erroneous. Cf. Ames 1. c. for discussion.

Dendrobium Convexum Lindl. apud Naves Novis. App. (1882) 231.

Dendrobium CRePidatum Lindl. apud Naves Novis. App. (1882) 232.

Dendrobium cumulatum Lindl. apud Kränzl. in Engl. Pflanzenreich IV.

50. II. B. 21 (1910) 115, quoad Philip.; Ames Orch. 5 (1915) 121.

The Philippine specimens referred here by Kränzlin represent Dendrobium orumenatum Sw. Cf. Ames 1. c.

DeNdRobium Cymbidioides Blume apud Naves Novis. App. (1882) $231=$ ? Dendrobium acuminatum Rolfe ex Ames.

Dendrobium dayanum Boxall ex Naves Novis. App. (1882) 234, nomen; Ceron Cat. Pl. Herb. Manila (1892) 166, nomen.

DeNDrobIUM DRACoNis Reichb. f. apud Naves Novis. App. (1882) 233.

Dendrobium Equitans Kränzl. in Engl. Pflanzenreich IV. 50. 11. B. 21 (1910) 228, quoad Philip. = Dendrobium aporoides (Lindl.) Merr.

This species is known only from Formosa.

Dendrobium erosum Lindl. apud Naves Novis. App. (1882) 233.

DeNDRoBium ERYTHRoXANTHUM Reichb. f. in Gard. Chron. II 2 (1874) 162; Boxall ex Naves Novis. App. (1882) $234=$ Dendrobium bullenianum Reichb. $f$.

Dendrobium euphuebium Reichb. f. ex Lindl, in Journ. Linn. Soc. 3 (1859)

7; Naves Novis. App. (1882) $232=$ Dendrobium spurium (Blume)

J. J. Sm.

Dendrobium Fimbriatum Hook. apud Naves Novis. App. (1882) 232.

Dendrobium Flabealum Reichb. f. apud Naves Novis. App. (1882) 232. 
Dendrobium Gemellum Lindl. apud Naves Novis. App. (1882) 233.

DendRobium GoLdSCHMIDTIANuM Kränzl. in Fedde Repert. 7 (1909) 40, in Engl. Pflanzenreich IV. 50. II. B. 21 (1910) 116.

"Provinz der Philippinen und Formosa."-Kränzlin. The specimer: probably originated in Formosa, not in the Philippines.

DENDROBIUM GouldI Reichb. f. apud Naves Novis. App. (1882) 234.

DendrobIum GRACILE Kränzl. in Engl. Pflanzenreich IV. 50. 11. B. 21 (1910)

230, quoad Philip; Ames Orch. 5 (1915) 125.

Kränzlin, in his monograph of the genus, refers Loher 4\%; to this species. What the Loher plant is is open to question, but no material referable to $D$. gracile has been seen in the collections received.

Dendrobium Grandiflorum Lindl. apud Naves Novis. App. (1882) 232.

Dendrobium HAENkEANum Steud. Nomencl. Bot. ed. 2, 1 (1840) $490=$ Geodorum nutans (Presl) Ames.

Dendrobium HasseltiI Blume apud Naves Novis. App. (1882) 232.

Dendrobium HeteroIdeum Blume apud Naves Novis. App. (1882) 231.

DENDROBIUM HYMENOPHYLLUM Lindl. apud Naves Novis. App. (1882) 233.

Dendrobium INDIVIsum Naves Novis. App. (1882) 231; Vidal Phan.

Cuming. Philip. (1885) 148, Rev. Pl. Vasc. Filip. (1886) 267;

Kränzl. in Engl. Pflanzenreich IV. 50. II. B. 21 (1910) 217, quoad

Philip.; Ames in Philip. Journ. Sci. 6 (1911) Bot. 52, non Miq. =

Dendrobium distichum (Presl) Reichb. f.

Dendrobium INTERMEdium Teijsm. \& Binn. apud Naves Novis. App. (1882) 232.

Dendrobium JAyanicum Lindl. apud Naves Novis. App. (1882) 234.

Dendrobium Johannis Reichb. f. apud Naves Novis. App. (1882) 233.

DFNDROBIUM KUHLII Lindl. apud Naves Novis. App. (1882) 233.

Dendrobium Labuanum Lindl. apud Naves Novis. App. (1882) 231.

Dendrobium Lamellatum Lindl. apud Kränzl. in Engl. Pflanzenreich IV.

50. II. B. 21 (1910) 269, quoad Philip.; Ames Orch. 5 (1915) ix.

Cuming 2074, referred here by Kränzlin, is probably Dendrobium platycaulon Rolfe; cf. Ames 1. c.

DENDROBIUM LILACINUM Reichb. f. apud Naves Novis. App. (1882) 233. DENDROBIUM LOBEII Lindl. apud Naves Novis. App. (1882) 231.

DendRobium LONGICOLle Lindl. in Bot. Reg. 26 (1840) Misc. 74; Ames Orch. 5 (1915) 127.

Diplocaulobium longicolle Kränzl. in Engl. Pflanzenreich IV. 50. 11. B. 21 (1910) 340.

The type was a specimen sent to Loddiges by Cuming from Singapore. where the species still occurs. There is no reason to consider it as Philippine. 
Dendrobium ludens Reichb. f. apud Naves Novis. App. (1882) 233.

Dendrobium Maccarthiae Thw. apud Naves Novis. App. (1882) 233.

DENDROBIUM MACRAEI Ames Orch. 1 (1905) 89, 2 (1908) 176, non Lindl. = Dendrobium plicatile Lindl.

DENDROBIUM MACRANTHUM Hook. in Bot. Mag. 69 (1842) $t$. 3970; Naves Novis. App. (1882) 233; Ceron Cat. Pl. Herb. Manila (1892) 166, non A. Rich. = Dendrobium anosmum Lindl.

DENDROBIUM MACROPHYLLUM Ames Orch. 2 (1908) 179, non A. Rich.; Kränzl. in Engl. Pflanzenreich IV. 50. II. B. 21 (1910) 244, quoad Philip. = Dendrobium ternatense J. J. Sm.

DendRoBIUM MACROPHYLLUM Lindl. in Bot. Reg. 25 (1839) Misc. 36, 28 (1842) Misc. 84, 30 (1844) Misc. 47, Sert. Orch. (1840) t. 35; Paxt. Mag. Bot. 8 (1841) 97, t.; Hook. Cent. Orch. Pl. (1851) t. 12; Planch. Hort. Donat. (1855) 156, Atlas t. 1; Reichb. f. in Linden Pescat. 1 (1860) 40, t. 40, non A. Rich. = Dendrobium anosmum Lindl.

Dendrobium Manillense Schauer in Nov. Act. Nat. Cur. 19, Suppl. 1 (1843) $430=$ Appendicula cornuta Blume.

DENDROBIUM METACHILINUM Reichb. f. in Bonpl. 3 (1855) 222, in Walp. Ann. 6 (1861) 290; Naves Novis. App. (1882) 234; Vidal Phan. Cuming. Philip. (1885) 148, Rev. Pl. Vasc. Filip. (1886) 267; Ames Orch. 5 (1915) 128.

The type of this species is Cuming $205 \%$, which was unquestionably from the Malay Peninsula, not from the Philippines. The species is known only from the Malay Peninsula. Cf. Ames 1. c.

Dendrobium minax Reichb, f. apud Naves Novis. App. (1882) 233.

DENDROBIUM MONILIFORMF Sw. apud Llanos ex F.-Vill. \& Naves in Blanco Fl. Filip. ed. 3, $4^{1}$ (1880) 100; Naves Novis. App. (1882) 233.

DENDROBIUM MONOPHyllum F. . Muell. apud Naves Novis. App. (1882) 232.

Dendrobium Nudum Lindl. apud Naves Novis. App. (1882) 232.

Dendrobium nutans Presl Rel. Haenk. 1 (1827) 102; Presl Symb. Bot. 1 (1832) 34, t. 23; Naves Novis. App. (1882) $234=$ Geodorum nutans (Presl) Ames.

DeNDROBIUM PARCIFLoRUM Kränzl. in Engl. Pflanzenreich IV. 50. 11. B. 21 (1910) 222, quoad Philip.; Ames in Philip. Journ. Sci. 7 (1912) Bot. 18, non Reichb. $f_{0}=$ Dendrobium marivelense Ames.

Dendrobium Parcum Reichb. f. apud Naves Novis. App. (1882) 233.

Dendrobium Paucilaciniatum J. J. Sm. apud Prain in Ind. Kew. Suppl. 3 (1908) 58.

Index Kewensis cites "Ins. Philip." as the locality; this is an error as the type was from Ternate.

Dendrobium PIERARDil Roxb. apud Naves Novis. App. (1882) 232. 
Dendrobium PSEUdACLinta Lindl. in Journ. Linn. Soc. 3 (1859) 9; Ames Orch. 5 (1915) $134=$ Dendrobium aclinia Reichb. $f$.

Dendrobium PUgioniforme A. Cunn. apud Naves Novis. App. (1882) 232. Dendrobium Pumilum Reichb. f. apud Vidal Phan. Cuming. Philip. (1885) 148.

Probably Dendrobium mumilum Roxb. was intended, as Reichenbach apparently described no such species. Vidal referred here Cuming 2102 as Philippine; the specimen in all probability came from the Malay Peninsula where the species is seemingly not uncommon.

Dendrobium Purpureum Blume apud Naves Novis. App. (1880) 233.

Dendrobium PUsillum Lindl. apud Naves Novis. App. (1882) 231.

Dendrobium Radians Reichb. f. apud Naves Novis. App. (1882) 233.

Dendrobium Ramosum Lindl. apud Kränzl. in Engl. Pflanzenreich IV. 50. II. B. 21 (1910) 53, quoad Philip.

This is an Indian species, Kränzlin giving its other range as "Philippinen (?)." There is no reason for considering it as a Philippine species.

Dendrobium Retusum Llanos in Mem. Real. Acad. Cienc. Madr. 4 (1857) 498; F.-Vill. \& Naves in Blanco Fl. Filip. ed. 3, $4^{1}$ (1880) $100=$ ? Dendrobium anosmum Lindl.

DENDROBIUM REVolutum Hook. f. Fl. Brit. Ind. 5 (1890) 732, quoad syn. $D$. uniflorum Griff. et spec. Malacca; Grant Orch. Burma (1895) 118, quoad syn. D. uniflorum Griff. et spec. Malacca; Ames Orch. 2 (1908) 184, quoad syn. D. uniflomm Griff. et spec., 5 (1915) 138; Kränzl. in Engl. Pflanzenreich IV. 50. II. B. 21 (1910) 185, quoad descr. (excl. bibliog. et spec.), non Lindl. = Dendrobium uniflom Griff.

DENDROBIUM RHOMBEUM Lindl. in Bot. Reg. 29 (1843) $t$. 17, 30 (1844) Misc. 56; Reichb. f. in Bot. Zeit. 1 (1843) 333; Lindl. in Paxt. Flow. Gard. 3 (1852-53) 52; Naves Novis. App. (1882) $233=$ Dendrobium heterocarpum Wall.

Dendrobium robinsonil Ames in Philip. Journ. Sci. 8 (1913) Bot. 425, Orch. 5 (1915) 138 = Dendrobium carinatum (Linn.) Willd.

DENDROBIUM RUCKERI Lindl, in Bot. Reg. 29 (1843) Misc. 25, t. 60, 30 (1844) Misc. 52; Reichb. f. in Walp. Ann. 6 (1861) 288; Naves Novis. App. (1882) 234; Ames Orch. 5 (1915) 138.

When first described this was thought to be of Philippine origin. $R$. A. Rolfe, in Orch. Rev. 4 (1896) 87, has shown it to be an Indian species. Cf. Ames 1. c.

DENDROBIUM SCABRILINGUe Lindl. apud Naves Novis. App. (1882) 232.

Dendrobium sculptum Reichb. f. apud Naves Novis. App. (1882) 233.

Dendrobium SERra Lindl. apud Naves Novis. App. (1882) 231.

Dendrobium sinuatum Schltr. in Perk. Frag. Fl. Philip. (1904) 43;

Kränzl. in Engl. Pflanzenreich IV. 50. II. B. 21 (1910) 216, quoad Philip., non Lindl. = Dendrobium cultratum Schltr. 
Dendrobitim striatum Hort. ex Gard. Chron. III 38 (1905) 382; Kränzl. in Engl. Pflanzenreich IV. 50. II. B. 21 (1910) $298=$ Dendrobium o’brienianum Kränzl.

Dendrobium striolatum Reichb. f. apud Naves Novis. App. (1382) 232.

Dendrobium subteres Lindl. apud Naves Novis. App. (1882) 231.

Dendrobium subulatum Blume apud Naves Novis. App. (1882) 232.

Dendrobium suPerbum Reichb. f. in Walp. Ann. 6 (1861) 282 ; Vidal Phan. Cuming. Philip. (1885) 149, Rev. Pl. Vasc. Filip. 11886) 267; Ames Orch. 1 (1905) 90, 2 (1908) 186, 5 (1915) $139=$ Dendrobium anosmum Lindl.

Dendrobium tenellum Blume apud Naves Novis. App. (1882) 2331.

DENDROBIUM TERES Blanco Fl. Filip. (1837) 638, non Roxb. = Luisia teretifolia Gaudich.

DENDRoBIUM TERETIFolium Blanco Fl. Filip. ed. 2 (1845) $4 \frac{1}{4}$, ed. 3,3 (1879) 34, non R. Br. = Luisia teretifolia Gaudich.

DENDROBIUM TERETIFOLIUM R. Br. apud Naves Novis. App. (1882) 232.

Dendrobium tetraedre Blume apud Naves Novis. App. (1882) 232.

Dendrobium tetragonum A. Cunn. apud Naves Novis. App. (1882) 232.

Dendrobium tMesipteris Lindl. apud Naves Novis. App. (1882) 232.

Dendrobium tuadenum Boxall ex Naves Novis. App. (1882) 234 , nomen.

Dendrobium unguiculatum Teijsm. \& Binn. in Tijdschr. Ned. Ind. 5 (1853) 941; Naves Novis. App. (1882) 231 = Dendrobium spurium (Blume) J. J. Sm.

Dendrobium vanoverberghil Ames in Philip. Journ. Sci. 8 (1913) Bot. 425, t. $13=$ Dendrobium papilio Loher.

Dendrobium veitchianum Lindl. apud Naves Novis. App. (1882) 231.

Dendrobium Veratrifolium Naves Novis. App. (1882) 233, non Lindl. = ? Dendrobium taurinum Lindl.

Dendrobium Xantholeucum Reichb. f. apud Naves Novis. App. (1882) 231. 65. ERIA Lindley

ERIA ALBOLUTEA Rolfe in Kew Bull. (1917) 80.

Described from cultivated specimens originating in the Philippines.

ERIA ANCEPS Leavitt in Philip. Journ. Sci. 4 (1909) Bot. 219, 237, fig. 18; Kränzl. in Engl. Pflanzenreich IV. 50. 11. B. $21^{2}$ (1911) 129; Ames Orch. 5 (1915) 146.

Negros (Occidental Negros), Palawan, Whitford 1601, F. B. 7275 Everett, B. S. 636 Foxworthy.

ERIA BARBIFRONS Kränzl. in Engl. Pflanzenreich IV. 50, II. B. 21" (1911) 52.

"Provinz der Philippinen und Süd-Formosa: Philippinen, vermuthlich Manila, von A. Loher gesandt." Known only from the description. 
ERIA BINABAYENSIS Ames in Philip. Journ. Sci. 2 (1907) Bot. 329; Leavitt in Philip. Journ. Sci. 4 (1909) Bot. 223, 242; Ames Orch. 5 (1915) 146.

Trichotosia binabayensis Kränzl. in Engl. Pflanzenreich IV. 50. 11. B. $21^{2}$ (1911) 141.

Luzon (Laguna), Mindoro, B. S. 16554 Ramos, Merrill 5661. Epiphyte in humid forest at $215 \mathrm{~m}$ altitude in Mindoro.

ERIA BONTOCEnsIS Ames in Philip. Journ. Sci. 8 (1913) Bot. 427, Orch. 5 (1915) 146.

Luzon (Bontoc), Vanoverbergh 1386.

ERIA BRACHYSTACHYA Reichb. f. in Bonpl. 3 (1855) 223; Lindl. in Journ. Linn. Soc. 3 (1859) 59; Miq. Fl. Ind. Bat. 3 (1859) 657; Reichb. f. in Walp. Ann. 6 (1861) 276; Naves Novis. App. (1882) 236; Vidal Phan. Cuming. Philip. (1885) 149, Rev. Pl. Vasc. Filip. (1886) 268; Leavitt in Philip. Journ. Sci. 4 (1909) Bot. 221, 241; Ames Orch. 2 (1908) 188, 5 (1915) 146; Kränzl. in Engl. Pflanzenreich IV. 50. II. B. $21^{2}$ (1911) 100.

Pinalia brachystachya Ktze. Rev. Gen. Pl. 2 (1891) 678.

Eria wenzelii Ames in Philip. Journ. Sci. 8 (1913) Bot. 428.

Babuyan Islands (Camiguin Island), Luzon (Kalinga, Rizal, Camarines, Sorsogon), Leyte, Mindanao (Agusan). A fairly common species of wide distribution. Epiphyte at sea level to $1,000 \mathrm{~m}$ altitude:

ERIA BRACTESCENS Lindl. in Bot. Reg. 27 (1841) Misc. 18, 30 (1844) t. 29; Paxt. Mag. Bot. 8 (1841) 118, 11 (1844) 116; Edit. in Gard. Chron. (1841) 231; Reichb. f. in Walp. Ann. 6 (1861) 277; Hemsl, in Gard. Chron. II 18 (1882) 468; Hook. f. Fl. Brit. Ind. 5 (1890) 796; Ridl. in Trans. Linn. Soc. II 3 (1893) 366; Grant Orch. Burma (1895) 135; Ridl. in Journ. Linn. Soc. 32 (1896) 293; King \& Pantl. in Ann. Roy. Bot. Gard. Calc. 8 (Orch. Sik.-Himal.) (1898) 120, t. 166; Williams in Bull. Herb. Boiss. II 4 (1904) 368; J. J. Sm. Orch. Amb. (1905) 74; Ridl. Mat. Fl. Mal. Penin. 1 (1907) 101; Ames Orch. 2 (1908) 189, 5 (1915) 147; Leavitt in Philip. Journ. Sci. 4 (1909) Bot. 209, 231, fig. 11; Kränzl. in Engl. Pflanzenreich IV. 50. II. B. $21^{\circ}$ (1911) 88; Ames in Merr. in Journ. Roy. Asiat. Soc. Straits Branch, Special No. (1921) 168.

Eria dillwynii Hook. in Bot. Mag. 71 (1845) t. 4163; Lindl. in Bot. Reg. 31 (1845) Misc. 63; F. Kl. in Bot. Zeit. 3 (1845) 550; Paxt. Mag. Bot. 12 (1846) 140; Lindl. in Journ. Linn. Soc. 3 (1859) 55; Naves Novis. App. (1882) 236; Kränzl. in Engl. Pflanzenreich IV. 50. II, B. $21^{2}$ (1911) 87.

Eria litoralis Teijsm. \& Binn. in Nat. Tijdschr. Ned. Ind. 24 (1862) 312.

Pinalia bractescens Ktze. Rev. Gen. Pl. 2 (1891) 679.

Luzon (Bataan, Rizal), Basilan, Samar, Mindanao (Zamboanga), Lyon 123, 150, F. B. 4017, 6562 Hutchinson, Whitford 1082, B. S. 3000, 6562, 17641 Ramos. Epiphyte, apparently at low altitudes. 


\section{ERIA CANDOONENSIS Ames Orch. 7 (1922) 101.}

Mindanao (Bukidnon), B. S. 38941 Ramos \& Edaño. Mt. Candoon, epiphyte at $1,650 \mathrm{~m}$ altitude.

\section{ERIA CARNICOLOR Ames Orch. 7 (1922) 152.}

Luzon (Bontoc), B. S. 37937 Ramos \& Edaño. Mt. Masapilid, epiphyte in mossy forest at $1,600 \mathrm{~m}$ altitude.

ERIA CLEMENSIAE Leavitt in Philip. Journ. Sci. 4 (1909) Bot. 220, 238, fig. 20; Kränzl. in Engl. Pflanzenreich IV. 50. II. B. $21^{2}$ (1911) 126; Ames Orch. 5 (1915) 147.

Luzon (Rizal), Panay (Antique), Leyte, Mindanao (Lanao, Bukidnon), Reillo s. n., B. S. 3219: McGregor, 15390 Ramos, 38891 Ramos \& Edaño, Wenzel 0393, 0495, M. S. Clemens 602. Epiphyte in forest at 500 to 1,000 $\mathrm{m}$ altitude.

ERIA COChleAtA Lindl. in Bot. Reg. 30 (1844) Misc. 14; Reichb. f. in Walp. Ann. 6 (1861) 269; Naves Novis. App. (1882) 236.

Imported from the Philippines by Loddiges. Known only from the original description in which Lindley states that it has the appearance of $E$. stellata which is a synonym of $E$. javanica (Sw.) Blume. Kränzlin does not treat $E$. cochleata in his monograph of the genus in Das Pflanzenreich.

ERIA COMPACTA Ames in Philip. Journ. Sci. 2 (1907) Bot. 329; Leavitt in Philip. Journ. Sci. 4 (1909) Bot. 208, 231; Kränzl. in Engl. Pflanzenreich IV. 50. II. B. $21^{2}$ (1911) 77, fig. 15 A-D; Ames Orch. 5 (1915) 147.

Mindoro, Merritt 4420. Mt. Halcon, at 1,415 m altitude.

ERIA COPELANDII Leavitt in Philip. Journ. Sci. 4 (1909) Bot. 203, 226, fig. 3; Ames Orch. 5 (1915) 147; Schltr. in Fedde Repert. Beihefte 4 (Orch. Sino-Jap. Prodr.) (1919) 217, in textu.

Eria formosana Kränzl. in Engl. Pflanzenreich IV. 50. II. B. 21" (1911) 47, non Rolfe.

Mindanao (Misamis, Bukidnon, Davao), F. B. 4596, 4600 Mearns \& Hutchinson, B. S. 38524 Ramos \& Edaño, Copeland 1423. Epiphyte at 1,560 to $3,060 \mathrm{~m}$ altitude.

Var. FUSIFORMIS Leavitt in Philip. Journ. Sci. 4 (1909) Bot. 204, 227.

Eria formosana Kränzl. var. fusiformis Kränzl. in Engl. Pflanzenreich IV. 50. II. B. $21^{2}$ (1911) 47.

Luzon (Lepanto, Benguet), Merrill 4590, Ramos \& Edaño 85, 106, 111, F. B. 5067 Curran. Epiphyte at 2,100 to $2,530 \mathrm{~m}$ altitude.

ERIA CURRANII Leavitt in Philip. Journ. Sci. 4 (1909) Bot. 210, 233, fig. 18; Kränzl. in Engl. Pflanzenreich IV. 50. II. B. 21: (1911) 79; Ames Orch. 5 (1915) 147.

Palawan, F. B. 3904 Curran.

ERIA CYLINDROSTACHYA Ames Orch. 2 (1908) 190, text cut, 5 (1915) 147; Leavitt in Philip. Journ. Sci. 4 (1909) Bot. 211, 234, fig. 15; Kränzl. in Engl. Pflanzenreich IV. 50. II. B. $21^{2}$ (1911) 69, fig. $51 L, M$. 
Luzon (Benguet, Rizal), Mindanao (Misamis, Bukidnon, Lavao), Joctrcher s. n., B. S. 29871, 38648, 390\%9 Ramos \& Edaño, H. L. 46132 M crom.; \& Hutchinson, Copeland 1424, Elmer 10632, 106324. Epiphyte at 100) to $1,560 \mathrm{~m}$ altitude.

ERIA CYMBIFORMIS J. J. Sm. in Rec. Trav. Bot. Néerl. 1 (1904) 152, text cut; Ames in Philip. Journ. Sci. 2 (1907) Bot. 330; Leavitt in Philip. Journ. Sci. 4 (1909) Bot. 224, 243, fig. 26; Kränzl. in Engl. Pflanzenreich IV. 50. II. B. $21^{2}$ (1911) 104; Schltr. in Engl. Bot. Jahrb. 45, Beibl. 104 (1911) 40; Ames Orch. 5 (1915) 147.

Luzon (Cagayan, Bataan, Rizal, Laguna, Tayabas, Mindoro, Negros (Oriental Negros), Leyte, Mindanao (Davao). A fairly common and widely distributed species. Epiphyte at 500 to $1,015 \mathrm{~m}$ altitude, in Sumatra.

ERIA DAGAMENSIS Ames Orch. 5 (1915) 147.

Luzon (Ifugao), Leyte, Sandkuhl 296, Wenzel 0123, 0305, 0308, 11314, $0315,0317,0318,0320,0321,0322,0323,0345,0346, B . S .42087$ Edaño, Epiphyte in forest at 500 to $600 \mathrm{~m}$ altitude in Leyte.

ERIA DAVAENSIS Ames in Elm. Leafl. Philip. Bot. 5 (1912) 1575, Orch. 5 (1915) 149.

Mindanao (Davao), Elmer 11821. Todaya, Mt. Apo; epiphyte in humid forest at $1,300 \mathrm{~m}$ altitude.

ERIA FASTIGATIFOLIA Ames Orch. 2 (1908) 191, text cut, 5 (1915) 149; Leavitt in Philip. Journ. Sci. 4 (1909) Bot. 221, 240, fig. 22.

Trichotosia fastigiatifolia Kränzl. in Engl. Pflanzenreich IV. 50. H. B. $21^{2}$ (1911) 162.

Luzon (Bontoc, Ifugao, Benguet, Rizal, Laguna, Tayabas, Sorsogon), Negros (Oriental Negros), Leyte. A fairly common species, especially in Luzon. Epiphyte at 300 to $1,300 \mathrm{~m}$ altitude.

ERIA FLORIBUNDA Lindl. in Wall. Cat. (1832) No. 7408, nomen, in Bot. Reg. 29 (1843) Misc. 43, 30 (1844) t. 20; Paxt. Mag. Bot. 11 (1844) 91; Lindl. in Journ. Hort. Soc. 3 (1848) 15, in Journ. Linn. Soc. 3 (1859) 60; Miq. Fl. Ind. Bat. 3 (1859) 661; Reichb. f. in Walp. Ann. 6 (1861) 276;. Hemsl. in Gard. Chron. II 18 (1882) 468; Hook. f. Fl. Brit. Ind. 5 (1890) 792; Ridl. in Trans. Linn. Soc. II 3 (1893) 366; Grant Orch. Burma (1895) 140; Ridl. in Journ. Linn. Soc. 32 (1896) 290; Williams in Bull. Herb. Boiss. II 4 (1904) 368; J. J. Sm. FI. Buitenz. 6 (Orch. Jav.) (1905) 400, Fig.-Atlas (1911) fig. \$03; Ridl. Mat. Fl. Mal. Penin. 1 (1907) 96; Leavitt in Philip. Journ. Sci. 4 (1909) Bot. 209, 231, fig. 10 (excl. syn. E. densa Ridl.); Kränzl. in Engl. Pflanzenreich IV. 50. II. B. $21^{2}$ (1911) 120, fig. 25 A-D; Winkler in Engl. Bot. Jahrb. 48 (1912) 101; Ames Orch. 5 (1915) 149, 6 (1920) 124, in Merr. in Journ. Roy. Asiat. Soc. Straits Branch, Special No. (1921) 170.

Eria leucostachya Lindl, in Journ, Hort. Soc. 3 (1848) 15.

Eria floribunda Lindl. var. lencostachya Lindl. in Paxt. Flow. Gard.

3 (1853) 116, fig. 288.

Pinalia floribunda Ktze. Rev. Gen. Pl. 2 (1891) 678. 
Palawan, Mindanao (Cotabato, Agusan, Bukidnon), B. S. 550, 633 Foxworthy, 38911, 39014, 39107 Ramos \& Edaño, Merrill 9617, Weber 152, 228. Epiphyte at 15 to $1,260 \mathrm{~m}$ altitude. In Burma, Malay Peninsula, Sumatra, Java, and Borneo.

ERIA FUsCA Blume Mus. Bot. Lugd.-Bat. 2 (1856) 183; Miq. Fl. Ind. Bat. 3 (1859) 658; Rolfe in Journ. Bot. 23. (1885) 215; Vidal Phan. Cuming. Philip. (1885) 149, Rev. Pl. Nasc. Filip. (1886) 268; J. J. Sm. Fl. Buitenz. 6 (Orch. Jav.) (1905) 388; Leavitt in Philip. Journ. Sci. 4 (1909) Bot. 222, 241; Ames Orch. 5 (1915) 149, in Merr. in Journ. Roy. Asiat. Soc. Straits Branch, Special No. (1921) 170.

? Eria hirta Blume Mus. Bot. Lugd.-Bat. 2 (1856) 184; Miq. Fl. Ind. Bat. 3 (1859) 658, fide Kränzl.

Trichotosia ciliata Teijsm. \& Binn. in Nat. Tijdschr. Ned. Ind. 5 (1853) 492.

Trichotosia fusca Teijsm. \& Binn. in Nat! Tijdschr. Ned. Ind. 5 (1853) 492; Kränzl. in Engl. Pflanzenreich IV. 50. II. B. $21^{2}$ (1911) 145.

Eria ciliata Miq. Fl. Ind. Bat. 3 (1859) 659; Reichb. f. in Walp. Ann. 6 (1861) 273.

Pinalia ciliata Ktze. Rev. Gen. Pl. 2 (1891) 678.

Pinalia fusca Ktze. Rev. Gen. P1. 2 (1891) 678.

? Pinalia hirta Ktze. Rev. Gen. Pl. 2 (1891) 678.

? Trichotosia hirta Blume in Herb. Mus. Lugd.-Bat. ex Kränzl. in Engl. Pflanzenreich IV. 50. 11. B. $21^{2}$ (1911) 145, in syn.

Negros (Occidental Negros), Leyte, F. B. 5582 Everett, Copeland 188, Wenzel 045, 0143, B. S. 40147 Lopez. Epiphyte in forest at $60 \mathrm{~m}$ altitude in Leyte. In Java and Borneo and, perhaps, Celebes.

ERIA GIGANTEA Ames Orch. 2 (1908) 192, text cut, 5 (1915) 149; Leavitt in Philip. Journ. Sci. 4 (1909) Bot. 218, 239, fig. 21; Kränzl. in Engl. Pflanzenreich IV. 50. II. B. $21^{2}$ (1911) 130; Ames in Addisoniá 6 (1921) 41, t. 213.

Luzon (Isabela, Bataan, Rizal, Laguna), Mindanao. (Misamis, Lanao), Lyon 146, Topping 472, Merrill 4110, B. S. 3430 Ramos, 18995 Brown, F. B. 4606 Mearns \& Hutchinson, M. S. Clemens s. n., 43\%. Terrestrial (?) at 660 to $1,300 \mathrm{~m}$ altitude.

ERIA GRACILISCAPA Rolfe ex Ames Orch. 1 (1905) 93, 2 (1908) 193, 3 (1908) 88, t. 57, 5 (1915) 149; Leavitt in Philip. Journ. Sci. 4 (1909) Bot. 205, 228; Kränzl. in Engl. Pflanzenreich IV. 50. II. B. $21^{2}$ (1911) 108.

Luzon (Kalinga, Bataan, Tayabas), Boettcher s. n., Whitford 307, Topping $474, F . B .795$ Borden, Elmer 7405. Epiphyte at 600 to $1,000 \mathrm{~m}$ altitude.

ERIA HALCONENSIS Ames in Philip. Journ. Sci. 2 (1907) Bot. 330; Ames Orch. 3 (1908) 85, t. 56, 5 (1915) 149; Leavitt in Philip. Journ. Sci. 4 (1909) Bot. 224, 241.

Trichotosia halconensis Kränzl. in Engl. Pfianzenreich IV. 50. 11. B. $21^{2}$ (1911) 149 . 
Mindoro, Panay (Capiz), Merrill 5510, 5742, B.S.30716 Rumos \& Edaño. Terrestrial and epiphytic at 330 to $2,000 \mathrm{~m}$ altitude.

ERIA HIRSUTIPETALA Ames Orch. 7 (1922) 102.

Mindanao (Bukidnon), B. S. 39124 Ramos \& Edaño. Mt. Candoon, epiphyte in mossy forest at $1,830 \mathrm{~m}$ altitude.

ERIA HUTChINSONIANA Ames in Philip. Journ. Sci. 2 (1907) Bot. 330; Leavitt in Philip. Journ. Sci. 4 (1909) Bot. 204, 227, fig. 4; Kränzl. in Engl. Pflanzenreich IV. 50. II. B. $21^{2}$ (1911) 80, fig. 17 $A-D$; Ames Orch. 5 (1915) 150.

Mindoro, Merrill 5514. Mt. Halcon, terrestrial on mossy ridge at 2,300 m altitude.

ERIA JARENSIS Ames Orch. 5 (1915) 150.

Luzon (Nueva Vizcaya, Laguna), Leyte, Mindanao (Surigao), Wenzel 0356, 035\%, 0358, 0355, 0.3\%7, Lyon 130, B. S. 20436 Ramos, 20090 McGregor. Fiphyte in forest at 500 to $750 \mathrm{~m}$ altitude.

ERIA JAVANICA (Sw.) Blume Rumphia 2 (1836) 23; Ames Orch. 2 (1908) 193; J. J. Sm. in Nova Guinea 8 (1909) 86, $8^{3}$ (1912) 577, $12^{4}$ (1 16) 352; Kränzl. in Engl. Pflanzenreich IV. 50. II. B. $21^{2}$ (1911) 26; Schltr. in Fedde Repert. 10 (1911) 91; J. J. Sm. Orch. Jav. Fig.-Atlas (1911) fig. 287; Ames in Merr. in Journ. Roy. Asiat. Sóc. Straits Branch, Special No. (1921) 171.

Dendrobium javanicum Sw. in Kongl. Svensk. Vet.-Akad. Nya Handl. 21 (1800) 247; Willd. Sp. Pl. 4 (1805) 137.

Dendrolirium rugosum Blume Bijdr. (July, 1825) 345.

Eria stellata Lindl. in Bot. Reg. 11 (August, 1825) $t .904$; Lindl. Gen. \& Sp̈. Orch. (1830) 67; Paxt. Mag. Bot. 2 (1836) 137, 4 (1838) 234; Hook. in Bot. Mag. 64 (1837) t. 3605; Reichb. f. in Bonp1. 5 (1857) 54; Miq. Fl. Ind. Bat. 3 (1859) 661; Reichb. f. in Walp. Ann. 6 (1861) 269; Hemsl. in Gard. Chron. II 18 (1882) 500 ; Naves Novis. App. (1882) 236; Ridl. in Trans. Linn. Soc. II 3 (1893) 366, in Journ. Linn. Soc. 31 (1896) 285, 32 (1896) 304; Kränzl. in K. Schum. \& Lauterb. Fl. Deutsch. Schutzgeb. (1901) 248; Usteri Beitr. Ken. Philip. Veg. (1905) 128; Ridl. Mat. Fl. Mal. Penin. 1 (1907) 98.

Octomeria stellata Spreng.. Syst. Veg. 4 Cur. Post (1827) 310.

Octomeria vaginata Breda Gen, et Sp. Orch. Jav. (1827) t. 5.

Eria rugosa Lindl. Gen. \& Sp. Orch. (1830) 66; Miq. Fl. Ind. Bat. 3 (1859) 661; Hemsl. in Gard. Chron. II 18 (1882) 500; J. J. Sm. Fl. Buitenz. 6 (Orch. Jav.) (1905) 378, Orch. Amb. (1905) 74; Leavitt in Philip. Journ. Sci. 4 (1909) Bot. 212, 235; Ames Orch. 5 (1915) 158.

Eria fragrans Reichb. f. in Bot. Zeit. 22 (1864) 415, in Trans. Linn. Soc. 30 (1874) 137; Hook. f. Fl. Brit. Ind. 5 (1890) 794; Grant Orch. Burma (1895) 140; King \& Pantl. in Ann. Roy. Bot. Gard. Calc. 8 (Orch. Sik.-Himal.) (1898) 122, t. 168; Krănzl。 in Engl. Pflanzenreich IV. 50. II. B. $21^{2}$ (1911) 24.

Eria striolata Reichb. f. in Gard. Chron. III 3 (May 5, 1888) 554, in Ill. Hort. 35 (May, 1888) 35, t. 48; Ridl. in Journ. Linn. Soc. $200823-24$ 
31 (1896) 285, 36 (1896) 304, Mat. Fl. Mal. Penin. 1 (1907) 99; Krånzl. in Engl. Pflanzenreich IV. 50. II. B. $21^{2}$ (1911) 25.

Tainia stellata Pfitz. in Engl. \& Prantl Nat. Pflanzenfam. 2 Abt. 6 (1888) 153.

Dendrobium perakense Hook. f. Fl. Brit. Ind. 5 (1890) 712, in Hook. Icon. Pl. 21 (1890) t. 2019.

Pinalia rugosa Ktze. Rev. Gen. Pl. 2 (1891) 679.

Pinalia fragrans Ktze. Rev. Gen. Pl. 2 (1891) 679.

Pinalia stellata Ktze. Rev. Gen. Pl. 2 (1891) 679.

Pinalia striolata Ktze. Rev. Gen. Pl. 2 (1891) 679.

Eria vaginata Benth. ex Jacks. Ind. Kew. (1893) 864.

Sarcopodium perakense Kränzl. in Engl. Pflanzenreich IV. 50. I. B. 21 (1910) 328.

Eria javanica Blume var. philippinensis Kränzl. in Engl. Pflanzenreich IV. 50. II. B. $21^{2}$ (1911) fig. $14 E-J$.

Luzon (Albay, Bontoc, Benguet, Rizal, Laguna), Leyte, Mindanao (Zamboanga, Lanao), Loher 6003, Vanoverbergh 3911, Lyon s. n., B. S. 15135 Ramos, 1790 s Broun, Wenzel 0100, F. B. 12364 Hutchinson, M. S. Clemens s. $n$. Up to $1,200 \mathrm{~m}$ altitude in Bontoc. In India, Burma, Malay Peninsula, Sumatra, Celebes, Amboina, Borneo, and New Guinea.

It seems probable that Eria micholitzii Kränzl. in Oesterr. Bot. Zeitschr. (1894) 459; Schltr. in Fedde Repert. Beihefte 1 (Orch. DeutschNeu-Guin.) (1912) 653, Eria pseudostellata Schitr. in K. Schum. \& Lauterb. Nachtr. Fl. Deutsch. Schutzgeb. (1905) 182, and Eria papuana Kränzl. in Engl. Pflanzenreich IV. 50. II. B. $21^{2}$ (1911) 25 are conspecific. Schlechter reduces his E. pseudostellata and E. papuana Kränzl. (to which Kränzlin reduces his E. micholitzii) to E. micholitzii Kränzl. $J$. J. Smith states that E. pseudostellata Schltr. is probably the same as E. javanica Blume, and Kränzlin, in Das Pfanzenreich, cites it as a synonym of that species.

ERIA LAGUNENSIS Ames Orch. 5 (1915) 150.

Luzon (Laguna), Serrato s. n. Epiphyte in thicket forest.

ERIA LAMELLATA Ames in Elm. Leaf. Philip. Bot. 5 (1912) 1576, Orch. 5 (1915) 152.

Biliran, Mindanao (Davao), B. S. 18942 MeGregor, Elmer 11715. Epiphyte in humid forest at 660 to $1,300 \mathrm{~m}$ altitude.

ERIA LEAVITTII Kränzl. in Engl. Pflanzenreich IV. 50. II. B. $21^{2}$ (1911) 64, fig. $17 \mathrm{G}, \mathrm{H}$.

Eria ventricosa Leavitt var. benguetensis Leavitt in Philip. Journ. Sci. 4 (1909) Bot. 212, 234.

Luzon (Benguet), Merrill 4849, B. S. 4986 Curan. Terrestrial and epiphytic in mossy forest up to $1,950 \mathrm{~m}$ altitude.

ERIA LEYTENSIS Ames in Philip. Journ. Sci. 8 (1913) Bot. 427, Orch. 5 (1915) 152.

Leyte, Negros, Wenzel 01\%,016\%, 06\%4, F. B. 190\%2 Curran. Epiphyte at 60 to $600 \mathrm{~m}$ altitude. 
ERIA LOHERIANA KränzI. in Fedde Repert. 17 (1921) 387.

Loher s. n. Known only from the original description; no material has been seen.

ERIA LONGIBRACTEATA Leavitt in Philip. Journ. Sici. 4 (1909) Bot. 219, 238, fig. 19; Kränzl. in Engl. Pflanzenreich IV. 50. II. B. $21^{2}$ (1911) 126; Ames Orch. 5 (1915) 152.

Luzon (Cagayan), Mindanao (Zamboanga, Misamis), F. L. J6735 Curran, 4728 Mearns \& Hutchinson, Merrill 8142. Up to $1,900 \mathrm{n}$ altituds in Misamis.

ERIA LONGICRURIS Leavitt in Philip. Journ. Sci. 4 (1909) Bot. 205, 228, fig. 6; Kränzl. in Engl. Pflanzenreich IV. 50. II. B. 21² (1911) 109; Ames Orch. 5 (1915) 152.

Mindanao (Misamis, Davao), F. B. 4609 Mearns \& Hutchinson, Elmer 11351. At $2,800 \mathrm{~m}$ in Misamis.

ERIA LONGILABRIS Lindl. in Bot. Reg. 27 (1841) Misc. 38, 30 (1844) sub t. 29, fig. 3 ; Reichb. f. in Walp. Ann. 6 (1861) 277; Naves Novis. App. (1882) 236; Leavitt in Philip. Journ. Sci. 4 (1909) Bot. 210, 232; Krånzl. in. Engl. Pflanzenreich IV. 50. II. B. $21^{2}$ (1911) 86; Ames Orch. 5 (1915) 152.

Panay, Cuming s. $n$. Known only from the description and the Cuming specimen.

As pointed out by Leavitt and by Ames this may be only a variety of $E$. bractescens Lindl., but for the present it is deemed best to maintain it as a separate species.

ERIA LYONII Leavitt in Philip. Journ. Sci. 4 (1909) Bot. 212, 234, fig. 17; Kränzl. in Engl. Pflanzenreich IV. 50. II. B. $21^{2}$ (1911) 87; Ames Orch. 5 (1915) 1 こ̌2.

Luzon (Tarlac), Lyon B, 116 in part (Luzon material). Terrestrial (?) near sea level.

ERIA MACERA Ames Orch. 7 (1922) 103.

Mindanao (Bukidnon), B. S. 38512 Ramos \& Edaño. Epiphyte on mossy forest slope at $2,130 \mathrm{~m}$ altitude.

ERIA MAJOR Ridl. ex Stapf in Trans. Linn. Soc. II 4 (1894) 237, in syn.; Ridl. in Journ. Linn. Soc. 31 (1896) 280, 32 (1896) 288, Mat. Fl. Mal. Penin. 1 (1907) 90; Leavitt in Philip. Journ. Sci. 4 (1909) Bot. 218, 239; Kränzl. in Engl. Pflanzenreich IV. 50. II. B. $21^{2}$ (1911) 128; Ames Orch. 5 (1915) 155, 6 (1920) 131, in Merr。 in Journ. Roy. Asiat. Soc. Straits Branch, Special No. (1921) 172.

Eria kingii Hook. f. Fl. Brit. Ind. 5 (1890) 790, in Hook. Icon. Pl. 21 (1891) t. 2066, non F. v. Muell.

Eria scortechinii Stapf in Trans. Linn. Soc. II 4 (1894) 237, non Hook. f.

Mindanao (Misamis), F. B. 4595 Mearns \& Hutchinson. Mt. Malindang at $1,750 \mathrm{~m}$ altitude. 
ERIA MAQUILINGENSIS Ames Orch. 5 (1915) 152.

Luzon (Laguna), B. S. 17909 Brown. Mt. Maquiling, epiphyte in forest at $700 \mathrm{~m}$ altitude.

ERIA MCGREGORII Ames Orch. 5 (1915) 153.

Luzon (Ifugao), B. S. 19876 McGregor. Mt. Polis.

ERIA MEARNSII Leavitt in Philip. Journ. Sci. 4 (1909) Bot. 216, 236; Kränzl. in Engl. Pflanzenreich IV. 50. 11. B. $21^{2}$ (1911) 37; Ames Orch. 5 (1915) 155.

Mindanao (Misamis, Agusan, Davao), F. B. 4729 Mearns \& Hutchinson, Elmer 11850, 13802. Epiphyte up to $1,700 \mathrm{~m}$ altitude in Misamis.

ERIA MERRILLII Ames in Philip. Journ. Sci. 2 (1907) Bot. 331; Leavitt in Philip. Journ. Sci. 4 (1909) Bot. 213, 235 ; Kränzl. in Engl. Pflanzenreich IV. 50. II. B. $21^{2}$ (1911) 56; Ames Orch. 5 (1915) 155.

Luzon (Rizal, Sorsogon), Mindoro, Leyte, Mindanao (Surigao), Lyon 116 in part, 5626, Elmer 15552, Merrill 5519, Wenzel 0104, 071\%. Epiphyte at 60 to $800 \mathrm{~m}$ altitude.

ERIA MERRITTII Ames in Philip. Journ. Sci. 2 (1907) Bot. 331; Leavitt in Philip. Journ. Sci. 4 (1909) Bot. 207, 230; Kränzl. in Engl. Pflanzenreich IV. 50, II. B. $21^{2}$ (1911) 107; Ames Orch. 5 (1915) 155.

Luzon (Rizal, Laguna), Mindoro, Leyte, B. S. 19205 Reillo, 15122 Ramos, F. B. 4537 Merritt. Epiphyte at 500 to $1,300 \mathrm{~m}$ altitude.

ERIA MICROCHILA Ames Orch. 7 (1922) 104.

Luzon (Bontoc), B. S. 37997 Ramos \& Edaño. Mt. Caual. Epiphyte in mossy forest at $1,530 \mathrm{~m}$ altitude.

ERIA MINDANAENSIS Ames in Elm. Leafl. Philip. Bot. 5 (1912) 1577, Crch. 5 (1915). 155, 6 (1920) 207.

Sibuyan, Panay (Capiz), Elmer 12271, B. S. 35379, 35633 Martelino \& Edaño. Epiphyte at 250 to $660 \mathrm{~m}$ altitude.

ERIA ODORIFERA Leavitt in Philip. Journ. Sci. 4 (1909) Bot. 223, 242, fig. 24; Ames Orch. 5 (1915) 156.

Trichotosia odorifera Kränzl. in Engl. Pflanzenreich IV. 50. II. B. $21^{2}$ (1911) 153.

Negros (Oriental Negros), Mindanao (Zamboanga, Davao), Elmer 9521, 9658, Copeland 1182, Merrill 8153. Epiphyte up to $1,600 \mathrm{~m}$ altitude in Davao.

ERIA ORNATA (Blume) Lindl. Gen. \& Sp. Orch. (1830) 66; De Vriese Ill. Orch. Ind. Néerl. (1854) t.; Reichb. f. in Bonpl. 5 (1857) 54; Lindl. in Journ. Linn. Soc. 3 (1859) 48 (excl. Indian and Burman plants); Miq. Fl. Ind. Bat. 3 (1859) 660 (excl. Indian and Burman plants); Reichb. f. in Walp. Ann. 6 (1861) 270; Naves Novis. App. (1882) 236; Vidal Phan. Cuming. Philip. (1885) 149, Rev. Pl. Vasc. Filip. (1886) 268; Ridl. in Journ. Linn. Soc. 31 (1896) 285, ? 32 (1896) 302; Schltr. in Perk. Frag. Fl. Philip. (1904) 43; J. J. Sm. Fl. Buitenz. 6 (Orch. Jav.) (1905) 380, Fig.-Atlas (1911) fig. 288; Ames Orch. 1 (1905) 94, 2 (1908) 195, 5 (1915) 
156; Leavitt in Philip. Journ. Sci. 4 (1909) Bot. 217, 237; Merr. \& Merritt in Philip. Journ. Sci. 5 (1910) Bot. 341; Kränzl. in Engl. Pflanzenreich IV. 50. II. B. $21^{2}$ (1911) 50, fig. 11 A, B; Ames in Merr. in Journ. Roy. Asiat. Soc. Straits Branch, Special No. (1921) 173.

Dendrolinium ornatum Blume Bijdr. (1825) 345.

Eria armeniaca Lindl. in Bot. Reg. 27 (1841) Misc. 38, t. 42.

Pinalia ornata Ktze. Rev. Gen. Pl. 2 (1891) 678.

Luzon (Bontoc, Benguet, Nueva Vizcaya, Zambales, Rizal), Negros (Occidental Negros), Mindanao (Zamboanga, Agusan). A fairly common species in the Philippines at 10 to $1,450 \mathrm{~m}$ altitude. In ? Siam, Sumatra, Java, and Borneo.

ERIA OVATA Lindl. in Bot. Reg. 30 (1844) sub t. 29; G. K. in Bot. Zeit. 3 (1845) 95; Reichb. f. in Walp. Ann. 6 (1861) 277; Naves Novis. App. (1882) 236; Vidal. Phan. Cuming. Philip. (1885) 149, Rev. Pl. Vasc. Filip. (1886) 268; Ames Orch. 2 (1908) 195, 5 (1915) 156; Leavitt in Philip. Journ. Sci. 4 (1909) Bot. 207, 230, fig. 8; Kränzl. in Engl. Pflanzenreich IV. 50. II. B. $21^{2}$ (1911) 76.

Eria elmeri Ames Orch. 1 (1905) 93, 2 (1908) 196, 5 (1915) 149; Leavitt in Philip. Journ. Sci. 4 (1909) Bot. 208, 230, fig. 10; Kränzl. in Engl. Pflanzenreich IV. 50. II. B. $21^{2}$ (1911) 74.

Luzon (Bontoc, Lepanto, Benguet, Bataan, Laguna), Negros (Oriental Negros), Leyte. A fairly common species, epiphyte at 500 to $2,550 \mathrm{~m}$ altitude.

ERIA PALAWANENSIS Ames in Elm. Leaf. Philip. Bot. 5 (1912) 1578, Orch. 5 (1915) 156.

Palawan, Elmer 13209. Mt. Pulgar.

ERIA PERSPICABILIS Ames Orch. 5 (1915) 156.

Luzon (Ifugao), B. S. 19733 McGregor. Mt. Polis.

ERIA PHILIPPINENSIS Ames Orch. 1 (1905) 94, 5 (1915) 157; Leavitt in Philip. Journ. Sci. 4 (1909) Bot. 204, 227, fig. 5; Ames in Philip. Journ. Sci. 4 (1909) Bot. 673; Merr. \& Merritt in Philip. Journ. Sci. 5 (1910) Bot. 341; Kränzl. in Engl. Pflanzenreich IV. 50. II. B. $21^{2}$ (1911) 66 .

Luzon (Abra, Bontoc, Lepanto, Benguet, Bataan, Laguna). A common species in Luzon. Fipiphyte in forest at relatively high altitudes.

ERIA POLYURA Lindl. in Bot. Reg. 27 (1841) Misc. 55, 28 (1842) t. 32; Paxt. Mag. Bot. 9 (1842) 142; Reichb. f. in Walp. Ann. 6 (1861) 279; Naves Novis. App. (1882) 236; Hemsl. in Gard. Chron. II 18 (1882) 500; Ames Orch. 1 (1905) 95, 2 (1908) 196, 5 (1915) 157; Leavitt in Philip. Journ. Sci. 4 (1909) Bot. 203, 226, fig. 2; Kränzl. in Engl. Pflanzenreich IV. 50. II. B. $21^{2}$ (1911) 115.

Pinalia polyura Ktze. Rev. Gen. Pl. 2 (1891) 679.

Luzon (Cagayan, Bontoc, Benguet, Bataan, Rizal, Laguna, Tayabas), Sibuyan, Panay (Antique), Leyte, Mindanao (Lanao, Surigao). A rather common and widely distributed species in the Philippines. Epiphyte at 60 to $2,050 \mathrm{~m}$ altitude. 
ERIA PROFUSA Lindl. in Bot. Reg. 28 (1842) Misc. 3; Paxt. Mag. Bot. 12 (1845) 46; Reichb. f. in Walp. Ann. 6 (1861) 271; Hemsl. in Gard. Chron. II 18 (1882) 500; Trim. Handb. Fl. Ceyl. 4 (1898) 168; Leavitt in Philip. Journ. Sci. 4 (1909) Bot. 210, 233, fig. 14; Kränzl. in Engl. Pflanzenreich IV. 50. II. B. $21^{2}$ (1911) 105.

Juzon (Isabela, Rizal), Negros (Oriental Negros), Mindanao (Agusan), Lyon F, 132, B. S. 3079, 3083 Ramos, F. B. 11237 Everett, Weber 111. Epiphyte at 100 to $700 \mathrm{~m}$ altitude.

ERIA PROPINQUA Ames Orch. 7 (1922) 105.

Mindanao (Bukidnon), B. S. 38939 Ramos \& Edaño. Mt. Candoon, on trees of forested slopes, at $1,650 \mathrm{~m}$ altitude.

ERIA PUGUAHAANENSIS Ames Orch. 5 (1915) 157.

Leyte, Wenzel 0221. Dagami, epiphyte in forest at $60 \mathrm{~m}$ altitude.

ERIA RACEMOSA Leavitt in Philip. Journ. Sci. 4 (1909) Bot. 206, 229; Kränzl. in Engl. Pflanzenreich IV. 50. II. B. $21^{2}$ (1911) 115; Ames Orch. 5 (1915) 158.

Luzon (Benguet), Mindanao (Misamis), Curran s. n., Sandhuhl $2 £ 5$, F. B. 4609 Mearns \& Hutchinson in part.

ERIA RAMOSA Ames nom. nov.

Eria vagans Ames Orch. 7 (1922) 106, non Schltr.

Luzon (Bontoc, Benguet), Vanoverbergh 2223, 3896, Elmer 8801.

ERIA RAMosII Leavitt in Philip. Journ. Sci. 4 (1909) Bot. 224, 243, fig. 25; Ames in Elm. Leafl. Philip. Bot. 5 (1912) 1579, Orch. 5 (1915) 158.

Trichotosia ramosii Kränzl. in Engl. Pflanzenreich IV. 50. II. B. $21^{2}$ (1911) 156.

Luzon (Rizal), Negros (Oriental Negros), Mindanao (Agusan), B. S. 1775 Ramos, 29731 Ramos \& Edaño, Elmer 9803, 13755, 15098, 16136. Epiphyte, forming dense mats, at $1,330 \mathrm{~m}$ altitude in Negros.

ERIA RETROFLEXA Lindl. in Journ. Linn. Soc. 3 (1859) 60; Miq. Fl. Ind. Bat. 3 (1859) 662; Naves Novis. App. (1882) 236; Vidal Phan. Cuming. Philip. (1885) 149, Rev. Pl. Vasc. Filip. (1886) 268; Ames Orch. 2 (1908) 197, 5 (1915) 158; Leavitt in Philip. Journ. Sci. 4 (1909) Bot. 206, 229, fig. \%; Kränzl. in Engl. Pflanzenreich IV. 50. II. B. $21^{2}$ (1911) 76.

Pinalia retroflexa Ktze. Rev. Gen. P1. 2 (1891) 679.

Luzon (Benguet, Bataan, Rizal, Tayabas), Negros (Oriental Negros), Leyte, Mindanao (Cotabato, Agusan). A not uncommon species, epiphyte at 60 to $860 \mathrm{~m}$ altitude.

ERIA RHODOPTERA Reichb. f. in Gard. Chron. II 18 (1882) 586; Rolfe in Orch. Rev. 17 (1909) 220, in Bot. Mag. 136 (1910), t. 8296; Kränzl. in Engl. Pflanzenreich IV. 50. U. B. $21^{2}$ (1911) 84; Ames Orch. 5 (1915) 158.

Eria laucheana Kränzl. in Gard. Chron. III 11 (1892) 809.

Eria bractescens Lindl. var. latipetala Leavitt in Philip. Journ. Sci. 4 (1909) Bot. 210, 232, fig. 12; Kränzl. in Engl. Pflanzenreich IV. 50. II. B. $21^{2}(1911) 88$. 
Luzon (Rizal), Palawan, B.S. 3000 Ramos, Weber 40\%. Epiphyte at, sea level to $600 \mathrm{~m}$ altitude.

ERIA RINGENS Reichb. f. in Bonpl. 3 (1885) 222; Iindl. in Journ. Linn. Soc. 4 (1859) 55 (erroneously referred to $E$. ovatu R. Br.); Reichb. f. in Walp. Ann. 6 (1861) 275, in Trans. Linn. Soc. 30 (1874) 137; Naves Novis. App. (1882) 236; Hook. f. Fl. Brit. Ind. 5 (1890) 793; Leavitt in Philip. Journ. Sci. 4 (1909) Bot. 207, 229;

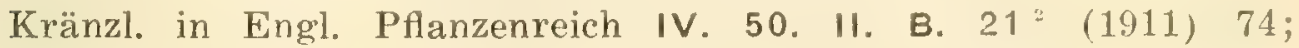
Ames in Elm. Leafl. Philip. Bot. 5 (1912) 1579, Orch. 5 (1915) 158.

Eria polyura Vidal Phan. Cuming. Philip. (1885) 149, Rev. Pl. Vasc. Filip. (1886) 268, non Lindl.

Pinalia ringens Ktze. Rev. Gen. Pl. 2 (1891) 679.

Negros (Oriental Negros), Cuming 2128, Elmer 10192. Cuernos Mountains. In Burma.

ERIA SENILIS Ames Orch. 5 (1915) 159.

Luzon (Sorsogon), Panay (Antique), Leyte, Mindanao (Lanao), Elmer 16375, B. S. 32575 McGregor, Wenzel 0339, 0335, 0348, 0455, 0859, 0890, 0892, 0894, 0897, 0898,0899, 0900, 0901, 0902, 0903, 0932, 0933, M.S. Ciemens s. $n$. Epiphyte in forest at 500 to $900 \mathrm{~m}$ altitude.

ERIA TAYLORII Ames Sched. Orch. 5 (1923) 31.

Luzon (Laguna), Taylor 25. Mt. Maquiling, epiphyte in mossy forest at $1,000 \mathrm{~m}$ altitude.

ERIA TRIDENS Ames in Elm. Leafl. Philip. Bot. 5 (1912) 1580, Orch. 5 (1915) 159.

Mindanao (Davao), Elmer 11850a. Todaya, Mt. Apo.

ERIA VANOVERBERGHII Ames in Philip. Journ. Sci. 7 (1912) Bot. 20, Orch. 5 (1915) 159.

Luzon (Kalinga, Bontoc, Tayabas), Boettcher s. n., Vanoverbergh 789 , 1340, B. S. 26523. Ramos \& Edaño. Epiphyte at 300 to $1,550 \mathrm{~m}$ altitude.

ERIA VENTRICosA Leavitt in Philip. Journ. Sci. 4 (1909) Bot. 211, 234, fig. 16; Merr. \& Merritt in Philip. Journ. Sci. 5 (1910) Bot. 341; Kränzl. in Engl. Pflanzenreich IV. 50. II. B. $21^{2}$ (1911) 63; Ames Orch. 5 (1915) 159.

Luzon (Bontoc, Ifugao, Benguet, Bataan, Tayabas), Mindoro, and possibly from Mindanao. A fairly common species in Luzon, epiphyte in forest at 1,650 to $2,400 \mathrm{~m}$ altitude.

ERIA VULPINA Reichb. f. in Bonpl. 3 (1855) 222; Lindl. in Journ. Linn. Soc. 3 (1859) 57; Miq. Fl. Ind. Bat. 3 (1859) 660; Reichb. f. in Walp. Ann. 6 (1861) 273; Naves Novis. App. (1882) 236; Vidal Phan. Cuming. Philip. (1885) 149, Rev. P1. Vasc. Filip. (1886) 268; Ames in Philip. Journ. Sci. 2 (1907) Bot. 332, Orch. 2 (1908) 198, 5 (1915) 160; Leavitt in Philip. Journ. Sci. 4 (1909) Bot. 222 241, fig. 23.

Eria vestita Lindl. in Bot. Reg. 30 (1844) Misc. 76, 31 (1845) sub t. 2 in textu, quoad spec. Cuming; Reichb. f. in Walp. Ann. 6 (1861) 274, quoad Philip.; Hook. f. in Bot. Mag. 95 (1868) sub t. 580 y in textu, quoad Philip.; Naves Novis. App. (1882) 236. 
Pinalia vulpina Ktze. Rev. Gen. Pl. 2 (1891) 679.

Trichotosia vulpina Kränzl. in Engl. Pflanzenreich IV. 50. II. B. $21^{2}$ (1911) 141.

Luzon (Bontoc, Pampanga, Rizal, Laguna, Tayabas, Sorsogon), Mindoro, Sibuyan, Negros (Oriental Negros), Leyte, Mindanao (Camiguin Island, Zamboanga), Basilan. A common and widely distributed species in the Philippines. Epiphyte at 60 to $1,600 \mathrm{~m}$ altitude.

ERIA WHITFORDII Leavitt in Philip. Journ. Sci. 4 (1909) Bot. 215; 236; Ames Orch. 5 (1915) 160.

Eria aeridostachya Reichb. f. apud Ames in Philip. Journ. Sci. 2 (1907) Bot. 329; Leavitt in Philip. Journ. Sci. 4 (1909) Bot. 213, nota; Kränzl. in Engl. Pflanzenreich IV. 50. 11. B. $21^{2}$ (1911) 35, quoad syn. E. whitfordii et quoad Philip.

Luzon (Isabela, Bataan, Laguna, Sorsogon), Mindoro, Negros (Oriental Negros), Leyte, Lyon 135, G, Whitford 1119, Merrill s. n., 5518, Reillo 10, Elmer 9731, 1645\%, Wenzel 0434, 0580. Epiphyte at 500 to $2,150 \mathrm{~m}$ altitude.

ERIA WOODIANA Ames in Philip. Journ. Sci. 2 (1907) Bot. 332; Leavitt in Philip. Journ: Sci. 4 (1909) Bot. 205, 228; Kränzl. in Engl. Pflanzenreich IV. 50. II. B. $21^{2}$ (1911) 105, fig. $14 \mathrm{~J}, \mathrm{~K}$.

Luzon (Laguna, Camarines), Mindoro, Leyte, B. S. 1707.2 Robinson, 42082 Edaño, Taylor 1, Merrill 5490, Wenzel 0324, 0864, 0924. Epiphyte at 500 to $1,500 \mathrm{~m}$ altitude.

ERIA ZAMBOANGENSIS Ames Orch. 5 (1915) 160.

Mindanao (Zamboanga, Bukidnon), Merrill 8143, B. S. 38686, 39123 Ramos \& Edaño. Epiphyte in mossy forest at 1,300 to $1,830 \mathrm{~m}$ altitude.

\section{EXCLUDED OR SYNONYMOUS SPECIES}

ERIA AERIdostachya Reichb. f. apud Ames in Philip. Journ. Sci. 2 (1907)

Bot. 239; Leavitt in Philip. Journ. Sci. 4 (1909). Bot. 213, nota; Kränzl. in Engl. Pflanzenreich IV. 50. II. B. $21^{2}$ (1911) 35, quoad syn. E. whitfordii et quoad Philip. = Eria whitfordii Leavitt.

For a discussion of the differences and identity of Eria aeridostachya Reichb. f. ex Lindl. cf. Leavitt. 1. c.

ERia ALBido-tomentosa Lindl. apud Naves Novis. App. (1882) 236.

ERIA AMICA Reichb. f. apud Naves Novis. App. (1882) 236.

ERIA APOROIDEs Lindl. in Journ. Linn. Soc. 3 (1859) 60; Miq. Fl: Ind. Bat. 3 (1859) 657; Naves Novis. App. (1882) 236; Merr. in Govt. Lab. Publ. (Philip.) 27 (1905) $84=$ Dendrobium aporoides' (Lindl.) Merr.

ERIA ARMENIACA Lindl, in Bot. Reg. 27 (1841) Misc. 38, t. $42=$ Eria ornata (Blume) Lindl.

ERIA BRACtescens Lindl. var. LAtipetala Leavitt in Philip. Journ. Scl. 4 (1909) Bot. 210, 232, fig. 12; Kränzl. in Engl. Pflanzenreich IV. 50. II. B. $21^{2}$ (1911) 88 = Eria rhodoptera Reichb. f.

ERIA Compressa Blume apud Naves Novis. App. (1882) 236. 
Eria DAsYPus Reichb. f. apud Naves Novis. App. (1882) 236.

ERIA DILlWYNII Hook. in Bot. Mag. 71 (1845) t. 4163; Lindl. in Bat. Reg. 31 (1845) Misc. 63; F. Kl. in Bot. Zeit. 3 (1845) 5500; Paxt. Mag. Bot. 12 (1846) 140; Lindl. in Journ. Linn. Soc. 3 (1859) 55; Naves Novis. App. (1882) 236; Kränzl. in Engl. Pflanzenreich IV. 50. II. B. $21^{2}$ (1911) 87 = Eria bractescens Lindl.

ERIA ELMERI Ames Orch. 1 (1905) 93, 2 (1908) 196, 5 (1915) 149; Leavitt in Philip. Journ. Sci. 4 (1909) Bot. 208, 230, fig. 10; Kränzl. in Engl. Pflanzenreich IV. 50. II. B. $21^{2}$ (1911) $74=$ Eria ovata Lindl.

ERIA ERECTA Lindl. apud Naves Novis. App. (1882) 236.

Eria flavescens Lindl. apud Naves Novis. App. (1882) 236; Vidal Rev. Pl. Vasc. Filip. (1886) 268; Ceron Cat. Pl. Herb. Manila (1892) 167.

This species is known only from Java. Vidal's identification of his 1959 was made with expressed doubt.

ERIA Formosana Kränzl. in Engl. Pflanzenreich IV. 50. II. B. $21^{2}$ (1911) 47, non Rolfe = Eria copelandii Leavitt.

Erra Formosana Kränzl. var. Fusiformis Kränzl. in Engl. Pflanzenreich IV. 50. II. B. $21^{2}$ (1911) $47=$ Eria copelandii Leavitt var. fusiformis Leavitt.

ERIa goldschmidtiana Schltr. apud Kränzl, in Engl. Pflanzenreich IV. 50. II. B. $21^{2}$ (1911) 83 .

"Provinz der Philippinen und Süd-Formosa."-Kränzlin. The type was from Formosa; the species has not been found in the Philippines.

ErIA LANICEPS Reichb. f. apud Naves Novis. App. (1882) 236.

Eria LaUcheanA Kränzl. in Gard. Chron. III 11 (1892) $809=$ Eria rhodoptera Reichb. f.

Eria monostachya Lindl. apud Naves Novis. App. (1882) 236.

Eria Mucronata Lindl, in Bot. Reg. 28 (1842) Misc. 27, in Journ. Linn. Soc. 3 (1859) 59; Miq. Fl. Iṇd. Bat. 3 (1859) 657; Naves Novis. App. (1882) 236.

Trichotosia mucronata Kränzl. in Engl. Pflanzenreich IV. 50. II. B. $21^{2}$ (1911) 157.

The type of this may or may not have been a Philippine plant. However, the collections of twenty years have not produced a specimen referable to it.

Eria polyura Vidal Phan. Cuming. Philip. (1885) 149, Rev. Pl. Vasc. Filip. (1886) 268, non Lindl. = Eria ringens Reichb. $f$.

ERIA PRoRePens Reichb. f. Otia Bot. Hamb. 1 (1878) 54, Xen. Orch. 3 (1878) 31; Naves Novis. App. (1882) $236=$ Phreatia prorepens Reichb. $f$.

ErIa PUSilla Lindl. apud Naves Novis. App. (1882) 236.

ERIA RetUSA Lindl. apud Naves Novis. App. (1882) 236. 
ERIA RUgosA Lindl. Gen. \& Sp. Orch. (1830) 66; Leavitt in Philip. Journ. Sci. 4 (1909) Bot. 212, 235; Ames Orch. 5 (1915) $158=$ Eria javanica (Sw.) Blume.

ERIA STELlata Lindl. in Bot. Reg. 11 (August, 1825) t. 904; Naves Novis. App. (1882) 236; Usteri Beitr. Ken. Philip. Veg. (1905) $128=$ Eria javanica (Sw.) Blume.

ERta VAGans Ames Orch. 7 (1922) 106, non Schltr. = Eria ramosa Ames.

ERIA ventricosa Leavitt var. Benguetensis Leavitt in Philip. Journ. Sci.

4 (1909) Bot. 212, 234 = Eria leavittii Kränzl.

ERIA vestita Lindl. in Bot. Reg. 30 (1844) Misc. 76, 31 (1845) sub $t .2$ in textu, quoad spec. Cuming; Reichb. f. in Walp. Ann. 6 (1861) 274, quoad Philip.; Hook. f. in Bot. Mag. 95 (1869) sub t. 5807, in textu, quoad Philip.; Naves Novis. App. (1882) $236=$ Eria vulpina Reichb. $\mathrm{f}$.

Lindley originally placed the Cuming specimen from Manila under Eria vestita, but in 1859 (in Journ. Linn. Soc. 3: 57) he corrected this error and gave the points of difference between the two species.

ERIA Wenzelit Ames in Philip. Journ. Sci. 8 (1913) Bot. $428=$ Ema brachystachya Reichb. $\mathrm{f}$.

\section{BULBOPHYLLUM Thouars}

BULBOPHYLLUM ADENOPETALUM Lindl, in Bot. Reg. 28 (1842) Misc. 85; Reichb. f. in Walp. Ann. 6 (1861) 255; Rolfe in Journ. Bot. 23 (1885) 215; Vidal Phan. Cuming. Philip. (1885) 149, Rev. Pl. Vase. Filip. (1886) 267; Hook. f. Fl. Brit. Ind. 5 (1890) 770, 6 (1890) 188; Ridl. in Journ. Linn. Soc. 32 (1896) 273, Mat. Fl. Mal. Penin. 1 (1907) 75; Ames in Philip. Journ. Sci. 2 (1907) Bot. 333, 7 (1912) Bot. 126, Orch. 2 (1908) 206, 5 (1915) 162.

Bulbophyllum penicillium Rolfe in Journ. Bot. 23 (1885) 215; Vidal Phan. Cuming. Philip. (1885) 149, Rev. Pl. Vasc. Filip. (1886) 267; Ames in Philip. Journ. Sci. 7 (1912) Bot. 128, non Par. \& Reichb. $f$.

Bulbophyllum ramosii Ames in Philip. Journ. Sci. 7 (1912) Bot. 25, 128, Orch. 5 (1915) 186.

Phyllorchis adenopetala Ktze. Rev. Gen. Pl. 2 (1891) 677.

Luzon (Apayao, Bontoc, Bataan, Rizal, Laguna, Tayabas), Mindoro, Leyte, Mindanao (Dapitan, Bukidnon). A fairly common species in the Philippines, epiphyte at 500 to $3,000 \mathrm{~m}$ altitude. In Malay Peninsula.

BULBOPHYLLUM AEOLIUM Ames in Philip. Journ. Sci. 8 (1913) Bot. 429, Orch. 5 (1915) 164.

Luzon (Rizal), Leyte, Mindanao (Bukidnon), Reillo s. n., B. S. 38678 Ramos \& Edaño, 15391 Ramos, Wenzel 0644, 0652, 0671, 0835. Epiphyte, in forest at 600 to about $1,300 \mathrm{~m}$ altitude.

BULBOPHYLLUM AESTIVALE Ames Orch. 5 (1915) 164.

Leyte, Wenzel 0395, 0505, 0506, 0568, 0626. Epiphyte in forest at 500 to $600 \mathrm{~m}$ altitude. 
BULBOPHYLLUM ALAGENSE Ames in Philip. Journ. Sci. 2 (1907) Bot. 333,7 (1912) Bot, 126, Oxch. 3 (1908) 54, t. 44, fig. 1. A, a, 5 (1915) 165.

Luzon (Rizal, Laguna), Mindanao, Leyte, Serrato s. n., B. S. 29736 Ramos \& Edaño, 16579 Ramos, Merrill 5495, Wenzel o77\%. Epiphyte in forest at 400 to $500 \mathrm{~m}$ altitude.

BULbophyLLUM ALboroseum Ames Orch. 7 (1922) 147.

Luzon (Benguet), Ramos \& Eduño 105. Mt. Lusong, epiphyte at 2,000 $m$ altitude.

BULBOPHYLLUM ALsiosum Ames in Elm. Leafl. Philip. Bot. 5 (1912) 1583, Orch. 5 (1915) 165.

Luzon (Rizal), Negros (Oriental Negros), Leyte, Reillo s. n., Elmer 9817, Wenzel 0642, 0643, 0666, 0672. Epiphyte in forest at 600 to 1,300 $m$ altitude.

BULBOPHYLLUM ANTENNIFERUM (Lindl.) Reichb. f. in Walp. Ann.

6 (1861) 248; Naves Novis. App. (1882) 235; Ames in Philip.

Journ. Sci. 7 (1912) Bot. 126, 138, Orch. 5 (1915) 165.

Cirrhopetalum antenniferum Lindl. in Bot. Reg. 29 (1843) sub t. 49.

Phyllorchis antennifera Ktze. Rev. Gen. P1. 2 (1891) 677.

Leyte, Mindanao (Lanao). Cuming s. n., Wenzel 0791, M. S. Clemens 445.

BULBOPHYLLUM ARRECTUM Kränzl. in Fedde Repert. 17 (1921) 384;

Ames Sched. Orch. 6 (1923) 82.

Philippines, probably Luzon, Loher s. $n$.

Very close to Bulbophyllum stellatum Ames, but material at hand is inadequate to determine its status more definitely.

BULBOPHYLLUM AURATUM (Lindl.) Reichb. f. in Walp. Ann. 6 (1861) 261; Naves Novis. App. (1882) 235; Ridl. Mat. Fl. Mal. Penin. 1 (1907) 82; Ames in Philip. Journ. Sci. 7 (1912) Bot. 126, 139, Orch. 5 (1915) 165.

Cirrhopetalum auratum Lindl. in Bot. Reg. 26 (1840) Misc. 50, 29 (1843) t. 61; G. K. in Bot. Zeit. 2 (1844) 103; Moore Ill. Orch. Pl. (1857) Cirrhopet. 5, t. 4; Ridl. in Journ. Linn. Soc. 32 (1896) 284.

Phyllorchis aurata Ktze. Rev. Gen. Pl. 2 (1891) 677.

Leyte, Mindanao, Butuan, Agusan, Davao), Cuming s. n., Lyon 139, Wenzel 0200, 0511, B. S. 12128 Celestino, Weber 106, 137, Black 8. Epiphyte at 5 to $500 \mathrm{~m}$ altitude. In Malay Peninsula.

BULBOPHYLLUM BATAANENSE Ames Orch. 1 (1905) 96, 2 (1908) 207, 5 (1915) 166, in Philip. Journ. Sci. 4 (1909) Bot. 599, 7 (1912) Bot. 126.

Luzon (Zambales, Bataan, Laguna), Mindanao (Lanao, Davao), B. S. 3028 Mrs. Lyon, 5001, 16576 Ramos, F. B. 8995, 9504 Merritt \& Cusran, 796 Borden, Whitford 121, 163, Topping 473, Merrill 5182, Black 10. 
BULBOPHYLLUM BLUMEI (Lindl.) J. J. Sm. Fl. Buitenz. 6 (Orch. Jav.) (1905) 459, Fig.-Atlas (1911) fig. 351; Ames in Philip. Journ. Sci. 7 (1912) Bot. 127, Orch. 5 (1915) 167.

Ephippium ciliatum Blume Bijdr. (1825)) 309, fig. 65, non Bulbophyllum ciliatum Lindl.

Cirrhopetalum blumei Lindl. Gen. \& Sp. Orch. (1830) 59, in Bot. Reg. 29 (1843) sub t. 49; Miq. Fl. Ind. Bat. 3 (1859) 654; Ridl. in Journ. Linn. Soc. 32 (1896) 285.

Cirrhopetalum maxillare Lindl.' in Bot. Reg. 29 (1843) sub t. 49.

Bulbophyllum cheiri Lindl. in Bot. Reg. 30 (1844) Misc. 44; Reichb. f. in Walp. Ann. 6 (1861) 246; Naves Novis. App. (1882) 235; Ames in Philip. Journ. Sci. 7 (1912) Bot. 127, Orch. 5 (1915) 169. Sarcopodium cheiri Lindl. Fol. Orch. Sarcopod. (1853) 4; Moore Ill. Orch. Pl. (1857) Sarcopod. 4.

Bulbophyllum maxillare Reichb. f. in Walp. Ann. 6 (1861) 248; Naves Novis. App. (1882) 235; Ames in Philip. Journ. Sci. 7 (1912) Bot. 127, Orch. 5 (1915) 181.

Bulbophyllum cuspidilingue Reichb. f. in Walp. Ann. 6 (1861) 264. Phyllorchis blumei Ktze. Rev. Gen. Pl. 2 (1891) 676.

Phyllorchis cheiri Ktze. Rev. Gen. Pl. 2 (1891) 677.

Phyllorchis maxillaris Ktze. Rev. Gen. Pl. 2 (1891) 677.

Bulbophyllum ephippium Ridl. Mat. Fl. Mal. Penin. 1 (1907) 78.

Leyte, Cuming s.n., Wenzel 050, 0107, 0736. Epiphyte at $60 \mathrm{~m}$ altitude. In Malay Peninsula and Java.

BULBOPHYLLUM BOLSTERI Ames in Philip. Journ. Sci. 7 (1912) Bot. 126, 139, Orch. 5 (1915) 168.

Bulbophyllum chryseum Ames in Philip. Journ. Sci. 6 (1911) Bot. 54 , quoad spec.

Luzon (Rizal), Mindanao (Lanao, Surigao), B. S. 3076 Ramos, $M: S$. Clemens s. n., Bolster 349 . Epiphyte.

BULBOPHYLLUM BONTOCENSE Ames in Philip. Journ. Sci. 7 (1912) Bot. 126, 135, Orch. 5 (1915) 168.

Luzon (Bontoc, Zambales), Vanoverbergh 705, 1366, 1382, 3681, F. B. 8135 Curran \& Merritt. Epiphyte at 1,450 to $1,700 \mathrm{~m}$ altitude.

BULBOPHYLLUM BRACCATUM Reichb. f. in Linnaea 41 (1877) 117;

Ames in Philip. Journ. Sci. 7 (1912) Bot. 126, Orch. 5 (1915) 168.

Philippines, probably Luzon, Wallis s. n. Probably conspecific with Bulbophyllum hortensoides Ames.

BulbophyLlum CANLAONENSE Ames in Philip. Journ. Sci. 7 (1912)

Bot. 126, 136, Orch. 5 (1915) 168.

Luzon (Benguet), Negros (Oriental Negros), Mindanao (Bukidnon), B. S. 38717 Ramos \& Edaño, 4314 Mearns, Elmer 9936, Merrill 7015. Eipiphyte at 1,300 to $2,300 \mathrm{~m}$ altitude.

BULBOPHYLLUM CATENULATUM Kränzl. in Fedde Repert. 17 (1921) 382.

Philippines, probably Luzon, Loher s. $n$. 
BULBOPHYLLUM CHRYSENDETUM Ames Orch. 5 (1915) 169.

Leyte, Wenzel 0219, 0621, 065\%. Dagami and Jaro, epiphyte in forest at 60 to $600 \mathrm{~m}$ altitude.

BULBOPHYLLUM CHRYSEUM (Kränzl.) Ames in Philip. Journ. Sci. 6 (1911) Bot. 54, (excl. spec.), 7 (1912) Bot. 127, Orch. 5 (1915) 170; J. J. Sm. in Bull. Jard. Bot. Buitenz. 8 (1912) 23.

Cirrhopetalum chryseum Kränzl. in Fedde Repert. 8 (1910) 97.

Luzon (Rizal), Leyte, Loher s. n., Lyon 152, Wenzel 09?, 0138, 0214. Epiphyte at low altitudes, $60 \mathrm{~m}$ in Leyte.

BULBophyLlum CLemensiAe Ames in Philip: Journ. Sci. 7 (1912) Bot. 127, 129, Orch. 5 (1915) 170.

Mindanao (Lanao), M. S. Clemens 440. Camp Keithley, Lake Lanao.

BULBOPHYLLUM COLUBRIMOdUM Ames Sched. Orch. 6 (1923) 82, fig. 12.

Leyte, Wenzel 0698. Epiphyte in forest at $600 \mathrm{~m}$ altitude.

BULBOPHYLLUM COPELANDII Ames Orch. 1 (1905) 97, 2 (1908) 5, t. 19, 5 (1915) 170, in Philip. Journ. Sci. 7 (1912) Bot. 127.

Luzon (Laguna), Mindanao (Davao), B. S. 20434 Ramos, Calica s. n., Copeland 1316, Weber 265.

BULBOPHYLLUM COSTATUM Ames Orch. 6 (1920) 299.

Leyte, Wenzel 0665. Epiphyte in forest at $600 \mathrm{~m}$ altitude.

BULBOPHYLLUM CUBICUM Ames Orch. 7 (1922) 83.

Mindanao (Bukidnon), B. S. 38633 Ramos \& Edaño. Mahilucot River, epiphyte in forest at $1,400 \mathrm{~m}$ altitude.

BULBOPHYLLUM CUMINGII (Lindl.) Reichb. f. in Walp. Ann. 6 (1861) 261; Naves Novis. App. (1882) 235; Ames in Philip. Journ. Sci. 6 (1911) Bot. 55, 7 (1912) Bot. 127, Orch. 5 (1915). 170.

Cirrhopetalum cumingii Lindl. in Paxt. Mag. Bot. 8 (1841) 16อ, in

Bot. Reg. 29 (1843) sub t. 49; G. K. in Bot. Zeit. 1 (1843) 791;

Moore Ill. Orch. Pl. (1857) Cirrhopet. 5; Hook. in Bot. Mag. 83 (1857) t. 4996; Miq. Fl. Ind. Bat. 3 (1859) 653; Veitch Man. Orch. Pl. pt. 3 (1888) 100; Rodigas in Ill. Hort. 39 (1892) 62, in textu.

Phyllorchis cumingii Ktze. Rev. Gen. Pl. 2 (1891) 677.

Luzon (Rizal, Laguna, Tayabas), Panay (Capiz), Negros (Occidental Negros), Cuming s. n., B. S. 13763 Ramos, 31273 Ramos \& Edaño, 7334 Ceiestino, Serrato s. n., F. B. 13070,13071 Curran, 5227 Danao \& Aspillera. Apparently at low altitudes.

BULBOPHYLLUM CUNEATUM Rolfe ex Ames Orch. 1 (1905) 98, 2 (1908) 207, 5 (1915) 127, in Philip. Journ. Sci. 4 (1909) Bot. 674, 7 (1912) Bot. 127.

Luzon (Bataan), Merrill 3730, B. S. 5183 Topping, R. S. Williams 431. Mt. Mariveles, at about $1,000 \mathrm{~m}$ altitude. 
BULBOPHYLLUM CURRANII Ames in Philip. Journ. Sci. 7 (1912) Bot. 127, 130, Orch. 5 (1915) 170.

Luzon (Benguet), F. B. 4953 Curran, Haight 7, Ramos \& Edaño 62. Epiphytic vine at about $3,000 \mathrm{~m}$ altitude.

BULBOPHYLLUM DAGAMENSE Ames Orch. 5 (1915) 170.

Leyte, Wenzel 05\%, 0912. Dagami and Jaro, epiphyte in forest at 60 to $500 \mathrm{~m}$ altitude.

BULBOPHYLLUM DASYPETALUM Rolfe ex Ames Orch. 1 (1905) 98, text cut, 2 (1908) 207, 5 (1915) 171; Ames in Philip. Journ. Sci. 2 (1907) Bot. 333, 7 (1912) Bot. 127.

Luzon (Bataan), Merrill 3720. Mt. Mariveles.

BULBOPHYLLUM DEAREI (Hort.) Reichb. f. in Flora 71 (April, 1888) 156; Veitch Man. Orch. Pl. pt. 3 (May or June, 1888) 95; Rolfe in Lindenia 8 (1892) t. 345; Cogn. in Dict. Icon. Orch. (1902) Bulbo. t. 2; Rolfe in Orch. Rev. 13 (1905) 197; Ames in Philip. Journ. Sci. 4 (1909) Bot. 674, 7 (1912) Bot. 127, Orch. 5 (1915) 171, 6 (1920) 174, in Merr. in Journ. Roy. Asiat. Soc. Straits Branch, Special No. (1921) 185.

Sarcopodium dearei Hort. ex Gard. Chron. II 20 (1883) 108, fig. 17; Flor. \& Pomol. 22 (1883) 108; Journ. Hort. III 24 (1892) 237; Kew Bull. Add. Ser. 4 (1900) 357.

Sarcopodium godseffanum Hort. ex Gard. Chron. III 7 (1890) 800, nomen.

Bulbophyllum godseffianum Weathers in Gardeners' Mag. 33 (1890) 540 , text cut.

Phyllorchis dearei Ktze. Rev. Gen. Pl. 2 (1891) 677.

Palawan, Mindanao (Lanao), Weber 7, M. S. Clemens s. n. Apparently at low altitudes. In Borneo.

BULBOPHYLLUM DECEPTUM Ames Orch. 5 (1915) 171.

Leyte, Wenzel 0148. Dagami, epiphyte in forest.

BULBOPHYLLUM DISSOLUTUM Ames in Philip. Journ. Sci. 8 (1913) Bot. 429, Orch. 5 (1915) 172.

Luzon (Laguna), B. S. 15069 Ramos, Reillo 5.

BULBOPHYLLUM DORYPHOROIDE Ames Orch. 5 (1915) 172.

Luzon (Ifugao), B. S. $198 \% 3$ McGregor. Mt. Polis.

BULBOPHYLLUM EBRACTEOLATUM Kränzl. in Ann. k. k. Nat. Hofmus. Wien 30 (1916) 58.

Philippines, probably Luzon, Loher s. $n$. Known only from the original description; no material of this species has been seen.

BULBOPHYLLUM ELMERI Ames in Philip. Journ. Sci. 7 (1912) Bot. 127, 136, Orch. 5 (1915) 173.

Luzon (Benguet), Elmer 6618. Baguio.

BULBOPHYLLUM ELONGATUM (Blume) Hassk. Cat. Hort. Bogor. (1844)

39; Miq. Fl. Ind. Bat. 3 (1859) 648; J. J. Sm. Fl. Buitenz. 6 (Orch. Jav.) (1905) 426, Fig.-Atlas (1911) fig. 321; Ames Orch. 5 (1915) 173, non Kränzl. 
Ephippium elongatum Blume Bijdr. (1825) 309.

Cirrhopetalum elongatum Lindl. in Bot. Reg. 29 (1843) sub t. 49;

Reichb. f. in Walp. Ann. 6 (1861) 264.

Bulbophyllum sceptrum Reichb. f. in Walp. Ann. 6 (1861) 254.

Phyllorchis elongata Ktze. Rev. Gen. Pl. 2 (1891) 677.

Phyllorchis sceptrum Ktze. Rev. Gen. Pl. 2 (1891) 678.

Bulbophyllum gigas Ridl. in Journ. Linn. Soc. 32 (1896) 277, Mat.

Fl. Mal. Penin. 1 (1907) 70, fide J. J. Sm.

Mindanao (Cotabato, Bukidnon), Weber 22\%, B. S. 38616 Ramos \& Edaño. Epiphyte in forest at 100 to $900 \mathrm{~m}$ altitude. In Malay Peninsula and Java.

BULBOPHYLLUM ERRATUM Ames Orch. 7 (1922) 85.

Mindanao (Bukidnon), B. S. 38685 Ramos \& Edaño. Mahilucot River, along stream in forest at about $1,400 \mathrm{~m}$ altitude.

BULBOPHYLLUM ESCRITORII Ames Orch. 5 (1915) 174.

Luzon (Benguet, Tayabas), Ramos \& Edaño 104, B. S.207.34 Escritor, F. B. 13088 Curran. Epiphyte up to $2,000 \mathrm{~m}$ in Benguet.

BULBOPHYLLUM EXILE Ames Orch. 2 (1908) 208, text cut, 5 (1915) 175, in Philip. Journ. Sci. 7 (1912) Bot. 127.

Mindanao (Davao), Copeland 1123, R. S. Williams 2563. Mt. Apo, epiphyte at 3,000 to $3,150 \mathrm{~m}$ altitude.

BULBOPHYLLUM EXQUISITUM Ames Sched. Orch. 6 (1923) 84.

Bulbophyllum macgregorii Ames in Philip. Journ. Sci. 7 (1912) Bot.

127, 137, Orch. 5 (1915) 180, non Schltr. (1911).

Luzon (Benguet), B. S. 8322 McGregor, 31900 Santos, Merrill 6624, Haight 1. Epiphyte in mossy forest at 2,100 to $2,400 \mathrm{~m}$ altitude.

BULBopHYLLUM FENIXII Ames in Philip. Journ. Sei. 8 (1913) Bot. 430, Orch. 5 (1915) 175.

Babuyan Islands (Camiguin Island), B. S. 4138 Fenix.

BULBOPHYLLUM FILICOIDES Ames Sched. Orch. 6 (1923) 84, fig. 11.

Mindanao (Agusan), Elmer 14200. Epiphyte at 1,500 $\mathrm{m}$ altitude.

BULBOPHYLLUM GIMAGAANENSE Ames in Philip. Journ. Sci. 7 (1912)

Bot. 127, 130, Orch. 5 (1915) 175.

Luzon (Laguna), Panay (Capiz), Negros (Occidental Negros), Leyte, Reillo 14, B. S. 22885 McGregor, 35462,35518 Martelino \& Edaño, Whitford 1610, Wenzel 0538, 0541, 0544, 0556, 0613, 0615, 0656. Epiphyte at 100 to $700 \mathrm{~m}$ altitude.

BULBOPHYLLUM GLANDULOSUM Ames Sched. Orch. 6 (1923) 87, fig. 12.

Luzon (Tayabas), B. S. 28787 Ramos \& Edaño. Epiphyte at $1,000 \mathrm{~m}$ altitude.

BULBOPHYLLUM GNOMONIFERUM Ames Orch. 2 (1908) 209, text cut,

5 (1915) 175, in Philip. Journ. Sci. 7 (1912) Bot. 127.

Mindanao (Davao), Copeland 1023. Mt. Apo, epiphyte at $1,500 \mathrm{~m}$ altitude. 
BULbophyllum goebelianum Kränzl. in Fedde Repert. 17 (1921) 385.

Philippines, probably Luzon, Loher $s . n$.

Allied to Bulbophyllum dearei (Hort.) Reichb. $f$.

BULBOPHYLLUM GRACILISCAPUM Ames \& Rolfe in Ames Orch. 5 (1915) 175.

Mindanao (Davao), Copeland 112\%. Mt. Apo, epiphyte at 2,000 m altitude.

BULBOPHYLLUM HALCONENSE Ames in Philip. Journ. Sei. 2 (1907)

Bot. 127, Orch. 3 (1908) 54, t. 44, fig. III. C, c, 5 (1915) 176.

Mindoro, Merrill 5832. Mt. Halcon, epiphyte in ridge forest at 1,500 to $2,250 \mathrm{~m}$ altitude.

\section{BULBOPHYLLUM HORTENSOIDES Ames Orch. 6 (1920) 300.}

Leyte, Wenzel 0649, 07r1. Epiphyte in forest at $600 \mathrm{~m}$ altitude.

Probably conspecific with Bulbophyllum braccatum Reichb. f.

BULBOPHYLLUM INVISUM Ames Orch. 7 (1922) 148.

Luzon (Benguet), Ramos \& Edaño 58. Mt. Pulogloco, epiphyte in mossy forest at $2,200 \mathrm{~m}$ altitude.

BULBOPHYLLUM JARENSE Ames Orch. 6 (1920) 301.

Leyte, Wenzel or61. Epiphyte in forest at $500 \mathrm{~m}$ altitude.

BULBOPHYLLUM LANCIFOLIUM Ames in Elm. Leafl. Philip. Bot. 5 (1912) 1583, Orch. 5 (1915) 176.

Negros, Mindanao (Bukidnon, Davao), F. B. 13662 Curran, Elmer 11352, B. S. 38954 Ramos \& Edaño. Epiphyte at about 2,600 m altitude in Davao.

\section{BULBOPHYLLUM LANCILABIUM Ames Orch. 5 (1915) 176.}

Luzon (Laguna), B. S. 20439 Ramos. Epiphyte at 1,000 m altitude.

BULBophyllum LANCIPETALUM Ames in Philip. Journ. Sci. 7 (1912)

Bot. 23, 127, Orch. 5 (1915) 177.

Mindanao (Dapitan, Davao), F. B. 4608 Mearns \& Hutchinson, Copeland $X$. At 2,000 to $2,400 \mathrm{~m}$ altitude.

BULbophylLum LAsioglossum Rolfe ex Ames Orch. 1 (1905) 100,

2 (1908) 3, 210, t. 18, 5 (1915) 177; Ames in Philip. Journ. Sci.

7 (1912) Bot. 127.

Luzon (Bataan), F. B. 797 Borden, Whitford s. n, 138, 1094, Copeland s. n. Epiphyte at about $1,000 \mathrm{~m}$ altitude.

BULBOPHYLLUM LASIOPETALUM Kränzl, in Ann. k. k. Nat. Hofmus. Wien 30 (1916) 57.

Philippines, probably Luzon, Loher s. $n$.

BULBOPHYLLUM LEIBERGII Ames \& Rolfe in Ames Orch. 5 (1915) 177.

Luzon (Bataan), Leiberg 6034, B. S. 702 Foxworthy. Mt. Mariveles at $\$ 00 \mathrm{~m}$ altitude. 
BULBOPHYLLUM LEPANTENSE Ames in Philip. Journ. Sci. 7 (1912)

Bot. 127, 131, Orch. 5 (1915) 178.

Luzon (Bontoc, Lepanto, Benguet), Vanoverbergh 1133, Merrill 4586. B. S. 31778 Santos. Epiphyte at 2,200 to $2,450 \mathrm{~m}$ altitude.

BULBOPHYLLUM LEPTOCAULON Kränzl. in Ann. k. k. Nat. Hofmus.

Wien 30 (1916) 59.

Philippines, probably Luzon, Loher s. $n$.

Known only from the original description and the fragment of the type.

BULbophyllum LeVAnAe Ames Orch. 5 (1915) 178, Sched. Orch, 6 (1923) 89.

Bulbophyllum nymphopolitanum Kränzl, in Ann. k. k. Nat. Hofmus. Wien 30 (1916) 60.

Luzon (Zambales, Sorsogon), Sibuyan, Leyte, B. S. 4985 Ramos, Elmer 12320, 15836, Wenzel 038\%. Epiphyte at $500 \mathrm{~m}$ altitude in Leyte.

BULBOPHYLLUM LEYTENSE Ames Orch. 5 (1915) 178.

Leyte, Mindanao (Agusan), Wenzel 098, Elmer 13805. Epiphyte.

BULBOPHYLLUM LIPENSE Ames Sched. Orch. 3 (1923) 22.

Mindanao (Bukidnon), B. S. 38582 Ramos \& Edaño. Epiphyte in damp forest at $1,300 \mathrm{~m}$ altitude.

BULBOPHYLLUM LOHERIANUM (Kränzl.) Ames comb. nov.

Cirrhopetalum loherianum Kränzl. in Ann. k. k. Nat. Hofmus. Wien 30 (1916) 61.

Philippines, probably Luzon, Loher.

Known only from the original description and the fragmentary type specimen.

BULBOPHYLLUM LONGIPETIOLATUM Ames in Elm. Leafi. Philip. Bot.

5 (1912) 1584, Orch. 5 (1915) 180.

Mindanao (Davao), Elmer 10883. Todaya, Mt. Apo, epiphyte in humid forest at about $1,300 \mathrm{~m}$ altitude.

BULBOPHYLLUM LUzonense Ames in Philip. Journ. Sci. 7 (1912) Bot. 127, 141, Orch. 5 (1915) 180, Sched. Orch. 6 (1923) 89.

Luzon (Cagayan, Nueva Vizcaya, Laguna), Leyte, Mindanao (Agusan), F. B. 16787 Curran, B. S. 20100 McGregor, 16557 Ramos, Serrato s. n.., Wenzel 084, 0167, 0405, 0765, Elmer 13623, 13908. Epiphyte, at 120 to $500 \mathrm{~m}$ altitude in Leyte.

BULBOPHYLLUM MACULOSUM Ames Orch. 5 (1915) 180.

Leyte, Wenzel 0151, 0199. Dagami, epiphyte in forest at $60 \mathrm{~m}$ altitude.

BULBOPHYLLUM MAKOYANUM Reichb, f. in Gard. Chron. II 11 (1879)

234; Ridl. Mat. Fl. Mal. Penin. 1 (1907) 81; Ames in Philip. Journ.

Sci. 6 (1911) Bot. 55, 7 (1912) Bot. 127, Orch. 5 (1915) 181.

Cirrhopetalum makoyanum Reichb. f. in Gard. Chron. II 11 (1879)

234; Hook. f. in Bot. Mag. 118 (1892) t. 7259; Ridl. in Journ.

Linn. Soc. 32 (1896) 284.

$200823-25$ 
Luzon (Ifugao, Rizal), Polillo, Busuanga, Mindanao (Agusan), B. S. 10438, 19990 McGregor, 19185 Reillo, Weber 37, 407 in part. Epiphyte, apparently at low altitudes. In Malay Peninsula.

BULBOPHYLLUM MARCIDUM Ames Sched. Orch. 6 (1923) 90, fig. 12.

Mindanao (Bukidnon), B. S. 38573 Ramos \& Edaño. Mt. Lipa, epiphyte at $1,630 \mathrm{~m}$ altitude.

BULbophyLLUM MARIVELENSE Ames in Philip. Journ. Sci. 7 (1912) Bot. 127, 132, Orch. 5 (1915) 181.

Luzon (Bataan), Merrill 3738. Mt. Mariveles, epiphyte on exposed ridge at $1,100 \mathrm{~m}$ altitude.

BULBOPHYLLUM MASAGANAPENSE Ames Orch. 6 (1920) 302.

Luzon (Rizal), Leyte, B. S. 29714 Ramos \& Edaño, 41632, 41639 Ramos, Wenzel 0681. Epiphyte at 200 to $1,300 \mathrm{~m}$ altitude.

BULBOPHYLLUM MEARNSII Ames in Philip. Journ. Sci. 8 (1913) Bot. 430, Orch. 5 (1915) 181.

Bulbophyllum carinatum Ames in Philip. Journ. Sci. 7 (1912) Bot. 126,140 , nec Naves nec Cogn.

Luzon (Sorsogon), Mindanao (Dapitan), Elmer 17039, F. B. 4593 Mearns \& Hutchinson. At $900 \mathrm{~m}$ altitude in Mindanao.

BULBOPHYLLUM MELANOGLOSSUM Kränzl, in Ann. k. k. Nat. Hofmus. Wien 30 (1916) 60.

Philippines, probably Luzon, Loher s. $n$.

BULBOPHYLLUM MERRITTII Ames in Philip. Journ. Sci. 2 (1907) Bot. 334, 7 (1912) Bot. 127, Orch. 5 (1915) 181.

Mindoro, F. B. 4398 Merritt. Mt. Halcon, at about $500 \mathrm{~m}$ altitude.

BULBOPHYLLUM MINDANAENSE Ames in Elm. Leafl. Philip. Bot. 5 (1912) 1585, Orch. 5 (1915) 181.

Leyte, Mindanao (Davao), Wenzel 0295, 0397, 0447, 0743, 0744, 0811. 0\$15, Elmer 11307, 116.25, 14160. Epiphyte in humid forest at 500 to $1,300 \mathrm{~m}$ altitude.

BULBOPHYLLUM MINDORENSE Ames in Philip. Journ. Sci. 2 (1907)

Bot. 334, 7 (1912) Bot. 127, Orch. 3 (1908) 58, t. 45, 5 (1915) 182.

Luzon (Camarines), Mindoro, Leyte, B. S. 22185 Ramos, Merrill 5796, Wenzel 0758. Epiphyte in damp forest at 500 to $1,000 \mathrm{~m}$ altitude.

BULBOPHYLLUM MONSTRABILE Ames Orch. 5 (1915) 182.

Leyte, Wenzel 0443. Jaro, epiphyte in forest at $500 \mathrm{~m}$ altitude.

BULBOPHYLLUM NEgRosianum Ames in Elm. Leafl. Philip. Bot. 5 (1912) 1586, Orch. 5 (1915) 182.

Negros (Oriental Negros), Elmer 9821. Cuernos Mountains, epiphyte in mossy woods at about $1,100 \mathrm{~m}$ altitude.

BULBOPHYLLUM ORTHOGLOSSUM Wendl. \& Kränzl. in Gard. Chron. III 19 (1896) 326; Ames in Philip. Journ. Sci. 7 (1912) Bot. 128, Orch. 5 (1915) 183. 
"In Sarangni insula det. W. Micholitz, Freder, Sander Verulamensis collecta indefessus." - Kränzlin. Probably Sarangani Island, off the southern end of Mindanao, is intended. Only a single flower and a sketch of the inflorescence have been seen.

BULBOPHYLLUM OTHONIS (Ktze.) J. J. Sm, in Bull. Gard. Bot. Buitenz. II 8 (1912) 26; Ames Orch. 5 (1915) 183.

Cirrhopetalum nutans Lindl. in Bot. Reg. 25 (1839) Misc. 71, 29 (1843) sub t. 49; Hook. in Bot. Mag. 75 (1849) t. 4418; Miq. Fl. Ind. Bat. 3 (1859) 653.

Bulbophyllum nutans Reichb. f. in Walp. Ann. 6 (1861) 260; Naves Novis. App. (1882) 235; Ames in Philip. Journ. Sci. 7 (1912) Bot. 24, 127, non Thou.

Phyllorchis othonis Ktze. Rev. Gen. Pl. 2 (1891) 677.

Luzon (Sorsogon), Polillo, Leyte, Elmer 16933, B. S. 9077 Robinson, Wenzel 0141, 0192. Epiphyte, apparently at low altitudes.

BULBOPHYLLUM PAMPANGENSIS Ames Sched. Orch. 6 (1923) 92.

Luzon (Pampanga), Merrill 11214.

BULBOPHYLLUM PAPILLIPETALUM Ames Orch. 7 (1922) 149.

Luzon (Benguet), Ramos \& Edaño 79. Mt. Boadan, epiphyte, on mossy forest slope at about $2,300 \mathrm{~m}$ altitude.

BULBOPHYLLUM PAUCIFLORUM Ames in Philip. Journ. Sci. 7 (1912) Bot. 127, 132, Orch. 5 (1915) 184.

Luzon (Bontoc), Vanoverbergh 1381. Epiphyte at $1,650 \mathrm{~m}$ altitude.

BULBOPHYLLUM PERAMOENUM Ames in Philip. Journ. Sci. 8 (1913)

Bot. 430, Orch. 5 (1915) 184.

Mindoro, Mindanao (Zamboanga), Merrill 5882, 8271.

BULBOPHYLLUM PHILIPPINENSE Ames Orch. 6 (1920) 303.

Leyte, Wenzel 0659. Jaro, epiphyte in forest at $500 \mathrm{~m}$ altitude.

BULBOPHYLLUM PLEUROTHALLOIDES Ames in Philip. Journ. Sci. 2

(1907) Bot. 335, 7 (1912) Bot. 128, Orch. 3 (1908) 55, t. 44, fig. II $B, b, 5$ (1915) 184 .

Mindoro, Merrill 6128. Mt. Halcon, epiphyte in mossy forest at 1,500 mi altitude.

BULBOPHYLLUM PLUMATUM Ames Orch. 5 (1915) 184.

Mindanao (Bukidnon), B. S. 21479 Escritor.

BULBOPHYLLUM PROFUSUM Ames in Philip. Journ. Sci. 7 (1912) Bot. 128, 137, Orch. 5 (1915) 185.

Luzon (Cagayan, Rizal, Sorsogon), Sibuyan, Leyte, Palawan, Basilan, Mindanao (Agusan, Butuan, Surigao). A not uncommon and widely distributed species. Epiphyte apparently at rather low altitudes.

BULBOPHYLLUM PUGUAHAANENSE Ames Orch. 5 (1915) 185.

Leyte, Wenzel 0128. Dagami, Puguahaan, epiphyte at $120 \mathrm{~m}$ altitude. BULbophYLLUM REILLOI Ames in Philip. Journ. Sci. 8 (1913) Bot. 431, Orch. 5 (1915) 186.

Luzon (Laguna), Reillo 24. 
BULBOPHYLLUM SANTOSII Ames Orch. 5 (1915) 186.

Luzon (Laguna, Camarines), Leyte, B. S. 20461 Ramos, Santos s. n., Wenzel 0269. Epiphyte, up to $750 \mathrm{~m}$ altitude in Leyte.

BULBOPHYLLUM SAPPHIRINUM Ames Orch. 5 (1915) 187.

Leyte, Wenzel 092, 0125, 0136, 0231, 0232. Dagami, epiphyte in forest at $60 \mathrm{~m}$ altitude.

BULBOPHYLLUM SAUROCEPHALUM Reichb. f. in Gard. Chron. II 26 (1886) 262; Rolfe in Orch. Rev. 11 (1903) 190, in textu, 13 (1905) 244; Ames in Philip. Journ. Sci. 7 (1912) Bot. 128, Orch. 5 (1915) 187.

No specimens of this species have been seen.

BULBOPHYLLUM SEMPITERNUM Ames Orch. 6 (1920) 304.

Leyte, Wenzel 0260, 0287, 0442, 0472, 0524, 0546, 0555, 0589, 0595, 0607, 0610, 0675, 0751, 0810, 0820, 0841, 0848, 0849, 0873, 0893, 0913. Epiphyte in forest at 500 to $750 \mathrm{~m}$ altitude.

BULBOPHYLLUM SENSILE Ames Orch. 5 (1915) 188.

Luzon (Bontoc), Vanoverbergh 1134, 2816, 3918. At 1,600 to 1,700 $m$ altitude.

BULBOPHYLLUM SIBUYANENSE Ames in Elm. Leafl. Philip. Bot. 5 (1912) 1587, Orch. 5 (1915) 189.

Sibuyan, Elmer 12140. Mt. Giting-giting, epiphyte in humid forest at about $400 \mathrm{~m}$ altitude.

BULBOPHYLLUM SIMULACRUM Ames Orch. 5 (1915) 189.

Leyte, Wenzel s. n., 0268, 0297, 0409, 0692, 0699, 0773, 0786, 0812. Epiphyte in forest at 500 to $750 \mathrm{~m}$ altitude.

BULBOPHYLLUM STELLATUM Ames in Philip. Journ. Sci. 7 (1912) Bot. 128, 141, Orch. 5 (1915) 190.

Luzon (Bontoc), Vanoverbergh 1360, 3914. Epiphyte at 1,650 to 1,750 $m$ altitude.

BULBOPHYLLUM STRAMINEUM Ames Orch. 5 (1915) 190.

Palawan, Merrill 956\%, F. B. 2157\% Danao. Epiphyte at 75 to $500 \mathrm{~m}$ altitude.

BULBOPHYLLUM SUBAEQUALE Ames Sched. Orch. 6 (1923) 93.

Luzon (Rizal), B. S. 29296 Ramos \& Edaño. Epiphyte at $300 \mathrm{~m}$ altitude.

BULBOPHYLLUM SYLLECTUM Kränzl. in Fedde Repert. 17 (1921) 383.

Philippines, probably Luzon, Loher s. $n$.

Owing to the scanty material at hand for study, this is maintained as a species, though it is apparently at best hardly more than a variety of Bulbophyllum luzonense Ames; the slight differences are presumably the result of cultivation. 
BULBOPHYLLUM TOPPINGII Ames in Philip. Journ. Sci. 8 (1913) Bot. 432, Orch. 5 (1915) 191.

Luzon (Benguet), Topping s. n., Merrill sor 5\%. Epiphyte at about 1,500 m altitude.

BULBOPHYLLUM TRIGONOSEPALUM Kränzl. in Fedde Repert. 17 (1921) 384.

Philippines, probably Luzon, Loher s. n.

From the material examined this appears to be a large variety of $B u l-$ bophyllum levanae Ames.

BULBOPHYLLUM TRISETUM Ames in Philip. Journ. Sci. 7 (1912) Bot. 128, 142, Orch. 5 (1915) 191:

Luzon (Benguet), F. B. 15899 Bacani. Itogan to Baguio, epiphyte.

BULBOPHYLLUM VAGANS Ames \& Rolfe in Philip. Journ. Sci. 2 (1907)

Bot. 335, 7 (1912) Bot. 128; Ames Orch. 2 (1908) 210, text cut, 5 (1915) 191.

Luzon (Benguet, Bataan, Laguna, Camarines), Mindanao (Camiguin Island, Agusan), F. B. 2205 Meyer, $15 \% 39$ Curran \& Merritt, Merrill s. n. (cult.), Whitford 1120, B. S. 14423 Ramos, 1636 Foxworthy, Santos s. n., Elmer 13699, Weber 30 . Vinelike epiphyte at 800 to $1,000 \mathrm{~m}$ altitude.

Var. ANGUSTUM Ames in Philip. Journ. Sci. 2 (1907) Bot. 210, 7 (1912) Bot. 128.

Bulbophyllum vagans Ames \& Rolfe var. linearifolium Ames Orch. 5 (1915) 191, nomen.

Mindoro, Merrill 6218. Mt. Halcon, epiphyte in mossy forest at $210 \mathrm{~m}$ altitude.

BULBOPHYLLUM VANOVERBERGHII Ames in Philip. Journ. Sci. 7 (1912) Bot. 133, Orch. 5 (1915) 191.

Luzon (Bontoc), Vanoverbergh $136 \%$.

BULBOPHYLLUM WEBERI Ames in Philip. Journ. Sci. 7 (1912) Bot. 128, 142, Orch. 5 (1915) 192.

Cirrhopetalum thouarsii Lindl. in Bot. Reg. 24 (1838) t. 11, non C. thovarsii Lindl. Gen. \& Sp. Orch. (1830) 58; Hook. in Bot. Mag. 72 (1846) t. 4237 (excl. syn.).

Bulbophyllum longiflorum Reichb. f. in Walp. Ann. 6 (1861) 260, quoad Philip.; Naves Novis. App. (1880) 235; Ames in Philip. Journ. Sci. 7 (1912) Bot. 127, non Thou.

Bulbophyllum baucoense Ames Orch. 5 (1915) 166.

Luzon (Bontoc, Rizal), Mindanao (Bukidnon, Davao), Vanoverbergh 2613, B. S. 19239 Reillo, 22253, 41917 Ramos, Weber 8, 194, 289. On rocks and in trees at 122 to $1,500 \mathrm{~m}$ altitude.

BULBOPHYLLUM WENZELII Ames in Philip. Journ. Sci. 8 (1913) Bot. 432, Orch. 5 (1915) 192.

Leyte, Wenzel s. n., 0162, 01\%8. Dagami, epiphyte in forest at about $60 \mathrm{~m}$ altitude. 
BULBOPHYLLUM. WHITFORDII Rolfe ex Ames Orch. 1 (1905) 100, 2 (1908) 211, 5 (1915) 192, in Philip. Journ. Sci. 7 (1912) Bot. 128.

Luzon (Bataan), Busuanga, Basilan, Mindanao (Davao), Whitford 61, Weber 403, s. n., F. B. 4021 Hutchinson, R. S. Williams 2915. Epiphyte.

BulbophylLUm ZAmbALense Ames in Philip. Journ. Sci. 7 (1912)

Bot. 134, 8 (1913) Bot. 433, Orch. 5 (1915) 192.

Luzon (Zambales), B. S. 4996 Ramos.

BUlbophylLUm zAmBoANGense Ames in Philip. Journ. Sci. 8 (1913) Bot. 433, Orch. 5 (1915) 192.

Mindanao (Zamboanga), F. B. 9165 Whitford \& Hutchinson. Epiphyte at $20 \mathrm{~m}$ altitude.

EXCLUDED OR SYNONYMOUS SPECIES

Bulbophyluum Aurecemum Boxall ex Naves Novis. App. (1882) 235, nomen.

BULbophyllum BALAENICEPS Reichb. f. in Hamb. Gartenz. 19 (1863) 280,

Xen. Orch. 2 (1870) 147, t. 15\%, fig. 1-6; Naves Novis. App. (1882)

234; Ames in Philip. Journ. Sci. 7 (1912) Bot. 126, Orch. 5 (1915) 165.

This species was described from material in the garden of Consul Schiller. The origin of the plant was unknown and it was doubtfully ascribed to the Philipines by Reichenbach. There seems no evidence that it occurs in the Philippines.

Bulbophyllum BAucoense Ames Orch. 5 (1915) $166=$ Bulbophyllum weberi Ames.

Bulbophyludm biflorum Teijsm. \& Binn, apud Naves Novis. App. (1882) 234.

Bulbophyllum CAREyANUm Spreng. apud Ames in Philip. Journ. Sci. 7 (1912) Bot. 126.

The type of the species was from Nepal, and there is no evidence to show that it occurs in the Philippines.

Bulbophyllum CARINAtum Ames in Philip. Journ. Sci. 7 (1912) Bot. 126, 140, nec Naves nec Cogn. = Bulbophyllum mearnsii Ames.

Bulbophyllum carinatum (Teijsm. \& Binn.) Naves Novis. App. (1882) 235.

BulbophylLum CHEIRI Lindl. in Bot. Reg. 30 (1844) Misc. 44; Reichb. f. in Walp. Ann. 6 (1861) 246; Naves Novis. App. (1882) 235; Ames in Philip. Journ. Sci. 7 (1912) Bot. 127, Orch. 5 (1915) $169=$ Bulbophyllum blumei (Lind1.) J. J. Sm.

Bulbophyllum chryseum Ames in Philip. Journ. Sci. 6 (1911) 54, quoad spec. $=$ Bulbophyllum bolsteri Ames.

BulbopHyllum COREYANUM Spreng. apud Llanos ex Naves \& F.-Vill. in Blanco Fl. Filip. ed. 3, $4^{1}$ (1880) 100; Naves Novis. App. (1882) 235. 
BulbophyLluM CUPREUM Lindl. in Bot. Reg. 24. (1838) Misc. 95; Reichb.

f. in Walp. Ann. 6 (1861) 257; Naves Novis. App. (1882) 235.

Lindley's statements in the original description that this species came from Manila and was collected by Cuming seem doubtful, as no specimens referable to it from the Philippines have been seen.

BUlbophyllum DAYANum Reichb. f. apud Naves Novis. App. (1882) 234.

Bulbophyllum DENSUM Spreng. apud Llanos ex Naves \& F.-Vill. in Blanco Fl. Filip. ed. 3, $4^{x}$ (1880) 100; Naves Novis. App. (1882) 235.

Bulbophyllum Echinochilum Kränzl. in Fedde Repert. 17 (1921) 385; Ames Sched. Orch. 6.(1923) 84.

Kränzlin, l. c., states: "Philippinen. Manila (Loher). Blüthe in kgl. botan. Garten zu München-Nymphenburg in Dezember 1916.

"Durch die Form und Behaarung des Labellums erinnert diese Art sehr stark an B. Calamaria Lindl. westafrikanischer Herkunft, * * *."

The record of the Philippine origin of this species is doubted; nothing of its alliance has been seen from the Philippines and its affinities are African, as pointed out by Kränzlin. Bulbophyllum calamaria Lindl. has a similar labellum, but its habit is very different.

BULBOPHyLLUM GODSEFFiANUM Weathers in Gardeners' Mag. 33 (1890) 540, text cut $=$ Bulbophyllum dearei (Hort.) Reichb. $\mathbf{f}$.

BulbopHyllum henschaliI Boxall ex Naves Novis. App. (1882) 235, nomen.

Bulbophyllum lasianthum Lindl. in Gard. Chron. (1855) 53; Reichb. f. in Walp. Ann. 6 (1861) 255; Naves Novis. App. (1882) 234; Ames in Philip. Journ. Sci. 7 (1912) Bot. 127.

The type of this was Sumatran; the living specimen examined by Lindley, said to. have come from Manila, imported by Loddiges, probably came from the Malay Peninsula or Sumatra. No record for this species as Philippine is known other than Lindley's original statement.

Bulbophylum lobbir Lindl. apud Naves Novis. App. (1882) 234.

BUlBophylium LONGIFLORUM Reichb. f. in Walp. Ann. 6 (1861) 260, quoad Philip.; Naves Novis. App. (1882) 235; Ames in Philip. Journ. Sci. 7 (1912) Bot. 127, non Thou. = Bulbophyllum weberi Ames.

Bulbophyllum MacGregoril Ames in Philip. Journ. Sci. 7 (1912) Bot. 127, 137, Orch. 5 (1915) 180, non Schltr. (1911) = Bulbophyllum exquisitum Ames.

Bulbophyllum maculatum Boxall ex Naves Novis. App. (1882) 235, nomen. BULBophyllum MAXILLARE Reichb. f. in Walp. Ann. 6 (1861) 248; Naves Novis. App. (1882) 235; Ames in Philip. Journ. Sci. 7 (1912) Bot. 127, Orch. 5 (1915) $181=$ Bulbophyllum blumei (Lindl.) J. J. Sm. BulbophyluUm MEdUSAe (Lindl.) Naves Novis. App. (1882) 235.

Bulbophyllum MEgalanthum Griff. apud Naves Novis. App. (1882) 235. Bulbophyllum mucronatum Lindl. apud Naves Novis. App. (1882) 234. 
Bulbophyllum Nutans Reichb. f. in Walp. Ann. 6 (1861) 260; Naves Novis. App. (1882) 235; Ames in Philip. Journ. Sci. 7 (1912) Bot. 24, 127, non Thou. = Bulbophyllum othonis (Ktze.) J. J. Sm.

BULBOPHYLLUM NYMPHOPOLITANUM Kränzl. in Ann. k. k. Nat. Hofmus. Wien 30 (1916) $60=$ Bulbophyllum levanae Ames.

Bulbophyllum odoratum Lindl. apud Naves Novis. App. (1882) 234.

BULBOPHYLLUM PENICILLIUM Rolfe in Journ. Bot. 23 (1885) 215; Vidal Phan. Cuming. Philip. (1885) 149, Rev. Pl. Vasc. Filip. (1886) 267; Ames in Philip. Journ. Sci. 7 (1912) Bot. 128, non Par. \& Reichb. f. = Bulbophyllum adenopetalum Lindl.

Bulbophyllum purpureum (Reichb. f.) Naves Novis. App. (1882) 234.

Bulbophyldum Ramosil Ames in Philip. Journ. Sci. 7 (1912) Bot. 25, 128, Orch. 5 (1915) $186=$ Bulbophyllum adenopetalum Lindl.

Bulbophyllum REgNeliI Reichb. f. apud Naves Novis. App. (1882) 234.

Bulbophyllum Reticulatum Boxall ex Naves Novis. App. (1882) 234, nomen.

Bulbophyllum teneluum Lindl. apud Naves Novis. App. (1882) 234.

BulbopHYLlum VAGans Ames \& Rolfe var. LINEARIFolium Ames Orch. 5 (1915) 191 , nomen $=$ Bulbophyllum vagans Ames \& Rolfe var. angustum Ames.

67. OCTARRHENA Thwaites

OCTARRHENA AMESIANA Schltr. in Fedde Repert. 9 (1911) 439; Ames Orch. 5 (1915) 192.

Phreatia parvula Ames Orch. 2 (1908) 204, non Benth. ex Hook. f. Mindanao (Davao), Copeland 1124, 1427, Elmer 10568, 11413, $1145 \%$. Mt. Apo, epiphyte at about $2,000 \mathrm{~m}$ altitude.

OCTARRHENA CAULESCENS Ames Orch. 5 (1915) 192.

Phreatia caulescens Ames Orch. 2 (1908) 200, text cut; Kränzl. in Engl. Pflanzenreich IV. 50. II. B. 23 (1911) 12.

Luzon (Bontoc, Lepanto, Ifugao, Benguet). A fairly common species in the provinces mentioned. Epiphyte in mossy forest at 1,650 to 2,300 $m$ altitude.

OCTARRHENA ELMERI Ames Orch. 5 (1915) 192.

Phreatia elmeri Ames in Elm. Leafl. Philip. Bot. 5 (1912) 1581.

Luzon (Camarines), Mindanao (Davao), B. S. 22184 Ramos, Elmer 10622. Epiphyte in forest at $2,000 \mathrm{~m}$ altitude in Mindanao.

OCTARRHENA ENSIFOLIA (Ames) Schltr. in Fedde Repert. 9 (1911) 217; Ames Orch. 5 (1915) 192.

Phreatia ensifolia Ames Orch. 2 (1908) 202, text cut; Kränzl. in

Engl. Pflanzenreich IV. 50. II. B. 23 (1911) 15.

Luzon (Bataan), Negros (Oriental Negros), Mindanao (Agusan), Whitford 153, Elmer 9733, 13851. Epiphyte at about 1,250 $\mathrm{m}$ altitude in Luzon. 
OCTARRHENA GEMMIFERA Ames Orch. 5 (1915) 193.

Leyte, Wenzel 0254, 0293, 0404, 0526, 054\%, 0592. Jaro, epiphyte at 500 to $700 \mathrm{~m}$ altitude.

\section{THELASIS Blume}

THELASIS CAPITATA Blume Bijdr. (1825) 386; Lindl. Gen. \& Sp. Orch. (1833) 253; Blume Mus. Bot. Lugd.-Bat. 2 (1856) 186 (excl. syn. Euphroboscis pygmaea Griff.) ; Reichb. f. in Bonpl. 5 (1857) 37; Blume Fl. Jav. Orch. (1858) 19 (Orch. Arch. Ind. 20) t. 7, fig. 1; Miq. Fl. Ind. Bat. 3 (1859) 700, Sumatra (1862) 275; Naves Novis. App. (1882) 244; Hook. f. Fl. Brit. Ind. 6 (1890) 86; Ridl. in. Trans. Linn. Soc. II 3 (1893) 376, in Journ. Linn. Soc. 32 (1896) 393; Schltr. in Mém. Herb. Boiss. 21 (1900) 69; J. J. Sna. F. Buitenz. 6 (Orch. Jav.) (1905) 493, Fig.-Atlas (1911) fig. 9\%2: Ridl. Mat. Fl. Mal. Penin. 1 (1907) 199; Ames Orch. 2 (1908) 212, 5 (1915) 194; Schltr, in Engl. Bot. Jahrb. 45, Beibl. 104 (1911) 54; Krärzl. in Engl. Pflanzenreich IV. 50. II. B. 23 (1911) 41: Ames in Merr. in Journ. Roy. Asiat. Soc. Straits Branch, Special No. (1921) 190.

Thelasis capitata Blume var. major Blume Fl. Jav. Orch. (1858) 19 (Orch. Arch. Ind. 20).

Thelasis ochreata Lindl. in Journ. Linn. Soc. 3 (1859) 63; Miq. Fl. Ind. Bat. 3 (1859) 701; Naves Novis. App. (1882) 244; Ridl. in Journ. Linn. Soc. 31 (1896) 303.

Thelasis elongata Blume var. major Ridl. in Journ. Linn. Soc. 32 (1896) 393, fide Ridl. in Mat. Fl. Mal. Penin. 1. c.

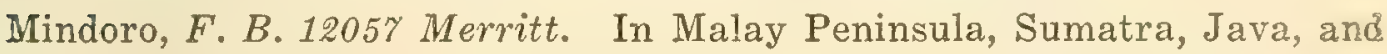
Borneo.

THELASIS CARINATA Blume Bijdr. (1825) 386; Lindl. Gen. \& Sp. Orch. (1833) 253, Mus. Bot. Lugd.-Bat. 2 (1856) 187, Fl. Jav. Orch. (1858) 21 (Orch. Arch. Ind. 24) t. 7, fig. 3; J. J. Sm. Fl. Buitenz. 6 (Orch. Jav.) (1905) 497, Fig.-Atlas (1912) fig. 375; Ames in Philip. Journ. Sci. 2 (1907) Bot. 336; Kränzl. in Engl. Pflanzenreich IV. 50. II. B. 23 (1911) 43; Ames Orch. 5. (1915) 194, in Merr. in Journ. Roy. Asiat. Soc. Straits Branch, Special No. (1921) 190.

Phreatia carinata Miq. Fl. Ind. Bat. 3 (1859) 655, Sumatra (1862) 274.

Eria carinata Reichb. f. in Seem. Fl. Vit. (1868) 301, in obs.

Thelasis elata Hook. f. Fl. Brit. Ind. 6 (1890) 87; Ridl. in Trans. Linn. Soc. II 3 (1893) 376; Hook. f. in Hook. Icon. P1. 22 (1893) t. 2156.

Oxyanthera elata Hook. f. in Hook. Icon. Pl. 22 (1893) sub t. 2156; Ridl. in Journ. Linn. Soc. 31 (1896) 303, 32 (1896) 394, Mato F1. Mal. Penin. 1 (1907) 200.

Oxyanthera carinata Schltr. in Mém. Herb. Boiss. 21 (1900) 76, in Fedde Repert. 10 (1911) 186.

Babuyan Islands (Camiguin Island), Luzon (Bataan, Laguna, Sorsogon), Mindoro, Negros, Leyte, Basilan, Mindanao (Zamboanga, Agusan). A fairly common and widely distributed species in the Philippines. Epi- 
phyte, apparently at low altitudes. In Malay Peninsula, Sumatra, Java, Celebes, and Borneo.

THELASiS MICRANTHA (Brongn.) J. J. Sm. Fl. Buitenz. 6 (Orch. Jav.) (1905) 495, Fig.-Atlas (1911) fig. 374; Ames Orch. 2 (1908) 213, 5 (1915) 194; Kränzl. in Engl. Pflanzenreich IV. 50. II. B. 23 (1911) 44; Ames in Merr. in Journ. Roy. Asiat. Soc. Straits Branch, Special No. (1921) 191.

Oxyanthera micrantha Brongn. in Duperr. Voy. Coquille Phan. (1834) 198, t. s7 B; Schltr. in Mém. Herb. Boiss. 21 (1900) 77; Ridl. Mat. Fl. Mal. Penin. 1 (1907) 200.

Thelasis contracta Blume Mus. Bot. Lugd.-Bat. 2 (1856) 187, Fl. Jav. Orch. (1858) 21 (Orch. Arch. Ind. 24) t. 5 B; Miq. Fl. Ind. Bat. 3 (1859) 701.

Thelasis carinata Reichb. f. in Trans. Linn. Soc. 30 (1874) 136; Grant. Orch. Burma (1895) 314, non Blume.

Thelasis decurva Hook. f. Fl. Brit. Ind. 6 (1890) 87; Ridl. in Trans. Linn. Soc. II 3 (1893) 376; Hook. f. in Hook. Icon. Pl. 22 (1893) t. $215 \%$.

Oxyanthera decurva Hook. f. in Hook. Icon. Pl. 22 (1893) sub t. 2157; Ridl. in Journ. Linn. Soc. 31 (1896) 303, 32 (1896) 394.

Luzon (Rizal, Laguna, Tayabas), Leyte, Mindanao (Zamboanga), B. S. 974, 15393, 23514 Ramos, Reillo s. n., 17083 Robinson, Weber 5, Wenzel $048, R$. S. Williams 2421, Elmer 7403. Epiphyte at 60 to $800 \mathrm{~m}$ altitude. In Malay Peninsula, Sumatra, Java, and Borneo.

THELASIS OBTUSA Blume Bijdr. (1825) 386; Lindl. Gen. \& Sp. Orch. (1833) 253; Blume Mus. Bot. Lugd.-Bat. 2 (1856) 186, Fl. Jav. Orch. (1858) 18 (Orch. Arch. Ind. 20); Schltr. in Mém. Herb. Boiss. 21 (1900) 72; J. J. Sm. Fl. Buitenz. 6 (Orch. Jav.) (1905) 492, Fig.-Atlas (1911) fig. 371; Ames in Philip. Journ. Sci, 4 (1909) Bot. 675; Kränzl. in Engl. Pflanzenreich IV. 50. II. B. 23 (1911) 43; Ames Orch. 5 (1915) 194.

Luzon (Bataan), Leiberg 6046. Mt. Mariveles, epiphyte at $900 \mathrm{~m}$ altitude. In Java.

THELASIS TRIPTERA Reichb. f. in Bonpl. 3 (1855) 219, in Walp. Ann. 6 (1864) 923; Naves Novis. App. (1882) 244; Hemsl. Bot. Chal. Exped. $1^{\circ}$ (1885) 248; Vidal Phan. Cuming. Philip. (1885) 151, Rev. Pl. Vasc. Filip. (1886) 272; Kränzl. in K. Schum. \& Lauterb. Fl. Deutsch. Schutzgeb. (1901) 249; Rolfe in Journ. Linn. Soc. 36 (1903) 39.

Thelasis elongata Blume Mus. Bot. Lugd.-Bat. 2 (1856) 187, Fl. Jav. Orch. (1858) 20 (Orch. Arch. Ind. 23) t. \%, fig. 2, $t .5$; Miq. Fl. Ind. Bat. 3 (1859) 701; Hook. f. Fl. Brit. Ind. 6 (1890) 86; Ridl. in Trans. Linn. Soc. II 3 (1893) 375, in Journ. Linn. Soc. 32 (1896) 393; Schitr. in Mém. Herb. Boiss. 21 (1900) 70; J. J. Sm. Fl. Buitenz. 6 (Orch. Jav.) (1905) 494, Fig.-Atlas (1911) fig. 373; Ridl. Mat. Fl. Mal. Penin. 1 (1907) 199; Ames Orch. 2 (1908) 212, 5 (1915) 194; Kränzl. in Engl. Pflanzenreich IV. 50. I1. B. 23 (1911) 41, fig. 5, T-X; Schltr. in Fedde Repert. Beihefte 1 (Orch. Deutsch-Neu-Guin.) (1913) 908; Ames in Merr. in Journ. Roy. Asiat. Soc. Straits Branch, Special No. (1921) 190. 
Thelasis zollingeri Reichb. f. in Bonpl. 5 (1857) 37.

Thelasis pygmaea Hance in Journ. Linn. Soc. 13 (1872) 127, F1. Hongk. Suppl. (1872) 39, non Lindt.

Thelasis hongkongensis Rolfe in Kew Bull. (1896) 199, in Journ. Linn. Soc. 36 (1903) 39; Schltr. in Fedde Repert. Beihefte 4 (Orch. Sino-Jap. Prodr.) (1919) 257.

Luzon (Bataan, Rizal, Sorsogon), Polillo, Panay (Antique), Leyte, Mindanao (Zamboanga, Cotabato, Agusan, Surigao, Davao), Cagayan Sulu. A fairly common and widely distributed species in the Philippines. Epiphyte at 10 to $1,500 \mathrm{~m}$ altitude. In Malay Peninsula, China, Sumatra, Java, Borneo, and the Admiralty Islands.

\section{EXCLUDED OR SYNONYMOUS SPECIES}

Thelasis elongata Blume Mus. Bot. Lugd.-Bat. 2 (1856) 187; Schltr. in Mém. Herb. Boiss. 21 (1900) 70; J. J. Sm. Fl. Buitenz. 6 (Orch. Jav.) (1905) 494, Fig.-Atlas (1911) fig. 373; Ames Orch. 2 (1908) 212, 5 (1915) 194; Kränzl. in Engl. Pflanzenreich IV. 50 II. B. 23 (1911) 41, fig. 5, T-X; Ames in Merr. in Journ. Roy. Asiat. Soc. Straits Branch, Special No. (1921) $190=$ Thelasis triptera Reichb. $f$.

Thelasis ochreata Lindl, in Journ. Linn. Soc. 3 (1859) 63; Naves Novis. App. (1882) $244=$ Thelasis capitata Blume.

\section{PHREATIA Lindley}

PHREATIA AMESI! Kränzl. in Engl. Pflanzenreich IV. 50. I1, B. 23 (1911) 16; Ames Orch. 5 (1915) 195, 6 (1920) 207.

Phreatia myosurus Ames Orch. 2 (1908) 203, quoad spec. (excl. syn.) non Lindl.

Luzon (Bontoc, Bataan, Sorsogon), Panay (Antique), Negros (Oriental Negros), Leyte, Mindanao (Agusan), Vanoverbergh 707, 1290a, 1477a, 3683, Merrill 3854, Whitford 1361, Leiberg 6061, B. S. 23390 Ramos, 32363 McGregor, Elmer 10354, 13804, Wenzel 0713, 0829. Epiphyte, at 300 to $1,600 \mathrm{~m}$ altitude. In Borneo.

PHREATIA ARISTULIFERA Ames Orch. 2 (1908) 199, text cut, 5 (1915)

195; Kränzl. in Engl. Pflanzenreich IV. 50. II. B. 23 (1911) 15.

Luzon (Bontoc, Lepanto, Ifugao, Benguet, Rizal, Tayabas). A reasonably common species in the mountainous regions of Luzon. Epiphyte at 300 to $2,500 \mathrm{~m}$ altitude.

PHREATIA DENSIFLORA (Blume) Lindl. Gen. \& Sp. Orch. (1830) 64; Miq. Fl. Ind. Bat. 3 (1859) 655; J. J. Sm. Fl. Buitenz. 6 (Orch. Jav.) (1905) 502, Fig.-Atlas (1912) fig. 380, Orch. Amb. (1905) 117; Ames Orch. 2 (1908) 201, 5 (1915) 195, 6 (1920) 208; Kränzl. in Engl. Pflanzenreich IV. 50. I.I. B. 23 (1911) 18; Rolfe in Gibbs in Journ. Linn. Soc. 42 (1914) 152; Ames in Merr. in Journ. Roy. Asiat. Soc. Straits Branch, Special No. (1921) 191.

Dendrolirium densiflorum Blume Bijdr. (1825) 350.

Eria myosurus Reichb. f. in Bonpl. 5 (1857) 54, non Reichb. f. in Seem. Fl. Vit. (1868) 300.

Phreatia myosurus Lindl. in Journ. Linn. Soc. 3 (1859) 61; Miq. Fl. Ind. Bat. 3 (1859) 655; Hook. f. Fl. Brit. Ind. 5 (1890) 810; Ridl. 
in Journ. Linn. Soc. 31 (1896) 285, 32 (1896) 307, Mat. Fl. Mal. Penin. 1 (1907) 106; Ames in Merr. in Journ. Roy. Asiat.

Soc. Straits Branch, Special No. (1921) 191.

Eria stachyurus Reichb. f. in Seem. Fl. Vit. (1868) 301, in obs.

Luzon (Bataan), Whitford 131, 1156. Mt. Mariveles, on rocks at about $900^{\circ} \mathrm{m}$ altitude. In Malay Peninsula, Sumatra, Java, Amboina, and Borneo.

PHREATIA INFUNDIBULIFORMIS Ames in Philip. Journ. Sci. 7 (1912) Bot. 20, Orch. 5 (1915) 195.

Mindanao (Dapitan), F. B. 4611 Mearns \& Hutchinson. Mt. Malindang at $1,900 \mathrm{~m}$ altitude.

PHREATIA LUZONIENSIS Rolfe ex Ames Orch. 1 (1905) 96, 2 (1908) 202, 5 (1915) 195; Kränzl. in Engl. Pflanzenreich IV. 50. II. B. 23 (1911) 25; Ames in Elm. Leafl. Philip. Bot. 5 (1912) 1581.

Luzon (Nueva Vizcaya, Bataan, Rizal, Laguna, Tayabas, Sorsogon), Negros (Oriental Negros), Leyte, Mindanao (Zamboanga, Cotabato, Agusan, Davao). A fairly common species of rather wide distribution; epiphyte at 10 to $2,000 \mathrm{~m}$ altitude.

PHREATIA MEARNSII Ames in Philip. Journ. Sci. 7 (1912) Bot. 21, Orch. 5 (1915) 195.

Luzon (Pampanga), Mindanao (Dapitan, Davao), F. B. 19313 Curran, 4592 Mearns \& Hutchinson, Elmer 10625. Epiphyte up to 1,900 $\mathrm{m}$ aititude in Dapitan.

PHREATIA NEGRosiANA Ames in Elm. Leafl. Philip. Bot. 5 (1912) 1582, Orch. 5 (1915) 195.

Negros (Oriental Negros), Elmer 10148. Cuernos Mountains, epiphyte.

PHREATIA PROREPENS Reichb. f. Otia Bot. Hamb. 1 (1878) 54, in textu; Ames in Philip. Journ. Sci. 2 (1907) Bot. 332, 6 (1911) Bot. 53, Orch. 2 (1908) 205, 3 (1908) 52, t. 43, 5 (1915) 195; Kränzl. in Engl. Pfianzenreich IV. 50. II. B. 23 (1911) 32.

Eria prorepens Reichb. f. Otia Bot. Hamb. 1 (1878) 54, Xen. Orch. 3 (1878) 31; Naves Novis. App. (1882) 236.

Pinalia prorepens Ktze. Rev. Gen. Pl. 2 (1891) 679.

Luzon (Abra, Kalinga, Bontoc, Ifugao, Benguet, Laguna), Mindoro, Negros, Leyte, Mindanao (Cotabato). A not uncommon species of wide distribution, originally collected on Mt. Mahaitai in Luzon by the Wilkes Expedition in 1838-1842. Epiphyte in mossy forest at 600 to $2,450 \mathrm{~m}$ altitude.

PHREATIA RAMOSII Ames in Philip. Journ. Sci. 7 (1912) Bot. 22, Orch. 5 (1915) 195.

Luzon (Abra), B. S. 7140 Ramos. Epiphyte in forest.

PHREATIA SECUNDA (Blume) Lindl. Gen. \& Sp. Orch. (1830) 64; Miq. Fl. Ind. Bat. 3 (1859) 656; Naves Novis. App. (1882) 235; J. J. Sm. Fl. Buitenz. 6 (Orch. Jav.) (1905) 500, Fig.-Atlas (1912) fig. 3r8 (excl. syn. P. microtidis Lindl.); Schltr. in Engl. Bot. Jahrb. 45, Beibl. 104 (1911) 53; Kränzl. in Engl. Pflanzenreich IV. 50. 
II. B. 23 (1911) 25 (excl. descr. p. p. et syn. P. microtidis Lindl.); J. J. Sm. in Winkler in Engl. Bot. Jahrb. 48 (1912) 102; Ames in Merr. in Journ. Roy. Asiat. Soc. Straits Branch, Special No. (1921) 191.

Dendrolirium secundum Blume Bijdr. (1825) 350.

Eria secunda Reichb. f. in Bonpl. 5 (1857) 54, quoad nom. et syn. sed forsan non quoad spec.

Phreatia minutiflora Lindl. in Journ. Linn. Soc. 3 (1859) 62; Miq. Fl. Ind. Bat. 3 (1859) 655; Naves Novis. App. (1882) 235; Hook. f. Fl. Brit. Ind. 5 (1890) 811; Ridl, in Journ. Linn. Soc. 31 (1896) 285, 32 (1896) 307; Kränzl. in Reinecke in Engl. Bot. Jahrb. 25 (1898) 607; Ridl. Mat. Fl. Mal. Penin. 1 (1907) 107; Ames Orch. 2 (1908) 205, 5 (1915) 195, 6 (1920) 208; Kränzl. in Engl. Pflanzenreich IV. 50. II. B. 23 (1911) 23, fig. $3 \mathrm{C}, a-g$; Ames in Merr. in Journ. Roy. Asiat. Soc. Straits Branch, Special No. (1921) 191.

Eria minutiflora Reichb. f. in Seem. Fl. Vit. (1868) 301, in obs.

Mindanao (Zamboanga), Copeland 1658. San Ramon River, epiphyte at about $200 \mathrm{~m}$ altitude. In Malay Peninsula, Sumatra, Java, and Borneo; also stated, by Kränzlin, to occur in the Celebes, Pacific Islands, and Samoa.

PHREATIA SUlCATA (Blume) J. J. Sm. Fl. Buitenz. 6 (Orch. Jav.) (1905) 505, Fig.-Atlas (1912) fig. 383; J. J. Sm. Orch. Amb. (1905) 118; Ames in Philip. Journ. Sci. 2 (1907) Bot. 332, 7 (1912) Bot. 22; Schltr. in Engl. Bot. Jahrb. 45, Beibl. 104 (1911) 55; Kränzl. in Engl. Pflanzenreich IV. 50. II. B. 23 (1911) 31; Ames Orch. 5 (1915) 195.

Dendrolirium sulcatum Blume Bijdr. (1825) 347.

Eria sulcata Lindl. Gen. \& Sp. Orch. (1830) 69; Miq. Fl. Ind. Bat. 3 (1859) 664.

Pinalia sulcata Ktze. Rev. Gen. Pl. 2 (1891) 679.

Phreatia bracteata Ames in Philip. Journ. Sci. 4 (1909) Bot. 674, non Schltr.

Luzon (Bontoc, Lepanto, Pampanga, Bataan, Rizal, Tayabas), Mindoro, Leyte, Mindanao (Agusan), Basilan. A fairly common species of wide distribution in the Philippines. Epiphyte in forest at 600 to $2,500 \mathrm{~m}$ altitude. In Sumatra and Java.

PHREAtIA VANOVERBERghII Ames in Philip. Journ. Sci. 7 (1912) Bot. 22, Orch. 5 (1915) 195.

Luzon (Abra, Kalinga, Bontoc, Ifugao, Benguet), B. S. 7145 Ramos, Boettcher s. n., Vanoverbergh 1115, 1493, 2571, 19885 McGregor, 31848 Santos. Epiphyte at 600 to $2,200 \mathrm{~m}$ altitude.

PHREATIA WENZELII Ames Orch. 5 (1915) 196.

Leyte, Wenzel 089. Ormoc, epiphyte in forest at $1,000 \mathrm{~m}$ altitude.

PHREATIA XANTHOLEUCA Kränzl. in Fedde Repert. 17 (1921) 386.

Philippines, probably Luzon, Loher $s \cdot n$.

This species was described from material which flowered at Nymphen. burg, Munich. No material has been seen. 
EXCLUDED OR SYNONYMOUS SPECIES

Phreatia Bracteata Ames in Philip. Journ. Sci. 4 (1909) Bot. 674, non Schltr. = Phreatia sulcata (Blume) J. J. Sm.

Phreatia Caulescens Ames Orch. 2 (1908) 200, text cut; Kränzl. in Engl. Pflanzenreich IV. 50. II. B. 23 (1911) 12 = Octarrhena caulescens Ames.

Phreatia Elmeri Ames in Elm. Leafl. Philip. Bot. 5 (1912) $1581=$ Octarrhena elmeri Ames.

Phreatia Ensifolia Ames Orch. 2 (1908) 202, text cut; Kränzl. in Engl. Pflanzenreich IV. 50. II. B. 23 (1911) 15 = Octarrhena ensifolia (Ames) Schltr.

Phreatia limenophylax Reichb. f. apud Naves Novis. App. (1882) 235.

Phreatia Minutiflora Lindl. in Journ. Linn. Soc. 3 (1859) 62; Naves Novis. App. (1882) 235; Ames Orch. 2 (1908) 205, 5 (1915) 195; Kränzl. in Engl. Pflanzenreich IV. 50. II. B. 23 (1911) 23, fig. \& $C, a-g$; Ames in Merr. in Journ. Roy. Asiat. Soc. Straits Branch, Special No. (1921) 191 = Phreatia secunda (Blume) Lindl.

Phreatia myosurus Ames Orch. 2 (1908) 203 (excl. syn.), non Lindl. = Phreatia amesii Kränzl.

Phreatia Parvula Ames Orch. 2 (1908) 204, non Benth. ex Hook. f. = Octarrhena amesiana Schltr.

Phreatia PUSilla Lindl. apud Naves Novis. App. (1882) 235.

70. ACRIOPSIS Reinwardt

ACRIOPSIS FLORIBUNDA Ames Orch. 6 (1920) 306.

Bucas Grande Island, B. S. 3.5112 Ramos \& Pascasio.

ACRIOPSIS INDICA Wight Icon. $5^{2}$ (1852) $20, t .1748$, fig. I; Reichb. f. in Walp. Ann. 6 (1863) 492; Hook. f. Fl. Brit. Ind. 6 (1890) 79; Grant Orch. Burma (1895) 313; Schltr. in Oesterr. Bot. Zeitschr. 50 (1900) 287; J. J. Sm. Fl. Buitenz. 6 (Orch. Jav.) (1905) 539, Fig.-Atlas (1912) fig. 411; Ridl. Mat. Fl. Mal. Penin. 1 (1907) 188; Ames Orch. 6 (1920) 307.

Luzon (Kalinga), Boettcher s. n. At $1,000 \mathrm{~m}$ altitude. In Burma, Malay Peninsula, and Java.

ACRIOPSIS JAVANICA Reinw. ex Blume Cat. Gew. Buitenz. (1823) 97; Blume Bijdr. (1825) 377, fig. 71; Lindl. Gen. \& Sp. Orch. (1832) 140 ; De Vriese Ill. Orch. Ind. Neerl. (1854) t. 3; Miq. Fl. Ind. Bat. 3 (1859) 709; Reichb. f. in Walp. Ann. 6 (1863) 492; Hook. f. Fl. Brit. Ind. 6 (1890) 79; Grant Orch. Burma (1895) 313; Ridl. in Journ. Linn. Soc. 32 (1896) 384; Schltr. in Oesterr. Bot. Zeitschr. 50 (1900) 286; J. J. Sm. Fl. Buitenz. 6 (Orch. Jav.) (1905) 537, Fig.-Atlas (1912) fig. 410; Ridl. Mat. Fl. Mal. Penin. 1 (1907) 187; J. J. Sm. in Nova Guinea 8 (1909) Bot. 119, $8^{3}$ (1912) Bot. 604; Schltr. in Fedde Repert. 10 (1911) 190; Ames Orch. 5 (1915) 197; J. J. Sm. in Philip. Journ. Sci. 12 (1917) Bot. 238. 
? Spathoglottis trivalvis Wall. Cat. (18:31) No. 3742, nomen; Lindl.

Gen. \& Sp. Orch. (1831) 120.

Acriopsis picta Lindl. in Bot. Reg. 29, (1843) Misc. 69.

Acriopsis crispa Griff. Notul. 3 (1851) 333 and Icon. Pl. Asiat. 3 (1851) t. 318.

Acriopsis griffithii Reichb. f. in Bonpl. 2 (1854) 92.

Acriopsis philippinensis Ames Orch. 2 (1908) 215, text cut.

Luzon (Sorsogon), Leyte, Mindanao (Misamis, Lanao, Davao), Elmer 1695\%, Wenzel 0818, 0845, Weber 270, 276, M. S. Clemens s. n., R. F. Black 15, R. S. Williams 2778. At 400 to $580 \mathrm{~m}$ altitude. In Burma, Malay Peninsula, Sumatra, Java, Borneo, Celebes, and New Guinea.

\section{EXCLUDED OR SYNONYMOUS SPECIES}

ACRIOPSIS PHILIPPINENSIS Ames Orch. 2 (1908) 215, text cut $=$ Acriopsis javanica Reinw. ex Blume.

\section{THECOSTELE Reichenbach $f$.}

Thecostele Alata (Roxb.) Par. \& Reichb. f. in Trans. Linn. Soc. 30 (1874) 135, 144, t. 29; J. J. Sm. Fl. Buitenz. 6 (Orch. Jav.) (1905) 541, Fig.-Atlas (1912) fig. 412; Rolfe in Orch. Rev. 20 (1912) 237; Ames Orch. 5 (1915) 197, 6 (1920) 215, in Merr in Journ. Roy. Asiat. Soc. Straits Branch, Special No. (1921) 183. Cymbiaium alatum Roxb. Hort. Beng. (1814) 63, nomen, Fl. Ind. ed. $2,3(1832) 459$.

Thecostele zollingeri Reichb. f. in Bonpl. 5 (1857) 37, Xen. Orch. 2 (1868) 133, t. 147; Hook. f. Fl. Brit. Ind. 6 (1890) 19; Grant Orch. Burma (1895) 232; Ridl. in Journ. Linn. Soc. 31 (1896) 299, 32 (1896) 385, Mat. Fl. Mal. Penin. 1 (1907) 190.

Pholidota elmeri Ames in Elm. Leafl. Philip. Bot. 5 (1912) 1557.

Thecostele elmeri Ames in Philip. Journ. Sci. 8 (1913) Bot. 434.

Leyte, Palawan, Wenzel 036, 0149, 0215, Elmer 12971. Epiphyte in forest at 60 to $80 \mathrm{~m}$ altitude.

\section{EXCLUDED OR SYNONYMOUS SPECIES}

Thecostele Elmert Ames in Philip. Journ. Sci. 8 (1913) Bot. $434=$ Thecostele alata (Roxb.) Par. \& Reichb. f.

\section{GRAMMATOPHYLLUM Blume}

GRAMMATOPHYLLUM SCRIPTUM (Linn.) Blume Rumphia 4 (1848) 48, Mus. Bot. Lugd.-Bat. 1 (1849) 47; Lindl. in Paxt. Flow. Gard. 2 (1852) 159; Reichb. f. in Walp. Ann. 3 (1852) 550, 6 (1863) 629; Miq. Fl. Ind. Bat. 3 (1859) 708; Teijsm. \& Binn. in Nat. Tijdschr. Ned. Ind. 27 (1864) 21; Naves Novis. App. (1882) 245; J. J.'Sm. Orch. Amb. (1905) 84, in Nova Guinea 8 (1909) Bot. 103, $12^{4}$ (1916) Bot. 428; Schltr. Orchideen (1914) 356, in Orchis 9 . (1915) 105, fig. 15; J. J. Sm. in Philip. Journ. Sci. 12 (1917) Bot. 258, in Merr. Interp. Rumph. Herb. Amb. (1917) 177.

Epidendrum scriptum Linn. Sp. Pl. ed. 2 (1763) 1351; Lam. Encycl. 1 (1783) 185. 
Cymbidium scriptum Sw. in Schrad. Journ. Bot. 2 (1799) 218, in Kongl. Svensk. Vet.-Akad. Nya Handl. 21 (1800) 237; Willd. Sp. Pl. 4 (1805) 100; Pers. Syn. Pl. 2 (1807) 515.

Vanda scripta Spreng. Syst. Veg. 3 (1826) 719.

Gabertia scripta Gaudich. in Freyc. Voy. Uranie et Physic. (1829) Bot. 425.

Grammatophyllum speciosum Lindl. Gen. \& Sp. Orch. (1833) 173, p. p. et quoad syn.; Veitch Man. Orch. Pl. pt. 9 (1893) 33, p. p. non Blume.

Ophrys cernua Blanco Fl. Filip. (1837) 639 (as Ophiris), ed. 2 (1845) 445 , ed. 3, 3 (1879) 35, t. 276, non Linn.

Grammatophyllum multiflorum Lindl. in Bot. Reg. 24 (1838) Misc. 46, 25 (1839) t. 65, in Paxt. Mag. 6 (1839) 217, t. \& text cut; Edit. in Allg. Berl. Gartenz. 8 (1840) 32; Lindl, in Paxt. Flow. Gard. 2 (1852) 160; Guidon in Journ. Soc. Imp. et Centr. Hort. Par. 4 (1858) 539; Reichb. f. Xen. Orch. 2 (1862) 16, in Walp. Ann. 6 (1863) 629; Naves Novis. App. (1882) 245; Vidal Phan. Cuming. Philip. (1885) 150, Rev. Pl. Vasc. Filip. (1886) 270; Veitch Man. Orch. Pl. pt. 9 (1893) 32; Ames Orch. 2 (1908) 217, 5 (1915) 198; Schltr. Orchideen (1914) 356, in Orchis 9 (1915) 104; Merr. Sp. Blancoanae (1918) 14.

Grammatophyllum multiflorum Lindl. var. tigrinum Lindl. in Bot.

Reg. 28 (1842) t. 69, in Paxt. Flow. Gard. 2 (1852) 160; Guidon in Journ. Soc. Imp. et Centr. Hort. Par. 4 (1858) 539; Veitch Man. Orch. PI. pt. 9 (1893) 32; Schltr. in Orchis 9 (1915) 105.

Grammatophyllum fenzlianum Reichb. f. Xen. Orch. 2 (1862) 16; Naves Novis. App. (1882) 245; Veitch Man. Orch. Pl. pt. 9 (1893) 31; Ames Orch. 5 (1915) 197, in nota.

Grammatophyllum rumphianum Miq. Ann. Mus. Bot. Lugd.-Bat. 4 (1869) 219, tt. 8, 9; Rolfe in Orch. Rev. 3 (1895) 208; Hook. f. in Bot. Mag. 122 (1896) t. 7507 ; Cogn. in Dict. Icon. Orch. (1899) Grammat. $t$. 1; Ames in Merr. in Journ. Roy. Asiat. Soc. Straits Branch, Special No. (1921) 192.

Grammatophyllum boweri F. v. Muell. in Wing South. Sci. Rec. (1883). Grammatophyllum leopardinum Reichb. f. in Flora 71 (1888) 151; Rolfe in Orch. Rev. 23 (1915) 7.

Grammatophyllum measuresianum Weathers in Gard. \& For. 2 (1889) 524; Edit. in Journ. Hort. III 23 (1891) 342, fig. 69; Stein Orchideenb. (1892) 267; Ames Orch. 2 (1908) 216, 5 (1915) 197; Schltr. in Orchis 9 (1915) 108.

Grammatophyllum seegerianum Hort. ex Gard. Chron. III 10 (1891) 49, nomen; Stein Orchideenb. (1892) 267; Rolfe in Orch. Rev. 3 (1895) 208, in textu.

Grammatophyllum fenzlianum Reichb. f. var. measuresianum Veitch Man. Orch. Pl. pt. 9 (1893) 32, text cut.

Grammatophyllum guilelmi-secundi Kränzl. in Gartenfl. 43 (1894) 114; K. Schum, in Notizbl. Bot. Gart. Berl. 1 (F1. New-Pomm.) (1898) 105;. This.-Dyer in Kew Bull. Add. Ser. 4 (1900) 194; 
Kränzl. in K. Schum. \& Lauterb. Fl. Deutsch. Schutzgeb. (1901) 250.

Grammatophyllum scriptum (Linn.) Blume var. boweri Schltr. in

Fedde Repert. Beihefte 1 (Orch. Deutsch-Neu-Guin.) (1913) 951.

Luzon (Cavite, Tayabas, Camarines, Sorsogon), Catanduanes, Mindorn, Leyte, Biliran, Palawan, Lumbucan. A very variable, common, and widely distributed species in the Philippines. Epiphyte, at sea level to $500 \mathrm{~m}$ altitude. In Amboina, Ternate, Celebes (fide J. J. Sm.), Borner, and New Guinea.

GRAMMATOPHYLLUM SPECIOSUM Blume Bijdr. (1825) 378, fig. 20 ; Lindl. Gen. \& Sp. Orch. (1833) 173, ex parte; Blume Rumphia 4 (1848) 47, t. 191, Mus. Bot. Lugd.-Bat. 1 (1849) 47; Lindl. in Paxt. Flow. Gard. 2 (1852) 157, 160, t. 69; Reichb. f. in Walp. Ann. 3 (1852) 550, 6 (1863) 628; Guidon in Journ. Soc. Imp. et Centr. Hort. Par. 4 (1858) 539 (excl. syn.); Miq. Fl. Ind. Bat. 3 (1859) 708; Hook. in Bot. Mag. 86 (1860) t. 5157; Van Houtte in Fl. des Serres 13 (1861) 177, $t$, 1386-7; Reichb. f. Xen. Orch.2 (1862) 16; Batem. Second Cent. Orch. Pl. (1867) t. 181; Edit. in Gard. Chron. II 10 (1878) 181, 183, fig. 36; Naves Novis. App. (1882) 245; Hook. f. Fl. Brit. Ind. 6 (1890) 18 (excl. syn. scripta et scriptum); Burb. in Gard. Chron. III 7 (1890) 287, 297, fig. 46; Stein Orchideenb. (1892) 267, figs. 86, 87; Ceron Cat. Pl. Herb. Manila (1892) 167; Veitch Man. Orch. Pl. pt. 9 (1893) 33 (excl. syn. scripta et scriptum); Grant Orch. Burma (1895) 263 (excl. syn. scripta et scriptum); Ridl. in Journ. Linn. Soc. 31 (1896) 290, 32 (1896) 335; Rolfe in Orch. Rev. 5 (1897) 268, 23 (1915) 6; Rendle in Journ. Bot. 39 (1901) 175; J. J. Sm. Fl. Buitenz. 6 (Orch. Jav.) (1905) 485; Ridl. Mat. Fl. Mal. Penin. 1 (1907) 142 (excl. syn. scripta et scriptum); Edit. in Gard. Chron. III 42 (1907) 168, figs. 69, \%0; Schltr. Orchideen (1914) 356, in Orchis 9 (1915) 101; Ames Orch. 5 (1915) 198; W. W. in Gard. Chron. III 64 (1918) 55, in textu; Ames in Merr. in Journ. Roy. Asiat. Soc. Straits Branch, Special No. (1921) 192; G. Wilson in Orch. Rev. 29 (1921) 83, 98.

Grammatophyllum fastuosum Lindl. in Paxt. Flow. Gard. 2 (1852) 159.

Pattonia macrantha Wight Icon. $5^{1}$ (1852) 21, $t .1750$.

Grammatophyllum macranthum Reichb. f. Xen. Orch. 2 (1862) 16, in Walp. Ann. 6 (1863) 628.

Grammatophyllum wallisii Reichb. f. in Linnaea 41 (1876) 107; Rolfe in Orch. Rèv. 23 (1915) 7; Ames Orch. 5 (1915) 198.

Grammatophyllum giganteum Blume apud Reichb. f. in Linnaea 41 (1876) 107, in textu.

Grammatophyllum sanderianum Hort. ex Edit. in Gard. Chron. III 14 (1893) 15, sphalm.

Mindanao (Surigao), Wallis s. n., de Veyra s. n. In Burma, Malay Peninsula, Cochin China (fide J. J. Sm.), Sumatra (fide J. J. Sm.), Java, and Borneo.

$200823-26$ 


\section{EXCLUDED OR SYNONYMOUS SPECIES}

GRAMMATOPHYLLUM FENZLIANUM Reichb. f. Xen. Orch. 2 (1862) 16; Naves Novis. App. (1882) 245; Veitch Man. Orch. Pl. pt. 9 (1893) 31; Ames Orch. 5 (1915) 197, in nota = Grammatophyllum scriptum (Linn.) Blume.

Grammatophyllum MeasuresianUm Weathers in Gard. \& For. 2 (1889) 524; Edit. in Journ. Hort. III 23 (1891) 342, fig. 69; Stein Orchideenb. (1892) 267; Ames Orch. 2 (1908) 216, 5 (1915) 197; Schltr. in Orchis 9 (1915) $108=$ Grammatophyllum scriptum (Linn.) Blume.

Grammatophyllum MULTifloRum Lindl. in Bot. Reg. 24 (1838) Misc. 46, 25 (1839) t. 65; Lindl. in Paxt. Mag. Bot. 6 (1839) 217, t. \& text cut; Edit. in Allg. Berl. Gartenz. 8 (1840) 32; Lindl. in Paxt. Flow. Gard. 2 (1852) 160; Guidon in Journ. Soc. Imp. et Centr. Hort. Par. 4 (1858) 539; Reichb. f. Xen. Orch. 2 (1862) 16, in Walp. Ann. 6 (1863) 629; Naves Novis. App. (1882) 245; Vidal Phan. Cuming. Philip. (1885) 150, Rev. Pl. Vasc. Filip. (1886) 270; Veitch Man. Orch. Pl. pt. 9 (1893) 32; Ames Orch. 2 (1908) 217, 5 (1915) 198; Schltr. Orchideen (1914) 356; Schltr. in Orchis 9 (1915) 104; Merr. Sp. Blancoanae (1918) $14=$ Grammatophyllum scriptum (Linn.) Blume.

GrammatophyllUM WALLISII Reichb. f. in Linnaea 41 (1876) 107; Rolfe in Orch. Rev. 23 (1915) 7; Ames Orch. 5 (1915) 198 = Grammatophyllum speciosum Blume.

\section{DIPODIUM R. Brown}

DIPODIUM PALUDOSUM (Griff.) Reichb. f. Xen. Orch. 2 (1862) 15, in Walp. Ann. 6 (1863) 648, in Gard. Chron. III 4 (1888) 91; Hook. f. Fl. Brit. Ind. 6 (1890) 19; Warner \& Will. Orch. Alb. 9 (1891) t. 422; Hook. f. in Bot. Mag. 122 (1896) t. 7464; Ridl. in Journ. Linn. Soc. 31 (1896) 290, 32 (1896) 339; This.-Dyer in Kew Bull. Add. Ser. 4 (1900) 161; Rendle in Journ. Bot. 39 (1901) 175; Ridl. Mat. Fl. Mal. Penin. 1 (1907) 142; Ames in Philip. Journ. Sci. 6 (1911) Bot. 55, Orch. 5 (1915) 199, in Merr. in Journ. Roy. Asiat. Soc. Straits Branch, Special No. (1921) 192.

Grammatophyllum paludosum Griff. Notul. 3 (1851) 344.

Grammatophyllum affine Griff. Icon. Pl. Asiat. 3 (1851) t. 323.

Wailesia paludosa Reichb. $f$. in Bonpl. 2 (1854) 93.

Sibuyan, Leyte, Mindanao (Surigao), Elmer 12435, Wenzel 0198, 0400, 0418, Bolster s. n., B. S. 34800 Ramos \& Pascasio. Epiphyte in forest at 60 to $600 \mathrm{~m}$ altitude. In Malay Peninsula and Bomeo; also reported from Cochin China.

\section{EXCLUDED OR SYNONYMOUS SPECIES}

Dipodium PICTUm Reichb. f. apud Naves Novis. App. (1882) 242.

Dipodium punctatum R. Br. apud Naves Novis. App. (1882) 242.

Dipodium Squamatum R. Br. apud Naves Novis. App. (1882) 242. 


\section{CYMBIDIUM Swartz}

CYMBIDIUM ATROPURPUREUM (Lindl.) Rolfe in Orch. Rev. 11 (1903) 190; Ames Orch. 2 (1908) 218, 5 (1915) 199.

Cymbidium pendulum Sw. var. atropurpureum Lindl. in Gard. Chron.

(1854) 287; Hook. f. in Bot. Mag. 94 (1868) t. 5710.

? Cymbidium pendulum Vidal Phan. Cuming. Philip. (1885) 150, p. p., Rev. Pl. Vasc. Filip. (1886) 269, p. p. non Sw.

Cymbidium finlaysonianum Lindl. var, atropurpureum Veitch Man. Orch. Pl. pt. 9 (1893) 16.

Cymbidium pendulum Sw. var. purpureum W. Wats. Orchids, ed. 2 (1895) 151, sphalm.

Luzon (Rizal), Leyte, Mindanao (Davao), Lyon 101, Copeland 648, Black 16, Wenzel 0103.

CYMBIDIUM FINLAYSONIANUM Lindl. in Wall. Cat. (1832) No. 7358, nomen, Gen. \& Sp. Orch. (1833) 164; Hook. f. Fl. Brit. Ind. 6 (1890) 11; Veitch Man. Orch. Pl. pt. 9 (1893) 16; Rendle in Journ. Bot. 39 (1901) 175; J. J. Sm. Fl. Buitenz. 6 (Orch. Jav.) (1905) 481, Fig.-Atlas (1911) fig. 366; Ridl. Mat. Fl. Mal. Penin. 1 (1907) 139; Ames Orch. 2 (1908) 218, 5 (1915) 199; Schltr. in Fedde Repert. 10 (1911) 190; Ames in Merr. in Journ. Roy. Asiat. Soc. Straits Branch, Special No. (1921) 192.

Cymbidium pendulum Blume Bijdr. (1825) 379; Miq. Fl. Ind. Bat. 3 (1859) 707; Lindl. in Bot. Reg. 26 (1840) t. 25 (excl. syn.); Reichb. f. in Walp. Ann. 6 (1863) 624, quoad spec.; Naves Novis. App. (1882) 245; Vidal Phan. Cuming. Philip. (1885) 150, p. p., Rev. P1. Vasc. Filip. (1886) 269, p. p.; Warner \& Will. Orch. Alb. 10 (1893) t. 437 (excl. syn. in part) non Sw.

Cymbidium aloifolium Lindl. in Wall. Cat. (1832) No. 7352, A \& B; Naves Novis. App. (1882) 245; Ridl. in Trans. Linn. Soc. II 3 (1893) 368, in Journ. Linn. Soc. 32 (1896) 333; Ames Orch. 1 (1905) 101; Merr. in Philip. Journ. Sci. 1 (1906) Suppl. 39, non Sw.

Cymbidium wallichii Lindl. Gen. \& Sp. Orch. (1833) 165; Miq. Fl. Ind. Bat. 3 (1859) 707; Naves Novis. App. (1882) 245.

Cymbidium pendulum Sw. var. brevilabre Lindl. in Bot. Reg. 28

(1842) Misc. 60, 30 (1844) t. 24; Paxt. Mag. Bot. 11 (1844) 115;

Reichb. f. in Walp. Ann. 6. (1863) 624.

Cymbidium tricolor Miq. Choix des P1. (1864) t. 19.

Luzon (Benguet, Bataan, Rizal, Tayabas), Polillo, Bohol, Palawan, Mindanao (Zamboanga, Surigao), Dinagat. Epiphyte at sea level to 250 $m$ altitude. In Burma (fide Hook. f.), Malay Peninsula, Java, Celebes, and Borneo; also reported from Sumatra and Cochin China.

\section{EXCLUDED OR SYNONYMOUS SPECIES}

Cymbidium Alorfolium Lindl. in Wall. Cat. (1832) No. 7352, A. \& B; Naves: Novis. App. (1882) 245; Ames Orch. 1 (1905) 101; Merr. in Philip. Journ. Sci. 1 (1906) Suppl. 39, non Sw. = Cymbidium fin. laysonianum Lindl. 
CYMBIDIUM FINLAYSONIANUM Lindl. var. Atropurpureum Veitch Man. Orch. PI. pt. 9 (1893) $16=$ Cymbidium atropurpureum (Lindl.) Rolfe.

Cymbidium Flavescens Llanos Fragm. Pl. Filip. (1851) 96; F.-Vill. \& Naves in Blanco Fl. Filip. ed. 3, $4^{1}$ (1880) 74; Merr. Sp. Blancoanae $(1918) 113=$ ? Oberonia iridifolia (Roxb.) Lindl.

Cymbidium huttoni Boxall ex Naves Novis. App. (1882) 245, nomen.

Cymbidium Imbricatum Roxb. Hort. Beng. (1814) 63, nomen, Fl. Ind. ed. 2, 3 (1832) 460 = Pholidota imbricata Lindl.

Cymbidium iridifolium Roxb. Hort. Beng. (1814) 63, nomen, Fl. Ind. ed. 2,3 (1832) 458 = Oberonia iridifolia (Roxb.) Lindl.

Cymbidium Pendulum Blume Bijdr. (1825) 379; Naves Novis. App. (1882) 245 ; Vidal Phan. Cuming. Philip. (1885) 150, p. p., Rev. Pl. Vasc. Filip. (1886) 269, p. p., non Sw. = Cymbidium finlaysonianum Lindl.

Cymbidium Pendulum Vidal Phan. Cuming. Philip. (1885) 150, p. p., Rev. Pl. Vasc. Filip. (1886) 269, p. p., non $\mathrm{Sw}=$ ? Cymbidium atropurpureum (Lindl.) Rolfe.

Cymbidium pendulum Sw, var. Atropurpureum Lindl, in Gard. Chron. (1854) 287; Hook. f. in Bot. Mag. 94 (1868) t. $5710=$ Cymbidium atropurpureum (Lindl.) Rolfe.

Cymbidium Pendulum Sw. var. Purpureum W. Wats. Orchids, ed. 2 (1895) 151, sphalm $=$ Cymbidium atropurpureum (Lindl.) Rolfe.

Cymbidium wallichil Lindl. Gen. \& Sp. Orch. (1833) 165; Naves Novis. App. (1882) $245=$ Cymbidium finlaysonianum Lindl.

\section{CHAMAEANTHUS Schlechter}

CHAMAEANTHUS WENZELII Ames Orch. 5 (1915) 200.

Luzon (Sorsogon), Leyte, Mindanao (Davao), Elmer 15\%67, 168\%, Wenzel 0110 A, 0190, Weber 260. Epiphyte at sea level to $60 \mathrm{~m}$ altitude.

\section{THRIXSPERMUM Loureiro}

THRIXSPERMUM ACUMINATISSIMUM (Blume) Reichb, f. Xen. Orch. 2 (1867) 121, in Flora 51 (1868) 53; J. J. Sm. Fl. Buitenz. 6 (Orch. Jav.) (1905) 569, Fig.-Atlas (1912) fig. 427; Schltr. in Orchis 5 (1911) 54; Ames Orch. 5 (1915) 201.

Dendrocolla acuminatissima Blume Bijdr. (1825) 288.

Aërides acuminatissimum Lindl. Gen. \& Sp. Orch. (1833) 240; Miq. Fl. Ind. Bat. 3 (1859) 696.

Sarcochilus acuminatissimus Reichb. f. in Walp. Ann. 6 (1863) 498. Sarcochilus notabilis Hook. f. Fl. Brit. Ind. 6 (1890) 42, in Hook.

Icon. Pl. 22 (1893) t. 2126.

Thrixspermum notabile Ridl. in Journ. Linn. Soc. 32 (1896) 379, Mat. Fl. Mal. Penin. 1 (1907) 183.

Luzon (Laguna), Leyte, Reillo 56, B. S. 16560, 20447, 20455 Ramos, Wenzel 060,0101,0163. Epiphyte at $60 \mathrm{~m}$ altitude in Leyte. In Maláy Peninsula and Java. 
THRIXSPERMUM AGUSANENSE Ames Orch. 5 (1915) 201.

Mindanao (Agusan), Weber 14\%. Veruela, Agusan River, epiphyte at $15 \mathrm{~m}$ altitude.

THRIXSPERMUM AMPLEXICAULE (Blume) Reichb. f. Xen. Orch. 2 (1867) 121, in Flora 51 (1868) 53; J. J. Sm. Fl. Buitenz. 6 (Orch. Jav.) (1905) 573, Fig.-Atlas (1912) fig. 430, Orch. Amb. (1905) 96; Schltr. in Orchis 5 (1911) 54; Ames in Philip. Journ. Sci. 8 (1913) Bot. 438, Orch. 5 (1915) 202; J. J. Sm. in Philip. Journ. Sci. 12 (1917) Bot. 259; Ames in Merr. in Journ. Roy. Asiat. Soc. Straits Branch, Special No. (1921) 194.

Dendrocolla amplexicaulis Blume Bijdr. (1825) 288.

Aërides amplexicaule Lindl. Gen. \& Sp. Orch. (1833) 239; Miq. Fl. Ind. Bat. 3 (1859) 696.

Sarcochilus lilacinus Griff. Notul. 3 (1851) 334, Icon. Pl. Asiat. 3 (1851) t. 320, fig. II; Reichb. f. in Walp. Ann. 6 (1863) 499; Hook. f. Fl. Brit. Ind. 6 (1890) 40; Ridl. in Trans. Linn. Soc. II 3 (1893) 371; Hook. f. in Bot. Mag. 127 (1901) t. 77.54.

Orsidice amplexicaulis Reichb. f. in Bonpl. 2 '(1954) 93.

Orsidice lilacina Reichb. f. in Bonpl. 2 (1854) 93.

Sarcochilus amplexicaulis Reichb. f. in Walp. Ann. 6 (1863) 499; Rolfe in Journ. Bot. 23 (1885) 215; Vidal Phan. Cuming. Philip. (1885) 150, Rev. Pl. Vasc. Filip. (1886) 271.

Thrixspermum Iilacinum Reichb. f. Xen. Orch. 2 (1867) 121, in Flora 51 (1868) 53; Ridl. in Journ. Linn. Soc. 31 (1896) 298, 32 (1896) 377, Mat. Fl. Mal, Penin. 1 (1907) 181.

Luzon (Nueva Vizcaya), Mindanao (Lanao), Cuming 2056, B. S. 11143 McGregor. M. S. Clemens 949. In bogs, twining on grasses. In Malay Peninsula, Sumatra, Java; Amboina, Celebes, and Banda Islands.

THRIXSPERMUM BICRISTATUM Ames Orch. 5 (1915) 202.

Leyte, Mindanao (Agusan), Wenzel 030, Weber 145. Epiphyte at 15 to $40 \mathrm{~m}$ altitude.

THRIXSPERMUM ELONGATUM Ames Orch. 5 (1915) 203.

Luzon (Bataan, Rizal, Laguna, Sorsogon), Polillo, Siquijor, Leyte, Palawan, Mindanao (Butuan, Agusan, Surigao, Davao). A fairly common and fairly widely distributed species. Epiphyte at sea level to $525 \mathrm{~m}$ altitude.

THRIXSPERMUM LINEARIFOLIUM Ames Orch. 5 (1915) 205.

Mindanao (Dapitan, Bukidnon), F. B. 4734 Mearns \& Hutchinson, B. S. 38499 Ramos \& Edaño. Mt. Malindang and Mt. Lipa. Epiphyte in mossy forest at 1,900 to $2,200 \mathrm{~m}$ altitude.

THRIXSPERMUM QUINQUELOBUM Ames Orch. 5 (1915) 206.

Luzon (Laguna), B. S. 10016, 16561, 16562, 20444 Ramos, Reillo 19, Serrato s. n. Epiphyte in forest.

THRIXSPERMUM ROBINSONII Ames Orch. 5 (1915) 207.

Luzon (Bataan, Laguna, Tayabas, Sorsogon), Negros (Occidental Negros), Leyte, Mindanao (Agusan), Whitford 13\%, 1508, B. S. 9447, 17090 Robinson, 17313 Robinson \& Brown, 16282 Brown, Elmer 13833, 17134, Wenzel 0408. Epiphyte at 600 to $2,150 \mathrm{~m}$ altitude. 
THRIXSPERMUM ROSTRATUM Ames Orch. 5 (1915) 208.

Panay, Lyon 159. Patolan, plant climbing.

THRIXSPERMUM SUBULATUM (Blume) Reichb. f. Xen. Orch. 2 (1867)

122, in Flora 51 (1868) 53; J. J. Sm. Fl. Buitenz. 6 (Orch. Jav.) (1905) 578, Fig.-Atlas (1912) fig. 434, Orch. Amb. (1905) 97;

Schltr. in Orchis 5 (1911) 57; J. J. Sm. in Bull. Jard. Buitenz. II

9 (1913) 102; Ames Orch. 7 (1922) 134.

Dendrocolla subulata Blume Bijdr. (1825) 291.

Aërides subulatum Lindl. Gen. \& Sp. Orch. (1833) 241; Miq. Fl. Ind.

Bat. 3 (1859) 698.

Sarcochilus subulatus Reichb. f. in Walp. Ann. 6 (1863) 500; Naves

Novis. App. (1882) 238.

Thrixspermum falcilobum Schltr. in Bull. Herb. Boiss. II 6 (1906) 469;

Schltr. in Orchis 5 (1911) 57 (fide J. J. Sm. in Bull. Jard. Buitenz.

1. c.).

Luzon (Bontoc), B. S. 37905 Ramos \& Edaño. Mt. Masapilid, epiphyte at $1,000 \mathrm{~m}$ altitude. In Sumatra, Java, and Amboina.

THRIXSPERMUM VANOVERBERghII Ames in Philip. Journ. Sci. 8 (1913) Bot. 438, Orch. 5 (1915) 209.

Luzon (Bontoc), Vanoverbergh 632, 1091, 1531, 1792. Epiphyte at about $1,400 \mathrm{~m}$ altitude.

THRIXSPERMUM WEBERI Ames Orch. 7 (1922) 134.

Mindanao (Agusan), Weber 187, 187X, 187X'. Cabadbaran, ephiphyte in swamp at sea level.

THRIXSPERMUM WENZELII Ames Orch. 5 (1915) 209.

Luzon (Laguna, Sorsogon), Leyte, Palawan, Mindanao (Agusan), Basilan. A not uncommon species of fairly wide distribution. Epiphyte at sea level to $60 \mathrm{~m}$ altitude.

\section{EXCLUDED OR SYNONYMOUS SPECIES}

THRIXSPERMUM MCGREGORII Ames in Philip. Journ. Sci. 2 (1907) Bot. 337 = Sarcochilus megregorii Ames.

THRIXSPERMum MindanAENSE Ames in Philip. Journ. Sci. 8 (1913) Bot. $436=$ Sarcochilus mindanaensis Ames.

THRIXSPERMUM PALLIDUM Reichb. f. Xen. Orch. 2 (1867) 122; Ames Orch. 2 (1908) 253 = Sarcochilus pallidus (Blume) Reichb. $f$.

ThRIXSPERMUM PHILIPPINENSE Ames in Philip. Journ. Sci. 8 (1913) Bot. 437 = Sarcochilus philippinensis Ames.

ThrixSPERMum UNGUICULATUm Reichb. f. Xen. Orch. 2 (1867) 122, in Flora 51 (1868) 53; Warner \& Will. Orch. Alb. 6 (1887) t. $266=$ Sarcochilus pallidus (Blume) Reichb. $f$.

77. SARCOCHILUS R. Brown

SARCOCHILUS APPENDICULATUS (Blume) J. J. Sm. Fl. Buitenz. 6 (Orch. Jav.) (1905) 564, Fig.-Atlas (1912) fig. 42.5.

Dendrocolla appendiculata Blume Bijdr. (1825) 289. 
Aërides pusillum Lindl. Gen. \& Sp. Orch. (1833) 241; Miq. Fl. Ind. Bat. 3 (1859) 697.

Sarcochilus pusillus Reichb. f. in Walp. Ann. 6 (1863) 500.

Grosourdya appendiculata Reichb. f. Xen. Orch. 2 (1867) 123; Naves

Novis. App. (1882) 239.

Thrixspermum appendiculatum Ktze. Rev. Gen. Pl. 2 (1891) 682.

Palawan, Weber 605. Alfonzo XIII, epiphyte at sea level. In Java.

J. J. Smith, Fl. Buitenz. 1. c., states that Sarcochilus hirtulus Hook. f. Fl. Brit. Ind. 6 (1890) 39, Hook. f. in Hook. Icon. Pl. 22 (1892) t. 2121: Ascochilus hirtulus Ridl. in Journ. Linn. Soc. 31 (1896) 375, Ridl. Mat. Fl. Mal. Penin. 1 (1907) 178 is perhaps conspecific.

SARCOCHILUS LEYTENSIS Ames Orch. 5 (1915) 211.

Leyte, ? Basilan, Wenzel s. n., 0109,? B. S. 16242 Reillo. Epiphyte at $60 \mathrm{~m}$ altitude.

SARCOCHILUS LONGICALCARUS Ames \& Rolfe in Ames Orch. 5 (1915) 212.

Luzon (Bontoc, Bulacan, Tayabas, Sorsogon, Camarines), Mindoro, Leyte, Mindanao (Butuan, Davao). A not uncommon species of wide distribution. Epiphyte at sea level to $500 \mathrm{~m}$ altitude.

SARCOCHILUS MCGREGORII Ames Orch. 5 (1915) 213.

Thrixspermam megregorii Ames in Philip. Journ. Sci. 2 (1907) Bot. 337.

Mindoro, McGregor 288. Balete, Baco River, epiphyte.

SARCOCHILUS MERRILLII Ames Orch. 5 (1915) 213.

Mindanao (Surigao), Merrill 5432. Baganga, epiphyte at near sea level.

SARCOCHILUS MINDANAENSIS Ames Orch. 5 (1915) 214.

Thrixspermum mindanaense Ames in Philip. Journ. Sci. 8 (1913) Bot. 436.

Luzon (Sorsogon), Sibuyan, Leyte, Mindanao (Agusan, Davao), Elmer 12530, 15361 in part, Wenzel 0110B, 0280, 0366, 0390, Weber 99, 139, 255. Epiphyte at sea level to $640 \mathrm{~m}$ altitude.

SARCOCHILUS PALAWANENSIS Ames Orch. 5 (1915) 214.

Palawan, Mindanao (Davao), Weber 614, Elmer 10515. Epiphyte at sea level in Palawan.

SARCOCHILUS PALLIDUS (Blume) Reichb. f. in Walp. Ann. 6 (1863) 500 ; Naves Novis. App. (1882) 238; J. J. Sm. Fl. Buitenz. 6 (Orch. Jav.) (1905) 559, Fig.-Atlas (1912) fig. 422, Orch. Amb. (1905) 92, in Winkler in Engl. Bot. Jahrb. 48 (1912) 104; Ames Orch. 5 (1915) 215, in Merr. in Journ. Roy. Asiat. Soc. Straits Branch, Special No. (1921) 196.

Dendrocolla pallida Blume Bijdr. (1825) 290.

Aërides pallidum Lindl. Gen. \& Sp. Orch. (1833) 241, in Paxt. Flow. Gard. 2 (1839) 143; Miq. Fl. Ind. Bat. 3 (1859) 697, nec Blume nec Roxb. 
Sarcochilus unguiculatus LindI. in Bot. Reg. 26 (1840) Misc. 67, 32 (1846) sub t. 19; Edit. in Allg. Berl. Gartenz. 14 (1846) 167; Reichb. f. in Walp. Ann. 6 (1863) 501, in Gard. Chron. II 15 (1881) 562; Naves Novis. App. (1882) 238; Rolfe in Orch. Rev. 2 (1894) 231, 14 (1906) 273, fig. 37; Ridl. in Journ. Linn. Soc. 31 (1896) 298, Mat. Fl. Mal. Penin. 1 (1907) 175; Ames in Merr. in Journ. Roy. Asiat. Soc. Straits Branch, Special No. (1921) 196. Aërides diurnum Teijsm. \& Binn. in Nat. Tijdschr. Ned. Ind. 27 (1864) 19.

Thrixspermum pallidum Reichb. f. Xen. Orch. 2 (1867) 122, in Flora 51 (1868) 53; Ames Orch. 2 (1908) 253.

Thrixspermum unguiculatum Reichb. f. Xen. Orch. 2 (1867) 122, in

Flora 51 (1868) 53; Warner \& Will. Orch. Alb. 6 (1887) $t .266$.

Phalaenopsis ruckeri Hort. ex Reichb, f. in Gard. Chron. II 15 (1881) 562 , in textu.

Phalaenopsis mekeriana Hort. ex Warner \& Will. Orch. Alb. 6 (1887) t. 266 , in syn.

Sarcochilus aureus Hook. f. Fl. Brit. Ind. 6 (1890) 35, in Ann. Roy.

Bot. Gard. Calc. 5 (Cent. Ind. Orch.) (1895) 42, t. 64; Ridl. in

Journ. Linn. Soc. 32 (1896) 373.

Thrixspermum aureum Ktze. Rev. Gen. Pl. 2 (1891) 682.

? Phalaenopsis fugax Kränzl. in Gard. Chron. III 14 (1893) 360;

Rolfe in Orch. Rev. 13 (1905) 230; cf. infra.

Sarcochilus unguiculatus Lindl. var. aureus Ridl. Mat. Fl. Mal. Penin. 1 (1907) 175.

Luzon (Cagayan, Benguet, Nueva Vizcaya, Rizal, ? Laguna, Tayabas, Sorsogon), Polillo, Mindoro, Leyte, Mindanao (Zamboanga, Agusan, Butuan, Davao), Basilan. A common and widely distributed species in the Philippines. Epiphyte apparently at low altitudes. In Malay Peninsula, Sumatra, Java, Amboina, Celebes, and Borneo.

J. J. Smith, Fl. Buitenz. 6 and Orch. Amb. 11. ce., reduces Phalaenopsis fugax Kränzl. to this species, whereas Ridley, Mat. Fl. Mal. Penin. 1 I. c., makes it a synonym of Sarcochilus caligaris Ridl. J. J. Smith also considers Sarcochilus cladostachys Hook. f. Fl. Brit. Ind. 6 (1890) 35, in Ann. Roy. Bot. Gard. Calc. 5 (1895) 43, t. 65 to be conspecific, but Ridley considers them as distinct species.

SARCOCHILUS PHILIPPINENSIS Ames Orch. 5 (1915) 215, non Vidal. Thrixspermum philippinense Ames in Philip. Journ. Sci. 8 (1913) Bot. 437.

Luzon (Apayao, Zambales, Rizal, Laguna, Sorsogon), Polillo, Leyte, Mindanao (Agusan). A fairly common species of wide distribution. Epiphyte occurring, as far as records show, at 3 to $60 \mathrm{~m}$ altitude.

SARCOCHILUS TRIPERCUS Ames Orch. 7 (1922) 125.

Leyte, Wenzel 0968. Tacloban, Tigbao, epiphyte in forest at sea leveí.

SARCOCHILUS ZAMBOANGENSIS Ames Sched. Orch. 5 (1923) 39.

Mindanao (Zamboanga), Merrill 11640. Flecha Point, epiphyte in forest at about $10 \mathrm{~m}$ altitude. 
Sarcochilus amplexicaulis Reichb. f. in Walp. Ann. 6 (1863) 499; Rolfe in Journ. Bot. 23 (1885) 215; Vidal Phan. Cuming. Philip. (1885) 150, Rev. Pl. Vasc. Filip. (1886) $271=$ Thrixspermum amplexicaule (Blume) Reichb. f.

Sarcochilus Calceolus Lindl. in Bot. Reg. 32 (1846) 1. 19; G. K. in Bot. Zeit. 4 (1846) 798; Paxt. Mag. Bot. 13 (1846) 93; Reichb. f. in Walp. Ann. 1 (1849) 781, 6 (1863) 501; Miq. Fl. Ind. Bat. 3 (1859) 689; Naves Novis. App. (1882) 238; Vidal Phan. Cuming. Philip. (1885) 150, Rev. Pl. Vasc. Filip. (1886) 271; Ridl. in Trans. Linn. Soc. II 3 (1893) 371; Ames Orch. 5 (1915) 210, in textu. Thrixspermum calceolus Reichb. f. Xen. Orch.2 (1867) 122, in Flora 51 (1868) 53; Ridl. in Journ. Linn. Soc. 32 (1896) 378, Mat. Fl. Mal. Penin. 1 (1907) 181.

This was originally described as Philippine, but there is every reason to believe that Cuming's specimen came from Singapore, where the species is common and where Cuming also collected botanical material; it has not appeared in any Philippine collections made since Cuming's visit (1836-1840); cf. Ames 1. c.

Sarcochilus centipeda Naves Novis. App. (1882) 238 (excl. syn.).

SARCochilus Compressus Reichb. f. apud Naves Novis. App. (1882) 238.

SARCochilus croceus Lindl. in Bot. Reg. 32 (1846) sub t. 19; Reichb. f. in Walp. Ann. 1 (1849) 781; Miq. Fl. Ind. Bat. 3 (1859) 689;

Naves Novis. App. (1882) 238; Ames Orch. 5 (1915) 210, in nota.

This was described from a single leaf and bud sent to Lindley by Messrs. Loddiges. No material from the Philippines referable to this species has been seen.

Sarcochilus falcatus R. Br. apud Naves Novis. App. (1882) 238.

SARCochilus NePAiEnsis Spreng. apud Llanos ex F.-Vill. \& Naves in Blanco F1. Filip. ed. 3, $4^{1}$ (1880) 99.

Sarcochilus olivaceus Lindl. apud Naves Novis. App. (1882) 238.

SARCochilus Parviflorus Lindl. apud Naves Novis. App. (1882) 238.

SARCochilus Philippinensis Vidal Phan. Cuming. Philip. (1885) 150, Rev. Pl. Vasc. Filip. (1886) $271=$ Camarotis phitippinensis Lindl.

SARCOCHILUS RHOPALORRHACHIS Reichb. f. apud Naves Novis. App. (1882) 238.

SaRCochilus subulatus Reichb. f. in Walp. Ann. 6 (1863) 500; Naves Novis. App. (1882) $238=$ Thrixspermum subulatum (Blume) Reichb. $f$.

Sarcochilus tenuifolius (Linn.) Naves Novis. App. (1882) 238.

SARCochilus teres Reichb. f. apud Naves Novis. App. (1882) 238.

Sarcochilus unguiculatus Lindl. in Bot. Reg. 26 (1840) Misc. 67, 32 (1846) sub t. 19; Edit. in Allg. Berl. Gartenz. 14 (1846) 167; Reichb. f. in Walp. Ann. 6 (1863) 501; Reichb. f. in Gard. Chron. 
II 15 (1851) 562; Naves Novis. App. (1882) 238; Rolfe in Orch. Rev. 2 (1894) 231, 14 (1906) 273, fig. 37; Ridl. in Journ. Linn. Soc. 31 (1896) 298, Mat. Fl. Mal. Penin. 1 (1907) 175; Ames in Merr. in Journ. Roy. Asiat. Soc. Straits Branch, Special No. (1921) 196 $=$ Sarcochilus pallidus (Blume) Reichb. $\mathbf{f}$.

SARCochilus usNeoldes Reichb. f. apud Naves Novis. App. (1882) 238.

\section{KINGIELLA Rolfe}

KINGIELLA PHILIPPINENSIS (Ames) Rolfe in Orch. Rev. 25 (1917) 197; Ames Orch. 6 (1920) 307.

Doritis philippinensis Ames Orch. 2 (1908) 235, text cut, 5 (1915) 215.

Luzon (Cagayan, Bataan, Bulacan, Rizal), Leyte, Palawan, Bancalan, Mindanao (Cotabato, Bukidnon, Agusan, Davao). A common species of wide distribution. Epiphyte at nearly sea level to $500 \mathrm{~m}$ altitude.

79. PHALAENOPSIS Blume

PHALAENOPSIS AMABILIS (Linn.) Blume Bijdr. (1825) 294, fig. 44; Lindl. Gen. \& Sp. Orch. (1833) 213; Bennett Pl. Jav. Rar. (1838) 28, t. 8; Blume Rumphia 4 (1848) 52, tt. 194, fig. 1, $199 \mathrm{~A}$ (excl. citat. Lindl.) ; Reichb. f. in Bot. Zeit. 10 (1852) 672, in Walp. Ann. 3 (1852) 561 (excl. pars Philip.); Miq. Fl. Ind. Bat. 3 (1859) 690 (excl. citat. Bot. Mag. et pars Philip.), Sumatra (1862) 274; Reichb. f. Xen. Orch. 2 (1862) 5; Duchartre in Journ. Soc. Imp. et Centr. Hort. Par. 8 (1862) 730; Lem. in Ill. Hort. 10 (1863) Misc. 13; Linden \& Rodigas in Lindenia 2 (1886) 65, quoad syn. in part; Rolfe in Gard. Chron. II 26 (1886) 212, fig. 43A; Kerchove in Rev. Hort. Belg. 16 (1890) 25, t. 3; Veitch Man. Orch. Pl. pt. 7 (1891) 22, t.; Stein Orchideenb. (1892) 504, fig. 153; Rolfe in l'Orchidoph. 13 (1893) 100; Will. Orch. Grow. Man. ed. 7 (1894) 660, text cut (excl. bibliog. in parte et quoad Philip.); Ridl. in Journ. Linn. Soc. 31 (1896) 292; J. J. Sm. Fl. Buitenz. 6 (Orch. Jav.) (1905) 549, Fig.-Atlas (1912) fig. 416 (excl. bibliog. in parte et quoad Philip.), Orch. Amb. (1905) 92 (excl. citat. Bot. Mag. t. 4297) ; Ames Orch. 1 (1905) 101, quoad syn. et descr. (excl. spec.), 2 (1908) 224; J. J. Sm. in Nova Guinea 8 (1909) Bot. 119 (excl. Philip.), $8^{3}$ (1912) Bot. 604, 9 (1913) Bot. 100, $12^{4}$ (1916) Bot. 455; Schltr. Orchideen (1914) 543, fig. 183; Rolfe in Gibbs in Journ. Linn. Soc. 42 (1914) 157; J. J. Sm. in Philip. Journ. Sci. 12 (1917) Bot. 258, in Merr. Interp. Rumph. Herb. Amb. (1917) 177; Ames in Merr. in Journ. Roy. Asiat. Soc. Straits Branch, Special No. (1921) 196.

Epidendrum amabile Linn. Sp. Pl. (1753) 953, ed. 2 (1763) 1351; Sw. in Nov. Act. Ups. 6 (1799) 67, in Schrad. Journ. Bot. 2 (1799) 210; Willd. Sp. Pl. 4 (1805) 115; Pers. Syn. Pl. 2 (1807) 518; Spreng. Syst. Veg. 3 (1826) 737.

Cymbidium amabile Roxb. Hort. Beng. (1814) 63, nomen, Fl. Ind. ed. 2,3 (1832) 457.

Phalaenopsis grandiflora Lind1. in Gard. Chron. (1848) 39, fig. 1; Reichb. f. in Walp. Ann. 3 (1852) 561; Moore Ill. Orch. Pl. (1857) Phalaenop. 7; Miq. Fl. Ind. Bat. 3 (1859) 690; Hook. in Bot. Mag. 86 (1860) t. 5184; Groenland in Rev. Hort. 32 (1860) 238, figs. 
53, 54; Gedney in Floral World (1876) 323; Puydt Orch. (1880) 307, t. 34; Burb. in The Garden 22 (1882) 118; Hemsl. Bot. Chal. Exped. 1 (1885) 199; Warner \& Will. Orch. Alb. 6 (1887) t. 277; Rolfe in Gard. Chron. III 5 (1889) 88; Gower in The Garden 35 (1889) 363; Rolfe in l'Orchidoph. 13 (1893) 100; Will. Orch. Grow. Man. ed. 7 (1894) 664.

Phalaenopsis aphrodite Lem. in Ill. Hort. 10 (1863) Misc. 14, quoad citat. Rumph., non Reichb. $\mathbf{f}$.

Phalaenopsis amabilis Blume var. grandiflora Batem. Second Cent. Orch. Pl. (1867) t. 114.

Phalaenopsis amabilis Blume var. rimestadtiana L. Linden in Lindenia 16 (1902) 35, t. 736; Edit. in Gard. Chron. III 32 (1902) 306, t.; Pauwels in Rev. Hort. Belg. 29 (1903) 88, figs. 22-24; Rolfe in Orch. Rev. 14 (1906) 233, fig. 27, 23 (1915) 254.

Phalaenopsis rimestadtiana "Argus" in Orch. Rev. 13 (1905) 260, in textu, nomen; Rolfe in Orch. Rev. 25 (1917) 151, in textu, nomen.

Although the species proper does not occur in the Philippines, it has been deemed advisable to cite a full synonymy and bibliography of it on account of the confusion arising from the inclusion in it, by Lindley and subsequently by others, of Philippine material. The species is represented in the Philippines by variety aphrodite (Reichb. f.) Ames and its variations.

Var. APHRODITE (Reichb. f.) Ames Orch. 2 (1908) 226, 5 (1915) 216.

Phalaenopsis amabilis Lindl. in Bot. Reg. 24 (1838) t. 34 (excl. syn.); Paxt. Mag. Bot. 5 (1838) 20, 162, 7 (1840) 49, text cut, t. (excl. syn.); Maund Botanist 3 (1839?) t. 133 (excl. syn.); Lem. in Fl. des Serres 1 (1845) 85, text cut, t. 36 (excl. syn.); Blanco F1. Filip. ed. 2 (1845) 592, ed. 3, 3 (1879) 41; Edit. in Allg. Berl. Gartenz. 13 (1845) 389, 15 (1847) 215 (excl. syn. in both); Hook. in Bot. Mag. 73 (1847) t. 4297 (excl. syn. in part); Blume Rumphia 4 (1848) 52, quoad citat. Lindl.; Lindl. in Gard. Chron. (1848) 39, fig. 2; Reichb. f. in Walp. Ann. 1 (1849) 790, 3 (1852) 561, quoad Philip.; Moore Ill. Orch. Pl. (1857) Phalaenop. 6, t. 1 (excl. syn.); Miq. Fl. Ind. Bat. 3 (1859) 690, quoad citat. Bot. Mag. et quoad Philip.; Gedney in Floral World (1876) 323; Reichb. f. in Gard. Chron. II 5 (1876) 654; Burb. in The Garden 22 (1882) 119; Naves Novis. App. (1882) 243; Vidal Phan. Cuming. Philip. (1885) 150, Rev. Pl. Vasc. Filip. (1886) 270 ; Linden \& Rodigas in Lindenia 2 (1886) 65, t. 79 (excl. syn. in parte); Gower in The Garden 35 (1889) 362, fig. 363; Roebelen in Gard. Chron. III 7 (1890) 459, in textu; Will. Orch. Grow. Man. ed. 7 (1894) 660, quoad bibliog. in parte et quoad Philip.; Merr. in Govt. Lab. Publ. (Philip.) 8 (1903) 57, 174, 27 (1905) 84; J. J. Sm. Fl. Buitenz. 6 (Orch. Jav.) (1905) 549, quoad bibliog. in parte et quoad Philip.; Ames Orch. 1 (1905) 101, quoad spec. (excl. syn. et descr.); Merr. Sp. Blancoanae (1918) 115, non Blume. Phalaenopsis aphrodite Reichb. f. in Hamb. Gartenz. 18 (1862) 35, Xen. Orch. 2 (1862) 6; Duchartre in Journ. Soc. Imp. et Centr. Hort. Par. 8 (1862) 730; Lem. in Ill. Hort. 10 (1863) Misc. 14 (excl. citat. Rumph.); Rolfe in Gard. Chron. II 26 (1886) 212. 
fig. 43 B; Pfitz. in Engl. \& Prantl Nat. Pflanzenfam. 2, Abt. 6 (1889) 211, fig. 230; Veitch Man. Orch. Pl. pt. 7 (1891) 24, t.; Stein Orchideenb. (1892) 505, fig. 155; Rolfe in Orch. Rev. 2 (1894) 209, fig. 30, 8 (1900) 135, 136, fig. 20, 13 (1905) 232, fig. 52, 25 (1917) 151, in textu; Cogn. in Dict. Icon. Orch. (1898) Phalaenop. t. 1; Edit. in Sem. Hort. 4 (1900) 392, fig. 132; Rolfe in Forbes \& Hemsl. in Journ. Linn. Soc. 36 (1903) 34; Matsum. Ind. Pl. Jap. 2 (1905) 257; Ames in Philip. Journ. Sci. 2 (1907) Bot. 336; Schltr. Orchideen (1914) 544 (excl. syn. sanderiana); Schltr. in Fedde Repert. Beihefte 4 (Orch. Sino-Jap. Prodr.) (1919) 277 (excl. syn. sanderiana).

Phalaenopsis ambigua Reichb. f. in Hamb. Gartenz. 18 (1862) 35, Xen. Orch. 2 (1862) 6; Duchartre in Journ. Soc. Imp. et Centr. Hort. Par. 8 (1862) 311, 731; Lem. in Ill. Hort. 10 (1863) Misc. 13; Naves Novis. App. (1882) 243; Rolfe in Gard. Chron. II 26 (1886) 212.

Phalaenopsis amabilis Blume var, ambigua Burb. in The Garden 22 (1882) 119.

Luzon (Ifugao, Rizal, Tayabas, Sorsogon), Samar, Palawan, Bancalan, Mindanao (Zamboanga, Lanao, Cotabato, Davao), Lumbucan. Fairly common and widely distributed in the Philippines. Epiphyte at sea level to $300 \mathrm{~m}$ altitude. No attempt has been made to segregate the varieties or forms in the distribution above given. In China and Japan.

The following variations of variety aphrodite have been noted and published as species, varieties, subvarieties, or forms, but are of little scientific value, as they are largely based on color and size:

Subvar. DAYANA (Hort. ex Warner \& Will.) Ames Orch. 2 (1908) 227.

Phalaenopsis amabilis Lindl. var. dayana Hort. ex Warner \& Will. Orch. Alb. 1 (1882) t. 11; Gower in The Garden 35 (1889) 363; Will. Orch. Grow. Man. ed. 7 (1894) 661.

Phalaenopsis aphrodite Reichb. f. var. dayana Veitch Man. Orch. Pl. pt. 7 (1891) 24; Stein Orchideenb: (1892) 505.

Subvar. ERUBESCENS (Burb.) Ames Orch. 2 (1908) 227.

Phalaenopsis amabilis Lindl. var. erubescens Burb. in The Garden 22 (1882) 119.

Subvar. GLORIOSA (Reichb. f.) Ames Orch. 2 (1908) 227.

Phalaenopsis gloriosa Reichb. f. in Gard. Chron. III 3 (1888) 554; Gower in The Garden 35 (1889) 362, t. 697; Will. Orch. Grow. Man. ed. 7 (1894) 664; Rolfe in Orch. Rev. 8 (1900) 136, in textu, 13 (1905) 228.

Phalaenopsis aphrodite Reichb. f. var. gloriosa Veitch Man. Orch. Pl. pt. 7 (1891) 25; Stein Orchideenb. (1892) 505.

Sulu Archipelago.

Subvar. SANDERIANA (Reichb. f.) Ames Orch. 2 (1908) 228.

Phalaenopsis sanderiana Reichb. f. in Flora 65 (1882) 466, in Gard. Chron. II 19 (1883) 607, 656, 20 (1883) 110; Burb. in The Garden 24 (1883) 270, t. 40\%; Edit. in l'Orchidoph. 3 (1833) 661; Godefr.-Lebeuf in l'Orchidoph. 5 (1885) 18, $t$.; Linden \& Rodigas in Lindenia 1 (1885) 51, $t$. 23; Will. Orch. Grow. Man. ed. 6 (1885) 535, ed. 7 (1894) 670; Rolfe in Gard. Chron. II 26 (1886) 
212; Warner \& Will. Orch. Alb. 5 (1886) t. 209; Gower in The Garden 35 (1889) 363; Roebelen in Gard. Chron. III 7 (1890) 459, in textu; Stein Orchideenb. (1892) 511; Kränzl. in Sander Reichenbachia II 2 (1894) 41, t. 68 (upper fig.); Rolfe in Orch. Rev. 8 (1900) 133, 15 (1907) 185, fig. 22; Cogn. in Dict. Icon Orch. (1903) Phalaenop. Hybr. t. 2; Rolfe in l'Orchidoph. 13 (1903) 101; G. Wilson in Orch. World 6 (1916) 266.

Forma ALBA (Veitch) Ames Orch. 2 (1908) 228.

Phalaenopsis sanderiana Reichb. f. subvar. alba Veitch Man. Orch. P1. pt. 7 (1891) 35, text cut.

Phalaenopsis sanderiana var. alba Stein Orchideenb. (1892) 511 G. Wilson in Orch. World 6 (1916) 266, text cut.

Phalaenopsis sanderiana Reichb. f. forma alba Will. Orch. Grow. Man. ed. 7 (1894) 670.

Forma MARMORATA (Reichb. f.) Ames Orch. 2 (1908) 228.

Phalaenopsis sanderiana Reichb. f. var. marmorata Reichb. f. in Gard. Chron. II 20 (1883) 812; Rolfe in Gard. Chron. II 26 (1886) 212: Stein Orchideenb. (1892) 511; Will. Orch. Grow. Man. ed. 7 (1894) 670.

Phalaenopsis sanderiana Reichb. f. subvar. marmorata Veitch Man. Orch. Pl.pt. 7 (1891) 35.

Forma PUNCTATA (O'Brien) Ames Orch. 2 (1908) 229.

Phalaenopsis sanderiana Reichb. f. var. punctata O'Brien in Gard. Chron. III 7 (1890) 78; Stein Orchideenb. (1892) 511; Will. Orch. Grow. Man. ed. 7 (1894) 670.

Phalaenopsis sanderiana Reichb. f. subvar. punctata Veitch Man. Orch. Pl.pt. 7 (1891) 35.

PHALAENOPSIS BOXALLII Reichb. f. in Gard. Chron. II 19 (1883) 274; Rolfe in Gard. Chron. II 26 (1886) 276; Veitch Man. Orch. Pl. pt. 7 (1891) 26; Ames Orch. 5 (1915) 216.

PHALAENOPSIS EQUESTRIS (Schauer) Reichb. f. in Linnaea 22 (1849) 864; Lind1. in Paxt. Flow. Gard. 2 (1852) 174; Reichb. f. in Walp. Ann. 3 (1852) 562, 6 (1864) 860; Miq. Fl. Ind. Bat. 3 (1859) 690; Reichb. f. in Hamb. Gartenz. 16 (1860) 116; Duchartre in Journ. Soc. Imp. et Centr. Hort. Par. 6 (1860) 869, 8 (1862) 727; Reichb. f. Xen. Orch. 2 (1862) 4; Naves Novis. App. (1882) 242; Ames Orch. 2 (1908) 229, 5 (1915) 216.

Siauroglottis equestris Schauer in Nov. Act. Acad. Nat. Cur. 19 Suppl. 1 (1843) 432.

Phalaenopsis rosea Lindl. in Gard. Chron. (1848) 671, text cut: Paxt. Mag. Bot. 16 (1849) 60, 189, text cut; Lindl. in Paxt. Flow. Gard. 2 (1852) 173, t. 79; Reichb. f. in Bot. Zeit. 10 (1852) 673; Moore Ill. Orch. Pl. (1857) Phalaen. 7; Hook, in Bot. Mag. 86 (1860) t. 5212; Lem. in Jard. Fleur. 3 (1853) t. 283, in Ill. Hort. 10 (1863) Misc. 11; Van Houtte in Fl. des Serres 16 (1866) t. 1645; Jennings Orch. (1875) t. 27; Burb. in The Garden 22 (1822) 119 (excl. var.) ; Vidal Phan. Cuming. Philip. (1885) 150, Rev. Pl. Vasc. Filip. (1886) 270; Rolfe in Gard. Chron. II 26 (1886) 276; Warner \& 
Will. Orch. Alb. 6 (1887) t. 268; Veitch Mari Oreh. Pl. pt. 7 (1891)

34; Ames Orch. 1 (1905) 102.

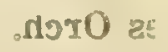

Phalaenopsis esmeralda Cogn. in Dict. Icon borch. (1898) Phalaen.

t. 3, non Reichb. f.

a

Luzon (Ilocos Norte, Bataan, Rizal, Laguna;1. Tayabas, Camárines), Panay (Capiz), Bohol, Mindanao (Camiguin Istotra :Lanao). A common and widely distributed species. Epiphyte at seartetel to $-300 \mathrm{~m}$ altitude.

Var. LeUCASPIS Reichb. f. in Gard. Chron. II 15 (1881) 688, in l'Orchidoph. 1 (1881) 50; Ames Orch. 2 (1908) 230.

Phalaenopsis rosea Lindl. var. leucaspis Rulfer in Gard. Chron.: II 26 (1886) 276; Veitch Man. Orch. Pl. pt. 34.

Var. LEUCOTANTHE Reichb. f. in l'Orchidoph (3) 490; Ames Orch. 2 (1908) 230.

PHALAENOPSIS FASCIATA Reichb. f. in Gard. Chroł' 18 (1882) 134; Rolfe in Orch. Rev. 13 (1905) 225; Ames breh. 25 (1915) 217.

No material of this species has been seen. Reichent ch gave the origin of the plant as Philippines, imported by Messrs. H: Low \& Co. The origin seems open to some doubt. Reichenbach statos that the species is near Phalaenopsis lueddemanniana Reichb. $\mathrm{f}$.

PHALAENOPSIS FUSCATA Reichb. f. in Gard. Chron. II 2 (1874) 6; Rolfe in Orch. Rev. 13 (1905) 226; Ames Orch: $5^{1}$ (1915) 216.

Phalaenopsis denisiana Cogn. in Gard. Chron. IIt 26 (1899) 82; Cogn. in Dict. Icon. Orch. (1899) Phalaenop. $t$. 6.

The origin of $P$. fuscata is given as the Malay Peninsula, that of $P$. denisiana as the Philippines. No material of this species has been seen.

PHALAENOPSIS LEUCORRHODA Reichb. f. in Gard. Chron. II 3 (March, 1875) 301, 366; W. G. Sm. in Floral Mag. 14 (June, 1875) 41, t. 166; Naves Novis. App. (1882) 243; Rolfe in Gard. Chron. II 26 (1886) 212; Veitch Man. Orch. Pl. pt. 7 (1891) 46; Stein Orchideenb. (1892) 507; Will. Orch. Grow. Man. ed. 7 (1894) 667; Rolfe in Orch. Rev. 13 (1905) 232, fig. 54.

This is a supposed natural hybrid between Phalaenopsis amabilis (Linn.) Blume var. aphrodite (Reichb. f.) Ames and $P$. schilleriana Reichb. f. Its origin is Philippine, but no specimens have been seen.

PHALAENOPSIS LINDENII Loher in Journ. des Orch. 6 (1895) 103; Rolfe in Orch. Rev. 13 (1905) 230, 15 (1907) 296; Ames in Philip. Journ. Sci. 4 (1909) Bot. 599, Orch. 5 (1915) 217; G. Wilson in Orch. Rev. 30 (1922) 354.

Luzon (Benguet), Lyon 39, F. B. 5121 Curran. Epiphyte.

PHALAENOPSIS LUEDDEMANNIANA Reichb. f. in Bot. Zeit. 23 (1865) 146, in Gard. Chron. (1865) 434; Moore in Flor. \& Pomol. (1865) 257, t. 254; Lem. in Ill. Hort. 12 (1865) Misc. 31; Edit. in Proc. Roy. Hort. Soc. 5 (1865) 137; Otto in Hamb. Gartenz. 21 (1865) 470; G. B. in Belg. Hort. 15 (1865) 229; Carr. in Rev. Hort. 44 (1872) 390, t.; Rolfe in Gard. Chron. II 26 (1886) 277, in Lindenia 2 (1886) 95, t. 94, 8 (1892) 63, t. 366; Veitch Man. Orch. Pl. pt. 
7 (1891) 30, ext cut; Cogn. in Dict. Icon. Orch. (1899) Phalaen. t. 9; Ames Orch. 2 (1908) 230, 5 (1915) 217.

Phalaenopsis! manii Boxall ex Naves Novis. App. (1882) 243, sphalm.

Luzon (Apayao, I eva Vizcaya, Bataan, Bulacan, Laguna, Tayabas, Sorsogon), Polillo, L a whst, Palawan, Mindanao (Bukidnon). A common and widely distributes epies, ephyte at 30 to $60 \mathrm{~m}$ altitude.

A number of varictic. of this species have been characterized from cultivated specimens:

Var. DELICATA Rachlo: f. in Gard. Chron. (1865) 434; Burb. in The Garden - 1882) 119; Rolfe in Gard. Chron. II 26 (1886) 277, in Linde (1892) 63, sub t. 366; Ames Orch. 2 (1908) 231.

Phalaen reddémanniana Batem, in Bot. Mag. 91 (1865) t. 5523; : : Toutte in Fl. des Serres 16 (1865) 53, t. 1636; Batem. Second (c. Jro Orch. Pl. (1867) t. 133, non Reichb. f.

Phalaenopsi deridemanniana subvar. delicata Veitch Man. Orch. .Pl. pt. 7 ( (\$91) 30.

Var. HIEROGLY 'CA Reichb. f. in Gard. Chron. III 2 (1887) 586; Edit. in '" sulidoph. 9 (1889) 197; Rolfe in Lindenia 8 (1892) 63, sub t. 366; Ames Orch. 2 (1908) 231.

Phalaenopsis lueddemanniana Reichb. f. subvar. hieroglyphica Veitch Man. Orch, Pl. pt. 7 (1891) 31.

Var. OCHRACEA Reichb. f. in Gard. Chron. (1865) 438; Carr. in Rev. Hort. 44 (1872) 391, fig. A; Burb. in The Garden 22 (1882) 119; Rolfe in Gard. Chron. II 26 (1886) 277, in Lindenia 8 (1892) 63, sub t. 366; Ames Orch. 2 (1908) 232.

Phalaenopsis lueddemanniana subvar. ochracea Veitch Man. Orch. P1. pt. 7 (1891) 31.

Lyon 48.

Var. PULCHRA Reichb. f. in Gard. Chron. II 4 (1875) 36; Burb. in The Garden 22 (1882) 119; Rolfe in Gard. Chron. II 26 (1886) 277, in Lindenia 8 (1892) 64, sub t. 366; Ames Orch. 2 (1908) 232.

Phalaenopsis lueddemanniana Reichb. f. subvar. pulchra Veitch Man. Orch. Pl. pt. 7 (1891) 31.

PHALAENOPSIS MARIAE Burb. in Warner \& Will. Orch. Alb. 2 (1883) t. 80 et sub t. 8\%; Rolfe in Gard. Chron. II 26 (1886) 277; Hook. f. in Bot. Mag. 113 (1887) t. 6964; Veitch Man. Orch. Pl. pt. 7 (1891) 32; Ridl. in Journ. Linn. Soc. 31 (1896) 292; Ames in Philip. Journ. Sci. 8 (1913) Bot. 434, Orch. 5 (1915) 217, in Merr. in Journ. Roy. Asiat. Soc. Straits Branch, Special No. (1921) 197.

Luzon (Nueva Vizcaya), Mindanao (Lanao, Cotabato, Bukidnon, Davao), B. S. 11136, 11141 McGregor, 21389 Escritor, M. S. Clemens 626, Weber 220, Loher 6011. Epiphyte up to $700 \mathrm{~m}$ altitude in Cotabato. In Borneo.

PHALAENOPSIS MICHOLITZII Rolfe in Gard. Chron. III 8 (1890) 187, in Journ. des Orch. 1 (1890) 198, in Orch. Rev. 13 (1905) 229; Ames Orch. 5 (1915) 217. 
Without locality indicated, Micholitz.

No technical description of this species has been published. From the material at hand it appears searcely distinct from Phalaenopsis lueddemanmiana Reichb. $f$.

PHALAENOPSIS REICHENBACHIANA Reichb. $f$. \& Sander in Gard. Chron. II 18 (1882) 586; Rolfe in Orch. Rev. 13 (1905) 226; Ames Orch. 5 (1915) 218.

According to Rolfe (in Orch. Rev. 1. c.) Micholitz stated that this species is a native of Mindanao. No material has been seen.

PHALAENOPSIS SCHILLERIANA Reichb. f. in Hamb. Gartenz. 16 (1860) 115, 116; Lindl. in Gard. Chron. (1860) 216, (1861) 617, (1862) 95; Duchartre in Journ. Soc. Imp. et Centr. Hort. Par. 7 (1861) 845, 8 (1862) 609, 730, t. 1\%; Warner Sel. Orch. Pl. I (1861) t. 1; Reichb. f. Xen. Orch. 2 (1862) 1, 5, t. 101; Lem. in Ill. Hort. 10 (1863) Misc. 13, t. 348; Lescuyer in Horticulteur Fr. (1863) 127, t. 11; Van Houtte in Fl. des Serres 15 (1864) 115, tt. 1559-60, 21 (1875) 142, text cut; Batem. in Bot. Mag. 91 (1865) $t .5530$, Second Cent. Orch. Pl. (1867) t. 1r1; Regel in Gartenfl. 17 (1868) 161, t. 581, 36 (1887) 57, fig. 21; Jennings Orch. (1875) t. 15; Edit. in Gard. Chron. II 4 (1875) 169, fig. 34, II 12 (1879) 301, fig. $47 \mathrm{D}-F$, III 3 (1888) 528, fig. 72; Gedney in Floral World (1876) 321, 323, t.; Puydt Orch. (1880) 309, t. 35; Burb. in The Garden 22 (1882) 118, 119, t. 348, fig. 2; Naves Novis. App. (1882) 243; Edit. in l'Orchidoph. 4 (1884) 279, t. 212; Rodigas in Ill. Hort. 35 (1888) 57, t. 56; Pfitz. in Engl. \& Prantl Pflanzenfam. 2. Abt. 6 (1889) 211, fig. 227 C; Rolfe in Lindenia 5 (1889) $t$. 227; Roebelen in Gard. Chron. III 7 (1890) 459; Veitch Man. Orch. Pl. pt. 7 (1891) 36, text cut; Rivois in Journ. des Orch. 3 (1892) 146, fig. 18; Rolfe in Orch. Rev. 2 (1894) 209, fig. 21, 8 (1900) 134-136, fig. 21, 13 (1905) 232, fig. 53; Grignan in Sem. Hort. 2 (1898) 46, fig. 214; Cogn. in Dict. Icon. Orch. (1899) Phalaen. t. 10; L. Linden in Sem. Hort. 4 (1900) 424, fig. 143; Ames Orch. 2 (1908) 232, 5 (1915) 218.

Luzon (Tayabas), Lyon 54, Merrill 4018, 4024. This is one of the most highly prized of the Philippine orchids and is extensively exported. A number of forms have been described from cultivated specimens.

Var. ADVENA Reichb. f. in Gard. Chron. II 23 (1885) 174; Edit. in 1'Orchidoph. 5 (1885) 200; Ames Orch. 2 (1908) 234.

Var. IMMACULATA Reichb. f. in Gard. Chron. II 3 (1875) 429; Rolfe in Gard. Chron. II 26 (1888) 212; Ames Orch. 2 (1908) 234.

Phalaenopsis schilleriana Reichb. f. var. delicata Dean in Floral Mag. 16 (1877) $t .25 \%$

Phalaenopsis schilleriana Reichb. f. subvar. immaculata Veitch Man. Orch. Pl. pt. 7 (1891) 37.

Var. MAJOR Rolfe in Gard. Chron. II 26 (1886) 212; Ames Orch. 2 (1908) 234. 
Var. PURPUREA Hort. ex Rolfe in Journ. des Orch.2 (1892) 358; Garnier in Journ. des Orch. 5 (1894) 9; O'Brien in Gard. Chron. III 15 (1894) 266; Ames Orch. 2 (1908) 234.

Var. SPLENDENS Hort. ex Carr. in Rev. Hort. 58 (1886) 396, t.; Warner Sel. Orch. Pl. III (?) t. 5; Ames Orch. 2 (1908) 234.

Var. VESTALIS Reichb. f. in Gard. Chron. II 17 (1882) 330; Edit. in l'Orchidoph. 2 (1882) 291; Rolfe in Gard. Chron. II 26 (1886) 212; Edit. in Gard. Chron. III 3 (1888) 75; Ames Orch. 2 (1908) 235.

Phalcenopsis schilleriana Reichb. f. var. alba Roebelen in Gard. Chron. III 7 (1890) 459; Rolfe in l'Orchidoph. 13 (1893) 101, in Orch. Rev. 8 (1900) 134, in textu.

Phalaenopsis schilleriana Reichb. f. subvar, vestalis Veitch Man. Orch. Pl. pt. 7 (1891) 37.

PHALAENOPSIS SCHILLERIANA-STUARTIANA Rolfe in Orch. Rev, 8 (1900) 94, 113, fig. 18, 13 (1905) 230, fig. 50; G. Wilson in Orch. Rev. 30 (1922) 77, text cut.

Said to be a natural hybrid of Philippine origin.

PHALAENOPSIS STUARTIANA Reichb. f. in Gard. Chron. II 16 (1881) 748, 753, fig. 149; Hook. f. in Bot. Mag. 108 (1882) t. 6622; Rodigas in Ill. Hort. 31 (1884) 175, t. 540; Rolfe in Gard. Chron. II 26 (1886) 212; Warner \& Will. Orch. Alb. 5 (1886) t. 237; Reichb. f. in Sander Reichenbachia I (1888) 95, $t$. 42; Veitch Man. Orch. Pl. pt. 7 (1891) 39, t. (opp. p. 29); Cogn. in Dict. Icon. Orch. (1898) Phalaen. t. 2; Ames Orch. 5 (1915) 218.

Philippines, without locality, Lyon s. n.

PHALAENOPSIS VEITCHIANA Reichb. f. in Gard. Chron. (1872) 935; Burb. in Floral Mag. 15 (1876) t. 213; Veitch Man. Orch. Pl. pt. 7 (1898) 47; Ames Orch. 5 (1915) 218; G. Wilson in Orch. Rev. 30 (1922) 346.

\section{EXCLUDED OR SYNONYMOUS SPECIES}

Phalaenopsis amabilis Lindl. in Bot. Reg. 24 (1838) $t .34$ (excl. syn.); Paxt. Mag. Bot. 5 (1838) 20, 162, 7 (1840) 49, text cut (excl. syn.); Maund Botanist 3 (1839?) t. 133 (excl. syn.); Lem. in Fl. des Serres 1 (1845) 85, text cut, t. 36 (excl. syn.); Blanco Fl. Filip. ed. 2 (1845) 592, ed. 3, 3 (1879) 41; Edit. in Allg. Berl. Gartenz. 13 (1845) 389, 15 (1847) 215 (excl. syn. in both); Hook. in Bot. Mag. 73 (1847) t. 4297 (excl. syn. in parte); Blume Rumphia 4 (1848) 52, quoad citat. Lindl.; Lindl. in Gard. Chron. (1848) 39, fig. 2; Reichb. f. in Walp. Ann. 1 (1849) 790, 3 (1852) 561, quoad Philip.; Moore Ill. Orch. Pl. (1857) Phalaenop. 6, t. 1 (excl. syn.) ; Miq. Fl. Ind. Bat. 3 (1859) 690, quoad citat. Bot. Mag. et quoad Philip.; Gedney in Floral World (1876) 323; Reichb. f. in Gard. Chron. II 5 (1876) 654; Burb. in The Garden 22 (1882) 119; Naves Novis. App. (1882) 243; Vidal Phan. Cuming. Philip. (1885) 150, Rev. Pl. Vasc. Filip. (1886) 270; Linden \& $200823-27$ 
Rodigas in Lindenia 2 (1886) 65, t. 79 (excl. syn. in parte); Gower in The Garden 35 (1889) 362, fig. 363; Roebelen in Gard. Chron. III 7 (1890) 459, in textu; Will. Orch. Grow. Man. ed. 7 (1894) 660, quoad bibliog. in parte et quoad Philip.; Merr. in Govt. Lab. Publ. (Philip.) 8 (1903) 57, 174, 27 (1905) 84; J. J. Sm. Fl. Buitenz. 6 (Orch. Jav.) (1905) 549, quoad bibliog. in parte et quoad Philip.; Ames Orch. 1 (1905) 101, quoad spec. (excl. syn. et descr.); Merr. Sp. Blancoanae (1918) 115, non Blume $=$ Phalaenopsis amabilis Blume var. aphrodite (Reichb. f.) Ames.

Phalaenopsis ambigua Reichb. $\mathrm{f}$. in Hamb. Gartenz. 18 (1862) 35, Xen. Orch. 2 (1862) 6; Duchartre in Journ. Soc. Imp. et Centr. Hort. Par. 2 (1862) 311, 731; Lem. in Ill. Hort. 10 (1863) Misc. 13; Naves Novis. App. (1882). 243; Rolfe in Gard. Chron. II 26 (1886) 212 = Phalaenopsis amabilis Blume var. aphrodite (Reichb. f.) Ames.

Phalaenopsis aPhrodite Reichb. $f$. in Hamb. Gartenz. 18 (1862) 35, Xen. Orch. 2 (1862) 6; Duchartre in Journ. Soc. Imp. et Centr. Hort. Par. 8 (1862) 730; Lem. in Ill. Hort. 10 (1863) Misc. 14 (excl. citat. Rumph.) ; Rolfe in Gard. Chron. II 26 (1886) 212, fig. $43 B$; Pfitz. in Engl. \& Prantl Nat. Pfianzenfam. 2, Abt. 6 (1889) 211, fig. 230; Veitch Man. Orch. Pl. pt. 7 (1891) 24, t.; Stein Orchideenb. (1892) 505, fig. 155; Rolfe in Orch. Rev. 2 (1894) 209, fig. 20, 8 (1900) 135, 136, fig. 20, 13 (1905) 232, fig. 52, 25 (1917) 151, in textu; Cogn. Dict. Icon. Orch. (1898) Phalaenop. t. 1; Edit. in Sem. Hort. 4 (1900) 392, fig. 132; Rolfe in Forbes \& Hemsl. in Journ. Linn. Soc. 36 (1903) 34; Matsum. Ind. Pl. Jap. 2 (1905) 257; Ames in Philip. Journ. Sci. 2 (1907) Bot. 336; Schltr. Orchideen (1914) 544 (excl. syn. sanderiana); Schltr. in Fedde Repert. Beihefte 4 (Orch. Sino-Jap. Prodr.) (1919) 277 (excl. syn. sanderiana) = Phalaenopsis amabilis Blume var. aphrodite (Reichb. f.) Ames.

Phalaenopsis casta Boxall ex Naves Novis. App. (1882) 243, nomen.

Boxall probably had in mind Phalaenopsis casta Reichb. f., which is now considered a form or variety of $P$. leucorrhoda Reichb. $f$.

Phalaenopsis Cornu-Cervi Blume apud Naves Novis. App. (1882) 243.

Phalaenopsis deliciosa Reichb. f. apud Naves Novis. App. (1882) 243.

Phalaenopsis denisiana Cogn. in Gard. Chron. III 26 (1899) 82; Cogn. in Dict. Icon. Orch. (1899) Phalaenop. t. $6=$ Phalaenopsis fuscata Reichb. f.

Phalaenopsis Devriesiana Reichb. f. apud Naves Novis. App. (1882) 243.

Phalaknopsis esmeralda Cogn. in Dict. Icon. Orch. (1898) Phalaenop. t. 3, non Reichb. $f_{0}=$ Phalaenopsis equestris (Schauer) Reichb. $\mathrm{f}$.

Phalaenopsis gloriosa Reichb. f. in Gard. Chron. III 3 (1888) 554; Gower in The Garden 35 (1889) 362, t. 697; Will. Orch. Grow. Man. ed. 7 (1894) 664; Rolfe in Orch. Rev. 8 (1900) 136, in textu, 
13 (1905) 228 = Phalaenopsis amabilis (Linn.) Blume subvar. gloriosa (Reichb. f.) Ames.

Phalaenopsis Hebe Reichb. f. apud Naves Novis. App. (188,2) 242.

Phalamopsis InTERmedia Lindl, apud Naves Novis. App. (1882) 243.

Phataenopsis INTERMEDIA Lindl. var. PORTEI Reichb. f. in Bot. Zeit. 21 (1863) 128; W. G. Sm. in Floral Mag. 14 (1875) t. 162; Naves Novis. App. (1882) 243; Warner Sel. Orch. Pl. II (1865-75) t. 2; Veitch Man. Orch. Pl. pt. 7 (1891) 45; Sander Reichenbachia II 2 (1894) 43, t. 68 (lower fig.).

Phalaenopsis intermedia Lindl. var. brymeriana Reichb. f. in Gard. Chron. II 5 (1876) 366; Dean in Floral Mag. 16 (1877) t. 263; Veitch Man. Orch. PI. pt. 7 (1891) 44; Warner \& Will. Orch. Alb. 9 (1891) t. 415.

Phalaenopsis portei Reichb. f. in Gard. Chron. II 5 (1876) 370, figs. 70, 71; Outram in Gard. Chron. II 15 (1881) 181.

There seems no valid authority for including this in the list of Philippine species.

Phalaknopsis LowII Reichb. f. apud Naves Novis. App. (1882) 243.

Phalaenopsis Lueddemanniana Batem. in Bot. Mag. 91 (1865) t. 5523; Van Houtte in Fl. des Serres 16 (1865) 53, $t .1636$; Batem. Second Cent. Orch. Pl. (1867) t. 133, non Reichb. f. = Phalaenopsis lueddemarnia Reichb. $f$. var. delicata Reichb. f.

Phalaenopsis Luddemanir Boxall ex Naves Novis. App. (1882) 243, sphalm $=$ Phalaenopsis lueddemanniana Reichb. $f$.

Phalaenopsis Paliens (Lindl.) Reichb. f. in Walp. Ann. 6 (1864) 932; Rolfe in Gard. Chron. II 26 (1886) 276, in Orch. Rev. 8 (1900) 327,13 (1905) 226.

Trichoglottis pallens Lindl. in Journ. Hort. Soc. 5 (1850) 34, in Paxt. Flow. Gard. 1 (1850) 15.

Stauropsis pallens Reichb. f. in Hamb. Gartenz. 16 (1860) 117, Xen. Orch. 2 (1862) 7; Naves Novis. App. (1882) 243.

For many years this species was ascribed to the Philippines. It does not occur in the Archipelago, and Rolfe, 1. c., has shown that the type could not have come from the Philippines.

Phalaenopsis Parishi Reichb. f. apud Naves Novis. App. (1882) 243.

Phalaenopsis Rosea Lindl. in Gard. Chron. (1848) 671, text cut; Paxt. Mag. Bot. 16 (1849) 60, 189, text cut; Lindl. in Paxt. Flow. Gard. 2 (1852) 173, t. 72; Reichb. f. in Bot. Zeit. 10 (1852) 673; Moore Ill. Orch. Pl. (1857) Phalaen. 7; Hook. in Bot. Mag. 86 (1860) t. 5212; Lem. in Jard. Fleur. 3 (1853) t. 283, in Ill. Hort. 10 (1863) Misc. 11; Van Houtte in Fl. des Serres 16 (1866) t. 1645; Jennings Orch. (1875) $i .2 \%$; Burb. in The Garden 22 (1882) 119 (excl. var.); Vidal Phan. Cuming. Philip. (1885) 150, Rev. Pl. Vasc. Filip. (1886) 270; Rolfe in Gard. Chron. II 26 (1886) 276; Warner \& Will. Orch. Alb. 6 (1887) t. 268; Veitch Man. Orch. Pl. pt. 7 (1891) 34; Ames Orch. 1 (1905) 102 = Phalaenopsis equestris (Schauer) Reichb. f. 
Phalaenopsis SANDERIANA Reichb. f. in Flora 65 (1882) 466, in Gard. Chron. II 19 (1883) 607, 656, 20 (1883) 110; Burb. in The Garden 24 (1883) 270, t. 407; Edit. in l'Orchidoph. 3 (1883) 661; Godefr.Lebeuf in l'Orchidoph. 5 (1885) 18, $t_{\text {.; }}$; Linden \& Rodigas in Lindenia 1 (1885) 51, t. 23; Will. Orch. Grow. Man. ed. 6 (1885) 535, ed. 7 (1894) 670; Rolfe in Gard. Chron. II 26 (1886) 212; Warner \& Will. Orch. Alb. 5 (1886) t. 209; Gower in The Garden 35 (1889) 363; Roebelen in Gard. Chron. III 7 (1890) 459, in textu; Stein Orchideenb. (1892) 511; Kränzl. in Sander Reichenbachia II 2 (1894) 41, t. 68 (upper fig.); Rolfe in Orch. Rev. 8 (1900) 133, 15 (1907) 185, fig. 22; Cogn. in Dict. Icon. Orch. (1903) Phalaenop. hybr. $t$. 2; Rolfe in l'Orchidoph. 13 (1903) 101; G. Wilson in Orch. World 6 (1916) $266=$ Phalaenopsis amabilis (Linn.) Blume subvar. sanderiana (Reichb. f.) Ames:

Phalaenopsis sumatrana Korth. apud Naves Novis. App. (1882) 242.

Phalaenopsis violacea Teijsm. \& Binn. apud Naves Novis. App. (1882) 243.

\section{AËRIDES Loureiro}

AËRIDES AUGUSTIANUM Rolfe in Lindenia 5 (1889)' 39, t. 210, in Gard. Chron. III 7 (1890) 9; Veitch Man. Orch. Pl. pt. 7 (1891) 65; Ames Orch. 5 (1915) 218.

Discovered in the Philippines by Auguste Linden; no specimen seen.

AËRIDES JARCKIANUM Schltr. in Orchis 9 (1915) 53 t. 5, figs. 15-21.

Luzon, Jarck. No specimen seen. Reported to have been collected near the "Lagune von Manila."

AËRIDES LAWRENCIAE Reichb. f. in Gard. Chron. II 20 (1883) 307 , 368,460 ; Warner \& Will. Orch. Alb. 6 (1887) t. 270; Gower in The Garden 35 (1889) 484, $t$. 702; Veitch Man. Orch. Pl. pt. 7 (1891) 71, text cut; Kränzl. Xen. Orch. 3 (1892) t. 252, fig. 5; Ames Orch. 2 (1908) 249, 5 (1915) 218, in Philip. Journ. Sci. 4 (1909) Bot. 600; G. Wilson in Orch. Rev. 31 (1923) 19.

Mindanao (Davao), R. F. Black s. n., Elmer 1078\%, Lyon $Y$ (without locality).

Var. AmEsiAnum Sander ex Kränzl, in Gard. Chron. III 10 (1891) 393; Kränzl. Xen. Orch. 3 (1892) 94, t. 252, figs. 1-4, 7-10; Ames Orch. 2 (1908) 250; G. Wilson in Orch. Rev. 31 (1923) 19, in textu.

Var. SANDERIANUM (Reichb. f.) Sander ex Kränzl. Xen. Orch. 3 (1892) 95, in textu, t. 252, fig. 6 (as var. sanderiana); Ames Orch. 2 (1908) 250; G. Wilson in Orch. Rev. 31 (1923) 19, in textu.

Aërides sanderianum Reichb. f. in Gard. Chron. II 22 (1884) 134; O'Brien in Gard. Chron. II 22 (1884) 460.

Aërides lawrenciae subvar. sander's Veitch Man. Orch. Pl. pt. 7 (1891) 72, text cut.

AËRIDES ODORATUM Lour. Fl. Cochinch 2 (1790) 525; Sw. in Kongl. Svensk. Vet.-Akad. Nya Handl. 21 (1800) 244; Lindl. Gen. \& Sp. Orch. (1833) 239; Knowles \& Westc. Floral Cab. 2 (1838) t. 75; Hook. in Bot. Mag. 71 (1845) t. 4139; Regel in Gartenfl. 6 
(1857) t. 272; Naves Novis. App. (1882) 239; Hook. f. Fl. Brit. Ind. 6 (1890) 47; Veitch Man. Orch. Pl. pt. 7 (1891) 76; Grant Orch. Burma (1895) 272; Ridl. in Journ. Linn. Soc. 31 (1896) 294, 32 (1896) 376; King \& Pantl. in Ann. Roy. Bot. Gard. Calc. 8 (Orch. Sik.-Himal.) (1898) 212, t. 282; J. J. Sm. Fl. Buitenz. 6 (Orch. Jav.) (1905) 581, Fig.-Atlas (1912) fig. 4.36; Duthie in Ann. Roy. Bot. Gard. Calc. 9 (Orch. North-west. Himal.) (1906) 143; Ridl. Mat. Fl. Mal. Penin. 1 (1907) 179; Ames Orch. 2 (1908) 250, 5 (1915) 218, 6 (1920) 220, in Merr。in Journ. Roy. Asiat. Soc. Straits Branch, Special No. (1921) 197.

Aërides cornutum Roxb. Hort. Beng. (1814) 63, nomen; Roxb. Fl. Ind. ed. 2,3 (1832) 472; Lindl, in Bot. Reg. 18 (1832) t. 1485.

Aërides virens Lindl. in Bot. Reg. 29 (1843) Misc. 41, 30 (1844)

t. 41; Miq. Fl. Ind. Bat. 3 (1859) 695; Naves Novis. App. (1882) 239; Warner \& Will. Orch. Alb. 4 (1885) t. 160; Veitch Man. Orch. Pl. pt. 7 (1891) 81.

Aërides suaveolens Blume Rumphia 4 (1848) 53, t. 193, fig. 1, Mus.

Bot. Lugd.-Bat. 1 (1849) 112, non Roxb.

Aërides suavissimum Lindl. in Journ. Hort. Soc. 4 (1849) 264, in

Paxt. Flow. Gard. 2 (1851-52) 141, t. 66; Hook. f. Fl. Brit. Ind. 6 (1890) 47; Veitch Man. Orch. Pl. pt. 7 (1891) 78, text cut; Rolfe in Lindenia 7 (1891) 41, t. 307; Ceron Cat. Pl. Herb. Manila (1892) 168.

Aërides flavidum Lindl. in Paxt. Flow. Gard. 2 (1851-52) 101.

Aërides reichenbachii J. Linden ex Reichb. f. in Koch. \& Fintelm.

Wochenschr. 1 (1858) 61; Reichb. f. Xen. Orch. 2 (1862) 11, $t$. 104; Naves Novis. App. (1882) 239; J. Linden in Lindenia 1 (1885) $7, t .1$.

Aërides jucundum Reichb. f. in Hamb. Gartenz. 16 (1860) 281; Morr. in Belg. Hort. 26 (1876) 289.

Aërides nobile Warner Sel. Orch. I (1862) t. 11; Regel in Gartenfl. 19 (1870) 40; Naves Novis. App. (1882) 239.

Aërides reichenbachii Linden var. cochinchinense Hort. ex Reichb. $f$. in Gard. Chron. II 14 (1880) 620.

Aërides rohanianum Reichb. f. in Gard. Chron. II 21 (1884) 206; Ames Orch. 5 (1915) 219.

Aërides wilsonianum Hort. ex Gard. Chron. II 23 (1885-) 705.

Aërides ballantinianum Reichb. f. in Gard. Chron. II 24 (1885) 198.

Mindoro, Lyon $P$. A very variable and widely distributed species.

Many horticultural varieties have been described and some field varieties, usually based on one of the specific names formerly in use. In India from the Himalayas to Sylhet, in Burma, Malay Peninsula, Cochin China, China, Java, and Borneo.

AËRIDES QUINQUEVULNERUM Lindl. Sert. Orch. (1838) t. 30 ; Paxt. Mag. Bot. 6 (1839) 167, 260, 8 (1841) 241, t. and text cut; Reichb. f. in Walp. Ann. 6 (1864) 899; Naves Novis. App. (1882) 239; Vidal Phan. Cuming. Philip. (1885) 150, Rev: Pl. Vasc. Filip. (1886), 271; J. Linden in Lindenia 4 (1888) 15, t. 150; Veitch Man. Oreh. Pl. pt. 7 (1891) 77; Warner \& Will. Orch. Alb. 11 (1897) t. 495; Ames Orch. 1 (1905) 104, 2 (1908) 252, 5 (1915) 219; Merr. Sp. Blancoanae (1918) 116. 
Aërides maculatum Llanos Frag. Fl. Filip. .(1851) 93; F.-Vill. \& Naves in Blanco Fl. Filip. ed. $3,4^{1}$ (1880) 72, t. 409, non Buch.Ham.

Aërides fenzlianum Reichb. f. in Hamb. Gartenz. 16 (1860) 282.

Aërides thibautianum Reichb. f. in Gard. Chron. (1866) 101.

Luzon (Bataan, Bulacan, Rizal, Cavite, Laguna, Tayabas). A common species in and about Rizal at relatively low altitude.

Var. FARMERI (Boxall) Stein Orchideenb. (1892) 63; Ames Orch. 2 (1908) 253.

Aërides farmeri Boxall ex Naves Novis. App. (1882) 239, nomen.

Aërides album Sander ex Stein Orchideenb. (1892) 63, in syn.

Var. MARGINATUM (Reichb. f.) Stein Orchideenb. (1892) 63; Ames Orch. 2 (1908) 253.

Aërides marginatum Reichb. f. in Gard. Chron. II 23 (1885) う̄33, 25 (1886) 170.

Var. PURPURATUM Reichb. f. in Gard. Chron. II 16 (1881) 230; Ames Orch. 2 (1908) 253.

Var. SCHADENBERGIANUM Stein Orchideenb. (1892) 63; Ames Orch. 2 (1908) 253.

Mindanao, Schadenberg (fide Stein).

\section{EXCLUDED OR SYNONYMOUS SPECIES}

Aërides Album Sander ex Stein Orchideenb. (1892) 63, in syn.

Aërides quinquevulnerum Lindl. var. farmeri (Boxall) Stein.

AËRIDES CYLINDRICUM Boxall ex Naves Novis. App. (1882) 239, nomen.

AËrIDES DIURNum Teijsm. \& Binn. in Nat. Tijdschr. Ned. Ind. 27 (1864) 19 = Sarcochilus pallidus (Blume) Reichb. f.

AËRIDES FARMERI Boxall ex Naves Novis. App. (1882) 239, nomen = Aërides quinquevulnerum Lindl. var. farmeri (Boxall) Stein.

AËrIDEs FENZLIANUM Reichb. f. in Hamb. Gartenz. 16 (1860) $282=$ Aërides quinquevulnerum Lindl.

AËrIDES LARPEnTA Boxall ex Naves Noyis. App. (1882) 239, nomen.

Ä̈rides Maculatum Llanos Frag. Pl. Filip. (1851) 93; F.-Vill. \& Naves in Blanco Fl. Filip. ed. $3,4^{7}(1880) 72, t$. 409 , non Buch.-Ham. = Aërides quinquevulnerum Lindl.

AËRIDES MARGARITACEUM Boxall ex Naves Novis. App. (1882) 239, nomen.

Ä̈RIDES MARgINATUM Reichb. f. in Gard. Chron. II 23 (1885) 533, 25

(1886) $170=$ Aërides quinquevulnerum Lindl. var. marginatum (Reichb. f.) Stein.

AËrides MENDELLII Boxall ex Naves Novis. App. (1882) 239, nomen.

AËRIDES NOBILE Warner Sel. Orch. Pl. I (1862) t. 11; Regel in Gartenf. 19 (1870) 40; Naves Novis. App. (1882) $239=$ Aërides odoratum Lour. 
AËRIDES ORTGESIANUM Reichb, f. in Gard. Chron. II 23 (1885) 501; Ames Orch. 5 (1915) 218.

No specimens from the Philippines have been seen. A near ally, probably a form, of Aërides quinquevulnerum Lindl.

AËRIDES REICHENBACHII J. Linden ex Reichb. $f$. in Koch \& Fintelm. Wochenschr. 1 (1858) 61; Naves Novis. App. (1882) 239; J. Linden in Lindenia 1 (1885) $t .1=$ Aërides odoratum Lour.

AËRIDES ROEBEIENII Reichb. f. in Gard. Chron. II 21 (1884) 510; Ames Orch. 5 (1915) 219.

No specimens from the Philippines have been seen. A near ally, probably a form, of Aërides quinquevulnerum Lindl.

AËRIDES ROHANIANUM Reichb. f. in Gard. Chron. II 21 (1884) 206; Ames Orch. 5 (1915) $219=$ Aërides odoratum Lour.

AËrides SAVAgeAnUM Sander ex Veitch Man. Orch. Pl. pt. 7 (1891) 78; Rolfe in Sander Reichenbachia II 2 (1894) 81, $t .81$; O'Brien in Gard. Chron. III 16 (1894) 562; Ames Orch. 5 (1915) 219.

Aërides shibatitiana Boxall ex Naves Novis, App. (1882) 239, nomen.

Perhaps an error for Aërides thibautianum Reichb. f., which is equal tc Aërides quinquevulnerum Lindl.

AËRIDES SPURIUm Lindl. Gen. \& Sp. Orch. (1833) $241=$ Dendrobium spurium (Blume) J. J. Sm.

Ä̈RIDES SUAVISSIMUM Lindl. in Journ. Hort. Soc. 4 (1849) 264; Ceron Cat. PI. Herb. Manila (1892) $168=$ Aërides odoratum Lour.

AËRIDES THIBAutianum Reichb. f. in Gard. Chron. (1866) $101=$ Aërides quinquevulnerum Lindl.

AËRIDES VIRENS Lindl. in Bot. Reg. 29 (1843) Misc. 41; Naves Novis. App. (1882) $239=$ Aërides odoratum Lour.

\section{RHYNCHOSTYLIS Blume}

RHYNCHOSTYLIS RETUSA (Linn.) Blume Bijdr. (1825) 286, fig. 49; Reichb. f. in Bonpl. 5 (1857) 40, in Walp.Ann. 6 (1864) 887 (and vars.); Rolfe in Journ. Bot. 23 (1885) 216; Vidal Phan. Cuming. Philip. (1885) 150, Rev. Pl. Vasc. Filip. (1886) 271; Hook. f. Fl. Brit. Ind. 6 (1890) 32; Veitch Man. Orch. Pl. pt. 7 (1891) 58, t. (and vars.); Stein Orchideenb. (1892) 536, fig. 164 (and vars. and forms); Grant Orch. Burma (1895) 290 (and vars.); Ridl. in Journ. Linn. Soc. 31 (1896) 294, 32 (1896) 356; King \& Pantl. in Ann. Roy. Bot. Gard. Calc. 8 (Orch. Sik.-Himal.) (1898) 213, t. 284; Trim. Handb. Fl. Ceyl. 4 (1898) 187; Rolfe in Orch. Rev. 9 (1901) 281, fig. 42 (var. guttata); Cogn. in Dict. Icon. Orch. (1902) Rhynchost. t. 1; J. J. Sm. Fl. Buitenz. 6 (Orch. Jav.) (1905) 629, Fig.-Atlas (1914) fig. 471; Duthie in Ann. Roy. Bot. Gard. Calc. 9 (Orch. North-west. Himal.) (1906) 143; Ridl. Mat. Fl. Mal. Penin. 1 (1907) 158; Ames Orch. 2 (1908) 255. 
5 (1915) 219; Merr. Sp. Blancoanae (1918) 117; Ames in Merr. in Journ. Roy. Asiat. Soc. Straits Branch, Special No. (1921) 197. Epidendrum retusum Linn. Sp. Pl. (1753) 953, ed. 2 (1763) 1351;

Poir. in Lam. Encycl. Suppl. 1 (1810) 384.

Aërides retusum Sw. in Schrad. Journ. Bot. 2 (1799) 233; Willd.

Sp. Pl. 4 (1805) 130; Pers. Syn. Pl. 2 (1807) 522; Spreng. Syst.

Veg. 3 (1826) 719; Grah. Cat. P1. Bomb. (1839) 204. Limodorum retusum Sw. in Nov. Act. Ups. 6 (1799) 80.

Aërides praemorsum Willd. Sp. Pl. 4 (1805) 130; Spreng. Syst. Veg.

3 (1826) 719; Grah. Cat. Pl. Bomb. (1839) 264.

Epidendrum indicum Poir. in Lam. Encycl. Suppl. 1 (1810) 384.

Aërides undulatum Sm. in Rees Cyclop. 39 (1819) No. 13.

Aërides spicatum D. Don Prodr. Fl. Nepal. (1825) 31.

Epidendrum hippium Buch.-Ham. ex D. Don Prod. Fl. Nepal (1825) 32 , in syn.

Rhynchostylis praemorsa Blume Bijdr. (1825) 286; Hassk. in Hoev. \& De Vriese Tijdschr. Nat. Gesch. 9 (1842) 143; Hassk. Pl. Jav. Rar. (1848) 128.

Sarcanthus guttatus Lindl. in Bot. Reg. 17 (1831) t. 1443.

Aërides guttatum Roxb. Fl. Ind. ed. 2, 3 (1832) 471; Regel in

Gartenfl. 12 (1863) 339, t. 415, figs. 8-12; Morr. in Belg. Hort. 26 (1876) 291.

Saccolabium guttatum Lindl. in Wall. Cat. (1832) No. 7308, nomen, Gen. \& Sp. Orch. (1833) 220 ; Paxt. Mag. Bot. 2 (1835) 141, 6 (1839) 214, 11 (1844) 211; Lindl. Sert. Orch. (1841) sub t. 47; Hook. in Bot. Mag. 70 (1844) t. 4108; Griff. Notul. 1 (1847) 22, Icon. Pl. Asiat. 1 (1847) tt. 8, 9; Wight Icon. $5^{1}$ (1852) 19, t. 1745-6; Harting. Parad. Vindob. 2 (1848-60) t. 13; De Vriese Ill. Orch. Ind. Neerl. (1854) t. 14; Lindl. in Journ. Linn. Soc. 3 (1859) 32; Miq. Fl. Ind. Bat. 3 (1859) 693; Dalz. \& Gibs. Bomb. Fl. (1861) 263; Thw. Enum. Pl. Zeyl. (1864) 303; Drury Handb. Ind. Fl. 3 (1869) 405; Puydt Orch. (1880) 227, text cut; Naves Novis. App. (1882) 241; Godefr.-Lebeuf in l'Orchidoph. 8 (1888) 273, t. (var. giganteum), 11 (1891) 11; Meylhand in Journ. des Orch. 2 (1892) 255; Will. Orch. Grow. Man. ed. 7 (1894) 701 and var.); Meylhand in Sem. Hort. 3 "(1899) 489.

Saccolabium praemorsum Lindl. Gen. \& Sp. Orch. (1833) 221; Paxt. Mag. Bot. 4 (1837) 94; Hassk. in Tijdschr. Nat. Gesch. 9 (1842) 143, Pl. Jav. Rar. (1848) 128; Edit. in Fl. des Serres 7 (1851-52) 92, text cut; Miq. Fl. Ind. Bat. 3 (1859) 693; Drury Handb. Ind. Fl. 3 (1869) 405; Meylhand in Journ. des Orch. 2 (1891) 255, in Sem. Hort. 3 (1899) 489, non Hook. f.

Saccolabium spicatum Lindl. Gen. \& Sp. Orch. (1833) 221.

Orchis lanigera Blanco Fl. Filip. (1837) 641, ed.2 (1845) 446, ed. 3,3 (1879) 37.

Saccolabium blumei Lindl. in Bot. Reg. 27 (1841) Misc. 55; Lindl. Sert. Orch. (1841) t. 47; Miq. Fl. Ind. Bat. 3 (1859) 693; Puydt Orch. (1880) 313, t. 27; Naves Novis. App. (1882) 241; Edit. in Gard. Chron. II 23 (1885) 572, fig. 105; Warner \& Will. Orch. Alb. 4 (1885) t. 169; Teran in Ill. Hort. 32 (1885) 125, t. 566; Meylhand in Journ. des Orch. 2 (1891) 254; Will. Orch. Grow. 
Man. ed. 7 (1894) 699, t.; E. L. in Sem. Hort. 2 (1898) 376, fig. 375; Meylhand in Sem. Hort. 3 (1899) 489, 516.

Saccolabium macrostachyum Lindl. Sert. Orch. (1841) sub t. 48.

Saccolabium retusum Voigt Hort. Sub. Calc. (1845) 630; Van Houtte in Fl. des Serres 14 (1861) t. 1463-4; Will. Orch. Grow. Man. ed. 7 (1894) 703.

Saccolabium rheedii Wight Icon. $5^{1}$ (1852) 19.

Saccolabium blumei Lindl. var. major Will. Orch. Grow. Man. (1852) 89, ed. 7 (1894) 699; J. Linden Pescat. 1 (1860) t. 21; Lem. in III. Hort. 15 (1868) t. 545; Meylhand in Journ. des Orch. 2 (1891) 254.

Rhynchostylis guttata Reichb. f. in Bonpl. 2 (1854) 93; Rolfe in Orch. Rev. 22 (1914) 281, fig. 33.

Saccolabium gurwalicum Lindl. in Journ. Linn. Soc. 3 (1859) 32; Reichb. f. in Gard. Chron. II 12 (1879) 102.

Rhynchostylis gurwalica Reichb. $\mathfrak{f}^{\circ}$ in Walp. Ann. 6 (1864) 888.

Rhynchostylis retusa (Linn.) Blume var. macrostachya Reichb. f. in Walp. Ann. 6 (1864) 888.

Saccolabium furcatum Hort. ex Will. Orch. Grow. Man. ed. 3 (1868) 201, ed. 7 (1894) 700.

Saccolabium holfordianum Hort. ex Warner Sel. Orch. PI. II (1874) t. 18.

Saccolabium littorale Reichb. f. in Gard. Chron. II 16 (1881) 198; Hook. f. Fl. Brit. Ind. 6 (1890) 33, cf. Ridl. in Journ. Linn. Soc. 32 (1896) 356.

? Saccolabium berkeleyi Reichb. f. in Gard. Chron. II 19 (1883) 814, Edit. in l'Orchidoph. 3 (1883) 693; Hook. f. Fl. Brit. Ind. 6 (1890) 33; Will. Orch. Grow. Man. ed. 7 (1894) 699.

Saccolabium heathii Hort. ex Gard. Chron. II 24 (1885) 369.

Saccolabium turneri Will. Orch. Grow. Man. ed. 6 (1885) 568, ed. 7 (1894) 703.

Saccolabium blumei Lindl, var. russellianum Will. in Warner \& Will. Orch. Alb. 5 (1886) t. 238; Will. Orch. Grow. Man. ed. 6 (1885) 564 , ed. 7 (1894) 699.

Gastrochilus blumei Ktze. Rev. Gen. P1. 2 (1891) 661.

Gastrochilus gorwalicus Ktze. Rev. Gen. Pl. 2 (1891) 661.

Gastrochilus retusus Ktze. Rev. Gen. Pl. 2 (1891) 661.

Gastrochilus rheedii Ktze. Rev. Gen. P1. 2 (1891) 661.

Gastrochilus spicatus Ktze. Rev. Gen. Pl. 2 (1891) 661.

? Rhynchostylis violacea (Lindl.) Reichb. f. var. berkeleyi Stein Orchideenb. (1882) 537.

Gastrochilus praemorsus Dur. \& Jacks. Ind. Kew. Suppl. 1 (1903) 178, non Ktze.

Rhyncostylis violacea Ames Orch. 1 (1905) 105, non Reichb. f.

Luzon (Bataan, Rizal), Lyon 13, Whitford 36, Merrill 4190, Sp. Blan. coance 1030, B. S. 13548 Ramos, Weber 10, Reillo 9. Epiphyte. In India, Ceylon, Burma, Malay Peninsula, Siam, Java, and Borneo.

A polymorphic species, which has been variously treated either as several distinct species or as one species with several varieties and forms. There are many varieties and forms based on extra-Philippine material 
that are not listed in the above synonymy unless as species. Cf. Stein Orchideenb. (1892) 536-537 for some of these variants.

\section{EXCLUDED OR SYNONYMOUS SPECIES}

Rhynchostylis violacea Ames Orch. 1 (1905) 105 (excl. syn.) non Reichb. $f_{.}$= Rhynchostylis retusa (Linn.) Blume.

Rhynchostylis VIolacea Reichb. f. in Bonpl. 2 (1854) 93; in Walp. Ann. 6 (1864) 888; Van Houtte in Fl. des Serres 17 (1869) 91; Stein Orchideenb. (1892) 537; Ames Orch. 5 (1915) $219=$ Anota violacea (Lindl.) Schltr.

\section{LUISIA Gaudichaud}

LUISIA FOXWORTHII Ames Orch. 2 (1908) 222, text cut, 5 (1915) 220.

Luzon (Bataan, Bulacan, Sorsogon), Polillo, B. S. 1679 Foxworthy, 9257 Robinson, F. B. 7191 Curran, Elmer 16251. Epiphyte at sea level to $830 \mathrm{~m}$ altitude.

LUISIA RAMOSII Ames in Philip. Journ. Sci. 6 (1911) Bot. 55, Orch. 5 (1915) 220.

Luzon (Cagayan, Zambales), B. S. 7970 Ramos, Boettcher s. n.

LUISIA TERETIFOLIA Gaudich. in Freyc. Voy. Uranie et Physic. (1829) Bot. 427, t. 37; Blume Rumphia 4 (1848) 50, t. 194, fig. 3, t. $197 \mathrm{D}$ (excl. syn. as to triste); Blume Mus. Bot. Lugd.-Bat. 1 (1849) 63 (excl. syn. Epidendrum triste Forst.); Reichb. f. in Walp. Ann. 3 (1852) 549, 6 (1863) 621 (excl. syn. Epidendrum triste Forst.); Lindl. Fol. Orch. Luisia (1853) 2 (excl. syn. Epidendrum triste Forst.) ; Miq. Fl. Ind. Bat. 3 (1859) 685; Naves Novis. App. (1882) 242; Hook. f. Fl. Brit. Ind. 6 (1890) 22; Grant Orch. Burma (1895) 236; Ridl. in Journ. Linn. Soc. $32 \cdot(1896)$ 349; King \& Pantl. in Ann. Roy. Bot. Gard. Calc. 8 (Orch. Sik.-Himal.) (1898) 203, t. 271; Trim. Handb. Fl. Ceyl. 4 (1898) 190; Rolfe in Forbes \& Hemsl. in Journ. Linn. Soc. 36 (1903) 33; J. J. Sm. Fl. Buitenz. 6 (Orch. Jav.) (1905) 545, Fig.-Atlas (1912) fig. 413, Orch. Amb. (1905) 91; Duthie in Ann. Roy. Bot. Gard. Calc. 9 (Orch. North-west. Himal.) (1906) 140; Ames in Philip. Journ. Sci. 8 (1913) Bot. 453, 9 (1914) Bot. 15, Orch. 5 (1915) 220; Schltr. in Fedde Repert. Beihefte 4 (Orch. Sino-Jap. Prodr.) (1919) 281.

Cymbium triste Roxb. Fl. Ind. ed. 2, 3 (1832) 461 (excl. syn.); Hook. in Bot. Mag. 65 (1838) t. 3648 (excl. syn. in part); Wight Icon. $3^{2}$ (1849) 10 (excl. t.); Griff. Notul. 3 (1851) 340, quoad Mergui planta, non Willd.

Dendrobium teres Blanco Fl. Filip. (1837) 638, non Roxb.

Dendrobium teretifolium Blanco Fl. Filip. ed. 2 (1845) 444, ed. 3, 3 (1879) 34, non R. Br.

Cymbidium tenuifolium Wight Icon. 5 (1852) t. 1689 (excl. text), non Willd.

Luisia burmanica Lindl. Fol. Orch. Luisia (1853) 3; Reichb. f. in Walp. Ann. 6 (1863) 621. 
Luisia zeylanica Lindl. Fol. Orch. Luisia (1853) 3; Reichb. f. Xen. Orch. 1 (1856) 204, in Walp. Ann. 6 (1863) 621; Thw. Enum. Pl. Zeyl. (1864) 302.

Luisia trichorrhiza Reichb. f. Xen. Örch. 1 (1856) t. 77, figs. 8-18, non Blume.

Luisia platyglossa Reichb. f. in Walp. Ann. 6 (1863) 622, in Trans. Linn. Soc. 30 (1874) 144.

Luisia tristis Ktze. Rev. Gen. Pl. 2 (1891) 672 (excl. syn.), non Hook. $\mathbf{f}$.

Luzon (Bontoc, Bulacan, Laguna, Sorsogon), Negros (Oriental Negros), Leyte, Palawan, Mindanao (Camiguin Island, Agusan, Surigao). A fairly common, variable, and widely distributed species. Epiphyte at 70 to $500 \mathrm{~m}$ altitude. In India, Ceylon, Burma, Malay Peninsula, Siam, China, Sumatra, Java, Amboina, Moluccas, Borneo (fide Ridley), and also the Marianne Islands and New Caledonia.

LUISIA VALIDA Reichb. f. in Bonpl. 10 (1862) 335, in Walp. Ann. 6 (1863) 621; Ames Orch. 5 (1915) 220.

According to Reichenbach (Bonpl. 1. c.) of Philippine origin. The description is vague, and it seems probable that the species is conspecific with Luisia teretifolia Gaudich.

\section{EXCLUDED OR SYNONYMOUS SPECIES}

Luisia Anteinnifera Blume apud Naves Novis. App. (1882) 242.

LUISIA BRACHYSTACHYS Blume apud Naves Novis. App. (1882) 242.

\section{ARACHNIS Blume}

ARACHNIS LYONII Ames Orch. 5 (1915) 221.

Luzon (Isabela), Mindanao (Lanao), Lyon 126, M. S. Clemens s. n. Terrestrial at $1,000 \mathrm{~m}$ altitude in Luzon.

\section{VANDOPSIS Pfitzer}

VANDOPSIS LEYTENSIS Ames Orch. 5 (1915) 222.

Luzon (Rizal, Sorsogon), Leyte, Lyon s. n., Elmer 16860, Wenzel 014, $0154,0172,0900 \mathrm{C}$. Epiphyte at 60 to $800 \mathrm{~m}$ altitude.

VANDOPSIS LISSOCHILOIDES (Gaudich.) Pfitz. in Engl. \& Prantl Nat. Pflanzenfam. 2, Abt. 6 (1889) 210, fig. 229; Kerchove Livre des Orch. (1894) 138, fig. 114; J. J. Sm. Orch. Amb. (1905) 102; Ames Orch. 2 (1908) 220, 5 (1915) 223; J. J. Sm. in Nat. Tijdschr. Ned. Ind. 72 (1913) 76 (reprint 6), in Merr. Interp. Rumph. Herb. Amb. (1917) 178.

Fieldia lissochiloides Gaudich. in Freyc. Voy. Uranie et Physic. (1829) Bot. 424, t. 36 ; Blume Rumphia 4 (1848) t. 194, fig. 2; Van Houtte in Fl. des Serres 17 (1869) 91; Reichb. f. Xen. Orch. 2 (1862) 38, in Walp. Ann. 6 (1864) 870.

Vanda lissochiloides Lindl. Gen. \& Sp. Orch. (1833) 216; Blume Rumphia 4 (1848) 49, Mus. Bot. Lugd.-Bat. 1 (1849) 63; Reichb. f. in Walp. Ann. 1 (1849) 790, 3 (1852) 565; Miq. Fl. Ind. Bat. 3 (1859) 680; Naves Novis. App. (1882) 240. 
Vanda batemanii Lindl. in Bot. Reg. 32 (1846) t. 59 (as batemanni): Paxt. Mag. Bot. 14 (1847) 21; Lindl. in Paxt. Flow. Gard. 2 (1851) 22; S. Ch. in Fl. des Serres 6 (1852) 332; Lindl. Fol. Orch. Vanda (1853) 2; Moore Ill. Orch. Pl. (1857) Vanda 10, t. 1; du Buyss in Fl. des Serres 18 (1870) 161, t. 1921-2.

Grammatophyllum pantherinum Herb. Zipp. ex Blume Rumphia 4 (1848) 49, in syn.

Stauropsis lissochiloides Benth. ex Pfitz. Vergl. Morph. Orch. (1882) 14; Veitch Man. Orch. Pl. pt. 7 (1891) 3; Cogn. in Journ. des Orch. 5 (1894) 291, fig. 80; Cogn. Dict. Icon. Orch. (1898) Stauropsis t. 1; G. Wilson in Orch. World 4 (1914) 227.

Stauropsis batemanii Nichols. Dict. Gard. 3 (1886) 493 (as batemanni).

Panay, Mindanao (Zamboanga, Bukidnon, Sigaboy Island), Loher 6025 (cult. in Manila), B. S. 117\%4 Robinson, 21434 Escritor, 19345 Wester, Merrill $831 \%$.

EXCLUDED OR SYNONYMOUS SPECIES

VANDOPSIS KUPPERIANA Kränzl. in Fedde Repert. 17 (1921) 390 = Staurochilus luzonensis Ames.

\section{RENANTHERA Loureiro}

RENANTHERA MONACHICA Ames Orch. 5 (1915) 224.

Luzon (Zambales), B. S. 3089 Curran.

RENANTHERA STORIEI Reichb. f. in Gard. Chron. II 14 (1880) 296, 16 (1881) 364; Warner \& Will. Orch. Alb. 11 (1897) t. 513; Cogn. in Dict. Icon. Orch. (1907) Renanth. t. 2; Ames Orch. 2 (1908) 219, 5 (1915) 225; Rolfe in Orch. Rev. 23 (1915) 279, 297, fig. 38; W. T. in The Garden 79 (1915) 445, text cut; G. Wilson in Orch. World 6 (1915) 16.

Vanda storiei Storie ex Reichb. f. in Gard. Chron. II 14 (1880) 296, in syn.; Hook. f. in Bot. Mag. 123 (1897) $t$. $753 \%$.

Luzon (Bataan, Rizal), Bucas Grande, Dinagat, F. B. 20110 Borden, Whitford 1336, Loher 6000, B. S. 35085, 35228 Ramos \& Pascasio, Lyon 136. Apparently at low altitudes (up to $130 \mathrm{~m}$ ).

EXCLUDED OR SYNONYMOUS SPECIES

Renanthera Amabilis Boxall ex Naves Novis. App. (1882) 241, nomen.

Renanthera ARACHNitis Lindl. apud Naves Novis. App. (1882) 240.

RenANTHERA COCcineA Lour. apud Naves Novis. App. (1882) 240.

Renanthera Elongata Ames Orch. 5 (1915) 224, non Lindl.

Renanthera matutina Lindl. in Bot. Reg. 29 (1843) $t .41$, quoad Philip.; G. K. in Bot. Zeit. 1 (1843) 760, quoad Philip.

Renanthera micrantha Blume Rumphia 4 (1848) 60, Mus. Bot. Lugd.Bat. 1 (1849) 60; Reichb. f. in Walp. Ann. 3 (1852) 566; Miq. Fl. Ind. Bat. 3 (1859) 698; Naves Novis. App. (1882) 240. 
Although this species, under the name Renanthera micrantha, is reported as being of Philippine origin, no material referable to it has been seen and it seems very doubtful that it cccurs in the Islands.

Renanthera Hookeriana Reichb. f. apud Naves Novis. App. (1882) 241.

ReNANTHERA LOWEI Reichb. f. apud Naves Novis. App. (1882) 241.

Renanthera matutina Lindl. in Bot. Reg. 29 (1843) t. 41, quoad Philip.; G. K. in Bot. Zeit. 1 (1843) 760, quoad Philip. = Renanthera elongata Ames, spec. excl.

Renanthera Micrantha Blume Rumphia 4 (1848) 60, Mus. Bot. Lugd.Bat. 1 (1849) 60; Reichb. f. in Walp. Ann. 3 (1852) 566; Miq. Fl. Ind. Bat. 3 (1859) 698; Naves Novis. App. (1882) $240=$ Renanthera elongata Ames, spec. excl.

Renanthera moluccana Blume apud Naves Novis. App. (1882) 240.

ReNANTHERA SUlingI Lindl. apud Naves Novis. App. (1882) 240.

86. ACAMPE Lindley

ACAMPE LOHERIANA Kränzl. in Fedde Repert. 17 (1921) 386.

Loher s. n.

Kränzlin states: "Philippinen. Ohne genaueren Standort."

The genus Acampe has not heretofore been reported from the Philippines, and field specimens are much desired to authenticate its habitat there. The material examined is inadequate and it is of garden origin.

\section{VANDA Jones}

VANDA CLITELLARIA Reichb. f. in Flora 55 (1872) 277; Ames Orch. 5 (1915) addend. opp. p. 258.

Sulu Archipelago (Mangsi Island), Wilkes Exped. (in Gray Herb.). of it.

Very closely allied to Vanda lamellata Lindl. and perhaps only a form

VANDA LAMELLATA Lindl. in Bot. Reg. 24 (1838) Misc. 66; Paxt. Mag. Bot. 6 (1839) 143; S. Ch. in Fl. des Serres 6 (1851) 332; Lindl. in Paxt. Flow. Gard. 2 (1851) 22, Fol. Orch. Vanda (1853) 9; Moore Ill. Orch. Pl. (1857) Vanda 12; Miq. Fl. Ind. Bat. 3 (1859) 680; Van Houtte in Fl. des Serres 17 (1867-68) 92; Reichb. f. in Walp. Ann. 6 (1864) 869; Naves in Blanco Fl. Filip. ed. 3, Laminas (1877-83) t. 465; Naves Novis. App. (1882) 240; Vidal Phan. Cuming. Philip. (1885) 150, Rev. Pl. Vasc. Filip. (1886) 271; Veitch Man. Orch. Pl. pt. 7 (1891) 99; Stein Orchideenb. (1892) 583; Schltr. in Perk. Frag. Fl. Philip. (1904) 43; Ames Orch. 1 (1905) 103, 2 (1908) 240, 5 (1915) 225.

Vanda cumingii Lindl. ex Paxt. in Loud. Hort. Brit. Suppl. 3 (1850) 654, nomen.

Luzon (Abra, Lepanto, Benguet, Zambales, Bataan, Cavite), Mindoro, Panay (Capiz), Palawan, Comiran. A fairly common species of wide distribution. On rocks or on trees at sea level to $400 \mathrm{~m}$ altitude. 
Var. BOXALLI Reichb. f. in Gard. Chron. II 13 (1880) 743, 15 (1881) 87, fig. 18, 16 (1881) 19, 786; Edit. in The Garden 19 (1881) $574, t .28 \%$, in l'Orchidoph. 5 (1885) 129; Warner \& Will. Orch. Alb. 8 (1889) t. 338; Veitch Man. Orch. Pl. pt. 7 (1891) 100, text cut; Stein Orchideenb. (1892) 583; Cogn. in Dict. Icon. Orch. (1898) Vanda t. 9; Ames Orch. 2 (1908) 241, 5 (1915) 225.

Vanda boxallii Reichb. f. in Gard. Chron. II 15 (1881) 336; Linden \& Rodigas in Lindenia 1 (1885) 69, t. 32.

Vanda superba Linden \& Rodigas in Lindenia 3 (1887) 83, t. 136. Luzon (Zambales), Merrill s. $n$. (cult. in Manila).

Var. CoBbIANA (Reichb. f.) Ames Orch. 2 (1908) 241.

Vanda boxallii Reichb. f. var. cobbiana Reichb. f. in Gard. Chron. II 16 (1881) 780; Edit. in l'Orchidoph. 1 (1882) 194.

VANDA LUZONICA Loher ex Rolfe in Orch. Rev. 23 (1915) 137, 371, fig. 12; Edit. in The Garden 79 (1915) 563, text cut; Rolfe in Bot. Mag. 143 (1917) t. 8709 ; Ames Orch. 6 (1920) 308.

Vanda tricolor Ames Orch. 2 (1908) 245, 5 (1915) 225, non Lindl. Luzon (Bulacan), Loher 6006.

VANDA ROEBLINGIANA Rolfe in Kew Bull. (1894) 365; Ames Orch. 2 (1908) 241, 5 (1915) 225; G. Wilson in Orch. Rev. 30 (1922) 96.

Luzon (Bontoc, Benguet), Vanoverbergh 125\%, 3939, F. B. 5059 Curran. Up to $1,600 \mathrm{~m}$ altitude in Bontoc. In Malay Peninsula.

VANDA SANDERIANA Reichb. f. in Gard. Chron. II 17 (1882) 588; Edit. in $1^{6}$ Orchidoph. 2 (1882) 318, 4 (1884) 109, $t$., in Gard. Chron. II 20 (1883) 402, 440, 446, figs. 67, 68, 22 (1884) 236; Warner \& Will. Orch. Alb. 3 (1884) t. 124; Moore in Gard. Chron. II 21 (1884) 9; Rodigas in Ill. Hort. 31 (1884) 139, t. 532; Carr. in Rev. Hort. 57 (1885) 372, t.; Hook. f. in Bot. Mag. 114 (1888) t. 6983; Sander Reichenbachia I 2 (1890) 29, t. 62; Veitch Man. Orch. Pl. pt. 7 (1891) 103, t.; Rolfe in Orch. Rev. 4 (1896) 358, frontisp., 19 (1911) 281, fig. 31, 23 (1915) 361, frontisp.; Cogn. in Dict. Icon. Orch. (1900) Vanda t. 12; Ames Orch. 2 (1908) 242; G. Wilson in Orch. World 2 (1911) 36, t., 5 (1915) 52, 201, text cuts, 6 (1916) 97, in Orch. Rev. 29 (1921) 79, text cut.

Esmeralda sanderiana Reichb. f. in Gard. Chron. II 17 (1882) 588, in textu; Kerchove Livre des Orch. (1894) 307, 355, figs. 214, 223; Will. Orch. Grow. Man. ed. 7 (1894) 398, text cut; Ames Orch. 5 (1915) . 222.

Vanda sanderiana Reichb. f. var. labello-viridi Linden \& Rodigas in Lindenia 1 (1885) 85, t. 40; Ames Orch. 2 (1908) 244.

Vanda sanderiana Reichb. f. var. albata Reichb. f. in Gard. Chron. III 2 (1887) 9; Edit. in l'Orchidoph. 8 (1888) 197; Reichb. f. in Sander Reichenbachia I 2 (1890) 29; Ames Orch. 2 (1908) 244. Esmeralda sanderiana Reichb. f. var, labello-viridi Will. Orch. Grow. Man. ed. 7 (1894) 400.

Esmeralda sanderiana Reichb. f. var. albata Will. Orch. Grow. Man. ed. 7 (1894) 400.

Vanda sanderiana Reichb. f. var. froebeliana Cogn. in Dict. Icon. Orch. (1903) Vanda t. 12A; Ames Orch. 2 (1908) 244. 
Euanthe sanderiana Schltr. Orchideen. (1914) 568, fi\%. 194; Ames Orch. 6 (1920) 308.

Mindanao (Davao), R. F. Black 20, Loher bo30 (cult. in Manila).

EXCLUDED OR SYNONYMOUS SPECIES

VANDA BATEMANII Lindl, in Bot. Reg. 32 (1846) /. 5! (as butemami); Paxt. Mag. Bot. 14 (1847) 21; S. Ch. in Fl. des Serres 6 (1851) 332; Lindl. in Paxt. Flow. Gard. 2 (1851) 22; Lindl. Fol. Orch. Vanda (1853) 2; Moore Ill. Orch. Pl. (1857) Vanda 10, t. 1; du Buyss in Fl. des Serres 18 (1870) 161, t. 1921-2 = Vandopsis lissochiloides (Gaudich.) Pfitz.

VANDA BOXauli Reichb. f. in Gard. Chron. II 15 (1881) 336; Linden \& Rodigas in Lindenia 1 (1885) 69, t. 32 = Vanda lamellata Lindl. var. boxallii Reichb. f.

VANDA CRISTATA Lindl. apud Boxall ex Naves Novis. App. (1882) 240; Ceron Cat. Pl. Herb. Manila (1892) 168.

VAnda Cumingir Lindl. ex Paxt. in Loud. Hort. Brit. Suppl. 3 (1850) 654 , nomen $=$ Vanda lamellata Lindl.

VANDA FURva Lindl. apud Naves Novis. App. (1882) 239.

VANDA Gigantea Lindl, apud Naves Novis. App. (1882) 240.

VANDA Helyola Bl. apud Naves Novis. App. (1882) 240.

VANDA HINDSII Lindl. apud Naves Novis. App. (1882) 239.

VANDA INSIGNIS Bl. apud Naves Novis. App. (1882) 239.

VANDA Limbata Bl. apud Naves Novis. App. (1882) 240.

VANDA LISSOCHILOIDES Lindl. Gen. \& Sp. Orch. (1833) 216; Naves Novis. App. (1882) $240=$ Vandopsis lissochiloides (Gaudich.) Pfitz.

VANDA Roxburghil R. Br. apud Naves Novis. App. (1882) 240.

VANdA STANGEana Reichb. f. apud Naves Novis. App. (1882) 240.

VANDA STORIEI Storie ex Reichb. f. in Gard. Chron. II 14 (1880) 296, in syn.; Hook. f. in Bot. Mag. 123 (1897) $t_{\text {。 }} 7537=$ Renanthera storiei Reichb. f.

VANDA SUPERBA Linden \& Rodigas in Lindenia 3 (1887) 83, t. $136=$ Vanda lamellata Lindl, var, boxallii Reichb, f.

VANDA TRICOLOR Ames Orch. 2 (1908) 245, 5 (1915) 225, non Lindl. = Vanda luzonica Loher ex Rolfe.

VANDA Undulata Lindl. apud Naves Novis. App. (1882) 240.

VANDA VIDALII Boxall ex Naves Novis. App. (1882) 240, nomen; Ceron Cat. Pl. Herb. Manila (1892) 168.

VANDA violacea Lindl. in Bot. Reg. 27 (1841) Misc. 12, 33 (1847) t. 30; Paxt. Mag. Bot. 9 (1842) 119; Van Houtte in Fl. des Serres 17 (1868-69) 92 = Anota violacéa (Lindl.) Schltr. 


\section{ANGRAECUM Bory}

ANGRAECUM PHILIPPINENSE Ames in Philip. Journ. Sci. 2 (1907) Bot. 336, 4 (1909) Bot. 597, Orch. 2 (1908) 246, 3 (1908) 69, t. 50, 5 (1915) 226.

Luzon (Bontoc, Nueva Vizcaya), Mindoro, Lyon (Curran) 121, F. B. 10860 Curran, Mervill 5698. On mountains, in the forest at 850 to $1,400 \mathrm{~m}$ altitude.

\section{SACCOLABIUM Blume}

SACCOLABIUM CONFUSUm Ames in Philip. Journ. Sci. 8 (1913) Bot. 438, Orch. 5 (1915) 227.

Luzon (Laguna, Sorsogon), Mindanao (Cotabato), Serrato s. n., Elmer 14713, Weber 221. Epiphyte, at $700 \mathrm{~m}$ altitude in Cotabato.

SACCOLABIUM ESCRITORII Ames Orch. 5 (1915) 227.

Luzon (Tayabas), B. S. 20733 Escritor.

SACCOLABIUM LOHERI Ames Orch. 5 (1915) 228.

Luzon (Zambales), Loher 6026.

SACCOLABIUM LUzONENSE Ames in Philip. Journ. Sci. 8 (1913) Bot. 435, Orch. 5 (1915) 229.

Luzon (Laguna, Sorsogon), B. S. 16556 Ramos, Serrato s. n., Elmer 14860, 17076. Epiphyte.

SACCOLABIUM SARCOCHILOIDES Schltr. in Orchis 5 (1911) 61, $t .8$; Ames Orch. 5 (1915) 230.

Saccolabium epichysiochilum Kränzl. in Ann. k. k. Nat. Hofmus. Wien 30 (1916) 63.

K. W. John (fide Schltr.), Loher s. $n$.

SACCOLABIUM TENELLUM Ames Orch. 6 (1920) 308, t. 99.

Leyte, Mindanao (Surigao), Wenzel 0988, 0103\%. Epiphyte at sea level to $150 \mathrm{~m}$ altitude.

SACCOLABIUM VANOVERBERGHII Ames Orch. 5 (1915) 230.

Luzon (Bontoc, Lepanto, Ifugao), Vanoverbergh 955, 1712, 2214, Ramos \& Edaño 91, B. S. 19634 McGregor. Epiphyte in mountain regions at 1,550 to $2,540 \mathrm{~m}$ altitude.

EXCLUDED OR SYNONYMOUS SPECIES

Saccolabium Asceindens Lindl. apud Naves Novis. App. (1882) 241.

Saccolablum bifidum Lindl. in Bot. Reg. 24 (1838) Misc. 3; Paxt. Mag. Bot. 6 (1839) 262; Reichb. f. in Walp. Ann. 6 (1864) 886; Naves Novis. App. (1882) $241=$ Sarcanthus bifidus (Lindl.) Ames.

SACColabium Blumei Lindl. in Bot. Reg. 27 (1841) Misc. 55, Sert. Orch. (1841) t. 47; Naves Novis. App. (1882) $241=$ Rhynchostylis retusa (Linn.) Blume.

Saccolabium Chionanthum Lindl. in Journ. Linn. Soc. 3 (1859) 35; Naves Novis. App. (1882) 241; Ames in Philip. Journ. Sci. 4 (1909) Bot. $675=$ Schoenorchis micrantha Blume. 
Saccolabium Compressum Lindl. in Bot. Reg. 26 (1840) Misc. 9, Sert. Orch. (1840) t. 32; Edit. in Allg. Berl. Gartenz. 8 (1840) 336; Reichb. f. in Walp. Ann. 6 (1864) 88?; Naves Novis. App. (1882) 241; Vidal Phan. Cuming. Philip. (1885) 150, Rev. Pl. Vasc. Filip. (1886) 271; Ames in Philip. Journ. Sci. 2 (1907) Bot. 396, Orch. 2 (1915). 238.

Gastrochilus compressus Ktze. Rev. Gen. Pl. 2 (1891) $661=$ Robi quetia compressa (Lindl.) Schltr.

SACCOLABIUM DENSIFLoRum Lindl. in Bot. Reg. 24 (18,8) Misc. 56, quoad Philip., non Lindl. in Wall. Cat.; Reichb. f. in Walp. Ann. 6 (1864) 885, quoad Philip.; Naves Novis. App. (1882) $241=$ Robiquetia pantherina (Kränzl.) Ames.

SaCcolabium ePICHysiochilum Kränzl. in Ann. k. k. Nat. Hofmus. Wien 30 (1916) $63=$ Saccolabium sarcochiloides Schltr.

Saccolabium guttatum Lindl. in Wall. Cat. (1832) No. 7308, nomen, Gen. \& Sp. Orch. (1833) 220; Naves Novis. App. (1882) 241; Ceron Cat. Pl. Herb. Manila (1892) 168 = Rhynchostylis retusa (Linn.) Blume.

SACColabium Hanissonil Boxall ex Naves Novis. App. (1882) 241, nomen.

Saccolabium macrostachyum Lindl. Sert. Orch. (1841) sub t. $47=$ Rhynchostylis retusa (Linn.) Blume.

SACCOLABIUM MiNLATUM Lindl. in Bot. Reg. 33 (1847) sub t. 26, t. 58; Ames Orch. 2 (1908) 239 (incl. var. citrinum) $=$ Ascocentrum miniatum (Lindl.) Schltr.

SACCOLABIUM PANTHERINUM Kränzl. in Fedde Repert. 17 (1921) $391=$ Robiquetia pantherina (Kränzl.) Ames.

SACCOLABIUM PUMIITO Reichb. f. in Walp. Ann. 6 (1864) 886; in Gard. Chron. II 4 (1875) 98; Ames Orch. 5 (1915) 229.

Saccolabium discolor Reichb. f. in Otia Bot. Hamb. 1 (1878) 42.

Reichenbach in Gardener's Chronicle (l. c.) states: "I obtained the novelty from $\mathrm{Mr}$. Bull, who introduced the plant from Manila." The species occurs in Sylhet, British India. Manila seems an error. No material from the Philippines referable to this species has been seen.

Saccolabium Reflexum Lindl. apud Naves Novis. App. (1882) 241.

SACColabium semiclausum Kränzl. in Ann. k. k. Nat. Hofmus. Wien 30 (1916) 63.

Said to have been sent from the Philippines by Loher. No material has been seen.

SACColabium TRICHRomum Reichb. f. apud Naves Novis. App. (1882) 241.

SACCOLABIUM VIOLACEUM Reichb. f. in Bonpl. 2 (1854) 93, in syn.; Wamer

Sel. Orch. 1 (1862-65) t. 14; Puydt Orch. (1880) 317, t.39; Naves

Noivis. App. (1882) 241; Veitch Man. Orch. Pl. pt. 7 (1891) 117;

Ceron Cat. Pl. Herb. Manila (1892) 168 = Anota violacea (Lindl.)

Schltr.

$200823-28$ 
90. GASTROCHILUS D. Don

GASTROCHILUS PHILIPPINENSIS Ames Orch. 5 (1915) 231.

Luzon (Benguet), Elmer 651\%, Baguio.

\section{OMOEA Blume}

OMOEA PHILIPPINENSIS Ames Orch. 5 (1915) 232.

Luzon (Pampanga, Bataan), Merrill 4208, B. S. 1669 Froxworthy. Epiphyte in mossy forest at 850 to $1,400 \mathrm{~m}$ altitude.

\section{ASCOCENTRUM Schlechter}

ASCOCENTRUM MINIATUM (Lindl.) Schltr, in Fedde Repert. Beihefte 1 (Orch. Deutsch-Neu-Guin.) (1913) 975; J. J. Sm. Orch. Jav. Fig.-Atlas (1914) fig. 483; Ames Orch. 5 (1915) 233.

Saccolabium miniatum Lindl. in Bot. Reg. 33 (1847) sub t. 26, t. 58;

Reichb. f. in Walp. Ann. 1 (1849) 791; Miq. Fl. Ind. Bat. 3 (1859) 692; Veitch Man. Orch. Pl. pt. 7 (1891) 117; C. K. in Journ. Hort. III 24 (1892) 449, fig. 77 ; Rolfe in Orch. Rev. 4 (1896) 178; J. J. Sm. Fl. Buitenz. 6 (Orch. Jav.) (1905) 648; Ridl. Mat. Fl. Mal. Penin. 1 (1907) 170; Ames Orch. 2 (1908) 239, non Hook. in Bot. Mag. 88 (1862) t. 5.226.

Saccolabium miniatum Lindl. var. citrinum Reichb. f. in Gard. Chron. II 21 (1884) 542; Ridl. Mat. Fl. Mal. Penin. 1 (1907) 170; Ames Orch. 2 (1908) 240.

Gastrochilus miniatus Ktze. Rev. Gen. Pl. 2 (1891) 661.

Luzon (Rizal), Mindanao (Lanao, Davao), B. S. 5649 Ramos, Weber 9, 3020 Mrs. Lyon, Copeland 68\%. Epiphyte at 180 to $750 \mathrm{~m}$ altitude. In India (fide Rolfe), Malay Peninsula, Siam (fide Ridley), and Java.

93. MALLEOLA J. J. Smith and Schlechter

MALLEOLA CONSTRICTA Ames Orch. 5 (1915) 234.

Luzon (Sorsogon), Leyte, Mindanao (Lanao, Agusan), Elmer 1395\$, 14831, 17199, 17342, Wenzel 055, 062, 0124, 0195, 0882, M. S. Clemens r02. Epiphyte at 60 to $700 \mathrm{~m}$ altitude in Leyte.

MALLEOLA LYONII Ames Orch. 5 (1915) 235.

Luzon?, Lyon s. $n$.

EXCLUDED OR SYNONYMOUS SPECIES

MALLEOLA MERRILLII Ames Orch. 5 (1915) $236=$ Robiquetia merrillii Ames.

\section{ROBIQUETIA Gaudichaud}

ROBIQUETIA COMPRESSA (Lindl.) Schltr, in Fedde Repert. Beihefte 1 (Orch. Deutsch-Neu-Guin.) (1913) 983; Ames Orch. 5 (1915) 237.

Saccolabium compressum Lindl. in Bot. Reg. 26 (1840) Misc. 9, Sert. Orch. (1840) t. 32; Edit. in Allg. Berl. Gartenz. 8 (1840) 336; Reichb. f. in Walp. Ann. 6 (1864) 883; Naves Novis. App. (1882) 241; Vidal Phan. Cuming. Philip. (1885) 150, Rev. Pl. Vasc. Filip. (1886) 271; Ames in Philip. Journ. Sci. 2 (1907) Bot. 336; Merr. \& 
Merritt in Philip. Journ. Sci. 5 (1910) Bot. 341; Ames Orch. 5 (1915) 238.

Gastrochilus compressus Ktze. Rev. Gen. Pl. 2 (1891) 661.

Luzon (Camarines, Sorsogon), Mindanao (Dapitan, Davao), $B . S$. 22081, 23679 Ramos, F. B. 4603 Mearns \& Hutchinson, Mearns s. n. At 550 to $3,000 \mathrm{~m}$ altitude.

ROBIQUETIA MERRILLII Ames Orch. 7 (1922) 123.

Malleola merrillii Ames Orch. 5 (1915) 236.

Luzon (Bontoc, Lepanto, Benguet, Bataan, Laguna, Camarines), Mindanao (Agusan), B. S. 5603 Worcester, Merrill 768.5, 31993 Santos, 17127 Robinson, 22178 Ramos, Taylor 3, Elmer 13838, F. B. 1596 Borden. Epiphyte at 350 to $3,200 \mathrm{~m}$ altitude.

ROBiquetia PANTHERINA (Kränzl.) Ames Sched. Orch. 6 (1923) 95. Cleisostoma spatulatum Blume apud Naves Novis. App. (1882) 238. Saccolabium densiflorum Lindl. in Bot. Reg. 24 (1838) Misc. 56, quoad Philip., non Lindl. in Wall. Cat.; Reichb. f. in Walp. Ann. 6 (1864) 885, quoad Philip.; Naves Novis. App. (1882) 241.

Robiquetia spathulata Ames Orch. 5 (1915) 237, non J. J. Sm.

Saccolabium pantherinum Kränzl. in Fedde Repert. 17 (1921) 391.

Leyte, Loher s. n., Wenzel 0180. Epiphyte in forest at $60 \mathrm{~m}$ altitude.

ROBIQUETIA RAMOSII Ames Orch. 7 (1922) 123.

Catanduanes, Panay (Antique), B. S. 30396 Ramos, 32592 McGregor. Epiphyte at 30 to $300 \mathrm{~m}$ altitude.

ROBIQUETIA VANOVERBERGHII Ames Orch. 5 (1915) 238.

Luzon (Bontoc, Benguet), Mindanao (Zamboanga), Vanoverbergh 147\%, R. S. Williams 1952, F. B. 5056 Curran, Ramos \& Edaño 65, 69, 102, Merrill 8075 .

EXCLUDED OR SYNONYMOUS SPECIES

Robiquetia spathulata Ames Orch. 5 (1915) 237, non J. J. Sm. = Robiquetia pantherina (Kränzl.) Ames.

\section{SCHOENORCHIS Blume}

SCHOENORCHIS MICRANTHA Blume Bijdr. (1825) 362; Lindl. Gen. \& Sp. Orch. (1833) 243 ; J. J. Sm. in Nat. Tijdschr. Ned. Ind. 72 (1912) 100 (reprint 30), Orch. Jav. Fig.-Atlas (1914) fig. 453; Ames Orch. 5 (1915) 239, 6 (1920) 224, in Merr. in Journ. Roy. Asiat. Soc. Straits Branch, Special No. (1921) 200.

Saccolabium chionanthum Lindl. in Journ. Linn. Soc. 3 (1859) 35; Miq. Fl. Ind. Bat. 3 (1859) 692; Naves Novis. App. (1882) 241; J. J. Sm. Fl. Buitenz. 6 (Orch. Jav.) (1905) 635; Ames in Philip. Journ. Sci. 4 (1909) Bot. 675.

Saccolabium perpusillum Hook. f. Fl. Brit. Ind. 6 (1890) 56, in Hook.

Icon. Pl. 22 (1893) t. 2129A; Ridl. in Journ. Linn. Soc. 32 (1896) 358, Mat. Fl. Mal. Penin. 1 (1907) 164.

Gastrochilus chionanthus Ktze. Rev. Gen. Pl. 2 (1891) 661.

Gastrochilus perpusillus Ktze. Rev. Gen. Pl. 2 (1891) 661. 
Luzon (Rizal), Leyte, Palawan, Mindanao (Agusan, Davao), B. S. 3055, 41637 Ramos, Wenzel 05, 0122, 0378, Weber 319,603, Copeland 685. Epiphyte at sea level to $1,300 \mathrm{~m}$ altitude. In Malay Peninsula, Sumatra, Java, and Borneo.

SCHOENORCHIS MINDANAENSIS Ames Orch. 5 (1915) 240.

Lużon (Sorsogon), Mindanao (Agusan), Elmer 16669, Weber 155. Epiphyte at $15 \mathrm{~m}$ altitude in Mindanao.

SCHOENORCHIS PHILIPPINENSIS Ames Orch. 5 (1915) 241.

Luzon (Nueva Vizcaya), Mindanao (Cotabato, Agusan), B. S. 20117 McGregor, Weber 212, Elmer 13969. Epiphyte at 550 to $1,000 \mathrm{~m}$ altitude.

SCHOENORCHIS VANOVERBERGHII Ames Orch. 5 (1915) 242.

Luzon (Cagayan, Bontoc, Pampanga, Bataan), F. B. 16781, 19307 Curran, Vanoverbergh 1761, 3644, Whitford 115 p. p. Epiphyte at 1,700 $m$ altitude in Bontoc.

\section{CAMAROTIS Lindley}

CAMAROTIS PHILIPPINENSIS Lindl. in Journ. Linn. Soc. 3 (1859) 37;

Miq. Fl. Ind. Bat. 3 (1859) 685; Naves Novis. App. (1882) 242;

Ames in Philip. Journ. Sci. 8 (1913) Bot. 438, Orch. 5 (1915) 244. Sarcochilus philippinensis Vidal Phan. Cuming. Philip. (1885) 150,

Rev. Pl. Vasc. Filip. (1886) 271.

Luzon (Laguna, Sorsogon), Leyte, Cuming s. n., B. S. 16582 Ramos, Serrato s. n., Wenzel 04, 0174, Elmer 17225. Epiphyte in forest.

CAMAROTIS UTRICULOSA Ames Orch. 5 (1915) 244.

Luzon, Mindanao (Cotabato), B. S. 5608 coll.? (flowered in Manila), 11701 Robinson.

EXCLUDED OR SYNONYMOUS SPECIES

Camarotis purpurea Boxall ex Naves Novis. App. (1882) 242, nomen.

\section{SARCANTHUS Lindley}

SARCANTHUS BIFIDUS (Lindl.) Ames Orch. 5 (1915) 245.

Saccolabium bifidum Lindl. in Bot. Reg. 24 (1838) Misc. 3; Paxt. Mag. Bot. 6 (1839) 262; Reichb. f. in Walp. Ann. 6 (1864) 886; Naves Novis. App. (1882) 241.

Gastrochilus bifidus Ktze. Rev. Gen. Pl. 2 (1891) 661.

Luzon (Apayao), Mindoro, Leyte, Mindanao (Cotabato, Agusan). A not uncommon species. Epiphyte at 5 to $500 \mathrm{~m}$ altitude.

SARCANTHUS DEALBATUS (Lindl.) Reichb. f. in Walp. Ann. 6 (1864) 892; J. J. Sm. in Nat. Tijdschr. Ned. Ind. 72 (1912) 85 (reprint 15); Ames Orch. 5 (1915) 247; Merr. Sp. Blancoanae (1918) 115. Cleisostoma dealbatum Lindl. in Bot. Reg. 29 (1843) Misc. 5.

Cypripedium lineari-subulatum Llanos Fragm. Pl. Filip. (1851) 99; F.-Vill. \& Naves in Blanco Fl. Filip. ed. 3, $4^{1}$ (1880) 76 ; Naves Novis. App. (1882) 244.

Luzon (Bontoc, Bulacan, Rizal, Camarines, Sorsogon), Leyte, Palawan, Bancalan, Mindanao (Lanao). A fairly common and widely distributed species. Epiphyte at sea level to $900 \mathrm{~m}$ altitude. 
SARCANTHUS MALLEIFER J. J. Sm. in Nat. Tijdschr. Ned. Ind. 72 (1912) 90 (reprint 19).

Cleisostoma sagittata Blume Bijdr. (1825) 363, fig. 27; Lindl. Gen. \& Sp. Orch. (1833) 226; Miq. Fl. Ind. Bat. 3 (1859) 682; Naves Novis. App. (1882) 238.

Sarcanthus sagittatus J. J. Sm. Fl. Buitenz. 6 (Orch. Jav.) (1905) 607, Fig.-Atlas (1914) fig. 451; Ames Orch. 5 (1915) 251, non King \& Pantl.

Palawan, Weber 601. At Alfonso XIII, epiphyte at sea level.

SARCANTHUS MICRANTHUS Ames Orch. 5 (1915) 248.

Luzon (Bataan, Laguna, Tayabas), Leyte, Mindanao (Lanao, Davao), Basilan. A not uncommon species of rather wide distribution. Epiphyte at sea level to $815 \mathrm{~m}$ altitude.

SARCANTHUS PACHYPHYLLUS Ames Orch. 5 (1915) 250.

Luzon (Albay, Rizal), Schultze s. n., Lyon 65.

SARCANTHUS RINGENS (Reichb. f.) J. J. Sm. in Nat. Tijdschr. Ned. Ind. 72 (1912) 93 (reprint 22); Ames Orch. 5 (1915) 251.

Cleisostoma ringens Reichb. f. in Gard. Chron. III 4 (1888) 724; Edit. in Kew Bull. (1889) 83.

Reichenbach states: "Ex insulis Philippinis." No specimens of this species have been seen.

SARCANTHUS STRIOLATUS Reichb. f. in Gard. Chron. II 18 (1882) 168; Ames in Philip. Journ. Sci. 2 (1907) Bot. 336 (excl. spec.), Orch. 5 (1915) 252.

Reichenbach states that this species was imported from the Philippines by Low. No specimens certainly referable to it have been seen.

SARCANTHUS WEBERI Ames Orch. 5 (1915) 252.

Luzon (Bataan), Palawan, Mindanao (Cotabato, Davao), B. S. 560\% Foxworthy, Weber 25.2, 604, Schultze s. n., F.B. 11825 Whitford. Epiphyte at sea level, from data at hand.

\section{EXCLUDED OR SYNONYMOUS SPECIES}

SARCANThus cumingit (Reichb. f.) J. J. Sm. in Nat. Tijdschr. Ned. Ind. 72 (1912) 85 (reprint 15).

Cleisostoma cumingii Reichb. f. in Allg. Berl. Gartenz. 24 (1856) 218.

The Cuming material on which this species was based may have originated in the Philippines, but no material referable to the descrpition has been seen in the Philippine collections of twenty years.

SARCANTHUS PUGIONIFormis Reichb. f. in Allg. Berl. Gartenz. 24 (1856) 219.

According to Dr. Rudolf Schlechter, in a letter, this species was in cultivation in Erlangen from material sent from the Philippines by Loher. No other record of its occurrence in the Islands is known.

SarCanthus sagittatus J. J. Sm. Fl. Buitenz. 6 (Orch. Jav.) (1905) 607, Fig.-Atlas (1914) fig. 451; Ames Orch. 5 (1915) 251, non King \& Pantl. = Sarcanthus malleifer J. J.Sm. 
Sarcanthus stcundus Griff. apud Usteri Beitr. Ken. Philip. Veg. (1905) 128.

Usteri refers a Guimaras specimen here on the basis of an identification made by Schlechter. There is no other evidence to show that this species occurs in the Philippines.

\section{ANOTA Schlechter}

ANOTA VIOLACEA (Lindl.) Schltr. Orchideen (1914) 588.

Vanda violacea Lindl. in Bot. Reg. 27 (1841) Misc. 12, 33 (1847) t. 30; Paxt. Mag. Bot. 9 (1842) 119; Van Houtte in Fl. des Serres 17 (1868-69) 92.

Rhynchostylis violacea Reichb. f. in Bonpl. 2 (1854) 93, in Walp. Ann. 6 (1864) 888; Van Houtte in Fl. des Serres 17 (1868-69) 91; Stein Orchideenb. (1892) 537; Ames Orch. 5 (1915) 219.

Saccolabium violaceum Reichb. $f$. in Bonpl. 2.(1854) 93, in syn.; Warner Sel. Orch. I (1862-65) t. 14; Puydt Orch. (1880) 317, $t$. 39; Naves Novis. App. (1882) 241; Veitch Man. Orch. Pl. pt. 7 (1891) 117; Ceron Cat. Pl. Herb. Manila (1892) 168.

Luzon (Nueva Ecija, Rizal), Lyon 50, Native collector s. n., B. S. 3090 Curran, 21334 Ramos.

\section{POMATOCALPA Breda}

POMATOCALPA BICOLOR (Lindl.) J. J. Sm. in Nat. Tijdschr. Ned. Ind. 72 (1912) 103 (reprint 33); Ames Orch. 5 (1915) 254.

Epidendrum lineare Blanco Fl. Filip. (1837) 644, ed. 2 (1845) 449, ed. 3,3 (1879) 44, non Linn.

Cleisostoma bicolor Lindl. in Paxt. Flow. Gard. 2 (1851-52) 99, fig. 185; Reichb. f. in Walp. Ann. 6-(1864) 889; Naves Novis. App. (1882) 239; Ames Orch. 2 (1908) 236; Merr. Fl. Manila (1912) 167, Sp. Blancoanae (1918) 115.

Luzon (Nueva Vizcaya, Bataan, Rizal, Laguna), Romblon, Negros (Occidental Negros), Bohol, Leyte, Palawan, Busuanga, Mindanao (Agusan, Butuan). A common and widely distributed species. Epiphyte or on rocks at sea level to $500 \mathrm{~m}$ altitude.

POMATOCALPA LATIFOLIUM (Lindl.) J. J. Sm. in Nat. Tijdschr. Ned. Ind. 72 (1912) 105 (reprint 35), Orch. Jav. Fig.-Atlas (1914) fig. 458; Ames Orch. 5 (1915) 254, in Merr. in Journ. Roy. Asiat. Soc. Straits Branch, Special No. (1921) 202.

Cleisostoma latifolium Lindl. in Bot. Reg. 26 (1840) Misc. 60; Reichb. f. in Walp. Ann. 6 (1864) 889; Hook. f. Fl. Brit. Ind. 6 (1890) 71 ; Ridl. in Trans. Linn. Soc. II 3 (1893) 370, in Journ. Linn. Soc. 32 (1896) 365; J. J. Sm. Fl. Buitenz. 6 (Orch. Jav.) (1905) 612 ; Ridl. Mat. Fl. Mal. Penin. 1 (1907) 165.

Luzon (Rizal), Palawan, Bancalan, Mindanao (Cotabato, Butuan), Lyon 23, 151, Reillo s. n., Weber 08, 600, B. S. 11702 Robinson, 12129 Celestino. Epiphyte apparently at or near sea level. In Malay Peninsula, Sumatra, Java, and Borneo.

POMATOCALPA MERRILLII Schltr. in Fedde Repert. Beihefte 1 (Orch. Deutsch-Neu-Guin.) (1913) 988; Ames Orch. 6 (1920) 309. 
Cleisostoma kunstleri Ames in Philip. Journ. Sci. 6 (1911) Bot. 54, non Hook. f.

Pomatocalpa kunstleri Ames Orch. 5 (1915) 254, non J. J. Sm.

Polillo, Negros, B.S. 10444 McGregor, F. B. 17409 Curran. Epiphyte at $460 \mathrm{~m}$ altitude in Negros.

POMATOcalpa Vitellinum (Reichb. f.) Ames comb. nov.

Cleisostoma vitellinum Reichb. f. in Linnaea 41 (1876) 76; J. J. Sm. in Nat. Tijdschr. Ned. Ind. 72 (1912) 112 (reprint 42); Ames Orch. 5 (1915) 253, in textu.

Cleisostoma expansum Reichb. f. Otia Bot. Hamb. 1 (1878) 52, Xen. Orch. 3 (1881) 29; Naves Novis. App. (1882) 239; Ames Orch. 2 (1908) 237.

Cleisostoma densum Schltr. in Perk. Frag. Fl. Philip. (1904) 43, non Ridl.

Pomatocalpa expansum J. J. Sm. in Nat. Tijdschr. Ned. Ind. 72 (1912) 103 (reprint 33); Ames Orch. 5 (1915) 254.

Luzon (Bataan, Bulacan, Rizal), Leyte, Busuanga, Palawan, Mindanao (Agusan, Davao). A common and rather widely distributed species. Epiphyte or in limestone at sea level to $1,000 \mathrm{~m}$ altitude.

\section{EXCLUDED OR SYNONYMOUS SPECIES}

Pomatecalpa Expansum J. J. Sm. in Nat. Tijdschr. Ned. Ind. 72 (1912) 103 (reprint 33); Ames Orch. 5 (1915) $254=$ Pomatocalpa vitellinum (Reichb. f.) Ames.

Pomatocalpa Kunstleri Ames Orch. 5 (1915) 254, non J, J, Sm. = Pomatocalpa merrillii Schltr.

PomatocalPa ? Roseum J. J. Sm. in Nat. Tijdschr. Ned. Ind. 72 (1912) 106 (reprint 36$)=$ Trichoglottis rosea (Lindl.) Ames.

\section{TRICHOGLOTTIS Reichenbach $f$.}

TRICHOGLOTTIS ATROPURPUREA Reichb. f. in Linnaea 41 (1876) 30; Ames Orch. 5 (1915) 254.

Reichenbach (1. c.) states: "Philippinens, insulae." No specimens have been seen.

TRICHOGLOTTIS BATAANENSIS Ames Orch. 1 (1905) 105, text cut, 5 (1915) 255.

Cleisostoma subviolaceum Reichb. f. in Bonpl. 10 (1862) 335; Ames Orch. 5 (1915) 253, in nota, non Trichoglottis subviolacea (Llanos) Merr.

Trichoglottis rigida Vidal Phan. Cuming. Philip. (1885) 150, Rev. Pl. Vasc. Filip. (1886) 271, nec Blume nec Naves.

Trichoglottis subviolacea Merr. Sp. Blancoanae (1918) 116, quoad planta (excl. syn.).

Luzon (Nueva Vizcaya, Pangasinan, Nueva Ecija, Zambales, Bataan, Rizal, Tayabas), Palawan. A common species, especially in Luzon. Epiphyte at about $200 \mathrm{~m}$ altitude. 
TRICHOGLOTTIS BRACHIATA Ames Orch. 7 (1922) 136.

Biliran, Mindanao (Agusan, Davao), B. S. 18931 McGregor, Weber 134, Elmer 12021.

TRICHOGLOTTIS LATISEPALA Ames in Philip. Journ. Sci. 4 (1909) Bot. 675, Orch. 5 (1915) 255.

Luzon (Nueva Vizcaya, Rizal, Tayabas), Palawan, Bancalan, Lumbucan, Mindanao (Zamboanga, Bukidnon, Agusan, Davao). A fairly common species of rather wide distribution. Epiphyte at sea level to $76 \mathrm{~m}$ altitude:

TRICHOGLOTTIS MINDANAENSIS Ames in Philip. Journ. Sci. 8 (1913)

Bot. 439, Orch. 5 (1915) 257.

Luzon (Camarines, Sorsogon), Panay (Capiz), Samar, Mindanao (Zamboanga, Lanao, Agusan, Surigao, Davao). A common species, epiphyte at 60 to $150 \mathrm{~m}$ altitude.

TRICHOGLOTTIS PHILIPPINENSIS Lindl, in Ann. \& Mag. Nat. Hist. 15 (1845) 386; Naves Novis. App. (1882) 243; Vidal Phan. Cuming. Philip. (1885) 150; Usteri Beitr. Ken. Philip. Veg. (1905) 128; Ames Orch. 7 (1922) 137.

Stauropsis philippinensis Reichb. f. in Hamb. Gartenz. 16 (1860) 117, Xen. Orch. 2 (1862) 8, "in Walp. Ann. 6 (1864) 882; Vidal Rev. Pl. Vasc. Filip. (1886) 270; Ames Orch. 2 (1908) 223, 5 (1915) 224.

Luzon (Bulacan, Rizal, Cavite, Camarines), Negros, Mindanao (Misamis, Surigao, Davao). A not uncommon species of fairly wide distribution, at sea level to $300 \mathrm{~m}$ altitude.

TRICHOGLOTTIS ROSEA (Lindl.) Ames comb. nov.

Cleisostoma rosea Lindl. in Bot. Reg. 24 (1838) Misc. 80; Paxt. Mag. Bot. 6 (1839) 189, 12 (1845) 165; Reichb. f. in Walp. Ann. 6 (1864) 888; Naves Novis. App. (1882) 239; Ames Orch. 5 (1915) 253 , in nota.

Trichoglottis flexuosa Rolfe ex Ames Orch. 1 (1905) 107, 2 (1908) 257,5 (1915) 255.

Pomatocalpa? roseum J. J. Sm. in Nat. Tijdschr. Ned. Ind. 72 (1912) 106 (reprint 36).

Luzon (Camarines), Panay (Capiz), Bohol, Leyte, Mindanao (Bukidnon, Agusan, Surigao), Cuming s.n., Loher 6012, 6029, Fortune s.n., Copeland 199, B. S. 38958 Ramos \& Edaño, 42085 Edaño, Weber 185, Bolster 230. Epiphyte at sea level to $1,300 \mathrm{~m}$ altitude.

TRICHOGLOTTIS SOLEREDERI Kränzl. in Fedde Repert. 8 (1910) 98; Ames Orch. 5 (1915) 257.

Philippines, probably Luzon, Loher $s, n$.

No specimens of this species have been seen. Kränzlin (1. c.) mentions its relationship to Trichoglottis bataanensis Ames.

TRICHOGLOTTIS WENZELII Ames in Philip. Journ. Sci. 8 (1913) Bot. 440, Orch. 5 (1915) 257.

Trichoglottis retusa Ames in Philip. Journ. Sci. 4 (1909) Bot. 676, non Blume. 
Leyte, Mindanao (Zamboanga, Agusan, Suirigan, Davao), Basilan, Lynn 127, Wenzel 015, 0176, Merrill 8206, Weber 189, Bolster 225, Bilack s. n., $F: B .6103$ Hutchinson. Epiphyte at sea-level to $60 \mathrm{~m}$ altitude.

EXCLUDED OR SYNONYMOUS SPECIES

TrichoglotTis BICRURis Kränzl. in Ann. k. k. Nat, Hofmus. Wien 30 (1916) 64.

Based on a Loher plant which Kränzlin questions as being of Philippine origin. No material seen.

Trichoglottis Bimae Reichb. f. apud Naves Novis. App. (1882) 243.

Trichoglottis flexuosa Rolfe ex Ames Orch. 1 (1905) 107, 2 (1908) 257, 5 (1915) 255 = Trichoglottis rosea (Lindl.) Ames.

Trichoglottis Ionosima J. J. Sm. in Nat. Tijdschr. Ned. Ind. 72 (1912) 108 (reprint 38) (excl. syn. Epidendrum lineare Blanco); Ames Orch. 5 (1915) $255=$ Staurochilus ionosma (Lindl.) Schltr.

Trichoglottis LANCEOLARIA Blume apud Naves Novis. App. (1882) 243.

TRICHogLotTIS LUZONENsis Ames Orch. 5 (1915) $255=$ Staurochilus luzonensis Ames.

Trichoglottis PALleins Lind1. in Journ. Hort. Soc. 5 (1850) 34, in Paxt. Flow. Gard. 1 (1850) $15=$ Phalaenopsis pallens (Lindl.) Reichb. f., spec. excl.

Trichoglottis pusilla Reichb. f. apud Naves Novis. App. (1882) 243.

Trichoglottis RetusA Ames in Philip. Joum. Sci. 4 (1909) Bot. 676, non Blume $=$ Trichoglottis wenzelii Ames.

Trichoglottis RetUSA Blume apud Naves Novis. App. (1882) 243.

Trichoglottis Rigida Blume apud Naves in Blanco Fl. Filip. ed. 3, Laminas (1877-83) t. 348; Naves Novis. App. (1882) 243.

Trichoglottis RIgIDA Vidal Phan. Cuming. Philip. (1885) 150, Rev. P1. Vasc. Filip. (1886) 271, nec Blume nec Naves = Trichoglottis bataanensis Ames.

Trichoglottis subviolacea (Llanos) Merr. Sp. Blancoanae (1918) 116, quoad syn. (excl. planta).

Trichogrottis subviolaceA Merr. Sp. Blancoanae (1918) 116, quoad planta (excl. syn.) $=$ Trichoglottis bataanensis Ames.

\section{STAUROCHILUS Ridley}

STAUROCHILUS IONOSMA (Lind1.) Schltr. in Fedde Repert. Beihefte 4 (Orch. Sino-Jap. Prodr.) (1919) 287 (excl. syn. Epidendrum lineare Blanco).

Cleisostoma ionosmum Lindl. in Bot. Reg. 33 (1847) t. 41; Paxt. Mag. Bot. 14 (1847) 164; G. K. in Bot. Zeit. 5 (1847) 633; Reichb. f. in Walp: Ann. 1 (1849) 791; Miq. Fl. Ind. Bat. 3 (1859) 682; Naves Novis. App. (1882) 238; Ames Orch. 1 (1905) 103, 2 (1908) 238 (excl. syn. Epidendrum lineare Blanco); Merr. in 
Philip. Journ. Sci. 1 Suppl. (1906) 39; Hayata Icon. Pl. Formosa 2 (1912) 134, 4 (1914) 96.

Trichoglottis ionosma J. J. Sm. in Nat. Tijdschr. Ned. Ind. 72 (1912) 108 (reprint 38) (excl. syn. Epidendrum lineare Blanco); Ames Orch. 5 (1915) 255.

Luzon (Bataan, Rizal, Tayabas, Camarines), Mindanao (Agusan), $F$. $B$. 737 Borden, Lyon 60, B. S. 20852 Escritor, 33626 Ramos \& Edaño, Elmer 139\%\%. Epiphyte at 330 to $1,300 \mathrm{~m}$ altitude. Possibly in Formosa; cf. Schltr. 1. c.

STAUROCHILUS LUZONENSIS Ames comb. nov.

Trichoglottis luzonensis Ames Orch. 5 (1915) 255.

Vandopsis kupperiana Kränzl. in Fedde Repert. 17 (1921) 390.

Luzon (Tayabas), Mindanao (Agusan), Loher s. n., B. S. 21199 Escritor, Weber 103. Epiphyte up to about $400 \mathrm{~m}$ altitude in Mindanao.

\section{MICROSACCUS Blume}

MICROSACCUS BREVIFOLIUS J. J. Sm. in Icon. Bogor. 3 (1906) 63, t. 225, in Bull. Dept. Agr. Ind. Néerl. 13 (1907) 77, in Bull. Jard.

Bot. Buitenz. II 26 (1918) 111; Ames Orch. 6 (1920) 309.

Mindanao (Davao), Wenzel 010003. Madaum, epiphyte in forest at sea level. In Java.

MICROSACCUS WENZELII Ames Orch. 5 (1915) 257.

Leyte, Wenzel 029, 0159. Dagami, epiphyte in forest at $60 \mathrm{~m}$ altitude. EXCLUDED OR SYNONYMOUS SPECIES

Microsaccus Javensis Blume apud Naves Novis. App. (1882) 244.

\section{TAENIOPHYLLUM Blume}

TAENIOPHYLLUM COMPACTUM Ames Orch. 2 (1908) 247, text cut, 5 (1915) 258.

Microtatorchis compacta Schltr. in Fedde Repert. 10 (1911) 209, in textu.

Luzon (Bontoc, Benguet), Negros, Vanoverbergh 2821, R. S. Williams 193\%, Merrill 7010. Epiphyte up to $1,700 \mathrm{~m}$ altitude in Bontoc.

TAENIOPHYLLUM COPELANDII Ames in Philip. Journ. Sci. 8 (1913) Bot. 436, Orch. 5 (1915) 258.

Mindanao (Davao), Copeland 339.

TAENIOPHYLLUM ELMERI Ames in Elm. Leafl. Philip. Bot. 5 (1912) 1588, Orch. 5 (1915) 258.

Luzon (Sorsogon), Negros (Oriental Negros), Elmer 10336, 16346. Epiphyte, apparently in mountain regions.

TAENIOPHYLLUM LEYTENSE Ames Orch. 5 (1920) 310.

Leyte, Wenzel 0998. Epiphyte at sea level.

TAENIOPHYLLUM MERRILLII Ames in Philip. Journ. Sci. 6 (1911) Bot. 56, Orch. 5 (1915) 258.

Luzon (Bataan), Merrill 3878, Whitford 117. Epiphyte at $800 \mathrm{~m}$ altitude. 
TAENIOPHYLLUM PHILIPPINENSIS Reichb. f. in Otia Bot. Hamb. 1 (1878) 53, Xen. Orch. 3 (1881) 29; Naves Novis. App. (1882) 237; Ames Orch. 2 (1908) 248, 5 (1915) 258.

Luzon (Apayao, Cagayan, Laguna), Leyte, Palawan, Mindanao (Zamboanga). A reasonably common species of wide distribution. Epiphyte at sea level to $130 \mathrm{~m}$ altitude.

\section{EXCLUDED OR SYNONYMOUS SPECIES}

Taeniophyllum AlWisil Lind1. apud Naves Novis. App. (1882) 237.

TAENIOPHyLum Hasselti Blume apud Naves Novis. App. (1882) 237.

Taeniophyllum obtusum Blume apud Naves Novis. App. (1882) 237.

\section{EXCLUDED OR SYNONYMOUS GENERA AND SPECIES}

Adenoncos virens Blume apud Naves Novis. App. (1882) 244.

Alismorchrs Thouars $=$ Calanthe R. Brown.

Alismorchis Furcata Ktze. Rev. Gen. Pl. 2 (1891) $650=$ Calanthe furcata Batem.

Alismorchis Lyroglossa Ktze. Rev. Gen. Pl. 2 (1891) $650=$ Calanthe lyroglossa Reichb. f.

AREThúsa GLUTinosa Blanco Fl. Filip. (1837) 641, ed. 2 (1845) 446, ed. 3,3 (1879) 38, t. 429bis = Geodorum nutans (Presl) Ames.

ARUndina PEntandra Reichb. f. apud Naves Novis. App. (1882) 246.

ARUNDINA SPECIOSA Blume apud Rolfe in Journ. Bot. 23 (1885) 215; Vidal Phan. Cuming. Philip. (1885) 150, Rev. Pl. Vasc. Filip. (1886) 269.

The Philippine record of this species was based on Cuming 2058 , but it is almost certain that Cuming's specimen was from the Malay Peninsula, where the species is common and where Cuming also collected. No representative of the genus has been found in the Philippines.

ARUNdiNa Wallichil Reichb. f. apud Naves Novis. App. (1882) 246.

Bartholina PeCtinata R. Br. apud Llanos ex F.-Vill. \& Naves in Blanco Fl. Filip. ed. $3,4^{\text {1 }}$ (1880) 100.

Bromeradia Palustris Lindl. apud Vidal Phan. Cuming. Philip. (1885) 150, Rev. P1. Vasc. Filip. (1886) 270.

The Philippine record was based on Cuming 2054, so labeled; the specimen was almost certainly from Singapore or the Malay Peninsula. CALLISTA Loureiro $=$ Dendrobium Swartz.

Callista Amethystoglossa Ktze. Rev. Gen. Pl. 2 (1891) $654=$ Dendrobium amethystoglossum Reichb. $\mathrm{f}$.

Callista ANosma Ktze. Rev. Gen. Pl. 2 (1891) 653 = Dendrobium anosmum Lindl.

Callista BURsigera Ktze. Rev. Gen. Pl. 2 (1891) $654=$ Dendrobium secundum Wall. 
Callista taurina Ktze. Rev. Gen. Pl. 2 (1891) $655=$ Dendrobium taurinum Lindl.

Cephalanthera ensifolia Llanos ex F.-Vill. \& Naves in Blanco Fl. Filip. ed. $3,4^{\text {I }}$ (1880) 100, non Spreng.

Ceratochilus Biglandulosus Blume apud Naves Novis. App. (1882) 244.

Ceratochilus micranthus Lindl, apud Naves Novis. App. (1882) 244.

CEstichis Pfitzer = Liparis L. C. Richard.

Cestichis Benguetensis Ames Orch. 1 (1905) 9, 75, t. 3, fig. I, 1-6, 2 (1908) $132=$ Liparis benguetensis (Ames) Schltr.

Cestichis CAespitosa Ames Orch. 2 (1908) 132, $t$. = Liparis caespitosa (Thou.) Lindl.

Cestichis ctemensiaf Ames in Philip. Journ. Sci. 4 (1909) Bot. $666=$ Liparis clemensiae Ames.

Cestichis compressa Ames Orch. 1 (1905) 76, text cut, 2 (1908) $134=$ Liparis compressa (Blume) Lindl.

Cestichis cumingiI Ames Orch. 1 (1905) 13, in Philip. Journ. Sci. 7 (1912) Bot. 7 = Liparis cumingii Ridl.

Cestichis disticha Pfitz. in Engl. \& Prantl Nat. Pflanzenfam. 2, Abt. 6 (1888) 131, quoad Philip.; Ames in Philip. Journ. Sci. 2 (1907) Bot. $320=$ Liparis propinqua Ames.

Cestichis ELMERI Ames Orch. 1 (1905) 10, 75, t. 3, fig. II, $7-12,2$ (1908) $135=$ Liparis elmeri (Ames) Schltr.

CestICHIS FORBESII Ames Orch. 2 (1908) 135 = Liparis forbesii Ridl.

Cestichis Fragilis Ames in Philip. Joum. Sci. 8 (1913) Bot. $412=$ Liparis fragilis Ames.

CESTICHIS GRACILIS Ames Orch. 2 (1908) 136, text cut, non Liparis gracilis Hook. f. = Liparis amesiana Schltr.

Cestichis Halconensis Ames in Philip. Journ. Sci. 2 (1907) Bot. 321, Orch. 3 (1908) 63, t. $47=$ Liparis halconensis Ames.

Cestichis linearifolia Ames in Elm. Leafl. Philip. Bot. 5 (1912) 1563 $=$ Liparis Linearifolia Ames.

Cestichis Longipes Ames Orch. 1 (1905) 75 (excl. Luzon specimen), 2 (1908) 137 = Liparis longipes Lindl.

Cestichis Lyonir Ames in Philip. Journ. Sci. 6 (1911) Bot. $47=$ Liparis lyonii Ames.

Cestichis MERRILlit Ames Orch. 1 (1905) 11, 75, t. 3, fig. III, 13-18, 2 (1908) 138, in Philip. Journ. Sci. 2 (1907) Bot. $321=$ Liparis merrillii (Ames) Schltr.

Cestichis nutans Ames in Philip. Journ. Sci. 4 (1909) Bot. $597=$ Liparis nutans Ames. 
Cestichis Philippinensis Ames Orch. 1 (1905) 7, 75, t. 2 2 , 2 (1908) 138, in Philip. Journ. Sci. 2 (1907) Bot. $321=$ Liparis philippinerisis (Ames) Schltr.

Cestichis TRICHOGLotTIS Ames Orch. 2 (1908) 139, text cut = Liparis trichoglottis (Ames) Schltr.

Cestichis vestita Ames Orch. 2 (1908) 139, p. p., non Liparis vestita Reichb. f. = Liparis confusa J. J. Sm.

CESTICHIS vestITA Ames Orch. 2 (1908) 139, p. p., non Liparis vestita Reichb. f. = Liparis parviflora (Blume) Lindl.

ChILopogon MERrILli Ames Orch. 5 (1915) $91=$ Appendicula merrillii Ames.

Chloidia confusa Ames Orch. 2 (1908) 67, text cut $=$ Corymborchis confusa Ames.

Cirrhopetalum Lindley $=$ Bulbophyllum Thouars.

CirRhopetalum Antenniferum Lindl. in Bot. Reg. 29 (1843) sub t. 49 $=$ Bulbophyllum antenniferum (Lindl.) Reichb. $\mathbf{f}$.

Cirrhopetalum auratum Lindl. in Bot. Reg. 26 (1840) Misc. 50, 29 (1843) t. 61; G. K. in Bot. Zeit. 2 (1844) 103; Moore Ill. Orch. Pl. (1857) Cirrhopet. 5, t. 4; = Bulbophyllum auratum (Lindl.) Reichb. f.

Cirrhopetalum Chryseum Kränzl. in Fedde Repert. 8 (1910) $97=$ Bulbophyllum chryseum (Kränzl.) Ames.

CrrRhopetalume Cumingir Lindl. in Paxt. Mag. Bot. 8 (1841) 165, in Bot. Reg. 29 (1843) sub t. 49; G. K. in Bot. Zeit. 1 (1843) 791; Moore Ill. Orch. Pl. (1857) Cirrhopet. 5; Hook. in Bot. Mag. 83 (1857) t. 4996; Miq. Fl. Ind. Bat. 3 (1859) 653; Veitch Man. Orch. Pl. pt. 3 (1888) 100; Rodigas in Ill. Hort. 39 (1892) 61, in textu = Bulbophyllum cumingii (Lindl.) Reichb. f.

CIRRHOPETALUM LOHERIANUM Kränzl. in Ann. k. k. Nat. Hofmus. Wien 30 (1916) $61=$ Bulbophyllum loherianum (Kränzl.) Ames.

Cirrhopetalum maxiluare Lindl. in Bot. Reg. 29 (1843) sub t. $49=$ Bulbophyllum blumei (Lindl.) J. J. Sm.

Cirrhopetalum Nutans Lindl. in Bot. Reg. 25 (1839) Misc. 71, 29 (1843) sub t. 49; Hook. in Bot. Mag. 75 (1849) t. 4418; Miq. Fl. Ind. Bat. 3 (1859) 653 = Bulbophyllum othonis (Ktze.) J. J. Sm.

Cirrhopetalum thouarsi Lindl. in Bot. Reg. 24 (1838) $t$. 11, non $C$. thouarsii Lindl. Gen. \& Sp. Orch. (1830) 58; Hook. in Bot. Mag. 72 (1846) t. $4237=$ Bulbophyllum weberi Ames.

Cleisostoma Amabile Teijsm. \& Binn. apud Naves Noris. App. (1882) 238. Cleisostoma bicolor Lindl. in Paxt. Flow. Gard. 2 (1851-52) 99, fig. 185; Reichb. f. in Walp. Ann. 6 (1861) 889; Naves Novis. App. (1882) 239; Ames Orch. 2 (1908) 236; Merr. Fl. Manila (1912) 167, 
Sp. Blancoanae (1918) $115=$ Pomatocalpa bicolor (Lindl. J. J. Sm.

CLEISOSTOMA BRACHYSTACHYUM Kränzl. in Ann. k. k. Nat. Hofmus. Wien 30 (1916) 62.

Based on a Loher plant said to have come from the Philippines. Kränzlin states that it is allied to Cleisostoma rosea Lindl., which is Trichoglottis rosea (Lindl.) Ames. No material of this species has been seen.

Cleisostoma chrysochilum Kränzl. in Fedde Repert. 8 (1910) 545; Ames Orch. 5 (1915) 253, in nota.

Probably a species of Pomatocalpa. Kränzlin (1. c.) refers the species to the alliance of Cleisostoma bicolor Lindl., which is Pomatocalpa bicolor (Lindl.) J. J. Sm. No specimens have been seen.

Clmisostoma cumingir Reichb. f. in Allg. Berl. Gartenz. 24 (1856) $218=$ Sarcanthus cumingii (Reichb. f.) J. J. Sm., spec. dub.

Cleisostoma deaibatum Lindl. in Bot. Reg. 29 (1843) Misc. 5 = Sar. canthus dealbatus (Lindl.) Reichb. $\mathbf{f}$.

Cleisostoma densum Schltr. in Perk. Frag. Fl. Philip. (1904) 43, non Ridl. = Pomatocalpa vitellinum (Reichb. f.) Ames.

Cleisostoma expansum Reichb. f. Otia Bot. Hamb. 1 (1878) 52, Xen. Orch. 3 (1881) 29 ; Naves Novis. App. (1882) 239; Ames Orch. 2 (1908) 237 = Pomatocalpa vitellinum (Reichb. f.) Ames.

Cleisostoma guibertil Lindl. apud Naves Novis. App. (1882) 238.

Cleisostoma ionosmum Lindl. in Bot. Reg. 33 (1847) t. 41; Paxt. Mag. Bot. 14 (1841) 164; G. K. in Bot. Zeit. 5 (1847) 633; Reichb. f. in Walp. Ann. 1 (1849) 791; Miq. Fl. Ind. Bat. 3 (1859) 682; Naves Novis. App. (1882) 238; Ames Orch. 1 (1905) 103, 2 (1908) 238 (excl. syn. Epidendrum lineare Blanco) = Stauro. chilus ionosma (Lindl.) Schltr.

Cleisostoma kUnstleari Ames in Philip. Journ. Sci. 6 (1911) Bot. 54, non Hook. f. = Pomatocalpa merrillii Schltr.

Cleisostoma longifolium Teijsm. \& Binn. apud Naves Novis. App. (1882) 238.

Cleisostoma RINGens Reichb. $f$. in Gard. Chron. III 4 (1888) 724; Edit. in Kew Bull. (1889) 83 = Sarcanthus ringens (Reichb. f.) J. J. Sm.

Cuetsostoma rosea Lindl. in Bot. Reg. 24 (1838) Misc. 80; Paxt. Mag. Bot. 6 (1839) 189, 12 (1845) 165; Reichb. f. in Walp. Ann. 6 (1864) 888; Naves Novis. App. (1882) 239; Ames Orch. 5 (1915) 253, in nota $=$ Trichoglottis rosea (Lindl.) Ames.

Cleisostoma sagrtTata Blume Bijdr. (1825) 363, fig. 27; Naves Novis. App. (1882) 238 = Sarcanthus malleifer J. J. Sm.

Ciersostoma spatulatum Blume apud Naves Novis. App. (1882) $238=$ Robiquetia pantherina (Kränzl.) Ames.

Cleisostoma suaveolens Blume apud Naves Novis. App. (1882) 238. 
Cleisostoma subviolaceum Reichb. f. in Bonpl. 10 (1862) 335; Ames Orch. 5 (1915).253, in nota, non Trichoglottis subviolacea (Llanos)

Merr. = Trichoglottis bataanensis. Ames.

Cleisostoma tridentatum Lindl: apud Naves Novis. App. (1882) 238.

Cleisostoma viteluinum Reichb. f. in Linnaea 41 (1876) 76; J. J. Sm. in Nat. Tijdschr. Ned. Ind. 72 (1912) 112 (reprint 42); Ames Orch. 5 (1915) 253, in textu = Pomatocalpa vitellinum (Reichb. f.) Ames.

CORYMBIS Thouars $=$ Corymborchis Thouars.

CoRYMBIS CONFusA Schltr. in Engl. Bot. Jahrb. 45 (1911) $395=$ Corymborchis confusa Ames.

Corymbis disticha Lindl. Fol. Orch. Corymbis (1854) 1, quoad Philip.; Thou. apud Miq. Fl. Ind. Bat. 3 (1859) 736, quoad Philip. = Corymborchis confusa Ames.

Corymbis Gracilis Vidal Rev. P1. Vasc. Filip. (1886) 272; Ceron Cat. Pl. Herb. Manila (1892) 168, non Blume = Ceratostylis subulata Blume.

Corymbis veratrifolia Reichb. f. apud Naves Novis. App. (1882) 248; Vidal Phan. Cuming. Philip. (1885) 151, Rev. Pl. Vasc. Filip. (1886) 168.

Probably referable to Corymborchis confusa Ames.

Corysanthes fornicata Lindl. apud Naves Novis. App. (1882) 249.

CORYSANTHES MERRILII Ames Orch. 1 (1905) 65, text cut = Corybas merrillii Ames.

Corysanthes PICTA Lindl, apud Naves Novis. App. (1882) 249.

Crinonia elmeri Ames Orch. 5 (1915) 67, nomen = Pholidota camea (Blume) Lindl.

Cymbidium flavescens Llanos Frag. Pl. Filip. (1851) 96; F.-Vill. \& Naves in Blanco Fl. Filip. ed. $3,4^{t}$ (1880) 74 ; Merr. Sp. Blancoanae (1918) $113=$ ? Oberonia iridifolia (Roxb.) Lindl.

CyPripedium as applicable to the Philippine flora = Cordula Raf.

CYPRIPEdIUM ARgus Reichb. f. in Gard. Chron. (1873) 608, (1874) 710, III 7 (1890) 259; Hook. f. in Bot. Mag. 101 (1875) t. 6175; Naves Novis. App. (1882) 251; Veitch Man. Orch. Pl.pt. 4 (1889) 11; Sander Reichenbachia I 2 (1890) 77, t. 8s; Ceron Cat. Pl. Herb. Manila (1892) 169; Kränzl. Orch. Gen. et Sp. 1 (1897) $56=$ Cordula argus (Reichb. f.) Rolfe.

CYPRIPEDIUM BARBATUM Lindl. in Bot. Reg. 27 (1841) Misc. 53; Vidal Phan. Cuming. Philip. (1885) 151, Rev. Pl. Vasc. Filip. (1886) $273=$ Cordula barbata (Lindl.) Rolfe.

CYPRIPEdum BUlbosum Blanco Fl. Filip. (1837) 637, ed. 2 (1845) 444, ed. 3, 3 (1897) 33; Merr. Sp. Blancoanae (1918) 117, non Linn.

This is indeterminable from the wholly inadequate description. Blanco's specimens may have been a Liparis or a Malaxis. 
CYPRIPEDIUM CANNARTIANUM Hort. ex L. Linden in Lindenia 3 (1887) 93, t. $141=$ Cordula phitippinensis (Reichb. f.) Rolfe.

CyprIPEdium CILIOLARE Reichb. f. in Gard. Chron. II 18 (1882) 488, II 20 (1883) 46; Rodigas in Ill. Hort. 31 (1884) 127, t. 530; Veitch Man. Orch. Pl. pt. 4 (1889) 16; Kränzl. Orch. Gen, et Sp. 1 (1897) $64=$ Cordula ciliolaris (Reichb. f.) Rolfe.

CYPRIPEDIUM ELLIOTTIANUM O'Brien in Gard. Chron. III 4 (1888) 501. Cf. Paphiopedilum rothschildianum (Reichb. f.) Stein, spec. excl.

CyPripedium FAIRIEANUm Lindl. apud Naves Novis. App. (1882) 251.

CYPRIPEDIUM HAYNALDIANUM Reichb. f. Xen. Orch. 2 (1874) 222, 3 (1881)

33 , t. 212, in Gard. Chron. II 7 (1877) 272, 752; Hook. f. in Bot.

Mag. 103 (1877) t. 6296; Naves Novis. App. (1882) 251; Veitch

Man. Orch. Pl. pt. 4 (1889) 28; Kränzl. Orch. Gen. et Sp. 1 (1897) $71=$ Cordula haynaldiana (Reichb. f.) Rolfe.

CYPRIPEDIUM HOOKERAE Reichb. f. apud Naves Novis. App. (1882) 251. CYPRIPEdIUM JAVANICUM Reinw, apud Naves Novis. App. (1882) 251.

CYPRipedium Laevigatum Batem. in Bot. Mag. 91 (1865) t. 5508; Dombrain in Floral Mag. 5 (1866) t. 29.8; Morr. in Belg. Hort. 17 (1867) 102, t. 6; Van Houtte in Fl. des Serres 17 (1869) 83, t. 1760-61: Batem. Second Cent. Orch. Pl. (1867) t. 101; Naves Novis. App. (1882) 251; Ceron Cat. Pl. Herb. Manila (1892) 169 = Cordula philippinensis (Reichb. f.) Rolfe.

CyPRIPEDIUM LINEARI-SUbUlatum Llanos Frag. Pl. Filip. (1851) 99; F.-Vill. \& Naves in Blanco Fl. Filip. ed. 3, $4^{1}$ (1880) 76 ; Naves Novis. App. (1882) $244=$ Sarcanthus dealbatus (Lindl.) Reichb. $f$.

CYPRIPEDIUM LowiI Lindl. apud Naves Novis. App. (1882) 251.

Paphiopedilum lowii Stein apud Ames Orch. 2 (1908) 21, 5 (1915.) 11 , in nota.

There is no satisfactory evidence that this is Philippine; it was described from Borneo.

CYPRIPEDIUM MOENSIANUM Hort. ex L. Linden in Lindenia 3 (1887) 69, t. $129=$ Cordula argus (Reichb. f.) Rolfe.

CyPRIPEDIUM PARDINUm Reichb. f. apud Boxall ex Naves Novis. App. (1882) 251.

CyPRIPEDIUM PHILIPPINENSE Reichb. f. in Bonpl. 10 (1862) 335; Veitch Man. Orch. Pl. pt. 4 (1889) 42, text cut; Kränzl. Orch. Gen. et Sp. 1 (1897) 46; Rolfe in Orch. Rev. 22 (1914) 253, fig. $29=$ Cordula phitippinensis (Reichb. f.) Rolfe.

Cypripedium Pitcherianum Manda in Am. Flor. 3 (1887) $178=$ Cordula argus (Reichb. f.) Rolfe.

Cypripedium purpuratum Lindl. apud Naves Novis. App. (1882) 251.

CYPRIPEDIUM RoEBBELENII Reichb. f. in Gard. Chron. II 20 (1883) 684; Kränzl. Xen. Orch. 3 (1893) 113, t. 265, Orch. Gen. et Sp. 1 (1897) $47=$ Cordula philippinensis (Reichb. f.) Rolfe. 
CyPripedium superbiens Reichb. f. apud Naves Novis. App. (1882) 251.

Cyrtopera Lindl. = Eulophia R. Brown.

Cyrtopera Aurea Boxall ex Naves Novis. App. (1882) 242, nomen.

Cyrtoper4 ENSIFORMIS Lindl. in Journ. Linn. Soc. 3 (1859) 31; Miq. Fl. Ind. Bat. 3 (1859) 686; Naves Novis. App. (1882) 242 = Eulophia stricta (Presl) Ames.

Cyrtopera Maculata Boxall ex Naves Novis. App. (1882) 242, nomen.

CYRTOPERA squalida Reichb. f. in Bonpl. 5 (1857) 38; Lindl, in Journ. Linn. Soc. 3 (1859) 31; Miq. Fl. Ind. Bat. 3 (1859) 686; Reichb. f. in Walp. Ann. 6 (1863) 668; Naves Novis. App. (1882) $242=$ Eulophia squalida Lindl.

Cyrtopodium enstforme Vidal Phan. Cuming. Philip. (1885) 80, 150, Rev. Pl. Vasc. Filip. (1886) 270; Schltr. in Perk. Frag. Fl, Philip. (1904) 43 = Eulophia stricta (Presl) Ames.

Cyrtopodium squalidum Vidal Phan. Cuming. Philip. (1885) 150, Rev. Pl. Vasc. Filip. (1886) $270=$ Eulophia squalida Lindl.

Cyrtosia JavaniCA Blume apud Naves Novis. App. (1882) 247.

Desmotrichum Blume = Dendrobium Swartz.

Desmotrichum Fimbriatum Blume apud Kränzl. in Engl. Pflanzenreich IV. 50. II. B. 21 (1910) 354, quoad Philip. = Dendrobium plicatile Lindl.

DESMOTRICHUM SCOPA Kränzl. in Engl. Pflanzenreich IV. 50. I1. B. 21 (1910) $349=$ Dendrobium scopa Lindl.

DienIA Lindl. = Malaxis Swartz.

DIENIA CARINATA Reichb. f. in Bonpl. 3 (1855) 223 = Malaxis acuminata D. Don var, biloba Ames.

Diploconchium INocephalum Schauer in Nov. Act. Nat. Cur. 19 Suppl. 1 (1843) 428, t. 12, fig. $A=$ Agrostophyllum inocephalum (Schauer) Ames.

DissorhynchiUM MURICATUM Schauer in Nov. Act. Nat. Cur. 19, Suppl. 1 (1843) $434, t .1$, fig. $A=$ Habenaria hystrix Ames.

Doritis as applicable to the Philippine flora $=$ Kingiella Rolfe. Cf. Ames Orch. 6 (1920) 307.

Doritis PHILIPPINENsis Ames Orch. 2 (1908) 235, text cut, 5 (1915) $215=$ Kingiella philippinensis (Ames) Rolfe.

Dossinia marmorata Morr. apud Naves Novis. App. (1882) 250.

Echioglossum Javanicum Blume apud Naves Novis. App. (1882) 244.

EPIDENDRUM AMEThystinum Reichb. f. apud Naves Novis. App. (1882) 237.

Epidendrum CARinatum Linn. Sp. Pl. (1753) 953, ed. 2 (1763) $1350=$ Dendrobium carinatum (Linn.) Willd. $200823-29$ 
EPIDENDRUM EQUITANS Blanco Fl. Filip. (1837) 645, ed. 2 (1845) 449, ed. 3, 3 (1879) 44, non Dendrobium equitans Kränzl. = Dendrobium aporoides (Lindl.) Merr.

EPIDENDRUM LINEARE Blanco Fl. Filip. (1837) 644, ed. 2 (1845) 449, ed. 3, 3 (1879) 44, non Linn. = Pomatocalpa bicolor (Lindl.) J. J. Sm.

EPIDENDRUM. RHABARBARUM REDOLENS Blanco Fl. Filip. ed. 3, 3 (1879) 45, t. $389=$ Dendrobium anosmum Lindl.

EPIDENDRUM RUCKERAE Reichb. f. apud Naves Novis. App. (1882) 237.

EPIDENDRUM RUIBARBARUM REDOLENS Blanco Fl. Filip. ed. 2 (1845) $593=$ Dendrobium anosmum Lindl.

EpIdendrum sophronitis Lindl. apud Naves Novis. App. (1882) 237.

Epidendrum ? SPatulatum Llanos in Mém. Real. Acad. Cienc. Madr. 4 (1857) 499; F.-Vill. \& Naves in Blanco Fl. Filip. ed. 3, $4^{2}$ (1880) 100 .

EPIdENDRUM vaNilla Blanco Fl. Filip. (1837) 643, non Linn. = Vanilla ovalis Blanco.

EPHIPPIUM CORNUTUM Blume apud Naves Novis. App. (1882) 244.

EPHiPPIUM LePIdiUm Blume apud Naves Novis. App. (1882) 244.

Esmeralda SANDERIANA Reichb. f. in Gard. Chron. II 17 (1882) 588, in textu; Kerchove Livre des Orch. (1894) 307, 335, figs. 214, 223; Will. Orch. Grow. Man. ed. 7 (1894) 398, text cut; Ames Orch. 5 (1915) $222=$ Vanda sanderiana Reichb. $\mathrm{f}$.

EUANTHE Schltr. = Vanda Jones.

EUANTHE SANDERIANA Schltr. Orchideen (1914) 568, fig. 194; Ames Orch. 6 (1920) $308=$ Vanda sanderiana Reichb. $f$.

Gastrochilus BIfidus Ktze. Rev. Gen. Pl. 2 (1891) 661 = Sarcanthus bifidus (Lindl.) Ames.

Gastrochilus compressus Ktze. Rev. Gen. Pl. 2 (1891) $661=$ Robiquetia compressa (Lindl.) Schltr.

Glossaspis tentaculata Spreng. apud Llanos ex F.-Vill. \& Naves in Blanco Fl. Filip. ed. 3, $4^{1}$ (1880) 100; Naves Novis. App. (1882) 250.

Gongora Philippica Llanos in Mém. Real. Acad. Cienc. Madr. 4 (1857) 498; F.-Vill. in Blanco Fl. Filip. ed. 3, $4^{1}$ (1880) 100; Merr. Sp. Blancoanae (1918) 117.

The description is very short and imperfect, and no data are given from which it is possible to determine the proper generic position of the species. It certainly is not Renanthera coccinea Lour. to which Naves reduced it. Cf. Merrill l. c.

GOODYERA R. Br. = Epipactis (Hallier) Boehmer.

Goodyera DAWsoniI Boxall ex Naves Novis. App. (1882) 248, nomen. 
Goodyera Grandis Blume Fl. Jav. Orch. (1858) 36 (Orch. Arch. Ind. 43): Naves Novis. App. (1882) $248=$ Epipactis rubimunda (Blume) A. A. Eaton.

GOODYERA LUzONENSIS Ames Orch. 5 (1915) $26=$ Ejripactis luzonensis Ames.

Goodyera ordiana Boxall ex Naves Novis. App. (1882) 248, nomen.

Goodyera PARViflora Lindl, apud Naves Novis. App. (1882) 248.

GoodyerA Philippinensis Schitr. in Engl. Bot. Jahrb. 45 (1911) 392; Ames Orch. 5 (1915) 27 = Epipactis philippinensis Ames.

Goodyera PICTA Boxall ex Naves Novis. App. (1882) 248, nomen.

GOODYERA PROCERA Hook. apud Naves Novis. App. (1882) 248.

GOODYERA RAMOsi Ames in Philip. Journ. Sci. 8 (1913) Bot. 409, Orch. 5 (1915) $27=$ Epipactis ramosii Ames.

Goodyera REPENS R. Br. apud Naves Novis. App. (1882) 248.

GOODYERA RUBICUNDA Lindl. in Bot. Reg. 25 (1839) Misc. 61, Gen. \& Sp. Orch. (1840) 493, in Journ. Linn. Soc. 1 (1857) 184; Miq. Fl. Ind. Bat. 3 (1859) 728; Naves Novis. App. (1882) 248; J. J. Sm. Fl. Buitenz. 6 (Orch. Jav.) (1905) 121, Fig.-Atlas (1909) fig. 88; Ames Orch. 5 (1915) $27=$ Epipactis rubicunda (Blume) A. A. Eaton.

Goodyera Rubrovenia Boxall ex Naves Novis. App. (1882) 248, nomen. GOODYERA Viridiflora Blume Fl. Jav. Orch. (1858) 34 (Orch. Arch. Ind. 41), t. 9c, fig. 2, 12-16; Ames Orch. 5 (1915) $27=$ Epipactis viridiflora (Blume) Ames.

Grosourdya APPEndiculata Reichb. f. Xen. Orch. 2 (1867) 123; Naves Novis. App. (1882) $239=$ Sarcochilus appendiculatus (Blume) J. J. Sm.

Grosourdya Hystrix Reichb. f. apud Naves Novis. App. (1882) 239.

Grosourdya zollingeri Reichb. f. apud Naves Novis. App. (1882) 239.

HaEmaria MerRilli Ames in Philip. Journ. Sci. 2 (1907) Bot. 315, Orch. 3 (1908) 21, t. $30=$ Kuhlhasseltia whiteheadii (Rendle) Ames.

HerPysma MERrilliI Ames in Philip. Journ. Sci. 2 (1907) Bot. $313=$ Erythrodes merrillii Ames.

Hexameria DistichA R. Br. in Benn. Pl. Jav. Rar. (1838) 26, t. 7; Schauer in Nov. Act. Nat. Cur. 19, Suppl. 1 (1843) $431=$ Podochilus serphyllifolius (Blume) Lindl.

Hylophila RUBRA Ames in Philip. Journ. Sci. 2 (1907) Bot. 315, nomen, Orch. 2 (1908) 65, text cut $=$ Dicerostylis rubra (Ames) Schltr.

IRIDORCHIS Thouars = Oberonia Lindley.

IRIDORCHIS ANCEPS Ktze. Rev. Gen. Pl. 2 (1891) $669=$ Oberonia anceps Lindl. 
IRIDORCHIS APOROPHYLLA Ktze. Rev. Gen. PI. 2 (1891) $669=$ Oberonia aporophylla Reichb. f.

IRIDORCHIS CYLINDRICA Ktze. Rev. Gen. Pl. 2 (1891) 669 = Oberonia cylindrica (Lindl.).

IRIDORCHIS THISBE Ktze. Rev. Gen. Pl. 2 (1891) 669 = Oberonia thisbe Reichb. f.

LEPTORCHIS Thouars = Liparis L. C. Richard.

LEPTORCHIS FORBESII Ktze. Rev. Gen. Pl. 2 (1891) 671 = Liparis forbesii Lindl.

LEPTORCHIS PECTINATA Ktze. Rev. Gen. Pl. 2 (1891) $671=$ Liparis pectinata Ridl.

Limatodes gracilis Lindl. apud Naves Novis. App. (1882) 247.

Limatodes Grata Miq. apud Naves Novis. App. (1882) 247.

MiCrostylis Nutt. = Malaxis Swartz.

Microstylis Alagensis Ames in Philip. Journ. Sci. 2 (1907) Bot. $318=$ Malaxis alagensis Ames.

Microstyis binabaYensis Ames in Philip. Journ. Sci. 2 (1907) Bot. 318 $=$ Malaxis binabayensis Ames.

Microstylis CARINATA Reichb. f. in Walp. Ann. 6 (1861) 207; Naves Novis. App. (1882) 230; Vidal Phan. Cuming. Philip. (1885) 184, Rev. Pl. Vasc. Filip. (1886) 266; Ridl. in Journ. Linn. Soc. 24 (1888) $338=$ Malaxis acuminata D. Don var. biloba (Lindl.) Ames.

Microstylis congesta Reichb. f. in Walp. Ann. 6 (1861) 206; Naves Novis. App. (1882) $230=$ Malaxis latifolia $\mathrm{Sm}$.

Microstrlis dentata Ames in Philip. Journ. Sci. 2 (July, 1907) Bot. 319 $=$ Malaxis dentata Ames.

Microstylis Hutchinsoniana Ames in Philip. Journ. Sci. 2 (1907) Bot. $319=$ Malaxis hutchinsoniana Ames.

Microstylis Merrilli Ames in Philip. Joum. Sci. 2 (1907) Bot. $319=$ Malaxis merrillii Ames.

Microstylis mindorensis Rendle in Journ. Bot. 34 (1896) 356 = Malaxis mindorensis (Rendle) Ames.

Mrcrostylis odorata Boxall ex Naves Novis. App. (1882) 230, nomen.

MicrostYlis PHILIPPINENSIS Kränzl. in Orchis 2 (Nov. 1907) $16=$ Malaxis dentata Ames.

Microstylis Quadridentata Ames in Philip. Journ. Sci. 2 (1907) Bot. $320=$ Malaxis quadridentata Ames.

Microstylis RHeEdil Lindl. apud Naves Novis. App. (1882) 230.

Microtatorchis compacta Schltr. in Fedde Repert. 10 (1911) 209, in textu $=$ Taeniophyllum compactum Ames.

Monochilus clandestinum Miq. apud Naves Novis. App. (1882) 250.

Monochilus zollingeri Reichb. f. apud Naves Novis. App. (1882) 250. 
OPHRYS CERnUA Blanco Fl. Filip. (18.37) 63.9) (as Ophiris), ed. 2 (1845) 445, ed. 3, 5 (1879) 35, t. 276, non Linn. = Grammatophyllum scriptum (Linn.) Blume.

OrChis LANIGERA Blanco Fl. Filip. (1837) 641, ed. 2 (1845) 446, ed. 3 , 3 .(1879) $37=$ Rhynchostylis retusa (Linn.) Blume.

Panisea bilamellata Reichb. f. in Walp. Ann. 6 (1861) 240 =: Coelogyne bilamellata Lindl.

PAPHIOPEDILUM as applicable to the Philippine flora $=$ Cordulu Raf.

Paphiopedilum ARgus Stein Orchideenb. (1892) 453; Pfitz. in Engl. Bot. Jahrb. 19 (1894) 40, in Engl. Pflanzenreich IV. 50 (1903) 90; Ames Orch. 1 (1905) 63, 2 (1908) 23 [incl. vars. moensii (Hort. ex Veitch) Stein and nigricans (Veitch) Hort. ex Stein], 5 (1915) 10 = Cordula argus (Reichb. f.) Rolfe.

Paphiopedilum barbatum Pfitz. in Pringsh. Jahrb. 19 (1888) 159; Ames Orch.2 (1908) 24, 5 (1915) 10 = Cordula barbata (Lindl.) Rolfe.

PAPhiopedlum cillolare Stein Orchideenb. (1892) 462, fig. 145; Pfitz. in Engl. Bot. Jahrb. 19 (1894) 40, in Engl. Pflanzenreich IV. 50 (1903) 89; Ames Orch. 2 (1908) 23, 5 (1915) 11, in Philip. Journ. Sci. 6 (1911) Bot. $39=$ Cordula ciliolaris (Reichb. f.) Rolfe.

PaPhiopedilum elliottianum Stein Orchideenb. (1892) 466. Cf. Paphiopedilum rothschildianum (Reichb. f.) Stein, spec. excl.

Paphiopedilum Haynaldianum Stein Orchideenb. (1892) 470; Pfitz. in Engl. Bot. Jahrb. 19 (1894) 41, in Engl. Pflanzenreich IV. 50 (1903) 66, fig. 31 A-C; Ames Orch. 2 (1908) 22, 5 (1915) 10, in Philip. Journ. Sci. 6 (1911) Bot. 39; Memml. in Orchis 14 (1920) 18 = Cordula haynaldiana (Reichb. f.) Rolfe.

Paphiopedilum laevigatum Pfitz. in Engl. \& Prantl Nat. Pflanzenfam. 2, Abt. 6 (1889) 84 = Cordula philippinensis (Reichb. f.) Rolfe.

PAPHIOPEDILUM Lowi Stein apud Ames Orch. 2 (1908) 21, 5 (1915) 11, in nota. Cf. Cypripedium lowii Lindl, apud Naves, spec. excl.

PAPHIOPEDILUM PHILIPPINENSE Stein Orchideenb. (1892) 480; Pfitz. in Engl. Bot. Jahrb. 19 (1894) 41, in Engl. Pflanzenreich IV. 50 (1903) 61, fig. 28 A-E; Ames Orch. 2 (1908) 19 (incl. vars.), 5 (1915) 10; Memml. in Orchis 14 (1920) $18=$ Cordula philippinensis (Reichb. f.) Rolfe.

PAPHioledilum Roebelenil Stein Orchideenb. (1892) 480, 482, in syn.; Pfitz. in Engl. Bot. Jahrb. 19 (1894) 41 (as röbbelenii), in Engl. Pflanzenreich IV. 50 (1903) 62 = Cordula philippinensis (Reichb. f.) Rolfe.

PAPHIOPEDILUM ROTHSCHILdAANUM (Reichb. f.) Stein Orchideenb. (1892) 482; Ames Orch. 2 (1908) 18, 5 (1915) 11, in nota.

Cypripedium elliottianum O'Brien in Gard. Chron. III 4 (1888) 501. Paphiopedilum elliottianum Stein Orchideenb. (1892) 466.

Paphiopedilum rothschildianum Stein var. elliottianum Pfitz. in Engl. Pflanzenreich IV. 50 (1903) 59. 
There is no satisfactory evidence that this species is Philippine; the type is from Borneo or from Sumatra. Cypripedium elliottianum O'Brien is supposed to have come from the Philippines.

PAXTONIA Lindl. = Spathoglottis Blume.

PAXTONIA ROSEA Lindl. in Bot. Reg. 24 (1838) Misc. 61, t. 60; Moore Ill. Orch. Pl. (1857) Paxtonia 4, t. 1; Reichb. f. in Walp. Ann. 6 (1862) 456; Naves Novis. App. (1882) 237 = Spathoglottis plicata Blume.

Pelexia ? Falcata Llanos Fragm. Pl. Filip. (1851) 95; F.-Vill. \& Naves in Blanco Fl. Filip. ed. 3, $4^{1}$ (1880) 73; Merr. Sp. Blancoanae (1918) 117.

This may be a species of Oberonia; the description of Llanos is very imperfect.

Prristylus GOOdYeroldes Lindl. Gen. \& Sp. Orch. (1835) 299; Ceron Cat. Pl. Herb. Manila (1892) 169; Kränzl. Orch. Gen. et Sp. 1 (1898) 503 (excl. syn. P. grandis Blume); J. J. Sm. Fl. Buitenz. 6 (Orch. Jav.) (1905) 32, Fig.-Atlas (1908) fig. $14=$ Habenaria goodyeroides D. Don.

PERISTYlUS WARBURgianus Kränzl. Orch. Gen. et Sp. 1 (1898) 519.

The type, Warburg 1498\%, is from southern Mindanao. The description suggests close affinity with Habenaria goodyeroides D. Don.

PHYLIORCHIS Thouars $=$ Bulbophyllum Thouars.

Phytiorchis ANTEN'NIFERA Ktze. Rev. Gen. Pl. 2 (1891) $677=$ Bulbophyllum antenniferum (Lindl.) Reichb. f.

Phyllorchis aurata Ktze. Rev. Gen. P1. 2 (1891) $677=$ Bulbophyllum auratum (Lindl.) Reichb. f.

Phyllorchis cheiri Ktze. Rev. Gen. Pl. 2 (1891) $676=$ Bulbophyllum blumei (Lindl.) J. J. Sm.

PHyLlorchis cumingII Ktze. Rev. Gen. Pl. 2 (1891) $677=$ Bulbophyllum cumingii (Lindl.) Reichb. f.

PHYLLORCHIS MAXILLARIS Ktze. Rev. Gen. Pl. 2 (1891) $677=$ Bulbophyllum blumei (Lindl.) J. J. Sm.

Phyllorchis othonis Ktze. Rev. Gen. Pl. 2 (1891) $677=$ Bulbophyllum othonis (Ktze.) J. J. Sm.

PhysURUS BLUmeI Lindl. apud Naves Novis. App. (1882) 250.

PrNalia Buch.-Ham. = Eria Lindley.

Pinalia APOROdes Ktze. Rev. Gen. Pl. 2 (1891) 678 = Dendrobium aporoides (Lindl.) Merr.

Pinalia bRAchystachya Ktze. Rev. Gen. Pl. 2 (1891) 678 = Eria brachystachya Reichb. f.

Pinalia Polyura Ktze. Rev. Gen. Pl. 2 (1891) 679 = Eria polyura Lindl.

PINAlia ProRepens Ktze. Rev. Gen. Pl. 2 (1891) $679=$ Phreatia prorepeng Reichb. $f$. 
Pinalia Retroflexa Ktze. Rev. Gen. Pl. 2 (1891) $679=$ Eria retroflexa Lindl.

Pinala vulpina Ktze, Rev. Gen, Pl。 2 (1891) $679=$ Eria vulpina Reichb. f.

Platanthera L. C. Richard = Habenaria Willdenow.

Platanthera Amesiana Schltr. in Fedde Repert, 9 (1911) $212=$ Habenaria angustata (Blume) Ktze.

Platanthera Angustata Lindl. Gen. \& Sp. Orch. (1835) 290; Naves Novis. App. (1882) $251=$ Habenaria angustata (Blume) Ktze.

Platanthera goodyeroides Vidal Phan. Cuming. Philip. (1885) $151=$ Habenaria goodyeroides D. Don.

Platanthera Gracilis (Blume) Lindl. apud Naves Novis. App. (1882) 251.

Platanthera Halconensis Schltr. in Fedde Repert. 9 (1911) $212=$ Habenaria halconensis Ames.

Platanthera Horsfieldi (Blume) Naves Novis. App. (1882) 251.

According to J. J. Smith Peristylus horsfieldii Blume, upon which Naves based his combination, is a synonym of Habenaria goodyeroides D. Don. This conclusion needs further examination before it can be accepted.

Piatanthera susannae Lindl. apud Naves Novis. App. (1882) 251.

Platyclinis Benth. = Acoridium Nees \& Meyen p. p. and Dendrochilum Blume p. p.

Platyclinis ARACHNites Rolfe in Kew Hand-list Orch. (1896) 189, nomen $=$ Dendrochilum arachnites Reichb. $\mathrm{f}$.

Platyclinis Bistorta Wendl. \& Kränzl. Xen. Orch. 3 (1900) 169, t. 299, fig. $I \quad 1-5=$ Dendrochilum bistortum (Wendl. \& Kränzl:) J. J. Sm., spec. excl.

Platyclinis cobbiana Hemsl. in Gard. Chron. II 16 (1881) 656; Veitch Man. Orch. Pl. pt. 5 (1889) $80=$ Dendrochilum cobbianum Reichb. f.

Platychinis cucumerina Rolfe in Kew Hand-list Orch. (1896) 189, nomen = Dendrochilum cucumerinum Reichb. f. spec. excl.

Platyclinis Filiformis Benth. ex Hemsl. in Gard. Chron. II 16 (1881) 656; Veitch Man. Orch. Pl. pt. 5 (1889) 80; Cogn. in Dict. Icon. Orch. (1902) Platyclinis $t .1=$ Dendrochilum filiforme Lindl.

Platyclinis GLumaceA Benth. in Journ. Linn. Soc. 18 (1881) 295, by inference only; ex Hemsl. in Gard. Chron. II 16 (1881) 656; Vidal Phan. Cuming. Philip. (1885) 148, Rev. Pl. Vasc. Filip. (1886) 267; Pfitz. in Engl. \& Prantl Nat. Pflanzenfam. 2, Abt. 6 (1888) 128, fig. 127 $B-F$; Veitch Man. Orch. Pl. pt. 5 (1889) 81, t.; Cogn. in Dict. Icon. Orch. (1902) Platyclinis t. 2; Ames Orch. 1 (1905) 74 $=$ Dendrochilum glumaceum Lindl. 
PLATYCLINIS IAATIFolia Hemsl. in Gard. Chron. II 16 (1881) 656; Rendle in Journ. Bot. 34 (1896) 355; Ames Orch. 1 (1905) $74=$ Dendrochilum latifolium Lindl.

Platyclinis microchila Schltr. in Bull. Herb. Boiss. II 6 (April, 1906) $302=$ Acoridium microchilum (Schltr.) Ames.

Platyclinis uncata Rolfe ex Vidal Phan. Cuming. Philip. (1885) 148; Rev. Pl. Vasc. Filip. (1886) 627 = Dendrochilum uncatum Reichb. $f$.

Pimone bilamellata Ktze. Rev. Gen. Pl. 2 (1891) $680=$ Coelogyne bila mellata Lindl.

Pleione sparsa Ktze. Rev. Gen. Pl. 2 (1891) $680=$ Coelogyne sparsa Reichb. f.

Pogonia A. Jussieu as applicable to the Philippine flora $=$ Nervilia Gaudichaud.

Pogonia Crispata Blume Mus. Bot. Lugd.-Bat. 1 (1849) 32; Naves Novis. App. (1882) $248=$ Nervilia discolor (Blume) Schltr.

Pogonia dilatata Blume Fl. Jav. Orch. (1858) 127 (Orch. Arch. Ind. 151) t. 10, fig. 4, $1 \& 2$; Ames Orch. 1 (1905) $66=$ Nervilia dilatata (Blume) Schltr.

Pogonia DIscolor Blume Mus. Bot. Lugd.-Bat. 1 (1849) 32; Naves Novis. App. (1882) $248=$ Nervilia discolor (Blume) Schltr.

Pogonia Flabelliformis Lindl. in Wall. Cat. (1832) No. 7400, nomen, Gen. \& Sp. Orch. (1840) 415; Naves Novis. App. (1882) $248=$ Nervilia aragoana Gaudich.

Pogonia Juliana Wall. apud Naves Novis. App. (1882) 248.

Pogonia Plicata Ames Orch. 2 (1908) 43, non Lindl. = Nervilia discolor (Blume) Schltr.

POGONIA PUDICA Ames Orch. 2 (1908) 44, text cut = Nervilia discolor (Blume) Schltr.

Pogonia PUnctata Blume apud Naves Novis. App. (1882) 248.

PTYchochilus SEPTEMNERVIS Schauer in Nov. Act. Nat. Cur. 19, Suppl. 1 (1843) 431, t. 12, fig. $B=$ Tropidia septemnervis (Schauer) Reichb. $\mathbf{f}$.

RHAMPHIDIA GRANDIFLORA Lindl. apud Naves Novis. App. (1882) 248.

Rhamphidia Tenuis Lindl. in Journ. Linn. Soc. 1 (1857) 182; Miq. Fl. Ind. Bat. 3 (1859) 730; Naves Novis. App. (1882) $248=$ Hetaeria oblongifolia Blume.

Salacistis Novembrilis Reichb. f. apud Naves Novis. App. (1882) 249.

SARCOPODIUM ACUminatum Rolfe in Orch. Rev. 18 (August, 1910) 239; Kränzl. in Engl. Pflanzenreich IV. 50. 11. B. 21 (November, 1910) 329, fig. 32 $D, E=$ Dendrobium acuminatum Rolfe.

SARCoPodium CHEIRI Lindl. Fol. Orch. Sarcopod. (1853) 4; Moore Ill. Orch. Pl. (1857) Sarcopod. $4=$ Bulbophyllum blumei (Lindl.) J. J. Sm. 
SARCoPodium CYMBIDIOIdes Kränzl. in Engl, Pflanzenreich IV. 50, H. B. 21 (1910) 325, quoad Philip. = Dendrobium acuminatum Rolfe.

SARCOPODIUM LYONII Rolfe in Orch. Rev. 18 (1910) 240; Ames in Philip. Journ. Sci. 6 (1911) Bot. 53, in Addisonia 8 (1923) 17, t. 265 $=$ Dendrobium lyonii Ames.

SARCOPODIUM STELLA-SILVAE Loher \& Kränzl. in Fedde Repert. 7 (1909) 40; Rolfe in Orch. Rev. 18 (August, 1910) 240; Kränzl. in Engl. Pflanzenreich IV. 50. II. B. 21 (November, 1910) 326, fig. 31 A-C; Ames in Philip. Journ. Sci. 8 (1913) Bot. $426=$ Dendrobium stella-silvae (Loher \& Kränzl.) Ames.

Schizmoceras Presl = Dendrobium Swartz.

Schizmoceras disticha Presl Rel. Haenk. 1 (1827) 96, t. 1.3, fig. $2=$ Dendrobium distichum (Presl) Reichb. $f$.

Semenipedum LaEvigatum May in Rev. Hort. 57 (1885) $301=$ Cordula philippinensis (Reichb. f.) Rolfe.

Stauroglottis equestris Schauer in Nov. Act. Acad. Nat. Cur. 19, Suppl. 1 (1843) 432 = Phalaenopsis equestris (Schaver) Reichb. $\mathrm{f}$.

STAUROPSIS BATEMANII Nichols. Dict. Gard. 3 (1886) 493 (as batemanni) $=$ Vandopsis lissochiloides (Gaudich.) Pfitz.

StaURopsis Padiens Reichb. f. in Hamb. Gartenz. 16 (1860) 117, Xen. Orch. 2 (1862) 7; Naves Novis. App. (1882) $243=$ Phalaenopsis pallens (Lindl.) Reichb. f., spec. excl.

STAUROPSIS PHILIPPINENSIS Reichb. f. in Hamb. Gartenz. 16 (1860) 117, Xen. Orch. 2 (1862) 8, in Walp. Ann. 6 (1864) 882; Vidal Rev. Pl. Vasc. Filip. (1886) 270; Ames Orch. 2 (1908) 223, 5 (1915) 224 = Trichoglottis philippinensis Lind1.

StAuropsis violacea Reichb. f. apud Naves Novis. App. (1882) 243.

STENORHYNCHUS VAgINATUS Spreng. apud Llanos ex F.-Vill: \& Naves in Blanco Fl. Filip. ed. 3, $4^{2}$ (1880) 99.

Synptera subviolaceA Llanos Frag. Pl. Filip. (1851) 98; F.-Vill. \& Naves in Blanco Fl. Filip. ed. $3,4^{1}$ (1880) 75 .

TAINIA Elmeri Ames in Elm. Leafl. Philip. Bot. 5 (1912) $1570=$ Ascotainia elmeri Ames.

TAINIA INAMOENA Kränzl. in Fedde Repert. 17 (1921) $387=$ Ascotainia elmeri Ames.

Trichotosia Blume = Eiria Lindley.

Trichotosia binabayensis Kränzl, in Engl. Pflanzenreich IV. 50. II. B. $21^{2}$ (1911) $141=$ Eria binabayensis Ames.

Trichotosia Fastigiatifolia Kränzl. in Engl. Pflanzenreich IV. 50. 11. B. $21^{2}$ (1911) $162=$ Eria fastigatifolia Ames.

TRIChotosia HALCONENSIS Kränzl. in Engl. Pflanzenreich IV. 50. I1. B. $21^{\circ}$ (1911) $149^{\prime}=$ Eria halconensis Ames. 
Trichotosia MUCRonata Kränzl. in Engl. Pflanzenreich IV. 50. II. B. $21^{3}$ (1911) 157 = Eria mucronata Lindl. spec. excl.

Trichotosia ODORIFEra Kränzl. in Engl. Pflanzenreich IV. 50, II. B. $21^{3}$ (1911) 153 = Eria odorifera. Leavitt.

Trichotosia RAMosir Kränzl. in Engl. Pflanzenreich IV. 50. II. B. $21^{2}$ (1911) 156 = Eria ramosï Leavitt.

Trichotosia vulPiNA Kränzl. in Engl. Pflanzenreich IV. 50. II. B. 21 = (1911) 141 = Evia vulpina Reichb. $\mathrm{f}$.

Tylostylis sp. Naves Novis. App. (1882) 247.

VANifolia ALbida Blume apud Naves Novis. App. (1882) 248.

Zeuxine sp. Vidal Rev. Pl. Vasc. Filip. (1886) 273 = Adenostylis strateumatica (Linn.) Ames.

Zeuxine Luzonensis Ames in Philip. Journ. Sci. 2 (1907) Bot. 314, nomen $=$ Adenostylis luzonensis Ames.

Zeuxine PURPUrascens Blume apud Naves Novis. App. (1882) 249.

ZEUXINE WHITEHEADII Rendle in Journ. Bat. 34 (1896) $358=$ Kuhlhassel tia whiteheadii (Rendle) Ames. 


\section{ERRATA}

Page,22, line 28, for Scirpiodendron ghaeri read Scirpodendron ghaeri.

Page 25, line 39, for Limnophyton obstusifolium Miq. read Lymnophyton obtusifolium Miq.

Page 33, line 23, for Brongh. read Brongn.

Page 41, line 22, for Vizcaya, read Vizcaya),

Page 71, line 44, for Vor. read Var.

Page 75, line 31, for CHAMAERAPHIS MURICATA (Linn. f.) comb. nov. read CHAMAERAPHIS SQUARROSA. (Linn. f.) comb nov.

Page 84, last line, for Hainan, Indo-China, read Hainan, Indo-China, Celebes, Ternate, Gilolo.

Page 116, after line 37, insert Fuirena philippinensis Gandoger in Bull. Soc. Bot. France 66 (1919) 197.

Page 132, line 10, for Lipironia read Lepironia.

Page 182, after line 6, insert Schismatoglottis plurivenia v. A. V. R. in Bull. Jard. Bot. Buitenzorg III 4 (1922) 209.

Page 187, line 27, for TYPHONIUM DIVARCATUM (Linn.) Decne, read TYPHONIUM DIVARICATUM (Linn.) Decne.

Page 198, line 42, for Tradescantia critata Linn. read Tradescantia cristata Linn.

Page 201, line 7, for Presl. read Presl

Page 215, line 36, for (Tag.), read (Tag.,

Page 277, line 21, for (Ames) Schltr.\& Ames. read (Ames) Ames \& Schltr. 



\section{INDEX}

I

Acampe, 429.

Acanthophippium. 330.

Acoridium, 284,

Acorus, 175.

Acostainia, 336

Acriopsis, 398.

Actinorhytis, 163.

Adelmeria, 236.

Adenostylis, 275.

Adonidia, 164.

Aërides, 420.

Agathis, 5.

Agave, 21\%.

Aglaonema, 182.

Agrostis, 81.

Agrostophyllum, 319.

Aletris, 207.

Alismaceae, 25.

Allium, 204.

Alloteropsis, 70 .

Alocasia, 184.

Aloe, 204.

Amaryllidaceae, 210 .

Amomum, 238.

Amorphophallus, 179.

Anadendron, 174.

Ananas, 193.

Andropogon, 42.

Aneilema, 196.

Angraecum, 432.

Aniselytron, 82.

Anoectochilus, 274.

Anota, 438.

Anthoxanthum, 78 .

Aphyllorchis, 264.

Apluda, 36.

Apostasia, 252.

Apostasiaceae, 252.

Appendicula, 323.

Araceae, 172.

Arachnis, 427.

Areca, 168.

Arenga, 159.

Arisaema, 188.

Aristida, 78 .

Arthraxon, 41.

Arundinaria, 94.

Arundinella, 52.

Ascocentrum, 434.

Asparagus, 206.

Asthenochloa, 49.

Atamosco, 210.

Avena, 83.
I3

Bambusa, 94.

Belamcanda, 220.

Blyxa, 27.

Boesenbergia, 243.

Boottia, 27.

Brachypodium, 93.

Bromeliaceae, 193.

Bromus, 92.

Bulbophyllum, 378 .

Bulbostylis, 127.

Burmannia, 251.

Burmanniaceae, 251.

\section{C}

Caladium. 187.

Calamagrostis, 82 .

Calamus, 147.

Calanthe, 332.

Camarotis, 436.

Canna, 247.

Cannaceae, 247.

Carex, 136.

Caryota, 158.

Cenchrus, 74 .

Centotheca, 91.

Centrolepidaceae, 191.

Centrolepis, 191.

Cephalostachyum, 97.

Ceratostylis, 317.

Chamaeanthus, 404.

Chamaeraphis, 75 .

Cheirostylis, 275.

Chionachne, 29.

Chloris, 84.

Chrysoglossum, 280.

Cladium, 129.

Cocos, 170.

Coelachne, 82.

Coelococcus, 145.

Coelogyne, 280.

Coix, 29.

Colocasia, 186.

Commelina, 195.

Commelinaceae, 194.

Cordula, 253.

Cordyline, 206.

Corybas, 261.

Corymborchis, 280.

Corypha, 143.

Costus, 246.

Crinum, 210.

Cryptocoryne, 189.

Cryptostylis, 261.
Curculigo, 213.

Curcuma, 243.

Cyanotis, 198.

Cycadaceae, 1.

Cycas, 1.

Cymbidium, 403.

Cymodocea, 24.

Cynodon, 83.

Cyperaceae, 102.

Cyperus, 103.

Cyrtosperma, 179.

Cystopus, 274.

Cystorchis, 273.

Dacrydium, 4.

Dactyloctenium, 86.

Daemonorops, 155.

Dendrobium, 342.

Dendrocalamus, 97.

Dendrochilum, 292.

Deschampsia, 83.

Dianella, 202.

Dicerostylis, 272.

Didymoplexis, 267.

Digitaria, 53.

Digly phosa, 280.

Dilochia, 317.

Dimeria, 30 .

Dinochloa, 100.

Dioscorea, 215.

Dioscoreaceae, 215.

Diplachne, 87.

Diplacrum, 136.

Diplanthera, 24.

Dipodium, 402.

Disperis, 260 .

Disporum, 206.

Donax, 248.

E

Eichornia, 200.

Elaeis, 170.

Eleocharis, 119.

Eleusine, 85.

Eleutherine, 220.

Enhalus, 27.

Epipactis, 271.

Epipogum, 265.

Epipremnopsis, $17 \%$.

Epipremnum, 177.

Eragrostis, 88.

Eremochloa, 39.

Eria, 364. 
Eriachne, 82.

Eriocaulon, 192.

Eriocaulonaceae, 192.

Eriochloa. 58.

Errata, 459.

Erythrodes, 273.

Eucharis. 211.

Eulophia, 339.

Eurycles, 211

F

Festuca, 92.

Fimbristylis, 121.

Flagellaria, 191.

Flagellariaceae, 190.

Floscopa, 199.

Forrestia, 197.

Freycinetia, 9.

Fuirena, 116.

\section{G}

Gahnia, 131

Galeola, 262.

Garnotia, 81.

Gastrochilus, 434.

Gastrodia, 268.

Geodorum, 341.

Gigantochloa, 96 .

Globba, 244.

Glomera, 320 .

Gnetaceae, 7.

Gnetum, ?

Gramineae, 28.

Grammatophyllum, 399

Guadua, 96.

\section{H}

Habenaria, 255.

Halophila, 25.

Hanguana, 191.

Hedychium, 241.

Heliconia, 228.

Herminium, 255 .

Hetaeria, 278.

Heterospathe, 162.

Hippeastrum, 212.

Hippeophyllum, 310 .

Homalomena, 180.

Hydrilla, 26.

Hydrocharitaceae, 25.

Hymenocallis, 211.

Hypolytrum, 102.

Hypoxis, 214.

Ichnanthus, 70 .

Imperata, 30.

Iphigenia, 202.

Iridaceae, 220 .

Isachne, 58 .

Ischaemum. 37 .
Joinvilleá, 190.

Juncaceae, 201.

Juncellus, 114.

Juncus. 201

\section{Ii}

Kaempfexia, 242.

Kingiella, 410 .

Kolowratia, 235.

Korthalsia, 146.

Kuhlhasseltia, 273.

Kyllinga, 114.

\section{L}

Languas, 230.

Lecanorchis. 264.

Leersia, 78.

Lemna, 190.

Lemnaceae, 190.

Lepidogyne, 272.

Leptaspis, 76.

Leptochloa, 86.

Leptosolena, 236 .

Licuala, 143.

Liliaceae, 202.

Lilium, 204.

Liparis, 310.

Lipocarpha, 102.

Liriope, 206.

Livistona, 144.

Lophatherum, 91.

Luisia, 426.

Luzula, 201.

\section{$\mathbf{M}$}

Macodes, 275.

Malaxis, 302.

Malleola, 434 .

Manisuris, 41.

Mapania, 131.

Maranta, 250.

Marantaceae, 248.

Mariscus, 112.

Metroxylon, 145.

Microchloa, 83.

Mierolaena, 78.

Microsaccus, 442 .

Microtis, 261.

Miscanthus, 31.

Monerma, 93.

Monochoria, 200.

Monophrynium, 250.

Monostachya, 92 .

Muehlenbergia, 80 .

Musa, 221.

Musaceae, 221.

Myrmechis, 277.

Najadaceae, 24.

Najas, 24.
Nephelaphyllum, 280 . Nervilia, 265.

Neuwiedia, 252.

Nypa, 170.

Oberonia, 307

Octarrhena, 392.

Omoea, 434.

Oncosperma, 162.

Ophiopogon, 207.

Ophiurus, 41.

Oplismenus, 71.

Orania, 161.

Orchidaceae, 253 .

Oryza, 77.

Ottelia, 27

\section{P}

Pachystoma, 338.

Palmae, 142.

Pancratium, 212.

Pandanaceae, 9.

Pandanus, 15.

Panicum, 61.

Paspalum, 56.

Patersonia, 220.

Pennisetum, 75 .

Perotis, 51.

Phacelophrynium, 250.

Phaeomeria, 241.

Phajus, 331.

Phalaenopsis, 410.

Phalaris, 78.

Philippinaea, 275.

Phoenix, 142.

Pholidota, 300.

Phragmites, 87.

Phreatia, 395.

Phrynium, 249.

Phyllocladus, 4.

Pinaceae, 5.

Pinanga, 164.

Pinus, 6.

Pistia, 189.

Plagiostachys, 237.

Plectocomia, 146.

Pleomele, 205.

Plocoglottis, 338.

Poa, 92.

Podocarpus, 2.

Podochilus, 320.

Pogonatherum, 35.

Polianthes, 212.

Pollia, 194

Pollinia, 33

Polystachya, 329.

Polytoca, 29.

Polytrias, 35.

Pomatocalpa, 438.

Pontederiaceae, 200.

Potamogeton, 22.

Potamogetonaceae, 22.

Pothoidium, 174. 
Pothos, 172.

Pseudacoridium, 284. Ptychoraphis, 163.

Pycreus, 110.

\section{$\mathbf{R}$}

Ramosia, 92.

Ravenala, 228.

Remirea, 130.

Renanthera, 428.

Rhaphidophora, 175.

Rhoeo, 199.

Rhynchostylis, 423.

Robiquetia, 434.

Rottboellia, 39.

Ruppia, 24.

Rynchospora, 130.

\section{$\mathbf{S}$}

Saccharum, 32. Saccolabium, 432.

Sagittaria, 25.

Sararanga, 22.

Sarcanthus, 436.

Sarcochilus, 406 .

Schismatoglottis, 181.

Schizostachyum, 97.

Schoenorchis, 435 .

Schoenus, 128.

Sciaphila, 28.

Scindapsus, 178.

Scirpodendron, 131.

Scirpus, 116.

Scleria, 133.
Setaria, 72.

Smilax, 207.

Spathiphyllum, 178.

Spathoglottis, 336.

Spinifex, 76.

Spiranthes, 268.

Spirodela, 190.

Sporobolus, 80.

Staurochilus, 441.

Stemona, 202.

Stemonaceae, 202.

Stenomeris, 219.

Stereosandra. 267.

\section{T}

Tacea, 214.

Taccaceae, 214.

Taeniophyllum, 442.

Taetsia, 205.

Taxaceae, 2.

Taxus, 5.

Thalassia, 27.

Thecostele, 399.

Thelasis, 393.

Thelymitra, 260.

Themeda, 50.

'Thoracostachyum, 132.

Thrixspermum, 404 .

Thuarea, 75 .

Thysanolaena, 53.

Thysanotus, 202.

Torulinium, 114.

Trichoglottis, 439.

Tripogon, 85.

Triticum, 93.
Triuridaceae, 28.

Tropidia, 279.

Typha, 8.

Typhaceae, 8.

Typhonium, 187.

T

Uncinia, 136.

Vallisneria, 26.

Vanda, 429.

Vandopsis, 427.

Vanilla, 263.

Vanoverberghia, 238.

Vrydagzynea, 278.

W

Wolffia, 190.

K.

Xyridaceae, 192.

Xyris, 192.

Y

Yucea, 204.

$\mathbf{Z}$

Zalacca, 146.

Zea, 28.

Zebrina, 199.

Zingiber, 228.

Zingiberaceae, 228.

Zoisia, 51. 



\title{
AN ENUMERATION OF PHILIPPINE FLOWERING PLANTS
}

\author{
BY \\ ELMER D. MERRILL \\ DIRECTOR AND BOTANIST, BUREAU OF SCIENCE \\ VOLUME 1, FASCICLE I
}

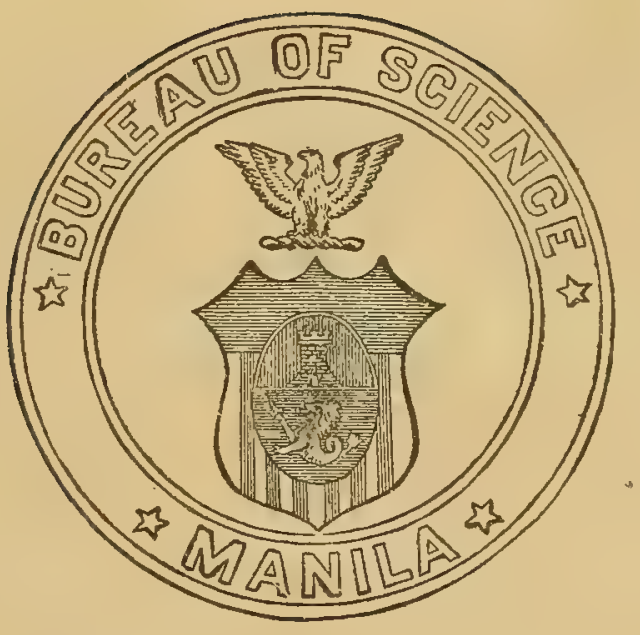




\section{BUREAU OF SCIENCE PUBLICATION No, 18 VOLUME 1, FASCICLE 1}

\section{EXPLANATORY NOTE}

The present enumeration is an attempt to summarize in convenient form for consultation our present knowledge of Philippine flowering plants, and represents a part of the knowledge accumulated by the author during a course of about twenty years' study of the problem. In the work an attempt has been made to account for all binomials accredited to the Philippine flora; to adjust the synonymy when necessary; to include all important references to the Philippine botanical literature and essential references to extra-Philippine literature; to give the habitat, altitudinal range, and Philippine and extra-Philippine distribution of each species; to cite illustrative specimens where expedient or necessary; and to record all available local names for each species.

It is proposed to issue the present work in fascicles, utilizing one volume for the gymnosperms and the monocotyledons and two volumes for the dicotyledons. It is planned to publish full introductory and explanatory matter in a general introduction to be prefixed to volume 1 when the entire enumeration is completed.

ELmer D. MerRILL.

Manila, P. I., August 25, 1921. 


\title{
AN ENUMERATION OF PHILIPPINE FLOWERING PLANTS
}

\author{
BY \\ ELMER D. MERRILL \\ DIRECTOR AND BOTANIST, BUREAU OF SCIENCE
}

VOLUME 1, FAscicle 2

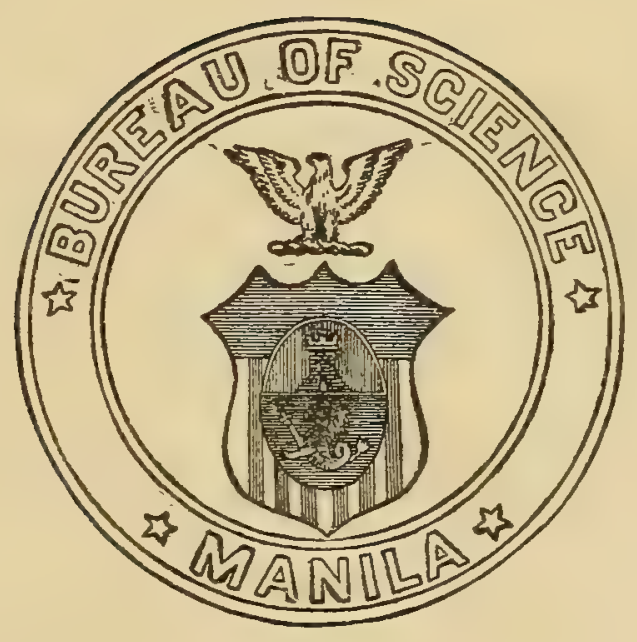


BUREAU OF SCIENCE PUBLICATION No. 18

VOLUME 1, FASCICLE 2

\section{EXPLANATORY NOTE}

The present enumeration is an attempt to summarize in convenient form for consultation our present knowledge of Philippine flowering plants, and represents a part of the knowledge accumulated by the author during a course of about twenty years' study of the problem. In the work an attempt has been made to account for all binomials accredited to the Philippine flora; to adjust the synonymy when necessary; to include all important references to the Philippine botanical literature and essential references to extra-Philippine literature; to give the habitat, altitudinal range, and Philippine and extra-Philippine distribution of each species; to cite illustrative specimens where expedient or necessary; and to record all available local names for each species.

It is proposed to issue the present work in fascicles, utilizing one volume for the gymnosperms and the monocotyledons and two volumes for the dicotyledons. It is planned to publish full introductory and explanatory matter in a general introduction to be prefixed to volume 1 when the entire enumeration is completed.

Manila, P. I., August 25, 1921.

ELMER D. MERRILL. 


\title{
AN ENUMERATION OF PHILIPPINE FLOWERING PLANTS
}

\author{
BY \\ ELMER D. MERRILL \\ DIRECTOR AND BOTANIST, BUREAU OF SCIENCE
}

VOLUME 1, FASCICLE 3

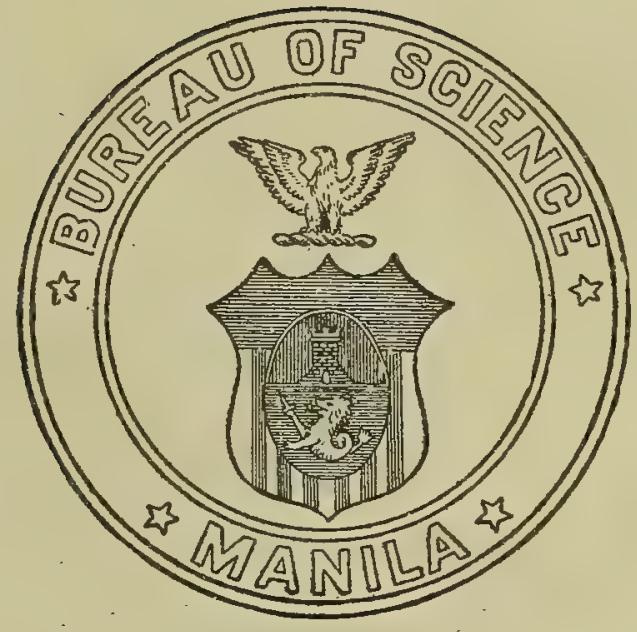


BUREAU OF SCIENCE PUBLICATION No. 18

VOLUME 1 , FASCICLE 3

\section{EXPLANATORY NOTE}

It was originally intended to issue this work in three volumes, the general introduction to be issued as a part of Volume 1 and the indices to form a part of Volume 3. Owing to the extent of the introduction, the bibliography, and the indices, it has been found best to issue all these data as Volume 4 .

\section{IMPORTANT}

In binding Volumes 2 and 3 discard the title-page issued with fascicle 5 of each of these yolumes and substitute therefor the revised title-pages found at the end of Volume 4.

ELMER D. MERRILL.

MaNILA, P. I., November 1, 1923. 


\title{
AN ENUMERATION OF PHILIPPINE FLOWERING PLANTS
}

\author{
BY \\ ELMER D. MERRILL \\ DIRECTOR AND BOTANIST, BUREAU OF SCIENCE
}

VOLUME 1. FASCICLE 4

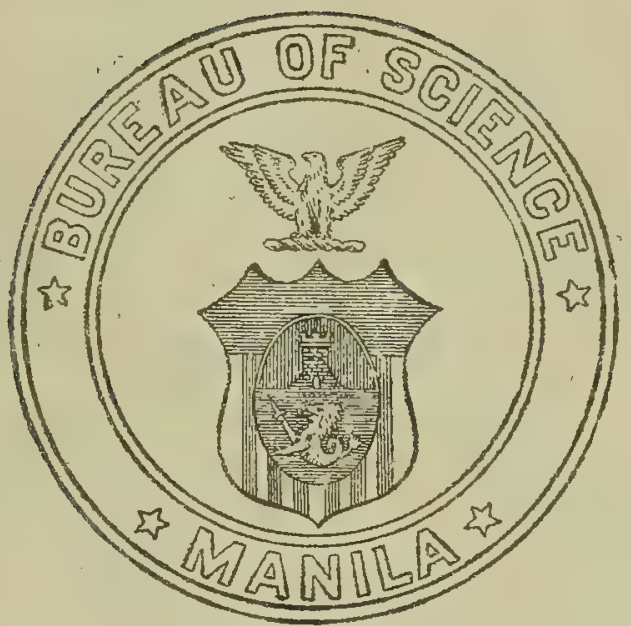

MANILA

BUREAU OF PRINTING 


\section{BUREAU OF SCIENCE PUBLICATION No. 18 VOLUME 1, FASCICLE 4}

\section{EXPLANATORY NOTE}

It was originally intended to issue this work in three volumes, the general introduction to be issued as a part of Volume 1 and the indices to form a part of Volume 3. Owing to the extent of the introduction, the bibliography, and the indices, it has been found best to issue all these data as Volume 4.

\section{IMPORTANT}

In binding Volumes 2 and 3 discard the title-page issued with fascicle 5 of each of these volumes and substitute therefor the revised title-pages found at the end of Volume 4.

MANILA, P. I, November $1,1923$.

ELMER D. MERRILL. 



$$
\frac{31}{4 x}
$$





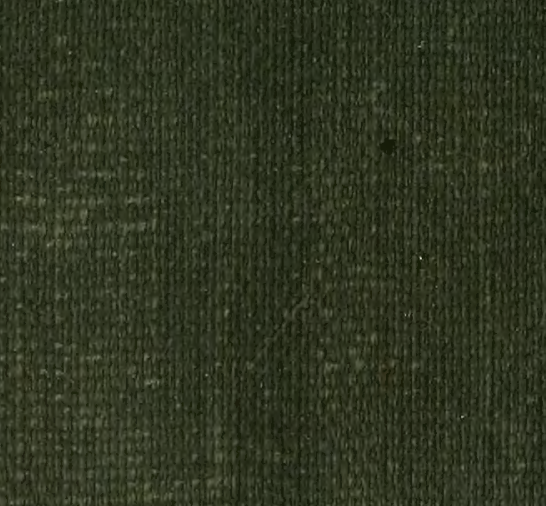

$$
\text { 19.5 }
$$

